fo sustainability

\title{
Assessment of \\ Socio-Economic \\ Sustainability \\ and Resilience \\ after COVID-19
}

Edited by

Idiano D'Adamo

Printed Edition of the Special Issue Published in Sustainability 


\section{Assessment of Socio-Economic Sustainability and Resilience after COVID-19}





\section{Assessment of Socio-Economic Sustainability and Resilience after COVID-19}

Editor

Idiano D'Adamo

MDPI $\bullet$ Basel $\bullet$ Beijing $\bullet$ Wuhan $\bullet$ Barcelona $\bullet$ Belgrade $\bullet$ Manchester $\bullet$ Tokyo $\bullet$ Cluj $\bullet$ Tianjin

$$
\text { MDPI }
$$


Editor

Idiano D'Adamo

Department of Computer,

Control and Management

Engineering

Sapienza - Università di Roma

Rome

Italy

Editorial Office

MDPI

St. Alban-Anlage 66

4052 Basel, Switzerland

This is a reprint of articles from the Special Issue published online in the open access journal Sustainability (ISSN 2071-1050) (available at: www.mdpi.com/journal/sustainability/special issues/ economic_resilience).

For citation purposes, cite each article independently as indicated on the article page online and as indicated below:

LastName, A.A.; LastName, B.B.; LastName, C.C. Article Title. Journal Name Year, Volume Number, Page Range.

ISBN 978-3-0365-2721-5 (Hbk)

ISBN 978-3-0365-2720-8 (PDF)

(C) 2021 by the authors. Articles in this book are Open Access and distributed under the Creative Commons Attribution (CC BY) license, which allows users to download, copy and build upon published articles, as long as the author and publisher are properly credited, which ensures maximum dissemination and a wider impact of our publications.

The book as a whole is distributed by MDPI under the terms and conditions of the Creative Commons license CC BY-NC-ND. 


\section{Contents}

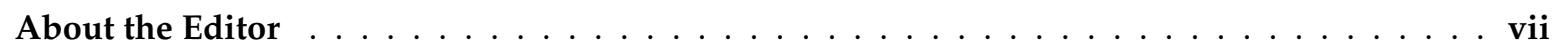

Preface to "Assessment of Socio-Economic Sustainability and Resilience after COVID-19" . . ix

Stefano Cianciotta and Idiano D'Adamo

The Evolution of Sustainability: The Automotive Supply Chain Opportunity in Southern Italy

Reprinted from: Sustainability 2021, 13, 10930, doi:10.3390/su131910930 . . . . . . . . . . . . . . 1

Idiano D'Adamo and Gianluca Lupi

Sustainability and Resilience after COVID-19: A Circular Premium in the Fashion Industry

Reprinted from: Sustainability 2021, 13, 1861, doi:10.3390/su13041861 . . . . . . . . . . . . . 7

Fabio Giudice, Rocco Caferra and Piergiuseppe Morone

COVID-19, the Food System and the Circular Economy: Challenges and Opportunities

Reprinted from: Sustainability 2020, 12, 7939, doi:10.3390/su12197939 . . . . . . . . . . . . . . .

Antonio Miceli, Birgit Hagen, Maria Pia Riccardi, Francesco Sotti and Davide Settembre-Blundo

Thriving, Not Just Surviving in Changing Times: How Sustainability, Agility and Digitalization Intertwine with Organizational Resilience

Reprinted from: Sustainability 2021, 13, 2052, doi:10.3390/su13042052 . . . . . . . . . . . . . .

Muhammad Jawad Sajid and Ernesto D. R. Santibanez Gonzalez

The Impact of Direct and Indirect COVID-19 Related Demand Shocks on Sectoral $\mathrm{CO}_{2}$ Emissions: Evidence from Major Asia Pacific Countries

Reprinted from: Sustainability 2021, 13, 9312, doi:10.3390/su13169312 . . . . . . . . . . . . . . .

Priom Mahmud, Sanjoy Kumar Paul, Abdullahil Azeem and Priyabrata Chowdhury

Evaluating Supply Chain Collaboration Barriers in Small- and Medium-Sized Enterprises

Reprinted from: Sustainability 2021, 13, 7449, doi:10.3390/su13137449 . . . . . . . . . . . . . . .

Sushil and Periyasami Anbarasan

Organization's Sustainable Operational Complexity and Strategic Overview: TISM Approach and Asian Case Studies

Reprinted from: Sustainability 2021, 13,9790, doi:10.3390/su13179790 . . . . . . . . . . . . . . 95

Francisco M. Baena-Moreno, Isabel Malico and Isabel Paula Marques

Promoting Sustainability: Wastewater Treatment Plants as a Source of Biomethane in Regions

Far from a High-Pressure Grid. A Real Portuguese Case Study

Reprinted from: Sustainability 2021, 13, 8933, doi:10.3390/su13168933 . . . . . . . . . . . . . . 127

Mattia Ferrari

Reflexive Governance for Infrastructure Resilience and Sustainability

Reprinted from: Sustainability 2020, 12, 10224, doi:10.3390/su122310224

Andrea Appolloni, Nathalie Colasanti, Chiara Fantauzzi, Gloria Fiorani and Rocco Frondizi

Distance Learning as a Resilience Strategy during Covid-19: An Analysis of the Italian Context Reprinted from: Sustainability 2021, 13, 1388, doi:10.3390/su13031388 . . . . . . . . . . . . . . 153 
Sara Alonso-Muñoz, Rocío González-Sánchez, Cristina Siligardi and Fernando E. García-Muiña

New Circular Networks in Resilient Supply Chains: An External Capital Perspective

Reprinted from: Sustainability 2021, 13, 6130, doi:10.3390/su13116130 . . . . . . . . . . . . . 165

Mohammad Faisal Khan, Asif Pervez, Umar Muhammad Modibbo, Jahangir Chauhan and Irfan Ali

Flexible Fuzzy Goal Programming Approach in Optimal Mix of Power Generation for Socio-Economic Sustainability: A Case Study

Reprinted from: Sustainability 2021, 13, 8256, doi:10.3390/su13158256 . . . . . . . . . . . . . . 183

Manel Arribas-Ibar, Petra A. Nylund and Alexander Brem

The Risk of Dissolution of Sustainable Innovation Ecosystems in Times of Crisis: The Electric Vehicle during the COVID-19 Pandemic

Reprinted from: Sustainability 2021, 13, 1319, doi:10.3390/su13031319 . . . . . . . . . . . . . . .

Idiano D'Adamo, Rocío González-Sánchez, Maria Sonia Medina-Salgado and Davide Settembre-Blundo

E-Commerce Calls for Cyber-Security and Sustainability: How European Citizens Look for a Trusted Online Environment

Reprinted from: Sustainability 2021, 13, 6752, doi:10.3390/su13126752 . . . . . . . . . . . . . .

Idiano D’Adamo, Rocío González-Sánchez, Maria Sonia Medina-Salgado and Davide Settembre-Blundo

Methodological Perspective for Assessing European Consumers' Awareness of Cybersecurity and Sustainability in E-Commerce

Reprinted from: Sustainability 2021, 13, 11343, doi:10.3390/su132011343 . . . . . . . . . . . . .

Matthias Klumpp and Dominic Loske

Sustainability and Resilience Revisited: Impact of Information Technology Disruptions on Empirical Retail Logistics Efficiency

Reprinted from: Sustainability 2021, 13, 5650, doi:10.3390/su13105650 . . . . . . . . . . . . . . 253

Angela Ivette Grijalba Castro and Leonardo Juan Ramírez López

Sustainability and Resilience of Emerging Cities in Times of COVID-19

Reprinted from: Sustainability 2021, 13, 9480, doi:10.3390/su13169480 . . . . . . . . . . . . . 273

Veronika Bikse, Inese Lusena-Ezera, Peteris Rivza and Baiba Rivza

The Development of Digital Transformation and Relevant Competencies for Employees in the Context of the Impact of the COVID-19 Pandemic in Latvia

Reprinted from: Sustainability 2021, 13, 9233, doi:10.3390/su13169233 . . . . . . . . . . . . . . 287

Ruiyu Sun, Siyao Zhang, Tianyu Wang, Jiarui Hu, Junhu Ruan and Junyong Ruan Willingness and Influencing Factors of Pig Farmers to Adopt Internet of Things Technology in Food Traceability

Reprinted from: Sustainability 2021, 13, 8861, doi:10.3390/su13168861 ․ . . . . . . . . . . . 305

Mohd Ashraf Zainol Abidin, Muhammad Nasiruddin Mahyuddin and Muhammad Ammirrul Atiqi Mohd Zainuri

Solar Photovoltaic Architecture and Agronomic Management in Agrivoltaic System: A Review Reprinted from: Sustainability 2021, 13, 7846, doi:10.3390/su13147846 . . . . . . . . . . . . . . . 325 


\section{Sungjo Hong and Seok-Hwan Choi}

The Urban Characteristics of High Economic Resilient Neighborhoods during the COVID-19 Pandemic: A Case of Suwon, South Korea

Reprinted from: Sustainability 2021, 13, 4679, doi:10.3390/su13094679 . . . . . . . . . . . . . . . 353

\section{Hui Wang, Qing Wang and Xia Sheng}

Does Corporate Financialization Have a Non-Linear Impact on Sustainable Total Factor Productivity? Perspectives of Cash Holdings and Technical Innovation

Reprinted from: Sustainability 2021, 13, 2533, doi:10.3390/su13052533 . . . . . . . . . . . . . . . 393

\section{Dongyong Zhang, Mengge Hao and Stephen Morse}

Is Environmental Sustainability Taking a Backseat in China after COVID-19? The Perspective of Business Managers

Reprinted from: Sustainability 2020, 12, 10369, doi:10.3390/su122410369 . . . . . . . . . . . . . . 411

Benito Umaña-Hermosilla, Hanns de la Fuente-Mella, Claudio Elórtegui-Gómez and Marisela Fonseca-Fuentes

Multinomial Logistic Regression to Estimate and Predict the Perceptions of Individuals and Companies in the Face of the COVID-19 Pandemic in the Nuble Region, Chile

Reprinted from: Sustainability 2020, 12, 9553, doi:10.3390/su12229553 . . . . . . . . . . . . . 435

Daniela Debone, Mariana V. da Costa and Simone G. E. K. Miraglia

90 Days of COVID-19 Social Distancing and Its Impacts on Air Quality and Health in Sao Paulo, Brazil

Reprinted from: Sustainability 2020, 12, 7440, doi:10.3390/su12187440 . . . . . . . . . . . . . . 455

Sarbast Moslem, Tiziana Campisi, Agnieszka Szmelter-Jarosz, Szabolcs Duleba, Kh Md Nahiduzzaman and Giovanni Tesoriere

Best-Worst Method for Modelling Mobility Choice after COVID-19: Evidence from Italy

Reprinted from: Sustainability 2020, 12, 6824, doi:10.3390/su12176824 . . . . . . . . . . . . . . . 473

Ángel Acevedo-Duque, Romel Gonzalez-Diaz, Elena Cachicatari Vargas, Anherys Paz-Marcano, Sheyla Muller-Pérez, Guido Salazar-Sepúlveda, Giulia Caruso and Idiano D'Adamo

Resilience, Leadership and Female Entrepreneurship within the Context of SMEs: Evidence from Latin America

Reprinted from: Sustainability 2021, 13, 8129, doi:10.3390/su13158129 . . . . . . . . . . . . . . . 493 



\section{About the Editor}

\section{Idiano D'Adamo}

Idiano D'Adamo is an Associate Professor at Sapienza University of Rome. He worked in the University of Sheffield, the National Research Council of Italy, Politecnico di Milano, University of L'Aquila and Unitelma Sapienza. In August 2015, he obtained the Elsevier Atlas Award. He received two Excellence Review Awards: Waste Management in 2017 and Resources Conservation and Recycling in 2018. During its academic career, Idiano D'Adamo published 90 papers in the Scopus database, reaching an h-index of 35 . His current research interests are bioeconomy, circular economy, renewable energy, sustainability and waste management. Idiano is among 100,000 Top Scientists for a global ranking in both 2019 and 2020. Idiano is Section Editor in Chief in Sustainability. He is Associate Editor in Resources, Conservation \& Recycling Advances, Global Journal of Flexible Systems Management and Frontiers in Sustainability. Idiano has participated in scientific research projects (Horizon 2020 "Star ProBio", Life "Force of the Future") and has collaborated with relevant national institutes (MATTM, CNBBSV, SVIMEZ). 



\section{Preface to "Assessment of Socio-Economic Sustainability and Resilience after COVID-19"}

This Special Issue, titled "Assessment of Socio-Economic Sustainability and Resilience after COVID-19", aims to propose the positive relationship between sustainability and resilience across multiple sectors.

Sustainability can promote a redistribution of people, since it is necessary to reduce the transfer of many young people to densely populated cities and, likewise, induce a reverse path. This can happen if two conditions are met: (i) attractive living conditions and (ii) present working conditions. It is indeed necessary to revitalize some territories and it is necessary to do this for future generations; for those who want to be protagonists in the social life giving a direct contribution. This society cannot remain deaf to this request because sustainability is the greatest opportunity for putting young people at the center of political action.

A special thank you to my family, my friends, and my students. My goal is to enable them to believe in dreams, and to set shareable goals that look not only at self-interest, but at developing social equity. I think of Pope Giovanni Paolo II's words during World Youth Day 2000: "You will defend life at every moment of its earthly development, you will strive with all your energy to make this earth more and more habitable for all."

This SI was made possible by the effort, talent and support of the Managing Editor, Ms Yichen Shen.

Idiano D'Adamo

Editor 



\title{
The Evolution of Sustainability: The Automotive Supply Chain Opportunity in Southern Italy
}

\author{
Stefano Cianciotta ${ }^{1}$ and Idiano $D^{\prime}$ Adamo ${ }^{2, *}$ (D) \\ 1 Abruzzo Sviluppo Spa, Corso Vittorio Emanuele 49, 65121 Pescara, Italy; \\ stefanocianciotta@abruzzosviluppo.it \\ 2 Department of Computer, Control and Management Engineering, Sapienza University of Rome, Via Ariosto \\ 25, 00185 Rome, Italy \\ * Correspondence: idiano.dadamo@uniroma1.it
}

check for updates

Citation: Cianciotta, S.; D'Adamo, I. The Evolution of Sustainability: The Automotive Supply Chain

Opportunity in Southern Italy. Sustainability 2021, 13, 10930. https://doi.org/10.3390/su131910930

Received: 20 September 2021 Accepted: 26 September 2021 Published: 30 September 2021

Publisher's Note: MDPI stays neutral with regard to jurisdictional claims in published maps and institutional affiliations.

Copyright: (c) 2021 by the authors. Licensee MDPI, Basel, Switzerland. This article is an open access article distributed under the terms and conditions of the Creative Commons Attribution (CC BY) license (https:// creativecommons.org/licenses/by/ $4.0 /)$.
This Special Issue titled "Assessment of Socio-Economic Sustainability and Resilience after COVID-19" aims to propose the positive relationship between sustainability and resilience across multiple sectors.

COVID-19 is not the only problem that needs to be addressed at this time, as the environment has clearly shown signs of weakness. Urban centers are responsible for $80 \%$ of the world's GDP, but they also produce $70 \%$ of the world's $\mathrm{CO}_{2}$ emissions and consume about $70 \%$ of the world's energy [1]. Moreover, it should be added that socio-economic inequality, unemployment, extreme poverty, unsustainable production and consumption patterns and environmental degradation demand urgent responses [2,3].

Europe has launched an ambitious plan towards the European Green Deal and, to this end, the Next Generation EU is a response to the socio-economic crisis caused by the pandemic. Some authors pointed out that it is necessary to develop the concept of the "sustainable hand", which is the set of actions that determine the social optimum within a market. This concept looks at the long term and can be implemented by virtue of new paradigms of social norms, models developed and implemented to help govern social interactions towards a sustainable future [4].

The manufacturing system highlights how innovation and sustainability can coexist, such that technological sustainability can be defined as a possible fourth dimension of sustainable development [5]. Similarly, it is also necessary to ensure a proper combination of sustainability and resilience [6]. This Editorial focuses on Italy, which will have a fundamental role within the Next Generation EU, since it is the recipient of about one third of the total available budget. In particular, it is necessary to overcome a significant problem that is the territorial disparity, in which the northern regions tend to show more performing results than the southern ones. In particular, these regions show potential for sustainability [7]. The adoption of digital technologies can enable competitiveness, but everything is linked to an effective collaboration between the different parties [8].

In addition, the analysis focuses on the automotive industry that presents very significant numbers in the European landscape through direct and indirect jobs amounting to employment for 13.8 million Europeans, which corresponds to about $6.1 \%$ of total employment. The European Union is among the world's largest producers of motor vehicles and the sector represents the largest private investor in research and development [9]. The performance of companies inferred from sustainability reports shows positive, but also negative performances. In particular, changes in organizational structures and appropriate policies have played an important role [10]. Sustainable manufacturing and green human resources are identified as critical success factors in the automotive sector [11].

The pandemic also affected the automotive sector, leading to a contraction in sales, however some companies have been able to absorb the contraction better. In particular, at the Sevel plant located in Val di Sangro (located in the Abruzzo Region (South Italy)) new hires were made in July 2020 and January 2021 [12]. Sevel was born in 1981 as the joint 
venture between Fiat and Peugeot, now united in Stellantis (Stellantis is a multinational company incorporated under Dutch law, born from the merger between the PSA and Fiat Chrysler Automobiles groups) and is considered to be the most important European production hub for light commercial vehicles. The company produces about 300 thousand vehicles per year. In particular, the Ducato is one of the most popular models globally and, with the shortening of production chains caused by the pandemic in the last year and a half, its production performance has increased significantly. In fact, the risks connected to production chains that are too geographically extensive are increasingly perceived by companies, just as the lack of production capacity in some areas (masks or vaccines themselves, for example) is perceived by public opinion to be an element of weakness of the entire system.

Sevel has always been defined as a model company where the production rate was so high that it required the use of people on loan from other plants in the group and staff on 'staff-leasing', i.e., on a temporary basis. The company therefore proved itself to be resilient to the pandemic, but the shortage of Bosch Abs control units from Malaysia has led to a slowing in the pace of work, with a reduction in production of commercial vehicles. This slowing down will lead to a reduction in work shifts (from $18 \mathrm{~h}$ to $15 \mathrm{~h}$ ), for a company in which work was done in shifts from Monday to Saturday and Sunday, including line maintenance operations. This reduction in shifts has had an impact on the reduction of personnel, which will involve laying off workers from other factories in the group and temporary workers whose contracts will not be renewed. In addition, another important element related to the company was the opening of a plant in Gliwice (Poland) in 2019. This was a choice dictated by reasons of industrial policy, since the production saturation of the Abruzzo plant of Sevel prevented it from responding to the new production levels [12]. In addition, the CEO of Stellantis, Carlos Tavares, announced that in the Termoli plant (located in the Molise Region (Southern Italy), which is about $70 \mathrm{~km}$ from Sevel) there will be one of the five gigafactories for the production of batteries of the Stellantis group. This decision will allow for the preservation of several jobs in the Termoli plant.

\section{Discussion and Policy Implications}

The pandemic has highlighted a sense of instability, with the closures of many businesses prompting significant changes that will need to be examined over time. One reflection we have come to is that the key role many businesses have taken on, the security systems already in place at these facilities, have enabled them not to close. However, lockdowns have also led to contractions in demand. Light vehicle production was not affected, but a long supply chain is responsible for the current crisis. Accordingly, the system has been proven to be resilient. Human resources are the fulcrum on which to develop a sustainable society. This is an area rich in students and potential, as highlighted by Luca De Meo, the Chairman of Renault [12]. The idea is to create a pole of sustainable automotive innovation in the Abruzzo and Molise cluster, using, first, all of the human resources acquired in recent years in the group's plants and then proceeding to a generational change. A challenge that must go beyond the automotive sector alone but must include all other sustainable spheres (e.g., production of renewable energy, energy efficiency, and re-use of materials) and digital; a team game that must involve all the surrounding industries, which represent a significant share of the local economy. Too often, short-sightedness leads to the simple replacement of people, forgetting the added value that they have acquired over time, regardless of the organizational position held. Within the school of human relations, Argyris pointed out that we must bear in mind that the people employed by the organization have their own personality. The individual evolves and seeks to achieve their own balances and is not only a subject of action, but also a subject of organizational learning. A necessary mutual balancing between personality and organization was therefore identified. The objective that is, therefore, posed in this work is twofold: (i) how to make this cluster globally competitive and (ii) how to create a virtuous model of industry-tourism coexistence. On this last aspect, Sergio Marchionne (Former CEO of the Fiat-Chrysler Automobiles 
group and in 2011 defined among the 100 most influential people in the world by Time magazine's ranking) focused on 9 July 2013, asking in his last speech in Abruzzo at Sevel, for investment in roads, development of commercial ports, implementation of broadband in industrial districts and also the design of tourism as a strategic asset.

The concept of a sustainable hand is based on making people part of the change, in which there are not only rights but also tasks, all of which are for the good of society as a whole. The binomial industry-tourism can call for appropriate lifestyles (e.g., diet, physical activity, stress reduction) which are possible in the territories examined, but sustainability must be achieved, i.e., leading companies and the entire supply chain must be able to compete on a global level. The challenge is by no means simple and requires the contribution of everyone in the formulation of ideas and strategies that can protect all the parties involved. This is a challenge in which everyone is called upon to make proposals, but also to take steps backwards. It is a matter of developing a stable and dynamic relationship between internal and external organization. Within this cluster, it is necessary to combine production with research and development in order to develop synergies and attract talent. In this context, the support that can be guaranteed by the universities in the area becomes fundamental.

The establishment of a true Abruzzo-Molise motor valley (specific automotive cluster, which in fact already exists because other 2500 people work in the Termoli plant today), would consolidate an industrial quadrilateral which, along with the Pomigliano D'Arco (Campania) and Melfi (Basilicata) plants, represents one of the most interesting production poles in Europe for the Stellantis Group.

The crisis caused by Covid-19 is confirming the centrality of integrated logistics, in light of the inevitable redefinition of industrial supply chains that will become increasingly proximate.

Logistics and industry, in fact, are the same thing, and it is no coincidence that Amazon has invested precisely in the South of Abruzzo, creating one of the most important logistics poles in Southern Europe.

In today's economy, industrial policy must favor processes of personnel training, technological and organizational innovation, reducing bureaucratic obstacles and making services available for efficient logistics.

Just the integrated logistics is a further factor of aggregation and development of the automotive quadrilateral of the Centre-South, which can be further strengthened by the establishment in Abruzzo of a Special Economic Zone (SEZ) - see Cianciotta [13], whose positive outcome will be determined by the value of integrated logistics and the quality of services provided.

The Special Economic Zone of Puglia is already in force and also includes Molise, while that of Abruzzo must wait a few more months because the Decree of appointment by the Government of the Commissioner Mauro Miccio is being registered at the Court of Auditors.

Our proposal, in order to increase the attractiveness of the entire Adriatic strip from Abruzzo to Puglia, is to work towards the creation of a single SEZ.

In fact, establishing a single Special Economic Zone of the Southern Adriatic would mean having several industrial clusters in strategic sectors for the relaunch of the economy (automotive and aerospace, for example), as well as having an integrated system of commercial port infrastructures (Vasto, Ortona, Brindisi and Bari), which would be particularly advantageous, both in terms of negotiations with multinationals already operating within this large area, and in terms of attracting investment.

If to the inevitable relapses on the territories, we add also that the Marche (to whose inside of the Harbor Authority figure also the Abruzzo) and the same Molise are regions in transition, this means that within a communitarian program, these regions will assign approximately $6 \%$ more of their resources to Italy, and it is estimated that, to these two regions, from $10 \%$ to $20 \%$ of greater contributions can be attributed. 
The Marche region itself is also working on obtaining a SEZ, which will have different criteria from those established with Government Decree No. 91 in 2017 that constituted the SEZs for the regions of Southern Italy, but which is still a novelty in terms of measures for attracting investment.

It is here, then, that the central-southern Adriatic would find itself united with a measure that could determine and increase its potential, especially in a strategic moment favorable to the resumption of maritime trade on the Mediterranean, which has returned to play an important role after the doubling of the Suez Canal and with the redefinition of the routes due to the pandemic (on the Mediterranean's contribution to economic development, see the monographic issue of Limes [14].).

Finally, sustainability can promote a redistribution of people, since it is necessary to reduce the transfer of many young people to densely populated cities and, likewise, induce a reverse path.

This can happen if two conditions are met: (i) attractive living conditions and (ii) present working conditions. It is indeed necessary to revitalize some territories and it is necessary to do this for future generations; for those who want to be protagonists in the social life giving a direct contribution. This society cannot remain deaf to this request because sustainability is the greatest opportunity for putting young people at the center of political action.

Author Contributions: Conceptualization, S.C. and I.D.; writing-Original draft preparation, S.C. and I.D.; writing-Review and editing, S.C. and I.D. All authors have read and agreed to the published version of the manuscript.

Funding: This research received no external funding.

Institutional Review Board Statement: Not applicable.

Informed Consent Statement: Not applicable.

Data Availability Statement: Not applicable.

Acknowledgments: This work is dedicated to all the young people who work in Sevel and in all the plants of the group, so that all their sacrifices are valued and new young people can become colleagues. The realization of young people's dreams is the compass of our action.

Conflicts of Interest: The authors declare no conflict of interest.

\section{References}

1. The World Bank Urban Development. Available online: https://www.worldbank.org/en/topic/urbandevelopment/overview (accessed on 27 August 2021).

2. D'Amico, G.; Taddeo, R.; Shi, L.; Yigitcanlar, T.; Ioppolo, G. Ecological indicators of smart urban metabolism: A review of the literature on international standards. Ecol. Indic. 2020, 118, 106808. [CrossRef]

3. D'Amico, G.; Arbolino, R.; Shi, L.; Yigitcanlar, T.; Ioppolo, G. Digital Technologies for Urban Metabolism Efficiency: Lessons from Urban Agenda Partnership on Circular Economy. Sustainability 2021, 13, 6043. [CrossRef]

4. D'Adamo, I.; Morone, P.; Huisingh, D. Bioenergy: A Sustainable Shift. Energies 2021, 14, 5661. [CrossRef]

5. Vacchi, M.; Siligardi, C.; Demaria, F.; Cedillo-González, E.I.; González-Sánchez, R.; Settembre-Blundo, D. Technological Sustainability or Sustainable Technology? A Multidimensional Vision of Sustainability in Manufacturing. Sustainability 2021, $13,9942$. [CrossRef]

6. D'Adamo, I.; Rosa, P. How Do You See Infrastructure? Green Energy to Provide Economic Growth after COVID-19. Sustainability 2020, 12, 4738. [CrossRef]

7. D'Adamo, I.; Falcone, P.M.; Imbert, E.; Morone, P. Exploring regional transitions to the bioeconomy using a socio-economic indicator: The case of Italy. Econ. Polit. 2020. [CrossRef]

8. Lepore, D.; Micozzi, A.; Spigarelli, F. Industry 4.0 Accelerating Sustainable Manufacturing in the COVID-19 Era: Assessing the Readiness and Responsiveness of Italian Regions. Sustainability 2021, 13, 2670. [CrossRef]

9. European Commission Automotive Industry. Available online: https:/ / ec.europa.eu/growth/sectors/automotive_en (accessed on 16 September 2021).

10. Sukitsch, M.; Engert, S.; Baumgartner, R.J. The Implementation of Corporate Sustainability in the European Automotive Industry: An Analysis of Sustainability Reports. Sustainability 2015, 7, 11504-11531. [CrossRef] 
11. Gedam, V.V.; Raut, R.D.; Lopes de Sousa Jabbour, A.B.; Narkhede, B.E.; Grebinevych, O. Sustainable manufacturing and green human resources: Critical success factors in the automotive sector. Bus. Strateg. Environ. 2021, 30, 1296-1313. [CrossRef]

12. Cianciotta, S. Per un Nuovo Ecosistema Industriale in Abruzzo; Textus Edizioni: L'Aquila, Italy, 2021.

13. Cianciotta, S. Il ruolo delle Zone economiche speciali in Italia: Alcuni casi di studio. In Ripartire dai Borghi per Cambiare le Città; FrancoAngeli Editore: Milano, Italy, 2020.

14. Limes L'Italia è il Mare; GEDI Gruppo Editoriale S.p.A.: Torino, Italy, 2020. 



\title{
Sustainability and Resilience after COVID-19: A Circular Premium in the Fashion Industry
}

\author{
Idiano D'Adamo ${ }^{1, *(1)}$ and Gianluca Lupi ${ }^{2}$ \\ 1 Department of Computer, Control and Management Engineering, Sapienza University of Rome, \\ Via Ariosto 25, 00185 Rome, Italy \\ 2 Accademia del Lusso, Via Montenapoleone 5, 20121 Milan, Italy; gianluca.lupi@gmail.com \\ * Correspondence: idiano.dadamo@uniroma1.it
}

check for

updates

Citation: D'Adamo, I.; Lupi, G. Sustainability and Resilience after COVID-19: A Circular Premium in the Fashion Industry. Sustainability 2021, 13, 1861. https://doi.org/ $10.3390 /$ su13041861

Academic Editor: Marc Rosen

Received: 2 February 2021

Accepted: 5 February 2021

Published: 9 February 2021

Publisher's Note: MDPI stays neutral with regard to jurisdictional claims in published maps and institutional affiliations.

Copyright: (c) 2021 by the authors. Licensee MDPI, Basel, Switzerland. This article is an open access article distributed under the terms and conditions of the Creative Commons Attribution (CC BY) license (https:/ / creativecommons.org/licenses/by/ $4.0 /)$.
COVID-19 has challenged so many of humanity's certainties, but it has also shown that we are able to react to serious threats. Moreover, it is possible to see a great opportunity: to create a real, sustainable renaissance. However, the challenge is so complex that it requires the involvement of as many categories of stakeholders as possible, and the implementation of low-carbon models in different production sectors [1].

A single closed-loop supply chain can be reconfigured as a multi-loop system, in which both reused and recycled materials from a previous life cycle are reintroduced into the market as new products and values. In particular, this editorial focuses on on the fashion industry, which unquestionably characterizes the lives of all citizens and identifies a potential circular premium.

\section{The Circular Economy Is the Cure that Can Give Oxygen to the Linear Economy and Consumption Model}

Large players in the fashion industry have the opportunity to introduce and reinforce sustainability obligations by accelerating and introducing structural changes such as reducing seasonal clothing in favor of a circular economic model. To address this type of change, they will need to reformulate their strategy by first identifying and then capturing the competitive advantage by having at their disposal sustainable policies that can foster greater resilience. As Einstein stated, " . . it is crisis that brings progress. It is in crisis that inventiveness, discovery and great strategy are born".

The time is right to enact a much-needed change to re-invent the fashion industry. A more resilient, anti-fragile and fair future can begin in the post-pandemic period. Circularity is the asset that can surely lead to an ethical and responsible improvement on both the production and consumption sides. It must be understood that an extremely high number of garments in one's closet certainly doesn't help anyone; if anything, it adds to the growth of waste and a greater consumption of both water and energy. It is not necessarily true that a garment purchased the previous year must be thrown away. Our system of consumerism and capitalism has educated us to accept an economic a model where every week trendy fashion groups propose new collections, triggering the need for novelty to the detriment of recently purchased garments, considered outdated and not fashionable.

Circularity serves to reduce the amount of waste by preventing it from ending up in landfills or being incinerated. In order to interrupt this wastefulness in the medium-long term, it is therefore necessary to make products with eco-sustainable materials from the very beginning of production. Linked to this is the problem of finding a solution for the immense amount of waste that already exists and will continue to exist in the transition to the circular economic model.

The need to design a future of well-being through sustainable design requires immediate action. Companies can adopt many strategies: (i) reorienting purchase methods towards renting; (ii) selling garments by subscription; and (iii) repairing or regenerating a garment, thereby creating a product with an infinite life cycle. It is interesting to note 
that in this alternative business model, productive attention is not directed solely at how a product is made but rather at how it is disassembled and recycled.

We could then make this assumption: the end of the product in strategic planning is the beginning of invention: upstream a new process will be developed through which materials will not become harmful or toxic waste. The circular economy can be the means to regenerate the linear model of production and consumption.

Fashion, as Oekom Research mentioned, is one of the most polluting industries in the world, causing enormous environmental damage. Half, or nearly half, of all the clothes produced in the world are made of polyester, a byproduct of crude oil. This certainly results in a durable product, but it makes the entire production process fragile. That is why it makes sense to use of not only classic, conventional raw materials (such as cotton, jute, and silk) but also the wide range of underrated sustainable fibers. The use of these natural resources for the production of clothing is found in many cultures. For example, in some countries the highest quality clothing is produced from lotus fiber, harvested from the lotus stem, and pineapple leaves are referred to as elite fibers. This is because normal cotton production process has a negative impact on environmental sustainability. In fact, high-intensity cultivation creates high water consumption, degrades soil fertility, and leaves a carbon footprint. For this reason, it is necessary to be aware that if there is an increase in demand for consumption after COVID-19, the fashion system will have to adopt resilient production models. A possible approach is to consider how agriculture can provide organic raw materials by diversifying primary products through crops of banana, pineapple, coffee, or bamboo. In this way, sustainable and superior quality products will be created, the agricultural method will regenerate the soil, and the carbon problem will be diminished or eliminated by improved biodiversity.

\section{Consumers and Businesses Directed to Follow a Green Path}

When analyzing and studying the circular economy in the fashion industry, it is useful to consider research that highlights how consumers and companies feel about sustainability. McKinsey conducted an important survey in April 2020 (Consumer Sentiment on Sustainability in Fashion) of 2000 British and German consumers. Two-thirds of the interviewees affirmed following issues: (i) the use of sustainable materials is an important driver towards the final purchase (67\%); (ii) brands should be totally transparent about sustainability (70\%); (iii) the delay of new collections is not a problem $(65 \%)$; (iv) there is a propensity to repair items in order to prolong their use (57\%); (v) there is a frequent purchase of durable fashion items (65\%); (vi) keeping items longer (71\%). The research also shows that the target audience of Generation $\mathrm{Z}$ and millennials has a strong propensity to purchase second-hand items.

Taking into account how purchasing habits are changing, and considering the latest report of LifeGate's National Observatory on Sustainable Lifestyle, we can see that if only $7 \%$ of respondents in 2016 claimed to buy natural clothing, this sample would have increased to $16 \%$ in three years. The growth of this change is mainly driven by the younger generations (as highlighted above). The state-of-fashion report prepared by BoF together with McKinsey showed that 31\% of consumers born after 1996 say they would be willing to pay more for products with a lower environmental impact. Of these, $26 \%$ were from the generation born between 1982 and 1995 and 17\% were from Generation X. This profound attention to the need for better, sustainable goods has further promoted the concept of buy less, buy better.

This change has also led to the management of large fashion companies to start following a greener path. Companies have understood how a sustainable model can produce a competitive advantage in reputation and differentiation. For this reason, the big fashion houses are committed to developing policies that encourage and improve sustainability.

During the pandemic period, this trend has greatly accelerated. The Kering Group, for example, to which Gucci, Bottega Veneta, Balenciaga and Yves Saint Laurent belong, was selected during the Word Economic Forum as seventh out of over 8000 companies 
for its commitment to green production. Several years ago, the group has also launched a product line composed mainly of renewable raw materials. In 2018, it also published the Kering Standards: industry-leading environmental and social requirements for its brands and suppliers. Maire-Claire Daveu, the Kering Group's head of sustainability said, "Our ambition is to redefine luxury to help influence and drive these positive changes."

The LVMH group, which includes Dior, Fendi and Loro Piana, has introduced the LIFE program (actions aimed at the environmental performance of LVMH and its maisons with the goal of reducing $\mathrm{CO}_{2}$ emissions. Prada, on the other hand, supports the sustainability manifesto for Italian fashion and is a member of the Commission for Sustainability, Ecology and the Environment. It has also contributed to the guidelines on eco-toxicological requirements for clothing, leather goods, footwear and accessories that introduce parameters for the use of chemicals to improve product safety and reduce pollution. Strong in this direction is also the action of Valentino, one of the first brands to join Greenpeace's Detox protocol, a campaign to eliminate harmful chemicals from the fashion industry production chain. "Being nature-positive means being business-positive," declares Marco Bizzarri, CEO of Gucci.

\section{A Circular Premium in the Fashion Industry}

Large companies also have the advantage of having bodies or guides that highlight what models to follow to create responsible and sustainable strategies. One of the most important authorities is the Global Fashion Agenda, which is proposed annually by the Copenhagen Fashion Summit. Abolishing the "take, make, dispose" economic model would mean reducing the problem of unsold goods, which is responsible for a good $73 \%$ of garments ending up in landfills. This institution has proposed as priorities several lines to be followed, among which we highlight: (i) traceability throughout the production chain; (ii) climate change conversion; (iii) efficient use of energy and water; (iv) a safe working environment that respects universal human rights; (v) investment in innovative and sustainable fibers; and (vi) a circular strategy.

Starting from this approach, Figure 1 proposes some lines of action that could be implemented to highlight how the sustainable transition, which will affects the fashion industry, will also have resilience in other sectors [2,3]. Within the fashion industry, the market analyses above have highlighted how new market segments are maturing and how necessary it is to capture this change to prevent the development of disruptive approaches by competitors.

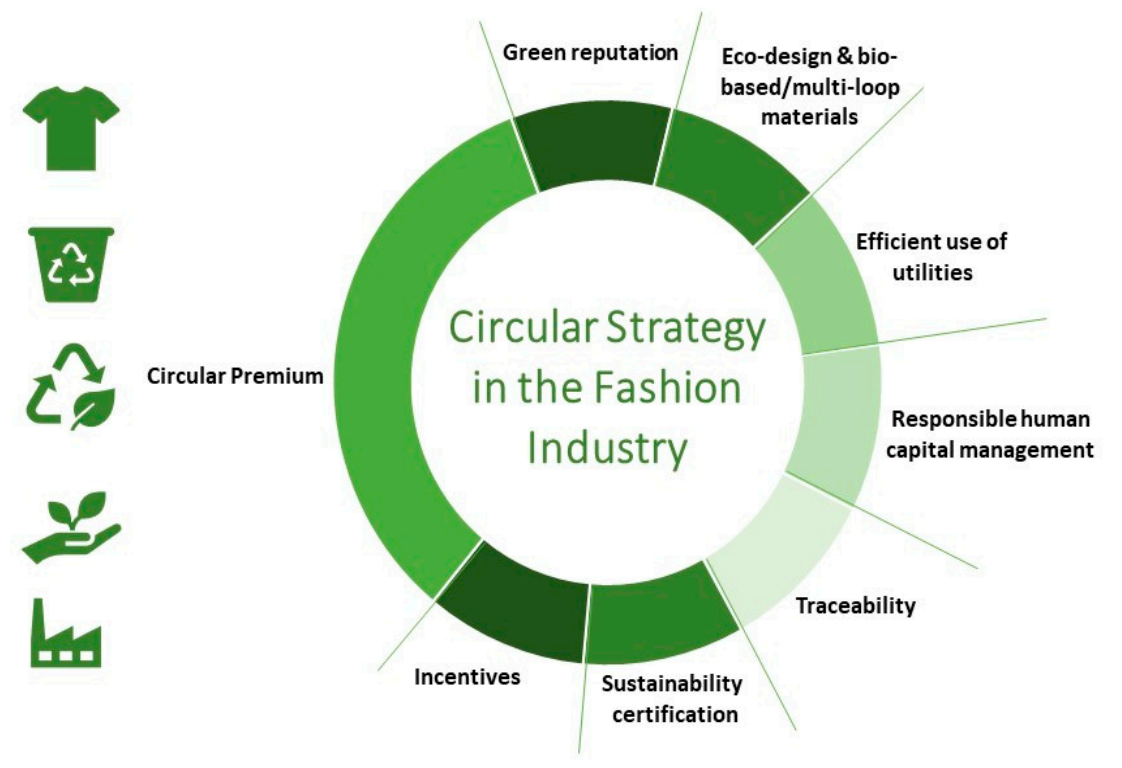

Figure 1. A circular strategy in the fashion industry. 
In order to promote a circular strategy, companies should be interested in increasing their green reputation, which not only means making changes to the supply chain, but also adopting a systemic change in which the company moves beyond its own sphere, to promote initiatives aimed at increasing community sensitivity towards the great challenge of climate change.

Regarding the specifics of the internal actions that need to be taken, eco-design is fundamental because a correct design at the beginning will allow a product to be disposed of properly at the end of its useful life. Changing the supply of raw materials is the next step because using bio-based materials will reduce the impact on other eco-systems much as the "reuse, recycle, recovery" of products already used can be re-introduced into the market to favor a multi-loop model.

The operational and distribution phases may require the excessive use of different utilities. To this end, the various textile production plants must be able to reduce water and energy consumption in addition to reducing the use and release of harmful chemicals. The action of energy efficiency can be supported by the use of energy produced from renewable sources, and the same can be done during the distribution phase through green fuels.

Human relations theory has highlighted how a mix of formal and informal factors can be identified in the organizational context. Some aspects such as health, safety, diversity and gender equality, should be ever-present requirements. This pandemic period has put the emphasis back on the relevance of human relations by fostering the concept of responsible human capital management.

Traceability makes it possible to identify where to concentrate resources, and this strategy of total transparency will allow consumers and investors to analyze the impact of their products. Digitization can aid traceability in a sector such as textiles, which is characterized by a fragmented structure. Traceability in the product realization phase is therefore very complex, but no less so for the consumer. Customer loyalty can take place in different ways, but in a circular perspective it is important to encourage the return of products that are no longer desired or usable. In the first case, the garment is given a new owner; in the second case, the materials are given a new life cycle. This can be done by returning them to stores, but also to specialized collection centers by means of agreements. Furthermore, it is necessary to investigate the relationship between traceability and e-commerce sales channels. In fact, the pandemic period has seen a propensity towards such use. Packaging plays a key role in a circular model, but future studies will need to explore the sustainability of this sales channel compared to the traditional one.

Companies called to redesign their production process to include the acquisition of natural raw materials could incur higher costs. Would consumers be willing to pay more? Thus, companies will have to balance return with risk because not making that choice could mean losing current customers as well as future ones. Consumers may be willing to accept that a circular price (i.e., the price required for a $100 \%$ bio-based product obtained with a sustainable approach) is different from the normal price (i.e., the price they currently pay for fossil fuel-based products). We can therefore introduce in the literature a new term called "the circular premium", which measures the difference between the circular price and the normal price. In addition, a further question to analyze is how to establish the relationship between the circular premium and the percentage of bio-based material in the products. The determining of this percentage will not be easy, but it is crucial to identifying which products (clothing) are truly sustainable. In addition, the circular premium may vary depending on the recipient (e.g., children) or use (e.g., underwear).

In this transition, a sustainability assessment is complex. The analysis must cover the life cycle of the whole product and be compared to a product manufactured under current conditions (totally fossil fuel-based or partially obtained from bio-based materials and renewable energies). However, the assessment cannot focus only on the environmental component; the social and human component cannot take a back seat. Sustainable certification must include these aspects in order to allow a fair comparison between companies. 
Furthermore, incentives are necessary to foster a circular model. The objective could be achieved through different but also complementary actions: producers would supply the raw materials and modify the production processes; consumers would encourage the collection of the product and do not dispose of it as undifferentiated; and third parties would set up reuse, recycling and recovery plants. Future policy perspectives will identify the most appropriate tool to foster the development of circular models. Good intentions are worthless if they are not translated into concrete actions.

"Sustainability is the organizing principle on which to build the future of the fashion industry, more resilient than ever,"declared Eva Kruse, CEO of Global Fashion Agenda. The pandemic period has caused severe socio-economic damage, but it is accompanied by environmental deterioration that can also affect economic opportunities and social equity. In the face of this double risk, future generations are ready to be resilient and make their contribution not only on the consumption side but also through their inclusion in fashion companies by bringing green and circular principles with them. Policy makers can also favor this choice. We think that human strength is resilient by overcoming shocks resulting from economic crises or natural disasters when wearing clothing made form sustainable material and with rainbow colors. Sustainability allows the fashion industry to determine value not only in the aesthetic direction but also in the ethical one.

Author Contributions: Conceptualization, I.D. and G.L.; writing-Original draft preparation, G.L.; writing-Review and editing, I.D. and G.L. All authors have read and agreed to the published version of the manuscript.

Funding: This research received no external funding.

Conflicts of Interest: The authors declare no conflict of interest.

\section{References}

1. D'Adamo, I.; Falcone, P.M.; Martin, M.; Rosa, P. A sustainable revolution: Let's go sustainable to get our globe cleaner. Sustainability 2020, 12, 4387. [CrossRef]

2. Elmqvist, T.; Andersson, E.; Frantzeskaki, N.; McPhearson, T.; Olsson, P.; Gaffney, O.; Takeuchi, K.; Folke, C. Sustainability and resilience for transformation in the urban century. Nat. Sustain. 2019, 2, 267-273. [CrossRef]

3. D'Adamo, I.; Rosa, P. How do you see infrastructure? Green energy to provide economic growth after COVID-19. Sustainability 2020, 12, 4738. [CrossRef] 



\title{
COVID-19, the Food System and the Circular Economy: Challenges and Opportunities
}

\author{
Fabio Giudice ${ }^{1}$, Rocco Caferra ${ }^{2}$ and Piergiuseppe Morone ${ }^{3, *(1)}$ \\ 1 Department of Law, Economics, Politics and Modern Languages, LUMSA University of Rome, \\ 00193 Rome, Italy; fabiogiudice@outlook.com \\ 2 Department of Economics, Management and Law, University of Bari, 70124 Bari, Italy; \\ rocco.caferra@gmail.com \\ 3 Bioeconomy in Transition Research Group (BiT-RG), University of Rome Unitelma Sapienza, \\ 00161 Rome, Italy \\ * Correspondence: piergiuseppe.morone@unitelmasapienza.it
}

Received: 4 August 2020; Accepted: 22 September 2020; Published: 25 September 2020

\begin{abstract}
This paper analyzes the causes and effects of the COVID-19 crisis, with a specific focus on the food system. Food consumption and production has not only been impacted by the crisis, but it may have also contributed to causing the pandemic. After providing a brief introductory framework, the paper presents the results of a pilot study on the link between COVID-19 and the food system, as indicated by the social media activity of selected European Union (EU) Twitter accounts, measured using an original "theme popularity" metric. Thereafter, a systematic review of the literature is proposed to identify the causes of the rise in popularity of a sustainable food system theme, the potential consequences of the COVID-19 crisis for the food system (targeting the production, consumption and waste disposal phases) and possible solutions, focusing on the circular economy. Challenges and opportunities for policymakers in the short and long term are discussed. A holistic approach is advocated, as the global food system is intimately connected with society and requires deep cooperation among nation states and economic actors.
\end{abstract}

Keywords: COVID-19; food system; circular economy; sustainability; EU; Twitter

\section{Introduction}

The COVID-19 pandemic has generated a huge economic crisis and exposed many of the fallacies of the current world economic system, including the food system [1]. The aim of this paper is twofold: first, it aims at identifying the rise and fall of specific narratives related to the food system during the pandemic by means of a content analysis of social media content. This analysis will show how issues associated with the food system gained centrality throughout the lockdown, raising questions regarding the (lack of) sustainability and resiliency of the food system. Subsequently, it will present the preliminary findings of a systematic literature review aimed at identifying possible solutions for improving the food system within the recent scholarly debate. These two objectives represent, in the authors' view, two faces of the same coin. By addressing them simultaneously, we seek to present a full picture of how discourse around the food system (in the context of the COVID-19 emergency) is being shaped and communicated in the interest of developing solutions.

Communication strategies are important, especially in social and political contexts, as they offer the possibility to introduce and attract attention to new problems. To this extent, the dissemination of information is crucial in tracking the path that society should follow, as well as raising public awareness of the importance of particular issues. Hence, it is important to investigate how experts and policymakers propose solutions and inform citizens about the food system. Their methods for doing 
so, as discussed in the current work, might influence subjects' attitudes, behaviors and beliefs about adopting more sustainable practices.

The present analysis starts by framing the sustainability issue against the contemporary backdrop of the health and economic crisis effected by COVID-19 (Section 2). As the pandemic is a contingent matter that has yet to unfold its deepest consequences, we will only seek to evaluate its possible economic repercussions. There are divergent opinions on this matter, but one certainty is that the crisis will leave a mark and question the global economic order, as never before. We will explore the deep causes of the pandemic and the connection between COVID-19 and the current food system, which has exposed the fallacies of the latter.

Subsequently, we will analyze the communication strategy adopted by particular social media accounts (Section 3). As mentioned, we will specifically investigate the dynamics of European Union (EU) communications related to the food system. Our social media content analysis will aim at assessing: (1) how the COVID-19 pandemic has re-shaped the EU's social media agenda with respect to the food system and the circular economy; and (2) how themes relating to the food system and the circular economy have evolved/co-evolved over the period of the pandemic and gained momentum amongst EU citizens. To this end, we will focus on Twitter posts, as these enable the re-construction of social networks, comprised of vertexes (i.e., people, institutions) and links (connections between accounts, people and institutions).

In Section 4, we will analyze possible solutions identified in the recent literature, placing particularly attention on how the European food system might be revolutionized by the introduction of circular economy principles, also in light of the COVID-19 crisis. We will focus on the potential for circular economy solutions to impact all three stages of the food system-production, consumption and waste disposal.

Finally, we will summarize the interconnections between COVID-19, the food system and the circular economy. While there remains much work to be done to facilitate the transition to a more sustainable food system, many instruments have already been set out for this purpose. Within the context of the current pandemic, a socio-economic and political international shift could ease the process towards achieving a more sustainable and circular food system.

\section{How COVID-19 Has Affected Our Lifestyles: The Main Picture}

In January 2020, a previously unknown virus (then named SARS-CoV-2) was identified in China. A few weeks later, an outbreak that would soon be defined by the WHO as a pandemic began to test the health care systems of both advanced and developing countries. Lockdown measures were enforced to slow the spread of the virus and the mortality of the infectious disease. As a result, people were forced to stay at home, with a significant impact on economic activity and daily lifestyles. Accordingly, a symmetric shock on both demand and supply unfolded, with consequences for the world economy.

The COVID-19 pandemic differs from many previous pandemics in its wide spread through many countries. While the outbreak started in China, the world's leading manufacturer, it soon spread across the world, significantly affecting the US, Japan, Germany, France and Italy—all G7 countries with a significant role in the world economy and global value chains [2]. The COVID-19 crisis is complex in many ways, and it will leave its mark not only on public health, but also on the globalized economy, as previously conceived. A recent study found that countries with higher levels of socio-economic globalization have been more severely exposed to the virus [3]. Indeed, as Baldwin and Di Mauro [2] point out, companies, individuals and governments are experiencing disruptions that may eventually lead to deglobalization, as companies are quickly learning the risks involved in global supply chains.

Moreover, as stated in Renzo et al. [4], COVID-19 represents a massive challenge for public health, since the forced isolation has generated noticeable changes in daily lifestyles [5]. In particular, following the main focus of this paper, such sudden lifestyle changes might have both positive and negative impacts on food consumption. Existing studies are controversial in this sense, since some studies—such as [4]—have found evidence of healthier lifestyles during the lockdown, while others 
have revealed problems emerging from unsafe lifestyles during quarantine [6]. The pandemic has also abruptly changed food consumption habits in the short term, and it remains to be seen whether these changes will persist over time. As for the immediate reaction to the crisis, when people first learned about the forced lockdown measures, they rushed to grocery stores to fill their pantries. In a report dated March 2020, the Institute of Services for the Agri-Food Market (ISMEA) showed that panic buying was people's first instinctive reaction [7]. Accordingly, global supermarket shelves emptied of key food items, such as pasta, rice, canned goods, flour, frozen foods and bottled water [8], raising questions about the overall sustainability and resilience of the food system.

As will be discussed in the following section, the impact of COVID-19 on the food system has been well reflected in the EU's social media discourse about the food system throughout the pandemic period.

\section{Evidence from Social Media: A Pilot Study}

In this pilot study, we detect the rise and fall of specific narratives related to the food system. On the one hand, by employing an open source dataset of Twitter posts (i.e., 'tweets') about COVID-19, we show the general increase in public attention related to food themes during the pandemic. On the other hand, we investigate how the EU has directed greater attention to the sustainability of the food system. Following a brief discussion of the role played by social networks in disseminating information (Section 3.1), we will introduce a metric of theme popularity (Section 3.2) and use this to analyze public interest (Section 3.3) in the food system and the emergence and dynamics of EU narratives around the food system (Section 3.4).

\subsection{How Social Networks Disseminate Information}

As discussed in several studies $[9,10]$, the information provided by the media is of fundamental importance for policymaking, as it signals which issues are gaining traction, which are falling out of favor, and which have been introduced as entirely new problems for the public to digest. During the Internet era, the birth of social media provided the opportunity-even in political contexts (see, e.g., Neuman et al. [11])—for information to be disseminated at a low cost [12]. Many studies on social media have focused on the growth in popularity of social media platforms during election seasons, by observing the communication of politicians and political parties [13]. Other studies have addressed the interaction and influence between political social media posts and the public [14], investigating how policy agendas are often settled as a result of the interrelation between interest groups. A complete review of how Twitter data have been exploited to analyze the public debate can be found in Korakakis et al. [15]. Interestingly, Gandy [12] focused on how political agendas are built and, as defined in their paper, the process by which the news determines what is publishable and the way in which elected officials shift issues on the policy agenda based on the media and, in turn, the public.

Different from traditional media data, Twitter data allow for the re-construction of social networks, comprised of vertexes (i.e., people, institutions) and links (connections between accounts, people and institutions). In particular, Twitter reveals the number of accounts observing the activity of a specific account (i.e., the latter's number of followers). Via this metric, one can identify the in-degree centrality [16] of an account, which can be exploited as a measure of user popularity. Using such data, studies have explored how central nodes influence behavior among followers (see, e.g., Chen et al. [17]). Beyond this, Twitter provides several tools for analyzing the accounts that disseminate information and the nature of that disseminated information.

As mentioned in the previous section, the COVID-19 pandemic has raised new challenges with respect to virtuous behavior and the transition to a more sustainable and resilient food system. On the one hand, it is worth analyzing the public's general increase in interest in this issue. On the other hand, it is also relevant to analyze how political actors set the tone for this issue via their social media communication by (i) communicating and disseminating information about actions, interests and 
progress relating to specific themes, and (ii) inducing their network of followers to pro-actively cooperate to achieve the proposed ends. While content analysis has been extensively used to analyze the ways in which actors influence public opinion on environmental themes [18,19], to the best of our knowledge, no prior study has performed a content analysis of social media to analyze themes relating to the food system.

It is common for political organizations to broadcast their interests and disseminate political information via Twitter. For instance, the EU owns and operates several Twitter accounts that deal with both general and specific themes. By observing online interactions with these accounts, it is possible to identify popularity levels relating to the accounts' communicated themes.

The empirical exercise engaged in here will provide a general overview of the possible growing interest in specific food themes during the COVID-19 outbreak, and if and how such themes-associated with select EU Twitter accounts-have demonstrated increased activity over this period. In particular, we will focus on the theme of food management, particularly with respect to the re-organization of the food management system to guarantee savings, security and safety.

\subsection{Metric of Theme Popularity}

In this subsection, we introduce our measure of theme popularity (p). Following Li et al. [20], we propose a measure of popularity based on the number of retweets. This measure may be considered a natural extension of the methodology proposed in Li et al. [20], which included a measure of tweet popularity based on the number of retweets per tweet. In particular, the authors introduced this measure to investigate the interplay between popularity and multimedia content; that is, to understand the public resonance and relevance of particular tweets. In our case, we extend the authors' approach by considering the total number of retweets per tweet pertaining to a specific theme. In this way, we move from measuring single tweet popularity to measuring broader theme popularity, collecting different messages on the same topic. However, we also consider individual tweets, in order to not overlook tweets dealing with a specific theme that do not have a positive number of retweets.

For example, let us consider a tweet vector at time $t, t w t$, of variable length $N$. Each element of $t w t$ is defined as twi,t and assumes a value of 1, indicating the presence of a tweet. Accordingly, $N=0$ defines a null vector and indicates the absence of a tweet at period $t$. As a proxy of popularity, we consider the number of retweets per post, rtwi,t. The number of retweets measures the increase (decrease) in popularity of a given tweet; hence, it is computed in addition to the presence of the tweet. In this way, the popularity of theme $j$ at time $t(p j, t)$ is represented by:

$$
p_{j, t}=\sum_{i}^{N} t w_{i j, t}\left(1+r t w_{i j, t}\right)
$$

Using this formula, it is possible to jointly identify (i) the presence of tweets on a given theme $j$ and (ii) the popularity of that theme. We may also observe the distribution of retweets per tweet, in order to analyze whether the dynamics of the virus might have affected the popularity of the theme at hand. In the following section, we provide an overview of the main descriptive statistics.

\subsection{Social Media Attention during COVID-19}

COVID-19 has attracted the attention of various advocacy groups, as well as media and political actors. Undoubtedly, the crisis is unprecedented, costing millions of lives and testing the social, health and economic systems of many countries. During this crisis, many fears have arisen, largely due to interruptions in the global supply chain. Among these, food shortages have been highly debated, as demonstrated by Laborde et al. in 2020 [21] suggesting the need to rethink global food supply chains. The topic has also been covered by various websites, which have discussed opportunities to create an environmentally friendly post-pandemic world, often framing the discourse in the perspective of the transition to a circular economy. 
In this section, we employ Kaggle data (https://www.kaggle.com/smid80/coronavirus-covid19tweets), comprised of tweets posted between 9 March and 30 April 2020. Drawing on the theme popularity metric $(\mathrm{p})$ introduced above, Figure 1 shows the growing interest in the topic of food security, as evidenced in Twitter hashtags as the virus spread. Hashtags allow users to apply dynamic, user-generated tagging that helps other users easily find messages relating to a specific theme. Data on worldwide daily COVID-19 cases were sourced by the European Center of Disease Prevention and Control.

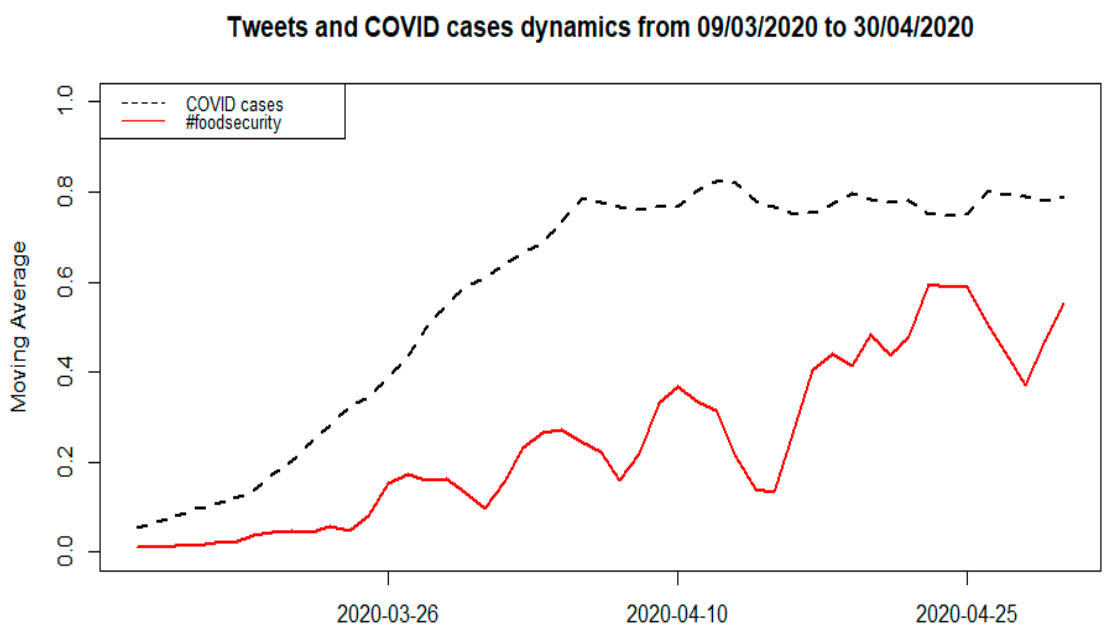

Figure 1. Distribution and dynamics of \#foodsecurity tweets and cases of COVID-19. Data were normalized and smoothed with a 5-day moving average. Maximum value $p=13,105$.

\subsection{EU Media Communication}

In this subsection, we introduce our analysis of the EU's social media agenda, as discussed at the beginning of this section. The official EU website devotes an entire section to the dissemination of information though social media. We selected the following six EU Twitter accounts for analysis (in brackets, we show the account ID and number of followers): (i) European Parliament (@Europarl_EN; 669,636), (ii) European Commission (@EU_Commission; 1,300,000), (iii) European Union Council (@EUCouncil; 507,691), (iv) European Food Safety Authority (@EFSA_EU; 35,831), (v) European Institute of Innovation \& Technology (@EITeu; 54,120) and (vi) European Economic and Social Committee Press (@EESC_PRESS1 5009). These accounts focus on general topics (as in the case of the European Parliament, European Council and European Commission accounts), as well as specific topics of innovation and development (as in the case of the European Food Safety Authority and European Institute of Innovation \& Technology accounts). We did not analyze out-of-topic accounts, since our proposed narrative did not apply to their social media content.

Here, we show the popularity of these accounts on the basis of their in-degree centrality, following $\mathrm{Li}$ et al. [20]. Figure 2 presents the in-degree distribution of the six accounts on a typical day of social media activity, identifying the level of centrality of the EU pages examined. Data refer to the \#covid19 hashtag (i.e., theme), and all analyses were conducted through the twitteR and rtweet packages in R, using a regular Twitter API. To provide a graphical overview, we plot the decumulative distribution function, whereby the y axis presents the log rank of pages, ordered in ascending order from most to least (followers), and the $x$ axis presents the number of followers. Hence, the bottom right of the plot displays the most popular accounts (i.e., those with the highest number of in-degrees). As emerges from Figure 2, the EU accounts play a prominent role in disseminating information via social media. 


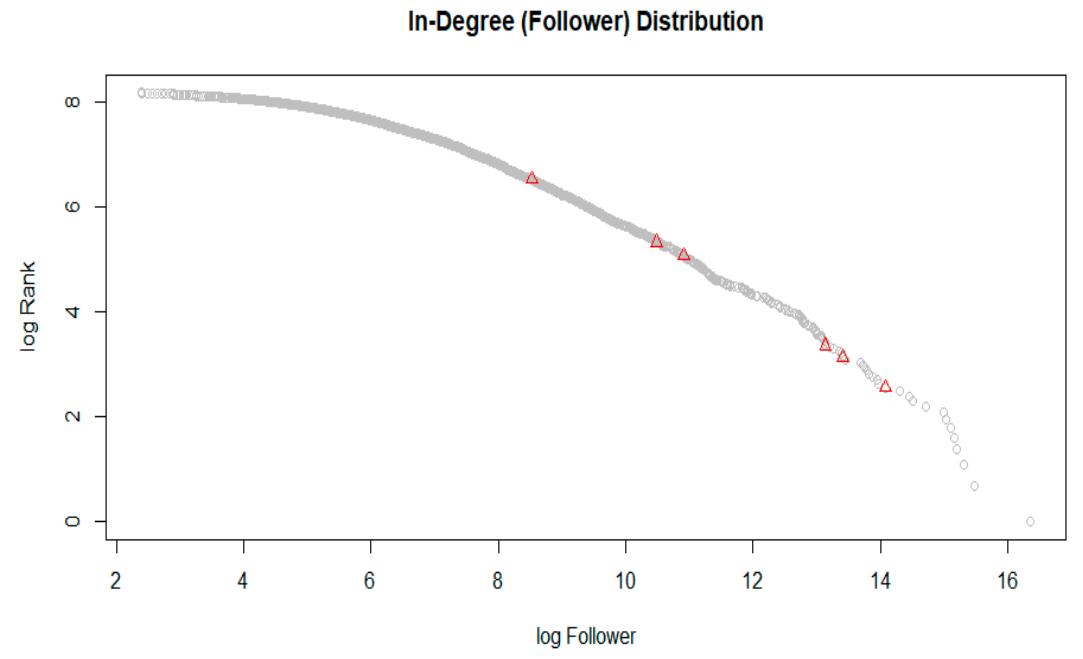

Figure 2. Account in-degree distribution, referring to social media activity on 26 June 2020 pertaining to the \#covid19 hashtag (theme). The data are comprised of 5000 tweets.

Moving onward, we track the timeline of account activity from 31 December 2019 to 26 June 2020. We consider the initial date in this period the day on which the first case of COVID-19 was detected. However, this date can be considered a pre-Covid stage in Europe, since the virus diffusion to Western societies is not thought to have begun until 11 March 2020. In particular, we are able to mine 534 tweets from the European Parliament account, 1862 from the European Commission account, 656 from the European Council account, 504 from the European Food Safety Agency account, 559 from the European Institute of Innovation \& Technology account and 655 from the European Economic and Social Committee account. From these tweets, we can observe the EU's stated position on food systems:

"The Green Deal is a key part of the EU's \#Covid19 recovery strategy for a greener, sustainable and inclusive Europe. Do you know what it tackles?" EU Parliament on Twitter, 2020-06-25

Thus, it seems that the EU's recovery communication strategy emphasizes the economic transition towards greater sustainability and resiliency.

In our mining exercise, we find 146 tweets relating to the food system. In order to find some important connections between the relevance and popularity of the food system and COVID-19, we split the sample into three timeframes:

- Pre-pandemic (pre): 1 January 2020 to 11 March 2020;

- Lockdown (during): from the WHO announcement of the pandemic (12 March 2020) to the partial relaxation of restrictive measures (30 April 2020);

- Post-lockdown (post): from 1 May 2020 to 1 July 2020.

As anticipated in Section 3.2, Table 1 provides a descriptive overview of the retweets distribution, distinguishing between the full sample (FS) (i.e., the initial set of mined tweets) and the sample of tweets pertaining to the food system (FSS). As is evident, the distribution of retweets for FSS was slightly lower than that of FS. However, it may also be noticed that FSS increased in popularity during the lockdown. 
Table 1. Descriptive statistics of the retweets distribution.

\begin{tabular}{|c|c|c|c|c|c|c|c|c|c|c|}
\hline \multirow{2}{*}{ Period } & \multicolumn{2}{|c|}{ Min rtw } & \multicolumn{2}{|c|}{ 1st Quartile } & \multicolumn{2}{|c|}{ Average rtw } & \multicolumn{2}{|c|}{ 3rd Quartile } & \multicolumn{2}{|c|}{ Max rtw } \\
\hline & FS & FSS & FS & FSS & FS & FSS & FS & FSS & FS & FSS \\
\hline Pre & 0 & 2 & 8 & 6 & 91.83 & 46.48 & 96 & 26 & 1925 & 649 \\
\hline During & 0 & 1 & 14 & 10.5 & 214.26 & 87.30 & 153 & 53.25 & 33,853 & 1043 \\
\hline Post & 0 & 0 & 10 & 2 & 88.97 & 26.59 & 76.25 & 23.5 & 3299 & 246 \\
\hline
\end{tabular}

By means of a manual content analysis, we can identify three main thematic areas:

- Food safety, defined as a measure of food health, ranging from the way it is produced to the way it is stored and consumed;

- Food security, defined as a measure of food availability and accessibility, including topics of food shortage, donation and wasting;

- Food sustainable management, defined as the system of incentives oriented towards guaranteeing the sustainability of the food supply chain.

The main results are presented in Figure 3. Here, we show the share of topic popularity, relative to the maximum possible popularity, over the reference period. To clarify, we consider the maximum share of popularity over the period as the sum of the respective total tweet popularities in the pre, during and post phases. In Figure 4, we illustrate the changes in discourse over time. It emerges that, in the pre phase, a large share of popularity was devoted to food safety themes. In line with the results shown in Figure 1, in the during phase, there was a marked interest in food security themes. Here, we found numerous tweets pertaining to food shortages and the importance of food donations, given the instability of the food system and the fear of not having enough supplies to meet basic needs. Tweets in the post period stressed the growing centrality of food sustainable management themes, stressing the importance of the development of an appropriate and sustainable food system in the post-pandemic world. As it seems, the resilience of the future economic system depends on the sustainability of its primary components. There is no question that the food system, which was under severe stress during the lockdown, is a crucial element of the system.

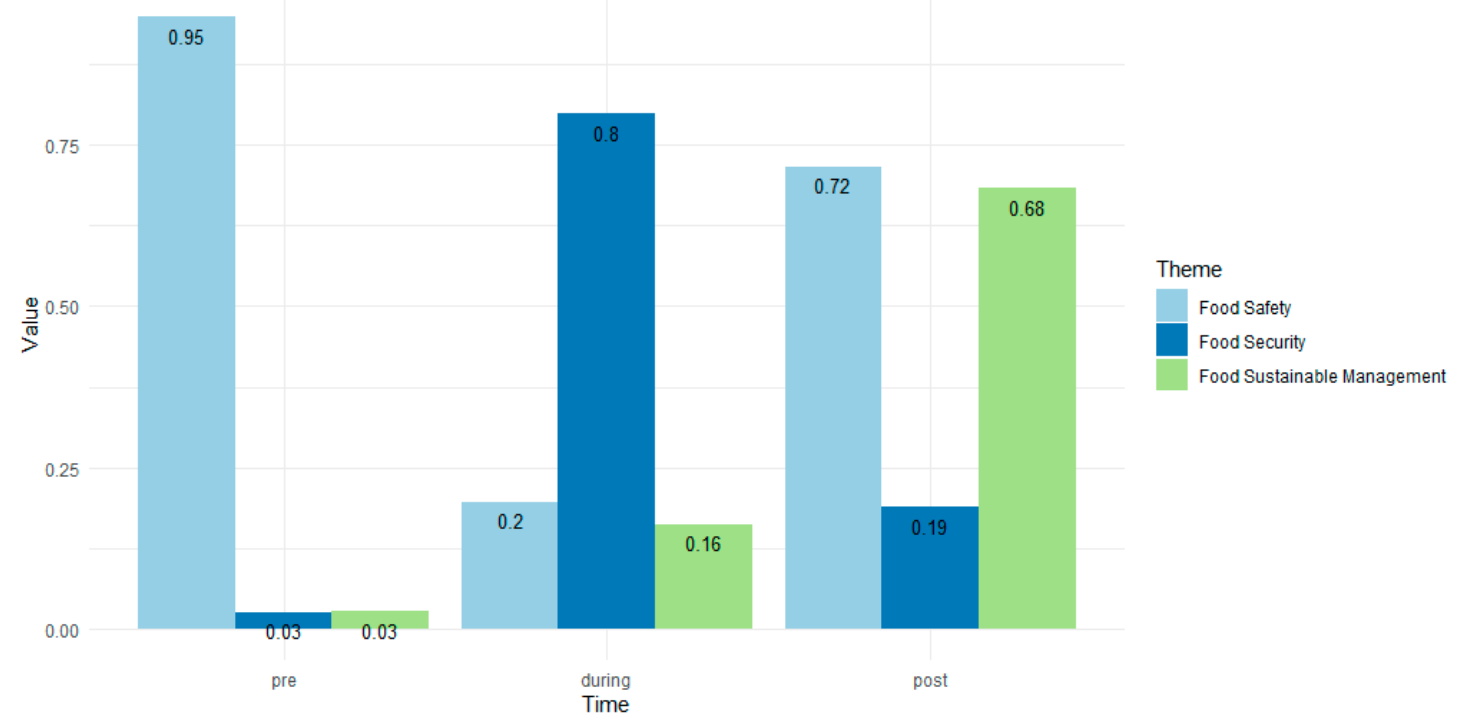

Figure 3. Popularity of EU tweet themes, normalized by the maximum value (1852 in the pre; 3179 in the during; 1959 in the post phase). 
Timeline of tweets- Some Examples

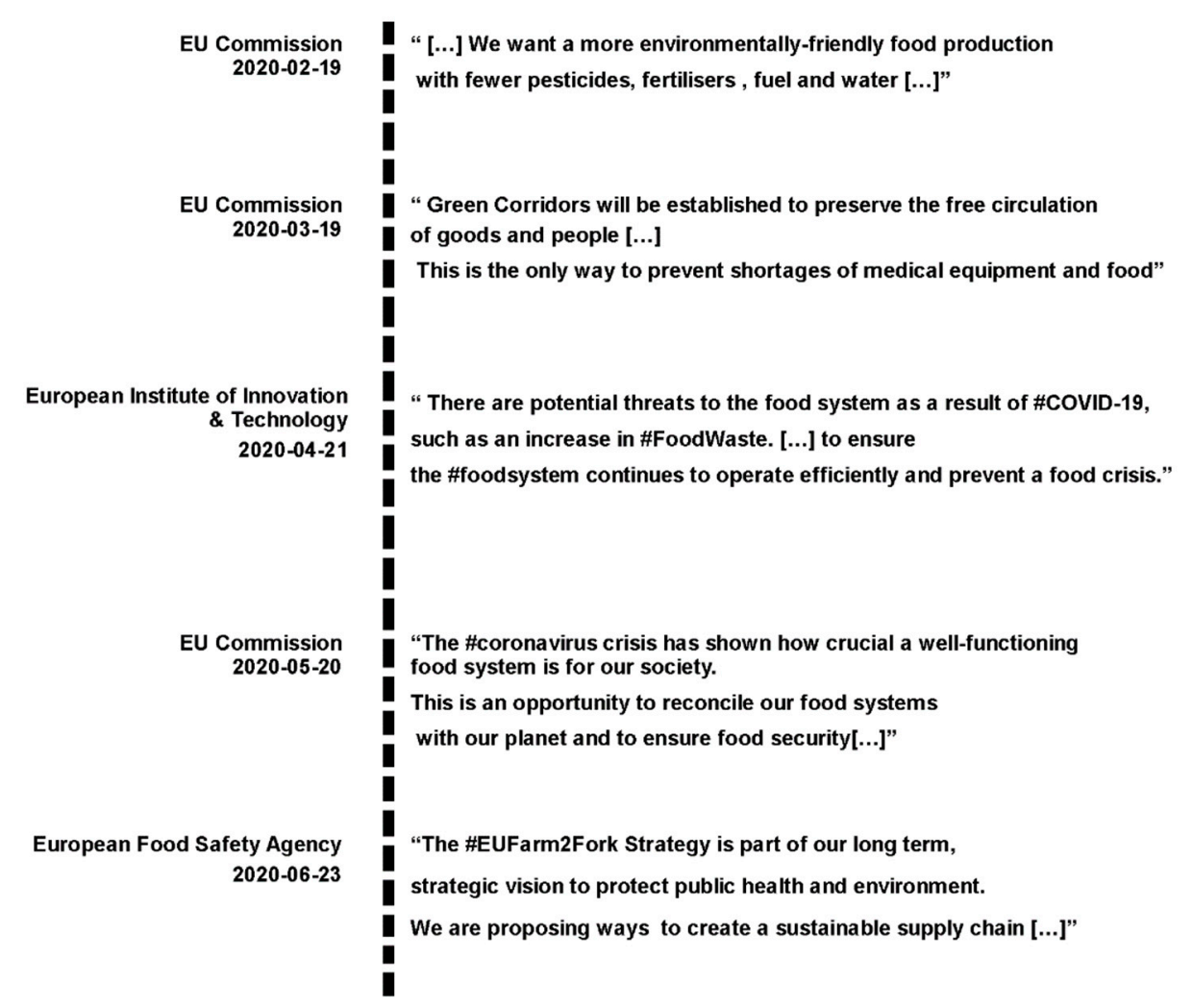

Figure 4. Timeline of example tweets showing the change in discourse over time.

Reflecting on these tweet patterns, it appears that the EU is suggesting that a green recovery must be at the center of the post-pandemic world. Focusing particularly on the food system, this is evident from the increase in popularity of the themes at hand. In our analysis, we consider the possibility that a tweet might simultaneously cover multiple topics; in such instances, we count the tweet towards different narratives on the basis of the precise topics covered. In particular, food safety and food sustainability are frequently jointly analyzed in the tweets, explaining the similar share of total popularity of these themes.

The EU effort towards achieving a sustainable food system began with the Farm2Fork (F2F) strategy, even before the spread of the virus. The F2F strategy is included in the European Green Deal set of actions aimed at mitigating climate change and related environmental disasters. The strategy focuses on the valorization of the food system, favoring the creation of a healthy and sustainable food environment. Indeed, as announced on the online EU portal of the F2F strategy:

"The COVID-19 pandemic has underlined the importance of a robust and resilient food system that functions in all circumstances, and is capable of ensuring access to a sufficient supply of affordable food for citizens $[\ldots]$ ]"

"Food systems cannot be resilient to crises such as the COVID-19 pandemic if they are not sustainable. We need to redesign our food systems $[\ldots .]$.

This narrative is in line with the tone set by the EU on Twitter. Figure 4 presents a timeline of sample tweets. Such an illustration is standard in narrative analysis, as it is useful in guiding readers toward the main conclusion of the analysis [18]. Here, it is evident that the food system is not only a health issue, but also critical to the renaissance of the global economic system. As suggested by the example 
tweets in the post phase, COVID-19 has highlighted the importance of sustainable food management by revealing the food system as a pivotal aspect of the sustainable supply chain.

\section{COVID-19 and the Food System: Causes, Consequences and Circular Solutions}

Having shown the increased importance placed on the food system in the EU's social media agenda, as well as the growing concerns around the sustainability and resilience of the food system, we shall now discuss how this topic has been integrated into the scholarly and practitioner debate over the circular economy. By extending our analysis beyond social media, we hope to achieve a more finely grained assessment of the nexus between the COVID-19 pandemic and the food system. At the same time, by assessing the link between the food system and the circular economy, we seek to propose some actionable-albeit preliminary-solutions.

As the German philosopher Feuerbach said, "We are what we eat." Perhaps this saying might even extend to COVID-19, as many have pointed out that our global food system (and economy) greatly increases our risk of experiencing a pandemic. In what follows, we will present the results of our comprehensive systematic literature review (drawing on Tranfield et al.'s [22] approach) to assess causes, consequences and circular solutions pertaining to the link between the food system and the COVID-19 pandemic.

Systematic reviews differ from traditional narrative reviews in their replicable, scientific and transparent process, aimed at minimizing bias through exhaustive literature searches of published and unpublished studies and providing an audit trail of reviewers' decisions, procedures and conclusions. Our review began with the definition of our goals: to find and integrate the most recent and relevant literature on the relationship between COVID-19, the food system and the circular economy. Subsequently, we analyzed and selected the most recent available literature on the topic, encompassing both scientific papers and gray literature, such as reports and plans from policymakers and international organizations. No subjective distinction was made between scientific papers and other documents, provided that they respected the rules described hereinafter.

The research was mainly conducted through the SCOPUS and Google Scholar public search engines. Forty-three references were selected and shortlisted on the basis of publication date (published in 2015 or later) and correspondence with specific keywords (i.e., "COVID-19," "food," "circular economy"), with the aim of generating collective insights through a theoretical synthesis of fields and subfields. The search was first conducted with the use of the "AND" Boolean operator, then expanded using the "OR" Boolean operator.

The data extraction process focused on synthesizing key information, based on the abovementioned goal of offering an up-to-date review of the current global food system and selecting the most recent and relevant solutions to enhance its sustainability and circularity.

\subsection{Causes}

The World Food Programme [23] recently confirmed that the devastating economic impacts of COVID-19 reinforce the need for investments to prevent future outbreaks of infectious diseases. In so doing, it emphasized the interconnections between people, animals, plants and their shared environment, as well as the need for stable and sustainable architecture to make economic growth feasible, while respecting the surrounding environment [24,25].

There are two primary issues with the current industrial food system. First, intensive livestock production amplifies the risk of disease, since it involves the confinement of large numbers of animals in small spaces, narrowing genetic diversity and fast animal turnover. Second, habitat destruction, unchecked urbanization and land grabbing lead to amplified human-wildlife interaction, which eventually leads to zoonotic spillover [1]. It is therefore clear that pandemics, like the COVID-19 one, are not random events, but the logical result of our current food system and, to a wider scale, our economic model. 
Another catalyst of pandemics is urbanization, as indicated above. Thirty-five years ago, more than $60 \%$ of the global population lived in rural areas; this figure has now dropped to $46 \%$, while the urban population is set to reach $68 \%$ by 2050 [26]. Cities are already consuming $75 \%$ of the world's natural resources and $80 \%$ of the global energy supply [27]. Urbanization impacts food consumption patterns by increasing demand for processed foods, animal-based foods, fruits and vegetables. Higher urban wages also tend to increase the opportunity costs of preparing food and favor food products that require a large amount of labor, such as fast food, store-bought convenience food and food that is prepared and sold by street vendors [26].

China, the alleged epicenter of this and several previous disease outbreaks, has one of the highest urbanization rates in the world, having doubled its level over the past 40 years (from $22.7 \%$ to $54.4 \%$ ) [28]. This urbanization has closely paralleled rising animal protein consumption (due to higher wages), increased land conversion and livestock production, higher zoonotic risk (due to closer contact with wild animals) and a more rapid spread of pathogens through the globalized channels of world economy.

\subsection{Consequences}

As mentioned above, when lockdown measures were first introduced, stockpiling behaviors prevailed, while governments reassured their residents about the resilience of food supply chains and business continuity in the agri-food sectors. In fact, there are diverging opinions on the actual solidity of the current food system: for some, empty grocery shelves are not just the result of the human tendency to hoard in times of danger, but also an important reminder that our food supply chains are easily disrupted and that many of our food systems lack resiliency and redundancy [29]. Many global regions rely on highly centralized food systems, at the expense of strong local and regional systems that could provide a better buffering capacity when needed [29]. However, other scholars have countered that if the number of importing countries has risen for most crops, so has the number of exports in many countries. This has made trade more resilient to swings in supply and demand. Supply lines may empty, but alternatives can be found. For instance, when Indian traders stopped signing new export contracts in April, Carrefour, a French supermarket group, found new rice suppliers in Pakistan and Vietnam and opened a beef import route from Romania [30]. Nonetheless, even the most optimist commentators acknowledge that the current food system has bottlenecks (as does every global supply chain) and that good harvests in 2019 were able to account for some of the resilience of the food supply chain in the face of COVID-19 [30].

Over the long term, consumer food habits might change along three main directions. First, the rapid growth in online grocery delivery services might continue. While many big companies were already implementing this service pre-pandemic, their systems struggled to cope with the sudden expansion in online orders during the lockdown, leaving long time lags before delivery slots were available [8]. The same could be said about food delivery systems, which mainly operate via mobile phone apps: since the pandemic hit, such apps have been increasingly used by restaurants, as in-person dining has been severely restricted in many countries. Therefore, to some extent, the crisis has dematerialized and "desocialized" the food sector, speeding up consumers' adoption of online services. The duration and degree of this trend is still uncertain, but the effect could be noticeable (depending on cultural factors) [8].

Second, consumers might demonstrate a revived interest in "local" food supply chains. In fact, interest in "local foods" was established prior to the pandemic, as people understood this food to offer economic, social, environmental and health benefits [31]. Local food is usually perceived as fresher and-particularly in the present context-more convenient, as it can be easily bought in smaller stores, allowing consumers to avoid long queues outside supermarkets. During the pandemic, consumers also expressed a desire to support the economic recovery of local small and medium enterprises (SMEs). Again, how rooted and long-lasting this effect will be is still unknown, also considering that local food chains are less cost efficient than global ones [8]. 
Third, the pandemic has forced people to significantly change their daily lifestyles, and these changes might persist over the long term. Staying home all day in what was previously a rushed, globalized society has tested people's resilience and led them to question their priorities. People have been forced to slow down their rhythms and rediscover new hobbies and passions (e.g., cooking, instead of buying processed food). It seems that waste recycling has benefitted from these changes [32], alongside a general decrease in waste production (due also to the economic slowdown) [33].

\subsection{Circular Solutions}

As discussed above, the pandemic has put the current food system-focused on a linear and globalized production and consumption model—under high stress. Tjisse Stelpstra of the European Committee of the Regions has said that the devastating situation created by COVID-19 must bring all policymakers together and be the wake-up call for a new economic model that places social wellbeing and environmental sustainability at the core of the EU's economic recovery [33]. The circular economy could be a pivotal element of this recovery plan [34].

According to an EU advisory scientific study [35], achieving a sustainable food system means "increasing or maintaining agricultural yields and efficiency while decreasing the environmental burden on biodiversity, soils, water and air; reducing food loss and waste; and stimulating dietary changes towards healthier and less resource-intensive diets". Jurgilevich et al. [36] summarized that the EU Commission have identified three main stages of the food system with reference to the circular economy: production, consumption and waste.

As for the first stage, the "localization" of the food system might represent a more resilient and sustainable solution: localized food systems reduce waste and favor nutrients [36]. Combining local and seasonal elements in short supply chains reduces storage and transportation, provides a better supply-demand balance, creates more transparency and tracking and contributes to waste reduction. In addition, consumers seem to place higher value on food purchased in local markets.

Another known issue regarding food production is packaging. Our current food system is based on single-use packaging, although recent trends have shown improvements in both the quantity and the quality of this packaging. Still, many recycling processes are insufficient, as is the case for light PET bottles and multilayer plastic (as opposed to mono-material plastic) [37]. In this vein, policymakers should continue to incentivize the reduced use of plastic, in favor of more durable or recyclable materials, such as paper, aluminum, steel and glass, even though these materials do not altogether prevent the accumulation of unwanted metal ions through repeated recycling [37]. For this reason, research and development $(R \& D)$ in materials science and engineering must be a priority.

As for consumption, policymakers should focus on making sustainable choices the easiest options and transferring costs to unsustainable food choices. One example of a sustainable choice is the avoidance and/or reduction of meat consumption. Through the lens of the circular economy, reduced meat consumption increases the efficiency of material flows within the food system by reducing the amount of energy, land and water used per calorie of food produced [38]. Furthermore, policymakers should invest more in food and nutrition education, in order to raise awareness not only amongst the younger generations, but also amongst the older ones, by disseminating information campaigns through both traditional and innovative media channels.

Besides these non-binding actions, more incisive ones (i.e., fiscal and regulatory measures) could force producers and consumers to improve their practices in support of greater sustainability. Policymakers might introduce bans, impose specific production and sourcing requirements, influence demand via public procurement and impose taxes or fees. These fiscal measures might encourage producers, suppliers and retailers to make sustainable choices and/or directly add costs to unhealthy or non-sustainable food for customers, in the form of a Pigouvian tax. Indeed, the SAPEA report [39] states that "examples of relatively imposing instruments that have become increasingly popular include the use of fiscal instruments (e.g., sugar and fat taxes), standard-setting (e.g., on the maximum amount of salt allowed in products), and outright bans (e.g., on trans fats)" (p. 98). 
The final stage of the food system, relating to waste, is perhaps where the circular economy can have the largest and most immediate impact. Indeed, as stated by the European Union [35], "food waste takes place all along the value chain: during production and distribution, in shops, restaurants, catering facilities, and at home. This makes it particularly hard to quantify" [par 5.2]. Within the larger food system, production accounts for approximately $24-30 \%$ of total waste, while the post-harvest stage accounts for $20 \%$ and consumption accounts for $30-35 \%$. Cereals account for $53 \%$ of the total waste; surprisingly, meat accounts for only $7 \%$-far less than the impact of meat production on the environment [40]. According to Stuart, $30-50 \%$ of material intended for consumption (including animal material that is fed to animals or discarded as a byproduct) is wasted in North America and the EU at different stages of the food system [41]. According to Bajzelj [42], the reduction of food waste is essential for achieving a resilient food system.

It is important to distinguish between edible and non-edible food waste, as only the latter is actually defined as waste. Edible food is potentially ready to be consumed, either by its owner or by another person. To reduce food waste, food labelling policies should be changed and harmonized, as "best before" labels are likely to generate unnecessary waste due to consumer misperceptions of food quality. Indeed, according to Borrello et al. [43], "Even when consumers try to follow indications of producers, $20 \%$ of food is thrown away because of the confusion generated by the dates on product labelling". [p. 2]. Policymakers should act to prevent these losses by imposing strict limitations on "best before" labels. In this vein, the EU Commission announced that it "will examine ways of promoting a better use and understanding of date marking by the various actors of the food chain. The EU [35] has also adopted measures to prevent edible fish being thrown back into the sea from fishing vessels" [par. 5.2].

Some authors warn that food sharing initiatives might facilitate upstream food waste, as such initiatives allow consumers to get rid of their waste without preventing its generation in the first place. Thus, they act as "short-term sticking plasters" that obscure entrenched issues of food poverty. Further research is needed to verify the real impact of these actions, which are very diverse and fragmented in their nature [39].

As regards non-edible food waste, this should remain in the system chain and be regarded as a precious resource-not only for the production of more food, but also for the production of new energy (which can be used as fuel in countries seeking to reduce their environmental footprint) and much more. Some policymakers promote "backyard composting" [44], or self-composting at home. More actions and incentives may be needed to promote this activity, considering that it also facilitates the possibility of growing fruits, vegetables and other plants at home. This would enhance household engagement with the production of clean local food and reduce demand for industrial agricultural products, thereby limiting the use of water and chemical fertilizers.

That being said, food waste can take on many other forms, thanks to "green chemistry" solutions within bio-refineries, which can generate biofuels, bio-chemicals, plastics, textiles, medicines and more from organic waste [27]. While a circular food system should primarily aim at transforming food waste into new food, where this is not possible, the system should reinvest these resources into new energy or material forms, which may be equally socio-economically beneficial.

The present analysis clearly shows that a circular food system should not be entirely self-contained, but it should incorporate a wider reconsideration of the current fossil-fueled, linear and unsustainable economic model towards one that is green, resilient and sustainable model—that is, a bioeconomy powered by circularity. Policymakers should therefore engage more with this transition, with the aim of creating a fertile ground for a more sustainable food system (and society) by:

- Reshaping food production via localized supply chains and improved packaging;

- Guiding consumption towards sustainable choices, through a mixture of tax and education policies;

- Focusing and investing in the conversion of non-edible food waste into energy and materials, via green chemistry and bio-refineries. 


\section{Conclusions}

This paper addressed two fundamental issues: first, it outlined the connections between the food system and the current pandemic, investigating how COVID-19 has affected discourse around the food system; second, it matched the requirement for change in the food industry with circular economy solutions. As regards the first issue, we conducted an analysis of the EU's social media agenda, focusing on six institutional Twitter accounts. This allowed us to re-construct the social networks surrounding the food system discourse, identifying the number of users observing the activity of specific accounts and interpreting this as a measure of popularity of specific accounts/tweets. A key finding of this analysis was the change in popularity over time of three specific themes related to the food system: food safety, food security and food sustainable management. The analysis examined three subsequent time periods: the pre-pandemic period, the lockdown period and the post-lockdown period. As it emerged, the dominant theme in the pre-pandemic period was food safety; during the lookdown period, social media attention shifted to food security; finally, in the post-lockdown period, the theme of food sustainable management gained momentum. Far from being conclusive, these preliminary findings suggest how the shock of the pandemic first catalyzed social media attention around issues related to food shortages, donation and wasting (likely associated with the immediate fear of not finding food in stores), and subsequently developed into a deeper reflection on the overall sustainability (and resilience) of the current food system.

As regards the second issue-connecting the need for an alternative food system with circular economy solutions-we found that the circular economy, far from being a panacea, could be an important starting point by dismantling the take-make-dispose system that rules the current world economy. By means of a systematic literature review, we complemented our initial social media analysis with a more finely grained assessment of the emerging debate in the academic and policy literature, proposing ideas and solutions that can be implemented by policymakers and economic actors to improve the food system across all three of its phases (i.e., production, consumption, waste). As it seems, a profound and holistic discussion is emerging around the question of how sustainable the present food system is and how prepared it is to face the kind of shock posed by the COVID-19 pandemic. Circular practices seem to hold the potential for a win-win solution, simultaneously enhancing sustainability throughout the entire value chain (from production to consumption and post-consumption) and improving its resilience through the introduction of localized supply chains that minimize waste and further promote sustainable production and consumption.

The EU Commission is working in this direction, though it should accelerate the transition to new economic solutions, taking advantage of the COVID-19 crisis to make investments in greener technologies. In fact, much work remains to be done, especially on an international scale, where multilateral cooperation is needed more than ever yet is struggling under the influence of foreign policies. Only time will tell if the next pandemic will be met with a renewed spirit and vision, and perhaps a more sustainable food system.

Author Contributions: Conceptualization, P.M. and F.G.; methodology, F.G. and R.C.; software, R.C.; formal analysis, F.G. and R.C.; writing—original draft preparation, F.G. and R.C.; writing—review and editing, P.M.; supervision, P.M. All authors have read and agreed to the published version of the manuscript.

Funding: This research received no external funding.

Conflicts of Interest: The authors declare no conflict of interest.

\section{References}

1. FAO. COVID-19 and the Crisis in Food Systems: Symptoms, Causes, and Potential Solutions. Available online: http://www.fao.org/agroecology/database/detail/en/c/1271231/ (accessed on 11 September 2020).

2. Baldwin, R.; Weder, B.; Mauro, D. Economics in the Time of COVID-19. Available online: https://voxeu.org/ content/economics-time-covid-19 (accessed on 11 September 2020). 
3. Farzanegan, M.R.; Feizi, M.; Gholipour, H.F. Globalization and Outbreak of COVID-19: An Empirical Analysis; CESifo Working Paper Series; CESifo: Munich, Germany, 2020. [CrossRef]

4. Di Renzo, L.; Gualtieri, P.; Pivari, F.; Soldati, L.; Attinà, A.; Cinelli, G.; Cinelli, G.; Leggeri, C.; Caparello, G.; Barrea, L.; et al. Eating Habits and Lifestyle Changes during COVID-19 Lockdown: An Italian Survey. J. Transl. Med. 2020, 18, 229. [CrossRef] [PubMed]

5. De Carvalho, P.M.; Moreira, M.M.; de Oliveira, M.N.A.; Landim, J.M.M.; Neto, M.L.R. The Psychiatric Impact of the Novel Coronavirus Outbreak. Psychiatry Res. 2020, 286, 112902. [CrossRef] [PubMed]

6. Mattioli, A.V.; Sciomer, S.; Cocchi, C.; Maffei, S.; Gallina, S. Quarantine during COVID-19 Outbreak: Changes in Diet and Physical Activity Increase the Risk of Cardiovascular Disease. Nutr. Metab. Cardiovasc. Dis. 2020, 39, 1409-1417. [CrossRef] [PubMed]

7. Emergenza Covid 19: Pubblicato il Rapporto ISMEA Sulla Domanda e L'offerta dei Prodotti Alimentari Nelle Prime Settimane di Diffusione del Virus_ISMEA. Available online: http://www.ismea.it/flex/cm/pages/ ServeBLOB.php/L/IT/IDPagina/10990 (accessed on 3 September 2020).

8. Hobbs, J.E. Food Supply Chains during the COVID-19 Pandemic. Can. J. Agric. Econ. Rev. Can. D'agroeconomie 2020, 68, 171-176. [CrossRef]

9. Kiousis, S.; McCombs, M. Agenda-Setting Effects and Attitude Strength. Commun. Res. 2004, 31, 36-57. [CrossRef]

10. Shapiro, M.A.; Hemphill, L. Politicians and the Policy Agenda: Does Use of Twitter by the U.S. Congress Direct New York Times Content? Policy Internet 2017, 9, 109-132. [CrossRef]

11. Russell Neuman, W.; Guggenheim, L.; Mo Jang, S.; Bae, S.Y. The Dynamics of Public Attention: Agenda-Setting Theory Meets Big Data. J. Commun. 2014, 64, 193-214. [CrossRef]

12. Gandy, O.H., Jr. Beyond Agenda Setting: Information Subsidies and Public Policy; Ablex Publishing Corporation: New York, NY, USA, 1982.

13. Chi, F.; Yang, N. Twitter Adoption in Congress. Rev. Netw. Econ. 2011, 10. [CrossRef]

14. Boutyline, A.; Willer, R. The Social Structure of Political Echo Chambers: Variation in Ideological Homophily in Online Networks. Political Psychol. 2017, 38, 551-569. [CrossRef]

15. Korakakis, M.; Spyrou, E.; Mylonas, P. A Survey on Political Event Analysis in Twitter. In Proceedings of the 12th International Workshop on Semantic and Social Media Adaptation and Personalization, Bratislava, Slovakia, 9-10 July 2017; Institute of Electrical and Electronics Engineers Inc.: Piscataway, NJ, USA, 2017; pp. 14-19. [CrossRef]

16. Matei, S. Analyzing Social Media Networks with NodeXL: Insights from a Connected World by Derek Hansen, Ben Shneiderman, and Marc A. Smith. Int. J. Hum. Comput. Interact. 2011, 27, 405-408. [CrossRef]

17. Chen, Z.Y.; Fan, Z.P.; Sun, M. Individual-Level Social Influence Identification in Social Media: A LearningSimulation Coordinated Method. Eur. J. Oper. Res. 2019, 273, 1005-1015. [CrossRef]

18. Rosenbloom, D.; Berton, H.; Meadowcroft, J. Framing the Sun: A Discursive Approach to Understanding Multi-Dimensional Interactions within Socio-Technical Transitions through the Case of Solar Electricity in Ontario, Canada. Res. Policy 2016, 45, 1275-1290. [CrossRef]

19. Tani, A.; Morone, P. Policy Implications for the Clean Energy Transition: The Case of the Boston Area. Energies 2020, 13, 2615. [CrossRef]

20. Li, J.; Qi, G.; Zhao, D.; Nejdl, W.; Zheng, H.-T. Semantic Web and Web Science; Springer: New York, NY, USA, 2013. [CrossRef]

21. COVID-19 Risks to Global Food Security. Available online: https://reference.medscape.com/medline/abstract/ 32732407 (accessed on 3 September 2020).

22. Tranfield, D.; Denyer, D.; Smart, P. Towards a Methodology for Developing Evidence-Informed Management Knowledge by Means of Systematic Review. Br. J. Manag. 2003, 14, 207-222. [CrossRef]

23. Joint Statement on COVID-19 Impacts on Food Security and Nutrition/World Food Programme. Available online: https://www.wfp.org/news/joint-statement-covid-19-impacts-food-security-and-nutrition (accessed on 3 September 2020).

24. D'Adamo, I.; Falcone, P.M.; Martin, M.; Rosa, P. A Sustainable Revolution: Let's Go Sustainable to Get Our Globe Cleaner. Sustainability 2020, 12, 4387. [CrossRef]

25. D'Adamo, I.; Rosa, P. How Do You See Infrastructure? Green Energy to Provide Economic Growth after COVID-19. Sustainability 2020, 12, 4738. [CrossRef] 
26. Food and Agriculture Organization of the United Nations (FAO). The Future of Food and Agriculture. Available online: http://www.fao.org/publications/fofa/en/ (accessed on 11 September 2020).

27. Food, Cities and the Circular Economy. Available online: https://www.ellenmacarthurfoundation.org/ explore/food-cities-the-circular-economy (accessed on 3 September 2020).

28. Wu, T.; Perrings, C.; Kinzig, A.; Collins, J.P.; Minteer, B.A.; Daszak, P. Economic Growth, Urbanization, Globalization, and the Risks of Emerging Infectious Diseases in China: A Review. Ambio 2017, 46, 18-29. [CrossRef]

29. Five COVID-19 Reflections from a Food System Perspective-And How We Could Take Action-The Rockefeller Foundation. Available online: https://www.rockefellerfoundation.org/blog/five-covid-19reflections-from-a-food-system-perspective-and-how-we-could-take-action/ (accessed on 3 September 2020).

30. The Economist. A Dangerous Gap: The Markets v the Real Economy. 9 May 2020. Available online: https://www.economist.com/weeklyedition/2020-05-09 (accessed on 3 September 2020).

31. Cranfield, J.; Henson, S.; Blandon, J. The Effect of Attitudinal and Sociodemographic Factors on the Likelihood of Buying Locally Produced Food. Agribusiness 2012, 28, 205-221. [CrossRef]

32. Rifiuti a Roma, Meno $12 \%$ a Marzo Aumenta la Differenziata LA GUERRA AL COVID-19-Corriere.It. Available online: https://roma.corriere.it/notizie/cronaca/20_aprile_06/rifiuti-meno-12percentomarzoaumenta-differenziata-a281dccc-7760-11ea-9a9a-6cb2a51f0129.shtml (accessed on 3 September 2020).

33. Coronavirus in Lombardia, Meno $27.5 \%$ di Rifiuti a Milano. Da Oggi Nuovo Ciclo di Sanificazione-La Repubblica. Available online: https://milano.repubblica.it/cronaca/2020/04/14/news/coronavirus_in_ lombardia_meno_27_5_di_rifiuti_a_milano_da_oggi_nuovo_ciclo_di_sanificazione-253954812/ (accessed on 3 September 2020).

34. D’Adamo, I. Adopting a Circular Economy: Current Practices and Future Perspectives. Soc. Sci. 2019, 8, 328. [CrossRef]

35. EU Commission. C. No. 98, 11th M. 2020, Para. 5. 2. EUR-Lex-52020DC0098-EN-EUR-Lex. Available online: https://eur-lex.europa.eu/legal-content/EN/TXT/?uri=COM:2020:98:FIN (accessed on 3 September 2020).

36. Jurgilevich, A.; Birge, T.; Kentala-Lehtonen, J.; Korhonen-Kurki, K.; Pietikäinen, J.; Saikku, L.; Schösler, H. Transition towards Circular Economy in the Food System. Sustainability 2016, 8, 69. [CrossRef]

37. Geueke, B.; Groh, K.; Muncke, J. Food Packaging in the Circular Economy: Overview of Chemical Safety Aspects for Commonly Used Materials. J. Clean. Prod. 2018, 193, 491-505. [CrossRef]

38. Mylan, J.; Holmes, H.; Paddock, J. Re-Introducing Consumption to the 'Circular Economy': A Sociotechnical Analysis of Domestic Food Provisioning. Sustainability 2016, 8, 794. [CrossRef]

39. SAPEA. A Sustainable Food System for the European Union. Available online: https://www.sapea.info/ topics/sustainable-food/ (accessed on 3 September 2020).

40. Vilariño, M.V.; Franco, C.; Quarrington, C. Food Loss and Waste Reduction as an Integral Part of a Circular Economy. Front. Environ. Sci. 2017, 5, 21. [CrossRef]

41. Stuart, T. Waste: Uncovering the Global Food Scandal; WW Norton \& Company: New York, NY, USA, 2009.

42. Bajželj, B.; Quested, T.E.; Röös, E.; Swannell, R.P.J. The Role of Reducing Food Waste for Resilient Food Systems. Ecosyst. Serv. 2020, 45, 101140. [CrossRef]

43. Borrello, M.; Caracciolo, F.; Lombardi, A.; Pascucci, S.; Cembalo, L. Consumers' Perspective on Circular Economy Strategy for Reducing Food Waste. Sustainability 2017, 9, 141. [CrossRef]

44. Illmer, P. Backyard Composting: General Considerations and a Case Study. In Microbiology of Composting; Springer: Berlin/Heidelberg, Germany, 2002; pp. 133-142. [CrossRef]

(C) 2020 by the authors. Licensee MDPI, Basel, Switzerland. This article is an open access article distributed under the terms and conditions of the Creative Commons Attribution (CC BY) license (http://creativecommons.org/licenses/by/4.0/). 



\title{
Thriving, Not Just Surviving in Changing Times: How Sustainability, Agility and Digitalization Intertwine with Organizational Resilience
}

\author{
Antonio Miceli ${ }^{1}$, Birgit Hagen ${ }^{1}$, Maria Pia Riccardi ${ }^{1,2} \mathbb{D}$, Francesco Sotti ${ }^{1}$ and Davide Settembre-Blundo ${ }^{1,3, *(\mathbb{D})}$ \\ 1 Department of Economics and Management, University of Pavia, Via San Felice 7, 27100 Pavia, Italy; \\ antonio.miceli01@universitadipavia.it (A.M.); birgit.hagen@unipv.it (B.H.); \\ mariapia.riccardi@unipv.it (M.P.R.); francesco.sotti@unipv.it (F.S.) \\ 2 Department of Earth and Environmental Sciences, University of Pavia, Via Ferrata 9, 27100 Pavia, Italy \\ 3 Gruppo Ceramiche Gresmalt, Via Mosca 4, 41049 Sassuolo, Italy \\ * Correspondence: davide.settembre@gresmalt.it
}

check for updates

Citation: Miceli, A.; Hagen, B.; Riccardi, M.P.; Sotti, F.; Settembre-Blundo, D. Thriving, Not Just Surviving in Changing Times: How Sustainability, Agility and Digitalization Intertwine with Organizational Resilience. Sustainability 2021, 13, 2052. https://doi.org/10.3390/su 13042052

Academic Editor: Idiano D'Adamo

Received: 27 January 2021

Accepted: 9 February 2021

Published: 14 February 2021

Publisher's Note: MDPI stays neutral with regard to jurisdictional claims in published maps and institutional affiliations.

Copyright: (c) 2021 by the authors. Licensee MDPI, Basel, Switzerland. This article is an open access article distributed under the terms and conditions of the Creative Commons Attribution (CC BY) license (https:// creativecommons.org/licenses/by/ $4.0 /)$.

\begin{abstract}
Nowadays, the buzzwords for organizations to be prepared for the competitive environment's challenges are sustainability, digitalization, resilience and agility. However, despite the fact that these concepts have come into common use at the level of both scholars and practitioners, the nature of the relation between sustainability and resilience has not yet been sufficiently clarified. Above all, there is still no evidence of what factors determine greater resilience to change in an organization that also wants to be more sustainable, especially in times of crisis and discontinuity. This research aims to explore from a theoretical point of view, through the construction of a conceptual model, how these dimensions interact to help the business to become strategically resilient by leveraging digitization and agility as enablers. A new view of resilience arises from the study, which goes beyond the well-known ability to absorb or adapt to adversity, to also include a strategic attribute that could help companies capture change-related opportunities to design new ways of doing business under stress. A key set of strategically agile processes, enabled by digitalization, creates strategic resilience that also includes a proactive, opportunity-focused attitude in the face of change. Strategic resilience to lead to organizational sustainability must be understood as a multi-domain concept quite similar to the holistic view of sustainability: environment, economy and society. Finally, the research offers a set of propositions and a theoretical framework that can be empirically validated.
\end{abstract}

Keywords: strategic resilience; multi-domain resilience; sustainability; strategic agility; digitalization; crisis; change

\section{Introduction}

Organizations to better adapt to change, they must some essential attributes, and nowadays the buzzwords become: sustainability, digitization, resilience and agility. In today's dynamic and interconnected world, organizations must be able to cope and thrive in conditions of crisis and change. Change can be permanent or discontinuous, incremental or radical, be expected or unexpected, reversible or irreversible and can come with varying intensity and at many different levels. This highlights the degree of complexity and multidimensionality characteristics of change [1] that influence the development and implementation of effective business strategies and solutions. In addition to occasional disruptions and continuous incremental changes, radical changes, discontinuities, crises or "crashes" are becoming increasingly important and frequent, all of which have a great impact on the operations of organizations [2]. The COVID-19 pandemic is only the most recent and dramatic example of a global crisis that highlighted, in a relatively short time, the far-reaching implications and challenges of change as well as the urgency of efficient and effective responses [3]. 
The crisis that developed globally following the spread of the COVID-19 pandemic has strongly highlighted the importance of sustainability, not only in the health aspect of its social dimension, but also from an economic and environmental point of view [4]. In fact, the health emergency immediately produced a crisis both on the offer side (blockage of supply chains, closure of factories, significant drop in employment) and on the demand side with the collapse of household consumption and business investments [5]. The growth of economies was, therefore, abruptly interrupted by the pandemic, also in the perspective of sustainable development as defined by Agenda 2030, putting an end to years of efforts by countries and the international community to achieve progress in the level of well-being of their populations [6]. It is, therefore, clear that future growth and development policies cannot ignore the ability of societies, organizations and individuals to face the complex and unpredictable risks and phenomena that continually afflict them. This capacity is commonly known as resilience, and the health crisis linked to COVID-19 has shown how it correlates with environmental and socio-economic sustainability. The nature and modalities of correlation between resilience and sustainability have not yet been appropriately investigated $[7,8]$.

Companies and organizations have also shown that they are not prepared for a global health emergency represented by the COVID-19 coronavirus pandemic [9]. This criticality has highlighted the need to develop a rapid capacity to adapt to change, quickly implementing effective actions to respond to the current situation [10]. In particular, companies need to be prepared to face the potential for prolonged impacts on operations, supply chains and the economy in general and, of course, on staff welfare resulting from a globally spreading infectious disease. Companies should take advantage of this critical moment to review their strategies and plans in response to the crisis to be well prepared for the potential impacts of the pandemic by increasing innovation capacity in a way that firms should become more resilient [11].

The term resilience has been widely used with the outbreak of the coronavirus emergency. Among the most recurrent statements is that resilient economies are the ones that will emerge best from the crisis, or that resilience is needed to restart. While governments and institutions talk about resilience with a view to building or maintaining it, it is not always clear what the exact meaning of this term is [12]. The term itself indicates the ability of a system to withstand any disturbance, organizing a response and returning to normal operation. However, resilience is not to be confused with resistance to change, since partial transformation of the parts is allowed. This ability stems mainly from the intrinsic characteristics of each system, which allow us to effectively overcome critical situations [13]. More specifically for an organization, elements such as knowledge, the ability to react to change, openness, availability of adequate resources, flexibility and a wide network of relationships allow an organization to be resilient [14].

Although resilience is a very topical concept that has received increasing attention from scholars over the past two decades in various research domains [15], nevertheless it has been conceptualized theoretically quite heterogeneously, resulting in the proliferation of different definitions, approaches, theories and interpretations [16]. The orientation of researchers has primarily focused on the conceptual meaning of resilience as an "absorptive" or "adaptive" capacity by borrowing definitions commonly used in systemic and evolutionary theory [17]. What is lacking in the body of research, however, is a theoretical discussion of the factors that make organizations more resilient, thus, able to successfully cope with change [18]. Likewise, there is a lack of theoretical exploration regarding how resilient organizations can prepare for and respond to change in a sustainable way [19].

Based on the foregoing, this research seeks to investigate the elements that identify, at least potentially, the resilience of an organization [20] also by relating it to other distinctive capabilities or enablers such as technological innovation or digitalization [21]. Secondly, it aims to fill conceptual gaps with describing the relationship between resilience and sustainability by designing a framework that also explores and maps ways to build them in the organization. 


\section{Methodology and Research Aim}

An exploratory conceptual research in order to fill the theoretical gaps highlighted in the previous section is the methodology followed in this study. This approach has already been widely applied in theoretical research in the organizational field [22,23]. Through a critical review of the literature, the existing knowledge of the relationship between resilience and sustainability in management practice was identified in order to design a conceptual model. The literature analysis was carried out through a bibliographic research of articles written in English and published in the Scopus repository and Google Scholar database. The keywords and terms used for the search were: "resilience", "sustainability", "digitalization" and "agility", combined with an "AND" and/or an "OR" [24].

The review of the literature thus has been conducted with the critical review approach, an appropriate method to address new topics in order to allow new interpretations and perspectives [25]. This approach differs from the systematic literature review, because it does not claim to include all published articles, but rather to combine viewpoints and insights from different fields of research to generate a novel conceptual framework or theory. Finally, the organizational framework of the critical review has been determined through the enunciation of constructs that help to make sense of the accumulated knowledge on the topics $[26,27]$. Below is the review of the literature organized into three sub-sections, which bring together the main concepts of the topics identified by the keywords.

\section{Towards a Conceptual Framework}

Two excellent and very recent systematic literature reviews [16,17] facilitated taking stock of the work on resilience in management, the discussion of resilience in sustainability literature instead helped in identifying and challenging some of the underlying assumptions [28,29].

\subsection{Clarifying the Concepts of Resilience and Sustainability}

3.1.1. Resilience

Resilience is an increasingly popular concept in both management practice and in scholarly research [30], encompassing not only the complexity of organizations, but also other contexts, for example, urban contexts [31,32]. It deals mainly with reactions to adversity and reflects the growing complexity and interdependence of socio-economic, financial and technological systems, the associated challenges for businesses and the need for solutions to deal with unexpected or unpredictable change [23]. According to $[16,17,33,34]$, academic inquiry has been undertaken at organizational level as a response to external threats, employee-level resilience and strengths, but also at business models or supply chain levels in order to explore adaptability or design principles that reduce vulnerabilities and disruptions. Thus, setting the boundaries is a formidable challenge, because of the many forces and types of change, the interdependencies and the levels of systems that come into play when discussing resilience.

According to the reviews [16,17], there are two dominant interpretations of resilience. One view relates to "absorption" or the system's ability to "bounce back". In this case, there is no real adaptation (i.e., development with change) to changing conditions. Such a "backup" system provides the same functionality to sustain the system exactly as it was before the change. Adaption instead, the second interpretation, includes a capacity to modify incrementally its functions in the face of change. In this way, the system "learns" and evolves with change [35]. Following this distinction, the understanding of the magnitude of "disturbance" that the system can tolerate [36] is important, as it will determine the response and the route of action in the face of change [37]. According to [38] and [39], it is essential not only to understand the nature of change, but also the system's degree of self-organization and the system's learning and adaptation capacities need to be understood.

What also remains to be discussed and clarified is whether the initial conditions and/or the new conditions are the (more) favorable conditions. In the first case, i.e., 
absorption, resilience work assumes that the initial conditions are more favorable than are the new ones- the aim is to bounce back; in the second case, adaptation, work seems to assume that the new conditions are more favorable than the initial ones. Under the same line of thought, we may add that neither absorption nor incremental and continuous adaptation may be an adequate response to changed conditions. With a view to thrive more than to survive, the organization may not only need but want to actively shape conditions, seek to prepare for and address change for long term growth and aim at transformation and renewal.

\subsubsection{Sustainability}

Sustainability and sustainable development are two terms that are mentioned more and more often and are linked to ever wider areas. Despite the great attentiveness, there is still a tendency to attribute different and sometimes discordant meanings to this term [40]. The generic definition of sustainability can be traced back to the 1980s with the drafting of the Brundtland Report by the World Commission on Environment and Development (WCED). In that document, sustainability was identified with the satisfaction of the needs of the present generation without compromising the ability of future generations to meet their own needs, ensuring a balance between economic growth, environmental protection and social well-being [41]. This is also the origin of the idea of sustainable development, as a way of progress that maintains this delicate balance today, without endangering the resources of tomorrow. It, therefore, refers to an approach that calls for the adoption of development strategies that take into account both the observable short-term effects (sustainability) and the long-term effects (sustainable development) [42]. A number of other concepts have since emerged from this foundation, such as the definition of environmental sustainability, which is one that emphasizes the preservation of biodiversity without sacrificing economic and social progress [43]; economic sustainability, which ensures that activities that seek environmental and social sustainability are profitable [44]; social sustainability, which seeks population cohesion and stability [45]. Thus, sustainability and sustainable development work on the principle that available resources cannot be used indiscriminately, that natural resources must be protected, and that all people must have access to the same opportunities.

Sustainability and sustainable development have become a central issue for companies, regardless of their size or industry [46]. Companies are called to seek a balance between economic, social and environmental benefits [47], an objective which is well illustrateted with the call for the triple $\mathrm{P}$ (people, profit and planet) bottom line, which defines sustainability as the intersection of environmental, social and economic value [48,49]. Environmental value can be achieved through the use of renewable resources and the reduction in waste and emissions [50]; the social value derives from the social development and the well-being generated by the organization [51]; while the economic value commonly is seen in terms of survival, growth or long-term performance of the firm. Frequently, economic value is seen as a consequence of organizational resilience [52].

\subsubsection{The Link between Resilience and Sustainability}

In times of uncertainty, commitment to sustainability is essential for companies that also want to be resilient [53]. The two concepts, although different, share the same goal: to achieve sustainable development [54]. Accompanying organizations and society as a whole towards a state of equilibrium that must be maintained as stable as possible is a challenging task [55]. A resilient system is one that has the ability to resist and recover from impacts and disruptions [56]. While a resilient development is one that adapts to changing conditions and can recover from extreme and adverse circumstances [57]. Therefore, the resilience of organizations helps to deal with the complexity of change, while preserving the capacity for development. The governance of these organizations in such complex scenarios should focus on achieving organizational resilience based on sustainability (economic, social and environmental) that ensures the achievement of their sustainable development goals [58]. 
The pandemic crisis of the COVID-19 has changed the paradigm of business performance evaluation [59]. While in the past the entrepreneur was responsible to the investor for the company's ability to generate profits [60], now all stakeholders (and not just shareholders) also want to know how those profits were generated. Therefore, they want to know if the environment (environment), the rights of workers and more generally the community (social) and the basic rules of corporate governance (governance) have been respected [61]. By now, we are consolidating the awareness that the value creation of companies is closely related to environmental, social and governance (ESG) performance even in times of crisis $[62,63]$. As a result, companies that are now more attentive to ESG factors, which in the past was only the prerogative of large listed companies, are also more resilient to risks produced by complex and unpredictable phenomena such as health emergencies. When talking about resilience and sustainability, it is important to remind us that there is a strong relationship between these two concepts, as they share many features [64] As a matter of fact, resilience and sustainability have in common many research methodologies, since both concepts are focused on the topic of system survivability [65]. According to the framework designed by Marchese [64], resilience can be considered as a component of the wider concept of sustainability; in fact, this point of view relies on a notion that assumes that an increase in the system's resilience leads to an increase in the system's sustainability [66]. The integration of sustainability and resilience allows to generate a comprehensive system approach, which is crucial to establish a sustainable decision making based on the consideration of industrial, social and ecological dynamics [67]. When discussing the ecological-economic system, it is important to consider that resilience is one of the elements that must be considered when planning the organization's strategies for sustainable management [68]. The connection between resilience and sustainability leads to the establishment of a new approach known as "resilient sustainability", which represents a driving mindset, allowing decision makers to find solutions to sustainability issues by leveraging on the adaptative capacities of the company in order to find the best strategies [69].

If sustainability is defined holistically, the required balance and the interplay of the three values (environmental, social, economic) necessitates a discussion of whether and how resilience can contribute to overall organizational sustainability, or, more in general, whether and how the two concepts are related [70]. As mentioned above, the dominant interpretations of resilience in the management context prescribe absorption, bouncing back to initial conditions, or adaptation towards reaching a new equilibrium without an explicit consideration of whether the initial conditions or the new conditions are more favorable or "sustainable" [71]. Thus, a system may be resilient but unsustainble [72]. Additional complexity is added by the fact that there may be a trade-off across the three values and that a balance is required. It follows that resilience, similarly to sustainability, must be broken down in sub-domains, since resilience in one system (economic, ecological, social) does not automatically lead to a positive impact in another system. An in-depth discussion of resilience - in terms of resilience of "what" to "what"- therefore, would help us to understand and qualify interconnections and responses better.

On the other hand, sustainability may be thought of as an element to build resilience. Companies whose practices and strategies include considerations of environmental, social and economic balance may also be more resilient to change [53]. For example, production processes or supply chains with lower environmental and social impact may immunize against environmental jolts or social upheaval. They may also bring benefit in terms of market positioning and, thus, contribute to resilience and value creation [73].

An in-depth discussion of the interdependencies and the causality between the broad view of sustainability and resilience goes beyond the scope of this work. Here, we see sustainability holistically, as a metaobjective and a basis for (normative) performance indicators of the resilient organization. Consequently, we postulate that:

Proposition 1 (P1). Sustainability and resilience can be viewed as two interdependent concepts. 
P1a. It remains to be clarified how resilience affects sustainability (understood in a holistic sense) and vice versa.

P1b. Resilience may induce positive outcomes in one of the sustainability domains (ecological, economic, social-EES), but it may not automatically induce the same positive effects in the other ones.

P1c. Sustainability may improve resilience, but the positive outcome experienced in one resilience domain does not automatically lead to a positive outcome in another one.

Proposition 2 (P2). If sustainability is understood as a multi-domain concept and ultimate meta objective of the organization, resilience should also be defined correspondingly to include economic, environmental and social resilience.

P2a. Establishing a multi-domain concept of resilience allows us to account for interconnections and trade-offs/synergies with regard to multi-domain sustainability. This implies that resilience must include a normative element regarding the desirable outcome and/or desirability of system conditions.

P2b. Resilience in one domain does not automatically lead to resilience in another domain.

P2c. Sustainability in one domain does not automatically lead to sustainability in another domain.

\subsection{Building the Strategically Resilient-Agile Organization}

According to [29], research so far has attempted to make sense of events in a given period to generate insights into how organizations (should) deal with adversity under a particular set of circumstances. Insights from the various research streams essentially point to the importance of slack and, thus, call for the accumulation of resources to build redundancy and resourcefulness (i.e., variety). The approach is in line with the ideas of evolutionary theory, which also builds the theoretical background for the conceptualization of resilience as adaptation. Additionally, the "structure" of the firm and its processes, for example in terms of losing control or flexibility are seen to promote resilience. These findings link to the supply chain work and emphasize facilitated resource reconfiguration [74], which is seen crucial to respond to changing conditions. Finally, a wide range of enablers in terms of information processing and communication, collaboration and networking is mentioned. Generally, the strategy of accumulating resources has been criticized not only for being inefficient and costly but also difficult in terms of "reconfiguration" for different purposes [75].

Overall, resilience research is yet to identify and understand the factors that build organizational resilience to future conditions [76]. Hamel and Välikangas [77] draw on insights from innovation research to propose that to become strategically resilient, the organization must address a cognitive, a strategic, a political and an ideological challenge. Their idea of resilience relates to a broad, strategic view of resilience in terms of an organization's renewal and transformation. The cognitive challenge relates to the idea that a company must build deep awareness of what is changing and be willing to consider how those changes will affect its current success. The strategic challenge consists of developing a range of new options that represent alternatives to dying strategies, while the political challenge relates to resource re-allocation to future programs. Finally, the ideology to be challenged is the quest for operational excellence and flawless execution. The approach proposed by [78] (i.e., to design strategically agile business processes to thrive in conditions of uncertainty and change) nicely fits with this line of thought, as it helps cope with these challenges and, thus, can be considered a driver of strategic resilience. The objective to thrive includes absorption, adaptation but also transformation and renewal to actively prepare for and achieve growth on the longer term. Importantly, it includes the notion of speed, which is, surprisingly, neglected in most management resilience work with the notable exception of the supply chain research strand $[79,80]$. The question of how quickly the organization can absorb, adapt or transform and renew is of huge importance, especially in a dynamically and quickly changing business landscape. 
In [78]'s view, designing flexible and responsive processes for (1) information search, (2) business development or innovation, (3) harmonization and coordination of the value chain and (4) resource mobilization and leverage make the firm strategically agile. According to the authors, such agile processes help the firm to move in conditions of unpredictability for which discontinuities or the consequence of change make a case. For example, in a situation where information is unavailable or contradictory, the authors propose to base information searches on close and regular contacts with a diverse range of customers and partners in order to help the organization read signals early, interpret them better and act on them efficiently and effectively. Customers and partners should also make part of the business development/innovation process, which is based on experimentation. The idea of experimentation for innovation is not new, but the idea to opt for multiple small experiments to develop strategic agility or resilience is [81]. In our context, such a way of approaching innovation has two major advantages: it prepares a variety of options in advance, while limiting the risk of "big" failure [82]. It, therefore, optimizes resource allocation instead of a potentially inefficient and costly accumulation of resources to guarantee slack. It is, thus, a flexible and strategic way of developing a variety of options, and developing them early, so that they can be put into use once the context is changing. Customers and partners should take part to the process so that resources and risk are shared, and the innovation is responsive to their needs [83]. At the same time, the innovation is tested and adapted early and quickly, reducing which again reduces the risk of lost investment while at the same time accelerating the innovation process and the response.

The third process, the coordination and harmonization of the value chain, is based on the idea of flexibility and responsiveness in terms of resource generation. Partners may compensate for the organization's lack of resources or contribute to their variety. Relationships, furthermore, may be more or less actively used under different conditions [84]. Importantly, the view of an internally and externally harmonized and coordinated value chain includes the notion that the various partners do act with the same "strategic agility" philosophy, so as to achieve coordinated and timely action, accommodating a "system view" and accounting for interdependence [85]. Finally, the process of resource mobilization links to the value chain and the idea that resources do not need to be developed and owned by the organization itself but can be mobilized externally or be co-developed and owned. Secondly, a creative use of resources, leveraging the resources at hand, involves creating synergies, for example regarding data collection, analysis and use, co-promotion, co-development of offerings, etc. [86]. Overall, designing and implementing processes with a view on strategic agility will guarantee a resource-conservative way to cope with the need for absorption, adaptation and renewal. At the same time, it ensures "preparedness" and a timely and effective response to unexpected change or, more in general, situations of uncertainty.

As is clear from the above, the concepts of strategic resilience and strategic agility are similar. What they have in common and what differentiates the concept from resilience, as it is predominantly discussed in management literature, is the view on change as opportunity and active, timely management of change towards renewal and long-term growth-as compared to survival and dealing successfully with adversity [87]. Finally, while the speed of response has received little attention in the overall body of resilience work, it is emphasized with the concept of agility. The dimension is also reinforced in the context of supply chains. Shekarian et al., for example [79], posit that above all the agile supply chain is fast in perceiving and responding to changing market needs and at the same time able to include the opportunity to improve delivery systems.

When resilience and agility are compared instead, again, the supply chain literature, which has discussed both concepts, may inform our work. Recently [88], thanks to an exhaustive analysis of the literature, have clarified the two concepts of agility and resilience in an integrated way by structuring differentiating and common features. In Figure 1, these dimensions are illustrated. 


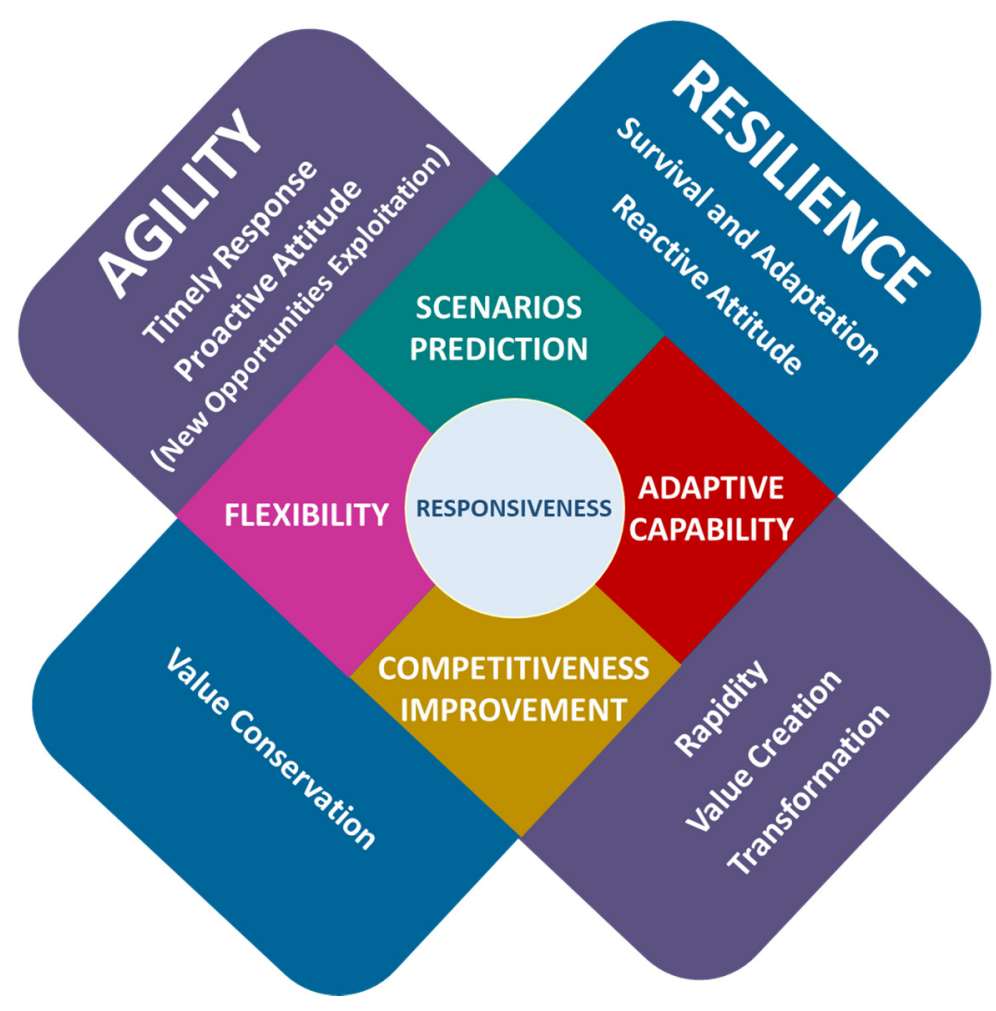

Figure 1. Distinctive and common features of resilience and agility (adapted from [88] and [89]).

The focus here, however, is not on discussing commonalities and differences but on the ways forward to combine the concepts in a way that allows an organization to build resilience strategically. Based on the above we propose.

Proposition 3 (P3). Agility builds a strategic dimension of resilience, i.e., the capability of an organization to manage change proactively, more effectively and efficiently with a view to transformation and renewal. It includes the notion of the speed of the organization's response to change.

\subsection{Digitalization}

Simply put, business digitalization consists of the implementation of digital tools and technologies as well as data, which together can make business processes more efficient [90] and effective. It is this aspect of digitalization that is of particular interest to our discussion of how to build strategically agile processes and, in turn, strategic resilience and sustainability. Digital technologies allow the collection of huge amounts of data, which are constantly increasing not only in quantity, but also in diversity [91]. Big data analytics for predictive analysis recognizes patterns that signal upcoming events and identifies measures to solve issues and improve outcomes $[92,93]$. Under the same line of thought, the contribution of artificial intelligence (AI) will be fundamental to analyze business data and provide a system that makes "complex thinking" easier and may build an improved basis for decision making [94] assisting both the identification of change as well as its management.

Digitalization also helps through an unprecedented potential of interconnection of business processes [95] and of stakeholders. Ensuing is the ability to better monitor activities to improve organization and coordination and quality of work [96] and to adapt more quickly to changing market conditions [97]. The basis of this "model" is the availability of all relevant information in real time that connects, through digital technologies, all stakeholders involved in the value chain, internally and externally. Digitalization also allows us to keep significant business processes operational when unexpected events occur, minimizing the economic impact and safeguarding the functioning of (production) 
processes in the medium and long term [98,99]. Digital technologies that enable remote collaboration, virtual process management and real-time connectivity provide the means to respond effectively and efficiently $[100,101]$.

In summary, digitalization has the potential, through processes, to mitigate the magnitude and reach of change by, at the same time, increasing the proactive stance and agility of business processes and the resilience of the organization. Based on this, we can argue:

Proposition 4 (P4). Digitalization, through data and technologies, promotes agility because it increases the flexibility and responsiveness of the organization's business processes, for example by identifying changes early and by enabling efficient and effective connection and coordination of business processes and partners.

Digitalization is also improving the sustainability of companies, enabling them to produce in a more environmentally friendly way [102]. Digital technologies increase the operational efficiency through the accessibility and collection of process data in real time, the management of energy and resource consumption and knowledge of the entire life cycle (design, manufacturing, distribution, maintenance and use) with the potential to eliminate discontinuities and inefficiencies [103]. The connection of processes and products, value chain and users allows the design of the product's manufacturing cycle together with that of its use in a logic of environmental and economic sustainability. In this way, it is possible to optimize the consumption of resources and reduce energy inefficiency and waste generated along the entire value chain [104]. Thereby, we can state:

Proposition 5 (P5). Digitalization, by itself, enables efficient use of resources, thus contributing positively to sustainability.

However, technology, including digitalization, is not neutral, especially when assessed from a socio-environmental-economic systems perspective [105]. Rebound effects and "turbulence", for example technological or industry disruption generated or triggered by digitalization, therefore, must be assessed. The ongoing discussion related to challenges and tensions around the Industry 4.0 illustrate the complexity and interdependence of the systems [106]. Therefore, we propose:

Proposition 6 (P6). Digitalization, by itself, triggers change and rebound effects may occur. Thus, it may have also a negative influence on:

P6a. Resilience and on

P6b. Sustainability.

In conclusion, we add the last proposition to the discussion on how to become a strategically resilient organization. One may propose that having built-in strategic resilience in the organization's processes and systems, this competence will be leveraged continually in the face of change. Under this line of thought, the strategically resilient organization establishes a virtuous cycle with regard to the management of change [107]. Thus, the following is being proposed.

Proposition 7 (P7). The strategically resilient organization continually leverages and strengthens its competences in change management to establish a virtuous circle.

\section{Designing a Conceptual Model}

This paragraph illustrates the design process of the conceptual model that aims to identify the characterizing elements and to formalize the links between them. The pivotal elements represented by the model are:

- The constructs that are the attributes of reality built through theoretical reasoning; 
- The propositions that are the concepts that relate the different constructs.

The Table 1 offers an overview of the propositions that illustrate the interplay between the key concepts of the conceptual framework.

Table 1. Propositions overview (own elaboration).

\begin{tabular}{|c|c|c|c|}
\hline & Proposition & Construct 1 & Construct 2 \\
\hline P1 & $\begin{array}{l}\text { Sustainability and resilience can be viewed as two } \\
\text { interdependent concepts. }\end{array}$ & \multirow{4}{*}{ Sustainability } & \multirow{4}{*}{ Resilience } \\
\hline$P 1 a$ & $\begin{array}{l}\text { It still remains to be clarified how resilience affects sustainabilty and vice } \\
\text { versa (understood in a holistic sense) and vice versa. }\end{array}$ & & \\
\hline$P 1 b$ & $\begin{array}{l}\text { Resilience may induce positive outcomes in one of the sustainability } \\
\text { domains (ecological, economic, social-EES), but it may not automatically } \\
\text { induce the same positive effects in the other ones. }\end{array}$ & & \\
\hline$P 1 c$ & $\begin{array}{c}\text { Sustainability may improve resilience, but the positive outcome } \\
\text { experienced in one resilience domain does not automatically lead to a } \\
\text { positive outcome in another one. }\end{array}$ & & \\
\hline P2 & $\begin{array}{l}\text { If sustainability is understood as a multi-domain concept and } \\
\text { ultimate meta objective of the organization, resilience should also } \\
\text { be defined correspondingly to include economic, environmental } \\
\text { and social resilience. }\end{array}$ & \multirow[t]{4}{*}{ Resilience } & \multirow[t]{4}{*}{ Sustainability } \\
\hline$P 2 a$ & $\begin{array}{c}\text { Establishing a multi-domain concept of resilience allows us to account for } \\
\text { interconnections and trade-offs/synergies with regard to multi-domain } \\
\text { sustainability. This implies that resilience must include a normative } \\
\text { element regarding the desirable outcome and/or desirability of } \\
\text { system conditions. }\end{array}$ & & \\
\hline$P 2 b$ & $\begin{array}{l}\text { Resilience in one domain does not automatically lead to resilience in } \\
\text { another domain. }\end{array}$ & & \\
\hline$P 2 c$ & $\begin{array}{l}\text { Sustainability in one domain does not automatically lead to sustainability } \\
\text { in another domain. }\end{array}$ & & \\
\hline P3 & $\begin{array}{l}\text { Agility builds a strategic dimension of resilience, i.e., the capability } \\
\text { of an organization to manage change proactively, more effectively } \\
\text { and efficiently with a view to transformation and renewal. It } \\
\text { includes the notion of the speed of the organization's response } \\
\text { to change. }\end{array}$ & Agility & Resilience \\
\hline $\mathrm{P} 4$ & $\begin{array}{l}\text { Digitalization, through data and technologies, promotes agility } \\
\text { because it increases the flexibility and responsiveness of the } \\
\text { organization's business processes, for example by identifying } \\
\text { changes early, and by enabling efficient and effective connection } \\
\text { and coordination of business processes and partners. }\end{array}$ & Digitalization & Agility \\
\hline P5 & $\begin{array}{l}\text { Digitalization, by itself, enables efficient use of resources, thus } \\
\text { contributing positively to sustainability. }\end{array}$ & Digitalization & Sustainability \\
\hline P6 & $\begin{array}{l}\text { Digitalization, by itself, triggers change and rebound effects may } \\
\text { occur. Thus, it may also have a negative influence on }\end{array}$ & \multirow{3}{*}{ Digitalization } & \multirow{3}{*}{$\begin{array}{l}\text { Resilience } \\
\text { Sustainability }\end{array}$} \\
\hline$P 6 a$ & resilience and on & & \\
\hline$P 6 b$ & sustainability. & & \\
\hline P7 & $\begin{array}{c}\text { The strategically resilient organization continually leverages and } \\
\text { strengthens its competences in change management to establish a } \\
\text { virtuous circle. }\end{array}$ & Resilience & $\begin{array}{l}\text { Change } \\
\text { Management }\end{array}$ \\
\hline
\end{tabular}

As it is possible to notice from the table, seven propositions have been formulated. The first one (P1) highlights the interdependence of the concepts of sustainability and resilience by pointing out that the effects of one on the other, and vice versa, can be both positive and negative. The second proposition (P2) calls for a multi-domain view 
of resilience, just as is the case for sustainability (environment, economy and society), to better capture the interdependencies between the two concepts. The third proposition (P3) states that agility represents the strategic dimension of resilience, that is, the responsiveness of an organization to change. The fourth proposition (P4) points to digitization as an enabler for agility because technologies speed up processes by making the organization more flexible, efficient and effective. The fifth proposition (P5) notes that digitization, by promoting more efficient use of resources, has a positive impact on sustainability. The sixth preposition (P6), on the other hand, emphasizes that digitization, by triggering change can also exert a negative rebound effect on both resilience and sustainability. Finally, the seventh proposition (P7) concludes that a strategically resilient organization leverages its capabilities to deal with change, while also strengthening them.

The conceptual model shown in Figure 2 provides an overview on the connections among the topics discussed in this research, thus showing the relationships between resilience, sustainability and digitalization. The seven propositions introduced above will represent the vectors used to connect the topics previously discussed. The model clearly shows the interdependence existing between sustainability and resilience (P1), and it is this correlation that justifies a multidimensional vision of resilience (P2) exactly as it occurs for sustainability that is articulated in the three pillars of environment, economy and society. In an organizational perspective, the multi-attribute gives resilience a strategic implication that is enabled by agility (P3), that is, the ability of organizations to (re)act on change flexibly and responsively. Conversely, digital technologies can play both a positive (P5) and negative (P6) role on both sustainability and resilience, all depending on whether or not organizations are able to leverage these attributes to grow through a virtuous cycle (P7).

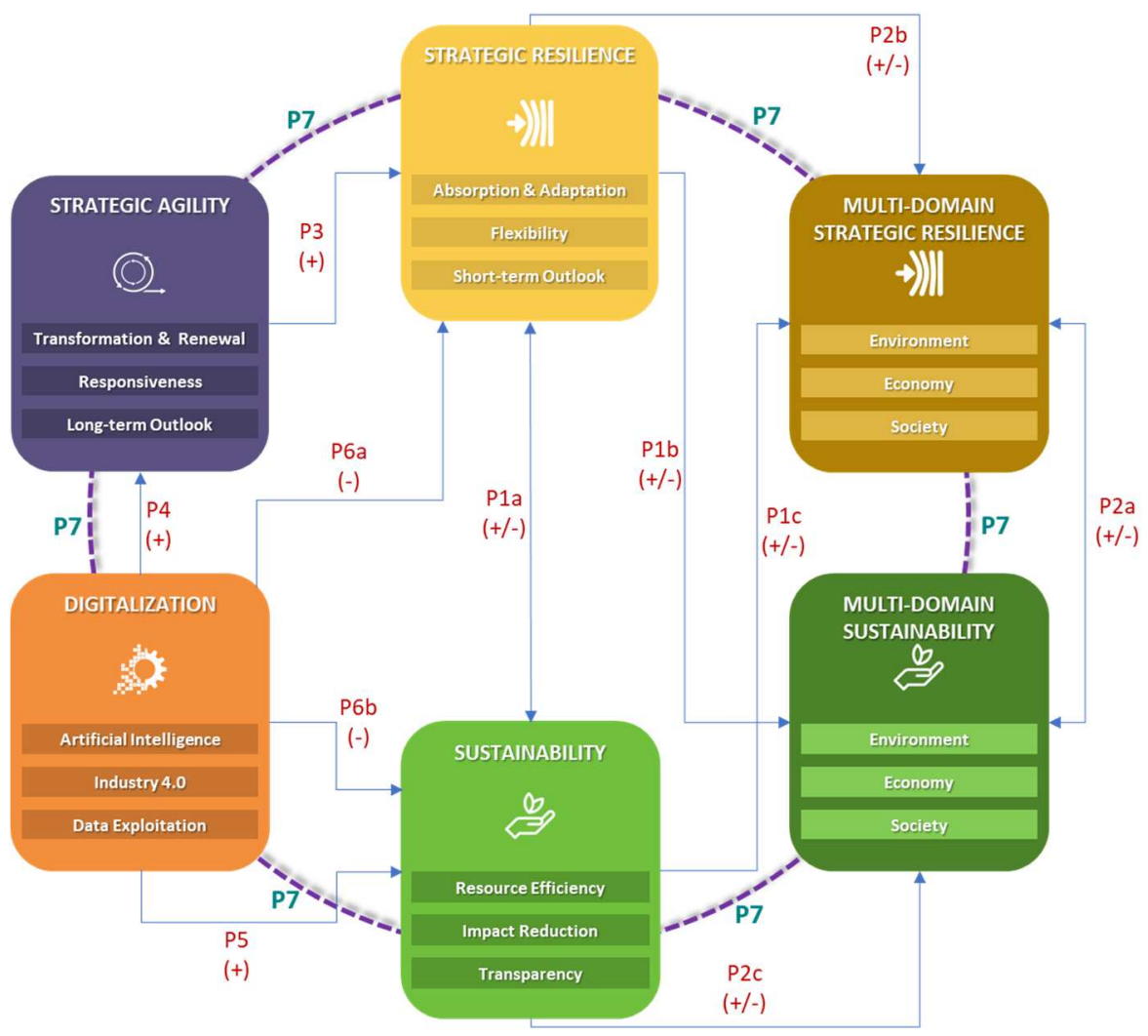

Figure 2. Conceptual model (own elaboration).

\section{Discussion and Contribution}

We set out to build, through a critical literature review, an explorative model to integrate resilience and sustainability. Through the combination of "isolated" areas, i.e., resilience work in management and sustainability literature, we identify and discuss key en- 
ablers and model a route to build strategic multi-domain resilience, which is hypothesized as a pillar of an organization's sustainability. In so doing, we account for the complexities and interdependencies between environment, social and economic sub-systems of the firm and show that resilience needs to be considered holistically, similar to sustainability, in order to understand its overall performance implications better. Systems may be resilient but unsustainable as [69] with the resilient but unsustainable dictatorship illustrate. Thus, the use of resilience concepts for organizational decision making requires the clarification of the resilience of "what" and the addition of performance measures. Along the same lines of thought, we propose that resilience work would benefit from a normative stance with regard to the desirability of initial or new system conditions.

Additionally, dominant conceptualizations such as "absorption" or "adaptation capability" fall short in providing a proactive, opportunity-focused view of change or simply neglect disturbance that exceeds thresholds for absorption or adaptation [108]. Our model provides a way forward to thrive more than to survive under conditions of change and uncertainty. It is not only the resilience "of what" we mentioned above, also the resilience "to what" needs attention. In other words, it is crucial to determine better the forces of change that are at play. Change comes with many facets-from discontinuous to incremental, from reversible to irreversible, etc.- which will influence the firm's response and organization in order to build resilience. Large-scale disturbances require quite different organizational response mechanisms compared with minor disturbances, or gradual change [72], and they usually exceed thresholds for adaptation [109].

However, when focusing on agile business processes, flexibility and responsiveness become an integral part of the firm's structure and strategy and, thus, should prepare it well for an effective and resource-conserving answer to expected change and surprises of all types. More than being a planned response or process, strategic agility is a way of operating that accommodates changes. Agility can solve the tension between "preparedness" and efficiency that extant work has evidenced in the discussion on building redundancy and resourcefulness. It allows for process and resource flexibility and responsiveness at the same time, while, at the same time, being a resource-conservative way of operating. Agility adds time considerations, a crucial element in (re)acting to change. Furthermore, and importantly, it also helps to build long-term or strategic resilience, as it goes beyond absorption and adaptation to include transformation and renewal of the organization.

As we show, digitalization enables strategically agile processes. Digitalization, for example, with big data analysis can help predict change, and due to its unprecedented interconnectivity, it can facilitate communication and coordination with various stakeholders. At the same time, digitalization, by itself, may have positive or negative effects on both sustainability and resilience. Big data while being beneficial in terms of early detection of change may have a negative impact with regard to privacy issues and, thus, be in conflict with social sustainability. Digitalization itself, through its production and logistics, also comes with a high carbon footprint [110], which may trade off a positive impact due to less waste in production.

\section{Conclusions and Future Research Directions}

Although exploratory, our model illustrates the complexities and interactions that organizations face when dealing with resilience and sustainability on firm level. The framework helps in understanding the dynamics and the interplay between the concepts, cascading effects, potential trade-offs and synergies. The multi-domain view that we propose makes interdependencies clear and transparent and can inform prioritization. With more work and with empirical tests of our framework and propositions, other elements will arise, and trade-offs and synergies will become clearer. We also present a viable option on how to build strategic resilience effectively and efficiently and, in turn, sustainability in organizations. Designing and implementing strategically agile processes is one proposal on how to create strategic resilience, the integration of research insights from others, however, will yield many more that may inform organizations. As mentioned above, strategically 
agile processes potentially allow us to solve the dilemma of efficiency versus efficacy in the organization's response to change. This comes close to the discussion of sets of capabilities, resources and structures that are necessary to create ambidextrous organizations, a body of research that is promising to further investigate the options for building strategic resilience. Additionally, literature on organizational adaptation presents a variety of approaches based on resource bases and capabilities [111] that describe how adaptation between the organization and the changing environment can be achieved, maintained or restored [112]. Another promising field for cross-fertilization is the research on business longevity or continuity, and the necessary ingredients, e.g., adaptability, flexibility, innovation [113,114] to it.

Even though our model accounts for interdependencies on the firm level, we neglect the interaction with the firm's environment, which definitely would merit close attention. Communities, networks and, more in general, ecosystems have been described to influence an organization's resilience. Setting boundaries, therefore, on the one hand helps us to manage complexity but on the other hand brings the disadvantage of omitting important relations. Future research should examine these interdependencies further. We have also assumed that sustainability, holistically, is an overarching objective of the organization, an assumption that may prove to be too optimistic. Additionally, in this context, internal versus external pressures and their interplay are promising areas to contribute to knowledge on resilience and sustainability of organizations.

Notwithstanding these limitations, we make a theoretical contribution through our framework and the strategic, multi-domain conceptualization of resilience. Additionally, we propose a novel approach to building strategic resilience via agile business processes, which presents a solution to tensions between efficiency and effectiveness of company response and strategy - a problem related to the current discussion on the creation of organizational resilience-and can be leveraged at the organizational level. Overall, we hope that the framework and the propositions help us to advance a step further towards resilience and truly sustainable organizations.

Author Contributions: Investigation, A.M and B.H.; conceptualization, A.M and B.H.; methodology, D.S.-B.; validation, B.H.; formal analysis, F.S.; data curation, F.S.; writing—original draft preparation, D.S.-B.; supervision, M.P.R.; project administration, M.P.R. All authors have read and agreed to the published version of the manuscript.

Funding: This research was co-funded by the Italian Ministry of Economic Development (D.M. 5 March 2018 - CHAPTER II-Call for Research and Development Projects within the application areas consistent with the National Strategy of Intelligent Specialization [SNSI]—Smart Factory), under the Project I.E.S.MAN. (Internet of Enterprise Sustainable Manufacturing) n. 211.

Institutional Review Board Statement: Not applicable.

Informed Consent Statement: Not applicable.

Data Availability Statement: Not applicable.

Conflicts of Interest: The authors declare no conflict of interest.

\section{References}

1. Ayres, R.U. On forecasting discontinuities. Technol. Forecast. Soc. Chang. 2000, 65, 81-97. [CrossRef]

2. Burt, G. Why are we surprised at surprises? Integrating disruption theory and system analysis with the scenario methodology to help identify disruptions and discontinuities. Technol. Forecast. Soc. Chang. 2007, 74, 731-749. [CrossRef]

3. Albach, H.; Meffert, H.; Pinkwart, A.; Reichwald, R. Management of Permanent Change-New Challenges and Opportunities for Change Management. In Management of Permanent Change; Springer Gabler: Wiesbaden, Germany, 2015; pp. 3-21.

4. Hakovirta, M.; Denuwara, N. How COVID-19 Redefines the Concept of Sustainability. Sustainability 2020, 12, 3727. [CrossRef]

5. Sarkis, J. Supply chain sustainability: Learning from the COVID-19 pandemic. Int. J. Oper. Prod. Manag. $2020,41,63-73$. [CrossRef]

6. Barbier, E.B.; Burgess, J.C. Sustainability and development after COVID-19. World Dev. 2020, 135, 105082. [CrossRef]

7. Jabbarzadeh, A.; Fahimnia, B.; Sabouhi, F. Resilient and sustainable supply chain design: Sustainability analysis under disruption risks. Int. J. Prod. Res. 2018, 56, 5945-5968. [CrossRef] 
8. Balugani, E.; Butturi, M.A.; Chevers, D.; Parker, D.; Rimini, B. Empirical Evaluation of the Impact of Resilience and Sustainability on Firms' Performance. Sustainability 2020, 12, 1742. [CrossRef]

9. Kraus, S.; Clauss, T.; Breier, M.; Gast, J.; Zardini, A.; Tiberius, V. The economics of COVID-19: Initial empirical evidence on how family firms in five European countries cope with the corona crisis. Int. J. Entrep. Behav. Res. 2020, 214, 26. [CrossRef]

10. Bartik, A.W.; Bertrand, M.; Cullen, Z.B.; Glaeser, E.L.; Luca, M.; Stanton, C.T. How Are Small Businesses Adjusting to Covid-19? Early Evidence from a Survey No. w26989; National Bureau of Economic Research: Cambridge, MA, USA, 2020.

11. Sneader, K.; Singhal, S. Beyond Coronavirus: The Path to the Next Normal; McKinsey \& Company: New York, NY, USA, 2020.

12. Takewaki, I. New Architectural Viewpoint for Enhancing Society's Resilience for Multiple Risks Including Emerging COVID-19. Front. Built Environ. 2020, 6, 143. [CrossRef]

13. de Oliveira Teixeira, E.; Werther, W.B., Jr. Resilience: Continuous renewal of competitive advantages. Bus. Horiz. 2013, 56, 333-342. [CrossRef]

14. Duchek, S. Organizational resilience: A capability-based conceptualization. Bus. Res. 2020, 13, 215-246. [CrossRef]

15. Lv, W.D.; Tian, D.; Wei, Y.; Xi, R.X. Innovation Resilience: A New Approach for Managing Uncertainties Concerned with Sustainable Innovation. Sustainability 2018, 10, 3641. [CrossRef]

16. Linnenluecke, M.K. Resilience in business and management research: A review of influential publications and a research agenda. Int. J. Manag. Rev. 2017, 19, 4-30. [CrossRef]

17. Conz, E.; Magnani, G. A dynamic perspective on the resilience of firms: A systematic literature review and a framework for future research. Eur. Manag. J. 2020, 38, 400-412. [CrossRef]

18. Mithani, M.A.; Gopalakrishnan, S.; Santoro, M.D. Does exposure to a traumatic event make organizations resilient? Long Range Plan. 2020, 102031. [CrossRef]

19. Carmeli, A.; Dothan, A.; Boojihawon, D.K. Resilience of sustainability-oriented and financially-driven organizations. Bus. Strategy Environ. 2020, 29, 154-169. [CrossRef]

20. D'Adamo, I.; Rosa, P. How do you see infrastructure? Green energy to provide economic growth after COVID-19. Sustainability 2020, 12, 4738. [CrossRef]

21. D'Adamo, I.; Falcone, P.M.; Martin, M.; Rosa, P. A Sustainable Revolution: Let's Go Sustainable to Get Our Globe Cleaner. Sustainability 2020, 12, 4387. [CrossRef]

22. Huang, L.; Knight, A.P. Resources and relationships in entrepreneurship: An exchange theory of the development and effects of the entrepreneur-investor relationship. Acad. Manag. Rev. 2017, 42, 80-102. [CrossRef]

23. Budak, J.; Rajh, E.; Slijepčević, S.; Škrinjarić, B. Conceptual Research Framework of Consumer Resilience to Privacy Violation Online. Sustainability 2021, 13, 1238. [CrossRef]

24. Harzing, A.W. Two new kids on the block: How do Crossref and Dimensions compare with Google Scholar, Microsoft Academic, Scopus and the Web of Science? Scientometrics 2019, 120, 341-349. [CrossRef]

25. Snyder, H. Literature review as a research methodology: An overview and guidelines. J. Bus. Res. 2019, 104, 333-339. [CrossRef]

26. Webster, J.; Watson, R.T. Analyzing the past to prepare for the future: Writing a literature review. MIS Q. 2002, $26,3$.

27. Saunders, M.N.; Rojon, C. On the attributes of a critical literature review. Coach. Int. J. Theory Res. Pract. 2011, 4, 156-162. [CrossRef]

28. Sandberg, J.; Alvesson, M. Ways of constructing research questions: Gap-spotting or problematization? Organization 2011, 18, 23-44. [CrossRef]

29. Alvesson, M.; Sandberg, J. The problematizing review: A counterpoint to Elsbach and Van Knippenberg's argument for integrative reviews. J. Manag. Stud. 2020, 57, 1290-1304. [CrossRef]

30. Xu, L.; Kajikawa, Y. An integrated framework for resilience research: A systematic review based on citation network analysis. Sustain. Sci. 2018, 13, 235-254. [CrossRef]

31. Sharifi, A. Urban resilience assessment: Mapping knowledge structure and trends. Sustainability 2020, 12, 5918. [CrossRef]

32. Elmqvist, T.; Andersson, E.; Frantzeskaki, N.; McPhearson, T.; Olsson, P.; Gaffney, O.; Takeuchi, K.; Folke, C. Sustainability and resilience for transformation in the urban century. Nat. Sustain. 2019, 2, 267-273. [CrossRef]

33. Skouloudis, A.; Tsalis, T.; Nikolaou, I.; Evangelinos, K.; Leal Filho, W. Small \& Medium-Sized Enterprises, Organizational Resilience Capacity and Flash Floods: Insights from a Literature Review. Sustainability 2020, 12, 7437.

34. Zavala-Alcívar, A.; Verdecho, M.J.; Alfaro-Saíz, J.J. A conceptual framework to manage resilience and increase sustainability in the supply chain. Sustainability 2020, 12, 6300. [CrossRef]

35. McCarthy, I.P.; Collard, M.; Johnson, M. Adaptive organizational resilience: An evolutionary perspective. Curr. Opin. Environ. Sustain. 2017, 28, 33-40. [CrossRef]

36. Holling, C.S.; Gunderson, L.H. Resilience and adaptive cycles. In Panarchy: Understanding Transformations in Human and Natural Systems; Island Press: St. Louis, MO, USA, 2002; pp. 25-62.

37. Scheffer, M.; Carpenter, S.; Foley, J.A.; Folke, C.; Walker, B. Catastrophic shifts in ecosystems. Nature 2001, 413, 591-596. [CrossRef]

38. Carpenter, S.; Walker, B.; Anderies, J.M.; Abel, N. From metaphor to measurement: Resilience of what to what? Ecosystems 2001, 4, 765-781. [CrossRef]

39. Nelson, D.R.; Adger, W.N.; Brown, K. Adaptation to environmental change: Contributions of a resilience framework. Annu. Rev. Environ. Resour. 2007, 32, 395-419. [CrossRef] 
40. Salas-Zapata, W.A.; Ortiz-Muñoz, S.M. Analysis of meanings of the concept of sustainability. Sustain. Dev. 2019, $27,153-161$. [CrossRef]

41. Keeble, B.R. The Brundtland report: 'Our common future'. Med. War 1988, 4, 17-25. [CrossRef]

42. Wu, L.; Subramanian, N.; Abdulrahman, M.D.; Liu, C.; Pawar, K.S. Short-term versus long-term benefits: Balanced sustainability framework and research propositions. Sustain. Prod. Consum. 2017, 11, 18-30. [CrossRef]

43. Moldan, B.; Janoušková, S.; Hák, T. How to understand and measure environmental sustainability: Indicators and targets. Ecol. Indic. 2012, 17, 4-13. [CrossRef]

44. Cardoni, A.; Zanin, F.; Corazza, G.; Paradisi, A. Knowledge management and performance measurement systems for SMEs' economic sustainability. Sustainability 2020, 12, 2594. [CrossRef]

45. Eizenberg, E.; Jabareen, Y. Social sustainability: A new conceptual framework. Sustainability 2017, 9, 68. [CrossRef]

46. Günther, K. Key Factors for Successful Implementation of a Sustainability Strategy. J. Appl. Leadersh. Manag. 2016, 4, 1-20.

47. Gundes, S. The use of life cycle techniques in the assessment of sustainability. Procedia Soc. Behav. Sci. 2016, 216, 916-922. [CrossRef]

48. KsiężaK, P.; FischBach, B. Triple Bottom Line: The Pillars of CSR. J. Corp. Responsib. Leadersh. 2017, 4, 95-110. [CrossRef]

49. Svensson, G.; Wagner, B. Implementing and managing economic, social and environmental efforts of business sustainability. Manag. Environ. Qual. Int. J. 2015, 26, 195-213. [CrossRef]

50. Alayón, C.; Säfsten, K.; Johansson, G. Conceptual sustainable production principles in practice: Do they reflect what companies do? J. Clean. Prod. 2017, 141, 693-701. [CrossRef]

51. Shou, Y.; Shao, J.; Lai, K.-H.; Kang, M.; Park, Y. The impact of sustainability and operations orientations on sustainable supply management and the triple bottom line. J. Clean. Prod. 2019, 240, 118280. [CrossRef]

52. Cancino, C.A.; La Paz, A.I.; Ramaprasad, A.; Syn, T. Technological innovation for sustainable growth: An ontological perspective. J. Clean. Prod. 2018, 179, 31-41. [CrossRef]

53. Gillespie-Marthaler, L.; Nelson, K.S.; Baroud, H.; Kosson, D.S.; Abkowitz, M. An integrative approach to concep-tualizing sustainable resilience. Sustain. Resilient Infrastruct. 2019, 4, 66-81. [CrossRef]

54. Cavaco, N.M.; Machado, V.C. Sustainable competitiveness based on resilience and innovation-an alternative ap-proach. Int. J. Manag. Sci. Eng. Manag. 2015, 10, 155-164.

55. Roostaie, S.; Nawari, N.; Kibert, C. Sustainability and resilience: A review of definitions, relationships, and their integration into a combined building assessment framework. Build. Environ. 2019, 154, 132-144. [CrossRef]

56. Frommer, B. Climate change and the resilient society: Utopia or realistic option for German regions? Nat. Hazards 2012, 67, 99-115. [CrossRef]

57. Virji, H.; Padgham, J.; Seipt, C. Capacity building to support knowledge systems for resilient development—approaches, actions, and needs. Curr. Opin. Environ. Sustain. 2012, 4, 115-121. [CrossRef]

58. Ortiz-De-Mandojana, N.; Bansal, P. The long-term benefits of organizational resilience through sustainable business practices Strat. Manag. J. 2016, 37, 1615-1631. [CrossRef]

59. Serafeim, G.; Rischbieth, A.M.; Koh, H.K. Sustainability, Business, and Health. JAMA 2020, 324, 147-148. [CrossRef] [PubMed]

60. Cyert, R.M.; DeGroot, M. Towards a Control Theory of the Firm. In Organizing Industrial Development; De Gruyter: Berlin, Germany, 1986. [CrossRef]

61. Kock, C.J.; Santaló, J.; Diestre, L. Corporate Governance and the Environment: What Type of Governance Creates Greener Companies? J. Manag. Stud. 2011, 49, 492-514. [CrossRef]

62. Demers, E.; Hendrikse, J.; Joos, P.; Lev, B.I. ESG Didn't Immunize Stocks Against the Covid-19 Market Crash. SSRN Electron. J. 2020. [CrossRef]

63. Ferriani, F.; Natoli, F. ESG risks in times of Covid-19. Appl. Econ. Lett. 2020, 1-5. [CrossRef]

64. Marchese, D.; Reynolds, E.; Bates, M.E.; Morgan, H.; Clark, S.S.; Linkov, I. Resilience and sustainability: Similarities and differences in environmental management applications. Sci. Total. Environ. 2018, 613, 1275-1283. [CrossRef]

65. Bocchini, P.; Frangopol, D.M.; Ummenhofer, T.; Zinke, T. Resilience and Sustainability of Civil Infrastructure: Toward a Unified Approach. J. Infrastruct. Syst. 2014, 20, 04014004. [CrossRef]

66. Fiksel, J. Sustainability and resilience: Toward a systems approach. Sustain. Sci. Pr. Policy 2006, 2, 14-21. [CrossRef]

67. Derissen, S.; Quaas, M.; Baumgärtner, S. The Relationship Between Resilience and Sustainable Development of Eco-Logical-Economic Systems (No. 146); Working Paper Series in Economics: Lower Saxony, Germany, 2009.

68. Winnard, J.; Adcroft, A.; Lee, J.; Skipp, D. Surviving or flourishing? Integrating business resilience and sustainability. J. Strat. Manag. 2014, 7, 303-315. [CrossRef]

69. Winnard, J.; Lee, J.; Skipp, D. Putting resilient sustainability into strategy decisions-case studies. Manag. Decis. 2018, 56, 1598-1612. [CrossRef]

70. Souza, A.A.A.; Alves, M.F.R.; Macini, N.; Cezarino, L.O.; Liboni, L.B. Resilience for sustainability as an eco-capability. Int. J. Clim. Chang. Strat. Manag. 2017, 9, 581-599. [CrossRef]

71. Dahles, H. The sustainability of small business resilience. The local tourism industry of Yogyakarta, Indonesia, a decade after the crisis. In Tourism, Resilience and Sustainability. Adapting to Social, Political and Eco-Nomic Change; Lew, A.A., Cheer, J., Eds.; Routledge: London, UK, 2018; pp. 150-163. 
72. Anderies, J.M.; Folke, C.; Walker, B.; Ostrom, E. Aligning Key Concepts for Global Change Policy: Robustness, Resilience, and Sustainability. Ecol. Soc. 2013, 18. [CrossRef]

73. Pal, R.; Westerlind, R.; Torstensson, H. Exploring the resilience development process by implementing the crisis strategic planning framework: A Swedish textile SME perspective. Int. J. Decis. Sci. Risk Manag. 2013, 5, 1-34. [CrossRef]

74. Parker, H.; Ameen, K. The role of resilience capabilities in shaping how firms respond to disruptions. J. Bus. Res. 2018, 88, 535-541. [CrossRef]

75. Linnenluecke, M.; Griffiths, A. Beyond Adaptation: Resilience for Business in Light of Climate Change and Weather Extremes. Bus. Soc. 2010, 49, 477-511. [CrossRef]

76. Sahebjamnia, N.; Torabi, S.A.; Mansouri, S.A. Building organizational resilience in the face of multiple disruptions. Int. J. Prod. Econ. 2018, 197, 63-83. [CrossRef]

77. Hamel, G.; Valikangas, L. The Quest for Resilience. Harv. Bus. Rev. 2003, 81, 52-63.

78. Hagen, B.; Zucchella, A.; Ghauri, P.N. From fragile to agile: Marketing as a key driver of entrepreneurial interna-tionalization. Int. Mark. Rev. 2019, 36, 260-288. [CrossRef]

79. Shekarian, M.; Nooraie, S.V.R.; Parast, M.M. An examination of the impact of flexibility and agility on mitigating supply chain disruptions. Int. J. Prod. Econ. 2020, 220, 107438. [CrossRef]

80. Mandal, S.; Sarathy, R.; Korasiga, V.R.; Bhattacharya, S.; Dastidar, S.G. Achieving supply chain resilience. Int. J. Disaster Resil. Built Environ. 2016, 7, 544-562. [CrossRef]

81. Vidmar, M.; Rosiello, A.; Golra, O. Resilience of New Space Firms in the United Kingdom During the Early Stages of COVID-19 Crisis: The Case for Strategic Agility. New Space 2020, 8, 172-178. [CrossRef]

82. Sabahi, S.; Parast, M.M. Firm innovation and supply chain resilience: A dynamic capability perspective. Int. J. Logist. Res. Appl. 2020, 23, 254-269. [CrossRef]

83. Teece, D.; Peteraf, M.; Leih, S. Dynamic Capabilities and Organizational Agility: Risk, Uncertainty, and Strategy in the Innovation Economy. Calif. Manag. Rev. 2016, 58, 13-35. [CrossRef]

84. Shekarian, M.; Parast, M.M. An Integrative approach to supply chain disruption risk and resilience management: A literature review. Int. J. Logist. Res. Appl. 2020, 1-29. [CrossRef]

85. Shams, R.; Vrontis, D.; Belyaeva, Z.; Ferraris, A.; Czinkota, M.R. Strategic agility in international business: A con-ceptual framework for "agile" multinationals. J. Int. Manag. 2020, 100737. [CrossRef]

86. Dubey, R.; Altay, N.; Gunasekaran, A.; Blome, C.; Papadopoulos, T.; Childe, S.J. Supply chain agility, adaptability and alignment. Int. J. Oper. Prod. Manag. 2018, 38, 129-148. [CrossRef]

87. Herbane, B. Rethinking organizational resilience and strategic renewal in SMEs. Entrep. Reg. Dev. 2019, 31, 476-495. [CrossRef]

88. Gligor, D.; Gligor, N.; Holcomb, M.; Bozkurt, S. Distinguishing between the concepts of supply chain agility and resilience. Int. J. Logist. Manag. 2019, 30, 467-487. [CrossRef]

89. Worley, C.G.; Williams, T.D.; Lawler, E.E., III. The Agility Factor: Building Adaptable Organizations for Superior Performance; John Wiley \& Sons: Hoboken, NJ, USA, 2014.

90. Parviainen, P.; Tihinen, M.; Kääriäinen, J.; Teppola, S. Tackling the digitalization challenge: How to benefit from digitalization in practice. Int. J. Inf. Syst. Proj. Manag. 2017, 5, 63-77.

91. Cherrington, M.; Lu, Z.J.; Xu, Q.; Airehrour, D.; Madanian, S.; Dyrkacz, A. Deep learning decision support for sus-tainable asset management. In Advances in Asset Management and Condition Monitoring; Springer: Cham, Germany, 2020; pp. 537-547.

92. Porter, M.E.; Heppelmann, J.E. How smart, connected products are transforming companies. Harv. Bus. Rev. 2015, 93, 96-114.

93. Hämäläinen, E.; Inkinen, T. Industrial applications of big data in disruptive innovations supporting environmental reporting. $J$. Ind. Inf. Integr. 2019, 16, 100105. [CrossRef]

94. Moşteanu, N.R.; Faccia, A.; Cavaliere, L.P.L. Digitalization and green economy-changes of business per-spectives. In Proceedings of the 2020 4th International Conference on Cloud and Big Data Computing, 26-28 August 2020; pp. 108-112.

95. Zhou, J.; Li, P.; Zhou, Y.; Wang, B.; Zang, J.; Meng, L. Toward New-Generation Intelligent Manufacturing. Engineering 2018, 4 , 11-20. [CrossRef]

96. Braña, F.-J. A fourth industrial revolution? Digital transformation, labor and work organization: A view from Spain. Econ. Politi Ind. 2019, 46, 415-430. [CrossRef]

97. Goldman, S.L.; Nagel, R.N.; Preiss, K. Agile Competitors and Virtual Organizations: Strategies for Enriching the Customer; Van Nostrand Reinhold: New York, NY, USA, 1995.

98. Ashrafi, A.; Ravasan, A.Z.; Trkman, P.; Afshari, S. The role of business analytics capabilities in bolstering firms' agility and performance. Int. J. Inf. Manag. 2019, 47, 1-15. [CrossRef]

99. Ungerman, O.; Dedkova, J.; Gurinova, K. The Impact of Marketing Innovation on the Competitiveness of Enterprises in the Context of Industry 4.0. J. Compet. 2018, 10, 132-148. [CrossRef]

100. Rolandsson, B.; Dølvik, J.E.; Hedenus, A.; Steen, J.R.; Ilsøe, A.; Larsen, T.P.; Hjelm, E. Digitalization in Nordic Manufacturing: Some Case-Study Illustrations; Nordic Future of Work Project 2017-2020: Working Paper; Fafo: Oslo, Norway, 2019.

101. Marinai, L.; David, R.; Di Sarra, A.; Escorcia, A.; Akhtar, M.M.J.; Al-Jefri, A.; Al-Hendasi, A. Digital Transformation of Production Governance and Assurance Process for Improving Production Efficiency. In Proceedings of the Abu Dhabi International Petroleum Exhibition \& Conference; Society of Petroleum Engineers (SPE), Abu Dhabi, United Arab Emirates, 9 November 2020. 
102. Niemimaa, M.; Järveläinen, J.; Heikkilä, M.; Heikkilä, J. Business continuity of business models: Evaluating the re-silience of business models for contingencies. Int. J. Inf. Manag. 2019, 49, 208-216. [CrossRef]

103. Felsberger, A.; Qaiser, F.H.; Choudhary, A.; Reiner, G. The impact of Industry 4.0 on the reconciliation of dynamic capabilities: Evidence from the European manufacturing industries. Prod. Plan. Control. 2020, 1-24. [CrossRef]

104. Yadav, G.; Kumar, A.; Luthra, S.; Garza-Reyes, J.A.; Kumar, V.; Batista, L. A framework to achieve sustainability in manufacturing organisations of developing economies using industry 4.0 technologies' enablers. Comput. Ind. 2020, 122, 103280. [CrossRef]

105. Balogun, A.-L.; Marks, D.; Sharma, R.; Shekhar, H.; Balmes, C.; Maheng, D.; Arshad, A.; Salehi, P. Assessing the Potentials of Digitalization as a Tool for Climate Change Adaptation and Sustainable Development in Urban Centres. Sustain. Cities Soc. 2020, 53, 101888. [CrossRef]

106. Ludbrook, F.; Michalikova, K.F.; Musova, Z.; Suler, P. Business models for sustainable innovation in industry 4.0: Smart manufacturing processes, digitalization of production systems, and data-driven decision making. J. Self Gov. Manag. Econ. 2019, 7, 21-26.

107. Chauhan, C.; Sharma, A.; Singh, A. A SAP-LAP linkages framework for integrating Industry 4.0 and circular economy. Benchmarking Int. J. 2019. [CrossRef]

108. Wilbanks, T.J.; Romero Lankao, P.; Bao, M.; Berkhout, F.; Cairncross, S.; Ceron, J.-P.; Kapshe, M.; Muir-Wood, R.; Zapata-Marti, R. Industry, settlement and society. In Climate Change 2007: Impacts, Adaptation and Vulnerability: Contribution of Working Group II to the Fourth Assessment Report of the Intergovernmental Panel on Climate Change; Parry, M.L., Canziani, O.F., Palutikof, J.P., van der Linden, P.J., Hanson, C.E., Eds.; Cambridge University Press: Cambridge, UK, 2007; pp. 357-390.

109. Gersick, C.J.G. Revolutionary change theories: A multilevel exploration of the punctuated equilibrium paradigm. Acad. Manag. Rev. 1991, 16, 10-36. [CrossRef]

110. Patsavellas, J.; Salonitis, K. The Carbon Footprint of Manufacturing Digitalization: Critical literature review and future research agenda. Procedia Cirp 2019, 81, 1354-1359. [CrossRef]

111. Hilty, L.M.; Bieser, J.C.T. Opportunities and Risks of Digitalization for Climate Protection in Switzerland; Universityof Zur-ich: Zurich, Switzerland, 2017. [CrossRef]

112. Lewin, A.Y.; Weigelt, C.D.; Emery, J.D. Adaptation and selection in strategy and change. In Handbook of Organizational Change and Innovation; Poole, M.S., van de Ven, A.H., Eds.; Oxford University Press: Oxford, UK, 2004; pp. 108-160.

113. Lengnick-Hall, C.A.; Beck, T.E. Adaptive fit versus robust transformation: How organizations respond to envi-ronmental change. J. Manag. 2005, 31, 738-757. [CrossRef]

114. Tàpies, J.; Moya, M.F. Values and longevity in family business: Evidence from a cross-cultural analysis. J. Fam. Bus. Manag. 2012, 2, 130-146. [CrossRef] 



\title{
The Impact of Direct and Indirect COVID-19 Related Demand Shocks on Sectoral $\mathrm{CO}_{2}$ Emissions: Evidence from Major Asia Pacific Countries
}

\author{
Muhammad Jawad Sajid ${ }^{1, *(\mathbb{D})}$ and Ernesto D. R. Santibanez Gonzalez ${ }^{2}$ \\ 1 School of Engineering Management, Xuzhou University of Technology, Xuzhou 221000, China \\ 2 Department of Industrial Engineering, CES4.0, Faculty of Engineering, University of Talca, Los Niches Km 1, \\ Curicó 74104, Chile; santibanez.ernesto@gmail.com \\ * Correspondence: jawad.jaws@outlook.com
}

check for updates

Citation: Sajid, M.J.; Gonzalez, E.D.R.S. The Impact of Direct and Indirect COVID-19 Related Demand Shocks on Sectoral $\mathrm{CO}_{2}$ Emissions: Evidence from Major Asia Pacific Countries. Sustainability 2021, 13, 9312. https://doi.org/ $10.3390 /$ su13169312

Academic Editors: Mohammad Aslam Khan Khalil and Idiano D'Adamo

Received: 30 July 2021

Accepted: 18 August 2021

Published: 19 August 2021

Publisher's Note: MDPI stays neutral with regard to jurisdictional claims in published maps and institutional affiliations.

Copyright: (c) 2021 by the authors. Licensee MDPI, Basel, Switzerland. This article is an open access article distributed under the terms and conditions of the Creative Commons Attribution (CC BY) license (https:// creativecommons.org/licenses/by/ $4.0 /)$.

\begin{abstract}
COVID-19's demand shocks have a significant impact on global $\mathrm{CO}_{2}$ emissions. However, few studies have estimated the impact of COVID-19's direct and indirect demand shocks on sectoral $\mathrm{CO}_{2}$ emissions and linkages. This study's goal is to estimate the impact of COVID-19's direct and indirect demand shocks on the $\mathrm{CO}_{2}$ emissions of the Asia-Pacific countries of Bangladesh, China, India, Indonesia, and Pakistan (BCIIP). The study, based on the Asian Development Bank's COVID19 economic impact scenarios, estimated the impact of direct and indirect demand shocks on $\mathrm{CO}_{2}$ releases using input-output and hypothetical extraction methods. In the no COVID-19 scenario, China emitted the most $\mathrm{CO}_{2}$ (11 billion tons (Bt)), followed by India (2 Bt), Indonesia (0.5 Bt), Pakistan $(0.2 \mathrm{Bt})$, and Bangladesh $(0.08 \mathrm{Bt})$. For BCIIP nations, total demand shocks forced a $1-2 \%$ reduction in $\mathrm{CO}_{2}$ emissions under a worst-case scenario. Given BCIIP's current economic recovery, a best or moderate scenario with a negative impact of less than $1 \%$ is more likely in coming years. Direct demand shocks, with a negative $85-63 \%$ share, caused most of the $\mathrm{CO}_{2}$ emissions decrease. The downstream indirect demand had only a $15-37 \%$ contribution to $\mathrm{CO}_{2}$ emissions reduction. Our study also discusses policy implications.
\end{abstract}

Keywords: Asia Pacific; COVID-19; $\mathrm{CO}_{2}$ emission; demand shock; hypothetical extraction method; input-output model; sectoral linkage; sustainability

\section{Introduction}

On 11 March 2020, the WHO declared the novel COVID-19 a pandemic [1]. Almost a year later, the WHO reports that more than 2.5 million people have died as a result of COVID-19, and approximately 114 million have been diagnosed [2]. Apart from the human cost, the COVID-19 pandemic has a massive economic cost, as lockdowns have halted production and logistics operations, as well as affected demand and supply of various products [3]. Obviously, the COVID-19 pandemic has a negative impact on global $\mathrm{CO}_{2}$ emissions [4]. COVID-19-related lockdowns reduced global $\mathrm{CO}_{2}$ emissions by nearly $7.9 \%$, with an annual reduction of 4-7\% expected during the pandemic [5].

Estimating the impact of COVID-19's direct and indirect demand shocks on $\mathrm{CO}_{2}$ emissions can help us understand how much of a target sector's direct demand and how much indirect demand for the target sector's products and services are responsible for COVID-19-related potential $\mathrm{CO}_{2}$ reductions. Given that emissions typically rise following a crisis [6], our estimate can assist policymakers in developing smart demand-side long-term fiscal and monetary policies by accounting for the direct and indirect effects of demand shocks on $\mathrm{CO}_{2}$ emissions from various industries. Several sectors have underlined the positive relationship between sustainability and resilience $[7,8]$. Thus, extending current emission reduction patterns and avoiding the anticipated increase in $\mathrm{CO}_{2}$ emissions as a result of the pandemic may aid in achieving long-term resilience, even after the $\mathrm{CO}_{2}$ 
reducing effects of COVID-19-related demand shocks fade in the long run and in the aftermath of the COVID-19 disaster, become more resilient by seizing the opportunity to build a genuine, sustainable resurrection (in terms of $\mathrm{CO}_{2}$ emissions reductions) [9].

A great deal of research has been done on the effects of COVID-19 demand shocks on the environment and $\mathrm{CO}_{2}$ emissions. Conversely, intermediate industrial environmental and carbon linkages have been extensively researched in order to estimate the direct and indirect sources of sectoral environmental and carbon impacts. The decomposition of COVID-19-related total demand shocks into direct and indirect demand shocks, on the other hand, has been rarely reported in the related literature. Domestic intermediate sectoral supply chain disruptions caused by demand shocks in intermediate sectoral linkages, in particular, have been rarely reported in the related literature. The goal of this study is to close these critical research gaps. This study accomplishes these goals by first categorizing intermediate sectoral linkages based on their direct and indirect carbon impact on the final demand of a specific sector. Following that, the study calculates the novel COVID-19related direct and indirect sectoral demand shocks' impacts on carbon emissions in key Asia-Pacific economies such as Bangladesh, China, India, Indonesia, and Pakistan (BCIIP). In this case, a target sector is isolated from the rest of the economy using the hypothetical extraction method (HEM) in order to understand the role of COVID-19-related direct and indirect demand shocks on total and sectoral-level $\mathrm{CO}_{2}$ emissions in the BCIIP countries.

The rest of the article is organized as follows: Section 2 delves into the literature on industrial carbon linkages, general COVID-19 demand shock literature, and COVID-19 CO emissions impacts. Section 3 introduces the material sources and explains the methodology of our research. Section 4 summarizes the findings. Section 5 discusses the findings in light of previous findings, presents policy implications, and discusses limitations and future research. Finally, Section 6 presents the conclusions of our study.

\section{Literature Review}

The literature review section presents some of the relevant literature on COVID19 's economic impacts. Furthermore, this section presents the most recent literature on COVID-19's impact on $\mathrm{CO}_{2}$ emissions. In addition, this section depicts the literature on the environmental and $\mathrm{CO}_{2}$ linkages conducted using the well-known hypothetical extraction method (HEM). The section does not go over the literature on the classical multiplier method, which is used in some studies to estimate intermediate sectoral linkages. This is because, when compared to HEM, the classical method has been shown in the literature to be a subpar approach.

\subsection{COVID-19 Related Supply Chain and Production Activity Distributions}

Because of the current COVID-19 pandemic, governments have been forced to restrict not only people's movement but also economic activity [10]. This has resulted in negative demand shocks, which have had a negative impact on all industrial operations. With the negative economic impact of COVID-19-related human activity restrictions in mind, many studies have concentrated on supply chain and production activity distributions. Chowdhury et al., for example, using a multiple-case-study approach, evaluated the effects of the COVID-19 pandemic on the food and beverage industry. The study estimated both the short-term and medium-to-long-term effects of the pandemic, as well as solutions for mitigating those effects [11]. Marimuthu et al. used a fuzzy-complex proportional assessment technique to quantify the impact of COVID-19 on mining activities in India [12]. Chen et al. calculated the impact of COVID-19-related consumer demand declines on the service industry. Based on a statistical survey of 940 firms in Hangzhou City, China, the authors created a risk factor analysis of business continuity management [13]. Cui et al. used a multi-sectoral computable general equilibrium model to examine the demand and supply-side effects of the COVID-19 pandemic on China's transportation sectors [14]. Aside from specific sectors, several studies have also focused on the impact of COVID-19 related disruptions on entire supply chains. For instance, Shaheed et al. created a mathematical 
model to manage COVID-19-related supply chain interruptions in a three-stage supply chain network comprising suppliers, manufacturers, and retailers [15]. Karmaker et al. explored the drivers of a sustainable supply chain to address COVID-19-related supply chain disruptions in such a pandemic in Bangladesh [16]. Chowdhury et al. conducted a comprehensive review of the 74 relevant articles on COVID-19 related supply chain disruptions. As per their findings, the main methodologies used in these 74 articles are as follows; 31 articles based on author's opinions; 27 articles focused on quantitative methodologies including simulation modelling, game-theoretical modelling, mixed-integer linear modelling, non-linear modelling, stochastic optimization, integrated mathematical and simulation model, principal component analysis and cluster analysis, and one study applied stepwise weight assessment ratio analysis; and 10 articles were focused on literature review.

\subsection{COVID-19's Environmental and $\mathrm{CO}_{2}$ Emissions Impact}

Furthermore, numerous studies have been conducted on the various environmental aspects of COVID-19. The effects of COVID-19 on air pollution [17-21], the circular economy [1,9,22], sustainability [4,23,24], waste management [25,26], water use [27], renewable and green energy [28,29], climate change [30], transportation [31], and public awareness [32] have been extensively studied. Several studies, in particular, have estimated the COVID-19 pandemic's impact on $\mathrm{CO}_{2}$ emission reductions. Turner et al., for example, estimated the observed impact of the COVID-19 lockdown on six counties in the United States' "San Francisco Bay" region [33]. Based on satellite observations, Zheng et al. calculated China's $\mathrm{CO}_{2}$ emissions during the COVID-19 pandemic [34]. Han et al. estimated the effect of COVID-19 on China's $\mathrm{CO}_{2}$ emissions using national economic data [35]. Using real-time activity data, Liu et al. estimated the impact of COVID-19 on global $\mathrm{CO}_{2}$ emissions from various sectors [36]. Quéré et al. estimated the temporary daily reductions in $\mathrm{CO}_{2}$ emissions caused by the COVID-19 pandemic using government policy and activity data [37]. Shan et al. estimated the effect of COVID-19 on global $\mathrm{CO}_{2}$ emissions and supply chains using a multi-regional input-output model [38]. Quéré et al. forecasted post-COVID-19 $\mathrm{CO}_{2}$ emissions from fossil fuels [6].

\subsection{Sectoral Environmental and Carbon Linkages}

Both classical and more recent HEM have been widely used to estimate intermediate sectoral environmental linkages including water, energy, air pollutant, and $\mathrm{CO}_{2}$ linkages within and across economies. The HEM is generally regarded as the superior option because it allows us to estimate the relative magnitude of an industry's (sector's) economic (environmental) impact by removing it from a specific economy [39]. The modified hypothetical extraction method (MHEM) [40] is the most commonly used type of HEM at the moment. Blanco et al. assessed cross-temporal direct and indirect water yield in the Spanish region of Castile and León using the MHEM modeling technique [41]. Deng et al. calculated China's intermediate sectoral water trade (linkages) using the HEM approach [42]. Duarte et al. estimated the sectoral water linkages in Spain using the MHEM [40]. Guerra and Sancho used the HEM to calculate the sectoral energy links in Spain [43]. He et al. calculated the air pollutant links in China using the MHEM technique [44]. Using the MHEM technique, Wang et al. quantified the air pollutant sectoral linkages in China [45].

The MHEM can assist us in estimating an economy's various net carbon linkages [46]. Several studies have employed the MHEM approach to estimate the sectoral $\mathrm{CO}_{2}$ linkages for different economies and sectors. For example, Sajid et al. estimated the sectoral $\mathrm{CO}_{2}$ linkages of India from different types of energy and non-energy uses using the original, Cella's [47], and modified HEM [39]. Sajid et al. used the MHEM to calculate the $\mathrm{CO}_{2}$ linkages of the transport sectors of the EU's top carbon emitting nations [46]. Using the MHEM, Bai et al. calculated China's industrial $\mathrm{CO}_{2}$ linkages [48]. Sajid et al. estimated Turkey's demand and supply-driven $\mathrm{CO}_{2}$ linkages using the original, modified, and hybrid HEM [49]. Zhao et al. estimated China's inter-regional sectoral $\mathrm{CO}_{2}$ linkages using the HEM [50]. Using the MHEM, Sajid et al. embedded Chinese industrial consumption- 
induced $\mathrm{CO}_{2}$ emissions into the household final demand [51]. Ali estimated Italy's sectoral $\mathrm{CO}_{2}$ linkages using the classical multiplier, Cella, and the original HEM approaches [52]. Sajid estimated the drivers of Chinese households' induced intermediate sectoral $\mathrm{CO}_{2}$ consumption emissions using MHEM in conjunction with structural decomposition and regional sensitivity analyses [53]. Sun et al. estimated China's weighted backward and forward linkages using the "absolute weighted measurement" technique [54]. Sajid et al. calculated the mining sector carbon linkages of the world's ten largest economies [55]. Sajid estimated Pakistan's $\mathrm{CO}_{2}$ linkages and the impact of final demand on sectoral $\mathrm{CO}_{2}$ linkages using the MHEM and hypothetical extraction of final demand (HEOFD) methods [56].

\subsection{Research Gaps and Significance}

Despite the fact that significant work has been done in general on COVID-19-related impacts on supply chain and production activities, as well as $\mathrm{CO}_{2}$ emissions, much research has focused on sectoral environmental and carbon linkages. However, the following significant research gaps remain in the related literature. (1) The literature on COVID19-related general economic activities and $\mathrm{CO}_{2}$ emission reductions does not usually divide total demand shocks into direct and indirect demand shocks. (2) The literature on the impact of COVID-19 on economic activities in general, and $\mathrm{CO}_{2}$ reductions in particular, does not typically classify the role of domestic intermediate sectoral supply chain disruptions based on the influence of direct and indirect demand shocks. (3) Traditionally, the literature on sectoral environmental and carbon linkages does not take into account the effects of direct and indirect demand shocks on intermediate sectoral linkages. (4) The case of the majority of BCIIP countries, with the exception of China, under COVID-19-related $\mathrm{CO}_{2}$ reductions has largely gone unstudied in the related literature.

This study addresses the aforementioned research gaps in the following ways. First, our study disaggregates COVID-19-demand-shock-related impacts into direct and indirect demand shock impacts within an economy, which are rarely investigated in the related literature (A sector's direct $\mathrm{CO}_{2}$ emissions can be further subdivided into internal and forward (downstream) emissions. Current assessments of COVID-19's impact on $\mathrm{CO}_{2}$ emissions overlook a critical factor: the extent to which indirect demand from other sectors can help a sector reduce its $\mathrm{CO}_{2}$ emissions, particularly when their demand is influenced by unexpected demand shocks such as the ongoing disaster of COVID-19). Second, the study categorizes intermediate sectoral supply chain disruptions based on the direct and indirect effects of sectoral final demand. These are not normally classified in both the general literature on sectoral environmental linkages and the COVID-19 economic and environmental impacts literature (Where a target sector's internal linkages are driven by their own demand, which means their value is directly dependent on their own final demand value or demand shocks. However, forward, or downstream linkages, are more complicated, as they are not driven by a sector's own demand but rather by the demand of the sector's downstream importing sectors. As a result, these forward $\mathrm{CO}_{2}$ linkages depict the effect of indirect industrial demand on a sector's $\mathrm{CO}_{2}$ emissions). Third, this study modifies the MHEM approach and introduces the impact of COVID-19-related direct and indirect demand shocks on intermediate sectoral linkages. Fourth, this study estimates the impact of COVID-19's direct and indirect demand shocks on $\mathrm{CO}_{2}$ emissions in the major developing Asian economies of Bangladesh, China, India, Indonesia, and Pakistan (BCIIP) using the single regional input-output model (SRIO) and the Asian Development Bank's (ADB) COVID-19 economic impact scenarios. COVID-19 has had the greatest impact on developing economies [4]. BCIIP countries are interesting cases not only because they are among the most important developing economies, but also because they are among the most populated, with approximately $45 \%$ of the world's population residing in BCIIP [57], and from an environmental standpoint, they are among the most polluted nations [58].

The estimation of COVID-19 pandemic effects on the world's most populated and polluted developing region can not only help with this region's future carbon policy but also serve as a model for other countries. Second, by estimating the effects of demand 
shocks on inter- and intra-sectoral $\mathrm{CO}_{2}$ linkages, this study advances sectoral linkage estimation methods, particularly the HEM. Third, the study clarifies the concept of direct and indirect demand and demand shocks in the context of a country's domestic economy. Appendix A Table A1 lists the full names of the sectoral abbreviations used in our study.

\section{Materials and Methods}

\subsection{Materials}

The data on the potential economic impact of COVID-19 on the various primary sectors of BCIIP countries were derived from the ADB's "COVID-19 Economic Impact Assessment Template" [59]. The data, updated on 10 March 2020, contain the most comprehensive information on the expected economic impact of COVID-19 under various scenarios. As a result, the March version was used in this study to estimate COVID-19's direct and indirect demand shock impact on $\mathrm{CO}_{2}$ emissions in BCIIP nations. In this version, the potential economic and sector-specific demand shock impact of the COVID-19 outbreak is presented in relation to the length of travel restrictions and steep decline in domestic demand. The ADB presents feasible scenarios for the best-case scenario for two months, the moderate case scenario for three months, the worst-case scenario for six months, and the hypothetical worst-case scenario for six months plus a three-month outbreak. The input-output (IO) data required to estimate the intermediate effects of COVID-19-related economic shocks were obtained from the EORA MRIO database's national IO tables [60]. The most recent year, 2015, tables and related $\mathrm{CO}_{2}$ emissions accounts from EDGAR were used as a proxy for current $\mathrm{CO}_{2}$ emissions. In order to correspond with the ADB's sectoral classification, the national IO tables were aggregated as shown in Supplementary Tables S1-S5. Other recent research on developing economies' sectoral $\mathrm{CO}_{2}$ linkages has also preferred the use of the EORA MRIO database [56].

\subsection{Methods}

\subsubsection{Environmentally Extended Input-Output Model}

The Wasley W. Leontief input-output model [61] is commonly used as the foundation of the HEM method, which is used in this study to estimate sectoral $\mathrm{CO}_{2}$ linkages. The following is the basic equation for the environmentally extended input-output model.

$$
C^{N}=t^{N}\left(I-A^{N}\right)^{-1} D^{N}
$$

where $C^{N}$ denotes the $\mathrm{CO}_{2}$ emissions of a specific nation, $N$. The intensity of the country's sectoral carbon emissions is denoted by $t^{N}$. I shows the appropriate size identity matrix. The country's intermediate technology matrix is represented by $A^{N}$. The Leontief inverse matrix of the country $N$ is represented by $L^{N}=\left(I-A^{N}\right)$. And $D^{N}$ is the country's final demand for sectoral products and services. The $t^{N}$ is simply calculated by dividing country $N^{\prime} s$ total $\mathrm{CO}_{2}$ emissions by country $N^{\prime} s$. total output.

$$
t_{r}^{N}=\frac{C_{r}^{N}}{X_{r}^{N}}
$$

where $t_{r}^{N}$ represents the intensity of $\mathrm{CO}_{2}$ emissions from sector $r$ in the country N. $C_{r}^{N}$ represents the total carbon emissions of sector $r$ from the country $N$. And $X_{r}^{N}$ is the total output of the country's sector $r$.

\subsubsection{Decomposition of the National Economy into the Target and Other Sectors}

To estimate the sectoral carbon linkages, an economy should be divided into two groups, one representing the target sector $r$ and the other representing the remaining sectors $-r$. 


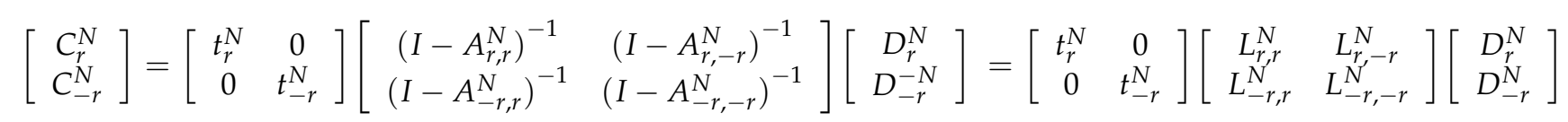

where $\left[\begin{array}{c}C_{r}^{N} \\ C_{-r}^{N}\end{array}\right]$ represents the total carbon emissions of the country $N^{\prime} s$ target sector $r$ and other sectors $-r .\left[\begin{array}{cc}t_{r}^{N} & 0 \\ 0 & t_{-r}^{N}\end{array}\right]$ displays the carbon emission intensity of the country's target and non-target sectors. $\left[\begin{array}{c}L_{r}^{N} \\ L_{-r}^{N}\end{array}\right]=\left[\begin{array}{cc}L_{r, r}^{N} & L_{r,-r}^{N} \\ L_{-r, r}^{N} & L_{-r,-r}^{N}\end{array}\right]$ depicts the Leontief inverse matrix for country $N^{\prime} s$ target and remaining sectors. And $\left[\begin{array}{c}D_{r}^{N} \\ D_{-r}^{-N}\end{array}\right]$ presents the final demand for the country's target and other sectors.

\subsubsection{Decomposition of Direct and Indirect Demand-Induced Emissions}

A sector's direct $\mathrm{CO}_{2}$ emissions are roughly equal to those induced by its internal and forward carbon links. Thus, direct $\mathrm{CO}_{2}$ emissions can be decomposed into intra-sectoral emissions resulting from direct final demand for a sector's products and services and the forward $\mathrm{CO}_{2}$ emissions embedded in the final demand of downstream purchasing sectors. After reclassifying a country's economy into target and non-target sectors, we can easily decompose the target sector's total direct emissions into internal emissions caused by the sector's own use, which is driven by final demand for its products or services and forward emissions caused by purchases by downstream importers of the sector's products or services, driven by the respective demands of various downstream importing sectors.

$$
\begin{gathered}
I C_{r}^{N}=t_{r}^{N}\left(I-A_{r, r}^{N}\right)^{-1} D_{r}^{N} \\
F C_{r}^{N}=t_{r}^{N} L_{r,-r}^{N} D_{-r}^{N} \\
C_{r}^{N}=I C_{r}^{N}+F C_{r}^{N}
\end{gathered}
$$

where $I C_{r}^{N}$ and $F C_{r}^{N}$ represent the country $N^{\prime} s$ internal and forward carbon linkages, respectively. $C_{r}^{N}$ represents the target sector's direct $\mathrm{CO}_{2}$ emissions. Forward carbon emissions can be further decomposed into sectoral destinations, i.e., the downstream indirect influencers of target sector $\mathrm{CO}_{2}$ emissions. Assume sector $d$ is one of the sectors in the group representing non-target sectors $-r$. The virtual carbon export from the target sector $r$ to the purchasing sector $d$ can then be represented by the following equation.

$$
F C_{r}^{N}=\sum_{d=0}^{n-1} F C_{r \rightarrow d}^{N}
$$

3.2.4. $\mathrm{CO}_{2}$ Emissions Estimations after Adjusting for Direct and Indirect Demand Shocks

The above-mentioned Equations (4) and (5) can be modified to present the new emissions after adjusting for the potential COVID-19-related sectoral demand shocks on sectoral carbon linkages under various scenarios.

$$
\begin{gathered}
\overline{I C_{r}^{N}}=t_{r}^{N}\left(I-A_{r, r}^{N}\right)^{-1} \overline{D_{r}^{N}} \\
\overline{F C_{r}^{N}}=t_{r}^{N} L_{r,-r}^{N} \overline{D_{-r}^{N}}
\end{gathered}
$$

where $\overline{I C_{r}^{N}}$ and $\overline{F C_{r}^{N}}$ represent the new internal and forward carbon linkages, respectively, after adjusting for negative demand shocks in the value of final demand. $\overline{D_{r}^{N}}$ and $\overline{D_{-r}^{N}}$ show the decrease in demand in the target and other sectors as a result of the COVID19-related lockdown and other measures. The ADB employs the "no-COVID baseline" 
scenario to estimate the relative decrease in demand in other COVID-19 impact scenarios. The authors used the ADB's COVID-19 related demand impact indicator for different scenarios to estimate the impact of decreases on the direct and indirect sectoral linkages of the BCIIP countries.

\subsubsection{Estimation of the Impact of Direct and Indirect Demand Shocks}

The total demand shock's impact on $\mathrm{CO}_{2}$ emissions can be expressed simply as the difference between the baseline no COVID-19 emissions and the emissions after adjusting for demand shocks under a specific scenario.

$$
\Delta C_{r}^{N}=\Delta I C_{r}^{N}+\Delta F C_{r}^{N}=F C_{r}^{N}-\overline{F C_{r}^{N}}=\left(I C_{r}^{N}-\overline{I C_{r}^{N}}\right)+\left(F C_{r}^{N}-\overline{F C_{r}^{N}}\right)
$$

The following equations can be used to estimate the percentage contribution of direct and indirect demand shocks to total direct $\mathrm{CO}_{2}$ reductions for a given scenario.

$$
\begin{gathered}
\% D D S_{r}=\frac{\Delta I C_{r}^{N}}{\Delta C_{r}^{N}} \\
\% I D S_{r}=\frac{\Delta F C_{r}^{N}}{\Delta C_{r}^{N}}
\end{gathered}
$$

where $\% D D S_{r}$ and $\% I D S_{r}$ represent the percentage contribution of direct and indirect demand shocks to total direct $\mathrm{CO}_{2}$ emissions reduction in a given scenario.

\section{Results}

\subsection{Direct $\mathrm{CO}_{2}$ Emissions under the Baseline (No COVID-19) Scenario}

Figure 1 depicts the direct sectoral $\mathrm{CO}_{2}$ emissions of the respective countries. As illustrated in Figure 1, China had the highest total $\mathrm{CO}_{2}$ emissions of all nations. It was followed by the countries of India, Indonesia, Pakistan, and Bangladesh. The MUC sector contributed the most to total national $\mathrm{CO}_{2}$ emissions in Bangladesh, China, India, Indonesia, and Pakistan, accounting for $61 \%$, 85\%, 82\%, 76\%, and 51\%, respectively. BTPS accounted for the second highest proportion of national $\mathrm{CO}_{2}$ emissions in Bangladesh, China, India, and Pakistan, accounting for 33\%, 7\%, 8\%, and 42\%, respectively. However, Indonesia's AMQ sector was the second highest emitter, accounting for nearly $10 \%$ of total national emissions.

\subsection{The Impact of Negative Total Demand Shocks on Country-Wide and Sectoral $\mathrm{CO}_{2}$ Releases}

Figure 2 depicts the effects of expected demand shocks on aggregate $\mathrm{CO}_{2}$ emissions by country under various scenarios. Appendix A Table A2 presents the effects of demand shocks on sectoral $\mathrm{CO}_{2}$ emissions under various scenarios. Apart from the worst-case scenario, no other scenario had a significant negative impact on $\mathrm{CO}_{2}$ emissions in Bangladesh, India, or Pakistan, as illustrated in Figure 2. However, when compared with the baseline scenario (no COVID-19), all other scenarios resulted in significant $\mathrm{CO}_{2}$ emission reductions for China and Indonesia. Whereas negative demand shocks had the greatest potential to reduce China's overall $\mathrm{CO}_{2}$ emissions in the worst-case and hypothetical worst-case scenarios, with an impact of approximately $-2 \%$. However, for Indonesia, the worst-case scenario with a negative demand shock of approximately $-1 \%$ had the greatest negative impact on $\mathrm{CO}_{2}$ emission reductions. When compared to the baseline scenario, negative demand shocks had the greatest impact on the BTPS and MUC sectors in Bangladesh and Pakistan. Under various scenarios, negative demand shocks had a significant impact on China and India's MUC sectors, as well as on Indonesia's MUC and HROS sectors. 


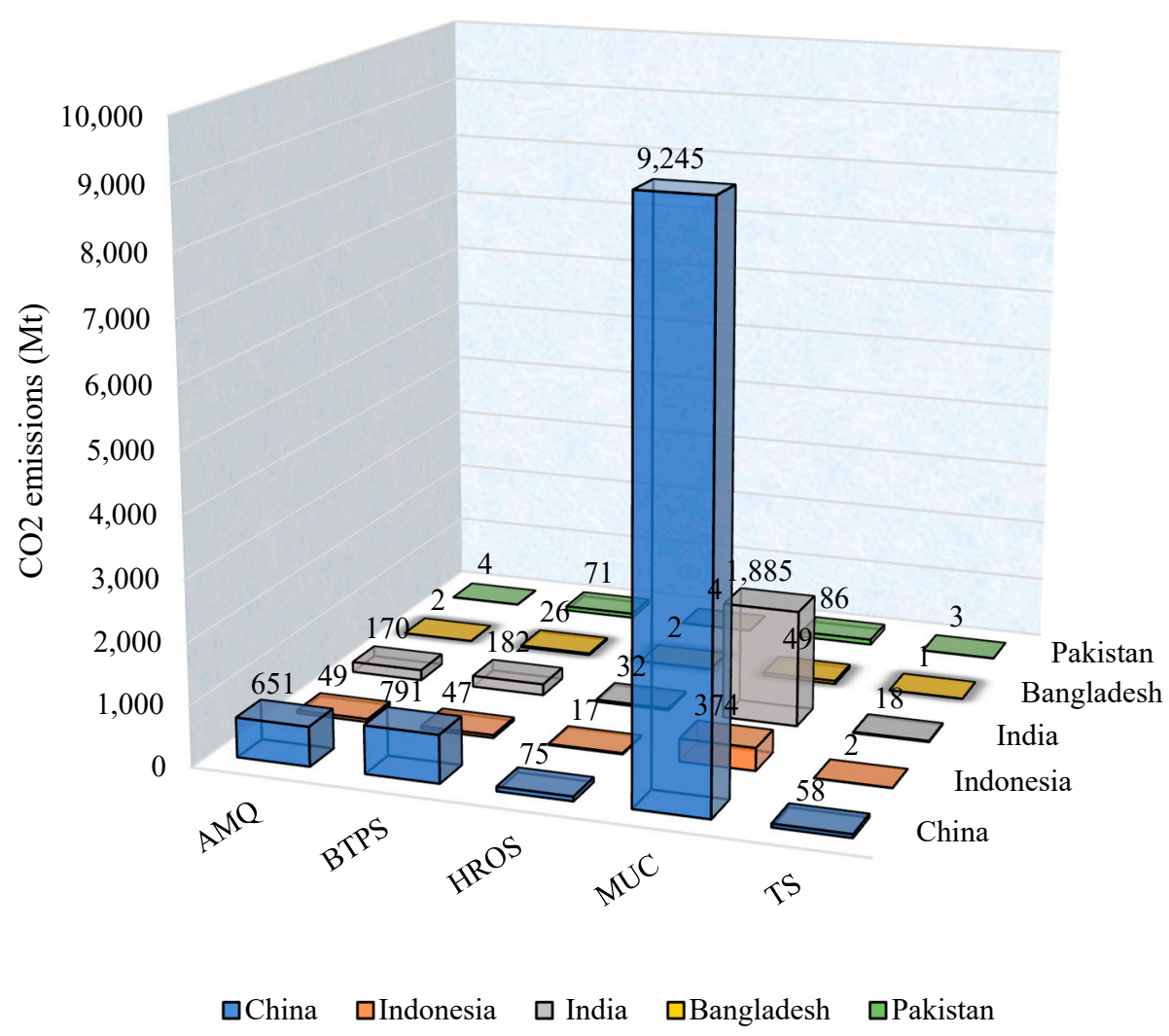

Figure 1. Direct $\mathrm{CO}_{2}$ emissions under the no COVID-19 baseline scenario. Source: Constructed by the authors.

\subsection{Decomposition of Impacts from Direct and Indirect Demand Shocks}

The impact of COVID-19 demand shocks on $\mathrm{CO}_{2}$ emissions can be further subdivided into direct and indirect demand shocks. The first type is driven by direct demand for a sector's products, while the second is driven by indirect demand, i.e., demand for a particular sector's downstream purchaser sector's products and services. Figure 3 represents the contribution of direct and indirect demand shocks to total $\mathrm{CO}_{2}$ emission reductions under various scenarios. The decomposed direct emissions are presented in the supplementary file. Figure 3 shows that direct demand shocks accounted for a large portion of the total $\mathrm{CO}_{2}$ reductions under various scenarios. For BCIIP countries, the impact of direct demand shocks on total reduction ranged from $85 \%$ to $63 \%$. Direct demand shocks with range values of $85-83 \%$ and $77-80 \%$, contributed the most to total $\mathrm{CO}_{2}$ reductions in Bangladesh and Pakistan, respectively. The contribution of indirect demand shocks to direct $\mathrm{CO}_{2}$ reductions ranged from $15 \%$ to $37 \%$. India and Indonesia had the greatest impact of indirect demand shocks on $\mathrm{CO}_{2}$ reductions, with range values of $31-37 \%$ and $28-41 \%$, respectively.

Figure 4 shows the sector-specific impact of direct and indirect demand shocks on total $\mathrm{CO}_{2}$ emissions reductions under various scenarios. As shown in Figure 5, the impact of direct demand shocks was greater than the impact of indirect demand shocks in the majority of sectors in BCIIP countries. However, in some sectors in different countries, the contribution of indirect demand shocks was generally greater than the contribution of direct demand shocks under different scenarios. The $\mathrm{CO}_{2}$ emissions reductions for AMQ (range $=49-83 \%$ ) from Bangladesh, TS (range $=47-63 \%$ ) from China, AMQ (range $=48-55 \%)$ and BTPS (range $=41-73 \%$ ) from Indonesia, and AMQ (range $=64-69 \%$ ) and TS (range $=50-75 \%$ ) from Pakistan, for example, were generally influenced more by indirect than direct demand shocks. 


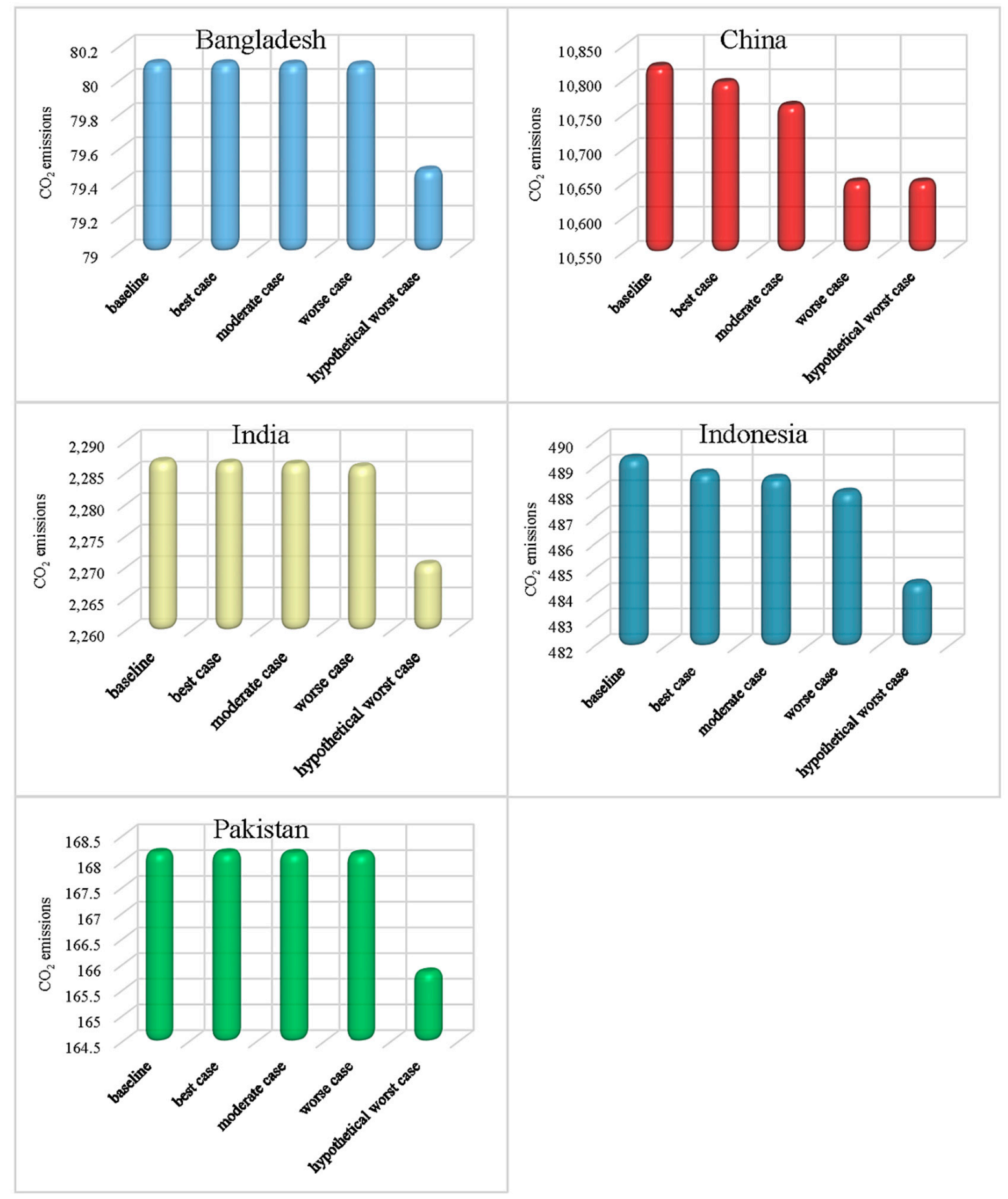

Figure 2. Country-wide aggregated emissions under different scenarios. Here, the base-line scenario presents $\mathrm{CO}_{2} \mathrm{emissions}$ under the assumption of no COVID-19 pandemic. Source: Constructed by the authors. 

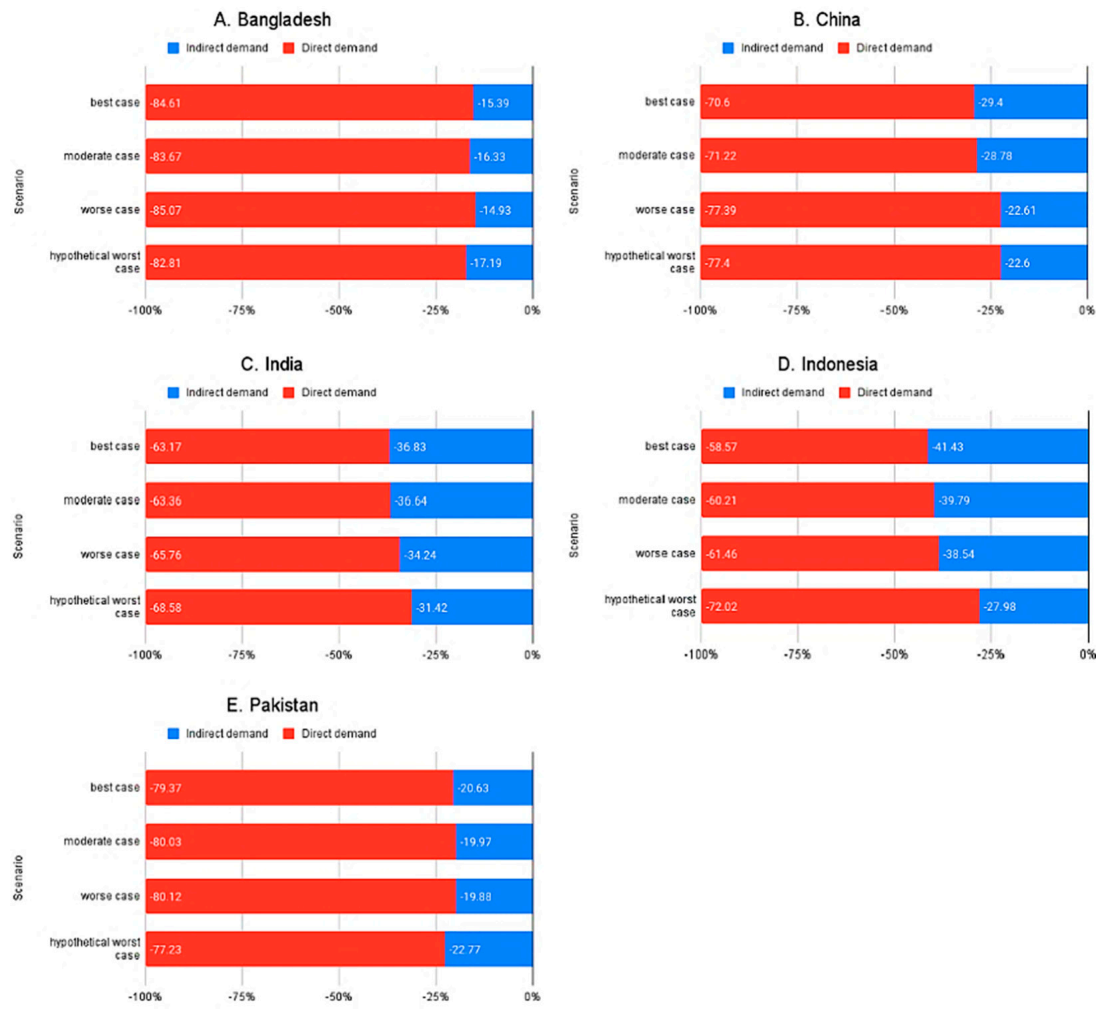

Figure 3. The contribution of direct and indirect demand shocks to $\mathrm{CO}_{2}$ emissions reduction under different scenarios. Source: Constructed by the authors.

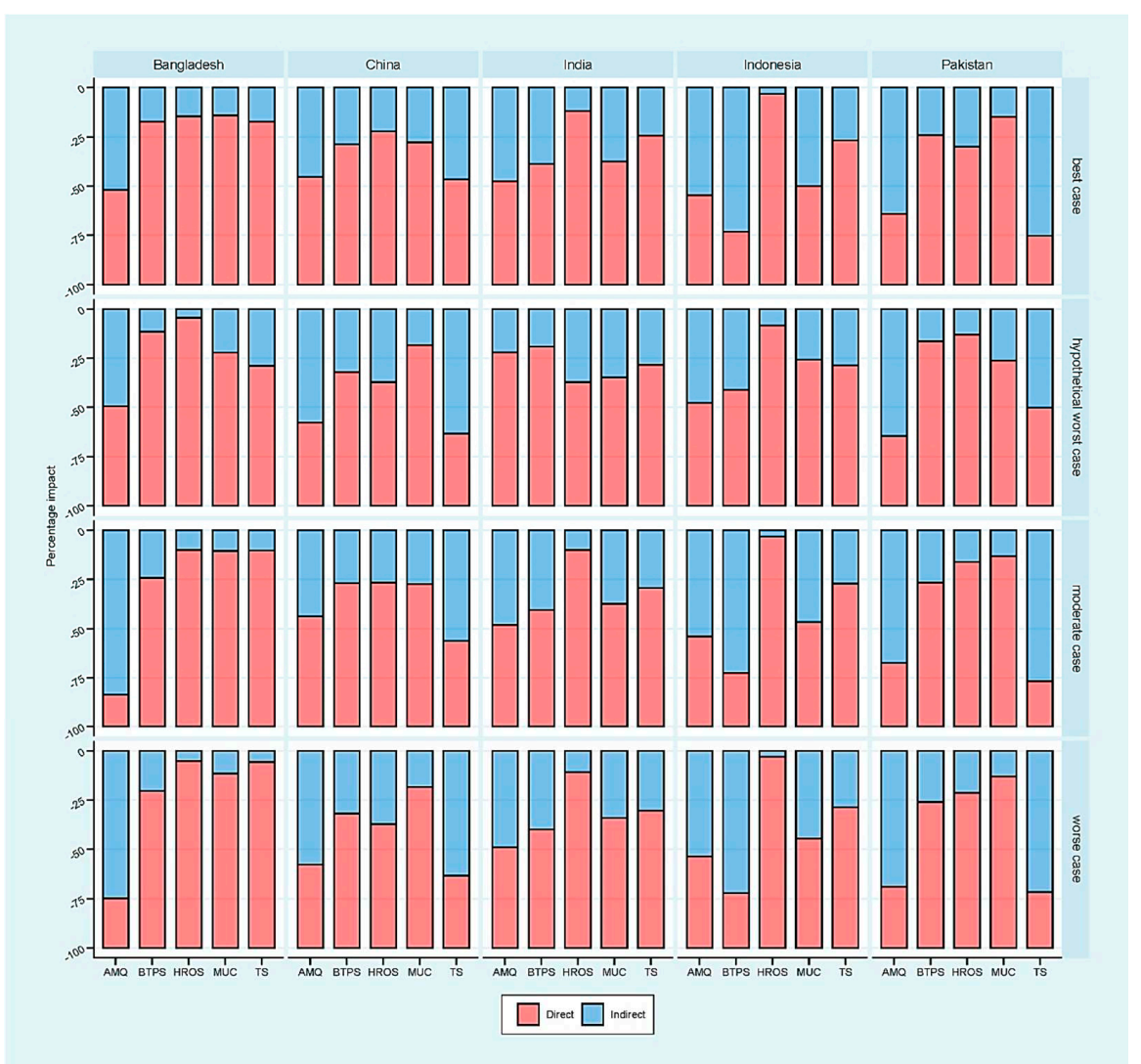

Figure 4. The sector-wide contribution of direct and indirect demand shocks to $\mathrm{CO}_{2}$ emissions reduction under different scenarios. Source: Constructed by the authors. 


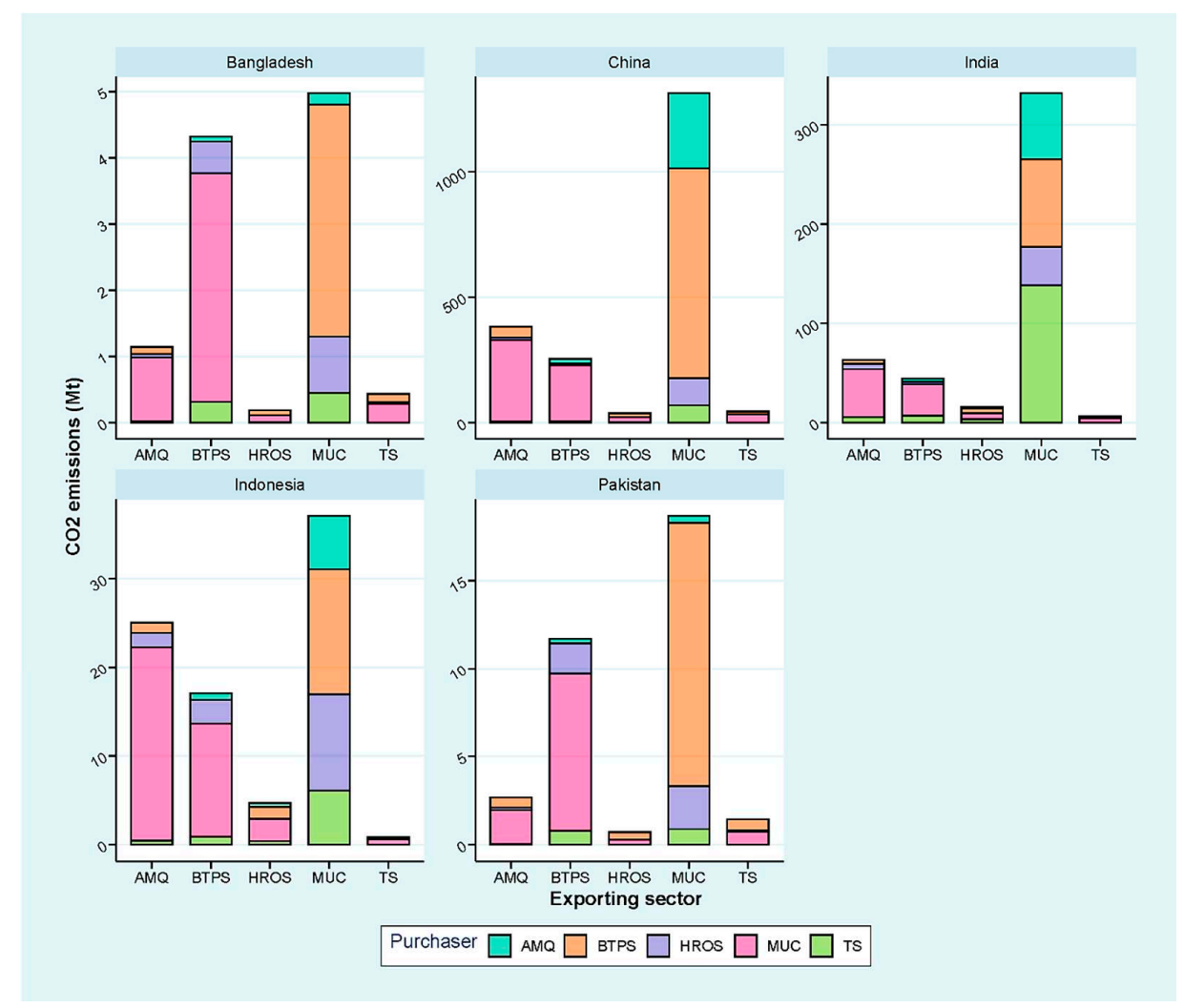

Figure 5. The sectoral sources of indirect emissions for BCIIP countries. Source: Constructed by the authors.

\subsection{The Sectoral Sources of Indirect Demand}

Although direct demand shocks were more important in reducing $\mathrm{CO}_{2}$ emissions under different scenarios, indirect demand shocks were also important in absolute terms, as shown above. Understanding the major sectoral sources of indirect demand can thus aid in the development of targeted mitigation policies. Figure 5 shows that demand for MUC, which had the highest forward $\mathrm{CO}_{2}$ emissions of all nations, received the majority of its indirect virtual $\mathrm{CO}_{2}$ demand from the $\mathrm{AMQ}$ for China, India, and Indonesia. However, BTPS was the source of the majority of MUC's indirect demand in Bangladesh and Pakistan. Similarly, for AMQ, the MUC sector's final demand was the largest driver of its downstream emissions in China, India, and Indonesia. While MUC was also the largest source of BTPS forward emissions in Bangladesh and Pakistan.

\section{Discussion}

\subsection{Discussion of the Results}

The COVID-19 pandemic has an impact on human activities, including energy consumption and $\mathrm{CO}_{2}$ emissions [10]. Many studies have been conducted on the effects of the novel COVID-19 pandemic on production [11-14] and supply chain disruptions [15,16]. Several studies have focused on $\mathrm{CO}_{2}$ emission reductions associated with COVID-19related economic activity disruptions at the same time. Many studies have concentrated on the effects of lockdown-related demand (consumption) reduction on $\mathrm{CO}_{2}$ emissions at the national [35], provincial/regional [33,34], and international levels [36-38]. Few studies, however, have quantified the impact of direct and indirect COVID-19-related demand shocks on sectoral production and supply chain disruptions in general, as well as $\mathrm{CO}_{2}$ emissions and linkages in particular. Furthermore, the role of consumer demand from an industry's downstream importers is an important but often overlooked aspect of the impact of COVID-19 demand shocks on sectoral $\mathrm{CO}_{2}$ emissions. This aspect is largely ignored in the literature on COVID-19's overall impacts, and particularly in the literature 
on COVID-19's effects on carbon and pollutant emissions. In this study, indirect demand shocks are defined as the effect of changes in demand for the target sector's downstream purchasers on $\mathrm{CO}_{2}$ emissions. This study addressed these research gaps by estimating the impact of direct and indirect demand shocks on BCIIP's important, densely populated, and polluted Asia-Pacific economies.

The study used MRIO [60] national input-output data and aggregated the inputoutput tables to correspond to the ADB [59] potential demand shock scenarios. Our findings revealed that the MUC sector was responsible for the greatest amount of emissions in all five countries. Aside from the worst-case scenario, no other scenario had a significant negative impact on $\mathrm{CO}_{2}$ emissions in Bangladesh, India, or Pakistan. However, when compared to the baseline (no COVID-19) scenario, all other scenarios showed a significant reduction in $\mathrm{CO}_{2}$ emissions for China and Indonesia. Direct demand shocks, on average, contributed more to total $\mathrm{CO}_{2}$ emissions reductions than indirect demand shocks. For all nations, the sectors with the highest emissions, such as the MUC, experienced the greatest reductions in emissions under various demand shock scenarios. Not only were these key sectors directly reducing emissions, but they were also indirectly driving a significant portion of other sectors' emissions through inter-sectoral imports.

The impact of direct and indirect demand shocks on intermediate industrial linkages is rarely estimated in the literature on sectoral carbon linkages. The limited literature either estimates the impact on final demand of intermediate sectoral ties by completely removing them through hypothetical extraction of a sector's final demand [56] or by embedding intermediate linkage emissions into various types of final demand [51]. The impact of direct and indirect demand shocks from disasters such as COVID-19, on the other hand, has not been considered in the related literature. As a result, the methodological approach used in this study is relatively new in the literature on sectoral (industrial) linkages, and thus it has implications that extend beyond the COVID-19 demand shock scenarios.

The long-term effects of the COVID-19 pandemic on emissions are unknown and are dependent on factors such as the success and rigor of public health programs, economic and human activity recovery, and long-term changes in human behavior [36]. Because of the uncertainties surrounding the duration, severity, and government lockdowns and restrictions associated with the current COVID-19 pandemic, various percentage $\mathrm{CO}_{2}$ emission reductions are presented in the related literature. According to Shan et al., compared to a no-pandemic baseline scenario, $\mathrm{CO}_{2}$ emissions for the 79 countries studied will decrease by $3.9 \%$ to $5.6 \%$ from 2020 to 2024 [38]. The low sensitivity test conducted by Quéré et al. predicted mid-point emissions reductions of $-2.6 \%,-6.7 \%,-5.1 \%$, and $-5.2 \%$ for China, the US, Europe (EU27 + UK), and India, respectively. However, their high sensitivity test predicted mid-point emissions reductions of $-5.6 \%,-11 \%,-8.5 \%$, and $-8.7 \%$ for these nations. Meanwhile, they forecast a $-5.7 \%$ decrease in global $\mathrm{CO}_{2}$ emissions by 2020 [37]. COVID-19-related confinement measures, according to Quéré et al., will reduce global emissions by approximately 7\% below 2019 levels by the end of 2020 [6]. According to our findings, in the worst-case scenario, $\mathrm{CO}_{2}$ emissions in Bangladesh, China, India, Indonesia, and Pakistan will be reduced by $-1 \%,-2 \%,-1 \%,-1 \%$, and $-1.4 \%$, respectively, in the coming years. However, given the current global GDP growth rate recovery and particularly significant improvements in GDP growth rates in all BCIIP countries in 2021 [62], it is more likely that COVID-19-related restrictions on global and BCIIP national $\mathrm{CO}_{2}$ emissions will have a minor impact in the future. As a result, the best and moderate case scenarios, which have a less than $1 \%$ impact on total national $\mathrm{CO}_{2}$ emissions reductions in BCIIP countries, are more likely for the coming years.

According to our findings, both direct and indirect negative demand shocks can play a significant role in decreasing direct $\mathrm{CO}_{2}$ emissions from respective sectors. The direct demand impact on $\mathrm{CO}_{2}$ reductions under different scenarios ranged from $85 \%$ to $63 \%$ for all BCIIP nations. While the impact of indirect demand shocks ranged from $15 \%$ to $37 \%$. As demonstrated in the introduction section, the COVID- $19 \mathrm{CO}_{2}$ emissions literature typically does not disaggregate the effects of direct and indirect demand shocks 
on national $\mathrm{CO}_{2}$ emissions. However, authors such as Shan et al. have examined the effect of national and global lockdown measures on $\mathrm{CO}_{2}$ emissions, concluding that for the United States of America, the effect of self-lockdown resulted in $76.4 \% \mathrm{CO}_{2}$ emission reductions in 2020, while disruptions in global supply chains resulted in $23.6 \% \mathrm{CO}_{2}$ emission reductions [38]. Our findings also indicate somewhat similar patterns, with direct sectoral demand shocks causing significantly more reductions than indirect demand shocks from the decline in downstream sectoral demand (downstream supply chain). At the sectoral level, the MUC sector, which includes manufacturing, utilities, and construction, is not only the largest emitter in each of the five BCIIP countries but it also had the greatest negative direct and indirect impact on the respective economies' sectoral $\mathrm{CO}_{2}$ emissions. These findings are consistent with those of other studies. Secondary industries, such as power generation and construction, have been shown to have the highest emissions in various economies $[35,49,63]$. The MUC sector has also been shown to have the greatest negative impact on direct $\mathrm{CO}_{2}$ emissions in a variety of other countries under various COVID-19 lockdown scenarios [35,38]. Furthermore, direct and indirect demand shocks had significant relative reductions in BTPS, AMQ and $\mathrm{HROS}$ total $\mathrm{CO}_{2}$ emissions under different scenarios. The subsequent decomposition of downstream sectoral emissions revealed a strong interdependence between the sectors with the greatest impacts. The downstream indirect demand for AMQ in China, India, and Indonesia (CII) was responsible for the majority of the indirect downstream emissions of MUC. Furthermore, downstream demand for BTPS accounted for the majority of MUC's forward emissions in Bangladesh and Pakistan (BP). The MUC's indirect demand accounted for the majority of indirect emissions for AMQ from CII countries and BTPS from BP countries.

\subsection{Limitations and Future Research}

It should be noted that final demand, or final demand shocks, can be classified into various categories, such as final demand from households, government, international trade, and capital formation. However, it is beyond the scope of this study to investigate the direct and indirect impact of various categories of final demand on the COVID-19 demand shocks associated with $\mathrm{CO}_{2}$ emissions. Furthermore, demand shocks can be studied in relation to various socioeconomic impacts on demand shocks and thus $\mathrm{CO}_{2}$ emissions. Further research into the role of different final demand categories and socioeconomic factors may yield some interesting findings on the direct and indirect effects of final demand shocks on sectoral $\mathrm{CO}_{2}$ emissions.

\section{Policy Implications}

The understanding of the impact of direct and indirect demand shocks on $\mathrm{CO}_{2}$ emissions in BCIIP countries can help policymakers develop long-term policies that go beyond the negative effects of COVID-19 demand shocks, which are diminishing over time. Policymakers can achieve this by improving key sectors' direct and indirect demand patterns and levels, as evidenced by the magnitude of their direct and indirect negative impacts under various COVID-19-related demand reduction scenarios. According to the patterns in our findings, for BCIIP countries, a significant portion of the major sector $\mathrm{CO}_{2}$ emissions reductions under different scenarios came from indirect downstream sector demand. As a result, in order to maintain current COVID-19-related reduction patterns, in addition to direct demand from key $\mathrm{CO}_{2}$ producing sectors, indirect demand from downstream industries must be considered. In this case, the intermediate virtual exporters of industrial $\mathrm{CO}_{2}$ emissions, downstream importers, and direct and indirect final demand sources (such as households and government) should be targeted through a mechanism of shared carbon taxes or carbon permits. As a result, a distributed but effective reduction in $\mathrm{CO}_{2}$ emissions can be achieved, with all stakeholders sharing the burden of $\mathrm{CO}_{2}$ mitigation. Numerous previous studies have also argued for the distribution of industrial $\mathrm{CO}_{2}$ emissions through various mechanisms. It has been argued, in particular, that transferring the traditional burden from industrial producers to various other stakeholders is not only just, but may also be 
more effective, due to the shredded burden of $\mathrm{CO}_{2}$ emissions responsibility [49,56]. Recent evidence suggests that environmental levies (such as carbon taxes) will have a significant long-term impact on carbon emissions reduction. As a result, a fair and effective carbon taxation policy is critical for long-term resilience by sustaining current reduction patterns.

COVID-19 $\mathrm{CO}_{2}$ emission reductions have mostly transitory effects. And, as time passes, these effects will fade. According to studies, when certain countries lift COVID-19related restrictions, emissions tend to spike [34]. Many studies have predicted that current $\mathrm{CO}_{2}$ emission reductions will be transient, with emissions returning to pre-COVID-19 levels if necessary steps are not taken $[35,36]$. As a result, capitalizing on the opportunity for long-term recovery would necessitate the adoption of low-carbon production models [9,64]. After the pandemic, the resurgence of both direct and indirect demand for various sectoral products and services to pre-COVID-19 levels, and possibly beyond, could offset any positive gains in sectoral $\mathrm{CO}_{2}$ emissions reductions achieved during the current pandemic. To avert a recession caused by the COVID-19 pandemic, the world's leading economies and economic blocs have contributed billions of dollars in monetary and fiscal stimulus $[65,66]$. There is enormous potential for sustaining and improving current $\mathrm{CO}_{2}$ emission reductions if policymakers consider the environment in addition to the economy when developing fiscal and monetary policies. Rather than the restrictions on human activities experienced during the COVID-19 pandemic [36], which are unsustainable in the long run, monetary and fiscal measures may be required to achieve long-run sustainable growth through demand control, which may aid in the reduction of economies' carbon intensity.

In this case, both long-term monetary and fiscal policies can be tailored to maintain current $\mathrm{CO}_{2}$ reduction trends in key $\mathrm{CO}_{2}$ emitting sectors. Monetary policy may, for example, include provisions for easy bank borrowing by lowering interest rates for industries and final consumers who agree to spend on green practices (such as renewable energy use, improved resource use efficiency, innovations [35,36,38], and investment in green (energy-efficient) infrastructure $[6,29,36])$. Similarly, long-term fiscal policy in the form of carbon taxes (both for producers and consumers [56]) and other COVID-19 economic recovery-related subsidies such as financial stimuli to sectors based on $\mathrm{CO}_{2}$ reductions, financial support to final consumers based on green behavior, and government investment in green infrastructure can aid in dealing with post-COVID- $19 \mathrm{CO}_{2}$ emissions. Shan et al. concluded that expected fiscal stimuli planned by various governments for economic recovery as a result of COVID-19 will either significantly increase $\mathrm{CO}_{2}$ emissions or help achieve net zero emissions by investing in clean energy sectors [38]. Taking into consideration the world's expanding energy consumption and the issue of fossil fuel depletion [67]. A favorable monetary and fiscal policy toward green practices will aid in the development of new technologies for energy consumption control and the transition from conventional to biofuels, which are necessary to meet energy demands while limiting $\mathrm{CO}_{2}$ emissions [67].

\section{Conclusions}

COVID-19 is a worldwide catastrophe of epic proportions. The COVID-19 pandemic has the greatest impact on developing economies. Many studies have been conducted to estimate the effects of COVID-19-related disruptions on production and supply chains in general, as well as on carbon emissions in particular. Many studies, in particular, have been conducted on the impact of related demand shocks on $\mathrm{CO}_{2}$ reductions. The impact of direct and indirect demand shocks on specific economies, on the other hand, has rarely been estimated. Furthermore, the role of domestic intermediate sectoral supply chain disruptions caused by direct and indirect demand shocks has received little attention in the literature. Moreover, in the related literature on both the sectoral linkages and the COVID-19 related impacts, the domestic intermediate sectoral linkages have not been classified based on their direct and indirect demand shocks. Our study filled these critical reset gaps by estimating the $\mathrm{CO}_{2}$ reduction impacts of COVID-19-related intermediate sectoral supply chain disruptions caused by direct and indirect demand shocks. The BCIIP developing economies case was considered due to their significant contribution 
to the global population and pollution. The study first differentiated a country's total emissions into those caused by intra-sectoral linkages and those caused by downstream forward sectoral linkages. The impact of total demand shocks was then disaggregated into intra-sectoral $\mathrm{CO}_{2}$ linkage reductions caused by direct demand shocks and inter-sectoral downstream $\mathrm{CO}_{2}$ linkages caused by indirect demand from a target sector's downstream sectoral importers. According to the findings, China had the highest total $\mathrm{CO}_{2}$ emissions of any nation under the no COVID-19 scenario, followed by India, Indonesia, Pakistan, and Bangladesh. Aside from the worst-case scenario, no other scenario significantly reduced $\mathrm{CO}_{2}$ emissions in Bangladesh, India, or Pakistan. All other scenarios, however, resulted in comparatively significant $\mathrm{CO}_{2}$ emission reductions for China and Indonesia when compared to the baseline scenario (no COVID-19). The impact of direct demand shocks on $\mathrm{CO}_{2}$ reduction was generally greater in $\mathrm{BCIIP}$ countries than the impact of indirect demand shocks. The MUC, which had the highest downstream $\mathrm{CO}_{2}$ emissions of any country, received the vast majority of its indirect virtual $\mathrm{CO}_{2}$ demand from the AMQ for China, India, and Indonesia. However, BTPS accounted for the vast majority of MUC indirect demand in Bangladesh and Pakistan. Given the current state of the BCIIP's economic recovery, a best or moderate scenario with a negative impact of less than $1 \%$ is more likely in the coming years. To be resilient in the face of COVID-19, current $\mathrm{CO}_{2}$ emission reductions must be sustained and improved over time. This can be accomplished through the development of a fair and effective carbon taxation policy that accounts for all intermediate sectoral and final demand sources, both direct and indirect. Furthermore, monetary and fiscal policies based on environmental impacts can help maintain or improve the COVID-19-related $\mathrm{CO}_{2}$ reduction pattern in the long run. When estimating the impact of direct and indirect demand shocks, our study did not take into account the distinct roles of different categories of final demand. Furthermore, the impact of various socioeconomic factors was not taken into account. Future research can thus consider both the role of final demand categories and socioeconomic factors in order to shed more light on the topic of $\mathrm{CO}_{2}$ reductions from COVID-19-related direct and indirect demand shocks.

Supplementary Materials: The following are available online at https: / www.mdpi.com/article/ $10.3390 /$ su13169312/s1, Table S1. The aggregation of Bangladesh's national input-output table. Table S2. The aggregation of China's national input-output table. Table S3. The aggregation of India's national input-output table. Table S4. The aggregation of Indonesia's national input-output table. Table S5. The aggregation of Pakistan's national input-output table. Appendix File. Decomposition of direct $\mathrm{CO}_{2}$ emissions from direct and indirect demand.

Author Contributions: Conceptualization, M.J.S.; methodology, M.J.S.; software, M.J.S.; validation, E.D.R.S.G.; formal analysis, M.J.S.; investigation, M.J.S.; resources, M.J.S. and E.D.R.S.G.; data curation, M.J.S.; writing-original draft preparation, M.J.S.; writing-review and editing, E.D.R.S.G.; visualization, M.J.S.; supervision, E.D.R.S.G.; project administration, E.D.R.S.G. All authors have read and agreed to the published version of the manuscript.

Funding: This research received no external funding.

Institutional Review Board Statement: Not applicable.

Informed Consent Statement: Not applicable.

Data Availability Statement: The data on the demand shocks under different scenarios are available on the Asian Development Bank's database at https:/ / data.adb.org/dataset/covid-19-economicimpact-assessment-template (accessed on 11 May 2021). The national input-output tables are available from the MRIO database at https: / worldmrio.com/countrywise/ (accessed on 1 January 2021). The aggregated input-output tables data and data depicted by the manuscript figures are available and can be provided upon reasonable request from the corresponding author.

Acknowledgments: The authors would like to thank the four anonymous reviewers and the guest editor, Idiano D'Adamo, for their insightful comments.

Conflicts of Interest: The authors declare no conflict of interest. 


\section{Appendix A}

Table A1. Full names of the sectoral abbreviations used in this study.

\begin{tabular}{cc}
\hline Sectoral Abbreviations & Full Names \\
\hline AMQ & "Agriculture, Mining and Quarrying" \\
BTPS & "Business, Trade, Personal, and Public Services" \\
HROS & "Hotel and Restaurants and Other Personal Services" \\
MUC & "Light/Heavy Manufacturing, Utilities, and \\
TS & Construction" \\
"Transport services"
\end{tabular}

Table A2. Sector-wide reduction in $\mathrm{CO}_{2}$ emissions in Mt compared to the no COVID-19 baseline scenario.

\begin{tabular}{|c|c|c|c|c|c|}
\hline Items $^{a}$ & Bangladesh $^{b}$ & China & India & Indonesia & Pakistan \\
\hline \multicolumn{6}{|c|}{ Best case } \\
\hline AMQ & $-3.18 \times 10^{-5}$ & -2.60 & -0.02 & -0.07 & 0.00 \\
\hline BTPS & $-5.85 \times 10^{-4}$ & -2.29 & -0.02 & -0.03 & 0.00 \\
\hline HROS & $-2.10 \times 10^{-4}$ & -0.66 & -0.03 & -0.24 & 0.00 \\
\hline MUC & $-1.29 \times 10^{-3}$ & -17.26 & -0.19 & -0.24 & -0.01 \\
\hline TS & $-1.42 \times 10^{-5}$ & -0.51 & 0.00 & -0.01 & 0.00 \\
\hline Total impact & $-2.13 \times 10^{-3}$ & -23.31 & -0.26 & -0.58 & -0.01 \\
\hline \multicolumn{6}{|c|}{ Moderate case } \\
\hline AMQ & $-7.22 \times 10^{-5}$ & -6.62 & -0.04 & -0.09 & 0.00 \\
\hline BTPS & $-1.15 \times 10^{-3}$ & -5.91 & -0.02 & -0.04 & -0.01 \\
\hline HROS & $-3.22 \times 10^{-4}$ & -1.27 & -0.06 & -0.30 & 0.00 \\
\hline MUC & $-2.82 \times 10^{-3}$ & -42.03 & -0.32 & -0.35 & -0.01 \\
\hline TS & $-2.62 \times 10^{-5}$ & -0.85 & -0.01 & -0.01 & 0.00 \\
\hline Total impact & $-4.39 \times 10^{-3}$ & -56.67 & -0.44 & -0.79 & -0.02 \\
\hline \multicolumn{6}{|c|}{ Worse case } \\
\hline AMQ & $-1.23 \times 10^{-4}$ & -11.17 & -0.06 & -0.16 & 0.00 \\
\hline BTPS & $-2.36 \times 10^{-3}$ & -13.34 & -0.05 & -0.07 & -0.01 \\
\hline HROS & $-6.41 \times 10^{-4}$ & -2.07 & -0.10 & -0.50 & 0.00 \\
\hline MUC & $-5.05 \times 10^{-3}$ & -139.71 & -0.66 & -0.60 & -0.02 \\
\hline TS & $-4.87 \times 10^{-5}$ & -1.77 & -0.01 & -0.01 & 0.00 \\
\hline Total impact & $-8.22 \times 10^{-3}$ & -168.06 & -0.88 & -1.34 & -0.04 \\
\hline \multicolumn{6}{|c|}{ Hypothetical worst case } \\
\hline AMQ & -0.02 & -11.18 & -2.75 & -0.60 & -0.07 \\
\hline BTPS & -0.30 & -13.34 & -2.01 & -0.57 & -1.04 \\
\hline HROS & -0.04 & -2.07 & -0.52 & -0.76 & -0.08 \\
\hline MUC & -0.25 & -139.82 & -10.82 & -2.90 & -1.09 \\
\hline TS & -0.01 & -1.77 & -0.22 & -0.04 & -0.05 \\
\hline Total impact & -0.63 & -168.18 & -16.32 & -4.88 & -2.33 \\
\hline
\end{tabular}

${ }^{a}$ Here, the above values in Mt present the difference between the sectoral values under different scenarios and the baseline scenario. ${ }^{\mathrm{b}}$ The scientific notation has been used to present the sector-wide impact of Bangladesh's $\mathrm{CO}_{2}$ emissions under different scenarios as the values are very small, and therefore not suitable for presentation in a normal format.

\section{References}

1. Ibn-Mohammed, T.; Mustapha, K.B.; Godsell, J.; Adamu, Z.; Babatunde, K.A.; Akintade, D.D.; Acquaye, A.; Fujii, H.; Ndiaye, M.M.; Yamoah, F.A.; et al. A critical analysis of the impacts of COVID-19 on the global economy and ecosystems and opportunities for circular economy strategies. Resour. Conserv. Recycl. 2021, 164, 105169. [CrossRef]

2. World Health Organization. WHO Coronavirus Disease (COVID-19) Dashboard (Data Last Updated: 2 March 2021, 6:09 pm CET). Available online: https:/ / covid19.who.int/ (accessed on 3 March 2021).

3. Singh, S.; Kumar, R.; Panchal, R.; Tiwari, M.K. Impact of COVID-19 on logistics systems and disruptions in food supply chain. Int. J. Prod. Res. 2020, 59, 1-16. [CrossRef]

4. Barbier, E.B.; Burgess, J.C. Sustainability and development after COVID-19. World Dev. 2020, 135, 105082. [CrossRef] [PubMed]

5. Zeng, N.; Han, P.; Liu, D.; Liu, Z.; Oda, T.; Martin, C.; Liu, Z.; Yao, B.; Sun, W.; Wang, P.; et al. Global to local impacts on atmospheric $\mathrm{CO}_{2}$ caused by COVID-19 lockdown. arXiv 2020, arXiv:2010.13025. 
6. Quéré, C.L.; Peters, G.P.; Friedlingstein, P.; Andrew, R.M.; Canadell, J.G.; Davis, S.J.; Jackson, R.B.; Jones, M.W. Fossil CO 2 emissions in the post-COVID-19 era. Nat. Clim. Chang. 2021, 11, 197-199. [CrossRef]

7. D’adamo, I.; González-Sánchez, R.; Medina-Salgado, M.S.; Settembre-Blundo, D. E-commerce calls for cyber-security and sustainability: How european citizens look for a trusted online environment. Sustainability 2021, 13, 6752. [CrossRef]

8. Acevedo-Duque, Á.; Gonzalez-Diaz, R.; Vargas, E.C.; Paz-Marcano, A.; Muller-Pérez, S.; Salazar-Sepúlveda, G.; Caruso, G.; D'Adamo, I. Resilience, Leadership and Female Entrepreneurship within the Context of SMEs: Evidence from Latin America. Sustainability 2021, 13, 8129. [CrossRef]

9. D'Adamo, I.; Lupi, G. Sustainability and resilience after COVID-19: A circular premium in the fashion industry. Sustainability 2021, 13, 1861. [CrossRef]

10. Amankwah-Amoah, J. Note: Mayday, Mayday, Mayday! Responding to environmental shocks: Insights on global airlines' responses to COVID-19. Transp. Res. Part E Logist. Transp. Rev. 2020, 143, 102098. [CrossRef]

11. Chowdhury, M.T.; Sarkar, A.; Paul, S.K.; Moktadir, M.A. A case study on strategies to deal with the impacts of COVID-19 pandemic in the food and beverage industry. Oper. Manag. Res. 2020. [CrossRef]

12. Marimuthu, R.; Sankaranarayanan, B.; Ali, S.M.; Karuppiah, K. Green recovery strategies for the mining industry of India: Lessons learned from the COVID-19 pandemic. J. Asia Bus. Stud. 2021. [CrossRef]

13. Chen, J.; Huang, J.; Su, W.; Štreimikienè, D.; Baležentis, T. The challenges of COVID-19 control policies for sustainable development of business: Evidence from service industries. Technol. Soc. 2021, 66, 101643. [CrossRef]

14. Cui, Q.; He, L.; Liu, Y.; Zheng, Y.; Wei, W.; Yang, B.; Zhou, M. The impacts of COVID-19 pandemic on China's transport sectors based on the CGE model coupled with a decomposition analysis approach. Transp. Policy 2021, 103, 103-115. [CrossRef]

15. Shahed, K.S.; Azeem, A.; Ali, S.M.; Moktadir, M.A. A supply chain disruption risk mitigation model to manage COVID-19 pandemic risk. Environ. Sci. Pollut. Res. 2021. [CrossRef]

16. Karmaker, C.L.; Ahmed, T.; Ahmed, S.; Ali, S.M.; Moktadir, M.A.; Kabir, G. Improving supply chain sustainability in the context of COVID-19 pandemic in an emerging economy: Exploring drivers using an integrated model. Sustain. Prod. Consum. 2021, 26, 411-427. [CrossRef] [PubMed]

17. Wang, P.; Chen, K.; Zhu, S.; Wang, P.; Zhang, H. Severe air pollution events not avoided by reduced anthropogenic activities during COVID-19 outbreak. Resour. Conserv. Recycl. 2020, 158, 104814. [CrossRef] [PubMed]

18. Acharya, P.; Barik, G.; Gayen, B.K.; Bar, S.; Maiti, A.; Sarkar, A.; Ghosh, S.; De, S.K.; Sreekesh, S. Revisiting the levels of Aerosol Optical Depth in south-southeast Asia, Europe and USA amid the COVID-19 pandemic using satellite observations. Environ. Res. 2021, 193, 110514. [CrossRef] [PubMed]

19. Dumka, U.C.; Kaskaoutis, D.G.; Verma, S.; Ningombam, S.S.; Kumar, S.; Ghosh, A. Silver linings in the dark clouds of COVID-19: Improvement of air quality over India and Delhi metropolitan area from measurements and WRF-CHIMERE model simulations. Atmos. Pollut. Res. 2020. [CrossRef]

20. Ranjan, A.K.; Patra, A.K.; Gorai, A.K. Effect of lockdown due to SARS COVID-19 on aerosol optical depth (AOD) over urban and mining regions in India. Sci. Total Environ. 2020, 745, 141024. [CrossRef]

21. Debone, D.; da Costa, M.V.; Miraglia, S.G.E.K. 90 days of COVID-19 social distancing and its impacts on air quality and health in Sao Paulo, Brazil. Sustainability 2020, 12, 7440. [CrossRef]

22. Alonso-Muñoz, S.; González-Sánchez, R.; Siligardi, C.; García-Muiña, F.E. New circular networks in resilient supply chains: An external capital perspective. Sustainability 2021, 13, 6130. [CrossRef]

23. Rume, T.; Didar-UlIslam, S.M. Environmental effects of COVID-19 pandemic and potential strategies of sustainability. Heliyon 2020, 6, e04965. [CrossRef] [PubMed]

24. Zhang, D.; Hao, M.; Morse, S. Is environmental sustainability taking a backseat in china after covid-19? The perspective of business managers. Sustainability 2020, 12, 369. [CrossRef]

25. Sharma, H.B.; Vanapalli, K.R.; Cheela, V.S.; Ranjan, V.P.; Jaglan, A.K.; Dubey, B.; Goel, S.; Bhattacharya, J. Challenges, opportunities, and innovations for effective solid waste management during and post COVID-19 pandemic. Resour. Conserv. Recycl. 2020, 162, 105052. [CrossRef]

26. Vanapalli, K.R.; Sharma, H.B.; Ranjan, V.P.; Samal, B.; Bhattacharya, A.; Dubey, B.K.; Goel, S. Challenges and strategies for effective plastic waste management during and post COVID-19 pandemic. Sci. Total Environ. 2021, 750, 141514. [CrossRef]

27. Kalbusch, A.; Henning, E.; Brikalski, M.P.; de Luca, F.V.; Konrath, A.C. Impact of coronavirus (COVID-19) spread-prevention actions on urban water consumption. Resour. Conserv. Recycl. 2020, 163, 105098. [CrossRef] [PubMed]

28. Khan, M.F.; Pervez, A.; Modibbo, U.M.; Chauhan, J. Flexible Fuzzy Goal Programming Approach in Optimal Mix of Power Generation for Socio-Economic Sustainability: A Case Study. Sustainability 2021, 13, 8256. [CrossRef]

29. D'Adamo, I.; Rosa, P. How do you see infrastructure? Green energy to provide economic growth after COVID-19. Sustainability 2020, 12, 4738. [CrossRef]

30. Forster, P.M.; Forster, H.I.; Evans, M.J.; Gidden, M.J.; Jones, C.D.; Keller, C.A.; Lamboll, R.D.; Quéré, C.L.; Rogelj, J.; Rosen, D.; et al. Current and future global climate impacts resulting from COVID-19. Nat. Clim. Chang. 2020, 10, 913-919. [CrossRef]

31. Arribas-Ibar, M.; Nylund, P.A.; Brem, A. The risk of dissolution of sustainable innovation ecosystems in times of crisis: The electric vehicle during the covid-19 pandemic. Sustainability 2021, 13, 1319. [CrossRef]

32. Rousseau, S.; Deschacht, N. Public Awareness of Nature and the Environment During the COVID-19 Crisis. Environ. Resour. Econ. 2020, 76, 1149-1159. [CrossRef] 
33. Turner, A.J.; Kim, J.; Fitzmaurice, H.; Newman, C.; Worthington, K.; Chan, K.; Wooldridge, P.J.; Köehler, P.; Frankenberg, C.; Cohen, R.C. Observed Impacts of COVID-19 on Urban $\mathrm{CO}_{2}$ Emissions. Geophys. Res. Lett. 2020, 47, e2020GL090037. [CrossRef]

34. Zheng, B.; Geng, G.; Ciais, P.; Davis, S.J.; Martin, R.V.; Meng, J.; Wu, N.; Chevallier, F.; Broquet, G.; Boersma, F.; et al. Satellite-based estimates of decline and rebound in China's $\mathrm{CO}_{2}$ emissions during COVID-19 pandemic. Sci. Adv. 2020, 6, eabd4998. [CrossRef]

35. Han, P.; Cai, Q.; Oda, T.; Zeng, N.; Shan, Y.; Lin, X.; Liu, D. Assessing the recent impact of COVID-19 on carbon emissions from China using domestic economic data. Sci. Total Environ. 2021, 750, 141688. [CrossRef] [PubMed]

36. Liu, Z.; Ciais, P.; Deng, Z.; Lei, R.; Davis, S.J.; Feng, S.; Zheng, B.; Cui, D.; Dou, X.; Zhu, B.; et al. Near-real-time monitoring of global $\mathrm{CO}_{2}$ emissions reveals the effects of the COVID-19 pandemic. Nat. Commun. 2020, 11, 5172. [CrossRef] [PubMed]

37. Quéré, C.L.; Jackson, R.B.; Jones, M.W.; Smith, A.J.P.; Abernethy, S.; Andrew, R.M.; De-Gol, A.J.; Willis, D.R.; Shan, Y.; Canadell, J.G.; et al. Temporary reduction in daily global $\mathrm{CO}_{2}$ emissions during the COVID-19 forced confinement. Nat. Clim. Chang. 2020, 10, 647-653. [CrossRef]

38. Shan, Y.; Ou, J.; Wang, D.; Zeng, Z.; Zhang, S.; Guan, D.; Hubacek, K. Impacts of COVID-19 and fiscal stimuli on global emissions and the Paris Agreement. Nat. Clim. Chang. 2021, 11, 200-206. [CrossRef]

39. Sajid, M.J.; Qingren, C.; Ming, C.; Shuang, L. Sectoral carbon linkages of Indian economy based on hypothetical extraction model. Int. J. Clim. Chang. Strateg. Manag. 2020, 12, 1756-8692. [CrossRef]

40. Duarte, R.; Sa, J.; Bielsa, J. Water use in the Spanish economy: An input-output approach. Ecol. Econ. 2002, 43, 71-85. [CrossRef]

41. Dionisio, C.; Blanco, P.; Thaler, T. An Input-Output Assessment of Water Productivity in the Castile and León Region (Spain). Water 2014, 6, 929-944. [CrossRef]

42. Deng, G.; Wang, L.; Xu, X. Linkage effect of virtual water trade in China's industrial products—Based on generalized hypothetical extraction method. Ecol. Indic. 2018, 93, 1302-1310. [CrossRef]

43. Guerra, A.I.; Sancho, F. Measuring energy linkages with the hypothetical extraction method: An application to Spain. Energy Econ. 2010, 32, 831-837. [CrossRef]

44. He, W.; Wang, Y.; Zuo, J.; Luo, Y. Sectoral linkage analysis of three main air pollutants in China's industry: Comparing 2010 with 2002. J. Environ. Manag. 2017, 202, 232-241. [CrossRef]

45. Wang, Y.; Lai, N.; Mao, G.; Zuo, J.; Crittenden, J.; Jin, Y.; Moreno-Cruz, J. Air pollutant emissions from economic sectors in China: A linkage analysis. Ecol. Indic. 2017, 77, 250-260. [CrossRef]

46. Sajid, M.J.; Cao, Q.; Kang, W. Transport sector carbon linkages of EU's top seven emitters. Transp. Policy 2019, 80, 24-38. [CrossRef]

47. Cella, G. The input-output measurement of interindustry linkages. Oxf. Bull. Econ. Stat. 1984, 46, 73-84. [CrossRef]

48. Bai, H.; Feng, X.; Hou, H.; He, G.; Dong, Y.; Xu, H. Mapping inter-industrial $\mathrm{CO}_{2}$ flows within China. Renew. Sustain. Energy Rev. 2018, 93, 400-408. [CrossRef]

49. Sajid, M.J.; Li, X.; Cao, Q. Demand and supply-side carbon linkages of Turkish economy using hypothetical extraction method. J. Clean. Prod. 2019, 228, 264-275. [CrossRef]

50. Zhao, Y.; Liu, Y.; Wang, S.; Zhang, Z.; Li, J. Inter-regional linkage analysis of industrial $\mathrm{CO}_{2}$ emissions in China: An application of a hypothetical extraction method. Ecol. Indic. 2016, 61, 428-437. [CrossRef]

51. Sajid, M.J.; Qiao, W.; Cao, Q.; Kang, W. Prospects of industrial consumption embedded final emissions: A revision on Chinese household embodied industrial emissions. Sci. Rep. 2020, 10, 1826. [CrossRef]

52. Ali, Y. Measuring $\mathrm{CO}_{2}$ emission linkages with the hypothetical extraction method (HEM). Ecol. Indic. 2015, 54, 171-183. [CrossRef]

53. Sajid, M.J. Structural decomposition and Regional Sensitivity Analysis of industrial consumption embedded emissions from Chinese households. Ecol. Indic. 2021, 122, 107237. [CrossRef]

54. Sun, C.; Chen, L.; $\mathrm{Xu}, \mathrm{Y}$. Industrial linkage of embodied $\mathrm{CO}_{2}$ emissions: Evidence based on an absolute weighted measurement method. Resour. Conserv. Recycl. 2020, 160, 104892. [CrossRef]

55. Sajid, M.J.; Shahni, N.; Ali, M. Calculating inter-sectoral carbon flows of a mining sector via hypothetical extraction method. J. Min. Environ. 2019, 10, 853-867. [CrossRef]

56. Sajid, M.J. Inter-sectoral carbon ties and final demand in a high climate risk country: The case of Pakistan. J. Clean. Prod. 2020, 269, 122254. [CrossRef]

57. Worldometer Countries in the World by Population. 2021. Available online: https://www.worldometers.info/world-population/ population-by-country/ (accessed on 1 January 2021).

58. Tiseo, I. Average PM 2.5 Levels in Most Polluted Countries Worldwide. 2019. Available online: https://www.statista.com/ statistics / 1135356/most-polluted-countries-in-the-world/\#: \{\}:text=Bangladesh\%20had\%20an\%20average\%20PM2,\%20 second $\% 20$ most $\% 20$ polluted $\% 20$ country\%2C\%20Pakistan (accessed on 6 March 2021).

59. Asian Development Bank COVID-19 Economic Impact Assessment Template. Available online: https://data.adb.org/dataset/ covid-19-economic-impact-assessment-template (accessed on 11 May 2021).

60. Eora Global MRIO Eora National IO Tables. Available online: https:/ / worldmrio.com/ countrywise/ (accessed on 1 January 2021).

61. Leontief, W.W. Quantitative Input and Output Relations in the Economic Systems of the United States. Rev. Econ. Stat. 1936, 18, 105-125. [CrossRef]

62. IMF Real GDP Growth. Available online: https://www.imf.org/external/datamapper/NGDP_RPCH@WEO/OEMDC/ADVEC/ WEOWORLD/IND/CHN/PAK/BGD/IDN (accessed on 26 July 2021).

63. Sajid, M.J.; Niu, H.; Xie, J.; Habib, M. Final consumer embedded carbon emissions and externalities: A case of Chinese consumers. Environ. Dev. 2021, 100642. [CrossRef] 
64. D'Adamo, I.; Falcone, P.M.; Martin, M.; Rosa, P. A sustainable revolution: Let's go sustainable to get our globe cleaner. Sustainability 2020, 12, 4387. [CrossRef]

65. Sajid, M.J. Economic consequences of resource trade-offs for special disaster-blessed industries: The case of COVID-19. In Proceedings of the 2020 International Conference on New Energy Technology and Industrial Development (NETID 2020), Dali, China, 18-20 December 2021; Volume 235, p. 02007.

66. MacSwan, A. Factbox: The Economic Remedies for the Coronavirus. Available online: https://www.reuters.com/ article/us-health-coronavirus-economy-policy-fac/factbox-the-economic-remedies-for-the-coronavirus-idUSKBN2121C4 (accessed on 3 April 2020).

67. Štreimikienè, D.; Mikalauskienè, A.; Atkočiūnienè, Z.; Mikalauskas, I. Renewable energy strategies of the Baltic States. Energy Environ. 2019, 30, 363-381. [CrossRef] 

Article

\title{
Evaluating Supply Chain Collaboration Barriers in Small- and Medium-Sized Enterprises
}

\author{
Priom Mahmud ${ }^{1}$, Sanjoy Kumar Paul ${ }^{2, * \mathbb{C}}$, Abdullahil Azeem ${ }^{1, *}$ and Priyabrata Chowdhury ${ }^{3}$ (D) \\ 1 Department of Industrial and Production Engineering, Bangladesh University of Engineering and Technology, \\ Dhaka 1000, Bangladesh; priom1996@gmail.com \\ 2 UTS Business School, University of Technology Sydney, Sydney, NSW 2007, Australia \\ 3 School of Accounting, Information Systems and Supply Chain, RMIT University, \\ Melbourne, VIC 3000, Australia; priyabrata.chowdhury@rmit.edu.au \\ * Correspondence: sanjoy.paul@uts.edu.au (S.K.P.); azeem@ipe.buet.ac.bd (A.A.)
}

check for updates

Citation: Mahmud, P.; Paul, S.K.;

Azeem, A.; Chowdhury, P. Evaluating Supply Chain Collaboration Barriers in Small- and Medium-Sized Enterprises. Sustainability 2021, 13, 7449. https://doi.org/10.3390/ su13137449

Academic Editor: Idiano D'Adamo

Received: 2 June 2021

Accepted: 29 June 2021

Published: 2 July 2021

Publisher's Note: MDPI stays neutral with regard to jurisdictional claims in published maps and institutional affiliations.

Copyright: (c) 2021 by the authors. Licensee MDPI, Basel, Switzerland. This article is an open access article distributed under the terms and conditions of the Creative Commons Attribution (CC BY) license (https:// creativecommons.org/licenses/by/ $4.0 /)$.

\begin{abstract}
The disruption has a significant impact on supply chain collaboration (SCC) which is an important task to improve performance for many enterprises. This is especially critical for small- and medium-sized enterprises (SMEs). We developed a decision-modeling framework for analyzing SCC barriers in SMEs for the emerging economy in Bangladesh. Through literature review and expert opinion survey, we have identified a comprehensive list of SCC barriers under four main categories, namely, information-related, communication-related, intra-organizational, and inter-organizational barriers. Then we applied the Grey DEMATEL and Fuzzy Best-Worst methods to evaluate these SCC barriers and compared the results. We also conducted a sensitivity analysis to assess the robustness of the proposed approach. The study reveals that lack of communication is the most crucial barrier in SCC, providing a model for assessing barriers in other emerging economies. This study contributes to the literature by analyzing SCC barriers and by comparing the results obtained from two different MCDM methods. The findings of this study can help decision-makers to plan for overcoming the most prioritized SCC barriers which ultimately contribute to improving the resilience and sustainability performances of SMEs.
\end{abstract}

Keywords: supply chain collaboration; resilience; small- and medium-sized enterprises; grey DEMATEL; fuzzy best-worst method

\section{Introduction}

Collaboration is a key factor for success in supply chain management, typically improving overall supply chain performance [1,2]. According to Whipple and Russel [3], collaboration is formed when two or more organizations work together to gain better efficiency, which is impossible to achieve by working alone. Supply chain collaboration (SCC) aligns plans and objectives of individual enterprises and is important for sustaining competitive advantage in today's competitive business era [4]. In this environment, firms are collaborating by combining the resources of suppliers and customers [5]. Supply chain partners are removing organizational barriers to ensure efficiency and responsiveness [6]. The significance of SCC for both large firms and small- and medium-sized enterprises (SMEs), described as enterprises having fewer than 250 employees [7], is already well studied and understood [2]. However, while large firms are well equipped to build collaborative relationships in a supply chain, SMEs face greater difficulties in achieving SCC. These difficulties arise from wide-ranging factors such as incompatible technology, lack of skilled personnel, poor networking with important supply chain players, and inadequate finance [7]. Moreover, the recent COVID-19 pandemic impacted supply chain collaboration practices of SMEs significantly [8]. Generally, SMEs lag behind in implementing SCC than large firms. The COVID-19 pandemic even impacted SMEs harder to maintain their SCC [9]. 
Although it is important to understand what makes SMEs lag behind, studies on SCC in the context of SMEs to date mostly focused on the importance and effect of SCC on various performance indicators. For example, Eyaa et al. [10] analyzed the effect of collaborative relationships on the supply chain performance of an individual SME in Uganda. Haji-Pakir and Alina [11] uncovered the minimal level of collaboration and its effects on Malaysian SMEs. Considering the importance of identifying the barriers of SCC for its successful implementation, Gumboh and Gichira [7] investigated and identified 11 collaboration barriers among SMEs in Kenya. However, as per the knowledge of the authors, none of the existing studies has put efforts to analyze the SCC barriers comprehensively to explore what are the most influential barriers. While SMEs across the world face some barriers in implementing SCC, the barriers might be different in a developed and an emerging economy, or at least their severity of the barriers is different. For example, SMEs in developed countries are more technologically advanced and receive more governmental support, whereas these are considered severe barriers to collaboration for SMEs in emerging countries [12]. In general, study on SCC in the context of SMEs in an emerging country is scarce. Aiming to contribute to the literature on SCC in the context of SMEs in an emerging economy, the study aims at answering the following specific questions:

Research Question 1 (RQ1). What are the SCC barriers to be considered in SMEs of an emerging country?

Research Question 2 (RQ2). What are the most influential SCC barriers in SMEs of an emerging country?

Research Question 3 (RQ3). What are the implications of the findings in supply chain resilience and sustainability?

The study used SMEs in Bangladesh as the subject of the investigation. The substantial contribution of SMEs to the economy of Bangladesh is the main reason for using Bangladesh as the context of this study. In Bangladesh, there are 177 SME clusters, which constitute about $90 \%$ of the industrial enterprises of Bangladesh [13]. They provide $80-85 \%$ of industrial employment and generate about 19\% of GDP (Gross Domestic Product) in the manufacturing and service sectors of Bangladesh [14]. Moreover, $75-80 \%$ of export earnings come from SMEs [15]. For answering the research questions, this study used an interview-based MCDM (Multi-Criteria Decision Making) methodology. While interviews are used to identify and finalize the list of barriers of SCC in SMEs, MCDM techniques are used to analyze the barriers to see what the most influential barriers are.

This study resolves the research gap by accumulating SCC barriers in various SME sectors of an emerging economy like Bangladesh and evaluating them by different MCDM techniques. This will help to differentiate the ways SMEs of emerging economies face barriers to implement SCC when compared to SMEs of developed countries. Moreover, the study analyzes the barriers to identify the most critical barriers in this regard. Previous studies in the context of various industries, both large and small, of developed economies already provided many crucial sets of barriers. Hudnurkar et al. [6] accumulate 28 factors affecting collaboration. Moreover, previous studies could not come to a consensus in deciding the most critical barriers of SCC for SMEs. For example, Eyaa et al. [10] report that lack of information sharing, decision desynchronization, and incentive misalignment are the most critical SCC barriers that affect supply chain performance. On the other hand, Zhang and Cao [5] suggest improper organizational culture as the most critical barrier of SCC. These divergent findings suggest the need for an in-depth analysis in the context of SMEs of the emerging economies to ensure that the findings truly reflect the SMEs and the context. Moreover, the findings can help the SMEs to improve their sustainability and resilience performances during a global pandemic by prioritizing and eradicating the SCC barriers. Many researchers showed the positive relationship between resilience and sustainability during the COVID-19 pandemic. As physical communication has been disrupted due to the COVID-19 pandemic, a distance communication strategy for enhancing SCC would help to attain resiliency [16]. The resilience strategies, in turn, 
can positively affect sustainability in the supply chain. In the literature, several studies explained the positive relationship between supply chain resilience and sustainability performance [17-19]. In this study, we aim to contribute to supply chain resilience and sustainability by analyzing the interrelations among SCC barriers and determining their priority ranking in the context of SMEs of Bangladesh.

The article is organized as follows. Section 2 presents a literature review. The decisionmaking framework for both MCDM methods (Fuzzy Best-Worst and Grey DEMATEL) is presented in Section 3. Decision criteria collection and evaluation of criteria by two methods are explained in Section 4. Section 5 presents the discussion on results and the sensitivity analysis. Finally, Section 6 concludes the paper.

\section{Literature Review}

Collaboration is one of the most important enablers in supply chain management. Barratt [20] identified major supporting elements of supply chain collaboration (SCC). Supply-side resilience can be gained by collaborative relationships [21]. There are many barriers and performance indices for evaluating supply chain performances in terms of collaboration [22-25]. For example, supply chain partnership and integration are crucial in the context of the apparel industry [23]. Coordination between departments, coordination with suppliers, and coordination with customers are essential when measuring the performance of SME supply chains [26]. Smooth supply chain practice in SMEs enables better supply chain performance in a large industry [27] as well as better business performance within a firm [28]. For smooth supply chain practice in SMEs, collaboration and coordination are mandatory [29]. Several studies identified barriers affecting SCC [6]. However, none of the studies thus far has specifically investigated SCC barriers for SMEs in an emerging economy.

\subsection{Information-Related Barriers}

Several information-related barriers disrupt SCC. Several studies consider different information-related barriers, including those related to reluctance to share information and poor information quality. Reluctance to share information increases coordination costs and leads to deterioration in performance [30]. Gunasekaran et al. [31] found that lack of information exchange among firms keeps supply chains from gaining competitive advantages. It weakens the foundation of the supply chain [32] and can create a bullwhip effect [33]. Lack of market-based information-sharing also causes difficulties in predicting customer demand [34], and creates disturbances in planning and controlling supply chains [35,36]. Inter-organizational relationships may be affected due to a lack of information-sharing [23]. Exchanged information that lacks accuracy, timeliness, credibility, and adequacy is labeled "poor information quality", which can affect trust and commitment [37]. Poor and obsolete technology also disrupts coordination in the supply chain [38].

\subsection{Communication-Related Barriers}

Current literature suggests a number of communication-related barriers, such as lack of communication and poor system connectivity, are already well identified. Lack of communication represents an insufficient exchange of messages and views among firms. Lack of parallel communication structure hinders integration [39]. Obsolete technology for communication and information exchange among firms can create poor system connectivity and discourages collaboration under a disruption like the COVID-19 pandemic [40,41]. Due to lack of communication information sharing between suppliers disrupts which results in a barrier to SCC [42]. Specifically, lack of communication with suppliers during a global crisis like the COVID-19 pandemic distorts the collaboration in a supply chain [43].

\subsection{Intra-Organizational Barriers}

These barriers include intra-organizational weak relationships, decision desynchronization, incentive obstacles, opposition to change, leadership deficit, and territoriality. 
Intra-organizational weak relationships represent those weaknesses in collaboration within an organization. Intra-organizational support is needed for successful SCC [20,44]. Decision desynchronization suggests a lack of collaborative decision-making among SC members in planning and operational aspects [35]. Incentives offered in different stages of SC create obstacles, increase supply variability, and reduce total supply chain profits [1,45]. Managers often oppose collaborative change. Opposition to change results from low trust [46]. Further, inadequate skills of senior executives towards collaboration result in leadership deficits and less collaboration [46]. Individual partners may focus on territorial benefit, neglecting overall benefit for the organization. SC collaborative performance collapses because of this kind of territoriality [46].

\subsection{Inter-Organizational Barriers}

These barriers include lack of trust, short-term relationships, behavioral uncertainty, cultural differences, different goals, non-standardized performance metrics, lack of commitment, small firms, governmental intervention, pricing obstacles, lack of resource sharing, lack of adaptation, and lack of commitment on delivery times. Trust is one of the important enablers of SCC [23]. Distrust creates a lack of information-sharing and asset investment [47]. It also affects resilience against disruptions in supply chain topology [48,49]. Information-sharing is mediated by trust [37]. Long-term relationships are another important enabler of SCC [23], whereas short-term relationships can create failures in collaboration [50]. Long-term supplier relationships are a key driver of integration [22]. Unpredictable behavior among partners negatively affects trust level as well as collaboration [37]. Chopra et al. [1] pointed out that behavioral uncertainty contributes to information distortion. Different norms, beliefs, and underlying values shared by supply chain partners create cultural differences, and these have impacts on trust and information exchange [20,36]. Collaborative culture also is a key antecedent of SCC [5]. Different goals are associated with different priorities among partners because of different competitive situations [51]; joint performance of supply chains will decrease because of it [39].

Lack of common performance measurement approaches creates non-standardized performance metrics [52]. It also creates ambiguity and becomes a barrier to collaboration [39]. Lack of commitment causes a lack of information-sharing and asset investment [47]. Desired collaborative breakthroughs cannot be achieved without commitment [23]. Besides, a lower level of commitment leads to performance deterioration [30]. Firm size is also an important barrier for collaboration, as supply chain members prefer large firms for collaboration [39]. Governments may impose policies against collaborative behavior under the COVID-19 pandemic [53]. This kind of government intervention has effects on collaborative planning [51] and information-sharing [36]. Inflexible pricing policies may result in uncertainty in placing orders for a product [45]. Distorted information from one end of a supply to the other can cause harmful inefficiencies which are known as the bullwhip effect [54]. Pricing obstacles cause the bullwhip effect [1]. Lack of resource sharing is also an essential barrier to collaboration. Without resource commitment, successful SCC cannot be achieved [20] and plans for meeting demand cannot be executed without it [34]. Adaptation suggests shifts in supplier behavior to make better use of the firm's resources. Lack of adaptation weakens the relationship between supplier and customer [55,56]. Firms may become inefficient in maintaining on-time delivery and this late delivery decreases reliability on firms; ultimately, collaboration may collapse [57]. Based on the literature review, a list of barriers to SCC is presented in Table 1.

\subsection{Different MCDM Techniques and Their Applications}

Many MCDM techniques have been used for analyzing barriers. These include: modeling sustainable supply chain complexities using Rough Set Theory (RST) [58]; analyzing critical barriers affecting the local sustainable development through adaptive reuse projects using Fuzzy-DEMATEL approach [59]; assessing barriers of circular supply chain using Fuzzy Analytical Network Process (FANP) [60]; ranking of solutions for reverse logistic 
barriers using Fuzzy AHP and Fuzzy TOPSIS [61]; prioritizing risk barriers involved in implementation of Product Life Management using integrated DEMATEL-based ANP and the Grey TOPSIS method [62]; evaluating barriers of sustainable supply chain management in the leather industry using Grey DEMATEL [63]; analyzing challenges for implementing Industry 4.0 using the BWM technique [64]; analyzing e-waste management barriers using ISM and DEMATEL approaches [65]; prioritizing software improvement success in global software development (GSD) using Fuzzy AHP [66]; assessing green innovation barriers in SMEs using BWM and Fuzzy TOPSIS [67]; prioritizing the drivers for integrated lean-green manufacturing for SMEs using Fuzzy TOPSIS [68]; and analyzing enablers and barriers in extension of useful life of automotive products through remanufacturing using Fuzzy ISM [69]. Table 2 summarizes the application areas of MCDM techniques.

Table 1. List of barriers from the literature review.

\begin{tabular}{ccc}
\hline Category & Barrier Name & Reference \\
\hline \multirow{2}{*}{ Information-related barriers } & Reluctance to share information & {$[23,30-36]$} \\
& Poor information quality & {$[37,38]$} \\
\hline \multirow{3}{*}{ Communication-related barriers } & Lack of communication & {$[39]$} \\
& Poor system connectivity & {$[46]$} \\
\hline & Intra-organizational weak relationships & {$[20,44]$} \\
& Decision desynchronization & {$[35]$} \\
Intra-organizational barriers & Incentive obstacles & {$[1]$} \\
& Opposition to change & {$[46]$} \\
& Territoriality & {$[46]$} \\
& Leadership deficit & {$[46]$} \\
\hline & Lack of trust & {$[23,37,47,48]$} \\
& Short-term relationships & {$[22,23,50]$} \\
& Behavioral uncertainty & {$[1,37]$} \\
& Cultural difference & {$[5,20,36]$} \\
& Different goals & {$[39,51]$} \\
& Lonter-organizational barriers & {$[39,52]$} \\
& Lack of commitment & {$[23,30,47]$} \\
& Small firms & {$[39]$} \\
& Governmental intervention & {$[36,51]$}
\end{tabular}

Table 2. MCDM techniques and their applications.

\begin{tabular}{ccc}
\hline Method & \multicolumn{1}{c}{ Applications } & References \\
\hline $\begin{array}{c}\text { DEMATEL, Grey DEMATEL, } \\
\text { and Fuzzy DEMATEL } \\
\text { fodeling interrelations among barriers } \\
\text { industrial symbiosis, adopting IoT in the } \\
\text { food supply chain, automotive parts } \\
\text { remanufacturing; analyzing challenges to } \\
\text { implementing sustainable manufacturing, } \\
\text { industry 4.0; identifying critical barriers } \\
\text { to the establishment of refueling stations }\end{array}$ & {$[59,63,70-74]$} \\
\hline Rough Set Theory & $\begin{array}{c}\text { Modeling sustainable supply } \\
\text { chain complexities }\end{array}$ & {$[58]$} \\
\hline ANP and Fuzzy ANP & $\begin{array}{c}\text { Analyzing drivers of green information } \\
\text { technology; prioritizing barriers of the } \\
\text { circular supply chain; assessing barriers } \\
\text { of sustainable shale gas revolution }\end{array}$ & {$[60,75]$} \\
\hline
\end{tabular}


Table 2. Cont.

\begin{tabular}{|c|c|c|}
\hline Method & Applications & References \\
\hline $\begin{array}{c}\text { Grey DEMATEL-ANP and } \\
\text { Grey TOPSIS }\end{array}$ & $\begin{array}{l}\text { Ranking of risk barriers of product } \\
\text { lifecycle management implementation; } \\
\text { analyzing criteria for Green } \\
\text { Strategic Sourcing }\end{array}$ & {$[62]$} \\
\hline BWM and Fuzzy BWM & $\begin{array}{l}\text { Analyzing barriers of green innovation in } \\
\text { SMEs, humanitarian supply chain; } \\
\text { assessing challenges of sustainable } \\
\text { manufacturing, evaluating performances } \\
\text { of the sustainable supply chain in the } \\
\text { leather industry; sustainable } \\
\text { supplier selection }\end{array}$ & [76-81] \\
\hline TOPSIS and Fuzzy TOPSIS & $\begin{array}{l}\text { Prioritizing barriers of supply chain } \\
\text { remanufacturing and their solutions, } \\
\text { ranking of drivers of lean-green } \\
\text { manufacturing; prioritizing challenges } \\
\text { and enablers of reverse logistics, green } \\
\text { manufacturing; analyzing barriers of } \\
\text { supply chain remanufacturing }\end{array}$ & {$[61,67,72,82,83]$} \\
\hline AHP and Fuzzy AHP & $\begin{array}{l}\text { Analyzing solutions for reverse logistics } \\
\text { barriers, prioritizing barriers to } \\
\text { renewable energy development, selecting } \\
\text { assembly machined parts }\end{array}$ & {$[61,84-88]$} \\
\hline
\end{tabular}

As reported in Table 2, no study has developed a decision-modeling framework to evaluate barriers of SCC using FBWM. But FBWM can generate better consistent results by handling triangular fuzzy numbers. It can also handle many criteria with no multi optimality [89]. The Grey DEMATEL method was used to analyze barriers for an automotive parts remanufacturer [73], to analyze critical success barriers for implementation of drones in logistics sectors [90], and to analyze interrelationships among barriers to sustainable supply chain management in the leather industry [63]. However, there is no such study that used Grey DEMATEL for analyzing SCC barriers. But this method can find critical factors with the help of an impact relation diagram. It can also solve uncertain and indeterminate problems with discrete incomplete information [91]. By considering the benefits, FBWM and Grey DEMATEL will be suitable for our analysis. Most notably, studies that have integrated FBWM and Grey DEMATEL methods are scarce in the literature.

We help fill this knowledge gap by working toward the following objectives:

i. To propose a framework to identify SCC barriers in the context of SMEs of Bangladesh,

ii. To analyze SCC barriers using the newly developed Fuzzy Best Worst (FBWM) and Grey DEMATEL methods and compare the results, and

iii. To outline implications for supply chain managers, as well as directions for future research, based on outcomes of the analysis.

\section{Decision-Modelling Framework}

Before the application of MCDM methods, a questionnaire survey was conducted among SMEs of Bangladesh for identifying potential SCC barriers. Barriers from both the literature review and the first survey were aggregated to conduct another questionnaire survey regarding inputs of the model methods. The decision-modeling framework is shown in Figure 1.

\subsection{Identification of SCC Barriers}

We first identified the barriers from the literature review as reported in Table 1. Then, a list of SCC barriers was sought based on the interviews with entrepreneurs of $5 \mathrm{SME}$ 
sectors. Then we finalized the SCC barriers from both interviews and literature review to contextualize for SMEs.

Identifying barriers for supply chain collaboration from literature

Collecting data on barriers from SMEs of Bangladesh

Finalizing the barriers to make a comprehensive list

Performing a survey among experts to collect suitable data for the MCDM

Applying the Fuzzy Best Worst and Grey DEMATEL methods to prioritize

Comparing results obtained from two MCDM techniques

Sensitivity analysis for checking robustness of results

Figure 1. Decision-modeling framework analyzing barriers of SCC.

\subsection{Analysis Using the Fuzzy Best Worst Method}

Fuzzy Best Worst Method (FBWM) eliminate the ambiguity of decision-maker by incorporating fuzzy linguistic scale instead of traditional scale, such as 1-9 scale. The consistency ratio will be high and more reliable results can be achieved for evaluating our SCC barriers [89]. We completed the following steps in our FBWM analysis:

Step 1: Building a list of decision-making criteria

An appropriate set of decision-making criteria was selected based on the opinions of SME experts consulted.

Step 2: Determining the most and least important criteria

This is not a quantitative step-rather, it involves selection by the experts consulted for this research.

Step 3: Computing fuzzy reference comparisons for the best criterion

The fuzzy preferences of the best barrier are identified using Table 3 [89], which includes the linguistic variables of decision-makers. This generates the best-to-others vector as:

$$
A_{B}=\left(a_{B 1}, a_{B 2}, a_{B 3}, \ldots, a_{B n}\right),
$$

Table 3. The linguistic scale for the respondents' assessment.

\begin{tabular}{cc}
\hline Linguistic Terms & Membership Function \\
\hline Equal importance (EI) & $(1,1,1)$ \\
Weakly important (WI) & $(2 / 3,1,3 / 2)$ \\
Fairly important (FI) & $(3 / 2,2,5 / 2)$ \\
Very important (VI) & $(5 / 2,3,7 / 2)$ \\
Absolutely important (AI) & $(7 / 2,4,9 / 2)$ \\
\hline
\end{tabular}


Here, $a_{B j}$ expresses the fuzzy significance of the most important criterion $B$ over criterion $j$,

Hence, $a_{B B}=(1,1,1)$.

Step 4: Computing pairwise comparisons between the other criteria and the worst criterion

The fuzzy preferences of all barriers are identified by using Table 3 . This generates the worst-to-others vector as:

$$
A_{W}=\left(a_{1 W}, a_{2 W}, \ldots, a_{n W}\right)^{T},
$$

Here, $a_{j W}$ expresses the fuzzy significance of criterion $j$ over the worst criterion $W$, and $a_{W W}=(1,1,1)$.

Step 5: Calculating the optimal fuzzy weights $\left(w_{1}{ }^{*}, w_{2}{ }^{*}, w_{3}{ }^{*}, \ldots, w_{n}{ }^{*}\right)$.

This aims to generate optimal weights of the selected indicators. For the ideal condition (that is, a fully consistent system) $\left|w_{B} / w_{j}-a_{B j}\right|$ and $\left|w_{j} / w_{w}-a_{j w}\right|$ should be equal to zero.

We need to minimize the maximum absolute differences to get closer to the ideal condition $\left\{\left|w_{B} / w_{j}-a_{B j}\right|,\left|w_{j} / w_{w}-a_{j w}\right|\right\}$, which creates the following model,

$$
\begin{gathered}
\underset{j}{\operatorname{minmax}}\left\{\left|\frac{w_{B}}{w_{j}}-a_{B j}\right|,\left|\frac{w_{j}}{w_{W}}-a_{j W}\right|\right\} \\
\text { s.t }\left\{\begin{array}{c}
\sum_{j=1}^{n} R\left(w_{j}\right)=1 \\
l_{j}^{W} \leq m_{j}^{W} \leq u_{j}^{W} \\
l_{j}^{W} \geq 0 \\
j=1,2, \ldots, n
\end{array}\right.
\end{gathered}
$$

Equation (1) can be transferred into the following nonlinear programming problem $\min \xi$ :

$$
\text { s.t }\left\{\begin{array}{c}
\left|\frac{w_{B}}{w_{j}}-a_{B j}\right| \leq \xi \\
\left|\frac{w_{j}}{w_{W}}-a_{j W}\right| \leq \xi \\
\sum_{j=1}^{n} R\left(w_{j}\right)=1 \\
l_{j}^{W} \leq m_{j}^{W} \leq u_{j}^{W} \\
l_{j}^{W} \geq 0 \\
j=1,2, \ldots, n
\end{array}\right.
$$

Let, $\xi^{*}=\left(k^{*}, k^{*}, k^{*}\right), k^{*} \leq l^{*} \leq m^{*} \leq u^{*}$, then Equation (2) can be rewritten as min $\xi^{*}$ :

$$
\text { s.t }\left\{\begin{array}{c}
\left|\frac{\left(l_{B}^{w}, m_{B}^{w}, u_{B}^{w}\right)}{\left(l_{j}^{w}, m_{j}^{w}, u_{j}^{w}\right)}-\left(l_{B j}, m_{B j}, u_{B j}\right)\right| \leq\left(k^{*}, k^{*}, k^{*}\right) \\
\left|\frac{\left(l_{j}^{w}, m_{j}^{w}, u_{j}^{w}\right)}{\left(l_{W}^{w w}, m_{W}^{w}, u_{W}^{w w}\right)}-\left(l_{j W}, m_{j W}, u_{j W}\right)\right| \leq\left(k^{*}, k^{*}, k^{*}\right) \\
\sum_{j=1}^{n} R\left(w_{j}\right)=1 \\
l_{j}^{W} \leq m_{j}^{W} \leq u_{j}^{W} \\
l_{j}^{W} \geq 0 \\
j=1,2, \ldots, n
\end{array}\right.
$$

By solving Equation (3), we can get optimal weights $\left(w_{1}{ }^{*}, w_{2}{ }^{*}, w_{3}{ }^{*}, \ldots, w_{n}{ }^{*}\right)$ and $\zeta^{*}$ thereby providing the consistency ratio. The value of $\xi^{*}$ defines the consistency level of the decision makers' opinions. Values close to zero indicate high levels of consistency and more reliable comparisons. Large values indicate problems in prediction or calculation, requiring re-assessment of the data. 


\subsection{Analysis Using the Grey DEMATEL Method}

The Grey DEMATEL method is a special kind of MCDM technique that considers interrelation among all barriers to evaluating decision criteria. Influential relation maps can be drawn in this method for visualizing relations among criteria. We have used this method in our analysis of barriers by considering its features.

The stepwise procedure of Grey DEMATEL is described as follows.

Step 1: Building the Grey direct relation matrices

Let the number of barriers be ' $n$ ' and the number of respondents be ' $l$ '. Each respondent $k$ evaluates the direct influence of criteria $i$ over criteria $j$ on the linguistic scale of Table 4 [92]. Thus, $l$ Grey direct relation matrices were constructed.

Table 4. Grey linguistic scale for decision makers' assessments.

\begin{tabular}{cc}
\hline Linguistic Terms & Grey Numbers \\
\hline No influence $(\mathrm{N})$ & $(0,0)$ \\
Very low influence $(\mathrm{VL})$ & $(0,0.25)$ \\
Low influence $(\mathrm{L})$ & $(0.25,0.5)$ \\
High influence $(\mathrm{H})$ & $(0.5,0.75)$ \\
Very high influence $(\mathrm{VH})$ & $(0.75,1)$ \\
\hline
\end{tabular}

Step 2: Computing the average Grey direct relation matrix

The average Grey direct matrix is established from $l$ Grey direct matrices,

$$
\begin{gathered}
{\left[\otimes y_{i j}^{k}\right] ; k=1,2, \ldots, l} \\
\otimes \widetilde{y_{i j}}=\left(\frac{\sum_{k} \otimes y_{i j}^{k}}{-}, \frac{\sum_{k} \bar{\otimes} y_{i j}^{k}}{l}\right)
\end{gathered}
$$

Step 3: Computing the crisp Grey direct relation matrix

The Grey values are crisped according to the following three-step modified-CFCS method [92],

(a) Normalization:

$$
\begin{aligned}
& \Delta_{\min }^{\max }={ }_{j}^{\max } \bar{\otimes} \widetilde{y_{i j}}-{ }_{j}^{\min } \otimes \widetilde{y_{i j}} \\
& \Delta \underline{\otimes} \dot{y_{i j}}=\frac{-\widetilde{y_{i j}}-{ }_{j}^{\min } \otimes \widetilde{y_{i j}}}{\Delta_{\min }^{\max }} \\
& \therefore \bar{\otimes} \dot{y_{i j}}=\frac{\bar{\otimes} \widetilde{y_{i j}}-{ }_{j}^{\min } \bar{\otimes} \widetilde{y_{i j}}}{\Delta_{\min }^{\max }}
\end{aligned}
$$

(b) Total Normalized Crisp Value:

$$
z_{i j}=\frac{\otimes \dot{y}_{i j}\left(1-\underline{\otimes} \dot{y_{i j}}\right)+\bar{\otimes} \dot{y}_{i j} * \bar{\otimes} \dot{y}_{i j}}{1-\underline{\otimes} \dot{y}_{i j}+\bar{\otimes} \dot{y_{i j}}}
$$

(c) Final Crisp Value:

$$
\begin{gathered}
z_{i j}^{*}=\min \underset{-}{\bigotimes \widetilde{y_{i j}}}+\left(z_{i j} * \Delta_{\min }^{\max }\right) \\
Z=\left[z_{i j}^{*}\right]
\end{gathered}
$$

Step 4: Computing the normalized direct relation matrix 
The normalized matrix $N$ is computed by identifying $s$ and multiplying $Z$ with $s$

$$
\begin{gathered}
s=\frac{1}{\max _{1 \leq i \leq n} \sum_{j=1}^{n} z_{i j}^{*}} \\
N=s Z
\end{gathered}
$$

Step 5: Computing the total relation matrix

The total relation matrix is calculated as below,

$$
T=N(I-N)^{-1}
$$

Step 6: Computing cause and effect parameters

The row sum $\left(r_{i}\right)$ and column sum $\left(c_{j}\right)$ values of $T$ are identified. Then prominence $\left(r_{i}+c_{j}\right)$ and relation $\left(r_{i}-c_{j}\right)$ values are calculated.

Step 7: Producing the prominence-causal DEMATEL graph

The horizontal axis of the graph includes the prominence values $\left(r_{i}+c_{j}\right)$ and the vertical axis contains the relation values $\left(r_{i}-c_{j}\right)$. This graph has four sections or quadrants including intertwined giver, autonomous giver, autonomous receiver, and intertwined receiver [93].

Step 8: Identifying relative weights of barriers

Importance weights are calculated based on prominence values $\left(r_{i}+c_{j}\right)$ through a normalization procedure as below,

$$
w_{i}=\frac{r_{i}+c_{j}}{\sum_{i=1}^{n}\left(r_{i}+c_{j}\right)}
$$

Global weights of the barriers are calculated after determining local weights from both methods.

\section{Data Collection and Analysis}

In this section, we identify and evaluate the barriers of SCC.

\subsection{Selection of Decision Criteria}

For identifying barriers for SCC, we took interviews of 30 entrepreneurs of $5 \mathrm{SME}$ sectors, such as,

1. Agro-processing

2. Leather and leather goods

3. Light engineering and metalworking

4. Plastic and other synthetics

5. Electrical and electronics

6. Fashionwear and handicrafts.

After collecting barriers from both literature and SMEs, we made a comprehensive list of the SCC barriers. A questionnaire-based survey was conducted to select the most appropriate barriers of SCC for SMEs in Bangladesh. We created a web-based form containing questions that included indicators identified from the relevant literature. The questionnaire was sent to targeted industrial managers (IMs) and academic experts. We have considered the same importance weight of the responses from each of the experts to ensure that they all have equal influence on the result. Respondents were asked whether the indicators were suitable (see Appendix A, Table A1) in Bangladesh. Through this survey process, 23 SCC barriers were selected as presented in Table 5 .

\subsection{Evaluation of Barriers Using the Fuzzy Best-Worst Method}

The 16 experts (Table 6), who responded, first selected the best and the worst categories and then the best and the worst barriers from the appropriate column (Appendix B, 
Tables A2-A12). Experts also completed the comparison matrix using the linguistic scale in Table 3. The optimal weight of the main categories and the barriers were calculated by solving models presented in Equation (3). The optimal weight of the main categories and the barriers and the consistency value of the results from the 16 experts are summarized in Table 7. By multiplying the main categories' weight with the barrier weight, a final normalized weight of each barrier was obtained. The normalized weight of each barrier and final ranking are shown in Table 8.

Table 5. Selected SCC barriers for application purposes.

\begin{tabular}{|c|c|c|}
\hline Category Name & Barrier Name & $\begin{array}{c}\text { Sources } \\
(\mathrm{LR}=\text { Literature Review })\end{array}$ \\
\hline \multirow{3}{*}{ Information-related barriers (A) } & Reluctance to share information (A1) & LR \\
\hline & Information flow disruption (A2) & survey \\
\hline & Poor information quality (A3) & LR + survey \\
\hline \multirow{3}{*}{ Communication-related barriers (B) } & Lack of communication (B1) & LR + survey \\
\hline & Poor systems connectivity (B2) & LR + survey \\
\hline & Vehicle routing problem (B3) & survey \\
\hline \multirow{5}{*}{ Intra-organizational barriers (C) } & Intra-organizational weak relationships (C1) & LR \\
\hline & Decision desynchronization $(\mathrm{C} 2)$ & LR + survey \\
\hline & Incentive obstacles (C3) & LR \\
\hline & Opposition to change (C4) & LR \\
\hline & Leadership deficit (C5) & LR + survey \\
\hline \multirow{12}{*}{ Inter-organizational barriers (D) } & Lack of trust (D1) & LR + survey \\
\hline & Short-term relationship (D2) & LR + survey \\
\hline & Behavioral uncertainty (D3) & $\mathrm{LR}+$ survey \\
\hline & Cultural difference (D4) & LR \\
\hline & Different goals (D5) & LR + survey \\
\hline & Non-standardized performance metrics (D6) & LR \\
\hline & Lack of commitment on quality (D7) & survey \\
\hline & Forecasting problem (D8) & survey \\
\hline & Reluctance to deal with small firms (D9) & LR + survey \\
\hline & Customer taste variations (D10) & survey \\
\hline & Governmental intervention (D11) & LR + survey \\
\hline & Lack of commitment on delivery time (D12) & LR + survey \\
\hline
\end{tabular}

Table 6. Experts and their professional roles.

\begin{tabular}{cccc}
\hline Type of Industry & Role of Respondents & Number of Respondents & Percentage of Respondents (\%) \\
\hline University & Academic & 3 & 18.75 \\
\hline Cigarette Company & $\begin{array}{c}\text { Executive, Production Department (1) } \\
\text { Manager, Supply Chain Management (1) }\end{array}$ & 2 & 12.5 \\
Garments Sector & $\begin{array}{c}\text { Officer, Production Department (4) } \\
\text { Manager, Supply Chain Management (2) } \\
\text { Chief Executive Officer (1) }\end{array}$ & 7 & 43.75 \\
\hline Food and Beverage Industry & Executive, Supply Chain Management & 6.25 \\
\hline $\begin{array}{c}\text { Paint and Coating } \\
\text { Manufacturing Company }\end{array}$ & $\begin{array}{c}\text { Manager, Supply Chain Management } \\
\text { Power Engineering Company }\end{array}$ & $\begin{array}{c}\text { Manager, Supply Chain Management } \\
\text { Chief Executive Officer }\end{array}$ & 1 \\
\hline $\begin{array}{c}\text { Furniture Manufacturing } \\
\text { Company }\end{array}$ & Supply Planner & 1 & 6.25 \\
\hline
\end{tabular}


Table 7. Weight of main categories and barriers.

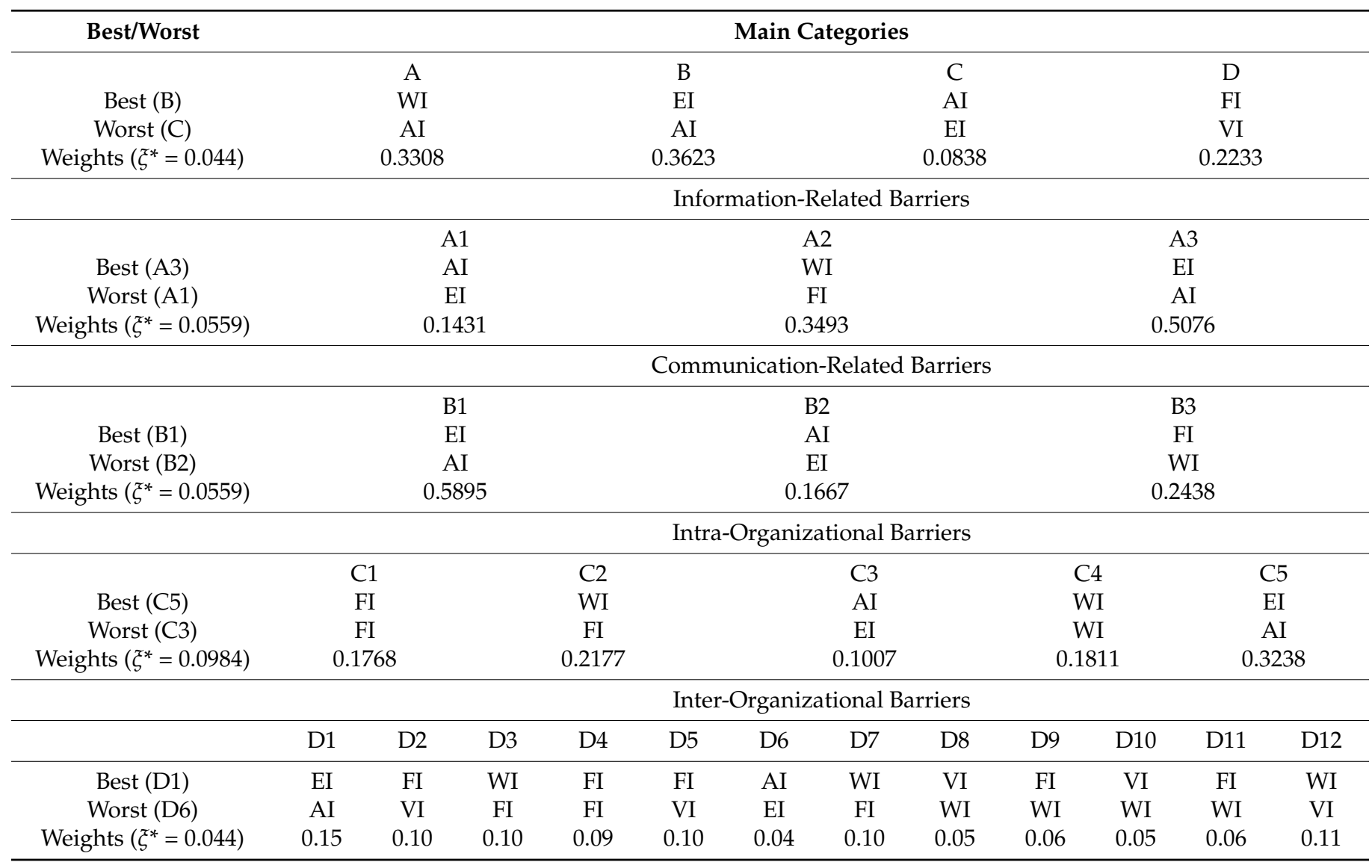

Table 8. Relative importance (weights) of categories and barriers using the fuzzy best-worst method.

\begin{tabular}{|c|c|c|c|c|}
\hline Category Name & Category Weights & Barrier Name & $\begin{array}{c}\text { Local Weights } \\
\text { of Barrier }\end{array}$ & $\begin{array}{c}\text { Global Weights } \\
\text { of Barrier }\end{array}$ \\
\hline \multirow{3}{*}{$\begin{array}{l}\text { Information-related } \\
\text { barriers }(\mathrm{A})\end{array}$} & \multirow{3}{*}{0.3308} & Reluctance to share information (A1) & 0.1431 & 0.0473 \\
\hline & & Information flow disruption (A2) & 0.3493 & 0.1155 \\
\hline & & Poor information quality (A3) & 0.5076 & 0.1679 \\
\hline \multirow{3}{*}{$\begin{array}{l}\text { Communication-related } \\
\text { barriers (B) }\end{array}$} & \multirow{3}{*}{0.3623} & Lack of communication (B1) & 0.5895 & 0.2136 \\
\hline & & Poor systems connectivity (B2) & 0.1667 & 0.0604 \\
\hline & & Vehicle routing problem (B3) & 0.2438 & 0.0883 \\
\hline \multirow{5}{*}{$\begin{array}{c}\text { Intra-organizational } \\
\text { barriers }(C)\end{array}$} & \multirow{5}{*}{0.0838} & Intra-organizational weak relation (C1) & 0.1768 & 0.0148 \\
\hline & & Decision desynchronization $(\mathrm{C} 2)$ & 0.2177 & 0.0182 \\
\hline & & Incentive obstacles (C3) & 0.1007 & 0.0084 \\
\hline & & Opposition to change (C4) & 0.1811 & 0.0152 \\
\hline & & Leadership deficit (C5) & 0.3238 & 0.0271 \\
\hline \multirow{12}{*}{$\begin{array}{c}\text { Inter-organizational } \\
\text { barriers }(\mathrm{D})\end{array}$} & \multirow{12}{*}{0.2233} & Lack of trust (D1) & 0.1453 & 0.0324 \\
\hline & & Short-term relationship (D2) & 0.1008 & 0.0225 \\
\hline & & Behavioral uncertainty (D3) & 0.0971 & 0.0217 \\
\hline & & Cultural difference (D4) & 0.0856 & 0.0191 \\
\hline & & Different goals (D5) & 0.1008 & 0.0225 \\
\hline & & Non-standardized performance metrics (D6) & 0.0401 & 0.0090 \\
\hline & & Lack of commitment on quality (D7) & 0.0969 & 0.0216 \\
\hline & & Forecasting problem (D8) & 0.0489 & 0.0109 \\
\hline & & Reluctance to deal with small firms (D9) & 0.0606 & 0.0135 \\
\hline & & Customer taste variations (D10) & 0.0489 & 0.0109 \\
\hline & & Governmental intervention (D11) & 0.0606 & 0.0135 \\
\hline & & Lack of commitment on delivery time (D12) & 0.1144 & 0.0255 \\
\hline
\end{tabular}

As, $a_{B W}=\mathrm{AI}=(7 / 2,4,9 / 2)$, the consistency index for this case is 8.04. [89].

As such, consistency ratio for the main categories $=0.3542 / 8.04=0.044$. 


\subsection{Evaluation of Barriers Using the Grey DEMATEL Method}

The Grey DEMATEL method was used to rank direct influences among barriers using the linguistic scale provided in Table 4. Experts individually constructed Grey direct relation matrices of categories and barriers. Some of those matrices obtained from 16 experts are provided in Appendix C (Tables A13-A17). The final Total Relation Matrices $(T)$ are computed using Equation (14).

Finally, the cause and effect parameters (prominence $\left(r_{i}+c_{j}\right)$ and relation $\left(r_{i}-c_{j}\right)$ values) and relative weights are computed from $T$. They are shown in Appendix C. Using the prominence and relation values, the prominence-causal DEMATEL graphs are formed for categories and the barriers as shown in Figure 2. Then we determined global weights using local weights; these are presented in Table 9.

Table 9. Relative importance (weights) of categories and barriers using the grey DEMATEL method.

\begin{tabular}{|c|c|c|c|c|}
\hline Category Name & Category Weights & Barrier Name & $\begin{array}{c}\text { Local Weights } \\
\text { of Barrier }\end{array}$ & $\begin{array}{c}\text { Global Weights } \\
\text { of Barrier }\end{array}$ \\
\hline \multirow{3}{*}{$\begin{array}{l}\text { Information-related } \\
\text { barriers }(\mathrm{A})\end{array}$} & \multirow{3}{*}{0.2713} & Reluctance to share information (A1) & 0.2750 & 0.0746 \\
\hline & & Information flow disruption (A2) & 0.3231 & 0.0877 \\
\hline & & Poor information quality (A3) & 0.4019 & 0.1090 \\
\hline \multirow{3}{*}{$\begin{array}{l}\text { Communication-related } \\
\text { barriers (B) }\end{array}$} & \multirow{3}{*}{0.2802} & Lack of communication (B1) & 0.4280 & 0.1199 \\
\hline & & Poor systems connectivity (B2) & 0.2624 & 0.0735 \\
\hline & & Vehicle routing problem (B3) & 0.3096 & 0.0867 \\
\hline \multirow{5}{*}{$\begin{array}{l}\text { Intra-organizational } \\
\text { barriers }(\mathrm{C})\end{array}$} & \multirow{5}{*}{0.2171} & Intra-organizational weak relation (C1) & 0.1919 & 0.0417 \\
\hline & & Decision desynchronization (C2) & 0.2102 & 0.0456 \\
\hline & & Incentive obstacles (C3) & 0.1679 & 0.0365 \\
\hline & & Opposition to change (C4) & 0.1950 & 0.0423 \\
\hline & & Leadership deficit (C5) & 0.2349 & 0.0510 \\
\hline \multirow{12}{*}{$\begin{array}{c}\text { Inter-organizational } \\
\text { barriers }(\mathrm{D})\end{array}$} & \multirow{12}{*}{0.2313} & Lack of trust (D1) & 0.1190 & 0.0275 \\
\hline & & Short-term relationship (D2) & 0.1050 & 0.0243 \\
\hline & & Behavioral uncertainty (D3) & 0.0948 & 0.0219 \\
\hline & & Cultural difference (D4) & 0.0891 & 0.0206 \\
\hline & & Different goals (D5) & 0.1027 & 0.0238 \\
\hline & & Non-standardized performance metrics (D6) & 0.0377 & 0.0087 \\
\hline & & Lack of commitment on quality (D7) & 0.0917 & 0.0212 \\
\hline & & Forecasting problem (D8) & 0.0493 & 0.0114 \\
\hline & & Reluctance to deal with small firms (D9) & 0.0851 & 0.0197 \\
\hline & & Customer taste variations (D10) & 0.0450 & 0.0104 \\
\hline & & Governmental intervention (D11) & 0.0730 & 0.0169 \\
\hline & & Lack of commitment on delivery time (D12) & 0.1075 & 0.0249 \\
\hline
\end{tabular}

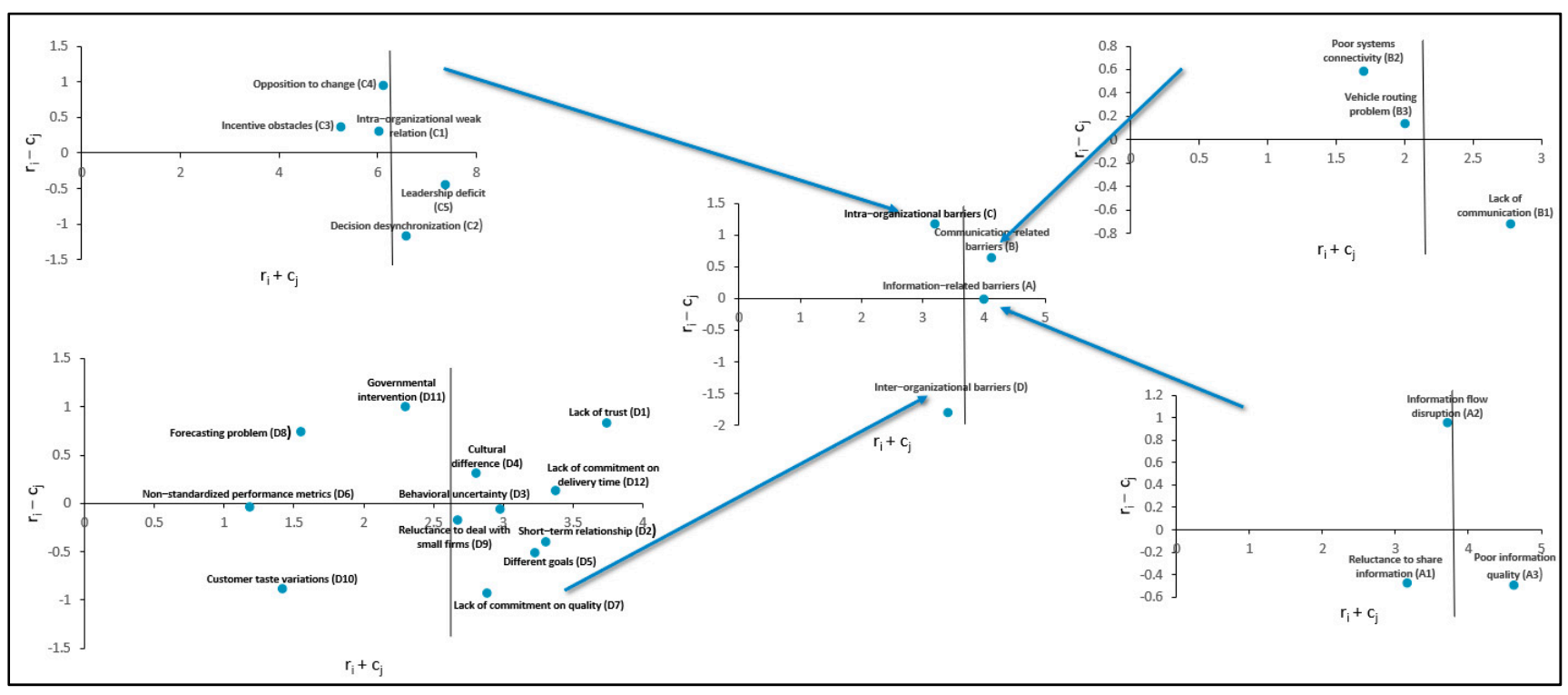

Figure 2. The prominence-causal DEMATEL graph for categories and barriers. 
Figure 2 connotes that communication-related barriers (B) is the core category or intertwined giver-it is the key category that affects all others. Intra-organizational barriers (C) is an autonomous giver or driving category. They have low prominence but high relation; it affects certain other categories. Inter-organizational barriers (D) is an autonomous receiver or independent category and has low prominence and relations. Information-related barriers (A) are intertwined receivers or an impact category. It has high prominence but low relation. It is affected by other categories and cannot be improved directly.

\section{Results and Discussion}

In this section, we discuss the results of our analysis. We also explain the validity of our results through sensitivity analysis. Finally, we discuss the practical implications and contributions of our work.

\subsection{Results and Discussion}

We have measured weights of four main barrier categories, as well as local weights of barriers using FBWM. The consistency ratios are all close to zero, ranging from 0.044 to 0.098 , which suggests the high reliability of the results. From Table 10, communicationrelated barriers (B) is the most important category, followed by information-related barriers (A) and inter-organizational barriers (D). Intra-organizational barriers (C) is the least important category. Poor information quality (A3) is the most significant informationrelated barrier. Among communication-related barriers, lack of communication (B1) plays the most important role. Leadership deficit (C5) plays the most significant role in creating intra-organizational barriers. Lack of trust (D1) is the most important inter-organizational barrier. Based on global weights, lack of communication (B1) is the most important barrier. It is followed by poor information quality (A3), Information flow disruption (A2), and Vehicle routing problems (B3).

Table 10. Importance ranking of the categories and barriers.

\begin{tabular}{|c|c|c|c|}
\hline Category & Barrier Name & $\begin{array}{l}\text { Importance Ranking } \\
\text { from FBWM }\end{array}$ & $\begin{array}{l}\text { Importance Ranking } \\
\text { from Grey-DEMATEL }\end{array}$ \\
\hline \multirow{3}{*}{ Information-related barriers (A) } & Reluctance to share information (A1) & 6 & 5 \\
\hline & Information flow disruption (A2) & 3 & 3 \\
\hline & Poor information quality (A3) & 2 & 2 \\
\hline \multirow{3}{*}{ Communication-related barriers (B) } & Lack of communication (B1) & 1 & 1 \\
\hline & Poor systems connectivity (B2) & 5 & 6 \\
\hline & Vehicle routing problem (B3) & 4 & 4 \\
\hline \multirow{5}{*}{ Intra-organizational barriers (C) } & Intra-organizational weak relation (C1) & 17 & 10 \\
\hline & Decision desynchronization (C2) & 15 & 8 \\
\hline & Incentive obstacles (C3) & 23 & 11 \\
\hline & Opposition to change (C4) & 16 & 9 \\
\hline & Leadership deficit (C5) & 8 & 7 \\
\hline \multirow{12}{*}{ Inter-organizational barriers (D) } & Lack of trust (D1) & 7 & 12 \\
\hline & Short-term relationship (D2) & 10 & 14 \\
\hline & Behavioral uncertainty (D3) & 12 & 16 \\
\hline & Cultural difference (D4) & 14 & 18 \\
\hline & Different goals (D5) & 10 & 15 \\
\hline & Non-standardized performance metrics (D6) & 22 & 23 \\
\hline & Lack of commitment on quality (D7) & 13 & 17 \\
\hline & Forecasting problem (D8) & 20 & 21 \\
\hline & Reluctance to deal with small firms (D9) & 18 & 19 \\
\hline & Customer taste variations (D10) & 20 & 22 \\
\hline & Governmental intervention (D11) & 18 & 20 \\
\hline & Lack of commitment on delivery time (D12) & 9 & 13 \\
\hline
\end{tabular}

Measuring weights using the Grey DEMATEL method, we got the highest value of " $r$ " for communication-related barriers (B), which means it had a high influence on other categories. Information flow disruption (A2) has the greatest influence on other information-related barriers. Poor systems connectivity (B2) affects lack of communi- 
cation (B1) and vehicle routing problems (B3). Opposition to change (C4) and lack of trust (D1) are the most effective barriers among intra- and inter-organizational barrier categories, respectively.

In the Grey DEMATEL method, " $c$ " for each barrier reflects the impact of other barriers on it. Inter-organizational barriers (D) is the most affected category. Poor information quality (A3) has the highest impact on other information-related barriers. All other communication-related barriers affect lack of communication (B1) most. Leadership deficit (C5) has the highest impact on other intra-organizational problems. Different goals (D5) is the most affected inter-organizational barrier.

The Prominence Factor $\left(r_{i}+c_{j}\right)$ shows the importance weight of a criterion in the DEMATEL method. Communication-related barriers (B) is the most important category, followed by information-related barriers (A) and inter-organizational barriers (D). Intraorganizational barriers (C) is the least important category. Poor information quality (A3) is the most significant information-related barrier. Among communication-related barriers, lack of communication (B1) plays the most important role. Leadership deficit (C5) plays the most significant role in creating intra-organizational barriers. Lack of trust (D1) is the most important inter-organizational barrier.

The vertical axis of the DEMATEL graph is "Relation" $\left(r_{i}-c_{j}\right)$ values. They categorize categories into cause groups and effect groups. The positive values stand for the cause group and negative values for the effect group. Communication-related barriers (B) and intra-organizational barriers $(\mathrm{C})$ are causal categories, whereas Information-related barriers (A) and inter-organizational barriers (D) are effect categories. A2 among informationrelated barriers; B2 and B3 under communication-related barriers; C1, C3, and C4; under intra-organizational barriers; and barriers D1, D4, D8, D11, D12 under inter-organizational barriers, are from the causal group which affects all other barriers. All other remaining barriers form the effect group which is affected by these barriers.

The difference in rankings of the barriers from both methods has been illustrated in Figure 3. Lack of communication (B1) is the most important barrier according to both methods. Poor information quality (A3), information flow disruption (A2), and vehicle routing problems (B3) are identified as the second, third, and fourth priorities, respectively, within all barriers. Other priority rankings are different among the applied methods for the following reasons:

- The Grey DEMATEL method considers interdependent relations among all barriers, whereas FBWM considers relationships of best and worst barriers.

- The Grey DEMATEL method contains prominence-causal maps for visualizing interrelations among barriers.

- FBWM does not consider cause and effect relations like the Grey DEMATEL method.

- In the Grey DEMATEL method, we used the Grey linguistic scale, which contains two numbers representing influence ratings, whereas we used triangular fuzzy numbers as linguistic variables in FBWM. They represent importance ratings.

\subsection{Sensitivity Analysis}

We performed a sensitivity analysis to ensure the robustness of our results. A sensitivity analysis is carried out by changing the weight of the top-ranked criterion and noting changes in the weights of other criteria $[94,95]$. The sensitivity analysis was also performed by adjusting the weights of the experts [96]. This testing helps filter out major changes during variation of the weights of experts or the top-ranked criterion. As the methodologies of the two proposed methods are different, the sensitivity analysis was conducted differently.

\subsubsection{Grey DEMATEL Method}

In our analysis, we considered equal weights for each expert. We have presented 16 scenarios by changing the weights of the experts and applying the Grey DEMATEL method for each scenario; results are tabulated in Appendix D (Tables A18-A20). The 
variation in rankings is illustrated in Figure 4. The rankings of A1, A2, B2, B3, and C1 are slightly different, but most of the rankings remain unchanged. So, the proposed model frameworks seem robust to small variations in input values.

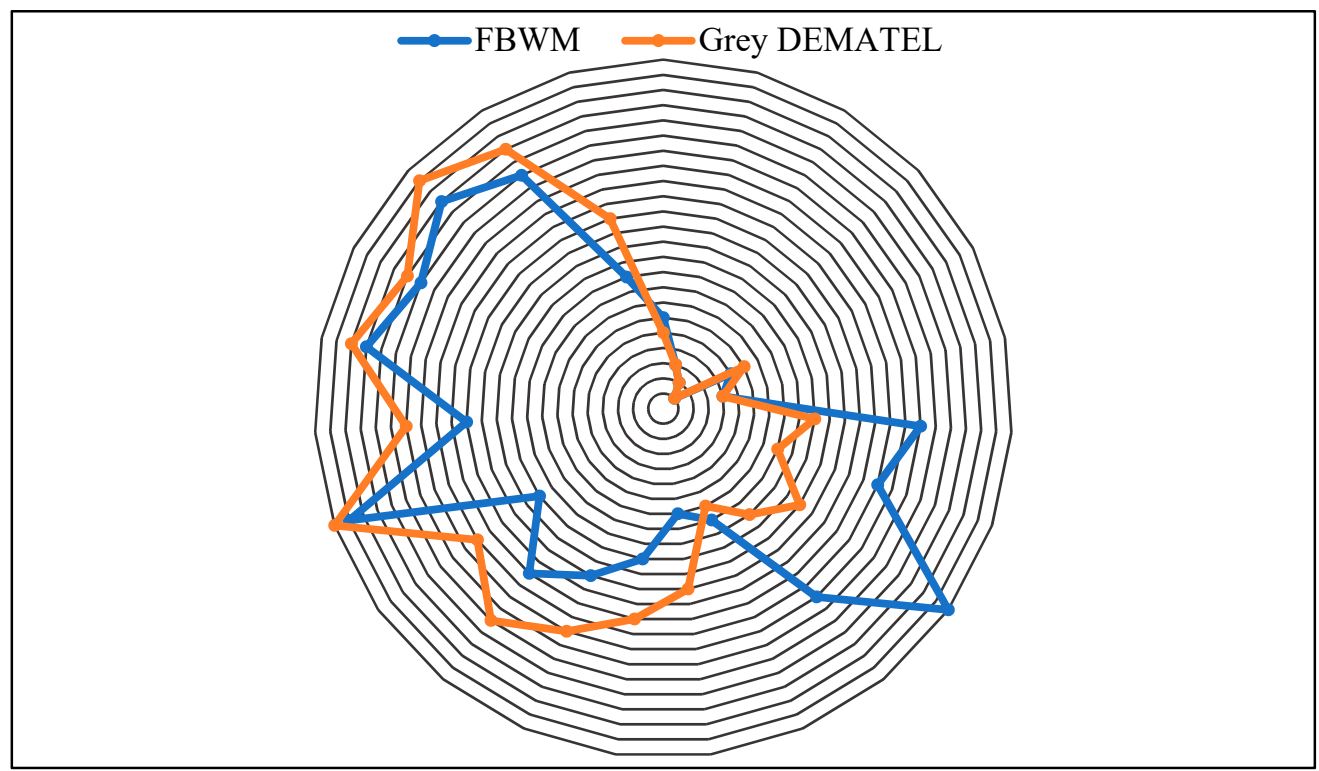

Figure 3. Radar chart for the ranking in terms of the FBWM and Grey DEMATEL methods.

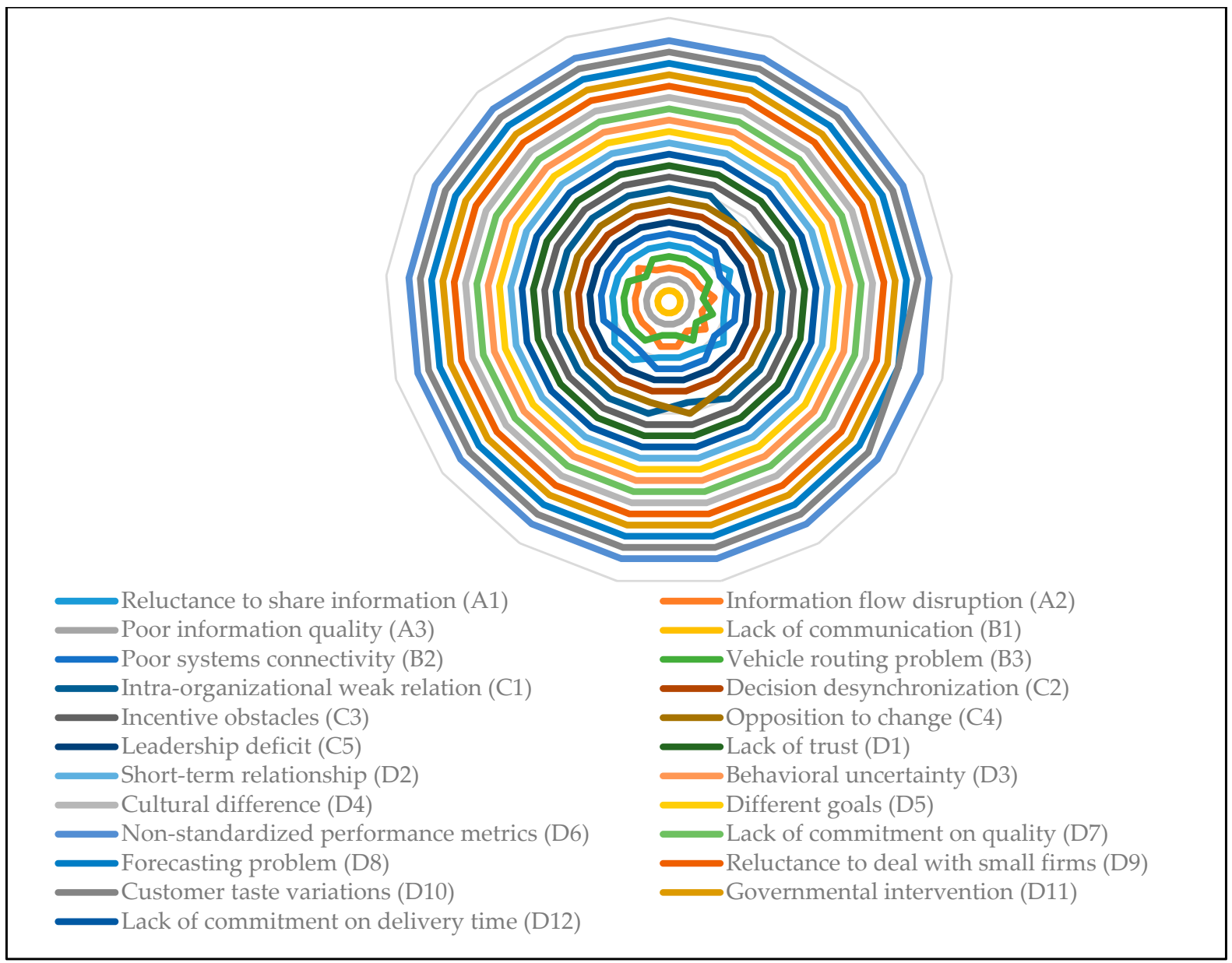

Figure 4. Ranking of barriers of supply chain collaboration during the sensitivity analysis. 


\subsubsection{Fuzzy Best Worst Method}

A top-ranked criterion capable of influencing other criteria is determined by sensitivity analysis, in which the weight of that criterion is changed [94]. Lack of communication (B1) is the most significant barrier according to FBWM. We have performed a sensitivity analysis by considering the incremental change in weights from 0.1 to 0.9 of B1. Appendix D shows different rankings of the barriers during different runs of the sensitivity analysis. The variation in the ranking of the barriers is illustrated in Figure 5. For a weight 0.9 of B1, rankings of some barriers have changed, but most not significantly, which is characteristic of a consistent system.

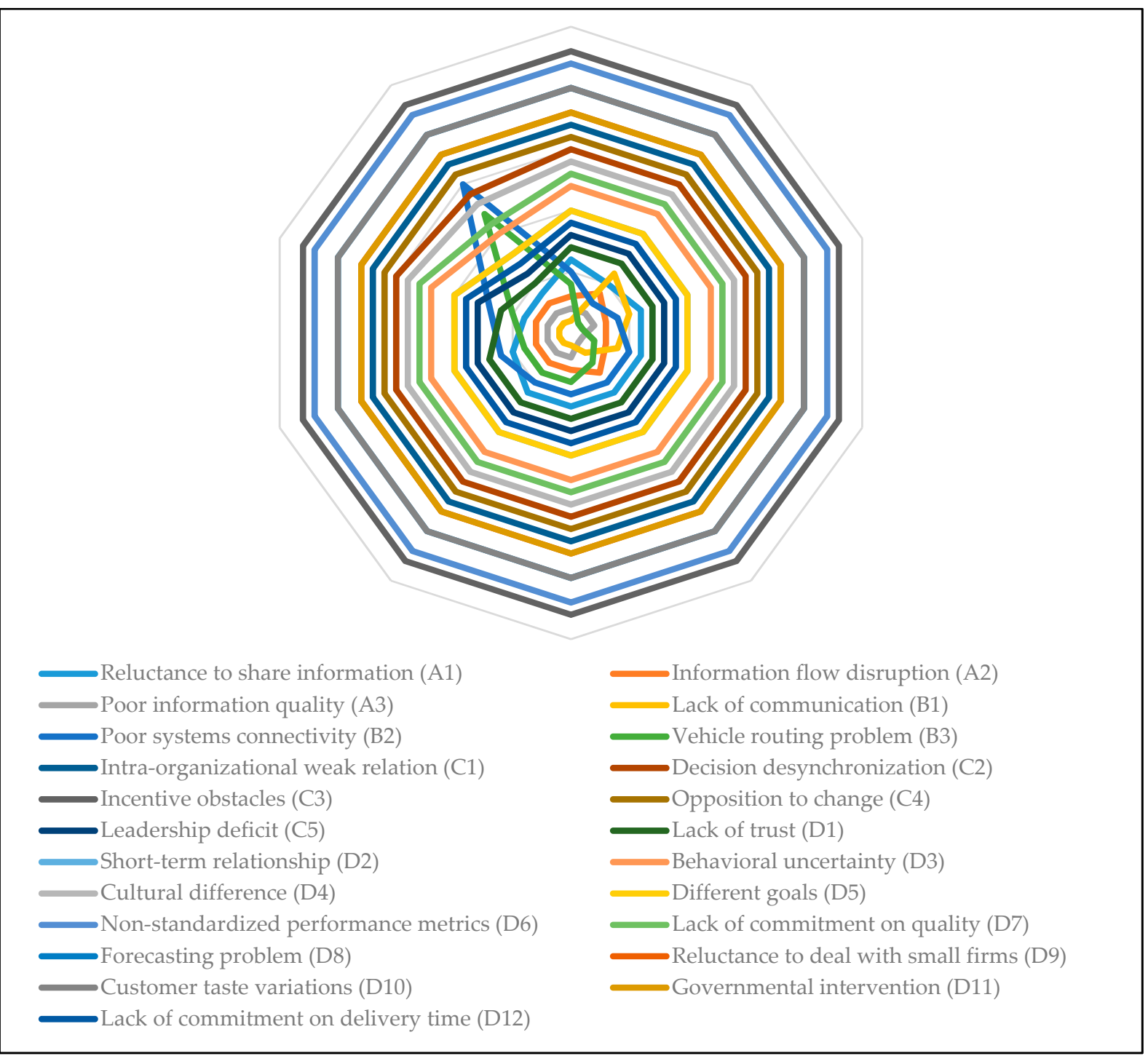

Figure 5. Ranking of barriers to supply chain collaboration during sensitivity analysis.

\subsection{Implications and Contributions}

This research identifies and ranks the barriers to SCC taking SMEs from various industries in Bangladesh. The findings will assist SME managers in understanding SCC barriers and hence formulate their strategies to improve supply chain resilience and sustainability. This is particularly important in the current situation as the COVID-19 pandemic has been taking a test of supply chain resilience for all supply chains across the globe. The classification of barriers under four main categories will enable SME managers to navigate the barriers they are facing. Since complete elimination of the barriers is not feasible, 
ranking of barriers will help in prioritizing the most crucial barriers. SMEs need to monitor continuously and devise strategies to eradicate the most critical barriers.

The finding of the study reveals the importance of communication and informationrelated barriers to SCC among SMEs of Bangladesh. Lack of communication is the most important barrier that SME managers should focus on (see Table 10). During the COVID-19 pandemic, the literature also identified that there is a significant lack of communication in supply chains $[8,43]$. Information-related barriers, in the form of poor information quality and information flow disruption, are the next focusing barriers for SMEs (Table 10). Especially, information integration is required amid the COVID-19 pandemic for smooth information flow [97]. Poor information flow increases lead time and thereby disrupts the collaborative supply chain during the COVID-19 pandemic [98]. Hence, SME managers need to ensure that the right information is shared among the supply chain partners at the right time consistently. Given that face-to-face interactions are found critical for SMEs in recent studies in the context of COVID-19 [8,9], SME managers need to seek avenues such as site visits and meeting at a colocation to improve such interactions. After working on these barriers, SME practitioners need to emphasize vehicle routing problems. Uncertainty in vehicle availability and increasing routing cost are common barriers during the COVID19 pandemic [99]. Poor systems connectivity and reluctance to share information are the next focusing barriers for SMEs. In this COVID-19 pandemic, information sharing is a must for SCC [100,101]. After that, SME practitioners should focus on the lack of trust and leadership deficit barriers. Trust issues are crucial during this pandemic [102]. The other SCC barriers should be in limelight according to the rankings. The management approaches to overcome SCC barriers will help to enhance the resilience and sustainability performances of SMEs. In this way, SME practitioners can eradicate the barriers according to their rankings and improve overall supply chain performance.

During this COVID-19 outbreak, collaboration in supply chains has been greatly disrupted. This study will help SME practitioners to build back a resilient supply chain by enhancing SCC practices. Accordingly, a resilient supply chain can positively impact sustainability performances [19]. For example, as lack of communication is the most crucial barrier, SME practitioners should focus on local suppliers and buyers rather than communicating with foreign buyers or suppliers [103]. Besides, flexible communication services and synchronized information systems are great supply chain resilient strategies for achieving SCC $[104,105]$. Vehicle routing problems will encourage supply chain partners to adopt resilient logistics facilities which will help to improve supply chain recovery. Financial supports from the government and incentives from suppliers will enhance to achieve economic sustainability [106]. Also, overcoming SCC barriers will enhance the supply chain recovery from the impacts of large-scale disruptions such as the COVID19 pandemic [107]. In this way, the barriers found from the study will encourage SME managers to establish various resilient strategies to attain sustainability.

This study makes several contributions to the existing literature. Several studies emphasized the importance of SCC for maintaining and improving the performance of SMEs $[10,11]$. However, there is a lack of studies that provided strategic directions on how SMEs can collaborate and what barriers they face in the process of collaboration. This study contributes to the literature on SCC in SMEs by providing the SCC barriers that these firms face in collaborating. Moreover, this study not only identifies the barriers to SCC but also analyzes and prioritizes them using MCDM methods. Besides, it focuses on SMEs of an emerging economy which is scarce at present in the literature. Another notable contribution of the study is that it used the data collected from multiple industries. Since the findings, SCC barriers and their ranking represent a wide variety of industries and these can be considered generalizable to various SME sectors.

\section{Conclusions}

Approximately $99 \%$ of formal business enterprises of Bangladesh are SMEs, which contribute 25 percent to the national GDP. Business decisions are dominated by the glob- 
alization of markets and increased competition among firms. To survive in this competitive world, SMEs must cooperate well regarding business structures and supply chain management. Most of the firms of Bangladesh are not aware of potential collaborative networks they could utilize. This paper enables the firms to identify the barriers of collaboration and helps them move past these barriers to improve their resilience and sustainability performances.

We found that lack of communication is the most important SCC barrier that restricts collaboration among firms. The identified barriers such as poor information quality, information flow disruption, and vehicle routing problems also are major barriers. This study extends the literature of supply chain management by developing a decision-modeling framework for analyzing SCC barriers in the context of SMEs of an emerging economy. This study also expands knowledge of MCDM by comparing the results obtained from FBWM and Grey DEMATEL methods. Most empirical data are vague, and decision-makers often feel confused when comparing different barriers. Linguistic variables can remedy this, by providing more reliable and consistent results. Both of the methods we employed contained linguistic scales, triangular fuzzy numbers, and Grey numbers. This helps mitigates ambiguity for decision-makers. Cause-effect relationships plotted in prominence-relation graphs enable managers to readily identify high relation and prominence (intertwined and giver) barriers. Using tools such as these, managers can take proactive measures to overcome SCC barriers and to improve the sustainability and resilience performance in their supply chains.

This research does have limitations. Although we focused on several SME sectors, our sample size is small. More barriers may be included by collecting data from more samples. Moreover, a future study could conduct a cross-sector comparison. Such a study would be valuable as it would provide more in-depth knowledge to understand how SMEs in various industries face challenges in collaboration. Finally, given that each MCDM method has certain shortcomings and benefits, several other methods can be combined to achieve more comprehensive results.

Author Contributions: Conceptualization, P.M., S.K.P.; methodology, P.M., S.K.P., A.A.; formal analysis, P.M.; investigation, A.A.; writing—original draft preparation, P.M., S.K.P., P.C.; writingreview and editing, S.K.P., A.A., P.C.; supervision, A.A.; project administration, S.K.P. All authors have read and agreed to the published version of the manuscript.

Funding: This research received no external funding.

Institutional Review Board Statement: Not applicable.

Informed Consent Statement: Informed consent was obtained from all subjects involved in the study.

Data Availability Statement: Not applicable.

Acknowledgments: This research work was carried out at the Department of Industrial and Production Engineering of Bangladesh University of Engineering and Technology (BUET) under the supervision of Abdullahil Azeem. The authors acknowledge the supports received from the Department of Industrial and Production Engineering of BUET to carry out the research work successfully.

Conflicts of Interest: The authors declare no conflict of interest.

\section{Appendix A}

Q.1. Which type of company/department are you working at?

Q.2. What is your designation and experience/role in your company/university?

Q.3. Are the listed supply chain collaboration barriers suitable in the context of SMEs of Bangladesh?

If the barrier is suitable for the lack of supply chain collaboration in the context of the

SMEs of Bangladesh, please write Yes and If the barrier is not relevant, please write No. Further, please mention your recommendation about any additional barrier if necessary. 
Table A1. SCC barriers for taking responses from respondents (experts).

\begin{tabular}{ccc}
\hline Barriers & Response (Yes/No) & Barriers \\
\hline Lack of commitment on quality & - & Cultural difference \\
Lack of communication & - & Unwillingness to deal with new firms \\
Poor systems connectivity & - & $\begin{array}{c}\text { Reluctance to deal with small firms } \\
\text { Governmental intervention }\end{array}$ \\
Intra-organizational weak relationships & - & Vehicle routing problem \\
Decision desynchronization & - & Customer taste variations \\
Incentive obstacles & - & Lack of adaptation \\
Lack of trust & - & Different goals \\
Leadership deficit & - & Non-standardized performance metrics \\
Territoriality & - & Demand fluctuations \\
Forecasting problem \\
Opposition to change & - & Lead time variations \\
Short term relationship & - & Reluctance to share information \\
Pricing obstacles & - & Information flow disruption \\
Lack of Resource sharing & - & Poor information quality \\
Behavioral uncertainty & - & -
\end{tabular}

\section{Appendix B}

Q.1 Please select the best (e.g., the most important) and the worst (e.g., the least important) barrier from the main group of barriers.

Select the best/worst criteria by putting tick marks beside the corresponding boxes of your selected barriers. Please select only one barrier as best and another one as worst.

Table A2. Selection of best and worst barriers.

\begin{tabular}{|c|c|c|c|c|c|}
\hline Category & Best & Worst & Barrier Name & Best & Worst \\
\hline \multirow{3}{*}{ Information related barriers (A) } & - & - & Reluctance to share information (A1) & - & - \\
\hline & - & - & Information flow disruption (A2) & - & - \\
\hline & - & - & Poor information quality (A3) & - & - \\
\hline \multirow{3}{*}{ Communication-related barriers (B) } & - & - & Lack of communication (B1) & - & - \\
\hline & - & - & Poor systems connectivity (B2) & - & - \\
\hline & - & - & Vehicle routing problem (B3) & - & - \\
\hline \multirow{5}{*}{ Intra-organizational barriers $(C)$} & - & - & Intra-organizational weak relationships (C1) & - & - \\
\hline & - & - & Decision desynchronization (C2) & - & - \\
\hline & - & - & Incentive obstacles (C3) & - & - \\
\hline & - & - & Opposition to change (C4) & - & - \\
\hline & - & - & Leadership deficit (C5) & - & - \\
\hline \multirow{12}{*}{ Inter-organizational barriers (D) } & - & - & Lack of trust (D1) & - & - \\
\hline & - & - & Short term relationship (D2) & - & - \\
\hline & - & - & Behavioral uncertainty (D3) & - & - \\
\hline & - & - & Cultural difference (D4) & - & - \\
\hline & - & - & Different goals (D5) & - & - \\
\hline & - & - & Non-standardized performance metrics (D6) & - & - \\
\hline & - & - & Lack of commitment on quality (D7) & - & - \\
\hline & - & - & Forecasting problem (D8) & - & - \\
\hline & - & - & Reluctance to deal with small firms (D9) & - & - \\
\hline & - & - & Customer taste variations (D10) & - & - \\
\hline & - & - & Governmental intervention (D11) & - & - \\
\hline & - & - & Lack of commitment on delivery time (D12) & - & - \\
\hline
\end{tabular}

Q.2. Please fill up the following comparison vectors by indicating the degree of importance between barriers.

Scales description:

EI: equally important WI: weakly important; FI: fairly important; VI: very important; AI: absolutely important

Table A3. Scaling of the best category over other categories (best-to-others pairwise comparison vectors).

\begin{tabular}{ccccc}
\hline Most Important Category & A & B & C & D \\
\hline- & - & - & - & - \\
\hline
\end{tabular}


Table A4. Scaling of other categories over the worst category (others-to-worst pairwise comparison vectors).

\begin{tabular}{cc}
\hline Other Categories & Least Important Category \\
\hline A & - \\
B & - \\
C & - \\
D & - \\
\hline
\end{tabular}

Table A5. Scaling of the best barrier over other barriers under Information related barriers (best-toothers pairwise comparison vectors).

\begin{tabular}{cccc}
\hline Most Important Barrier & A1 & A2 & A3 \\
\hline- & - & - & - \\
\hline
\end{tabular}

Table A6. Scaling of other barriers over the worst barrier under Information related barriers (othersto-worst pairwise comparison vectors).

\begin{tabular}{cc}
\hline Other Barriers & Least Important Barrier \\
\hline A1 & - \\
A2 & - \\
A3 & - \\
\hline
\end{tabular}

Table A7. Scaling of the best barrier over other barriers under Communication-related barriers (best-to-others pairwise comparison vectors).

\begin{tabular}{cccc}
\hline Most Important Barrier & B1 & B2 & B3 \\
\hline- & - & - & - \\
\hline
\end{tabular}

Table A8. Scaling of other barriers over the worst barrier under Communication-related barriers (others-to-worst pairwise comparison vectors).

\begin{tabular}{cc}
\hline Other Barriers & Least Important Barrier \\
\hline B1 & - \\
B2 & - \\
B3 & - \\
\hline
\end{tabular}

Table A9. Scaling of the best barrier over other barriers under Intra-organizational barriers (best-toothers pairwise comparison vectors).

\begin{tabular}{cccccc}
\hline Most Important Barrier & C1 & C2 & C3 & C4 & C5 \\
\hline- & - & - & - & - & - \\
\hline
\end{tabular}

Table A10. Scaling of other barriers over the worst barrier under Intra-organizational barriers (others-to-worst pairwise comparison vectors).

\begin{tabular}{cc}
\hline Other Sub Barriers & Least Important Barrier \\
\hline C1 & - \\
C2 & - \\
C3 & - \\
C4 & - \\
C5 & - \\
\hline
\end{tabular}


Table A11. Scaling of the best barrier over other barriers under Inter-organizational barriers (best-to-others pairwise comparison vectors).

\begin{tabular}{|c|c|c|c|c|c|c|c|c|c|c|c|c|}
\hline Most Important Barrier & D1 & D2 & D3 & D4 & D5 & D6 & D7 & D8 & D9 & D10 & D11 & D12 \\
\hline- & - & - & - & - & - & - & - & - & - & - & - & - \\
\hline
\end{tabular}

Table A12. Scaling of other barriers over the worst barrier under Inter-organizational barriers (others-to-worst pairwise comparison vectors).

\begin{tabular}{cccc}
\hline Other Barriers & Least Important Barrier & Other Barriers & Least Important Barrier \\
\hline D1 & - & D7 & - \\
D2 & - & D8 & - \\
D3 & - & D9 & - \\
D4 & - & D10 & - \\
D5 & - & D11 & - \\
D6 & - & D12 & - \\
\hline
\end{tabular}

\section{Appendix C}

Table A13. Grey Direct Relation Matrix for the categories by Expert 1.

\begin{tabular}{ccccc}
\hline Categories & A & B & C & D \\
\hline A & $(0,0)$ & $(0.5,0.75)$ & $(0.25,0.5)$ & $(0.5,0.75)$ \\
B & $(0.75,1)$ & $(0,0)$ & $(0.25,0.5)$ & $(0.75,1)$ \\
C & $(0.75,1)$ & $(0.25,0.5)$ & $(0,0)$ & $(0.5,0.75)$ \\
D & $(0,0.25)$ & $(0.25,0.5)$ & $(0,0.25)$ & $(0,0)$ \\
\hline
\end{tabular}

Table A14. Grey Direct Relation Matrix for the categories by Expert 2.

\begin{tabular}{ccccc}
\hline Categories & A & B & C & D \\
\hline A & $(0,0)$ & $(0.5,0.75)$ & $(0.25,0.5)$ & $(0.5,0.75)$ \\
B & $(0.75,1)$ & $(0,0)$ & $(0,0.25)$ & $(0.75,1)$ \\
C & $(0.75,1)$ & $(0.25,0.5)$ & $(0,0)$ & $(0.5,0.75)$ \\
D & $(0,0.25)$ & $(0.25,0.5)$ & $(0,0.25)$ & $(0,0)$ \\
\hline
\end{tabular}

Table A15. Average Direct Relation Matrix for the categories from 16 experts' feedback.

\begin{tabular}{ccccc}
\hline Categories & A & B & C & D \\
\hline A & $(0,0)$ & $(0.5,0.75)$ & $(0.25,0.5)$ & $(0.5,0.75)$ \\
B & $(0.75,1)$ & $(0,0)$ & $(0.17,0.42)$ & $(0.75,1)$ \\
C & $(0.67,0.92)$ & $(0.25,0.5)$ & $(0,0)$ & $(0.5,0.75)$ \\
D & $(0,0.25)$ & $(0.08,0.33)$ & $(0.25,0.5)$ & $(0,0)$ \\
\hline
\end{tabular}

Table A16. Total Relation Matrix $\left(T=N(I-N)^{-1}\right)$ for the categories.

\begin{tabular}{ccccc}
\hline Categories & A & B & C & D \\
\hline A & 0.3774 & 0.5627 & 0.3259 & 0.7297 \\
B & 0.7402 & 0.4069 & 0.3332 & 0.9039 \\
C & 0.6940 & 0.5099 & 0.2158 & 0.7671 \\
D & 0.1909 & 0.2650 & 0.1382 & 0.2069 \\
\hline
\end{tabular}


Table A17. Cause/effect parameters and relative weights for the categories and the barriers.

\begin{tabular}{|c|c|c|c|c|c|}
\hline $\begin{array}{c}\text { Categories/ } \\
\text { Barriers }\end{array}$ & Row Sum $\left(r_{i}\right)$ & Column Sum $\left(c_{j}\right)$ & $r_{i}+c_{j}$ & $r_{i}-c_{j}$ & $\begin{array}{c}\text { Relative Degrees of } \\
\text { Interactions (Weights), } \\
\qquad w_{i}=\frac{r_{i}+c_{j}}{\sum_{i=1}^{n}\left(r_{i}+c_{j}\right)}\end{array}$ \\
\hline A & 1.9957 & 2.0025 & 3.9982 & -0.0068 & 0.2713 \\
\hline $\mathrm{B}$ & 2.3843 & 1.7446 & 4.1289 & 0.6397 & 0.2802 \\
\hline $\mathrm{C}$ & 2.1868 & 1.0130 & 3.1998 & 1.1738 & 0.2171 \\
\hline $\mathrm{D}$ & 0.8010 & 2.6077 & 3.4087 & -1.8067 & 0.2313 \\
\hline A1 & 1.3462 & 1.8101 & 3.1563 & -0.4640 & 0.2750 \\
\hline A2 & 2.3322 & 1.3767 & 3.7089 & 0.9554 & 0.3231 \\
\hline A3 & 2.0612 & 2.5527 & 4.6139 & -0.4915 & 0.4019 \\
\hline B1 & 1.0251 & 1.7454 & 2.7705 & -0.7203 & 0.4280 \\
\hline B2 & 1.1429 & 0.5555 & 1.6983 & 0.5874 & 0.2624 \\
\hline B3 & 1.0683 & 0.9353 & 2.0036 & 0.1329 & 0.3096 \\
\hline $\mathrm{C} 1$ & 3.1561 & 2.8595 & 6.0155 & 0.2966 & 0.1919 \\
\hline $\mathrm{C} 2$ & 2.7126 & 3.8772 & 6.5897 & -1.1646 & 0.2102 \\
\hline C3 & 2.8179 & 2.4461 & 5.2640 & 0.3718 & 0.1679 \\
\hline $\mathrm{C} 4$ & 3.5270 & 2.5852 & 6.1122 & 0.9419 & 0.1950 \\
\hline C5 & 3.4583 & 3.9039 & 7.3622 & -0.4457 & 0.2349 \\
\hline D1 & 2.2816 & 1.4578 & 3.7394 & 0.8239 & 0.1190 \\
\hline D2 & 1.4501 & 1.8484 & 3.2985 & -0.3983 & 0.1050 \\
\hline D3 & 1.4598 & 1.5173 & 2.9771 & -0.0575 & 0.0948 \\
\hline D4 & 1.5572 & 1.2430 & 2.8002 & 0.3142 & 0.0891 \\
\hline D5 & 1.3606 & 1.8658 & 3.2265 & -0.5052 & 0.1027 \\
\hline D6 & 0.5717 & 0.6114 & 1.1831 & -0.0397 & 0.0377 \\
\hline D7 & 0.9721 & 1.9073 & 2.8794 & -0.9352 & 0.0917 \\
\hline D8 & 1.1404 & 0.4096 & 1.5501 & 0.7308 & 0.0493 \\
\hline D9 & 1.2506 & 1.4221 & 2.6727 & -0.1715 & 0.0851 \\
\hline D10 & 0.2652 & 1.1497 & 1.4149 & -0.8846 & 0.0450 \\
\hline D11 & 1.6456 & 0.6482 & 2.2938 & 0.9973 & 0.0730 \\
\hline D12 & 1.7511 & 1.6253 & 3.3764 & 0.1258 & 0.1075 \\
\hline
\end{tabular}

\section{Appendix D}

Table A18. Weights assigned for experts during sensitivity analysis.

\begin{tabular}{|c|c|c|c|c|c|c|c|c|c|c|c|c|c|c|c|c|}
\hline \multirow{2}{*}{ Scenarios } & \multicolumn{16}{|c|}{ Expert No. } \\
\hline & 1 & 2 & 3 & 4 & 5 & 6 & 7 & 8 & 9 & 10 & 11 & 12 & 13 & 14 & 15 & 16 \\
\hline 1 & 0.1 & 0.06 & 0.06 & 0.06 & 0.06 & 0.06 & 0.06 & 0.06 & 0.06 & 0.06 & 0.06 & 0.06 & 0.06 & 0.06 & 0.06 & 0.06 \\
\hline 2 & 0.06 & 0.1 & 0.06 & 0.06 & 0.06 & 0.06 & 0.06 & 0.06 & 0.06 & 0.06 & 0.06 & 0.06 & 0.06 & 0.06 & 0.06 & 0.06 \\
\hline 3 & 0.06 & 0.06 & 0.1 & 0.06 & 0.06 & 0.06 & 0.06 & 0.06 & 0.06 & 0.06 & 0.06 & 0.06 & 0.06 & 0.06 & 0.06 & 0.06 \\
\hline 4 & 0.06 & 0.06 & 0.06 & 0.1 & 0.06 & 0.06 & 0.06 & 0.06 & 0.06 & 0.06 & 0.06 & 0.06 & 0.06 & 0.06 & 0.06 & 0.06 \\
\hline 5 & 0.06 & 0.06 & 0.06 & 0.06 & 0.1 & 0.06 & 0.06 & 0.06 & 0.06 & 0.06 & 0.06 & 0.06 & 0.06 & 0.06 & 0.06 & 0.06 \\
\hline 6 & 0.06 & 0.06 & 0.06 & 0.06 & 0.06 & 0.1 & 0.06 & 0.06 & 0.06 & 0.06 & 0.06 & 0.06 & 0.06 & 0.06 & 0.06 & 0.06 \\
\hline 7 & 0.06 & 0.06 & 0.06 & 0.06 & 0.06 & 0.06 & 0.1 & 0.06 & 0.06 & 0.06 & 0.06 & 0.06 & 0.06 & 0.06 & 0.06 & 0.06 \\
\hline 8 & 0.06 & 0.06 & 0.06 & 0.06 & 0.06 & 0.06 & 0.06 & 0.1 & 0.06 & 0.06 & 0.06 & 0.06 & 0.06 & 0.06 & 0.06 & 0.06 \\
\hline 9 & 0.06 & 0.06 & 0.06 & 0.06 & 0.06 & 0.06 & 0.06 & 0.06 & 0.1 & 0.06 & 0.06 & 0.06 & 0.06 & 0.06 & 0.06 & 0.06 \\
\hline 10 & 0.06 & 0.06 & 0.06 & 0.06 & 0.06 & 0.06 & 0.06 & 0.06 & 0.06 & 0.1 & 0.06 & 0.06 & 0.06 & 0.06 & 0.06 & 0.06 \\
\hline 11 & 0.06 & 0.06 & 0.06 & 0.06 & 0.06 & 0.06 & 0.06 & 0.06 & 0.06 & 0.06 & 0.1 & 0.06 & 0.06 & 0.06 & 0.06 & 0.06 \\
\hline 12 & 0.06 & 0.06 & 0.06 & 0.06 & 0.06 & 0.06 & 0.06 & 0.06 & 0.06 & 0.06 & 0.06 & 0.1 & 0.06 & 0.06 & 0.06 & 0.06 \\
\hline 13 & 0.06 & 0.06 & 0.06 & 0.06 & 0.06 & 0.06 & 0.06 & 0.06 & 0.06 & 0.06 & 0.06 & 0.06 & 0.1 & 0.06 & 0.06 & 0.06 \\
\hline 14 & 0.06 & 0.06 & 0.06 & 0.06 & 0.06 & 0.06 & 0.06 & 0.06 & 0.06 & 0.06 & 0.06 & 0.06 & 0.06 & 0.1 & 0.06 & 0.06 \\
\hline 15 & 0.06 & 0.06 & 0.06 & 0.06 & 0.06 & 0.06 & 0.06 & 0.06 & 0.06 & 0.06 & 0.06 & 0.06 & 0.06 & 0.06 & 0.1 & 0.06 \\
\hline 16 & 0.06 & 0.06 & 0.06 & 0.06 & 0.06 & 0.06 & 0.06 & 0.06 & 0.06 & 0.06 & 0.06 & 0.06 & 0.06 & 0.06 & 0.06 & 0.1 \\
\hline
\end{tabular}


Table A19. Ranking of barriers of supply chain collaboration during sensitivity analysis for Grey DEMATEL method.

\begin{tabular}{|c|c|c|c|c|c|c|c|c|c|c|c|c|c|c|c|c|c|}
\hline \multirow{2}{*}{ Barriers } & \multicolumn{17}{|c|}{ Scenarios } \\
\hline & Normal & 1 & 2 & 3 & 4 & 5 & 6 & 7 & 8 & 9 & 10 & 11 & 12 & 13 & 14 & 15 & 16 \\
\hline A1 & 5 & 5 & 5 & 6 & 5 & 5 & 6 & 5 & 5 & 5 & 6 & 6 & 5 & 5 & 5 & 5 & 5 \\
\hline $\mathrm{A} 2$ & 3 & 3 & 3 & 3 & 4 & 3 & 4 & 3 & 4 & 4 & 3 & 3 & 3 & 3 & 3 & 4 & 3 \\
\hline A3 & 2 & 2 & 2 & 2 & 2 & 2 & 2 & 2 & 2 & 2 & 2 & 2 & 2 & 2 & 2 & 2 & 2 \\
\hline $\mathrm{B} 1$ & 1 & 1 & 1 & 1 & 1 & 1 & 1 & 1 & 1 & 1 & 1 & 1 & 1 & 1 & 1 & 1 & 1 \\
\hline B2 & 6 & 6 & 6 & 5 & 6 & 6 & 5 & 6 & 6 & 6 & 5 & 5 & 6 & 6 & 6 & 6 & 6 \\
\hline B3 & 4 & 4 & 4 & 4 & 3 & 4 & 3 & 4 & 3 & 3 & 4 & 4 & 4 & 4 & 4 & 3 & 4 \\
\hline $\mathrm{C} 1$ & 10 & 10 & 9 & 10 & 10 & 10 & 10 & 10 & 9 & 10 & 10 & 10 & 10 & 10 & 10 & 10 & 10 \\
\hline $\mathrm{C} 2$ & 8 & 8 & 8 & 8 & 8 & 8 & 8 & 8 & 8 & 8 & 8 & 8 & 8 & 8 & 8 & 8 & 8 \\
\hline C3 & 11 & 11 & 11 & 11 & 11 & 11 & 11 & 11 & 11 & 11 & 11 & 11 & 11 & 11 & 11 & 11 & 11 \\
\hline $\mathrm{C} 4$ & 9 & 9 & 9 & 9 & 9 & 9 & 9 & 9 & 10 & 9 & 9 & 9 & 9 & 9 & 9 & 9 & 9 \\
\hline C5 & 7 & 7 & 7 & 7 & 7 & 7 & 7 & 7 & 7 & 7 & 7 & 7 & 7 & 7 & 7 & 7 & 7 \\
\hline D1 & 12 & 12 & 12 & 12 & 12 & 12 & 12 & 12 & 12 & 12 & 12 & 12 & 12 & 12 & 12 & 12 & 12 \\
\hline D2 & 14 & 14 & 14 & 14 & 14 & 14 & 14 & 14 & 14 & 14 & 14 & 14 & 14 & 14 & 14 & 14 & 14 \\
\hline D3 & 16 & 16 & 16 & 16 & 16 & 16 & 16 & 16 & 16 & 16 & 16 & 16 & 16 & 16 & 16 & 16 & 16 \\
\hline D4 & 18 & 18 & 18 & 18 & 18 & 18 & 18 & 18 & 18 & 18 & 18 & 18 & 18 & 18 & 18 & 18 & 18 \\
\hline D5 & 15 & 15 & 15 & 15 & 15 & 15 & 15 & 15 & 15 & 15 & 15 & 15 & 15 & 15 & 15 & 15 & 15 \\
\hline D6 & 23 & 23 & 23 & 23 & 23 & 23 & 23 & 23 & 23 & 23 & 23 & 23 & 23 & 23 & 23 & 23 & 23 \\
\hline D7 & 17 & 17 & 17 & 17 & 17 & 17 & 17 & 17 & 17 & 17 & 17 & 17 & 17 & 17 & 17 & 17 & 17 \\
\hline D8 & 21 & 21 & 21 & 21 & 21 & 21 & 21 & 21 & 21 & 21 & 21 & 21 & 21 & 21 & 21 & 21 & 21 \\
\hline D9 & 19 & 19 & 19 & 19 & 19 & 19 & 19 & 19 & 19 & 19 & 19 & 19 & 19 & 19 & 19 & 19 & 19 \\
\hline D10 & 22 & 22 & 22 & 22 & 22 & 21 & 22 & 22 & 22 & 22 & 22 & 22 & 22 & 22 & 22 & 22 & 22 \\
\hline D11 & 20 & 20 & 20 & 20 & 20 & 20 & 20 & 20 & 20 & 20 & 20 & 20 & 20 & 20 & 20 & 20 & 20 \\
\hline D12 & 13 & 13 & 13 & 13 & 13 & 13 & 13 & 13 & 13 & 13 & 13 & 13 & 13 & 13 & 13 & 13 & 13 \\
\hline
\end{tabular}

Table A20. Ranking of barriers of supply chain collaboration during sensitivity analysis for Fuzzy Best-Worst method.

\begin{tabular}{|c|c|c|c|c|c|c|c|c|c|c|}
\hline \multirow{2}{*}{ Barriers } & \multicolumn{10}{|c|}{ Changing Weights of "Lack of Communication (B1)" } \\
\hline & Normal & 0.1 & 0.2 & 0.3 & 0.4 & 0.5 & 0.6 & 0.7 & 0.8 & 0.9 \\
\hline A1 & 6 & 5 & 6 & 6 & 6 & 6 & 6 & 5 & 4 & 4 \\
\hline A2 & 3 & 4 & 3 & 3 & 4 & 3 & 3 & 3 & 3 & 3 \\
\hline A3 & 2 & 2 & 2 & 1 & 1 & 2 & 2 & 2 & 2 & 2 \\
\hline B1 & 1 & 6 & 5 & 4 & 2 & 1 & 1 & 1 & 1 & 1 \\
\hline B2 & 5 & 3 & 4 & 5 & 5 & 5 & 5 & 6 & 7 & 15 \\
\hline B3 & 4 & 1 & 1 & 2 & 3 & 4 & 4 & 4 & 5 & 12 \\
\hline C1 & 17 & 17 & 17 & 17 & 17 & 17 & 17 & 17 & 17 & 17 \\
\hline C2 & 15 & 15 & 15 & 15 & 15 & 15 & 15 & 15 & 15 & 14 \\
\hline C3 & 23 & 23 & 23 & 23 & 23 & 23 & 23 & 23 & 23 & 23 \\
\hline $\mathrm{C} 4$ & 16 & 16 & 16 & 16 & 16 & 16 & 16 & 16 & 16 & 16 \\
\hline C5 & 8 & 8 & 8 & 8 & 8 & 8 & 8 & 8 & 8 & 6 \\
\hline D1 & 7 & 7 & 7 & 7 & 7 & 7 & 7 & 7 & 6 & 5 \\
\hline D2 & 10 & 10 & 10 & 10 & 10 & 10 & 10 & 10 & 10 & 8 \\
\hline D3 & 12 & 12 & 12 & 12 & 12 & 12 & 12 & 12 & 12 & 10 \\
\hline D4 & 14 & 14 & 14 & 14 & 14 & 14 & 14 & 14 & 14 & 13 \\
\hline D5 & 10 & 10 & 10 & 10 & 10 & 10 & 10 & 10 & 10 & 8 \\
\hline D6 & 22 & 22 & 22 & 22 & 22 & 22 & 22 & 22 & 22 & 22 \\
\hline D7 & 13 & 13 & 13 & 13 & 13 & 13 & 13 & 13 & 13 & 11 \\
\hline D8 & 20 & 20 & 20 & 20 & 20 & 20 & 20 & 20 & 20 & 20 \\
\hline D9 & 18 & 18 & 18 & 18 & 18 & 18 & 18 & 18 & 18 & 18 \\
\hline D10 & 20 & 20 & 20 & 20 & 20 & 20 & 20 & 20 & 20 & 20 \\
\hline D11 & 18 & 18 & 18 & 18 & 18 & 18 & 18 & 18 & 18 & 18 \\
\hline D12 & 9 & 9 & 9 & 9 & 9 & 9 & 9 & 9 & 9 & 7 \\
\hline
\end{tabular}




\section{References}

1. Chopra, S.; Meindl, P.; Kalra, D.V. Supply Chain Management: Strategy, Planning, and Operation; Pearson: Boston, MA, USA, 2013.

2. Liao, S.-H.; Hu, D.-C.; Ding, L.-W. Assessing the influence of supply chain collaboration value innovation, supply chain capability and competitive advantage in Taiwan's networking communication industry. Int. J. Prod. Econ. 2017, 191, 143-153. [CrossRef]

3. Whipple, J.M.; Russell, D. Building supply chain collaboration: A typology of collaborative approaches. Int. J. Logist. Manag. 2007, 18, 174-196. [CrossRef]

4. Allaoui, H.; Guo, Y.; Sarkis, J. Decision support for collaboration planning in sustainable supply chains. J. Clean. Prod. 2019, 229, 761-774. [CrossRef]

5. Zhang, Q.; Cao, M. Exploring antecedents of supply chain collaboration: Effects of culture and interorganizational system appropriation. Int. J. Prod. Econ. 2018, 195, 146-157. [CrossRef]

6. Hudnurkar, M.; Jakhar, S.; Rathod, U. Factors Affecting Collaboration in Supply Chain: A Literature Review. Procedia Soc. Behav. Sci. 2014, 133, 189-202. [CrossRef]

7. Gumboh, J.; Gichira, R. Supply Chain Collaboration among SMEs in Kenya: A Review of Collaboration Barriers. Int. J. Humanit. Soc. Sci. 2015, 5, 223-229.

8. Chowdhury, P.; Paul, S.K.; Kaisar, S.; Moktadir, M.A. COVID-19 pandemic related supply chain studies: A systematic review. Transp. Res. Part. E Logist. Transp. Rev. 2021, 148, 102271. [CrossRef]

9. Wen, W.; Yang, S.; Zhou, P.; Gao, S. Impacts of COVID-19 on the electric vehicle industry: Evidence from China. Renew. Sustain. Energy Rev. 2021, 144, 111024. [CrossRef]

10. Eyaa, S.; Ntayi, J.M.; Namagembe, S. Collaborative relationships and SME supply chain performance. World J. Entrep. Manag. Sustain. Dev. 2010, 6, 233-245. [CrossRef]

11. Haji-Pakir, M.I.; Alina, S. Level of supply chain collaboration of Malaysian SME manufacturers. In Proceedings of the 2010 IEEE International Conference on Management of Innovation \& Technology, Singapore, 2-5 June 2010; Institute of Electrical and Electronics Engineers (IEEE): Piscataway, NJ, USA, 2010; pp. 169-174.

12. Roy, S.; Das, M.; Ali, S.M.; Raihan, A.S.; Paul, S.K.; Kabir, G. Evaluating strategies for environmental sustainability in a supply chain of an emerging economy. J. Clean. Prod. 2020, 262, 121389. [CrossRef]

13. Acma, M.Q. Productivity and performance evaluation of SME sector in Bangladesh: Evidence from the historical data. J. Islam. Financ. Bus. Res. 2015, 3, 14-22.

14. Abdin, M.J. Development of SMEs: Replicating Top Foreign Models. Dev. SMEs Replicating Top Foreign Model. 2014. [CrossRef]

15. Uddin, M.T. A Study on Financing of SMEs in Bangladesh. J. Econ. Sustain. Dev. 2014, 5, 161-168.

16. Appolloni, A.; Colasanti, N.; Fantauzzi, C.; Fiorani, G.; Frondizi, R. Distance Learning as a Resilience Strategy during Covid-19: An Analysis of the Italian Context. Sustainability 2021, 13, 1388. [CrossRef]

17. D'Adamo, I.; Rosa, P. How Do You See Infrastructure? Green Energy to Provide Economic Growth after COVID-19. Sustainability 2020, 12, 4738. [CrossRef]

18. Miceli, A.; Hagen, B.; Riccardi, M.P.; Sotti, F.; Settembre-Blundo, D. Thriving, Not Just Surviving in Changing Times: How Sustainability, Agility and Digitalization Intertwine with Organizational Resilience. Sustainability 2021, 13, 2052. [CrossRef]

19. D'Adamo, I.; Lupi, G. Sustainability and Resilience after COVID-19: A Circular Premium in the Fashion Industry. Sustainability 2021, 13, 1861. [CrossRef]

20. Barratt, M. Understanding the meaning of collaboration in the supply chain. Supply Chain Manag. Int. J. 2004, 9, 30-42. [CrossRef]

21. Fan, Y.; Stevenson, M.; Li, F. Supplier-initiating risk management behaviour and supply-side resilience: The effects of interpersonal relationships and dependence asymmetry in buyer-supplier relationships. Int. J. Oper. Prod. Manag. 2020, 40, 971-995. [CrossRef]

22. Prajogo, D.; Olhager, J. Supply chain integration and performance: The effects of long-term relationships, information technology and sharing, and logistics integration. Int. J. Prod. Econ. 2012, 135, 514-522. [CrossRef]

23. Anbanandam, R.; Banwet, D.K.; Shankar, R. Evaluation of supply chain collaboration: A case of apparel retail industry in India. Int. J. Product. Perform. Manag. 2011, 60, 82-98. [CrossRef]

24. Bahinipati, B.K.; Kanda, A.; Deshmukh, S. Horizontal collaboration in semiconductor manufacturing industry supply chain: An evaluation of collaboration intensity index. Comput. Ind. Eng. 2009, 57, 880-895. [CrossRef]

25. Cai, Y.-J.; Choi, T.-M. A United Nations' Sustainable Development Goals perspective for sustainable textile and apparel supply chain management. Transp. Res. Part. E Logist. Transp. Rev. 2020, 141, 102010. [CrossRef]

26. Kumar, R.; Singh, R.K. Coordination and responsiveness issues in SME supply chains: A review. Benchmarking Int. J. 2017, 24, 635-650. [CrossRef]

27. Hong, P.; Jeong, J. Supply chain management practices of SMEs: From a business growth perspective. J. Enterp. Inf. Manag. 2006, 19, 292-302. [CrossRef]

28. Zhou, H.; Li, L. The impact of supply chain practices and quality management on firm performance: Evidence from China's small and medium manufacturing enterprises. Int. J. Prod. Econ. 2020, 230, 107816. [CrossRef]

29. Quayle, M. A study of supply chain management practice in UK industrial SMEs. Supply Chain Manag. Int. J. 2003, 8, 79-86. [CrossRef]

30. Huo, B.; Zhang, C.; Zhao, X. The effect of IT and relationship commitment on supply chain coordination: A contingency and configuration approach. Inf. Manag. 2015, 52, 728-740. [CrossRef] 
31. Gunasekaran, A.; Subramanian, N.; Papadopoulos, T. Information technology for competitive advantage within logistics and supply chains: A review. Transp. Res. Part. E Logist. Transp. Rev. 2017, 99, 14-33. [CrossRef]

32. Lotfi, Z.; Mukhtar, M.; Sahran, S.; Zadeh, A.T. Information Sharing in Supply Chain Management. Procedia Technol. 2013, 11, 298-304. [CrossRef]

33. Jiang, Q.; Ke, G. Information sharing and bullwhip effect in smart destination network system. Ad Hoc Networks 2019, 87, 17-25. [CrossRef]

34. Kumar, G.; Banerjee, R.N. Supply chain collaboration index: An instrument to measure the depth of collaboration. Benchmarking Int. J. 2014, 21, 184-204. [CrossRef]

35. Simatupang, T.M.; Sridharan, R. The collaboration index: A measure for supply chain collaboration. Int. J. Phys. Distrib. Logist. Manag. 2005, 35, 44-62. [CrossRef]

36. Cai, S.; Jun, M.; Yang, Z. Implementing supply chain information integration in China: The role of institutional forces and trust. J. Oper. Manag. 2009, 28, 257-268. [CrossRef]

37. Chen, J.V.; Yen, D.C.; Rajkumar, T.; Tomochko, N.A. The antecedent factors on trust and commitment in supply chain relationships. Comput. Stand. Interfaces 2011, 33, 262-270. [CrossRef]

38. Linton, J.D. Open innovation/integration versus disintermediation/disintegration. Technovation 2018, 78, 1-3. [CrossRef]

39. Forslund, H.; Jonsson, P. Obstacles to supply chain integration of the performance management process in buyer-supplier dyads. Int. J. Oper. Prod. Manag. 2009, 29, 77-95. [CrossRef]

40. Tareq, S.; Rahman, T.; Hossain, M.; Dorrington, P. Additive manufacturing and the COVID-19 challenges: An in-depth study. J. Manuf. Syst. 2021. [CrossRef]

41. Wang, X.V.; Wang, L. A literature survey of the robotic technologies during the COVID-19 pandemic. J. Manuf. Syst. 2021. [CrossRef] [PubMed]

42. Mzougui, I.; Carpitella, S.; Certa, A.; El Felsoufi, Z.; Izquierdo, J. Assessing Supply Chain Risks in the Automotive Industry through a Modified MCDM-Based FMECA. Processes 2020, 8, 579. [CrossRef]

43. Ilyas, M.; Carpitella, S.; Zoubir, E. Designing supplier selection strategies under COVID-19 constraints for industrial environments. Procedia CIRP 2021, 100, 589-594. [CrossRef]

44. Butt, A.S. Guanxi and intra-organizational conflicts: Evidence from Chinese logistics industry. Manag. Res. Rev. 2019, 42, 495-505. [CrossRef]

45. Taqi, H.M.M.; Ahmed, H.N.; Paul, S.; Garshasbi, M.; Ali, S.M.; Kabir, G.; Paul, S.K. Strategies to Manage the Impacts of the COVID-19 Pandemic in the Supply Chain: Implications for Improving Economic and Social Sustainability. Sustainability 2020, 12, 9483. [CrossRef]

46. Fawcett, S.E.; McCarter, M.W.; Fawcett, A.M.; Webb, G.S.; Magnan, G.M. Why supply chain collaboration fails: The sociostructural view of resistance to relational strategies. Supply Chain Manag. Int. J. 2015, 20, 648-663. [CrossRef]

47. Kwon, I.G.; Louis, S.; Louis, S. Factors Affecting the Level of Trust and Commitment in Supply Chain Relationships. J. Supply Chain Manag. 2004, 40, 4-14. [CrossRef]

48. Hou, Y.; Wang, X.; Wu, Y.J.; He, P. How does the trust affect the topology of supply chain network and its resilience? An agent-based approach. Transp. Res. Part. E Logist. Transp. Rev. 2018, 116, 229-241. [CrossRef]

49. Paul, S.K.; Sarker, R.; Essam, D. Managing risk and disruption in production-inventory and supply chain systems: A review. J. Ind. Manag. Optim. 2015, 12, 1009-1029. [CrossRef]

50. Ramanathan, U.; Gunasekaran, A. Supply chain collaboration: Impact of success in long-term partnerships. Int. J. Prod. Econ. 2014, 147, 252-259. [CrossRef]

51. Wu, L.; Chiu, M.-L. Examining supply chain collaboration with determinants and performance impact: Social capital, justice, and technology use perspectives. Int. J. Inf. Manag. 2018, 39, 5-19. [CrossRef]

52. Shaw, S.; Grant, D.B.; Mangan, J. A supply chain practice-based view of enablers, inhibitors and benefits for environmental supply chain performance measurement. Prod. Plan. Control. 2021, 32, 382-396. [CrossRef]

53. Weible, C.M.; Nohrstedt, D.; Cairney, P.; Carter, D.; Crow, D.A.; Durnová, A.P.; Heikkila, T.; Ingold, K.; McConnell, A.; Stone, D. COVID-19 and the policy sciences: Initial reactions and perspectives. Policy Sci. 2020, 53, 225-241. [CrossRef]

54. Lee, H.L.; Padmanabhan, V.; Whang, S. The bullwhip effect in supply chains. Sloan Manag. Rev. 1997, 38, 93-102. [CrossRef]

55. Fynes, B.; Voss, C.; de Búrca, S. The impact of supply chain relationship quality on quality performance. Int. J. Prod. Econ. 2005, 96, 339-354. [CrossRef]

56. Walter, A. Relationship-specific factors influencing supplier involvement in customer new product development. J. Bus. Res. 2003, 56, 721-733. [CrossRef]

57. Tan, E.N.; Smith, G.; Saad, M. Managing the global supply chain: A SME perspective. Prod. Plan. Control 2006, 17, 238-246. [CrossRef]

58. Bai, C.; Sarkis, J. Honoring complexity in sustainable supply chain research: A rough set theoretic approach (SI: ResMeth). Prod. Plan. Control 2018, 29, 1367-1384. [CrossRef]

59. Vardopoulos, I. Critical sustainable development factors in the adaptive reuse of urban industrial buildings. A fuzzy DEMATEL approach. Sustain. Cities Soc. 2019, 50, 101684. [CrossRef]

60. Ozkan-Ozen, Y.D.; Kazancoglu, Y.; Mangla, S.K. Synchronized barriers for circular supply chains in industry $3.5 /$ industry 4.0 transition for sustainable resource management. Resour. Conserv. Recycl. 2020, 161, 104986. [CrossRef] 
61. Sirisawat, P.; Kiatcharoenpol, T. Fuzzy AHP-TOPSIS approaches to prioritizing solutions for reverse logistics barriers. Comput. Ind. Eng. 2018, 117, 303-318. [CrossRef]

62. Singh, S.; Misra, S.C.; Kumar, S. Identification and ranking of the risk factors involved in PLM implementation. Int. J. Prod. Econ. 2020, 222, 107496. [CrossRef]

63. Moktadir, M.A.; Ali, S.M.; Rajesh, R.; Paul, S.K. Modeling the interrelationships among barriers to sustainable supply chain management in leather industry. J. Clean. Prod. 2018, 181, 631-651. [CrossRef]

64. Moktadir, A.; Ali, S.M.; Kusi-Sarpong, S.; Shaikh, A.A. Assessing challenges for implementing Industry 4.0: Implications for process safety and environmental protection. Process. Saf. Environ. Prot. 2018, 117, 730-741. [CrossRef]

65. Kumar, A.; Dixit, G. An analysis of barriers affecting the implementation of e-waste management practices in India: A novel ISM-DEMATEL approach. Sustain. Prod. Consum. 2018, 14, 36-52. [CrossRef]

66. Kiliç, B.; Ucler, C. Stress among ab-initio pilots: A model of contributing factors by AHP. J. Air Transp. Manag. 2019, 80, 101-106. [CrossRef]

67. Gupta, H.; Barua, M.K. A framework to overcome barriers to green innovation in SMEs using BWM and Fuzzy TOPSIS. Sci. Total. Environ. 2018, 633, 122-139. [CrossRef]

68. Gandhi, N.S.; Thanki, S.J.; Thakkar, J.J. Ranking of drivers for integrated lean-green manufacturing for Indian manufacturing SMEs. J. Clean. Prod. 2018, 171, 675-689. [CrossRef]

69. Chakraborty, K.; Mondal, S.; Mukherjee, K. Critical analysis of enablers and barriers in extension of useful life of automotive products through remanufacturing. J. Clean. Prod. 2019, 227, 1117-1135. [CrossRef]

70. Kamble, S.S.; Gunasekaran, A.; Parekh, H.; Joshi, S. Modeling the internet of things adoption barriers in food retail supply chains. J. Retail. Consum. Serv. 2019, 48, 154-168. [CrossRef]

71. Kumar, A.; Dixit, G. Evaluating critical barriers to implementation of WEEE management using DEMATEL approach. Resour. Conserv. Recycl. 2018, 131, 101-121. [CrossRef]

72. Nilashi, M.; Samad, S.; Manaf, A.A.; Ahmadi, H.; Rashid, T.A.; Munshi, A.; Almukadi, W.; Ibrahim, O.; Ahmed, O.H. Factors influencing medical tourism adoption in Malaysia: A DEMATEL-Fuzzy TOPSIS approach. Comput. Ind. Eng. 2019, 137, 106005. [CrossRef]

73. Xia, X.; Govindan, K.; Zhu, Q. Analyzing internal barriers for automotive parts remanufacturers in China using grey-DEMATEL approach. J. Clean. Prod. 2015, 87, 811-825. [CrossRef]

74. Sharma, S.K.; Singh, R.; Matai, R. Force field analysis of Indian automotive strategic sourcing risk management enablers and barriers. Meas. Bus. Excel. 2018, 22, 258-275. [CrossRef]

75. Ren, J.; Tan, S.; Goodsite, M.; Sovacool, B.; Dong, L. Sustainability, shale gas, and energy transition in China: Assessing barriers and prioritizing strategic measures. Energy 2015, 84, 551-562. [CrossRef]

76. Dixit, A.; Routroy, S.; Dubey, S.K. A systematic literature review of healthcare supply chain and implications of future research. Int. J. Pharm. Health Mark. 2019, 13, 405-435. [CrossRef]

77. Lind, L.; Pirttilä, M.; Viskari, S.; Schupp, F.; Kärri, T. Working capital management in the automotive industry: Financial value chain analysis. J. Purch. Supply Manag. 2012, 18, 92-100. [CrossRef]

78. Omrani, H.; Alizadeh, A.; Emrouznejad, A. Finding the optimal combination of power plants alternatives: A multi response Taguchi-neural network using TOPSIS and fuzzy best-worst method. J. Clean. Prod. 2018, 203, 210-223. [CrossRef]

79. Liao, H.; Mi, X.; Yu, Q.; Luo, L. Hospital performance evaluation by a hesitant fuzzy linguistic best worst method with inconsistency repairing. J. Clean. Prod. 2019, 232, 657-671. [CrossRef]

80. Sahebi, I.G.; Arab, A.; Moghadam, M.R.S. Analyzing the barriers to humanitarian supply chain management: A case study of the Tehran Red Crescent Societies. Int. J. Disaster Risk Reduct. 2017, 24, 232-241. [CrossRef]

81. Wu, Q.; Zhou, L.; Chen, Y.; Chen, H. An integrated approach to green supplier selection based on the interval type-2 fuzzy best-worst and extended VIKOR methods. Inf. Sci. 2019, 502, 394-417. [CrossRef]

82. Ansari, Z.N.; Kant, R.; Shankar, R. Prioritizing the performance outcomes due to adoption of critical success factors of supply chain remanufacturing. J. Clean. Prod. 2019, 212, 779-799. [CrossRef]

83. De Silva, U.S.K.; Paul, A.; Paul, S.K.; Ali, S.M.; Chakrabortty, R.K. Examining risks and strategies for the spice processing supply chain in the context of an emerging economy. Int. J. Emerg. Mark. 2021, 1-23, in press. [CrossRef]

84. Ansari, Z.N.; Kant, R.; Shankar, R. Evaluation and ranking of solutions to mitigate sustainable remanufacturing supply chain risks: A hybrid fuzzy SWARA-fuzzy COPRAS framework approach. Int. J. Sustain. Eng. 2020, 13, 473-494. [CrossRef]

85. Garg, C.P.; Kashav, V. Evaluating value creating factors in greening the transportation of Global Maritime Supply Chains (GMSCs) of containerized freight. Transp. Res. Part. D Transp. Environ. 2019, 73, 162-186. [CrossRef]

86. Ghimire, L.P.; Kim, Y. An analysis on barriers to renewable energy development in the context of Nepal using AHP. Renew. Energy 2018, 129, 446-456. [CrossRef]

87. Khan, A.A.; Shameem, M.; Kumar, R.R.; Hussain, S.; Yan, X. Fuzzy AHP based prioritization and taxonomy of software process improvement success factors in global software development. Appl. Soft Comput. 2019, 83, 105-148. [CrossRef]

88. Saivaew, N.; Butdee, S. Decision making for effective assembly machined parts selection using fuzzy AHP and fuzzy logic. Mater. Today Proc. 2020, 26, 2265-2271. [CrossRef]

89. Guo, S.; Zhao, H. Fuzzy best-worst multi-criteria decision-making method and its applications. Knowl. Based Syst. 2017, $121,23-31$. [CrossRef] 
90. Raj, A.; Sah, B. Analyzing critical success factors for implementation of drones in the logistics sector using grey-DEMATEL based approach. Comput. Ind. Eng. 2019, 138, 106118. [CrossRef]

91. Si, S.-L.; You, X.-Y.; Liu, H.-C.; Zhang, P. DEMATEL Technique: A Systematic Review of the State-of-the-Art Literature on Methodologies and Applications. Math. Probl. Eng. 2018, 2018, 1-33. [CrossRef]

92. Fu, X.; Zhu, Q.; Sarkis, J. Evaluating green supplier development programs at a telecommunications systems provider. Int. J. Prod. Econ. 2012, 140, 357-367. [CrossRef]

93. Hwang, W.; Hsiao, B.; Chen, H.-G.; Chern, C.-C. Multiphase Assessment of Project Risk Interdependencies: Evidence from a University ISD Project in Taiwan. Proj. Manag. J. 2016, 47, 59-75. [CrossRef]

94. Mangla, S.K.; Kumar, P.; Barua, M.K. An integrated methodology of FTA and fuzzy AHP for risk assessment in green supply chain. Int. J. Oper. Res. 2016, 25, 77. [CrossRef]

95. Prakash, C.; Barua, M.K. Integration of AHP-TOPSIS method for prioritizing the solutions of reverse logistics adoption to overcome its barriers under fuzzy environment. J. Manuf. Syst. 2015, 37, 599-615. [CrossRef]

96. Rajesh, R.; Ravi, V. Modeling enablers of supply chain risk mitigation in electronic supply chains: A Grey-DEMATEL approach. Comput. Ind. Eng. 2015, 87, 126-139. [CrossRef]

97. Yin, S.; Zhang, N.; Dong, H. Preventing COVID-19 from the perspective of industrial information integration: Evaluation and continuous improvement of information networks for sustainable epidemic prevention. J. Ind. Inf. Integr. 2020, $19,100157$. [CrossRef]

98. McMaster, M.; Nettleton, C.; Tom, C.; Xu, B.; Cao, C.; Qiao, P. Risk Management: Rethinking Fashion Supply Chain Management for Multinational Corporations in Light of the COVID-19 Outbreak. J. Risk Financial Manag. 2020, 13, 173. [CrossRef]

99. Singh, S.; Kumar, R.; Panchal, R.; Tiwari, M.K. Impact of COVID-19 on logistics systems and disruptions in food supply chain. Int. J. Prod. Res. 2021, 59, 1993-2008. [CrossRef]

100. Sodhi, M.S.; Tang, C.S. Supply Chain Management for Extreme Conditions: Research Opportunities. J. Supply Chain Manag. 2021, 57, 7-16. [CrossRef]

101. Chowdhury, T.; Sarkar, A.; Paul, S.K.; Moktadir, A. A case study on strategies to deal with the impacts of COVID-19 pandemic in the food and beverage industry. Oper. Manag. Res. 2020, 1-13. [CrossRef]

102. Kumar, S.; Raut, R.D.; Narwane, V.S.; Narkhede, B.E. Applications of industry 4.0 to overcome the COVID-19 operational challenges. Diabetes Metab. Syndr. Clin. Res. Rev. 2020, 14, 1283-1289. [CrossRef]

103. Sarkis, J. Supply chain sustainability: Learning from the COVID-19 pandemic. Int. J. Oper. Prod. Manag. 2020, 41, 63-73. [CrossRef]

104. Ivanov, D.; Dolgui, A. Viability of intertwined supply networks: Extending the supply chain resilience angles towards survivability. A position paper motivated by COVID-19 outbreak. Int. J. Prod. Res. 2020, 58, 2904-2915. [CrossRef]

105. Paul, S.K.; Chowdhury, P. Strategies for Managing the Impacts of Disruptions During COVID-19: An Example of Toilet Paper. Glob. J. Flex. Syst. Manag. 2020, 21, 283-293. [CrossRef]

106. Karmaker, C.L.; Ahmed, T.; Ahmed, S.; Ali, S.M.; Moktadir, M.A.; Kabir, G. Improving supply chain sustainability in the context of COVID-19 pandemic in an emerging economy: Exploring drivers using an integrated model. Sustain. Prod. Consum. 2021, 26, 411-427. [CrossRef]

107. Paul, S.K.; Chowdhury, P. A production recovery plan in manufacturing supply chains for a high-demand item during COVID-19. Int. J. Phys. Distrib. Logist. Manag. 2021, 51, 104-125. [CrossRef] 
Article

\title{
Organization's Sustainable Operational Complexity and Strategic Overview: TISM Approach and Asian Case Studies
}

\author{
Sushil (D) and Periyasami Anbarasan *(D) \\ Department of Management Studies, Indian Institute of Technology Delhi, New Delhi 110016, India; \\ sushil@dms.iitd.ac.in \\ * Correspondence: smz168453@iitd.ac.in or jeevakarunyam@gmail.com
}

check for updates

Citation: Sushil; Anbarasan, P. Organization's Sustainable Operational Complexity and Strategic Overview: TISM Approach and Asian Case Studies. Sustainability 2021, 13, 9790. https://doi.org/10.3390/ su13179790

Academic Editor: Idiano D’Adamo

Received: 9 August 2021

Accepted: 25 August 2021

Published: 31 August 2021

Publisher's Note: MDPI stays neutral with regard to jurisdictional claims in published maps and institutional affiliations.

Copyright: (c) 2021 by the authors. Licensee MDPI, Basel, Switzerland. This article is an open access article distributed under the terms and conditions of the Creative Commons Attribution (CC BY) license (https:// creativecommons.org/licenses/by/ $4.0 /)$.

\begin{abstract}
As a region, Asia comprises communist China, democratic India and many small quasidemocratic and authoritarian states. Both China and India play a significant role in maintaining multilateral world order. Asia's regional power remains with its enormous potential of resources for domestic markets and per capita purchasing power parity. Hence, the economic and the business aspects of the Asian region require comprehensive study. Sustainable operational excellence is a notion carried by an organisation's sustainable economic development and other values. This study incorporates the multiple case study method. Twelve case organisations such as Tata Motors, Samsung, Nissan, Indigo, Mitsubishi, Huawei, Wilmar, Canon, NTPC, Hitachi, Singapore Airlines, and L\&T were chosen to study their sustainability values, and operational and strategic strands. TISM (total interpretive structural modelling) method is used for model building; four variables such as operating activities, investing activities, financing activities, and SVE (Social value expenditures) are taken for empirical analysis. Based on the available secondary data, the study incorporated panel data regression analysis. The result shows that SVE positively and significantly explains operational activities that proxy with sustainable business practices. The study concludes with a Paux strategy framework for discussion and managerial implications.
\end{abstract}

Keywords: sustainable operations; case studies; the Asian region; resilience decisions

\section{Introduction}

Organisational business activities are the central axis of the economic development of a particular region, and their performance is related to the financial position of the region. The state as an actor provides land, resources, and bureaucratic support to conduct business activities. In turn, business activities are expected by the state to cater to economic and regional development in particular. Business activities are an influential and essential source for the generation of income. Hence, business operations and operations strategy decide the opportunities and operational excellence of a particular enterprise.

In the competitive world, to sustain the market enterprise operational performance and order of merit are the pillars to maintain their status quo in the domestic and international markets. The company's position in local markets decides their equity returns and income generation, such as shareholder capital and subsidies from financial institutions. Focus on market demands, introducing new products, international market expansion with quality, and price-sensitive products are essential for operational excellence.

Asia is a unique continent with passionate philosophers such as Confucius, Gautama Buddha, and many enlightened masters fertilised the local knowledge system. Similarly, within a region, many cultural groups practise various faiths and knowledge dimensions. The Confucius ideology in China, the wide prevalence of Buddhism in the far east and south-east Asian countries, and Hinduism in India make Asia an oriental region in world politics. After globalisation, India opened its market economy in 1999, which initiated its economic growth. According to Dittmer [1], "Distance and other geographic barriers 
have diminished in power and cost, permitting people, things, and ideas to move more freely from place to place. However, the globalisation trend is not new, and there was greater demographic mobility than today in the 19th century and the early 20th centuries." The East Asian region is considered an anthropological paradise by experts. Throughout human history, historical belief and faith had built many cultural institutions or political institutions. Concerning the Asian region, familism, universalism, and cultural relativism are considered as Asian values [2].

Although those values are not confined to a particular region, Asia is a multi-cultural region with strong cultural institutions. Such sentimental attachments towards cultural institutions led to the formation of quasi-democracy and soft- authoritarian governments. However, in response to the recent crisis and disaster in east and south-east Asia leads to re-examination of their human rights standards [3]. Different philosophical belief systems, different languages with numerous dialects and these variations lead to differences in market structure, distribution systems, income groups, and uncertain stores [4]. Likewise, India's impressive eco-centre growth could centre Asia's growth and integration progressively. However, the Asian situation remains complex and challenging as the bitter history still haunts many countries in Asia [5]. "While on the other side, both south Asian nations remain caught in a vicious cycle of poverty, deprivation, and underdevelopment. Economic deprivation, illiteracy and unemployment provide a fertile ground for intolerance and extremism, which in turn promotes conflict and violence within the south Asian societies." [6].

From the strategic point of view, [7] explains four factors that add turmoil in regionalism: non-traditional security threats, territorial conflicts, arms race, and international trade negotiations; these lead to strategic uncertainty. On the other hand, [8] the south Asian context views globalisation as a boon that facilitates the availability of transportation, upliftment of the poor from a vicious circle of poverty, creation of free trade, and ultimately draws the countries closer. Therefore, fewer studies apply scientific inquiry on developing regional-based studies focused on organisational sustainability values and model building. Hence, the objective of the study is to evolve a sustainable business model for Asian regional case studies, analyse the operational complexities in their management discussions, and study what contributes towards sustainable business practices. The study applies quantitative and qualitative research methods such as multiple case studies and interpretive research methods. This study includes testing of the framework study, Pareto chart analysis, histogram analysis, and panel data analysis secondary data that are available in the chosen firms' annual reports and finally concluding with limitations and future scope of the study. The study is confined to an Asian regional analysis and the time frame of the study is 2013-2017.

\subsection{Regional Complexities: An Overview}

On 12 June 2002, China reported its first SARS (severe acute respiratory syndrome) case; such diseases are caused to people living in rural areas where global standards do not apply. SARS is attributed with 'Drama' due to its nature of sudden appearance and unfamiliarity [9]. Many questions remain unanswered concerning SARS, such as the source of origin and pathogenesis. During the peaks of the SARS pandemic, Hong Kong, Mainland China, and Singapore cancelled $50 \%$ of their airline movements. SARS provided a warning of its severe impact on health and the economy [10]. Between 2002-2003 in the SARS pandemic, 19\% of FDI (foreign direct investment) was lowered; two issues are insisted as significant outbreaks, such as governance and the need for risk diversification [11]. However, careful planning remains a vital human factor [12].

The second significant factor in regional complexity is a natural disaster. According to Frankenberg et al. [13], "Disasters are threats to population well-being that derail socioeconomic progress, strain social safety nets, and require complex assistance and recovery interventions. Over the last decade alone, Indonesia, Sri Lanka, Pakistan, China, Haiti, and Japan have experienced natural disasters with death tolls in the tens of thousands." 
Such disasters are related to human behaviour and deeply associated with cultural, social, and political contexts [14]. Natural disasters also affect the labour market and growth in income and have an undeniable effect on regional development [15].

On the other side of the socio-economic development, disaster brings numerous health challenges; public health requires safe drinking water, medical facilities, vector control, food, and shelter [16]. The existing market structure cannot separate the labour market and the public from a service point of view. The sound development policies lead to a strong labour market. Therefore, sustainability and resilience can provide a new design to regional studies that contribute to the advancement of the knowledge paradigm. Quasi-democracy, an authoritarian form of government, and bureaucratic corruption were significant contributors to the Asian financial crisis, and functions as a third important factor for regional complexity. As an integral part of society, enterprises or organisations had to oblige to the regional tension and pressure groups. Since the Asian market is a potentially promising market for investors, the concept of resilience is very much applicable in Asian regional developmental studies.

\subsection{Context of the Study}

According to United Nations ESCAP [17], "Asia-Pacific share in global FDI inflows dropped from $45 \%$ in 2018 to $35 \%$ in 2019 . However, the region remains the largest source of global outflows for the second consecutive year. The FDI is expected to remain low and below pre-crisis level throughout 2021". The gap between the rich and poor is increasing, and inequality of opportunity is also prevailing in Asia [18]. In terms of regional integration, a national income and neighbourhood are positively associated with regional integration in Asia. A low level of regional integration is found in geographically disadvantaged countries [19].

\subsection{Rationale of Asian Regional Study}

According to Claessens et al. [20], sudden shifts in market expectations and confidence were the primary source of financial turmoil. Some macro-economic fundamentals have worsened in the middle of the 1990s. Others argue that the crisis reflected structural and policy distortions [21]. Like Thailand, they had done in 1997 that developed the US dollar against the local currency. China, too attempted to devaluate the US dollar. Although the East-Asian economy was called as tiger economy, it lacked security regulations and import restrictions to develop domestic industries. Studies show that tiger economies have relatively recovered well. The IMF (International Monetary Fund) explains that the financial reason for the crisis is that the region accumulated large external deficits, property, and stock market bubbles. The same phenomenon is showing in the recent analysis of market absorption, with their increasing purchasing power. China's economic presence and 'red giant' effect in the Asian region is another reason to choose for the regional study. Japan's dominant position in automobiles and far-east proficiency on IoT (Internet of Things) in communication and progress are expected for another Asian miracle or the persistence of plaque. Such a combination of opportunities and challenges fabricated with Asian regional studies and the political dilemmas and modernisation process are the reason for choosing the Asian regional case study.

\section{Literature Review}

The study follows theme-based literature review that is focused on organizational complexity in a regional perspective. The study identifies four themes such as

(i) Imperatives of operational excellence;

(ii) Organizational Strategic Inheritance;

(iii) Resilience and regional development;

(iv) Theoretical background: Institutionalist approach. 


\subsection{Imperatives of Operational Excellence}

According to Wisner and Fawcett [22], "The greatest problem associated with traditional performance criteria is their failure to provide sufficient guidance in the formation of tactical decisions". Mere financial information on performance is insufficient; how much a firm's turnover and opportunity loss and tax loss an organisation underwent are part of financial performance. In contrast, imperatives for business excellence and performance are the essentials that firms incorporate to sustain their business in a competitive market. When the firm is under administrative changes or external changes such as regional political factors, disturbance in regional peace, and bureaucratic corruption affects sustainable operational excellence. Hence, firms' influential culture and capability to adjust incrementally over a period [23] are essential. Little evidence shows a direct connection between trust and civic cooperation towards economic performance [24]. This evidence is applicable in operational decision making. Such organisational complexity affects profits negatively. However, that might provide advantage information in certain circumstances [25].

Market competitiveness cannot be averted. Hence, only enterprise elements are essential; despite accelerated macro competitiveness, vast gaps exist, which remains an opportunity [26]. Internal enterprise capabilities to be developed to understand the opportunities available externally. Furthermore, strategic vision is essential to utilise the growing economic opportunities [27]. Although strategy and the company's magic recipes are not to be discussed or disclosed, certain universal imperatives are widely discussed by researchers and academicians that are considered essential for sustainable operational excellence practices. Organisations have to assimilate various dimensions of performance and its interconnectived elements [28].

Similarly, Calori [29] insists on the importance of managers and planners coming together in understanding creative tensions and the existence of ambivalence in organisations. Such ambivalence can be answered by employees' participation and moving from the base of the pyramid. That leads to information sharing to improve the social categorisation process in corporations [30,31]. Additionally, such associated activities introduce bias and are open to few alternatives [32].

\subsection{Strategic Organizational Inheritance}

Exogenous environments, such as the industrial environment, play a critical role in an organisation's strategic choice, financial performance, and resource heritage [33,34]. Similarly, Device and Carañana et al. [35] say that an assimilation strategy enhances the chances of international joint ventures performances. Further, various stakeholders' value systems, power, and influence in pluralistic settings bestow organisations' strategic change and development, leading to won dissolution [36]. Lack of visibility on the world scale and viewing through a historical point of view [37] may provide a monochromatic belief system in operational performances.

\subsection{Resilience and Regional Development}

Although resilience is more allied with psychology and ecological concepts, organisational scientists incorporate resilience in terms of organisation strategy and their capabilities to cope with emerging challengeable global scenarios. The path and pattern of economic growth are destabilised by regional crisis, disaster, and shocks [38]. The stability domain is a constantly changing parameters, prolonged crisis or pandemic situations, and unattended disasters led to the system's collapse or may enter another stability domain [39]. In India, the pandemic situation widely affected regular business, production system, education, and good governance [40]. Therefore, understanding how far resilience helps in regional development and change seems essential [41]. Jones et al. [42] say there are conceptual and methodological hurdles in measuring resilience. However, organisations are slowly assuming the importance of sustainability models that provide competitive advantage and reputation [43]. 


\subsection{Theoretical Background: Institutionalist Approach}

Regional development is triggered by economic and non-economic factors and collective consumption, including education facilities, health, power distribution, and political stratification. Additionally, in all these endogenous conditions there is a 'circular causation'. This is what Myrdal [44] called an institutional approach. Preston [45] states that Myrdal argued that the third world must consistently work towards socio-economic, political and cultural problems. Once the direction is set, it will continue further, which requires proper planning and development approach. The power distribution reflects the complexity of institutional arrangements among different groups and their influence in the market either directly or through a network of government regulations and political processes [46]. Likewise, Sushil [47] says any managerial context consists of the situation (time frame), actors (potential claimants), and process and resilience is necessary to cope with the situation. The doctrine of balanced growth, what Myrdal called 'holistic' in the institutionalist approach, stresses unwanted production is not production [48]. In terms of political institutionalization, statelike (structured) polities enjoy long term developmental advantages over less institutionalized polities [49] that remain a question.

\section{The Methodology of the Study}

The phenomenon involved studying with operational excellence and complexity included within the concept of sustainability. The literature review is conducted on the imperatives and attributes perspectives. For case study analysis, twelve major firms were considered: Tata Motors, Samsung, Nissan, Indigo, Mitsubishi, Huawei, Wilmar, Canon, NTPC, Hitachi, Singapore Airlines, and L\&T, focusing on the Asian region. Based on the twelve case studies, factors were collected for total interpretive structural modelling (TISM) and for validation panel data analysis. The methodology of the study is shown in Figure 1.

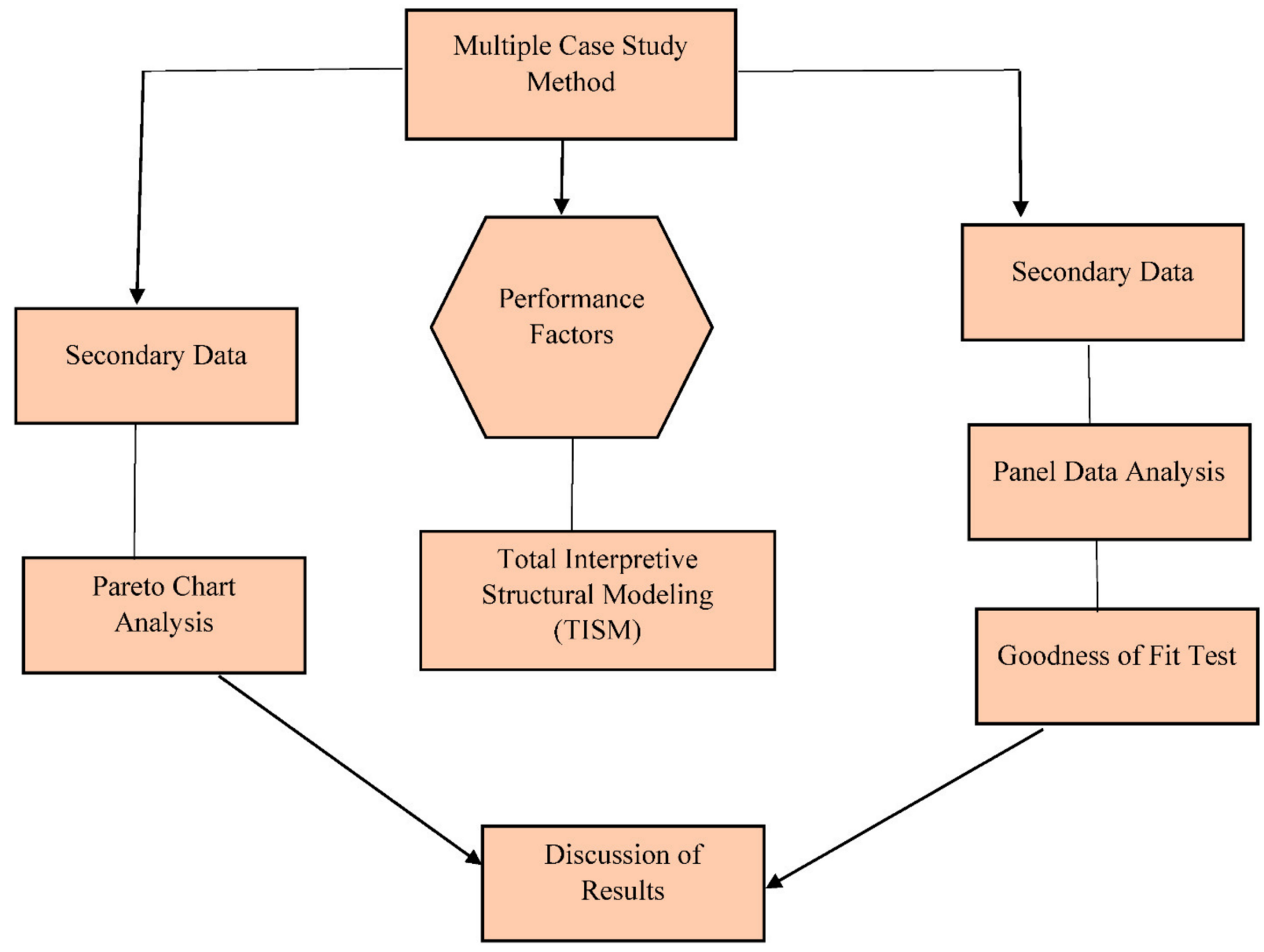

Figure 1. Methodology of the study. 


\subsection{Case Study Method}

Case studies rely on an observational technique that requires hard, deep and holistic analysis of organisational studies [50]. The case study method remains a potential strategic research framework; this particular method clears the susceptible researchers to advance towards framing knowledge body and provides theoretical and the current trend in knowledge paradigm. Although the validation requires both causal and empirical studies, the results tend to be dynamic and holistic.

Case studies provide a distinguishable body of knowledge with various decisions and what it implies [51]. Another important aspect is the generalisation of inferences obtained through the case study research method; this could be validated through regional studies, clustering the case organisations or impartial towards research approach. Hence, the ambiguity persists with the quantitative technique, the conceptual framework and factors involved within the framework will restrict the deviation of the scope of research. The most challenging aspect of the case study strategy is investigating from 'what happens' to the worth being claimed [52].

A case study enhances the researcher's observation technique and the need for an appropriate approach; it is the most popular qualitative research strategy that generates valuable theoretical insights [53]. This method may vary along several dimensions, including internal validity and precise influence related to ambiguity [54]. A case study is an essential research track in organisational science for testing and generating theory [55]. If a case paper stands and sustains within the conceptual framework, it will view the challenges involved with a case-based approach [56]. Some of the required guidelines for the case study are internal validity, external validity, and experimental reliability [57].

Multiple case studies require a careful specification of the study's nature to extend the claim and degree of generalisation [58]. Multiple case study is an adequate research strategy for when and why questions are being posed [59]. This case study method helps in research when conducting external validations [60], which cannot be possible in a single case study or comparative case study. External validation in multiple case studies means demarcating the domain and generalising the inferences [57].

\subsection{Total Interpretive Structural Modeling}

The interpretive research method is knowledge action that involves social actors in the human domain more related to critical realism [61]. Total interpretive structural modelling helps create a knowledge base based on interpretive logic and allows policymakers and decision analysts by providing transitive links and decision links [62-64]. According to Dhir and Dhir [65], "paired comparison is used to reduce the cognitive overload of elements in model building." Modified TISM helps in studying and analysing the antecedent that is suitable to the context [66]. The process of identification of relationships is repeated until the establishment of complete transitivity [67]. Modified TISM need not compare the pair of factors with transitive links [68]. There are many possibilities of permutations and combinations of relationships, and some may result in transitive and others may not [63].

\subsection{Poisson Situation}

According to Consul and Jain [69], Poisson distribution is explained as "It has also observed that in a population, supposed to be Poissonian the probability of the occurrence of an event does not remain constant. Additionally, changes with time or previous occurrences, resulting in unequal mean and variance in the data, for example, for the increase in the suicide rate, vary from time to time and particularly concerning depression period". It is used to explain the observed pattern. The Poisson situation is considered, as within a given internal time, when the event happens with a fixed period. Similarly, Thorndike [70] explains Poisson distribution as the possible number of occurrences is much larger than the average of occurrences through series of discrete points. 


\subsection{Goodness of Fit Test}

Panel data regression analysis has three different models: the fixed effect, random effect, and Ordinary Least Square (OLS). When the panel data is fed into Stata 14 software, it can obtain all three models. However, model fit relevant and appropriate to the study can be obtained only by conducting a goodness of fit test. According to Hsiao [71], "panel data could have more complicated clustering or hierarchical structure; however, in certain cases, the panel data simplifies computation and inference." Panel data provides a variety of models and general references to standards over the years [72]. Based on the obtained data, the time frame, and the variables, the models may vary. Some studies obtain a result from the fixed-effect model and random-effect model, under such circumstances, the Hausman specification test need to be conducted. Similarly, OLS regression and random effect model yield results, then the LM test need to be undertaken. That shows which model is appropriate for this study. In Asian case cluster analysis, both OLS regression and random effect model are to be conducted. The goodness of fit test (LM test) shows that the random effect model is appropriate for the study with the Prob $>$ chibar2 $=0.0000$.

\subsection{Data}

The secondary data is collected from the balance sheets of twelve firms: Tata Motors, Samsung, Nissan, Indigo, Mitsubishi, Huawei, Wilmar, Canon, NTPC, Hitachi, Singapore Airlines, and L\&T. These firms are located in the Asian region. The time frame covered five years, i.e., from 2013 to 2017. Four variables were taken for studying their operational performance, such as operating activities, investing activities, financing activities, and social value expenditure. Operating expenditure is defined as cash generated or spent on goods and services. Such as money is coming from and how it is spent, chosen variables provide such insights.

Operating activities show the cash entering into the firm through their manufacturing, distribution of goods and services, the cash position that shows spending on plants and machinery considered investing activities, similarly cash provided for debt, equity, and funding as financing activities.

Three essential investments are considered when calculating the social value expenditure, such as administrative expenses or employee benefit expenditure $(X)$, other expenses or doubtful returns $(\mathrm{Y})$, and tax expenses $(\mathrm{Z})$. Collectively referred as $\mathrm{X}+\mathrm{Y}+\mathrm{Z}=$ social value expenditure. We collected Data from 12 case organisations balance sheets of consolidated cash flow statements and social value expenditure taken from profit loss statements from the company's balance sheet between 2012-2013 to 2016-2017.

\section{Case Diagnostic Criteria and Background for Case Studies}

According to Woodside and Wilson [73], "a case study is an empirical inquiry investigating a contemporary phenomenon within its real-life context, especially when the boundaries between the phenomenon and context are not evident". Like human action, the research is also familiarised with philosophical notions. Removing the philosophical notion in case study research could seriously affect the quality of the management research [74]. Good case studies are new, interesting and theoretically relevant. Any research aims to find a new way to observe, examine new ideas, and check the theory's accuracy [75].

The case studies are taken from the Asian region, committed to sustainable values in conducting their business. All those twelve firms have had a sustainability report for more than five years. Some have as an integrated report combined as annual report and sustainability report. The background of chosen case organisations is as follows:

(1) Hitachi is committed to society by developing original technology and products. The Hitachi sustainability approach initiated the social innovation business aspires to find solutions through digital technology in social, power, finance, and health care.

(2) L\&T is one of the leading construction enterprises that focus on construction engineering and smart cities. It is committed to social development through soil conserva- 
tion structures, providing necessary infrastructure such as toilet blocks and midday meal kitchens.

(3) Nissan aims to conduct fair and efficient business activities and sustainable economic growth; it also practised ethical and environmentally sound actions in all supply chain stages.

(4) Mitsubishi Corporation is committed to contributing to society through a firmly rooted business in integrity and fairness.

(5) Huawei provides communication service across all geographic areas; it also develops supporting network stability and security, especially at critical times.

(6) Canon contributes to solving problems faced by global communities and working for sustainable societies focusing on commercial printing, network camera, health care, and industrial equipment.

(7) Wilmar has been producing a sustainability report from 2009 onwards; they are committed to reporting their palm oil and sugar productions, operations and are committed to United Nations Sustainable Development Goals (UNSDG's).

(8) InterGlobe Aviation Limited (IndiGo) is India's largest and most profitable passenger aviation company and fastest-growing carrier company with low fare as a strategy in ticketing. Indigo reach programme focuses on three themes such as children education, women empowerment, and environment.

(9) Samsung established itself as a leader in home appliances and later evolved as a prospectus player in consumer electronics, mobile communication, and the life care business. As a part of the United Nations sustainable developmental goal practices, Samsung is working towards global corporate citizenship and promote sustainable economic growth.

(10) Singapore Airlines has a sustainability strategic working committee (SSWC) that monitors the company's sustainability initiative. The airline strongly believes that its sustainability commitments are one of the main reasons for its success.

(11) NTPC (National Thermal Power Corporations) is India's largest energy-producing enterprise, committed to generating reliable power through multiple energy sources. NTPC sustainability policy is inclusive growth with a focus on neighbourhood operations.

(12) Tata Motors is a part of the Tata Group founded by Jamsetji Tata in 1868; the company is significantly contributing to India's auto-motors. Tata Motors is practising good corporate citizenship. Tata motors widely focusing on education, health, clean water, community development, and skill development.

Asian case organisations' industries and sustainability values are shown in Table 1. Table 2 shows the obtained factors that are sensitive towards sustainability and operational complexities from the case studies.

It is evident that these firms are committed to sustainable developmental values, and they make an effort to envision sustainable development goals. The study is intended to understand the complexity involved in operational decision making while bringing sustainable principles along with long-term enterprise goals regarding the planning process. The case studies provide some insights towards their sustainability understandings from a regional perspective. The questions are "What comprises sustainable development principles and what attributes are stick towards operational decisions" are vital aspects to follow in this study. 
Table 1. Asian case organisations industries and sustainability values.

\begin{tabular}{|c|c|c|}
\hline Case Organizations (Country) & Industry & Sustainability Values \\
\hline 1. Tata Motors (India) & Automotive & $\begin{array}{l}\text { Holistic approach and strategic initiative towards } \\
\text { sustainability practices }\end{array}$ \\
\hline 2. Samsung (South Korea) & Conglomerate & $\begin{array}{l}\text { Operating in an ethical manner; certified products with } \\
\text { environmental labels }\end{array}$ \\
\hline 3. Nissan (Japan) & Automotive & Listening to society and solid understanding towards stakeholders \\
\hline 4. Indigo (India) & Airlines & $\begin{array}{l}\text { Low cost and customer satisfaction and employment } \\
\text { opportunities in small cities are the fundamentals for business }\end{array}$ \\
\hline 5. Mitsubishi (Japan) & Automotive & $\begin{array}{l}\text { Create sustainable corporate value and contributing towards } \\
\text { economic development as responsible corporate citizen }\end{array}$ \\
\hline 6. Huawei (China) & Telecommunications & $\begin{array}{l}\text { creating more social values through ICT (information } \\
\text { communication technology) by providing scientific solutions }\end{array}$ \\
\hline 7. Wilmar (Singapore) & Food Processing & Strengthen labour practices and sustainability certification \\
\hline 8. Canon (Japan) & Electronics & $\begin{array}{l}\text { A growth strategy based on social and industrial shift; creating } \\
\text { new values and solving social issues }\end{array}$ \\
\hline 9. NTPC (India) & Electric Utility & $\begin{array}{l}\text { Sound corporate practices based on openness, soundness and } \\
\text { fairness }\end{array}$ \\
\hline 10. Hitachi (Japan) & Conglomerate & $\begin{array}{l}\text { Integrating sustainability into their management and } \\
\text { business practices }\end{array}$ \\
\hline 11. Singapore Airlines (Singapore) & Airline & $\begin{array}{l}\text { From farm to plane; supporting local communities and } \\
\text { sustainable operation with limited resources }\end{array}$ \\
\hline 12. L\&T (India) & Conglomerate & $\begin{array}{l}\text { Creating value in a socially responsible manner through newer } \\
\text { technologies focused on customer requirements }\end{array}$ \\
\hline
\end{tabular}

Source: Author.

Table 2. The obtained factors from the case studies.

\begin{tabular}{|c|c|c|c|c|}
\hline $\begin{array}{l}\text { 1-New Synergies for } \\
\text { Growth }\end{array}$ & 2-Business Tie-ups & $\begin{array}{l}\text { 3-Price Sensitive } \\
\text { Consumers }\end{array}$ & $\begin{array}{l}\text { 4-Technology } \\
\text { Integration }\end{array}$ & 5-Purposeful Design \\
\hline (i) Certified products & $\begin{array}{l}\text { (i) Growth strategy; } \\
\text { Industrial shift }\end{array}$ & (i) Low cost & $\begin{array}{l}\text { (i) Social values through } \\
\text { ICT }\end{array}$ & (i) Certified products \\
\hline $\begin{array}{l}\text { (ii) Sustainable } \\
\text { operations }\end{array}$ & $\begin{array}{l}\text { (ii) Corporate value and } \\
\text { economic development }\end{array}$ & (ii) Fairness in approach & $\begin{array}{l}\text { (ii) Social responsibility } \\
\text { through newer } \\
\text { Technologies }\end{array}$ & $\begin{array}{l}\text { (ii) New products and } \\
\text { listening to society }\end{array}$ \\
\hline $\begin{array}{l}\text { 6-Customer } \\
\text { Connectivity }\end{array}$ & 7-Channel Enhancement & 8-Functional Training & 9-Opportunity Loss & 10-Urban Infrastructure \\
\hline (i) Low cost & (i) Strategic initiatives & (i) Labour practices & (i) Openness & $\begin{array}{l}\text { (i) Supporting local } \\
\text { communities }\end{array}$ \\
\hline \multirow{2}{*}{$\begin{array}{l}\text { (ii) Customer } \\
\text { connectivity } \\
\text { (iii) Attending customer } \\
\text { needs }\end{array}$} & \multirow[t]{2}{*}{ (ii) Growth strategy } & \multirow[t]{2}{*}{$\begin{array}{l}\text { (ii) Integrating } \\
\text { sustainability }\end{array}$} & \multirow[t]{2}{*}{$\begin{array}{l}\text { (ii) Sound corporate } \\
\text { practices }\end{array}$} & (ii) Creating value \\
\hline & & & & $\begin{array}{l}\text { (iii) Socially responsible } \\
\text { manner }\end{array}$ \\
\hline
\end{tabular}

Source: Author.

\subsection{Attributes of Sustainable Operational Decisions}

Firms are committed to their functional values, such as answering customer needs, responding to global changes, and, at the same time, keeping their market value through meeting their fluctuating product demands. The customer needs change according to their differences in lifestyle, and needs are diverse in socio-economic conditions. Individual needs and affordability continue to remain as a driving component in customer-based business. The linear series of demand for a particular product may not continue for a long time; that sort of monologue in order may be due to any of the market phenomenon of customer curiosity. Therefore, continuous knowledge of the market and customer is essential. Global economic and political uncertainty has a significant role in the present 
political and global economic structure. Hence, the operational decisions are crucial in conducting business and sustaining profit motives.

Success in the domestic market and exploring new ventures for the international market deserve much bolstering effort and operational decisions to achieve the global market share, particularly about emerging economies. Although a new market flourishing creates positive signals, it is important to consider the infrastructure problems, local insurgency problems, inefficient bureaucracy crisis, and local currency value. The elements of sustainable operational decisions will certainly be based on a few of those mentioned above, which are shown in Table 3.

\subsection{Macro-Economic Criteria: Business Opportunities}

According to Denoon and Colbert [76], “The 1967 Bangkok Declaration called for joint endeavours to accelerate economic growth. It was not until 1976 at the Bali Summit that ASEAN committed itself to specific economic activities. These included efforts to improve global market access, cooperative approaches to international commodity issues, and cooperation in establishing region-wide industries". The critical business element of these regions is the outward-looking economies in orientation, and they have three times trade share with the world economy. Specific emphasis regarding ASEAN is rapid economic growth, open for structural change, and steady social indicators improvement [77]. The ASEAN +3 had proposed to pursue co-operation with China, Japan, and South Korea [78]. Therefore, the regional macro-economic condition provides business opportunities.

"At the Male summit in 1997, member countries agreed to work towards forming a South Asian Free Trade Area (SAFTA) by 2001. South Asian Free Trade Association (SAFTA) can treat as the third step of South Asian economic integration. South Asian Customs Union (SACU) is to be established by 2015 as the second stage, and the South Asia Economic Union (SAEU) is to be established by 2020 as the final stage of economic union" [79].

Searching for new markets both in local and international spheres requires macroeconomic insights. Where is the enterprise aspiring for channel enhancement? That particular geographic sphere's culture, individual purchasing power parity, and political structure are essentials for business performance. For example, India's newly introduced GST (goods and services tax) has a compelling impact on individual purchasing choices over a product's design and extra features. The board capital might have been inclined to open extra channels in the southern part of India. In contrast, the new bureaucratic structure might insist enterprises open an outlet in an economically backward region or economic up shooting regions. Therefore, such bureaucratic adjustments and compulsions will affect business performances and significantly impact operational performances. 
Table 3. Elements of sustainable operational decisions shown from Asian case studies.

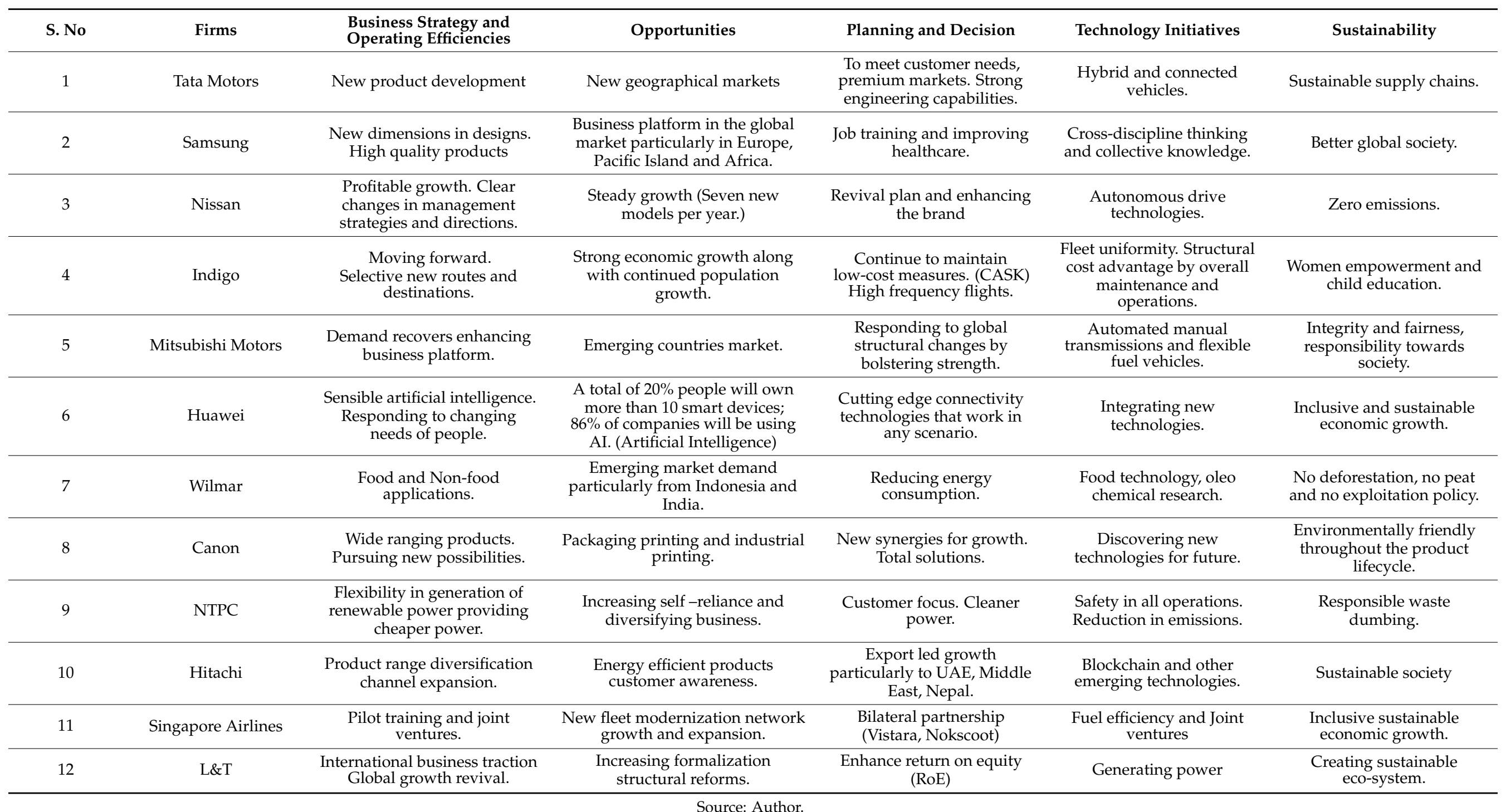




\subsection{Poisson Situation}

Operational excellence of 12 groups (firms) captured between the time trend of 20132017. Asia is an emerging economy and shows much potential for regional development and shaping the world economy. In the 1980s and early 1990s, East Asian economies (newly industrialised countries) had considered Asian tigers because of their double-digit economic growth rate, educated labour force, and potential of economic growth. However, the currency devaluation issue created the financial crisis in 1991 and became an Asian plague. The situation turned from an East Asian miracle to an Asian plague. The economic development of 'takeoff' happened due to government and IMF intervention to avoid capital flight. However, financial institutions continue to remain silent due to the lack of appropriate procedures [80]. The head of the Indonesian chamber of commerce had already warned that 25,000 Indonesian businessmen had already fled the country with an estimated USD 500 million in their briefcases [81]. The business CEO and board capital had pledged this towards the board's of chambers of commerce in terms of investments tax and duties. Therefore, corporations have to invest a considerable amount in supporting activities. The growth is expected not through knowledge intensity of technology intensity. However, their labour forces educated, indigenous improvement is limited [82]; therefore, the East Asian economies may focus on "catch up" syndrome through involvement in R\&D and expanding domestic market. The past lesson on the financial crisis and economic meltdown taught the importance of regional economic cooperation and its necessities to institutionalise interdependence. The specific situation is pictured in Figure 2.

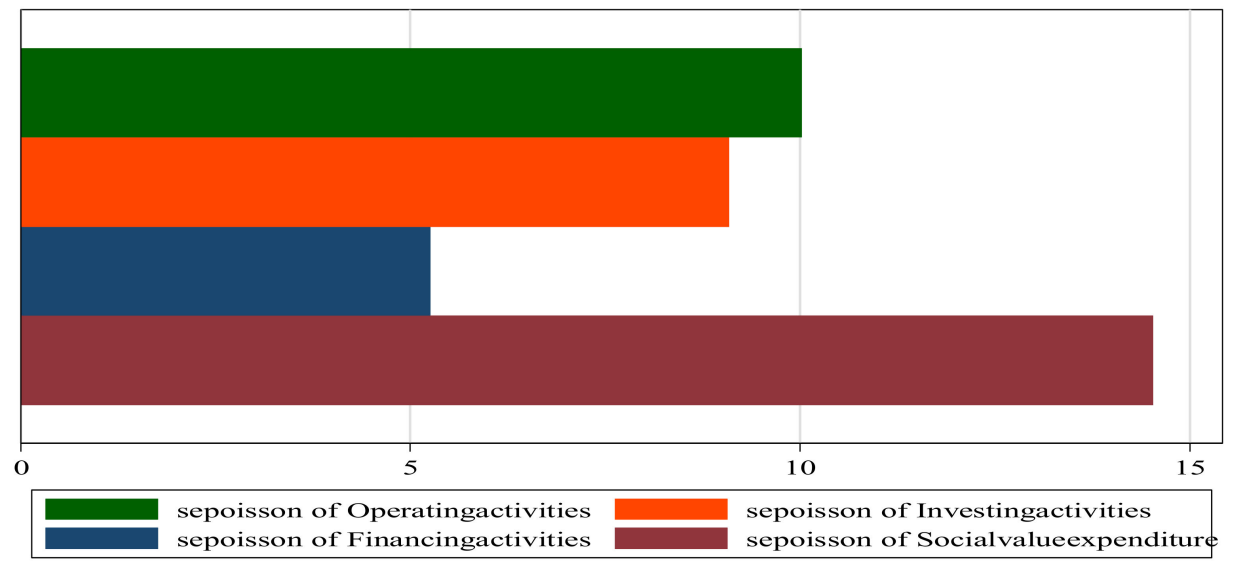

(A)

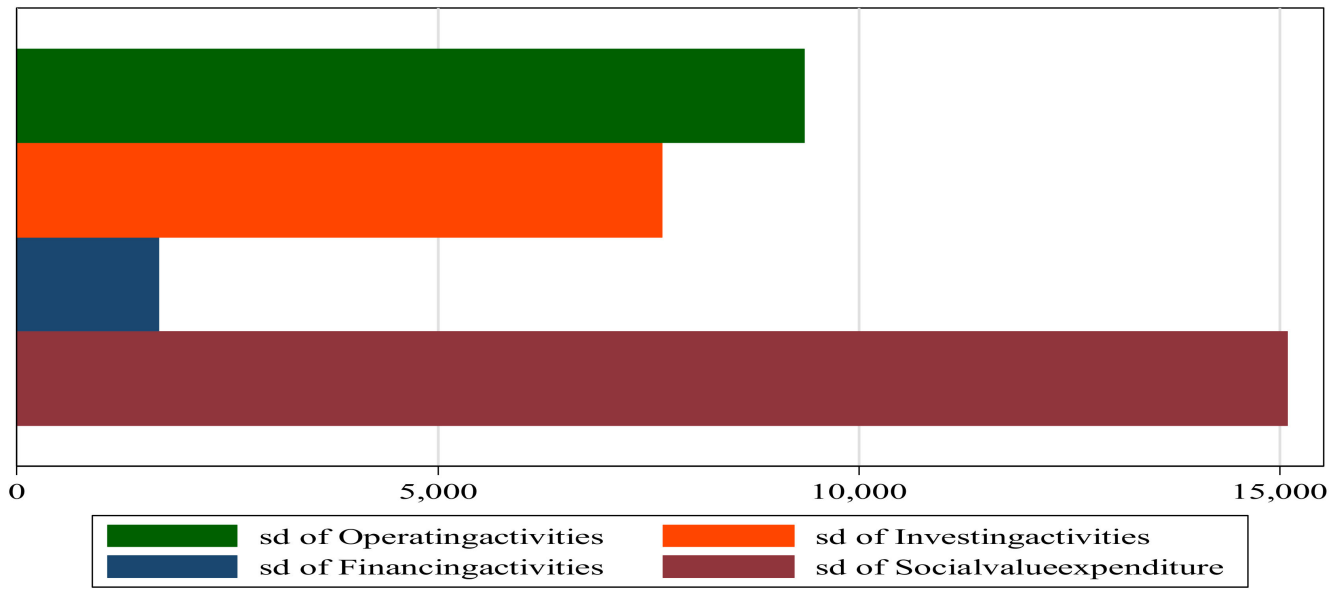

(B)

Figure 2. Fluctuations are shown in Poisson distribution from obtained case data. (A) shows the data structure with standard deviation of four factors. (B) shows the data structure with Poisson distribution of four factors. 
The Poisson situation here is: within five years (2013-2017), there were a capital push in the investing and financing (funding) activities of chosen 12 observation groups. Hence, the data fit the Poisson expectation closely and something other than random occurrence is at work. Considerable expansion on plants and machinery spending is shown in the second subfigure Figure 1 that explains the Poisson situation of twelve identified firms. Such significant financial investments and investing activities are an answer to solve the plague of earlier financial crisis or a laying stone for regional economic interdependence?

\subsection{Overall Customer Needs}

The customer needs change according to their differences in lifestyle. Flight travel is often unavoidable due to laissez-faire communication circumstances. In emerging economies like India, travel fare is also an important criterion; hence, economical and affordable travel fare extensively supports Indigo's price-sensitive consumer's policy. Similarly, Singapore Airlines is involved in onboard comfort through taking pre-orders and increasing passenger's carriages to $16 \%$. Likewise, Canon understood the customer value as a potential element in unlocking new growth potential; therefore, Canon strives to achieve production reforms through cost reductions [83]. For a successful business, Nissan figured out that customer trust is an essential element. Huawei supports customers through supporting the energy sector, transportation, and manufacturing.

\subsection{Channel Enhancement}

The successful movement of goods in domestic markets enhance enterprise opportunities to expand their fortune towards international markets. Hence, channel enhancement is an essential factor to succeed in business ventures. Channel enhancement is adjacent to expanding markets and business opportunities; it introduces new products with new features and sophisticated technologies. Choosing a location for an outlet for sustainable transportation of goods involves channel enhancement. Nissan also continues to launch attractive new products and expand new technologies [84]. Singapore Airlines explains the importance of channel enhancement in their 2012-2013 annual report: "In light of continued economic difficulties in southern Europe, reduced frequencies to Milan and Barcelona to five times weekly. While the management had suspended Athens services, a codeshare agreement with Aegean Airlines allows SIA customers to travel to Athens via London, Frankfurt, Milan and Munich. Consequently, Istanbul flights no longer linked to Athens" [85]. Channel enhancement is a one-way process and involves two-way communication that involves international market health. Similarly, L\&T says it reduces business dependence from the middle east, focusing on Egypt and African regions. Moore [86] says channel strategy is a plan that requires a product to move towards consumers through various chains of commerce. Hence, assessing sustainability in any supply chain is essential [87]. However, supply chains are becoming circular and closed loops [88]. A green supply chain helps to achieve the sustainable growth of a company [89].

\subsection{Functional Training}

Safety at work is an essential focus for L\&T business operations; L\&T practises initiatives such as digital monitoring, recording, and reviewing safety practices in construction sites. The frontline staff provides opportunities to learn leadership and professional skills, front office management, new training programmes that facilitate customer connectivity, and various functional areas of sales and marketing, reservations and ticketing, and airport operations [85]. Tata Motors focuses on 10th- and 12th-grade dropouts by giving them vocational training to prepare a 'ready supply of workforce'. The company also started training programmes in collaboration with the automotive skill development council (ASDC); the programme aims for training about the shop floor to service centre [90]. 


\subsection{Technology Integration}

Data comes from more sources and in more forms, from personal and consumption data to video and technical data. During this digital transformation, enterprises face the challenge of deploying and integrating new technologies. These platforms enable to harness new technologies and agilely innovate to deal with changes and new competitive forces [91]. Nissan introduced intelligent seat belts that increase security, comfort, and hospitality; the secure systems function helps the driver and the front passengers secure feeling in skids or abrupt manoeuvres. Samsung's 28FDS process technology combines high performance and low power consumption with responsiveness to power management design techniques and promises modern mobile and consumer multimedia chips.

\subsection{Opportunity Loss}

The course of action did not permit the investor to profit due to strategic choice or a specific asset. The opportunity loss may happen due to other factors such as the decline of demand for specific traditional brands related to competitors' introduction of new products. In the case of Nissan, demand shifts from mature markets to growing markets faster than Nissan's mid-term planning assumptions. In the NTPC case, the generation of power from the coal-based unit could have been higher but reduced due to less generation schedule.

\subsection{Urban Infrastructure}

Huawei supports urban infrastructure by developing ubiquitous connectivity that provides network connectivity in three main areas: individuals, homes, and organisations. Nissan is introducing advanced safety technologies and autonomous driving technologies such as ProPILOT to advance the ultimate goal of reducing fatalities involving Nissan vehicles to zero [84]. L\&T continues to remain as one of a few leaders in construction, providing building solutions, affordable housing, hospitals, smart cities, intelligent traffic management, and logistic systems, which are some of L\&T's milestones.

\subsection{Purposeful Design}

Product design determines the company's success and turnover. A purposeful design must be portable, cost-effective, and scientific. What product definition that the top management is visualised; its purpose is essential that is characterised by the ability to repeatedly employ problem-solving, creativity and decision-making in a controlled and efficient manner to reach an adequate product definition [92].

\section{Application of TISM}

Total interpretive structural modelling (TISM) is a technique that facilitates researchers thinking process into systematic and structured knowledge. Table 4 shows the factors that are derived for strategic intent. In the transitive reachability matrix, factors numbered as ten vertical and ten linear, and diagonal matrices remain one shown in Table 5. When the definite significant answer had marked as one, and the inverse marked as zero. Additionally, in the next step, the transitivity is checked; further, Table 6 shows level partitioning obtained that shows the hierarchical positions of factors. The successive pairwise comparison of both decision and transitive links is shown in Figure 3. Based on the obtained level, partitioned factor's ISM (Interpretive structural modelling) is developed that shown in Figure 4. From the ISM, the fully developed TISM model, along with transitive links, are shown in Figure 5. 
Table 4. Strategic intend of the Asian case study.

\begin{tabular}{|c|c|c|c|}
\hline Element No. & Identified Factors & Contextual Relationship & Interpretation \\
\hline $\begin{array}{l}\mathrm{O} 1 \\
\mathrm{O} 2 \\
\mathrm{O} 3 \\
\mathrm{O} 4 \\
\mathrm{O} 5\end{array}$ & $\begin{array}{l}\text { New synergies for growth } \\
\text { Business tie-ups } \\
\text { Price sensitive consumers } \\
\text { Technology integration } \\
\text { Purposeful design }\end{array}$ & $\mathrm{A} \rightarrow \mathrm{B}$ means $\mathrm{A}$ will help achieve $\mathrm{B}$ & In what way A will help to achieve B \\
\hline $\begin{array}{l}\text { O6 } \\
\text { O7 } \\
\text { O8 } \\
\text { O9 } \\
\text { O10 }\end{array}$ & $\begin{array}{l}\text { Customer connectivity } \\
\text { Channel enhancement } \\
\text { Functional training } \\
\text { Opportunity loss } \\
\text { Urban Infrastructure }\end{array}$ & & \\
\hline
\end{tabular}

Table 5. Transitive reachability matrix.

\begin{tabular}{ccccccccccc}
\hline & O1 & O2 & O3 & O4 & O5 & O6 & O7 & O8 & O9 & O10 \\
\hline O1 & $\mathbf{1}$ & 1 & 0 & 0 & 0 & $1^{*}$ & 1 & 0 & 0 & 1 \\
O2 & 1 & $\mathbf{1}$ & 0 & 0 & 0 & 1 & 1 & 0 & 0 \\
O3 & $1^{*}$ & 0 & 1 & 1 & 1 & $1^{*}$ & 1 & 1 & 1 \\
O4 & 1 & $1^{*}$ & 0 & $\mathbf{1}$ & 0 & 1 & $1^{*}$ & 0 & 1 & 0 \\
O5 & $1^{*}$ & $1^{*}$ & 1 & 1 & $\mathbf{1}$ & 1 & 1 & 1 & $1^{*}$ & $1^{*}$ \\
O6 & 0 & 0 & 0 & 0 & 0 & $\mathbf{1}$ & 1 & 0 & 0 \\
O7 & 0 & 0 & 0 & 0 & 0 & 1 & $\mathbf{1}$ & 0 & 0 & 0 \\
O8 & $1^{*}$ & 0 & 0 & 1 & 0 & $1^{*}$ & 1 & $\mathbf{1}$ & 1 \\
O9 & 0 & 1 & 0 & 1 & 0 & 1 & 1 & 0 & 0 \\
O10 & 0 & 0 & 0 & 0 & 0 & 0 & 1 & 0 & 0 & 0 \\
\hline
\end{tabular}

1* Transitive links O -Operational factors.

Table 6. Hierarchical partitioning of the Asian case studies TISM.

\begin{tabular}{|c|c|c|c|c|}
\hline E. No. & Reachability & Antecedent & Intersection & Level \\
\hline O1 & $1,2,6,7,10$ & $1,2,3,4,5,8$ & 1,2 & \\
\hline $\mathrm{O} 2$ & $1,2,6,7,10$ & $1,2,4,9$ & 1,2 & \\
\hline O3 & $1,3,4,5,6,7,8,9$ & 3,5 & 3,5 & \\
\hline O4 & $1,2,4,6,7,9$ & $3,4,5,9$ & 4,9 & \\
\hline $\mathrm{O} 5$ & $1,2,3,4,5,6,7,8,9,10$ & 3,5 & 3,5 & \\
\hline O6 & 6,7 & $1,2,3,4,5,6,7,8,9$ & 6,7 & I \\
\hline O7 & 6,7 & $1,2,3,4,5,6,7,8,9,10$ & 6,7 & I \\
\hline O8 & $1,4,6,7,8,9$ & $3,5,8$ & 8 & \\
\hline O9 & $2,4,6,7,9$ & $3,4,9$ & 4,9 & \\
\hline O10 & 7,10 & $1,2,10$ & 10 & \\
\hline $\mathrm{O} 1$ & $1,2,10$ & $1,2,3,4,5,8$ & 1,2 & \\
\hline $\mathrm{O} 2$ & $1,2,10$ & $1,2,4,9$ & 1,2 & \\
\hline O3 & $1,3,4,5,8,9$ & 3,5 & 3,5 & \\
\hline $\mathrm{O} 4$ & $1,2,4,9$ & $3,4,5,9$ & 4,9 & \\
\hline O5 & $1,2,3,4,5,8,9,10$ & $3,4,5,9$ & 4,9 & \\
\hline $\mathrm{O} 8$ & $1,4,8,9$ & $3,5,8$ & 8 & \\
\hline O9 & $2,4,9$ & $3,4,8,9$ & 4,9 & \\
\hline 010 & 10 & 10 & 10 & II \\
\hline O1 & 1,2 & $1,2,3,4,5,8$ & 1,2 & III \\
\hline $\mathrm{O} 2$ & 1,2 & $1,2,4,9$ & 1,2 & III \\
\hline O3 & $1,3,4,5,8,9$ & 3,5 & 3,5 & \\
\hline $\mathrm{O} 4$ & $1,2,4,9$ & $3,4,5,9$ & 4,9 & \\
\hline O5 & $1,2,3,4,5,8,9$ & 3,5 & 3,5 & \\
\hline O8 & $1,4,8,9$ & $3,5,8$ & 8 & \\
\hline O9 & $2,4,9$ & $3,4,8,9$ & 4,9 & \\
\hline $\mathrm{O} 3$ & $3,4,5,8,9$ & 3,5 & 3,5 & \\
\hline $\mathrm{O} 4$ & 4,9 & $3,4,5,9$ & 4,9 & IV \\
\hline O5 & $3,4,5,8,9$ & 3,5 & 3,5 & \\
\hline O8 & $4,8,9$ & $3,5,8$ & 8 & \\
\hline O9 & 4,9 & $3,4,8,9$ & 4,9 & IV \\
\hline $\mathrm{O} 3$ & $3,5,8$ & 3,5 & 3,5 & \\
\hline O5 & $3,5,8$ & 3,5 & 3,5 & \\
\hline O8 & 8 & $3,5,8$ & 8 & $\mathrm{~V}$ \\
\hline O3 & 3,5 & 3,5 & 3,5 & VI \\
\hline O5 & 3,5 & 3,5 & 3,5 & VI \\
\hline
\end{tabular}




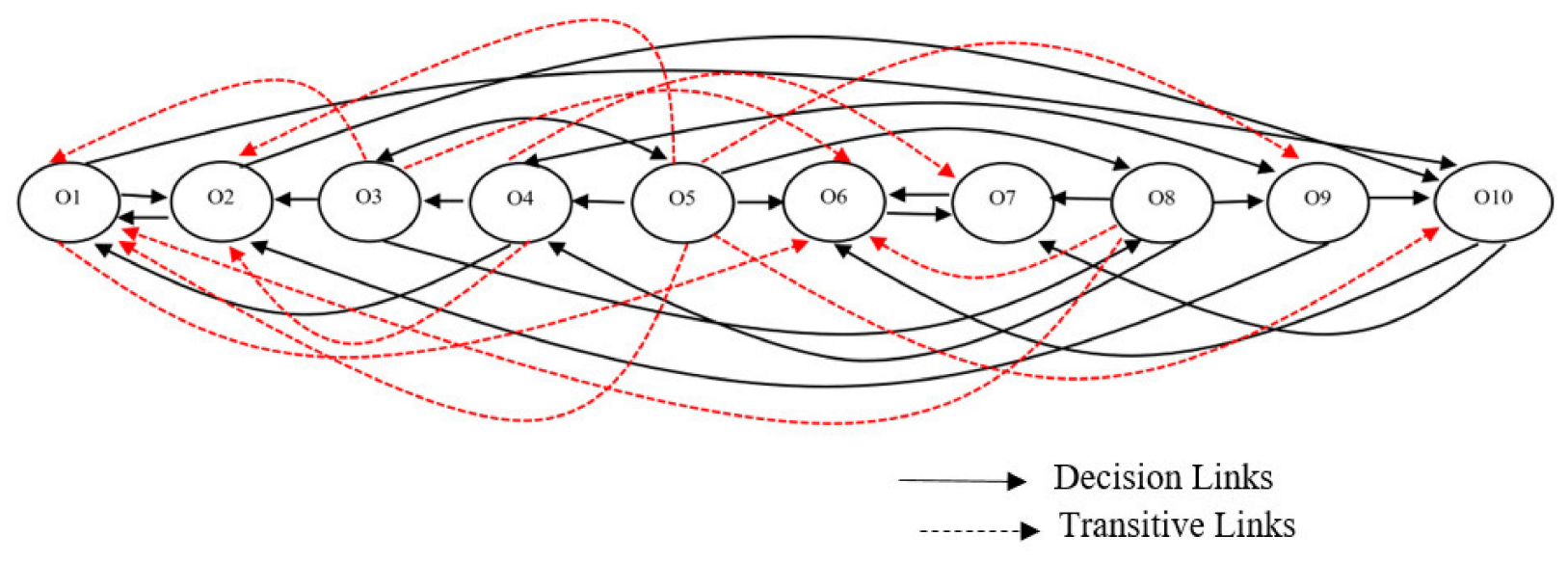

Figure 3. The successive pairwise comparison of both decision and transitive links of Asian case studies.

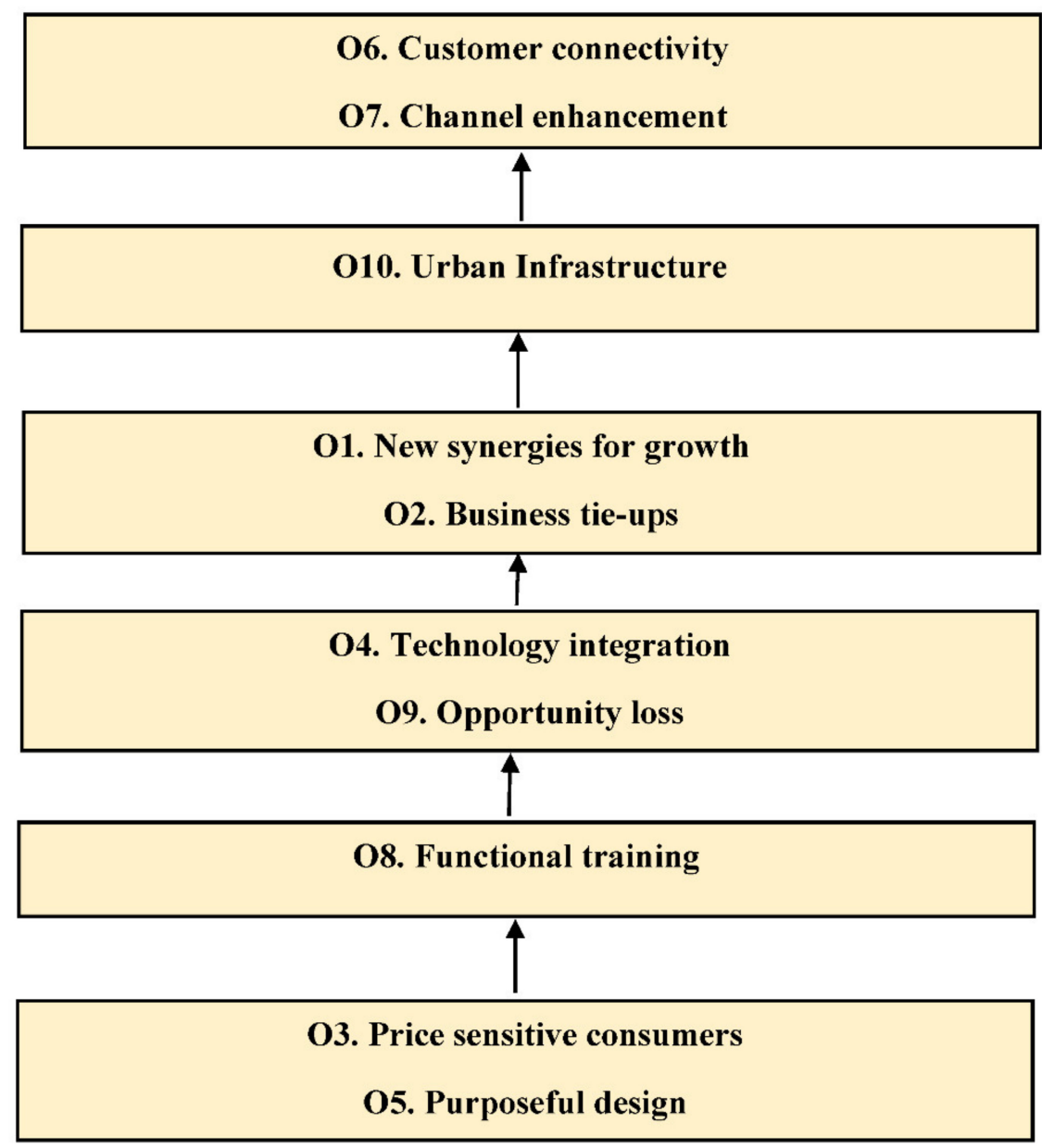

Figure 4. Interpretive structural modelling (ISM) of the case studies. 


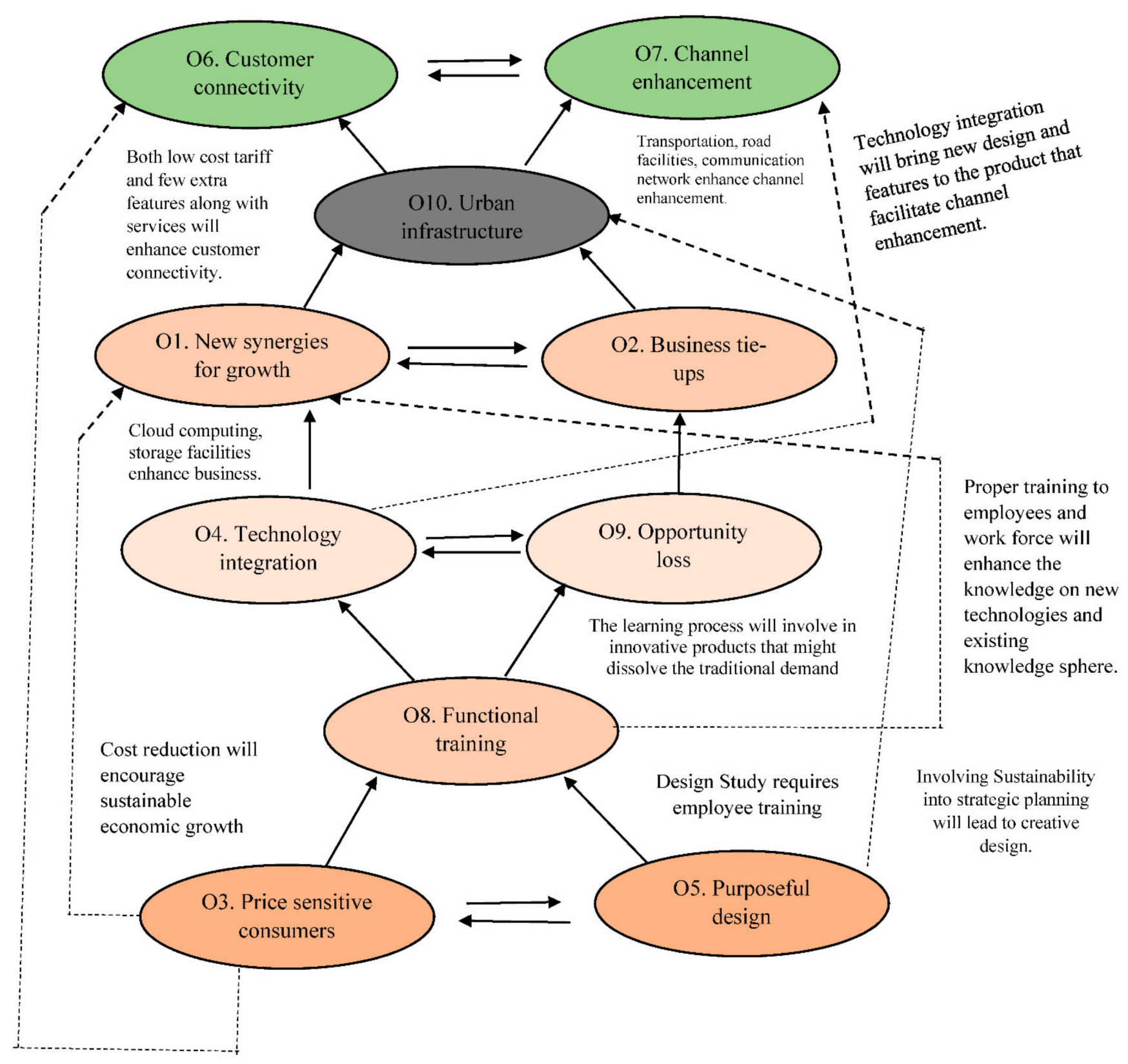

$\longrightarrow$ Enhances

Transitive

Figure 5. Total interpretive structural modelling of case studies.

The ten factors are taken from case studies collectively and combined with the joint discussion similar to the case organisations. Channel enhancement and customer connectivity remain as high-intensity factors for the case organisations. The firms are interested in fulfilling customer needs, and they are operating and strategizing to expand their channels from the domestic market to the international market. Technology integration remains a transitive link for channel enhancement. Urban infrastructure enhances the channel enhancement and customer connectivity process. New synergies for growth and business tie-ups enhance urban infrastructure; when there are more business activities, infrastructure comes up in the geographical areas, road constructions, network connectivity, and transportation facilities are part of urban infrastructure. Such new synergies of growth are possible through technology integration. At the same time, the enterprise has to be aware 
of opportunity loss; every business tie-up does not yield success, as explained in the TISM model in Figure 5.

Functional training remains a driving factor for technology integration and opportunity loss. Proper training on soft skills and technical skills supports the technology integration that avoids unnecessary workforce stigmas. Similarly, lack of knowledge on the market, new technology, and technical and soft skills leads to opportunity loss. Purposeful design and price-sensitive consumers remain as driving factors of the model. Low-cost strategy and purposeful structure of the front office, product design seems to be pushing element in case discussions. Hence, the model explains that Asian firms are framing business strategies to utilise the emerging economies market and business opportunities. At the same time, they are aware of fluctuating macro-economic situations. The interpretations are derived from case studies for both decision and transitive links are shown in Tables 7 and 8.

Table 7. Decision links obtained from Asian case studies.

\begin{tabular}{|c|c|c|c|}
\hline S. No & Element No. & Paired Comparison of Reachability Links & Case Organizations \\
\hline 1 & $\mathrm{O} 1-\mathrm{O} 2$ & $\begin{array}{l}\text { Under the philosophy of Kyosei (Excellent global corporation plan) } \\
\text { company emphasis on financial health and business reforms. }\end{array}$ & Canon \\
\hline 2 & O1-O7 & $\begin{array}{l}\text { Expanding business operation through diversification; -The sales } \\
\text { and profit grew steadily each year. }\end{array}$ & Canon \\
\hline 3 & O1-O10 & $\begin{array}{l}\text { Canon institute for global studies draws strategic policies for } 3 \\
\text { major areas such as macroeconomic, energy and foreign affairs. }\end{array}$ & Canon \\
\hline 4 & $\mathrm{O} 2-\mathrm{O} 1$ & $\begin{array}{l}\text { Mitsubishi work with business partners in Japan and overseas that } \\
\text { have technology advancement, global capability in terms of IT } \\
\text { services, including consulting and system development. }\end{array}$ & Mitsubishi \\
\hline 5 & $\mathrm{O} 2-\mathrm{O} 6$ & $\begin{array}{l}\text { Value based on consumer needs by providing safe and stable } \\
\text { supply of living essentials. }\end{array}$ & Mitsubishi \\
\hline 6 & $\mathrm{O} 2-\mathrm{O} 7$ & Focus on Asia and continued to sell new vehicles. & Mitsubishi \\
\hline 7 & $\mathrm{O} 2-\mathrm{O} 10$ & Business in public with focus on infrastructure transactions. & Mitsubishi \\
\hline 8 & $\mathrm{O} 3-\mathrm{O} 4$ & Price sensitive consumers will enhance technology integration & Indigo \\
\hline 9 & O3-O5 & $\begin{array}{l}\text { Accommodating and tracking of customers are important in } \\
\text { purposeful design }\end{array}$ & Indigo \\
\hline 10 & O3-O7 & $\begin{array}{l}\text { Low-cost strategy will enhance channel enhancement by } \\
\text { connecting more domestic routes. }\end{array}$ & Indigo \\
\hline 11 & $\mathrm{O} 3-\mathrm{O} 8$ & $\begin{array}{l}\text { Consumers needs are managed and promptly answered through } \\
\text { functional training of staffs and front office team. }\end{array}$ & Indigo \\
\hline 12 & O3-O9 & Unattended consumers' needs will lead to opportunity loss & Indigo \\
\hline 13 & O4-O1 & $\begin{array}{l}\text { New technologies including cloud computing, big data, will } \\
\text { enhance business opportunities. }\end{array}$ & Huawei \\
\hline 14 & O4-O5 & $\begin{array}{l}\text { Huawei is working towards developing top smart brand preferred } \\
\text { and trusted by consumers. }\end{array}$ & Huawei \\
\hline 15 & $\mathrm{O} 4-\mathrm{O} 6$ & Technology integration will enhance customer connectivity & Huawei \\
\hline 16 & O4-O9 & $\begin{array}{l}\text { In technology integration; cost loss, new knowledge will further } \\
\text { delay the immediate progress. }\end{array}$ & Huawei \\
\hline 17 & O5-O3 & Purposeful design will enhance price sensitive consumers & Samsung \\
\hline 18 & $\mathrm{O} 5-\mathrm{O} 4$ & $\begin{array}{l}\text { New product with integrated features in appliances is a part of } \\
\text { technology integration activity. }\end{array}$ & Samsung \\
\hline 19 & O5-O6 & $\begin{array}{l}\text { Attractive features and combat products will enhance customer } \\
\text { base. }\end{array}$ & Samsung \\
\hline 20 & O5-O7 & $\begin{array}{l}\text { Beautiful designs and purposeful features will cater more demands } \\
\text { in markets that will lead to channel enhancement. }\end{array}$ & Samsung \\
\hline
\end{tabular}


Table 7. Cont.

\begin{tabular}{|c|c|c|c|}
\hline S. No & Element No. & Paired Comparison of Reachability Links & Case Organizations \\
\hline 21 & O5-O8 & $\begin{array}{l}\text { The market knowledge, design trends and technological } \\
\text { advancements are learned through functional trainings. }\end{array}$ & Samsung \\
\hline 22 & O6-O7 & $\begin{array}{l}\text { Successful customer connectivity will enhance channel } \\
\text { enhancement }\end{array}$ & Singapore Airlines \\
\hline 23 & O7-O6 & $\begin{array}{l}\text { Opening outlets and expanding domestic markets will familiarize } \\
\text { the products to the customers }\end{array}$ & Hitachi \\
\hline 24 & $\mathrm{O} 8-\mathrm{O} 4$ & Functional training will enhance technology integration & Tata Motors \\
\hline 25 & O8-O7 & Training and knowledge will help & Tata Motors \\
\hline 26 & O8-O9 & Functional training will enhance opportunity loss & Tata Motors \\
\hline 27 & $\mathrm{O} 9-\mathrm{O} 2$ & Identifying right alliances will yield positive outcome. & NTPC \\
\hline 28 & $\mathrm{O} 9-\mathrm{O} 4$ & $\begin{array}{l}\text { Novel technology and clear technology will have a significant } \\
\text { impact over opportunity loss }\end{array}$ & NTPC \\
\hline 29 & O9-O6 & Wide customer base will be affected by opportunity loss & NTPC \\
\hline 30 & O9-O7 & $\begin{array}{l}\text { More business opportunities and market share will be affected by } \\
\text { opportunity loss. }\end{array}$ & NTPC \\
\hline 31 & O10-O7 & $\begin{array}{l}\text { Proper network connectivity, transportations, road connectivity } \\
\text { will improve channel enhancement. }\end{array}$ & L\&T \\
\hline
\end{tabular}

Source: Author.

Table 8. Transitive Links Related to Asian Case Studies.

\begin{tabular}{cclc}
\hline S. No & Element No. & Paired Comparison of Transitive Links & Case Organizations \\
\hline 1 & O1-O6 & $\begin{array}{l}\text { New and sophisticated products will } \\
\text { enhance customer connectivity }\end{array}$ & Canon \\
\hline 2 & O3-O1 & $\begin{array}{l}\text { The need for flight carriage in emerging } \\
\text { economies will provide new synergies for } \\
\text { growth }\end{array}$ & Indigo \\
\hline 3 & O3-O6 & $\begin{array}{l}\text { Price sensitive consumers enhance customer } \\
\text { connectivity }\end{array}$ & Huawei \\
\hline 5 & O4-O2 & $\begin{array}{l}\text { Utilizing technology in network connectivity } \\
\text { and safety will enhance business tie-ups }\end{array}$ & Huawei \\
\hline 6 & O4-O7 & $\begin{array}{l}\text { Technology upgradation will make product } \\
\text { and service more feasible to market reach }\end{array}$ & Samsung \\
\hline 8 & O5-O1 & $\begin{array}{l}\text { Purposeful features, new designs will favour } \\
\text { business growth }\end{array}$ & Tata motors \\
\hline
\end{tabular}

Source: Author.

\section{Testing of the Framework Study}

How does the study empirically analyse the current position and shortcomings of chosen case organisations? The answer lies in selecting the appropriate variables that are related to the study. The amount received from operating activities and money spent on investing in machines and plants is directly associated with the manufacturing process. Similarly, money returned as credit pay and debt took as variables for conducting panel data 
analysis along with social value expenditure. Operating activities remain the dependent variable, whereas investing activities, financing activities, and social value expenditure remain explanatory variables. The study conducted panel data analysis to understand the model fit are shown in Tables 9 and 10. The empirical analysis based on secondary data is shown in Tables A1 and A2 in Appendix A. The model fit of the empirical analysis is performed through the goodness of fit test is shown in Table 11.

Table 9. Pooled OLS model.

\begin{tabular}{|c|c|c|c|c|c|c|}
\hline & & & \multicolumn{4}{|c|}{$\begin{array}{c}\text { Number of obs }=60 \\
F(3,56)=282.05 \\
\text { Prob }>F=0.0000 \\
\text { R- squared }=0.9379 \\
\text { Adj } R \text {-squared }=0.9346 \\
\text { Root MSE }=2391.5\end{array}$} \\
\hline Source & \multicolumn{2}{|c|}{ SS } & \multicolumn{2}{|c|}{$\mathrm{df}$} & \multicolumn{2}{|c|}{ MS } \\
\hline Model & \multicolumn{2}{|c|}{$4.8392 \times 10^{9}$} & \multicolumn{2}{|c|}{3} & \multicolumn{2}{|c|}{$1.6131 \times 10^{9}$} \\
\hline Residual & \multicolumn{2}{|c|}{$320,270,129$} & \multicolumn{2}{|c|}{56} & \multicolumn{2}{|c|}{$5,719,109.45$} \\
\hline Total & \multicolumn{2}{|c|}{$5.1595 \times 10^{9}$} & \multicolumn{2}{|c|}{59} & \multirow{2}{*}{\multicolumn{2}{|c|}{$\begin{array}{c}87,449,072.6 \\
{[95 \% \text { conf. Interval] }}\end{array}$}} \\
\hline Operating Activities & Coef. & Std. Err. & $\mathrm{t}$ & $P>|t|$ & & \\
\hline Investing Activities & 0.5525548 & 0.0978834 & 5.65 & 0.000 & 0.3564708 & 0.7486387 \\
\hline Financing activities & 0.4058251 & 0.2249273 & 1.80 & 0.077 & -0.0447585 & 0.8564086 \\
\hline Social value expenditure & 0.3060674 & 0.0496878 & 6.16 & 0.000 & 0.2065308 & 0.4056039 \\
\hline _cons & -1273.329 & 463.3307 & -2.75 & 0.008 & -2201.492 & -345.1664 \\
\hline
\end{tabular}

Table 10. Random-effect Model.

\begin{tabular}{|c|c|c|c|c|c|c|}
\hline \multicolumn{3}{|c|}{$\begin{array}{c}\text { Random-effects GLS regression } \\
\text { Group Variable: Firm_id } \\
\text { R-sq: } \\
\text { within }=0.4472 \\
\text { between }=0.9535 \\
\text { overall }=0.9345 \\
\text { corr }(\mathrm{u} \text { _i, } x b)=0 \text { (assumed) }\end{array}$} & \multicolumn{4}{|c|}{$\begin{array}{c}\text { Number of observations }=60 \\
\text { Number of groups }=12 \\
\text { Observation per group: } \\
\min =5 \\
\operatorname{avg}=5.0 \\
\max =5 \\
\text { Wald chi } 2(4)=248.97 \\
\text { prob }>\text { chi } 2=0.0000\end{array}$} \\
\hline Operating Activities & Coef. & Std. Err. & $\mathrm{z}$ & $P>|z|$ & {$[95 \% \mathrm{c}$} & nterval] \\
\hline Investing Activities & 0.3898477 & 0.0888032 & 4.39 & 0.000 & 0.2157965 & 0.5638988 \\
\hline Financing activities & 0.5678415 & 0.2003188 & 2.83 & 0.005 & 0.1752239 & 0.960459 \\
\hline Social value expenditure & 0.3787422 & 0.0542567 & 6.98 & 0.000 & 0.2724011 & 0.4850833 \\
\hline _cons & -1655.192 & 797.5347 & -2.08 & 0.038 & -3218.331 & -92.05232 \\
\hline sigma_u & \multicolumn{2}{|c|}{2005.1502} & & & & \\
\hline sigma_e & \multicolumn{2}{|c|}{1536.4836} & & & & \\
\hline rho & 0.6 & 321 & & & \multicolumn{2}{|c|}{ (Fraction of variance due to $\mathrm{u} \_\mathrm{i}$ ) } \\
\hline
\end{tabular}

The significance of the study is shown in colour.

Table 11. Goodness of fit test estimated result.

\begin{tabular}{lcc}
\hline & Var & sd = sqrt (Var) \\
\hline operati $\sim \mathrm{s}$ & $8.74 \times 10^{7}$ & 9351.421 \\
$\mathrm{e}$ & 2360782 & 1536.484 \\
$\mathrm{u}$ & 4020628 & 2005.15 \\
\hline
\end{tabular}

$\mathrm{u}$ is the random -error component, $\mathrm{e}$ is overall error component. Test: $\operatorname{Var}(\mathrm{u})=0$. chibar2 $(01)=33.44$. Prob $>$ chibar2 $=0.0000$.

Both pooled OLS regression and random effect GLS regression were conducted to understand the model fit for this study. Further Breush Page (LM) test undertaken to understand the goodness of fit that shows random effect model is appropriate for the study shown in Table 11. 
Twelve firms were considered as twelve groups and sixty observation points throughout 2013-2017. The data analysis supports the study by showing that social value expenditure and investing activities positively and significantly explain operating activities. It implies that social value expenditure needs explanation and clarifications in understandings. In this study, employees benefit expenditure, pension allowances, administrative expenses, and other least expected returns by management and board capitals are considered social value expenditures (SVE). An increase in SVE increase firms operating activities. This observation positively implies that SVE positively and significantly contributes to enterprise sustainable operational performances. Organisations' investment in sophisticated technology, efficient plant and machinery and less energy-consuming machinery led to an increase in the firm's operating activities that had explained in the model as investing activities positively and significantly explaining firms operating activities.

\section{Asian Regional Study Result Analysis}

In this section, for Asian regional analysis is conducted with a Pareto chart. According to Grosfeld-Nir [93], "Statistically, a Pareto chart is simply a frequency block diagram displaying the relative frequency of different attributes in descending order. This classification is an essential step that must precede taking the corrective measures of differentiation and allocation". A Pareto chart is widely used to gather information on minor attributes that cause significant problems. This technique is a decisive decision-making criterion in quality that explains that the bulk of the issues result from few sources [94]. This bar graph analysis in this regional study aims to identify the amount of expenses and analyse their performance differences among twelve regional firms collectively.

From the bar diagram shown in Figure 6, it is evident that three firms such as Samsung, Hitachi, and Canon, are spending significantly towards social value expenditure. The exponential line shows that SVE is higher than the operating expenses. This observation conveys important regional information that operating activities are facilitated by SVE (social value expenditures). Such an approach can be a part of their operational strategy or business tactics to utilise the available market opportunities and absorbing capacities. All the first three firms are electronic and communication equipment focused firms, there may be situational growth, wide presence of growth opportunities, and their competitive parity may slow down. However, the analysis shows that SVE enhances operational activities.

In the case of Samsung, their design strategy continues to remain as their business cutting edge. Although yet to firmly establish in the global market, according to Yoo and Kim [95], "The bold designs of its televisions often defy conventional style. With its Galaxy Note series, Samsung introduced a new category of smartphones-the phabletwidely copied by competitors. Design is now so much of its corporate DNA that top leaders rely on designers to help visualise the entire company's future. They incorporate empathy, visualisation and experimentation". Another reason for Samsung's growth is the 'opportunity based' approach. The opportunity for channel enhancement exists because other known brands slow down the process or lack knowledge towards the emerging market scenario. However, statistically, the ratio between SVE and operating activities are 3:1 that is shown in Figure 7 two of the exponential line of SVE and operating activities. 


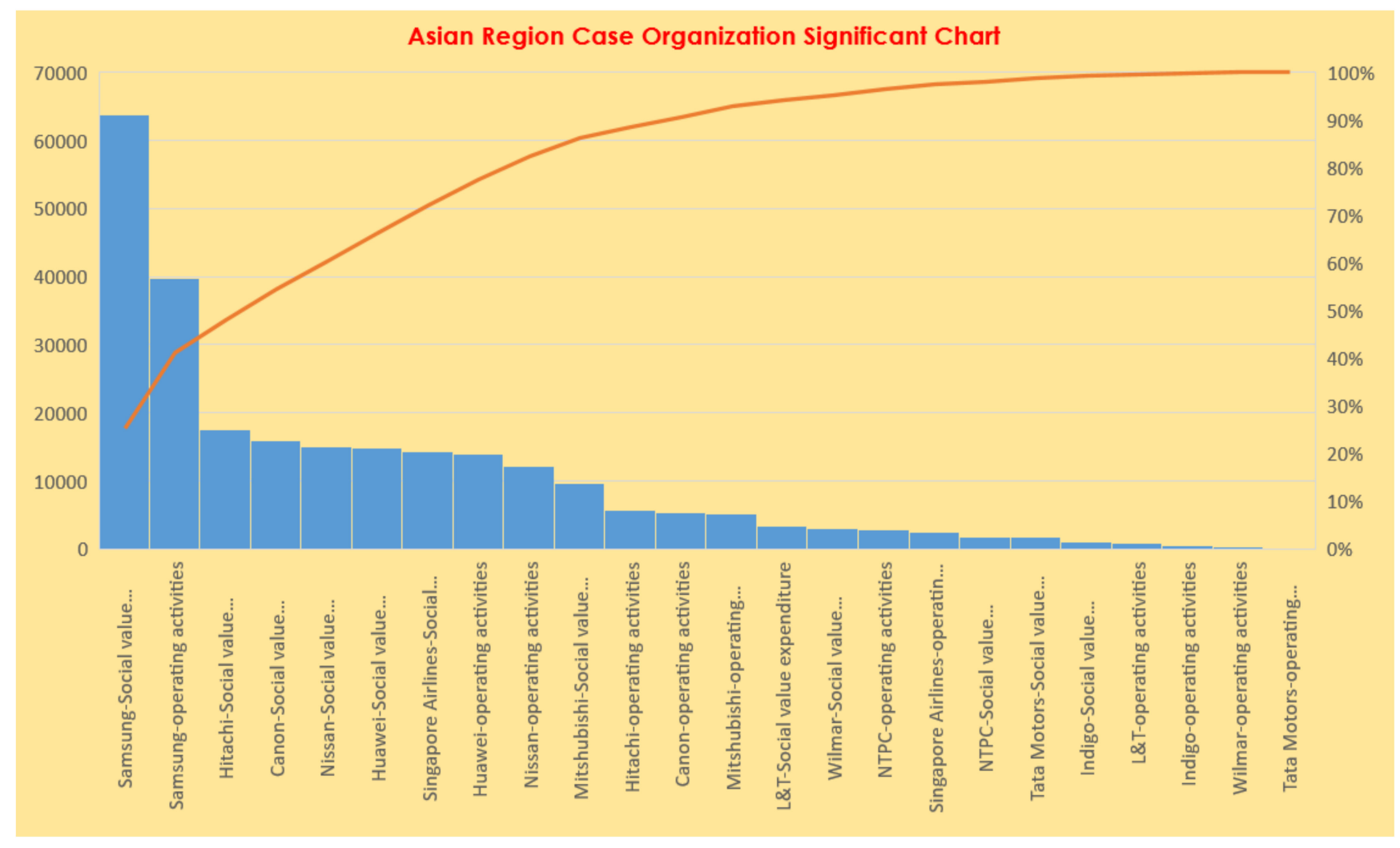

Figure 6. The bar diagram shows the Asian firms expenditure activities. Source: Author.

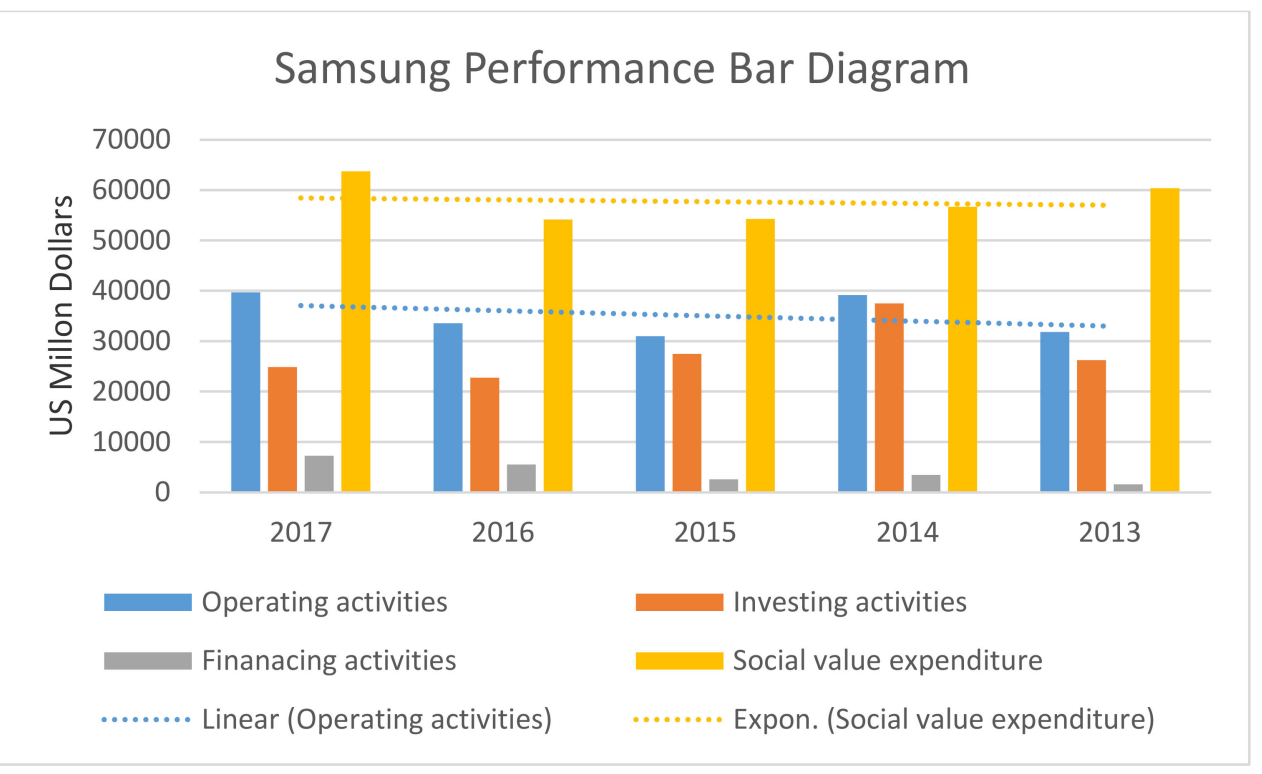

Figure 7. Samsung's performance with exponential lines. Source: Author.

Hitachi is known for its best service delivery, highest quality and advanced technology, and it skilfully uses the workforce to get the maximum out of the system [96]. Hitachi exponential line is shown in Figure 8 and Canon in Figure 9. 


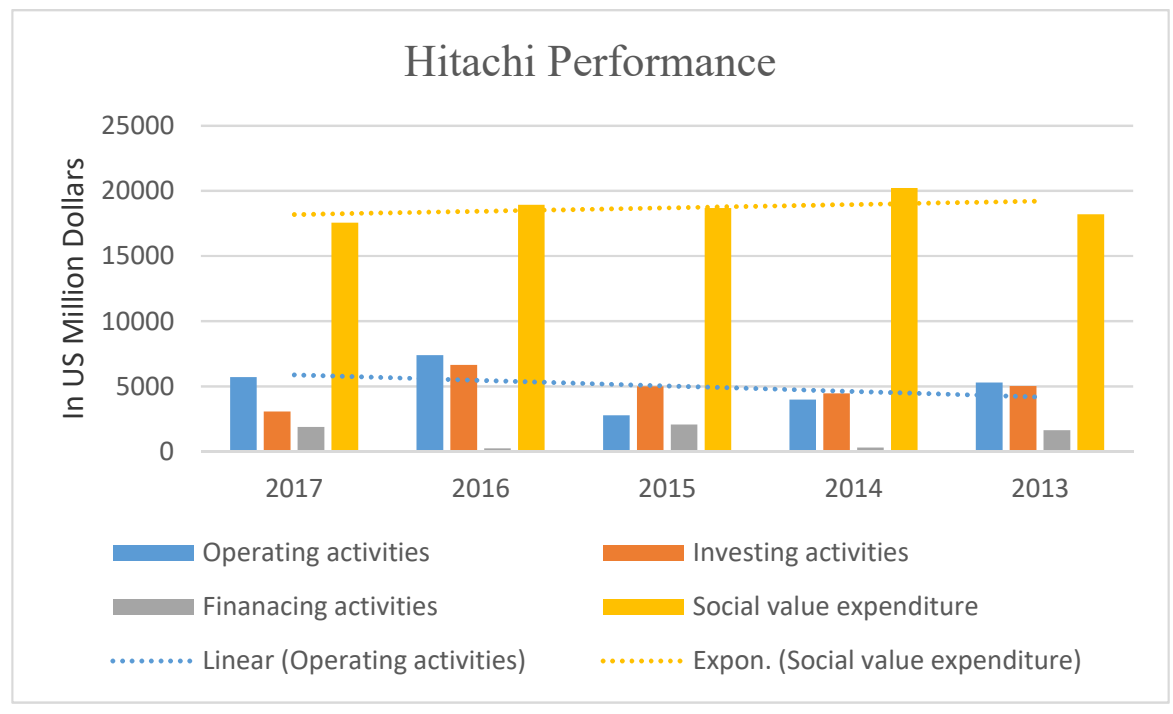

Figure 8. Hitachi's performance with exponential lines. Source: Author.

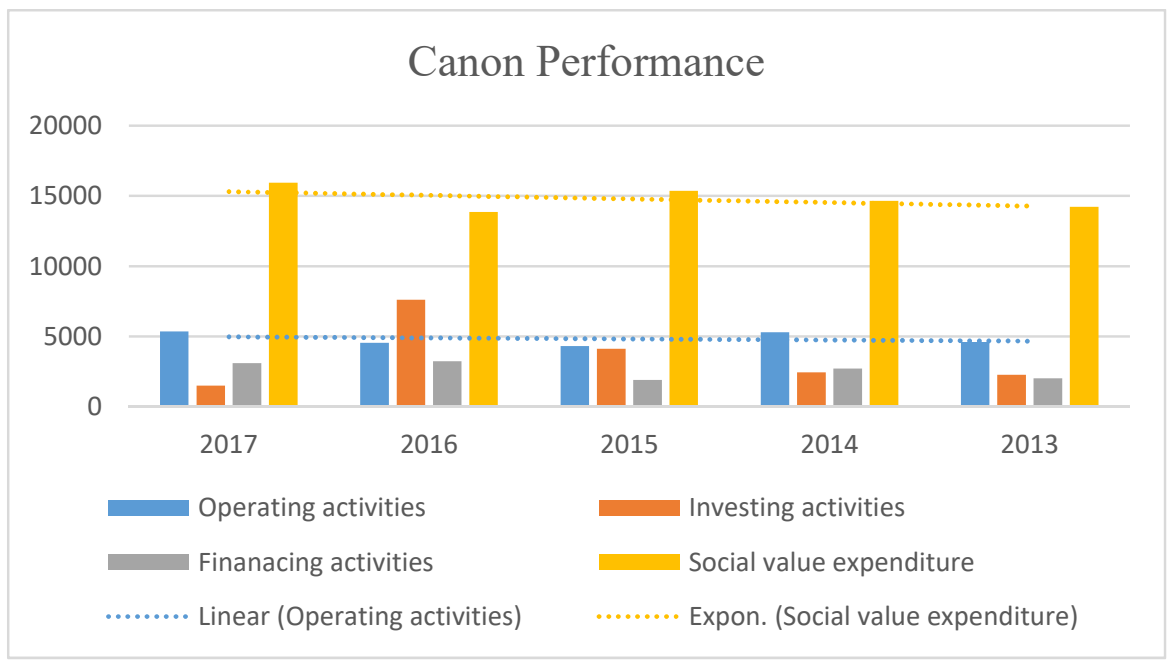

Figure 9. Canon's performance with exponential lines. Source: Author.

Canon used original technology that did not violate any of the 600 patents; until then, Canon was concentrated in its camera industry, as a part of diversification, canon started a plain paper copier. Canon viewed the market as a fresh and positive rather than matured market in technology [97].

\section{Implications}

After a broader understanding that derived from Asian regional case studies, the generalised implications are:

- Social value expenditure positively signifies the operating activities of an organisation.

- Efficient machinery and plants in manufacturing activities had positively signified enterprise operation excellence.

- Be it profit, total turnover, or total sales, such returns are an outcome of enterprise operational excellence practices cultivated over time through experience and R\&D practices. Hence, both workforce (SVE) and investing activities (plant and machinery) are visible as both sides of a single coin to achieve enterprise sustainable operational excellence. 
Enterprise financing activities shows moderate observation as credit and loans need to be considered in enterprise cash flow activities.

\subsection{Sustainability Implications from Asian Case Studies}

Based on the case organisations sustainability practices and their effort in sustainability principles, the following sustainability implications are:

- Environmentally friendly products and services (Operating activities) provide a competitive advantage.

- Incorporating sustainability in employee training (Social Value Expenditure) will increase the organisation internal knowledge.

- Customer connectivity (Social Value Expenditure) through network communications will improve the sustainable operation chances.

\subsection{Managerial Implications}

Globalisation is a period of both challenges and opportunities. As a proverbial English story of an ant and grasshopper explains the importance of climate and effort, Europe underwent an industrialisation era in the 16th century that is the involved metaphor. Similarly, globalisation is one of such inevitable events. Hence, when a situation requires more effort, a positive perk for the workforce must facilitate firms' production activities. The government focuses on supportive measures such as providing water, electricity, and infrastructure facilities to the MNC's and domestic enterprises. On the other hand, service is expected to the respective regional people and regional economic development. Structured government and well-informed consumers attract investment activities that significantly affect operational activities in Asian regional case studies. Hence, careful framing of planning and strategic directions are essential in sustainable business practices.

\section{Discussion}

Does sustainable operational excellence depend on the firm's manufacturing decisions or strategic decisions? What leads to enterprise sustainable operational excellence? Such questions had comprehended sustainability issues about decision making. However, there is limited evidence that the strategic decision-making process influences decision effectiveness; the same way, the link between strategic decision process and efficacy has not been convincingly demonstrated $[98,99]$. Some of the most fundamental decisions are made by top management through a strategic decision-making process; managers need to be keenly aware of assumptions, heuristics, and biases in decision making [100].

Business success, turnover, and pitfalls in profit-making are intensely related to board capital decision making and the planning process. Board capitals generally follow few important thumb rules in decision making; otherwise, they are visibly flexible and listening towards constructive criticism. They are concerned with manufacturing, business operations, and channel enhancement, mostly towards existing macroeconomic circumstances in domestic and international markets. Local government tax policies, land utilisation policies, and workforce policies are considered in the decision-making process. When the turnover lies in billion dollars and the enterprise is actively involving itself in technology integration, board capital expects that may provide leverage in the existing competitive market, it is crucial in the discussion. Under those circumstances, it is always suggestible to rely on past experiences, past knowledge sources and local knowledge. In terms of planning and decisions, it is obligatory to rely on data and its inference results.

There are no established rules in strategic process or strategic decision making. It is an instrument that facilitates and conveniently drives towards the established target or goal set ahead of a particular department or planning team. Business is all about profit-making both for enterprise and shareholders and equity holders. The rest of the intangible aspects follows the firm positive performance. It is obvious in the balance sheet of any enterprise that says if at all other expenditures exist that can calculate otherwise, it remains mute. Therefore, manufacturing decisions strengthened by strategic decisions through managerial 
actions are considered a core value in strategic decision making. The study developed the Paux strategy framework to explain this complex phenomenon and show how four elements accumulate together in a strategic planning process, as shown in Figure 10.

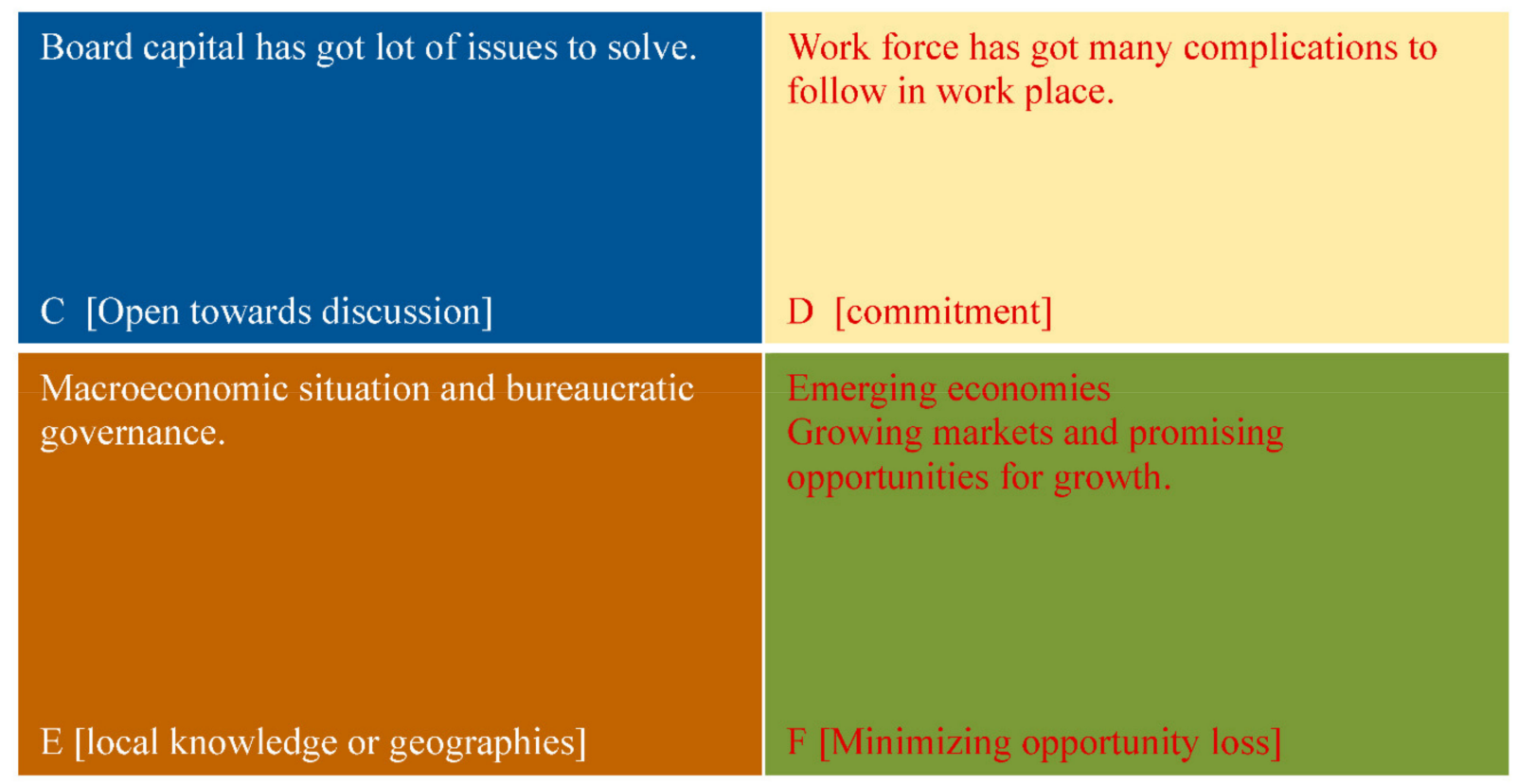

Figure 10. Paux Strategy Framework. Source: Author.

One frame accepts that board capitals have problems or issues to discuss; similarly, the workforce is obliged to accept new changes in practices and training. For both board capital and workforce macro-economic situation remain the same. However, managerial tactics and skills are used to resolve the problem and reduce opportunity loss through strategic choices and decisions.

In this framework, there is a potential to stimulate a synergic and converging effort to address health, social and needed green transition [101]. Environmental concerns, recurring oil crises and market weaknesses, combined with the availability of power from natural resources and resulting possibilities for job creation and energy independence, have all pushed developed and developing countries towards new energy strategies [102]. The application of multi-criteria analysis can support this initiative, in which a panel of experts can determine the weight. The importance of providing a quantitative study that aims to reduce subjectivity during policy choices and increase models of reflexive governance is evident $[103,104]$. Asset creation, market regulation, and developing a sound labour market will further increase sustainability and resilience through capability enhancement for domestic and MNC's in the Asian region.

\section{Relationship with Conceptual Framework}

Many firms incorporate the concept of sustainability and sustainable business practices into their group strategy. The board capital started to view sustainability as an integrated part of their business practices. Some firms even designed sustainability committees to evaluate their practices. From the employee perspective, their human rights, safety and training program are covered in this framework. The governance aspect is once again directly related to the group strategy of sustainable enterprise. Macroeconomic situation and regional economics are defined.

\section{Concluding Remarks}

The study aimed to focus on the Asian region through multiple case study research methods. Two aspects are concerned, such as operational complexities and their strategic 
overview. Some of the research notions are that despite being rich in cultural and social values, the Asian region remains a developing region. The role of business is identified as an influential factor in uplifting regional development with a promising domestic market and investment opportunities. Hence, the managerial discussion and governance need scholarly attention.

To generalise any findings or conclusion, the point of observation in the empirical study has to be significant. Such observations provide a clearer picture of the study. Since TISM (total interpretive structural modelling) is one of those of kind methods, the study has incorporated the TISM method for model building. Similarly, for empirical research, panel data analysis is applied, which shows SVE (social value expenditure) positively and significantly contributes to operational activities. Such an observation shows that sustainable business practices enhance firms' commitment towards sustainability values. The employee benefit expenses contribute towards a healthy and well-informed workforce. The tax expenses offer organisations social obligations, respect for the constitution, and follow the rule of law. The administrative expenses enhance the working atmosphere and conducive working culture that further improve firms' productivity.

Observations from the Pareto chart show that leading firms are significantly investing in social value expenditure and histogram analysis shows as the social value expenditure increases; operational activities show an increasing trend. The observations are made from the secondary data available in their respective annual reports. Although this is a preliminary attempt to study Asian regional firms using an index, there can be many other significant factors such as geography, political environment, domestic governance regulations, and bureaucratic pressures. However, business activities have to be carried out for any successful enterprise.

Various firms with multiple foci on strategy and operational practices; some want to expand their market share, some want to regain their traditional positions, another set of firms want to bring out cost reduction in new products. Airline industries focus mainly on customer satisfaction and customer connectivity and training and development in front office management; all these discrete approaches in operational excellence practising by multiple sectors show diversification in modes of operation and board capital expectations. Because investing, expenditures, and manufacturing are directly concerned with board capitals. What kind of technology innovation is expected by the workforce? The purchasing capacity of an enterprise, increasing pension allowances and cutting other expenses related to operational decisions. Hence, sustainable operational excellence is interwoven with organisation cash flows and investing priorities on par with derived strategic decisions.

The study is confined to the Asian region and the chosen case studies committed to sustainable developmental values. Although social value expenditure (SVE) is catered for in this study, how much is invested in value creation? Other expenses have to explain whether those expenses are socially inclined or administrative related. The annual reports repeat the context with changes in the numerical figures of every year: the cross-sectional analysis and regional comparative study of similar firms or similar sectors. What remains a row between decision making and implementation of inboard capital while discussing sustainable business operational practices is the future scope of the study.

Author Contributions: S., Investigation and supervision; P.A., conceptualization and Methodology. Both authors have read and agreed to the published version of the manuscript.

Funding: This research received no external funding.

Institutional Review Board Statement: Not Applicable.

Informed Consent Statement: Not Applicable.

Data Availability Statement: Data are collected from the financial statements of the annual reports of respective firms for the particular study time frame. The links are provided in the Appendix.

Conflicts of Interest: The authors declare no conflict of interest. 


\section{Appendix A}

Table A1. Shows the chosen variables for the study. (In million USD).

\begin{tabular}{|c|c|c|c|c|c|}
\hline Operating Activities & Investing Activities & Financing Activities & Social Value Expenditure & Firm_id & Year \\
\hline 196.72 & 389.9 & 159.38 & 1767.23 & 1 & 2017 \\
\hline 334.1 & 416.66 & 10.13 & 1600.37 & 1 & 2016 \\
\hline 364.92 & 85.69 & 374.73 & 1712.83 & 1 & 2015 \\
\hline 350.8 & 363.53 & 716.81 & $10,066.09$ & 1 & 2014 \\
\hline 321.6 & 141.19 & 576.11 & 8020.3 & 1 & 2013 \\
\hline $39,699.92$ & $24,848.18$ & 7263.36 & $63,716.792$ & 2 & 2017 \\
\hline $33,563.93$ & $22,761.3$ & 5507.32 & $54,145.441$ & 2 & 2016 \\
\hline $30,978.16$ & $27,485.36$ & 2561.26 & $54,258.115$ & 2 & 2015 \\
\hline $39,131.35$ & $37,489.25$ & 3466 & $56,656.721$ & 2 & 2014 \\
\hline $31,813.96$ & $26,241.72$ & 1562.51 & $60,376.108$ & 2 & 2013 \\
\hline $12,139.45$ & $12,522.62$ & 2914.34 & $15,052.82$ & 3 & 2017 \\
\hline 8426.55 & $11,174.16$ & 4823.21 & $15,724.2$ & 3 & 2016 \\
\hline 6297.07 & 9290.21 & 2235.19 & $15,064.98$ & 3 & 2015 \\
\hline 6618.64 & 9820.98 & 3608.05 & $12,966.63$ & 3 & 2014 \\
\hline 3553.25 & 8700.38 & 4141.65 & $10,366.84$ & 3 & 2013 \\
\hline 538.57 & 432.59 & 199.53 & 1052.14 & 4 & 2017 \\
\hline 441.31 & 188.85 & 173.72 & 823.42 & 4 & 2016 \\
\hline 339.47 & 133.93 & 186.28 & 664.58 & 4 & 2015 \\
\hline 227.2 & 414.92 & 182.96 & 484.68 & 4 & 2014 \\
\hline 247.96 & 269.09 & 5.14 & 390.18 & 4 & 2013 \\
\hline 5205 & 1603 & 6716 & 9650.13 & 5 & 2017 \\
\hline 6251 & 4499 & 3255 & 9913.4 & 5 & 2016 \\
\hline 6652 & 1290 & 2544 & $10,991.19$ & 5 & 2015 \\
\hline 3705 & 2917 & 1154 & $10,563.27$ & 5 & 2014 \\
\hline 4291 & 8005 & 4273 & 8174.52 & 5 & 2013 \\
\hline $13,941.96$ & 3568.41 & 2451.01 & $14,760.85$ & 6 & 2017 \\
\hline 7122.94 & 4128.06 & 1570.38 & $14,074.12$ & 6 & 2016 \\
\hline 7136.97 & 324.76 & 2860.14 & $10,292.64$ & 6 & 2015 \\
\hline 6042.87 & 3793.02 & 1505.98 & 7836.09 & 6 & 2014 \\
\hline 3264.06 & 1163.13 & 1031.29 & 6812.81 & 6 & 2013 \\
\hline 386.372 & 936.594 & 917.723 & 2917.874 & 7 & 2017 \\
\hline 1123.61 & 810.723 & 271.594 & 3052.674 & 7 & 2016 \\
\hline 2232.126 & 1312.999 & 1593.744 & 2984.032 & 7 & 2015 \\
\hline 1973.343 & 1228.279 & 1281.248 & 2848.142 & 7 & 2014 \\
\hline 2161.055 & 1432.14 & 527.311 & 2776.031 & 7 & 2013 \\
\hline 5368.16 & 1499.94 & 3094.82 & $15,954.8$ & 8 & 2017 \\
\hline 4547.57 & 7609.47 & 3233.24 & $13,857.67$ & 8 & 2016 \\
\hline 4315.24 & 4123.4 & 1910.74 & $15,372.92$ & 8 & 2015 \\
\hline 5307.9 & 2447.92 & 2735.05 & $14,653.34$ & 8 & 2014 \\
\hline 4614.47 & 2274.43 & 2019.63 & 14,230 & 8 & 2013 \\
\hline 2890.92 & 3519.96 & 456 & 1782.22 & 9 & 2017 \\
\hline 2065.3 & 2623.39 & 631.74 & 1374.87 & 9 & 2016 \\
\hline 2027.02 & 2073.71 & 267.44 & 1277.85 & 9 & 2015 \\
\hline 2240.26 & 1990.71 & 471.2 & 1627.54 & 9 & 2014 \\
\hline 2206.51 & 1996.01 & 107.14 & 1654.64 & 9 & 2013 \\
\hline 5722.9 & 3072.01 & 1904.68 & $17,553.41$ & 10 & 2017 \\
\hline 7383.13 & 6642.96 & 240.59 & $18,946.04$ & 10 & 2016 \\
\hline 2788.6 & 5001.13 & 2080.16 & $18,686.07$ & 10 & 2015 \\
\hline 3994.2 & 4466.49 & 299.68 & $20,217.44$ & 10 & 2014 \\
\hline 5304.09 & 5030.92 & 1640.25 & $18,207.64$ & 10 & 2013 \\
\hline 2532.9 & 2943.5 & 224.6 & $14,322.4$ & 11 & 2017 \\
\hline 3005.5 & 2699.7 & 1321.4 & $14,678.1$ & 11 & 2016 \\
\hline 2067.2 & 1605.2 & 137.1 & $15,192.3$ & 11 & 2015 \\
\hline 2098.1 & 1822.7 & 435.8 & $15,041.1$ & 11 & 2014 \\
\hline 1854.4 & 1146.2 & 338.6 & $14,909.4$ & 11 & 2013 \\
\hline 887.84 & 1387.01 & 502.26 & 3307.26 & 12 & 2017 \\
\hline 459.8 & 588.4 & 1066.36 & 2568.65 & 12 & 2016 \\
\hline 95.25 & 777.87 & 1123.64 & 111.83 & 12 & 2015 \\
\hline 149.13 & 172.92 & 71.78 & 1200.98 & 12 & 2014 \\
\hline 209.65 & 93.52 & 472.23 & 1075.44 & 12 & 2013 \\
\hline
\end{tabular}

(Source: Annual report of the respective 12 firms) Note: Firm id: 1-Tata motors; 2-Samsung; 3-Nissan; 4-Indigo; 5-Mitshubishi; 6-Huawei; 7-Wilmar; 8-Canon; 9-NTPC; 10-Hitachi; 11-singapore Airlines; 12-L\&T). 
Table A2. Social value expenditure.

\begin{tabular}{|c|c|c|c|c|c|c|}
\hline $\begin{array}{c}\text { Administrative } \\
\text { Expenses/Employee } \\
\text { Benefit Expenses (X) }\end{array}$ & $\begin{array}{c}\text { Other } \\
\text { Expenses/Non- } \\
\text { operating } \\
\text { Expenses/Doubtful } \\
\text { Receivables (Y) }\end{array}$ & $\begin{array}{c}\text { Income } \\
\text { Tax/Tax } \\
\text { Expenses (Z) }\end{array}$ & $\begin{array}{l}\text { Social Value } \\
\text { Expenditure } \\
\text { (Sum of XYZ) }\end{array}$ & $\begin{array}{l}\text { Social Value } \\
\text { Expenditure } \\
\text { (In USD } \\
\text { Millions) }\end{array}$ & Firm_id & Year \\
\hline 3558.52 & 8697.42 & 59.22 & $12,315.16$ & 1767.23 & 1 & 2017 \\
\hline 3026.75 & 8041.81 & 83.84 & $11,152.4$ & 1600.37 & 1 & 2016 \\
\hline 3091.46 & 8080.39 & 764.23 & $11,936.08$ & 1712.83 & 1 & 2015 \\
\hline $21,556.42$ & $43,825.77$ & 4764.79 & $70,146.98$ & $10,066.09$ & 1 & 2014 \\
\hline $16,584.05$ & $35,535.58$ & 3770.99 & $55,890.62$ & 8020.30 & 1 & 2013 \\
\hline $50,075,918$ & $1,255,130$ & $12,385,744$ & $63,716,792$ & $63,716.792$ & 2 & 2017 \\
\hline $45,134,348$ & $2,124,281$ & $6,886,812$ & $54,145,441$ & $54,145.441$ & 2 & 2016 \\
\hline $44,866,898$ & $3,291,288$ & $6,099,929$ & $54,258,115$ & $54,258.115$ & 2 & 2015 \\
\hline $50,253,744$ & $2,146,611$ & $4,256,366$ & $56,656,721$ & $56,656.721$ & 2 & 2014 \\
\hline $51,370,553$ & $1,529,468$ & $7,476,087$ & $60,376,108$ & $60,376.108$ & 2 & 2013 \\
\hline $1,555,262$ & 105,290 & - & $1,660,552$ & $15,052.82$ & 3 & 2017 \\
\hline $1,599,243$ & 135,372 & - & $1,734,615$ & $15,724.20$ & 3 & 2016 \\
\hline $1,544,305$ & 117,588 & - & $1,661,893$ & $15,064.98$ & 3 & 2015 \\
\hline $1,348,092$ & 82,322 & - & $1,430,414$ & $12,966.63$ & 3 & 2014 \\
\hline $1,083,372$ & 60,246 & - & $1,143,618$ & $10,366.84$ & 3 & 2013 \\
\hline $20,481.90$ & $47,986.24$ & 4851.52 & $73,319.66$ & 1052.14 & 4 & 2017 \\
\hline $17,899.23$ & $38,393.71$ & 1088.37 & $57,381.31$ & 823.42 & 4 & 2016 \\
\hline $11,886.91$ & $30,876.97$ & 3548.59 & $46,312.47$ & 664.58 & 4 & 2015 \\
\hline 9289.40 & $24,480.46$ & 5.61 & $33,775.47$ & 484.68 & 4 & 2014 \\
\hline 6971.33 & $19,015.34$ & 1202.25 & $27,189.92$ & 390.18 & 4 & 2013 \\
\hline 932,607 & 10,581 & 121,366 & 1064,554 & 9650.13 & 5 & 2017 \\
\hline $1,015,968$ & 37,787 & 39,841 & 1093,596 & 9913.40 & 5 & 2016 \\
\hline 998,751 & 45,411 & 168,331 & $1,212,493$ & $10,991.19$ & 5 & 2015 \\
\hline 952,898 & 66,794 & 145,595 & $1,165,287$ & $10,563.27$ & 5 & 2014 \\
\hline 889,955 & 5827 & 5990 & 901,772 & 8174.52 & 5 & 2013 \\
\hline 92,681 & 573 & 8673 & 101,927 & $14,760.85$ & 6 & 2017 \\
\hline 86,442 & 3737 & 7006 & 97,185 & $14,074.12$ & 6 & 2016 \\
\hline 62,281 & 3715 & 5077 & 71,073 & $10,292.64$ & 6 & 2015 \\
\hline 47,468 & 1455 & 5187 & 54,110 & 7836.09 & 6 & 2014 \\
\hline 38,943 & 3942 & 4159 & 47,044 & 6812.81 & 6 & 2013 \\
\hline $699,678+1,814,478$ & 112,842 & 290,876 & $2,917,874$ & 2917.874 & 7 & 2017 \\
\hline $680,675+1,806,434$ & 359,271 & 206,294 & $3,052,674$ & 3052.674 & 7 & 2016 \\
\hline $696,461+1,677,771$ & 315,756 & 294,044 & $2,984,032$ & 2984.032 & 7 & 2015 \\
\hline $673,816+1,668,882$ & 191,770 & 313,674 & $2,848,142$ & 2848.142 & 7 & 2014 \\
\hline $681,313+1,619,993$ & 90,032 & 384,693 & $2,776,031$ & 2776.031 & 7 & 2013 \\
\hline $1,661,212$ & 98,024 & 818 & $1,760,054$ & $15,954.80$ & 8 & 2017 \\
\hline $1,444,967$ & 82,681 & 1061 & $1,528,709$ & $1,3857.67$ & 8 & 2016 \\
\hline $1,579,174$ & 116,105 & 584 & $1,695,863$ & $15,372.92$ & 8 & 2015 \\
\hline $1,497,983$ & 118,000 & 500 & $1,616,483$ & $14,653.34$ & 8 & 2014 \\
\hline $1,461,144$ & 108,088 & 550 & $1,569,782$ & $14,230.00$ & 8 & 2013 \\
\hline 4324.60 & 5092.38 & 3002.64 & $12,419.62$ & 1782.22 & 9 & 2017 \\
\hline 3609.32 & 5787.39 & 184.24 & 9580.95 & 1374.87 & 9 & 2016 \\
\hline 3669.78 & 4979.31 & 255.79 & 8904.88 & 1277.85 & 9 & 2015 \\
\hline 3867.99 & 4543.85 & 2929.91 & $11,341.75$ & 1627.54 & 9 & 2014 \\
\hline 3360.12 & 4211.22 & 3959.24 & $11,530.58$ & 1654.64 & 9 & 2013 \\
\hline $1,792,278$ & 19,014 & 125,112 & $1,936,404$ & $17,553.41$ & 10 & 2017 \\
\hline $1,940,363$ & 27,594 & 122,075 & $2,090,032$ & $18,946.04$ & 10 & 2016 \\
\hline $1,887,901$ & 26,913 & 146,540 & $2,061,354$ & $18,686.07$ & 10 & 2015 \\
\hline $2,000,028$ & 26,107 & 204,152 & $2,230,287$ & $20,217.44$ & 10 & 2014 \\
\hline $1,875,052$ & 26,707 & 106,816 & $2,008,575$ & $18,207.64$ & 10 & 2013 \\
\hline $14,245.7$ & - & 76.7 & $14,322.4$ & $14,322.4$ & 11 & 2017 \\
\hline $14,557.5$ & - & 120.6 & $14,678.1$ & $14,678.1$ & 11 & 2016 \\
\hline $15,156.1$ & - & 36.2 & $15,192.3$ & $15,192.3$ & 11 & 2015 \\
\hline $14,984.6$ & - & 56.5 & $15,041.1$ & $15,041.1$ & 11 & 2014 \\
\hline $14,869.0$ & - & 40.4 & $14,909.4$ & $14,909.4$ & 11 & 2013 \\
\hline 7045.50 & $13,853.07$ & 2148.55 & $23,047.12$ & 3307.26 & 12 & 2017 \\
\hline 6146.68 & 9204.84 & 2548.48 & 17,900 & 2568.65 & 12 & 2016 \\
\hline 1997.11 & 4150.84 & 1645.04 & 7792.99 & 111.83 & 12 & 2015 \\
\hline 1932.03 & 4662.37 & 1774.78 & 8369.18 & 1200.98 & 12 & 2014 \\
\hline 2085.66 & 3860.93 & 1547.80 & 7494.39 & 1075.44 & 12 & 2013 \\
\hline
\end{tabular}

(Source: Profit loss statement / income statement from the balance sheet of 12 firms). 


\section{Data Source}

Tata Motors Annual Report. (2018). https:/ / www.tatamotors.com/wp-content/uploads / 2018/07/12115930/Annual-Report-2017-2018.pdf Retrieved on 18 November 2019

Singapore Airlines Annual Report. (2018). https:/ / www.singaporeair.com/saar5/pdf/ Investor-Relations / Annual-Report/annualreport1819.pdf Retrieved on 18 November 2019

Samsung Annual Report. (2018). https: / /images.samsung.com/is / content/samsung/ p5/global/ir/docs/2018_Business_Report_vF.pdf Retrieved on 18 November 2019

Nissan Annual Report. 2018. Nissan motor corporation annual report, available online: Nissan https: / / www.nissan-global.com/EN/IR/LIBRARY/AR/2018/. Retrieved on 11 May 2019.

Indigo Annual Report. (2018). https: / / www.goindigo.in/content/dam/goindigo/ investor-relations / annual-report/2018-19/ Annual-Report-and-Notice-InterGlobe-AviationLimited-2018-19.pdf Retrieved on 18 November 2019

Huawei Annual Report. (2018). https:/ / www-file.huawei.com/-/media/corporate/ pdf/annual-report/annual_report2018_en.pdf?la=en Retrieved on 18 November 2019

Hitachi Annual Report. (2018). https://www.hitachi.com/IR-e/library/integrated/ 2018/index.html Retrieved on 18 November 2019

Canon Annual Report. (2018). Canon annual report, available online https:/ /global. canon/en/ir/annual/2018/canon-annual-report-2018.pdf. Retrieved on 11 May 2019.

L\&T Annual Report. (2018). https:/ / investors.larsentoubro.com/AnnualReports.aspx Retrieved on 18 November 2019

Mitsubishi Annual Report. (2018). https://www.mitsubishicorp.com/jp/en/ir/ library/ar/pdf/areport/2018/all.pdf Retrieved on 18 November 2019

NTPC Annual Report. (2018). https:/ / www.ntpc.co.in/en/investors/annual-reports Retrieved on 18 November 2019

Wilmar Annual Report. (2018). https:/ / www.wilmar-international.com/annualreport2 018 / Retrieved on 18 November 2019.

\section{References}

1. Dittmer, L. Globalization and the Asian financial crisis. Asian Perspect. 1999, 23, 45-64. [CrossRef]

2. Kim, S.Y. Do Asian values exist? Empirical tests of the four dimensions of Asian values. J. East Asian Stud. 2010, 10, 315-344. [CrossRef]

3. Kausikan, B. Asia's different standard. Foreign Policy 1993, 92, 24. [CrossRef]

4. Schmitt, B. The "new wave" in studying Asian markets and consumers. Mark. Lett. 2015, 26, 261-264. [CrossRef]

5. Cheow, E.T. Strategic relevance of Asian economic integration. Econ. Polit. Wkly. 2005, 40, 3960-3967.

6. Rana, A. Challenges to strategic stability in south Asia. Strateg. Stud. 2018, 38, 1-20.

7. He, K.A. Strategic Functional Theory of Institutions and Rethinking Asian Regionalism: When Do Institutions Matter? Asian Surv. 2014, 54, 1184-1208. [CrossRef]

8. Johnston, T. The Strategic Risks of East Asia's Slowing Economies. Australian Strategic Policy Institute. August 2017. Available online: https:/ / ethz.ch/content/dam/ethz/special-interest/gess/cis/center-for-securities-studies / resources / docs / ASPI-SI1 20\%20The\%20strategic\%20risks\%20of\%20East\%20Asias\%20slowing\%20economies.pdf (accessed on 24 August 2019).

9. Biao, X.; Wong, T. SARS: Public Health and Social Science Perspectives. Econ. Polit. Wkly. 2003, 38, $2480-2483$.

10. Lam, W.K.; Zhong, N.S.; Tan, W.C. Overview on SARS in Asia and the World. Respirology 2003, 8, S2-S5. [CrossRef] [PubMed]

11. Hanna, D.; Huang, Y. The Impact of SARS on Asian Economies. Asian Econ. Pap. 2004, 3, 102-112. [CrossRef]

12. Chan-Yeung, M.; Seto, W.H.; Sung, J.J.Y. Severe acute respiratory syndrome: Patients were epidemiologically linked. BMJ 2003, 326, 1393. [CrossRef]

13. Frankenberg, E.; Sikoki, B.; Sumantri, C.; Suriastini, W.; Thomas, D. Education, Vulnerability, And Resilience After a Natural Disaster. Ecol. Soc. J. Integr. Sci. Resil. Sustain. 2013, 18, 16. [CrossRef] [PubMed]

14. Gaillard, J.-C.; Clavé, E.; Vibert, O.; Azhari, D.; Denain, J.-C.; Efendi, Y.; Grancher, D.; Liamzon, C.C.; Sari, D.R.; Setiawan, R. Ethnic groups' response to the 26 December 2004 earthquake and tsunami in Aceh, Indonesia. Nat. Hazards 2008, 47, 17-38. [CrossRef]

15. Kellenberg, D.; Mobarak, A.M. The Economics of Natural Disasters. Annu. Rev. Resour. Econ. 2011, 3, 297-312. [CrossRef]

16. Low, W.-Y.; Binns, C. Disasters and Public Health Concerns. Asia Pac. J. Public Health 2011, 23, 277-279. [CrossRef] [PubMed]

17. United Nations ESCAP. Available online: https://www.unescap.org/sites/default/d8files/knowledge-products/APTIT\%20FDI. pdf (accessed on 19 August 2021).

18. OECD. Available online: https://www.oecd.org/development/asia-challenges.htm (accessed on 19 August 2021). 
19. ABD. Available online: https://www.adb.org/sites/default/files/publication/674421/asian-economic-integration-report-20 21.pdf (accessed on 19 August 2021).

20. Claessens, S.; Djankov, S.; Xu, L.C. Corporate Performance in the East Asian Financial Crisis. World Bank Res. Obs. 2000, 15, 23-46. [CrossRef]

21. Silva, C.A.; Ordeñana, X.; Vera-Gilces, P.; Jiménez, A. Global Imbalances: The Role of Institutions, Financial Development and FDI in the Context of Financial Crises. Sustainability 2021, 13, 356. [CrossRef]

22. Wisner, J.D.; Fawcett, S.E. Linking Firm Strategy to Operating Decisions Through Performance Measurement. Prod. Inventory Manag. J. 1991, 32, 5.

23. Sørensen, J.B. The Strength of Corporate Culture and the Reliability of Firm Performance. Adm. Sci. Q. 2002, 47, 70. [CrossRef]

24. Knack, S.; Keefer, P. Does Social Capital Have an Economic Payoff? A Cross-Country Investigation. Q. J. Econ. 1997, 112, 1251-1288. [CrossRef]

25. Larsen, M.M.; Manning, S.; Pedersen, T. The ambivalent effect of complexity on firm performance: A study of the global service provider industry. Long Range Plan. 2019, 52, 221-235. [CrossRef]

26. Momaya, K.S.; Bhat, S.; Lalwani, L. Institutional Growth and Industrial Competitiveness: Exploring the Role of Strategic Flexibility Taking the Case of Select Institutes in India. Glob. J. Flex. Syst. Manag. 2017, 18, 111-122. [CrossRef]

27. Bhattacharya, S.; Momaya, K.S.; Iyer, K.C. Strategic Change for Growth: A Case of Construction Company in India. Glob. J. Flex. Syst. Manag. 2012, 13, 195-205. [CrossRef]

28. Dosi, G.; Faillo, M.; Marengo, L. Organizational Capabilities, Patterns of Knowledge Accumulation and Governance Structures in Business Firms: An Introduction. Organ. Stud. 2008, 29, 1165-1185. [CrossRef]

29. Calori, R. Essai: Philosophizing on Strategic Management Models. Organ. Stud. 1998, 19, 281-306. [CrossRef]

30. Van de Ven, A.H.; Rogers, R.W.; Bechara, J.P.; Sun, K. Organizational diversity, integration and performance. J. Organ. Behav. 2008, 29, 335-354. [CrossRef]

31. Yström, A.; Agogué, M.; Rampa, R. Preparing an Organization for Sustainability Transitions-The Making of Boundary Spanners through Design Training. Sustainability 2021, 13, 8073. [CrossRef]

32. Mueller, G.C.; Mone, M.A.; Barker, V.L. Formal Strategic Analyses and Organizational Performance: Decomposing the Rational Model. Organ. Stud. 2007, 28, 853-883. [CrossRef]

33. Luo, Y.; Tan, J.J.; Shenkar, O. Strategic Responses to Competitive Pressure: The Case of Township and Village Enterprises in China. Asia Pac. J. Manag. 1998, 15, 33-50. [CrossRef]

34. Colpan, A.M. Are strategy-performance relationships contingent on macroeconomic environments? Evidence from Japan's textile industry. Asia Pac. J. Manag. 2007, 25, 635-665. [CrossRef]

35. Damanpour, F.; Devece, C.; Chen, C.C.; Pothukuchi, V. Organizational culture and partner interaction in the management of international joint ventures in India. Asia Pac. J. Manag. 2010, 29, 453-478. [CrossRef]

36. Abdallah, C.; Langley, A. The Double Edge of Ambiguity in Strategic Planning. J. Manag. Stud. 2013, 51, 235-264. [CrossRef]

37. Lasserre, P. Corporate strategic management and the overseas Chinese groups. Asia Pac. J. Manag. 1988, 5, 115-131. [CrossRef]

38. Martin, R.; Sunley, P. On the notion of regional economic resilience: Conceptualization and explanation. J. Econ. Geogr. 2014, 15, 1-42. [CrossRef]

39. Lele, S. Resilience, sustainability environmentalism. Environ. Dev. Econ. 1998, 3, 221-262. [CrossRef]

40. Khan, M.; Pervez, A.; Modibbo, U.; Chauhan, J.; Ali, I. Flexible Fuzzy Goal Programming Approach in Optimal Mix of Power Generation for Socio-Economic Sustainability: A Case Study. Sustainability 2021, 13, 8256. [CrossRef]

41. Martin, R. Regional economic resilience, hysteresis and recessionary shocks. J. Econ. Geogr. 2012, 12, 1-32. [CrossRef]

42. Jones, L.; Constas, M.A.; Matthews, N.; Verkaart, S. Advancing resilience measurement. Nat. Sustain. 2021, 4, 288-289. [CrossRef]

43. D'Adamo, I.; Lupi, G. Sustainability and resilience after COVID-19: A circular premium in the fashion industry. Sustainability 2021, 13, 1861. [CrossRef]

44. Myrdal, G. What is development? J. Econ. Issues 1974, 8, 729-736. [CrossRef]

45. Preston, P.W. Development Theory: An Introduction; Blackwell Publishers Ltd.: Oxford, UK, 1999; pp. 169-173.

46. Gordon, R.A. Rigor and relevance in a changing institutional setting. Am. Econ. Rev. 1976, 66, 1-14.

47. Sushil. SAP-LAP Framework. Glob. J. Flex. Syst. Manag. 2001, 2, 51-55.

48. Streeten, P. Unbalanced growth. Oxf. Econ. Pap. 1959, 11, 167-190. [CrossRef]

49. Gerring, J.; Ziblatt, D.; Van Gorp, J.; Arevalo, J. An institutional theory of direct and indirect rule. World Polit. 2011, 63, 377-433. [CrossRef]

50. McClintock, C.C.; Brannon, D.; Maynard-Moody, S. Applying the logic of sample surveys to qualitative case studies: The case cluster method. Adm. Sci. Q. 1979, 24, 612. [CrossRef]

51. Lowi, T.J. American business, public policy, case-studies, and political theory. World Polit. 1964, 16, 677-715. [CrossRef]

52. Rowley, J. Using case studies in research. Manag. Res. News 2002, 25, 16-27. [CrossRef]

53. Welch, C.; Piekkari, R.; Plakoyiannaki, E.; Paavilainen-Mäntymäki, E. Theorising from case studies: Towards a pluralist future for international business research. J. Int. Bus. Stud. 2010, 42, 740-762. [CrossRef]

54. Kazdin, A.E. Drawing valid inferences from case studies. J. Consult. Clin. Psychol. 1981, 49, 183. [CrossRef]

55. Patton, E.; Appelbaum, S.H. The case for case studies in management research. Manag. Res. News 2003, 26, 60-71. [CrossRef]

56. Siggelkow, N. Persuasion with case studies. Acad. Manag. J. 2007, 50, 20-24. [CrossRef] 
57. Kitchenham, B.; Pickard, L.; Pfleeger, S. Case studies for method and tool evaluation. IEEE Softw. 1995, 12, 52-62. [CrossRef]

58. Stewart, J. Multiple-case study methods in governance-related research. Public Manag. Rev. 2012, 14, 67-82. [CrossRef]

59. Kompier, M.A.; Cooper, C.L.; Geurts, S.A. A multiple case study approach to work stress prevention in Europe. Eur. J. Work. Organ. Psychol. 2000, 9, 371-400. [CrossRef]

60. Bandara, W.; Gable, G.G.; Rosemann, M. Factors and measures of business process modelling: Model building through a multiple case study. Eur. J. Inf. Syst. 2005, 14, 347-360. [CrossRef]

61. Walsham, G. Doing interpretive research. Eur. J. Inf. Syst. 2006, 15, 320-330. [CrossRef]

62. Sushil. Interpretive matrix: A tool to aid interpretation of management and social research. Global J. Editor. Flex. Syst. 2005, 6, 27-30.

63. Sushil. Modified ISM/TISM process with simultaneous transitivity checks for reduced direct pair comparisons. Glob. J. Flex. Syst. Manag. 2017, 18, 331-351. [CrossRef]

64. Sushil. How to check correctness of total interpretive structural models? Ann. Oper. Res. 2018, 270, 473-487. [CrossRef]

65. Dhir, S.; Dhir, S. Modeling of strategic thinking enablers: A modified total interpretive structural modeling (TISM) and MICMAC approach. Int. J. Syst. Assur. Eng. Manag. 2020, 11, 175-188. [CrossRef]

66. Singh, S.; Dhir, S. Modified total interpretive structural modelling of innovation implementation antecedents. Int. J. Prod. Perform. Manag. 2021. [CrossRef]

67. Agrawal, A. Modified Total Interpretive Structural Model of Corporate Financial Flexibility. Glob. J. Flex. Syst. Manag. 2020, 21, 369-388. [CrossRef]

68. Rajan, R.; Rana, N.P.; Parameswar, N.; Dhir, S.; Sushil; Dwivedi, Y.K. Developing a modified total interpretive structural model (M-TISM) for organizational strategic cybersecurity management. Technol. Forecast. Soc. Chang. 2021, 170, 120872. [CrossRef]

69. Consul, P.C.; Jain, G.C. A generalization of the Poisson distribution. Technometrics 1973, 15, 791-799. [CrossRef]

70. Thorndike, F. Applications of Poisson's probability summation. Bell Syst. Tech. J. 1926, 5, 604-624. [CrossRef]

71. Hsiao, C. Panel data analysis—advantages and challenges. Test 2007, 16, 1-22. [CrossRef]

72. Plümper, T.; Troeger, V.E.; Manow, P. Panel data analysis in comparative politics: Linking method to theory. Eur. J. Polit. Res. 2005, 44, 327-354. [CrossRef]

73. Woodside, A.G.; Wilson, E.J. Case study research methods for theory building. J. Bus. Ind. Mark. 2003, 18, 493-508. [CrossRef]

74. Amaratunga, D.; Baldry, D. Case study methodology as a means of theory building: Performance measurement in facilities management organisations. Work. Study 2001, 50, 95-105. [CrossRef]

75. Stiles, W.B. Theory-building case studies of counselling and psychotherapy. Couns. Psychother. Res. 2007, 7, 122-127. [CrossRef]

76. Denoon, D.B.H.; Colbert, E. Challenges for the Association of Southeast Asian Nations (ASEAN). Pac. Aff. 1998, 71, 505. [CrossRef]

77. Hill, H. ASEAN economic development: An analytical survey-The state of the field. J. Asian Stud. 1994, 53, 832-866. [CrossRef]

78. Raipuria, K. ASEAN and SAARC: Select Futuristic Scenarios. Econ. Polit. Wkly. 2002, 37, 3581-3582.

79. Bandara, J.S.; Yu, W. How Desirable is the South Asian Free Trade Area? A Quantitative Economic Assessment. World Econ. 2003, 26, 1293-1323. [CrossRef]

80. Park, J.H. The East Asian Model of Economic Development and Developing Countries. J. Dev. Soc. 2002, 18, 330-353. [CrossRef]

81. Moghadam, M.R.; Samavati, H.; Dilts, D.A. An Examination of Capital Flight from East Asian Emerging Economies: Paradise Lost. J. Asia-Pac. Bus. 2003, 5, 33-49. [CrossRef]

82. Kim, J.-I.; Lau, L.J. The Sources of Economic Growth of the East Asian Newly Industrialized Countries. J. Jpn. Int. Econ. 1994, 8, 235-271. [CrossRef]

83. Canon Annual Report. 2018. Available online: https://global.canon/en/ir/annual/2018/canon-annual-report-2018.pdf (accessed on 11 May 2019).

84. Nissan Annual Report. Nissan Motor Corporation Annual Report. 2018. Available online: https://www.nissan-global.com/EN/ IR/LIBRARY/AR/2018/ (accessed on 11 May 2019).

85. Singapore Airlines Annual Report. Annual Report and Sustainability Report. 2018. Available online: https://www.singaporeair. com/en_UK/us/about-us/information-forinvestors/annual-report/ (accessed on 11 May 2019).

86. Moore, J. Channel Strategy. 2021. Available online: https://searchitchannel.techtarget.com/definition/channel-strategy (accessed on 19 August 2021).

87. Bappy, M.M.; Ali, S.M.; Kabir, G.; Paul, S.K. Supply chain sustainability assessment with Dempster-Shafer evidence theory: Implications in cleaner production. J. Clean. Prod. 2019, 237, 117771. [CrossRef]

88. Dulia, E.F.; Ali, S.M.; Garshasbi, M.; Kabir, G. Admitting risks towards circular economy practices and strategies: An empirical test from supply chain perspective. J. Clean. Prod. 2021, 317, 128420. [CrossRef]

89. Rabbi, M.; Ali, S.M.; Kabir, G.; Mahtab, Z.; Paul, S.K. Green Supply Chain Performance Prediction Using a Bayesian Belief Network. Sustainability 2020, 12, 1101. [CrossRef]

90. Economic Times. Tata Motors Aims to Provide Skill Training to 40k in 3 Years. 2017. Available online: https: / / economictimes. indiatimes.com/industry/tata-motors-aims-to-provide-skill-training-to-40k-in-3-years/articleshow/58912005.cms (accessed on 28 May 2019).

91. Huawei Annual Report. 2018. Available online: https://www.huawei.com/en/press-events/annual-report (accessed on 11 May 2019). 
92. Lutters, E.; Van Houten, F.J.; Bernard, A.; Mermoz, E.; Schutte, C.S. Tools and techniques for product design. CIRP Ann. 2014, 63, 607-630. [CrossRef]

93. Grosfeld-Nir, A.; Ronen, B.; Kozlovsky, N. The Pareto managerial principle: When does it apply? Int. J. Prod. Res. 2007, 45, 2317-2325. [CrossRef]

94. Radson, D.; Boyd, A.H. The pareto principle and rate analysis. Qual. Eng. 1997, 10, 223-229. [CrossRef]

95. Yoo, Y.; Kim, K. How Samsung Became a Design Powerhouse. Harv. Bus. Rev. 2015, 93, 73-78.

96. Lincoln, J.R.; Ahmadjian, C.L.; Mason, E. Organizational Learning and Purchase-Supply Relations in Japan: Hitachi, Matsushita, and Toyota Compared. Calif. Manag. Rev. 1998, 40, 241-264. [CrossRef]

97. Nonala, I.; Kenney, M. Towards a new theory of innovation management: A case study comparing Canon, Inc. and Apple Computer, Inc. J. Eng. Technol. Manag. 1991, 8, 67-83. [CrossRef]

98. Dean, J.W., Jr.; Sharfman, M.P. Does Decision Process Matter? A Study of Strategic Decision-Making Effectiveness. Acad. Manag. J. 1996, 39, 368-392.

99. Ma, H.; Park, S. Relationship between Corporate Sustainability Management and Sustainable Tax Strategies. Sustainability 2021, 13, 7429. [CrossRef]

100. Das, T.K.; Teng, B.-S. Cognitive Biases and Strategic Decision Processes: An Integrative Perspective. J. Manag. Stud. 1999, 36, 757-778. [CrossRef]

101. D'Adamo, I.; Gastaldi, M.; Morone, P. The post COVID-19 green recovery in practice: Assessing the profitability of a policy proposal on residential photovoltaic plants. Energy Policy 2020, 147, 111910. [CrossRef] [PubMed]

102. Cucchiella, F.; D'Adamo, I.; Gastaldi, M. Financial analysis for investment and policy decisions in the renewable energy sector. Clean Technol. Environ. Policy 2015, 17, 887-904. [CrossRef]

103. D'Adamo, I.; Rosa, P. How Do You See Infrastructure? Green Energy to Provide Economic Growth after COVID. Sustainability 2020, 12, 4738. [CrossRef]

104. Margherita, E.G.; Braccini, A.M. Industry 4.0 Technologies in flexible manufacturing for sustainable organizational value: Reflections from a multiple case study of italian manufacturers. Inf. Syst. Front. 2020, 1-22. [CrossRef] 
Article

\title{
Promoting Sustainability: Wastewater Treatment Plants as a Source of Biomethane in Regions Far from a High-Pressure Grid. A Real Portuguese Case Study
}

\author{
Francisco M. Baena-Moreno ${ }^{1,2, * \mathbb{D}}$, Isabel Malico ${ }^{2,3, *(\mathbb{D})}$ and Isabel Paula Marques 4 \\ 1 Chemical and Environmental Engineering Department, Technical School of Engineering, University of Seville, \\ 41092 Sevilla, Spain \\ 2 Department of Mechatronics Engineering, University of Évora, 7000-671 Évora, Portugal \\ 3 Instituto Superior Técnico (IDMEC), Universidade de Lisboa, 1049-001 Lisboa, Portugal \\ 4 Laboratório Nacional de Energia e Geologia (LNEG), 1649-038 Lisboa, Portugal; isabel.paula@lneg.pt \\ * Correspondence: fbaena2@us.es (F.M.B.-M.); imbm@uevora.pt (I.M.)
}

Citation: Baena-Moreno, F.M.;

Malico, I.; Marques, I.P. Promoting Sustainability: Wastewater Treatment Plants as a Source of Biomethane in Regions Far from a High-Pressure Grid. A Real Portuguese Case Study. Sustainability 2021, 13, 8933.

https://doi.org/10.3390/su13168933

Academic Editor: Idiano D’Adamo

Received: 8 July 2021

Accepted: 5 August 2021

Published: 10 August 2021

Publisher's Note: MDPI stays neutral with regard to jurisdictional claims in published maps and institutional affiliations.

Copyright: (c) 2021 by the authors. Licensee MDPI, Basel, Switzerland. This article is an open access article distributed under the terms and conditions of the Creative Commons Attribution (CC BY) license (https:/ / creativecommons.org/licenses/by/ $4.0 /)$.

\begin{abstract}
Wastewater treatment plants (WWTP) located in regions far from a high-pressure grid can produce renewable biomethane, which can partially substitute the natural gas locally consumed. However, the economic viability of implementing biomethane plants in WWTP has to be guaranteed. This paper uses the discount cash flow method to analyze the economic viability of producing biomethane in a WWTP located in Évora (Portugal). The results show that, under the current conditions, it is unprofitable to produce biomethane in this WWTP. Since selling the $\mathrm{CO}_{2}$ separated from biogas may result in an additional income, this option was also considered. In this case, a price of $46 \mathrm{EUR} / \mathrm{t} \mathrm{CO}_{2}$ has to be paid to make the project viable. Finally, the impact of potential government incentives in the form of feed-in premia was investigated. Without selling $\mathrm{CO}_{2}$, the project would only be profitable for feed-in premia above $55.5 \mathrm{EUR} / \mathrm{MWh}$. If all the $\mathrm{CO}_{2}$ produced was sold at $30 \mathrm{EUR} / \mathrm{t} \mathrm{CO}_{2}$, a premium price of $20 \mathrm{EUR} / \mathrm{MWh}$ would make the project profitable. This study shows that the economic attractiveness of producing biomethane in small WWTP is only secured through sufficient financial incentives, which are vital for developing the biomethane market with all its associated advantages.
\end{abstract}

Keywords: biomethane; natural gas grid; bioenergy; biogas; gas supply decarbonization; incentives

\section{Introduction}

The search for alternative energy sources is a present challenge for societies [1]. The need to find a replacement for fossil fuels emerged both because of the scarcity of known non-renewable fuel reserves and because of the environmental problems caused by greenhouse gas (GHG) emissions [2,3]. In this context, renewable energies have become important in the last decades mainly because of the sources from where they come [4,5]. Indeed, the global total primary renewable energy supply in the world reached around $80 \mathrm{EJ}$ in 2018 , with an average annual growth rate of $2.0 \%$ since 1990 [6]. Renewable energies can also improve the relationship between sustainability and resilience, an important aim if we look at how COVID-19 quickly changed our lifestyle [7]. Among the available renewable energy sources, renewable waste has a double benefit to societies [8]. Waste-to-energy solutions reduce the amount of waste that needs to be treated or that is disposed of and at the same time produce energy that can replace conventional fossil fuels, hence promoting the evolution towards sustainable paths [9]. Clear examples of such solutions involve the sludge produced in wastewater treatment plants (WWTP), which has a huge potential to be converted into energy or fuels [10], but in many cases still ends up in landfills [11,12]. Nowadays, the most used waste-to-energy solution in WWTP is the conversion of the produced sludge into biogas in digesters [13] and then to electricity and heat in combined 
heat and power (CHP) systems [14-16] (Figure 1). Biogas is mainly composed of $\mathrm{CH}_{4}$ (50-75\%) and $\mathrm{CO}_{2}(25-45 \%)$ [17], and when it is burned with air, mainly $\mathrm{CO}_{2}$, water vapor and nitrogen are released [18]. $\mathrm{CO}_{2}$ is an important GHG; however, $\mathrm{CHP}$ systems are a better solution than emitting $\mathrm{CH}_{4}$ during the anaerobic decomposition of sludge [19]. Another alternative to the onsite conversion of biogas to electricity and heat (presented in Figure 1) is to upgrade it [20], thus removing $\mathrm{CO}_{2}$ and producing a high purity $\mathrm{CH}_{4}$ stream, which is called "biomethane" and can replace traditional natural gas [21]. Biomethane is very versatile. It can be injected into a natural gas grid, where it is mostly consumed for the production of heat [22]. Moreover, it can be used as a transport fuel [20]. Due to its benefits, biogas upgrading techniques for the production of biomethane are increasing in presence at industrial levels $[23,24]$. Indeed, many studies focused on making the process more affordable have recently been presented $[25,26]$.

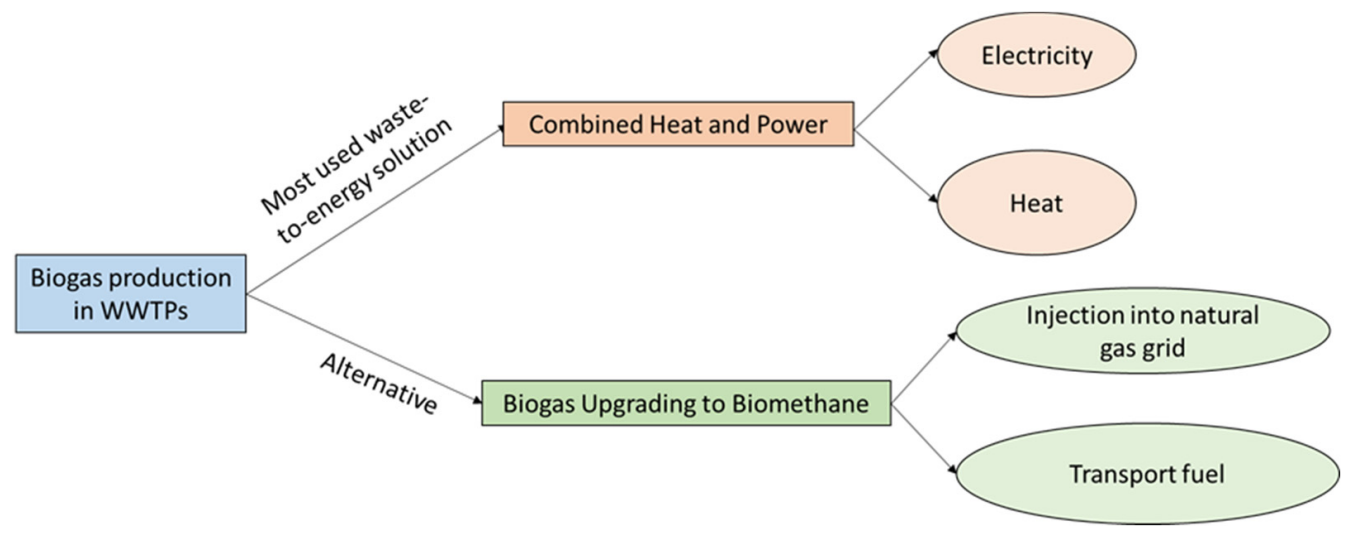

Figure 1. Process scheme of alternatives to valorize biogas.

The production of biomethane presents itself as a really interesting option for regions with no natural gas reserves and that are far from a high-pressure natural gas grid. The natural gas consumed in those regions needs to be brought by road tankers, hence increasing the overall costs and the environmental impacts caused by the transport. The consumption of locally produced biomethane instead of traditional natural gas would avoid these two issues. Moreover, this alternative prevents the consumption of natural gas, therefore extending the life span of the reserves of this kind of fossil fuel. On the other hand, the upgrading stage needed for removing $\mathrm{CO}_{2}$ from biogas and the transportation of the final biomethane to a delivery point would add an important cost to the operation of the WWTP. Therefore, economic feasibility studies are needed to assess the real benefits of upgrading the biogas produced in WWTP into biomethane and its potential profitability for regions far from high-pressure natural gas grids. There are studies that have already addressed the profitability of biogas/biomethane production plants sourced by various substrates in general $[27,28]$ and by sewage sludge in particular $[15,29-31]$. For example, Venkatesh et al. compared several routes for energy recovery from sewage sludge [29]. Among the several options, they considered biogas upgrading to biomethane for transport. Other examples are the study of Mills et al., which compared producing biomethane for grid injection with other options [15], of Collet et al., which compared (among others) biogas upgrading with biomethane injection into the grid with and without $\mathrm{CO}_{2}$ conversion into methane via methanation [30], or of Michailos et al., who studied the techno-economic feasibility of coupling biomethanation with digestate gasification [31]. These studies show that the choice of the most financially and environmentally attractive option depends on several factors, such as the electricity grid carbon intensity, existence of nearby users for the surplus heat produced by CHP systems, fuel and energy vector prices, the weighting factors used to combine the environmental and economic results, incentives, region, etc. For the Portuguese scenario and for regions far from the high-pressure natural gas grid, no studies dealing with the profitability of biomethane production from WWTP have been 
found. This work arises as a study for closing the gap herein explained. To the best of the authors' knowledge, no works have been presented to date dealing with profitability studies of the replacement of natural gas by biomethane as a solution for regions far from the high-pressure natural gas grid.

In this work, a real case study approach is used to analyze the economic feasibility of upgrading the biogas produced in the WWTP of Évora (Portugal). Évora was chosen as a real example of a city that is far from the high-pressure natural gas grid and to which the natural gas is supplied by road transportation. To meet the objective proposed, this work is organized as follows. First, the current status of biogas and biomethane production in Portugal is analyzed, followed by a description of the case study selected. The scenarios considered are also explained in this section. The economic model and the main assumptions of this work are explained in the method section. The results obtained are then presented and analyzed, followed by a discussion section. Finally, the conclusion section summarizes the main achievements of our work.

\section{Biogas and Biomethane in Portugal}

In 2020, biomethane from biogas was produced Europe-wide in 729 plants in 18 countries [32,33]. The production has been steadily growing, and so has the size of the biomethane plants [32]. The shift from CHP to upgrading biogas to biomethane that occurs in Europe has various reasons: developments in biogas upgrading technologies, low costeffectiveness of electricity biogas plants and the new opportunities for biomethane use in the transport sector [34]. In 2017, a total of 1.94 billion cubic meters of biomethane were produced in Europe; with Denmark, Sweden and Germany having the greatest production per capita [32]. Water scrubbing and membrane separation are the most used upgrading techniques [32]. According to Terlouw et al. [35], the European biomethane potential is 95 billion cubic meters by 2050, so the current production is still far away from its potential. Most of the European biomethane is combusted in CHP systems, but its use as a transport fuel has been increasing [36]. In 10 of the EU (European Union) member states, biomethane is injected into the natural gas grid [34]. The European renewable energy directive imposes $14 \%$ of renewable energy in the transport sector by 2030 , with a sub-target of $3.5 \%$ of advanced biofuels and biogas in this sector [37]. This is a political drive towards the implementation of biomethane plants, and it is expected that the sector will develop in Europe in the next decade [38].

In Portugal, most of the biogas comes from landfills and is used in the production of electricity [32]. In 2017, the number of biogas plants in the country was 64 [32], a number that was lower than the one of 1998, 103 [36]. However, this decrease is not reflected in primary biogas production, as shown in Figure 2.

From 1997 to 2018, there was a shift in the main source of biogas from pig slurry residues [39] (included in "other" in Figure 2) to municipal solid wastes [12]. Till 2007, the Portuguese electricity feed-in-tariff favored landfill gas to the detriment of biogas from other sources, which lead to investments in landfill gas systems [40]. Decree-Law 225/2007 of 31 May matched the electricity feed-in-tariff for all biogas sources but did not produce a large impact. One reason for this may be the economic crisis that immediately followed the change in the legislation, and that hindered the investments in the renewable energy sector.

The Portuguese biogas market is not mature in terms of biogas plants installed [41]. There is still untapped potential for the production of biogas in Portugal that needs to be uncovered [40], and new investments are needed to implement biogas systems for the recycling of organic effluents. More than half of this potential comes from municipal wastes, mostly from the organic fraction of municipal solid wastes. The potential of sewage sludge for the production of biogas in the country was estimated as $1.42 \mathrm{PJ} /$ year (considering it is used for CHP) [40]. Less than $20 \%$ of this potential was realized in 2018, despite the fact that most of the big WWTP in the country already produce biogas and sell electricity to the grid [36]. 


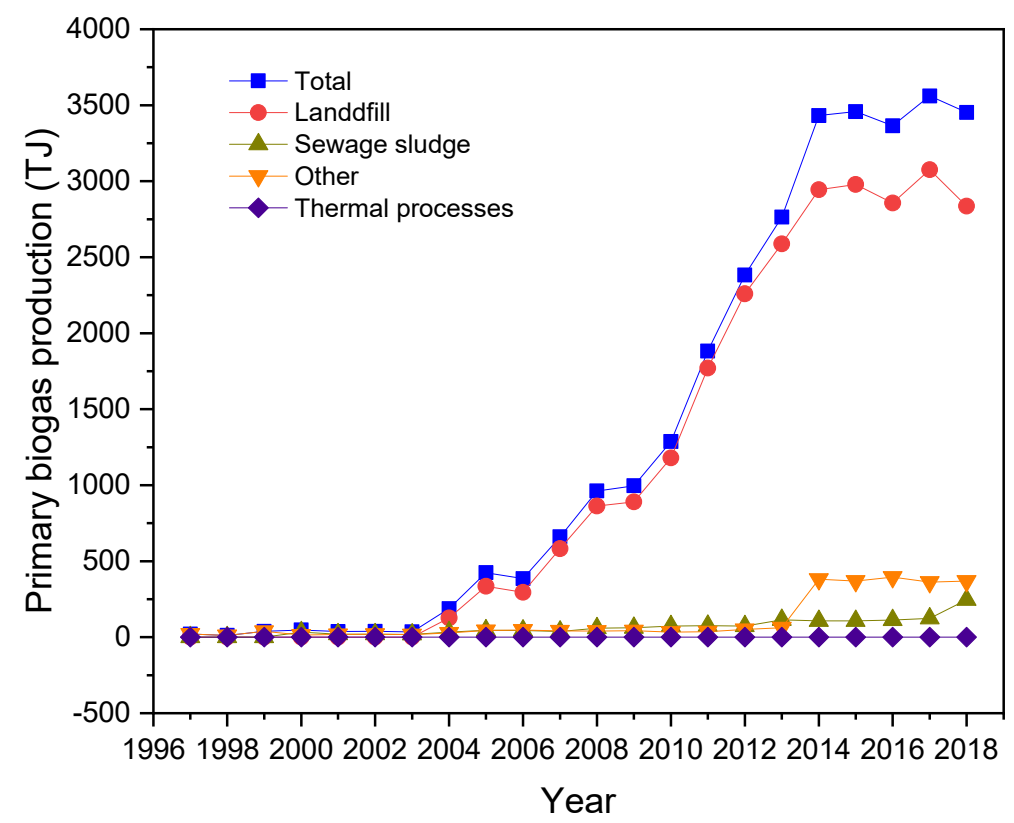

Figure 2. Primary biogas production in Portugal from 1997 to 2018 (Data source: [12]).

To date, there is no biogas upgrading plant in the country, even though the potential exists [36]. The injection of biomethane into the Portuguese natural gas grid would be an interesting option since it could be a partial replacement for the natural gas consumed in the country, which is all imported. Furthermore, it would use the current infrastructure and take advantage of the investments already made in the Portuguese natural gas grid. In line with what has been said, the recent National Energy and Climate Plan [42] lists several actions to be taken for promoting biomethane in Portugal. One of these is the creation of specific regulations for the injection of biomethane into the natural gas grid. Additionally, the plan states that targets for the incorporation of renewable gases will be set. Other than the creation of technical regulations and targets, incentive mechanisms, such as the ones implemented in Sweden, the United Kingdom, Italy, the Netherland or Germany, can be established so that biomethane is attractive for uses other than power production [36].

\section{Case Study}

\section{1. Évora}

Évora $\left(38^{\circ} 34^{\prime} 0^{\prime \prime} \mathrm{N}, 7^{\circ} 54^{\prime} 0^{\prime \prime} \mathrm{W}\right)$ is a Portuguese city located in a rural region in Southwestern Iberia. The municipality of Évora occupies an area of $1307 \mathrm{~km}^{2}$ and hosts circa 57,000 inhabitants. It was selected as a case study on the profitability of upgrading biogas produced in WWTP and injecting it into a local natural gas grid since it is representative of a town in the interior of the country that is not connected to the high-pressure grid. Many other cities are in this situation, and the natural gas that they consume is supplied by road tankers. The consumption of natural gas in Évora in 2018 was $5.048 \times 10^{6} \mathrm{Nm}^{3}$ [43] (services: 37\%; industry: 37\%; households: $26 \%$ ), $67 \%$ more than ten years before.

\subsection{The Évora Wastewater Treatment Plant}

The Évora WWTP has an anaerobic biodigester, which receives the primary and secondary sludge that results from the wastewater treatment and produces biogas. Currently, this biogas is burned in a spark-ignition engine that produces electricity and heat. This energy is used internally in the plant; with the heat being used to maintain the digestion process temperature under mesophilic conditions, and the electricity being utilized in the operation of the WWTP. Even though the onsite energy valorization of the biogas produced in the biodigester lowers the electricity consumed in the treatment of the wastewater and helps to reduce the energy consumption from the grid, the main energy vector substituted 
is electricity supplied by the national grid, which has an already high incorporation of renewable energies. In 2018, in Portugal, the share of energy from renewable energy sources in electricity, heating and cooling and transport was, respectively, $52 \%, 41 \%$ and $9 \%$ [12]. In this context, it is interesting to look for sustainable alternatives for the valorization of the biogas produced in the WWTP.

The WWTP of Évora serves a population equivalent of 47,702 inhabitants and had in 2009 an average volumetric flow rate of effluents of $9987 \mathrm{~m}^{3}$ /day and of sludge to digest of $87 \mathrm{~m}^{3}$ /day (74 and $13 \mathrm{~m}^{3}$ / day of primary and secondary sludge, respectively) [44]. The average BOD5 (biochemical oxygen demand) was $357 \mathrm{mg} / \mathrm{L}$, and its load in the effluent was $104,079 \mathrm{~kg} / \mathrm{month}$ [44]. The Évora WWTP also receives the scum, oil and fat from other WWTP of the region [44]. The bioreactor produces on average $24,917 \mathrm{~m}^{3}$ of biogas per month [44]. The Évora WWTP CHP system started operating in 2007 and has $180 \mathrm{kWe}$ capacity [44]. It produces an average of $38,564 \mathrm{kWh} / \mathrm{month}$, which represents $26 \%$ of the energy consumed in the WWTP [44]. The electricity consumption of the WWTP places it in the consumer band-IC, which corresponded to an electricity price with all taxes and levies included of $0.1440 \mathrm{EUR} / \mathrm{kWh}$ in 2018 [12].

\subsection{The Évora Regasification Unit}

Évora is a region that does not have access to the high-pressure natural gas network [45]. Therefore, the natural gas distributed within the city comes from an autonomous regasification unit that receives liquefied natural gas (LNG) arriving in road tankers coming from the LNG terminal of Sines, typically three per week [46]. The tankers transport 19 to $20.5 \mathrm{t}$ of LNG at average thermodynamic conditions of $-162{ }^{\circ} \mathrm{C}$ and $1 \mathrm{bar}$ [46]. The gasification unit is located in the South of the city (such as the WWTP), $1.2 \mathrm{~km}$ away from the WWTP. It contains a reservoir with a capacity of $120 \mathrm{~m}^{3}$ of LNG (53.4 $\mathrm{t}$ of LNG at a temperature of $-155^{\circ} \mathrm{C}$ and a pressure of 4 bar) [46].

\subsection{Scenarios Considered}

The main motivation to define the scenarios analyzed in this work is considering the shift from producing electricity and heat from the biogas currently generated in the WWTP of Évora to upgrading biogas to biomethane, which enables the replacement of fossil fuels by a renewable gas in applications where other renewable sources are scarcer. The biomethane injected in the natural gas grid would mainly be used for heating purposes (services, industry and households). To fulfill the aforementioned purpose, three scenarios were defined. All of them consider the replacement of the CHP system that is currently working in the WWTP by a biomethane upgrading unit and the construction of a piping system that transports the biomethane to the Évora regasification unit. The main points that differentiate the scenarios are the existence of government incentives for biomethane grid injection and the sale of the $\mathrm{CO}_{2}$ separated in the upgrading stage.

- Scenario 1: this case was selected as the baseline scenario for the proper comparison with the two actions expressed above. Therefore, in this scenario, no $\mathrm{CO}_{2}$ is sold, and no incentives for producing biomethane were considered. The different revenues and costs necessary to install and run the biogas upgrading unit are herein analyzed.

- Scenario 2: this case examines the dependence of the economic viability indicators on the prices for selling $\mathrm{CO}_{2}$. A discussion on the $\mathrm{CO}_{2}$ price needed to make the project profitable in comparison with the realistic $\mathrm{CO}_{2}$ selling price is also included.

- Scenario 3: the last scenario considered in this work includes both the effect of biomethane incentives offered by the government and the sale of $\mathrm{CO}_{2}$. Furthermore, a comparison between the biomethane incentives needed to make the project profitable with and without selling the $\mathrm{CO}_{2}$ was carried out.

\section{Methods}

The discount cash flow method was chosen to assess the profitability of upgrading biomethane in the Évora WWTP under the conditions specified in the aforementioned 
scenarios. This method is widely used for the profitability analysis of engineering projects, and it mainly evaluates the difference between revenues and costs (in terms of cash inflows and cash outflows). Furthermore, in this method, the effect of time is taken into account by the discount rate parameter $\left(r_{\mathrm{d}}\right)$. The indicators usually used to conclude if a project is profitable (enough) or not are the net present value (NPV), discounted payback time (DPBT), internal rate of return (IRR) and profitability index (PI). These indicators are calculated by means of Equation (1) to Equation (4). NPV establishes the difference between the present value of cash inflows and cash outflows over a period of time. DPBT refers to the years needed to recover the initial expenditure considering the time value of money. IRR is the discount rate that makes the NPV of all cash flows of a project equal to zero. Finally, PI indicates the amount of value created per money unit invested.

$$
\begin{gathered}
\mathrm{NPV}=\sum_{t=0}^{n} \frac{I_{t}-O_{t}}{\left(1+r_{\mathrm{d}}\right)^{t}} \\
\sum_{t=0}^{\mathrm{DPBT}} \frac{I_{t}-O_{t}}{\left(1+r_{\mathrm{d}}\right)^{t}}=0 \\
\sum_{t=0}^{n} \frac{I_{t}-O_{t}}{(1+\mathrm{IRR})^{t}}=0 \\
\mathrm{PI}=\frac{\sum_{t=0}^{n} \frac{I_{t}-O_{t}}{\left(1+r_{\mathrm{d}}\right)^{t}}}{C_{\mathrm{inv}}}
\end{gathered}
$$

In the above equations, $I_{t}$ and $O_{t}$ are, respectively, the cash inflow and outflow in the period of time $t, C_{\text {inv }}$ the investment cost and $n$ the project lifetime.

Cash inflows are calculated by Equation (5).

$$
I_{t}=R_{\text {biomethane }}+R_{\mathrm{CO}_{2}}+R_{\mathrm{CHP} \text { avoided cost }}
$$

The yearly revenues obtained by selling biomethane to the grid are calculated by Equation (6) and are based on the average quantity of biomethane produced ( $Q_{\text {biomethane }}$ ) and its unit selling price $\left(p_{\mathrm{NG}}\right)$ plus the potential incentives that may be provided by the Portuguese government ( $\left.p_{\text {premium }}\right)$. The quantity of biomethane was calculated assuming a complete separation of $\mathrm{CH}_{4}$ from the average yearly biogas produced in the WWTP and considering that $60 \%$ of the biogas is methane [47,48]. It was considered that the biomethane could be sold to the operator of the regasification unit at $0.0263 \mathrm{EUR} / \mathrm{kWh}$, which was, in the second semester of 2018, the price excluding taxes and levies of the natural gas to a consumer in band I5 (consumption between $1 \times 10^{6}$ and $4 \times 10^{6} \mathrm{GJ}$ ) [12].

$$
R_{\text {biomethane }}=Q_{\text {biomethane }} \times\left(p_{\mathrm{NG}}+p_{\text {premium }}\right)
$$

The revenues obtained by selling $\mathrm{CO}_{2}$ to other industries (Equation (7)) are obtained by the multiplication of the amount of $\mathrm{CO}_{2}$ produced yearly $\left(\mathrm{Q}_{\mathrm{CO}_{2}}\right)$ and the unitary $\mathrm{CO}_{2}$ selling price $\left(p_{\mathrm{CO}_{2}}\right)$. It was considered that $40 \%$ of the biogas is $\mathrm{CO}_{2}$ and that this gas can be completed separated from the biogas.

$$
R_{\mathrm{CO}_{2}}=Q_{\mathrm{CO}_{2}} \times p_{\mathrm{CO}_{2}}
$$

The money currently spent for the operation of the CHP unit would be saved if it would be replaced by a biomethane plant. This is included in the model as a revenue that corresponds to the yearly avoided costs for not using the CHP unit for cogeneration purposes. It is calculated by multiplying the unitary cost for maintaining and operating the $\mathrm{CHP}$ unit $\left(\mathrm{C}_{\mathrm{u}, \mathrm{CHP}}\right)$ by the average electricity produced by the $\mathrm{CHP}$ system monthly $\left(Q_{\mathrm{e}, \mathrm{CHP}}\right)$ and by the number of months in a year (Equation (8)). According to Monte [44], the WWTP would spend between 0.0075 and $0.015 \mathrm{EUR} / \mathrm{kWh}$ for the operation and management of 
the cogeneration system. An average value of $0.01125 \mathrm{EUR} / \mathrm{kWh}$ was chosen to perform the analysis.

$$
R_{\mathrm{CHP} \text { avoided cost }}=\mathrm{C}_{\mathrm{u}, \mathrm{CHP}} \times Q_{\mathrm{e}, \mathrm{CHP}} \times 12
$$

Cash outflows are calculated by Equation (9). This equation includes a set of costs that are computed in Equation (10) to Equation (16) and that relate to two different stages: biogas upgrading stage (noted by the subscript 1) and biomethane transport to the regasification tank (noted by the subscript 2).

$$
\begin{aligned}
\mathrm{O}_{\mathrm{t}}=\left(C_{\mathrm{loan}, 1}\right. & \left.+C_{\mathrm{il}, 1}+C_{\mathrm{om}, 1}+C_{\mathrm{df}, 1}+C_{\mathrm{ins}, 1}\right) \\
& +\left(C_{\text {loan }, 2}+C_{\mathrm{il}, 2}+C_{\mathrm{om}, 2}\right)+C_{\mathrm{e}}+C_{\text {lab }}
\end{aligned}
$$

The costs considered for biogas upgrading were chosen in agreement with previous studies $[27,49]$. These costs refer to: loan needed to cover the investment to construct the upgrading unit $\left(C_{\text {loan,1 }}\right)$, the interests on this loan $\left(C_{\mathrm{il}, 1}\right)$, yearly operation and maintenance (O\&M) of the upgrading stage $\left(C_{\mathrm{om}, 1}\right)$, depreciation $\left(C_{\mathrm{df}, 1}\right)$ and insurance $\left(C_{\mathrm{ins}, 1}\right)$. The costs of transporting biomethane to the regasification tank are related to: the loan needed to cover the investment in the transport infrastructure $\left(C_{\text {loan,2 }}\right)$, the interests on this loan $\left(C_{i 1,2}\right)$ and operation and maintenance of the infrastructure $\left(C_{\mathrm{om}, 2}\right)$. Moreover, labor costs $\left(C_{\text {lab }}\right)$ are considered, as is the electricity needed to run the upgrading unit and the electricity to be bought from the grid because the CHP system is replaced by the biomethane upgrading unit $\left(C_{\mathrm{e}}\right)$.

Loans generate a yearly cash outflow calculated by dividing the amount of money needed for the investment $\left(C_{\mathrm{inv}, i}\right)$ by the number of years to repay the investment $\left(n_{1}\right)$ (Equation (10)).

$$
C_{\text {loan }, i}=\frac{C_{\text {inv }, i}}{n_{1}}
$$

where the subscript $i$ refers to one of the two stages needed to deliver biomethane to the local grid (it takes a value of 1 for the investment in the biogas upgrading stage and 2 for the investment in the infrastructure needed to transport the biomethane to the regasification unit). The investment costs were calculated based on typical unitary costs $C_{\mathrm{u}, \text { inv }, i}$ taken from the literature and reported in Table 1. It was considered that the loan would be repaid in 15 years [49].

The interests on the loans were expressed as previously done by other authors [49] (Equation (11)).

$$
C_{\mathrm{il}, i}=\left[C_{\mathrm{inv}, i}-C_{\text {loan }, i} \times(t+1)\right] \times r_{\mathrm{int}}
$$

where time $(t)$ and interest rate $\left(r_{\text {int }}\right)$ play a key role. A 3\% interest rate was considered, based on the SME financing costs in Portugal (average of the median reported in the period between 2014 and the 1st semester of 2019 [50]).

O\&M and insurance costs were calculated as a percentage of the investment costs ( $p_{\mathrm{mo}, i}$ and $p_{\mathrm{ins}}$, respectively, for O\&M and insurance). Similarly, the depreciation costs were calculated as a percentage of the loan $\left(p_{\mathrm{df}}\right)$ (Equations (12)-(14)).

$$
\begin{aligned}
C_{\mathrm{om}, i} & =C_{\mathrm{inv}, i} \times p_{\mathrm{om}, i} \\
C_{\mathrm{df}, 1} & =C_{\mathrm{loan}, 1} \times p_{\mathrm{df}} \\
C_{\mathrm{ins}, 1} & =C_{\mathrm{inv}, 1} \times p_{\mathrm{ins}}
\end{aligned}
$$

The cost of the electricity is the sum of two terms: (i) the cost of electricity spent to upgrade the biogas produced, calculated from the amount of biogas that is produced by the biodigester monthly $\left(Q_{\text {biogas }}\right)$, the consumption of electricity per unit of biogas upgraded $\left(C_{\mathrm{u}, \mathrm{e}}\right)$, and the electricity price $\left(p_{\mathrm{e}}\right)$; and (ii) the electricity that would not be produced by 
the CHP system and, therefore, would need to be purchased. The latter depends on the electricity produced by the CHP system $\left(Q_{\mathrm{e}, \mathrm{CHP}}\right)$ and the electricity unit price.

$$
C_{\mathrm{e}}=12 \times Q_{\text {biogas }} \times C_{\mathrm{u}, \mathrm{e}} \times p_{\mathrm{e}}+12 \times Q_{\mathrm{e}, \mathrm{CHP}} \times p_{\mathrm{e}}
$$

Additionally, the labor cost $\left(C_{\mathrm{lab}}\right)$ was calculated by multiplying the number of extra operators needed to run the upgrading unit $\left(n_{\mathrm{op}}\right)$ by the annual cost of an operator $\left(C_{\text {lab }, \mathrm{u}}\right)$. The latter was based on the Portuguese yearly national minimum wage (8400 EUR/year [12]), plus the mandatory social security contributions (1995 EUR/year [51], and 1154 EUR/year [45]).

$$
C_{\text {lab }}=C_{\text {lab }, \mathrm{u}} \times n_{\mathrm{op}}
$$

It is worth mentioning that an additional compression stage following biogas upgrading is not needed since it was assumed that the natural gas tank operates at a similar pressure to that of the biomethane produced [52]. It was considered that the lifetime of the project is 20 years [53] and that the discount rate $\left(r_{\mathrm{d}}\right)$ is $6 \%$. This value was calculated by summing the Portuguese inflation rate in $2019(0.3 \%$ [12]), the SME financing costs in Portugal in the first semester of 2019 (median, 1.85\% [50]) and a term accounting for the risk $(3.85 \%)$. A list of the model inputs is presented in Table 1.

\begin{tabular}{|c|c|c|}
\hline Variable & Value & Reference \\
\hline$p_{\mathrm{NG}}(\mathrm{EUR} / \mathrm{MWh})$ & 27.3 & [12] \\
\hline$C_{\mathrm{u}, \text { inv }, 1}\left(\mathrm{EUR} / \mathrm{m}^{3}\right)$ & 6000 & {$[27,49,54]$} \\
\hline$C_{\mathrm{u}, \mathrm{inv}, 2}(\mathrm{EUR} / \mathrm{km})$ & 237,500 & [28] \\
\hline$n_{1}(\mathrm{y})$ & 15 & [55] \\
\hline$r_{\text {int }}(\%)$ & 3 & [50] \\
\hline$p_{\mathrm{om}, 1}(\%)$ & 10 & [28] \\
\hline$p_{\mathrm{om}, 2}(\%)$ & 10 & [56] \\
\hline$p_{\mathrm{df}}(\%)$ & 20 & [28] \\
\hline$p_{\text {ins }}(\%)$ & 1 & [49] \\
\hline$C_{\text {u.e }}\left(\mathrm{kWh} / \mathrm{m}^{3}\right)$ & 0.29 & [54] \\
\hline$p_{\mathrm{e}}(\mathrm{EUR} / \mathrm{kWh})$ & 0.144 & [57] \\
\hline$C_{\text {lab,u }}$ (EUR/year/worker) & 11,549 & [27] \\
\hline$n_{\mathrm{op}}$ (worker) & 1 & [49] \\
\hline$n_{\mathrm{wh}}(\mathrm{h} /$ year) & 8000 & [58] \\
\hline$r_{\mathrm{d}}(\%)$ & 6 & - \\
\hline$Q_{\text {biogas }}\left(\mathrm{m}^{3} /\right.$ month $)$ & 24,917 & [44] \\
\hline$Q_{\mathrm{e} C H P}(\mathrm{kWh} / \mathrm{month})$ & 38,564 & [44] \\
\hline$Q_{\mathrm{u}, \mathrm{CHP}}(\mathrm{EUR} / \mathrm{KWh})$ & 0.01125 & [44] \\
\hline
\end{tabular}

Table 1. Economic variables used as input for the profitability study.

\section{Results}

\subsection{Baseline Scenario Results}

Table 2 shows the results obtained for the baseline scenario. As it can be seen, the project herein proposed is not feasible under the conditions imposed, revealing the great challenge ahead in the path towards more sustainable societies with improved resilience. From the profitability analysis, a negative NPV of EUR $-1325 \mathrm{k}$ was obtained. Other parameters to highlight are the long DPBT obtained (more than 20 years, in agreement with the negative value obtained for NPV) and a PI of -2.23 . These results would be very hard to overcome by optimizing plant parameters and increasing the number of years to recover the investment. Indeed, as it can be seen in Figure 3A, the poor economic performance is a consequence of the project presenting much higher total yearly costs than total yearly revenues. The relationship between yearly cash inflows (revenues, in green) and outflows (cost, in red) is not constant throughout the lifetime of the project, but, for example, in the first year, the costs are EUR $183 \mathrm{k}$ and the revenues EUR $51 \mathrm{k}$. In these circumstances, if the 
project lifetime was increased, the NPV would only evolve towards higher negative values over time.

Table 2. Results obtained for the baseline case.

\begin{tabular}{cc}
\hline Indicator (Units) & Value \\
\hline NPV (k EUR) & -1325 \\
DPBT (years) & $>20$ \\
IRR (\%) & n.d. \\
PI (-) & -2.23 \\
\hline
\end{tabular}

To have a complete picture of the cash outflows and to find a profitable proposal that may be attractive for investors, costs were disaggregated and analyzed (Figure 3B).
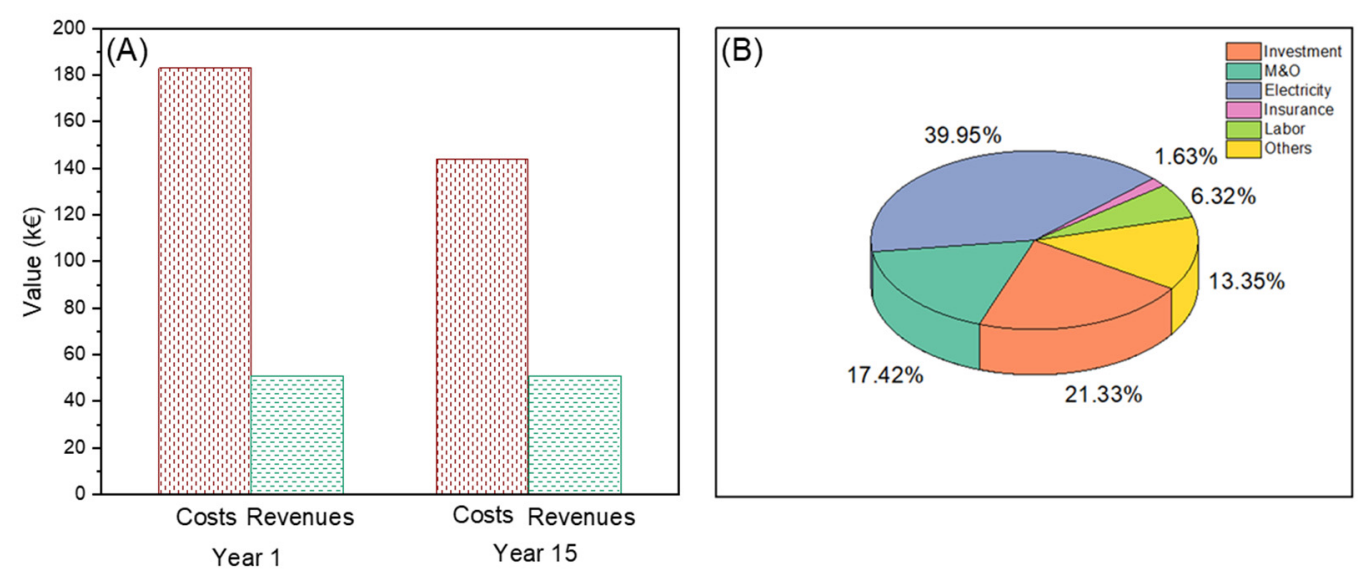

Figure 3. Analysis of results obtained for the baseline scenario. (A) Annual costs and revenues for the baseline scenario. (B) Total cost disaggregation.

Electricity has the highest share of the costs. Most of the electricity costs (approximately $85 \%$ of the electricity share) refer to the electricity that needs to be bought to the national grid because the CHP system stops working. The rest of the electricity costs (approximately 15\% of the electricity share) refer to the electricity consumption for biogas upgrading. One can see that the fact that the WWTP stops the production of electricity from biogas in the CHP unit to start upgrading biogas is strongly impacting the profitability of the project. This result was expected because electricity is much more expensive than natural gas. Other relevant costs are related to total investment, labor and O\&M and should be considered further. The former could be partially covered by incentives in the form, for example, of investment subsidies, which could be granted by the Portuguese government as a percentage of the initial investment costs. The other two costs mentioned are not easy to reduce since it could directly affect the day-to-day operation of the biomethane plant.

It seems clear that, under the impositions introduced by the baseline scenario, there is not a chance to obtain profitability. To improve the baseline scenario, two extra revenues can be considered to balance the economic performance of the project: the $\mathrm{CO}_{2}$ separated from the biogas stream could be sold, and government incentives for the production of biomethane could be granted. The impact of these two options will be analyzed below.

\subsection{Impact of Selling $\mathrm{CO}_{2}$ on the Profitability of the Biomethane Unit Proposed}

In this section, the economic feasibility of replacing the CHP system currently in use in the Evora WWTP by a biomethane upgrading unit and by the transport infrastructure to inject biomethane into the local natural gas grid was analyzed assuming that all the $\mathrm{CO}_{2}$ produced is sold. In order to properly examine the dependence of the project feasibility on the $\mathrm{CO}_{2}$ selling price, a wide range of prices was considered in the analysis (from 10 to $70 \mathrm{EUR} / \mathrm{t} \mathrm{CO}_{2}$ ). The commercial price of $\mathrm{CO}_{2}$ depends greatly on the region and industry 
and ranges from 3 to $360 \mathrm{EUR} / \mathrm{t}$ [59]. The lowest prices correspond to long-term contracts for $\mathrm{CO}_{2}$ from ammonia producers, the highest to small amounts of $\mathrm{CO}_{2}$ for lab purposes with a high degree of purity. Considering the capacities treated in this study, a price in the range of 10-70 EUR/t of $\mathrm{CO}_{2}$ was assumed. Figure 4 shows the results obtained for the NPV (Figure 4A) and PI (Figure 4B) as a function of the $\mathrm{CO}_{2}$ price considered.
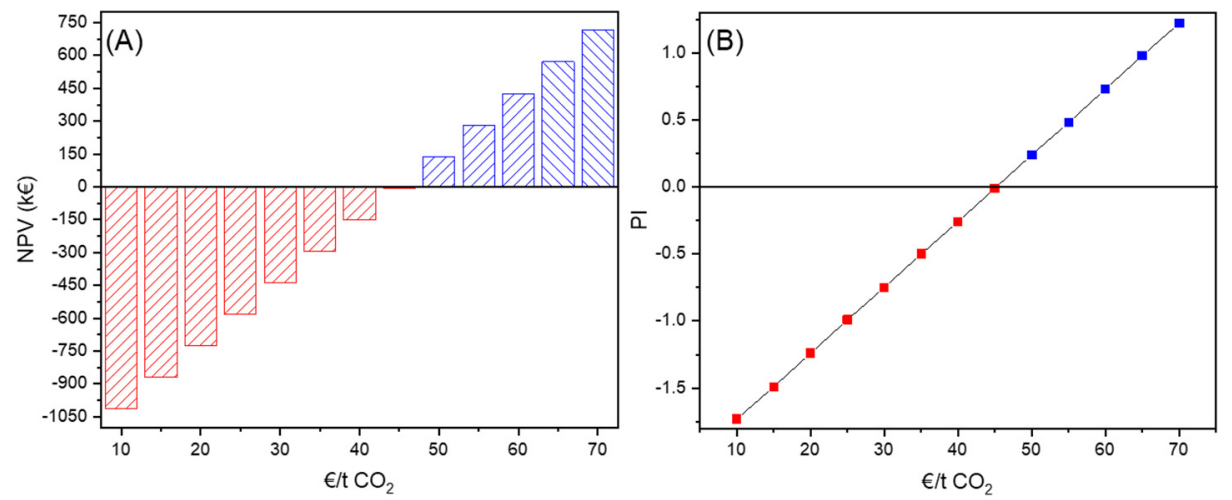

Figure 4. Economic results as a function of the $\mathrm{CO}_{2}$ selling price. (A) NPV; (B) PI.

At $30 \mathrm{EUR} / \mathrm{t} \mathrm{CO}$, the price considered as the reference price for $\mathrm{CO}_{2}$ in this study (see Section 5.3), an NPV value of EUR $-437 \mathrm{k}$ was obtained, which is still not attractive for investors. In agreement with Figure $4 \mathrm{~A}$, a zero NPV would be obtained at around $46 \mathrm{EUR} / \mathrm{t}$ $\mathrm{CO}_{2}$, which is probably a too high commercial price. Even $55 \mathrm{EUR} / \mathrm{t} \mathrm{CO}_{2}$ would produce little benefits (EUR $282 \mathrm{k} \mathrm{NPV}$ ) in comparison with other investment options. Figure 4B shows similar behavior for the PI parameter. Therefore, selling all the $\mathrm{CO}_{2}$ produced is not enough to pay for the investment of transforming an existing biogas/CHP plant into a biomethane plant if we assume a realistic selling price for the $\mathrm{CO}_{2}$.

At this point, it is worth mentioning that the rationale underlying the consideration of selling the $\mathrm{CO}_{2}$ that inevitably results from the upgrading process is not making a profit with the production of $\mathrm{CO}_{2}$ but to give a use to this GHG. The objective of the biogas/biomethane unit should always be optimizing the $\mathrm{CH}_{4}$ fraction in the biogas stream, and hence its energy content. This point will be further discussed in Section 6 .

\subsection{Impact of Incentives for Producing Biomethane on the Profitability of the Biomethane Unit Proposed}

In this section, incentives for biomethane production will be considered assuming two scenarios: one where all the $\mathrm{CO}_{2}$ produced is sold at a price of $30 \mathrm{EUR} / \mathrm{t} \mathrm{CO}$, and another where the $\mathrm{CO}_{2}$ is not valorized. It is assumed that the payment structure of the feed-in tariff policy is based on a premium price. Figures 5 and 6 present the NPV and PI results obtained for different biomethane premium prices, when $\mathrm{CO}_{2}$ is not sold (Figure 5A,B) and when it is sold (Figure 6A,B). As it can be observed, the difference is noticeable. No profitable scenarios were found in those cases in which $\mathrm{CO}_{2}$ sales were not considered for feed-in premium values below 55 EUR/MWh. Indeed, the first feed-in premium value which shifts the profitability sign is $55.5 \mathrm{EUR} / \mathrm{MWh}$. On the other hand, if $\mathrm{CO}_{2}$ was sold in the market, around $20 \mathrm{EUR} / \mathrm{MWh}$ of government incentives would be necessary to achieve profitability. The first NPV positive value would be obtained if the government offered a feed-in premium of 18.67 EUR/MWh. At this value, the DPBT would be 19 years, which is quite high for this kind of investment and still not very attractive. IRR and PI would be $9 \%$ and 0.0003 , respectively. 

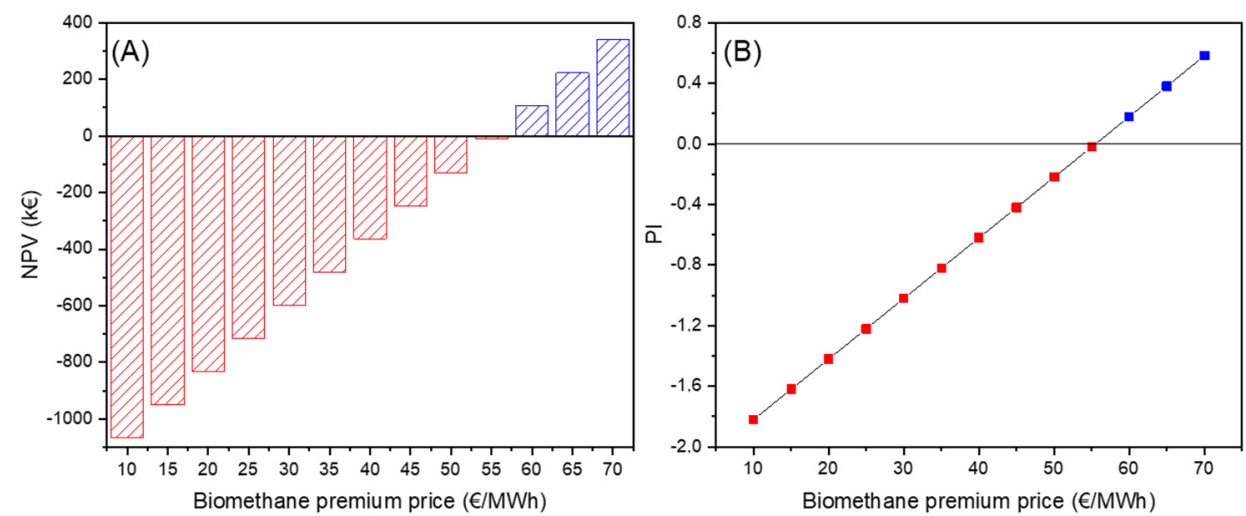

Figure 5. Economic results dependence on the biomethane premium price (no $\mathrm{CO}_{2}$ is sold). (A) NPV; (B) PI.
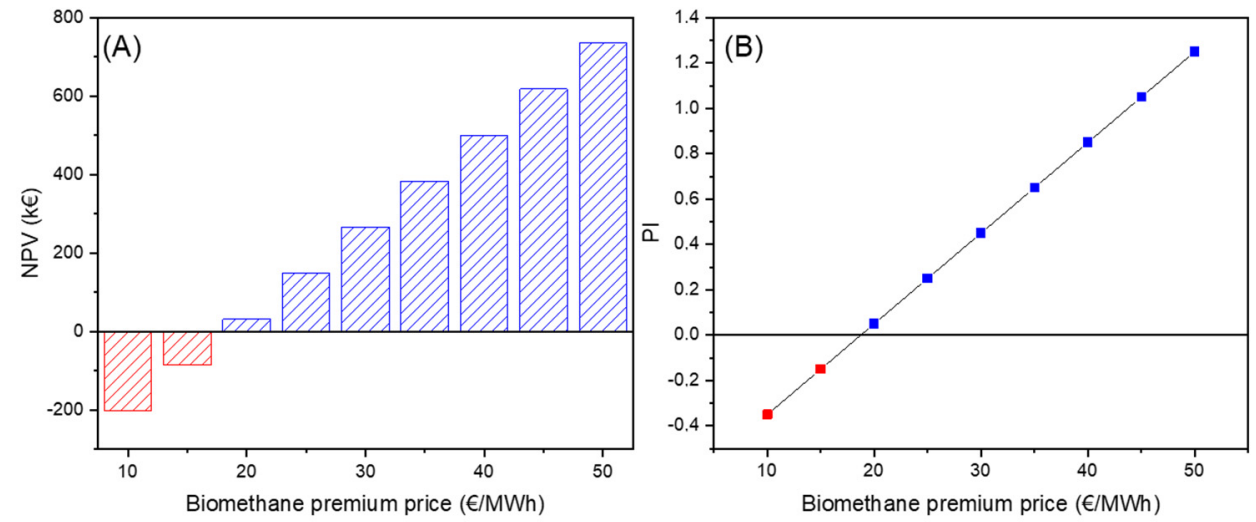

Figure 6. Economic results dependence on the biomethane premium price $\left(\mathrm{CO}_{2}\right.$ sold at $\left.30 \mathrm{EUR} / \mathrm{t}\right)$. (A) NPV; (B) PI.

\section{Discussion}

From the results presented above, one can conclude that, under the current circumstances, it is not economically viable to replace the existent CHP unit with a plant that upgrades biogas to biomethane at the Évora WWTP. The capital expenditure is too high, the revenues would not be enough, and the investment would only be feasible if there were support measures for the development of the biomethane market in Portugal. One possibility in the context of a circular economy would be to investigate the opportunities of selling the $\mathrm{CO}_{2}$ that inevitably would be separated from the methane. But even if this could be done, the present results show that economic viability was only obtained if the WWTP would simultaneously receive a feed-in premium for the biomethane injected in the local grid. Alone, the current $\mathrm{CO}_{2}$ market price is not enough to make the investment profitable. $\mathrm{CO}_{2}$ is needed for a wide range of industrial applications; the most important of them are described below. In the metal industry, $\mathrm{CO}_{2}$ can be used to improve the hardness of casting molds [60]. For construction purposes, $\mathrm{CO}_{2}$ is also used as dry ice pellets for removing extra paint. Within the chemical-oil industry, methanol industrial manufacturing also employs $\mathrm{CO}_{2}$ in considerable quantities, as well as it is used for enhanced oil recovery (EOR) in oil wells [61]. In the food and beverages industry, $\mathrm{CO}_{2}$ is typically used to carbonate soft drinks, beers and wine. In the production processes, it can also be used as supercritical fluid $[62,63]$. The fertilizer industry is another important $\mathrm{CO}_{2}$ consumer [59]. Even though $\mathrm{CO}_{2}$ can be used in the aforementioned applications, the necessities are not high when compared to the world's $\mathrm{CO}_{2}$ emissions. Globally, around $230 \mathrm{Mt}$ of $\mathrm{CO}_{2}$ are used each year industrially; with the fertilizer industry (i.e., urea) being the largest consumer (130 Mt $\mathrm{CO}_{2}$ /year). The oil and gas sector consumes around 70-80 Mt yearly for EOR activities. Yearly global $\mathrm{CO}_{2}$ emissions are over $36,000 \mathrm{Mt}$ nowadays, and this value is expected to 
increase during the forthcoming years [64]. Thus, only a small percentage of $\mathrm{CO}_{2}$ total emissions are currently used for industrial purposes. In this context, selling $\mathrm{CO}_{2}$ should not be seen as a means of investments in biomethane upgrading units reaching profitability.

Under the current market conditions, the replacement of the CHP unit with an upgrading plant is not recommended. However, the existing CHP unit is already 14 years old and when it needs to be replaced or stops working, considering its substitution by another technology would be interesting. If the CHP stopped working today and was not substituted, the NPV of the investment on upgrading and transporting biomethane would be EUR $-643 \mathrm{k}$ without government incentives and without valorizing the $\mathrm{CO}_{2}$ produced. Under these premises, 27.5 EUR/MWh of feed-in premium would be needed to render the project profitable. In the scenario where all the $\mathrm{CO}_{2}$ could be sold at $30 \mathrm{EUR} / \mathrm{t}$, EUR $220 \mathrm{k}$ of NPV would be obtained with this government support. This would allow for the replacement of the $\mathrm{CHP}$ unit by a biogas upgrading plant.

The existence of a stable and reliable legal and political framework and effective support schemes is the greatest driver for the development of the biomethane market [41]. To date, the biomethane sector does not have a lot of support in EU member states, and the existent support is focused on the transport sector [41]. Portugal, having no specific regulations for biomethane injection into the grid and no specific support scheme for biomethane yet does not promote the conversion of wastes into this energy source. In fact, not even the conversion of wastes in biogas is promoted in the country. If the 2030 targets defined in the National Climate and Energy Plan are to be reached, the promotion of biogas and biomethane is an important step. Renewable gases are one of the ways for renewable energies to penetrate into the heating and transport sectors. These (especially the latter) are the sectors where the market uptake of renewable energies has been more difficult. Decarbonizing the Portuguese energy system will require decarbonizing the gas industry, and biomethane produced from waste has important environmental advantages. For the scenarios studied in this work, the injection of biomethane into the Évora gas grid could replace around $4 \%$ of fossil fuel.

To put the results of the present work into context, the feed-in premium that is necessary to make biomethane production in the Évora WWTP profitable (with and without selling $\mathrm{CO}_{2}$ ) is lower than the feed-in premium currently offered by the Italian government for biomethane production, which is $61 \mathrm{EUR} / \mathrm{MWh}$ [65]. In Italy, biogas is well-established as a renewable energy source, but only a few biomethane plants exist [65]. With the objective of increasing the production of biomethane and advanced biofuels for transport, a new incentive scheme based on a biofuel certificate system came into effect in 2018 [66]. If the Portuguese government supported biomethane in a similar way, it would be profitable for the Évora WWTP to upgrade the biogas it produces to biomethane even without selling $\mathrm{CO}_{2}$. This type of incentive is important for developing the biomethane market in the first stage. However, other types of support schemes need to be designed so that there is a market for biomethane beyond the end of this kind of financial support. One possibility is the establishment of quotas for biomethane in the gas that is supplied by natural gas grids or an increase in the price for emission allowances [67].

Another chance of improving the profitability of the project herein presented would be the production of a bigger biogas stream. This could be achieved by receiving in the WWTP the sludge of other nearby regions. Nevertheless, this option would require equipment with much more capacity, trucks that would bring the sludge to Évora or the construction of facilities to transport them, and higher labor costs to accomplish the different tasks. Inasmuch as that the scenario herein assumed would change drastically, this idea opens new windows for further research in future works.

The present results were obtained for an existent WWTP, the Évora WWTP; however, they can serve as an indication of the viability of biogas upgrading in other WWTP in regions far from the national natural gas grid. In the country, several other regasification units with different distances to the high-pressure natural gas grid exist [68-70]. Investi- 
gating the profitability of implementing biomethane plants close to WWTP would be an interesting future work.

In connection with the recent COVID-19 pandemic, the investment in renewable energy production plants is a need to boost the sustainability and the resilience of our society. As recently claimed by some authors, air quality improved considerably after three months of the pandemic, revealing that our energy sector must shift towards a more sustainable one [71]. However, this is not a task only for the energy sector but a global effort of our society, including other sectors, such as the transport [72] or food industry [73].

\section{Conclusions}

This study shows that, under the current conditions, producing biomethane from biogas in the Évora WWTP is unprofitable without the existence of support measures. Indeed, the analysis reveals that a $55.5 \mathrm{EUR} / \mathrm{MWh}$ feed-in premium would be needed to reach profitability without selling $\mathrm{CO}_{2}$. If $\mathrm{CO}_{2}$ was sold at $30 \mathrm{EUR} / \mathrm{t}$, the feed-in premium needed would be decreased to $20 \mathrm{EUR} / \mathrm{MWh}$. However, there is a high uncertainty that the WWTP would be capable of selling $\mathrm{CO}_{2}$ at this price. Additionally, selling the $\mathrm{CO}_{2}$ should not be seen as a means of making the investments in biomethane plants profitable. In any case, the goal of a biogas/biomethane plant should be to optimize $\mathrm{CH}_{4}$ production to the detriment of $\mathrm{CO}_{2}$ production.

The results herein presented invite the reflection upon the need for new policies to boost the presence of biomethane in regions far from a high-pressure grid. In this sense, the consumption of biomethane would not only avoid the consumption of fossil resources but would also minimize the external dependence of many regions on natural gas, which is currently supplied by road transport. As proved in our analysis, the evolution towards a bio-economy society needs large economic efforts. Thus, the Portuguese government should play an important role in the development of biomethane production plants in the coming years.

Author Contributions: Conceptualization, F.M.B.-M., I.M. and I.P.M.; methodology, F.M.B.-M. and I.M.; validation, I.M. and I.P.M.; formal analysis, F.M.B.-M. and I.M.; investigation, F.M.B.-M., I.M. and I.P.M.; resources, F.M.B.-M. and I.M.; data curation, F.M.B.-M. and I.M.; writing-original draft preparation, F.M.B.-M., I.M. and I.P.M.; writing-review and editing, F.M.B.-M., I.M. and I.P.M.; visualization, F.M.B.-M., I.M. and I.P.M.; supervision, I.M. and I.P.M.; project administration, I.M. and I.P.M.; funding acquisition, F.M.B.-M. and I.M. All authors have read and agreed to the published version of the manuscript.

Funding: This work was supported by the University of Seville through V PPIT-US and by FCT Fundação para a Ciência e Tecnologia, Portugal (project UIDB/50022/2020).

Institutional Review Board Statement: Not applicable.

Informed Consent Statement: Not applicable.

Data Availability Statement: Not applicable.

Acknowledgments: This work was supported by the University of Seville through V PPIT-US and by FCT_Fundação para a Ciência e Tecnologia, Portugal (project UIDB/50022/2020).

Conflicts of Interest: The authors declare no conflict of interest.

\section{List of Abbreviations}

$\begin{array}{ll}\text { Abbreviation } & \text { Name } \\ \text { CHP } & \text { Combined heat and power } \\ \text { COVID-19 } & \text { Disease caused by severe acute respiratory syndrome coronavirus 2 (SARS-CoV-2) } \\ \text { EOR } & \text { Enhanced oil recovery } \\ \text { GHG } & \text { Greenhouse gas } \\ \text { LNG } & \text { Liquefied natural gas } \\ \text { O\&M } & \text { Operation and maintenance } \\ \text { WWTP } & \text { Wastewater treatment plant }\end{array}$




\section{Nomenclature}

\begin{tabular}{|c|c|c|}
\hline Symbol & Name & Units \\
\hline BOD5 & Biochemical Oxygen Demand & $\mathrm{mg} / \mathrm{L}$ \\
\hline$C_{\mathrm{df}}$ & Depreciation Cost & EUR \\
\hline$C_{e}$ & Electricity Cost & EUR \\
\hline$C_{\mathrm{il}}$ & Interest of Loan Cost & EUR \\
\hline$C_{\text {ins }}$ & Insurance Cost & EUR \\
\hline$C_{\text {inv }}$ & Investment Cost & EUR \\
\hline$C_{\text {lab }}$ & Labor Cost & EUR \\
\hline$C_{\text {labu }}$ & Unitary Labor Cost & EUR/worker \\
\hline$C_{\text {loan }}$ & Cost of Loan & EUR \\
\hline$C_{\mathrm{om}}$ & Maintenance \& Overhead Cost & EUR \\
\hline $\mathrm{C}_{\mathrm{u}, \mathrm{CHP}}$ & Unitary Cost for Combined Heat and Power & $\mathrm{EUR} / \mathrm{kWh}$ \\
\hline$C_{\mathrm{u}, \mathrm{e}}$ & Unitary Cost for Electricity & $\mathrm{EUR} / \mathrm{kWh}$ \\
\hline$C_{\mathrm{u} \text {,inv }}$ & Unitary Investment Cost & $\mathrm{EUR} / \mathrm{m}^{3}$ \\
\hline$C_{\text {ueBU }}$ & Unitary Electricity Consumption for Biogas Upgrading & $\mathrm{kWh} / \mathrm{m}^{3}$ biogas \\
\hline DPBT & Discounted Payback Time & years \\
\hline IRR & Internal Rate of Return & $\%$ \\
\hline$I_{t}$ & Cash Inflow at year $t$ & EUR \\
\hline$N$ & Number of Years & years \\
\hline$n_{1}$ & Loan Years & years \\
\hline$n_{\mathrm{op}}$ & Number of workers & workers \\
\hline NPV & Net Present Value & EUR \\
\hline$n_{\mathrm{wh}}$ & Working hours & $\mathrm{h} / \mathrm{y}$ \\
\hline $\mathrm{O}_{t}$ & Cash Outflow at year $t$ & EUR \\
\hline$p_{\mathrm{df}}$ & Depreciation Percentage & $\%$ \\
\hline$p_{\mathrm{e}}$ & Electricity Price & $\mathrm{EUR} / \mathrm{kWh}$ \\
\hline PI & Profitability Index & EUR/EUR \\
\hline$p_{\text {ins }}$ & Insurance Percentage & $\%$ \\
\hline$p_{\mathrm{mo}}$ & Maintenance \& Overhead Percentage & $\%$ \\
\hline$p_{\text {ng }}$ & Natural Gas Price & EUR/MWh \\
\hline$p_{\mathrm{CO} 2}$ & Carbon Dioxide Price & $\mathrm{EUR} / \mathrm{t}$ \\
\hline$p_{\text {premium }}$ & Incentives price & EUR/MWh \\
\hline$Q_{\text {biogas }}$ & Biogas Flow & $\mathrm{m}^{3} / \mathrm{h}$ \\
\hline$Q_{\text {biomethane }}$ & Biomethane Flow & $\mathrm{m}^{3} / \mathrm{h}$ \\
\hline$Q_{\mathrm{CO} 2}$ & Carbon Dioxide Flow & $\mathrm{m}^{3} / \mathrm{h}$ \\
\hline$Q_{e, C H P}$ & Average Electricity Produced by Combined Heat and Power & $\mathrm{kWh} / \mathrm{month}$ \\
\hline$R_{\text {biomethane }}$ & Biomethane Revenues & EUR \\
\hline$R_{\mathrm{CHP}}$ avoided cost & Avoided Cost for Combined Heat and Power & EUR \\
\hline$R_{\mathrm{CO} 2}$ & Carbon Dioxide Revenues & EUR \\
\hline$r_{\mathrm{d}}$ & Discount Rate & $\%$ \\
\hline$r_{\text {int }}$ & Interest rate & $\%$ \\
\hline$t$ & Time & years \\
\hline
\end{tabular}

\section{References}

1. Pestana, C.; Barros, L.; Scuri, S.; Barreto, M. Can HCI Help Increase People's Engagement in Sustainable Development? A Case Study on Energy Literacy. Sustainability 2021, 13, 7543. [CrossRef]

2. Baena-Moreno, F.M.; Sebastia-Saez, D.; Pastor-Pérez, L.; Ramirez-Reina, T. Analysis of the potential for biogas upgrading to syngas via catalytic reforming in the United Kingdom. Renew. Sustain. Energy Rev. 2021, 144, 110939. [CrossRef]

3. Maraqa, M.A.; Albuquerque, F.D.B.; Alzard, M.H.; Chowdhury, R.; Kamareddine, L.A.; El Zarif, J. GHG Emission Reduction Opportunities for Road Projects in the Emirate of Abu Dhabi: A Scenario Approach. Sustainability 2021, 13, 7367. [CrossRef]

4. Tsiakiri, E.P.; Mpougali, A.; Lemonidis, I.; Tzenos, C.A.; Kalamaras, S.D.; Kotsopoulos, T.A.; Samaras, P. Estimation of Energy Recovery Potential from Primary Residues of Four Municipal Wastewater Treatment Plants. Sustainability 2021, $13,7198$. [CrossRef]

5. Rosas, J.G.; Gómez, N.; Cara-Jiménez, J.; González-Arias, J.; Olego, M.Á.; Sánchez, M.E. Evaluation of joint management of pine wood waste and residual microalgae for agricultural application. Sustainability 2020, 13, 53. [CrossRef] 
6. International Energy Agency. Renewables Information: Overview. 2019. Available online: https://iea.blob.core.windows.net/ assets /6959bcb0-d298-404c-80e1-2afaa784798e/Renewables_Information_2019_Overview.pdf (accessed on 4 July 2021).

7. D'Adamo, I.; Rosa, P. How do you see infrastructure? Green energy to provide economic growth after COVID-19. Sustainability 2020, 12, 4738. [CrossRef]

8. González-Arias, J.; Carnicero, A.; Sánchez, M.E.; Martínez, E.J.; López, R.; Cara-Jiménez, J. Management of off-specification compost by using co-hydrothermal carbonization with olive tree pruning. Assessing energy potential of hydrochar. Waste Manag. 2021, 124, 224-234. [CrossRef]

9. Zhang, J.; Mao, L.; Nithya, K.; Loh, K.C.; Dai, Y.; He, Y.; Wah Tong, Y. Optimizing mixing strategy to improve the performance of an anaerobic digestion waste-to-energy system for energy recovery from food waste. Appl. Energy 2019, 249, 28-36. [CrossRef]

10. Ali, S.M.H.; Lenzen, M.; Sack, F.; Yousefzadeh, M. Electricity generation and demand flexibility in wastewater treatment plants: Benefits for $100 \%$ renewable electricity grids. Appl. Energy 2020, 268, 114960. [CrossRef]

11. Kacprzak, M.; Neczaj, E.; Fijałkowski, K.; Grobelak, A.; Grosser, A.; Worwag, M.; Rorat, A.; Brattebo, H.; Almås, Å.; Singh, B.R. Sewage sludge disposal strategies for sustainable development. Environ. Res. 2017, 156, 39-46. [CrossRef]

12. Eurostat. 2020. Available online: https:/ / ec.europa.eu/eurostat/data/database (accessed on 4 March 2020).

13. Ning, C.; You, F. Data-driven Wasserstein distributionally robust optimization for biomass with agricultural waste-to-energy network design under uncertainty. Appl. Energy 2019, 255, 113857. [CrossRef]

14. Schopf, K.; Judex, J.; Schmid, B.; Kienberger, T. Modeling the bioenergy potential of municipal wastewater treatment plants. Water Sci. Technol. 2018, 77, 2613-2623. [CrossRef] [PubMed]

15. Mills, N.; Pearce, P.; Farrow, J.; Thorpe, R.B.; Kirkby, N.F. Environmental \& economic life cycle assessment of current \& future sewage sludge to energy technologies. Waste Manag. 2014, 34, 185-195. [CrossRef]

16. Vasco-Correa, J.; Khanal, S.; Manandhar, A.; Shah, A. Anaerobic digestion for bioenergy production: Global status, environmental and techno-economic implications, and government policies. Bioresour. Technol. 2018, 247, 1015-1026. [CrossRef] [PubMed]

17. Baena-Moreno, F.M.; Rodríguez-Galán, M.; Vega, F.; Vilches, L.F.; Navarrete, B.; Zhang, Z. Biogas upgrading by cryogenic techniques. Environ. Chem. Lett. 2019, 17, 1251-1261. [CrossRef]

18. Baena-Moreno, F.M.; le Saché, E.; Pastor-Pérez, L.; Reina, T.R. Membrane-based technologies for biogas upgrading: A review. Environ. Chem. Lett. 2020, 18, 1649-1658. [CrossRef]

19. Hobson, J. $\mathrm{CH}_{4}$ and $\mathrm{N}_{2} \mathrm{O}$ Emissions from Waste Water Handling. Good Practice Guidance and Uncertainty Management in National Greenhouse Gas Inventories; Intergovernmental Panel on Climate Change (IPCC) Publications: Geneve, Switzerland, 2000.

20. Baena-Moreno, F.M.; Rodríguez-Galán, M.; Ramirez-Reina, T.; Zhang, Z.; Vilches, L.; Navarrete, B. Understanding the effect of $\mathrm{Ca}$ and $\mathrm{Mg}$ ions from wastes in the solvent regeneration stage of a biogas upgrading unit. Sci. Total Environ. 2019, 691, 93-100. [CrossRef]

21. Nguyen, L.N.; Kumar, J.; Vu, M.T.; Mohammed, J.A.H.; Pathak, N.; Commault, A.S.; Sutherland, D.; Zdarta, J.; Tyagi, V.K.; Nghiem, L.D. Biomethane production from anaerobic co-digestion at wastewater treatment plants: A critical review on development and innovations in biogas upgrading techniques. Sci. Total Environ. 2020, 765, 142753. [CrossRef] [PubMed]

22. Awe, O.W.; Zhao, Y.; Nzihou, A.; Minh, D.P.; Lyczko, N. A Review of Biogas Utilisation, Purification and Upgrading Technologies. Waste Biomass Valoriz. 2017, 8, 267-283. [CrossRef]

23. Calderón, C.; Colla, M.; Jossart, J.-M.; Hemelleers, N.; Martin, A.; Aveni, N.; Caferri, C. European Bioenergy Outlook 2019; Biogas: Brussels, Belgium, 2019.

24. Prussi, M.; Padella, M.; Conton, M.; Postma, E.D.; Lonza, L. Review of technologies for biomethane production and assessment of EU transport share in 2030. J. Clean. Prod. 2019, 222, 565-572. [CrossRef]

25. Baena-Moreno, F.M.; Reina, T.R.; Rodríguez-Galán, M.; Navarrete, B.; Vilches, L.F. Synergizing carbon capture and utilization in a biogas upgrading plant based on calcium chloride: Scaling-up and profitability analysis. Sci. Total Environ. 2021, 758, 143645. [CrossRef]

26. Baena-Moreno, F.M.; Rodríguez-Galán, M.; Vega, F.; Ramirez-Reina, T.; Vilches, L.; Navarrete, B. Understanding the influence of the alkaline cation $\mathrm{K}^{+}$or $\mathrm{Na}^{+}$in the regeneration efficiency of a biogas upgrading unit. Int. J. Energy Res. 2019, 43, 1578-1585. [CrossRef]

27. Cucchiella, F.; D'Adamo, I.; Gastaldi, M.; Miliacca, M. A profitability analysis of small-scale plants for biomethane injection into the gas grid. J. Clean. Prod. 2018, 184, 179-187. [CrossRef]

28. Ferella, F.; Cucchiella, F.; D’Adamo, I.; Gallucci, K. A techno-economic assessment of biogas upgrading in a developed market. J. Clean. Prod. 2019, 210, 945-957. [CrossRef]

29. Venkatesh, G.; Elmi, R.A. Economic-environmental analysis of handling biogas from sewage sludge digesters in WWTPs (wastewater treatment plants) for energy recovery: Case study of Bekkelaget WWTP in Oslo (Norway). Energy 2013, 58, 220-235. [CrossRef]

30. Collet, P.; Flottes, E.; Favre, A.; Raynal, L.; Pierre, H.; Capela, S.; Peregrina, C. Techno-economic and Life Cycle Assessment of methane production via biogas upgrading and power to gas technology. Appl. Energy 2017, 192, 282-295. [CrossRef]

31. Michailos, S.; Walker, M.; Moody, A.; Poggio, D.; Pourkashanian, M. Biomethane production using an integrated anaerobic digestion, gasification and $\mathrm{CO}_{2}$ biomethanation process in a real waste water treatment plant: A techno-economic assessment. Energy Convers. Manag. 2020, 209, 112663. [CrossRef]

32. EBA. European Biogas Association Statistical Report for Year 2019; European Biogas Association: Brussels, Belgium, 2020. 
33. GIE. EBA European Biomethane Map. Available online: https://www.europeanbiogas.eu/eba-gie-biomethane-map/ (accessed on 4 July 2021).

34. Scarlat, N.; Dallemand, J.F.; Fahl, F. Biogas: Developments and perspectives in Europe. Renew. Energy 2018, 129, 457-472. [CrossRef]

35. Terlouw, W.; Peters, D.; van Tilburg, J.; Schimmel, M.; Berg, T.; Cilhar, J.; Rehman Mir, G.U.; Spöttle, M.; Staats, M.; Lejaretta, A.V.; et al. Gas for Climate. The Optimal Role for Gas in a Net-Zero Emissions Energy System; Navigant Consulting Inc.: Utrecht, The Netherlands, 2019; Volume 231.

36. Cabrita, I.; Silva, L.M.; Marques, I.P.R.; Di Berardino, S.; Gírio, F.M. Avaliação do Potencial e Impacto do Biometano em Portugal; LNEG: Lisbon, Portugal, 2015.

37. European Union. Directive (EU) 2018/2001 of the European Parliament and of the Council on the promotion of the use of energy from renewable sources. Off. J. Eur. Union 2018, 2018, 1-128.

38. Europe Union. Orientations towards the First Strategic Plan for Horizon Europe. 2019. Available online: https: / / ec.europa.eu/info/sites/default/files/research_and_innovation/strategy_on_research_and_innovation/documents / ec_rtd_orientations-he-strategic-plan_122019.pdf (accessed on 4 July 2021).

39. Cabrita, I.; Pinto, F.; Lopes, H.; Oliveira, F.; Marques, I.P. Resíduos Industrias; INETI, DGEG: Lisbon, Portugal, 2000.

40. Ferreira, M.; Marques, I.P.; Malico, I. Biogas in Portugal: Status and public policies in a European context. Energy Policy 2012, 43, 267-274. [CrossRef]

41. Kampman, B.; Leguijt, C.; Scholten, T.; Tallat-Kelpsaite, J.; Brückmann, R.; Maroulis, G.; Lesschen, J.P.; Meesters, K.; Sikirica, N.; Elbersen, B. Optimal Use of Biogas from Waste Streams-An Assessment of the Potential of Biogas from Digestion in the EU beyond 2020; European Commission: Luxembourg, 2017. [CrossRef]

42. PNEC Plano Nacional de Energia e Clima. Available online: https://apambiente.pt/_zdata/Alteracoes_Climaticas/Mitigacao/ PNEC/PNECPT_TemplateFinal201930122019.pdf (accessed on 23 January 2020).

43. DGEG. Consumo de Gás Natural no Mercado Interno em 2018 (Provisório). Available online: http:/ / www.dgeg.gov.pt/ (accessed on 4 July 2021).

44. Monte, M.M.D. Contributo para o Estudo da Valorização Energética de Biogás em Estações de Tratamento de Águas Residuais. Master's Thesis, Nova University of Lisbon, Lisbon, Portugal, 2010.

45. Assembleia da República. Diário da República; Lei n.o 88/2017; Assembleia da República: Lisbon, Portugal, 2017; pp. 3190-3228.

46. CertiTecna. Avaliação de Compatibilidade de Localização; Dianagás; Unidade Autónoma de Gás Natural (UAG); CertiTecna: Lisbon, Portugal, 2016.

47. Costa, C.M.d.E. Estudo de Melhorias em ETAR com Produção de Biogás e Geração de Eletricidade ETAR Municipal de Abrantes. Master's Thesis, Universidade da Beira Interior, Covilhã, Portugal, 2014.

48. Baena-Moreno, F.M.; Rodríguez-Galán, M.; Vega, F.; Vilches, L.F.; Navarrete, B. Review: Recent advances in biogas purifying technologies. Int. J. Green Energy 2019, 16, 401-412. [CrossRef]

49. Cucchiella, F.; D'Adamo, I. Technical and economic analysis of biomethane: A focus on the role of subsidies. Energy Convers. Manag. 2016, 119, 338-351. [CrossRef]

50. European Central Bank (ECB). Survey on the Access to Finance of Enterprises in the Euro Area-April to September 2019; ECB: Frankfurt am Main, Germany, 2019.

51. Segurança Social Segurança Social. Available online: www.seg-social.pt/ (accessed on 4 July 2021).

52. Baena-Moreno, F.M.; Malico, I.; Rodríguez-Galán, M.; Serrano, A.; Fermoso, F.G.; Navarrete, B. The importance of governmental incentives for small biomethane plants in South Spain. Energy 2020, 206, 118158. [CrossRef]

53. Kraussler, M.; Pontzen, F.; Müller-Hagedorn, M.; Nenning, L.; Luisser, M.; Hofbauer, H. Techno-economic assessment of biomassbased natural gas substitutes against the background of the EU 2018 renewable energy directive. Biomass Convers. Biorefinery 2018, 8, 935-944. [CrossRef]

54. Bortoluzzi, G.; Gatti, M.; Sogni, A.; Consonni, S. Biomethane production from agricultural resources in the Italian scenario: Techno-Economic analysis of water wash. Chem. Eng. Trans. 2014, 37, 259-264. [CrossRef]

55. D'Adamo, I.; Falcone, P.M.; Ferella, F. A socio-economic analysis of biomethane in the transport sector: The case of Italy. Waste Manag. 2019, 95, 102-115. [CrossRef] [PubMed]

56. Cucchiella, F.; D'Adamo, I.; Gastaldi, M. Profitability analysis for biomethane: A strategic role in the Italian transport sector. Int. J. Energy Econ. Policy 2015, 5, 440-449.

57. PORDATA. Electricity Prices for Households and Industrial Users (Euro/ECU). Available online: https://www.pordata.pt/en/ Europe/Electricity+prices+for+households+and+industrial+users+(Euro+ECU)-1477 (accessed on 18 July 2019).

58. Skorek-Osikowska, A.; Martín-Gamboa, M.; Iribarren, D.; García-Gusano, D.; Dufour, J. Thermodynamic, economic and environmental assessment of energy systems including the use of gas from manure fermentation in the context of the Spanish potential. Energy 2020, 200, 117452. [CrossRef]

59. International Energy Agency. Putting CO2 to Use. Creating Value from Emissions; International Energy Agency: Paris, France, 2019.

60. Universal Industrial Gases CO2 Industrial Uses. Available online: http:/ / www.uigi.com/carbondioxide.html (accessed on 4 July 2021). 
61. Baena-moreno, F.M.; Rodríguez-galán, M.; Vega, F.; Alonso-fariñas, B.; Arenas, L.F.V.; Navarrete, B. Carbon capture and utilization technologies: A literature review and recent advances. Energy Sources Part A Recover. Util. Environ. Eff. 2019, 41, $1403-1433$. [CrossRef]

62. Yan, J.; Zhang, Z. Advances in carbon capture, utilization and storage. Appl. Energy 2020, 278, 115627. [CrossRef]

63. Zhang, Z.; Pan, S.Y.; Li, H.; Cai, J.; Olabi, A.G.; Anthony, E.J.; Manovic, V. Recent advances in carbon dioxide utilization. Renew. Sustain. Energy Rev. 2020, 125, 109799. [CrossRef]

64. Ritchie, H.; Roser, M. $\mathrm{CO}_{2}$ and Greenhouse Gas Emissions Resource; Our World in Data: Oxford, UK, 2020.

65. Marc, A.; Carole, M. Biogas and Biomethane in Europe: Lessons from Denmark, Germany and Italy; Ifri: Paris, France, 2019.

66. Cucchiella, F.; D'Adamo, I.; Gastaldi, M. Sustainable Italian cities: The added value of biomethane from organic waste. Appl. Sci. 2019, 9, 2221. [CrossRef]

67. Horschig, T.; Adams, P.W.R.; Gawel, E.; Thrän, D. How to decarbonize the natural gas sector: A dynamic simulation approach for the market development estimation of renewable gas in Germany. Appl. Energy 2018, 213, 555-572. [CrossRef]

68. Entidade Reguladora dos Serviços Energéticos ERSE. Available online: https:/ / www.erse.pt/ (accessed on 5 March 2020).

69. GALP. Available online: https://galpgasnaturaldistribuicao.pt/ (accessed on 5 March 2020).

70. Sonorgás. Available online: https://www.sonorgas.pt/ (accessed on 5 March 2020).

71. Debone, D.; da Costa, M.; Miraglia, S. 90 Days of COVID-19 Social Distancing and Its Impacts on Air Quality and Health in Sao Paulo, Brazil. Sustainability 2020, 12, 7440. [CrossRef]

72. Moslem, S.; Campisi, T.; Szmelter-Jarosz, A.; Duleba, S.; Nahiduzzaman, K.; Tesoriere, G. Best-Worst Method for Modelling Mobility Choice after COVID-19: Evidence from Italy. Sustainability 2020, 12, 6824. [CrossRef]

73. Giudice, F.; Caferra, R.; Morone, P. COVID-19, the Food System and the Circular Economy: Challenges and Opportunities. Sustainability 2020, 12, 7939. [CrossRef] 



\title{
Reflexive Governance for Infrastructure Resilience and Sustainability
}

\author{
Mattia Ferrari 1,2 \\ 1 European Institute for Innovation-Technology e.V., 73525 Schwäbisch Gmünd, Germany; \\ mat.ferrari2@gmail.com \\ 2 Centre for Studies on European Economy (AIM), Azerbaijan State University of Economics (UNEC), \\ Baku 1001, Azerbaijan
}

Received: 25 October 2020; Accepted: 3 December 2020; Published: 7 December 2020

\begin{abstract}
Infrastructure development is one of the areas most in need of climate-resilient and friendly investments. The COVID-19 pandemic will increase government spending in this direction. This paper demonstrates how the principles of reflexive governance are key to unlock the full potential of such investments. By establishing an adaptive and redundant institutional capacity in the provision of public services, reflexive governance can enable a successful path towards climate resilience and sustainability.
\end{abstract}

Keywords: reflexive governance; climate change; infrastructure; urban resilience; sustainability

\section{Introduction}

Nowadays infrastructure networks are at an increasing risk of disruption due to extreme and unpredictable weather events caused by climate changes. These infrastructures must provide a continuous, safe and reliable performance to satisfy the demand coming from several stakeholders [1]. Due to the high degree of network interdependencies and market connectivity [2], the United Nations Office for Disaster Risk Reduction (UNDDR) estimated that the economic and environmental damages due to service disruptions could reach USD 415 billion within the next 15 years [3].

Financing climate-resilient infrastructure is particularly relevant in the least developed countries as this is essential to achieve inclusive economic growth [4]. The Global Climate Risk Index 2015 indicated how 9 out of the 10 most-affected countries by climate events between 1994 and 2013 were low-income or middle-income countries [5]. A study from the World Bank reported how investments in climate-resilient hydropower infrastructures in Africa could lead to an increase in revenues of 20 to 140 per cent; on the other hand, inadequate planning could result in revenue losses ranging from 5 to 60 per cent [6].

The COVID-19 pandemic will increase government spending for economic recovery plans, which will include infrastructure development. This offers the opportunity to smooth the path towards 2030 Sustainable Development Goals (SDGs), also by investing in climate-resilient and sustainable infrastructure provision [7]. For instance, the required financing for transport infrastructure is estimated to be USD 440 billion annually to achieve the 2030 SDGs [8].

The principles of reflexive governance are key to unlock the potential of such investments. Reflexive governance is defined as the ability to go through complex processes of socio-technical change by developing innovative approaches, in a perspective of participation, probing and collective learning [9]. An example is a collaborative approach to environmental risk management, such as waste banks in developing countries [10]. This paper demonstrates how reflexive governance can establish an adaptive and redundant institutional capacity in the provision of public services, enabling a successful 
path towards climate resilience and sustainability. The close relationship between resilience and sustainability was highlighted by previous literature (see Elmqvist et al. [11] for a literature review).

The types of infrastructure considered for the analysis are those network components operating at the community level (distribution of electricity and water, collection and recycling of waste and local roads) for two reasons. First, the principles of collaboration, probing and collective learning are highly applicable at a local scale. Second, cities are responsible for 70 per cent of global greenhouse gas emissions, and 90 per cent of urban areas are located on climate-vulnerable coastlines [11].

This paper contributes to the discussion on the lack of adequate governance arrangements in the face of future global crises. In particular, it tries to provide an answer to the question posed by Brousselle et al. [12] of which elements of recovery plans are capable to reduce future vulnerabilities. This paper proposes reflexive governance as a tool to sustain resilient, sustainable and inclusive development in the upcoming decades. Supporting reflexive, resilient and inclusive societies is a prerequisite to achieving sustainable development [13-15].

Section 2 introduces the concept of resilience applied to infrastructure. It focuses on critical infrastructure because this category of assets is considered the most in need of a climate-resilient policy. Critical infrastructure can serve as a reference for investigating resilience interventions in the area of infrastructure. Section 3 explains the notion of reflexive governance, introducing the model of transition management (TM) and the concept of better regulation. Section 4 illustrates the application of reflexive governance in structuring resilient infrastructure service provision. Section 5 highlights the positive implications for sustainable and inclusive growth. Section 6 discusses the applicability of such recommendations.

\section{Critical Infrastructure Resilience}

The concept of critical infrastructure has been defined differently over time and across institutions. Overall, critical infrastructures are identified as those networks (or components) which are strategic for the provision of basic public services and for national security (such as energy, transport, water, telecommunications and waste management). At the EU level, this definition has been expanded to account for the increasing network interconnectivity across Member States. A European critical infrastructure (ECI) exists when the shock has a significant impact on at least two Member States [16].

The notion of critical infrastructure resilience refers to the buffering capacity of such a network to absorb a disturbance while retaining essentially the same functions as before the disruptive shock. It also refers to the network capacity to limit the duration of service interruption, hence minimising the recovery time. The recovery process does not necessarily mean resuming to exactly the prior state before the shock but may involve changing and adapting to new conditions [17,18]. Thus, infrastructure resilience has replaced the traditional concept of infrastructure protection, which was characterized exclusively by preventive and protective actions [19].

Governments play a central role in setting the stage for the development of resilient critical infrastructure [20]. Sectoral regulation, through the implementation of financial incentives (compensation to end-users) and non-financial incentives (transparency requirements), must ensure the establishment of acceptable standards of both risk and resilience [2]. For example, imposing compensation mechanisms to end-users in case of service disruption can incentivize operators to invest properly in resilience, while providing them with flexibility in the choice. Transparency requirements could instead create reputational concerns upon operators in case of service disruption.

Governments must also implement mitigation and adaptation policies to reduce the likelihood of the shock and to minimize its magnitude and duration. Such policies must be adopted throughout the whole life cycle of the infrastructure project, from its design and operational phase to retrofitting interventions [21]. The innovation process towards decentralized or autonomous networks (for example, renewable energy production and smart grids, artificial intelligence, big data, etc.) allows for a flexible response to negative events [22]. In order to develop mitigation and adaptation policies, it is necessary to adopt a multi-hazards, multi-sectoral and cross-border approach [23]. In addition, the cascading 
impact effect stemming from the large-scale network interdependency across countries requires sound cooperation (and information sharing) among various stakeholders, including local governments and private operators [22].

In order to ensure fair competition and to avoid opportunistic behaviours, it is necessary to guarantee a fair redistribution of the costs for resilience across stakeholders, requiring agreements on acceptable risks, required investments and compensations [24]. Nowadays, this is difficult because of the presence of complex procurement contracts and ownership structures, such as concession contracts and public-private partnerships (PPPs) [25]. However, the large financing gap in infrastructure provision, tied with increasing costs for resilience, requires the involvement of private capitals [26-28].

\section{The Notion of Reflexive Governance}

Infrastructure sectors have identified their own governance, i.e., the capacity to shape and transform itself, as a major concern [9]. As a response, the notion of reflexive governance was proposed to tackle challenges such as the transition to clean and/or decentralized energy production [29]. Reflexive governance becomes concerned with its own conditions, perspectives, expectations, knowledge, strategies and dynamics, in order to avoid the assumption of full knowledge in advance [30]. Nowadays, this assumption cannot be valid due to extreme and unexpected weather events. Reflexive governance implies the acknowledgment of participation, deliberation, probing and collective learning as key elements for inducing and navigating complex processes of socio-technical change [31]. Some authors have underlined that the notion of reflexive governance is recalled by the term resilience itself as the ability to thrive on and to adapt to shocks by developing new approaches $[32,33]$.

This dynamic and polycentric model of governance may lead to more effective and sustainable provision of public services [34]. Reflexive governance is widely applied in the context of sustainability as it aims to solve socio-ecological vulnerabilities. To contrast environmental degradation, reflexive governance proposes renewed forms of analysis and design of environmental policy and planning [35], as well as collaborative climate risk management [36,37]. The importance of a collaborative approach to environmental risk governance was recognized by the Aarhus Convention (1999), which established rights to access environmental information and legitimated public participation in environmental decision-making [38].

The model of transition management represents a suitable tool for the adoption of innovative approaches within complex socio-technical systems such as network infrastructures, natural resources and waste, agriculture and housing [39]. TM aims at "influencing the direction and speed of transitions by coordinating and enabling the processes that occur at different levels in a more systemic and evolutionary way" [40]. Rotmans et al. [38] defined TM in a perspective of incrementalism planning by adopting long-term system thinking, back-casting and forecasting. The objectives of TM are to achieve desirable social goals, to avoid serious pitfalls, i.e., strengthening resilience, and to adopt institutional reforms to cope with unfolding patterns of change [39].

The concept of better regulation, adopted by the European Commission, seems an adequate instrument to adopt the notions of reflexive governance and TM through the implementation of concrete actions. These include (i) evidence-based policymaking, (ii) legislative simplification for fit-for-purpose law, (iii) extensive planning, risk and impact assessment, (iv) improved stakeholders consultation and coordination to strengthen mutual capability and (v) transparency [41].

\section{Governance for Resilience}

At present, there are barriers to deliver climate-resilient infrastructure. First, resilient criteria in procurement contracts are not yet the norm. Second, when those criteria are present, they tend to increase the overall costs of an infrastructure project [2]. Furthermore, according to McPhearson [42], the traditional governance for infrastructure resilience has proved to be narrow-minded and not socially optimal, causing local inequity and injustice, driving gentrification, displacing minority and vulnerable groups, etc. Other concerns refer to poor institutional capacity. For example, limited 
administrative and jurisdictional scales, political and sectoral divisions, dysfunctional collaboration, opaque interests and autonomous actors with limited resources [43].

This section proposes reflexive governance as an instrument to establish an adaptive and redundant institutional capacity in order to structure resilient infrastructure service provision. This renewed form of institutional capacity is expected to increase resilience through easily changing and adapting to new conditions and through coordinating the service provision at different levels in an evolutionary way. Given the long-term, large-scale and social nature of infrastructure, enhanced participation of stakeholders and local end-users are capable to trigger self-adjustment mechanisms in case of disruptive shocks, i.e., adaptation policies, in order to change and to adapt to new conditions [44]. This allows to minimize the recovery time of service provision after the negative event and, by definition, improves the overall resilience.

This proposed approach is in line with the works of Kumaraswamy et al. [44] and Junqi [45], who introduced the term public-private-people partnership (4P). The term $4 \mathrm{P}$ was further proposed in the context of urban development [46], sustainable waste management and post-disaster reconstruction [47]. According to Sundararajan and Suriyagoda [27], due to the increasing unpredictability of climate events, it is necessary to introduce an "active" model of governance for risk management in the context of infrastructure development. This implies working proactively together to continuously "collect, identify and assess the likelihood and impacts" of climate events in order to intervene effectively. It can help in the "informed development and implementation of actions/responses through learning" [27].

A practical example is waste banks in developing countries, a collaborative and innovative approach to sustaining a climate-resilient and sustainable waste management infrastructure. This is done through an institutional capacity-building strategy at the local level, involving environmental communication strategy, education and training and partnerships [10]. Community-led initiatives towards recycling can prevent adversities such as homes being buried by landslides of waste dumps, clogging drains causing flood-related disruption of infrastructures, heavy reliance on waste transportation and incineration, waste dispersion due to winter storms and health hazards caused by waste degradation in case of heat waves [48,49].

These collaborative approaches to environmental risk management allow adopting individual or community-based responses. Existing strategies include (i) risk prevention, such as spatial planning and monitoring of watercourse or roads, (ii) protection, such as the construction of flood defence infrastructure and identification of vulnerable groups, (iii) preparedness through emergency response plans and (iv) recovery phase, including insurance schemes and redistribution of reconstruction tasks [34]. The input from local end-users can vary between co-funding or co-delivery of material and intellectual resources (time, technical skills, knowledge and machinery) [50]. In addition, flexible contracts for the co-management of urban commons have been proposed [51,52]. In line with the idea of knowledge co-production [12], ICT (Information and Communications Technology) can shape a new role for citizens in risk management activities, for instance, in the building of citizen observatories [53]. Global movements in this direction are already present, such as the Global Resilient Cities Network, which implements social innovation and urban experiments to bridge the gap in urban resilience strategies [54].

All the above supports the mandate of multilateral development banks (MDBs) in facilitating global compliance with the climate-resilient infrastructure agenda [55]. Such institutions are in the position to develop frameworks of agreement with national, regional and local stakeholders to promote the principles of reflexive governance. MDBs are currently at the forefront of the use of climate risk screening tools [2]. The International Finance Corporation (IFC), focusing on the private sector in the least developed countries, defines private sector responsibilities for managing environmental and climate risks. It also identifies appropriate mitigation and adaptation policies, including the sustainable use of natural resources [56]. The World Bank's 2013 Urban Risk Assessment includes recommendations for local stakeholders, such as cities, for a detailed assessment of institutional 
gaps in addressing climate risks [56]. Kreditanstalt für Wiederaufbau (KfW) operates a climate risk screening tool, analysing whether the adaptive capacity (or resilience) of people and eco-systems can be improved [56].

\section{Governance for Sustainability and Inclusive Growth}

This work proposes reflexive governance as an instrument to establish an adaptive and redundant institutional capacity in order to structure resilient infrastructure service provision. The central feature of any resilient network is redundancy [11]. For example, a parallel back-up system must be available in case of network disruption. However, this renewed form of institutional capacity can allow increasing resilience when desired and reducing it when not, according to climate events. This solves an inherent problem of sustainable infrastructure systems, which often aim at system/process maximization and at avoiding resource inefficiencies linked to redundancy [11]. This can enable a successful path towards sustainable development because it avoids unnecessary costs for constructing parallel back-up systems. Therefore, the analysis indicates reflexive governance as essential to unlock the full potential of infrastructure investments following the COVID-19 crisis.

This renewed form of governance also has positive implications for inclusive growth. First, it can improve the access conditions to financial insurance markets by private investors, increasing their confidence in engaging in infrastructure projects in those regions most vulnerable to severe climate events. Lack of access to financial insurance markets is considered a major obstacle in attracting private capital in the least developed countries [27]. Second, in those countries, input from local communities can help in finding a fair balance between investment in resilience and investment in service provision. Indeed, a large financing gap with regard to infrastructure provision still exists, creating a tradeoff in investment decision-making [57].

\section{Discussion}

The analysis carried out in this paper holds true for those countries (both advanced economies and developing countries) implementing economic recovery plans from the COVID-19 crisis, with a focus on infrastructure development. As developing countries are the most vulnerable to climate change, they should take these recommendations carefully. Of course, the implementation of the principles of reflexive governance will be different according to the country's means and resources.

While Hepburn et al. [58] did not identify governance arrangements as a priority in the path towards sustainability within the G20 fiscal recovery packages, existing literature for developing countries does. Bakare [59], analysing the post-COVID-19 infrastructure sector in Nigeria, suggests the human agency theory and to invest in adaptive institutions to cope with future sector vulnerabilities. For South Asia, Madhurima et al. [60] proposed a multi-agents model for environmental risk management and infrastructure resilience. In contrasting future global vulnerabilities, they recommended to consider critical resilient infrastructure as a common public goods and to foster regional cooperation.

Finally, Brousselle et al. [12] corroborated the policy recommendations advanced in this study. In the path towards climate resilience, they suggested to foster innovation in public administrations and to restructure government-civil society relationships. Governance models will need to be adaptive to account for place-specific climate vulnerabilities. This requires leadership from mayors, with the risk of implementing policies conflicting with central governments. Indeed, while climate change hits at the local level, the measures to contrast it are common responsibilities [12].

To summarize, further research is needed to understand how to best conjugate a human-centred model of governance with global macro challenges, such as climate resilience and sustainability. Pioneering case studies would help in providing best practices in the field of governance for climate resilience at the community level, focusing on infrastructure service provision, for instance, following the positive experience of waste banks in developing countries. Case studies will help to identify (i) which actions were taken to promote reflexive governance, (ii) to which extent reflexive governance was applied, (iii) what the positive implications for the provision of public service at the community 
level were, (iv) how infrastructure resilience was strengthened, and (v) if this will lead to positive economic, social and environmental externalities.

Funding: This research received no external funding.

Acknowledgments: I would like to express gratitude to the managing editors and the three anonymous reviewers for their precious feedback.

Conflicts of Interest: The author declares no conflict of interest.

\section{References}

1. Val, D.V.; Yurchenko, D.; Nogal, M.; O'Connor, A. Climate Change-Related Risks and Adaptation of Interdependent Infrastructure Systems. In Climate Adaptation Engineering; Elsevier: Amsterdam, The Netherlands, 2019; pp. 207-242.

2. Vallejo, L.; Mullan, M. Climate-Resilient Infrastructure: Getting the Policies Right; OECD Environment Working Papers, No. 121; OECD Publishing: Paris, France, 2017.

3. United Nations Office for Disaster Risk Reduction (UNDDR). Urgent Need for Disaster Resilient Infrastructure; UNISDR: Geneva, Switzerland, 2018.

4. Bowen, A.; Cochrane, S.; Fankhauser, S. Climate change, adaptation and economic growth. Clim. Chang. 2012, 113, 95-106. [CrossRef]

5. Kreft, S.; Eckstein, D.; Junghans, L.; Kerestan, C.; Hagen, U. Global Climate Risk Index 2015; Briefing Paper; Germanwatch: Berlin, Germany, 2014.

6. Cervigni, R.; Liden, R.; Neumann, J.E.; Strzepek, K.M. (Eds.) Enhancing the Climate Resilience of Africa's Infrastructure: The Power and Water Sectors; The World Bank: Washington, DC, USA, 2015; ISBN 1464804664.

7. D'Adamo, I.; Falcone, P.M.; Martin, M.; Rosa, P. A sustainable revolution: Let's go sustainable to get our globe cleaner. Sustainability 2020, 12, 4387. [CrossRef]

8. OECD. Enhancing Connectivity through Transport. Infrastructure: The Role of Official Development Finance and Private Investment, The Development Dimension; OECD Publishing: Paris, France, 2018.

9. Voß, J.-P.; Borneman, B. The politics of reflexive governance: Challenges for designing adaptive management and transition management. Ecol. Soc. 2011, 16, 9. [CrossRef]

10. Asteria, D.; Santoso, T. Local Action for Waste Bank Management Through an Environmental Communication Strategy and a Collaborative Approach for the Sustainability of Villages. In Competition and Cooperation in Social and Political Sciences; Routledge Taylor and Francis Group: London, UK, 2018.

11. Elmqvist, T.; Andersson, E.; Frantzeskaki, N.; McPhearson, T.; Olsson, P.; Gaffney, O.; Takeuchi, K.; Folke, C. Sustainability and resilience for transformation in the urban century. Nat. Sustain. 2019, 2, 267-273. [CrossRef]

12. Brousselle, A.; Brunet-Jailly, E.; Kennedy, C.; Phillips, S.D.; Quigley, K.; Roberts, A. Beyond COVID-19: Five commentaries on reimagining governance for future crises and resilience. Can. Public Adm. 2020, 63, 369-408. [CrossRef] [PubMed]

13. D'Adamo, I.; Rosa, P. How do you see infrastructure? Green energy to provide economic growth after COVID-19. Sustainability 2020, 12, 4738. [CrossRef]

14. Glass, L.-M.; Newig, J. Governance for achieving the sustainable development goals: How important are participation, policy coherence, reflexivity, adaptation and democratic institutions? Earth Syst. Gov. 2019, 2, 100031. [CrossRef]

15. European Commission. Decision C(2020)6320 of 17 September 2020. Europe in a Changing World-Inclusive, Innovative and Reflective Societies. Horizon 2020, Work Programme 2018-2020. Off. J. Eur. Union 2020, L345-L376. Available online: https://ec.europa.eu/research/participants/data/ref/h2020/wp/2018-2020/main/ h2020-wp1820-societies_en.pdf (accessed on 6 December 2020).

16. European Council. Council Directive 2008/114/EC of 8 December 2008 on the Identification and Designation Of European Critical Infrastructures and the Assessment of the Need to Improve Their Protection. Off. J. Eur. Union 2008, L345-L375. Available online: https://eur-lex.europa.eu/LexUriServ/LexUriServ.do?uri=OJ:L: 2008:345:0075:0082:EN:PDF (accessed on 6 December 2020).

17. Tvaronavičienè, $\mathrm{M}$. Towards internationally tuned approach towards critical infrastructure protection. J. Secur. Sustain. Issues 2018, 8, 143-149. [CrossRef] 
18. Forzieri, G.; Bianchi, A.; e Silva, F.B.; Marin Herrera, M.A.; Leblois, A.; Lavalle, C.; Aerts, J.C.J.H.; Feyen, L. Escalating impacts of climate extremes on critical infrastructures in Europe. Glob. Environ. Chang. 2018, 48, 97-107. [CrossRef]

19. Pursiainen, C. Critical infrastructure resilience: A Nordic model in the making? Int. J. Disaster Risk Reduct. 2018, 27, 632-641. [CrossRef]

20. Murdock, H.J.; de Bruijn, K.M.; Gersonius, B. Assessment of critical infrastructure resilience to flooding using a response curve approach. Sustainability 2018, 10, 3470. [CrossRef]

21. Yang, Y.; Ng, S.T.; Xu, F.J.; Skitmore, M. Towards sustainable and resilient high density cities through better integration of infrastructure networks. Sustain. Cities Soc. 2018, 42, 407-422. [CrossRef]

22. OECD. Good Governance for Critical Infrastructure Resilience, OECD Reviews of Risk Management Policies; OECD Publishing: Paris, France, 2019.

23. Baubion, C. System Thinking for Critical Infrastructure Resilience and Security. In Proceedings of the Joint OECD-JRC Workshop Report, Paris, France, 24-25 September 2018.

24. Christopher, H. Bovis risk in public-private partnerships and critical infrastructure. Eur. J. Risk Regul. 2017, 6, 200-207.

25. Wojewnik-Filipkowska, A.; Wȩgrzyn, J. Understanding of public-private partnership stakeholders as a condition of sustainable development. Sustainability 2019, 11, 1194. [CrossRef]

26. Miller, A.; Swann, S. How to Make Infrastructure Climate Resilient; EMCompass, Brief Paper No. 14; International Finance Corporation: Washington, DC, USA, 2016.

27. Sundararajan, S.; Suriyagoda, N. Climate Risks and Resilience in Infrastructure PPPs: Issues to be Considered; Issue Brief; Public Private Infrastructure Advisory Facility (PPIAF): Washington, DC, USA, 2016.

28. Karanasios, K.; Parker, P. Tracking the transition to renewable electricity in remote indigenous communities in Canada. Energy Policy 2018, 118, 169-181. [CrossRef]

29. Wegrzyn, J.; Gluszak, M.; Telega, A. Infrastructure endowment, financial constraints and willingness to engage in PPPs: The case of Poland. Public Money Manag. 2019, 39, 132-138. [CrossRef]

30. Meadowcroft, J. What about the politics? Sustainable development, transition management, and long term energy transitions. Policy Sci. 2009, 42, 323-340. [CrossRef]

31. Voß, J.P.; Smith, A.; Grin, J. Designing long-term policy: Rethinking transition management. Policy Sci. 2009, 42, 275-302. [CrossRef]

32. Boin, A.; McConnell, A. Editorial: Unravelling the Puzzles of Critical Infrastructures. J. Conting. Crisis Manag. 2007, 15, 1-3. [CrossRef]

33. Crichton, M.T.; Ramsay, C.G.; Kelly, T. Enhancing organizational resilience through emergency planning: Learnings from cross-sectoral lessons. J. Conting. Crisis Manag. 2009, 17, 24-37. [CrossRef]

34. Goldthau, A. Rethinking the governance of energy infrastructure: Scale, decentralization and polycentrism. Energy Res. Soc. Sci. 2014, 1, 134-140. [CrossRef]

35. Feindt, P.H.; Weiland, S. Reflexive governance: Exploring the concept and assessing its critical potential for sustainable development. Introduction to the special issue. J. Environ. Policy Plan. 2018, 20, 661-674. [CrossRef]

36. Mees, H.; Alexander, M.; Gralepois, M.; Matczak, P.; Mees, H. Typologies of citizen co-production in flood risk governance. Environ. Sci. Policy 2018, 89, 330-339. [CrossRef]

37. Westling, E.L.; Sharp, L.; Rychlewski, M.; Carrozza, C. Developing adaptive capacity through reflexivity: Lessons from collaborative research with a UK water utility. Crit. Policy Stud. 2014, 8, 427-446. [CrossRef]

38. Koester, V. The Convention on Access to Information, Public Participation in Decision-Making and Access to Justice in Environmental Matters (Aarhus Convention). In Making Treaties Work: Human Rights, Environment and Arms Control; Cambridge University Press: Cambridge, UK, 2007; ISBN 9780511494345.

39. Rotmans, J.; Loorbach, D.; Kemp, R. Transition Management: Its Origin, Evolution and Critique. In Proceedings of the Workshop on Politics and Governance in Sustainable Socio-Technical Transitions, Berlin, Germany, 19-21 September 2007.

40. Kemp, R.; Loorbach, D. Transition Management: A Reflexive Governance Approach. In Reflexive Governance for Sustainable Development; Edward Elgar: Cheltenham, UK, 2006.

41. Decision of the President of the European Commission on the Appointment of Members to the Task Force on Subsidiarity, Proportionality and "Doing Less More Efficiently", C(2018) 406; European Commision: Brussels, Belgium, 2017. 
42. McPhearson, T. Urban Futures: Transforming Cities for Resilience and Sustainability. 2018. Available online: https: //www.elon.edu/u/news/2019/11/05/mcphearson-brings-climate-warnings-scientific-progress-to-elon/ (accessed on 1 October 2020).

43. Bai, X.; McAllister, R.R.J.; Beaty, R.M.; Taylor, B. Urban policy and governance in a global environment: Complex systems, scale mismatches and public participation. Curr. Opin. Environ. Sustain. 2010, 2, $129-135$. [CrossRef]

44. Kumaraswamy, M.; Zou, W.; Zhang, J. Reinforcing relationships for resilience-by embedding end-user 'people' in public-private partnerships. Civ. Eng. Environ. Syst. 2015, 32, 119-129. [CrossRef]

45. Junqi, Z. Developing public private people partnership (4P) for post disaster infrastructure procurement. Int. J. Disaster Resil. Built Environ. 2015, 6, 468-484. [CrossRef]

46. Majamaa, W. The 4th P-People-in Urban Development Based on Public-Private-People Partnership. Ph.D. Thesis, Helsinki University of Technology, Helsinki, Finland, 2008.

47. Jahangiri, K.; Izadkhah, Y.O.; Tabibi, S.J. A comparative study on community-based disaster management in selected countries and designing a model for Iran. Disaster Prev. Manag. 2011, 20, 82-94. [CrossRef]

48. The World Bank. What a Waste: An Updated Look into the Future of Solid Waste Management. 2018. Available online: www.worldbank.org/en/news/immersive-story/2018/09/20/what-a-waste-an-updatedlook-into-the-future-of-solid-waste-management (accessed on 1 October 2020).

49. Winne, S.; Horrocks, L.; Kent, N.; Miller, K.; Hoy, C.; Benzie, M.; Power, R. Increasing the Climate Resilience of Waste Infrastructure. Final Report under Defra Contract ERG 1102. Available online: https://assets.publishing.service.gov.uk/government/uploads/system/uploads/attachment_data/file/ 183933/climate-resilience-full.pdf (accessed on 6 December 2020).

50. Bovaird, T.; Loeffler, E. The Role of Co-Production for Better Health and Wellbeing: Why We NEED to Change; Governance International: Birmingham, UK, 2013; ISBN 9780957253322.

51. Colding, J.; Barthel, S. The potential of "Urban Green Commons" in the resilience building of cities. Ecol. Econ. 2013, 86, 156-166. [CrossRef]

52. Buijs, A.E.; Mattijssen, T.J.; Van der Jagt, A.P.; Ambrose-Oji, B.; Andersson, E.; Elands, B.H.; Steen Møller, M. Active citizenship for urban green infrastructure: Fostering the diversity and dynamics of citizen contributions through mosaic governance. Curr. Opin. Environ. Sustain. 2016, 22, 1-6. [CrossRef]

53. Wehn, U.; Rusca, M.; Evers, J.; Lanfranchi, V. Participation in flood risk management and the potential of citizen observatories: A governance analysis. Environ. Sci. Policy 2015, 42, 187-198. [CrossRef]

54. Global Resilient Cities Network. Available online: www.rockpa.org/project/global-resilient-cities-network/ (accessed on 1 October 2020).

55. Runde, D.F.; Ramanujam, S.R. Financing and Implementing the Quality Infrastructure Agenda: Leveraging the United States-Japan Partnership to Ensure a High.-Quality Option and Other Next Steps; Report; Center for Strategic and International Studies: Washington, DC, USA, 2018.

56. Ricardo-AEA. Climate Related Standards and Measures for Assessing Investments in Infrastructure Projects; Ricardo-AEA: Harwell, UK, 2013.

57. OECD Development Center. Quality Infrastructure for Development; OECD Development Center: Paris, France, 2018.

58. Hepburn, C.; O'Callaghan, B.; Stern, N.; Stiglitz, J.; Zenghelis, D. Will COVID-19 fiscal recovery packages accelerate or retard progress on climate change? Oxf. Rev. Econ. Policy 2020, 36, S359-S381. [CrossRef]

59. Bakare, H.O. Regional Planning for Resilient And Sustainable Post-Covid-19 Recovery and Transport Infrastructure Transformation in Nigeria. In Proceedings of the Special Virtual Conference on Covid-19 Theme: Geographers and Post Covid-19, Held via Zoom, 29-30 June 2020.

60. Madhurima, S.; Sanjay, S. When COVID-19 and Natural Hazards Collide: Building Resilient Infrastructure in South Asia; Issue Brief; No. 413; Observer Research Foundation (ORF): New Delhi, India, 2020.

Publisher's Note: MDPI stays neutral with regard to jurisdictional claims in published maps and institutional affiliations. 


\title{
Distance Learning as a Resilience Strategy during Covid-19: An Analysis of the Italian Context
}

\author{
Andrea Appolloni ${ }^{1,2, * \mathbb{C}}$, Nathalie Colasanti ${ }^{1}$, Chiara Fantauzzi ${ }^{1}$, Gloria Fiorani ${ }^{1}$ and Rocco Frondizi ${ }^{1} \mathbb{D}$ \\ 1 Department of Management and Law, University of Rome Tor Vergata, 00133 Rome, Italy; \\ nathalie.colasanti@uniroma2.it (N.C.); chiara.fantauzzi@uniroma2.it (C.F.); gloria.fiorani@uniroma2.it (G.F.); \\ rocco.frondizi@uniroma2.it (R.F.) \\ 2 Institute for Research on Innovation and Services for Development (IRISS), National Research Council (CNR), \\ 80134 Naples, Italy \\ * Correspondence: andrea.appolloni@uniroma2.it
}

Citation: Appolloni, A.; Colasanti, N.; Fantauzzi, C.; Fiorani, G.; Frondizi, R. Distance Learning as a Resilience Strategy during Covid-19: An Analysis of the Italian Context. Sustainability 2021, 13, 1388. https:// doi.org/10.3390/su13031388

Academic Editor: Idiano D'Adamo

Received: 30 December 2020

Accepted: 25 January 2021

Published: 29 January 2021

Publisher's Note: MDPI stays neutral with regard to jurisdictional claims in published maps and institutional affiliations.

Copyright: (c) 2021 by the authors. Licensee MDPI, Basel, Switzerland. This article is an open access article distributed under the terms and conditions of the Creative Commons Attribution (CC BY) license (https:// creativecommons.org/licenses/by/ $4.0 /)$.
Abstract: The purpose of this paper is to analyze the strategic model of distance learning adopted by Italian higher education, showing how the health emergency due to Covid-19 has transformed it from an "optional" for traditional universities to the only means to ensure public health protection and continuity in education programs. Comparing two situations (before and during the pandemic), the aim is to identify best practices that, even after the end of the emergency, can be adopted by Italian higher education institutions to boost their digital supply and compete in an international context. After a general context analysis, aimed to underline benefits and risks connected to the development of distance learning, the case of the Italian higher education system has been analyzed. Data were collected through a documentary analysis, looking at what Italian higher education institutions disclosed through their official websites and documents: every form of communication about digital strategy was taken into account. Then, they were analyzed qualitatively, in order to individuate which platforms have been combined to ensure quality in education provided. Research findings demonstrate the resilience of the Italian higher education, able to react and to re-organize itself in only one week: the results of the pandemic may be a stronger university, able to combine quality in education with the potential of technological devices and to compete at the international level. Distance learning represents a complex field, still characterized by separated understandings and in a context where limited attention has been dedicated to its development for what concerns the Italian context, the choice to examine it represents the originality of this paper.

Keywords: higher education; digitalization; distance learning; Covid-19 outbreak; resilience

\section{Introduction}

Among different transformations that took place during the last years, digitalization has changed the ways to work and to do things, and also to teach and learn [1]. According to Westerman et al. [2], digitalization can be defined as the use of different digital devices to change existing business models, improving services and facilitating trade and activities. Therefore, in a context where education is seen as one of the solutions to many of today's problems, such as unemployment, world peace, and poverty, the aim of universities is not just to deliver education, but to become more digital learning institutions updating their strategy in order to meet the new requests and expectations of students and other stakeholders.

According to Drucker [3], now and hereafter, knowledge represents the key to success, and higher education institutions have to satisfy the needs of their students even after the end of their educational path in order to ensure continuously new and updated knowledge during the course of their career [4,5].

In a globalized society, oriented to international competition and characterized by the spread of internet and other technology devices [6], a growing number of individuals 
has been inspired to ask for further new learning opportunities, available anywhere and anytime, without requiring physical presence in universities.

The answer has been the rise of distance learning, which can be seen as an evolution of lifelong learning, through the appearance of online universities, able to offer distance courses by exploiting new communication devices and to deliver academic qualifications recognized by the State in which they operate. Given the rise of these online universities, perceived as new competitors by the traditional ones, higher education institutions have been forced to update their strategy and modify their supply.

The real value of digitalization and distance learning has been underlined during the Covid-19 emergency: it is during such occasions that distance learning, previously perceived as a simple answer provided by traditional universities to the recent rise of the online ones, has become the only means to perform education and training paths for all levels and all kinds of institution, at the same time ensuring health protection.

More precisely, it is during the health emergency that distance learning assumed the form of emergency remote education (ERE), allowing students, teachers, and lecturers to continue their learning and teaching activities $[7,8]$.

On the basis of these considerations, the aim of this work is to investigate the strategic model of distance learning adopted by Italian higher education, showing how the pandemic has accelerated its growth and evolution. The aim is to present emergency remote education as a sustainable and resilience strategy for higher education, in order to support the rebirth after Covid-19 [9]. Indeed, if according to Gallagher et al. [10], sustainability is based on the triple bottom line-people, profit, and planet-it is clear that today distance learning represents the instrument to foster its social dimension. Furthermore, distance learning could represent a way to foster the environmental dimension of sustainability: using sharing platforms, it allows to create value in a circular way, building new communities and providing students with more affordable and convenient access to education, the first step towards the achievement of green campuses [11,12].

Identifying specific best practices that, even after the end of the emergency, can be adopted by all Italian higher education institutions, the aim is to answer the following research questions: how has the Italian higher education reacted to Covid-19 outbreak? Which lessons can be learned from the pandemic experience?

Findings suggest that before the pandemic emergency, Italian higher education institutions started to open themselves to such innovation, showing a huge delay if compared to other international universities.

The pandemic has turned upside down all the education systems over the world, including the Italian one, forcing all institutions to interrupt their traditional activities and then to experiment emergency remote education in order to ensure continuity for all education programs.

So the methodology of case study has been adopted, with the Italian higher education system representing the object of the analysis. It has been chosen not because it is extreme or unusual, but because researchers are members of it, providing additional longitudinal elements from their personal experience. The Italian case has never distinguished itself for its capacity to evolve and to welcome digitalization, but the velocity in which it has translated to online justifies the originality of the paper. Furthermore, it has been analyzed in a very extraordinary time (Covid-19 outbreak).

The paper is the output of the first phase of our research, since it analyzes distance learning as emergency remote education, so as an instrument adopted to provide answers in a time of emergency. On the basis of evidence emerged, the second phase of the research will point out the evolution of distance learning in Italian universities, analyzing it as a usual and structured practice.

The paper is structured as follows. Section 2 analyzes the development of digitalization in higher education, with a specific focus on distance learning, underlining the main features and limits of such a new way to provide education. 
Section 3 is dedicated to explaining the methodology adopted to collect and analyze data, and findings are presented in Section 4, with the aim of pointing out the model of distance learning adopted in Italy, before and during the health emergency; a brief introduction will be dedicated to present the measures enacted by the Italian government with the aim of ensuring public safety and education provision. Discussions are presented in Section 5 and preliminary conclusions in the final one, hypothesizing the future of distance learning in the Italian context.

\section{Digitalization in Higher Education: The Development of Distance Learning}

Today, digitalization is everywhere around the world, changing operations within organizations and enabling far-reaching social and political changes [1]. It represents a transformation process, which requires the implementation of a specific strategy, aimed to better satisfy customers' wishes and preferences and, sometimes, able to ensure competitive advantage [13].

Digitalization represents one of the main challenges for all kinds of institutions [14], including universities, that have been called to become more digital and to assist students in coping and leading such transitions [1]. Indeed, in a context characterized by the spread of the internet and other technology devices, the main need for higher education is not just for capturing knowledge, but also for exploiting new technologies in order to "teach to learn" and "learn to learn" independently [15]. The market has become global everywhere, and higher education institutions have started to compete globally: it is in such a context that they have to leverage new digital potential to benefit in the digital era [16,17]. As a consequence, universities, previously seen as "ivory towers" [18], have been called to update their strategy, in order to ensure accessible and affordable learning opportunities for all [19], adapting themselves to the needs of individuals and providing education in a variety of settings and forms, even outside of the school system [20]. They have been called to develop a new e-learning strategy, with the aim to provide answers to the growing demand for new and different learning experiences [21], fostering the development of distance learning [22].

In this sense, the development of distance learning turns out to be strictly connected with the diffusion of new technologies, but, in reality, it has arisen through the introduction of printers and printed books, less expensive and more accessible for a wider audience [23]. Therefore, the evolution of distance learning can be divided into three sections: the first one was characterized by the use of material printed exclusively for students, the second one by the use of television in order to deliver courses, and the last one dominated by internet and other technologies. During the last section (1994-2000), universities have started to perform e-learning or blended learning experiences, and in 2008 they developed "massive open online courses" (MOOCs) in order to offer a possible solution to a rapidly increasing need for education worldwide [24].

Therefore, in this sense, the development of distance learning can be seen as the direct consequence of digitalization in higher education, enabling both the societies and education organizations to create new opportunities to grow, improve, change, and renew themselves [25].

Of course, digitalization brings several opportunities, increasing interactions and collaborations with stakeholders [26], but, at the same time, it could be characterized by negative aspects, such as the risk of losing customers who are used to traditional services [27], and when problems occur, ordinary employees are unable to solve them, requiring the involvement of IT experts (an additional cost for organizations) [28].

Likewise, according to Veletsianos and Kimmons [29], distance education solutions could be analyzed in terms of strengths and weaknesses: while they offer open access, open education, open teaching, and network participation, on the other hand, they are associated with the problem of low completion rates, poor quality assurance, and lack of adequate assessment tools. Furthermore, high workloads, challenging course content, lack of time, lack of pressure, lack of a sense of community, and social influence are the potential 
reasons why learners are not satisfied [30-32] with the assessment of learning that remains one of the main challenges [33].

Of course, distance learning has changed the role of teachers in the traditional learning system [34] and their relationship with learners. The main benefit is represented by a greater flexibility in terms of access to learning resources [35], but the instrumentalization of educational activities is left to the students themselves, rather than to an expert figure, and they are not always capable of structuring their learning path.

In the struggle for competition and international visibility, distance learning can be seen as a great opportunity for higher education institutions, in order to strengthen their brand and to widen the supply for their students.

Thus, learning is not merely the transfer of knowledge from teacher to learner in a single environment, but it derives from the transformation and the transfer of knowledge through the interaction of people, both online and offline [36].

Generally, distance learning has developed to fulfill additional needs, but in a time when students are expecting to learn and to be taught through methods in accordance with their personal preferences (implementing modern technologies) [37], universities, especially the traditional ones, have to look at online courses in order to develop their new digital strategy in a quick and effective way [38]. However, to achieve successful digital transformation, higher education institutions have to be digitally well-equipped, and students, staff, and academics have to be prepared to study, work, and teach with digital devices and techniques, driving innovation and disruption approaches [39].

Distance learning represents a complex field, and there is no unified understanding of how to plan, practice, and evaluate it, but different national and scientific traditions of educational science and philosophy can influence its development. According to Bates [40], distance learning is a broader concept, including online learning and remote learning. According to Bozkurt et al. [8], another branch of distance learning is represented by emergency remote education, which can be defined as way of "surviving in a time of crisis with all resources available, including offline and/or online resources" [7]. It is clear that, while distance learning represents an option, based on planning, theoretical and practical competences, and consolidated models, emergency remote education is the need of changing to react in an emergency time.

Of course, several education systems more used to facing challenges, learning, and evolving themselves would be more ready to welcome and develop distance formats, while other systems, still focused on their bureaucratic and static structures, could appear more reluctant to do it. Today, academic institutions have to continually update and advance their management and learning processes, fostering connectivity among students, staff, and departments. Digital approaches require skills, knowledge, and confidence to use new technologies, but not all universities and faculty members are ready to welcome such changes: so far, the Italian higher education system has never distinguished itself for its capacity to innovate and evolve, characterized by top down approaches and a limited autonomy [41]. Consequently, poor attention has been dedicated to the potential of distance learning and its development in the national context.

With the Covid-19 outbreak, education systems have been forced to move to online platforms, with the aim to ensure continuity in education and training paths. More in depth, they have been forced to develop and improvise blended solutions, combining elements coming from online and remote learning: if they had to completely translate their activity online, they did not have time to plan and organize it.

As anticipated by Cleveland-Innes and Lim [42,43], tutor/learner-readiness is regarded as the main risk connected to the development of distance learning: of course, it has represented the main difficulty faced by the Italian education system, effectively surpassed after an initial phase of uncertainty.

Distance learning, previously perceived as "optional", has been adopted as the only means to provide education, highlighting a lack of flexibility, resources, and competences for many institutions. In this sense, three different assets emerged as crucial in order to 
achieve success in such a new experience: a clear and strong leadership, able to assume relevant decisions even in crisis situations; effective communication to better engage stakeholders (internal and external); and concrete support provided by administrative staff, ensuring continuity for what concerns new settings and formats [44].

Covid-19 changed the lifestyle for citizens all over the world, generating a considerable number of deaths and unquantifiable damages in social and economic terms, but the resilience capacity of systems can reduce such damages [9], with knowledge that maintains its leading role in the provision of opportunities to face the emergency.

Covid-19 can be seen as the beginning of a new era for higher education and distance learning; especially for those systems, like the Italian one, that are shy in opening themselves to the exploitation of new technologies, it can be seen as an opportunity to change and to evolve. Nevertheless, digitalization for the university has to be seen not as a purpose per se, but it helps in order to make universities' products and services better, also from a sustainable perspective, but there are aspects, such as face-to-face meetings, working in groups, and learning from each other, that have to stay.

\section{Research Methodology}

As already declared, the aim of the paper is to investigate the strategic model of distance learning characterizing higher education in Italy, showing the effects generated by the diffusion of the health emergency. More precisely, the aim is to identify specific best practices that, even after the end of the emergency, can be adopted by all Italian higher education institutions in order to boost their digital supply and compete in an international context, evolving distance learning from emergency remote learning to a usual and structured practice within universities.

\section{Research Design}

After a theoretical background on the digitalization of higher education, aimed at pointing out benefits and risks connected with the development of distance learning, in order to achieve the scope previously mentioned, the method of case study will be adopted. Generally, the term "case" refers to a specific location, community, or organization; in this sense, the Italian higher education system will be the object of the research, representing an exemplifying case [45]. It has been chosen not because it is extreme or unusual, but because it epitomizes a broader category of cases, allowing the researchers to examine such key progress. In this sense, the Italian case is apt to generate new theory out of the findings, allowing the observation of the evolution of distance learning within higher education institutions: if during the health emergency it has been adopted in the form of emergency remote education, we expect it to become a more usual and structured practice, enabling the development of green campuses [11,12]. As suggested by Bryman [46], researchers are members of the system analyzed, so they can provide additional longitudinal elements.

The Italian higher education system represents the starting point of this analysis; it is composed of 98 higher education institutions and, among these, 67 are state universities, while 31 are non-state universities. The term "non-state" does not mean "private": indeed, among the 31 non-state universities, eight do not belong to the State, but to other public entities, while the other 23 are effectively "private". Among state universities, three are technical universities, completely focused on engineering and architecture; six are schools for advanced studies, specialized in postgraduate studies; and two are for foreigners, aiming to foster the advancement and dissemination of the Italian language, culture, and literature.

Among non-state institutions, three are characterized by a religious orientation; 11 are online universities, specialized in e-learning and distance programs; and only one, the University for Foreigners Dante Alighieri, is dedicated to foreign students.

On the basis of the number of students, there are 12 large universities, with more than 40,000 students; 29 middle universities, with a number of students between 15,000 and 40,000; and 56 small universities, with less than 15,000 students. The system enrolls about 
$1,700,000$ students; $90 \%$ of them attend state institutions, with only $10 \%$ attending non state ones, and among them, only $3.5 \%$ are enrolled in online universities [47].

The study will be structured in two main parts: the first one aims to provide a general view about distance learning in the national context before the pandemic, considering all the Italian higher education institutions, while the second one is exclusively focused on the behavior adopted by traditional higher education institutions (basically less used to operate online) during the health emergency, taking into account all the legislative measures enacted by the Italian government. Therefore, for what concerns the second part of the study, the sample will be composed of only 87 universities, excluding the online ones.

Data were collected by taking into account official statements provided by the Italian National Agency for the Evaluation of the University and Research Systems (ANVUR) and by institutions investigated. The process was not easy, since not all universities publicly share their digital strategy, communicating with students by mails, even during the pandemic. In this sense, the initial strategy was to look at universities' official websites in order to find specific sections dedicated to the pandemic outbreak and the strategy to face it, but then, given the lack of data, every form of communication about digital strategy was taken into account. Then, data were analyzed qualitatively: for what concerns the second phase of the study, they were organized in an excel file, aimed to highlight the number and the type of online platforms used by each institution to provide education. The aim was to understand how different platforms can be combined in order to enhance the quality of services provided.

Through a more in-depth analysis, based on the official measures enacted by universities' rectors, the effort was to understand the future of the Italian distance learning, answering these research questions: how has Italian higher education reacted to Covid19 outbreak? Which lessons can be learned from the pandemic experience? At the end, findings were presented with the aim to provide answers to such questions.

\section{Findings}

The following section is divided in two different paragraphs. Paragraph 4.1 will present the situation of distance learning in Italy before the health emergency, taking into account the entire higher education system (composed of traditional and online institutions). Then, paragraph 4.2 aims to show changes that have occurred in universities' behavior in response to the health emergency. After a brief resume of the measures enacted to regulate education during the emergency, it will be focused exclusively on traditional universities, pointing out their new e-learning strategy, in order to individuate a new distance learning model (on the basis of the best practices) for the future of the Italian university system.

\subsection{The Italian Distance Learning before the Covid-19 Outbreak}

By referring to the period before the health emergency, in a context where all higher education institutions over the world have started to think and to apply more sustainable learning experiences, exploiting technology, the Italian system turned out to be still concentrated on restrictive bureaucratic regulations.

In this sense, if compared to the international scenario, Italy showed a large delay (more or less 15 years) [48]. Of course, the delay was due to the poor autonomy that has characterized the Italian higher education system during the last years, with universities that started to think about their specific strategic plan only in 2009 [49], but then there were other two specific causes. The first one regarded a negative perception about the introduction of technology in education: new devices were considered dangerous, generating alienation in the traditional teaching relationship. The other one derived from the habit of associating online courses with private online universities, perceived as providers of a lower quality education. Indeed, based on the provision of virtual exams and a weak assessment process for what concerns students' progress, these institutions are able to ensure the achievement of degrees in exchange of large payments. 
Nevertheless, digital innovation has become a need and has started to spread through Italian universities, even despite the opinions of the education system leadership and people's traditional aversions.

Students have started to use the web as a support to traditional lectures: they downloaded teaching materials recommended or created by professors or looked for further information in order to know more about a specific topic of the course or a specific area of interest. On the other hand, professors have started to require their students to produce digital documents.

In this sense, the weight of distance learning has grown from $2.3 \%$ to $4.2 \%$ in ten years: while in 2011, 100 courses were provided online, in 2019, they became 195. Of course, most (about 60\%) distance learning initiatives belonged to online universities, with traditional ones that started to open themselves to new technologies (the University of Turin provided eight online degree paths, the University of Foggia and the University of Rome Tor Vergata had seven). At the same time, beyond the 11 online universities, no traditional institution communicated to diversify its supply through distance learning solutions. They preferred to share and advertise the quality of their activities for what concerns teaching, research, and, sometimes, social engagement, increasing traditional people's conviction about their superiority over online universities.

In this sense, in Italy, the digital revolution in the education system had started, but it appeared in a preliminary stage, characterized by a bottom up path and based on the adoption of non-institutionalized good practices that just aimed to support traditional courses, rarely to replace them. A relevant attempt through which traditional Italian higher education institutions tried to react to the changes occurred in the education market was represented by a design and delivery platform founded by 17 public Italian universities (Aldo Moro University of Bari, Polytechnic of Bari, Bolzano, Catania, Ferrara, Foggia, Genova, LUMSA, Marche, Milano-Bicocca, Modena and Reggio Emilia, Padova, Parma, Perugia, University of Salento, Salerno, and Ca' Foscari University) and aimed to offer distance learning courses for all (students, workers, and citizens who want to learn); it was financed by the Ministry of Education.

On the basis of this evidence, for what concerns the Italian higher education before Covid-19 outbreak, it was characterized by the lack of specific regulations defining the provision of distance learning, its attractiveness, its effectiveness, and its composition of digital resources [50].

\subsection{Higher Education's Response to Covid-19 Outbreak}

During the health emergency, a series of legislative measures has been enacted in order to re-organize the Italian education system. The aim has always been to ensure public health protection and, at the same time, the right to study for all.

The first measure was enacted on 23 February 2020, addressed to all the education institutions (including universities) located in the most affected regions by the virus (Emilia Romagna, Lombardia, and Veneto) and consisting in mere advice to suspend teaching activities, soliciting the autonomy of every institution. A more effective measure was represented by the DPCM (President of the Council of Ministers' decree) enacted on the 1 March 2020, according to which every education institution (for all levels) was obliged to suspend its activity, even referring to the most affected regions.

Through the following DPCM, enacted on 4 March 2020, the previous measures were extended to the national territory, so every education institution in Italy had to suspend its teaching activity, providing distance learning solutions. In this context, universities, by observing security measures, could develop their research activity. The following DPCMs and law decrees were enacted with the aim to extend the suspension of teaching.

Then, further measures were enacted, and while on May 4 the aim was to present indications for what concerns the "second and third" phase of lockdown, ensuring safety for all and continuity for what concerns universities' activity (education programs, research activity, and assistance for local needs), the decree of May 13 was about the provision of 
62 million euros in order to bridge the digital gap and to evenly facilitate the access to distance learning for students. On May 17, instead, the suspension of teaching activities and the need to develop distance learning solutions was confirmed for every education system.

At the end, the Government left to every institution the autonomy to organize written tests and graduations in presence in July, stimulating confusion among academic communities.

After the end of the summer, universities tried to re-start, providing blended learning solutions, especially for what concerns students of the first year, in order to better introduce them to the education path, but the diffusion of the second wave of the virus forced the Government to enact further DPCMs (on 18 and 24 October 2020). Through such measures, higher education institutions were invited to adopt distance learning and smart working solutions, reducing the contagion risk.

Therefore, in this context, traditional universities have been called to define the way to operate until the end of the emergency, on the basis of their decision-making autonomy.

In the sample, composed of 87 traditional universities (excluding the online ones), 15 do not share the number and the type of online platforms used, and one of them communicates to students exclusively through emails.

Then, 38 universities have decided to exploit only one online platform to provide education and perform exams and graduation exams; 26 have adopted two different platforms (generally one for teaching and oral exams and another one for written tests); six institutions have implemented three online platforms; only one institution, the Sapienza University of Rome, has used four online platforms; and another one, the University of Milan "Statale", has adopted six different platforms. Focusing on this institution, every platform is aimed to a specific activity: Microsoft Teams and Zoom are used to provide lessons and for oral exams, Moodle SEB and exam.net are dedicated to written exams, Proctoring to propose quiz, and UNIMIA to written exams on paper. Furthermore, this institution is the only one to provide an "Ariel Plan" to help professors with distance instruments, without saying anything about its future distance learning.

Generally, Microsoft Teams seems the most exploited; it has been adopted by 36 institutions, even if 22 of them have decided to accompany Teams with another platform, especially for what concerns written exams, while nine institutions have decided to use their own e-learning platforms (two of them combine them with another).

Moodle has been chosen by 20 institutions, but for most of them it represents a support to perform written exams; for five universities it is the only platform exploited.

As previously mentioned, every institution has been called to organize its activity independently during the following phases of the emergency, so rectors have started to enact temporary measures.

Thirty-nine Italian universities do not communicate their arrangements on their websites, for 17 of them there is no arrangement enacted by rector on the website (probably they use other communication means), while the others 22 just communicate to operate online, without specifying further information about time: some of them declare to continue until new alerts, others until the end of the emergency.

Thirty-two higher education institutions specify to adopt distance learning until the end of the academic year, even if with different deadlines.

Nine universities appeared braver, having anticipated the end of the measures enacted in late May or in June; only the University of Trieste declared to operate in distance until the end of July, but to experiment blended opportunities for exams and graduations starting from June 15. Only five universities communicated to exploit distance learning even during the first semester 2020/2021, and only one university, the University of Genoa, explicitly declared the intention to maintain its identity as a traditional university. With the spread of the second wave, a time of uncertainty was opened for Italian higher education institutions: they translated again to their distance learning, except for medical disciplines, without specifying which will be the duration of the measure, strictly related to the trend of the 
pandemic emergency and to the need to ensure public health for their students, of course, even with the hope to come back to a "normal life" as soon as possible.

\section{Discussion}

In a knowledge-based society, globalization and the spread of internet and other technology devices have encouraged the need for new learning formats [6] and the diffusion of digitalization, also in higher education. Distance learning is seen as an evolution of lifelong learning, through the appearance of online universities and several forms of elearning and blended learning. Distance learning could help universities in their transition from closed education systems to mass ones, ensuring open access, open education, open teaching, and network participation, but, at the same time, it could represent a risk for what concerns the quality of education and the possibility to lose customers (students) during their educational path. Especially through its configuration of emergency remote education, distance learning can help in responding to an emergency, allowing continuity in education programs.

The Italian context, previously characterized by a negative perception about technological devices in education processes and the quality of education provided by online universities, has been always characterized by a large delay in terms of education digitalization [48] if compared with the international scenario. Starting from such a delay, it has represented the object of this work.

Digitalization enabled new opportunities in every field, also in higher education, increasing interactions and collaborations with stakeholders [26], but also the possibility to create value in a new, circular, and sustainable way [11]. Nevertheless, not all higher education institutions have welcomed such processes [1] and while some of them decided to find their identity on the exploitation of digital devices (online universities), others shared doubts on the introduction of technology in higher education.

In a time when the emergency has not yet ended, on the basis of these considerations, the study underlines how the pandemic has forced the Italian higher education to evolve and to experiment with a new strategic behavior. Comparing two different situations, the situation before Covid-19 outbreak and that characterized by the need to face it, the aim was to observe and describe the resilience of the Italian education system. More precisely, the focus was on the strategy of emergency remote education adopted by universities, hypothesizing how the future of the Italian higher education could be (with distance learning that will probably become a usual and structured practice).

Indeed, after the first and timid attempts to exploit technologies in education performed by Italian universities, it is during the pandemic crisis that distance learning has been used as emergency remote education and has been transformed from a support for traditional means to the only instrument to ensure continuity in education and, at the same time, public health protection for all.

Of course, it brings a series of disadvantages, represented by the risk students or points in education quality [27], confirming the value of real interactions and the need, for universities, to remain a place of sociality and growth for students.

The Italian university case was investigated through a documentary analysis, looking at what universities communicate in their official websites and documents. The data collection process was not easy, characterized by fragmentation for what concerns information shared by every institution and a multitude of measures enacted by the Italian government to regulate the necessary lockdown, firstly addressed to the most affected regions and then extended to all the national territory. In this sense, the most relevant decrees have been the DPCM enacted on 4 March 2020, through which every education institution stopped its teaching activity and started to provide distance learning solutions, and the last ones, enacted in October 2020, through which distance learning has been highly recommended and traditional universities have been called to define the way to operate up to the end of the emergency, on the basis of their decision-making autonomy. 
Every Italian university has dedicated a temporary space on its website to Covid-19, some of them just to remind readers of good and hygienic practices, others to offer updates on the virus trend, and others to share the rector's decisions with their academic community.

In this sense, for 15 universities, it has not been possible to analyze the distance learning platform used; 17 universities do not share their rector's decision, and another 22 declare to operate online, without offering further and more precise indications.

Looking at available information, research findings demonstrate the resilience capacity of the Italian higher education system, able to react and to evolve, re-organizing itself in only one week.

Indeed, while before the pandemic the Italian university system registered only timid attempts to become more digital, looking at distance solutions as a mere support to traditional learning and teaching tools, it is during the emergency that distance learning has become the only means able to ensure the right to study and health protection for all. It is on the basis of such evolution observed that we believe in a new era for higher education, characterized by a structured use of digital devices and, consequently, more attention to sustainable approaches [11,12].

The main risk connected to the pandemic is to register a drop in the number of university students, and to avoid it, the Ministry of the University ensures the right to study for all through three main measures: reduction on fees, increase in scholarships, and incentives to face the digital divide.

Exams, especially written tests, and graduations continue to represent hard proofs for universities: mutual trust is needed, because if institutions have to respect students' privacy, on the other hand students must be serious.

\section{Preliminary Conclusions}

Distance learning has been an important solution, an effective answer to an unpredictable situation, but universities have always been a place of meeting for students, and the intention is to come back to normality as soon as possible, considering distance learning as a support instrument to manage off-site and foreign students, performing blended learning opportunities, and enabling the idea of green campuses. Of course, thanks to emergency remote education, the Italian university system has never stopped its activity, and students have continued to take exams and to graduate, but looking at the future, it does not intend to change its peculiarity.

Distance learning has been a challenge for students not used to learning in an online environment, but also for teachers, taking time and preparation to quickly adapt traditional and in presence lectures to a virtual format. In this sense, the University of Milan "Statale" can be seen as a good example, performing an ad hoc program to sustain professors in this drastic transition. This institution is at the forefront even for what concerns the organization of distance teaching: it exploits six different platforms, with the aim to ensure the best in all aspects.

Of course, each institution has developed its distance learning strategy in its own way, but in general, Italian higher education has demonstrated how resilience is needed in order to react, ensuring continuity for its teaching and research activity.

The practical implications are related to the potential of technology, presented as a crucial instrument in a time of crisis, when social distance has become the golden rule, but also for the future, ensuring new and sustainable ways to create value [12].

Looking at lessons learned from the pandemic experience, three different dimensions appeared as fundamental for distance learning [44]; they are represented first of all by a clear and strong leadership, able to transmit a sense of management for the entire situation; effective communication, in order to engage with a larger audience and to share a sense of belonging to a specific community; and then administrative support in order to ensure continuity for what concerns distance services.

If the paper was dedicated to the first phase of the emergency, in a future perspective, the aim would be to compare the Italian case in the international context, individuating 
the impact generated by different models of distance education, that will be considered as ordinary practices and not as extraordinary ones.

The results of the pandemic may be a stronger university, aware of digital potential and the relevance of distance instruments, but even a physical community; maybe it will be a new university, halfway between a traditional and an online one. In this sense, the pandemic has represented the beginning of a new era for Italian universities: it has demonstrated that technological devices and distance learning represent a way to do more and not less, fostering a new sustainable sensitivity, because if the knowledge is the key to success, universities must be the place to re-start from.

Author Contributions: Conceptualization: R.F. and C.F.; methodology: N.C.; validation: A.A., G.F., N.C., and R.F.; formal analysis: C.F.; investigation: C.F. and R.F.; resources: R.F.; data curation: C.F.; writing-original draft preparation: C.F.; writing-review and editing: C.F., N.C, R.F., and A.A.; supervision: A.A. and G.F. All authors have read and agreed to the published version of the manuscript.

Funding: This research was supported and funded by the University of Rome Tor Vergata, Department of Management and Law by the research project named "Sustainable Development" and by the Master Program in "Reporting Innovation Sustainability".

Institutional Review Board Statement: Not Applicable.

Informed Consent Statement: Not Applicable.

Data Availability Statement: Not Applicable.

Conflicts of Interest: The authors declare that they have no conflict of interest.

\section{References}

1. Kooskora, M. The Role of an Entrepreneurial Mindset in Digital Transformation-Case Study of the Estonian Business School. In Digital Entrepreneurship: Impact on Business and Society; Soltanifar, M., Hughes, M., Gocke, L., Eds.; Springer: Cham, Switzerland, 2021; pp. 143-164.

2. Westerman, G.; Bonnet, D.; McAfee, A. Leading Digital: Tuning Technology into Business Transformation; Harvard Business Review: Brighton, MA, USA, 2014.

3. Drucker, P.F. Post-Capitalist Society; Harper Bus: New York, NY, USA, 1993.

4. Boucouvalas, A. Self-Directed Learning: Critical Practice; Routledge: Abingdon, UK, 1981.

5. Jarvis, P. Adult Education and Lifelong Learning: Theory and Practice; Routledge: Abingdon, UK, 1986.

6. Dan, M.C. The Third Mission of Universities in the Development Strategy of Vienna City. Inform. Econ. 2012, 16, 49-56.

7. Giacosa, A. Can Emergency Remote Education Make our Universities "Smarter"? Some Reflections Based on Students" Perceptions. In Proceedings of the DIDAMATiCA 2020: “Smarter School for SmartCities", Trieste, Italy, 6-8 May 2020. Available online: https://www.researchgate.net/publication/346038670_Can_Emergency_Remote_Education_make_our_ universities_smarter_Some_reflections_based_on_students $\backslash T 1 \backslash$ textquoteright_perceptions (accessed on 10 July 2020).

8. Bozkurt, A.; Xiao, F.; Jung, I.; Vladimirshi, V. A global outlook to the interruption of education due to COVID-19 Pandemic: Navigating in a time of uncertainty and crisis. Asian J. Distance Educ. 2020, 15, 1-126.

9. D'Adamo, I.; Rosa, P. How Do You See Infrastructure? Green Energy to Provide Economic Growth after COVID-19. Sustainability 2020, 12, 4387.

10. Gallagher, V.C.; Hrivnak, M.W.; Valcea, S.; Mahoney, C.B.; LaWong, D. A comprehensive three- dimensional sustainability measure: The 'missing $\mathrm{P}^{\prime}$ of 'people' - A vital stakeholder in sustainable development. Corp. So Responsib. Environ. Manag. 2018, 25, 772-787. [CrossRef]

11. Sonetti, G.; Lombardi, P.; Chelleri, L. True Green and Sustainable University Campuses? Toward a Clusters Approach. Sustainability 2016, 8, 83. [CrossRef]

12. Tudorie, C.A.-M.; Vallés-Planells, M.; Gielen, E.; Arroyo, R.; Galiana, F. Towards a Greener University: Perceptions of Landscape Services in Campus Open Space. Sustainability 2020, 12, 6047.

13. Pagani, M. Digital business strategy and value creation: Framing the dynamic cycle of control points. MIS Q. 2013, 37, 617-632. [CrossRef]

14. Gronroos, M.G. Mahdollisuuden Aika kohti Virtuaalista Organisaatiota; Tammer-Paino Oy: Tampere, Finland, 2006.

15. Zaborova, E.N.; Glazkova, I.G.; Markova, T.L. Distance learning: Students' perspective. Sociol. Stud. 2017, 2, 131-139.

16. PwC. The 2018 University-Making the Right Choices, Making It Happen. 2015. Available online: https://www.pwc.com/mx/ es/publicaciones/c2g/231_2018-university-making-the-right-choices-making-it-happen.pdf (accessed on 23 June 2020).

17. MicKinsey. A Labour Market that Works: Connecting Talent with Opportunity in the Digital Age; McKinsey Global Institute: New York, NY, USA, 2015. 
18. Etzkowitz, H. The norms of entrepreneurial science: Cognitive effects of the new university-industry linkages. Res. Policy 1998, 27, 823-833. [CrossRef]

19. Jokhan, A.; Sharma, B.; Singh, S. Early warning system as a predictor for student performance in higher education blended courses. Stud. High. Educ. 2018, 44, 1900-1911. [CrossRef]

20. Darkenwald, G.G.; Merriam, S.B. Adult Education: Foundations of Practice; Harper and Row: New York, NY, USA, 1982.

21. Derounian, J.G. Active Learning in Higher Education. SAGE J. 2017, 13, 9-21.

22. Kaplan, A.M.; Haenlein, M. Higher education and the digital revolution: About MOOCs, SPOCs, social media, and the Cookie Monster. Bus. Horiz. 2016, 59, 441-450. [CrossRef]

23. Gottschalk, T. Print tools in distance education. In Distance Education: Strategies and Tools; Wills, B., Ed.; Educational Technology Publications: Englewood Cliffs, NJ, USA, 1994.

24. Buhl, M.; Andreasen, L.B. Learning potentials and educational challenges of massive open online courses (MOOCs) in lifelong learning. Int. Rev. Educ. 2018, 64, 151-160. [CrossRef]

25. Ilmarinem, V.; Koskela, K. Digitalisaatio; Talentum: Helsinki, Finland, 2015.

26. Berman, S.J. Digital transformation: Opportunities to create new business models. Strategy Leadersh. 2012, 40, 16-24. [CrossRef]

27. Matzler, K.; Strobl, A.; Thurner, N.; Fuller, J. Switching experience, customer satisfaction and switching costs in the ICT industry. J. Serv. Manag. 2015, 26, 117-136. [CrossRef]

28. Bentley, P.J. Digitized; Oxford University Press: New York, NY, USA, 2012.

29. Veletsianos, G.; Kimmons, R. Assumption and challenges of open scholarship. Int. Rev. Res. Open Distance Learn. 2012, 13, 166-189.

30. Hone, K.S.; El Said, G.R. Exploring the factors affecting MOOC retention: A survey study. Comput. Educ. 2016, 98, 157-168. [CrossRef]

31. Zheng, S.; Rosson, M.B.; Shih, P.C.; Carroll, J.M. Understanding student motivation, behaviours and perceptions in MOOCs. In Proceedings of the 18th ACM Conference on Computer Supported Cooperative Work \& Social Computing, Vancouver, BC, Canada, 14-18 March 2015; pp. 1882-1895.

32. Singh, A.B. Learning through Massive Open Online Courses (MOOCs): A Case of the First International MOO Offered by University of Oslo in 2015. Master's Thesis, Department of Education, University of Oslo, Oslo, Norway, 2016.

33. Xiong, Y.; Suen, H.K. Assessment approaches in massive open online courses: Possibilities, challenges and future directions. Int. Rev. Educ. 2018, 64, 241-263. [CrossRef]

34. Dale, E.L. Pedagogisk Profesjionalitet [Pedagogical Professionalism]; Gyldendal: Oslo, Norway, 1989.

35. Buhl, M.; Andreasen, L.B. MOOCs-The promise of meeting the need of flexibility for the adult learner? In Proceedings of the 15th European Conference on e-Learning, Prague, Czech Republic, 27-28 October 2016; Novotna, J., Jarcarik, A., Eds.; Academic Conferences and Publishing International Limited: Reading, UK, 2016; pp. 98-104.

36. Kop, R. The challenges to connectivist learning on open online networks: Learning experiences during a massive open online course. Int. Rev. Res. Open Distrib. Learn. 2011, 12, 19-38.

37. Kirkwood, A.; Price, L. Examining some assumptions and limitations of research on the effects of emerging technologies for teaching and learning in higher education. Br. J. Educ. Technol. 2013, 44, 536-543.

38. Berger, T.; Frey, B. Digitalisation, Jobs and Convergence in Europe: Strategies for Closing the Skills Gap; Oxford Martin School: Oxford, UK, 2016.

39. Khalid, J.; Ram, B.R.; Soliman, M.; Ali, A.J.; Khaleel, M.; Islam, M.S. Promising digital university: A pivotal need for higher education transformation. Int. J. Manag. Educ. 2018, 12, 264-275. [CrossRef]

40. Bates, A. Teaching in a Digital Age, 2nd ed. 2019. Available online: https://pressbooks.bccampus.ca/teachinginadigitalagev2/ (accessed on 23 June 2020).

41. Vaira, M. La Costruzione Della Riforma Universitaria e Dell'autonomia Didattica: Idee, Norme, Pratiche, Attori; LED Edizioni Universitarie: Milano, Italy, 2011.

42. Cleveland-Innes, M. Teaching in an online community of inquiry: Institutional and individual adjustment in the new higher education. In Educational Communities of Inquiry: Theoretical Framework, Research and Practice; Akyol, Z., Garrison, R., Eds.; IGI Global: Hershey, PA, USA, 2013.

43. Lim, C. Cultivating higher order thinking dispositions in undergraduate students. Int. J. Learn. Teach. 2017, 3, 154-159. [CrossRef]

44. Agasisti, T.; Soncin, M. Higher education in troubled times: On the impact of Covid-19 in Italy. Stud. High. Educ. 2020, 46, 86-95. [CrossRef]

45. Yin, R.K. Case Study Research: Design and Methods, 4th ed.; Sage: Los Angeles, CA, USA, 2009.

46. Bryman, A. Social Research Methods; University Press: Oxford, UK, 2012.

47. ANVUR. Rapporto Biennale Sullo Stato del Sistema Universitario e Della Ricercar; ANVUR: Roma, Italy, 2018.

48. OECD. Review of the Italian Strategy for Digital Schools; OECD Publishing: Paris, France, 2013.

49. Giusepponi, K.; Tavoletti, E. Vision and Mission Statements in Italian Universities: Results of an Empirical Investigation on Strategic Orientation. J. Knowl. Econ. 2015, 9, 301-328. [CrossRef]

50. Walker, L.; Loch, B. Academics' perceptions on the quality of MOOCs: An empirical study. Int. J. Innov. Qual. Learn. 2014, 22, 53-63. 
Article

\title{
New Circular Networks in Resilient Supply Chains: An External Capital Perspective
}

\author{
Sara Alonso-Muñoz ${ }^{1, *(\mathbb{D})}$, Rocío González-Sánchez ${ }^{1} \mathbb{D}$, Cristina Siligardi $^{2} \mathbb{D}$ and Fernando E. García-Muiña ${ }^{1} \mathbb{D}$ \\ 1 Department of Business Administration (ADO), Applied Economics II and Fundaments of \\ Economic Analysis, Rey-Juan-Carlos University, 28032 Madrid, Spain; rocio.gonzalez@urjc.es (R.G.-S.); \\ fernando.muina@urjc.es (F.E.G.-M.) \\ 2 Department of Engineering Enzo Ferrari, University of Modena and Reggio Emilia, 41125 Modena, Italy; \\ cristina.siligardi@unimore.it \\ * Correspondence: sara.alonso@urjc.es
}

Citation: Alonso-Muñoz, S.; González-Sánchez, R.; Siligardi, C.; García-Muiña, F.E. New Circular Networks in Resilient Supply Chains: An External Capital Perspective. Sustainability 2021, 13, 6130. https:// doi.org/10.3390/su13116130

Academic Editor: Idiano D’Adamo

Received: 2 May 2021

Accepted: 27 May 2021

Published: 29 May 2021

Publisher's Note: MDPI stays neutral with regard to jurisdictional claims in published maps and institutional affiliations.

Copyright: (c) 2021 by the authors. Licensee MDPI, Basel, Switzerland. This article is an open access article distributed under the terms and conditions of the Creative Commons Attribution (CC BY) license (https:// creativecommons.org/licenses/by/ $4.0 /)$.

\begin{abstract}
The pandemic caused by COVID-19 has had an impact on the relationships established between different actors in organisations. To deal with these changes, it is necessary to develop a resilience capacity that allows for the establishment of different patterns of relationships through a new management model. The application of circularity principles implies a radical change in stakeholder relations, breaking with the "end-of-life" concept existing in linear economies. Furthermore, circular economy can ensure resilience in supply chains, and it can be considered as a tool in uncertain environments. Therefore, the objective of this study is to analyse the association between the customer-supplier relationships with circular supply chains based on the intellectual capital-based view theory. External capital is a crucial factor for organisations, and it helps with building remarkable capabilities for the whole supply chain due to collaboration and cooperation. This research contributes with a systematic revision of the literature regarding circular supply chains and customer-supplier external capital, providing an exploratory model. Establishing a closer and effective relationship with customers and suppliers supposes a differentiating value and competitive advantages. Actors involved in the supply chain are essential in the implementation of circularity in organisations for reducing waste production and returning resources to the production cycle. Therefore, circular networks related to customers' behaviour, sustainable supplier election and IT tools play a key factor in improving resilience in supply chains.
\end{abstract}

Keywords: resilient supply chains; external capital; customer-supplier relationship; circular network

\section{Introduction}

The COVID-19 pandemic has had an unprecedented impact on the global industry at all levels around the world [1-3]. Effective crisis management is required in order to restore the confidence between socioeconomic actors. However, beyond the consideration of the negative effects of this type of historical crisis, this new scenario can be a source of opportunities for beneficial change. The concept of resilience is incorporated into the management of organisations to guide this change. "Resilience is the capacity of a system to anticipate, adapt, and reorganize itself under conditions of adversity in ways that promote and sustain its successful functioning" [4] (p. 1).

One of the organisational processes most affected by the current health crisis involves activities related to the supply chain that have been affected by severe ruptures and dysfunctions not experienced in previous pandemics [5-12]. This is because this involves a network of stakeholders and a means of distribution involved in the different global processes and activities. Supply managers have had to deal with a variety of problems such as: (1) mobility restrictions and border closures, (2) shortages of raw materials and workforce, (3) the maintenance of social distance, (4) the radical increase in certain demands for both raw materials and final products and (5) the diversion of certain raw materials for 
the manufacture of products considered to be of greater need to combat COVID-19 [11]. As a result, they have had to adapt quickly to a situation where strategic planning did not anticipate this new scenario. Organisational resilience involves a capacity to adapt to turbulent environments through routines that enable rapid and appropriate responses to change. Thus, the association of resilience and the supply chain has attracted the attention of researchers, although we are still at a basic stage of development.

A resilient supply chain (RSC) enables greater manageability for dealing with disruptions in uncertain business environments, such as the situation caused by COVID-19 [9-12]. The severe impact of the COVID-19 pandemic on supply chains leads us to conclude that it is necessary to establish new patterns of relationships in order to solve scenarios in which social distance and mobility restrictions must be maintained [6,10-14].

One of the factors that is attracting the most attention with regard to supply chain management at this stage of the health crisis is related to the new rules that must regulate relations with the different stakeholders [10,11]. Establishing a new framework for more sustainable relationships with suppliers and customers in the postpandemic period would improve value creation. As an example, the automotive industry has relied on global-scale supply chains based on maximum efficiency and just-in-time parts supply, processes that have been blown apart by COVID-19-related restrictions. With a high dependence on components from China, the industry has to diversify its supply chains in order to reduce the risk of stock shortages. However, in the Spanish automotive sector, a significant number of component suppliers are located in the local area of the manufacturers. Therefore, during this crisis, the occasional component shortage was quickly resolved early in the pandemic.

The crisis recovery plan should be based on flexible production strategies involving external and internal resources and capacities. Therefore, achieving a resilient supply chain requires both logistics and relationship network redesign to reduce vulnerability [15]. This study aims to contribute to the establishment of a framework of relationships that will enable the supply chain to be resilient in the face of crises of global impact. Expanding the number of suppliers to cooperate, finding suppliers in proximity to production locations, establishing tools to facilitate communication and involving customers in the return of used products would all contribute to the adaptation to the new business reality [16].

Although many organisations have prioritised survival over investment in other "nonpriority activities" [11], this crisis is an opportunity to consider sustainability as a priority investment in the future as well as a differentiating element. Sustainability is positively related to resilience [17]. The application of sustainability principles to the management and development of the supply chain would make it possible to achieve a more efficient operation with a more engaged ecosystem. It is necessary to incorporate a new framework that regenerates the relationships in the most sustainable way possible. In this way, the application of the principles of circularity would facilitate this transformation [18-20].

The circular economy represents a further step forward in the field of sustainability by breaking with the linear production model with substantial modifications in both operations and relationships [20]. The principles of circularity can provide the supply chain with greater resilience and a more flexible response to future disasters [21]. The functioning of a circular system depends to a high degree on the establishment of well-founded partnerships, mainly with suppliers and customers, which requires the application of a variety of disciplines at different levels [22].

The customer-supplier relationship mechanisms linked with circular economy and supply chains have not been studied enough in the extant literature [23]. To manage this from a resilience perspective, it would be useful to incorporate a theoretical framework to facilitate a developmental model. In this regard, this study is based on the intellectual capital-based view theory (ICV). This theory proposes an efficient management of knowledge through its relationships among external capital, human resources, human capital, organisational design and structural capital. Knowledge management in the supply chain is a topic that is still understudied in the literature, and mainly from a quantitative 
approach [24]. Recent studies highlight the importance of resilient supply chains according to their intellectual capital [13].

In addition, intellectual capital (IC) enables the development of sustainability by generating synergies between stakeholders through novel combinations of their different utilities [25]. Currently, the number of articles about sustainability and intellectual capital has increased, even coining the term "green intangible capital" $[25,26]$, but there is a gap in the research between circular economy and intellectual capital.

External capital is an intangible asset that focuses on the establishment of superior relationships with stakeholders through the alignment of different interests. These stakeholders' interests include concerns about green problems [26]. Through external capital, intellectual capital literature has addressed the study of these aspects and evaluated their strategic potential. Hence, the integration of circular economy and intellectual capital theoretical frameworks can advance developing sustainable and flexibly functioning relationships in turbulent environments.

A resilient supply chain is crucial to financial and economic survival $[9,12,13]$, highlighting the importance of collaborative relationships with customers and suppliers, which could minimise and mitigate disruptions and the negative impacts of the COVID-19 pandemic on supply chains. Our research aims to contribute to the construction of a CI-based theoretical framework that enables the development of circular networks in a resilient supply chain. Specifically, the research questions to be answered are:

1. How does the circular network enable the development of resilience routines in the supply chain based on the intellectual capital-based view theory?

2. How do circular networks in resilient supply chains support sustainability?

This study aims to contribute theoretically to the foundations of resilient supply chains through a systematic literature review. For this purpose, we follow the following structure. After the introduction, the research methodology is presented. In Section 3, the theoretical framework is elaborated. Firstly, the extant literature about the term intellectual capital is explored related to different models, and its three more accepted categories in the current literature are explained. Secondly, the circular economy and circular supply chains concepts are defined, and they are studied with a resilience perspective. In Section 4, the linkage between intellectual capital, particularly external capital, and circularity in supply chains is exposed in a conceptual model, which is developed in Section 5, with seven propositions highlighting the key role of customer-supplier relationships among supply chains towards implementing more circular practices. Finally, in Section 6, conclusions, contributions, limitations, and future research are exposed.

\section{Research Methodology}

The method applied in this study is based on exploratory and conceptual research analysing the link between circular supply chains and external capital. The lack of results obtained in the searches for 'circular economy' or 'circular supply chain' and the concept of 'external capital' have highlighted the gaps to fill in this research.

Systematic reviews are used to synthesise findings in a systematic, reproducible and transparent way [27]. Their use in business research is increasing [28]. This type of review is a research method and process in which the data analysed and collected is assessed and identified. The purpose of a systematic review is to detail and recognise pre-established criteria in the literature to answer the research question suggested. In order to reach reliable results to make decisions and draw conclusions. Qualitative systematic reviews compare results from qualitative studies, compiling articles and assessing quality. The strength of this type of analysis is to study whether an effect is repeated or constant across studies and which studies show this [29].

The information has been extracted from the Web of Science database. The literature review was conducted between January 2021 and April 2021. The search protocol defined is as follows: firstly, the terms were selected, and the keywords were combined using the Boolean operators 'AND' and 'OR' in order to refine the information found. The keywords 
used in the search were: 'circular economy' OR 'circular supply chain' AND 'intellectual capital' AND/OR 'external capital'. Whilst the terms associated were 'suppliers' and 'customers'. Secondly, the results obtained were recorded filtering them by subject and title. Table 1 shows the most researched topics.

Table 1. Most researched topics.

\begin{tabular}{lcc}
\hline \multicolumn{2}{c}{ Topics Researched } & Number of Articles \\
\hline Circular economy/Circular supply chain & Customer relationship & 8 \\
\hline Circular economy/Circular supply chain & Customer behaviour & 31 \\
\hline Circular economy/Circular supply chain & Supplier relationship & 9 \\
\hline Circular economy/Circular supply chain & Supplier behaviour & 7 \\
\hline
\end{tabular}

The total number of articles available for review were 408 . The searches were sorted by journal, selecting the articles that had been published in high impact journals (Journal Citation Report, (JCR) Q1-Q2 and Scimago Journal \& Country Rank, (SJR) Q1, resulting 122 articles). The main journals are the following: Business Strategy and the Environment; Ecological Economics; Environment Development and Sustainability; Journal of Cleaner Production; Journal of Economics \& Management Strategy; Journal of Environmental Management; Journal of Industrial Ecology; Journal of Intellectual Capital; Journal of Knowledge Management; Production Planning \& Control; Renewable \& Sustainable Energy Reviews; Resources, Conservation and Recycling; Sustainability Science; Sustainable Development and Sustainable Production and Consumption. Then, the searches were sorted by author, date of publication, title and abstract reading, excluding 67 articles. The most relevant papers were selected, gathering a core sample of 55 papers, taking into account the purpose of this analysis. In Figure 1, this review process is represented.

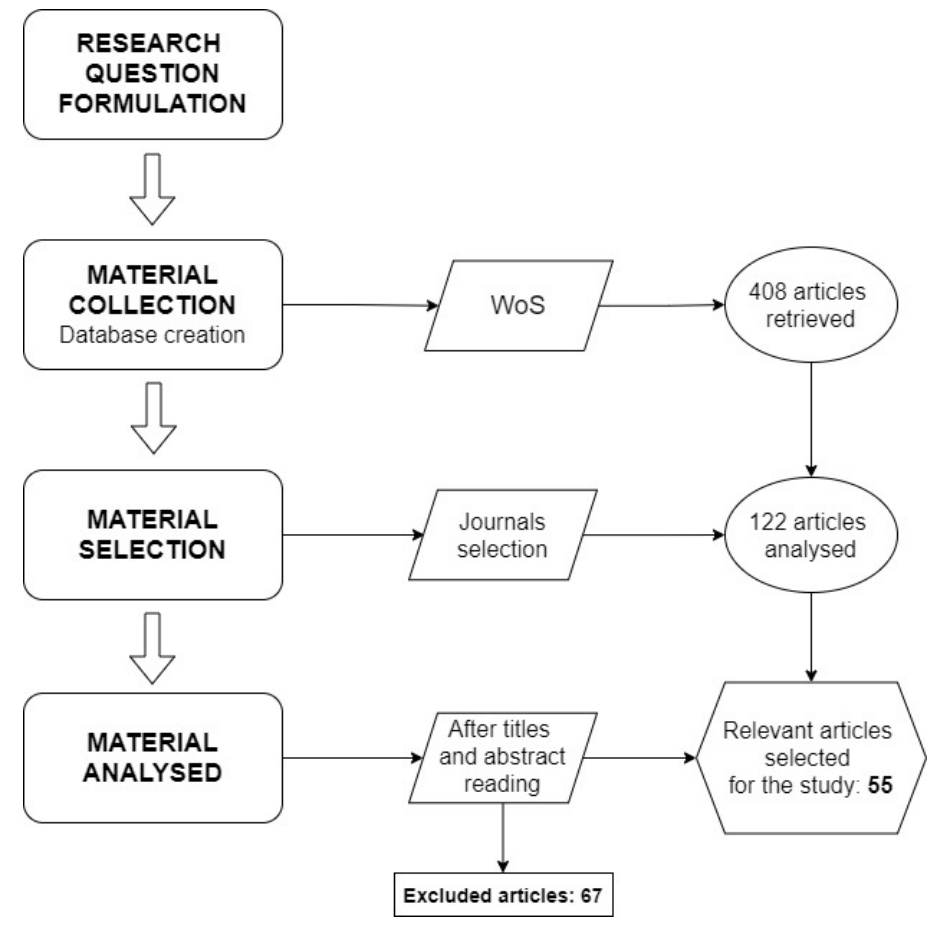

Figure 1. Review process.

Subsequently, once the methodology used in this paper has been explained, the following section will show the theoretical background, conceptualising the main terms studied in this article: resilient supply chain; circular economy and supply chains; and intellectual capital. 


\section{Theoretical Background}

\subsection{Resilient Supply Chain}

Resilience is the capacity for an enterprise to adapt, reorganise its system and keep surviving and growing when a change is undergoing, whilst disturbance is absorbing [30]. RSC implies "the ability of a supply chain to return to normal operating performance, within an acceptable period, after being disturbed" [31] (p.2). The concept of resilience in supply chains refers to mitigating and anticipating disruptions and disturbances throughout adapted techniques for decreasing vulnerabilities in uncertain environments [30].

Operations in the supply chain are constantly under change and conditions of uncertainty; thus, resilience is a key factor. Supply chains must be prepared to respond to unexpected events [32]. Collaboration, supply chain reengineering, agility, innovation, flexibility, visibility, sharing and trust are the main principles for supply chain resilience [31]. Researchers have argued for the importance of some factors for enhancing resilience in supply chains, highlighting integration, cooperation and communication. The capacity to return more quickly to equilibrium after a period of disturbance is called stability [32]. Visibility is also a crucial element of risk reduction in supply chains.

The complexity in supply chain networks demands more resilience. It plays a critical role in the adaptiveness of networks, their interdependencies, their interactions throughout the whole system and their ability to change its behaviour. Designing resilience, collaboration between suppliers and customers, agility and following a risk management culture are general principles for resilience in supply chains networks [30]. Logistics capabilities, such as low-cost distribution, reliability, delivery speed and responsiveness towards dynamic integration, enables resilience in the supply chain for a competitive advantage. Resilience is a requirement in order to achieve sustainable development, and thus a transition to circular networks [32].

\subsection{Circular Economy and Circular Networks}

Environments change rapidly, which is why companies depend on their capabilities to reconfigure, integrate and build their internal and external competencies. Organisations must be able to achieve competitive advantages in innovative ways [33]. Based on this theory, in order to implement new circular models, organisations must rely on their dynamic capabilities. This would enable them to make the transition to more sustainable and circular processes and products, reconfiguring the structure and transforming their relationships in closed-loop chains.

Supply chains can be open or closed loop [34]. Open-loop chains imply that third parties recover materials. Closed-loop chains recover products from customers, returning them to the producer for recovering the value and being able to reuse it as a whole or in part. The aim of both chains is to reuse and recover the value of products and resources, maximising their life cycle and preventing waste by incorporating their return over time [35]. Effective implementation of closed loops depends on the involvement and participation of stakeholders in the supply chain, with the alignment of technical, economic and environmental elements. Relationships with suppliers and customers enable adaptation and resilience to changes in the environment [36].

The circular economy is a restorative and regenerative economic model that breaks with the linear economy, replacing the concept of "end-of-life" by seeking to maintain resources and their value for as long as possible. It seeks to reduce the negative effects on the environment by reducing and eliminating waste [37]. This system aims to minimise the emission and leakage of energy by tightening energy and material loops, following the principle of material balance based on recycling, reuse, refurbishment and remanufacturing [38].

Circular supply chains can bring resources back into the production cycle and reduce waste and residues. The reverse supply chain involves an adaptation of circular economy principles to supply chain management, including product design, activities to maximise value creation and product recovery, among others. In this way, damaged products are 
brought back into operation through the logistics network by means of reconditioning and remanufacturing [39]. Supply chain actors play an essential role in the implementation of the circular economy to promote circular thinking in organisations and rethink the production system, achieving greener and more efficient processes [38]. This circular approach involves expanding the number of partnerships, expanding the number of collaborators and reaching more stable agreements with stakeholders in different industries, mainly suppliers and customers [23]. For this, traditional structures and organisational processes must be renewed with innovations.

\subsection{Conceptualising Intellectual Capital}

The concept of "intellectual capital" was first introduced by Galbraith in 1969, who defined it as an element that produces value for the organisation. A knowledge-based view concerns strategic choice and competitive advantage, taking into account the organizational structure, the nature of coordination and the theory of innovation, and allowing for the renovation of traditional processes and structures in organisations [40]. Thus, intangible resources and assets enable organisations to create value. Intellectual capital (IC) is often synonymous with intangible assets [41]. In this sense, IC is linked to the theory of resources and capabilities, understood as the set of intangible assets and capabilities that a company possesses to generate value to the company. Capabilities lie in processes, and its competitive advantage is based on a collection of skills, complementary assets and routines that are complicated for competitors to imitate.

Sustained competitive advantage depends on the alternatives of competitors to duplicate an organisation's attributes that imply an advantage [42]. Imperfections in transferability imply that resources are not freely transferable and are not available to everyone, because they are heterogeneous and scarce. Competitors cannot imitate valuable organisational resources, which is called "imperfectly imitability". Organisations seek causal ambiguity between their competitive advantages and the resources they possess. Therefore, the advantage is not fully understood by competitors, and for that reason, it is difficult for them to duplicate it.

There is consensus among the three main categories that encompasses intellectual capital: human capital, organisational (or internal) capital and external capital [43].

Human capital is defined as the individual knowledge stock of firms that is represented by workers and their capacity to generate and learn it. According to [44], it can be measured based on training, skills and knowledge.

External capital, also known as relational capital, refers to the network with external stakeholders, such as customers and suppliers, social responsibility activities and customer satisfaction and loyalty [45]. External capital is defined as the values, behavioural rules, codes and common objectives between customers and suppliers that result from collaboration and integration, thanks to an alignment of vision and organisational culture [46].

Establishing a distinctive and nonimitable combination of the resources and capabilities that make up these capitals is a generator of competitive advantages for organisations. The relevance of external capital with the supply chain, highlighting the relationship that organisations have between customers and suppliers, is the main reason for selecting this kind of capital in the current study. This analysis focuses on the role that external capital plays in the adaptive capacity of supply chains, considering the principles of circularity.

\section{Building the Circular Network in the Resilient Supply Chain}

The transition to a sustainability-based approach requires a shared vision among all stakeholders along with a collaborative approach. Manufacturing companies must care more about how to minimise environmental damage, implementing circularity into supply chains and stimulating their innovation, efficiency and cooperation between actors implied by such suppliers and customers [47]. Most studies on supply chain coordination do not take into consideration product quality from an environmental point of view and consumers' environmental concerns [48]. 
Vertical and horizontal cooperation in closed-loop supply chains decreases emissions and costs [49]. Based on the principles of circularity, collaboration networks are established with suppliers and customers on a broad basis, both in terms of number and in terms of the activities involved. This enables the incorporation of new raw materials and new processes and the recovery of end-of-life products into the manufacturing and distribution processes. To this end, connection and trust must be fostered through more active and transparent knowledge and information flows [20].

This requires establishing routines that allow actors to be aware of and take responsibility for the different practices related to technical, social or administrative aspects. External capital is a crucial factor for organisations, and it helps for building remarkable capabilities for the whole supply chain [47].

In terms of external capital, organisations can create competitive advantages by accessing, using and acquiring resources and developing capacities in their supply chains [47]. Regarding supplier-related routines, the focus should be on working towards the establishment of long-term relationships with a large number of suppliers.

\subsection{The Establishment of Knowledge Flows through Supply Chain Intelligence Integraton (SCII)}

Supply chain relationships and interactions strive to improve the environmental collaboration between buyers and suppliers [50]. The implementation of the circular economy requires the approval of all actors in the supply chain [18]. The aim of circularity is to achieve waste reduction and return resources to the production cycle. Coordination and cooperation with suppliers are crucial to supply restorative, recoverable and regenerative raw materials, with less negative effects on the environment [34].

Establishing a closer relationship with customers and suppliers implies a differentiator advantage. External capital in supply chains promotes common actions, and it allows forming stronger relationships. Routines that collect and process information and integrate innovation play a key role to achieve effectiveness and efficiency in external coordination with suppliers and customers. Engineering design choices must be linked, on the one hand, to coordination with suppliers and their components and factories and, on the other hand, to the experience of customers [51]. These dynamic capabilities are necessary to achieve better coordination and cooperation with suppliers and customers, and that is why all agents involved in the supply chain must work in line with each other to succeed in implementing circular models.

External capital implies the accessibility to external knowledge embedded in interorganizational relationships and in networks within suppliers and customers [46]. Good relationships with these external agents improves communication along the supply chain and facilitates the integration of diverse knowledge [52]. In order to leverage the benefits of knowledge incorporated in the supply chain network, organisations must invest in their external capital [46]. Furthermore, external capital and collaboration in supply chains increase the innovation [53] and process redesign, a key element for implementing circular practices, promoting interdependence of resources, information and communication between the companies and parties engaged. In a transaction, trust is the ability to believe and trust the other party, which is crucial for purchase intention [54]. So-called "green trust" involves consumers' confidence that it will be an environmentally sustainable product. To achieve this, it is essential to establish two-way knowledge flows between the organisation and the stakeholders involved in the supply and distribution process [20].

Organisations enhance their collaboration and relationships with supply chain partners, suppliers and customers when they possess valuable knowledge resources. The longterm partnership that takes place with supply chain agents is based on the exchange of knowledge, resources and information through fluid communication between the parties. Shared values and culture, satisfaction, commitment, trust, coordination and collaboration are key points [55].

External capital plays a key role in achieving transparency in the relationship between actors implied in the supply chain [56]. Transparency provides information about the 
interaction and relationship of both parties that can create value and enhance competitive advantage, commitment and satisfaction between the agents. Cooperation along the supply chain enhances partnerships [57]. The relationship between supply chain actors is called partnership and follows transparency, as it involves a close long-term relationship with open communication, mutual coordination of efforts and joint planification [58].

Suppliers and customers' knowledge are crucial as external sources in addition to applying internal knowledge to conduct innovation practices in organisations [59]. The knowledge obtained from suppliers and customers could improve a firm's customer satisfaction and operational and green performance [60]. The knowledge embedded in supply chain networks is called supply chain intelligence (SCI), whilst the application and absorption of knowledge from supply chain collaborators is a process denoted as supply chain intelligence integration (SCII) [61]. SCII integrates knowledge application from organisations and knowledge absorption by supply chain partners [40]. External capital supports SCII knowledge processing [62], which is crucial for an organisation's innovation practices. Supplier intelligence integration promotes a more efficient application and a rapid absorption of knowledge from suppliers, which helps to adjust productivity when environments change quickly [63]. Likewise, customer intelligence integration analyses a greater understanding of customer demands, needs and expectation from the market [59].

Consequently, we postulate that:

Proposition 1 (P1). Supply chain intelligence integration (SCII) positively affects circular networks in resilient supply chains by supporting and sharing knowledge between suppliers and customers.

\subsection{The Establishment of Greener Logistics and Intelligent Transport through Industry 4.0}

In terms of pollution, suppliers have a high environmental impact, which is why cooperation and coordination with them is necessary to achieve a greener product [64]. Organisations have the option to select suppliers with environmental and social standards and who have implemented so-called "reverse logistics" based on remanufacturing and waste management. This is key to the development of reverse logistics, the consideration of waste and how it is managed [23]. In reverse logistics, resources that have become obsolete are moved between companies in order to provide for their favourable disposal or to recapture their value [65]. The right choice of suppliers can boost material circularity and reduce environmental damage [64].

Industry 4.0 plays an essential role in sustainability in organisations, highlighting the improvement of logistics and intelligent transport management. It could have an impact on promoting greater monitoring of sustainable principles, "green" consumer behaviour, increasing product visibility throughout its life cycle and decreasing operational and development costs [66]. Industry 4.0 encompasses artificial intelligence, 5G networking, the Internet of things (IoT), robotics, blockchain, augmented reality and 3D printing, among others [67]. Currently, there are numerous programmes to make logistics management greener through distributed manufacturing systems and self-driving vehicles [68] Furthermore, external capital can be related to the right information and communications technology (ICT) management, and R\&D investments are influenced by industry 4.0 [69]. As [66] point out in their study, Industry 4.0 makes the exchange of knowledge and information along the supply chain more transparent and improves decision making between different parties. Digital technologies offer opportunities to integrate and implement the circular economy in supply chains [66]. Consequently, we postulate that:

Proposition 2 (P2). Industry 4.0 positively affects circular networks in resilient supply chains by achieving greener logistics and intelligent transport. 


\subsection{The Optimisation of the Supplier Selection Process through Ethical Codes and Multicriteria Tools}

The composition of certain products supposes that when discarded at the end of their lifespan, they are major pollutants that harm human health, which is why environmental collaboration in the supply chain is important. The most crucial elements for a more circular supply chain are low carbon emissions, sustainability, and green suppliers [70]. The selection of suppliers affects the behaviour of environmentally responsible organisations [71].

Suppliers should be selected on the basis of their sustainable or green performance, taking into account aspects such as sustainable product packaging, use of renewable energy, recycled items and reduction of emissions associated with manufacturing and transportation [72]. To optimise the supply chain network, it is important to detail the most efficient location of facilities and the necessary connections in order to reduce carbon emissions and associated costs [73]. Reducing transport and promoting local supplier relationships is one of the recommendations to implement more circular practices [74]. Recent practical examples support this trend. Permanent magnets, a component used in various technological products such as mobile phones and cars, are made from rare earth that is mined in developing countries without any environmental safeguards. In the circular current, the use of recycled raw materials and a manufacturing change of raw materials are being encouraged, and consequently, a search for more environmentally friendly suppliers available in nearby markets is also being encouraged. These sustainable practices by suppliers are recognised through environmental certifications, such as ISO 14000, which allow the most ecoefficient suppliers to be identified [74]. The circular economy encompasses new ethical relationships and moral requirements throughout the supply chain, according to the sustainable and circular value principles. New production methods were initiated based on ethical codes and attitudes which respect the environment. This requires a circular economy development model to establish new production ethical codes guidelines, such as emission reductions, cleaner production and resource conservation, integrating long-term interests within suppliers [75,76].

It is therefore recommended that ethical codes be established for the selection of suppliers, setting out the principles and features they must comply with in order to be part of the network.

Supply chain partnerships have improved due to globalisation and computerisation [70]. A better supplier selection can be achieved using IT tools such as big data or data mining to study the environmental impact of suppliers. Several green supplier ranking tools exist, such as the linguistic entropy weighting method, LEWM, which evaluates the different possibilities. The authors of [77] conducted a study in which they take into account environmental issues to put pressure on actors involved in supply chains. Multicriteria decision-making approaches, MCDM, are used to select suppliers through evaluation [74], and another method of note is the analytic hierarchy process, AHP [78]. Ecodesign capabilities, compliance with legislation, codification, pollution control, green competencies, product recycling, environmental efficiency, use of clean energy and materials and green image, among others, are the main selection criteria [74].

Thus, the following is being proposed:

Proposition 3 (P3). Ethical codes and multicriteria tools positively affect circular networks in resilient supply chains by improving the supplier selection process.

\subsection{The Establishment of a Strong Relationship Based on Supplier's Trust and Commitment}

One of the most important criteria when engaging with a supplier is to establish an appropriate level of commitment [77]. Sustainable and circular supply chain practices and collaboration within the supply chain are facilitated by suppliers' trust in the organisations they engage with [79]. Trust is a good mechanism for organisational control and for improving supply chain performance and fostering interfirm relationships [80]. 
Supplier trust, information and knowledge sharing and collaboration and communication are key aspects of supplier relationships. Providing support, motivation, training, assistance and active participation by allowing suggestions or feedback gives suppliers confidence in organisations. In addition, reward mechanisms have a positive effect on engagement [79].

Proposition 4 (P4). Assistance, active participation and reward mechanisms positively affect circular networks in resilient supply chains by improving the suppliers' commitment.

\subsection{The Establishment of Customer Acceptance through Warranty Policies, Additional Services and New Service-Oriented Contracts}

Collaborating with consumers in process and product innovation is essential for companies for applying new knowledge and customers' preferences that are changing rapidly [81]. Companies can be more adaptative to the changes because of the supplier and customer partnership and the transferring of knowledge [82]. This implies a key factor towards circularity, e.g., in terms of customer's green behaviour, for recycling or remanufacturing. Repair extends the lifespan of many products, which can be designed to have a long service life or can be extended through repair, refurbishment and remanufacturing. Furthermore, through recycling it is possible to close the loop [83].

Market acceptance of remanufacturing is low as consumers think of lower quality products; when customers understand that remanufactured products use less energy, are less resource intensive and have ecological benefits, customer acceptability will increase [84]. Experience and satisfaction with previous purchases also play an important role [85]. The marketing of remanufactured products implies a challenge for organisations, highlighting the low incentives to buy remanufactured products [86].

Refurbishment is an environmentally beneficial strategy that contributes to the circular economy by bringing back used products and repairing or replacing components [87]. The concept of refurbishment is often unclear to consumers, and the lack of attractiveness of these products and their unavailability in all markets are the main barriers [88].

It is important to provide information to raise consumer awareness; the use or purchase of remanufactured products can be encouraged through new ways of consuming a product based on use rather than purchase, complementarity through additional services or by extending the warranty against malfunctions $[88,89]$. Product price is affected by warranty duration and reliability and plays a determining role in the efficiency and profit of the supply chain. The performance of the closed-loop supply chain is affected by the warranty period, which in turn fixes the reliability of products and perceived value [90]. A strong relationship with customers is imperative to engage in reuse, recycling and take-back activities and to incorporate new business models such as collaborative consumption [91]. The leasing contract allows the customer to use an asset in exchange for the payment of lease rentals with the option to buy the leased asset, return it or renew the contract.

Hence, we formulate:

Proposition 5 (P5). Warranty policies, additional services and new service-oriented contracts positively affect circular networks in resilient supply chains by increasing customer acceptability of remanufactured or refurbished products.

4.6. The Establishment of Customer Participation and Purchase Promotion through Enviromental Education, Rewards and Discounts

Product-service systems (PSS) enable resource recovery, reuse, and recycling. Several studies report how circularity influences consumer behaviour by changing their role to a more active one in closed-loop systems [92]. Effectively collected resources can be used again in their life cycle; at this point, consumers play a crucial role, because the quality of revalued goods is improved by the key activities of separation, sorting, storage, collection and disposal [93]. In the linear economy, consumers were the last link in the supply chain, 
playing a passive role, but in the circular economy they are actively involved in the recovery and recycling of products and waste [94].

Customers can follow more sustainable purchasing behaviours by purchasing products that are less harmful to the environment, thus lowering environmental and social impacts [95]. Raising awareness of circular consumption through policies is imperative [23]. Consumers are key stakeholders in the return of products when they become obsolete and determine the success of circular systems [96].

Encouraging consumer proactivity and efforts to reflect green thinking in their consumption patterns is critical to the successful implementation of circular relationships [97]. Through environmental education campaigns or programmes, customers can understand the importance of sustainable purchasing behaviour [98]. It is important to make consumers aware of the quality and environmental impact of the products they buy by providing them with additional information. Translating environmental data into understandable information influences sustainable consumption [99].

Consumer awareness is defined as the level of consumer awareness of their responsibility towards the environment and of information about the alternatives they have in the market. However, there are barriers that can interfere with the positive effect that awareness has on changes in consumer behaviour. For example, there may be a lack of alternatives or price increases for ecoresponsible products [100].

Individual consumer behaviour can be measured and predicted based on their purchase intention, according to the theory of reasoned action (TRA), although factors can change attitudes and interfere [101]. The company should look for factors that encourage consumers to buy or participate in certain circular processes. These tools would facilitate processes such as the establishment of rewards or discounts.

To improve purchasing behaviour, organisations could apply financial rewards or discounts on future purchases to motivate consumers to participate, for example, in sending their products to collection or recycling points, among other measures [98]. On-site collection services and exchange of used products for a reduction in the purchase price of new products are other incentive measures that circular organisations can use in the development of their external capital [102]. It is necessary for companies to explore the incentives they can use with their consumers to change their purchase intention and their motivations to participate in product returns [103].

The willingness to pay a higher price for a product that has been produced in a more environmentally friendly way is a critical point to address. However, by raising consumer awareness, consumers can be made willing to pay more for a product produced in a more ecoefficient way, which reduces the environmental and social impact [95]. In order to make consumers willing to pay a higher price for a product resulting from the implementation of a circular system, an awareness or educational process is required. However, in the early stages of developing environmental awareness, it is key that green products are priced competitively in order to be attractive [104]. Some studies [105] argue that the higher the discounts are for returning used products, the more effect on customers' decisions.

According to the literature, we can postulate that:

Proposition 6 (P6). Environmental education, rewards and discounts positively affect circular networks in resilient supply chains by increasing customer purchase promotion and participation in product recovery processes.

\section{Designing a Conceptual Model}

This section illustrates the design of the conceptual model where the links between the elements studied are identified. The model proposed seeks to fill the gap in the literature by taking into account the importance between the key role of customer-supplier relationships in order to implement more circular and resilient supply chains.

Six main propositions have been suggested. The first (P1) outlined that supply chain intelligence integration (SCII) positively affects circular networks in resilient supply chains 
by supporting and sharing knowledge between suppliers and customers. The second proposition (P2) argues that Industry 4.0 positively affects circular networks in resilient supply chains by achieving greener logistics and intelligent transport. The third proposition (P3) notes that ethical codes and multicriteria tools positively affect circular networks in resilient supply chains by improving the supplier selection process. The fourth proposition (P4) indicates that assistance, active participation and reward mechanisms positively affect circular networks in resilient supply chains by improving the suppliers' commitment.

The fifth proposition (P5) highlights that warranty policies, additional services and new service-oriented contracts positively affect circular networks in resilient supply chains by increasing customer acceptability with remanufactured or refurbished products. Finally, the sixth proposition (P6) states that environmental education, rewards and discounts positively affect circular networks in resilient supply chains by increasing customer purchase promotion and participation in product recovery processes.

The conceptual model (Figure 2) offers an overview on the connections among the topics studied and their linkage between the customer-supplier relationship and circular supply chains. The model demonstrates the connection between external capital and circularity in order to achieve more resilience in supply chains. Transparency plays a key role in providing information about the interactions. Relationships with suppliers and customers imply the access to the external knowledge embedded, SCII [61]. SCII supposes a rapid absorption of knowledge from suppliers and a better understanding of customers' needs, highlighting the importance of an appropriate level of commitment with suppliers and taking into account that motivation, support, assistance and training improve confidence in organisations.

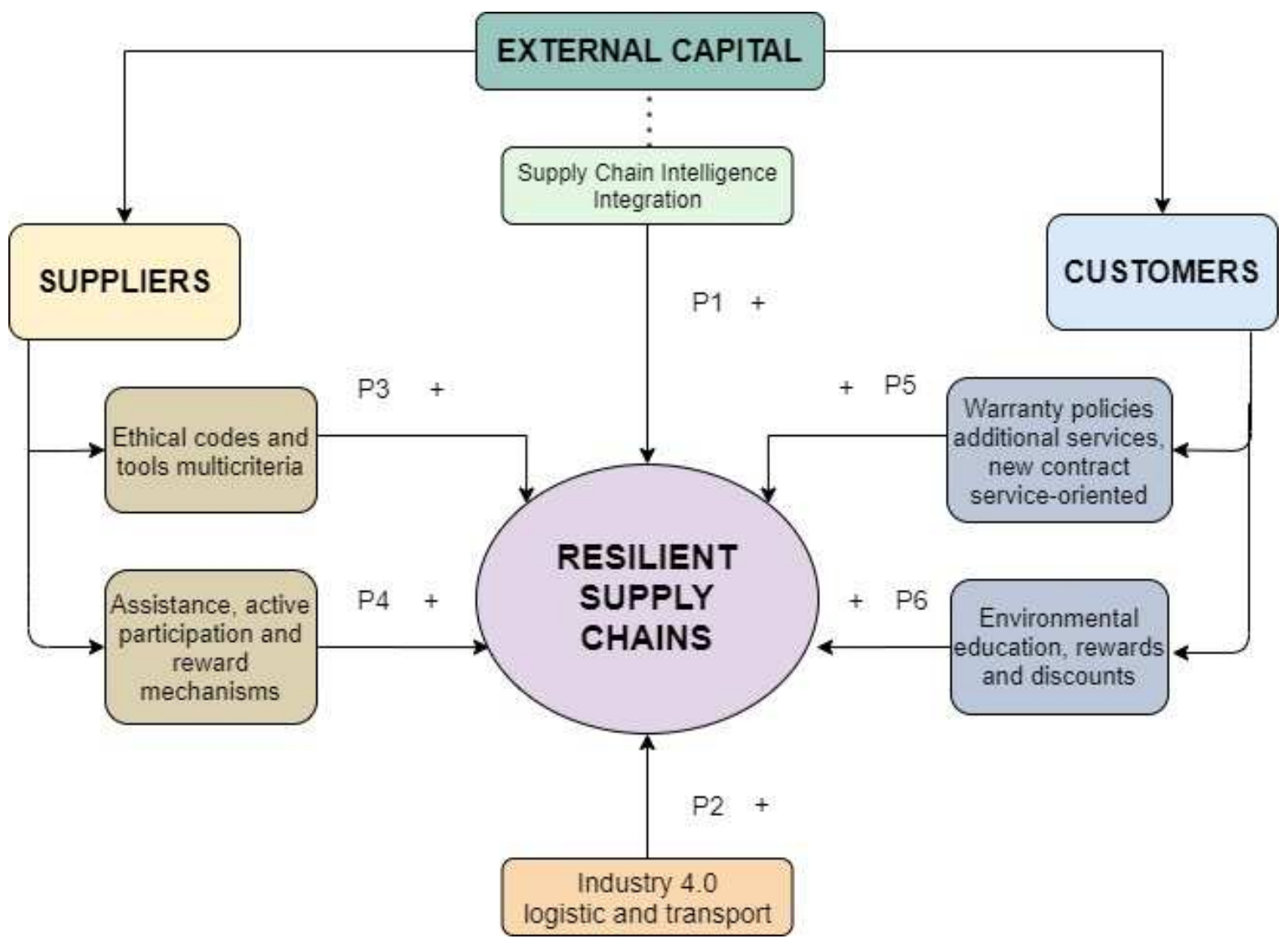

Figure 2. Conceptual model.

Organisations must select those suppliers with environmental concerns and those that develop reverse logistics. The implementation of logistics and intelligent transport management is possible thanks to Industry 4.0, which makes the exchange of knowledge and 
information throughout the supply chain more transparent between parties. For instance, multicriteria tools such as MCDM or AHP resulted in a greater supplier's selection [74,78].

On the other hand, providing information to raise consumer awareness is key. Warranty periods and leasing options increase customer acceptability with refurbished and remanufactured products $[89,106]$. Applying financial rewards or discounts on future purchases motivates customers to return used products according to circularity practices. Furthermore, educational environment campaigns encourage customers to participate actively in product recovery [107].

\section{Conclusions}

The COVID-19 pandemic has affected the functioning of supply chains in a way never seen before [11]. This has meant the disruption of organisational operations due to a lack of supply and mobility problems that have prevented the development of transport for a prolonged period. Organisations need to be resilient and adapt the functioning of their networks $[3,36]$. Many sectors are moving towards sustainable practices achieving resilience, such as automotive production and the technology components industry. Sustainability is an element that could build resilience, and both concepts pursue sustainable development [108].

The implementation of sustainable strategies can facilitate such changes, as it can have economic, social and environmental benefits. Therefore, applying the principles of circularity is a step forward on the road to sustainability for organisations. The circular economy is a revolution at a relational, production and technological level. This implies the establishment of new knowledge flows, which requires the establishment of routines and tools to facilitate them [20].

Based on the intellectual capital-based view theory (ICV), this study aims to provide an operational framework for the circular relationships that are established in circular supply chains. In particular, the external capital that facilitates and optimises knowledge flows with actors outside the organisation has been analysed [24]. Applying the principles of circularity to the supply chain allows new rules to be established with suppliers and customers. It increases the number of actors with an active role in greener operations. A long-term partnership between customers and suppliers is fundamental to achieve social and environmental solutions [109]. The design of new networks in supply chains needs to be further analysed to achieve resilience, effectiveness and efficiency through circularity [18].

The contributions in this article can be differentiated between theoretical and practical contributions.

\subsection{Theoretical Contribution}

Several current studies, mainly empirical, focus on the productive and technological aspects of the circular economy. Throughout this paper, we aim to contribute to the construction of a theoretical basis that focuses on the management of circular network relationships. Intellectual capital-based view theory (ICV) provides us a framework for action that favours knowledge flows in supply chain relationships [24]. We therefore focus on external capital. Although there is literature linking external capital to sustainability, there is a significant gap in the relationship between external capital and circularity.

Even though this conceptual model is exploratory, what is developed in this research provides theoretical contributions to the circular supply chain the literature, thanks to the linkage presented between customer-supplier relationships and circularity, filling this gap in the literature.

\subsection{Practical Contribution}

External capital proposes the establishment of routines and tools that facilitate the flow of knowledge from actors outside the organisation. In this sense, our study postulates the use of this framework in order to favour relations with suppliers and customers 
under the principles of circularity [47,51]. Organisations must take advantage of the necessary investments and organisational changes that must be made to adapt to the new postpandemic scenario to make a real commitment to sustainability [47].

It is essential to establish a climate of trust with both suppliers and customers. The development of training and information programs in the target markets modifies the behaviour and consumption patterns of customers by considering the principles of sustainability [79]. However, the effects of these programs are not immediate, so complementary tools must be put in place to build customer confidence.

To this end, the use of specific guarantees and the offer of complementary services are proposed. In addition, companies must adapt to new consumption patterns that focus on use rather than possession. Therefore, the offer of companies must be adapted to this. Accompanying a new offer, the use of discounts would also allow the customer to buy or carry out circular activities [105].

To build trust with suppliers, activities are proposed to enable the organisation to maintain a closer relationship with them. Through a reward system, if certain standards are met and advisory and support services are established, suppliers will be more likely to adopt circularity principles in their value chain $[79,107]$. In addition, the establishment of ethical codes and supplier selection systems through multicriteria tools will help to ensure that suppliers are more likely to adopt circularity principles in their value chain $[75,76]$.

\subsection{Limitation and Future Recommendation}

In the literature review, we have found a majority of papers that consider intangible capital with sustainability. This shows that it is still a developing field of research. Our research has focused on the application of external capital, given the importance of relationships in supply chain development, with much of the research focused on the operational and technological part of the process [110]. However, in future research, it would be interesting to study the joint effect of the three types of intellectual capital. Similarly, their practical application in different sectors would allow us to establish useful empirical evidence to adapt or extend the proposal offered.

Author Contributions: Conceptualization, R.G.-S. and S.A.-M.; methodology C.S. and F.E.G.-M.; investigation, C.S. and F.E.G.-M.; writing-original draft preparation, R.G.-S. and S.A.-M.; writingreview and editing, C.S. and F.E.G.-M.; supervision, R.G.-S. and F.E.G.-M. All authors have read and agreed to the published version of the manuscript.

Funding: This research was funded by the European Union under the LIFE Program, grant number: LIFE16ENV/IT/000307 (LIFE Force of the Future).

Institutional Review Board Statement: Not applicable.

Informed Consent Statement: Not applicable.

Data Availability Statement: Not applicable.

Conflicts of Interest: The authors declare no conflict of interest.

\section{References}

1. Zenker, S.; Kock, F. The coronavirus pandemic_A critical discussion of a tourism research agenda. Tour. Manag. 2020, 81, 104164. [CrossRef]

2. Giudice, F.; Caferra, R.; Morone, P. COVID-19, the food system and the circular economy: Challenges and opportunities. Sustainability 2020, 12, 7939. [CrossRef]

3. Siagian, H.; Tarigan, Z.J.H.; Jie, F. Supply chain integration enables resilience, flexibility, and innovation to improve business performance in COVID-19 era. Sustainability 2021, 13, 4669. [CrossRef]

4. Ungar, M. Systematic resilience: Principles and processes for a science of change in contexts of adversity. Ecol. Soc. 2018, 23, 34. [CrossRef]

5. Singh, S.; Kumar, R.; Panchal, R.; Tiwari, M.K. Impact of COVID-19 on logistics systems and disruptions in food supply chain. Int. J. Prod. Res. 2021, 59, 1993-2008. [CrossRef]

6. Golan, M.S.; Jernegan, L.H.; Linkov, I. Trends and applications of resilience analytics in supply chain modeling: Systematic literature review in the context of the COVID-19 pandemic. Environ. Syst. Decis. 2020, 1. [CrossRef] [PubMed] 
7. Remko, V.H. Research opportunities for a more resilient post-COVID-19 supply chain-Closing the gap between research findings and industry practice. Int. J. Oper. Prod. Manag. 2020, 40, 341-355. [CrossRef]

8. Govindan, K.; Mina, H.; Alavi, B. A decision support system for demand management in healthcare supply chains considering the epidemic outbreaks: A case study of coronavirus disease 2019 (COVID-19). Transp. Res. Part. E Logist. Transp. Rev. 2020, 138, 101967. [CrossRef]

9. Farooq, M.U.; Hussain, A.; Masood, T.; Habib, M.S. Supply chain operations management in pandemics: A state-of-the-art revire inspired by COVID-19. Sustainability 2021, 13, 2504. [CrossRef]

10. Chowdhury, T.; Sarkar, A.; Sanjoy, K.P.; Moktadir, A. A case study on strategies to deal with the impacts of COVID-19 pandemic in the food and beverage industry. Oper. Manag. Res. 2020. [CrossRef]

11. Chowdhury, P.; Sanjoy, K.P.; Kaisar, S.; Moktadir, A. COVID-19 pandemic related supply chain studies: A systematic review. Transp. Res. Part E 2021, 148, 102271. [CrossRef]

12. Paul, S.K.; Chowdhury, P. A production recovery plan in manufacturing supply chains for a high-demand item during COVID-19. Int. J. Phys. Distrib. Logist. Manag. 2021, 51, 104-125. [CrossRef]

13. Mubarik, M.S.; Bontis, N.; Mubarik, M.; Mahmood, T. Intellectual capital and supply chain resilience. J. Intellect. Cap. 2021, 1469-1930. [CrossRef]

14. Centobelli, P.; Cerchione, R.; Ertz, M. Managing supply chain resilience to pursue business and environmental strategies. Bus. Strategy Environ. 2020, 29, 1215-1246. [CrossRef]

15. Queiroz, M.M.; Ivanov, D.; Dolgui, A.; Wamba, S.F. Impacts of epidemic outbreaks on supply chains: Mapping a re-search agenda amid the COVID-19 pandemic through a structured literature review. Ann. Oper. Res. 2020. [CrossRef] [PubMed]

16. Sharma, G.; Volgman, A.S.; Michos, E.D. Sex differences in mortality from COVID-19 pandemic: Are men vulnerable and women protected? Case Rep. 2020, 2, 1407-1410.

17. D'Adamo, I.; Rosa, P. How do you see infraestructure? Green energy to provide economic growth after COVID-19. Sustainability 2020, 12, 4738. [CrossRef]

18. Muñoz-Torres, M.J.; Fernández-Izquierdo, M.A.; Rivera-Lirio, J.M.; Ferrero-Ferrero, I.; Escrig-Olmedo, E. An Assessment Tool to Integrate Sustainability Principles into the Global Supply Chain. Sustainability 2018, 10, 535. [CrossRef]

19. D'Adamo, I.; Lupi, G. Sustainability and resilience after COVID-19: A circular premium in the fashion industry. Sustainability 2021, 13, 1861. [CrossRef]

20. Alonso-Muñoz, S.; González-Sánchez, R.; Siligardi, C.; García-Muiña, F.E. Building exploitation routines in the circular supply chain to obtain radical innovations. Resources 2021, 10, 22. [CrossRef]

21. Choi, T.Y.; Roger, D.; Vakil, B. Coronavirus Is a Wake-Up Call for Supply Chain Management. 2020. Available online: https: //hbr.org/2020/03/coronavirus-is-awake-up-call-for-supply-chain-management (accessed on 10 February 2021).

22. Brown, P.; von Daniels, C.; Bocken, N.M.P.; Balkenende, A.R. A process model for collaboration in circular oriented innovation. J. Clean. Prod. 2021, 286, 125499. [CrossRef]

23. Farooque, M.; Zhang, A.; Thürer, M.S.; Qu, T.; Huisingh, D. Circular supply chain management: A definition and structured literature review. J. Clean. Prod. 2019, 228, 882-900. [CrossRef]

24. Cerchione, R.; Esposito, E. A systematic review of supply chain knowledge management research: State of the art and research opportunities. Int. J. Prod. Econ. 2016, 182, 276-292. [CrossRef]

25. Minoja, M.; Romano, G. Managing intelectual capital for sustainability: Evidence from a remunicipalized, publicly owned waste management firm. J. Clean. Prod. 2021, 279, 123213. [CrossRef]

26. Yong, J.Y.; Yusliza, M.Y.; Ramayah, T.; Fawehinmi, O. Nexus between green intellectual capital and green human resource management. J. Clean. Prod. 2019, 215, 364-374. [CrossRef]

27. Davis, J.; Mengersen, K.; Bennett, S.; Mazerolle, L. Viewing systematic reviews and meta-analysis in social research through different lenses. Springer Plus 2014, 3, 511. [CrossRef]

28. Snyder, H.; Witell, L.; Gustafsson, A.; Fombelle, P.; Kristensson, P. Identifying categories of service innovation: A review and synthesis of the literature. J. Bus. Res. 2016, 69, 2401-2408. [CrossRef]

29. Snyder, H. Literature review as a research methodology: An overview and guidelines. J. Bus. Res. 2019, 104, 333-339. [CrossRef]

30. Souza, V.; Bloemhof-Ruwaard, J.; Borsato, M. Towards Regenerative Supply Networks: A design framework proposal. J. Clean. Prod. 2019, 221, 145-156. [CrossRef]

31. Christopher, M.; Peck, H.; Rutherford, C.; Juttner, U. Supply Chain Resilience, Cranfield Centre for Logistics and Supply Chain Management. Cranfield Sch. Manag. 2003. Available online: https://www.cranfield.ac.uk/som/masters-courses/logistics-andsupply-chain-management (accessed on 10 February 2021).

32. Serhiy, Y.; Ponomarov, M.; Holcomb, C. Understanding the concept of supply chain resilience. Int. J. Logist. Manag. 2009, 20, 124-143. [CrossRef]

33. Teece, D.J.; Pisano, G.; Shuen, A. Dynamic capabilities and strategic management. Strategy Manag. J. 1997, 18, 509-533. [CrossRef]

34. Genovese, A.; Acquaye, A.A.; Figueroa, A.; Koh, S.L. Sustainable supply chain management and the transition towards a circular economy: Evidence and some applications. Omega 2017, 66, 344-357. [CrossRef]

35. Yang, M.; Smart, P.; Kumar, M.; Jolly, M.; Evans, S. Product-service systems business models for circular supply chains. Prod. Plan. Control 2018, 29, 498-508. [CrossRef]

36. Borgatti, S.P.; Li, X. On social network analysis in a supply chain context. J. Supply Chain Manag. 2009, 45, 5-22. [CrossRef] 
37. Charonis, G. Degrowth, Steady State Economics and the Circular Economy: Three Distinct yet Increasingly Converging Alternative Discourses to Economic Growth for Achieving Environmental Sustainability and Social Equity. World Economic Association Sustainability Conference. 2012. Available online: https://sustainabilityconference2012.weaconferences.net/ papers / degrowth-steady-state-economics-and-the-circular-economy-three-distinct-yet-increasingly-converging-alternativediscourses-to-economic-growth-for-achieving-environmental-sustainability-and-social-eq/ (accessed on 4 February 2021).

38. Geissdoerfer, M.; Savaget, P.M.P.; Bocken, N.; Hultink, E.J. The circular economy: A new sustainability paradigm? J. Clean. Prod. 2017, 143, 757-768. [CrossRef]

39. Cui, Y.Y.; Guan, Z.; Saif, U.; Zhang, L.; Zhang, F.; Mirza, J. Close Loop Supply Chain Network Problem with Uncertainty in Demand and Returned Products: Genetic Artificial Bee Colony Algorithm Approach. J. Clean. Prod. 2017, 162, 717-742. [CrossRef]

40. Grant, R.M. A resource based theory of competitive advantage. Calif. Manag. Rev. 1991, 3, 114-135. [CrossRef]

41. Brooking, A. Intellectual Capital: Core Asset for the Third Millennium Enterprise; Thomson Business Press: London, UK, 1997.

42. Barney, J.B. Firm resources and sustained competitive advantage. J. Manag. 1991, 17, 99-120. [CrossRef]

43. Daou, A.; Joseph, J.; Yousif, D.S.; Fathallah, R.; Reyes, G. Intellectual capital and resilience in torn societies. J. Intellect. Cap. 2019, 20, 598-618. [CrossRef]

44. Bontis, N. World Congress on Intellectual Capital Reading; Butterworth-Heinemann: Boston, MA, USA, 2002.

45. Alipour, M. The effect of intellectual capital on firm performance: An investigation of Iran insurance companies. Meas. Bus. Excell. 2012, 16, 53-66. [CrossRef]

46. Shou, Y.; Hu, W.; Xu, Y. Exploring the role of intellectual capital in supply chain intelligence integration. Ind. Manag. Data Syst. 2018, 118. [CrossRef]

47. Wu, R.; Huo, B.; Yu, Y.; Zhang, Z. Quality and green management for operational and environmental performance: Relational capital in supply chain management. Int. J. Logist. Res. Appl. 2020. [CrossRef]

48. Zhang, W.; He, Y. Optimal policies for new and green remanufactured shortlife-cycle products considering consumer behavior. J. Clean. Prod. 2019, 214, 483-505. [CrossRef]

49. Li, W.; Chen, J.; Chen, B. Supply chain coordination with customer returns and retailer's store brand product. Int. J. Prod. Econ. 2018, 203, 69-82. [CrossRef]

50. Woo, C.; Kim, M.G.; Chung, Y.; Rho, J.J. Suppliers' communication capability and external green integration for green and financial performance in Korean construction industry. J. Clean. Prod. 2016, 112, 483-493. [CrossRef]

51. Garvin, D.A. The Processes of Organization and Management. MIT Sloan Manag. Rev. 1998. Available online: https://sloanreview. mit.edu/article/the-processes-of-organization-and-management/ (accessed on 28 February 2021).

52. Acedo, F.J.; Barroso, C.; Galan, J.L. The resource-based theory: Dissemination and main trends. Strategy Manag. J. 2006, 27, 621-636. [CrossRef]

53. Anh, N.T.M.; Hui, L.; Khoa, V.D.; Mehmood, S. Relational capital and supply chain collaboration for radical and incremental innovation: An empirical study in China. Asia Pac. J. Mark. Logist. 2019, 31, 1076-1094. [CrossRef]

54. Moorman, C.; Deshpande, R.; Zaltman, G. Factors affecting trust in market research relationships. J. Mark. 1993, 57, 81-101. [CrossRef]

55. Boddy, D.M.; Wagner, B. Implementing collaboration between organizations: An empirical study of supply chain partnering. J. Manag. Stud. 2000, 37, 1003-1018. [CrossRef]

56. Kang, S.C.; Snell, S.A. Intellectual capital architectures and ambidextrous learning-A framework for human resource management. J. Manag. Stud. 2009, 46, 65-92. [CrossRef]

57. Eggert, A.; Helm, S. Exploring the impact of relationship transparency on business relationships: A crosssectional study among purchasing managers in Germany. Ind. Mark. Manag. 2003, 32, 101-108. [CrossRef]

58. Tuten, T.; Urban, D. An expanded model of business-to-business partnership formation and success. Ind. Mark. Manag. 2001, 30, 149-164. [CrossRef]

59. Carbonell, P.; Rodríguez-Escudero, A.I.; Pujari, D. Customer involvement in new service development: An examination of antecedents and outcomes. J. Prod. Innov. Manag. 2009, 26, 536-550. [CrossRef]

60. Du, L.; Zhang, Z.; Feng, T. Linking green customer and supplier integration with green innovation performance: The role of internal integration. Bus. Strategy Environ. 2018, 27, 1583-1595. [CrossRef]

61. Schoenherr, T.; Swink, M. The roles of supply chain intelligence and adaptability in new product launch success. Decis. Sci. 2015, 46, 901-936. [CrossRef]

62. Jones, O.; Macpherson, A. Inter-organizational learning and strategic renewal in SMEs: Extending the 4I framework. Long Range Plan. 2006, 39, 155-175. [CrossRef]

63. Wowak, K.D.; Craighead, C.W.; Ketchen, D.J.; Hult, G.T.M. Supply chain knowledge and performance: A meta-analysis. Decis. Sci. 2013, 44, 843-875. [CrossRef]

64. Govindan, K.; Mina, H.; Esmaeili, A.; Gholami-Zanjani, S.M. An Integrated Hybrid Approach for Circular supplier selection and Closed loop Supply Chain Network Design under Uncertainty. J. Clean. Prod. 2020, 242. [CrossRef]

65. Souza, G.C. Closed-loop supply chains: A critical review, and future research. Decis. Sci. J. 2013, 44, 7-38. [CrossRef]

66. Esmaeiliana, B.; Sarkis, J.; Lewis, K.; Behdad, S. Blockchain for the future of sustainable supply chain management in Industry 4.0. Resour. Conserv. Recycl. 2020, 163, 105064. [CrossRef] 
67. Rayome, A.D. Top 10 Emerging Technologies of 2019. TechRepublic 2019. Available online: https://www.techrepublic.com/ article/top-10-emerging-technologies-of-2019/ (accessed on 3 March 2021).

68. Prause, G. Sustainable business models and structures for Industry 4.0. J. Secur. Sustain. Issues 2015, 5. [CrossRef]

69. Davenport, T.H.; Prusak, L. Working Knowledge: How Organizations Manage What They Know; Harvard Business School Press: Boston, MA, USA, 1997. [CrossRef]

70. Feng, H.J.; Gong, Z. Integrated linguistic entropy weight method and multi-objective programming model for supplier selection and order allocation in a circular economy: A case study. J. Clean. Prod. 2020, 277, 122597. [CrossRef]

71. Zou, Z.; Wang, J.; Deng, G.; Chen, H. Third-party remanufacturing mode selection: Outsourcing or authorization? Transport. Res. E Logist. Transport. Rev. 2016, 87, 1-19. [CrossRef]

72. Yu, Y.; Zhang, M.; Huo, B. The impact of relational capital on green supply chain management and financial performance. Prod. Plan. Control 2020. [CrossRef]

73. Zohal, M.; Soleimani, H. Developing an ant colony approach for green closed-loop supply chain network design: A case study in gold industry. J. Clean. Prod. 2016, 133, 314-337. [CrossRef]

74. Kannan, D.; Khodaverdi, R.; Olfat, L.; Jafarian, A.; Diabat, A. Integrated fuzzy multi criteria decision making method and multi-objective programming approach for supplier selection and order allocation in a green supply chain. J. Clean. Prod. 2013, 47, 355-367. [CrossRef]

75. Qiao, F.; Qiao, N. Circular economy: An ethical and sustainable economic development model. Prakseologia 2013, 154, $253-272$.

76. Salvioni, D.M.; Astori, R.; Cassano, R. Corporate sustainability and ethical codes effectiveness. J. Mod. Acc. Audit. 2014, 10, 969-982. [CrossRef]

77. Mishra, A.R.; Rani, P.; Pardasani, K.R.; Mardani, A. A novel hesitant fuzzy WASPAS method for assessment of green supplier problem based on exponential information measures. J. Clean. Prod. 2019, 238. [CrossRef]

78. Haq, A.N.; Kannan, G. Fuzzy Analytical Hierarchy Process for evaluating and selecting a vendor in a Supply Chain model. Int. J. Adv. Manuf. Technol. 2006, 29, 826-835. [CrossRef]

79. Mokhtar, A.R.M.; Genovese, A.; Brint, A.; Kumar, N. Improving reverse supply chain performance: The role of supply chain leadership and governance mechanisms. J. Clean. Prod. 2019, 216, 42-55. [CrossRef]

80. Dyer, J.H.; Chu, W. The determinants of trust in supplier-automaker relationships in the U.S., Japan, and Korea. J. Int. Bus. Stud. 2000, 31, 259-285. [CrossRef]

81. Onofrei, G.; Nguyen, H.M.; Zhang, M.; Fynes, B. Building supply chain relational capital: The impact of supplier and customer leveraging on innovation performance. Bus. Strategy Environ. 2020, 1-13. [CrossRef]

82. Dahlmann, F.; Roehrich, J.K. Sustainable supply chain management and partner engagement to manage climate change information. Bus. Strategy Environ. 2019, 28, 1632-1647. [CrossRef]

83. Ertz, M.; Durif, F.; Arcand, M. Life after death? Study of goods multiple lives practices. J. Consum. Mark. 2017, 34, 108-118. [CrossRef]

84. Hazen, B.T.; Overstreet, R.E.; Jones-Farmer, L.A.; Field, H.S. The role of ambiguity tolerance in consumer perception of remanufactured products. Int. J. Prod. Econ. 2012, 135, 781-790. [CrossRef]

85. Kuah, A.T.H.; Wang, P. Circular economy and consumer acceptance: An exploratory study in East Southeast Asia. J. Clean. Prod. 2020, 247, 119097. [CrossRef]

86. Laitala, K.; Klepp, I.G.; Haugronning, V.; Throne-Holst, H.; Strandbakken, P. Increasing repair of household appliances, mobile phones and clothing: Experiences from consumers and the repair industry. J. Clean. Prod. 2021, 282, 125349. [CrossRef]

87. Ellen Macarthur Foundation. Towards the Circular Economy Vol. 2: Opportunities for the Consumer Goods Sector. 2013. Available online: https:/ / www.ellenmacarthurfoundation.org/publications/towards-the-circular-economy-vol-2-opportunitiesfor-the-consumer-goods-sector (accessed on 3 February 2021).

88. Van Weelden, E.; Mugge, R.; Bakker, C. Paving the way towards circular consumption: Exploring consumer acceptance of refurbished mobile phones in the Dutch market. J. Clean. Prod. 2016, 113, 743-754. [CrossRef]

89. Jain, N.; Rathore, A.P.S.; Jain, R.; Yadav, O.P. Maintenance planning based on reliability assessment of multi-state multi-component system. In Proceedings of the IEEE International Conference on Industrial Engineering and Engineering Management, Bangkok, Thailand, 16-19 December 2018; pp. 262-267. [CrossRef]

90. Lei, Y.; Liu, Q.; Shum, S. Warranty pricing with consumer learning. Eur. J. Oper. Res. 2017, 263, 596-610. [CrossRef]

91. Kazancoglu, Y.; Kazancoglu, I.; Sagnak, M. A new holistic conceptual framework for green supply chain management performance assessment based on circular economy. J. Clean. Prod. 2018, 195. [CrossRef]

92. Mugge, R.; Jockin, B.; Bocken, N. How to sell refurbished smartphones? An investigation of different customer groups and appropriate incentives. J. Clean. Prod. 2017, 147, 284-296. [CrossRef]

93. Nassour, A.; Hemidat, S.; Lemke, A.; Elnaas, A.; Nelles, M. Separation by manual sorting at home: State of the art in Germany. In Source Separation and Recycling. The Handbook of Environmental Chemistry; Maletz, R., Dornack, C., Ziyang, L., Eds.; Springer: Cham, Switzerland, 2017; pp. 67-87. [CrossRef]

94. Ghisellini, P.; Cialani, C.; Ulgiati, S. A review on circular economy: The expected transition to a balanced interplay of environmental and economic systems. J. Clean. Prod. 2016, 114, 11-32. [CrossRef]

95. Taghikhah, F.; Voinov, A.; Shukla, N. Extending the supply chain to address sustainability. J. Clean. Prod. 2019, $229,652-666$. [CrossRef] 
96. Bocken, N.M.P.; Bakker, C.; Pauw De, I. Product design and business model strategies for a circular economy. J. Ind. Prod. Eng. 2016, 1015, 20. [CrossRef]

97. Sarkis, J.; Zhu, Q.; Lai, K. An organizational theoretic review of green supply chain management literature. Int. J. Prod. Econ. 2011, 130, 1-15. [CrossRef]

98. Wang, Z.H.; Zhang, B.; Yin, J.H.; Zhang, X. Willingness and behavior towards ewaste recycling for residents in Beijing city, China. J. Clean. Prod. 2011, 19, 977-984. [CrossRef]

99. Laurenti, R.; Martin, M.; Stenmarck, A. Developing adequate communication of waste footprints of products for a circular economy-A stakeholder consultation. Resources 2018, 7, 78. [CrossRef]

100. Ketelsen, M.; Janssen, M.; Hamm, U. Consumers' response to environmentally friendly food packaging-A systematic review. J. Clean. Prod. 2020, 254, 120123. [CrossRef]

101. Young, W.; Hwang, K.; McDonald, S.; Oates, C.J. Sustainable consumption: Green consumer behavior when purchasing products. Sustain. Dev. 2010, 18, 20-31. [CrossRef]

102. Wang, Z.; Akbar, M.; Akbar, A. The interplay between working capital management and a firm's financial performance across the corporate life. Sustainability 2020, 12, 1661. [CrossRef]

103. Jiménez-Parra, B.; Rubio, S.; Vicente-Molina, M.A. Key drivers in the behavior of potential consumers of remanufactured products: A study on laptops in Spain. J. Clean. Prod. 2014, 85, 488-496. [CrossRef]

104. Coskun, S.; Ozgur, L.; Polat, O.; Gungor, A. A model proposal for green supply chain network design based on consumer segmentation. J. Clean. Prod. 2016, 110, 149-157. [CrossRef]

105. Taleizadeh, A.A.; Alizadeh-Basban, N.; Akhavan Niaki, S.T. A closed-loop supply chain considering carbon reduction, quality improvement effort, and return policy under two remanufacturing scenarios. J. Clean. Prod. 2019, 232, 1230-1250. [CrossRef]

106. Patricia, V.L.; van Wassenhove, L.N. Assessing the economic and environmental impact of remanufacturing: A decision support tool for OEM suppliers. Int. J. Prod. Res. 2018, 56, 1662-1674. [CrossRef]

107. Saphores, J.-D.M.; Ogunseitan, O.A.; Shapiro, A.A. Willingness to engage in a proenvironmental behavior: An analysis of e-waste recycling based on a national survey of U.S. Households. Resour. Conserv. Recycl. 2012, 60, 49-63. [CrossRef]

108. Miceli, A.; Hagen, B.; Riccardi, M.P.; Sotti, F.; Settembre-Blundo, D. Thriving, not just surviving in changing times: How sustainability, agility and digitalization intertwine with organizational resilience. Sustainability 2021, 13, 2052. [CrossRef]

109. Hofman, P.S.; Blome, C.; Schleper, M.C.; Subramanian, N. Supply chain collaboration and eco-innovations: An institutional perspective from China. Bus. Strategy Environ. 2020, 29, 2734-2754. [CrossRef]

110. González-Sánchez, R.; Settembre-Blundo, D.; Ferrari, A.M.; García-Muiña, F.E. Main dimensions in the building of the circular supply chain: A literature review. Sustainability 2020, 12, 2459. [CrossRef] 


\title{
Flexible Fuzzy Goal Programming Approach in Optimal Mix of Power Generation for Socio-Economic Sustainability: A Case Study
}

\author{
Mohammad Faisal Khan ${ }^{1}\left(\mathbb{D}\right.$, Asif Pervez $^{2}$, Umar Muhammad Modibbo ${ }^{3,+} \mathbb{D}^{\mathbb{D}}$, Jahangir Chauhan 4 \\ and Irfan Ali ${ }^{5, * \text { (D) }}$ \\ 1 Department of Basic Science, College of Science and Theoretical Studies, Saudi Electronic University, \\ Riyadh 11673, Saudi Arabia; f.khan@seu.edu.sa \\ 2 Centre for Distance and Online Education, Jamia Millia Islamia, New Delhi 110025, India; \\ asifpervez10@jmi.ac.in \\ 3 Department of Statistics \& Operations Research, Modibbo Adama University, P.M.B. 2076, Yola, Nigeria; \\ umarmodibbo@mautech.edu.ng \\ 4 Department of Commerce, Aligarh Muslim University, Aligarh 202002, India; jchauhan.cm@amu.ac.in \\ 5 Department of Statistics \& Operations Research, Aligarh Muslim University, Aligarh 202002, India \\ * Correspondence: irfii.st@amu.ac.in \\ + Current address: Department of Statistics \& Operations Research, Aligarh Muslim University, \\ Aligarh 202002, India.
}

check for

updates

Citation: Khan, M.F.; Pervez, A.; Modibbo, U.M.; Chauhan, J; Ali, I. Flexible Fuzzy Goal Programming Approach in Optimal Mix of Power Generation for Sustainable Development: A Case Study. Sustainability 2021, 13, 8256. https:// doi.org/10.3390/su13158256

Academic Editor: Idiano D'Adamo

Received: 15 June 2021

Accepted: 18 July 2021

Published: 23 July 2021

Publisher's Note: MDPI stays neutral with regard to jurisdictional claims in published maps and institutional affiliations.

Copyright: (C) 2021 by the authors Licensee MDPI, Basel, Switzerland. This article is an open access article distributed under the terms and conditions of the Creative Commons Attribution (CC BY) license (https:// creativecommons.org/licenses/by/ $4.0 /)$.

\begin{abstract}
The demand for cost-efficient and clean power energy cannot be overemphasised, especially in a developing nation like India. COVID-19 has adversely affected many nations, power sector inclusive, and resiliency is imperative via flexible and sustainable power generation sources. Renewable energy sources are the primary focus of electricity production in the world. This study examined and assessed the optimal cost system of electricity generation for the socio-economic sustainability of India. A sustainable and flexible electricity generation model is developed using the concept of flexible fuzzy goal programming. This study is carried out with the aim of achieving the government's intended nationally determined contribution goals of reducing emission levels, increasing the capacity of renewable sources and the must-run status of hydro and nuclear, and technical and financial parameters. The result shows an optimal cost solution and flexibility in how increased electricity demand would be achieved and sustained via shifting to renewable sources such as solar, wind and hydro.
\end{abstract}

Keywords: renewable energy; sustainable electricity production; socio-economic sustainability; sustainable development goals; emission level; levelized cost; gross domestic product

\section{Introduction}

Environmental sustainability focuses on minimizing the negative environmental impacts of generating electricity based on conventional resources. It can be achieved by increasing production based on renewable energy sources (RES). Therefore, it is composed of several criteria by which power sources have a direct impact on human life, ecological balance, and the environment [1]

The ever-increasing $\mathrm{CO}_{2}$ emissions and the rapid degradation of the environment globally affects environmental sustainability adversely. As a result, policy-makers and researchers are developing interest in, and shifting to, greener manufacturing and the production of electricity via renewable energy sources. Developing countries like India suffer the most from environmental issues due to rapid population growth and lack of adequate resources to harness the potential of RES. Recently, a study was conducted to identify, analyze and rank the predominant barriers restricting India from implementing green manufacturing practices in its small and medium-sized enterprises [2]. The study 
identified 25 barriers and used different multi-criteria decision-making (MCDM) frameworks to analyze and rank the barriers. The study advocated eco-friendly design in the manufacturing system.

As well as the environmental issue, the COVID-19 pandemic poses challenges globally, especially when developing nations are at a higher risk of damage. The pandemic disrupted regular businesses, supply chain networks, production systems, educational systems and, above all, good governance. Recently, the effects of COVID-19 on the e-commerce of European countries in terms of cyber-security have been analyzed using MCDM tools [3]. The countries' sensitivity to cyber-security and e-commerce performance during the pandemic has been identified and ranked. The study suggests digital transformation to policymakers as a framework for a sustainable environment. Similarly, strategies for managing the adverse effects of the COVID-19 pandemic on the educational sector have been evaluated flexibly using MCDM techniques [4].

Several kinds of research have been ongoing regarding the disruption of the supply chain of food and services during the COVID-19 pandemic. Recently, the challenges and opportunities of the food system and circular economy concerning the COVID-19 pandemic have been studied to pave the way for, and aid, policy designers in enacting environmental sustainability policies [5-7]. In all cases, electricity consumption is unavoidable hence the need to devise an optimal mix for the sustainable production of power energy for environmental sustainability development.

In modern times, electricity is among the most important inventions of science for humanity. From home appliances such as fans and toasters, to modern communications and transportation, to the heavy machines used for production in industries, we cannot do without electricity-based technology. India had a population of 1.353 billion people in 2018 alone. It is positioned as the second most populated country globally and the seventh largest economy with a GDP of 2.726 trillion USD in 2018 [8]. However, the electricity consumption per capita was $1122 \mathrm{Kwh}$ in 2017 [9], which is much lower than that of many countries. Electricity shortages are one of South Asia's most significant barriers to achieving development. The power distortion in South Asia causes a four to seven percent lower GDP a year [10]. As of March 2017, Asia's total installed electricity generation capacity, both from utilities and non-utilities, was 377,122 MW and the gross electricity generation was $1,432,358 \mathrm{GWh}$.

The gross import and export of electricity during 2016-2017 was 5617 GWh and $6710 \mathrm{GWh}$, respectively. In 2016-2017, electricity available for supply was $1168,317 \mathrm{GWh}$ in, and the estimated electricity consumption was 1,066,268 GWh [9]. The enhancement of India's power sector would be essential to the growth of its economy. Many studies have shown the association between the electricity consumption and the GDP of a country [11-13]. The importance of electricity is understandable as electricity consumption serves as an indicator for the socio-economic development of countries $[14,15]$. With the growth of an economy, electricity demand also grows [See Figure 1]. Because of the scarce fuel resources available to satisfy the demand, additional optimal capacity must be planned [16].

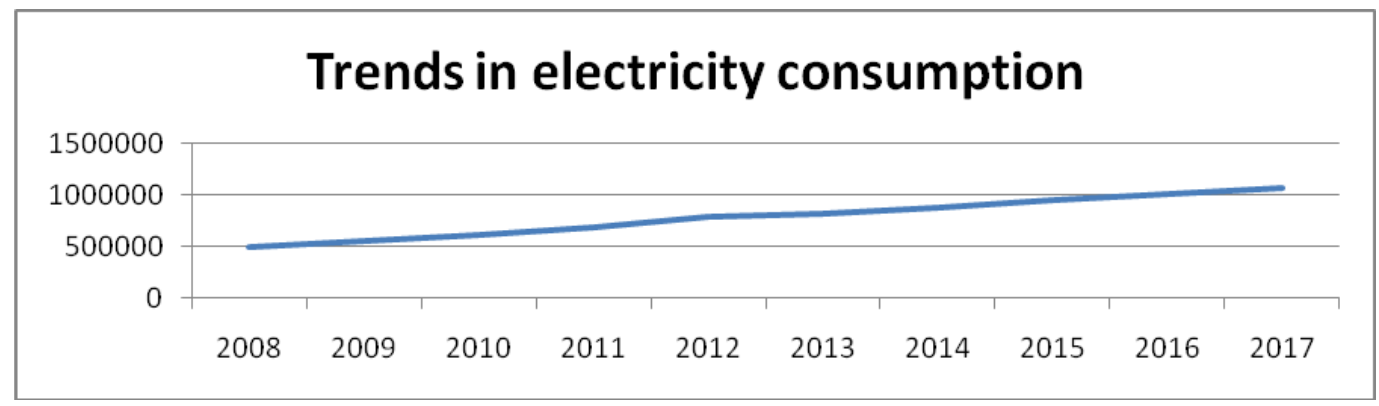

Figure 1. Trends in electricity consumption. source: Energy Statistics 2018, CSO [9]. 
Recently, a two-stage optimization problem was modeled to address hydropower systems and wind parks [17]. The study used mixed-integer linear programming to maximize the system production profits and minimize the imbalances caused by profit reduction penalties. Similarly, wind power production plants have some uncertainties in their production due to the stochastic nature of the operating system during transmission. As such, an optimization model was developed to address the congestions by re-dispatching various cascaded hydropower plants [18]. The study formulated mixed-integer programming to maximize the profit from selling the energy and using a hybrid of quadratic and chance-constrained programming to minimize possible congestions due to re-dispatching the cascaded hydropower plants. More recently, a cooling system with thermodynamic and thermo-economic assessments has been investigated, and energy cost was discovered to be dominant for a single-phase cooling system with a future minimum carbon cost for both systems [19]. Similarly, dynamic mode decomposition has been used to predict the thermal performance of a battery surface [20].

The costs and environmental effects of fossil and nuclear fuels are enormous. Therefore, the need for an optimal mix from various technologies for generating power at a minimum cost cannot be overemphasized. This study tried to assess the cost-optimal additional capacity required by the end of 2021-2022 from conventional and non-conventional energy sources. The study uses flexible fuzzy goal programming to analyze various power generation scenarios for India's sustainable development. This study addresses the UN sustainable development goal 7 (SDG7) related to ensuring affordable, reliable, sustainable and modern energy for all. The goal is interconnected and has synergy with several SDGs such as no poverty (SDG 1), good health and well-being (SDG 3), quality education (SDG 4), clean water and sanitation (SDG 6), decent work and economic growth (SDG 8), sustainable cities and communities (SDG 11), and climate action (SDG 13), among others [21-23]. According to [22], "decisions about SDG7 affect humanity's ability to: realize aspirations of greater welfare and well-being, build physical and social infrastructures for sustainable development, and achieve sustainable management of the natural environment." Therefore, achieving SDG 7 will help to realize socio-economic and environmental sustainability. Some benefits of the techniques employed in this study are discussed briefly in the next section.

\subsection{Benefit of Flexible Fuzzy Goal Programming}

Flexible Fuzzy goal programming is one of the distance-based methods. It is an extension of conventional goal programming. One of the significant advantages of such approaches is their computational efficiency. While dealing with multiobjective optimization problems, flexible fuzzy goal programming allows us to stay within an efficient linear programming computational environment. In this method, each objective's aspiration level is taken as unity, regarding their highest degree of achievement goal. The technique helps to solve multiobjective optimization problems with imprecise parameters in a decision-making environment.

Additionally, the approach uses tolerance values, making it more flexible for decisionmakers to realize the range of the solutions they can operate within. In this approach, instead of measuring the achievement of fuzzy objective values directly, achieving membership values of objectives to the highest degree (unity) by minimizing under-deviations is taken into account in a solution search process. All these are incorporated in this research.

\subsection{Paper Organization}

The paper is organized as follows: Section 1 introduces the study background and presents some benefits of the technique used in the study. In Section 2, the relevant literature in the subject area is reviewed, and the research gap established. Section 3 discusses the general multiobjective optimization model followed by the flexible goal programming technique, which the study uses for modeling and solving the problem. The stepwise procedure of the solution method is presented as well. Models related to the levelized cost 
of energy and its components, such as capital costs, operational costs, and fuel costs, are presented and discussed. Section 4 briefly discusses the sustainable development goals (SDGs) and identifies the SDG 7-goal related to energies and emissions that are crucial to environmental sustainability in the Indian context, based on which the study is carried out. In this section, the Indian Intended Nationally Determined Contribution (INDC), the current scenario of the Indian power sector, the installed capacity of power generation, the Indian electricity generation and consumption, its import and export, and the renewable energy scenarios are all discussed. This section further discusses the state-wise scenario of the power sector and the projections of electricity demand. Section 5 presents the modeling of electrical energy production. The necessary parameters and the system constraints, such as capacity additional targets, renewable energy sources, reserve margin, emissions limits, current energy mixed, and so forth, are discussed. Section 6 presents the results, analysis and discussions, and the article is concluded in Section 7 with research implications and recommendations for decision-makers to implement the findings.

\section{Literature Review}

Over the years, researchers have been engaged in optimization, studying and investigating, proposing new methodologies and strategies for finding alternative solutions to the existing and newly emerging problems of human endeavors, for the betterment of the universe. Studies related to the sustainability and development agendas, with respect to India, have been intensive and ongoing. For instance, Rathi [24] and Ghose [25] studied how to promote clean production in the industrial set up of India; Mukherjee [26] considered barriers to the use of energy and the control of pollution with the aim of preserving the environment by the use of cleaner production technologies. The study discovered inconsistency in technology parameters and that they were not reliable for optimizing the eco-friendly casting production problem. Pal et al. [27] studied the process of a device for effectively controlling pollution, developed by the SDC-TERI partnership in India, and discussed measures to replicate and improve the technology for energy efficiency. Narayanaswamy and Scott [28] discussed the lessons derived from cleaner production in textile industries, related to urban and rural environmental interdependency in India.

Unnikrishnan and Hedge [29] analyzed industrial training imparted with the goal of cleaner production. Affordability for cleaner water production was studied by Annala et al. [30], where they used "Reverse Osmosis (RO)" technology to investigate the low cost of water filters in Indian households. The study found that there is active participation in the frugal innovation process by the citizens. Nomani et al. [31] analyzed Indian vision 2030 using the concept of FFGP. Khatun and Ahamad [32] discussed the energy situation in Bangaladesh and analyzed the gap between energy production and usage over 38 years, linking it to the economic growth of the country. McCollum et al. [33] extensively reviewed the linkage between energy and its counterparts related to SDGs.

Similarly, Hassan and Garg [34] studied a system approach for water resource development. Robust optimization techniques have recently been used in sustainability studies [35,36]. Multi-criteria goal programming was used by Gupta et al. [1,37] to analyze the SDGs of India. Recently, a critical review of the application of optimization techniques to the United Nation's SDGs has been conducted [38]. Globally, researchers and decision-makers tend to investigate and proffer solutions to the problem of environmental sustainability [39]. For instance, Yang et al. [40] investigated factors influencing urban sustainability in Beijing and Shanghai in China, and found that service industries have the most substantial consumption of energy and water and $\mathrm{CO}_{2}$ emissions.

Other similar environmental and electricity regulations have been studied and documented in [39-47]. Recently, green energy has been used for providing sustainable economic growth [48,49]. A compact summary of the related work concerning energy studies and environmental sustainability is shown in Table 1 . The next section discusses the methodology of this research. 
Table 1. A summary of closely related literature and the present work.

\begin{tabular}{|c|c|c|c|}
\hline Authors & Optimization Type & Description & Solving Method \\
\hline Ali et al. [1] & Multiobjective & modeling India's SDGs & Classical Goal Programming \\
\hline Karuppiah et al. [2] & - & Ranked barriers to implementing greener manufacturing in India & Fuzzy MCDM (DEMATEL, ANP, TOPSIS) \\
\hline DAdamo et al. [3]. & - & Ranking e-commerce in European countries amidst pandemic & MCDA and a Likert scale survey \\
\hline Giudice et al. [5]. & - & Cause-effect analysis of COVID-19 on food security & theme popularity metric. \\
\hline Mahmud et al. [7] & - & $\begin{array}{c}\text { Evaluating Supply Chain Collaboration Barriers in Small-and } \\
\text { Medium-Sized Enterprises. }\end{array}$ & MCDM (Grey DEMATEL and Fuzzy Best-Worst methods ) \\
\hline Zhang et al. [15] & - & $\begin{array}{l}\text { Study of the relationship between electricity access and } \\
\text { social-economic factors }\end{array}$ & Bayesian Model Averaging \\
\hline Knežević et al. [17] & Biobjective & modeling hydroelectric system and wind parks & Mixed integer linear programming \\
\hline Fekete et al. [18] & Biobjective & $\begin{array}{c}\text { Addressing congestion problem in the transmission network of } \\
\text { hydropower plants }\end{array}$ & $\begin{array}{l}\text { Mixed integer linear programming, Quadratic and } \\
\text { Chance-constrained programming }\end{array}$ \\
\hline Mukherjee [26] & - & $\begin{array}{c}\text { Evaluation of operational performances of cupola and pollution } \\
\text { Control system for optimizing energy use }\end{array}$ & Descriptive statistics and Factor analysis \\
\hline Nomani et al. [31] & Multiobjective & Analysis of the sustainable development goals of India & Fuzzy goal programming \\
\hline $\begin{array}{l}\text { Khatun and } \\
\text { Ahamad [32] }\end{array}$ & - & $\begin{array}{l}\text { Examination of the causal relationship between FDI in the energy } \\
\text { and power sector, and economic growth in Bangladesh }\end{array}$ & Empirical study \\
\hline McCollum et al. [33] & - & Study of the interconnectivity of the UN SDGs & Descriptive statistics \\
\hline Modibbo et al. [38] & Multiobjective & modeling and analysis of Nigeria's SDGs & AHP, Fuzzy goal programming \\
\hline AlArjani et al. [42] & Multiobjective & A framework for SDGs in Saudi Arabia & Fuzzy goal programming \\
\hline Yang et al. [40] & - & $\begin{array}{l}\text { Investigating energy-water-carbon nexus of urban sectors in } \\
\text { Shanghai and Beijing. }\end{array}$ & Environmental input-output model. \\
\hline Wang et al. [43] & - & $\begin{array}{l}\text { Evaluation of the relationship between environmental regulation } \\
\text { and eco-efficiency }\end{array}$ & De-linking and re-linking tool \\
\hline Yabar et al. [45] & - & $\begin{array}{c}\text { Study on the impact of environmental policy on technological } \\
\text { innovation }\end{array}$ & Patent data analysis \\
\hline Curtis and Lee [46] & - & $\begin{array}{l}\text { Study of Onsite industrial electricity generation, energy efficiency } \\
\text { and policy instruments }\end{array}$ & Survey \\
\hline English et al. [47] & - & $\begin{array}{c}\text { Examining balancing requirements in a decarbonizing electricity } \\
\text { system. }\end{array}$ & Capacity expansion and dispatch model \\
\hline D'Adamo et al. [48] & - & $\begin{array}{c}\text { An economic assessment of a } 3 \mathrm{~kW} \text { plant in the context of several } \\
\text { policy scenarios during a pandemic }\end{array}$ & Descriptive statistics \\
\hline Hondo [50] & - & $\begin{array}{c}\text { A life cycle analysis of greenhouse gas emissions from power } \\
\text { generation systems }\end{array}$ & Framework and descriptive statistics \\
\hline Present work & Multiobjective & Optimal mix of various technology for electricity generation & Flexible fuzzy goal programming \\
\hline
\end{tabular}




\section{Methodology}

In this section, the techniques used for modeling the optimization problem and calculating the levelized cost of energy are discussed and presented. First, a general multiobjective optimization problem is discussed, followed by the specific techniques employed in the study.

\subsection{Multiobjective Optimization Model}

A problem is said to exist if there is a discrepancy between what is and what should be in a real-life situation. Optimization, in simple terms, is finding the best possible desired result(s) out of many available solutions. In an optimization problem, the objective could be single or multiple. A multi-objective problem has more than one objective or goal that is desired to be achieved. It can be a linear or nonlinear function(s) with some constraints or limitations, which can also be linear or nonlinear. For instance, the problem can be about minimizing a certain quantity (say cost) or maximizing a particular value (say profit), or a combination of both. An optimal solution is possible in a single objective optimization depending on the nature of the problem; however, if there is more than one objective, it is a multi-objective optimization problem (MOOP). Naturally, in MOOP, it is impossible to obtain an optimal solution for all the objectives since they could be conflicting. Therefore, a Pareto or a compromise solution is possible. There are different types of models and solutions obtainable in MOOP. The MOOP can be linear or nonlinear depending on the problem's nature and constraints. However, the general MOOP model is presented below:

Let a multiobjective programming problem (MOPP) with $j$ objectives functions be given as:

$$
\begin{aligned}
& \text { Optimize }\left(Z_{1}(X), Z_{2}(X), \ldots Z_{j}(X)\right) \\
& \text { subject to; } \\
& g_{i}(x)(\leq,=, \geq) b_{i}, \quad i=1,2, \ldots, m ; x \geq 0,
\end{aligned}
$$

where $Z_{j}$ is the set of objectives, $g_{i}(x)(\leq,=, \geq) b_{i}$ are $m$ sets of constraints for which $b_{i}$ is the $i$ th resource. Many techniques and approaches exist for solving the MOPP model Equation (1), one of which is flexible goal programming.

\subsection{Flexible Fuzzy Goal Programming with Tolerance Functions}

Fuzzy set theory is a concept to which flexible fuzzy goal programming (FFGP) applies. Fuzzy sets describe the imprecise goals of a decision-maker. These goals are flexible and can be associated with an objective function or constraints. They can reflect a weighting with a value from zero to one or a range of "goal achievement" possibilities. An FFGP allows decision-makers who cannot define goals in a precise manner to express them in a weighting structure, which is not limited. The decision is generally made under four different environments with various conditions. The decision-making takes place in an environment where the DM either has ultimately no knowledge about the environment (ignorance), has complete knowledge (certainty), has little or no knowledge (uncertainty), but can be assigned probabilities and environments in which the DM is competing with the state of nature. Some decisions are simple, while others are very complicated. It is simple when there is precision of the boundaries in the environment, while it is very complicated when the environment is full of uncertainties and vagueness. Fuzzy set theory with imprecise boundaries, developed by Zadeh [51], can handle such vagueness and uncertainty. Zimmermann [52] proposed a fuzzy programming concept for solving multiobjective DM problems, in which both the objectives and the constraints of the problem are considered to be a fuzzy set, a characteristic function (membership) in that set assigns some grades (real values) of membership between one and zero to each of the objectives or goals of the DM. A generalized model for this type of problem (FFGP) can be stated as:

Find

$$
X=\left(x_{1}, x_{2}, \ldots, x_{n}\right)^{T},
$$


such that

$$
\begin{aligned}
& Z_{k}(X)(\succeq, \simeq, \preceq) g_{k}, \quad k=1,2,3, \ldots, K . \\
& A X \leq b_{i}, \quad i=1,2, \ldots, m \\
& X \geq 0,
\end{aligned}
$$

where $g_{k}$ is the various anticipated future goals, $b_{i}$ is the vector of available resources at hand, and $A$ is the technological coefficient. The symbol $\succeq$ is the fuzzy-max type, meaning that $Z_{k}(X)$ should be approximately more than or exactly the same as the level of aspiration $\mathrm{g}_{k}$; this implies that it can be satisfied by the DM even if it is less than $\mathrm{g}_{k}$ at a certain level. The symbol $\preceq$ stands for fuzzy-min, meaning that $Z_{k}(X)$ should be less than or exactly the same as the level of aspiration $g_{k}$ approximately, up to an allowable limit (tolerance), while the symbol $\simeq$ stands for fuzzy-equal and implies that $Z_{k}(X)$ should be within the level of aspiration $g_{k}$, which means that it can be satisfied by the DM even if it is less than or greater than $g_{k}$ to a certain level of tolerance. The k-th fuzzy objective is denoted by $Z_{k}$, and the $\mathrm{n}$-dimensional vector for decision variables is represented by $X$.

For multi-objective fuzzy goal programming, let $\mathrm{g}_{k}$ be the aspiration level set by DM for the k-th objective value $Z_{k}(X)$. Thus, using the method developed by Zimmermann [52], for a maximization problem fo the fuzzy-goal type $Z_{k}(X) \succeq \mathrm{g}_{k}$, the membership function for fuzzy-max goals is given as: “

$$
\lambda_{k}\left(Z_{k}(X)\right)= \begin{cases}1, & \text { if } Z_{k}(X) \geq \mathrm{g}_{k} \\ \frac{Z_{k}(X)-L_{k}}{\mathrm{~g}_{k}-L_{k}}, & \text { if } L_{k} \leq Z_{k}(X) \leq \mathrm{g}_{k} \\ 0, & \text { if } Z_{k}(X) \leq L_{k} .\end{cases}
$$

While the constraint of a fuzzy model is a subset of vector $X$ with a membership characteristic function $\lambda_{a_{i j}}\left(x_{j}\right): x \longrightarrow[0,1]$, given by

$$
\lambda_{\Sigma a_{i j} x_{j} \lesssim \curvearrowright b_{i}}=\left\{\begin{array}{l}
1, \text { if } \sum_{j=1}^{n} a_{i j} x_{j}=b_{i}, i=1,2, \ldots, m \\
\frac{\sum_{j=1}^{n} a_{i j} x_{j}-b_{i}+T * b_{i}}{T * b_{i}}, \\
\text { if } b_{i}-T * b_{i}<\sum_{j=1}^{n} a_{i j} x_{j} \leq b_{i}, i=1,2, \ldots, m \\
\frac{b_{i}+T * b_{i}-\sum_{j=1}^{n} a_{i j} x_{j}}{T * b_{i}}, \\
\text { if } b_{i}<\sum_{j=1}^{n} a_{i j} x_{j} \leq b_{i}+T * b_{i}, i=1,2, \ldots, m \\
0, \quad \text { Otherwise. }
\end{array}\right.
$$

The Flexible Fuzzy Goal Programming Model can be written as:

Find $x \in X$

such that it will Maximize $\lambda$

subject to : 


$$
\begin{cases}\lambda \leq \frac{Z_{k}(x)-L_{k}}{\mathrm{~g}_{k}-L_{k}}, & \text { if } Z_{k}(x) \gtrsim \mathrm{g}_{k} \\ \lambda \leq \frac{U_{k}-Z_{k}(x)}{U_{k}-g_{k}}, & \text { if } Z_{k}(x) \lesssim g_{k} \\ \lambda \leq \frac{\left(b_{i}+T * b_{i}\right)-\sum_{i=1}^{m} a_{i j}\left(x_{j}\right)}{T * b_{i}}, & \text { if } \sum_{i=1}^{m} a_{i j}\left(x_{j}\right) \succeq b_{i} \\ \lambda \leq \frac{\left(\sum_{i=1}^{m} a_{i j}\left(x_{j}\right)-\left(b_{i}+T * b_{i}\right)\right.}{T * b_{i}}, & \text { if } \sum_{i=1}^{m} a_{i j}\left(x_{j}\right) \preceq b_{i} \\ x_{j} \geq 0, & j=1,2, \ldots, n \\ \lambda \geq 0, & \end{cases}
$$

where $T$ is the tolerance interval."

\subsection{Stepwise Solution Procedure for MOPP}

We define four linear functions in our study as a multi-objective optimization problem and the following step-wise algorithms are employed for solving the model.

Step 1: Formulate the problem at hand as a multi-objective mathematical model.

Step 2: Obtain the individual optimal solution of the model using any available package, considering one objective at a time.

Step 3: Formulate a pair-wise comparison matrix using the solutions in Step 2, given as:

$$
\left[\begin{array}{cccc}
Z_{1}^{*}\left(x^{1}\right) & Z_{2}\left(x^{1}\right) & \cdots & Z_{j}\left(x^{1}\right) \\
Z_{1}\left(x^{2}\right) & Z_{2}^{*}\left(x^{2}\right) & \cdots & Z_{j}\left(x^{2}\right) \\
\vdots & \vdots & \ddots & \vdots \\
Z_{1}\left(x^{j}\right) & Z_{2}\left(x^{j}\right) & \cdots & Z_{j}^{*}\left(x^{j}\right) .
\end{array}\right]
$$

Step 4: Identify the lowest and highest value of each column in Step 3 obtained from Step 2 above and set them as a lower and upper goal, respectively.

Step 5: Construct the membership functions using the FFGP models in Equation (5).

Step 6: Construct a function that will maximize the overall linear additive model of the auxiliary variables defined from the membership function in Step 5 above.

Step 7: Solve for the function in Step 6 using a suitable optimization package and obtain the goal achievement value.

\section{4. levelized Cost of Energy}

The "Levelized Cost" of electricity generation can be defined as "the ratio of the net present value of total capital cost and the total operating cost of a particular plant to the net present value of the net electricity generated by that plant over its operating life" [53,54]. It has not been a reasonable way to quantify the cost economics by simply comparing the electricity generating cost of various RES, such as wind or solar, with that of "conventional sources" such as coal, nuclear or natural gas. Present Net Value (NPV) is one of the critical parameters used to judge the financial viability of the technology; it is the current investment value considering the cost of capital, fuel, as well as other operating and maintenance costs. The LCOE model is given in Equation (6).

$$
L C O E=\frac{\sum \frac{I_{t}+(O M)_{t}+F_{t}}{(1+r)^{t}}}{\sum \frac{E_{t}}{(1+r)^{t}}},
$$


where LCOE = levelized cost of energy, $I_{t}=$ initial investment, $(O M)_{t}=$ operating and maintenance charge, $F_{t}=$ fuel cost $\mathrm{r}=$ discount rate, $E_{t}=$ system energy yield, and $\mathrm{t}=$ year .

The NPV is calculated by determining the annual cash flows from the investment and discounting them to the present time with a specific discount rate. Thus, to compare the costs of generating technologies, the total costs and the load factor for each technology are first considered, and the net present value analysis is then performed. That is the only logical way to evaluate power generation technologies.

\subsubsection{Component of levelized Costs}

The levelized cost of energy helps with the economic assessment and comparison of different power generation technologies with unequal plant life, capital costs, capacity factor, and fuel costs. Different methods of power generation incur different costs, which include the initial capital cost, operation and maintenance cost and fuel cost (see Table 2).

Table 2. Parameters used for the study.

\begin{tabular}{|c|c|c|c|c|c|c|c|c|c|}
\hline Technology & Hydro & Coal & Gas & Nuclear & $\begin{array}{c}\text { Solar } \\
\text { Thermal }\end{array}$ & Solar PV & Wind & Biomass & $\begin{array}{l}\text { Small } \\
\text { Hydro }\end{array}$ \\
\hline $\begin{array}{c}\text { Capital } \\
\text { Cost } \\
\text { (Lakh/MW) }\end{array}$ & 667.38 & 700.27 & 523 & 1600 & 1200 & 530 & 575 & 605.6 & 846.5 \\
\hline $\begin{array}{l}\text { Operation } \\
\text { \& maint. } \\
\text { (Lakh/MW) }\end{array}$ & 27.44 & 20.43 & 28.61 & 32 & 16.8 & 7.42 & 10 & 40 & 29.86 \\
\hline $\begin{array}{l}\text { Fuel Price } \\
\text { (Rs) } \\
\text { Specific }\end{array}$ & 0 & 3 & 5.71 & 4228 & 0 & 0 & 0 & 3.2 & 0 \\
\hline $\begin{array}{l}\text { Fuel con- } \\
\text { sumption } \\
\text { (Kg/Kwh) }\end{array}$ & 0 & 0.627 & 0.46 & 0.00025 & 0 & 0 & 0 & 1.25 & 0 \\
\hline $\begin{array}{c}\text { Fuel cost } \\
(\mathrm{Lakh} / \mathrm{MW})\end{array}$ & 0 & 98.86 & 69.02 & 62.03 & 0 & 0 & 0 & 245.3 & 0 \\
\hline $\begin{array}{l}\text { Capacity } \\
\text { factor }\end{array}$ & 35 & 60 & 30 & 67 & 23 & 19 & 29 & 70 & 45 \\
\hline $\begin{array}{l}\text { Auxiliary } \\
\text { consump- } \\
\text { tion }\end{array}$ & 1.2 & 5.25 & 5.25 & 7.8 & 1 & 1 & 0.5 & 10 & 1 \\
\hline Plant life & 40 & 25 & 25 & 40 & 25 & 25 & 25 & 20 & 35 \\
\hline
\end{tabular}

\subsubsection{Capital Costs}

Capital costs are the overnight construction costs, including mechanical equipment supply and installation, civil and structural costs, project indirect costs, electrical and instrumentation and control and owners costs [53]. They also include waste disposal and decommissioning costs in the case of nuclear power plants [54]. These costs are lower for gas, wind and solar PV and higher for coal, solar thermal and nuclear.

\subsubsection{Operation and Maintenance Cost}

Power plants' operating costs include labor and maintenance costs. Unlike the capital costs, the operation and maintenance costs of the plant can vary with the electricity produced. It is low for solar PV, solar thermal and wind, and high for biomass plants.

\subsubsection{Fuel Costs}

These costs are high for coal, gas and biomass plants, low for nuclear power plants, and zero for many renewable energy sources. Fuel costs can vary arbitrarily over the life of the plants, due to political and other factors such as inflation; therefore, for the present study fuel cost was inflated at $5.72 \%$ on a year on year basis.

To calculate the overall cost of the production of electricity from different technologies, various streams of costs are discounted by a discounting factor to net present value. Similarly, yearly energy units produced by different methods are discounted back to 
net present value. In this study, a discount factor of 10 percent has been used to calculate the net present value of streams of cash outflows and energy units produced. Capital costs, operating and maintenance and fuel costs are applied in Lakh per megawatt installed capacity while electrical energy produced is applied in million $\mathrm{U}$.

\section{Sustainable Development Goals}

Member states of the United Nations (UN) adopted the seventeen Sustainable Development Goals (SDGs) in 2015. The SDGs serve as a global action against poverty, hunger, AIDS and discrimination, and to ensure that all people enjoy peace and prosperity by 2030 for balanced and sustainable social, economic and environmental development [55]. The SDG 7 agenda is determined to ensure the affordability, reliability, and sustainability of energy for the benefit of all. Secure energy access is linked with various social and economic development goals such as alleviating poverty, education, health, improving industrialisation, providing infrastructure for communication, and mitigating climate changes. In India, NITI Aayog is saddled with the responsibility of ensuring the SDG implementation, while the "Ministry of Statistics and Programme Implementation (MoSPI) is evolving the related national indicators" (Economic survey, 2017).

\subsection{Intended Nationally Determined Contribution (INDC)}

India endorsed the Copenhagen Accord in 2010 and intended to reduce $\mathrm{CO}_{2}$ intensity by at least 20 to 25 percent of that of 2005 levels by 2020. In October 2015, with the view of eradicating poverty and adopting low carbon clean technologies, India also submitted its "Intended Nationally Determined Contribution" (INDC) to UNFCCC (Government of India 2016). The actual contents of the reports include:

i. reduce the emissions intensity of its GDP by 33 to 35 percent by 2030 from 2005 levels.

ii. achieve about 40 percent cumulative electric power installed capacity from non-fossil fuel-based energy resources by 2030, with the help of the transfer of technology and low-cost international finance including from the Green Climate Fund (GCF).

iii. create an additional carbon sink of 2.5 to 3 billion tonnes of $\mathrm{CO}_{2}$ equivalent through additional forest and tree cover by 2030.

Therefore, GOI needed to enrich the existing policies and intended to introduce more efficient and cleaner technologies, promote renewable energy, reduce carbon emissions from different sources, promote energy efficiency in the economy, develop resilient climate infrastructure, Implement programmes of afforestation, enhance climate resilience and reduce vulnerability to climate change.

\subsection{Current Scenario of the Indian Power Sector}

In India, electricity generation, transmission, distribution and trading are currently governed by the Electricity Act of 2003. The act promotes the development of the power industry by promoting and encouraging competition, protecting consumers' interests, ensuring electricity supply, electricity bill rationalisation, transparent subsidies policies, and promoting efficient electricity policies. The Central Electricity Authority (CEA) advises the government on policy matters regarding the country's electricity system. It has a constitution under section 3(1) of the "Electricity Supply Act 1948", which was superseded by section 70(1) of the "Electricity Act 2003". The central electricity regulatory commission is a statutory body with "quasi-judicial status," functioning under section 76 of the Electricity Act 2003 for rationalisation of the electricity tariff. Many state electricity regulatory commissions are also working for the development of power sector in the respective states. The Appellate Tribunal for Electricity APTEL was established in 2005 to appeal against the orders of the arbitrating officer or central and state electricity regulatory commissions under the Electricity Act 2003. In collaboration with the states, CEA (the 'Central Electricity Regulatory Commission') and other stakeholders, the Government of India (GOI) issued a revised tariff policy in 2016. The electricity is generated from conventional sources of energy such as coal and lignite, hydro, nuclear and natural gas power generation as well as 
from non-conventional RES such as solar, wind, biomass, small hydro, tidal, geothermal, waste to energy and hydrogen/ fuel cells, among others. The CEA, the Ministry of Power and the GOI consider the principles of sustainable development in the power sector and the development of generation capacity to meet the demand pattern, varying demand, efficient use of resources, availability of fuel and integration of Non-Dispatchable Renewable Energy Sources (NDRES) like wind and solar, during the planning process for electricity generation capacity addition [56].

\subsubsection{Installed Capacity of Power Generation}

Figure 2 depicts the electricity installed capacity in the country as of March 31, 2017. India, the third-largest electricity producer and consumer in the world [57], has installed a generation capacity of $377,122 \mathrm{MW}$, including $326,833 \mathrm{MW}$ in utilities and 50,289 MW in non-utilities. In $326,833 \mathrm{MW}$ of the total installed capacity from utilities, thermal accounted for 218,330 MW (66.80\%), followed by RES with 57,244 MW (17.51\%), nuclear $6780 \mathrm{MW}$ $(2.07 \%)$ and hydro $44,478 \mathrm{MW}(13.60 \%)$, as of March 2017. The generation capacity of power from utilities in India increased from 143,061 MW in 2008 to 326,833 MW in 2017 (8.61\% CAGR), as shown in Figure 3.

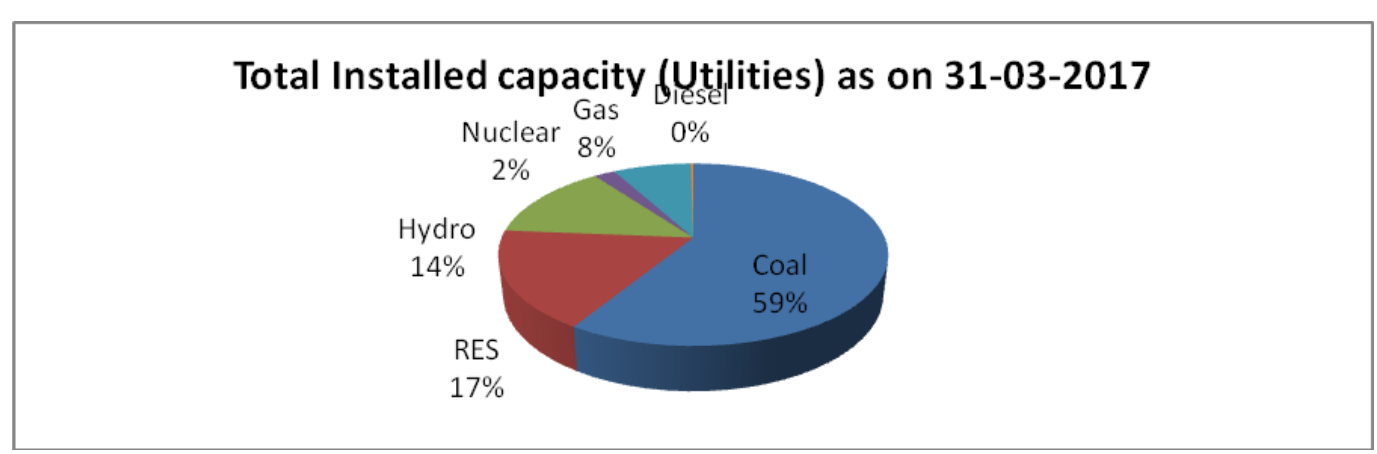

Figure 2. Total installed capacity of electricity from utilities. source: Energy Statistics 2018, CSO [9].

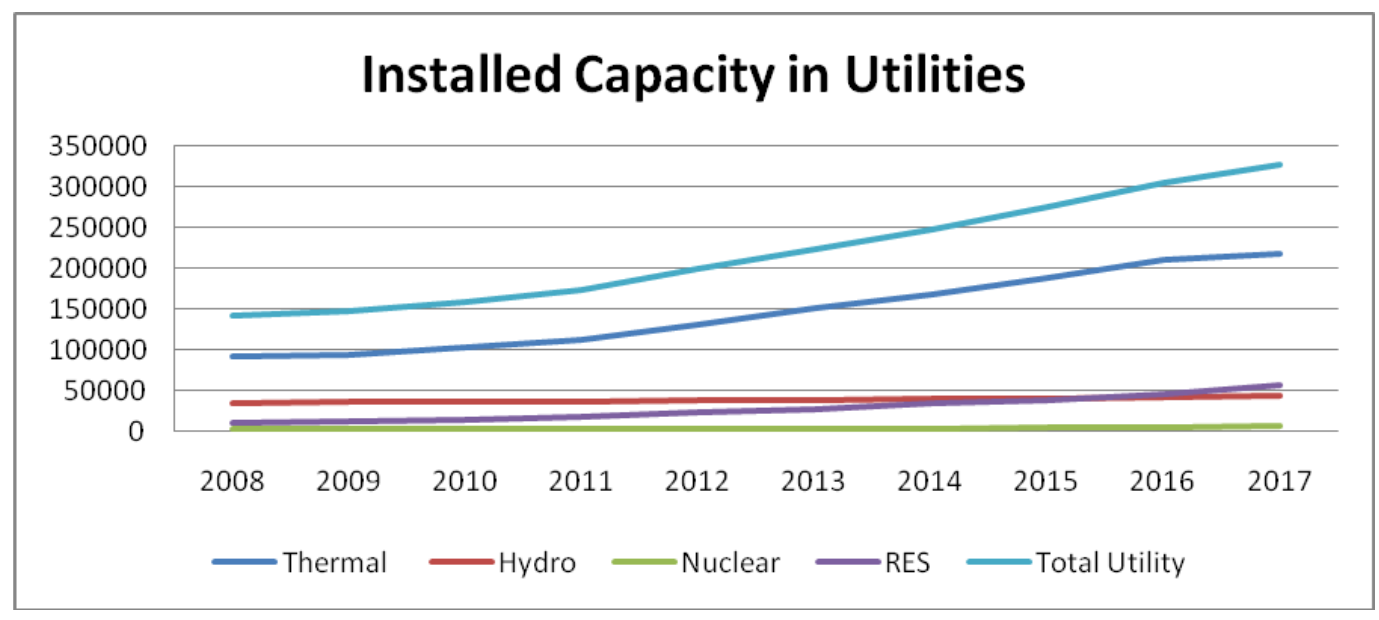

Figure 3. Installed capacity in utilities, from 2008 to 2017. source: Energy Statistics 2018, CSO [9].

\subsubsection{Generation of Electricity}

The gross generation of electricity from utilities was 1,235,358 GWh during 20162017, in which 993,516 GWh was generated from thermal, 122,378 GWh from hydro and $37,916 \mathrm{GWh}$ from nuclear, respectively (Figure 4). The non-utilities total output was 197,000 GWh. It rose from 722,625 GWh during 2007-2008 to 1,235,358 GWh during 2016-2017. 


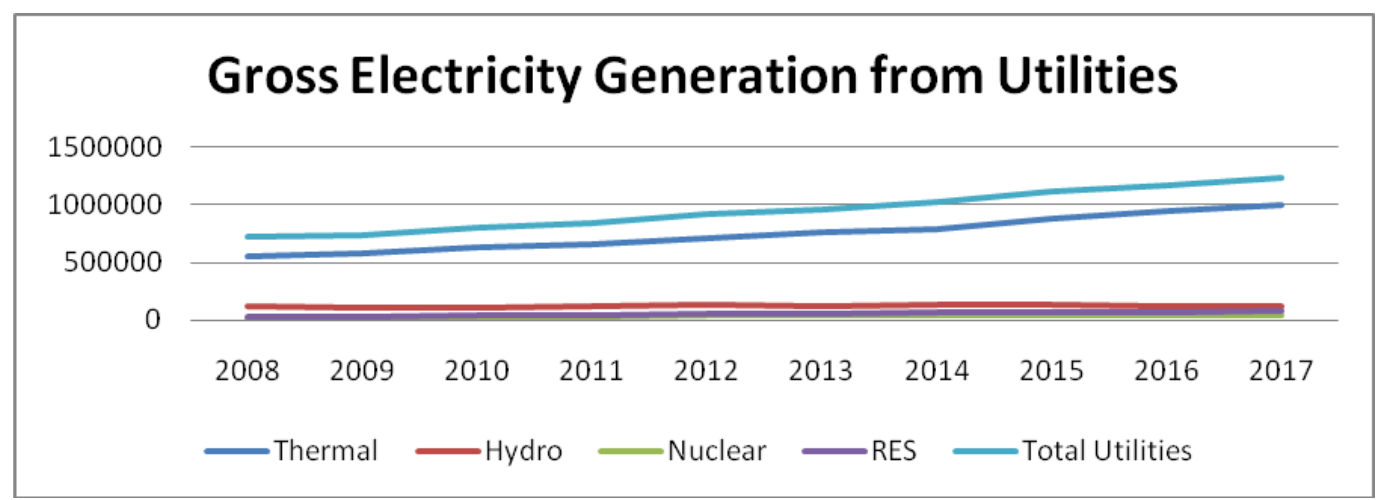

Figure 4. Gross electricity generation from utilities from 2008 to 2017. source: Energy Statistics 2018, $\mathrm{CSO}[9]$.

\subsubsection{Import and Export of Electricity}

India is gradually becoming an electricity exporting country. The gross importation of electricity decreased from 5897 GWh in 2008-2009 to 5617 GWh in 2016-2017. Similarly, the exportation of electricity has increased from 58 GWh in 2008-2009 to 6710 GWh in 2016-2017 [9] as depicted in Figure 5. India exports electricity to Bangladesh, Nepal and Myanmar, while Bhutan is the only power supplier to India. This trade of electricity takes place under bilateral Memorandum of Understandings and power Trade Agreements. The Ministry of Power issued import/export (Cross Border) guidelines for electricity in 2018 [58]. After adding the net import and purchase of electricity from non-utilities, the electricity available for supply in 2016-2017 was 1,168,317 GWh, while the loss of electricity due to transmission was $21.30 \%$, a much higher loss rate than other countries.

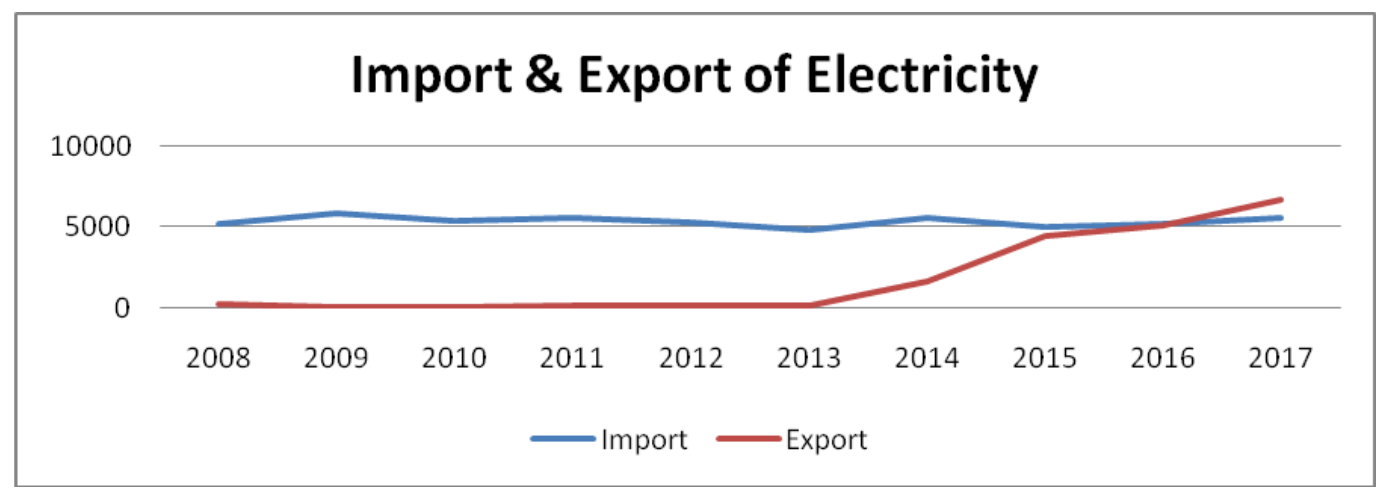

Figure 5. Import and export of electricity from 2008 to 2017. Data source: Energy Statistics 2018, $\mathrm{CSO}[9]$.

\subsubsection{Consumption of Electricity}

According to the report of [58,59], India has become the third-largest producer as well as consumer of electricity in the world. In 2016-2017, the estimated electricity consumption was 1,066,268 GWh. However, the average consumption of electricity per capita in India was only $1122 \mathrm{Kwh}$ in 2017 [9], which is much lower than that of the world's average per capita electricity consumption (see Figure 6). Despite having a lower tariff, the per capita electricity consumption of India is much lower compared to many other countries. Of India's electricity consumption, industry consumed 40.01 percent of the total, followed by the domestic sector which consumed 24.32 percent, agriculture with a consumption of 18.33 percent and the commercial sectors with 9.22 percent, respectively as shown in Figure 7. 


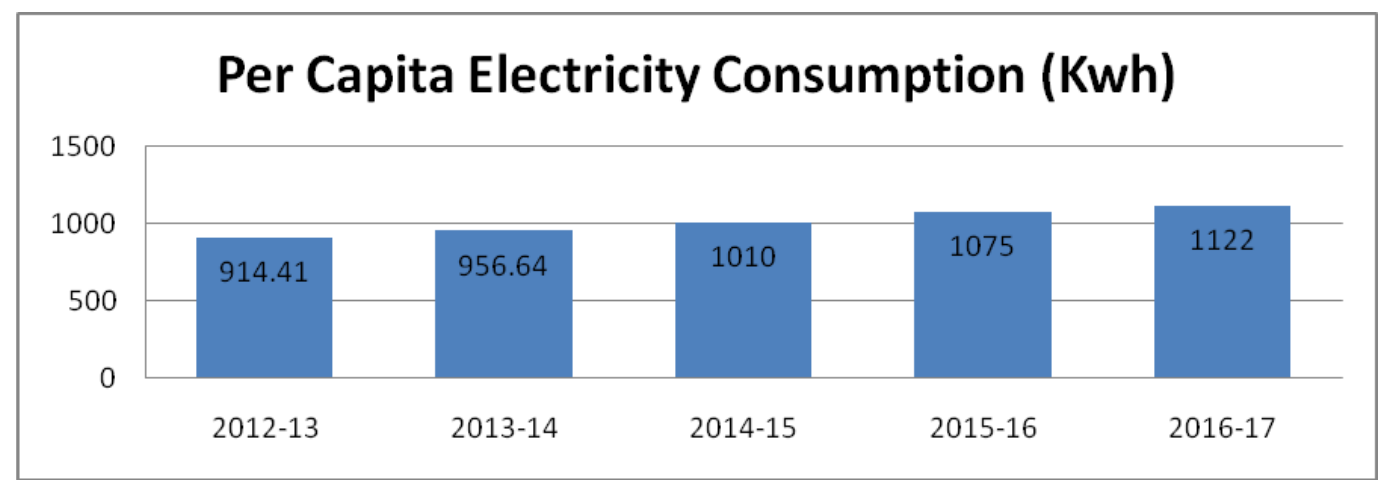

Figure 6. Per capita electricity consumption. Data source: National Electricity Plan, CEA [16].

\section{Others Sector-wise power consumption}

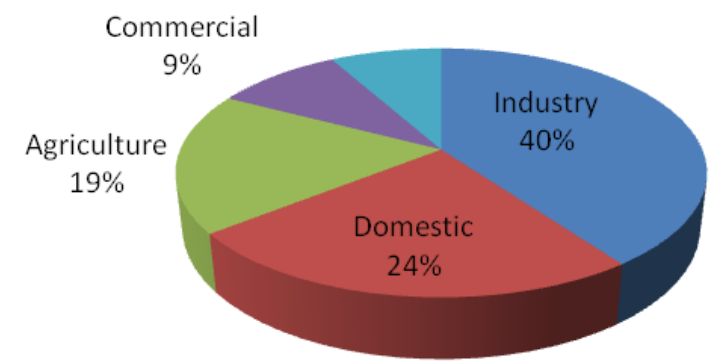

Figure 7. Sector-wise power consumption. Data source: Energy Statistics 2018, CSO [9].

\subsubsection{Renewable Energy in India}

India is among the countries with the most significant production of energy from renewable sources. Including hydro, as of March 2017, renewable energy accounts for 31.12 percent of the total installed capacity from the utility in the country, while renewable energy sources other than hydro accounted for 57,244 MW, which is 17.51 percent of the total installed capacity. Wind power capacity was 32,280 MW, solar accounted for 12,288.83 MW, biopower accounted for 8295.78, and the small hydro plant has a capacity of $4379.86 \mathrm{MW}$ (see Figure 8). A total of $175 \mathrm{GW}$ has been targeted by the Government of India for installed capacity from RES by March 2022. The additional capacity anticipated from RES during 2022-2027 has been considered to be 100,000 MW, of which 50,000 MW will be from solar, 40,000 MW from wind, $7000 \mathrm{MW}$ from biomass and $3000 \mathrm{MW}$ from small hydro, respectively, to reach a target of 175 GW RES by 2021-2022 and 275 MW by 2027. There is a huge potential for power generation in India from RES. The total potential for generating renewable power in India in 2017 was projected to be 1,001,132 MW. These include 649,342 MW from solar, 302,251 MW from wind, 21,134 MW from small-hydro, 18,601 MW from biomass, 7260 MW from "bagasse-based" cogeneration in sugar mills and $2554 \mathrm{MW}$ from waste to energy. The detailed estimated potential for renewable energy in India is presented in Section 4.3.

\subsection{State-Wise Scenario of the Power Sector}

The installed state-wise and region-wise capacity of power generation as of 31st March 2017 and the per capita power consumption during 2016-2017 have been reported. Furthermore, the state-wise and region-wise projected energy requirement and peak demand for 2021-2022 and 2026-2027, and the estimated potential of renewable power, were also investigated. Dadra and Nagar Haveli have the highest electricity consumption per capita with 15,783 Kwh, while Bihar has the lowest consumption in the country, with 272 Kwh for 
2016-2017. In India, the per capita power consumption was 1122 Kwh in 2017, far lower than many developed countries. Maharashtra has the highest projected energy requirement and peak demand for 2021-2022 and 2026-2027, followed by Uttar Pradesh, Tamil Nadu and Gujarat. Rajasthan has the highest share of the estimated potential of renewable power generation, at about 16.21 percent (i.e.,162,326 MW), followed by Gujarat with 12.17 percent (i.e., 121,791 MW) and Jammu and Kashmir with 11.27 percent (i.e., 112,800 MW), mainly from solar power potential.

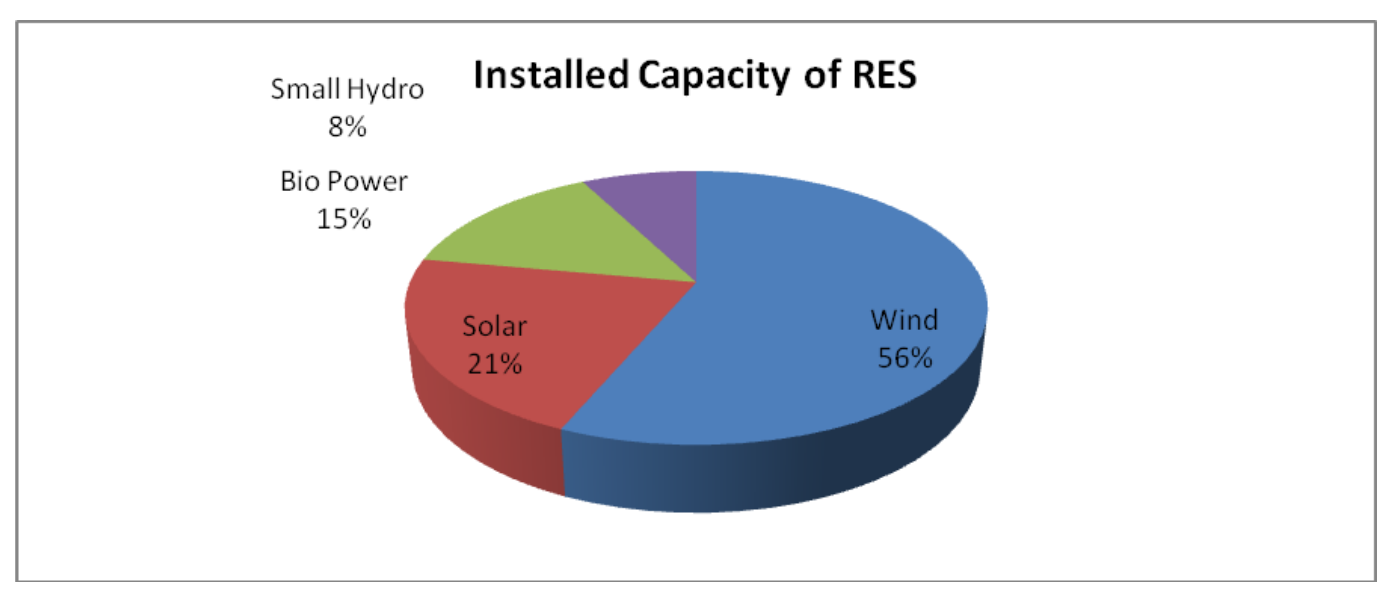

Figure 8. Installed capacity of Renewable Energy Sources (utilities). Data source: National Electricity Plan, CEA [16].

The North region accounted for 26 percent of the total installed generating capacity of electricity. In the North Region, per capita consumption for Uttar Pradesh is well below the national average at $585 \mathrm{Kwh}$. Other states are above the national average, with Punjab having the highest per capita power consumption of $2028 \mathrm{Kwh}$, followed by Haryana (1975 Kwh), Delhi (1574 Kwh), Uttarakhand (1454 Kwh), Himachal Pradesh (1340 Kwh), Jammu and Kashmir (1282 Kwh), Rajashthan (1166 Kwh) and Chandigarh (1128 Kwh). The average per capita power consumption for the North region was $1003 \mathrm{Kwh}$ in 2016-2017. The North region has the second-highest projected energy requirement and peak demand for 2021-2022 and 2026-2027. This region has a very high potential of 373,398.48 MW power generation from renewable sources.

The Western region accounted for the highest share of 34 percent of the total installed generating capacity of electricity. The average per capita power consumption for the West region is above the national average, with a per capita consumption of $1533 \mathrm{Kwh}$. The per capita power consumption of Madhya Pradesh is below the national average, at $989 \mathrm{Kwh}$. In the West region, Dadra and Nagar Haveli has the highest consumption of 15,783 Kwh, followed by Daman and Diu (7965 Kwh), Goa (2466 Kwh), Gujarat (2279 Kwh), Chhattisgarh (2016 Kwh), Maharashtra (1307 Kwh) and Madhya Pradesh (989 Kwh). The Western region has the highest projected energy requirement and peak demand for 20212022 and 2026-2027. This region has a high potential of 248,616.79 MW power generation from renewable sources.

The South region accounted for 27 percent of the total installed generating capacity of electricity. In the South Region, per capita consumption for Kerala and Lakshadweep is well below the national average at $763 \mathrm{Kwh}$ and $633 \mathrm{Kwh}$, respectively. Other states are above the national average, with Tamil Nadu having the highest per capita power consumption of $1847 \mathrm{Kwh}$, followed by Puducherry (1784 Kwh), Telangana (1551 Kwh), Karnataka (1367 Kwh) and Andhra Pradesh (1319 Kwh). The average per capita power consumption for the South region was $1432 \mathrm{Kwh}$, above the national average in 2016-2017. The South region has the third-highest projected energy requirement and peak demand for 2021-2022 and 2026-2027. This region has a potential of 225,985.37 MW power generation from renewable sources. 
The Eastern region accounted for 12 percent of the total installed generating capacity of electricity. The average per capita power consumption for the Eastern region is well below the national average at $694 \mathrm{Kwh}$, with Bihar having the lowest per capita consumption of $272 \mathrm{Kwh}$. Odisha is the only state in the Eastern region with per capita power consumption above the national average at $1622 \mathrm{Kwh}$. It has the highest per capita consumption in the region, followed by Jharkhand (915 Kwh), Sikkim (806 Kwh), West Bengal (665 Kwh), Andaman and Nicobar Islands ( $370 \mathrm{Kwh}$ ) and Bihar ( $272 \mathrm{Kwh}$ ). The Eastern region has the fourth-highest projected energy requirement and peak demand for 2021-2022 and 2026-2027. This region has a potential of 73,198.25 MW power generation from renewable sources.

The Northeast region accounted for only one percent of the total installed generating capacity of electricity. Per capita consumption for the Northeast region is well below the national average at $392 \mathrm{Kwh}$. Meghalaya has the highest per capita power consumption of $832 \mathrm{Kwh}$, followed by Arunachal Pradesh (648 Kwh), Mizoram (523 Kwh), Tripura (470 Kwh), Nagaland (345 Kwh), Assam (339 Kwh), and Manipur (326 Kwh). The Northeast region has the fifth-highest projected energy requirement and peak demand for 2021-2022 and 2026-2027. This region has a potential of 60,873.45 MW power generation from RES.

\subsection{Electricity Demand Projections for India}

Various agencies and investigators have made projections for energy demands in India; these reports have a substantial spread in energy demand forecasts. According to the World Bank, with expected GDP growth at an average of 7 percent every year, demand for electricity in India would be almost tripled between 2018 and 2040 [11]. In another report, nine electricity demand projections were generated for three scenarios of GDP growth and three levels of energy efficiency. Aggregate demand could grow from 949 TWh in 2015 to 2338 TWh in 2030 [60]. The Energy Resources Institute (TERI) presented the future electricity mix in its report in 2017, based on two scenarios: a 'High Renewables Scenario (HRES)' and a 'Low Renewables Scenario (LRES)'. In the HRES, the renewable energy capacity increases by $125 \mathrm{GW}$ in 2021-2022, 225 GW in 2025-2026 and 803 GW in 2029-2030 from $50 \mathrm{GW}$, respectively. The LRES is based on a lower trajectory of renewables; here, capacity addition was taken to be $75 \mathrm{GW}$ during the first five years and $100 \mathrm{GW}$ in the five years after that [61]. The aggregate projected electricity energy requirement in MU and peak demand in megawatt are presented in Table 3.

Table 3. Estimated demand as per 19th EPS.

\begin{tabular}{ccc}
\hline Year & $\begin{array}{c}\text { Electrical Energy } \\
\text { Requirement (MU) }\end{array}$ & $\begin{array}{c}\text { Peak Electricity } \\
\text { Demand (MW) }\end{array}$ \\
\hline $2021-2022$ & $1,566,023$ & 225,751 \\
$2026-2027$ & $2,047,434$ & 298,774 \\
\hline
\end{tabular}

The national electricity plan surveyed the periods of 2021-2022 and 2026-2027 to identify the optimal capacity mix based on the demand of electricity, considering various initiatives by GOI, such as RES capacity targets by 2022 with committed capacity. The present study suggests an optimization model intending to minimize the cost of generation considering various other constraints.

\section{Electrical Energy Production Modeling}

In this section, we discuss the constraints and parameters of modeling the electrical energy system.

\subsection{Constraints for Electric Energy Production}

The optimum mix of electricity generation can be viewed as an optimisation problem, where the objective is to minimise the operating cost of the existing plants and levelized 
cost of capital and operating new generating stations satisfying a different set of constraints or limitations in the system, which include: "

i. Renewable capacity addition targets fixed by Government;

ii. Must Run Status for Renewable Energy Sources;

iii. Loss of Load Probability;

iv. Energy Not Served;

v. Provision of Reserve Margin;

vi. International commitments by the country;

vii. Emission limits if any; and

viii. Current Energy mix.

"The various decision variables of the problem are as follows:

$X_{1}=$ Installed Capacity of Hydro

$X_{2}=$ Installed Capacity of Coal

$X_{3}=$ Installed Capacity of gas

$X_{4}=$ Installed Capacity of nuclear

$X_{5}=$ Installed Capacity of solar thermal

$X_{6}=$ Installed Capacity of solar PV

$X_{7}=$ Installed Capacity of Wind

$X_{8}=$ Installed Capacity of Biomass

$X_{9}=$ Installed Capacity of Small Hydro.

\subsubsection{Renewable Capacity Addition Targets Fixed by Government}

The GOI recently set a target to achieve 175 GW capacity installed from RES by March 2022 (see Table 4). Additional capacity anticipated from RES during 2022-2027 has been considered to be 100,000 MW, of which 50,000 MW would be from solar, 40,000 MW from wind, $7000 \mathrm{MW}$ from biomass and $3000 \mathrm{MW}$ from small hydro, respectively, to reach a target of 175 GW RES by 2021-2022 and 275 MW by 2027 (see Table 4).

Table 4. Renewable energy target in India.

\begin{tabular}{|c|c|c|c|}
\hline $\begin{array}{c}\text { RES } \\
\text { Category }\end{array}$ & $\begin{array}{c}\text { Target RES } \\
\text { IC as on } \\
31 \text { March } 2022\end{array}$ & $\begin{array}{l}\text { RES Installed } \\
\text { Capacity as on } \\
31 \text { March } 2017\end{array}$ & $\begin{array}{l}\text { Expected RES } \\
\text { Capacity Addition } \\
\text { from 2017-2022 }\end{array}$ \\
\hline Solar & 100,000 & 12,289 & 87,711 \\
\hline Wind & 60,000 & 32,280 & 27,720 \\
\hline Biomass & 10,000 & 8295 & 1705 \\
\hline Small Hydro & 5000 & 4380 & 620 \\
\hline Total & 175,000 & 57,244 & 117,756 \\
\hline
\end{tabular}

National Electricity Plan, CEA [16]

\subsubsection{Must Run Status for Renewable Energy Sources}

Must Run Renewable Energy Sources, such as solar, wind, nuclear and hydro projects, followed by gas being given priority, are considered the "must run projects" based on their potential. During 2017-2022, the additional capacity of hydro is estimated to be about $6823 \mathrm{MW}$, and that of nuclear about $3300 \mathrm{MW}$, while hydro is projected to total 12,000 MW, and the additional capacity of nuclear is projected to total $6800 \mathrm{MW}$ in the years 2022-2027 (see Table 5). Renewable capacity is also considered a "must-run" capacity. The expected import of hydro during 2021-2022 is $4356 \mathrm{MW}$, and during 2026-2027 it is 21,600 MW. Projected capacity after addition of hydro in 2017-2022 is 51,301.42 MW; for nuclear it is $10,080 \mathrm{MW}$ and for gas it is 25,735.38 MW. For 2022-2027, the projected capacity of hydro is $63,301.42 \mathrm{MW}$, for nuclear is $16,880 \mathrm{MW}$, and for gas it is 25,735.38 MW. 
Table 5. Committed capacity addition.

\begin{tabular}{cccccc}
\hline Year & Hydro & Nuclear & Gas & $\begin{array}{c}\text { Committed } \\
\text { RES\& } \\
\text { Retirement of } \\
\text { Capacity } \\
\text { (MW) }\end{array}$ & Coal(MW) \\
\hline (MW) & (MW) & (MW) & (MW & 175,000 & 22,716 \\
$2017-2022$ & 6823 & 3300 & 0 & 275,000 & 25,572 \\
\hline Source: National Electricity Plan, CEA [16]. & 6800 & &
\end{tabular}

Source: National Electricity Plan, CEA [16].

\subsubsection{Loss on Load Probability and Energy Not Served}

This is the probability of an electricity system failing to serve the peak load. It can be described as the proportion of days or hours in a year when the available capacity generated is insufficient to meet the peak demand. Energy Not Served can be expressed as a fraction of the total energy required, which is expected not to be supplied to the consumers by the electricity system. It is the unmet energy demand in the number of hours in a year. LOLP and ENS are used as reliability criteria for electricity systems. LOLP of 0.2 percent and ENS of 0.05 percent are adopted for electricity planning in India.

\subsubsection{Provision for Reserve Margin}

Future electricity demand is challenging to forecast with accuracy, therefore, as a simple strategy, a capacity with more supply than may be required is maintained as it would take years to build new power generation capacity. "Reserve margin = (Capacity - Demand)/Demand, where capacity is the expected maximum available supply and demand expected peak demand". A $5 \%$ spinning reserve for conventional plants is required as per the National Electricity Plan in India.

\subsubsection{International Commitments by India}

The Indian Government is committed to achieving "energy autonomy and to provide clean, affordable, reliable and sustainable power for all". The GOI has made the international commitment (INDC) to have at least 40 percent electric power capacity installed from non-fossil fuel-based sources cumulatively by the year 2030 and to reduce the intensity of its GDP emissions by 33 percent to 35 percent by the year 2030 from the levels in 2005 . The non-fossil fuel energy sources include hydro, nuclear and RES. The Government of India recently set a target of achieving $175 \mathrm{GW}$ installed electricity capacity from renewable energy sources (RES) by March 2022. More emphasis is given to developing a non-fossil fuel-based generation of power, that is, hydropower, to the greatest extent possible, shifting towards more efficient supercritical technologies for coal-based power plants.

\subsubsection{Emission Limits}

The Indian government has made an international commitment (INDC) to reduce its GDP emission intensity by 33 to 35 percent by 2030 from the levels in 2005. Therefore, the installed capacity of thermal sources (coal, gas, diesel and ignite) of electricity should be those with smaller emissions than the prescribed emission limit for the government's INDC goal for emissions intensity. The estimate of the total emission of $\mathrm{CO}_{2}$ from the "grid-connected" power stations during 2005 was 462 million tonnes. The emission of $\mathrm{CO}_{2}$ resulting from the power sector was estimated to reach 1026 million tonnes at the end of 2021-2022 and 1173 million tonnes at the end of the year 2026-2027, respectively. Emission intensity is likely to reduce by 40.51 percent and 53.65 percent, respectively, at the end of 2021-2022 and 2026-2027 from the 2005 level [62]. In the year 2005, the emission intensity in India was $0.015548 \mathrm{~kg} \mathrm{CO}_{2} /$ GDP. The GDP in 2005 was Rs 2971464 crore at factor cost $[1,31,37]$. For the present study, 2005 has been taken as the base year, and GDP at factor cost for 2021-2022 and 2026-2027 has been projected, assuming an annual GDP growth rate of 7 percent. Available GDP of 2013-2014 at the base price of 2005 was Rs 5,741,791 crore [63]. Projected GDP for 2021-2022 is Rs 9,865,466 crore, and for 2026-2027 it is Rs 
13,836,826 crore (see Table 6). Emission intensity in 2005 was $0.015548 \mathrm{~kg} \mathrm{CO}_{2} / \mathrm{Rs}$ GDP [64]. The Government of India has made an international commitment (INDC) to reduce the emissions intensity of its GDP by 33 to 35 percent by the year 2030 from the 2005 level. For the present study, we have assumed achievement of this target by 2026-2027. Therefore, in 2026-2027 the emission intensity should reduce to $0.010062 \mathrm{~kg} \mathrm{CO}_{2} / \mathrm{Rs}$ GDP. Therefore, total allowed emissions should be $0.010062 * 98,654,660,000,000=992,663,188,920 \mathrm{~kg}$ for the year 2021-2022 and $0.010062 * 138,368,260,000,000=1,392,261,432,120 \mathrm{~kg}$ for the year 2026-2027.

Table 7 shows $\mathrm{CO}_{2}$ emission factors for different power generation systems [50,64]. A coal-based electricity generation system has a substantial $\mathrm{CO}_{2}$ emission factor. As the installed capacity of power generation is primarily coal-based, coal is a significant source of emitting carbon dioxide in India. Therefore, if RES usage increased and thermal efficiency improved, the $\mathrm{CO}_{2}$ emissions in India would be reduced significantly.

Table 6. Gross Domestic Product.

\begin{tabular}{ccc}
\hline Serial Number & Financial Year & $\begin{array}{c}\text { GDP at Constant 2004-2005 } \\
\text { Prices (Rupees Crore) }\end{array}$ \\
\hline 1 & $2004-2005$ & $2,971,464$ \\
2 & $2005-2006$ & $3,253,073$ \\
3 & $2006-2007$ & $3,564,364$ \\
4 & $2007-2008$ & $3,896,636$ \\
5 & $2008-02009$ & $4,158,676$ \\
6 & $2009-2010$ & $4,516,071$ \\
7 & $2010-2011$ & $4,918,533$ \\
8 & $2011-2012$ & $5,247,530$ \\
9 & $2012-2013$ & $5,482,111$ \\
10 & $2013-2014$ & $5,741,791$ \\
11 & $2021-2022$ & $9,865,466$ \\
\hline
\end{tabular}

Table 7. Various technologies emission factors.

\begin{tabular}{ccc}
\hline S/N & Technology & $\begin{array}{c}\mathbf{C O}_{2} \text { Emission Factor } \\
\text { (tCO2/Mwh) }\end{array}$ \\
\hline 1 & Coal & 0.98 \\
2 & Diesel & 0.59 \\
3 & Gas & 0.45 \\
4 & Lignite & 1.38 \\
5 & Hydro & 0.011 \\
6 & Nuclear & 0.0242 \\
7 & Wind & 0.0295 \\
8 & Solar & 0.0534 \\
\hline
\end{tabular}

Source: Latest CERC orders, CERC Tariff Regulations for FY 2014-19 \& 2019-24 [50,64].

\subsubsection{Current Energy Mix in India}

The installed capacity of electricity in India as of 31 March 2017 was 326,833 MW. Of the 326,833 MW of total installed capacity, coal accounted for 192,163 MW, nuclear $6780 \mathrm{MW}$, hydro 44,478 MW, diesel accounted for $838 \mathrm{MW}$, and gas accounted for 25,329 MW. India is among the largest producers of energy from renewable sources. RES accounted for $57,244 \mathrm{MW}$ with wind power capacity accounting for 32,280 MW, solar accounting for $12,288.83 \mathrm{MW}$, biopower accounting for 8295.78 , and the small hydro plant has a capacity of 4379.86 MW (see Table 8). Installed capacity of coal up until 31 March 2017 was 192,163 MW, 22,716 MW to be retired until 2022 (see Table 5) while 47,855 MW are under-construction and to be completed during 2017-2022 [62]. Therefore, the likely capacity of coal would be $217,302 \mathrm{MW}$ in 2021-2022. 
Table 8. Installed Capacity utilities (MW) as of 31 March 2017.

\begin{tabular}{ccc}
\hline S/N & Technology & Installed Capacity (MW) \\
\hline 1 & Coal & 192,163 \\
2 & Diesel & 838 \\
3 & Gas & 25,329 \\
4 & Hydro & 44,478 \\
5 & Nuclear & 6780 \\
6 & Solar & 12,288 \\
7 & Wind & 32,280 \\
8 & Bio Power & 8295 \\
9 & Small Hydro & 4379 \\
\hline
\end{tabular}

The various cost components of electricity production are the capital cost, operation and maintenance cost, and fuel cost. The cost of electricity production also depends upon specific fuel consumption, capacity factor, auxiliary consumption and plant life (see Table 2). In the current study, the technology-specific and overall cost of electricity was computed with the help of a levelized energy formula Equation (6).

\subsection{Mathematical Model Formulation}

In this section, we formulate three models with the objectives of minimizing the levelized cost of electricity, minimizing the total present value of the cost of energy, and maximizing the present value of total energy produced. In order to optimise the system cost, we introduce installed capacities as variables with the respective costs of the generation technologies and specified constraints. LCOE is calculated by dividing all expected "technology lifetime costs by the total energy production" throughout its lifetime. The present value of the cost of energy is computed as the ratio of the present value of the cost of the installed capacity to the present value of energy produced over the lifetime of the plants, while the last objective is calculated as the present value of total energy produced over the lifetime of different plants. The complete mathematical formulation is presented in Equatinos (7)-(20).

$$
\begin{aligned}
& \min Z_{1}=37.674 X_{1}+52.838 X_{2}+81.569 X_{3}+61.399 X_{4}+73.698 X_{5}+39.479 X_{6}+ \\
& 29.172 X_{7}+89.380 X_{8}+35.082 X_{9}
\end{aligned}
$$

$\min Z_{2}=\left(1228.431 X_{1}+2629.385 X_{2}+2029.527 X_{3}+3576.471 X_{4}+1471.684 X_{5}+\right.$ $\left.649.9936 X_{6}+736.7164 X_{7}+4622.645 X_{8}+1422.595 X_{9}\right) /\left(32.60631 X_{1}+49.76247 X_{2}+\right.$ $24.88098 X_{3}+58.25021 X_{4}+19.96949 X_{5}+16.46369 X_{6}+25.25431 X_{7}+51.71852 X_{8}+$ $\left.40.55516 X_{9}\right)$

$$
\begin{aligned}
& \max Z_{3}=32.60631 X_{1}+49.76247 X_{2}+24.88098 X_{3}+58.25021 X_{4}+19.96949 X_{5}+ \\
& 16.46369 X_{6}+25.25431 X_{7}+51.71852 X_{8}+40.55516 X_{9}
\end{aligned}
$$

subject to:

$$
\begin{aligned}
& 3.031184 X_{1}+4.98385 X_{2}+2.4919 X_{3}+5.41512 X_{4}+2.0 X_{5}+1.648885 X_{6}+ \\
& 2.52929 X_{7}+5.52258 X_{8}+3.905259 X_{9} \geq 1,566,023 \\
& 33.75 X_{1}+5154.4 X_{2}+788.94 X_{3}+142.13 X_{4}+88.94\left(X_{5}+X_{6}\right)+74.99 X_{7}+ \\
& 613.62 X_{8}+43.39 X_{9} \leq 992,663,189
\end{aligned}
$$




$$
\begin{gathered}
X_{1}+X_{4}+X_{5}+X_{6}+X_{7}+X_{8}+X_{9} \geq 0.40\left(X_{1}+X_{2}+X_{3}+X_{4}+X_{5}+\right. \\
\left.X_{6}+X_{7}+X_{8}+X_{9}\right) \\
12,289 \leq X_{5}+X_{6} \leq 100,000 \\
44,478.42 \leq X_{1} \leq 51,301.42 \\
169,447 \leq X_{2} \leq 217,302 \\
26,167.01 \leq X_{3} \leq 26,573.01 \\
6780 \leq X_{4} \leq 10,080 \\
32,280 \leq X_{7} \leq 60,000 \\
8295 \leq X_{8} \leq 10,000 \\
4380 \leq X_{9} \leq 5000 .
\end{gathered}
$$

Equation (7) is related to optimizing the levelized costs of energy by individual technologies. Equation (8) is related to optimizing the levelized costs of energy by all technologies. Equation (9) is related to optimizing the present value of energy by all technologies. These Equations (7)-(9) are derived from Table 2.

The constraints of the above optimization model are explained as follows:

Constraint Equation (10) is related to the unit production of electricity in a year derived from Tables 2 and 3. Constraint Equation (11) is related to $\mathrm{CO}_{2}$ emissions limit during a year. The coefficient of the constraint is derived from Section 5.1.6, Tables 2 and 7. Constraint Equation (12) is related to non-fossil fuel derived from Section 5.1.5. Constraint Equation (13) is related to solar installed capacity derived from Table 4. Constraint Equation (14) is related to hydro installed capacity derived from Tables 5 and 8. Constraint Equation (15) is related to coal installed capacity derived from Section 5.1.6. Constraint Equation (16) is related to gas installed capacity derived from Tables 5 and 8, respectively. Constraint Equation (17) is related to nuclear-installed capacity derived from Tables 5 and 8, respectively. Constraint Equation (18) is related to projected wind installed capacity derived from Table 4. Constraint Equation (19) is related to projected bio-power plant capacity derived from Table 4. Finally, constraint Equation (20) is related to projected small hydro capacity derived from Table 4 .

\section{Results Analysis and Discussions}

This study was carried out using the flexible fuzzy goal programming (FFGP) approach to find out the optimum cost solution for electricity "system expansion" for the study period from 2016-2017 to 2021-2022. The model proposed determines the optimal cost expansion that will guarantee the optimal mix of the capacity generation for all the years until 2021-2022, considering all the input parameters (financial/technical) for the study period. The optimal mix of installed capacity by the end of the year 2021-2022 is given in Table 9. 
Table 9. Optimal installed capacity.

\begin{tabular}{cccc}
\hline S/N & Technology & $\begin{array}{c}\text { Capacity } \\
\text { (MW) }\end{array}$ & $\begin{array}{c}\text { Percentage } \\
\text { Mix (\%) }\end{array}$ \\
\hline 1 & Hydro & $51,301.42$ & 11.48 \\
2 & Coal & $184,073.40$ & 41.17 \\
3 & Gas & $26,573.01$ & 5.94 \\
4 & Nuclear & 10,080 & 2.25 \\
5 & Solar Thermal & $35,938.05$ & 8.04 \\
6 & Solar PV & $64,061.95$ & 14.33 \\
7 & Wind & 60,000 & 13.42 \\
8 & Biomass & 10,000 & 2.24 \\
9 & Small Hydro & 5000 & 1.12 \\
& Total & $\mathbf{4 4 7 , 0 2 7 . 8 3}$ & $\mathbf{1 0 0}$ \\
\hline
\end{tabular}

The FFGP model has selected the target installed capacity of hydro, nuclear, solar, wind, biomass and small hydro due to the reduction in cost and $\mathrm{CO}_{2}$ emissions. The model does not select any new coal power plants apart from the existing projects. It can be observed that RES installed capacity, including solar and wind, will become 160,000 GW by the end of 2021-2022, which is more than 35 percent of the total installed capacity of $447,027.83 \mathrm{GW}$. In contrast, energy from non-fossil sources will be $236,381.4 \mathrm{GW}$ by the end of 2021-2022, which is more than 52 percent of the total installed capacity of 447,027.83 GW. Capacity expansion for coal-based plants is not significant as compared to the solar and wind capacity addition.

The optimal gross electricity generation during the year 2021-2022 is 1,597,715 MU, comprised of 983,611 MU from thermal, 177,507 MU from solar, 151,757 MU from wind, 155,504 MU from hydro, 54,584 MU from nuclear and 55,226 MU from biomass and 19,526 MU from small hydro, as shown in Table 10. It can be noticed from the results above that the installed capacity of non-fossil fuel-based plants, including solar, wind, biomass, hydro and nuclear-based, is likely to be about 50 percent of the total installed capacity. It contributes around 35 percent of the gross electricity generation in the year 2021-2022. The levelized cost of electricity at this optimal solution would be Rs 51.53 lakh per MU or Rs. 5.15 per Kwh.

Table 10. Optimal generation of electricity by different technologies.

\begin{tabular}{cccc}
\hline S/N & Technology & $\begin{array}{c}\text { Generation } \\
\text { (MWH) }\end{array}$ & $\begin{array}{c}\text { Percentage } \\
\text { Mix (\%) }\end{array}$ \\
\hline 1 & Hydro & 15,550 & 9.73 \\
2 & Coal & 917,394 & 57.42 \\
3 & Gas & 66,217 & 4.14 \\
4 & Nuclear & 54,584 & 3.42 \\
5 & Solar Thermal & 71,876 & 4.50 \\
6 & Solar PV & 105,631 & 6.61 \\
7 & Wind & 151,757 & 9.50 \\
8 & Biomass & 55,226 & 3.46 \\
9 & Small Hydro & 195,26 & 1.22 \\
\end{tabular}

Furthermore, we introduce different tolerance values to the FFGP model to obtain the optimum cost solution for electricity system expansion for the study period of 2016-2017 to 2021-2022. We considered both technical and financial input parameters and a tolerance of one percent to ten percent for increasing the projected demand and installed capacity of solar, hydro, wind and small hydro. The optimal mix of installed capacity with tolerance level is given in Table 11. 
Table 11. The optimal mix of installed capacity at different tolerance levels.

\begin{tabular}{|c|c|c|c|c|c|c|c|c|c|c|}
\hline Technologies & Tol $=1 \%$ & Tol $=2 \%$ & Tol = 3\% & $\mathrm{Tol}=4 \%$ & Tol $=5 \%$ & Tol $=6 \%$ & Tol $=7 \%$ & Tol $=8 \%$ & Tol $=9 \%$ & Tol $=10 \%$ \\
\hline Hydro & $51,686.49$ & $51,932.12$ & $52,102.44$ & $52,227.48$ & $52,325.34$ & $52,467.24$ & $52,651.13$ & $52,833.09$ & $53,013.37$ & $53,192.17$ \\
\hline Coal & $178,135.4$ & $180,485.2$ & $183,004.70$ & $184,277.40$ & $184,138.40$ & $183,982.30$ & $183,967.60$ & $183,953.10$ & $183,938.60$ & $183,924.20$ \\
\hline Gas & $26,167.25$ & $26,167.01$ & $26,167.01$ & $26,167.01$ & $26,573.01$ & $26,167.01$ & $26,167.01$ & $26,167.01$ & $26,167.01$ & $26,167.01$ \\
\hline Nuclear & 10,080 & 10,080 & 10,080 & 10,080 & 10,080 & 10,080 & 10,080 & 10,080 & 10,080 & 10,080 \\
\hline $\begin{array}{l}\text { Solar } \\
\text { Thermal }\end{array}$ & $80,283.14$ & $66,046.33$ & $51,809.30$ & $40,907.75$ & $31,162.40$ & $25,697.84$ & $24,173.95$ & $22,683.91$ & $21,223.47$ & $19,789.04$ \\
\hline Solar PV & $20,966.25$ & $36,724.26$ & $52,629.30$ & $65,287.11$ & $76,841.70$ & $84,029.66$ & $87,195.12$ & $90,330.46$ & $93,439.49$ & $96,525.39$ \\
\hline Wind & $60,450.37$ & $59,438.06$ & $57,188.34$ & $56,389.40$ & $56,762.99$ & $54,135.70$ & $53,103.53$ & $52,061.21$ & $51,010.02$ & $49,951.03$ \\
\hline Biomass & 8295 & 8295 & 8295 & 8295 & 8295 & 10,000 & 10,000 & 10,000 & 10,000 & 10,000 \\
\hline $\begin{array}{l}\text { Small } \\
\text { Hydro }\end{array}$ & 5037 & 5061.47 & 5078.07 & 5090.25 & 5099.79 & 5113.62 & 5131.54 & 5149.28 & 5166.85 & 5184.27 \\
\hline
\end{tabular}

As evident from Table 11, the projected demand and installed capacity of renewable sources of electricity increased by one percent to ten percent tolerance throughout the solution cases. The optimal solutions give increased installed renewable sources of electricity that is hydro, solar and small hydro. In contrast, it does not increase non-renewable sources such as gas, nuclear and biomass. The optimal solution also indicated a slight increase in the installed capacity of coal; however, it does not exceed the existing and under-construction plants, implying that no new plant is required.

Table 12 shows that the levelized cost for an optimal electricity system at a different level of tolerance, ranging from one percent to ten percent, decreases as the demand increases. This implies that increased electricity demand can be met with renewable energy sources by increasing hydro, solar PV and small hydro, which have lower levelized costs than non-renewables such as gas, nuclear and biomass, which have a higher levelized cost.

Table 12. levelized Cost at Different Tolerance and Emissin values.

\begin{tabular}{ccc}
\hline Tolerance & Electricity Demand & Levelized Cost (lakh/MU) \\
\hline 0.01 & 1581683 & 53.02 \\
0.02 & 1597343 & 52.46 \\
0.03 & 1613004 & 51.95 \\
0.04 & 1628664 & 51.52 \\
0.05 & 1644324 & 51.16 \\
0.06 & 1659984 & 51.17 \\
0.07 & 1675645 & 51.12 \\
0.08 & 1691305 & 51.07 \\
0.09 & 1706965 & 51.03 \\
0.10 & 1722625 & 50.98 \\
\hline
\end{tabular}

\section{Managerial and Practical Implications}

Results of the study indicate that flexible fuzzy goal programming can be implemented to meet energy demand in the future, at the same time achieving various national goals and international commitments. The results and methodology of the study can be used by researchers and governments to further the research in the field of the cost-production optimization of energy. There are various sources of energy generation; however, some are costly and have a highly negative impact on environmental sustainability. Therefore, from the managerial perspective, such sources are not the most desired. Currently, most of the energy produced by the GOI is based on hydro and coal, and their installed capacity can meet the Indian demands up to 2022. However, from 2022 onwards, there will be a high demand for electricity power due to the increasing population. As such, this study suggests shifting to renewable energy sources. The study further suggests the rates of shifting from non-renewable to renewable sources at every step. The tolerance values shown in Table 11 provide the optimal mix for electricity generation from the various technologies. This concept will help the government and policymakers to gain insight into what amount is required from every technology to optimally generate electricity for the sustainable development of India. The study balanced the three tiers of the SDGs-social, economic and environmental issues. 


\section{Conclusions and Recommendations}

India is determined to ensure the affordability of clean energy for the benefit of all and intends to reduce $\mathrm{CO}_{2}$ intensity by adopting low-carbon technologies, as evident from the INDC submitted to the UNCC and their various targets for RES. It is observable from Tables 11 and 12 that, with an increase of projected demand from one percent to ten per cent, the capacity expansion for "coal-based" plants is not significant as compared to renewable energy capacity addition; also the levelized cost decreases as the electricity demand increases. Although India has made sound progress towards a mix of cleaner energy as recognized by the international community [65], the following recommendations are vital for accelerating India's progress towards attaining cost-efficient and clean electricity generation while simultaneously achieving its international commitments and financial and technical constraints.

- $\quad$ The optimal allocation of installed capacity among different technologies in Table 9 can be adopted to meet the projected demand for electricity;

- For any further increase in demand, the allocation of different electricity plants can be made based on the optimal solution in Table 11;

- Increasing electricity demand should be made by shifting allocations towards renewable energy sources, especially solar, wind and hydro;

- A less-costly decommissioning method can be adopted for plants, especially for the nuclear plant;

- Government should install solar panels on the roof-tops of government offices and encourage individuals to use a solar panel for electricity generation for their personal use;

- Government should reduce the dependency on costly imported coal and explore and use domestic coal reserves for running existing coal plants.

This research studied energy policy choices and addressed issues related to electricity generation (SDG 7), and will enhance the achievement of several SDGs for environmental sustainability in many ways.

- $\quad$ Shifting from fossil fuels to renewable energy sources may lower carbon dioxide emissions, which furthers climate change mitigation goals (SDG13);

- Ensuring efficient energy access to poorer citizens and deploying large scale renewable sources will positively impact the SDG1 goal of alleviating poverty and all its ramifications;

- $\quad$ Energy efficiency will help achieve the sustainability of cities (SDG11), and with smart cities, road traffic accident risks will reduce drastically, improving peoples' health (SDG3);

- $\quad$ The provision of access to efficient and affordable energy will create employment opportunities for men and women who will be engaging in hairdressing and digital services (SDG5). It will also improve the quality of education via access to laboratories, internet facilities and modern technologies, helping the necessary flourishing of interdisciplinary research (SDG 10).

Author Contributions: Conceptualization, M.F.K., A.P., and I.A.; methodology, I.A., and U.M.M.; software, I.A., and U.M.M.; validation, M.F.K., A.P., U.M.M., J.C., and I.A.; formal analysis, A.P., U.M.M. and J.C.; investigation, A.P.; resources, M.F.K., A.P., and J.C.; writing-original draft preparation, M.F.K., A.P., U.M.M., and J.C.; writing-review and editing, I.A.; visualization, A.P., and U.M.M.; supervision, I.A.; funding acquisition, M.F.K. All authors have read and agreed to the published version of the manuscript.

Funding: The first author is thankful to Saudi Electronic University, Riyadh, Saudi Arabia, for providing a financial assistance to carry out this research.

Acknowledgments: This work was supported by the Deanship of Scientific Research, College of Science and Theoretical Studies, Saudi Electronic University, Riyadh, Saudi Arabia. All authors are thankful to the Special Issue editor D'Adamo Idiano and the anonymous reviewers. 
Conflicts of Interest: The authors have no known conflict of interest regarding the authorship and publication of this paper.

\section{Abbreviations}

The following abbreviations are used in this manuscript:

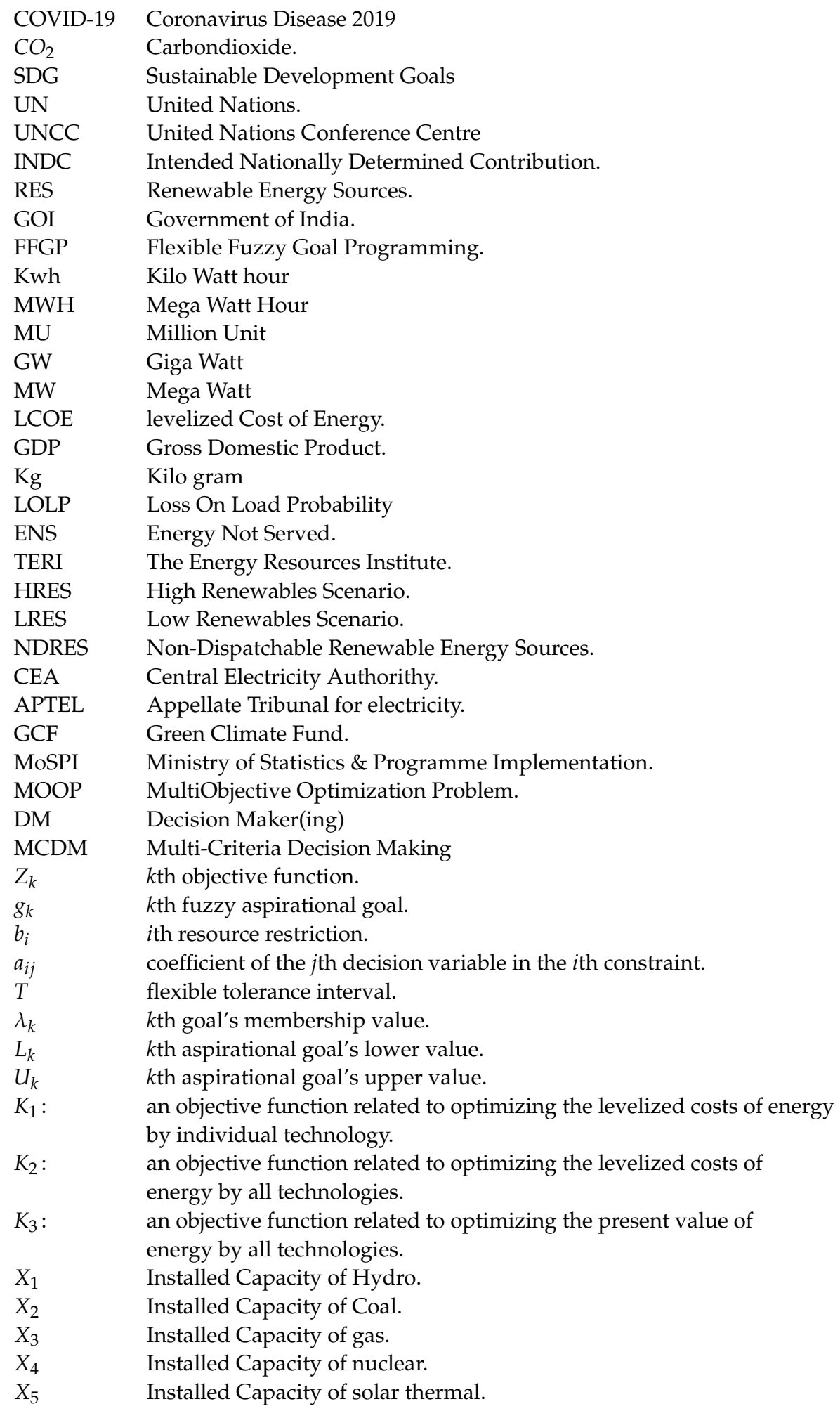




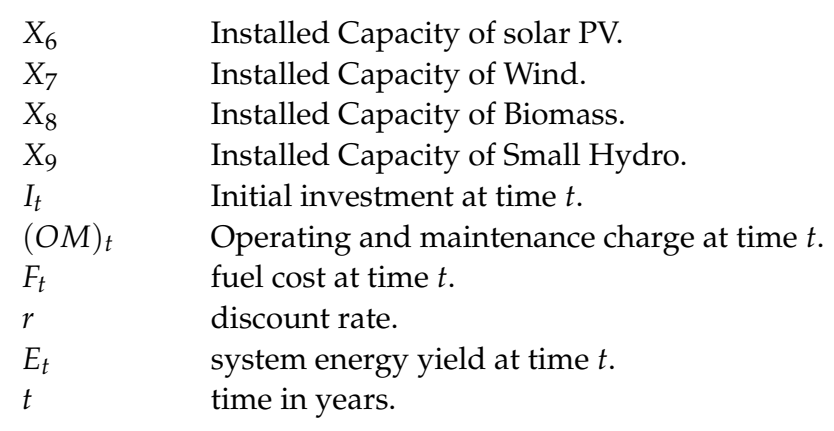

\section{References}

1. Ali, I.; Modibbo, U.M.; Chauhan, J.; Meraj, M. An integrated multi-objective optimization modeling for sustainable development goals of India. Environ. Dev. Sustain. 2021, 2, 3811-3831. [CrossRef]

2. Karuppiah, K.; Sankaranarayanan, B.; Ali, S.M.; Chowdhury, P.; Paul, S.K. An integrated approach to modeling the barriers in implementing green manufacturing practices in SMEs. J. Cleaner Prod. 2020, 265, 121737. [CrossRef]

3. D'Adamo, I.; González-Sánchez, R.; Medina-Salgado, M.S.; Settembre-Blundo, D. E-Commerce Calls for Cyber-Security and Sustainability: How European Citizens Look for a Trusted Online Environment. Sustainability 2021, 13, 6752. [CrossRef]

4. Ahmed, S.; Taqi, H.M.M.; Farabi, Y.I.; Sarker, M.; Ali, S.M.; Sankaranarayanan, B. Evaluation of Flexible Strategies to Manage the COVID-19 Pandemic in the Education Sector. Global J. Flexible Syst. Manag. 2021. [CrossRef]

5. Giudice, F.; Caferra, R.; Morone, P. COVID-19, the food system and the circular economy: Challenges and opportunities. Sustainability 2020, 12, 7939. [CrossRef]

6. Ali, I.; Fügenschuh, A.; Gupta, S.; Modibbo, U.M. The LR-Type Fuzzy Multi-Objective Vendor Selection Problem in Supply Chain Management. Mathematics 2020, 8, 1621. [CrossRef]

7. Mahmud, P.; Paul, S.K.; Azeem, A.; Chowdhury, P. Evaluating Supply Chain Collaboration Barriers in Small-and Medium-Sized Enterprises. Sustainability 2021, 13, 7449. [CrossRef]

8. The World Bank. 2019. Available online: https://data.worldbank.org/country/india (accessed on 3 September 2019).

9. Energy Statistics 2019 (Twenty-Fifth Issue) Central Statistics Office, Ministry of Statistics and Programme Implementation, Government of India New Delhi. Available online: http:/ / mospi.nic.in/sites/default/files/publication-reports/EnergyStatistics2 019-finall.pdf (accessed on 3 September 2019).

10. Zhang, F. How much do power sector distortions cost to South Asia. In Proceedings of the 2nd IAEE Eurasian Conference, Energy in Eurasia: Economic Perspectives on Challenges, Risks and Opportunities, International Association for Energy Economics, Zagreb, Croatia, 12-14 October 2017.

11. Devarajan, S.; Nabi, I. Economic growth in South Asia: promising, unequalising, sustainable? Econ. Pol. Wkl. 2006, 41, 3573-3580.

12. Holmes. F. Unmasking the Asian Giant I Financial Sense. 5 July 2012. Available online: https://www.financialsense.com/ contributors / frank-holmes / unmasking-the-asian-giant (accessed on 2 September 2019).

13. Andrews. R. Electricity and the Wealth of Nations I Energy Matters. 22 November 2015. Available online: http:/ / euanmearns. com/electricity-and-the-wealth-of-nations / (accessed on 2 September 2019).

14. Burke, P.J.; Stern, D.I.; Bruns, S. B. The impact of electricity on economic development: a macroeconomic perspective. Int. Rev. Environ. Resour. Econ. 2018, 12, 85-127. [CrossRef]

15. Zhang, T.; Shi, X.; Zhang, D.; Xiao, J. Socio-economic development and electricity access in developing economies: A long-run model averaging approach. Energy Policy 2019, 132, 223-231. [CrossRef]

16. Central Electricity Authority. Committee on Optimal Energy Mix in Power Generation on Medium and Long Term Basis; Ministry of Power, Government of India: New Delhi, India, 2018.

17. Knežević, G.; Topić, D.; Jurić, M.; Nikolovski, S. Joint market bid of a hydroelectric system and wind parks. Comput. Electr. Eng. 2019, 74, 138-148. [CrossRef]

18. Fekete, K.; Nikolovski, S.; Klaić, Z.; Androjić, A. Optimal re-dispatching of cascaded hydropower plants using quadratic programming and chance-constrained programming. Energies 2019, 12, 1604. [CrossRef]

19. Kanbur, B.B.; Wu, C.; Fan, S.; Duan, F. System-level experimental investigations of the direct immersion cooling data center units with thermodynamic and thermoeconomic assessments. Energy 2021, 217, 119373. [CrossRef]

20. Kanbur, B.B.; Kumtepeli, V.; Duan, F. Thermal performance prediction of the battery surface via dynamic mode decomposition. Energy 2020, 201, 117642. [CrossRef]

21. Kroll, C.; Warchold, A.; Pradhan, P. Sustainable Development Goals (SDGs): Are we successful in turning trade-offs into synergies? Palgrave Commun. 2019, 5, 140. [CrossRef]

22. Nerini, F.F.; Tomei, J.; To, L.S.; Bisaga, I.; Parikh, P.; Black, M.; Borrion, A.; Spataru, C.; Broto, V.C.; Anandarajah, G.; et al. Mapping synergies and trade-offs between energy and the Sustainable Development Goals. Nat. Energy 2018, 3, 10-15. [CrossRef]

23. Bisaga, I.; Parikh, P.; Tomei, J.; To, L.S. Mapping synergies and trade-offs between energy and the sustainable development goals: A case study of off-grid solar energy in Rwanda. Energy Policy 2021, 149, 112028. [CrossRef] 
24. Rathi, A.K.A. Promotion of cleaner production for industrial pollution abatement in Gujarat (India). J. Clean. Prod. 2003, 11, 583-590. [CrossRef]

25. Ghose, M.K. Promoting cleaner production in the Indian small-scale mining industry. J. Clean. Prod. 2003, 11, 167-174. [CrossRef]

26. Mukherjee, D.P. Barriers towards cleaner production for optimizing energy use and pollution control for foundry sector in Howrah, India. Clean Technol. Environ. Policy 2011, 13, 111-123. [CrossRef]

27. Pal, P.; Sethi, G.; Nath, A.; Swami, S. Towards cleaner technologies in small and micro enterprises: a process-based case study of foundry industry in India. J. Clean. Prod. 2008, 16, 1264-1274. [CrossRef]

28. Narayanaswamy, V.; Scott, J.A. Lessons from cleaner production experiences in Indian hosiery clusters. J. Clean. Prod. 2001, 9, 325-340. [CrossRef]

29. Unnikrishnan, S.; Hegde, D.S. Environmental training and cleaner production in Indian industry-A micro-level study. Resour. Conserv. Recycl. 2007, 50, 427-441. [CrossRef]

30. Annala, L.; Sarin, A.; Green, J.L. Co-production of frugal innovation: Case of low cost reverse osmosis water filters in India. J. Clean. Prod. 2018, 171, S110-S118. [CrossRef]

31. Nomani, M.A.; Ali, I.; Fügenschuh, A.; Ahmed, A. A fuzzy goal programming approach to analyse sustainable development goals of India. Appl. Econ. Lett. 2017, 24, 443-447. [CrossRef]

32. Khatun, F; Ahamad, M. Foreign direct investment in the energy and power sector in Bangladesh: Implications for economic growth. Renew. Sustain. Energy Rev. 2015, 52, 1369-1377. [CrossRef]

33. McCollum, D.L.; Echeverri, L.G.; Busch, S.; Pachauri, S.; Parkinson, S.; Rogelj, J.; Riahi, K. Connecting the sustainable development goals by their energy inter-linkages. Environ. Res. Lett. 2018, 13, 033006. [CrossRef]

34. Hassan, Q.; Garg, N.K. Systems approach for water resources development. Global J. Flexible Syst. Manag. 2007, 8, 29-43. [CrossRef]

35. Kaur, H.; Singh, S.P.; Glardon, R. An integer linear program for integrated supplier selection: A sustainable flexible framework. Global J. Flex. Syst. Manag. 2016, 17, 113-134. [CrossRef]

36. Khorasani, S.T. A robust optimization model for supply chain in agile and flexible mode based on variables of uncertainty. Global J. Flex. Syst. Manag. 2018, 19, 239-253. [CrossRef]

37. Gupta, S.; Fügenschuh, A.; Ali, I. A multi-criteria goal programming model to analyze the sustainable goals of India. Sustainability 2018, 10, 778. [CrossRef]

38. Modibbo, U.M.; Raghav, Y.S.; Hassan, M.; Mijinyawa, M. A Critical Review on the Applications of Optimization Techniques in the UN Sustainable Development Goals. In Proceedings of the 2021 2nd International Conference on Intelligent Engineering and Management (ICIEM), London, UK, 28-31 April 2021; pp. 572-576. [CrossRef]

39. Modibbo, U.M.; Ali, I.; Ahmed, A. Multi-objective optimization modeling for analysing sustainable development goals of Nigeria: Agenda 2030. Environ. Dev. Sustain. 2021, 23, 9529-9563. [CrossRef]

40. Yang, X.; Wang, Y.; Sun, M.; Wang, R.; Zheng, P. Exploring the environmental pressures in urban sectors: An energy-water-carbon nexus perspective. Appl. Energy 2018, 228, 2298-2307. [CrossRef]

41. Ahmadini, A.A.H.; Modibbo, U.M.; Shaikh, A.A.; Ali, I. Multi-objective optimization modeling of sustainable green supply chain in inventory and production management. Alex. Eng. J. 2021, 60, 5129-5146. [CrossRef]

42. AlArjani, A.; Modibbo, U.M.; Ali, I.; Sarkar, B. A new framework for the sustainable development goals of Saudi Arabia. J. King Saud Univ.-Sci. 2021, 33, 101477. [CrossRef]

43. Wang, Y.; Liu, J.; Hansson, L.; Zhang, K.; Wang, R. Implementing stricter environmental regulation to enhance eco-efficiency and sustainability: A case study of Shandong Province's pulp and paper industry, China. J. Clean. Prod. 2011, 19, 303-310. [CrossRef]

44. Christainsen, G.B.; Haveman, R.H. The contribution of environmental regulations to the slowdown in productivity growth. J. Environ. Econ. Manag. 1981, 8, 381-390. [CrossRef]

45. Yabar, H.; Uwasu, M.; Hara, K. Tracking environmental innovations and policy regulations in Japan: case studies on dioxin emissions and electric home appliances recycling. J. Clean. Prod. 2013, 44, 152-158. [CrossRef]

46. Curtis, E.M.; Lee, J.M. When do environmental regulations backfire? Onsite industrial electricity generation, energy efficiency and policy instruments. J. Environ. Econ. Manag. 2019, 96, 174-194. [CrossRef]

47. English, J.; Niet, T.; Lyseng, B.; Keller, V.; Palmer-Wilson, K.; Robertson, B.; Rowe, A. Flexibility requirements and electricity system planning: Assessing inter-regional coordination with large penetrations of variable renewable supplies. Renew. Energy 2020, 145, 2770-2782. [CrossRef]

48. D'Adamo, I.; Gastaldi, M.; Morone, P. The post COVID-19 green recovery in practice: Assessing the profitability of a policy proposal on residential photovoltaic plants. Energy Policy 2020, 147, 111910. [CrossRef] [PubMed]

49. D'Adamo, I.; Rosa, P. How Do You See Infrastructure? Green Energy to Provide Economic Growth after COVID-19. Sustainability 2020, 12, 4738. [CrossRef]

50. Hondo, H. Life cycle GHG emission analysis of power generation systems: Japanese case. Energy 2005, 30, 2042-2056. [CrossRef]

51. Zadeh, L.A. Fuzzy sets. Inf. Control. 1965, 8, 338-353. [CrossRef]

52. Zimmermann, H.J. Fuzzy programming and linear programming with several objective functions. Fuzzy Sets Syst. 1978, 1, 45-55. [CrossRef] 
53. U.S. Energy Information Administration. Capital Cost Estimates for Utility Scale Electricity Generating Plants. 2016. Available online: https://www.eia.gov/analysis/studies/powerplants/capitalcost/pdf/capcost/assumption.pdf (accessed on 4 September 2019).

54. Heptonstall, P. A review of Electricity Unit Cost Estimates. 2007. Available online: http:/ /www.ukerc.ac.uk/publications/areview-of-electricity-unit-cost-estimates.html (accessed on 7 September 2019).

55. United Nation Development Programme (n.d) Sustainable Development Goals. Available online: https://www.undp.org/ content/undp/en/home/sustainable-development-goals.html (accessed on 7 September 2019).

56. Government of India, "Nationally Determined Contirbution," Working towards Climate Justice. 2016, pp. 1-38. Available online: https:/ / www4.unfccc.int/sites/ndcstaging/PublishedDocuments/India\%20First/INDIA\%20INDC\%20TO\%20UNFCCC. pdf (accessed on 3 September 2019).

57. Dudley, B. BP Statistical Review of World Energy. 2019. Available online: https://www.bp.com/content/dam/bp/businesssites/en/global/corporate/pdfs/energy-economics/statistical-review/bp-stats-review-2019-full-report.pdf (accessed on 5 September 2019).

58. Ministry of Power, Government of India. Guidelines on Cross Border Trade of Electricity-2018. 2018. pp. 1-9. Available online: https://powermin.nic.in/en/content/guidelines-importexport-cross-border-electricity-2018 (accessed on 6 September 2019).

59. Tripathi, B. Now, India is the third largest electricity producer ahead of Russia, Japan. Business Standard News. 26 March 2018. Available online: https:/ / www.business-standard.com/article/economy-policy/now-india-is-the-third-largestelectricityproducerahead-of-russia-japan \-118032600086-1.html (accessed on 4 September 2019).

60. Ali, S. The Future of Indian Electricity Demand: How much, by Whom, and under What Condition? 2018. Available online: https: / / www.brookings.edu/research/the-future-of-indian-electricity-demand-how-much-by-whom-and-under-whatconditions / (accessed on 6 September 2019).

61. Pathak, S.; Saxena, P.; Ray, A.K.; Großmann, H.; Kleinert, R. Irradiation based clean and energy efficient thermochemical conversion of biowaste into paper. J. Clean. Prod. 2019, 233, 893-902. [CrossRef]

62. Central Electricity Authority. National Electricity Plan. 2018. Available online: www.cea.nic.in/reports/committee/nep/nep/ jan/2018 (accessed on 3 September 2019).

63. Central Statistical Organization, MOSPI, Government of India. New Series of National Accounts Statistics. 2010. Available online: mospi.nic.in/default/files/publication/reports/brochure/2004-05 (accessed on 6 September 2019).

64. Bhawan, S.; Puram, R.K. $\mathrm{CO}_{2}$ Baseline Database for the Indian Power Sector; Central Electricity Authority, Ministry of Power, Government on India: New Delhi, India, 2011.

65. Srikanth, R. India's sustainable development goals-Glide path for India's power sector. Energy Policy 2018, 123, 325-336. [CrossRef] 



\title{
The Risk of Dissolution of Sustainable Innovation Ecosystems in Times of Crisis: The Electric Vehicle during the COVID-19 Pandemic
}

\author{
Manel Arribas-Ibar ${ }^{1, *}$, Petra A. Nylund ${ }^{2}$ and Alexander Brem ${ }^{2,3}$ (D) \\ 1 Faculty of Business and Communication Studies, University of Vic-Central University of Catalonia, \\ Sagrada Família 7, 08500 Vic Barcelona, Spain \\ 2 Institute of Entrepreneurship and Innovation Science, University of Stuttgart, Pfaffenwaldring 19, \\ 70569 Stuttgart, Germany \\ 3 The Mads Clausen Institute, University of Southern Denmark, Alsion 2, 6400 Sønderborg, Denmark \\ * Correspondence: manuel.arribas@uvic.cat
}

Citation: Arribas-Ibar, M.; Nylund, P.A.; Brem, A. The Risk of Dissolution of Sustainable Innovation Ecosystems in Times of Crisis: The Electric Vehicle during the COVID-19 Pandemic.

Sustainability 2021, 13, 1319.

https://doi.org/10.3390/su13031319

Academic Editor: Idiano D'Adamo

Received: 11 December 2020

Accepted: 21 January 2021

Published: 27 January 2021

Publisher's Note: MDPI stays neutral with regard to jurisdictional claims in published maps and institutional affiliations.

Copyright: (C) 2021 by the authors Licensee MDPI, Basel, Switzerland. This article is an open access article distributed under the terms and conditions of the Creative Commons Attribution (CC BY) license (https:// creativecommons.org/licenses/by/ $4.0 /)$.

\begin{abstract}
Innovation ecosystems evolve and adapt to crises, but what are the factors that stimulate ecosystem growth in spite of dire circumstances? We study the arduous path forward of the electric vehicle (EV) ecosystem and analyse in depth those factors that influence ecosystem growth in general and during the pandemic in particular. For the EV ecosystem, growth implies outcompeting the less sustainable internal combustion engine (ICE) vehicles, thus achieving a transition towards sustainable transportation. New mobility patterns provide a strategic opportunity for such a shift to green mobility and for EV ecosystem growth. For innovation ecosystems in general, we suggest that a crisis can serve as an opportunity for new innovations to break through by disrupting prior behavioural patterns. For the EV ecosystem in particular, it remains to be seen if the ecosystem will be able to capitalize on the opportunity provided by the unfortunate disruption generated by the pandemic.
\end{abstract}

Keywords: innovation ecosystem; innovation strategy; sustainability; electric vehicle; dominant design; crisis; pandemic

\section{Introduction}

The green economy can become an engine for economic recovery after COVID-19 [1], and the electric vehicle (EV) ecosystem is one of the central pillars in the quest for reducing our reliance on fossil fuels. Only about 17,000 electric cars were on the world's roads in 2010, but by 2019, that number had swelled to 7.2 million, $47 \%$ of which were in the People's Republic of China. However, electric cars only accounted for $2.6 \%$ of global car sales and about $1 \%$ of global car stock in 2019. At the same time, only nine countries had more than 100,000 electric cars on the road, and only about 20 countries reached market shares above 1\% [2]. Like most other new technologies, EV sales grow along a traditional S-curve [3,4] and they are still in the stage of slow start with oversupply [5]. It is not clear when the EV market will enter in the next life-cycle stage, with a fast growth and supply sometimes unable to keep up with demand, since the automotive industry is a sector that has never had to deal with truly disruptive changes regarding its products, processes, or value network structure [6].

The automotive industry has been considered as an innovative industry driven by strong competitive pressure and constant technological progress, typically with huge investments $[7,8]$. Nevertheless, this innovation has been incremental rather than breakthrough or radical because it has been focused on optimizing existing products for existing customers and processes [9]. It has been during the last 15 years that this situation has started to change due to the rise of different car engine alternatives to the internal com- 
bustion engine (ICE) and new complementary technologies, e.g., artificial intelligence for autonomous driving [10].

The industry is currently in the process of being disrupted additionally by connected, autonomous, and shared driving, causing an unprecedented technology and business model transformation. Competition thus no longer takes place only between firms, but also between entire innovation ecosystems, in which loosely connected entities interact and coevolve to generate and profit from innovation [11-13]. The new competition dynamics in the automotive industry are not limited to a zero-sum game where all competitors compete for a market of a given size [14,15], but are instead focused on how each one of these ecosystems can meet customer and social needs $[15,16]$. The sustainable innovation ecosystem of the EV is hence trying to outcompete the less sustainable ICE ecosystem. Competition between innovation ecosystems can therefore drive internal competition within firms that produce both ICE vehicles and EVs. On the other hand, firms that compete in some arenas might also collaborate in others, e.g., by influencing policy or strengthening supporting infrastructure [3].

Amid this transformation, the COVID-19 outbreak has put additional stress on the industry [17]. Quarantined workforces, widespread shutdown of business, disrupted global supply chains, and decreasing demand have undermined the viability of the automotive industry [18]. Due to the supply-chain disruptions related to COVID-19, many important firms in the automotive industry, e.g., Tesla, Toyota, Hyundai, and Volkswagen, had to cease operations in several production plants, thus leading to the further compromising of the automotive industry [19]. Such multinational enterprises can play a major role in leading entire innovation ecosystems towards more sustainable practices [20] and can thus foment EV emergence. However, the pandemic has brought the aspirations and main projects of the major automobile companies to a grinding halt [21]. The COVID19 pandemic will affect global EV markets, although to a lesser extent than the overall passenger car market, which was estimated to contract by 15\% in 2020 relative to 2019 . The International Energy Agency expects that the EV sales for passenger and commercial vehicles will remain broadly at 2019 sales levels and will represent $3 \%$ of global car sales in 2020 [2]. These predictions can change due to the effects of the second and third waves, which will slow and weaken the expected economic recovery [22]. According to Eurometal, the second wave of COVID-19 could drive the recovery of the automotive sector into 2022 instead of the mild recovery currently forecast for 2021 [23]. These predictions and the evolution of the third wave are expanding the uncertainty that car manufacturers are facing. For the participants of this ecosystem, it is difficult to know how long the recovery will take and predict what the next normal will look like. The pandemic is accelerating and reconfiguring existing trends in the economy [24].

Nonetheless, the pandemic has not only caused the emergence of new threats, but also new opportunities that the sector must analyse carefully [25]. Both types of influences lead to profound changes in the macroeconomic and microeconomic environment of this ecosystem; they are driving the emergence of, for example, new consumer behaviours, new regulatory trends, and new technologies. For this reason, it is vital to identify additional factors that are directly affecting the current trends of the ecosystem. In this paper, we therefore ask which factors associated with a severe crisis influence the evolution of innovation ecosystems.

\section{Methodology}

We seek the answer to this research question through a detailed inductive case study, which aims to identify relevant factors [26]. The inductive case study method is a qualitative research method particularly suitable for identifying unknown factors or mechanisms, which is what we aim to do in this article [27]. This method is therefore adequate for the analysis of emerging fields, e.g., innovation ecosystems, where qualitative research is needed to identify mechanisms and relationships before these can be tested quantitatively [28]. We follow the current tendency to use secondary data when studying innovation 
ecosystems [29,30]. This is warranted by the analysis of complex relations involving a multitude of actors, which requires multiple data sources.

The studied case is the evolution of the EV innovation ecosystem during the crisis generated by COVID-19. The considerable impact of the pandemic on EV evolution makes this particular innovation ecosystem suitable for identifying factors that may affect ecosystems in general. In the following section, we analyse the evolution of the EV innovation ecosystem. We then examine factors affecting this evolution related to the pandemic. Finally, we discuss these findings and their impact on research, policy, and practice.

\section{Results}

\subsection{Barriers to the Evolution of the EV Sustainable Innovation Ecosystem}

Even though the EV has many advantages compared to ICE vehicles, e.g., sustainability, simplicity, reliability, compact dimensions, and fewer moving parts of electric motors requiring less maintenance, whether or not EVs are superior to ICE vehicles throughout their entire life cycle is still subject to debate [31]. More research is necessary to understand the energy performance of EVs [32]. There is still a divergence of opinions and assumptions that confuse the consumer, and above all, there is a lack of compelling business cases that can be presented to the consumer. Additionally, it is necessary to make this comparison from the perspective of a life-cycle assessment to avoid problem shifting or rebound effects and to quantify the environmental impact from raw-material extraction to the end-of-life [33]. According to this view, it seems that EVs have already reached cost parity with ICE cars from a total cost perspective, including upfront payment, maintenance, depreciation, and fuel costs. The performance of the EV is not robust enough and depends on a great variety of interconnected factors, such as duty cycles of the electric engine, driving conditions, and traffic situations [2]. Moreover, the environmental performance of EVs changes greatly depending on the electricity sources.

Another impediment to EV diffusion is that the industry has not yet converged towards a dominant design of the electric car that would lay down a co-aligned structure within the EV ecosystem to set shared technological compatibility standards [15,34,35]. For example, there is not yet convergence on core powertrain design of the EV. There are different battery-cell designs with different geometries, along with multiple chemical compositions, and there is a large variance in the design approach for thermal management with four battery-cooling solutions. The lack of a dominant design reduces incremental innovation to refine the product [36]. Due to this lack of a standardized and shared design and architecture, there is a great variability of EV performance attributes between the different design solutions that have been developed and adapted in parallel. For example, the environmental performance of EVs is strongly influenced by the size of the battery, the energy required in the battery production phase, and how that energy is produced [37-40].

The EV is not an isolated product, which makes its performance dependent on a combination of several factors that have a distinct nature and exert a greater or lesser influence. These factors are controlled by public and private participants of the EV ecosystem, producing different effects in terms of not only the performance of the electric car, but also the degree of acceptance of the EV by the consumer. This expresses the co-dependence that exists within this ecosystem and between these actors and stakeholders, brought about by their mutual co-specialization [41-43]. For example, since the performance of EVs depends on driving styles, weather, traffic, infrastructure, etc. [32], it is necessary to add in the EV's complementary services of support to the driver. These services may include navigation services, vehicle support services, advanced charging services, shared mobility services, or insurance. For instance, beyond delivering a car with superior performance-as-developed, the EV ecosystem also entails the emergence of sufficiently robust complements, e.g., charging infrastructure [3]. Thus, in addition to the battery performance and the charging time, the availability of charging infrastructure is somewhat associated with the driving range performance, which is one of the attributes that influence the adoption of EVs $[44,45]$. In this sense, fast and smart charging stations are expected to propel the growth of electric 
vehicles; the slow charging times of the EV are viewed as a liability when compared with the simplicity of filling up at a gas station [46]. In addition, the inclusion of different pricing and technical charging options of time-of-use pricing will encourage consumers to move their charging from peak to off-peak periods.

The rise and fast development of new technologies with in-vehicle systems and applications are constantly transforming the value propositions brought by the EV. This is due to the rise of the affordability and quality of the properties of these new technologies, which are emerging through the new interactions between drivers, EVs, and these technologies [47]. These major innovations are driving automotive firms into more disruptive innovations that are game changing in the sector and are creating new businesses, new models, and major new categories that are completely redefining the competitive environment $[48,49]$. For example, the advances in communication and digitalization have transformed EVs into mobile digital devices or platforms that enable and foster new kinds of interactions with the Internet, people, other cars, road infrastructure, etc., by integrating different hardware and software systems as well as support devices such as sensors, cameras, and radar for different purposes (e.g., active safety, driving assistance, and entertainment). The application of these developments is improving and adding new product and service attributes to EVs and delivering new experiences to both drivers and users. These new features are transforming the concept of a car and demand new kinds of co-specialization and collaborative arrangements within and outside the ecosystem with other related ecosystems that also require new forms of governance and new structures. This also generates new opportunities for firms and entails an underlying competition. For example, the global race to be the first company to bring a fully autonomous vehicle to the marketplace depends on a number of components and subsystems coming together that need to be integrated [50]. These new technologies also allow the creation of new business models with new complementarities, such as car sharing services, which are more cost effective and beneficial to society since they reduce traffic and decrease the demand for parking [51,52]. The introduction of these services is driving different usage patterns of car sharing and private EVs. For example, within these new systems of car-sharing services, new technologies allow firms to introduce pay-per-use systems.

The introduction of these radical innovations and new perspectives is shaking up the established order of the automotive ecosystem and is introducing disorders that are constraining the ability of all actors to achieve a clear, deep, and immediate understanding of the new and upcoming complex problems, challenges, or situations that are about to emerge in the transformation of this ecosystem. The constant development and implementation of these changes are now transforming all ecosystem participants, their relationships, and value-creation processes. These new drivers of ecosystem interaction are blurred by the traditional perception of these ecosystem participants of their environment, hence amplifying the value gap and generating a blind spot in the ecosystem [53]. These blurred perceptions also inhibit the gathering of all actors' insights, especially through intuitive apprehension and a lack of understanding of the upcoming crucial relationships within the ecosystem. At the same time, the evolution of the traditional automotive ecosystem is typically viewed as the evolution of more traditional, linear value chains $[3,12,35]$, which has implied a supply- and production-centric perspective to value creation, where the role of the end user is generally reduced to that of a more or less passive recipient buying the system-level orchestrated offering [54]. Thus, the evolution of the EV sustainable innovation ecosystem is altering and disrupting the structure of the car industry, while at the same time questioning century-old assumptions of technological supremacy as the sole differentiator [55].

Table 1 summarizes the barriers to the development of the EV sustainable innovation ecosystem that have been identified in this section, together with the level of innovation required to overcome each barrier. Issues regarding a lack of standardization, infrastructure, efficient business models, and ecosystem structure all require ecosystem-level 
innovation, and thus need to be solved through widespread collaboration and coevolution of ecosystem participants.

Table 1. Main barriers hindering the evolution of the EV sustainable innovation ecosystem.

\begin{tabular}{cc}
\hline Barrier & Title 2 \\
\hline Price & Firm \\
Performance & Firm \\
Standardization & Ecosystem \\
Infrastructure & Ecosystem \\
Business model & Ecosystem \\
Ecosystem structure & Ecosystem \\
\hline
\end{tabular}

\subsection{The impact of COVID-19 on the EV Ecosystem}

Before 2020, the EV innovation ecosystem was already struggling to achieve a dominant design and widespread diffusion of the EV. We have identified a number of trends associated with the pandemic that have influenced this struggle (Table 2).

Table 2. Trends associated with the pandemic that have influenced the evolution of the EV innovation ecosystem.

\begin{tabular}{cc}
\hline Trend & Impact on EV Evolution \\
\hline Work from home & Decreased mobility and less need for vehicles \\
Private transportation & Increased need for private vehicle ecosystem \\
Decreased spending & EVs are considered too expensive \\
Active travel & Decreased need for vehicles \\
Technology adoption & Increasing inclination to adopt EVs \\
Changing mobility patterns & Uncertainty about future mobility needs \\
Stimulus spending by states & Higher adoption through lower purchase costs \\
\hline
\end{tabular}

\subsubsection{Working from Home}

During the period of the pandemic, automotive consumers and users, as all humans, have been subjected to unprecedented psychological and survival pressures and environment-imposed constraints [56] that have led them to learn and improvise innovative forms to cope with new and blurred boundaries of work, leisure, and education. This has resulted in less commuting to work and other activities. It is quite probable that after the pandemic situation, many meetings will also be held online instead of in person. Thus, there might be a decrease not only in the private demand for vehicles, but also the demand associated with business travel [25].

\subsubsection{Private Transportation}

During the COVID-19 pandemic, there has been a tendency for people to switch to a different transport mode that reduces the risk of infection, but the exact shifts largely depend on their pre-COVID-19 habits [57]. There is a significant shift from public transport to private transport and non-motorized modes [58,59]. For example, people who own a private vehicle will use it increasingly, while those who previously relied on public transport might switch to another mode, such as biking or walking. Some governments encouraged people returning to work to travel by active means or private car instead of using public transport. According to a survey of the consultancy firm McKinsey [57] about the current consumer sentiment and the anticipated future behaviour related to mobility as economies find a next normal, one third of consumers value constant access to a private vehicle more than before COVID-19, especially amongst younger consumers.

Due to the lockdown, internet searches for used cars for sale in the UK have increased [60], and prices have risen to record levels. [61]. Even if there is no clear guarantee that such results will translate into actual purchases, at the very least they suggest a shift in 
opinion [62]. People are more concerned about using private vehicles to travel to/from work, contradicting pre-COVID policy to encourage a modal shift towards more sustainable active and public modes of transport [63].

\subsubsection{Decreased Spending}

People are inclined to spend less on their car, due to economic effects of the COVID- 19 pandemic situation [64]. This can delay the switch to EVs, since the consumer wants to take fewer risks. However, planned spending on vehicles has increased across all geographies vs. previous waves, and this indicates that in some cases EVs may be financially preferable where there are subsidies and tax exemptions in place due to the pandemic effects [64].

\subsubsection{Active Travel}

Many people have switched to new forms of active travel like walking and cycling, alone or with members of a single household. Active travel encompasses all healthy journeys that demand some form of physical exertion on behalf of the individual [65]. Despite their offering a healthy break during the lockdown, they are also feasible alternatives to the private car or public transport for short journeys [63]. This has been taken as a great opportunity by public authorities to rapidly reconfigure and redesign transportation infrastructures in towns and cities, at relatively low cost, to accommodate active travel in order to improve public health and deliver cleaner air [66]. Active travel is the most sustainable form of transport. It does represent a threat to the EV, but cycling is not accessible to all, and inclement weather and cultural and social barriers continue to limit the number of cyclists who are women and ethnic minorities $[67,68]$.

\subsubsection{Technology Adoption}

The pandemic has strengthened the role of new technologies as vital complementarities within the EV. Due to the pandemic, consumers have had to rapidly learn to use and adopt new technologies, thus positively affecting their perceptions and acceptance of new technologies and their added value within the EV as modular offerings that encompass inputs from different sources $[35,69]$. Such acceptance of improved technology due to the pandemic means that EVs are becoming more relevant and competitive. For example, the autonomous and connected EV, if approved for on-road use, could see higher-thanexpected demand, since these vehicles enable physical distancing [70]. Some consultancy firms like Accenture consider that the adoption of the megatrends in the automobile sector (connected, autonomous, shared, and electric driving) will remain unchanged as trends will continue to drive the industry's evolution going forward, but the speed of adoption might slow down due to the pandemic [17].

\subsubsection{Changing Mobility Patterns}

Individual mobility, compared to public transport, leads to higher consumption of natural resources. Hence, there has been a recent trend toward more sustainable behaviours through the use of public transportation, like trains or buses. This is important since sustainable behaviour is not only vital on an institutional level, but also on an individual level [71]. Behaviour during the pandemic is a sign that people adapt quickly to new mobility and driving needs, constraints, and patterns [57,72]. For example, government measures for combating the pandemic, such as movement restriction regulations [73] and their side effects, like panic buying and its time interventions and pressures, have affected consumer behaviours [74]. The population has learned a new skill, i.e., staying at home, which has interfered with individual needs for autonomy, connection, and competence [73]. Faced with this new equilibrium, the consumer has had to adapt by developing and adjusting to new mobility and driving routines, for example, using new routes, new schedules, new mobility purposes, and new destinations. For example, in the UK, "click to car" has become the latest pandemic-friendly way to shop [75]. During this time, the consumer has been experiencing and evaluating these new routines and has been adapting 
them to their needs and convenience. We do not know which of these new routines and spatial and temporal changes in mobility [73] will remain as the new normal or if new ones will arise as a result of those that have emerged during the pandemic.

\subsubsection{Stimulus Spending by States}

As indicated earlier, planned spending on vehicles has increased, and due to the pandemic effects, governmental programs have provided financial support towards the purchase of EVs. Hence, with subsidies and tax exemptions in place [64], a higher adoption through lower purchase costs might also foster EV diffusion. Lower prices might also attract entrepreneurial action, with further competition in the future [76].

For instance, Germany has now overtaken, in terms of EV sales, California, the home of Tesla, due to recently introduced state-funded subsidies [77].

\subsection{Long-Term Influence of the Crisis}

McKinsey believes that policy makers react differently across regions, since some might view the crisis as an opportunity to reconfigure future transport policy and practice for the benefit of the global environment and individual citizens alike, while others might loosen regulatory mandates to prop up their automotive industries [70]. For example, if physical distancing continues, governments might relax regulations for private mobility, at least over the short term, because people feel less vulnerable to infection in individually owned vehicles [70]; this contradicts pre-COVID-19 policies about the sustainability of public modes of transport. On the contrary, due to the new human mobility behaviours, policy makers might also revise the local mobility regulations to give more space to pedestrians and cyclists. Governments should analyse and develop localised movement policies and regulations [73]. The design of incentives, e.g., green mobility incentives, should also be aligned with such regulations and policies. Previous approaches and policies to mitigate transport noise, emissions, congestion, etc., such as smart mobility, active travel initiatives, and tax reductions, on their own will be inadequate in a postCOVID-19 world because they don't take into account the relevant knowledge about the new needs and customs within individual and corporate travel behaviour [63].

\subsubsection{The COVID-19 Pandemic as a Strategic Opportunity}

The COVID-19 shutdown is an opportunity to reconfigure future transport policy and practice for the benefit of the global environment and individual citizens alike [63]. EV firms should now focus on resource optimization and standardization, new growth segments, and cost rationalization to overcome slowdown [78], and this will facilitate their transition to the mass market. As an additional strategic opportunity, the pandemic represents a testing ground for EV firms and governments alike, as they can measure the effects on consumers' perceptions of the different decisions made in terms of the introduction and further development of new technologies within the EV, the design of new regulations, and incentives for EVs. This will enhance the framing of more reliable strategic visions and more appealing value propositions for the consumers of EVs, which can accelerate the transition to EVs over ICE vehicles.

\subsubsection{Automotive Supply Chain Resilience to the COVID-19 Outbreak}

The countermeasures against the pandemic have caused increased border restrictions and complete nationwide lockdowns, leading to important disruptions to international trade and global supply chains [25], especially in the automobile markets. For example, the number of EV models might be reduced to cut costs. Previous strategies related to global supply-chain efficiency have made the supply chain vulnerable to this disruption [17]. These negative consequences have pushed firms to rethink their strategies regarding supply chain resilience (SCR), which refers to the supply chains' ability to prevent and absorb changes as well as regain or improve the initial performance level after an unexpected disturbance [79]. The pandemic has revealed that many companies were focused only 
on the quantification of the resilience level and the resulting consequences, rather than the development of both response and recovery strategies [80-82], thereby limiting the capacity of recovering from disruptions [82]. The global analytics firm Crisil has identified the automobile industry as having been highly impacted by the COVID-19 pandemic due to the industry's low resilience [83]. Industry firms can now reconfigure their supply chain resilience strategies in order to predict, be prepared for, and understand the extent of the impact of a future disruption by devising adequate strategies to respond to and cope quickly with the consequences of a disruption and reconfiguring their resources to strengthen competencies and adapt to the consequent effects $[21,84]$. Such resilient post-COVID-19 strategies require increasing organizational frugality and adapting strategy processes to the new normal [76]. While the resilience of the automobile supply chain has attracted significant attention in recent times, the existing literature lacks empirical investigation into building predictive, receptive, and preventative supply chain resilience strategies and has not addressed the global supply chain impact $[85,86]$.

\section{Discussion}

When analysing the case of the EV ecosystem during the pandemic, we find that a crisis can serve as an opportunity for new innovations to break through established barriers by disrupting prior behavioural patterns. In the case of the EV, these patterns are mainly related to mobility, but other industries may experience similar disruptions to other patterns. Ecosystem innovation requires aligning ecosystem participants, and a crisis can serve as the necessary impetus that motivates actors towards a joint objective. While posing many challenges, a crisis also offers opportunities. A thorough analysis of the interactions within the ecosystem will render the opportunities presented by this disruption applicable for other innovation ecosystems. Future research can also quantitatively measure and test the factors identified in this study.

The coronavirus pandemic has highlighted the importance of further developing current and new product attributes in response to the new trends and personal protective issues generated with the advent of the pandemic [17]. For example, EV manufacturers are encouraged to shift towards health and wellness solutions in vehicles as part of the new value propositions [78]. Vehicle manufacturers are reconfiguring the internal layout of seats and circulation spaces on buses, taxis, etc., and are installing contactless door sensors and hand-sanitizer dispensers as well as clear screens between seats to provide a physical barrier to airborne aerosols [87]; however, the efficacy and levels of public acceptance of these new configurations are unknown [63].

The COVID-19 pandemic is affecting oil demand and supply, since it has helped trigger a dramatic fall in oil prices due to coordinated massive production cuts to offset the collapse in oil demand [88]. As a consequence, previously planned oil exploration and production may be abandoned on cost grounds and the perceived weakness or uncertainty of demand. The forces of the pandemic will permit slow recovery of the oil demand, thereby curbing major oil price rises for at least three or four years [88]. Some experts have suggested that this could hinder the perceptions of drivers regarding EVs as they look to capitalise on the cost savings associated with lower fuel costs [62]. These factors slow down the pace of transition to more sustainable modes of transportation.

The pandemic has reconfigured the demand for, as well as the role and mobility of, light commercial vehicles (LCVs) due to their role during COVID-19. Panic buying in supermarkets was quickly replaced with overwhelming demand for online food ordering and delivery and retail deliveries as consumers tried to avoid going outside [89]. For some logistics providers, this might mean increasing the number of LCVs in their fleet to cope with a greater number of deliveries. COVID-19 has become a sudden catalyst for change within strategic fleet management because logistic operators have been conveyed to reconfigure and renovate their value propositions. This is a great opportunity for EV manufacturers that can extend their product portfolios to new models of electric LCVs. It 
is fundamental that these companies collaborate with logistics companies and with rental companies for commercial fleets.

There is an apparent contradiction between the post-COVID priorities of economic growth needed for a fast economic recovery and the environmental safeguarding and protection priorities through top-down interventions [63]. Restarting the global economy will inevitably require the increased mobility of people and movement of goods, but this contradiction generates a knowledge gap, since all actors in an ecosystem need to align themselves in order to find a delicate new equilibrium and shared new vision; this is not easy to configure between strategic demands and within a period of transition to a wider and mass technological acceptance. This coherent shared vision among participants may therefore reduce the gap of uncertainty and lower the threshold of complements necessary to invest in this emerging ecosystem of the EV [54,90] New business models, e.g., for car sharing, indicate the additional potential of the EV for less costly and more sustainable modes of ownership and transportation. Future research could push this line of investigation further by developing and testing even more sustainable models, such as those based on a circular economy, with recycling and repurposing of vehicles and their parts [91].

Political action is fundamental for EV uptake since, if policy support is lacking, EV sales will slow down [92-94]. EV adoption requires policy interventions as it is a technological change that is faced with market, system, and institutional failures [95]. Current EV adoption rates are generally low in countries with no or weak policy interventions in this area and higher in countries with strong policies $[93,96,97]$, which suggests that policy interventions can contribute to changing behaviour [98]. The policy environment provides an important set of contextual factors for consumers [99], and even if it does not affect consumer EV adoption directly, it interacts with psychological factors, moderating their relationships with EV adoption [100]. For example, perceived behavioural control may lead to high EV purchase intentions only when financial policy instruments sufficiently reduce the price gap between EVs and ICE vehicles [101]. There is still an important research gap regarding empirical analysis on the effect of policies on EVs [102]. It has been suggested that the hybrid data-driven models that combine both macroeconomic and microeconomic variables are preferable to other methodologies (e.g., agent-based) that have delivered biased predictions; however, there is no unanimity on which method is the most appropriate [103].

Innovation policy can support the investment of research and development funds and the improvement of innovation capabilities for entire innovation ecosystems [102]. The "double credit policy" uses different reward and punishment mechanisms simultaneously to block the development of the ICE vehicle industry and promote the development of new energy vehicles [103]. Another possibility, especially for emerging countries, is public investment in the domestic automotive industry, such as favourable financing or requiring local manufacturing to qualify for subsidies; this has proven effective in the development of EVs that meet the needs of domestic populations [104].

The effectiveness and efficiency of different policy instruments may be similar depending on their design and robustness from a purely economic viewpoint, but also on their political feasibility and their effects on public opinion [105]. Pull policies, e.g., subsidies, attract more public support than push measures, e.g., fuel taxes and travel restrictions. In addition, there is considerable political room to manoeuvre for more ambitious pull measures, such as the large-scale expansion of public charging infrastructure.

\section{Conclusions}

A crisis tends to foment the emergence of a dominant design in science-based industries [106]. However, we have analysed factors and circumstances that both support the EV innovation ecosystem as a whole, and slow down ecosystem growth. To increase the pace of transition to EVs, countries with key markets must shape and implement jointly common and synchronized policy packages to enhance policy synergies and effects between 
countries [107]. Although different prediction models have been designed for the diffusion of the electric car at the national level, no truly global diffusion model has been agreed upon and developed to investigate EV uptake [107]. The identified factors demonstrate that this sector still lacks a full perspective, structure, and ecosystem governance, since the coordination of policies requires cooperation not only from different public national and international authorities but also between the different stakeholders and participants in the ecosystem. These negotiations require the full commitment of the global players, including governments and EV manufacturers. An extended charging infrastructure for EVs is thus equal in importance to the institutional infrastructure supporting the resilience of the EV innovation ecosystem. Finally, the sustainability of the EV innovation ecosystem depends on whether it's fuelled by green energy. Carbon-intensive electricity sources imply little improvement compared to ICE vehicles, and the use of climate-friendly energy, e.g., biogas and biomass, is crucial to make the EV ecosystem part of climate action [108]. The support of such underlying energy infrastructure hence defines the climate impact of the EV ecosystem.

Author Contributions: M.A. initially conceptualized this article, collected and analysed data, and drafted/revised the manuscript; P.A.N. and A.B. provided conceptual input and comments and contributed to writing/revising main parts of the article. All authors have read and agreed to the published version of the manuscript.

Funding: This research received no external funding.

Institutional Review Board Statement: Not applicable.

Informed Consent Statement: Not applicable.

Data Availability Statement: Data is contained within the article.

Acknowledgments: The authors would like to thank Lena Krebs for her support with proofreading of the article.

Conflicts of Interest: The authors declare no conflict of interest.

\section{References}

1. D'Adamo, I.; Rosa, P. How Do You See Infrastructure? Green Energy to Provide Economic Growth after COVID-19. Sustainability 2020, 12, 4738. [CrossRef]

2. IEA. Global EV Outlook. Global Electric Car Stock, 2010-2019. 2020. Available online: https:/ /www.iea.org/reports/global-evoutlook-2020 (accessed on 20 November 2020).

3. Adner, R.; Kapoor, R. Innovation ecosystems and the pace of substitution: Reexamining technology s-curves. Strateg. Manag. J. 2016, 37, 625-648. [CrossRef]

4. Utterback, J.M. Mastering the Dynamics of Innovation; Harvard Business School Press: Boston, MA, USA, 1994.

5. Harrop, P. Electric Vehicles Enter Phase of Fastest Growth. 2019. Available online: https://www.idtechex.com/fr/researcharticle/electric-vehicles-enter-phase-of-fastest-growth/17044 (accessed on 23 September 2020).

6. Wollschlaeger, D.; Foden, M.; Cave, R.; Stent, M. Digital Disruption and the Future of the Automotive Industry. Automotive RevolutionPerspective Towards 2030; IBM Corp: Armonk, NY, USA, 2015.

7. Holweg, M. The evolution of competition in the automotive industry. In Build to Order; Springer: London, UK, 2008; pp. 13-34.

8. Gerhard, D.; Brem, A.; Voigt, K.I. Product development in the automotive industry: Crucial success drivers for technological innovations. Int. J. Technol. Mark. 2008, 3, 203-222. [CrossRef]

9. Nagji, B.; Tuff, G. Managing Your Innovation Portfolio. Harv. Bus. Rev. 2012, 90, 66-73.

10. Sodenkamp, M.A.; Wenig, J.; Thiesse, F.; Staake, T. Who can drive electric? Segmentation of car drivers based on longitudinal GPS travel data. Energy Policy 2019, 130, 111-129. [CrossRef]

11. Iansiti, M.; Levien, R. Strategy as ecology. Harv. Bus. Rev. 2004, 82, 68-78.

12. Adner, R.; Kapoor, R. Value creation in innovation ecosystems: How the structure of technological interdependence affects firm performance in new technology generations. Strateg. Manag. J. 2010, 31, 306-333. [CrossRef]

13. Teece, D.J. Profiting from innovation in the digital economy: Enabling technologies, standards, and licensing models in the wireless world. Res. Policy 2018, 47, 1367-1387. [CrossRef]

14. Priem, R.L. A consumer perspective on value creation. Acad. Manag. Rev. 2007, 32, 219-235. [CrossRef]

15. Thomas, L.; Autio, E.; Oxford Research Encyclopedia of Business and Management. Innovation Ecosystems in Management: An Organizing Typology. 2020. Available online: https:/ / oxfordre.com/business/view/10.1093/acrefore/9780190224851.001.0001 /acrefore-9780190224851-e-203 (accessed on 20 November 2020). 
16. Cennamo, C.; Santalo, J. Platform competition: Strategic trade-offs in platform markets. Strateg. Manag. J. 2013, 34, 1331-1350. [CrossRef]

17. Accenture Strategy. The Transforming Mobility Land Scape. Industry Insights Mobility. 2020. Available online: https://www. accenture.com/us-en/insights/automotive/transforming-mobility-landscape (accessed on 25 November 2020).

18. Kaitwade, N. COVID-19 shatters global automotive industry; sales of metal powder take a nosedive amid wavering demand. Met. Powder Rep. 2020. [CrossRef]

19. Araz, O.M.; Choi, T.; Olson, D.; Salman, F. Data Analytics for Operational Risk Management. Decis. Sci. 2020, 51, 1316-1319. [CrossRef]

20. Nylund, P.A.; Brem, A.; Agarwal, N. Innovation ecosystems for meeting sustainable development goals: The evolving roles of multinational enterprises. J. Clean. Prod. 2021, 281, 125329. [CrossRef]

21. Belhadi, A.; Kamble, S.; Jabbour, C.J.C.; Gunasekaran, A.; Ndubisi, N.O.; Venkatesh, M. Manufacturing and service supply chain resilience to the COVID-19 outbreak: Lessons learned from the automobile and airline industries. Technol. Forecast. Soc. Change 2020, 163, 120447. [CrossRef]

22. Arnold, M. Europe's New COVID Outbreaks Raise Threat of Double-Dip Recession. Financial Times, 18 October 2020.

23. Eurometal. Second COVID-19 Wave Could Delay Automotive Sector Recovery. Available online: https:/ / eurometal.net/secondCOVID-19-wave-could-delay-automotive-sector-recovery / (accessed on 16 June 2020).

24. Strauss, S.D. Some Emerging Hypotheses on the Economic Opportunities and Challenges of the Post-Pandemic World; Princeton University-Woodrow Wilson School of Public and International Affairs: Princeton, NJ, USA, 2020.

25. Brem, A.; Viardot, E.; Nylund, P.A. Implications of the coronavirus (COVID-19) outbreak for innovation: Which technologies will improve our lives? Technol. Forecast. Soc. Chang. 2021, 163, 120451. [CrossRef]

26. Eisenhardt, K.M.; Graebner, M.E.; Sonenshein, S. Grand challenges and inductive methods: Rigor without rigor mortis. Acad. Manag. J. 2016, 59, 1113-1123. [CrossRef]

27. Eisenhardt, K.M. Building theories from case study research. Acad. Manag. Rev. 1989, 14, 532-550. [CrossRef]

28. Yin, R.K. Case Study Research: Design and Methods; Sage: Thousand Oaks, CA, USA, 1994.

29. Beltagui, A.; Rosli, A.; Candi, M. Exaptation in a digital innovation ecosystem: The disruptive impacts of 3D printing. Res. Policy 2020, 49, 103833. [CrossRef]

30. Gifford, E.; McKelvey, M.; Saemundsson, R. The evolution of knowledge-intensive innovation ecosystems: Co-evolving entrepreneurial activity and innovation policy in the West Swedish maritime system. Ind. Innov. 2020, 1-26. [CrossRef]

31. Wilken, D.; Oswald, M.; Draheim, P.; Pade, C.; Brand, U.; Vogt, T. Multidimensional assessment of passenger cars: Comparison of electric vehicles with internal combustion engine vehicles. Procedia CIRP 2020, 90, 291-296. [CrossRef]

32. Donkers, A.J.; Yang, D.; Viktorović, M. Influence of driving style, infrastructure, weather and traffic on electric vehicle performance. Transp. Res. D-transp. Environ. 2020, 88, 102569. [CrossRef]

33. Egede, P.; Dettmer, T.; Herrmann, C.; Kara, S. Life Cycle Assessment of Electric Vehicles—A Framework to Consider Influencing Factors. Procedia CIRP 2015, 29, 233-238. [CrossRef]

34. Adner, R. Ecosystem as structure: An actionable construct for strategy. J. Manag. 2017, 43, 39-58. [CrossRef]

35. Jacobides, M.G.; Cennamo, C.; Gawer, A. Towards a theory of ecosystems. Strateg. Manag. J. 2018, 39, 2255-2276. [CrossRef]

36. Brem, A.; Nylund, P.A.; Schuster, G. Innovation and de facto standardization: The influence of dominant design on innovative performance, radical innovation, and process innovation. Technovation 2016, 50, 79-88. [CrossRef]

37. Ellingsen, L.; Hung, C.; Stromman, A.H. Identifying key assumptions and differences in life cycle assessment studies of lithium-ion traction batteries with focus on greenhouse gas emissions. Transp. Res. D-transp. Environ. 2017, 55, 82-90. [CrossRef]

38. Peters, J.F.; Baumann, M.; Zimmermann, B.; Braun, J.; Weil, M. The environmental impact of Li-Ion batteries and the role of key parameters-A review. Renew. Sustain. Energy Rev. 2017, 67, 491-506. [CrossRef]

39. Cox, B.; Mutel, C.; Bauer, C.; Beltrán, A.M.; Vuuren, D.V. Uncertain Environmental Footprint of Current and Future Battery Electric Vehicles. Environ. Sci. Technol. 2018, 52, 4989-4995. [CrossRef]

40. Schmidt, T.; Beuse, M.; Zhang, X.; Steffen, B.; Schneider, S.; Pena-Bello, A.; Bauer, C.; Parra, D. Additional Emissions and Cost from Storing Electricity in Stationary Battery Systems. Environ. Sci. Technol. 2019, 53, 3379-3390. [CrossRef]

41. Teece, D.J. Profiting from technological innovation: Implications for integration, collaboration, licensing. Res. Policy 1986, 15, 285-305. [CrossRef]

42. Alexy, O.; George, G.; Salter, A. Cui Bono? The Selective Revealing of Knowledge and Its Implications for Innovative Activity. Acad. Manag. Rev. 2013, 38, 270-291. [CrossRef]

43. Kapoor, R.; Lee, J.M. Coordinating and competing in ecosystems: How organizational forms shape new technology investments. South. Med. J. 2013, 34, 274-296. [CrossRef]

44. Leviäkangas, P.; Kinnunen, T.; Kess, P. The Electric Vehicles Ecosystem Model: Construct, Analysis and Identification of Key Challenges. Manag. Glob. Trans. 2014, 12, 253-277.

45. Kim, S.; Lee, J.; Lee, C. Does driving range of electric vehicles influence electric vehicle adoption? Sustainability 2017, 9 , 1783. [CrossRef]

46. Gnann, T.; Funke, S.Á.; Jakobsson, N.; Plötz, P.; Sprei, F.; Bennehag, A. Fast charging infrastructure for electric vehicles: Today's situation and future needs. Transp. Res. D-transp. Environ. 2018, 62, 314-329. [CrossRef]

47. Gibson, J.J. The Ecological Approach to Perception; Houghton Mifflin: London, UK, 1979. 
48. Birkinshaw, J.; Bouquet, C.; Barsoux, J.-L. The 5 Myths of Innovation. MIT Sloan Manag. Rev. 2011, 52, 43-50.

49. Goffin, K.; Mitchell, R. Innovation Management, 3rd ed.; Red Globe Press: London, UK, 2017.

50. Faisal, A.; Yigitcanlar, T.; Kamruzzaman, M.; Paz, A. Mapping Two Decades of Autonomous Vehicle Research: A Systematic Scientometric Analysis. J. Urban Technol. 2020, 1-30. [CrossRef]

51. Conner-Simons, A. How Ride-Sharing Can Improve Traffic, Save Money, and Help the Environment. 2017. Available online: http:/ / news.mit.edu/2016/how-ride-sharing-can-improve-traffic-save-money-and-help-environment-0104 (accessed on 5 October 2020).

52. Kiron, D. How Next Gen Car Sharing Will Transform Transportation. MIT Sloan Manag. Rev. 2013, 54, 1.

53. Thaler, R. Misbehaving: The Making of Behavioral Economics, 1st ed.; W.W. Norton and Company: New York, NY, USA, 2015.

54. Autio, E.; Thomas, L.W. Value co-creation in ecosystems: Insights and research promise from three disciplinary perspectives. In Handbook of Digital Innovation; Edward Elgar Publishing: Cheltenham, UK, 2019.

55. Ferràs-Hernández, X.; Tarrats-Pons, E.; Arimany-Serrat, N. Disruption in the automotive industry: A Cambrian moment. Bus. Horiz. 2017, 60, 855-863. [CrossRef]

56. Sofi, S.A.; Mir, F.A.; Baba, M.M. Cognition and affect in consumer decision making: Conceptualization and validation of added constructs in modified instrument. Futur. Bus. J. 2020, 6, 1-20. [CrossRef]

57. McKinsey \& Co. Moving Forward: How COVID-19 Will Affect Mobility in the United Kingdom. 2020. Available online: https://www.mckinsey.com/ \{\}/media/McKinsey/Industries/Automotive\%20and\%20Assembly/Our\%20Insights / Moving\%20forward\%20How\%20COVID\%2019\%20will\%20affect\%20mobility\%20in\%20the\%20United\%20Kingdom/Movingforward-How-COVID-19-might-affect-mobility-in-the-United-Kingdom-vF.pdf (accessed on 24 November 2020).

58. Abdullah, M.; Dias, C.; Muley, D.; Shahin, M. Exploring the impacts of COVID-19 on travel behaviour and mode preferences. Transp. Res. Interdiscip. Perspect. 2020, 8, 100255.

59. Moslem, S.; Campisi, T.; Szmelter-Jarosz, A.; Duleba, S.; Nahiduzzaman, K.M.; Tesoriere, G. Best-worst method for modelling mobility choice after COVID-19: Evidence from Italy. Sustainability 2020, 12, 6824. [CrossRef]

60. Kirwan, J.; Motortrader.com. Used Car Prices Rise with Demand Exceeding Supply. Available online: https:/ /www.motortrader. $\mathrm{com}$ / motor-trader-news/automotive-news/used-car-prices-rise-demand-exceeding-supply-18-09-2020 (accessed on 18 September 2020).

61. Financial Times. UK Lockdown Measures Drive Used Car Prices to Record Growth. Available online: https://www.ft.com/ content/6617dc19-6302-42b6-9c98-94faecb0dd8c (accessed on 2 October 2020).

62. Oxford Business Group. Can the Automotive Industry Adapt to a COVID-19 World? Available online: https:// oxfordbusinessgroup.com/news / can-automotive-industry-adapt-COVID-19-world (accessed on 30 June 2020).

63. Budd, L.; Ison, S. Responsible Transport: A post-COVID agenda for transport policy and practice. Transp. Res. Interdiscip. Perspect. 2020, 6, 100151.

64. Furcher, T.; Grühn, B.; Huber, I.; Tschiesner, A. COVID-19 Auto and Mobility Insights. 2020. Available online: https: //www.mckinsey.com/business-functions/marketing-and-sales/our-insights/how-consumers-behavior-in-car-buying-andmobility-changes-amid-COVID-19 (accessed on 2 November 2020).

65. Sport England. Active Travel and Physical Activity Evidence Review. 2019. Available online: https: / / www.sportengland.org/ know-your-audience/demographic-knowledge/active-travel?section=our_research (accessed on 12 October 2020).

66. Greenpeace, Manifesto for a Green Recovery. 2020. Available online: https://www.greenpeace.org.uk/resources/green-recoverymanifesto/ (accessed on 4 June 2020).

67. Corcoran, J.; Li, T.; Rohde, D.; Charles-Edwards, E.; Mateo-Babiano, D. Spatio-temporal patterns of a Public Bicycle Sharing Program: The effect of weather and calendar events. J. Transp. Geogr. 2014, 41, 292-305. [CrossRef]

68. Goodman, A.; Aldred, R. Inequalities in utility and leisure cycling in England, and variation by local cycling prevalence. Transp. Res. 2018, 56, 381-391. [CrossRef]

69. Reynolds, P.; Bosma, N.; Autio, E.; Hunt, S.; De Bono, N.; Servais, I.; Lopez-Garcia, P.; Chin, N. Global entrepreneurship monitor: Data collection design and implementation 1998-2003. Small Bus. Econ. 2005, 24, 205-231. [CrossRef]

70. Hausler, S.; Heineke, K.; Hensley, R.; Möller, T.; Schwedhelm, D.; Shen, P.; McKinsey. The Impact of COVID-19 on Future Mobility Solutions. 2020. Available online: https://www.mckinsey.com/ \{\}/media/McKinsey/Industries/Automotive \%20and\%20 Assembly /Our\%20Insights/The\%20impact\%20of\%20COVID19\%20on \%20future \%20mobility \%20solutions/The-impact-ofCOVID-19-on-future-mobility-solutions-vF.ashx (accessed on 3 December 2020).

71. Brem, A.; Puente-Díaz, R. Are you acting sustainably in your daily practice? Introduction of the Four-S model of sustainability. J. Clean. Prod. 2020, 267, 122074. [CrossRef]

72. Sheth, J. Impact of COVID-19 on consumer behavior: Will the old habits return or die? J. Bus. Res. 2020, 117, 280-283. [CrossRef]

73. Drake, T.M.; Docherty, A.B.; Weiser, T.G.; Yule, S.; Sheikh, A.; Harrison, E.M. The effects of physical distancing on population mobility during the COVID-19 pandemic in the UK. Lancet Digit. Health 2020, 2, 385-387. [CrossRef]

74. Prentice, C.; Chen, J.; Stantic, B. Timed intervention in COVID-19 and panic buying. J. Retail. Consum. Serv. 2020, 57, 102203. [CrossRef]

75. Eccles, L. COVID-Conscious Can Go Shopping without Leaving Their Own Car. The Sunday Times, 20 September 2020.

76. Giones, F.; Brem, A.; Pollack, J.M.; Michaelis, T.L.; Klyver, K.; Brinckmann, J. Revising entrepreneurial action in response to exogenous shocks: Considering the COVID-19 pandemic. J. Bus. Ventur. Insights 2020, 14, e00186. [CrossRef] 
77. Bloomberg. Germany's Electric-Car Market Is Poised to Overtake California's, William Wilkes. Available online: https://www. bloomberg.com/news/articles/2020-12-03/germany-s-electric-car-market-is-poised-to-overtake-california-s (accessed on 4 December 2020).

78. Research and Markets. COVID-19 Growth Impact Assessment for the Automotive Industry. 2020. Available online: http: / / www.researchandmarkets.com (accessed on 3 December 2020).

79. Hendry, L.; Stevenson, M.; MacBryde, J.; Ball, P.; Sayed, M.; Liu, L. Local food supply chain resilience to constitutional change: The Brexit effect. Int. J. Oper. Prod. Manag. 2019, 39, 429-453. [CrossRef]

80. Hosseini, S.; Ivanov, D. Resilience assessment of supply networks with the ripple effect considerations: A Bayesian network approach. Ann. Oper. Res. 2019, 278,1-27.

81. Graveline, N.; Grémont, M. Measuring and understanding the microeconomic resilience of businesses to lifeline service interruptions due to natural disasters. Int. J. Disaster Risk Reduct. 2017, 24, 526-538. [CrossRef]

82. Ivanov, D.; Dolgui, A.; Sokolov, B.; Ivanova, M. Literature review on disruption recovery in the supply chain *. Int. J. Prod. Res. 2017, 55, 6158-6174. [CrossRef]

83. Crisil Research. Sector Report: Automotive Components. Available online: https://www.crisil.com/en/home/our-analysis/ reports /2017/09/ sector-report-automotive-components.html (accessed on 21 September 2020).

84. Elleuch, H.; Dafaoui, E.M.; Elmhamedi, A.; Chabchoub, H. Resilience and Vulnerability in Supply Chain: Literature review. IFAC PapersOnLine 2016, 49, 1448-1453. [CrossRef]

85. Scavarda, L.F.; Ceryno, P.S.; Pires, S.; Klingebiel, K. Supply chain resilience analysis: A brazilian automotive case. Rev. Adm. Empresas 2015, 55, 304-313. [CrossRef]

86. Bevilacqua, M.; Ciarapica, F.E.; Marcucci, G. Supply Chain Resilience research trends: A literature overview. IFAC PapersOnLine 2019, 52, 2821-2826. [CrossRef]

87. Paton, G.; The Times. Contactless Doors and Visors are the Future for Rail. Available online: https://www.thetimes.co.uk/ article/coronavirus-contactlessdoors-and-visors-are-the-future-for-rail-qgsnd6p08 (accessed on 26 May 2020).

88. Jefferson, M. A crude future? COVID-19s challenges for oil demand, supply and prices. Energy Res. Soc. Sci. 2020, 68, 101669. [CrossRef] [PubMed]

89. Hanbury, M.; Business Insider. UK Grocery Chains Add Hundreds of Thousands of Delivery Slots for Online Orders but Admit that They Still Can't Keep Up with Demand. Available online: https: / /www.businessinsider.com/tesco-ocado-sainsburys-cantkeep-up-with-surging-demand-amid-coronavirus-4? $\mathrm{r}=\mathrm{US} \& \mathrm{IR}=\mathrm{T}$ (accessed on 8 April 2020).

90. Dattée, B.; Alexy, O.; Autio, E. Maneuvering in poor visibility: How firms play the ecosystem game when uncertainty is high. Acad. Manag. J. 2018, 61, 466-498. [CrossRef]

91. Wurster, S.; Heß, P.; Nauruschat, M.; Jütting, M. Sustainable Circular Mobility: User-Integrated Innovation and Specifics of Electric Vehicle Owners. Sustainability 2020, 12, 7900. [CrossRef]

92. Lévay, P.Z.; Drossinos, Y.; Thiel, C. The effect of fiscal incentives on market penetration of electric vehicles: A pairwise comparison of total cost of ownership. Energy Policy 2017, 105, 524-533. [CrossRef]

93. Hardman, S. Understanding the impact of reoccurring and non-financial incentives on plug-in electric vehicle adoption-A review. Transp. Res. A-policy Pract. 2019, 119, 1-14. [CrossRef]

94. Nykvist, B.; Sprei, F.; Nilsson, M. Assessing the progress toward lower priced long range battery electric vehicles. Energy Policy 2019, 124, 144-155. [CrossRef]

95. Weber, K.M.; Rohracher, H. Legitimizing research, technology and innovation policies for transformative change: Combining insights from innovation systems and multi-level perspective in a comprehensive 'failures' framework. Res. Policy 2012, 41, 1037-1047. [CrossRef]

96. Sierzchula, W.; Bakker, S.; Maat, K.; Van Wee, B. The influence of financial incentives and other socio-economic factors on electric vehicle adoption. Energy Policy 2014, 68, 183-194. [CrossRef]

97. Rietmann, N.; Lieven, T. How policy measures succeeded to promote electric mobility-Worldwide review and outlook. J. Clean. Prod. 2019, 206, 66-75. [CrossRef]

98. Tummers, L. Public Policy and Behavior Change. Public Adm. Rev. 2019, 79, 925-930. [CrossRef]

99. Zhang, X.; Bai, X.; Zhong, H. Electric vehicle adoption in license plate-controlled big cities: Evidence from Beijing. J. Clean. Prod. 2018, 202, 191-196. [CrossRef]

100. Steg, L.; Vlek, C. Encouraging pro-environmental behaviour: An integrative review and research agenda. J. Environ. Psychol. 2009, 29, 309-317. [CrossRef]

101. Huang, X.; Ge, J. Electric vehicle development in Beijing: An analysis of consumer purchase intention. J. Clean. Prod. 2019, 216, 361-372. [CrossRef]

102. Hu, Y.; Wang, Z.; Li, X. Impact of policies on electric vehicle diffusion: An evolutionary game of small world network analysis. J. Clean. Prod. 2020, 265, 121703. [CrossRef]

103. Jochem, P.; Vilchez, J.J.; Ensslen, A.; Schäuble, J.; Fichtner, W. Methods for forecasting the market penetration of electric drivetrains in the passenger car market. Transp. Rev. 2018, 38, 322-348. [CrossRef]

104. Government of India. Minutes of the Meeting of Committee for Finalization of Demand and Supply Side Incentives for Promotion of Electric Mobility Held on 22nd February 2018. 2018. Available online: https://dhi.nic.in/writereaddata/UploadFile/ Demand\%20Supply\%20side\%20Incentives636663000191442326.pdf (accessed on 22 September 2020). 
105. Brückmann, G.; Bernauer, T. What drives public support for policies to enhance electric vehicle adoption. Environ. Res. Lett. 2020, 15, 094002. [CrossRef]

106. Brem, A.; Nylund, P.; Viardot, E. The impact of the 2008 financial crisis on innovation: A dominant design perspective. J. Bus. Res. 2020, 110, 360-369. [CrossRef]

107. Gómez Vilchez, J.; Jochem, P.; Fichtner, W. Interlinking major markets to explore electric car uptake. Energy Policy 2020, 144, 111588. [CrossRef]

108. Karmaker, A.K.; Hossain, M.; Manoj Kumar, N.; Jagadeesan, V.; Jayakumar, A.; Ray, B. Analysis of Using Biogas Resources for Electric Vehicle Charging in Bangladesh: A Techno-Economic-Environmental Perspective. Sustainability 2020, 12, 2579. [CrossRef] 


\title{
E-Commerce Calls for Cyber-Security and Sustainability: How European Citizens Look for a Trusted Online Environment
}

\author{
Idiano D'Adamo ${ }^{1, *(1)}$, Rocío González-Sánchez ${ }^{2}\left(\mathbb{D}\right.$, Maria Sonia Medina-Salgado ${ }^{2} \mathbb{C}$ \\ and Davide Settembre-Blundo ${ }^{2}$ (D) \\ 1 Department of Computer, Control and Management Engineering, Sapienza University of Rome, \\ 00185 Rome, Italy \\ 2 Department of Business Administration (ADO), Applied Economics II and Fundamentals of Economic \\ Analysis, Rey Juan Carlos University, 28032 Madrid, Spain; rocio.gonzalez@urjc.es (R.G.-S.); \\ sonia.medina@urjc.es (M.S.M.-S.); davide.settembre@urjc.es (D.S.-B.) \\ * Correspondence: idiano.dadamo@uniroma1.it
}

check for updates

Citation: D'Adamo, I.; González-Sánchez, R. Medina-Salgado, M.S.; Settembre-Blundo, D. E-Commerce Calls for Cyber-Security and Sustainability: How European Citizens Look for a Trusted Online Environment. Sustainability 2021, 13, 6752. https://doi.org/10.3390/ su13126752

Academic Editor: Giuseppe Ioppolo

Received: 17 May 2021

Accepted: 12 June 2021

Published: 15 June 2021

Publisher's Note: MDPI stays neutral with regard to jurisdictional claims in published maps and institutional affiliations.

Copyright: (c) 2021 by the authors. Licensee MDPI, Basel, Switzerland. This article is an open access article distributed under the terms and conditions of the Creative Commons Attribution (CC BY) license (https:/ / creativecommons.org/licenses/by/ $4.0 /)$.
Abstract: The pandemic has changed the citizens' behavior, inducing them to avoid any real contact. This has given an incredible impulse to e-commerce; however, the complexity of the topic has not yet been adequately explored in the literature. To fill this gap, this study has a twofold purpose: (1) to investigate how European countries comparatively perform in e-commerce, and (2) to describe what are the most important challenges for the further expansion of e-commerce. To this end, we adopted a hybrid methodology based on multi-criteria decision analysis (MCDA) and a Likert scale survey. The first method allows to us rank the e-commerce performance of different European countries, while the second one looks at the problems and barriers that characterize online shopping. The results of the study show that European countries have different sensitivities to the issue of cyber-security, and among them it is possible to identify three groups with different levels of attention to the critical issues of e-commerce. The Netherlands, Sweden and Denmark belong to the group of countries most responsive to e-commerce. This request is part of a broader framework of transition toward sustainable development, i.e., a reliable digital environment where citizens and businesses can exercise their rights and freedoms in complete security. Finally, from a theoretical perspective, this paper adds a new baseline to the literature on the state of the art of e-commerce in Europe that addresses the effects of the pandemic. From a managerial point of view, decision makers can find in the results of this analysis a support for the setting of business strategies for the expansion of firms in certain markets and guidance for public authorities when defining regulatory policies for e-commerce.

Keywords: cyber-security; e-commerce; Europe; sustainability

\section{Introduction}

Since the 2000s, the sudden development of digital technologies and the exponential spread of the Internet Economy have revolutionized people's lives due to the simultaneous and progressive increase in bandwidth for web connections, first on fixed locations and then on mobile ones [1]. All this has generated a great paradigm change in society, which has influenced users' purchasing habits and the companies' way of selling products or services [2]. Thus, the evolution of cyber-security has followed the ongoing progress of communication technologies [3]. In this new environment, cyber-security is gaining special relevance, just like the innovations that are rapidly appearing in this new digital market. Speed, mobility, data and information exchange are also exploited by those who seek to profit from them fraudulently, the so-called cyber-criminals [4]. Among these fraudulent activities, the theft of personal data, phishing, attempted fraud or the blocking of web services are the order of the day when it comes to cyber-security [5]. E-commerce, one of the sectors that moves the most business on the Internet, is therefore one of the most exposed 
to the risks of cyber-attacks. So, taking cyber-risks and data protection into consideration is crucial when setting up an e-commerce business [6].

This clearly highlights the need to investigate the relationship between resilience and sustainability, and the positive link between them has been verified in several areas [7-9]. The goal is to contribute to a sustainable revolution [10].

The recent pandemic crisis affecting the world's economies has forced organizations to redesign their operating models, leveraging digital technologies to ensure the continuity of current operations even remotely [11]. This change has affected mainly the employees of companies who have seen their work habits radically transformed [9,12], but the impact has also affected consumer behavior [13]. Individual habits of selecting and purchasing a product or service online have changed, forcing manufacturers and retailers to adapt their offers to new demand requirements, especially by leveraging the widespread use of technology and customer data [14].

Thus, the COVID-19 crisis has forced companies to respond quickly to critical operational issues and new business needs with technology, inevitably increasing the likelihood of becoming the victims of cyber-attacks, as cyber-criminals have exploited the uncertainty of this unpredictable scenario [15]. The many effects of the pandemic include a huge increase in e-commerce; lockdown measures have caused consumers to increasingly turn to online retailers, seeking security and convenience [16]. This trend was confirmed by data published by Salesforce in its Shopping Index report for the first quarter of 2021 [17]. According to this study, in the first quarter of 2021, global e-commerce grew 58\% year-overyear compared to $17 \%$ in the first quarter of 2020 . In detail, the report shows that traffic on shopping sites increased by $28 \%$ on PC and $29 \%$ on mobile devices.

Figure 1, clearly shows how e-commerce has grown significantly throughout 2021, driven by the closure of physical stores due to the pandemic. It is also evident how in the same period some European countries (Italy, France, Great Britain and The Netherlands) have grown in e-commerce more than the United States and the global level. A direct result of this incredible trend, the online sales volume increase, is the rise in the number of cyberattacks on online retailers by cyber-criminals taking advantage of changing shopping habits.

\section{E-commerce expansion 2020-21}

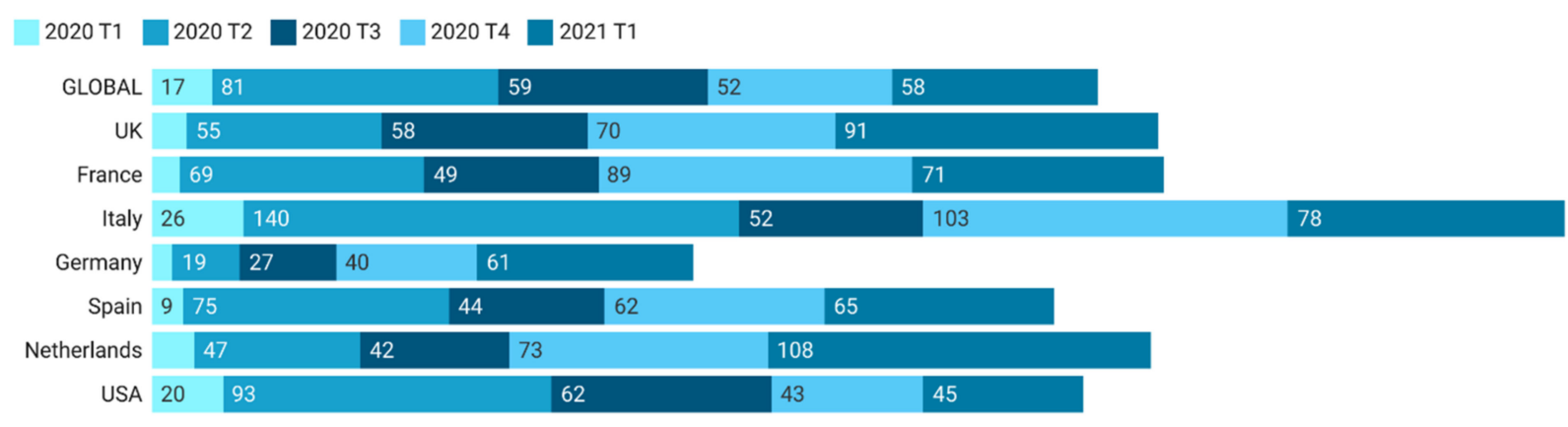

Created with Datawrapper

Figure 1. Online shopping growth in 2020-21 by quarter (Source: Own elaborations on Salesforce's Q1 Shopping Index data).

These phenomena also exert a serious effect on consumer behavior in the process of buying a good or service online. Some studies have shown how individual behavior is influenced by the perceived level of security in the exchange of private data at the time of the transaction $[18,19]$. In fact, it is unlikely that consumers will turn for their online purchases to an organization that has recently suffered a cyber-attack. However, it remains to be investigated whether consumers' increased awareness of the vulnerability of ecommerce platforms can affect their purchasing decisions. In addition, the literature has not yet sufficiently explored how the pandemic period oriented consumers' intention 
toward online purchasing [20]. Along with this, the literature also highlights how crossnational and cross-cultural studies on online consumer behavior are scarce and how most of them are based on data collected in a single country [21]. Thus, it becomes clear how important it is to delve into the advantages and criticalities of e-commerce, not only because the data evidence shows a trend of great growth of this sales channel, but also because the scientific literature demands that this gap be filled [22-24]. In addition, a cross-country methodological approach is needed to better exploit the potential of the predictive dimension of the analysis. In fact, the effectiveness of this predictive feature has been proved in recent research studies $[25,26]$.

Therefore, based on the above discussion, the following research questions can be stated:

- RQ1: How do European countries perform comparatively in e-commerce?

- RQ2: What are the most important challenges to the further expansion of e-commerce?

\section{Materials and Methods}

The literature proposes a mixed approach, collecting data from websites and evaluating the users' experience [27]. MCDA was proposed to evaluate a comparison among European countries in order to highlight the different performances (see RQ1-Section 2.1), while a Likert Scale Survey was proposed to investigate both the problems encountered by individuals and the perceived barriers to buying/ordering over the Internet (see RQ2 Section 2.2).

\subsection{Multicriteria Analysis}

The MCDA is a well-known methodology in the literature, which is useful to compare multiple and conflictual alternatives [28]. It is based on both the score associated with each alternative (i.e., the scoring criterion) and the weight assigned to the relevance of each criterion. One difficulty in this analysis relates to data acquisition. To this end, a very useful database is that of Eurostat, which facilitates the harmonization of statistical methods among the various member states (MSs). Data provided by Eurostat are used in the literature $[29,30]$ and typically used to compare several MSs [25,31].

In this research, we analyzed a set of criteria proposed by Eurostat for a specific topic, "Science, technology, digital Society", that was subdivided in (i) science and technology and ii) digital economy and society(t_isoc). Within this second group, five sub-topics were identified: i) ICT usage in households and by individuals (t_isoc_i), (ii) ICT usage in enterprises (t_isoc_e), (iii) digital skills (t_isoc_sk), iv) ICT sector (t_isoc_se) and v) digital economy and society-historical data. Within the first sub-topic, several items are proposed, including the one related to the core topic of this research. Table 1 proposes several criteria associated to e-commerce, proposing six categories.

Table 1. List of criteria.

\begin{tabular}{ll}
\hline Category & Criteria \\
\hline & Last online purchase: in the last 3 months \\
Last online purchase: in the 12 months \\
Frequency of online purchases in the last 3 months: 1 or 2 times \\
Frequency of online purchases in the last 3 months: 3 to 5 times \\
Frequency of online purchases in the last 3 months: 6 to 10 times \\
Frequency of online purchases in the last 3 months: more than 10 times
\end{tabular}


Table 1. Cont.

\begin{tabular}{|c|c|}
\hline Category & Criteria \\
\hline Internet purchases-origin of sellers & $\begin{array}{l}\text { Online purchases ( } 3 \text { months): from national sellers } \\
\text { Online purchases ( } 3 \text { months): from sellers from other EU countries } \\
\text { Online purchases ( } 3 \text { months): from sellers of the rest of the world (non-EU } \\
\text { countries) } \\
\text { Online purchases ( } 3 \text { months): from sellers from unknown countries } \\
\text { Online purchases ( } 3 \text { months): from sellers from other countries (EU or non-EU) }\end{array}$ \\
\hline Internet purchases—collaborative economy & $\begin{array}{l}\text { Online purchases ( } 3 \text { months) from private persons: any physical goods } \\
\text { Online purchases ( } 3 \text { months) from private persons: household services } \\
\text { Online purchases ( } 3 \text { months) from a private person: transport service } \\
\text { Online purchases ( } 3 \text { months) from a private person: rented accommodation } \\
\text { Online purchases ( } 3 \text { months) from a private person: goods, household services, } \\
\text { transport services or rented accommodation }\end{array}$ \\
\hline Internet purchases-money spent & $\begin{array}{l}\text { Online purchases in the last } 3 \text { months for less than } 50 \text { euro } \\
\text { Online purchases ( } 3 \text { months) for between } 100 \text { and } 499 \text { euro } \\
\text { Online purchases ( } 3 \text { months) for between } 500 \text { and } 999 \text { euro } \\
\text { Online purchases ( } 3 \text { months) for } 1000 \text { euro or more }\end{array}$ \\
\hline Financial activities over the Internet & $\begin{array}{l}\text { Online purchases ( } 3 \text { months): insurance policies, including travel insurance, also } \\
\text { as a package together with, e.g., a plane ticket } \\
\text { Online purchases ( } 3 \text { months): took a loan or a mortgage or arranged credit from } \\
\text { banks or other financial providers } \\
\text { Online purchases ( } 3 \text { months): bought or sold shares, bonds, units in funds or } \\
\text { other financial assets } \\
\text { Online purchases ( } 3 \text { months): at least one of the financial activities (I_BFIN_SH1, } \\
\text { I_BFIN_IN1,I_BFIN_CR1) }\end{array}$ \\
\hline
\end{tabular}

The criteria chosen were all of those available on Eurostat and referred to 2020 as the latest year available. All data were reported as percentages and are thus comparable to each other. Finally, the panel data set was always expressed as a percentage and therefore the different criteria were comparable to each other. The choice of alternatives was associated with the number of MSs and, in this case, they were equal to twenty-five out of twentyseven, as data from two countries, such as Italy and France, were absent.

The multi-criteria analysis consists of two distinct phases in which values and weights are calculated.

$$
\mathrm{PV}_{(\mathrm{MS})}=\sum_{\mathrm{J}=1}^{\mathrm{N}} \mathrm{RV}_{(\mathrm{MS})} * \mathrm{CV}
$$

where the result of the MCDA analysis is the calculation of a performance value (PV), obtained by multiplying a row vector $(R V)$, representing the values of the criteria $(\mathrm{J})$, and a column vector $(\mathrm{CV})$, representing the weights of the criteria. The PV is calculated for each alternative, which, as mentioned earlier, is represented by the MS aggregating $\mathrm{N}$ criteria.

Regarding the assessment of RV, several values are normalized [25]. Starting from the set of data available for each of the 30 criteria proposed in Table 1, the maximum value was identified and assigned a value of 1 . Subsequently, an intermediate value calculated as a function of the maximum value was identified for all 24 remaining values (associated with the MSs). It should be noted that a minimum value, to which the value of 0 would be associated, was not calculated, in order not to accentuate the negative performance. The results are therefore objective, since they are exactly those which Eurostat has found, but transformed to make them congruent with an MCDA.

Concerning the assessment of $\mathrm{CV}$, a subjectivity criterion was used for the assignment of the weights. The literature typically proposes well-established methods to define the weights of the criteria, such as Analytic Hierarchy Process [32], Promethee [33] or Delphi analysis [34]. These methods are not chosen for three main reasons: i) the need to identify new approaches; ii) the nature of the criteria analyzed, which tend to be sometimes alternative and not complementary; and iii) the topic under investigation in this work. Thus, one is aware that a pairwise comparison analysis can be seen as a more robust method to compare countries [25], but the topic of e-commerce finds its origin in the network. Consequently, the method most suitable to evaluating these criteria is one in which the weights are defined according to the number of views of the individual criteria on the Google search engine. A similar approach was also used by some authors to provide insights into population behavior [35]. In addition, we integrated the assessment of CV 
with a method well consolidated in the literature, which is based on the calculation of local priority and global priority [36]. Initially, a weight was assigned to each category, and then within each category a weight was assigned to the individual criteria. This weight is called the local priority, and the global priority is obtained by multiplying the local priority by the category priority. This method has the advantage of comparing a significant number of criteria. This work considers two scenarios:

- Different weights (DW) scenario, in which both the local and the category priority were based on the number of views in the Google search engine.

- Hybrid equal weights (HEW) scenario, in which only the local priority was defined as in the previous scenario, while the same weight was assigned to all category priorities.

An Equal weights (EW) scenario, in which all criteria have the same relevance, was not considered in this work because it was not representative of reality. All input data used in this work are available at Supplementary Materials.

\subsection{A Likert-Scale-Based Survey}

Within the Eurostat database there were not only the criteria proposed in Table 1 under the heading "e-commerce" but also both of the problems encountered by individuals (Table 2) and the perceived barriers (Table 3) to buying/ordering over the Internet. However, the data were not available for all countries. Within this framework, an alternative method was to use a panel of experts. In particular, the aim was not to propose the impact for individual countries but to build a framework from which to discuss the findings and potential policy implications.

Table 2. Problems encountered by individuals when buying/ordering over the Internet.

\begin{tabular}{ll}
\hline $\mathbf{N}^{\circ}$ & $\begin{array}{l}\text { Individuals Who Encountered the Following Problem When Making Purchases } \\
\text { over the Internet: }\end{array}$ \\
\hline 1 & difficulties concerning guarantees \\
2 & speed of delivery longer than indicated \\
3 & delivery costs higher than indicated \\
4 & final price higher than indicated \\
5 & delivery costs or final price higher than indicated \\
6 & wrong goods delivered \\
7 & damaged goods delivered \\
8 & wrong or damaged goods delivered \\
9 & wrong or damaged good/services delivered \\
10 & lack of security of payments \\
11 & problems with fraud \\
12 & complaints and redress were difficult \\
13 & no satisfactory response received after complaint \\
14 & complaints and redress were difficult or no satisfactory response received \\
15 & other \\
16 & no problems \\
17 & technical failure \\
18 & difficulties finding information concerning guarantees, other legal rights \\
19 & for private use \\
20 & foreign retailer did not sell in my country \\
\hline
\end{tabular}


Table 3. Perceived barriers to buying/ordering over the Internet.

\begin{tabular}{ll}
\hline $\mathbf{N}^{\circ}$ & Individuals Who Have Not Ordered Goods or Services over the Internet, Because \\
\hline 1 & they have no need \\
2 & they prefer to shop in person, they like to see the product, loyalty to shops or force of \\
3 & habit \\
4 & for their own private use (relevant information about goods difficult to find on website) \\
5 & they lack the necessary skills \\
6 & It is too expensive \\
7 & of too long delivery times \\
8 & of problems receiving the ordered goods at home \\
9 & of too long delivery times/due to problems in receiving the ordered goods at home \\
10 & for their own private use (payment security concerns) \\
11 & for their own private use (privacy concerns) \\
12 & of security concerns, they are worried about giving credit card details over the Internet \\
13 & of privacy concerns, they are worried about giving personal details over the Internet \\
14 & I am worried about giving credit card or personal details over the Internet \\
15 & of trust concerns about receiving or returning goods, complaint/redress \\
16 & they do not have a payment card \\
17 & the speed of the Internet connection is too low \\
18 & of other reasons \\
\hline
\end{tabular}

A well-established method in the literature is the Likert-scale-based survey [37], with five levels of assessment that were defined as follows: $1=$ totally disagree, $2=$ disagree, $3=$ neither agree nor disagree, $4=$ agree and $5=$ completely agree [38].

The choice of experts was based on an announcement published on the LinkedIn social network in which the required characteristics were indicated. Having at least 10 years of experience in the sector, interest in participating in a survey for the purpose of a scientific publication and propensity to participate in a video-call through Skype or Google Meet to discuss the topic e-commerce. The announcement also stated that the maximum interview time would be one hour and that only 10 experts would be selected according to [39].

The number of applications received was significant and led us to post a new message stating that the 10 experts had been chosen. After acceptance they were sent an official invitation e-mail in which the two RQs were explained and the Excel file containing the questions proposed in Tables 2 and 3 was sent. It was specified that the questions had been identified by Eurostat and for this reason they were not asked to validate them at the beginning. All respondents sent their input via e-mail, and then the video-call was used to initially gather feedback on what they had compiled in Excel and then to discuss the topic in general (from March to April 2021). Table 4 proposes the list of experts.

Table 4. List of experts.

\begin{tabular}{llll}
\hline $\mathbf{N}^{\circ}$ & Role & Country & No. Years \\
\hline 1 & Marketing manager & Spain & 12 \\
2 & Operations manager & France & 11 \\
3 & Account manager & Sweden & 21 \\
4 & Consultant & United Kingdom & 15 \\
5 & Marketing manager & Italy & 18 \\
6 & Operations manager & Germany & 20 \\
7 & Consultant & Denmark & 12 \\
8 & Account manager & Finland & 18 \\
9 & Marketing manager & Spain & 15 \\
10 & Consultant & Italy & 13 \\
\hline
\end{tabular}




\section{Results}

The theme of e-commerce has become crucial in the pandemic period, as the fear of infection, the lockdown and the closure of many commercial activities has led consumers to choose this channel of purchase. In this section, the main results obtained from the analyses that focused on both the MCDA and the Likert scale are offered.

\subsection{A Comparison among European Countries}

A cross-country comparison has the advantage of highlighting certain critical points and characteristics. However, comparing each individual criterion has the limitation of focusing just on a single aspect and the criteria may also conflict with each other. To this end, it is important to adopt a methodology capable of synthesis, as MCDA. The development of these indicators is by no means simple. Aggregating values and weights based on both Eurostat and Google data yields a single indicator, based on the contribution of the six reference categories (Table 5) considering the DW scenario.

Table 5. Results of multicriteria analysis. Key: I (Internet purchases-collaborative economy), II (Internet purchases-money spent), III (Financial activities over the Internet), IV (Internet purchasesgoods or services), V (Internet purchases_origin of sellers) and VI (Internet purchases by individuals). Italy and France were not considered because of absent data in Eurostat.

\begin{tabular}{|c|c|c|c|c|c|c|c|}
\hline & I & II & III & IV & $\mathbf{V}$ & VI & Total \\
\hline EU 27 & 0.025 & 0.022 & 0.194 & 0.081 & 0.064 & 0.027 & 0.413 \\
\hline Belgium & 0.030 & 0.026 & 0.145 & 0.083 & 0.075 & 0.032 & 0.392 \\
\hline Bulgaria & 0.005 & 0.012 & 0.086 & 0.018 & 0.017 & 0.009 & 0.147 \\
\hline Czechia & 0.026 & 0.018 & 0.129 & 0.062 & 0.058 & 0.030 & 0.323 \\
\hline Denmark & 0.034 & 0.022 & 0.379 & 0.147 & 0.090 & 0.039 & 0.712 \\
\hline Germany & 0.017 & 0.022 & 0.210 & 0.120 & 0.086 & 0.037 & 0.492 \\
\hline Estonia & 0.011 & 0.027 & 0.521 & 0.057 & 0.068 & 0.029 & 0.713 \\
\hline Ireland & 0.018 & 0.017 & 0.359 & 0.083 & 0.078 & 0.032 & 0.586 \\
\hline Greece & 0.008 & 0.014 & 0.097 & 0.038 & 0.038 & 0.019 & 0.214 \\
\hline Spain & 0.024 & 0.028 & 0.164 & 0.067 & 0.069 & 0.027 & 0.380 \\
\hline Croatia & 0.008 & 0.022 & 0.243 & 0.045 & 0.052 & 0.023 & 0.393 \\
\hline Cyprus & 0.001 & 0.007 & 0.063 & 0.034 & 0.045 & 0.017 & 0.167 \\
\hline Latvia & 0.006 & 0.030 & 0.539 & 0.035 & 0.047 & 0.020 & 0.678 \\
\hline Lithuania & 0.008 & 0.024 & 0.242 & 0.041 & 0.050 & 0.022 & 0.386 \\
\hline Luxembourg & 0.040 & 0.021 & 0.237 & 0.089 & 0.075 & 0.035 & 0.497 \\
\hline Hungary & 0.027 & 0.031 & 0.145 & 0.055 & 0.059 & 0.025 & 0.341 \\
\hline Malta & 0.023 & 0.026 & 0.277 & 0.081 & 0.079 & 0.027 & 0.512 \\
\hline $\begin{array}{l}\text { The } \\
\text { Netherlands }\end{array}$ & 0.027 & 0.030 & 0.453 & 0.146 & 0.089 & 0.038 & 0.784 \\
\hline Austria & 0.006 & 0.013 & 0.126 & 0.082 & 0.066 & 0.028 & 0.322 \\
\hline Poland & 0.008 & 0.025 & 0.098 & 0.053 & 0.041 & 0.024 & 0.249 \\
\hline Portugal & 0.008 & 0.020 & 0.145 & 0.047 & 0.041 & 0.018 & 0.280 \\
\hline Romania & 0.001 & 0.010 & 0.047 & 0.026 & 0.020 & 0.012 & 0.117 \\
\hline Slovenia & 0.028 & 0.028 & 0.145 & 0.046 & 0.061 & 0.026 & 0.334 \\
\hline Slovakia & 0.029 & 0.026 & 0.129 & 0.055 & 0.053 & 0.025 & 0.317 \\
\hline Finland & 0.025 & 0.020 & 0.509 & 0.078 & 0.064 & 0.031 & 0.727 \\
\hline Sweden & 0.027 & 0.023 & 0.465 & 0.123 & 0.078 & 0.036 & 0.753 \\
\hline
\end{tabular}

The results obtained show how the distribution of weights determines that the category of financial activities over the Internet (item III as showed in Table 5) has a considerable influence on comparisons between the various performances of European countries. In fact, it appears that its weight is equal to around $59.80 \%$ of the total value, followed by the category Internet purchases-goods or services, with $15.20 \%$ (item IV), and the category Internet purchases-origin of sellers, with $11.40 \%$ (item V). The other three categories have a weight that varies between $4.00 \%$ and $5.40 \%$. 
An analysis of the performance of individual countries shows that Denmark leads the way in items VI, V and VI, Hungary in item II and Luxembourg in item I. As far as item III is concerned, it is Latvia that comes first, with 0.539 , equal to about $80 \%$ of the total value. As mentioned above, it is this item that determines the final results. It follows that the first six countries in the ranking (Table 6) occupy the first six positions for the item Financial activities over the Internet. It should also be noted that the countries that follow Latvia in the ranking show a significant percentage weight that tends to decrease. This is an expected result, but it is significant to look at the differences that are equal to $27 \%$ of the total computation. In fact, we have Estonia with 0.521 (73\% of the total), Finland with 0.509 (70\%), Sweden with $0.465(62 \%)$, The Netherlands with $0.453(58 \%)$ and Denmark with $0.379(53 \%)$. The same is not verified for Ireland, which is the seventh in this category, with 0.359 , but has a weight on the total of $61 \%$.

Table 6. Ranking of European countries in 2020.

\begin{tabular}{|c|c|c|c|c|c|}
\hline \multicolumn{3}{|c|}{ Different Weights (DW) Scenario } & \multicolumn{3}{|c|}{ Hybrid Equal Weights (HEW) Scenario } \\
\hline No. & Country & Value & No. & Country & Value \\
\hline 1 & The Netherlands & 0.784 & 1 & The Netherlands & 0.780 \\
\hline 2 & Sweden & 0.753 & 2 & Denmark & 0.755 \\
\hline 3 & Finland & 0.727 & 3 & Sweden & 0.705 \\
\hline 4 & Estonia & 0.713 & 4 & Luxembourg & 0.627 \\
\hline 5 & Denmark & 0.712 & 5 & Germany & 0.612 \\
\hline 6 & Latvia & 0.678 & 6 & Finland & 0.604 \\
\hline 7 & Ireland & 0.586 & 7 & Belgium & 0.571 \\
\hline 8 & Malta & 0.512 & 8 & Estonia & 0.569 \\
\hline 9 & Luxembourg & 0.497 & 9 & Malta & 0.567 \\
\hline \multirow{2}{*}{10} & Germany & 0.492 & 10 & Ireland & 0.558 \\
\hline & EU27 & 0.413 & 11 & Spain & 0.518 \\
\hline 11 & Croatia & 0.393 & & EU27 & 0.513 \\
\hline 12 & Belgium & 0.392 & 12 & Hungary & 0.495 \\
\hline 13 & Lithuania & 0.386 & 13 & Slovenia & 0.486 \\
\hline 14 & Spain & 0.380 & 14 & Latvia & 0.481 \\
\hline 15 & Hungary & 0.341 & 15 & Slovakia & 0.471 \\
\hline 16 & Slovenia & 0.334 & 16 & Czechia & 0.466 \\
\hline 17 & Czechia & 0.323 & 17 & Austria & 0.410 \\
\hline 18 & Austria & 0.322 & 18 & Croatia & 0.401 \\
\hline 19 & Slovakia & 0.317 & 19 & Lithuania & 0.395 \\
\hline 20 & Portugal & 0.280 & 20 & Poland & 0.368 \\
\hline 21 & Poland & 0.249 & 21 & Portugal & 0.333 \\
\hline 22 & Greece & 0.214 & 22 & Greece & 0.284 \\
\hline 23 & Cyprus & 0.167 & 23 & Cyprus & 0.223 \\
\hline 24 & Bulgaria & 0.147 & 24 & Bulgaria & 0.171 \\
\hline 25 & Romania & 0.117 & 25 & Romania & 0.165 \\
\hline
\end{tabular}

This analysis at the category level should be conducted at the level of each individual criterion to understand which criteria primarily determine these final values. To this end, item III is composed of four criteria which, as shown in the data, have different weights. The criterion Online purchases ( 3 months): at least one of the financial activities (I_BFIN_SH1, I_BFIN_IN1, I_BFIN_CR1) has a local weight of 0.727 and a global weight of 0.435 . The countries present an order that mirrors that which was presented for the category in general.

However, an alternative method (HEW scenario) could be to consider a weight in which the local weight was always calculated using the same approach as before, while it was assumed that the weights of the six categories were the same (Table 6). These analyses are useful for evaluating the trends to understand how much impact the weights have on the final indicator. For example, the criterion Online purchases ( 3 months): at least one of the financial activities (I_BFIN_SH1, I_BFIN_IN1, I_BFIN_CR1) has always a local weight 
of 0.727 , while the global weight is now 0.121 (since the weight of category III is 0.167 ).

Figure 2 shows the difference between two different (DW and HEW) scenarios.

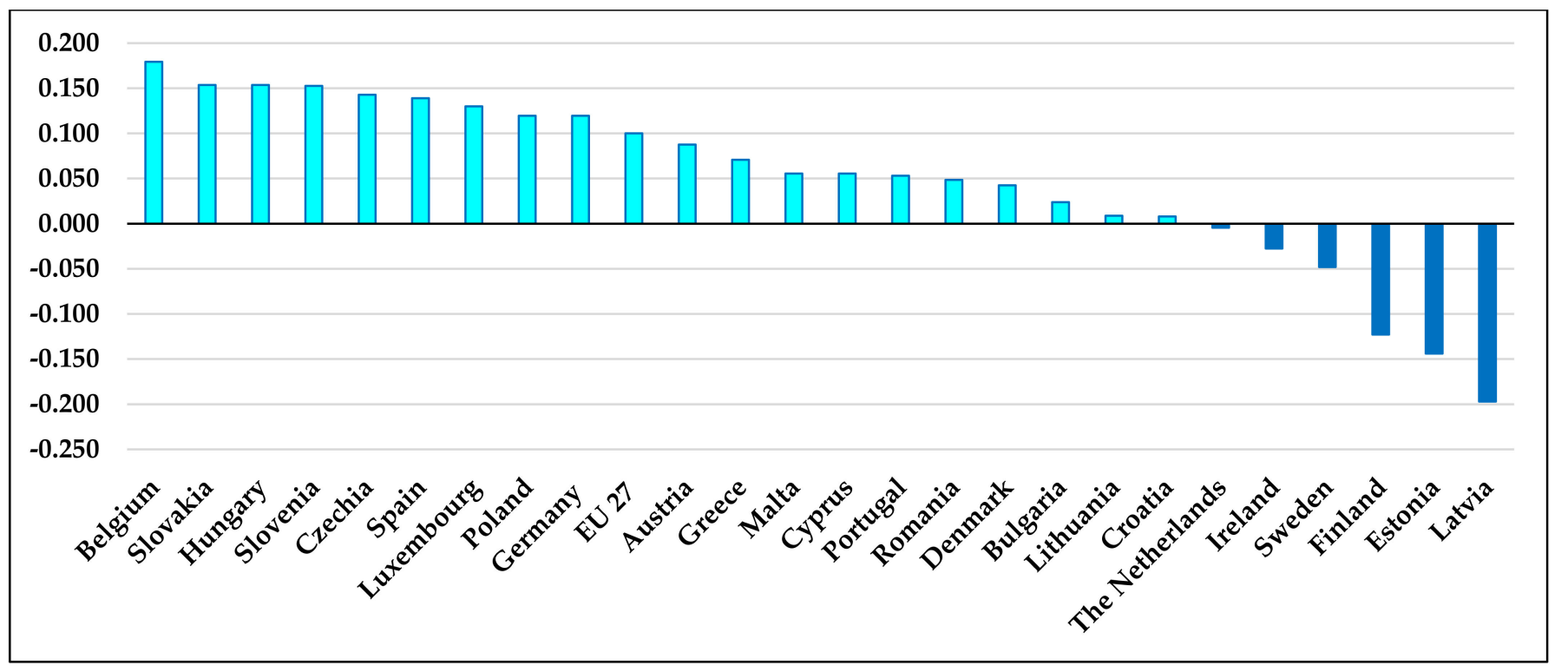

Figure 2. Delta between HEW and DW scenarios.

The results are influenced by the value of the weights, that brings the EU 27 from a total value of 0.413 in the DW scenario to 0.513 in the HEW scenario, recording an increase (HEW-DW scenario) of 0.100. The advantages of the alternative scenarios are to provide results depending on the proposed point of view. In fact, this research had chosen the number of views as the reference method, considering it strategic to evaluate the perspectives of a greater number of stakeholders. Similarly, a panel of experts from specific stakeholder categories could provide valuable information, but the simpler method of equal weights also provided important insights. The HEW model represents an intermediate point between a DW and an EW scenario.

In the DW scenario, the top seven countries are those identified by the most relevant criterion, Online purchases (3 months): at least one of the financial activities (I_BFIN_SH1, I_BFIN_IN1, I_BFIN_CR1), but the order of the ranking changes. This means that this category is relevant but not decisive. In particular, The Netherlands leads with 0.784, followed by Sweden (0.753) and Finland (0.727). At the same time, it is also logical that these seven countries are the ones that present the greatest deviation when considering the HEW scenario, where it is Latvia that has a reduction of 0.197.

The latter is the only country that, when passing from the DW scenario to the HEW scenario, no longer has a value higher than the European average, while Belgium and Spain move in the opposite direction and acquire a value higher than the European average. More precisely, Belgium increases its indicator by 0.179 . The Netherlands remains in first place in the alternative scenario, with 0.780, followed by Denmark (0.755) and Sweden (0.705). On the other hand, it can be seen that the lowest ranking is held by Greece, Cyprus, Bulgaria and Romania in both scenarios.

These data should be monitored over time, perhaps integrating them with the specific results of Italy and France to evaluate temporal trends that are always suitable for understanding how a topic evolves. However, compared to a simple subdivision of the European countries above or below the European average, it is possible to use a range to distinguish those countries that have a significantly higher/lower value compared to those that have a more limited value (Figure 3). The delta considered always depends on the case study being analyzed, and in this case a percentage weight of $20 \%$ was chosen. Consequently, those countries that have a value greater than 0.496 in the DW scenario and/or 0.616 in the 
HEW scenario will be virtuous. On the other hand, those countries that have a value of less than 0.330 in the DW scenario and/or 0.410 in the HEW scenario will be defined as laggard. Finally, other countries will be defined as in-between.

\section{DIFFERENT WEIGHTS}
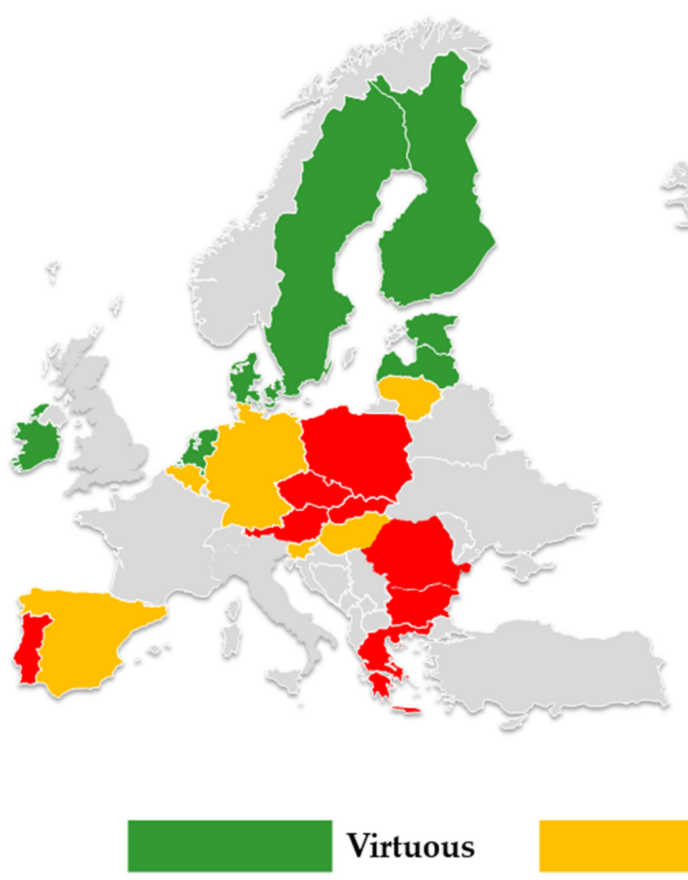

\section{HYBRID EQUAL WEIGHTS}

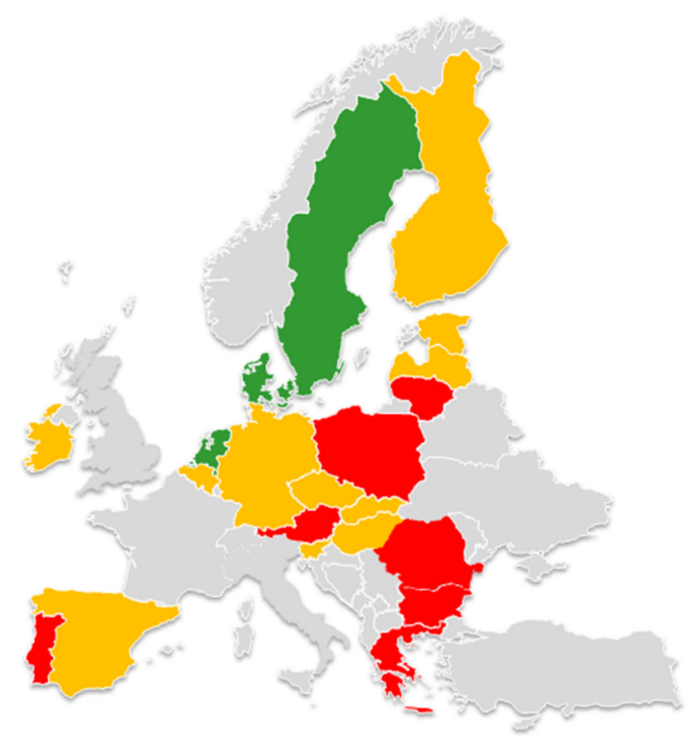

In-between
Laggard

Figure 3. The subdivision of countries in three groups. Data not available for Italy and France.

The results show that there are only three countries (The Netherlands, Sweden and Denmark) that are virtuous and seven countries (Austria, Poland, Portugal, Greece, Cyprus, Bulgaria and Romania) that are laggards in both scenarios. These values indicate that, regardless of the weights considered, the target values proposed by Eurostat make it possible to rank the European countries and then to classify them into groups. This summary analysis is necessary in a context where the multiplicity of data is significant, and methodologies are proposed for assigning weights that vary according to the specificity of the topic under analysis.

\subsection{Expert Assessment}

The panel of experts could be seen as subjective because only certain categories of stakeholders are involved in the analysis and because the individual respondents may not have a global view. However, the Likert scale is an established method in the literature, and the previous section demonstrates precisely that its use, as an evaluation system in surveys, is a generally accepted method. Experts provide their judgment by looking not at a personal perspective but at the overall perspective that has been formed over time through years of experience. Figure 4 proposes the ranking related to problems encountered during the purchasing phases, while Figure 5 illustrates the ranking related to perceived barriers. 


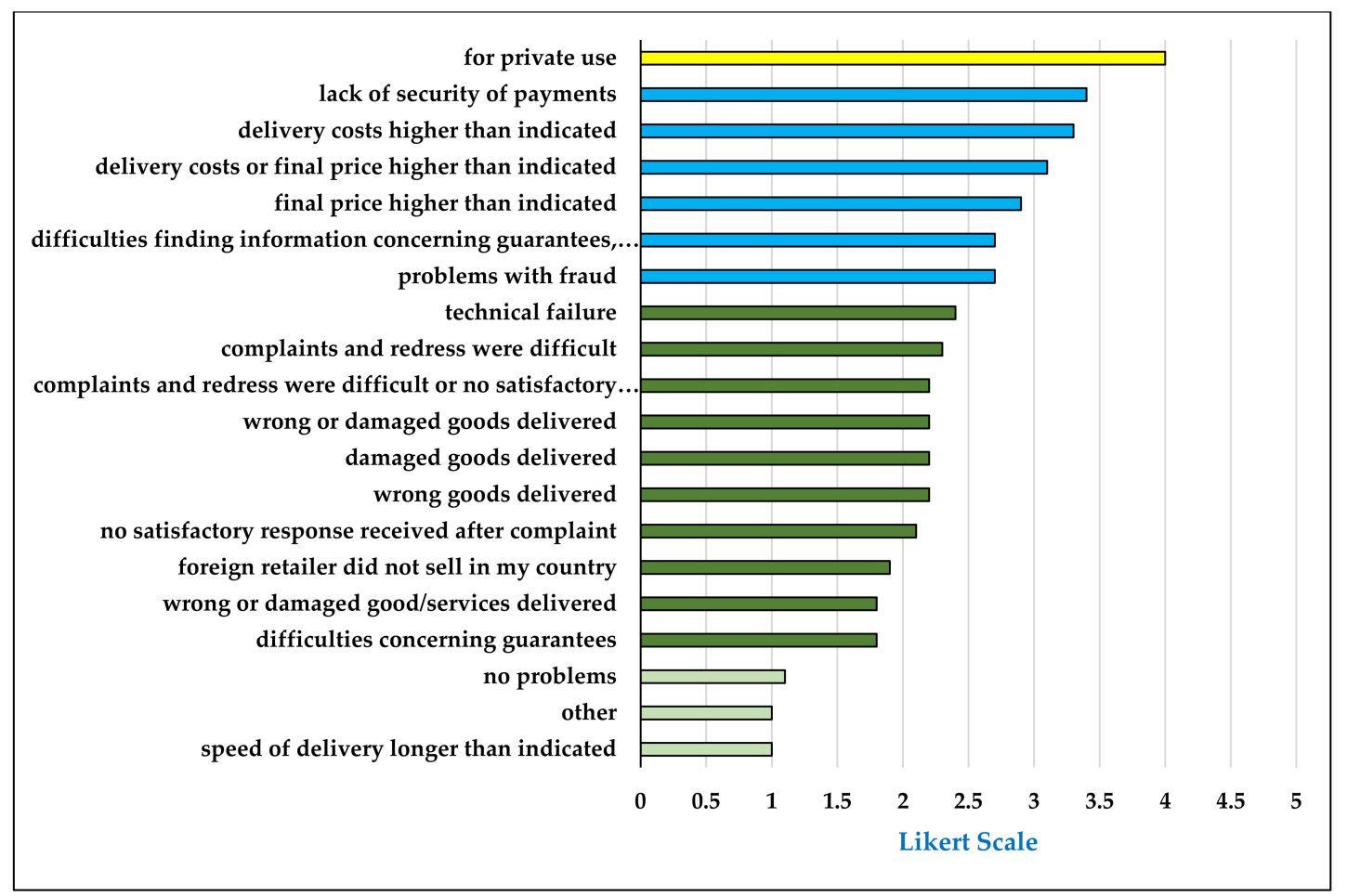

Figure 4. Results of a Likert-scale-based survey (Problems encountered by individuals). The following color bars are used: totally disagree (pink), disagree (orange), neither agree nor disagree (blue), agree (yellow) and completely agree (green).

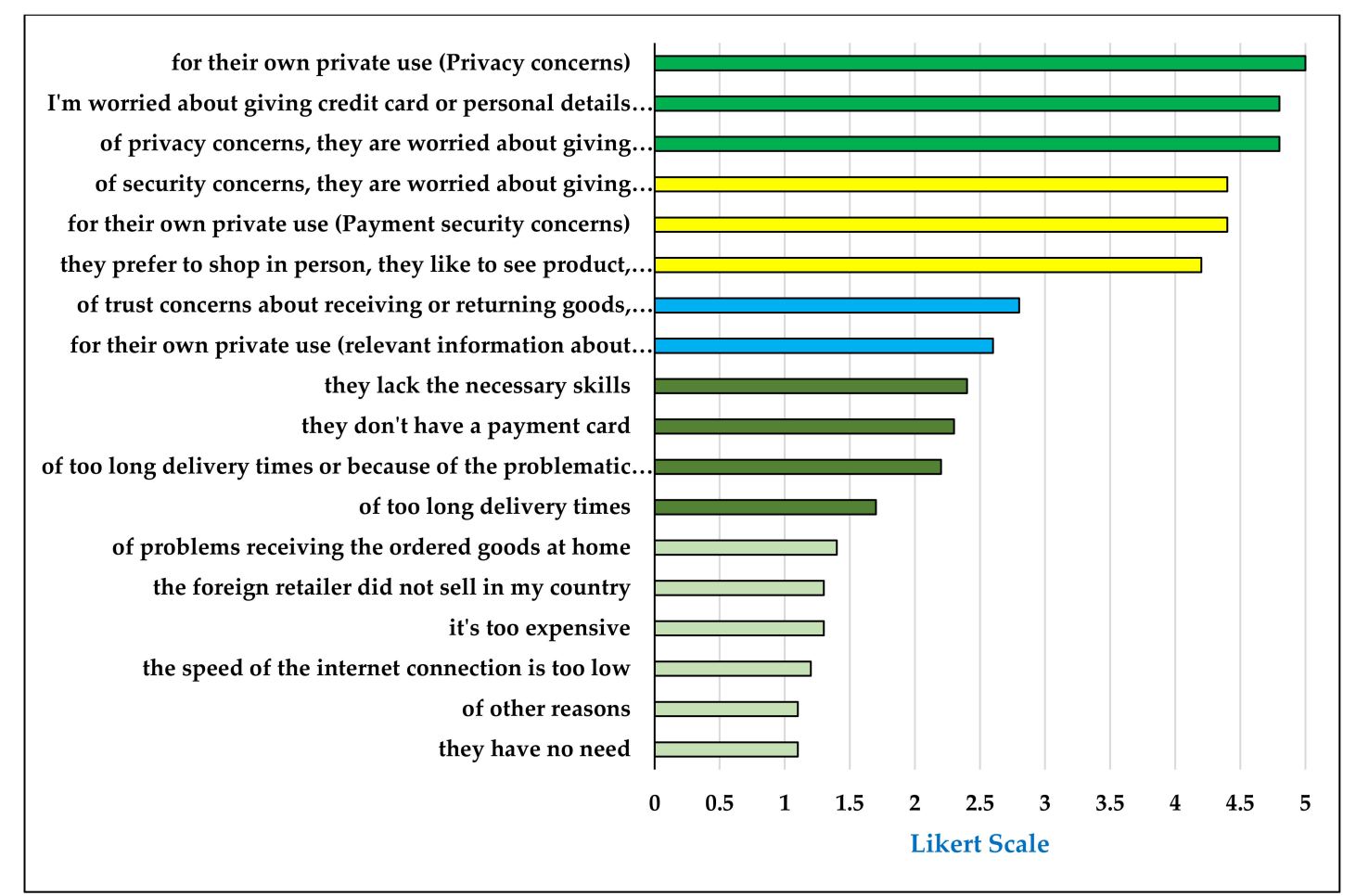

Figure 5. Results of a Likert-scale-based survey (Perceived barriers). The following color bars are used: totally disagree (pink), disagree (orange), neither agree nor disagree (blue), agree (yellow) and completely agree (green).

The results show that the problems encountered by individuals when buying/ordering over the Internet are not considered relevant by the expert panel. No item received a significant relevance, while the item related to private use always collected a value of 4 and 
is the only one that presents a moderate relevance. Next in the ranking appears the fear associated with the security of payment (average value of 3.4). It emerges, therefore, that e-commerce is no longer seen as an alternative to face-to-face shopping but has now entered the habits of citizens. The pandemic period has widened this phenomenon. Moreover, this consideration emerges not only from the assignment of Likert Scale values, but is a concept reiterated by all respondents. Those who buy through this channel have a clear idea of what they want to buy. Indeed, more than problems they see opportunities, and can compare the price they have seen in a store with the price they can quickly see on a cell phone or a tablet. At the same time, delivery costs are slightly more critical than the selling price (3.3 vs. 2.9). Protection in terms of privacy and fraud is considered neither relevant nor irrelevant (average value 2.7).

The situation changes when the experts are asked to identify potential barriers. In fact, it emerges that there are three items that are considered very relevant and, in particular, all the experts have assigned a value of 5 to private use (confirming the previous figure). However, the privacy concerns component is given the highest value, while payment security concerns are given a value of 4.4. Privacy concerns are also considered very relevant when talking specifically about concerns about personal details (average value of 4.8). Similarly, when we talk specifically about security concerns in terms of credit cards, the value of 4.4 is confirmed. Finally, the preference to purchase in person is also moderately significant (4.2).

\section{Discussion}

\subsection{Nexus between e-Commerce, Cyber-Security and Sustainability}

The theme of e-commerce is viewed with great concern by some operators, as they fear serious economic repercussions. Still, the definition of a relationship between demand and offer is an extremely complex matter, and those operators that are able, more than others, to fulfill demands of the market can be deemed successful. The present work aims to place the accent on a topic that is debated in the literature, focusing on aspects that are still insufficiently explored. For instance, how is it possible to manage the amount of data that Eurostat makes available? In addition to the results proposed in the previous parts of the paper, the interviews with the experts also covered other topics. It emerged that the experts would have liked to investigate further aspects of this topic, and this clearly represents a limitation of this work. In particular, this work was not directed at investigating the topic of sustainability. However, the expert panel underlined the importance of this topic and the need to further investigate the relationship between e-commerce and sustainability. If the consumer buys the final product directly from the producer, the transport could be about the same as between the producer and the point of sale and between a point of sale and the consumer. The same could apply to the packaging, even if, in this case, the mono-product delivery is notoriously more impacting than the delivery of a stock that is quantitatively more meaningful. The answer to this question is to apply the 'polluter pays' principle. The sustainable revolution calls for the calculation of the cost of pollution per unit of product, and it is then up to the logic of the market to see how it will be shared between the producer and the consumer. In this context, the use of green fuels and reused, recycled and recovered materials can lead to a significant reduction in the environmental impact associated with a single product. However, another major theme of analysis cannot go unnoticed: the sustainability of digitization. Similarly, experts highlight how Europe is moving toward the combination of sustainability and resilience. The reading that is given is that of creating competitive conditions. Investing in the opportunities of natural resources and building dynamic models capable of reacting to unpredictability are basic components for companies positioned in a global context.

Furthermore, e-commerce can also be seen in terms of an expansion through which some small businesses can find space in larger markets without paying expensive rents in a big city, in the most luxurious neighborhood. They can reach customers who are not used to traveling and therefore would not have been able to purchase such products. The openings 
of distribution points of large international players in local contexts are seen as positive for direct employment outlets but are then identified as negative because in some cases they lead to the closure of some activities. In this context, local products can gain momentum because a product is made available beyond its usual borders: exporting is what can make the difference in times of crisis. One of the problems of e-commerce is the loss of human contact. To solve this problem, support services can make the consumer feel that he or she has not been left to his or her own devices to deal with any doubts that may arise during the purchasing process. Many companies practice a zero-cost return service, and this has been a market move that has represented a tsunami for companies that used to use only traditional channels. Within other realities (e.g., Media World), an internal competition is created between the two different sales channels, characterized by specific offers.

Our interviewees paid attention to the cyber-security issues that could be generated in this new context. The main concern of consumers is to lose part of their privacy and to share personal data even if they do not want to. Also of concern is the traceability that results in the many e-mails and phone calls that are received daily. In short, the time that could be saved through e-commerce could be reduced for these phenomena, and a still more incisive element is that not all the consumers are social, that is, they are not so prone to sharing their life habits on the net. To these aspects we must add the problems connected to the security of payments. There can be no resilience when one's personal data have been used by others or when one's money has been stolen.

Economic growth and sustainability are aspects of sustainable development that are increasingly present in the policies and strategies of European countries. Digitalization and cyber-security represent two of the main enabling factors of sustainable development, and for this reason they play a primary role in the Next Generation EU (NGEU). The NGEU aims to support the positive relationship between sustainability and resilience, and this work underlines the need of new studies in the context of e-commerce able to demonstrate their relative advantages.

This study has highlighted how European citizens increasingly benefit from digital infrastructure and applications through which they exercise their fundamental rights and freedoms, such as the process of purchasing goods or services online. For this reason, the NGEU also provides the enhancement and strengthening of public and private technological infrastructure to create a safe online environment for consumers and businesses. The results of the survey provide important insights into how the NGEU will be implemented. Each European country, when defining its digital transformation policies and strategies, should plan investments in cyber-security that include actions aimed at orienting organizational, process and technological issues, also thanks to the adoption of a systematic approach to risk management. As a direct consequence, the importance of considering the issue of cyber-security in a comprehensive and systematic way arises. In fact, it is imperative to appropriately protect the information of citizens and businesses that is collected and processed through digital channels. These actions are able to generate a positive relationship between sustainability and resilience.

Finally, it is necessary to underline the impact of the impetuous growth of e-commerce on the environmental dimension of sustainability. The closure of physical stores during the pandemic and the subsequent growth of e-commerce has resulted in an increase in shipping and packaging waste. Nevertheless, operators in e-commerce supply chains, having fewer middlemen, can exercise greater control over packaging solutions and delivery logistics. In addition, there is a trend among consumers to support those companies that operate responsibly toward the environment, also thanks to the action exerted, especially among the younger generations, by social media and eco-influencers. Therefore, the demand for eco-friendly products is high, especially among Millennials and Generation Z, who are becoming the most sensitive customers to sustainability issues. So, also for e-commerce organizations, it has become necessary to design and implement sustainability policies that communicate to consumers in a transparent way the actual actions taken to reduce the environmental impact of the product, packaging and delivery system. For e-commerce 
organizations, this means adopting green marketing strategies that expand the consumer market and increase sales.

\subsection{Featured Application}

Through this research it was possible to build a benchmark able to identify and correlate the different items that represent the topic of e-commerce within Eurostat. These data are normally used by European countries mainly to carry out decisions, so a synthesisbased benchmark can help decision makers. In this perspective, the results of this study have both theoretical and managerial implications, as described below.

\subsection{Theoretical Contribution}

This study contributes to the literature by analyzing the revolution in e-commerce development caused by the current health crisis. Pre-pandemic studies need to be updated because e-commerce has changed the playing field. This provides a homogeneous starting point for future studies. Using an expert opinion panel, based on a Likert scale survey, a less biased overall view of e-commerce challenges has been achieved. The use of hybrid methodologies allows the study to be carried out according to the research objectives.

\subsection{Managerial Contribution}

From an organizational management perspective, the study facilitates decision-making at two levels. The development stages of different countries have been mapped through different indicators, both at the individual and aggregate level. Businessmen can use this information to develop their expansion strategies in the countries whose development levels best match their characteristics. In a complementary way, the concerns and preferences of consumers have been studied in depth, which is fundamental for organizations to design their products and services. In addition, various activities in their value chain, such as post-sales activities, can be refined. This contribution is essential for making future investments efficiently, which can provide competitive advantages at a global level.

The importance for consumers of the processing of private data and the security of their transactions should be underlined. On a second level, particular attention should be paid to the management of costs associated with deliveries or transparency in pricing. However, consumers do not express concern about the extension of delivery times, possibly due to a greater understanding of the situation generated by the pandemic.

The results also enable governments to design infrastructure and regulatory policies to provide technological support and legal protection for the development of online commerce. It should be considered that certain actions should be carried out at the European or global level. E-business involves overcoming physical and geographic barriers, so new regulations are required to provide guarantees to companies and consumers in different countries. The European Union can provide a frame of reference in this respect.

\section{Conclusions}

Mobility restrictions and social distancing have been the main tools that governments have used to combat the Covid-19 pandemic. This situation has led to an accelerated implementation of e-commerce that requires a new approach for both governments and the business environment.

The new situation needs to be studied from different perspectives. At the governmental level, it is crucial to know the level of development of e-commerce in different countries in relation to their counterparts in a European context. Establishing comparisons between countries would allow the different European organizations to apply policies to promote the least developed areas. At the business level, it is essential to understand the demands and concerns of consumers in their use of e-commerce from an overall perspective.

Regardless of the pandemic period, e-commerce is now a new reality that must be confronted, and companies that fail to grasp this challenge will risk not gaining potential market share. However, this cannot lead to a maximization of production because it does 
not optimize the balance between eco-systems. The productive system, even after traumatic events, can react better when it has a greater number of sales channels and a number of shared and non-dedicated resources.

The results of the analysis provide a clear answer to the research questions behind this study. In particular, in response to RQ1, a different behavior of European countries toward the issue of cyber-security emerges. Based on the scenarios considered, The Netherlands, Sweden and Denmark can be considered virtuous, while Austria, Poland, Portugal, Greece, Cyprus, Bulgaria and Romania are in a less performing situation. This shows that, compared to the target values proposed by Eurostat, countries have very different perceptions of the e-commerce experience. Finally, in response to RQ2, the results make it very clear that the big challenge for e-commerce after the pandemic period is to include cyber-security and sustainability in its processes, because this is what European citizens and consumers are asking for. A more efficient and secure supply and delivery chain not only helps to reduce the environmental impact, but also makes the system more resilient. A resilient supply chain can only become a factor of competitive advantage for operators that can communicate in a more transparent way to their customers a real value proposition.

This study also has some limitations that may be resolved through further research:

- The Eurostat database provides a powerful tool for the analysis of time series. However, despite the detailed process of data mining and validation, the dataset is only partially complete for the aims of this study. The lack of data for Italy and France, which are important European countries where e-commerce plays an important role in purchasing processes, represents a limitation.

- The set of questions proposed to the experts allowed us to explore some aspects related to the problems and barriers that characterize Internet purchases, but it is necessary to move from a macro to a micro analysis considering different sectors in order to obtain more specific data.

- The results of the analysis are stakeholder-dependent because consumer behaviors varies significantly depending on the socio-cultural segment to which they belong.

E-commerce is set to grow, and this puts pressure on policy makers to favor choices that balance growth with sustainability.

Supplementary Materials: All input data are available online at http:/ / doi.org/10.5281/zenodo.4769642 (accessed on 7 May 2021).

Author Contributions: Conceptualization, D.S.-B.; methodology, I.D.; data curation, R.G.-S. and M.S.M.-S.; writing—original draft preparation, I.D., R.G.-S., M.S.M.-S. and D.S.-B.; writing—review and editing, I.D., R.G.-S., M.S.M.-S. and D.S.-B.; supervision, I.D. All authors have read and agreed to the published version of the manuscript.

Funding: This research received no external funding.

Institutional Review Board Statement: Not applicable.

Informed Consent Statement: Not applicable.

Data Availability Statement: Not applicable.

Conflicts of Interest: The authors declare no conflict of interest.

\section{References}

1. Calzada, J.; Tselekounis, M. Net Neutrality in a hyperlinked Internet economy. Int. J. Ind. Organ. 2018, 59, 190-221. [CrossRef]

2. Treiblmaier, H.; Mirkovski, K.; Lowry, P.B.; Zacharia, Z.G. The physical internet as a new supply chain paradigm: A systematic literature review and a comprehensive framework. Int. J. Logist. Manag. 2020, 31, 239-287. [CrossRef]

3. Etemadi, N.; Van Gelder, P.; Strozzi, F. An ISM Modeling of Barriers for Blockchain/Distributed Ledger Technology Adoption in Supply Chains towards Cybersecurity. Sustainability 2021, 13, 4672. [CrossRef]

4. Dupont, B.; Lusthaus, J. Countering Distrust in Illicit Online Networks: The Dispute Resolution Strategies of Cybercriminals. Soc. Sci. Comput. Rev. 2021. [CrossRef]

5. Benz, M.; Chatterjee, D. Calculated risk? A cybersecurity evaluation tool for SMEs. Bus. Horiz. 2020, 63, 531-540. [CrossRef] 
6. Girsang, M.J.; Candiwan; Hendayani, R.; Ganesan, Y. Can Information Security, Privacy and Satisfaction Influence The ECommerce Consumer Trust? In Proceedings of the 2020 8th International Conference on Information and Communication Technology (ICoICT), Yogyakarta, Indonesia, 24-26 June 2020; pp. 1-7.

7. D'Adamo, I.; Lupi, G. Sustainability and Resilience after COVID-19: A Circular Premium in the Fashion Industry. Sustainability 2021, 13, 1861. [CrossRef]

8. D'Adamo, I.; Rosa, P. How do you see infrastructure? Green energy to provide economic growth after COVID-19. Sustainability 2020, 12, 4738. [CrossRef]

9. Miceli, A.; Hagen, B.; Riccardi, M.P.; Sotti, F.; Settembre-Blundo, D. Thriving, Not Just Surviving in Changing Times: How Sustainability, Agility and Digitalization Intertwine with Organizational Resilience. Sustainability 2021, 13, 2052. [CrossRef]

10. D'Adamo, I.; Falcone, P.M.; Martin, M.; Rosa, P. A sustainable revolution: Let's go sustainable to get our globe cleaner. Sustainability 2020, 12, 4387. [CrossRef]

11. Keenan, J.M. COVID, resilience, and the built environment. Environ. Syst. Decis. 2020, 40, 216-221. [CrossRef]

12. Huertas-Valdivia, I.; Ferrari, A.M.; Settembre-Blundo, D.; García-Muiña, F.E. Social Life-Cycle Assessment: A Review by Bibliometric Analysis. Sustainability 2020, 12, 6211. [CrossRef]

13. Amicarelli, V.; Tricase, C.; Spada, A.; Bux, C. Households' Food Waste Behavior at Local Scale: A Cluster Analysis after the COVID-19 Lockdown. Sustainability 2021, 13, 3283. [CrossRef]

14. Grewal, D.; Gauri, D.K.; Roggeveen, A.L.; Sethuraman, R. Strategizing Retailing in the New Technology Era. J. Retail. 2021, 97, 6-12. [CrossRef]

15. Lallie, H.S.; Shepherd, L.A.; Nurse, J.R.C.; Erola, A.; Epiphaniou, G.; Maple, C.; Bellekens, X. Cyber security in the age of COVID-19: A timeline and analysis of cyber-crime and cyber-attacks during the pandemic. Comput. Secur. 2021, 105, 102248. [CrossRef]

16. Villa, R.; Monzón, A. Mobility Restrictions and E-Commerce: Holistic Balance in Madrid Centre during COVID-19 Lockdown. Economies 2021, 9, 57. [CrossRef]

17. Salesforce Salesforce Shopping Index Q1 2021. Available online: https://public.tableau.com/profile/salesforcecommercecloud\# !/vizhome/SalesforceShoppingIndex/SalesforceShoppingIndex (accessed on 7 May 2021).

18. Aggarwal, A.; Rahul, M. The effect of perceived security on consumer purchase intensions in electronic commerce. Int. J. Public Sect. Perform. Manag. 2018, 4, 1-20. [CrossRef]

19. Valdez-Juárez, L.E.; Gallardo-Vázquez, D.; Ramos-Escobar, E.A. Online Buyers and Open Innovation: Security, Experience, and Satisfaction. J. Open Innov. Technol. Mark. Complex. 2021, 7, 37. [CrossRef]

20. Tran, L.T.T. Managing the effectiveness of e-commerce platforms in a pandemic. J. Retail. Consum. Serv. 2021, 58, 102287. [CrossRef]

21. Hallikainen, H.; Laukkanen, T. Trustworthiness in e-commerce: A replication study of competing measures. J. Bus. Res. 2021, 126, 644-653. [CrossRef]

22. Jiang, Y.; Lai, P.; Chang, C.-H.; Yuen, K.F.; Li, S.; Wang, X. Sustainable Management for Fresh Food E-Commerce Logistics Services. Sustainability 2021, 13, 3456. [CrossRef]

23. Kleisiari, C.; Duquenne, M.-N.; Vlontzos, G. E-Commerce in the Retail Chain Store Market: An Alternative or a Main Trend? Sustainability 2021, 13, 4392. [CrossRef]

24. Wang, J.; Gao, X.; Wang, Z. Sustainable Supply Chain Decisions under E-Commerce Platform Marketplace with Competition. Sustainability 2021, 13, 4162. [CrossRef]

25. D'Adamo, I.; Gastaldi, M.; Rosa, P. Assessing Environmental and Energetic Indexes in 27 European Countries. Int. J. Energy Econ. Policy 2021, 11, 417-423. [CrossRef]

26. Ferrari, A.M.; Volpi, L.; Settembre-Blundo, D.; García-Muiña, F.E. Dynamic life cycle assessment (LCA) integrating life cycle inventory (LCI) and Enterprise resource planning (ERP) in an industry 4.0 environment. J. Clean. Prod. 2021, 286, 125314. [CrossRef]

27. Vila, T.D.; González, E.A.; Vila, N.A.; Brea, J.A. Indicators of Website Features in the User Experience of E-Tourism Search and Metasearch Engines. J. Theor. Appl. Electron. Commer. Res. 2021, 16, 18-36. [CrossRef]

28. Marović, I.; Perić, M.; Hanak, T. A Multi-Criteria Decision Support Concept for Selecting the Optimal Contractor. Appl. Sci. 2021, 11, 1660. [CrossRef]

29. Crecente, F.; Sarabia, M.; del Val, M.T. Sustainable Entrepreneurship in the 2030 Horizon. Sustainability 2021, 13, 909. [CrossRef]

30. Ronzon, T.; Piotrowski, S.; Tamosiunas, S.; Dammer, L.; Carus, M.; M'barek, R. Developments of economic growth and employment in bioeconomy sectors across the EU. Sustainability 2020, 12, 4507. [CrossRef]

31. Castillo-Giménez, J.; Montañés, A.; Picazo-Tadeo, A.J. Performance and convergence in municipal waste treatment in the European Union. Waste Manag. 2019, 85, 222-231. [CrossRef]

32. Gompf, K.; Traverso, M.; Hetterich, J. Using Analytical Hierarchy Process (AHP) to Introduce Weights to Social Life Cycle Assessment of Mobility Services. Sustainability 2021, 13, 1258. [CrossRef]

33. Koliouska, C.; Andreopoulou, Z. A Multicriteria Approach for Assessing the Impact of ICT on EU Sustainable Regional Policy. Sustainability 2020, 12, 4869. [CrossRef]

34. Al-Qawasmi, J. Selecting a Contextualized Set of Urban Quality of Life Indicators: Results of a Delphi Consensus Procedure. Sustainability 2021, 13, 4945. [CrossRef] 
35. Caruso, G.; Fortuna, F. Mediterranean diet Patterns in the Italian Population: A functional data analysis of Google Trends. In Decisions and Trends in Social Systems, Innovative and Integrated Approaches of Care Services; Springer: Cham, Switzerland, 2020; pp. 1-10.

36. Brudermann, T.; Mitterhuber, C.; Posch, A. Agricultural biogas plants-A systematic analysis of strengths, weaknesses, opportunities and threats. Energy Policy 2015, 76, 107-111. [CrossRef]

37. Falcone, P.M.; Imbert, E. Social life cycle approach as a tool for promoting the market uptake of bio-based products from a consumer perspective. Sustainability 2018, 10, 1031. [CrossRef]

38. Czeczotko, M.; Górska-Warsewicz, H.; Laskowski, W. Towards Sustainable Private Labels—What is the Consumer Behavior Relating to Private Labels in the UK and Poland? Sustainability 2020, 12, 6035. [CrossRef]

39. D'Adamo, I.; Gastaldi, M.; Rosa, P. Recycling of end-of-life vehicles: Assessing trends and performances in Europe. Technol. Forecast. Soc. Chang. 2020, 152, 119887. [CrossRef] 



\title{
Methodological Perspective for Assessing European Consumers' Awareness of Cybersecurity and Sustainability in E-Commerce
}

\author{
Idiano D'Adamo ${ }^{1, *(1)}$, Rocío González-Sánchez ${ }^{2}\left(\mathbb{D}\right.$, Maria Sonia Medina-Salgado ${ }^{2} \mathbb{C}$ \\ and Davide Settembre-Blundo ${ }^{2}$ (D) \\ 1 Department of Computer, Control and Management Engineering, Sapienza University of Rome, Via Ariosto \\ 25, 00185 Rome, Italy \\ 2 Department of Business Administration (ADO), Applied Economics II and Fundamentals of Economic \\ Analysis, Rey Juan Carlos University, 28032 Madrid, Spain; rocio.gonzalez@urjc.es (R.G.-S.); \\ sonia.medina@urjc.es (M.S.M.-S.); davide.settembre@urjc.es (D.S.-B.) \\ * Correspondence: idiano.dadamo@uniroma1.it
}

check for

updates

Citation: D'Adamo, I.; GonzálezSánchez, R.; Medina-Salgado, M.S.; Settembre-Blundo, D. Methodological Perspective for Assessing European Consumers' Awareness of Cybersecurity and Sustainability in E-Commerce. Sustainability 2021, 13, 11343. https://doi.org/10.3390/ su132011343

Academic Editor: Giuseppe Ioppolo

Received: 26 September 2021

Accepted: 11 October 2021

Published: 14 October 2021

Publisher's Note: MDPI stays neutral with regard to jurisdictional claims in published maps and institutional affiliations.

Copyright: (c) 2021 by the authors. Licensee MDPI, Basel, Switzerland. This article is an open access article distributed under the terms and conditions of the Creative Commons Attribution (CC BY) license (https:// creativecommons.org/licenses/by/ $4.0 /)$.

\begin{abstract}
The increasing European consumer awareness of cybersecurity and sustainability issues in e-commerce is raising key methodological concerns. In a field like this, still unexplored by scholars, it is crucial to identify reliable data as well as to choose the modalities of combining primary and secondary data. As the robustness of the outcome of a study on a complex issue is highly sensitive to the quality of the data used, this paper is a communication that aims to complement and support previously published empirical research. This communication describes the methodological path to collect, integrate, and process data from different sources to interpret European consumers' attitudes towards cybersecurity and sustainability during e-commerce. In fact, COVID-19 has driven many users to shop online, raising concerns especially about the security of digital transactions. Scientific studies analyzing these effects are still lacking in the literature and therefore this paper aims to fill this gap. The limits of a rigid dataset such as Eurostat's are overcome by integrating the results of European citizens' behavior during online purchases, with a survey that involved some experts appropriately selected by the research team. The combination of primary and secondary sources of data and the application of a hybrid methodological approach using MCDA (multi-criteria decision analysis) and a Likert scale allowed new information that fills some gaps in the literature to be extracted from the data. How European citizens correlate e-commerce with cybersecurity and sustainability emerged, thus providing important insights for decision-makers.
\end{abstract}

Keywords: cybersecurity; sustainability; e-commerce; consumers' awareness; methodology

\section{Summary}

This communication aims to complement and support previously published empirical research [1]. E-commerce was already a relevant phenomenon before COVID-19 [2], but its growing trend has become more evident due to the blocking situation the world has been experiencing [3]. The increase in the number of online commercial exchanges has become more widespread globally, and with it, the security risks of online transactions have also grown [4], also causing impacts on the sustainability of supply chains [5]. As highlighted in the work associated with this data descriptor [1], the effect of the pandemic on the evolution of e-commerce is a topic unexplored by scholars, particularly in aspects concerning cybersecurity and sustainability of e-commerce supply chains. The issue of integrated logistics is as crucial for large as for small and medium-sized enterprises, and the pandemic has highlighted the importance of having raw materials available [6-8].

This new reality forces companies to adapt their business models and governments to quickly understand regulatory needs. A better understanding of the current state of e-commerce is therefore not only necessary for scientific knowledge, but also a critical need for decision-making by public authorities and businesses $[9,10]$. This raises at least two 
questions: what this trend has been like in the European context and whether there are the same or new challenges limiting its further development, e.g., security of transactions and privacy.

To answer these questions, Eurostat has been identified as an essential source of open data. Moreover, its use should be promoted to achieve better descriptive cross-sectional studies in Europe. However, the information is incomplete in terms of getting a better picture of the problems and obstacles of online shopping. Consequently, primary data were collected through experts. To reduce subjectivity bias and to achieve judgements from a global rather than a particular perspective, experts with 10 years of experience were chosen later for the interviews. The analysis presented in this paper aims to fill the previously highlighted literature gap and therefore represents a new contribution to the current body of literature on e-commerce.

In terms of methodology, this study proposes a multi-criteria decision analysis (MCDA) and a Likert-scale survey with post-interviews. The multi-criteria analysis provides a comparability model that can be easily fed with Eurostat annual data, which is very useful for data-driven decision-making on e-commerce in Europe. With the information obtained in the survey, we can provide important insights into the importance of digitization and cybersecurity for the sustainable development of e-commerce in Europe.

The pandemic period has prompted researchers to investigate the relationship between sustainability and resilience, and there are many areas in which the relationship between these two topics is investigated [11-13]. The study that this communication intends to integrate [1] contributed to this direction by focusing on the context of e-commerce by formulating two research questions:

- RQ1: How do European countries perform comparatively in e-commerce?

- RQ2: What are the most important challenges to the further expansion of e-commerce?

In this context, this communication instead aims to emphasize the methodological approach that made it possible to answer the RQs, explaining the implications underlying the selection, processing, and integration of heterogeneous data from both primary and secondary sources. The hybrid methodological approach adopted in this research has already been tested in other studies $[14,15]$ to collect data from suitable web-based databases for their processing in order to investigate user behavior. The MCDA allowed for a comparison between the performances of different European countries (to answer RQ1), whereas the Likert scale survey was useful for clarifying the impediments and barriers perceived by consumers when shopping online (to answer RQ2). In this way, this hybrid method can be applied to process other datasets on European countries or regions made available by public or private databases such as Eurostat.

\section{Data Description}

Some academics might ask how you can write a good scientific article without a literature gap. Others might ask how you write one without identifying a good data set. In the approach of this e-commerce-focused line, it was obvious that this is a fast-growing topic $[16,17]$. The data proposed by Salesforce in its Shopping Index report for the first quarter of 2021 are significant: $+58 \%$ compared to the first quarter of 2020 [3]. An analysis of individual countries showed $+111 \%$ for Canada, followed by the Netherlands $(+108 \%)$, UK $(+91 \%)$, Italy $(+78 \%)$, and France $(+71 \%)$. However, our literature analysis showed that the topic of cybersecurity in e-commerce is an up-and-coming issue that has not yet produced a sufficiently solid theoretical body to unambiguously highlight knowledge gaps [1].

Given this scarcity of data, we thought of using Eurostat, which promotes the process of harmonization of statistical methodology among the states themselves. This tool was used in the literature $[18,19]$ and was useful for comparing different countries [20,21]. On the Eurostat search engine, we looked for e-commerce as a keyword and we identified the following cascade process: within the topic "Science, technology, digital society" is "digital economy and society," which in turn presents "ICT usage in households and by 
individuals," and here we came to our keyword. There were 30 criteria divided into six categories (Figure 1):

- Internet purchases by individuals is proposed in the file "isoc_ec_ib20.xls" (provided as a supplementary file http:/ / doi.org/10.5281/zenodo.4769642).

- Internet purchases-goods or services is proposed in the file "isoc_ec_ibgs.xls" (provided as a supplementary file http://doi.org/10.5281/zenodo.4769642).

- Internet purchases_origin of sellers is proposed in the file "isoc_ec_ibos.xls" (provided as a supplementary file http://doi.org/10.5281/zenodo.4769642).

- Internet purchases—collaborative economy is proposed in the file "isoc_ec_ce_i.xls" (provided as a supplementary file http://doi.org/10.5281/zenodo.4769642).

- Internet purchases-money spent is proposed in the file "isoc_ec_ibm.xls" (provided as a supplementary file http:/ / doi.org/10.5281/zenodo.4769642).

- Financial activities over the Internet-money spent is proposed in the file "isoc_ec_ifize.xls" (provided as a supplementary file http://doi.org/10.5281/zenodo.4769642).

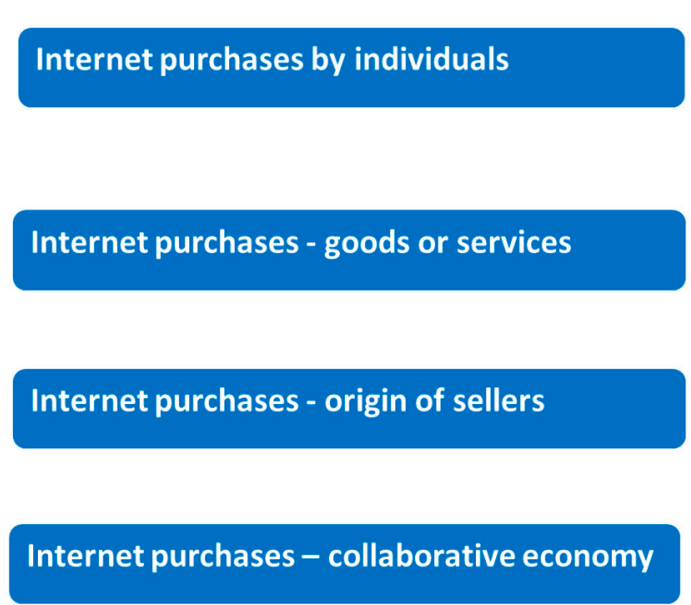

Internet purchases - money spent
Last online purchase: in the last 3 months

Last online purchase: in the 12 months

Frequency of online purchases in the last 3 months: 1 or 2 times

Frequency of online purchases in the last 3 months: 3 to 5 times

Frequency of online purchases in the last 3 months: 6 to 10 times

Frequency of online purchases in the last 3 months: more than 10 times

Online purchases (3 months): clothes (including sport clothing), shoes or accessories

Online purchases (3 months): furniture, home accessories or gardening products

Online purchases ( 3 months): printed books, magazines or newspapers

Online purchases ( 3 months): deliveries from restaurants, fast-food chains, catering services

Online purchases ( 3 months): music as a streaming service or downloads

Online purchases (3 months): films or series as a streaming service or downloads

Online purchases (3 months): from national sellers

Online purchases ( 3 months): from sellers from other EU countries
- Online purchases ( 3 months): from sellers of the rest of the world (non-EU countries)

Online purchases ( 3 months): from sellers from other countries (EU or non-EU)

- Online purchases (3 months) from private persons: any physical goods

Online purchases (3 months) from private persons: household services

Online purchases (3 months) from a private person: transport service

Online purchases ( 3 months) from a private person: rented accommodation

Online purchases (3 months) from a private person: goods, household services, transport services or rented accommodation

Online purchases in the last 3 months for less than 50 euro

Online purchases (3 months) for between 100 and 499 euro

Online purchases (3 months) for between 500 and 999 euro

Online purchases (3 months) for 1000 euro or more

Financial activities over the internet

Online purchases ( 3 months): insurance policies, including travel insurance, also as a package together with e.g. a plane ticket

- Online purchases ( 3 months): took a loan, mortgage or arranged credit from banks or other financial providers Online purchases ( 3 months): bought or sold shares, bonds, units in funds or other financial assets Online purchases (3 months): at least one of the financial activities (I_BFIN_SH1, I_BFIN_IN1, I_BFIN_CR1)

Figure 1. List of criteria.

Eurostat is a database in which data are proposed in multiple ways, and this is clearly an advantage. We highlighted the following hypothesis during our analysis:

- The presence of data for 2020 is a positive since the data are as recent as possible, but this was not the case for all items. There were no values for previous years, so we could not perform a trend analysis.

- The average value of EU 27 is proposed, but after analyzing the list of all countries there were some absences. These are very significant because both Italy and France, as shown above, had very significant values. However, these absences did not affect the results of the other countries, considering the presence of the Netherlands, which had an even more significant margin of growth, or how the intermediate values were calculated according to the most significant performance (associated with the leading country). 
- All data are reported in percentages, which allows for comparability across criteria. This led to two distinct observations. The first concerns the fact that the values are expressed in such a way as not to make distinctions based on the population numbers that characterize the individual countries. In an alternative case, the values could be divided according to specific variables (e.g., the number of inhabitants, the gross domestic product). The second concerns the unit of measurement, since if there were multiple, a way would have had to be found to make them comparable. The solution was in the normalization process. However, this choice was made because we wanted to give a replicable model in other contexts.

The aggregation of these data allowed for the calculation of values to measure the performance of all criteria for each of the 25 European countries examined. Within the same Excel files proposed above, the weights associated with all criteria are also reported. Unlike the values, these data were not identified through Eurostat. The procedure used was to calculate the number of views through the Google search engine. The keywords used from time to time were the specific names of the different criteria. More details will be provided below in the methodology section. Given that the different criteria were not aggregated all together but were evaluated on a category-by-category basis, the specific names of the different categories were used as keywords in this phase as well. This choice was motivated as follows:

- The definition of weights can typically be achieved by collecting data from a panel of respondents, who are chosen on the basis of their experience. In this context, experts' knowledge plays a key role. In some contexts, it is appropriate to choose specific categories of stakeholders. This approach is based on a scenario constructed to obtain the required data. Alternatively, another approach is one in which already available data are captured. It is the approach used in this work that is based on the number of views. However, the number of views is not always a positive element and therefore a feedback operation is necessary. In our case study, no hostile factors were detected.

- The definition of weights should be based on multiple approaches. Too many times in the literature there are works that analyze only a baseline scenario in which the output is proposed as a function of the chosen inputs. This has the limitation of not considering the variability of the results achieved. The construction of alternative scenarios allows, instead, relevance to be given to demonstrate how the output varies as a function of changes in inputs. This led to the identification of an alternative scenario, in which the weights of the categories were all considered of equal relevance.

- The surveys refer to April 2021. Changing the time frame can obviously change the number of views.

The data proposed above were identified to analyze RQ1. The next step was to identify the data useful for investigating RQ2. The identification of problems and barriers affecting Internet shopping could be gleaned from the literature or from field experiments designed to capture the critical issues highlighted by people. Based on the same method used in the previous step of the research, it was found that these aspects were already present in Eurostat and it seemed logical to use the same source. Consequently, all the potential responses that were proposed in the database were chosen, both for what concerns the problems that people have encountered when shopping on the Internet (Figure 2), and for what concerns the barriers, that is, the motivations that do not push people to make purchases on the Internet (Figure 3). 
INDIVIDUALS WHO ENCOUNTERED THE FOLLOWING PROBLEM WHEN MAKING PURCHASES OVER THE INTERNET:

Difficulties concerning guarantees

Speed of delivery longer than indicated

Delivery costs higher than indicated

Final price higher than indicated

Delivery costs or final price higher than indicated

\section{Wrong goods delivered}

Damaged goods delivered

Wrong or damaged goods delivered

\section{Wrong or damaged good/services delivered}

Lack of security of payments
Problems with fraud

Complaints and redress were difficult

No satisfactory response received after complaint

Complaints and redress were difficult or no satisfactory response received

Other

No problems

Technical failure

Difficulties finding information concerning guarantees, other legal rights

For private use

Foreign retailer did not sell in my country

Figure 2. Problems encountered by individuals when buying/ordering over the Internet.

\section{INDIVIDUALS WHO HAVEN'T ORDERED GOODS OR SERVICES OVER THE INTERNET, BECAUSE:}

They have no need

They prefer to shop in person, they like to see product, loyalty to shops or force of habit

For their own private use (relevant information about goods difficult to find on website)

They lack the necessary skills

It's too expensive

Of too long delivery times

Of problems receiving the ordered goods at home

Of too long delivery times / due to the problematic to receive the ordered goods at home

For their own private use (Payment security concerns)
For their own private use (Privacy concerns)

Of security concerns, they are worried about giving credit card details over the internet

\section{Of privacy concerns, they are worried about giving} personal details over

I'm worried about giving credit card or personal details over the internet

Of trust concerns about receiving or returning goods, complaint/redress

\section{They don't have a payment card}

The speed of the internet connection is too low

Of other reasons

The foreign retailer did not sell in my country

Figure 3. Perceived barriers to buying/ordering over the Internet.

At this stage of the work, it emerged that the data were proposed as a time trend and the latest reference year was 2019; however, several data were missing. Clearly this was generating an imbalance. For this reason, the hypothesis of comparing the different 
answers at the level of a single country was no longer valid and a panel of experts was used. Below are some hypotheses:

- RQ2 could have declined not in a general way, but at the level of each individual country. This output would have been useful because that way it would have been possible to highlight possible links with the results obtained in RQ1.

- The set of answers proposed by Eurostat turned out to be complete because it considered 20 possible answers to potential problems encountered when buying on the Internet and 18 possible answers relative to barriers. In the same way as the previous point, this analysis should not have turned out to be the same for all the markets. This leads to future directions of work, where the different answers proposed in this work can be complemented by specificities of individual sectors.

Our dataset concerns the responses that experts assigned by applying a Likert scale and are proposed in the following files:

- Problems encountered by individuals when buying/ordering over the Internet is proposed in the file "Problems.xlsx" (provided as a supplementary file http:/ / doi. org/10.5281/zenodo.4769642).

- Perceived barriers to buying/ordering over the Internet is proposed in the file "Barriers.xlsx" (provided as a supplementary file http:// doi.org/10.5281/zenodo.4769642).

To share our datasets, we used Zenodo, the multidisciplinary Open Access archive of research publications and data, the result of the collaboration between Cern and the OpenAire project [22]. It allows for self-archiving also for researchers of institutions that do not have institutional repositories. Each dataset is assigned a DOI, a standard identifier that associates any intellectual property object to its reference data (metadata).

\section{Methods}

The work was built on a cross-mix of methodologies (Figure 4). The first used was MCDA, which is widely used both in the literature and in the decision-making processes of the private and public sectors $[23,24]$. Related to this, some authors have employed a multi-criteria decision analysis model integrating both user preferences and expert opinions to support consumption decisions in an e-commerce environment [25]. Its strength is represented by the ability to make a synthesis from a multiplicity of data. It can compare multiple and conflicting alternatives, but it is also well suited to assess alternatives represented by territorial realities, such as countries, regions, and cities. The best alternative is the one that has the highest score, since each alternative has an associated score that is obtained through a product between the values associated with the individual criteria and the weights combined with the same criteria.

MCDA results are represented by the calculation of a performance value (PV), obtained as product between a row vector (RV), composed by the values of the criteria $(\mathrm{J})$, and a column vector $(\mathrm{CV})$, composed by the weights of the criteria. PV is calculated for each alternative (represented by single member states (MSs)) aggregating $\mathrm{N}$ criteria.

$$
\mathrm{PV}_{(\mathrm{MS})}=\sum_{\mathrm{J}=1}^{\mathrm{N}} \mathrm{RV}_{(\mathrm{MS})} * \mathrm{CV}
$$

The definition of the values, as explained in the previous section, saw the data defined based on Eurostat. The definition of the weights, on the other hand, was assumed to be based on the number of views. Here, however, it is worth focusing on the choice of calculating a global priority associated with each criterion that was calculated as the product of a local priority and a category priority [26]. This work considered two scenarios: (i) different weights (DW), in which the number of views in the Google search engine determined both local and category priority, and (ii) hybrid equal weights (HEW), in which all category priorities had the same weight, whereas local priority was calculated as in the DW scenario. 


\section{E-COMMERCE}

RQ1: A comparison among European countries

$$
\text { MCDA }
$$

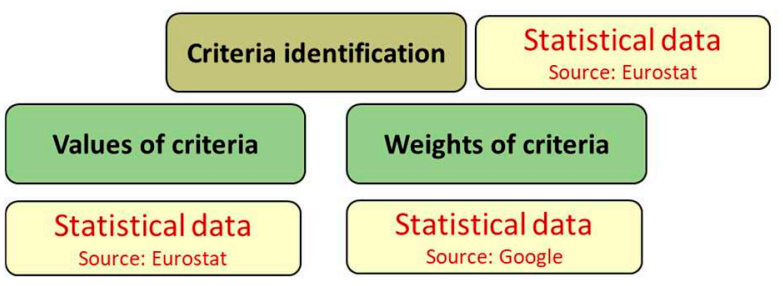

Ranking of European countries

Aggregated data
RQ2: Assessment of problems and barriers

Likert Scale Survey

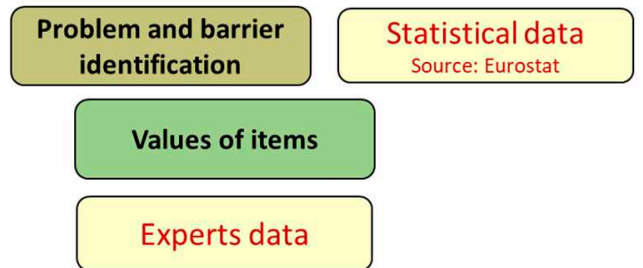

Ranking of items

Aggregated data

Sustainability
TOPIC

IDENTIFICATION

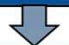

RQS DEFINITION

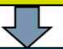

METHODOLOGY

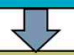

INPUT
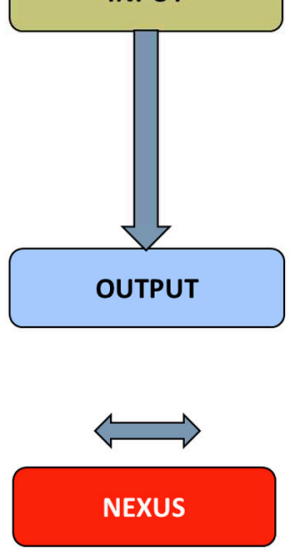

Figure 4. General framework.

Regarding the DW scenario, the first phase consisted of evaluating for each category a weight assigned to the various criteria that characterized it (called local priority). The second phase consisted of evaluating the various categories and the following weights were recorded (called category priority) based on the number of views: (i) Internet purchases by individuals $(4.0 \%)$, (ii) Internet purchases-goods or services (15.2\%), (iii) Internet purchases-origin of sellers (11.4\%), (iv) Internet purchases-collaborative economy (5.4\%), (v) Internet purchases-money spent (4.2\%), and (vi) financial activities over the Internet $(59.8 \%)$. Concerning the HEW scenario, there was no difference among these categories.

The second methodology used was the Likert-scale survey evaluated in the range of 1-5: 1 = totally disagree, $2=$ disagree, $3=$ neither agree nor disagree, $4=$ agree, and 5 = completely agree. A critical step in this methodology is represented by the choice of experts. Considering the subject matter, managers were chosen as the category of stakeholders, and it was therefore preferred to focus on practical expertise. The tool used to establish connections with the experts was LinkedIn, a social network mainly used in the development of professional contacts. These phases of the work required time, which was optimized when the goal to be achieved and the knowledge of the people needed were clear. The experts contributed by assigning an evaluation and giving their ideas on the topic analyzed. This part certainly represents a value-added element and is the one that for all 10 experts represented the most time spent on the interview (carried out in March-April 2021). To encourage this approach, the material was sent by email before the interview.

\section{Discussion and Conclusions}

The analysis of this work is part of a larger research project [1]. Specifically, the need to develop additional work stems from the significance associated with defining the methodology and data collection in detail. This aspect is gaining increasing relevance in the literature landscape. We are in a historical phase in which the pandemic has eradicated many certainties. Research has regained a central role. The pandemic has changed many lifestyles, leading consumers to rely even more on online purchases, and in the same way, remote work has become more important. The issue of the relationship between 
sustainability and cybersecurity must be carefully considered. In particular, life cycle analyses demonstrating beneficial effects are required. Digitization certainly saves time, but climate change requires tough choices. Consequently, digital development must be aligned with sustainable development.

In particular, this research project focuses on those aspects of e-commerce that show a greater propensity on the part of European citizens to use this method of purchasing. This work allows a framework to be built that can be replicated in other contexts. The description of the data of a work turns out to be very relevant because it shows us how the results were obtained. On the one hand, the availability of data is fundamental because it allows analysts to interpret such data and identify possible relationships. On the other hand, a methodology applied following a scientific method assigns robustness to the results obtained. The availability of data makes it possible to assess correlations and to identify how variables are related to each other. This is crucial for developing a business strategy in which e-commerce is set to play a crucial role as a sales channel. Moreover, it also induces changes at an organizational level.

In particular, e-commerce is a hot topic and is a preferred channel for the younger generation. In the same way, these very generations are the ones who demand more rights and more sustainability. Sustainable consumption models are not based on approaches that slow economic growth, but on those that combine consumer needs with available resources. In some ways the underlying idea is to minimize waste, an idea that is very well present in production models. However, as a result of crony capitalism, even these models have been seen to focus solely on the economic sphere. E-commerce should not favor production models in places where some workers' rights are not respected or where environmental regulations are less stringent. However, these features would also be potential risks in traditional sales conditions. This is therefore a change of perspective. Likewise, it should not penalize emerging countries, but rather offer them a way to grow economically while respecting social and environmental principles.

This communication makes it possible to propose a method such as the MCDA method, which is well known in the literature because of its flexibility and fields of application. For many, experience in the field is synonymous with knowledge, and the approach proposed in this paper totally agrees. However, the right product is not always sold, and many times the product that is fashionable is sold. On the topic of sustainability, just look at a few years ago, when there were few consumers reading labels and looking for sustainable components. Today everything has changed. However, the strong attention of consumers towards the environment is not enough if the propensity does not translate into an act of purchase. This methodology considers the number of views and of manifestation of interest towards some items. Obviously, this approach cannot be used all the time. For this reason, methods must always be innovative, new ways of assigning weights must be identified, and expert evaluations must be integrated with young talent. Each analysis requires an appropriate choice because there is no universal solution to the various methods. Therefore, the final message of this communication is to provide in detail the framework with which this analysis was carried out in order to be able to replicate it and include it as a methodology in which the selection of weights is attributed to search-engine research. Sustainability has a big task: to provide answers to current problems. This means that e-commerce cannot simply be seen as a mode of sales that can reduce the sales space of some local markets. It is necessary to take note of a change that has already taken place. The data proposed in this paper highlight a significant trend in the European sector, and micro analysis must be conducted at the product level to understand how business strategies must change in order to respond to this external change. Competitiveness also inevitably passes through digital purchasing.

Author Contributions: Conceptualization, D.S.-B.; methodology, I.D.; data curation, R.G.-S. and M.S.M.-S.; writing-original draft preparation, I.D., R.G.-S., M.S.M.-S. and D.S.-B.; writing-review and editing, I.D., R.G.-S., M.S.M.-S. and D.S.-B.; supervision, I.D. All authors have read and agreed to the published version of the manuscript. 
Funding: This research received no external funding.

Institutional Review Board Statement: Not applicable.

Informed Consent Statement: Not applicable.

Data Availability Statement: Not applicable.

Conflicts of Interest: The authors declare no conflict of interest.

\section{References}

1. D’Adamo, I.; González-Sánchez, R.; Medina-Salgado, M.S.; Settembre-Blundo, D. E-Commerce Calls for Cyber-Security and Sustainability: How European Citizens Look for a Trusted Online Environment. Sustainability 2021, 13, 6752. [CrossRef]

2. Calzada, J.; Tselekounis, M. Net Neutrality in a hyperlinked Internet economy. Int. J. Ind. Organ. 2018, 59, 190-221. [CrossRef]

3. Salesforce Salesforce Shopping Index Q1 2021. Available online: https:/ / public.tableau.com/profile/salesforcecommercecloud\# !/vizhome/SalesforceShoppingIndex/SalesforceShoppingIndex (accessed on 7 May 2021).

4. Dupont, B.; Lusthaus, J. Countering Distrust in Illicit Online Networks: The Dispute Resolution Strategies of Cybercriminals. Soc. Sci. Comput. Rev. 2021, 0894439321994623.

5. Settembre-Blundo, D.; González-Sánchez, R.; Medina-Salgado, S.; García-Muiña, F.E. Flexibility and Resilience in Corporate Decision Making: A New Sustainability-Based Risk Management System in Uncertain Times. Glob. J. Flex. Syst. Manag. 2021, $1-26$.

6. Mahmud, P.; Paul, S.K.; Azeem, A.; Chowdhury, P. Evaluating Supply Chain Collaboration Barriers in Small- and Medium-Sized Enterprises. Sustainability 2021, 13, 7449. [CrossRef]

7. Loizia, P.; Voukkali, I.; Chatziparaskeva, G.; Navarro-Pedreño, J.; Zorpas, A.A. Measuring the Level of Environmental Performance on Coastal Environment before and during the COVID-19 Pandemic: A Case Study from Cyprus. Sustainability 2021, 13, 2485. [CrossRef]

8. Paul, S.K.; Moktadir, M.A.; Sallam, K.; Choi, T.-M.; Chakrabortty, R.K. A recovery planning model for online business operations under the COVID-19 outbreak. Int. J. Prod. Res. 2021, 1-23. [CrossRef]

9. Amicarelli, V.; Tricase, C.; Spada, A.; Bux, C. Households' Food Waste Behavior at Local Scale: A Cluster Analysis after the COVID-19 Lockdown. Sustainability 2021, 13, 3283. [CrossRef]

10. Keenan, J.M. COVID, resilience, and the built environment. Environ. Syst. Decis. 2020, 40, 216-221. [CrossRef]

11. D'Adamo, I.; Rosa, P. How do you see infrastructure? Green energy to provide economic growth after COVID-19. Sustainability 2020, 12, 4738. [CrossRef]

12. D'Adamo, I.; Lupi, G. Sustainability and Resilience after COVID-19: A Circular Premium in the Fashion Industry. Sustainability 2021, 13, 1861. [CrossRef]

13. Miceli, A.; Hagen, B.; Riccardi, M.P.; Sotti, F.; Settembre-Blundo, D. Thriving, Not Just Surviving in Changing Times: How Sustainability, Agility and Digitalization Intertwine with Organizational Resilience. Sustainability 2021, 13, 2052. [CrossRef]

14. Vila, T.D.; González, E.A.; Vila, N.A.; Brea, J.A. Indicators of Website Features in the User Experience of E-Tourism Search and Metasearch Engines. J. Theor. Appl. Electron. Commer. Res. 2021, 16, 18-36. [CrossRef]

15. Ferrari, A.M.; Volpi, L.; Settembre-Blundo, D.; García-Muiña, F.E. Dynamic life cycle assessment (LCA) integrating life cycle inventory (LCI) and Enterprise resource planning (ERP) in an industry 4.0 environment. J. Clean. Prod. 2021, $286,125314$. [CrossRef]

16. Kleisiari, C.; Duquenne, M.-N.; Vlontzos, G. E-Commerce in the Retail Chain Store Market: An Alternative or a Main Trend? Sustainability 2021, 13, 4392. [CrossRef]

17. Wang, J.; Gao, X.; Wang, Z. Sustainable Supply Chain Decisions under E-Commerce Platform Marketplace with Competition. Sustainability 2021, 13, 4162. [CrossRef]

18. Crecente, F.; Sarabia, M.; del Val, M.T. Sustainable Entrepreneurship in the 2030 Horizon. Sustainability 2021, 13, 909. [CrossRef]

19. Ronzon, T.; Piotrowski, S.; Tamosiunas, S.; Dammer, L.; Carus, M.; M'barek, R. Developments of economic growth and employment in bioeconomy sectors across the EU. Sustainability 2020, 12, 4507. [CrossRef]

20. D'Adamo, I.; Gastaldi, M.; Rosa, P. Assessing Environmental and Energetic Indexes in 27 European Countries. Int. J. Energy Econ. Policy 2021, 11, 417-423. [CrossRef]

21. Castillo-Giménez, J.; Montañés, A.; Picazo-Tadeo, A.J. Performance and convergence in municipal waste treatment in the European Union. Waste Manag. 2019, 85, 222-231. [CrossRef] [PubMed]

22. Sicilia, M.-A.; García-Barriocanal, E.; Sánchez-Alonso, S. Community Curation in Open Dataset Repositories: Insights from Zenodo. Procedia Comput. Sci. 2017, 106, 54-60. [CrossRef]

23. Sharma, R.K.; Singh, P.K.; Sarkar, P.; Singh, H. A hybrid multi-criteria decision approach to analyze key factors affecting sustainability in supply chain networks of manufacturing organizations. Clean Technol. Environ. Policy 2020, 22, 1871-1889. [CrossRef]

24. Voukkali, I.; Zorpas, A.A. Evaluation of urban metabolism assessment methods through SWOT analysis and analytical hierocracy process. Sci. Total Environ. 2021, 39, 150700. 
25. Karanik, M.; Bernal, R.; Peláez, J.I.; Gomez-Ruiz, J.A. Combining user preferences and expert opinions: A criteria synergy-based model for decision making on the Web. Soft Comput. 2019, 23, 1357-1373. [CrossRef]

26. Brudermann, T.; Mitterhuber, C.; Posch, A. Agricultural biogas plants-A systematic analysis of strengths, weaknesses, opportunities and threats. Energy Policy 2015, 76, 107-111. [CrossRef] 
Article

\title{
Sustainability and Resilience Revisited: Impact of Information Technology Disruptions on Empirical Retail Logistics Efficiency
}

\author{
Matthias Klumpp ${ }^{1,2,3, *(\mathbb{D})}$ and Dominic Loske ${ }^{2,4}$ (D) \\ 1 Fraunhofer Institute for Material Flow and Logistics IML, 44227 Dortmund, Germany \\ 2 Institute for Logistics and Service Management, FOM University of Applied Sciences, 45130 Essen, Germany; \\ dominic.loske@fom-net.de \\ 3 Department of Business Administration, University of Göttingen, 37073 Göttingen, Germany \\ 4 Faculty of Business and Law, UCAM Universidad Católica San Antonio de Murcia, 30107 Guadalupe, Spain \\ * Correspondence: matthias.klumpp@uni-goettingen.de
}

Citation: Klumpp, M.; Loske, D. Sustainability and Resilience Revisited: Impact of Information Technology Disruptions on Empirical Retail Logistics Efficiency. Sustainability 2021, 13, 5650. https:// doi.org/10.3390/su13105650

Academic Editor: Andrea Appolloni

Received: 27 February 2021

Accepted: 11 May 2021

Published: 18 May 2021

Publisher's Note: MDPI stays neutral with regard to jurisdictional claims in published maps and institutional affiliations.

Copyright: (c) 2021 by the authors. Licensee MDPI, Basel, Switzerland. This article is an open access article distributed under the terms and conditions of the Creative Commons Attribution (CC BY) license (https:// creativecommons.org/licenses/by/ $4.0 /)$.

\begin{abstract}
The increasing use of information technology (IT) in supply chain management and logistics is connected to corporate advantages and enhanced competitiveness provided by enterprise resource planning systems and warehouse management systems. One downside of advancing digitalization is an increasing dependence on IT systems and the negative effects of technology disruption impacts on firm performance, measured by logistics efficiency, e.g., with data envelopment analysis (DEA). While the traditional DEA model cannot deconstruct production processes to find the underlying causes of inefficiencies, network DEA (NDEA) can provide insights into resource allocation at the individual stages of operations. We apply an NDEA approach to measure the impact of IT disruptions on the efficiency of operational processes in retail logistics. We compare efficiency levels during IT disruptions, as well as ripple effects throughout subsequent days. In the first stage, we evaluate the efficiency of order picking in retail logistics. After handing over the transport units to the outgoing goods department of a warehouse, we assess the subsequent process of truck loading as a second stage. The obtained results underline the analytical power of NDEA models and demonstrate that the proposed model can evaluate IT disruptions in supply chains better than traditional approaches. Insights show that efficiency reductions after IT disruptions occur at different levels and for diverse reasons, and successful preparation and contingency management can support improvements.
\end{abstract}

Keywords: supply chain resilience; IT disruptions; efficiency measurement; warehouse logistics; DEA; economic sustainability

\section{Introduction}

Disruptions to the supply chain and transportation processes comprise an important field of research that can help us to understand their causes and effects, as well as to develop mitigation and coping strategies [1-5]. In many cases, such disruptions are identified in connection with supply chain management and transportation, information technology, computer science and process areas [6-10]. However, the COVID-19 pandemic, which has caused multiple interruptions to production and transportation processes all over the world, has a special impact on supply chains and provides further motivation to study disruption situations and management [11-13]. It is of high interest within supply chain research and business management to understand the processes and implications regarding transportation process interruptions more in detail, as they are relevant to any form of global, digital and sustainable supply chains [14-16].The specific case of computer system disruptions as a common cause of problems in warehouse and transportation logistics has seldom been analyzed empirically, though they are deemed highly relevant, even for, e.g., quantified shareholder value [17]. This study is one of the first quantitative and efficiency-based papers dedicated to computer system disruptions on operational processes in warehouse logistics, for example, regarding software or hardware failures, electricity blackouts and hacking incidents, including ransomware. This 
approach relates to two perspectives regarding the effect of computer system disruptions on warehouse logistics for retailing [18], which connects to the field of industrial ecology and economic sustainability in closed-loop concepts for supply chain management. From a static process point of view, the first research question $\left(R_{2}\right)$ is: What is the impact of a supply chain computer system disruption in the first sub-process on the subsequent sub-process in a supply chain and vice versa, and how does this affect the efficiency of the entire warehouse system? From a dynamic longitudinal point of view, the second research question is directed at the time-series effects of computer system disruptions $\left(\mathrm{RQ}_{2}\right)$ : What is the impact of a computer system disruption on the efficiency of operational processes in warehouse logistics at the time of occurrence and in the aftermath in subsequent periods? The relevance of this question becomes prominent when considering the rapid and versatile advances in computer systems and automation for warehouse logistics. This article aims to shed light on the phenomenon of cross-sectoral effects of computer system-related supply chain disruptions.

The contributions of this paper are threefold: (1) elaborating, justifying and applying network data envelopment analysis (NDEA) as a method of performance measurement for intralogistics processes in retail warehouse logistics for supply chain disruptions due to technical failure; (2) identifying and weighting key factors influencing the efficiency levels from technical disruptions; and (3) deriving implications for supply chain management. We used empirical data obtained from a large German food retailing company. The dataset contains 17 days' worth of data on five warehouses, with 9.3 million stock-keeping units (SKUs) picked in $42,100 \mathrm{~h}$. This paper is structured as follows: The second section provides a theoretical framework regarding the impacts of supply chain and transport process interruptions. The third section outlines the data and sample setup, while the fourth section describes the method used, alongside its justification. The fifth section presents the specific use of the network DEA (NDEA) approach (model formulation). The sixth section outlines the empirical results obtained. The seventh section provides a discussion regarding the results and their implications, as well as their limitations, and the eighth section presents the conclusions.

\section{Theoretical Framework: Supply Chain Disruptions}

The theoretical framework regarding interruptions to supply chain and transportation processes can be structured according to Figure 1: Eight areas of management science research can be identified regarding supply chain interruptions, making up most of the research and practically relevant questions in relation to this subfield of supply chain management. As the eight relevant areas found from a literature analysis as described below, it can be recognized that the area of internal causes is under-represented and therefore under-researched. Our research is positioned exactly within this field and research gap and has the objective to further increase the knowledge about internal causes for supply chain disruptions and their effects. In order to achieve this, we analyze the specific internal case of computer system failures and the impacts on downstream supply chain processes. Additionally, the examined disruptions with internal causes take an explorative research perspective and do not focus on the impact of IT disruptions. Hence, we aspire to contribute to this research stream through our quantitative explanative research design.

From the above figure, we outline the described elements and levels in detail, as follows.

Preparation (Supply Chain Resilience, SCR): Resilience refers to the ability of supply chains to withstand disruptions and unexpected events, such as supply chain interruptions. This is exemplified, for example, by Chen, Dui and Zhang [5], suggesting a quantitative cost-based measure for overall supply chain resilience. This team of authors also discussed this aspect from different customer perspectives. Specific measures, e.g., repair capacities to increase resilience, are analyzed by Goldbeck, Angeloudis and Ochieng [19]. A dedicated network approach is presented by $\mathrm{Li}$, Zobel, Seref and Chatfield [20] regarding a holistic supply chain resilience approach. This holistic supply chain resilience perspective is also addressed, for example, by Jabbarzadeh, Fahimnia, Sheu and Moghadam [3]. 


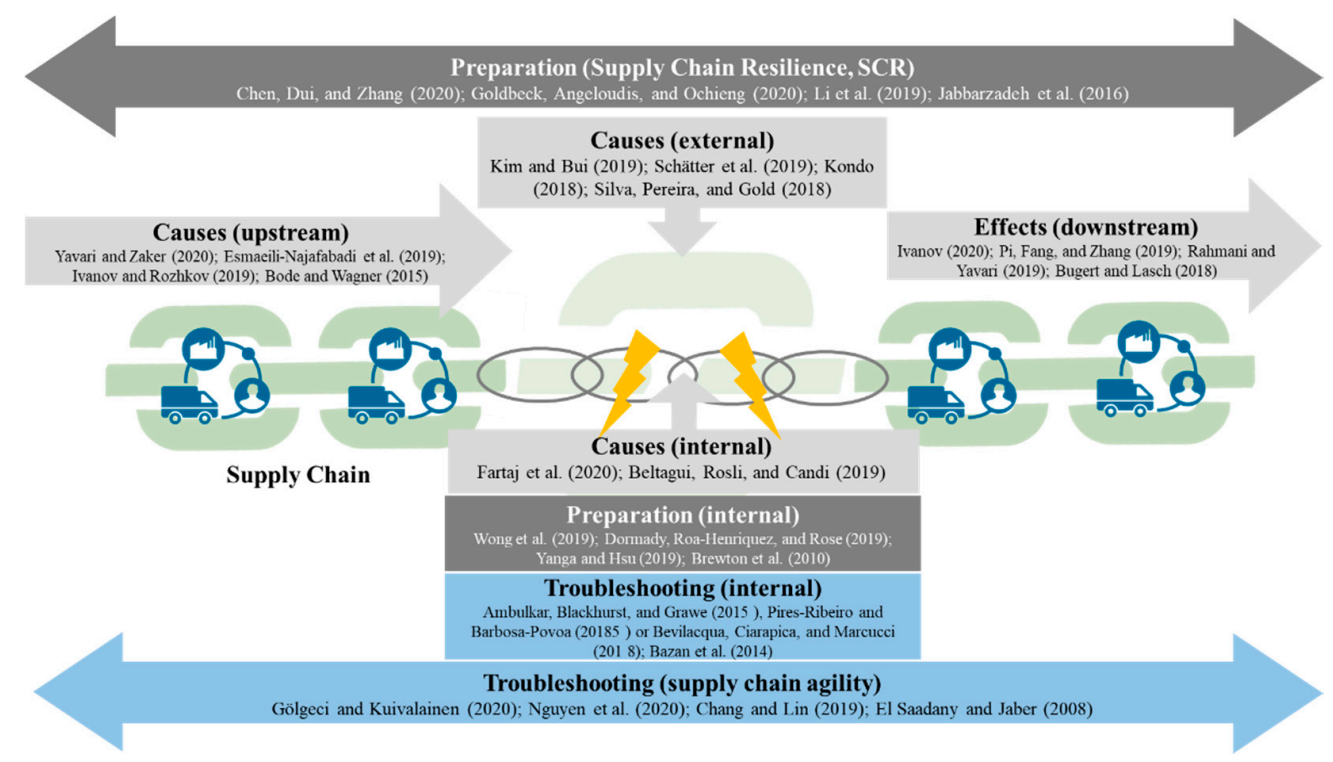

Figure 1. Theoretical framework structure for supply chain interruption analysis and management.

Preparation (internal): Wong, Lirn, Yang and Shang [4], for example, provide a vivid example of the questions related to firm-level preparation analysis and management approaches. They propose the application of organizational information process theory for preparing individual firms for supply chain disruptions. Furthermore, this research shows under which circumstances firm-level preparation and resilience pay off regarding specific performance measures. This is also connected to the production theory of individual forms, for example, in the work of Dormady, Roa-Henriquez and Rose [21]. Research outputs also address specific industries and sectors, for example, the maritime business sector [22]. A specific application of this form perspective to family-owned forms is presented by Brewton, Danes, Stafford and Haynes [23].

Causes (upstream) The upstream structure of supply chains can be the source of many disruption potentials in global value chains, as outlined, for example, by Bode and Wagner [24]. This is especially true for a supply chain with increased complexity, as shown in this research based on prediction models. Many publications shed light on the reasons for ripple effects and disruptions along the value chain, e.g., Esmaeili-Najafabadi, Fallah Nezhad, Pourmohammadi, Honarvar and Vahdatzad [25]; Ivanov and Rozhkov [26]; and Yavari and Zaker [27].

Causes (external): Discussions of external causes of supply chain disruptions mainly concentrate on grave events such as natural disasters and other "force majeure" events regarding supply chain management. This is shown, for example, by Kondo [28] for a powerful earthquake in Japan. Similar approaches are presented, e.g., by Silva, Pereira and Gold [29] for Brazil; Kim and Bui [30] for Puerto Rico; and Schätter, Hansen, Wiens and Schultmann [31] with regard to a general decision management approach to unexpected external events.

Causes (internal): Fartaj, Kabir, Eghujovbo, Ali and Paul [32] show for the automotive industry, transportation processes and also production logistics that internal causes can be a major source of process and supply chain disruptions. Nevertheless, disruptions not only stem from negative process deviations, but might also be caused by positive innovation changes, as Beltagui, Rosli and Candi [33] show for the introduction of 3D printing processes in firms.

Effects (downstream): A multitude of research works address the effects of interruptions and outages on supply chain management from a downstream perspective. For the 2020 COVID-19 crisis, this is exemplified by Ivanov [12] from a global perspective. Further research outputs highlight the role of demand disruptions in the context of supply 
chain resilience, e.g., Pi, Fang and Zhang [34]; Rahmani and Yavari [35]; and Bugert and Lasch [36].

Troubleshooting (internal): Some research contributions explore and show the benefits of firm-level mitigation measures for process and supply chain interruptions. A typical example is Bazan et al. (2014), who analyze the positive effects of production restoration operations under such critical conditions. This is further detailed by other publications such as Ambulkar, Blackhurst and Grawe [37]; Pires Ribeiro and Barbosa-Povoa [38]; and Bevilacqua, Ciarapica and Marcucci [39].

Troubleshooting (Supply Chain Agility): Regarding mitigation efforts after supply chain disruptions, El Saadany and Jaber [1], for example, describe how production interruptions can be managed in a two-tier setting. This aims to increase supply chain agility for disruption cases. Similar approaches are presented by Gölgeci and Kuivalainen [40] in the marketing domain; Nguyen, Sharkey, Wheeler, Mitchell and Wallace [41], proposing quantitative indicators; and Chang and Lin [42], regarding lead-time.

\section{Data and Sample}

To investigate the impact of information technology disruptions in retail logistics, we chose an empirical single-case study research design as one part of our field-based research. Additionally, we wished to delimit our approach from experimental investigations, as we did not influence variables, but rather observed their development within a real-world setting. Herein, we examine five warehouses belonging to a large German brick-and-mortar grocery retailer. While the retailer is operating 18 of these distribution centers, each of the observed warehouses is responsible for supplying between 341 and 522 grocery stores per day.

Wollenburg et al. (2018) elaborate several typologies for retailers operating within offline and online retailing channels [43]. According to their framework, we assigned the observed retail company to the type where offline and online operations are separated on the operational level and distribution centers solely pick and deliver orders for grocery stores. This is also commonly referred to as traditional brick-and-mortar grocery logistics. Therefore, we focus on the impact of information technology disruptions during the order fulfillment of offline demand and within the context of stationary grocery retailing.

In our case study, every distribution center receives goods from suppliers, stores them within the warehouse, picks orders that are sent to the supermarket stores and delivers picked stock keeping units (SKU) to the assigned grocery shops using transportation aids. Parallel to this material flow, the information flow is ensured through a warehouse management system and a route-planning system, as well as a track-and-trace system.

Taking the perspective of Porter's value chain, we analyze the impact of information technology disruptions on the activity level of outbound logistics. Each of the examined warehouses has two major steps for order fulfillment: (1) order picking and (2) transport logistics. Although there are sub-units for each of these process steps, we choose the aggregation level of these two inter-organizational units, referred to as (1) the order-picking sector and (2) the transport sector, with the key processes being (1) warehouse logistical process and (2) truck loading, respectively.

The order-picking sector is responsible for complete, on-time order compiling based on the orders of the shops and uses a pick-by-voice technology where all order pickers wear a headset connected to a small wearable computer. The picking system names the storage location and the number of SKUs to pick. Successful picking operations are confirmed when pre-defined confirmation codes on the storage locations are correctly given back to the picking system, e.g., through linguistic input that can be identified by a speech recognition software running on the wearable computer, or by scanning barcode labels at the storage locations. Rolling cages and pallets are utilized as transportation aids. All data assigned to this process can be obtained through the company's warehouse management system.

After the order-picking process, the rolling cages with SKUs are buffered at a shipping area and ready for distribution to the grocery stores. The transport sector is responsible for delivering the full amount of transport units within a given time window. All truck 
drivers use a mobile device to receive their work tasks, e.g., to load a certain number of containers for a grocery store and deliver them within a predefined time window. In order to fulfill the task, truck drivers have to scan all relevant 1D barcodes, which are attached to the containers, load them into the trucks, and record differences between the data provided by the mobile device and the condition of transported goods. All data assigned to this process can be obtained through the company's track-and-trace system.

Due to the high level of digitalization and automation, the IT department is permanently measuring the functionality of the logistics systems. Errors that affect the operational logistics processes are reported by users or IT specialists through a management information system, which documents the strength of the computer system disruption, the number of affected employees and the duration of the disruption. Thus, computer system disruptions can be matched with the efficient progression of the warehouse processes during these outages.

In this paper, we investigate computer system disruptions related to the order picking sector, as well as disruptions related to the transport sector. The order picking sector is referred to as the warehouse logistics process and is understood as the first node in the network of relevant retail logistics operations. After this step, the transport logistics processes of truck loading represent the second node as an equivalent process step.

Our observations include the evaluation of efficiency for computer system disruptions regarding (1) the network as a whole and (2) the two nodes as isolated processes. The object of investigation is a computer system disruption within node 1 in the context of case 1 (C1) and a computer system disruption within node 2 in the context of $C 2$. In each case, the computer system disruptions affect the core information system of the main process for one hour.

In $\mathrm{C} 1$, the picking system broke down for one hour, while, in C2, the track-and-trace system broke down for the same duration. During the breakdown, the employees tried to fulfill their work tasks without digital work equipment and fell back on paper-based picking and truck loading. The following chapter introduces and justifies the application of DEA as a key research method for measuring the efficiency impact of computer system disruptions in retail logistics.

\section{Methodology}

\subsection{Method Selection}

In general, DEA is a non-parametric optimization method of mathematical programming for measuring the relative efficiency of decision-making units (DMUs) that have multiple inputs and outputs. A basic model was introduced by Charnes, Cooper and Rhodes (1978) and is based on Pareto's definition of economic efficiency [44] and Koopmans' activity analysis concept [45], together with the publications of Debreu and Farrell, which deal with radial efficiency measurement [46,47].

Four characteristics of computer system disruptions justify the application of DEA as a key research method: (1) The impact is not predictable and is not yet examined from an efficiency-based point of view. As a result, there is no a priori knowledge about the functional relationships of new technologies towards humans. (2) Because computer system disruptions influence the human workforce, the theory of work systems is applied as a theoretical framework within the case analyses. As the achievement of work objectives requires inputs and produces outputs, a method that enables the integration of several inand output factors along with the possibility of factor enhancement is needed. (3) Without the existence of a benchmark value for the level of efficiency in computer system disruption scenarios, the analysis has to compare the performance of the different empirical observations. (4) As it is unclear whether computer system disruptions spawn an immediate or gradual development of efficiency, the progress of efficiency in retail logistics is illustrated with an empirical curve progression.

Therefore, the results of the analysis have to be comparable between several periods. As DEA does not require a priori information (requirement 1 ), considers multiple measures (requirement 2) [48,49], compares only the different empirical observations (requirement 3) [50] 
and has comparable results when factors are constant (requirement 4) [48,49], it is the method of choice.

\subsection{CCR and BCC Model}

The optimization method can be based on constant returns to scale (CRS) in the CCR model named by its authors Charnes, Cooper and Rhodes [50] or variable returns to scale (VRS) in the BCC model named by its authors Banker, Charnes and Cooper [51], as well as each case with an input or output orientation. The mathematical formulation of the CCR model is [50]:

$$
\begin{gathered}
\text { maximize efficiency score }=\frac{\sum_{r=1}^{t} u_{r} y_{r j}}{\sum_{i=1}^{m} v_{i} x_{i j}} \\
\text { subject to }=\frac{\sum_{r=1}^{t} u_{r} y_{r j}}{\sum_{i=1}^{m} v_{i} x_{i j}} \leq 1, j=1, \ldots, n \\
u_{r}, v_{i} \geq \forall r \text { and } i
\end{gathered}
$$

where:

$u_{r}=$ the weight given to the output $r$;

$y_{r j}=$ amount of output $\mathrm{r}$ produced by DMU $j$;

$v_{i}=$ the weight given to input $i$;

$x_{i j}=$ amount of input $i$ used by DMU $j$;

$n=$ the number of DMUs;

$t=$ the number of outputs; $m=$ the number of inputs;

$\varepsilon=$ a small positive number.

The basic idea is to calculate an efficiency frontier that is used as a best practice inputoutput combination for the underlying production scenario. A score of 1.0 indicates that a DMU is efficient and positioned on the efficiency frontier, whereas the relative inefficiency of other DMUs can be determined by measuring the distance between individual DMU performance and the efficiency frontier. Measuring efficiency under the assumption of CRS is known as overall technical efficiency (OTE). This includes the determination of (in)efficiency based on (1) the input/output transformation, meaning pure technical efficiency (PTE), as well as (2) the size of operations, meaning scale efficiency (SE). This decomposition is possible under VRS [51-53].

$$
\begin{gathered}
O T E=P T E \times S E \\
S E=\frac{\text { eff } C C R}{\text { eff BCC }}
\end{gathered}
$$

As $S E$ is a number without a unit between 0 and 1 , an $S E$ value of 1 means that the DMU is operating with the optimal operation size, and a difference of 1-SE determines the extent of the inefficiency arising from the non-optimal size of operations for a single DMU. A DMU that is efficient under CRS and VRS operates under the most productive scale size (MPSS), which is also used as a measure for the optimal size of the operation for all other DMUs. The BCC model can be mathematically expressed as:

$$
\begin{gathered}
\text { maximize efficiency score }=\sum_{r=1}^{t} u_{r} y_{r j}+\omega \\
\text { subject to }=\sum_{i=1}^{m} v_{i} x_{i j}=1, \quad j=1, \ldots, n \\
\sum_{r=1}^{t} u_{r} y_{r j}-\sum_{i=1}^{m} v_{i} x_{i j}+\omega \leq 0 \\
u_{r}, v_{i} \geq \varepsilon, \forall r \text { and } i
\end{gathered}
$$




$$
\omega=\text { free (unconstrained in sign) }
$$

\subsection{NDEA and Multi-Stage NDEA}

Conventional DEA models like those explained in the previous section, as well as methodological advancements, e.g., super efficiency to rank DMUs in DEA [54], DEA window analysis [55-57] and the DEA Malmquist index [58] for time series analysis, and fuzzy DEA for imprecise or vague input and output measures [59-61], use the black box assumption for describing DMUs. Therein, the internal structure of DMUs is ignored, and their performance is explained as a function resulting from the transformation of its input and output measures. This paradigm was dissolved by Färe and Primont [62], who presented a DEA model for multi-plant firms and applied it to a dataset of nineteen production plants from four firms operating coal-fired steam electric generating plants. For the first time, the internal structure of the company was not ignored, but was deconstructed into several plants. Over the years, Färe worked on several extensions for DEA models, evaluating the efficiency of DMUs with their known internal structure [63-67]. Thereby, Färe [64] was the first approach to deal with intermediate input variables that are used in several nodes of a network. Based on these approaches, NDEA was applied to measure the efficiency of banks, as a frequently used object of research in the DEA literature, by separating services and sales as two components of a banking system [68]. As the above-presented introduction of intermediate variables to the DEA methodology cannot be equated with establishing the logic of sequential nodes within one network, the development of two-stage DEA models is understood as a parallel stream of research and is of central interest in the course of this paper. Wang, Gopal and Zionts [69] developed a DEA model to assess the impact of IT on firm performance using two inputs, $x_{1}$ and $x_{2}$, for node 1 . Connected with intermediate variable I, which acts as an output for node 1 and an input for node 2, the second stage has one output measure, y. Figure 2 illustrates the structure of the two-stage network DEA model applied in this paper.

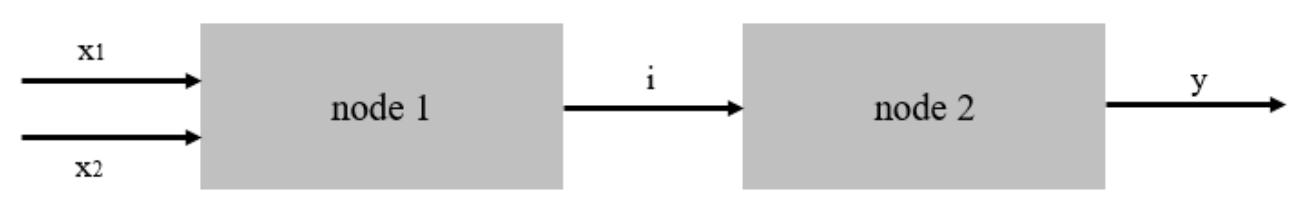

Figure 2. Structure of the two-stage network data envelopment analysis (NDEA) model (according to Wang, Gopal and Zionts [69]).

A similar approach was presented by Seiford and Zhu [70], examining the performance of the top 55 U.S. commercial banks via a two-stage production process that separates profitability and marketability. NDEA and multi-stage NDEA is often applied in current research approaches for performance evaluation in supply chain management and logistics [71-76].

\section{Formulation of a Multi-Stage NDEA Model}

\subsection{Specifications of Input-Output Measures}

The specification of the DEA model begins with the selection of appropriate input and output measures that are used to calculate aggregated efficiency values per DMU and period, as well as per node in the network and for the network as a whole. Within the efficiency analysis, we evaluated the performance of five distribution centers that are used as DMUs. Since the efficiency analysis aspires to measure the impact of computer system disruptions on the operational processes in warehouse logistics, we treated the two key processes-order picking and transportation-as two separate nodes. As a first step, we define the input and output values for the order-picking process. The following input measures are applied to the order-picking efficiency model:

- Total picking time, $\mathrm{I}_{1}$ : As order picking is a laborious and time-intensive warehouse process, the sum of total picking hours represents the human resources invested in 
the picking process. Focusing exclusively on the core process picking, this measurand indicates how well human resources utilize their work equipment. For the DEA model, the period of time between (a) receiving general order data from a picking system and (b) finishing a batch through transfer to the next workstation is considered. The data were extracted from the warehouse management system. Process delays through computer system disruptions could possibly occur and result in an extension of the total picking time and, as a consequence, be quantified by this input factor.

- Total number of batches, $\mathrm{I}_{2}$ : Orders from the grocery stores are aggregated into batches through the warehouse management system, which is primarily premised on product groups and the assigned transportation aid. Hence, the total number of batches is an input factor that expresses the work amount for the picking department. On the other hand, computer system disruptions can lead to problems in replenishing storage locations through forklifts. This, in turn, generates a high number of additional batches that will significantly lower the key SKU figure per batch. Problems that may occur in relation to storage replenishment can therefore be quantified with this input measure. - Total number of incoming pallets, $\mathrm{I}_{3}$ : Supplies to the warehouse are measured as incoming pallets and can be understood as the primary resource for order picking. The total number of pallets is an input factor that expresses the workload for a warehouse and is independent of computer system disruptions. It is used as a control variable to ensure that the volatile demands of grocery stores can be considered when measuring the efficiency of a warehouse in retail logistics.

On the output side, the individual performance of order pickers can be measured by one indicator.

- Total SKUs picked, $\mathrm{O}_{1}$ : Because the most important output of the order-picking process is the physically compiled orders, the units picked by the individual order picker are used as an output. However, as the total amount of picked units correlates with the first input (total picking time of order picker), which is inadmissible when applying DEA, the number of targeted storage locations is used.

Within the NDEA model, the intralogistics processes of order picking and truck loading are divided into two parts: the first with the objective of transforming picking time, batches, as well as incoming pallets into picked SKUs as an intermediate variable, and the second with the objective of transforming these intermediate variables into outputs of the truck loading process. Thus, the intermediate total SKUs picked connect the order picking and the truck loading process by process by acting as $\mathrm{O}_{1}$ in the upstream and as $\mathrm{I}_{1}$ in the downstream process. Furthermore, the following input and output measures are applied to the loading process efficiency model:

- Total loading time, $\mathrm{I}_{2}$ : After registering at the responsible dispatcher in the logistics center, the professional truck drivers receive their route with the grocery stores to deliver to, as well as the loading gate, loading lane, time window, and the number of transportation aids on a mobile device. A handheld scanner is directly connected to the retailer's track-and-trace system and is mainly used to digitally document the loading process by scanning all 1D barcodes on the assigned load carriers. The total loading time includes the period of time between scanning the first $\left(\mathrm{t}_{0}\right)$ and the last $\left(\mathrm{t}_{1}\right)$ load carrier.

- Total on-time deliveries to stores, $\mathrm{O}_{1}$ : As one main goal of the transport unit is the on-time delivery to all customers, the total amount of stores that receive their goods punctually is a vital variable to monitor the success of the process. Furthermore, obstructions through computer system disruptions can extend process times in warehouse logistics, which can result in delayed deliveries. This makes $\mathrm{O}_{1}$ an important measurand for the network itself, as well as for the impacts of any computer system disruptions that may occur.

- Lost transport units, $\mathrm{O}_{2}$ : The second goal of the transport unit is the complete delivery of all assigned transport units for the defined order date. As transportation aids are 
labeled with a 1D barcode and scanned at every relevant node of the warehouse material flow, lost transport units are rare in ordinary business. However, several kinds of operational distortions can interrupt the information flow of the warehouse. This leads, e.g., to transport units without information about the gate and lane at the end of the order-picking process, transport units sent to the wrong gates, or transport units that cannot be scanned during the loading process. The results of these exceptional cases are that the units cannot be found, are recorded as lost transportation units, and are delivered on the next delivery date. As DEA would consider a large number of lost transport units as a large output and, therefore, as highly efficient, $\mathrm{O}_{2}$ is integrated as an undesirable output.

The statistically equal distribution of these data attributes is essential, as significant inequalities could affect the quantitative analysis. Furthermore, the size of the warehouses, expressed through the number of batches, the number of picking hours, the total number of SKUs picked and loaded, and the total number of delivered customers, is of central importance. Table 1 summarizes the key attributes of the dataset by applying descriptive statistics. Furthermore, as the measures can only be applied if they are not highly correlated, a correlation matrix was calculated. The results indicate that there is no linear statistical relationship between the applied input and output measures.

Table 1. Attributes of the dataset and correlation matrix for applied input and output measures.

\begin{tabular}{cccccccc}
\hline & $\mathbf{N}_{\mathbf{1}}, \mathbf{I}_{\mathbf{1}}$ & $\mathbf{N}_{\mathbf{1}}, \mathbf{I}_{\mathbf{2}}$ & $\mathbf{N}_{\mathbf{1}}, \mathbf{I}_{\mathbf{3}}$ & $\mathbf{O}_{\mathbf{1}}, \mathbf{I}_{\mathbf{1}}$ & $\mathbf{N}_{\mathbf{2}}, \mathbf{I}_{\mathbf{2}}$ & $\mathbf{N}_{\mathbf{2}}, \mathbf{O}_{\mathbf{1}}$ & $\mathbf{N}_{\mathbf{2}}, \mathbf{O}_{\mathbf{2}}$ \\
\hline Min. & 697.60 & 422.00 & 1633.00 & $87,397.00$ & 2125.35 & 255.00 & 0 \\
\hline Max & 986.72 & 651.02 & 2749.51 & $130,959.70$ & 7669.39 & 288.20 & 55.27 \\
\hline Mean & 801.94 & 516.34 & 2059.80 & $105,608.95$ & 3683.24 & 272.53 & 11.73 \\
\hline Std. Dev & 59.30 & 52.68 & 256.04 & $12,408.72$ & 1173.22 & 7.72 & 12.49 \\
\hline r: $\mathrm{N}_{1}, \mathrm{I}_{1}$ & 1.00 & 0.38 & 0.13 & 0.09 & 0.50 & -0.09 & 0.39 \\
\hline r: $\mathrm{N}_{1}, \mathrm{I}_{2}$ & & 1.00 & 0.45 & 0.01 & 0.39 & -0.17 & 0.28 \\
\hline r: $\mathrm{N}_{1}, \mathrm{I}_{3}$ & & 1.00 & 0.01 & 0.51 & 0.26 & 0.37 \\
\hline r: $\mathrm{O}_{1}, \mathrm{I}_{1}$ & & & 1.00 & 0.31 & 0.13 & 0.05 \\
\hline r: $\mathrm{N}_{2}, \mathrm{I}_{2}$ & & & & 1.00 & -0.06 & 0.70 \\
\hline r: $\mathrm{N}_{2}, \mathrm{O}_{1}$ & & & & & 1.00 & -0.13 \\
\hline r: $\mathrm{N}_{2}, \mathrm{O}_{2}$ & & & & & & 1.00 \\
\hline
\end{tabular}

\subsection{Model Orientation, Type of Intermediate Variable, and Window Width}

For an efficiency-oriented analysis of supply chain disruptions, we use the previously elaborated factors, whereby the DEA model aspires to (1) maximize desirable outputs, (2) minimize undesirable outputs, (3) maximize desirable inputs, or (4) minimize normal inputs [77]. In the course of this paper, reducing inputs is suitable for $I_{1}$ and $I_{2}$ in node 1 , but not for $I_{3}$. As we use the retailer's warehouses as DMUs, it is doubtful that the warehouses can reduce the number of incoming pallets $\left(\mathrm{I}_{3}\right)$ through warehouse optimization. Incoming goods result from the retailer's orders to suppliers and, hence, are not reducible through operational warehouse processes. Maximizing the outputs, on the other hand, is suitable for node 1, as well as for node 2. Figure 3 illustrates the formulated NDEA model. 


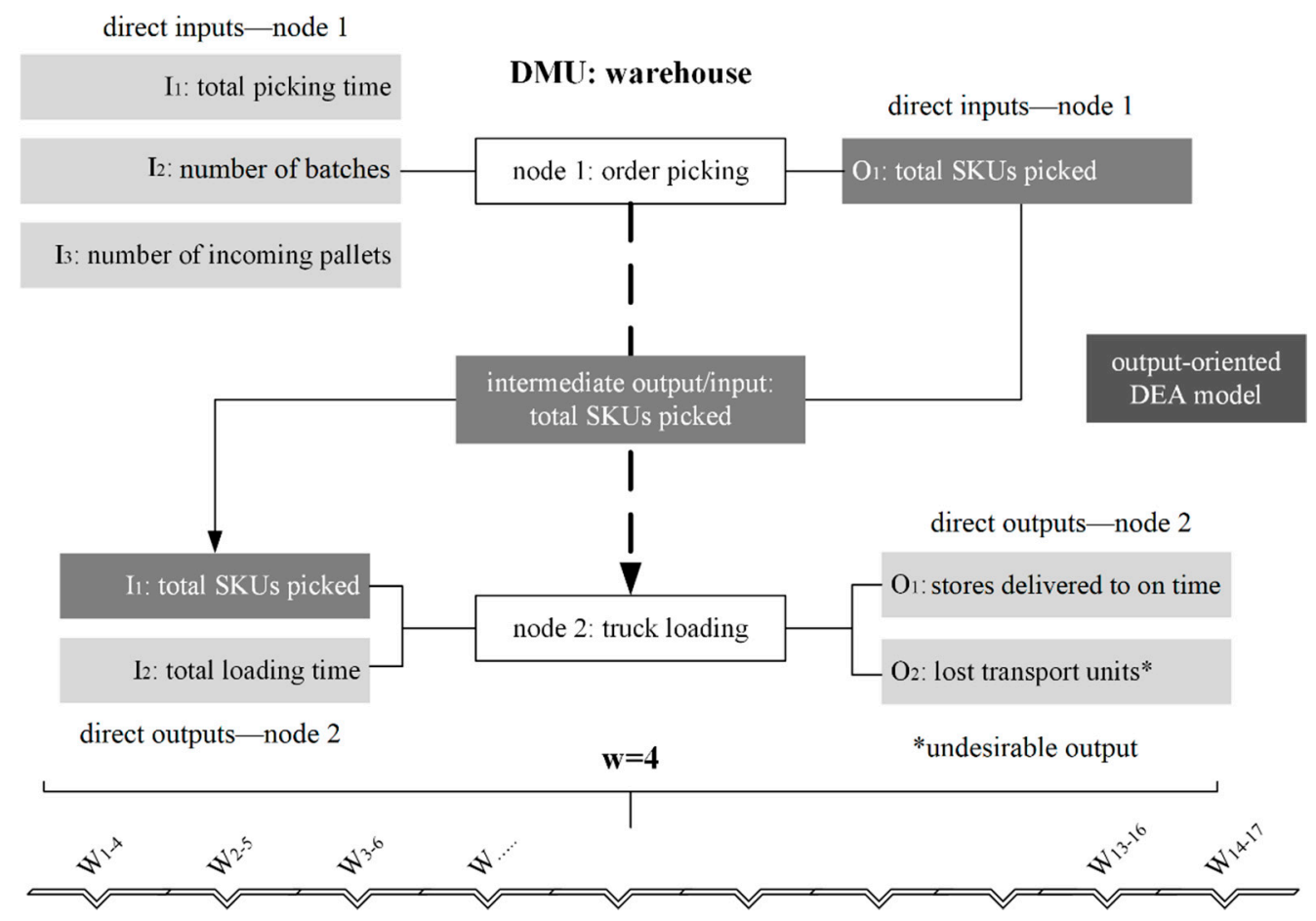

Figure 3. Summary of the NDEA model with nodes and applied input/output factors.

Regarding the warehouse processes in node 1 , it is important to mention that the warehouse can operate on day $n$ with batches assigned to day $n$ and day $n+1$. As a result, the total number of SKUs to pick is controllable and can be influenced by operational logistical processes. For node 2, the number of stores delivered to on time, $\mathrm{O}_{1}$, as well as the lost transport units, $\mathrm{O}_{2}$ (treated as an undesirable output), can be maximized by internal optimizations of the operational transport logistics processes. We chose an outputoriented DEA model. A third possibility would be a non-oriented model that allows inputincreasing and output-decreasing options for a DMU to reach the constructed efficiency frontier. However, as we already excluded the input orientation, a non-oriented DEA is not applicable. Furthermore, an NDEA model can apply several assumptions regarding the orientation of the intermediate variable. It can operate as a free, fixed, non-increasing, non-decreasing, or item-specific variable. These settings can also be found in MaxDEA Pro. As the number of total SKUs picked changes during the transition from node 1 to node 2, we assume that the intermediate variable has a fixed orientation. Since we are aspiring to evaluate the impact of computer system disruptions based on panel data, we combined the formulated NDEA model with a DEA window analysis. Hence, the question of a suitable window width arises. As the analysis includes 17 days, which are treated as 17 periods within the panel dataset, whereby the computer system disruptions take place on day 5 , we chose a window width of $\mathrm{w}=4$. The first window $\left(\mathrm{W}_{1-4}\right)$ includes periods 1 to 4 and includes unobstructed operational processes, while the following periods are impacted by a computer system disruption.

To evaluate the impact of computer system disruptions on operational processes, we chose the investigation of panel data through a DEA window analysis. We included the day before the computer system disruption as $t_{-n}$ to compare the efficiency during the regular business to possible variations, the point in time of the computer system disruption as $\mathrm{t}_{0}$, and the consequential days as $t_{+n}$ to quantify the possible consequences for the operational efficiency.

\section{Empirical Results and Analysis}

\subsection{C1: Computer System Disruptions in Order-Picking System Affecting Node 1}

With the aim of quantifying the efficiency impact of computer system disruptions on operational processes in warehouse logistics, the first part of the analysis was carried out 
concerning the scale efficiency development of each DMU within the defined windows. To find out if CRS or VRS needed to be applied, CCR and BCC versions of the DEA model proposed earlier were calculated. The Table 2 summarizes the mean efficiency scores of the DEA model calculated with CRS and VRS, as well as the SE scores for the whole network and per DMU.

Table 2. Mean efficiency scores and scale efficiency (SE) per decision-making unit (DMU) for the network (node1 + node2).

\begin{tabular}{|c|c|c|c|c|c|c|c|c|c|c|c|c|c|c|c|}
\hline \multirow{2}{*}{ W } & \multicolumn{3}{|c|}{ DMU1 } & \multicolumn{3}{|c|}{ DMU2 } & \multicolumn{3}{|c|}{ DMU3 } & \multicolumn{3}{|c|}{ DMU4 } & \multicolumn{3}{|c|}{ DMU5 } \\
\hline & CRS & VRS & SE & CRS & VRS & SE & CRS & VRS & SE & CRS & VRS & SE & CRS & VRS & SE \\
\hline $1-4$ & 0.88 & 0.94 & 0.94 & 0.86 & 0.97 & 0.88 & 0.79 & 0.96 & 0.83 & 0.87 & 0.95 & 0.92 & 0.80 & 0.90 & 0.89 \\
\hline $2-5$ & 0.78 & 0.91 & 0.85 & 0.83 & 0.97 & 0.86 & 0.77 & 0.96 & 0.80 & 0.83 & 0.95 & 0.88 & 0.78 & 0.90 & 0.87 \\
\hline $3-6$ & 0.67 & 0.87 & 0.76 & 0.83 & 0.96 & 0.86 & 0.80 & 0.98 & 0.82 & 0.78 & 0.93 & 0.84 & 0.76 & 0.91 & 0.83 \\
\hline $4-7$ & 0.56 & 0.82 & 0.67 & 0.79 & 0.92 & 0.85 & 0.79 & 0.97 & 0.81 & 0.78 & 0.92 & 0.85 & 0.73 & 0.90 & 0.80 \\
\hline $5-8$ & 0.47 & 0.82 & 0.57 & 0.87 & 0.96 & 0.90 & 0.87 & 0.99 & 0.88 & 0.83 & 0.95 & 0.87 & 0.82 & 0.92 & 0.89 \\
\hline $6-9$ & 0.49 & 0.86 & 0.57 & 0.83 & 0.95 & 0.88 & 0.87 & 0.97 & 0.89 & 0.80 & 0.96 & 0.83 & 0.77 & 0.93 & 0.82 \\
\hline $7-10$ & 0.55 & 0.88 & 0.62 & 0.78 & 0.94 & 0.83 & 0.83 & 0.97 & 0.85 & 0.78 & 0.96 & 0.81 & 0.77 & 0.93 & 0.82 \\
\hline 8-11 & 0.56 & 0.90 & 0.62 & 0.82 & 0.95 & 0.86 & 0.81 & 0.98 & 0.83 & 0.74 & 0.95 & 0.78 & 0.79 & 0.96 & 0.82 \\
\hline 9-12 & 0.56 & 0.90 & 0.62 & 0.87 & 0.98 & 0.89 & 0.83 & 0.98 & 0.85 & 0.71 & 0.96 & 0.74 & 0.79 & 0.95 & 0.83 \\
\hline 10-13 & 0.58 & 0.90 & 0.64 & 0.84 & 0.97 & 0.87 & 0.85 & 0.98 & 0.87 & 0.81 & 0.97 & 0.83 & 0.83 & 0.94 & 0.89 \\
\hline 11-14 & 0.59 & 0.89 & 0.67 & 0.81 & 0.98 & 0.83 & 0.80 & 0.98 & 0.82 & 0.76 & 0.96 & 0.79 & 0.82 & 0.96 & 0.86 \\
\hline $12-15$ & 0.62 & 0.89 & 0.70 & 0.77 & 0.93 & 0.82 & 0.77 & 0.93 & 0.82 & 0.77 & 0.96 & 0.80 & 0.81 & 0.95 & 0.86 \\
\hline $13-16$ & 0.68 & 0.90 & 0.75 & 0.73 & 0.93 & 0.78 & 0.73 & 0.92 & 0.80 & 0.79 & 0.95 & 0.83 & 0.82 & 0.95 & 0.86 \\
\hline 14-17 & 0.73 & 0.89 & 0.81 & 0.73 & 0.92 & 0.79 & 0.78 & 0.92 & 0.86 & 0.81 & 0.96 & 0.84 & 0.81 & 0.94 & 0.87 \\
\hline
\end{tabular}

As the average SE score for all DMUs and in all windows is $<1$, the results indicate that the DMUs are not operating under MPSS. This becomes particularly clear when we consider that the individual warehouses can change their order picking and transport volume limits within a certain bound. As a result, a BCC model under the assumption of VRS must be applied within the methodological framework of a DEA window analysis. When considering the efficiency scores, the DMUs without computer system disruptions are constant, with a standard deviation of 0.02 (DMU2), 0.02 (DMU3), 0.01 (DMU4), and 0.02 (DMU5). DMU 1, on the other hand, has a standard deviation of 0.03 among all windows and 0.05 for $\mathrm{W}_{1-4}$ without a computer system disruption and $\mathrm{W}_{4-7}$ immediately after the computer system disruption. Besides the efficiency of the whole network, the developments of the single notes are of special interest when answering the research question. MaxDEA Pro enables the calculation of isolated efficiency values per DMU and node. The following figure visualizes, summarizes, and contrasts the efficiency scores per DMU and window for (1) the whole network, (2) the warehouse processes as node 1, and (3) the transport logistical processes as node 2.

The results illustrated in Figure 4 show that the computer system disruption in the warehouse logistics process takes place on day five and is therefore included in the efficiency score since $W_{2-5}$ significantly reduces the efficiency of the whole network. The lowest efficiency level is reached in $\mathrm{W}_{4-7}$ and $\mathrm{W}_{5-8}$ immediately after the outage, so it is important to mention that the network efficiency of DMU 1 does not return to the original level before the disruption. Considering the single nodes, the warehouse logistics process is mainly influenced, which is not surprising in itself, as it was the epicenter of the computer system disruption. An interesting development can be found for the transport logistical process (node 2): (1) Although this subsequent process is not directly influenced by the disruption, the efficiency score drops at the same time as in the warehouse process; (2) the efficiency loss is not as grave as in node 1 ; and (3) node 2 recovers significantly faster to a higher and more stable efficiency level than node 1. 

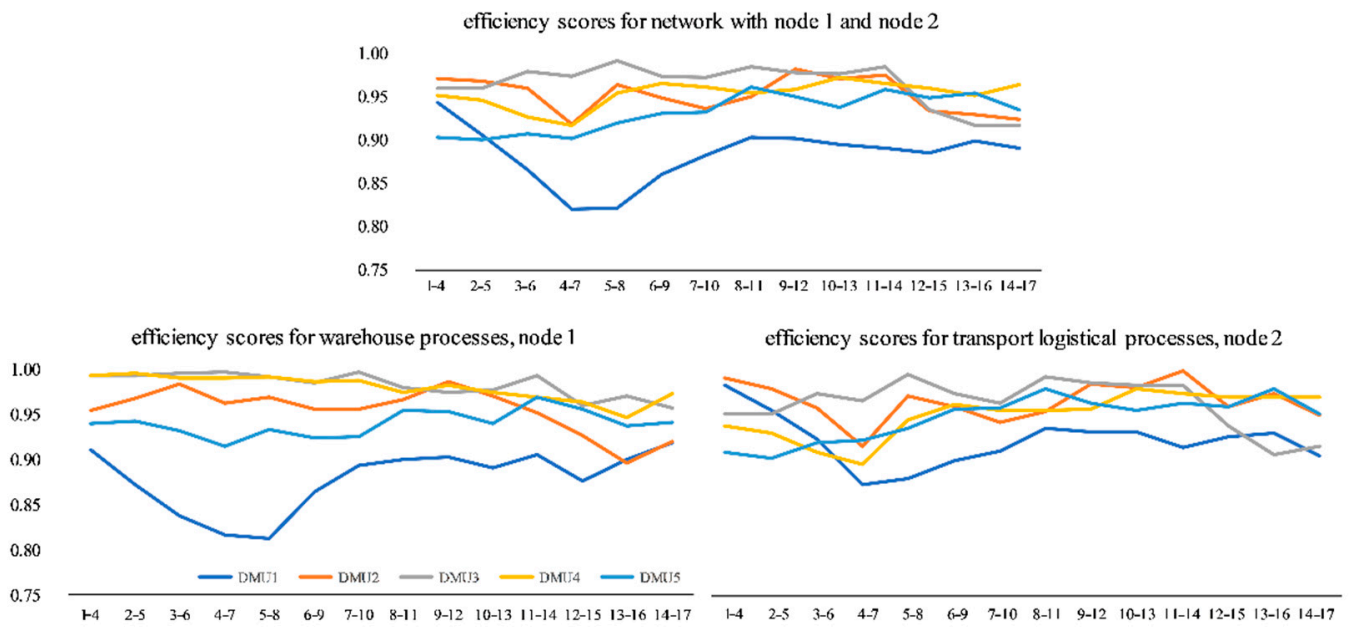

Figure 4. C1, Summary of efficiency progression for the network and per node.

\subsection{C2: Computer System Disruptions in Track-and-Trace Systems Affecting Node 2}

Considering that the decision to apply CRS or VRS was already discussed in the previous section, the investigation of a computer system disruption in the track-and-trace system affecting node 2 uses the same DEA model as C1. Figure 5 visualizes, summarizes, and contrasts the efficiency scores per DMU and window for (1) the whole network, (2) the warehouse processes as node 1, and (3) the transport logistical processes of node 2.
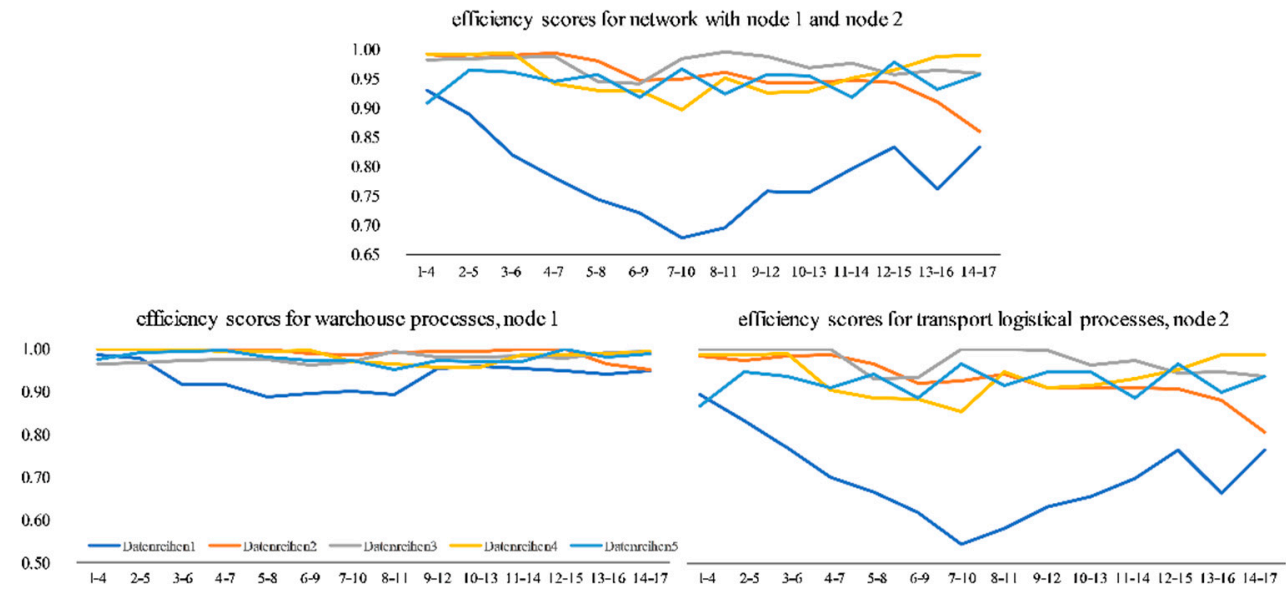

Figure 5. C2, Summary of efficiency progression for the network and per node.

The curve progression of the total network evaluation in Figure 5 indicates a significantly decreasing efficiency level after the computer system disruption on day five over the course of the first week. The bottom is reached in $W_{7-10}$ with an average efficiency score of 0.67 after starting at 0.93 in $W_{1-4}$. A significant decrease is especially notable after the computer system disruption in $\mathrm{W}_{5-8}, \mathrm{~W}_{6-9}$, and $\mathrm{W}_{7-10}$. By considering each node, it can be observed that the transport logistical process was mainly influenced, which is not surprising as it is the epicenter of the computer system disruption, with a drop from 0.87 in $\mathrm{W}_{2-5}$ to 0.46 in $\mathrm{W}_{7-10}$. Two circumstances deserve closer attention: (1) the length and duration of the curve drop are identical for the entire network and for node 1 , and (2) a surprising finding can be stated when considering the efficiency progression of the warehouse processes. This upstream process is directly affected by the disruption of the track-and-trace system, although there is no material or information flow in the upstream direction. The efficiency drop is statistically not as severe as in the directly affected process, but is still noticeable, with a decline from 0.99 in $\mathrm{W}_{1-4}$ to 0.89 in $\mathrm{W}_{5-8}$. 


\section{Discussion}

To provide a deeper understanding of the reasons for inefficiencies during computer system disruptions in retail logistics, we investigated the contribution of all input and output factors on the given efficiency scores. We thereby analyzed the number of non-zero input and output slacks, which is frequently applied in the context of DEA methodology after presenting a radial efficiency score improvement [78]. Depending on the orientation of the DEA model, slackbased measurements quantify the possibility of DMUs maximizing output values or minimizing input values that go beyond the radial projection of the envelopment model (CCR or BCC model). For the specific cases analyzed in $\mathrm{C} 1$ and $\mathrm{C} 2$, slack-based measurement can provide in-depth insights into which input or output factor is mainly responsible for inefficiencies. It is essential to mention that the slack-based measurement is calculated for the whole network of the NDEA model and not for single nodes. The following figure illustrates the results of the slack-based measurement for the computer system disruption in node 1 ( $\mathrm{C} 1$, colored in grey) and in node 2 ( $\mathrm{C} 2$, colored in blue).

The pie chart in Figure 6 on the left illustrates the proportion of possible optimizations per input and output factor for the case of a computer system disruption in the orderpicking system (node 1 ). Therein, it is surprising that only $28 \%$ of the possible optimizations may come from the epicenter of the computer system disruption, namely the order-picking system (grey). Most of the inefficiencies result from the subsequent process, the transport logistical work system of truck loading. Increasingly high loading times and high numbers of lost transport units are, therefore, the main drivers of inefficiencies after a breakdown in the order-picking system. This deviation becomes clear when remembering the processes described in the data and sample section. An outage within the order-picking system may result in transportation aids with incomplete, wrong, or missing 1D barcodes. This leads to a media disruption of the digital workflow, consisting of scanning barcodes for the information flow. At this point, one finding deserves special attention: as the computer system disruption in node 1 leads to high inefficiencies regarding the $\mathrm{I}_{2}$ and $\mathrm{O}_{2}$ of node 2, the information workflow that is based on digital technology has a major impact on the efficiency of the retail operational processes in warehouse logistics. As seen in the right pie chart, the computer system disruption in node 2 leads to situations in which mainly $\mathrm{I}_{2}$ and $\mathrm{O}_{2}$ of node 2 could be minimized / maximized in order to reach the efficiency frontier. This also supports the efficiency progression in the previous chapter, which highlighted the low impact of the computer system disruption in node one on the previous order-picking process. However, it may be surprising that disruptions in the track-and-trace system lead to inefficiencies caused by the total picking time $\left(\mathrm{I}_{1}\right.$, node 1$)$. In operational logistics, a massively slowed loading process results in a significantly lower material flow of all goods leaving the warehouse. Consequently, the continuous working material flow of picked SKUs collides with the space that is still blocked in the outgoing goods department. This consequently slows down the order picker when moving at rack ends or changing aisles.
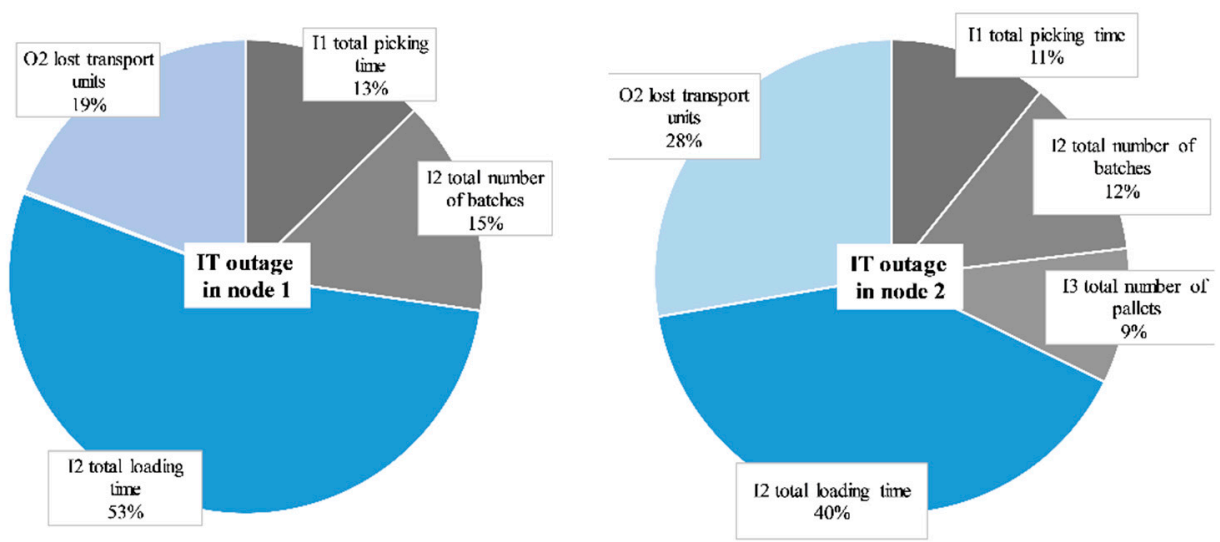

Figure 6. Share of optimizations per input or output factor from slack-based measurement. 
The complex and versatile interaction of warehouse and transport logistical processes during computer system disruptions is summarized in a system dynamics framework. The system dynamics methodology, developed by Jay W. Forrester in the 1950s as industrial dynamics, initially aimed to solve problems related to top management [79]. The methodology includes "[ ... ] a perspective and set of conceptual tools that enable us to understand the structure and dynamics of complex systems" [80], p. VII. As management problems contain various elements in several systems and sub-systems interacting with one another, system dynamics abstracts these elements, takes an aggregated view, and captures the dynamic behavior of a system over time by mathematical modeling and visualization. Causal loop diagrams have been applied to shed light on the interplay and resulting mechanisms of various variables and levels in complex systems by visualizing a reference model [80]. The variables are connected to influence lines, forming causal chains and indicating whether the affected variable is influenced positively $(+)$ or affected negatively $(-)$. One example of a causal chain is as follows: A computer system disruption in the track-and-trace system leads to an increasing number of errors when scanning barcodes, which, as a consequence, requires a post-processing step for non-scannable barcodes. This additional process enlarges the loading time and decreases the number of units delivered on time. Finally, this has a negative impact on the efficiency of transport logistical processes.

We augment our quantitative and mostly static DEA methodology with the flexible system dynamics approach as an additional qualitative method to summarize the complex problem of computer system disruptions, consisting of various explanations on multiple levels. This allows us to integrate additional variables and factors that we were not able to include in our multivariate DEA approach, which may be due to data availability or restrictions of the applied methodology. Furthermore, the combination of these methodologies enables a further and deeper discussion of the problem structure and aims to provide new insights. As a basis for further deviations, we use the quantitative findings of our DEA model regarding the disruptions in different nodes of the logistics system. For further research, the system dynamics approach may again serve as a basis for an additional quantitative analysis of the examined system, as the relationships mapped in our framework can be transformed into mathematical equations.

These results link to existing research outputs and publications, for example, in the domain of resilience [81]. In particular, the link between sustainability and resilience is explored by these authors. Another example of where this paper connects to the existing literature is the interrelation between economic sustainability and resilience as the ability to withstand disruptions with adequate resources [82]. Furthermore, this links to resilience and even growth options in times of crisis due to critical infrastructure being a basic resource in relation to individual corporate capacities for change [83].

We can also connect the sustainability dimension of the resilience provided by corporate actors, vice versa, to their ability to establish long-term sustainability $[84,85]$. This, again, is connected to the public resource of resilience management, as outlined in [86].

Taking the results from the presented NDEA analysis as well as the systems dynamics model in Figure 7 towards a comparison with established resilience research, we arrive at the following observations: 


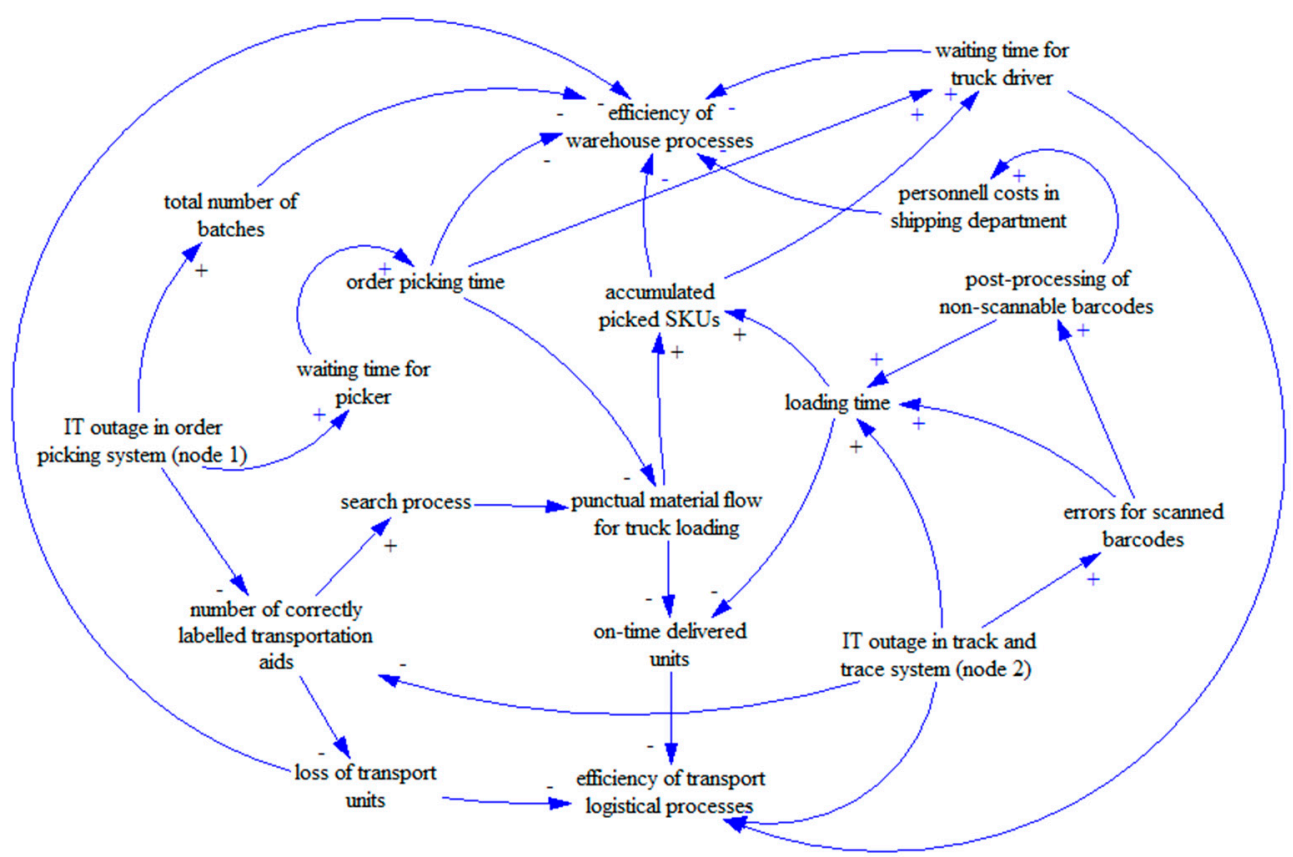

Figure 7. System dynamics framework for the impact of computer system disruptions on operational logistics.

First, ripple and subsequent effects of disruptions are a major field of analysis like for example described by Hosseini and Ivanov in 2020 [87]. In this paper, we report that in a quantitative (NDEA) and qualitative (system dynamics) was for such ripple or process effects. For example, in the above figure, we can recognize that disruptions in many cases lead to increased waiting times of workers (order pickers, truck drivers), increasing the operational and efficiency impact of disruptions originating, for example, from IT systems. This connects also to the observation by Gölgeci and Kuivalainen (2020) that social capital could be an important mediating and mitigating factor in resilience and disruption management [40].

Second, the "quantitative push" in resilience research is followed in our paper with the reference to the NDEA efficiency analysis to evaluate supply chain disruptions; this also represents the final factor in the qualitative system dynamics perspective ("efficiency of warehouse/transport processes"). Other research papers apply, for example, value-at-risk analytics like by Dixit et al. 2020 [88], financial impact measures like with Yu et al. in 2019 [89] or the general overview by Aldrighetti et al. in 2021 regarding costs of disruptions and low resilience levels in supply chains [90]. The most general and generic view on metrics for resilience analysis and management is provided by Behzadi et al. in 2020 [91].

Third, technology use is discussed as a driver as well as mitigation perspective for supply chain disruptions and resilience as was the original starting question for this paper: Gu et al. provide 2021 a similar approach [92], whereas Lohmer et al. in 2020 see, for example, blockchain technology as a possible moderator in supply chain disruptions and resilience [93]. Finally, Al-Talib et al. in 2020 describe how to apply digital IoT technologies to improve supply chain resilience [94].

In total, there is a considerate body of literature where the presented paper fits well into the main discussion streams with the presented messages and results. From a general perspective [95], focusing on specialized areas such as retail [96] up to the challenge of a generalized theory contribution in this field [4] -all aspects are addressed. This is the basis for further interactions and research addressing the challenges of supply chain resilience.

\section{Conclusions}

The quantitative results from this study showed specific but diverse efficiency impacts by technical computer system disruptions in the two stages of the operational retail logistics process. 
Considering performance measurement in supply chain management, Agrell and HatamiMarbini [97] differentiate between (1) performance measurement that provides productivity estimations in the sense of prediction, e.g., yields, or demands, and (2) providing targets for improvement in the case of poor performance as a normative paradigm.

The findings of this paper can be assigned to a normative rather than a predictive paradigm, as they provide a deeper understanding of decreasing performance after computer system disruptions in operational retail logistics processes. For the research questions raised at the beginning, the findings show that supply chain disruptions are operationalizable on a micro-level and through a multi-variable approach. The quantitative DEA methodology applied in this paper takes an a posteriori evaluation perspective, which is a counterbalance to recent research in supply chain management focusing on the a priori simulations of disruption, e.g., related to COVID-19. Especially for logistics and supply chain managers, these evaluation approaches are valuable for scenarios, where disruptions may occur more than once. In most cases, technological disruptions are recurring. Therefore, our approach can be used to estimate costs and benefits for IT system reliability.

From a theoretical viewpoint, our results can be connected to the research results of Fartaj, Kabir, Eghujovbo, Ali and Paul [32] or Beltagui, Rosli and Candi [33] for internal causes of supply chain disruptions. In this sense, our research adds a further internal cause analysis regarding computer system breakdowns as internal disruptions of supply chain processes. Therefore, the existing theory and body of knowledge is expanded for this specific topical area.

The limitations of this study include its use of specific input and output types in one particular retail logistics setting. In addition, a specified setting in Germany is addressed; other countries' cases and datasets could increase the geographical reach of such efficiency perspectives on supply chain disruptions. From a methodological point of view, we applied a traditional VRS-based DEA approach. As this requires the availability of exactly known values for the specified input and output measures, it is a deterministic method. HatamiMarbini, Agrell, Fukuyama, Gholami and Khoshnevis [98] argue that this kind of model is susceptible to changes or errors in data values. As the data in real-world problems are sometimes imprecise or vague, they propose the application of fuzzy DEA as a more probabilistic model [61].

This study can be the basis for further research directed at showing similar effects for further processes in the supply chain and logistics management using the NDEA methodology. Additional analyses could also be directed at identifying new relevant explaining factors for the severity of efficiency losses and capacities for recovering (resilience). This could complement the predominantly qualitative research on supply chain resilience in the future.

From a practitioner's point of view, this paper is dedicated to a non-parametric evaluation model to assess ex-post effects on the technical efficiency of operational processes in warehouse and transport logistics as a result of computer system disruptions. A potential ex ante approach could positively impact the decision-making process of logistic managers when it comes to the specification of the degree of availability [99], as well as the maximum degree of failure for computer system disruptions in logistics [100]. However, this is often a decision based on (rather short-term) cost considerations and seldom based on the overall long-term efficiency of operational production and logistics systems [101]. Therefore, further research is warranted in this interesting field, as highlighted by the disruptions to many supply chain processes caused by the COVID-19 pandemic.

Author Contributions: Conceptualization, M.K.; methodology, D.L.; software, D.L.; validation, M.K.; formal analysis, D.L.; investigation, D.L.; data curation, D.L.; writing—original draft preparation, D.L., M.K.; writing—review and editing, M.K.; visualization, D.L.; supervision, M.K. All authors have read and agreed to the published version of the manuscript.

Funding: This research received no external funding.

Institutional Review Board Statement: Ethical review and approval were waived for this study, due to the fact that we used anonymous data that was not retractable to individuals at any time. 
Informed Consent Statement: Patient consent was waived due to the fact that we used anonymous data that was not retractable to individuals at any time.

Data Availability Statement: No data provided.

Conflicts of Interest: The authors declare no conflict of interest.

\section{References}

1. El Saadany, A.M.; Jaber, M.Y. Coordinating a two-level supply chain with production interruptions to restore process quality. Comput. Ind. Eng. 2008, 54, 95-109. [CrossRef]

2. Bazan, E.; Jaber, M.Y.; Zanoni, S.; Zavanella, L.E. Vendor Managed Inventory (VMI) with Consignment Stock (CS) agreement for a two-level supply chain with an imperfect production process with/without restoration interruptions. Int. J. Prod. Econ. 2014, 157, 289-301. [CrossRef]

3. Jabbarzadeh, A.; Fahimnia, B.; Sheu, J.B.; Moghadam, H.S. Designing a supply chain resilient to major disruptions and supply/demand interruptions. Transp. Res. Part B Methodol. 2016, 94, 121-149. [CrossRef]

4. Wong, C.W.; Lirn, T.C.; Yang, C.C.; Shang, K.C. Supply chain and external conditions under which supply chain resilience pays: An organizational information processing theorization. Int. J. Prod. Econ. 2020, 226, 107610. [CrossRef]

5. Chen, L.; Dui, H.; Zhang, C. A resilience measure for supply chain systems considering the interruption with the cyber-physical systems. Reliab. Eng. Syst. Saf. 2020, 199, 106869. [CrossRef]

6. Gaur, J.; Amini, M.; Rao, A.K. The impact of supply chain disruption on the closed-loop supply chain configuration profit: A study of sourcing policies. Int. J. Prod. Res. 2020, 58, 5380-5400. [CrossRef]

7. Jahani, H.; Abbasi, B.; Hosseinifard, Z.; Fadaki, M.; Minas, J.P. Disruption risk management in service-level agreements. Int. J. Prod. Res. 2021, 59, 226-244. [CrossRef]

8. Liu, M.; Liu, Z.; Chu, F.; Zheng, F.; Chu, C. A new robust dynamic Bayesian network approach for disruption risk assessment under the supply chain ripple effect. Int. J. Prod. Res. 2021, 59, 265-285. [CrossRef]

9. Macdonald, J.R.; Zobel, C.W.; Melnyk, S.A.; Griffis, S.E. Supply chain risk and resilience: Theory building through structured experiments and simulation. Int. J. Prod. Res. 2018, 56, 4337-4355. [CrossRef]

10. Han, J.; Shin, K. Evaluation mechanism for structural robustness of supply chain considering disruption propagation. Int. J. Prod. Res. 2016, 54, 135-151. [CrossRef]

11. Dong, E.; Du, H.; Gardner, L. An interactive web-based dashboard to track COVID-19 in real time. Lancet Infect. Dis. 2020, 20, 533-534. [CrossRef]

12. Ivanov, D. Predicting the impacts of epidemic outbreaks on global supply chains: A simulation-based analysis on the coronavirus outbreak (COVID-19/SARS-CoV-2) case. Transp. Res. Part E Logist. Transp. Rev. 2020, 136, 101922. [CrossRef] [PubMed]

13. Kaplan, E.H. Containing 2019-nCoV (Wuhan) coronavirus. Health Care Manag. Sci. 2020, 23, 311-314. [CrossRef] [PubMed]

14. Ivanov, D.; Dolgui, A. Low-Certainty-Need (LCN) supply chains: A new perspective in managing disruption risks and resilience. Int. J. Prod. Res. 2019, 57, 5119-5136. [CrossRef]

15. Jabbarzadeh, A.; Fahimnia, B.; Sabouhi, F. Resilient and sustainable supply chain design: Sustainability analysis under disruption risks. Int. J. Prod. Res. 2018, 56, 5945-5968. [CrossRef]

16. Ralston, P.; Blackhurst, J. Industr. 4.0 and resilience in the supply chain: A driver of capability enhancement or capability loss? Int. J. Prod. Res. 2020, 58, 5006-5019. [CrossRef]

17. Zsidisin, G.A.; Petkova, B.N.; Dam, L. Examining the influence of supply chain glitches on shareholder wealth: Does the reason matter? Int. J. Prod. Res. 2016, 54, 69-82. [CrossRef]

18. Pires, M.; Camanho, A.; Amorim, P. Solving the grocery backroom sizing problem. Int. J. Prod. Res. 2020, 58, 5707-5720. [CrossRef]

19. Goldbeck, N.; Angeloudis, P.; Ochieng, W. Optimal supply chain resilience with consideration of failure propagation and repair logistics. Transp. Res. Part E: Logist. Transp. Rev. 2020, 133, 101830. [CrossRef]

20. Li, Y.; Zobel, C.W.; Seref, O.; Chatfield, D. Network characteristics and supply chain resilience under conditions of risk propagation. Int. J. Prod. Econ. 2020, 223, 107529. [CrossRef]

21. Dormady, N.; Roa-Henriquez, A.; Rose, A. Economic resilience of the firm: A production theory approach. Int. J. Prod. Econ. 2019, 208, 446-460. [CrossRef]

22. Yang, C.-C.; Hsu, W.-L. Evaluating the impact of security management practices on resilience capability in maritime firms-A relational perspective. Transp. Res. Part A Policy Pract. 2019, 110, 220-233. [CrossRef]

23. Brewton, K.E.; Danes, S.M.; Stafford, K.; Haynes, G.W. Determinants of rural and urban family firm resilience. J. Fam. Bus. Strategy 2010, 1, 155-166. [CrossRef]

24. Bode, C.; Wagner, S.M. Structural drivers of upstream supply chain complexity and the frequency of supply chain disruptions. $J$. Oper. Manag. 2015, 36, 215-228. [CrossRef]

25. Esmaeili-Najafabadi, E.; Nezhad, M.S.F.; Pourmohammadi, H.; Honarvar, M.; Vahdatzad, M.A. A joint supplier selection and order allocation model with disruption risks in centralized supply chain. Comput. Ind. Eng. 2019, 127, 734-748. [CrossRef]

26. Ivanov, D.; Rozhkov, M. Disruption tails and post-disruption instability mitigation in the supply chain. IFAC-PapersOnLine 2019, 52, 343-348. [CrossRef] 
27. Yavari, M.; Zaker, H. Designing a resilient-green closed loop supply chain network for perishable products by considering disruption in both supply chain and power networks. Comput. Chem. Eng. 2020, 134, 106680. [CrossRef]

28. Kondo, A. The effects of supply chain disruptions caused by the Great East Japan Earthquake on workers. Jpn. World Econ. 2018, 47, 40-50. [CrossRef]

29. Silva, M.E.; Pereira, S.C.; Gold, S. The response of the Brazilian cashew nut supply chain to natural disasters: A practice-based view. J. Clean. Prod. 2018, 204, 660-671. [CrossRef]

30. Kim, K.; Bui, L. Learning from Hurricane Maria: Island ports and supply chain resilience. Int. J. Disaster Risk Reduct. 2019, 39, 101244. [CrossRef]

31. Schätter, F.; Hansen, O.; Wiens, M.; Schultmann, F. A decision support methodology for a disaster-caused business continuity management. Decis. Support Syst. 2019, 118, 10-20. [CrossRef]

32. Fartaj, S.R.; Kabir, G.; Eghujovbo, V.; Ali, S.M.; Paul, S.K. Modeling transportation disruptions in the supply chain of automotive parts manufacturing company. Int. J. Prod. Econ. 2020, 222, 107511. [CrossRef]

33. Beltagui, A.; Rosli, A.; Candi, M. Exaptation in a digital innovation ecosystem: The disruptive impacts of 3D printing. Res. Policy 2019, 49, 103833. [CrossRef]

34. Pi, Z.; Fang, W.; Zhang, B. Service and pricing strategies with competition and cooperation in a dual-channel supply chain with demand disruption. Comput. Ind. Eng. 2019, 138, 106130. [CrossRef]

35. Rahmani, K.; Yavari, M. Pricing policies for a dual-channel green supply chain under demand disruptions. Comput. Ind. Eng. 2019, 127, 493-510. [CrossRef]

36. Bugert, N.; Lasch, R. Effectiveness of responsive pricing in the face of supply chain disruptions. Comput. Ind. Eng. 2018, 124, 304-315. [CrossRef]

37. Ambulkar, S.; Blackhurst, J.; Grawe, S. Firm's resilience to supply chain disruptions: Scale development and empirical examination. J. Oper. Manag. 2015, 33-34, 111-122. [CrossRef]

38. Pires Ribeiro, J.; Barbosa-Povoa, A. Supply Chain Resilience: Definitions and quantitative modelling approaches-A literature review. Comput. Ind. Eng. 2018, 115, 109-122. [CrossRef]

39. Bevilacqua, M.; Ciarapica, F.E.; Marcucci, G. A modular analysis for the Supply Chain Resilience Triangle. IFAC-PapersOnLine 2018, 51, 1528-1535. [CrossRef]

40. Gölgeci, I.; Kuivalainen, O. Does social capital matter for supply chain resilience? The role of absorptive capacity and marketingsupply chain management alignment. Ind. Mark. Manag. 2020, 84, 63-74. [CrossRef]

41. Nguyen, H.; Sharkey, T.C.; Wheeler, S.; Mitchell, J.E.; Wallace, W.A. Towards the development of quantitative resilience indices for Multi-Echelon Assembly Supply Chains. Omega 2021, 99, 102199. [CrossRef]

42. Chang, W.-S.; Lin, Y.-T. The effect of lead-time on supply chain resilience performance. Asia Pac. Manag. Rev. 2019, 24, 298-309. [CrossRef]

43. Wollenburg, J.; Hübner, A.; Kuhn, H.; Trautrims, A. From bricks-and-mortar to bricks-and-clicks: Logistics networks in omnichannel grocery retailing. Int. J. Phys. Distrib. Logist. Manag. 2018, 48, 415-438. [CrossRef]

44. Pareto, V. Cours d'économie Politique; Librairie Droz: Paris, France, 1897.

45. Koopmans, T.C. An analysis of production as an efficient combination of activities. In Activity Analysis of Production and Allocation; Koopmans, T.C., Ed.; Wiley: London, UK, 1951; pp. 33-97.

46. Farrell, M.J. The measurement of productive efficiency. J. R. Stat. Soc. 1957, 120, 253-290. [CrossRef]

47. Debreu, G. The coefficient of resource utilization. Econometrica 1951, 19, 273-292. [CrossRef]

48. Cooper, W.W.; Seiford, L.M.; Tone, K. Data Envelopment Analysis: A Comprehensive Text with Models, Applications, References and DEA-Solver Software; Springer: Boston, MA, USA, 2007.

49. Cooper, W.W.; Seiford, L.M.; Zhu, J. Data Envelopment Analysis: History, Models, and Interpretations. In Handbook on Data Envelopment Analysis; Cooper, W.W., Seiford, L.M., Zhu, J., Eds.; Springer: Boston, MA, USA, 2011; pp. 1-39.

50. Charnes, A.; Cooper, W.W.; Rhodes, E. Measuring the efficiency of decision making units. Eur. J. Oper. Res. 1978, 2, 429-444. [CrossRef]

51. Banker, R.D.; Charnes, A.; Cooper, W.W. Some models for estimating technical and scale inefficiencies in Data Envelopment Analysis. Manag. Sci. 1984, 30, 1078-1092. [CrossRef]

52. Banker, R.D. Estimating most productive scale size using data envelopment analysis. Eur. J. Oper. Res. 1984, 17, 35-44. [CrossRef]

53. Banker, R.D.; Cooper, W.W.; Seiford, L.M.; Zhu, J. Returns to scale in DEA. In Handbook on Data Envelopment Analysis; Cooper, W.W., Seiford, L.M., Zhu, J., Eds.; Springer: Boston, MA, USA, 2011; pp. 41-70.

54. Andersen, P.; Petersen, N.C. A Procedure for Ranking Efficient Units in Data Envelopment Analysis. Manag. Sci. 1993, 39, 1261-1264. [CrossRef]

55. Charnes, A.; Clark, C.T.; Cooper, W.W.; Golany, B. A developmental study of data envelopment analysis in measuring the efficiency of maintenance units in the U.S. air forces. Ann. Oper. Res. 1985, 2, 95-112. [CrossRef]

56. Halkos, G.E.; Tzeremes, N.G. Exploring the existence of Kuznets curve in countries' environmental efficiency using DEA window analysis. Ecol. Econ. 2009, 68, 2168-2176. [CrossRef]

57. Jia, T.; Yuan, H. The application of DEA (Data Envelopment Analysis) window analysis in the assessment of influence on operational efficiencies after the establishment of branched hospitals. BMC Health Serv Res 2017, 17, 265. [CrossRef] [PubMed]

58. Malmquist, S. Index numbers and indifference surfaces. Trab. De Estad. 1953, 4, 209-242. [CrossRef] 
59. Sengupta, J.K. A fuzzy systems approach in data envelopment analysis. Comput. Math. Appl. 1992, 24, 259-266. [CrossRef]

60. Sengupta, J.K. Measuring efficiency by a fuzzy statistical approach. Fuzzy Sets Syst. 1992, 46, 73-80. [CrossRef]

61. Hatami-Marbini, A.; Emrouznejad, A.; Tavana, M. A taxonomy and review of the fuzzy data envelopment analysis literature: Two decades in the making. Eur. J. Oper. Res. 2011, 214, 457-472. [CrossRef]

62. Färe, R.; Primont, D. Efficiency measures for multiplant firms. Oper. Res. Lett. 1984, 3, 257-260. [CrossRef]

63. Färe, R.; Grosskopf, S. Network DEA. Socio-Econ. Plan. Sci. 2000, 34, 35-49. [CrossRef]

64. Färe, R. Measuring Farrell efficiency for a firm with intermediate inputs. Acad. Econ. Pap. 1991, 19, 329-340.

65. Färe, R.; Whittaker, G. An Intermediate Input Model of Dairy Production Using Complex Survey Data. J. Agric. Econ. 1995, 46, 201-213. [CrossRef]

66. Färe, R.; Grosskopf, S. Intertemporal Production Frontiers: With Dynamic DEA; Springer: Dordrecht, The Netherlands, 1996.

67. Färe, R.; Grosskopf, S. Productivity and intermediate products: A frontier approach. Econ. Lett. 1996, 50, 65-70. [CrossRef]

68. Cook, W.D. Multicomponent Efficiency Measurement and Shared Inputs in Data Envelopment Analysis: An Application to Sales and Service Performance in Bank Branches. J. Product. Anal. 2000, 14, 209-224. [CrossRef]

69. Wang, C.H.; Gopal, R.D.; Zionts, S. Use of Data Envelopment Analysis in assessing Information Technology impact on firm performance. Ann. Oper. Res. 1997, 73, 191-213. [CrossRef]

70. Seiford, L.M.; Zhu, J. Profitability and Marketability of the Top 55 U.S. Commercial Banks. Manag. Sci. 1999, 45, 1270-1288. [CrossRef]

71. Tavana, M.; Mirzagoltabar, H.; Mirhedayatian, S.M.; Saen, R.F.; Azadi, M. A new network epsilon-based DEA model for supply chain performance evaluation. Comput. Ind. Eng. 2013, 66, 501-513. [CrossRef]

72. Badiezadeh, T.; Saen, R.F.; Samavati, T. Assessing sustainability of supply chains by double frontier network DEA: A big data approach. Comput. Oper. Res. 2018, 98, 284-290. [CrossRef]

73. Ang, S.; Zhu, Y.; Yang, F. Efficiency evaluation and ranking of supply chains based on stochastic multicriteria acceptability analysis and data envelopment analysis. Int. Trans. Oper. Res. 2019, 16, 113. [CrossRef]

74. Huang, H.; Li, S.; Yu, Y. Evaluation of the allocation performance in a fashion retail chain using data envelopment analysis. J. Text. Inst. 2019, 110, 901-910. [CrossRef]

75. Kalantary, M.; Farzipoor Saen, R. Assessing sustainability of supply chains: An inverse network dynamic DEA model. Comput. Ind. Eng. 2019, 135, 1224-1238. [CrossRef]

76. Li, Y.; Abtahi, A.-R.; Seyedan, M. Supply chain performance evaluation using fuzzy network data envelopment analysis: A case study in automotive industry. Ann. Oper. Res. 2019, 275, 461-484. [CrossRef]

77. Hatami-Marbini, A.; Agrell, P.J.; Tavana, M.; Khoshnevis, P. A flexible cross-efficiency fuzzy data envelopment analysis model for sustainable sourcing. J. Clean. Prod. 2016, 142, 2761-2779. [CrossRef]

78. Morita, H.; Hirokawa, K.; Zhu, J. A slack-based measure of efficiency in context-dependent data envelopment analysis. Omega 2005, 33, 357-362. [CrossRef]

79. Forrester, J.W. Industrial Dynamics; MIT Press: Cambridge, MA, USA, 1961.

80. Sterman, J.D. Business Dynamics: Systems Thinking and Modeling for a Complex World; Irwin/McGraw-Hill: Boston, MA, USA, 2000.

81. Miceli, A.; Hagen, B.; Riccardi, M.P.; Sotti, F.; Settembre-Blundo, D. Thriving, Not Just Surviving in Changing Times: How Sustainability, Agility and Digitalization Intertwine with Organizational Resilience. Sustainability 2021, 13, 2052. [CrossRef]

82. Klumpp, M.; Loske, D. Order Picking and E-Commerce: Introducing Non-Parametric Efficiency Measurement for Sustainable Retail Logistics. J. Appl. Electron. Commer. Res. 2021, 16, 846-858. [CrossRef]

83. D'Adamo, I.; Rosa, P. How Do You See Infrastructure? Green Energy to Provide Economic Growth after COVID-19. Sustainability 2020, 12, 4738. [CrossRef]

84. Klumpp, M. Do Forwarders Improve Sustainability Efficiency? Evidence from a European DEA Malmquist Index Calculation. Sustainability 2017, 9, 842. [CrossRef]

85. Klumpp, M. How to Achieve Supply Chain Sustainability Efficiently? Taming the Triple Bottom Line Split Business Cycle. Sustainability 2019, 10, 397. [CrossRef]

86. Ferrari, M. Reflexive Governance for Infrastructure Resilience and Sustainability. Sustainability 2020, 12, 10224. [CrossRef]

87. Hosseini, S.; Ivanov, D. Bayesian networks for supply chain risk, resilience and ripple effect analysis: A literature review. Expert Syst. Appl. 2020, 161, 113649. [CrossRef]

88. Dixit, V.; Verma, P.; Tiwari, M.K. Assessment of pre and post-disaster supply chain resilience based on network structural parameters with CVaR as a risk measure. Int. J. Prod. Econ. 2020, 227, 107655. [CrossRef]

89. Yu, W.; Jacobs, M.A.; Chavez, R.; Yang, J. Dynamism, disruption orientation, and resilience in the supply chain and the impacts on financial performance: A dynamic capabilities perspective. Int. J. Prod. Econ. 2019, 218, 352-362. [CrossRef]

90. Aldrighetti, R.; Battini, D.; Ivanov, D.; Zennaro, I. Costs of resilience and disruptions in supply chain network design models: A review and future research directions. Int. J. Prod. Econ. 2021, 235, 108103. [CrossRef]

91. Behzadi, G.; O'Sullivan, M.J.; Olsen, T.L. On metrics for supply chain resilience. Eur. J. Oper. Res. 2020, 287, 145-158. [CrossRef]

92. Gu, M.; Yang, L.; Huo, B. The impact of information technology usage on supply chain resilience and performance: An ambidexterous view. Int. J. Prod. Econ. 2021, 232, 107956. [CrossRef]

93. Lohmer, J.; Bugert, N.; Lasch, R. Analysis of resilience strategies and ripple effect in blockchain-coordinated supply chains: An agent-based simulation study. Int. J. Prod. Econ. 2020, 228, 107882. [CrossRef] 
94. Al-Talib, M.; Melhem, W.Y.; Anosike, A.I.; Garza, A.; Reyes JA, G.; Nadeem, S.P.; Kumar, A. Achieving resilience in the supply chain by applying IoT technology. Procedia Cirp 2020, 91, 752-757. [CrossRef]

95. Belhadi, A.; Kamble, S.; Jabbour CJ, C.; Gunasekaran, A.; Ndubisi, N.O.; Venkatesh, M. Manufacturing and service supply chain resilience to the COVID-19 outbreak: Lessons learned from the automobile and airline industries. Technol. Forecast. Soc. Chang. 2021, 163, 120447. [CrossRef] [PubMed]

96. Alikhani, R.; Torabi, S.A.; Altay, N. Retail supply chain network design with concurrent resilience capabilities. Int. J. Prod. Econ. 2021, 234, 108042. [CrossRef]

97. Agrell, P.J.; Hatami-Marbini, A. Frontier-based performance analysis models for supply chain management: State of the art and research directions. Comput. Ind. Eng. 2013, 66, 567-583. [CrossRef]

98. Hatami-Marbini, A.; Agrell, P.J.; Fukuyama, H.; Gholami, K.; Khoshnevis, P. The role of multiplier bounds in fuzzy data envelopment analysis. Ann. Oper. Res. 2017, 250, 249-276. [CrossRef]

99. Chakraborty, T.; Chauhan, S.S.; Ouhimmou, M. Mitigating supply disruption with a backup supplier under uncertain demand: Competition vs. cooperation. Int. J. Prod. Res. 2020, 58, 3618-3649. [CrossRef]

100. Sawik, T. A portfolio approach to supply chain disruption management. Int. J. Prod. Res. 2017, 55, 1970-1991. [CrossRef]

101. Sawik, T. Two-period vs. multi-period model for supply chain disruption management. Int. J. Prod. Res. 2019, 57, 4502-4518. [CrossRef] 
Article

\title{
Sustainability and Resilience of Emerging Cities in Times of COVID-19
}

\author{
Angela Ivette Grijalba Castro ${ }^{1, *}$ [C and Leonardo Juan Ramírez López ${ }^{2} \mathbb{C}$ \\ 1 Engineering Faculty, Nueva Granada Military University, Bogotá 250240, Colombia \\ 2 TIGUM Research Group, Nueva Granada Military University, Bogotá 250247, Colombia; \\ leonardo.ramirez@unimilitar.edu.co \\ * Correspondence: est.angela.grijalba@unimilitar.edu.co; Tel.: +57-3118471127
}

Citation: Grijalba Castro, A.I.; Ramírez López, L.J. Sustainability and Resilience of Emerging Cities in Times of COVID-19. Sustainability 2021, 13, 9480. https://doi.org/ $10.3390 /$ su13169480

Academic Editor: Idiano D'Adamo

Received: 10 June 2021

Accepted: 12 August 2021

Published: 23 August 2021

Publisher's Note: MDPI stays neutral with regard to jurisdictional claims in published maps and institutional affiliations.

Copyright: (C) 2021 by the authors. Licensee MDPI, Basel, Switzerland. This article is an open access article distributed under the terms and conditions of the Creative Commons Attribution (CC BY) license (https:// creativecommons.org/licenses/by/ $4.0 /)$.

\begin{abstract}
The organization of a territory relies on a group of transformations produced by economic, environmental, and social emergencies, generating disruptions along with history. Furthermore, every new scenario generates a considerable impact, which makes it more difficult to recover from increasing urban ecological footprints. COVID-19-emergence-aware cities face new challenges that will test their resilience. This new outline constitutes a study regarding urban planning from an environmental and resilience perspective within this new pandemic state of emergency. It contains four main topics: emergent cities, natural resources, sustainability, and resilience. The document shows a case study carried out in a Colombian town named Cajicá, where a bibliometric inquiry conducted with PRISMA (Preferred Reporting Items for Systematic Reviews and Meta-Analyses) adjustments was managed, tested on forty-one scientific papers; all the above were verified by VOSviewer software tools. The study reveals the creation and visualization of several keyword networks and relations retrieved from all the selected articles, along with the use of eight additional documents for all relation analyses. Sustainability and resilience are the main findings, supported as a process of functionality within urban planning. Sustainability findings' results are prioritized, along with resilience analysis processes, which are both frameworks used during the COVID-19 pandemic; they constitute the main argument within this set of changes, building on alterations of lifestyle and behavioral situations within the main cities.
\end{abstract}

Keywords: resilience; emerging cities; sustainability; COVID-19

\section{Introduction}

When we examine urban planning, the structure of a territory rests on a group of transformations produced by economic crises and the reduction of natural resources due to inappropriate administrations, leading to economic, social, and environmental disruptions across history. Furthermore, every new scenario generates a considerable impact, which is difficult to recover from. As a consequence, this increases the urban footprint [1]. COVID19-emergence-aware cities face new challenges aimed to test their resilience competence, revealing with this several issues related to infrastructure losses, public utility purveyance, urban planning, and governance; all the above lead to considerable social, economic, and environmental impacts [2], demonstrating the inadequacy of sustainable development objectives (SDO). As a result, we present the following inquires: What kind of changes has the COVID-19 pandemic generated regarding sustainability and resilience in emergent cities? What is, in this case, the main challenge these emergent cities have to deal with?

To answer these questions, after all quarantine and curfew intervals, citizens from metropolitan areas considered rural lands with lower population denseness, along with medium cities, as ideal zones to safeguard themselves from the virus outbreak. This raised their mental health, generating an unexpected departure without considering that all these areas could not meet their basic needs. All the above produces conflict among a rural community that tries to maintain a virus-free environment [3]. The Inter-American 
Development Bank (IDB) categorized these emergent cities into new forms of metropolitan areas. Inside the case study, we will examine a place (Cajicá, Colombia) located inside a strategic location within the Colombian National Road System.

In the ongoing COVID-19 scenario, government organizations have taken restrictive measures to decrease the infection rate, without ignoring local problems, which has generated a considerable impact on the administration of vital natural resources that can support this crisis. Thereby, it is necessary to address this analysis by considering two factors: one concerning the main actions to control the emergency (e.g., the high demand and use of potable water for handwashing), and other factors produced by habits and lifestyles (e.g., the use of chemical substances for household cleaning) [4].

In summary, it is relevant to understand the sustainability concept provided by the United Nations (UN), enclosed in Sustainable Development, which presents two criteria. The first aims to meet all needs across generations; the second specifies present and future generations, where time acts as a key variable within the sustainability concept. In conclusion, we can define sustainability as an ideal goal, whereas sustainable development is the procedure we must follow by considering all social, economic, and environmental areas.

Thus, a bioregional approach towards territory planning is appropriate, perceived as a region delimited by geographical limits and determined by ecological systems, both allowing for ecological processes, satisfying the territorial demands for the preservation of native species, understanding factors regarding biological resources, and social cohesion $[5,6]$. Consequently, the importance of connections between rural and urban areas is reflected, turning this into a complex symbiotic network that connects goods and services, playing a determining role in the perspective of the territory as promoters of sustainable management.

In this uncertain scenario created by the pandemic, increasing our capacity for improvement and adaptation is urgent. Resilience is the result of a process where organizations work to anticipate and respond to ongoing external threats $[7,8]$. It is not a concern about leaving behind principles of order and logic; instead, it is about integrating them into a deep and complete scheme, thus allowing adaptive agents to emerge that exhibit more complex structures and actions that permit evolutionary resilience, which leads to taking advantage of the diversity of factors existing in a region and benefiting from the cooperation between territories to guarantee the sustainability of exchanges between them, preventing the development of one territory/area at the expense of the other and seeking to relieve inequalities or territorial polarization in terms of economy or job opportunities, among others [5,9].

\section{Method}

The present research started by looking at existing research available in multidisciplinary scientific journals, focusing specifically on the availability of information associated with conceptualization in conjunction with different viewpoints used for territorial management from sustainability and resilience perspectives. Further assessment of the progress was made by various researchers worldwide, ranking and considering peer-reviewed journal articles. Figure 1 shows the analysis process carried out in four phases:

a. A compilation of the information was carried out in several scientific database search engines (Scopus, Web of Science, and Google Scholar) through the use of Boolean operators "AND" and "OR"; the inclusion of documents whose titles or abstracts contained the words listed below, from 2006 to 2021, appear in the following list:

TITLE-ABS-KEY ("sustainability") AND
TITLE-ABS-KEY ("resilient cities") AND
TITLE-ABS-KEY (“COVID-19") OR
TITLE-ABS-KEY ("emerging cities").




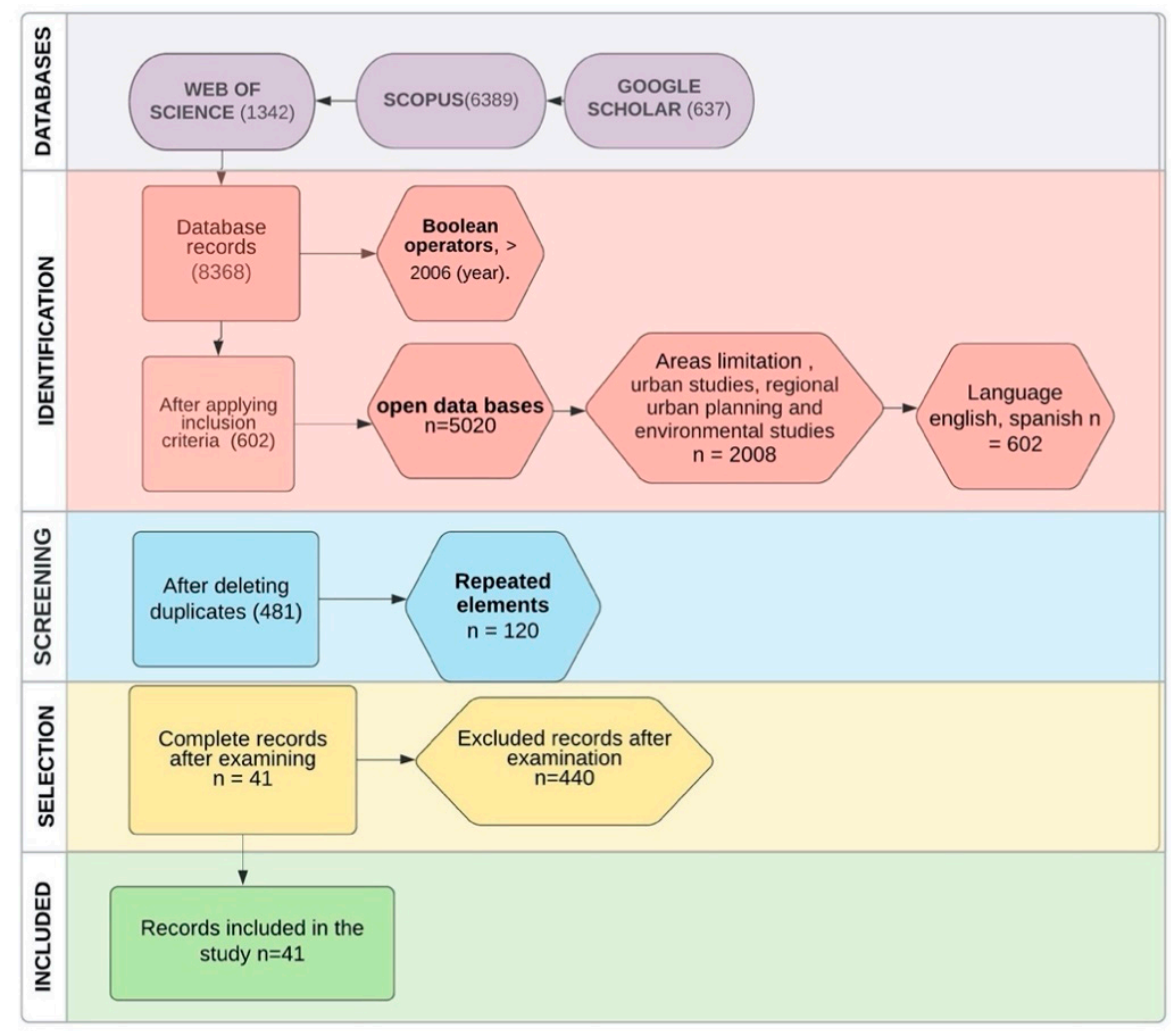

Figure 1. Analysis method.

The inclusion criteria used were open data investigations limited to the following areas of interest: urban studies, regional urban planning, and environmental studies. In addition, the research included articles and books written in English and Spanish.

b. Identification of duplicates was required, as well as some articles that appeared in more than one database.

c. For the quality evaluation, the PRISMA tool (Preferred Reporting Items for Systematic Reviews and Meta-Analyses) was used for each article, providing an objective comparison between the articles and their classification.

d. Finally, grouping was applied through the use of VOSviewer ${ }^{\circledR}$, applying to the latter a viewer of keywords where they were correlated, giving a weight to each word according to the number of times it was mentioned in the selected information.

By this approach, the analysis of both similarities and differences between sustainability, resilient cities, COVID-19, and emerging cities allow the mark of crucial elements used for their conceptualization. To achieve this, eight documents were included, among which were reports, guides, and conferences performed by agencies such as the United Nations (UN), United Nations Environment Program (UNEP), and the Center for the Implementation of Public Policies Promoting Equity and Growth (CIPPEC, by the Spanish acronym) for a total of 49 documents.

e. All in all, results, and further analysis regarding this research, were carried out in Cajicá, a town located in Cundinamarca (Colombia), considering this area as a place that fulfills the requirements established by the Inter-American Development Bank (IDB) regarding emerging cities. Moreover, we will analyze chronological data regarding potable water and solid waste consumption and generation rates by 20192021; We retrieve the mentioned data from "Empresa de Servicios Públicos EPC Cajicá" company. We identify monthly potable water record volumes (Measured in $\mathrm{m}^{3}$ ), as well as a town's provision and exploitation of solid waste (Measured in Tons) report. All the above allowed us to establish a monthly consumption-exploitation 
average data, thereby this method allows us to explain all upcoming situations before and after the COVID-19 pandemic [10].

\section{Co-Occurrence Analysis}

To better understand the approach towards sustainability and resilience in cities in the COVID-19 scenario, we used the method stated above in 49 documents from 1992 to 2021 included the Boolean formulation, building keyword, and co-occurrence networks. Figure 2 shows the dominant position using the VOSviewer ${ }^{\circledR}$ tool: 12 terms out of 122 in total linked inside the topics studied

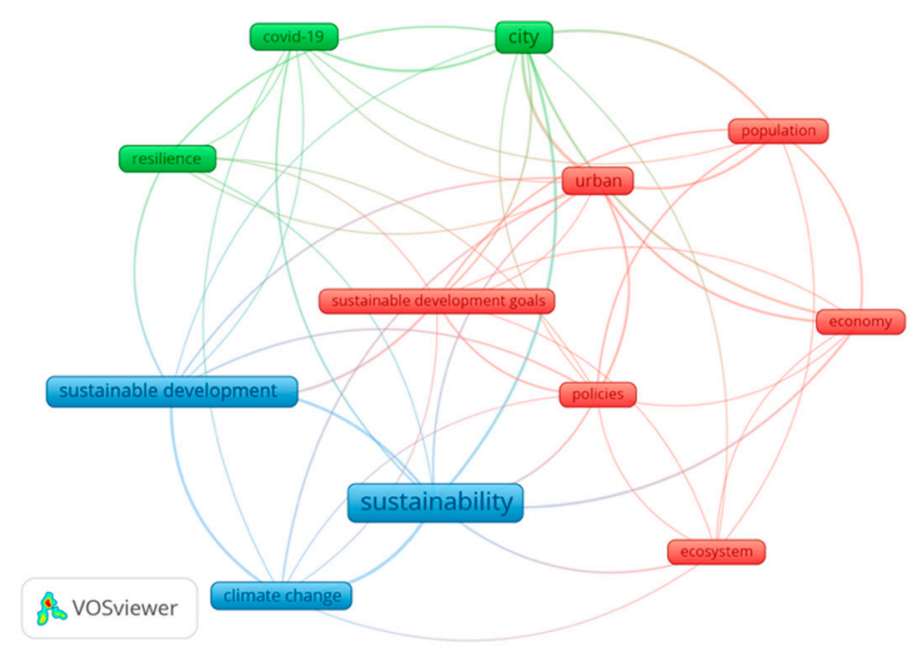

Figure 2. Keyword co-occurrence analysis.

Figure 2 shows the constitution of three clusters, which showed the following predominant behaviors: red connections refer to the term sustainable development goals, generating 5 co-occurrences; green connections refer to the term city making 12 co-occurrences; blue connections refer to the term sustainability generating 20 co-occurrences. The domain of the approach of the formulation was confirmed: city, sustainable development, resilience, urbanism, and sustainability.

In the discourse of sustainability and resilience in cities inside the COVID-19 scenario, as observed in Figure 2, the words with the highest appearance weight were urban, sustainable development, city, and sustainability. A perceived necessity of academics to connect the resilience concept with policies and ecosystems using sustainable development goals is evident.

Additionally, Figure 2 highlights that concepts such as urban, sustainable development, city, and sustainability are linked with the idea of resilience. All articles and documents with clear connections among these concepts, highlighting sustainability and resilience, depending on the approach found in the studies, referring those principally to the acquisition of different practices and policies. These results confirm that there is still a vision of the territory as an immovable and utilitarian structure leaving aside its conception as a complex system, without considering internal processes as adaptive cycles, thus moving away from implementing sustainable territorial planning.

\section{COVID-19's Challenges for Sustainable and Resilient Cities}

\subsection{Emerging Cities}

According to historical facts, the structure inside the territory enclosed political milestones and environmental crises. As a result, Latin America and the Caribbean (LAC) must face a new challenge due to the COVID-19 pandemic, revealing facts such as the deficit of essential equipment that guarantees the minimum of vital resources for inhabitants and the lack of integration among the different government actors that shows faults within urban planning, demonstrating how cities are still far from being resilient and sustainable [1]. 
In the last two decades, urban settlements and economic growth have become solid in medium-sized cities, creating a new expansion model, as well as tremendous challenges for them. For this reason, the Inter-American Development Bank described these areas as emerging cities, classifying those urban areas as intermediate based on the total amount of residents inside an environment of social stability and governability; additionally, they are growing economically and demographically above the national average [11].

According to the United Nations (2019), intermediate or emerging cities are growing at higher rates compared to a metropolis, showing that $75 \%$ of the world population lives in these, notably in rural areas or urban settlements of less than 500,000 inhabitants [12,13]. Borja states that "Urbanization without quality and the agglomeration/dispersion of populations causes unsustainable dynamics; the future of humanity matters" [14]; therefore, factors such as the occupation and inequality of the urban-rural, the degeneration of natural resources, including loss of biodiversity, high poverty rates, and vulnerability to natural phenomena consequently increase the urban footprint [15].

These problems produced a domino effect that troubles the efficient administration of the urban environment. This effect, coupled with the presence of COVID-19 in LAC, had an immediate impact because of the nearness of metropolitan areas towards the so-called emerging cities, generating a challenge for public health because of the percentage of the elderly vulnerable populations that live in these areas, with low-income wages as well as lack of access to health goods and services [3].

After lockdown and curfew periods, citizens of the metropolitan areas considered rural areas with less densification, as well as intermediate cities, as ideal spaces to protect themselves from the virus outbreak and enhance their mental health, creating an unexpected exodus without considering that all these areas could not meet their basic needs. Generating conflicts among a rural community that tries to maintain their environment virus-free, whereas metropolitan inhabitants try to seek a safe place [3].

To sum up, it applies to assess the risks involving these areas and the new criteria driving towards a design of sustainable and resilient cities [2]. This migration event in the actual scenario can be associated with the capital city and the impacts across the nearby areas located around the Bogotá city limits (Sabana de Bogotá), specifically related to Cajicá town. This place with a population of approximately 80,000 inhabitants can be found inside the region that shapes the inner-central area within Bogotá city limits; Figure 3 shows its strategic position in the national corridor, responsible for articulating this region of the country. The economy is supported by the manufacturing industry, transportation, commerce, agribusiness, and tourism.

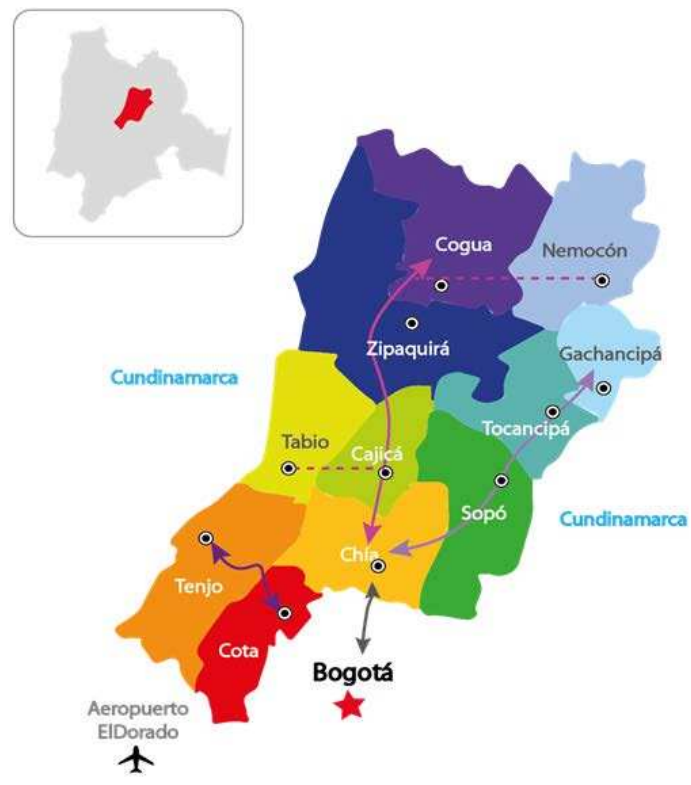

Figure 3. Map of the central savanna (central region) [16]. 


\subsection{The Importance of Natural Resources in the Pandemic}

The population-growth phenomena had their first research in 1798 when Thomas Malthus published a study referring to the population principle; this opinion predicts that food production would not be at the same rate as the population growth rate, which results in a catastrophe [17]. Although this theory failed in its apocalyptic outcome, its approach regarding how complicated it will be to feed a larger population with existing resources is not too far from reality. According to the $\mathrm{UN}$ report on indicators of urban population growth, which concluded that by 2050, at least two-thirds of the world population will live in cities [18], in either case, the increase in population and consumption will increase the demand for natural resources, generating a tremendous impact on the ecosystem. Thus, in 2050, world agricultural production will increase by $60 \%$ compared to $2005-2007$ production to ensure supplies for about 9700 million people [18].

This same case scenario affects water resources because of the increasing deficit rate; $40 \%$ of violent conflicts within the last 60 years are associated with the use of natural resources, placing climate change as one major threat that amplifies this scenario [19].

The COVID-19 pandemic scenario has caused enormous social and economic effects on both a local and global scale, where all restriction measures countries adapt to stop the infection rate, along with local issues, create a higher risk of impact on the availability of crucial natural resources that support and control this crisis. The COVID-19 pandemic and natural resources generate connections addressed from two factors: one given by the main actions to deal with the current crisis (for instance, the high demand and use of potable water for handwashing), as well as other factors produced by habits and lifestyles (e.g., the use of chemical substances for household cleaning) [4].

Some measures to control the crisis and minimize the infection rate are access and use of potable water for handwashing, face masks' mandatory use, plus power and electricity supplies for maintenance/use of intensive care units (ICU) in hospitals. Otherwise, the new lifestyle habits resulting from the quarantine measures demand high pressure on agricultural activities. All the above preserve nutritional well-being. In addition, an increased consumption rate of electricity in households and urban expansions because of the population departure from large metropolitan areas towards rural areas also occur (Figure 4). Despite this, there has also been a decrease in the use of fossil fuels as well as a decrease in atmospheric emissions [4].

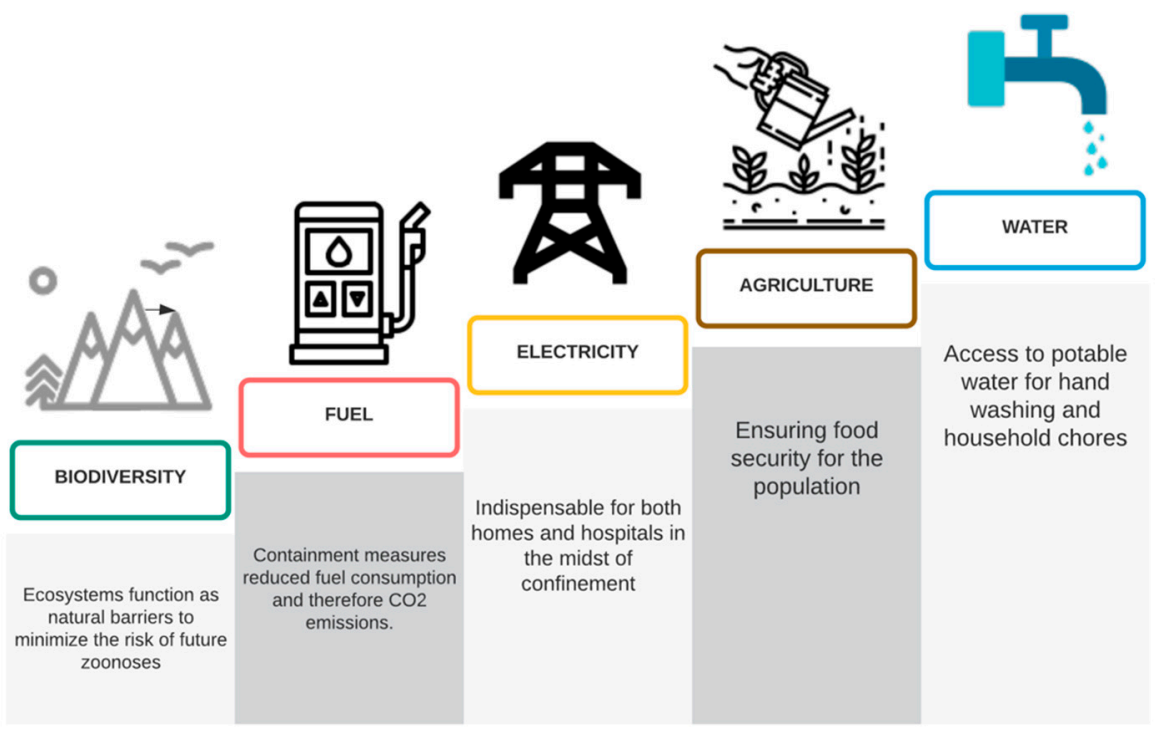

Figure 4. Role of natural resources [4].

Therefore, communities must have sufficient physical and administrative structures to supply basic needs and thus fulfill all care and immediate response measures enclosed on 
the sanitary emergency because of the COVID-19 pandemic. The United Nations Economic Commission for Latin America and the Caribbean (ECLAC) considers the basic needs under the following categories: (i) to have home access assuring minimum standards of habitable conditions; (ii) to have access to vital services enclosed on an adequate sanitary level; (iii) to have sufficient literacy and economic capacity to reach minimum levels of consumption [20].

In the case of Colombia, the Food and Agriculture Organization of the United Nations (FAO) identified 213 municipalities in which 85\% of their population has Unsatisfied Basic Needs (UBN); as a result, one in five town areas lacks quality life standards [21]. Nevertheless, in this case study, the UBN results in an optimal range of quality life standards [22]. Cajicá has a public utilities provider company (ESP, by the Spanish acronym) whose objective is to control, operate, and maintain domestic water supply, sewerage, and cleaning services according to the provisions enclosed in the Colombian legal framework. The ESP of Cajicá town reports that the percentage of potable water coverage is $95 \%$, and the rate of solid waste collection coverage is $99 \%$, being an outstanding service compared to other populations in the country.

According to pandemic measures (lockdowns/curfew), there has been an increase in the consumption of potable water and the production of solid waste, forcing the ESP to extend measures regarding the separation of the solid waste directly from the source, as well as the efficient use of potable water. In summary, by 2019, Cajicá produced 1600 tons of waste per month (as shown in Figure 5); this number increased by $20 \%$ in 2020, and by February 2021, it exceeded by 5\% the 2019 average value. These phenomena could have occurred due to the modification of consumption habits such as food preparation and single-use items such as facemasks and plastics.

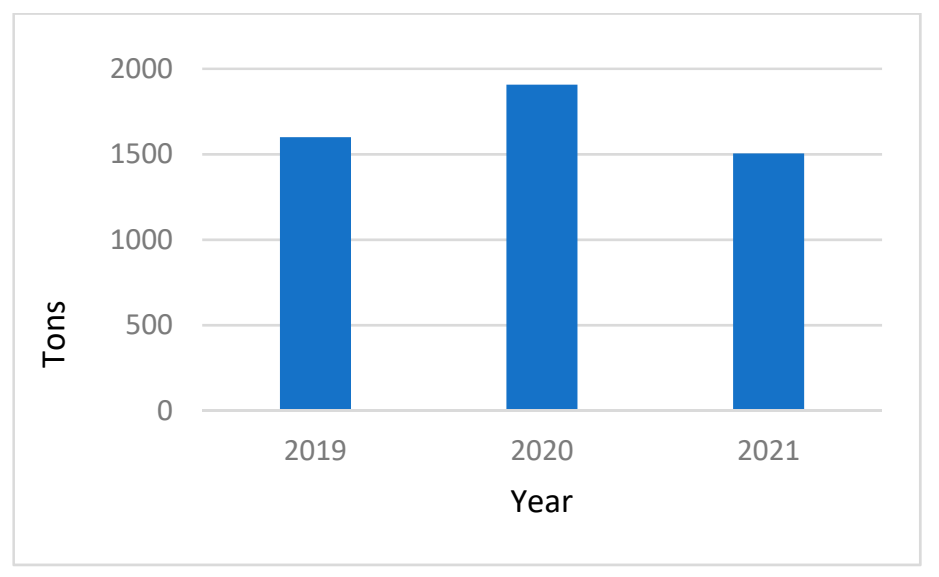

Figure 5. Solid waste production 2019-2021 (Average tons/month).

Likewise, water consumption had similar behavior, as shown in Figure 6, showing that in 2020 there was an increase of $13.17 \%$ compared to the previous year, while in 2021, February had the average consumption of the year 2020 exceeded by $22.15 \%$. A potential reason for this pattern is related to domestic activities, such as the use of showers and toilets.

In terms of strategic location, the town of Cajicá became a housing alternative for those looking for safety against the virus in rural or low-density populated areas, leading to a $9 \%$ growth in building permits, classifying the town as the "the golden mile".

The influence over the ecosystem may harm the ecological integrity and biodiversity, affecting the structure and property, not to mention all terrestrial and aquatic biogeochemical cycles, which leads to the necessity for the conservation of biodiversity and the ecosystem services. It is necessary to take urgent measures to protect human health against the virus (directly or indirectly). In addition, people settle in high agricultural production areas, which may place elements such as food security at risk [23]. 
It is a noteworthy fact that even when households have access to water utilities, most of the ESP does not offer a full-time service, which affects rural and peri-urban areas in specific, reflecting the lack of necessary infrastructure to guarantee the service. All of this makes it hard to comply with the Sustainable Development Goals (SDG) especially No. 11 on sustainable cities and communities.

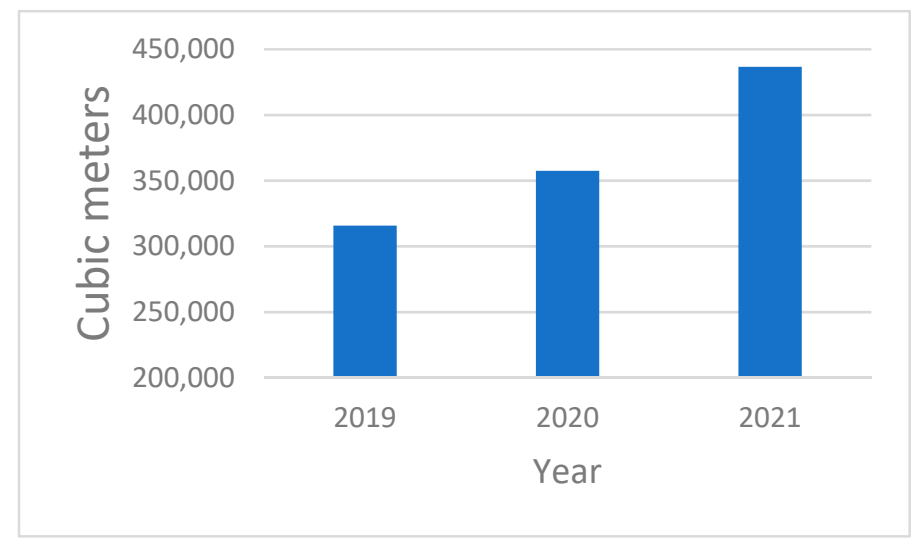

Figure 6. Drinking water consumption 2019-2021 (average $\mathrm{m}^{3} / \mathrm{month}$ ).

\subsection{Sustainability in Times of Pandemic}

Environmental sustainability takes center stage from the Brundtland Report, prepared by different nations in 1987 for the UN. The document, originally called our Common Future, confronts the position of current economic development in contrast to environmental sustainability [24], addressing the concern regarding global warming and its devastating consequences, plus the degradation of natural resources and loss of biodiversity, high rates of poverty, and vulnerability to natural phenomena. All the above still demands a quick course of action.

The concept of sustainable development is then adapted, allowing us to meet the actual needs without jeopardizing the ability of future generations to fulfill their needs [25]. Therefore, the continuous examination for a territorial approach makes notable the meaning of bioregion, where the maintenance of essential ecological processes, life-support systems, and the sustainable use of species according to their carrying capacity is absolute [26].

The human being is no longer the center, which forces Urban Planning to reevaluate the territorial planning as an instrument that harmonizes ecosystem dynamics and allows the assessment of its limits to guarantee long-term planning.

The idea of a bioregion originated in 1996 from the studies of William Rees and Mathis Wackernagel, which tried to base an ecological language that would allow for quantifying the sustainability of the lifestyles of humanity and its direct relationship along with the capacity to renew ecosystems [27]. Everything aforementioned uses the concepts of human carrying capacity and natural resources to develop an assessment framework on each city's ecological footprint.

The prevailing economic assumptions regarding urbanization and the sustainability of cities dictate that the situation must be revised with aims of global ecological change [28], making way for models based on a green economy or sustainable economy [29].

The UN Program UNEP launched the Green Economy Initiative (GEI) in 2008, where they state: the green economy in not a substitute for sustainable development; this is conceived as the results in improved human well-being and social equity, while significantly reducing environmental risks and ecological scarcities [30] strengthens the circular economy model.

In this way, it opens a field to the fulfillment proposed in the 2030 Agenda of the Sustainable Development Goals for SDG 11: Sustainable Cities and Communities, which promotes the responsible use of resources and the environment and the improvement of the well-being of the citizens. The above, based on the general principles of governance, 
empowerment, education, health, and mobility, help define the objectives and strategies for sustainable development.

However, it is important to highlight that each territory is not only the result of its history, but must also be resilient in the face of the situations it currently faces, such as the pandemic, which has conditioned the future of emerging cities and the way in which these were understood, which leads us to reflect on, and not overlook that although globalization allowed the opening of great benefits, not all populations have the same capacity to face the changes that COVID-19 has raised to date, demanding a rapid response to health emergencies. Thus, it is of vital importance to strengthen the resilience of territories through practices that reduce the subjectivity of public politics and consolidate reflective governance and the implementation of new infrastructure, generating the possibility of economic development in terms of sustainable development [29].

Cajicá Mayor's office has been promoting for about 10 years the culture of separation and use of solid waste among citizens; as a result, in 2017, the town was awarded by the UN as one of the five towns leading waste and contamination control actions in the world [31]. This culture allowed Cajicá to withstand the increasing volume of solid wastes in a resilient way. However, the measure was not enough since, by 2020, the average volume of reused solid waste reached just $23 \%$ across the municipality.

In addition, economic reactivation measures have incited mixed feelings within the municipality; this town being a place for tourists, the number of visitors from Bogotá increased significantly, generating a considerable volume of solid waste, as well as producing discomfort in the citizens due to crowds, traffic jams, and the fear of provoking new infection cases, a case scenario that the town is not prepared to handle. In summary, there is still a long way to go towards the use and implementation of the circular economy

To summarize, connections between rural and urban areas build up interest; both areas form a complex symbiotic network where goods and services are connected. In addition, these play a significant role in the perspective of territory as promoters of sustainable management. Therefore, this network could be associated with the definition of urban ecosystems that indicates that there is a symbiosis between natural and spatial scenarios, assuming that systems must remain in balance, along with the constant need for permanent flows of energy, matter, and information, throughout a complex holistic approach, where the agglomerations have a rhizomatic behavior [32]. The linearity of exposure is not substantial because, in the rhizome, some aspects form a connection where dynamics showing its singularity are born, developing under its principles of connection and heterogeneity, multiplicity, assigning rupture, cartography, and decalcomania [33].

\subsection{Urban Resilience}

Resilient cities are replacing traditional ideas of urbanism and have become a key strategy for sustainable development, determining how to improve the capacity of the urban system to cope with uncertain factors and improve the predictability and orientation of urban planning, which has gradually become a popular topic in the field of urban planners, as announced in Kobe, Hyogo, Japan, at the World Conference on Disaster Reduction, where special emphasis was placed on the fact that increasing the resilience of nations and communities to natural disasters, leads to a reduction in the loss of human life and social, economic, and environmental assets [34,35].

Similarly, the UN-Habitat report defines urban resilience as the capacity of urban systems to recover rapidly from any event caused by disruptive phenomena of natural or human origin. Its purpose is to prevent an event from evolving into a disaster [36]. Terms such as threat, exposure, and vulnerability are frequently used for risk management, understood as a characteristic of the community that makes it susceptible to being damaged by a threat [37]. However, such risk assessment involves the analysis of disruptive agents that can cause changes to a system in a positive or negative way.

Therefore, cities are seen as connecting elements between urban processes that allow increasing resilience as a result of their interactions [38], from which later "new properties 
emerge and characterize the city as a collective entity" [39]. This ensures that relationships are the key factor in understanding resilience. For example, once the lockdown stage is over, it has been possible to show how some of these settings, especially rural ones and public spaces, have been valued as necessary areas for safe social contact; it is for this reason that it is imperative to identify the risks to which these places are exposed and to rewrite the main criteria for the design of a sustainable and resilient city [2].

The city of Cajicá, similar to other regions of the country, had a substantial collapse in its economy as a result of the lockdown and social distancing measures that began in March 2020, which forced the administrative authorities to create action plans that allowed for compliance with the isolation measures for four months. Once the measure was lifted and with the obligation to adapt to the "new normal" under the guidelines and security protocols, the economic uncertainty continued. Without an evident capacity to face it, the municipality carried out initiatives, with the support of the municipal council, related to tax relief for entrepreneurs and contractors, temporary subsidies for public services for the most vulnerable populations, and economic incentives for the reactivation of small and medium-sized companies.

\section{Discussion}

\subsection{Emerging Cities}

The new population and economic growth patterns at the urban level occur principally in cities classified as intermediate, creating expansion and agglomeration phenomena. These urban areas are classified as intermediate, involving populations within a social stability and governance context, according to the provisions of the IDB [13,40]. In addition, those towns become especially important because of the transformation into stationary or temporary towns, which tends to be notably attractive for those who seek a nearby rural ambiance as well as an improvement in their quality of life, even when this implies long drives to job sites inside metropolitan cities. However, these emerging cities struggle to respond to the high housing demand, guaranteeing public services while reducing rural areas.

Therefore, this indicates a socioenvironmental challenge for cities since their growth rates are higher than the values obtained from the metropolitan cities, home to $75 \%$ of the world population, being these rural areas or urban settlements. It estimates that they consume between $60 \%$ and $80 \%$ of energy, produce $80 \%$ of the global GDP, and this being a stationary town, around $7 \%$ of greenhouse gases are emitted [41]. This growth occurs in a disorganized way, demonstrating poor-quality urbanization and generating unsustainable dynamics that lead to further inequity gaps.

The current scenario caused by the pandemic produces notable displacements of people from metropolitan areas to these intermediate cities, not only for housing but also because those are ideal spaces to protect themselves from the virus outbreak and improve their mental health, playing a remarkable role in the development and territorial cohesion compared to metropolitan cities [3,42]. In effect, this would be an improvement for economic reactivation; however, this idea loses importance rapidly due to traffic jams, overcrowding, inequity gaps, and the transformation in the original population basis, producing fragmentation and a change in lifestyles.

\subsection{The Importance of Natural Resources in the Pandemic}

Natural resources are essential components within human survival; the lack of agricultural resources, fertile lands, water, and vegetation have generated violent scenarios and conflicts during the last 60 years, and climate change issues further complicate this scenario [43]. Additionally, in times of COVID-19, the loss of confidence in food safety measures established by government entities, because of the upcoming politics that these may have, increases non-conformity and affects governance; on the other hand, the lack of water and land can influence variations in food prices as well as access and quality. 
It is relevant to understand in-depth the concept of carrying capacity, understood as "Communities/Species that can reside indefinitely in a specific habitat without producing permanent damage to the ecosystem on which they depend." For human beings, this is the maximum consumption of resources and waste creation that a region can sustain indefinitely without altering the ecosystem functions. With this overview, we must reflect on the tremendous impact we, as human beings, provoke on the urban footprint as a product of the excessive use of resources to mitigate the pandemic outbreak effects, even though cities nurture their resources and productivity from places located on the outskirts and their political limits. As a result, the use and primary consumption of food, fuel, waste processing, and water use, among others, depend directly on inhabitants' lifestyles [28]. For this reason, it is necessary to promote a proactive citizen culture in environmental matters, encouraging awareness, attitude, and interest in caring for the environment, including spaces that allow discussing and proposing alternatives to provide solutions to environmental challenges.

\subsection{Sustainability in Times of Pandemic}

The Brundtland Summit established the concept of sustainable development as the one that meets the needs of present generations without compromising the capacity of those in future scenarios [25,44], outlining two ideas: the first in the environmental footprint, and the second in the capacity load assigned to a time variable. The current conditions we have as a result of the COVID-19 pandemic are compelling us to reinforce the biocentric vision where the human being is no longer the center, which forces urban planners to reevaluate the territorial planning as an instrument that harmonizes with the dynamics of an ecosystem and allows evaluating limits that guarantee long-term planning.

Whereas regions fulfill urban building processes, the role of cities gains importance in the sustainability results since the urban footprint is linked to the paradigm of sustainable development, moving forward the science of sustainability. The urban form determines the variation of the ecological footprint inside the territory, based on changes regarding the land use acts $[45,46]$; therefore, the study of this variation will allow an analysis of sustainable management tasks within cities that will provide an accurate understanding of the demands on environmental services that these inflict on a territory.

One of the effects of the COVID-19 pandemic was to test the performance of the elements inside supply chains as well as global demands, which resulted in a shocking outcome. In an effort to isolate cases and limit the virus's transmission rate, countries implemented severe measures such as national and border closures, demonstrating once again their dependence on external actors and the lack of self-sufficiency. Then, an approach regarding the appropriation of circular economy models and how these could satisfy economic needs, separating economic growth from resource consumption and waste management, was considered in this study [47]. As a result, government entities can find in this model an opportunity for green economic reactivation, where the fundamental challenge aims the transformation of the traditional linear economy towards a model of industrial practices that allow the user to reuse or recycle a product, increasing its lifespan period and consequently generating a lower amount of land waste disposal found in dumps.

Tools such as environmental footprints and circular economy models can provide a guideline basis for public policies to contain the pressures placed on ecosystems and promote sustainable development of cities. These are crucial elements for the compliance with the Habitat Agenda, and the thoughts coming from the circular economy will also allow us to focus on recognizing innovations in favor of the general well-being of the population [45,47]. In this manner, an approach to SDG 11: "Sustainable Cities and Communities" is possible with local and regional governance and associated socioeconomic transformations. Since it is useless to establish public policies if we do not carry out training processes inside the community, all the above are regarding a settling-territory vision. 


\subsection{Urban Resilience}

Urban resilience is usually associated with natural disasters and their alterations in intra-urban processes, considering it as a complex network of relationships between its internal components and external networks [48], thus functioning as a gear in which each component of its extension it fulfills a function to the extent that it harmonizes with the network. This function is magnified with the common goal of making this complex system work effectively with a multilevel approach at the local and regional level. Therefore, the city is conceived as a connecting element between inter-urban and intra-urban processes that allows for increasing resilience as a result of their interactions.

So, in the uncertainty resulting from the pandemic, our capacity for recovery and adaptation becomes urgent; resilience is the result of a process by which organizations work to anticipate and respond to continuous external threats $[7,8]$. It is not about leaving behind the principles of order and logic, but about integrating them into a more complete scheme, thus allowing for adaptive agents to emerge, exhibiting more complex structures and behaviors and allowing for the passage to evolutionary resilience.

On the other hand, emerging cities have had to face situations related to security, economic and public health crises, and political and social tensions unleashing problems such as the hoarding of basic supplies, the spread of fear, generating a latent risk for the instability of the cities. It will take a long time and much effort to restore the trust of citizens [49], and for this reason, characteristics of cities that were not considered important at first emerge strongly. It is thus necessary to envision the city as a collective entity, which includes the perception of the actors involved and strengthens social cohesion. Additionally, the design of protocols that not only focus on natural disasters, but also take into account scenarios such as pandemics, for which cities were clearly not prepared, is imperative.

Even so, the current situation shows that sustainability can have a positive and lasting impact on resilience, building "resilient systems that promote radical innovation in economic policy, corporate strategy and in the social sphere, systems and public governance", and it should be proposed as a general policy flag and improvement of social innovation [48]. In other words, sustainability and resilience are two concepts that will allow for a strategic functionality in urban planning, since while the first prioritizes results, the second analyzes processes.

\section{Conclusions}

Emerging cities should be the new matter of study for urban planners, bearing in mind that these will be the ones that receive a new departure of people interested in improving their quality of life, becoming in a notable dependency for regional connectivity. Moreover, there is still time to plan its growth and ensure urban and environmental sustainability for all inhabitants.

A community's behavior based on the principle of shared responsibility for environmental sustainability in cities is fundamental because it is necessary to promote a proactive citizen culture in ecological topics, encouraging awareness, attitude, and interest in caring for the environment; therefore, spaces are required that allow discussing and proposing alternatives to provide solutions to environmental challenges.

Tools such as environmental footprints, urban footprints, and circular economy models can provide a baseline for public policies that promote sustainable development of cities, generating an approach to SDG 11: "Sustainable Cities and Communities" together with a settling-territory vision.

Sustainability can contribute a positive and lasting impact on resilience, bringing biocentric thinking and transforming the traditional vision of urbanism; in this case, sustainability prioritizes results while resilience analyzes processes. These are two concepts that will allow for a strategic functionality within urban planning. It is relevant to consider that urban studies should focus on understanding the natural dynamics of the territory, conceiving it as a complex adaptive system that requires stages of transformation to achieve evolutionary resilience, enclosed in nature-based solutions. 
The case study showed that Cajica is a typical emerging town in which it is possible to visualize the consequences of the pandemic and the effects of deficient administrative directives regarding urban planning. However, the crises should also mark an opportunity to consider the COVID-19 pandemic as a pause to rethink the planning of cities as main contributors within the ecological crisis and, in this way, propitiate an encounter between the natural and the human to fulfill the goal of implementing sustainability and resilience to guard the wellbeing of present and future generations.

Author Contributions: Conceptualization, L.J.R.L. and A.I.G.C.; methodology, L.J.R.L. and A.I.G.C.; software, L.J.R.L. and A.I.G.C.; validation, L.J.R.L. and A.I.G.C.; formal analysis, L.J.R.L. and A.I.G.C.; investigation, L.J.R.L. and A.I.G.C.; resources, L.J.R.L. and A.I.G.C.; data curation, L.J.R.L. and A.I.G.C.; writing-original draft preparation, L.J.R.L. and A.I.G.C.; writing-review and editing, L.J.R.L. and A.I.G.C. All authors have read and agreed to the published version of the manuscript.

Funding: This research has not received external funding.

Acknowledgments: The authors want to thank Nueva Granada Military University for the availability of databases in the Ph.D. in Applied Sciences.

Conflicts of Interest: The authors declare no conflict of interest.

\section{References}

1. Lopez, L.J.R.; Castro, A.I.G. Sustainability and resilience in smart city planning: A review. Sustainability 2020, 13, 181. [CrossRef]

2. Gastelú, N.F.S.; Albán, M.A.S.; Yanez, D.G.M.; Poveda, T.V.P.; Vizcarra, G.C.A. Ciudad en pandemia. Una aproximación desde la escala humana a las prioridades urbanas. Eídos 2020, 16, 71-86.

3. David, L. Ciudades, vínculos rurales-urbanos y COVID-19: La necesidad de repensar lo urbano. Rimis 2020, 10, 1-6.

4. Suzán, G. El rol de los Recursos Naturales Ante la Pandemia por el COVID-19 en América Latina y el Caribe. CEPAL. 2020. Available online: https:/ / www.cepal.org/es/enfoques/rol-recursos-naturales-la-pandemia-covid-19-america-latina-caribe (accessed on 19 August 2021).

5. De la Republica, B. Capacidad de Carga de un Ecosistema-Enciclopedia. Available online: https://enciclopedia.banrepcultural. org /index.php?title=Capacidad_de_carga_de_un_ecosistema\&mobileaction=toggle_view_desktop\&printable=yes (accessed on 18 March 2021).

6. Reguero, B.G.; Beck, M.W.; Losada, I.J.; Narayan, S. Uniendo ingeniería y ecología: La protección costera basada en ecosistemas. Ribagua 2017, 4, 41-58. [CrossRef]

7. Bryce, C.; Ring, P.; Ashby, S.; Wardman, J.K. Resilience in the face of uncertainty: Early lessons from the COVID-19 pandemic. J. Risk Res. 2020, 23, 880-887. [CrossRef]

8. Shi, Y.; Zhai, G.; Xu, L.; Zhou, S.; Lu, Y.; Liu, H.; Huang, W. Assessment methods of urban system resilience: From the perspective of complex adaptive system theory. Cities 2021, 112, 103141. [CrossRef]

9. Orgnización Naciones Unidas. Los Objetivos de Desarrollo Sostenible; United Nations: New York, NY, USA, 2018.

10. Yin, R. How to do Better Case Studies: (With Illustrations from 20 Exemplary Case Studies). In The SAGE Handbook of Applied Social Research Methods; SAGE Publications, Inc.: New York, NY, USA, 2014; pp. 254-282.

11. Banco Interamericano de Desarrollo. Guía Metodológica del Programa de Ciudades Emergentes y Sostenibles; IDB: Washington, DC, USA, 2004.

12. UN. Perspectivas de la Población Mundial. 2019. Available online: https://www.un.org/es/global-issues/population (accessed on 30 April 2021).

13. Terraza, H.; Blanco, D.R.; Vera, F. De ciudades emergentes a ciudades sostenibles. Educ. Siglo XXI 2014, 32, $287-290$.

14. Borja, J.; Carrión, F.; Corti, M. Ciudades resistentes. Ciudad. Posibles 2017, 14, 1-350.

15. García, M.D.M.H. Las ciudades como objetivo de desarrollo sostenible. IEEE 2017, 3, 10.

16. Cámara de Comercio de Bogotá; Corporación PRODENSA. Libro Naranja De La Rinn-Sabana Centro Región De Innovación 1 ¿Hacia Dónde Debemos Ir? Camara de Comercio de Bogota: Bogotá, Colombia, 2016.

17. D'Adamo, I.; Rosa, P. How do you see infrastructure? Green energy to provide economic growth after COVID-19. Sustainability 2020, 12, 4738. [CrossRef]

18. Manuel Ordorica. Una Mirada al Futuro Demográfico de México; El Colegio de México: Tlalpan, Mexico, 2015.

19. Banco Interamericano de Desarrollo. Sostenibilidad urbana en América Latina. BID 2011, 70. Available online: https:// publications.iadb.org/es/publicacion/16383/sostenibilidad-urbana-en-america-latina-y-el-caribe (accessed on 19 August 2021).

20. Denier, L.; Scherr, S.; Shames, S.; Chatterton, P.; Hovani, L. Stam, El Pequeño Libro sobre los Paisajes Sostenibles 2017. Available online: http:/ / ecoagriculture.org (accessed on 19 August 2021).

21. Mancero, X.; Feres, J.C. El método de las necesidades básicas insatisfechas (NBI) y sus aplicaciones en America Latina. CEPAL 2001, 8, 52.

22. FAO América. 100 Territorios Libres de Pobreza y Hambre; FAO: Rome, Italy, 2020; pp. 1-6. 
23. DANE. Necesidades Básicas Insatisfechas (NBI). 2018. Available online: https://www.dane.gov.co/index.php/estadisticas-portema/pobreza-y-condiciones-de-vida/necesidades-basicas-insatisfechas-nbi (accessed on 29 April 2021).

24. Gottdenker, N.L.; Streicker, D.G.; Faust, C.L.; Carroll, C.R. Anthropogenic Land Use Change and Infectious Diseases: A Review of the Evidence. EcoHealth 2014, 11, 619-632. [CrossRef]

25. Zillman, J.W. Historia de las actividades en torno al clima. Terc. Conf. Mund. Sobre Clima 2009, 58, 141-150.

26. López, I.G. Desarrollo Sostenible. 2020. Available online: https://unstats.un.org/sdgs/report/2020/progress-chart-2020 _Spanish.pdf (accessed on 21 August 2021).

27. Cuberos, R. Cambios de paradigma en la ordenación del territorio. In Planificación Territorial y Desarrollo Sostenible; $2017 ;$ p. 15. Available online: https:/ / repositorio.cepal.org/bitstream/handle/11362/44731/1/S1900439_es.pdf (accessed on 22 August 2021).

28. Luis, P.; Burguera, J.; Huella ecológica: Reto intergeneracional. Tres Advertencias. 2012. Available online: https:/ / docplayer.es/ 11956054-Huella-ecologica-reto-intergeneracional-tres-advertencias-introduccion.html (accessed on 22 August 2021).

29. Rees, W.E. Ecological footprints and appropriated carrying capacity: What urban economics leaves out. Environ. Urban 1992, 4, 121-130. [CrossRef]

30. UNEP. Towards a Green Economy: Pathways to Sustainable Development and Poverty Eradication; UNEP: New York, NY, USA, 2011.

31. UNEP. Solid Approach to Waste: How 5 Cities Are Beating Pollution; UNEP: New York, NY, USA, 2017.

32. Gorgolas, P. Estrategias de Actuación para La Promoción de una Ecociudad: Una Experiencia Marroquí. El Caso de Estudio de La Comuna de Benslimane. In Regeneración Y Planeamiento Para Ciudades Sostenibles; Experiencias En América, Marruecos Y España; 2020. Available online: https:/ / dialnet.unirioja.es/servlet/libro?codigo=765437 (accessed on 21 August 2021).

33. Castrillón, L.F.; Zapata, R.C. Fantasmagorías y disposiciones en el Ordenamiento Territorial. Discursividades Rizomáticas de la ciudad de Manizales. Antropol. Sociol. Virajes 2017, 19, 33-62.

34. Nieto, K.R.T.; Potes, L.R. Hábitat sostenible: Adaptación y mitigación frente al cambio climático hacia los territorios resilientes. Módulo Arquit. CUC 2018, 21, 63-96. [CrossRef]

35. UNISDR; WMO. Disaster Risk and Resilience. UN System Task Team on the Post-2015 UN Development Agenda. United Nations Office for Disaster Risk Reduction. 2012. Available online: http:/ /www.un.org/en/development/desa/policy/untaskteam_ undf/thinkpieces/3_disaster_risk_resilience.pdf. (accessed on 29 April 2021).

36. Hábitat, O. Guía de Resiliencia Urbana. Secr. Desarro. Agrar. Territ. y Urbano—SEDATU 2016, 1-57. Available online: https: //www.gob.mx/cms/uploads/attachment/file/179708/Guia_de_Resiliencia_Urbana_2016.pdf\%0A (accessed on 22 August 2021).

37. PNUD. Manual Técnico de Resiliencia Urbana. Instrucción Metodológica Resiliencia Urbana; PNUD: New York, NY, USA, 2018.

38. Davalos, J.; Pérez, A.R. Ciudades sostenibles, inclusivas y resilientes: Gobiernos locales y participación ciudadana en la implementación de las agendas globales para el desarrollo. INNOVA Res. J. 2017, 2, 116-131. [CrossRef]

39. Folke, C. Resilience: The emergence of a perspective for social-ecological systems analyses. Glob. Environ. Chang. 2006, 16, 253-267. [CrossRef]

40. Giglio, A.; Adriana, M.; Werther, H. Sostenibilidad de distintas formas de crecimiento en ciudades emergentes sudamericanas: Experiència colaborativa para la medición comparativa de indicadores aplicados al hábitat urbano local. Dep. d'Urbanisme Ord. Territ. Univ. Politècnica Catalunya 2018, 4, 120-138.

41. González, A. Cadenas globales de valor sostenibles cómo promover buenas prácticas de comercio. Integr. Comer. 2017, 41, 120-138.

42. Molina, L. Esto es una Migración de la Ciudad al Campo' Cambios en las Dinámicas Sociales, Económicas y Ambientales de la Sabana de Bogotá. Caso de Estudio de Cajicá-Cundinamarca; Universidad Externado de Colombia: Bogotá, Colombia, 2017.

43. Vesco, P.; Dasgupta, S.; de Cian, E.; Carraro, C. Natural resources and conflict: A meta-analysis of the empirical literature. Ecol. Econ. 2020, 172, 106633. [CrossRef]

44. Castiblanco-Prieto, J.J.; Aguilera-Martínez, F.A.; Sarmiento-Valdés, F.A. Principios, criterios y propósitos de desarrollo sustentable para la redensificación en contextos urbanos informales. Rev. Arquit. 2019, 21, 21-33. [CrossRef]

45. Zambon, I.; Serra, P.; Salvati, L. The (Evolving) urban footprint under sequential building cycles and changing socio-demographic contexts. Environ. Impact Assess. Rev. 2019, 75, 27-36. [CrossRef]

46. Rees, W.; Wackernagel, M. Urban Ecological Footprints: Why Cities Cannot Be Sustainable-and Why They Are a Key to Sustainability. In Urban Ecology: An International Perspective on the Interaction between Humans and Nature; Springer: Boston, MA, USA, 2008; pp. 537-555.

47. Ibn-Mohammed, T.; Mustapha, K.B.; Godsell, J.M.; Adamu, Z.; Babatunde, K.A.; Akintade, D.D.; Acquaye, A.; Fujii, H.; Ndiaye, M.M.; Yamoah, F.A.; et al. A critical review of the impacts of COVID-19 on the global economy and ecosystems and opportunities for circular economy strategies. Resour. Conserv. Recycl. 2021, 164, 105169. [CrossRef]

48. Carreño Meléndez, F.; Iglesias Piña, D.; Sánchez Barreto, R.F.; Carrasco Aquino, R.J.; Andrés Calderón, H.; Platas López, F. Discusión Epistemológica Entorno a la Sustentabilidad y el Desarrollo; Universidad Autónoma del Estado de México: Mexico, Mexico, 2018.

49. Allam, Z.; Jones, D.S. Pandemic stricken cities on lockdown. Where are our planning and design professionals [now, then and into the future]? Land Use Policy 2020, 97, 104805. [CrossRef] [PubMed] 


\title{
The Development of Digital Transformation and Relevant Competencies for Employees in the Context of the Impact of the COVID-19 Pandemic in Latvia
}

\author{
Veronika Bikse ${ }^{1}$, Inese Lusena-Ezera ${ }^{1}$, Peteris Rivza ${ }^{2}$ and Baiba Rivza ${ }^{3, *(D)}$ \\ 1 Institute of Management Science, Liepaja University, 14 Liela Street, LV-3401 Liepaja, Latvia; \\ vbikse@lu.lv (V.B.); inese.lusena-ezera@liepu.lv (I.L.-E.) \\ 2 Department of Computer Systems, Latvia University of Life Sciences and Technologies, 2 Liela Street, \\ LV-3001 Jelgava, Latvia; peteris.rivza@llu.lv \\ 3 Institute of Economics and Regional Development, Latvia University of Life Sciences and Technologies, \\ 2 Liela Street, LV-3001 Jelgava, Latvia \\ * Correspondence: baiba.rivza@llu.lv
}

Citation: Bikse, V.; Lusena-Ezera, I.; Rivza, P.; Rivza, B. The Development of Digital Transformation and Relevant Competencies for Employees in the Context of the Impact of the COVID-19 Pandemic in Latvia. Sustainability 2021, 13, 9233. https://doi.org/10.3390/su13169233

Academic Editor: Idiano D'Adamo

Received: 19 June 2021

Accepted: 12 August 2021

Published: 17 August 2021

Publisher's Note: MDPI stays neutral with regard to jurisdictional claims in published maps and institutional affiliations.

Copyright: (C) 2021 by the authors Licensee MDPI, Basel, Switzerland. This article is an open access article distributed under the terms and conditions of the Creative Commons Attribution (CC BY) license (https:/ / creativecommons.org/licenses/by/ $4.0 /)$.

\begin{abstract}
The current period describes the impact of the global COVID-19 pandemic and the ensuing economic crisis on businesses and the lives of citizens. It has accelerated digital transformation in all areas. The work and learning of many individuals have moved to the digital environment. In order to use digital technologies, employees need to acquire new knowledge and skills. The aim of this research study is to perform an analysis of the development of digital transformation and relevant competencies for employees and to identify the opportunities and challenges in Latvia. The research methodology applied for this research study is based on examining relevant theoretical concepts and publications of the EU regarding digital transformation. A survey method was used to find out the opinions of Latvian employers regarding the importance of digital transformation and relevant competencies for employees. The analysis of the research indicated that the majority of the respondents surveyed rated the level of implementation of digital transformation as high or medium-high, which shows that this is a good trend, and the digitalization process continues to progress. However, about a third of enterprises are only at the early stage of digitalization, while some have not yet begun it. The problem is the development of human capital competencies and digital skills. This is a specific research study that expands and provides insights into the situation in Latvia on the possibilities of implementation of digital transformation, which is closely linked with the development of human capital competencies and digital skills. This requires maintaining a holistic approach to targeted digital transformation management.
\end{abstract}

Keywords: COVID-19; competences; digitization; digitalization; digital transformation; sustainability

\section{Introduction}

Today, the greatest challenge is the COVID-19 pandemic and the resultant global economic crisis that has significantly changed people's habits of communication by means of information and communication technologies (ICT). The application of ICT has become the norm everywhere: at work, while learning, and for everyday transactions. Moreover, in particular, it has far-reaching implications for workers as it most likely has accelerated the transformation process of jobs [1]. Already, many organizations have successfully transformed their workflows. A very rapid transition to teleworking has occurred. Online work is becoming increasingly popular, and the number of employees working remotely tends to increase. In Latvia for example, only $4.8 \%$ of employees worked remotely before the COVID-19 pandemic crisis, while in 2020, it was already 39\%, i.e., almost eight times more than in 2019 [2]. This is also evidenced by the results of surveys conducted by the Latvian Information and Communication Technology Association, which showed that $43 \%$ of enterprises provided opportunities for their employees to work remotely, and $45 \%$ 
of enterprises used the possibility to receive and send e-invoices on a daily basis, while only a quarter used e-signatures (26\%) [3]. Consequently, the coronavirus pandemic has forced enterprises to adopt digital transformation and change how they create, deliver, and capture value to their customers [4]. Moreover, in the near future, enterprises will face the additional challenge of big data management. If they do not master it, their competitive position will seriously weaken if it becomes the starting point of a genuine industrial revolution based on converging technologies [5].

In this context, more action is needed to implement the digital transformation in all areas. At the same time, to use the digital technology in different situations and for different purposes, employees will require and demand to acquire relevant competencies: new knowledge and skills, work organization and management skills, and other characteristics that become an important component of the development and competitiveness of individuals and enterprises. With digital transformation processes deepening, the most important issue is an appropriate, flexible education system to enable the development of competences and new skills. The mentioned aspects are closely interrelated: the better a company is technologically equipped and the more appropriate competencies and skills to use technologies employees have, the greater the opportunity to increase the competitiveness of enterprises and for the country to gain economic, social, environmental, and consumer benefits. However, it should be noted that a large part of the Latvian and EU population, $58 \%$ of EU individuals and $41 \%$ of Latvia's individuals, still lack basic digital skills and continue to build them up slowly [3]. In this context, an important research problem is that the digital transformation processes are not closely linked with the development of human capital competencies.

Therefore, the aim of the research is to perform an analysis of the development of digital transformation and relevant competencies for employees and to identify the opportunities and challenges in Latvia. However, digital transformation plays a critical role in an organization's ability to create new growth opportunities for businesses and improve the lives of citizens.

The questions addressed in this paper are as follows:

- What does digital transformation mean and what competences have to be developed to prepare new professionals?

- Are the digital transformation and the development of relevant competences in employees successfully carried out in all sectors of the national economy in Latvia?

\section{Research Methodology}

In order to achieve the aim set, the present research performed a review of scientific literature, examining publications and documents of the European Commission regarding the digital transformation. To obtain a deeper insight into the research problem, the implementation of digital transformation in the EU and Latvia is analyzed in this research. A survey method was used to find out the opinions of Latvian employers regarding the importance of digital transformation and relevant competencies for employees.

The survey was conducted on the Internet by filling out a specially designed online questionnaire at docs.google.com/forms. The questionnaire consisted of 10 questions. The structure of the questionnaire comprised open and closed questions. Several questions were formed based on the Likert Scale, allowing the respondents to provide their answers to several statements. A total of 162 respondents from Latvia participated in the survey. Replies were received from 161 employers or $99.4 \%$ of the total. In the survey $(n=161)$, the distribution of the employers by field of economic activity was as follows: $26.1 \%$ from the goods production sector, $14.3 \%$ from the services sector, $5.6 \%$ from the public sector, and $54.0 \%$ employers represented other fields, mostly through small and medium-sized enterprises.

According to the Latvian Statistical Database in the percentage distribution of economically active enterprises, in 2019, the production sectors (30.5\%), business services (18.3\%), public sectors $(7.2 \%)$, and others, mostly small and medium-sized enterprises such as trade 
and accommodation, real estate activities, as well as agriculture (54.0\%) of all businesses in Latvia, were represented. Thus, all the questionnaires were considered suitable for the purposes of this survey. Most of the employers were relatively young people: $27.3 \%$ were under 30 years of age, $27.3 \%$ of the total were $31-50$ years old, and $45.4 \%$ were over 50 years and older. The distribution of the employers by field of economic activity and age is shown in Figure 1.

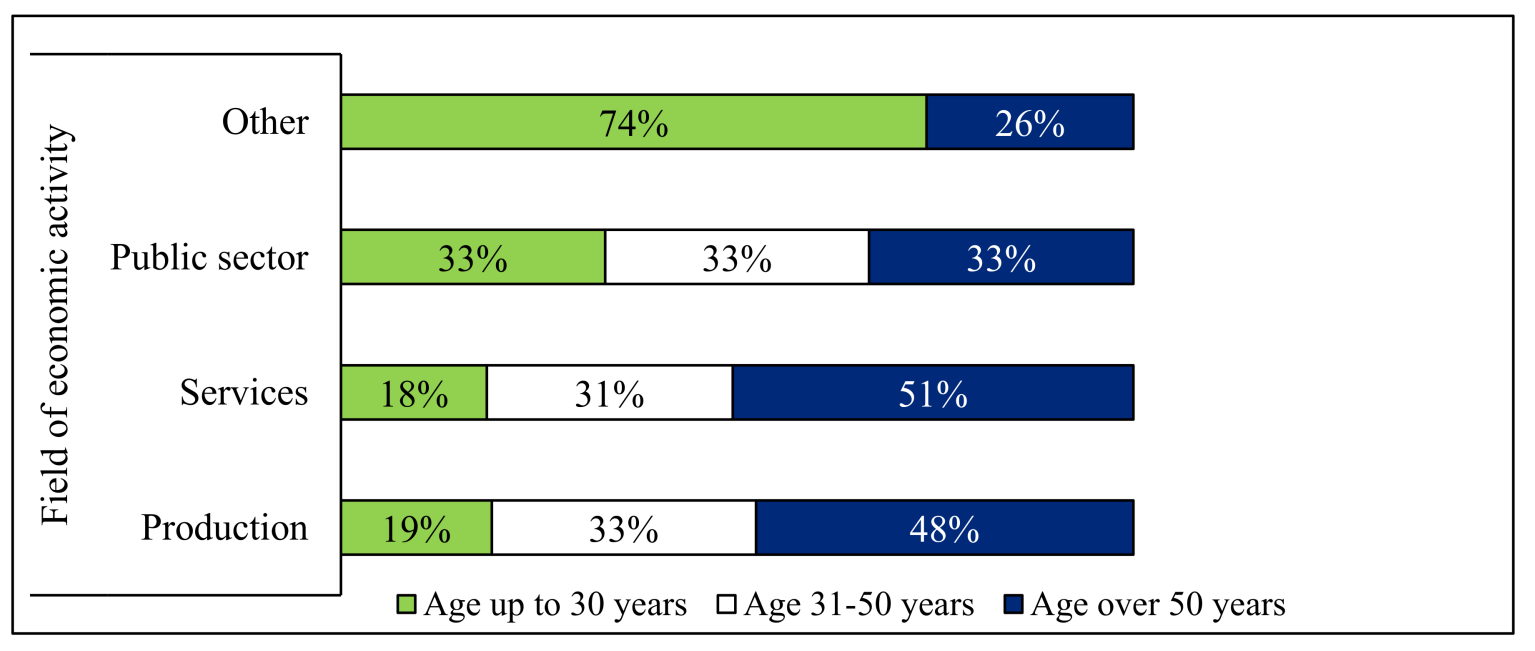

Figure 1. Distribution of the respondents by field of economic activity and age $(\mathrm{n}=161)$.

The survey was carried out during the period between January and May 2020. The number of respondents was selected using a simple random sample. The survey results were analyzed and the data processed by the authors of the paper within the present study by applying methods of descriptive statistics (frequencies, central tendency, and crosstabs analysis), data visualization methods, and a nonparametric method-the Kruskal Wallis H-Test. SPSS software (26 version) and MS Excel 2016 were used to analyze the statistical data.

\section{Results}

3.1. What Does Digital Transformation Mean and What Competences Have to Be Developed to Prepare New Professionals?

The concept of digital transformation is used together with other concepts-digitization and digitalization. In this context, we should look at the relation between these concepts and the competences that have to be developed to prepare new professionals according to the challenges of the 21st century. This leads us to analyze the theoretical background of these concepts, which would then form the basis of the practical solution of the research problem. In this section, we present a general overview of these concepts.

\subsubsection{Digitization and Digitalization}

Digital transformation goes beyond digitization and digitalization by including the whole organization. The lowest stage of digital transformation is digitization. A number of different definitions reviewed (studied) revealed that the concept of digitization is associated with the process of changing data into a digital form that can be easily read and processed by a computer [6]. According to Gartner's IT Glossary, digitization takes an analog process and changes it to a digital form without any different-in-kind changes to the process itself [7]. A similar description of the concept of digitization with small modifications mainly focusing on the process of changing data into a digital form has been given by [8,9]. For example, Wieberneit [8] defines digitization as the process of converting analog information into a computer-readable format with the goal of improving existing processes. 
However, other authors, for example, Bloomberg [10], consider that it is the information being digitized, not the processes, is where digitalization comes in. Digitization essentially refers to taking analog information and encoding it into zeroes and ones so that computers can store, process, and transmit such information. Accordingly, the authors of the paper also consider that the concept of digitization does not relate to processes, while digitalization refers to the process in which digital technologies and digitalized data are used to create new processes to focus on potential changes in the processes beyond the mere digitizing of existing processes and forms [11].

\subsubsection{The Concept of Digital Transformation}

In the last years in the specialized literature, policy-related papers, and reports, much attention has been paid to digital transformation. For example, Verina and Titko [12] summarized a number of publications devoted to digital transformation based on the findings of various authors' papers from the Web of Science and SCOPUS scientific databases from 1995 to 2018. The authors point out that there are plenty of definitions provided by academicians, government authorities, and business experts and more than 3000 relevant publications, the number of which is increasing every year.

The literature review shows that there are various approaches to defining the concept of digital transformation. Therefore, digital transformation has a definition problem [13]. Some authors define digital transformation by mainly focusing on the transformation of existing digital technologies to create new ones [14-17]. For instance, Duncin [15] defines the digital transformation as the process of using digital technologies to create new-or modify existing - business processes, culture, and customer experiences to meet changing business and market requirements.

In a broader sense, digital transformation is presented as the change of organizational processes. Wieberneit [8] indicated in their research that a digital transformation is an organizational transformation that covers organization, values, culture, mission, and vision, using an outside-in view. It is enabled by computer technology.

Most other authors $[9,11]$ consider that digital transformation is more relevant to individuals, not digital technology. For example, Talin [9] pointed out that it is important to understand that digital transformation is never triggered by technology; it is always about solving a problem or providing a new approach to customers. The customer-centric solution is always the start of the digital transformation, not the technology. (Do not create problems by looking first at tech and then only focusing on a solution for this technology).

In addition, other authors $[12,18,19]$ have analytically summarized essential definitions from different sources and concluded that the given definitions allow categorizing the digital transformation (DT) into three distinct elements: technological, where DT is based on the use of new digital technologies such as social media, mobile, analytics, or embedded devices; organizational, where DT requires a change of organizational processes or the creation of new business models; and social, where DT is influencing all aspects of human life. In this regard in their research, Verina and Titko [12] pointed out that digital transformation is not about the implementation of IT solutions only. However, it should be viewed in a broader context as "organizational change", "cultural transformation", and "moving toward a customer-centric approach". According to Bloomberg [10], "digitization and digitalization are essentially about technology, but the digital transformation is not. Digital transformation is about the customer". In this regard, the element "people" becomes essential and even more important than anything else. Therefore, we can conclude that the concept of digital transformation is more comprehensive than the concepts of digitization and digitalization. In order to ensure digital transformation, it requires maintaining a holistic approach to ensuring the implementation of all the above-mentioned elements, digital technologies and organizational solutions together with the human element. According to the European Commission, [20] "machines and humans can do together". Moreover, the increasing use of information technologies requires addressing the development of human capital: adequate knowledge, skills, and specific competences, without which one 
cannot fully benefit from digital transformation. It makes us seek answers to the questions of how to explain what competences and personal traits must be developed for digital transformation and prepare new professionals according to the challenges of the labor market.

\subsubsection{Human Capital Competencies and Digital Skills}

Based on the academic literature and the review of policy documents in the field of developing relevant skills and competences for digital transformation, one can find that some authors mainly focused on developing digital skills. Others emphasized the importance of digital competences and related skills in the implementation of digital transformation [21-24]. The importance of digital competencies/skills and their development is well-recognized in most European Commission policy documents. For example, the Digital Education Action Plan states that Member States should pay special attention to increasing and improving the level of digital competences across all segments of the population [25].

It is evident that for digital transformation, it is important for everyone to build up relevant competencies and digital skills that are required when using ICT and digital media because, first, during digital transformation, to be able to deal and work more effectively with the latest available technologies, one should have a high level of professionalism with advanced digital skills. This was also evidenced by the survey of residents of Latvia conducted by SKDS in 2020, in which more than 1000 respondents aged 18-75 participated. Most or $72 \%$ of respondents believed that they needed to improve their digital skills. Additionally, $80 \%$ of respondents had done something to improve their digital skills in the last year [26].

Second, it is important to develop not only skills but also digital competences, as the concept of digital competence is much broader than the concept of digital skills. Digital competence is a combination of knowledge, skills, and attitudes, including the development of soft skills such as problem solving, collaboration, and creativity. Consequently, digital skills are an important component of digital competence. Moreover, OECD [27] documents stress that the concept of digital competence must be focused on a broader approach, as in the digital economy era, ICT skills will not be enough, and other complementary skills will be needed. as Additionally, the European Commission policy documents states that "in addition to digital skills, the digital economy requires also complementary skills such as adaptability, communication and collaboration skills, problem solving, critical thinking, creativity, entrepreneurship and readiness to learn" [25] (p.13).

However, it should also be noted that during the COVID-19 pandemic, individuals are in a completely different environment, mainly working, doing daily activities, and learning remotely. Everyone who must deal with remote activities becomes like a "self-manager" person. S/he should be ready to work independently, plan his/her working time, make optimal decisions, organize his/her work, continuously acquire new knowledge, and tackle other problems and must be a high-qualified specialist in a certain field. Moreover, each individual person has to become the subject of social changes and must be able to understand the complicated processes of societal development and influence them. This means that the role of individuals in the production process, learning, studies, and daily life is significantly modified, and the focus is placed on a competent individual as the most important precondition for competitiveness. Therefore, the European Commission developed a framework of eight key competences as part of their lifelong learning strategies that have to be developed by everyone, beginning from childhood and throughout their entire life. These competences are considered equally important for life and work in a knowledge-based society [28].

Accordingly, the authors of the paper consider that the process of digital transformation will require developing the individual's key competences. It envisages implementing competence-based education together with digital education, thus integrating the elements of digital education in all study programs (courses) focused on building key competences. This will encompass a range of skills, from technical, academic, sectoral, and digital skills to 
softer skills like problem solving, creative and design thinking, communication, emotional intelligence, multicultural openness, leadership, and managerial and interaction skills [29]. It means that the digital economy will require all people to build up a range of new knowledge, abilities, and different digital skills needed not only in traditional STEM occupations and ICT professionals but also in nearly all job sectors where ICT complements existing tasks [30].

3.2. Are the Digital Transformation and the Development of Relevant Competences in Employees Successfully Carried out in All Sectors of the National Economy in Latvia?

To answer these questions, an analysis of the implementation of digital transformation in the EU and Latvia, the employers' ratings of the importance of digital transformation, the relevant competencies for employees in their organizations, and the challenges they were facing was carried out.

\subsubsection{Implementation of Digital Transformation in the EU and Latvia}

The importance of implementing the digital transformation in all areas is highlighted in multiple European Union (EU) strategy and policy documents. One of the main political priorities of the European Commission for 2019 to 2024 is to shape the EU so that it is fit for the digital age and empower its citizens with a new generation of technologies [31]. Furthermore, according to the European Commission forecasts for 2019 to 2030, one of the five key global broad trends in the EU is a revolution in technologies, and digitization transforms all aspects of society, such as politics, governance, education, science, lifestyles, collective intelligence networks, the setting-up of open systems, and health, including the transformation of the human genome. Divisions between education, work, leisure, and retirement phases will be less clear-cut than today, and training will be life-long for many [5].

An assessment of the current state of implementation of digital transformation in the EU and Latvia is based on the data from the database of the Digital Economy and Society Index (DESI, 2020). It provides a much-needed, integrated information source on Europe's overall digital performance, tracks the progress of EU countries in digital competitiveness, and is a solid decision-making basis for policy development [32].

The Digital Economy and Society Index (DESI, 2020) shows that across the EU Member States, there was impressive progress in digital transformation over the last 5 years. Finland, Sweden, Denmark, and the Netherlands have the most advanced digital economies and lead the ranking of all the EU Member States (Figure 2).

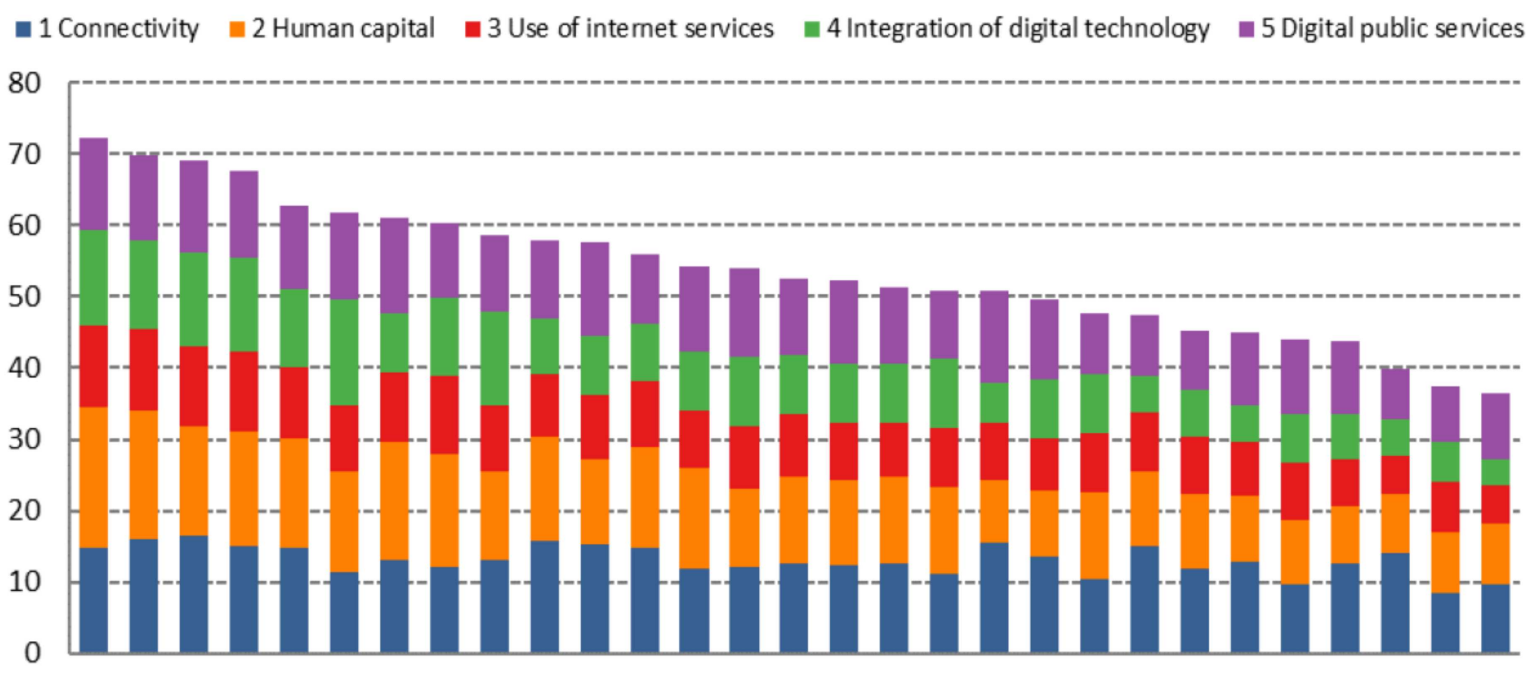

FI SE DK NL MT IE EE UK BE LU ES DE AT LT EU FR SI CZ LV PT HR HU SK PL CY IT RO EL BG

Figure 2. Rankings of EU Member States on the Digital Economy and Society Index in 2020 based on 2019 data [32]. 
Figure 2 shows that Latvia was ranked 18th among the 28 EU Member States. This position of Latvia could be influenced by the low values of indicators in the area of integration of digital technology. Bulgaria, Greece, Romania, and Italy had the lowest scores on the index.

It is important to underline that according to the rankings (DESI, 2020), large enterprises were becoming more and more digitized: $38.5 \%$ of them relied already on advanced cloud services and $32.7 \%$ were using big data analytics. The top EU performers in the digitization of businesses are Ireland, Finland, Belgium, and the Netherlands. The quality and usage of digital public services also increased: $67 \%$ of internet users who submitted forms to their public administration now use online channels (up from 57\% in 2014). The top performers in this area were Estonia, Spain, Denmark, Finland, and Latvia. Throughout the past years, there has been an improvement in Internet user skills, with 58\% of individuals having at least basic digital skills, 33\% having above-basic digital skills, and $61 \%$ of individuals having at least basic software skills [32].

Similarly, in Latvia, significant progress has also been made in the digital transformation of some areas in recent years. For example, Latvia ranked second in the world in terms of mobile Internet usage. During the last three years, the use of the Latvian Mobile Phone (LMT) network has increased three times, and during the pandemic by another third [33]. Latvia is among the top EU Member States with the main public services reachable online for citizens and businesses. According to the Digital Economy and Society Index (DESI, 2020), Latvia ranked fifth in terms of e-government performance: e-signature, electronic documents, and digital mail. The introduction of automatically partially completed forms on the service portals of public institutions has been particularly successful, reaching an index value of 86 against the EU average of 59. In this field, Latvia had the highest score (ranked fourth).

The digital transformation is being successfully carried out in the banking and insurance sector, as well as in several large enterprises. Many enterprises have been able to respond quickly and reorient their business in the changing environment by digitizing the services provided and even creating and offering new, innovative products and solutions to the market. The results of a RAIT GROUP survey "Use of Telecommunication Services by Enterprises" conducted in May-July 2020 show that there was a significant increase in the use of various digital solutions by 10 percentage points compared with the previous year. The importance of websites has grown - without them, no company can do businessand the amount of data stored remotely increased, while the domain of the company has become $12 \%$ more important. The use of Office 365 in office and corporate work as well as record keeping has tripled, while accounting software in the form of cloud services has also been actively used by small and medium-sized enterprises (SMEs) [33].

Despite the progress, the most essential problem of digital transformation and developing relevant competences pertains to the small and medium-sized business segment because, during the COVID-19 pandemic, many Latvian SMEs were not ready for change and could not adapt quickly to the changing market conditions in order to modernize their production processes and promote the integration of digital technologies. For example, the European Investment Bank EIBIS survey that gathers information on investment activities among small and medium-sized enterprises (SMEs) and larger corporations showed that they expected to use digital technologies in the long term, and Latvia's SMEs had a lower score than the EU average (22\% and $43 \%$, respectively). As for the implementation of digital technologies across sectors, Latvia lagged behind the EU averages: firms in the manufacturing sector (18\% versus 55\%) and services and infrastructure sectors (18\% versus $49 \%$ ). Only firms in the construction sector had a relatively higher score compared with the EU average (55\% versus 37\%) [32,34-36].

In this context, according to the DESI 2020, Latvia had one of the lowest scores on the index of digitization of businesses and e-commerce compared with the EU average. The leading countries were Ireland, Finland, Belgium, the Netherlands, Denmark, and Sweden, with scores greater than 55 points (out of 100). At the other end of the scale, 
Bulgaria, Romania, Hungary, Poland, Greece, and Latvia lagged behind with scores less than 35 points, significantly below the EU average of 43 points. Moreover, only $8 \%$ of Latvian enterprises used big data, $11 \%$ used cloud computing services, and $11 \%$ had web sales to customers [32].

In addition, developing human capital competencies and digital skills is a problem because no one can fully benefit from digital technologies. According to the human capital dimension of the DESI 2020, the level of digital skills has continued to grow slowly, and among Latvia's individuals, at least $41 \%$ had basic digital skills and $24 \%$ had above-basic digital skills. In terms of digital skills, Latvia ranked the lowest in both sub-dimensions of human capital, followed by Greece, Bulgaria, Romania, and Italy. However, specialists in the field of information and communication technologies made up only $1.7 \%$ of the total number of employees in Latvia. This was almost half of the EU average of 3.9\% [32].

Consequently, it has to be mentioned that the situation in the EU was very diverse. Digital transformation and developing relevant competences for employees occurred in some countries successfully, whereas in others slowly. In addition, digital solutions for Latvian enterprises and organizations are very different. The solutions were of much lower quality in the private sector and small and medium-sized enterprises than those in larger enterprises and the public sector. Therefore, in the following sections, we will describe employers' ratings of the importance of the above-mentioned issues in their organizations and the challenges they were facing and the levers executives can use to drive digital transformation in Latvia.

\subsubsection{Employers' Ratings of the Importance of Digital Transformation}

As part of the survey, the employers were asked to rate the implementation of digital transformation in their organizations on a scale from 0 to 10 ( 0 -no digitalization in their enterprises at all; 10—-the highest). The respondents' ratings are summarized in Figure 3.

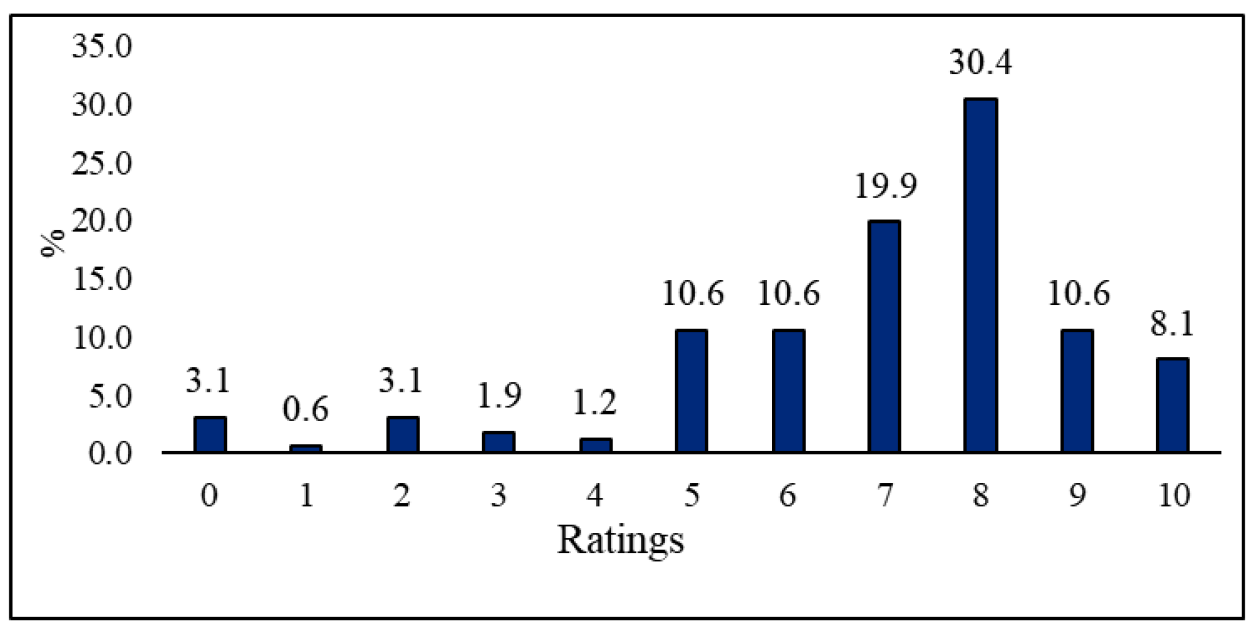

Figure 3. Percentage breakdown of the respondents' ratings of implementation of digitalization in their organizations $(n=161)$.

As shown in Figure 3, the majority of the respondents surveyed rated the implementation of digital transformation in their organizations as high or medium-high in the range from 7 to 10 . However, almost a third rated it as relatively low. Only 3.1\% indicated that there was no digitalization in their enterprises at all; however, a total of $5.6 \%$ of employers rated the implementation as unsuccessful (scale from 1-3). This could relate to SMEs, which were newly established, had relatively young leaders, and were at the early stage of digitalization.

The mean rating of implementation of digitalization by the employers was $6.9(\mathrm{SD}=2.2)$, while the most frequent rating given by the employers was 8 (Table 1). As shown by the central tendency indicators of the ratings of implementation of digitalization summarized 
in Table 1 by field of economic activity and employer age, it is not observed that in any of the fields of economic activities and employers' age groups, the average rating would be significantly higher or lower. Comparing the rating results using a Kruskal Wallis test, no statistically significant difference in the ratings of implementation of digitalization was found between the sectors of goods and services, the public sector and other fields $(p=0.149)$, and the age groups of employers $(p=0.939)$ (Table 2).

Table 1. Ratings of implementation of digitalization by field of economic activity and employer's age: statistics of central tendency.

\begin{tabular}{cccccccc}
\hline & & \multicolumn{3}{c}{ Implementation of Digitalization in Organization } \\
& & Mean & Median & Mode & Max & Min & Standard Deviation \\
\hline & Production & 6.5 & 7.0 & 8.0 & 10.0 & 0 & 2.5 \\
Field of Economic Activity & Services & 7.2 & 7.0 & 8.0 & 10.0 & 1.0 & 1.9 \\
& Public sector & 7.7 & 8.0 & 8.0 & 10,0 & 2.0 & 2,3 \\
& Other & 6.5 & 7.0 & 8.0 & 10.0 & 0 & 2.6 \\
& Total & 6.9 & 7.0 & 8.0 & 10.0 & 0 & 2.2 \\
\hline \multirow{2}{*}{ Employers Age } & Up to 30 years & 6.8 & 7.5 & 8.0 & 10.0 & 0 & 2.7 \\
& 31-50 years & 6.7 & 7.0 & 8.0 & 10.0 & 0 & 2.4 \\
& Over 50 years & 7.1 & 7.0 & 8.0 & 10.0 & 2.0 & 1.8 \\
& Total & 6.9 & 7.0 & 8.0 & 10.0 & 0 & \\
& & & & & & & 2.2 \\
\hline
\end{tabular}

Table 2. Implementation of digitalization in organization by field of economic activity: Kruskal Wallis test results.

\begin{tabular}{ccc}
\hline & Test Statistics $^{\text {a }}$ & \\
\hline & Field of Economic Activity & Employer's Age \\
\hline Kruskal Wallis H & 5.326 & 0.126 \\
df & 3 & 2 \\
Asymp. Sig. & 0.149 & 0.939 \\
\hline
\end{tabular}

a. Kruskal Wallis test.

Recent studies on the digital transformation potential of SMEs (150 SMEs surveyed) also showed that most SMEs in Latvia are at the first stage of digital transformation (digitization) [36]. SMEs still devote $64 \%$ of their activities to the provision of analog information, calling and sending letters to customers, as well as the circulation of paper documents. However, an assessment of the use of technologies by SMEs reveals that the degree of digitalization is $38 \%$. The most popular technologies are cloud computing and social media with a utilization rate of $66 \%$. The least popular was robotics with $21 \%$ [36]. The results of the present research are considerably consistent with the DESI 2020 and the European Investment Bank EIBIS survey, which showed that the implementation of digital technologies across sectors in Latvia lagged behind EU averages and had one of the lowest scores on the index of digitization of businesses and e-commerce [32,34-36].

Even though the ratings of implementation of digitalization did not differ significantly across the age group of employers, the analysis of how the employers of different ages rated the level of digitalization in their organizations shows that the employers in the age group over 50 gave much higher ratings (Figure 4). To our knowledge, this could be because a significant number of employers represented areas where the digital transformation was already being carried out successfully, such as the banking and insurance sector, as well as several large enterprises.

To identify to what extent digitalization was developed in the organizations represented by the employers, the questionnaires sent to them gave them the opportunity to rate the different levels of digitalization processes implemented. Each of the levels mentioned in the questionnaire was rated by the employers based on the number of digital processes implemented as follows: 0 -no digitalization was implemented; one to three processes 
were digitalized; three to five processes were digitalized; and more than five processes were digitalized. The employers' ratings are summarized in Table 3. An analysis of the employers' total ratings of the level of digital transformation in their organizations shows that despite the fact that about a third of enterprises (32.9\%) were only at the early stage of digitalization, implementing one to three processes, the majority of them $(63.3 \%)$ had digitalized from three processes to more than five processes. It was found that the level of digital transformation differed between the sectors of economic activity $(p=0.026)$ (Table 4). The results of the comparative analysis in Table 3 show that the digitalization of more than five processes has been implemented more by the public sector $(66.7 \%)$, while in the goods sector, it has been implemented by $21.4 \%$ of enterprises, and in the sector of services by $32.2 \%$ of enterprises.

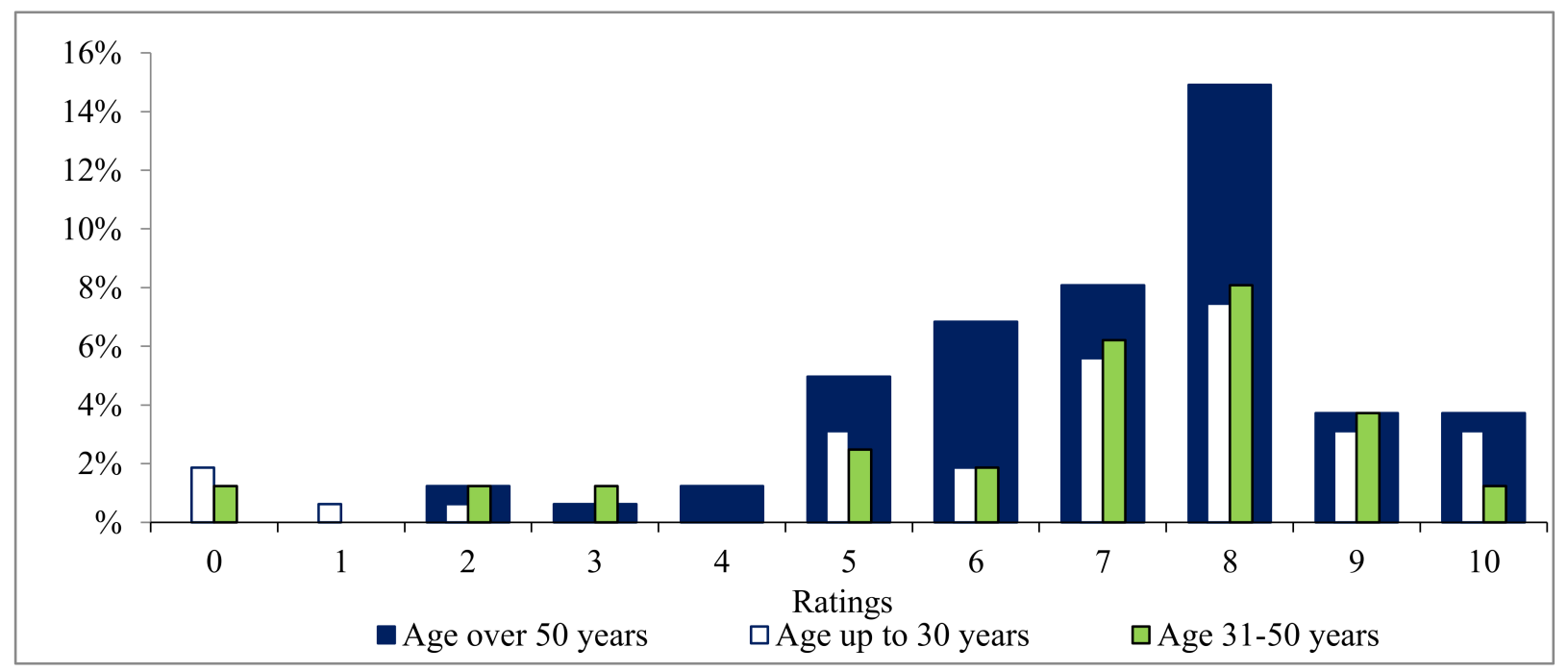

Figure 4. Percentage breakdown of the employers' ratings of implementation of digital transformation in their organizations by age. Calculated from the total percentage of the ratings $(n=161)$.

Table 3. Percentage breakdown of the employers' ratings of the level of digital transformation in their organizations by field of economic activity $(\mathrm{n}=161)$.

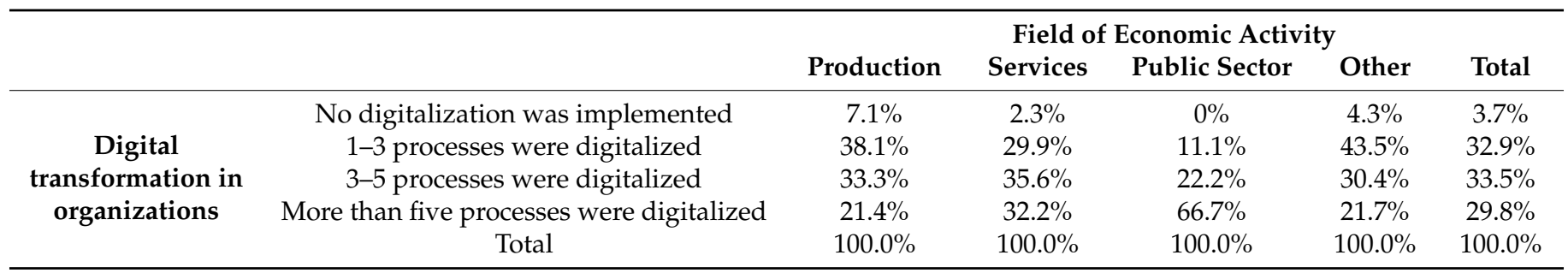

Table 4. Ratings of the level of digital transformation in organizations by field of economic activity: Kruskal Wallis test results $(\mathrm{n}=161)$.

\begin{tabular}{cc}
\hline & Test Statistics $^{\mathbf{a}, \mathbf{b}}$ \\
& Digital Transformation in Organizations \\
\hline Kruskal Wallis H & 9.237 \\
df & 3 \\
Asymp. Sig. & 0.026 \\
\hline
\end{tabular}

a. Kruskal Wallis test. ${ }^{\text {b. }}$ grouping variable: field of economic activity.

As it turned out, three to five processes and more than five processes were digitalized in the enterprises. In this respect, the employers were asked to assess whether their 
employees and enterprises were ready for digital transformation. A data analysis of the survey indicates that the employees' readiness for digitalization had a mean rating of $6.6(\mathrm{SD}=2.5)$ and the enterprises' readiness for digitalization had a mean rating of 6.4 $(\mathrm{SD}=2.8)$, which means that the employers rated the readiness from medium to high (on a scale from 0 to 10) (Table 5). Comparing the results between the sectors of economic activity and between the age groups of employers, it was revealed that there were statistically significant differences in the ratings of employees' readiness $(p=0.002)$ and enterprises readiness for digitalization $(p=0.010)$ between the sectors of economic activity (Table 6). The results of the comparative analysis summarized in Table 5 show that among the sectors of economic activity, the lowest mean rating was given by the employers from the goods sector both for the readiness of their employees $(5.3(\mathrm{SD}=3))$ and the readiness of the enterprises $(5.4(\mathrm{SD}=3.2)$ ) themselves for digitalization. An analysis of the results shows that the average rating of the public sector for the readiness of the employees was 7.7 $(\mathrm{SD}=2)$ and $8(\mathrm{SD}=2.1)$ for the readiness of the enterprises (Table 5).

Table 5. Employers' ratings of their employees' and enterprises' readiness for digital transformation by employer's age and field of economic activity: central tendency $(n=161)$.

\begin{tabular}{|c|c|c|c|c|c|c|c|c|c|}
\hline & & \multicolumn{3}{|c|}{ Age } & \multicolumn{5}{|c|}{ Field of Economic Activity } \\
\hline & & $\begin{array}{l}\text { Up to } \\
30 \text { Years }\end{array}$ & $\begin{array}{l}31-50 \\
\text { Years }\end{array}$ & $\begin{array}{c}\text { Over } 50 \\
\text { Years }\end{array}$ & Production & Services & Public Sector & Other & Total \\
\hline \multirow{6}{*}{$\begin{array}{l}\text { Employees' } \\
\text { readiness for } \\
\text { digitalization }\end{array}$} & Mean & 6.7 & 6.7 & 6.4 & 5.3 & 7.1 & 7.7 & 6.3 & 6.6 \\
\hline & Median & 7.0 & 7.0 & 7.0 & 6.0 & 7.0 & 8.0 & 7.0 & 7.0 \\
\hline & Mode & 7.0 & 7.0 & 7.0 & 7.0 & 7.0 & 8.0 & 7.0 & 7.0 \\
\hline & Max & 10.0 & 10.0 & 10.0 & 100 & 10.0 & 10.0 & 10.0 & 10.0 \\
\hline & Min & 0 & 0 & 0 & 0 & 0 & 3.0 & 0 & 0 \\
\hline & Standard Deviation & 2.7 & 2.3 & 2.5 & 3.0 & 2.0 & 2.0 & 3.0 & 2.5 \\
\hline \multirow{6}{*}{$\begin{array}{l}\text { Enterprises' } \\
\text { readiness for } \\
\text { digitalization }\end{array}$} & Mean & 6.7 & 5.8 & 6.5 & 5.4 & 6.7 & 8.0 & 6.3 & 6.4 \\
\hline & Median & 7.5 & 7.0 & 7.0 & 6.0 & 7.0 & 8.0 & 8.0 & 7.0 \\
\hline & Mode & 7.0 & 70 & 7.0 & 7.0 & 7.0 & 8.0 & 8.0 & 7.0 \\
\hline & $\operatorname{Max}$ & 10.0 & 10.0 & 10.0 & 10.0 & 10.0 & 10.0 & 10.0 & 10.0 \\
\hline & Min & 0 & 0 & 0 & 0 & 0 & 3.0 & 0 & 0 \\
\hline & Standard Deviation & 3.2 & 3.0 & 2.5 & 3.2 & 2.5 & 2.1 & 3.4 & 2.8 \\
\hline
\end{tabular}

Table 6. Employers' ratings of their employees' and enterprises' readiness for digital transformation by employers age and field of economic activity: Kruskal Wallis test results $(n=161)$.

\begin{tabular}{ccccc}
\hline & & \multicolumn{2}{c}{ Test Statistics a } & \multicolumn{2}{c}{ Field of Economic Activity } \\
\hline & Employees' readiness & Enterprises' readiness & Employees' readiness & Enterprises' readiness \\
for digitalization & for digitalization & for digitalization & 1.56 \\
for digitalization & 1.328 \\
Kruskal Wallis H & 1.106 & 4.204 & 3 & 3 \\
df & 2 & 2 & 0.002 & 0.010 \\
\hline
\end{tabular}

a. Kruskal Wallis test.

Based on the analysis of the surveys of employers' ratings of the importance of digital transformation, we can conclude that the majority of the employers surveyed rated the implementation of digital transformation as medium-high and that from three to five processes and more than five processes were digitalized in their organizations as well as that their employees and enterprises were ready for digital transformation in general. This is a positive trend, which shows that digitalization processes continue to develop, the majority of organizations are ready for changes in the field of digitalization, and one can hope for positive changes in the future. However, about a third of enterprises are only at the early stage of digitalization, while some have not yet begun implementing it.

\subsubsection{Employers' Ratings of the Importance of Relevant Competencies for Employees}

As mentioned above, the digital transformation and the impact of the COVID-19 pandemic on businesses and on the lives of citizens significantly modifies the role of 
individuals. Under these conditions, the development of human capital might be explained in a much broader sense as the development of the individual's key competences, including digital competence/skills. It is built through a process of acquiring knowledge, skills, and experience.

To our knowledge, the development of human capital could be viewed in two senses: in a broader sense, it is oriented toward developing a certain set of personal traits and soft skills, which are important in any field of activity and in life, without directly associating it with how to use ICT, while in a narrow sense, it involves developing specific skills and acquiring knowledge of and experience in how to use ICT. Within this context, the answers of the respondents to the open question about how they rate the importance of relevant competencies for employees associated with digital transformation are also grouped in Table 7. on the one hand as specific skills, while on the other hand as soft skills.

Table 7. Employers' ratings of the importance of relevant competencies for employees associated with digital transformation.

\begin{tabular}{|c|c|}
\hline \multicolumn{2}{|r|}{ Comments } \\
\hline Specific skills & $\begin{array}{c}\text { Ability to work with technology; knowledgeable, competent } \\
\text { Programmer with good communication skills } \\
\text { Ability to achieve better results with fewer resourcess } \\
\text { Loyal, trustworthy } \\
\text { Accuracy at work, honesty } \\
\text { Competent in the field } \\
\text { Ability to speak several languages; a good partner, competent in the industry } \\
\text { Ability to improve the e-environment }\end{array}$ \\
\hline $\begin{array}{l}\text { Personal traits and } \\
\text { soft skills }\end{array}$ & $\begin{array}{l}\text { Ability to work in a team } \\
\text { Innovative; ability to make decisions, ability to co-operate with others } \\
\text { Ability to listen and help with solving problems } \\
\text { Rich in initiative, creative, ready for change, ability to adapt to new conditions and technologies, focused on } \\
\text { cooperation } \\
\text { Willingness to work, good communication and organizational skills } \\
\text { Knowledgeable and willing to develop and grow } \\
\text { Supportive, enthusiastic, rich in ideas } \\
\text { Flexible, self-motivated } \\
\text { Willing and able to work independently and effectively, motivated } \\
\text { Communicative with new ideas, with a desire to implement them, good communication skills } \\
\text { Wish to keep up with the times, learn and apply the acquired knowledge in practice } \\
\text { Ability to control emotions, act rationally in a stressful situation, take responsibility, think outside the box }\end{array}$ \\
\hline
\end{tabular}

As shown in the table, the respondents indicated in their comments that, along with the development of specific digital skills, it is essential that individuals also have personal talents, traits, and abilities such as the ability to cooperate with others, work in a team and have tolerance, start new activities, show initiative and enthusiasm, put forward real aims and try to achieve them, and act creatively, as well as other personal qualities. Similar answers were given to the question of what the most important problems in their organization with regard to digital transformation are. The employers stressed the need to develop specific digital skills such as the ability to work with digital devices, be competent in the latest technologies, and work in difficult conditions, as well as soft skills. The employers' replies are summarized on a scale from 1 to 10 (1-the least important; 10 -the most important) in Figure 5.

The analysis of the survey data revealed that these problems in organizations with regard to digital transformation did not differ significantly between the sectors of economic activity $(p>0.05)$ and between the ages of employers $(p>0.05)$ (Table 8$)$. 


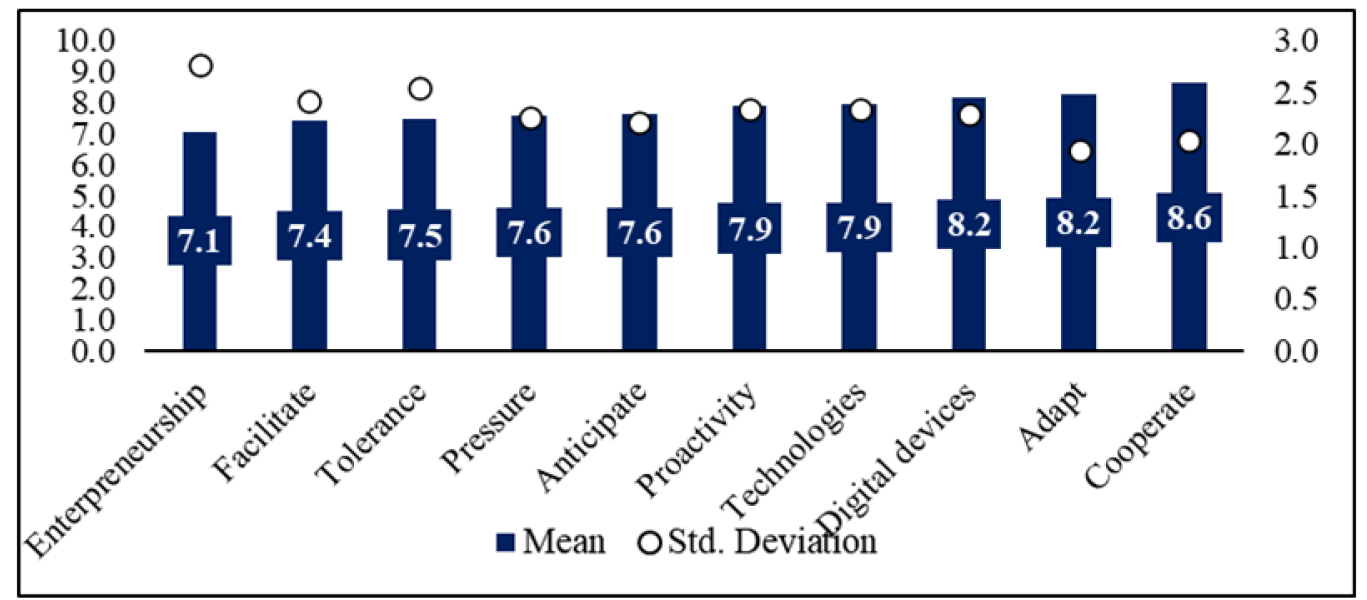

Figure 5. Employers' ratings (on a scale of 1 to 10) of the most important problems associated with digital transformation.

Table 8. Employers' ratings of the most important problems associated with digital transformation by field of economic activity and employers' age: Kruskal Wallis test results.

Test Statistics ${ }^{a}$

Field of Economic Activity

\begin{tabular}{|c|c|c|c|c|c|c|}
\hline & & \\
\hline & Kruskal-Wallis H & $\mathrm{df}$ & Asymp. Sig. & Kruskal-Wallis H & $\mathrm{df}$ & Asymp. Sig. \\
\hline Digital devices & 4952 & 3 & 0.175 & 0.146 & 2 & 0.930 \\
\hline Technologies & 0.734 & 3 & 0.865 & 0.555 & 2 & 0.758 \\
\hline Pressure & 0.327 & 3 & 0.955 & 0.482 & 2 & 0.786 \\
\hline Adapt & 3.215 & 3 & 0.360 & 0.048 & 2 & 0.976 \\
\hline Cooperate & 3.476 & 3 & 0.324 & 1.866 & 2 & 0.393 \\
\hline Facilitate & 3.015 & 3 & 0.389 & 1.292 & 2 & 0.524 \\
\hline Anticipate & 5.183 & 3 & 0.159 & 5.114 & 2 & 0.078 \\
\hline Proactivity & 0.584 & 3 & 0.900 & 0.917 & 2 & 0.632 \\
\hline Entrepreneurship & 2.681 & 3 & 0.444 & 1.458 & 2 & 0.482 \\
\hline Tolerance & 0.313 & 3 & 0.958 & 5.402 & 2 & 0.067 \\
\hline
\end{tabular}

a. Kruskal Wallis test.

As shown in Figure 5, the highest rating of $8.6(\mathrm{SD}=2.0)$ showed that the employers believed that the greatest challenge was to develop employees' abilities to communicate with others, as well as to work with digital devices and adapt (8.2, respectively) to digital transformation. The fact that the employers considered the development of employees' soft skills to be one of the most important problems, to our knowledge, related to the need for a faster transition to remote work. It does not require a permanent presence in the enterprise or institution. Job functions can also be performed from home via the Internet. Under the new circumstances, job duties are increasingly performed based on self-organization at the workplace, the ability to work independently and simultaneously coordinate one's actions with other partners of a united team, and to choose an optimal solution in multi-variant situations. This, in turn, requires the activation of abilities and skills such as planning, making decisions, communication, the desire to take up responsibility, independence, and the ability to see (find) a certain problem and solve it.

\section{Discussion and Future Research Recommendations}

The literature review shows that there are various approaches to defining the concept of digital transformation. Some authors define digital transformation by mainly focusing on the transformation of existing digital technologies to create new [14-17]. Other authors $[9,11]$ consider that digital transformation is more relevant to individuals, not digital technology. 
Our position on the main elements of digital transformation identified by various authors could be supported in general. At the same time, the authors consider that the elements of digital transformation they developed are more authentic and more practical $[12,18,19]$. It has been argued that the concept of digital transformation is more comprehensive than the concepts of digitization and digitalization. Digital transformation (DT) includes three main elements: technological, organizational, and social. In order to ensure digital transformation, it requires maintaining the holistic approach to ensuring the implementation of all the above-mentioned elements-digital technologies and organizational solutions together with the human element. The authors consider that a mentioned approach to the concept of digital transformation is essential for the practical solution of the research problem and is a great opportunity for sustainable economic development.

Today, individuals are in a completely different environment, mainly working, doing daily activities, and learning remotely. This means that the role of individuals in the production process, learning, studies, and daily life is significantly modified, and the focus is placed on a competent individual and the development of human capital: adequate knowledge, skills, and specific competences, without which one cannot fully benefit from digital transformation. Moreover, the authors of the paper consider that the process of digital transformation will require developing not only digital skills or digital competences $[21,24]$ but also the individual's key competences. In order to develop key competences, personal specific skills, and qualities in an individual, it is necessary to provide the individual with an opportunity to acquire new knowledge and relevant competencies to be able to live and work in the new digital environment. In this respect, an analysis of the views of the respondents on the open question "What additional competencies do employees need to be ready for the digital age?" shows that it is important for their employees to be trained in the use of specific equipment and technologies, be able to understand and work with the latest technologies, acquire IT knowledge, and be ready for continuous learning and development, as well as learn several foreign languages, thus expanding their personal fields of competences. At the same time, according to a survey of residents of Latvia conducted by SKDS, businesspersons and entrepreneurs did not take sufficient care of the further education of their employees, as the majority or $62 \%$ of respondents had independently tried to acquire knowledge and improve their skills in working with technologies during the last year. Only $17 \%$ of respondents indicated that training opportunities were provided by their employers [33]. This is not a positive trend, and as mentioned earlier, the main problem in Latvia with regard to the implementation of digital transformation was a lack of qualified specialists and the fact that a large segment of the population lacks even basic digital skills. This is indicative of the growing role of employers and education to focus on developing human capital as well as competencies and digital skills because, without them, one cannot implement the digital transformation to gain economic, social, environmental, and consumer benefits [37,38] and to ensure sustainable economic development [39].

\section{Conclusions}

\subsection{Contributions to Theory}

From a theoretical point of view, this research adds a new baseline to the specialized literature on the possibilities of implementation of digital transformation, which is closely linked to the development of human capital competencies and digital skills with a focus on sustainability.

The research findings show that the concept of digital transformation (DT) is more comprehensive than the concepts of digitization and digitalization. DT includes three main elements: technological, where DT is based on the use of new digital technologies such as social media, mobile, analytics, or embedded devices; organizational, where DT requires a change of organizational processes or the creation of new business models; and social, where DT is influencing all aspects of human life. In order to ensure digital transformation, it requires maintaining the holistic approach to ensuring the implementation of all the above-mentioned elements. 


\subsection{Contributions to Practice}

From a practical point of view, this is a specific research study that expands and provides insights into the situation in Latvia: employers can find in the results of this analysis support for the setting of strategies for the implementation of digital transformation and guidance for public authorities when defining regulatory policies for DT, as well as for educators to implement competence-based education together with digital education, thus integrating the elements of digital education in all study programs (courses) focused on developing the individual's key competences.

According to the DESI 2020, there is impressive progress in digital transformation across the EU Member States. Finland, Sweden, Denmark, and the Netherlands have the most advanced digital economies and lead the ranking of all the EU Member States. However, progress in the implementation of digital transformation in the EU and Latvia was very diverse. Some Member States were successful, while the others were not. In Latvia, too, digital solutions for enterprises and organizations were very different. Latvia is among the top EU Member States with the main public services reachable online for citizens and businesses. The digital transformation is being successfully carried out in the banking and insurance sector, as well as in several large enterprises. At the same time, in terms of the use of digital technologies, Latvia's SMEs had a lower score than the EU average. In addition, the problem is the development of human capital competencies and digital skills.

The analysis of the surveys of employers' ratings of the importance of digital transformation in Latvia allows us to conclude that the majority of the respondents surveyed rated the implementation of digital transformation in their organizations as medium-high, and in their organizations from three to five processes and more than five processes were digitalized; moreover, this was most specific to the public sector. This is a good trend, which shows that the digitalization process continues to progress, the majority of them are ready for change in the field of digitalization, and one can hope for positive changes in the future. However, about a third of enterprises are only at the early stage of digitalization, while some have not yet begun it, and the lowest ratings of the readiness of employees and enterprises for digitalization were found in the goods sector. This is particularly true of the private sector's small and medium-sized enterprises.

The research findings show that one of the most significant problems associated with digital transformation is human capital and the importance for employees to acquire relevant competencies and digital skills. Along with the development of specific digital skills, it is essential that individuals also build up soft skills such as the ability to communicate with others, work in a team and have tolerance, be able to start new activities, show initiative and enthusiasm, put forward real aims and try to achieve them, and act creatively, as well as other personal qualities. At the same time, according to a survey of residents of Latvia, businesspersons and entrepreneurs did not take sufficient care of the further education of their employees. This is indicative of the growing role of employers and education in focusing on developing human capital as well as competencies and digital skills because, without them, one cannot implement the digital transformation in all areas.

\subsection{Limitations}

Because in the last years in the specialized literature, much attention has been paid to explaining the concept of digital transformation, there are plenty of definitions provided by various authors, yet the present research is limited to and focuses on the general overview of the concepts and on an analysis of the implementation of digital transformation and the development of appropriate competences in employees. These limitations may be resolved through further research.

\subsection{Future Research Recommendations}

As regards the implementation of digital transformation in Latvia, in the future, it is advisable to conduct in-depth research on (1) the possibilities of implementing digital 
transformation in the private sector's small and medium-sized enterprises, adapting to remote work and developing employers' competencies and digital skills; (2) how to provide the necessary infrastructure associated with digital transformation in order to be able to implement remote work in the long term; and (3) how investments made in the development of human capital competencies and digital skills facilitate digital transformation.

Author Contributions: Conceptualization V.B. and B.R.; methodology I.L.-E.; software P.R.; validation, P.R.; formal analysis P.R.; investigation B.R.; resources B.R.; data curation B.R.; writing-original draft preparation V.B. and B.R.; writing-review and editing, V.B. and B.R.; visualization I.L.-E.; supervision B.R.; project administration B.R.; funding acquisition B.R. All authors have read and agreed to the published version of the manuscript.

Funding: This research was funded by Latvia Ministry of Education and Science, State research programme INTERFRAME-LV, grant number VPP-IZM-2018/1-0005.

Institutional Review Board Statement: Not applicable.

Informed Consent Statement: Not applicable.

Acknowledgments: The research was supported by the National Research Programme "Latvian Heritage and Future Challenges for the Sustainability of the State", project "Challenges for the Latvian State and Society and the Solutions in International Context (INTERFRAME-LV)".

Conflicts of Interest: The authors declare no conflict of interest.

\section{References}

1. Guillermo, B.; Roberto, F.J.; Rishabh, S.; Michael, M.; Rekha, S.A. Going Viral: COVID-19 and the Accelerated Transformation of Jobs in Latin America and the Caribbean. World Bank Group: Washington, DC, USA, 2020; p. 111.

2. Central Statistical Bureau of Latvia. Available online: http:/ / data1.csb.gov.lv/ (accessed on 18 February 2021).

3. Digitālā Latvija-Ārkārtējās Situācijas Laika Izaicinājumi un Iespējas (Digital Latvia: Challenges and Opportunities during the Emergency Situation). Available online: https://www.pkc.gov.lv/ (accessed on 18 January 2021).

4. Lugtu, R. Dual Transformation: Now Is the Right Time. 2020. Available online: https:/ /www.institutefordigitaltransformation. org/dual-transformation-now-is-the-right-time/ (accessed on 20 February 2021).

5. Global Trends to 2030: Can the EU meet the challenges ahead? An Inter-Institutional EU Project with the Participation of the European Parliament, the Council of the European Union, the European Commission and the European External Action Service. European Strategy and Policy Analysis System. 2015. Available online: https:/ / espas.secure.europarl.europa.eu/orbis/ sites/default/files/generated/document/en/espas\%20report\%202015.pdf (accessed on 18 January 2021).

6. Oxford Learner's Dictionary. Available online: https://www.oxfordlearnersdictionaries.com/ (accessed on 25 November 2020).

7. Gartner's IT Glossary. Available online: https:/ /www.gartner.com/en/glossary (accessed on 25 November 2020).

8. Wieberneit, T. Digitization, Digitalization, Digital Transformation-A Stake in the Ground. 2021. Available online: https: / / customerthink.com/digitalization-digital-transformation-a-stake-in-the-ground/ (accessed on 14 March 2021).

9. Talin, B. Digitalization vs. Digital Transformation-What's the Difference? 2021. Available online: https://morethandigital.info/ en/digitalization-vs-digital-transformation-whats-the-difference/ (accessed on 14 March 2021).

10. Bloomberg, J. Digitization, Digitalization, and Digital Transformation: Confuse Them at Your Peril. 2018. Available online: https:/ / www.forbes.com/sites/jasonbloomberg/2018/04/29/digitization-digitalization-and-digital-transformation-confusethem-at-your-peril/ (accessed on 15 October 2020).

11. Mergel, I.; Edelmann, N.; Haug, N. Defining digital transformation: Results from expert interviews. Gov. Inf. Q. 2019, 36, 1-16. [CrossRef]

12. Verina, N.; Titko, J. Digital transformation: Conceptual framework. In Proceedings of the International Scientific Conference Contemporary Issues in Business, Management and Economics Engineering, Vilnius, Lithuania, 9-10 May 2019 ; pp. 719-727.

13. Atkinson, R. IT Excellence and Digital Transformation. 2020. Available online: https:/ /www.institutefordigitaltransformation. org/it-excellence-and-digital-transformation/ (accessed on 14 March 2021).

14. Westerman, G.; Calméjane, C.; Bonnet, D.; Ferraris, P.; McAfee, A. Digital transformation: A roadmap for billion-dollar organizations. MIT Cent. Digit. Bus. Capgemini Consult. 2011, 1, 1-68.

15. Duncan, A. Why Is Digital Transformation So Hard in China? 2020. Available online: https://www.campaignasia.com/article/ why-is-digital-transformation-so-hard-in-china/465877 (accessed on 17 January 2021).

16. Digital Transformation. What It Is and Why It Matters. Available online: www.graycelltech.com/digital-transformation-what-itis-and-why-it-matters / (accessed on 16 January 2021).

17. The Enterprisers Project. A Community Helping CIOs and IT Leaders Solve Problems. Available online: https:// enterprisersproject.com/what-is-digital-transformation (accessed on 18 December 2020). 
18. Reis, J.; Amorim, M.; Melão, N.; Matos, P. Digital transformation: A literature review and guidelines for future research. In Trends and Advances in Information Systems and Technologies; Rocha, Á., Adeli, H., Reis, L.P., Costanzo, S., Eds.; Springer: Cham, Switzerland; Berlin/Heidelberg, Germany, 2018; pp. 411-421. [CrossRef]

19. Jurgielewicz, K. Digital Transformation Theoretical Backgrounds of Digital Change. Available online: https://www.academia. edu/40717849/Digital_transpormation_teoretical_backgraund (accessed on 12 December 2020).

20. Shaping Digital Transformation in Europe; Final Report; A Study Prepared for the European Commission DG Communications Networks, Content and Technology. European Commission. 2020. Available online: https://www.ospi.es/export/sites/ospi/ documents/documentos/Sstudy_Shaping_the_digital_transformation_in_Europe_Final_report_202009.pdf (accessed on 16 December 2020).

21. Gallardo-Echenique, E.E.; de Oliveira, J.M.; Marqués-Molias, L.; Esteve-Mon, F. Digital competence in the knowledge society. MERLOT J. Online Learn. Teach. 2015, 11, 1-16.

22. Justenhoven, R. A Digital Competency Framework for the New Digital Workforce. 2018. Available online: https://insights. humancapital.aon.com/talent-assessment-blog/a-digital-competency-framework-for-the-new-digital-workforce (accessed on 18 January 2021).

23. Ilomäki, L.; Kantosalo, A.; Lakkala, M. What is digital competence? In Linked Portal; European Schoolnet: Brussels, Belgium, 2011. Available online: http:/ /linked.eun.org/web/guest/in-depth3 (accessed on 10 February 2021).

24. Ilomäki, L.; Paavola, S.; Lakkala, M.; Kantosalo, A. Digital competence-An emergent boundary concept for policy and educational research. Educ. Inf. Technol. 2016, 21, 655-679. [CrossRef]

25. Digital Education Action Plan 2021-2027; Resetting Education and Training for the Digital Age; Communication from the Commission to the European Parliament, the Council, the European Economic and Social Committee and the Committee of the Regions. European Commission. COM (2020) 624 Final. Available online: https://eur-lex.europa.eu/legal-content/EN/TXT/ PDF/?uri=CELEX:52020DC0624 (accessed on 15 February 2021).

26. Accenture un SKDS Aptauja (Accenture and SKDS Survey). 2020. Available online: https://forbesbaltics.com/lv/zinas/raksts/ accenture-digitalas-prasmes (accessed on 21 February 2021).

27. Skills for a Digital World. 2016. Available online: http://www.oecd.org/els/emp/Skills-for-a-Digital-World.pdf (accessed on 9 November 2020).

28. European Commission. Recommendation of the European Parliament and of the Council of 18 December 2006 on key competences for lifelong learning. Off. J. Eur. Union 2006, 30, 10-18.

29. European Commission. Digital Transformation Soreboard 2018: EU Businesses Go Digital: Opportunities, Outcomes and Uptake; Publications Office of the European Union: Luxembourg, 2018. [CrossRef]

30. Carnevale, A.P.; Smith, N.; Melton, M. STEM: Science Technology Engineering Mathematics. 2011. Available online: https: / / files.eric.ed.gov/fulltext/ED525297.pdf (accessed on 24 June 2020).

31. Digital Markets Act. Impact Assessment Support Study Executive Summary and Synthesis Report; Directorate-General for Communications Networks, Content and Technology; Publications Office of the European Union: Luxembourg, 2020. [CrossRef]

32. European Commission. Digital Economy and Society Index DESI 2020; Thematic Chapters; Final Report; European Union. 2021. Available online: https: / digital-strategy.ec.europa.eu/en/library/digital-economy-and-society-index-desi-2020 (accessed on 13 April 2021).

33. Binde, J. 21 Gadsimta Darvinisms: Digitalizējies vai Mirsti (21st Century Darwinism: Digitalize or Die). 2020. Available online: https://ir.lv (accessed on 3 April 2021).

34. European Investment Bank. Country overview: Latvia. In EIB Group Survey on Investment and Investment Finance 2020; European Investment Bank: Luxembourg, 2020. Available online: https://www.eib.org/attachments/efs/eibis_2020_latvia_en.pdf (accessed on 5 February 2021).

35. European Union Overview. In EIB Group Survey on Investment and Investment Finance 2020; European Investment Bank: Luxembourg, 2020. Available online: https:/ / www.eib.org/attachments/efs/eibis_2020_european_union_en.pdf (accessed on 5 February 2021).

36. Rupeika-Apoga, R. MVU Digitālo Transformāciju Bremzē Finansējuma Trūkums. (Digital Transformation of SMEs Is Hindered by a Lack of Funds). 2021. Available online: https:/ /www.delfi.lv/bizness/versijas/ (accessed on 18 March 2021).

37. D'Adamo, I.; Gonzalez-Sanchez, R.; Medina-Salgado, M.S.; Settembre-Blundo, D. E-commerce calls for cyber-security and sustainability: How European citizens look for a trusted online environment. Sustainability 2021, 13, 6752. [CrossRef]

38. Klumpp, M.; Loske, D. Sustainability and resilience revisited: Impact of information technology disruptions on empirical retail logistics efficiency. Sustainability 2021, 13, 5650. [CrossRef]

39. D'Adamo, I.; Rosa, P. How do you see infrastructure? Green energy to provide economic growth after COVID-19. Sustainability 2020, 12, 4738. [CrossRef] 



\title{
Willingness and Influencing Factors of Pig Farmers to Adopt Internet of Things Technology in Food Traceability
}

\author{
Ruiyu Sun ${ }^{1}$, Siyao Zhang ${ }^{1}$, Tianyu Wang ${ }^{1}$, Jiarui Hu ${ }^{1}$, Junhu Ruan ${ }^{1}{ }^{\circledR}$ and Junyong Ruan ${ }^{2, *}$ \\ 1 College of Economics and Management, Northwest A\&F University, Xianyang 712100, China; \\ sunruiyu@nwafu.edu.cn (R.S.); jiayouzsy@nwafu.edu.cn (S.Z.); wty@nwsuaf.edu.cn (T.W.); \\ hjrljgxy@nwafu.edu.cn (J.H.); rjh@nwsuaf.edu.cn (J.R.) \\ 2 Medical Engineering Department, Qingdao Special Servicemen Recuperation Center of PLA Navy, \\ Qingdao 266071, China \\ * Correspondence: ruanjunyongqdy1@163.com
}

check for updates

Citation: Sun, R.; Zhang, S.; Wang, T.; $\mathrm{Hu}, \mathrm{J}$.; Ruan, J.; Ruan, J. Willingness and Influencing Factors of Pig Farmers to Adopt Internet of Things Technology in Food Traceability. Sustainability 2021, 13, 8861. https:// doi.org/10.3390/su13168861

Academic Editor: Idiano D'Adamo

Received: 1 July 2021

Accepted: 5 August 2021

Published: 8 August 2021

Publisher's Note: MDPI stays neutral with regard to jurisdictional claims in published maps and institutional affiliations.

Copyright: (c) 2021 by the authors. Licensee MDPI, Basel, Switzerland. This article is an open access article distributed under the terms and conditions of the Creative Commons Attribution (CC BY) license (https:// creativecommons.org/licenses/by/ $4.0 /)$.

\begin{abstract}
The Internet of Things technology (IoT) in food traceability provides new ideas to solve the problem of smart production and offers new ideas for the formation of safe and high-quality markets for meat products. However, scholars have studied the combination of blockchain and IoT technology. There is a lack of research on the combination of IoT and food traceability technology. Moreover, previous studies focused on the application of IoT traceability technology, taking farmers' adoption willingness as an exogenous variable while ignoring its endogeneity. Therefore, it is essential to study farmers' willingness to adopt IoT traceability technology and find the factors that influence farmers' adoption intention. Based on survey data from 264 pig farmers in Shaanxi Province, this paper discussed the factors which influence pig farmers' adoption of the technology by using the Unified Theory of Acceptance and Use of Technology (UTAUT). The results showed that farmers' adoption intention was influenced by a combination of farmers' performance expectancy, effort expectancy, social influence, personal innovation, and perceived risk. Personal innovation played a mediating role in effort expectancy and adoption willingness and perceived risk played a moderating role in personal innovation and adoption willingness.
\end{abstract}

Keywords: pig farmers; adoption willingness of IoT traceability technology; Unified Theory of Acceptance and Use of Technology; Latent Moderate Structural Equations

\section{Introduction}

With the development of the economy, people's quality of life is constantly improving, and the proportion of meat food in people's daily dietary needs is increasingly high. At the same time, the transmission of COVID-19 has created unquantifiable damage. The economy has been destroyed by this virus and immediate action is required. D'Adamo et al. [1] pointed out that the availability of infrastructure was necessary to generate economic growth and social opportunities without compromising environmental protection. Moreover, infrastructure could influence, directly or indirectly, about $72 \%$ of the targets in terms of the Sustainable Development Goals [2]. Following this approach, D'Adamo et al. [1] suggested that favoring digitalization could be implemented in order to improve our lives. Therefore, the application of IoT traceability technology in food as a digital infrastructure should be emphasized.

Traceability is the ability to follow the movement of food products throughout food supply chains [3]. When people find that there are quality or safety issues in food, they can locate the problem and in turn the cause based on the product traceability system. The IoT traceability technology refers to the technology that realizes the function of food traceability through the Internet of Things. Through the use of the Internet of Things and various sensors, such as the global positioning system (GPS), geographic information system (GIS), near-field communication (NFC), radio frequency identification (RFID) and 
temperature and humidity sensors, monitoring and information capturing can be improved in various processes, such as production, processing, storage, distribution, and retail [4]. However, due to the cost of applying IoT traceability technology, farmers' perception, technology acceptance and production privacy, farmers' willingness to adopt IoT traceability technology is different. Therefore, it is of great practical significance to analyze farmers' willingness and influencing factors to adopt IoT traceability technology and identify the key influencing factors for solving the food safety problems facing China and connecting farmers to the modernized large market.

China has a huge pork market and is the largest pork producer in the world. Affected by the African swine fever epidemic, the proportion of pork in the total meat market has dropped sharply. However, based on past consumption habits, there is still more space for callback in the pork market in the future [5]. The application of IoT technology in pig farming can reduce labor costs and improve production efficiency, which is of great importance in large-scale pig production [6]. Therefore, this study selected pig farmers as the subjects to illustrate the influencing factors of pig farmers' adoption willingness of IoT traceability technology, and accordingly proposes policy recommendations, which are important for further promoting the application of IoT traceability technology in pig farming.

Existing research on IoT traceability technologies is mainly characterized by the following.

Firstly, most scholars have studied blockchain, IoT technology, and the combination of blockchain and traceability technology. For example, Reyna et al. [7] pointed out that blockchain could enrich the IoT by providing a trusted sharing service, where information was reliable and could be traceable. Data sources could be identified at any time and data remained immutable over time, which increased its security. Therefore, the use of blockchain could complement the IoT with reliable and secure information. It is considered that in future research, the block structure should be studied to improve data retrieval efficiency by combining the characteristics of IoT engineering. Previous studies explored the methods of using blockchain for traceability system construction in various daily food and dual-use foods, and explored the deeper promotion role of block chain technology in food traceability system construction. Furthermore, Kamilaris et al. [8] proposed that blockchain was a promising technology towards a transparent supply chain of food, but many barriers and challenges still existed, which hindered its wider popularity among farmers and food supply systems. The challenges involved accessibility, governance, technical aspects, policies, and regulatory frameworks.

Secondly, existing studies focused on the application of IoT traceability technology, taking farmers' adoption willingness as an exogenous variable while ignoring its endogeneity. For example, Ma Peng [9] proposed several methods and measures for the construction of the traceability system of plateau summer vegetables based on IoT technology by combining the current situation of planting and sales of a variety of summer vegetable agricultural products enterprises, such as agricultural cooperatives and plateau summer vegetable sales enterprises in Yuzhong County, Lanzhou City. Moreover, based on a practical application case, which was a city's Food and Drug Administration using quick response code ( $Q R$ code), integrated circuit card (IC card), and traceability code as the carrier to collect and record the traceability information of each link for the construction of the city's food safety traceability system, and realized the complete information traceability of food in production, circulation, storage, and consumption links, researchers proposed that with the support of information technology such as cloud computing, big data and mobile Internet, the core of improving supervision efficiency, and the implementation of the main responsibility of food safety of production operators as the landing point, the government should take the lead and enterprises should be responsible for establishing a scientific, complete, and efficient food safety traceability system in order to fully protect food safety for the general public.

In summary, through the collation of existing studies, it was found that scholars have made great academic achievements in the study of IoT traceability technology, which has 
important theoretical reference value for this study. However, there is still a need for improvement in at least the following aspects. Firstly, as the previous literature mainly studied the combination of IoT and blockchain, there is a lack of research on applying IoT to food traceability. Therefore, it is necessary to study the combination of IoT and traceability technology, especially food traceability technology. Secondly, most of the literature on IoT traceability technology ignored farmers' adoption intention which is an important endogenous variable, so we should emphasize the influence of farmers' adoption willingness of IoT traceability technology in promotion and application of this technology. Furthermore, Jurgilevich et al. [10] summarized that the European Union Commission has identified three main stages of the food system with reference to the circular economy: production, consumption, and waste. The research of farmers' willingness to adopt the technology is to ensure the circularity of food system in the production stage. As mentioned above, this paper takes Shaanxi Province as an example and explores the factors influencing pig farmers' willingness to adopt IoT traceability technology from the microscopic perspective of pig farmers based on the innovative Unified Theory of Acceptance and Use of Technology (UTAUT) model, and provides a theoretical basis for the formulation of relevant policies to increase the popularity of IoT traceability technology in rural areas.

\section{Theoretical Models and Research Hypotheses}

\subsection{Theoretical Model}

The Technology Acceptance Model (TAM) was mostly used in previous studies. The TAM model was proposed by Davis et al. (1989) based on the Theory of Reasoned Action (TRA model) with reference to self-efficacy theory, input-output theory, and other related theories. The TAM model was mainly used to predict and explain users' perceived acceptance of a new information system after using it for a period of time, the purpose of which was to find out the reasons why people accepted or rejected new information systems. The TAM model assumes that the actual usage behavior of users for a specific information system in an organization is determined by their usage intention, which is determined by both users' usage attitude and perceived usefulness, while users' usage attitude will be determined by both users' perceived usefulness and perceived ease of use [11]. Finally, users' perceived usefulness and perceived ease of use are influenced by external factors. The external factors are composed of system characteristics, user characteristics, organizational characteristics, and other factors. The specific model is shown in Figure 1.

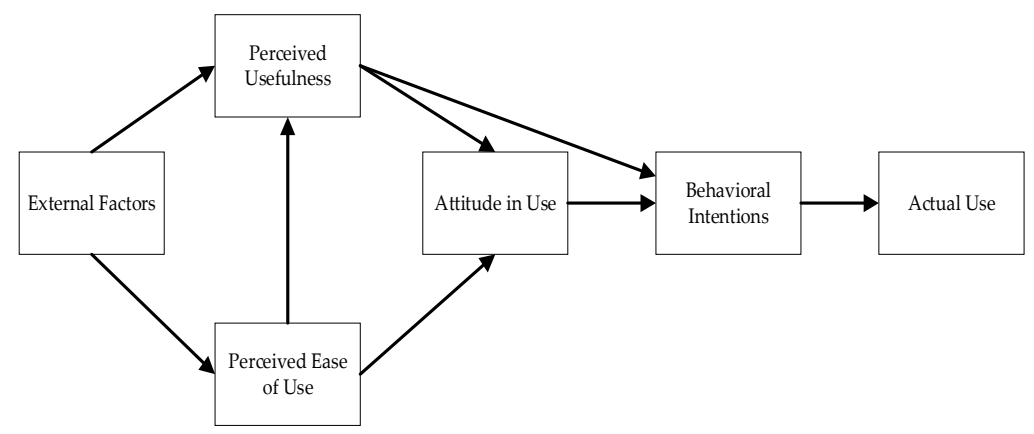

Figure 1. TAM model.

Since the TAM model was proposed, scholars have always had different opinions on the relationship between perceived usefulness, perceived ease of use, intention to use, and attitudes toward the use of new systems. For example, Venkatesh and Davis (1996) stated that usage attitudes were only users' preferences for information technology, which was reflected in their emotions, and could not fully convey the influence of useful and easyto-use perceptions on behavioral intentions. In addition, the TAM model and expanded TAM were used to explain the acceptance of new technologies and new systems. In previous studies of extended TAM, there have been few secondary constructions abstracted from perceived usefulness and perceived ease of use to explore new technologies. This 
indicates that the TAM model has many shortcomings in identifying the reasons for people's acceptance of new information systems and needs to be revised according to the specific situation.

Venkatesh and Davis et al. [12] proposed the behavioral model Unified Theory of Acceptance and Use of Technology (UTAUT) by integrating eight behavioral theoretical models which were Theory of Reasoned Action (TRA), Technology Acceptance Model (TAM), Model of PC Utilization (MPCU), Theory of Planned Behavior (TPB), Innovation Diffusion Theory (IDT), Social Cognition Theory (SCT), composite TAM and TPB model, and Motivation Model. They extracted four factors from them that influence users' acceptance motivation, namely, effort expectation, performance expectation, social impact, and contributing factor. They also extracted four moderating variables which were age, gender, experience, and voluntary. The Theory of Reasoned Action (TRA) shows that the personal perception and prevailing perceptions of the society in which one lives are important determinants of a person's attitudes and values. Individual attitudes and values determine a person's motivation to adopt a particular behavior, and motivation ultimately determines whether a behavior is adopted by a person. The Theory of Planned Behavior (TPB) shows that factors influencing behavioral willingness include behavioral beliefs, which have a potential influence on individual attitudes to perform the behavior, and normative beliefs, which are subjective norms that influence individual behavior. This means that information influences willingness and subsequent behavior through attitudes and subjective norms [13]. Innovation Diffusion Theory (IDT) is defined as a rational contemplation that seeks to clarify how, why, and to what degree new ideas and technologies are being spread [14]. Based on the recognition that individuals have subjective motivation, the Social Cognitive Theory (SCT) systematically reveals the process of generating individual behavior from individual cognition. In SCT, human behavior is extensively motivated and regulated by the ongoing exercise of self-influence. SCT conceives individuals as being goal-directed and actively engaged in developing thought processes and behaviors to meet their goals. It highlights the interaction between personal goals, cognition, and contextual factors in regulating motivated behavior [15].

The UTAUT model extracts the important factors that can predict people's use of a particular technology. Due to the integration of various theories and models, the explanatory effect is better and more realistic in predicting individuals' acceptance behaviors of information technology compared to the TAM model [12]. In addition, the model achieves the highest explanatory validity for usage behavior. Therefore, it is widely used in many fields such as e-commerce and information technology, and the validity of the UTAUT model is about $10 \%$ higher than TAM in explaining individual behavior [16].

For example, Hoque et al. [17] studied the key factors influencing elderly users' intention to adopt and use the mHealth services by developing a theoretical model based on UTAUT model. Akinnuwesi et al. [18] investigated factors affecting users' intention to use biometric technology (BT) in a developing country based on the modified version of the UTAUT model. Moreover, Alalwan et al. [19] explained the key factors influencing Jordanian customers' intention and adoption of Internet banking by using the extended UTAUT model.

The UTAUT behavioral model is shown in Figure 2.

Do all of these variables have a significant effect on farmers' adoption intention? Based on prior knowledge, all survey areas in this study had network coverage and all villagers in the area had access to the network. There was no influence of the contributing factor, so the factor was deleted and replaced by "personal innovation". In addition, because most pig farmers were $40-50$ years old in the pre-investigation, the moderating variables of the original model were deleted and an innovative UTAUT model was constructed as the research method. How do these factors affect the willingness of farmers' intention to adopt IoT traceability technology? What is the relationship between them? Considering the factors that influence pig farmers' adoption willingness of IoT traceability technology are complex and diverse, it was assumed that the adoption willingness is influenced by 
a combination of performance expectancy, effort expectancy, social influence, personal innovation, and perceived risk. Accordingly, a hypothetical model of pig farmers' adoption willingness of IoT traceability technology is proposed, as shown in Figure 3.

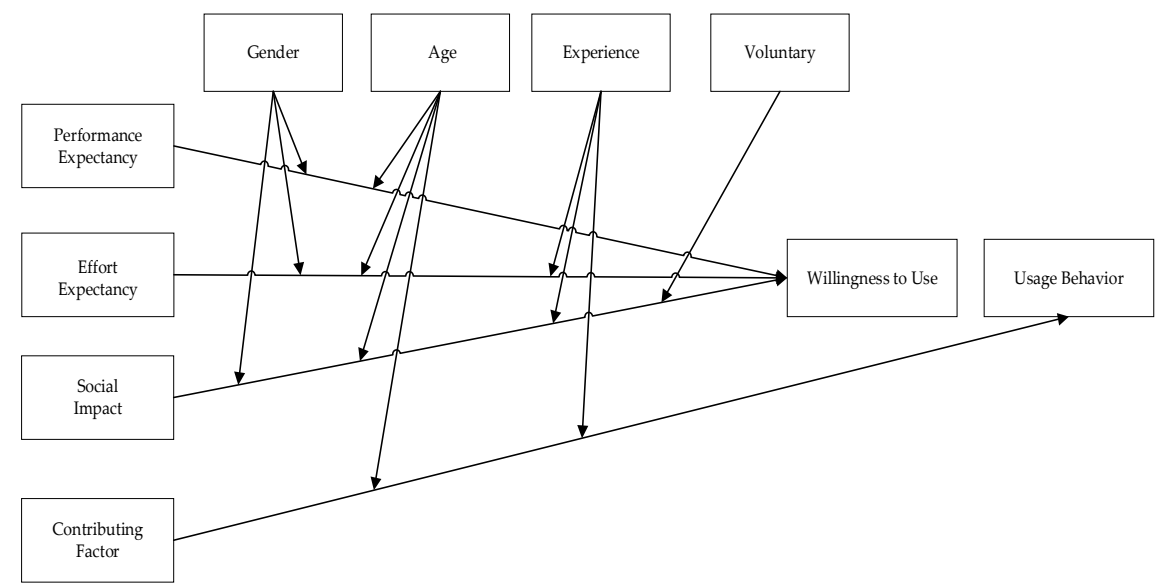

Figure 2. Unified Theory of Acceptance and Use of Technology (UTAUT).

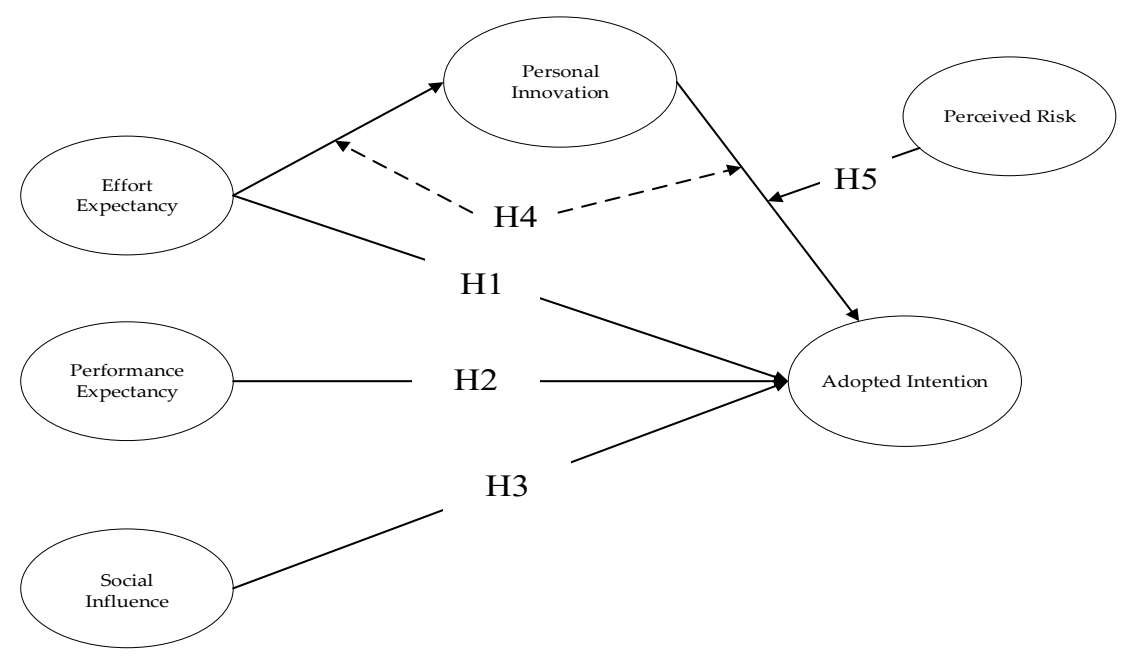

Figure 3. Latent variables and related hypotheses.

\subsection{Research Hypotheses}

In this study, effort expectancy refers to the ease of use and whether farmers perceive the IoT traceability technology to be simple to master. Knutsen's study found that effort expectancy had a significant impact on the adoption and use of emerging technologies. A study provided evidence that the more the system was perceived as effortless, the more likely it would be adopted by the individuals [20]. Therefore, this paper proposes the following hypothesis.

Hypothesis 1 (H1). Farmers' effort expectations have a positive effect on the adoption willingness of IoT traceability technology.

In this study, performance expectancy represents the extent to which farmers subjectively believe that the adoption of IoT traceability technology will bring improvements to their farming and marketing process. When performance expectancy is at a high level, users have a positive attitude towards using the system. For agriculture, studies found the importance of performance expectancy on the intentions of farmers to adopt mobile-based technologies for agricultural information. This implies that farmers' intentions to use apps 
will be strengthened if they believe that the apps will result in greater performance in their daily agricultural activities [21]. Internet of Things is one of the most popular subjects today, where sensors and smart devices facilitate the provision of information and communication. The supporting role of IoT traceability technology on farming influences the adoption of IoT technology in agricultural quality traceability systems [22]. Accordingly, the following hypothesis is proposed.

Hypothesis 2 (H2). Performance expectations of farmers have a positive effect on adoption willingness of IoT traceability technology.

Social influence refers to the extent to which farmers perceive whether others think they should adopt IoT traceability technology. The positive impact of the social influence on the behavioral intentions sheds light on the convincing effect of the farmers' coworkers and farmhands in persuading them to use IoT in farming [23]. The more the farmer's neighbors, relatives, friends, and village cadres support the farmer in adopting the new technology, the higher the farmer's willingness is. In this study, if the number of relatives and friends around the surveyed farmers adopt IoT traceability technology is in a large proportion or recommend them to adopt the technology, it will naturally have a positive impact on farmers' adoption willingness. Therefore, the following hypothesis is proposed in this study.

Hypothesis 3 (H3). Social influence has a positive impact on farmers' adoption willingness of IoT traceability technology.

Personal innovation refers to the degree of farmers' personal acceptance of new things and it was found that personal innovation affects users' adoption willingness of a new technology. In this study, personal innovation represents the personal characteristics of the farmers in terms of actively exploring new and unknown things and the stronger personal innovation of farmers, the higher degree of initiative in exploring new things, which implies the stronger initiative in understanding and adopting IoT traceability technology. Dewi et al. [24] proposed that personal innovation had a crucial role in innovation adoption and also had a strong and direct effect on consumers' decision to adopt new technology because individuals became aware of new technology based on personal traits such as personal innovation. It can be concluded that the more innovative the consumers are, the higher the behavioral intention will be. Therefore, when the degree of initiative to explore something new is higher, the willingness of farmers to adopt IoT traceability technology will be stronger. Meanwhile, in the process of field research, the author found that farmers were more likely to take the initiative to learn about the IoT traceability technology when they perceived that the technology was easy to grasp. For example, after the team members explained the operation related to the technology, farmers showed higher acceptance level of the technology than before, which implied they showed strong personal innovation. Therefore, the following hypothesis is proposed in this paper.

Hypothesis 4 (H4). Personal innovation is a mediating variable between farmers' effort expectations and adoption willingness.

Perceived risk explains the extent to which individual farmers believe that there is a potential for adverse consequences from using IoT traceability technology networks. In this study, perceived risk represents the potential dangers that farmers perceive in using IoT traceability technology, such as the farming costs and the threats of personal information. In this paper, the latent variable of perceived risk is extracted by combining perceived risk with personal innovation. Wu et al. [25] classified perceived risk into four aspects, which were technology, function, behavior, and economy, and found that perceived risk negatively affected users' adoption willingness. At the same time, the higher the farmers' 
perceived risk to new technology, the lower their acceptance of the technology. Therefore, this study puts forward the following assumption.

Hypothesis 5 (H5). The mediating effect of perceived risk on farmers' personal innovation acts as a moderator.

\section{Materials and Methods}

\subsection{Data Preparation}

The data used in this paper were obtained from field research in Bailiang Village, Shuangzhao Office, Qinhan New City, Xixian New District, Shaanxi Province; Podi Village, Junma Town, Liquan County, Xianyang City, Shaanxi Province; and Xinfeng Town, Lintong Area, Xi'an City, Shaanxi Province. Researchers randomly selected 90 pig farmers within each sample from July to October 2020 to form a data sample of 270 pig farmers. In order to make the researched farmers understand the content of the questionnaire more specifically, on the one hand, several trainings were given to the participants of the research. On the other hand, a video explanation was provided for farmers to understand the meaning of the terminologies such as IoT traceability technology and questions in the questionnaire. A total of 270 questionnaires were distributed, excluding some questionnaires with incomplete or wrong information. Finally, 264 valid questionnaires were obtained with an efficiency rate of $97 \%$.

\subsection{Variable Settings and Descriptive Statistics}

Firstly, the variable indicators affecting adoption willingness were constructed according to previous studies, then the content of the questionnaire items was adjusted according to the pre-investigation, and the specific content of the formal questionnaire was determined. The participants of the pre-investigation were pig farmers in Bailiang Village, Shuangzhao Office, Qinhan New City, Xixian New Area, Shaanxi Province. The pre-investigation was conducted to test whether the questionnaire scale was applicable to the study of pig farmers' adoption willingness of IoT traceability technology, in which 49 questionnaires were collected. After that, researchers made the item analysis and exploratory factor analysis on the collected data. According to the results of the test, the items with factor loading less than 0.5 were excluded, and finally six groups of 46 items were obtained for formal research. The Likert scale method was used for the measurement of this paper, with values 1 to 5 corresponding to "strongly disagree", "somewhat disagree", "neutral", "somewhat agree", and "strongly agree", respectively.

\subsubsection{Dependent Variable}

The Internet of Things (IoT) is a dynamic global network infrastructure with selforganization capabilities based on standard and interoperable communication protocols, in which virtual "things" have identities, physical properties, virtual characteristics, and intelligent interfaces, and are integrated seamlessly with information networks [26]. In 2007, the first traceability system in China with Universal Signage System began to be piloted in Carrefour Supermarket in Beijing. Professionals pointed out that consumers could scan the barcode or QR code on the outer package of the food bought in this supermarket with their smart phones. They could promptly find out all the information about the place of production, production date, supplier, and production raw materials of the food. Thus, the traceability of food safety was carried out in this place. If the food was found to have safety problems, consumers could quickly get the traceability information of the food. Food safety sectors could identify and deal with the food in time to reduce unnecessary losses at the same time. 
For pig breeding, IoT traceability technology mainly refers to the application of a pig breeding traceability management system by placing ID cards on piglets. The Radio Frequency Identification (RFID) technology is used to scan the electronic tag to store all the data of the breeding stages from breeding to birth, including management information such as medicine and vaccination. In addition, the IoT traceability technology may detect and control the environmental conditions in the breeding process in real time, such as the temperature, humidity, ventilation conditions of piggery, and the amount of cleaning and maintenance in the processing workshop [27]. as follows.

The measure of pig farmers' adoption willingness was divided into two indicators

AI1: I am very willing to adopt the existing IoT traceability technology; and

AI2: I am willing to take the initiative to understand and consider adopting IoT traceability technology if there is an opportunity.

Both indicators are based on the Likert scale with values 1 to 5 corresponding to "strongly disagree", "somewhat disagree", "neutral", "somewhat agree", and "strongly agree".

\subsubsection{Independent Variables}

(1) Performance expectancy. The variable is described by the following three indicators. Participants believe that the use of IoT traceability technology can largely improve the efficiency of pig farming. Participants believe that pig sales can be helped to a great extent through the use of IoT traceability technology. Participants believe that the use of the Internet is a great improvement to life.

(2) Effort expectancy. The variable is described by the following three indicators. After learning about IoT traceability technology, participants think it is easy to master. If there is a simpler IoT traceability technology, participants are very likely to use it. Participants find the Internet is very convenient.

(3) Social influence. The variable is described by the following four indicators. Participants have heard many people talk about IoT traceability technology. Participants have been recommended IoT traceability technology by slaughterhouse staff, wholesalers, and consumers many times. Participants have been recommended using IoT traceability technology by many family members and friends. Participants have been recommended to use IoT traceability technology by many people from governmental regulatory departments and quarantine departments.

\subsubsection{Mediating Variable: Personal Innovation}

This variable is described by the following two indicators. Participants are willing to take the initiative to learn about new food safety technologies. Participants strongly believe in the policy information promoted in the village.

\subsubsection{Moderating Variable: Perceived Risk}

This variable is described by the following two indicators. Participants are very worried that the IoT traceability technology will cause loss to their profit. Participants are very distrustful of the detection capability of the IoT traceability technology.

The indicators for the specific questions are shown in Table 1. 
Table 1. Scale indicators.

\begin{tabular}{|c|c|c|}
\hline Variables & Indicators & Indicator Content \\
\hline \multirow{3}{*}{$\begin{array}{c}\text { Performance } \\
\text { expectancy (PE) }\end{array}$} & PE1 & $\begin{array}{c}\text { The use of IoT traceability technology can greatly improve the } \\
\text { efficiency of pig farming. }\end{array}$ \\
\hline & PE2 & $\begin{array}{l}\text { The use of IoT traceability technology can largely help to complete } \\
\text { the pig sales. }\end{array}$ \\
\hline & PE3 & The use of the Internet can make a big difference to life. \\
\hline \multirow{3}{*}{$\begin{array}{l}\text { Effort expectancy } \\
\text { (EE) }\end{array}$} & EE1 & $\begin{array}{l}\text { After learning about IoT traceability technology, I think IoT } \\
\text { traceability technology is easy to master. }\end{array}$ \\
\hline & EE2 & $\begin{array}{l}\text { If there is a simpler IoT traceability technology, I am very likely to } \\
\text { use it. }\end{array}$ \\
\hline & EE3 & I find the Internet is very convenient. \\
\hline \multirow{4}{*}{ Social impact (SI) } & SI1 & I have heard a lot of people talk about IoT traceability technology. \\
\hline & SI2 & $\begin{array}{l}\text { Slaughterhouse staff, wholesalers, and consumers have } \\
\text { recommended I use IoT traceability technology many times. }\end{array}$ \\
\hline & SI3 & $\begin{array}{l}\text { Many family members and friends have recommended I use IoT } \\
\text { traceability technology. }\end{array}$ \\
\hline & SI 4 & $\begin{array}{l}\text { People from governmental regulatory departments and quarantine } \\
\text { departments have recommended I use IoT traceability technology. }\end{array}$ \\
\hline \multirow{2}{*}{$\begin{array}{l}\text { Personal innovation } \\
\text { (PI) }\end{array}$} & PI1 & $\begin{array}{l}\text { I am very willing to take the initiative to learn about new food } \\
\text { safety technologies. }\end{array}$ \\
\hline & PI2 & I strongly believe in the policy information promoted by the village. \\
\hline \multirow{2}{*}{ Perceived risk (PR) } & PR1 & $\begin{array}{l}\text { I am very worried about the loss of my profits from using IoT } \\
\text { traceability technology. }\end{array}$ \\
\hline & PR2 & $\begin{array}{l}\text { I am very distrustful of the detection capabilities of IoT traceability } \\
\text { technology. }\end{array}$ \\
\hline \multirow{2}{*}{$\begin{array}{l}\text { Adoption intention } \\
\text { (AI) }\end{array}$} & AI1 & \multirow{2}{*}{$\begin{array}{l}\text { I am very willing to adopt the existing IoT traceability technology. } \\
\text { If given the opportunity, I would like to learn about and consider } \\
\text { adopting IoT traceability technology. }\end{array}$} \\
\hline & AI2 & \\
\hline
\end{tabular}

\subsubsection{Descriptive Statistical Analysis of Variables}

Table 2 summarizes the basic information of the respondents.

Table 2. Descriptive statistics of the variables influencing the adoption willingness of IoT traceability technology.

\begin{tabular}{|c|c|c|c|}
\hline $\begin{array}{c}\text { Statistical } \\
\text { Characteristics }\end{array}$ & Classification Indicators & Number of People & Share $(\%)$ \\
\hline \multirow{2}{*}{ Gender } & Male & 207 & $78.41 \%$ \\
\hline & Female & 57 & $21.59 \%$ \\
\hline \multirow{4}{*}{ Age } & $1=20 \sim 35$ years old & 9 & $3.41 \%$ \\
\hline & $2=35 \sim 50$ years old & 113 & $42.81 \%$ \\
\hline & $3=50 \sim 65$ years old & 128 & $48.48 \%$ \\
\hline & $4=65 \sim 80$ years old & 14 & $5.30 \%$ \\
\hline \multirow{5}{*}{ Education level } & $1=$ Never went to school & 22 & $8.34 \%$ \\
\hline & $2=$ Primary school and below & 67 & $25.38 \%$ \\
\hline & $3=$ Junior high school & 120 & $45.45 \%$ \\
\hline & $4=$ High school/secondary vocational school & 49 & $18.56 \%$ \\
\hline & $5=$ Bachelor $/$ higher vocational school & 6 & $2.27 \%$ \\
\hline \multirow{2}{*}{ Internet usage } & Yes & 221 & $83.71 \%$ \\
\hline & No & 43 & $16.29 \%$ \\
\hline
\end{tabular}

Firstly, the proportion of men in the total number of the respondents is larger than that of women, which is mainly due to the influence of pig farming environment and the fact that most jobs are manual labor, which requires the help of men.

Secondly, in the age distribution, the proportion of farmers under 35 years old is relatively low, which is mainly because this group of farmers has less experience in keeping pigs and prefer to go out to work, fewer farmers in this age group are engaged in pig farming. The largest number of respondents, between the ages of 35 and 65 years old, accounts for $91 \%$. This may be because this group of farmers are more experienced and 
adaptable to the environment and more willing to engage in pig farming. Therefore, the number of farmers in this age range accounts for the largest percentage of respondents.

Thirdly, in terms of the education level of the respondents, the largest proportion of farmers with junior high school education level and below is over $80 \%$. This indicates that most pig farmers are not highly educated and have limited ability to accept new technology and knowledge.

Fourthly, in terms of Internet use, the vast majority of farmers use the Internet. This is related to the fact that the Internet has become very popular in rural areas in recent years. Through the above analyses, we can find that most of the researched subjects were men, who were older, less educated, and more likely to use the Internet. The above characteristics are consistent with the basic situation of rural pig farmers at present.

\subsection{Methods}

The moderated mediation model implies that the independent variable $X$ influences the dependent variable $Y$ through the mediating variable $M$, and the mediation process $(\mathrm{X} \rightarrow \mathrm{M} \rightarrow \mathrm{Y})$ is moderated by the moderating variable $\mathrm{Z}$ [28]. The existing moderated mediation effect test methods are based on multiple linear regression analysis of the explicit variables [29]. The most important shortcoming of the multiple linear regression analysis of mediating and moderating effects is the assumption that all variables are measured without measurement error, which results in an underestimation of the mediating and moderating effects. The biggest advantage of establishing the Structural Equation Model (SEM) for the analysis of moderated mediation effect is that it is a better way to set latent variables, effectively control measurement errors, and accurately estimate the values of mediating and moderating effects.

Although the analysis of moderated mediation effects based on the Structural Equation Model has obvious advantages, the application of this method is not common in practice [30]. Wang [31] suggested that one possible reason for this was that the current analysis of moderated mediation effects based on structural equation model requires the use of product-indicator approaches, which required the use of product indicator as the index for the potential moderator. The product-indicator approaches had two major shortcomings. First, the generation of product indicators was complex, and there were multiple strategies for generating indicators, which were not easy to be mastered by general researchers. Different strategies for generating product indicators might produce different parameter estimates, which might cause confusion for the researchers in understanding and interpreting. Second, the product terms were non-normally distributed, which made the parameter estimation results based on the assumption of normal distribution produce bias and had problems of robustness. Fang et al. [32] pointed out that a feasible solution was to use the Latent Moderate Structural equations (LMS) method to perform the analysis of moderated mediation effects based on SEM because the LMS method did not require the use of the product indicator and avoided the problem of the product indicator. They also explored how to use the LMS method to perform the analysis of moderated mediation effects based on SEM. Facing the analysis of moderated mediation effects based on SEM tasks, Fang et al. [32] summarized a set of analysis processes as follows.

(1) Judge whether the baseline SEM model is acceptable or not; if not, the analysis is finished, otherwise go to Step 2.

(2) Judge whether the moderated mediation effects based on SEM model is acceptable or not; if not, the analysis is finished, otherwise go to Step 3.

(3) Use the coefficient multiplication method to analyze the moderated mediation effects, if the bootstrap confidence interval excludes 0 , it means that the moderated mediation effects are significant, as shown in Figure 4. 


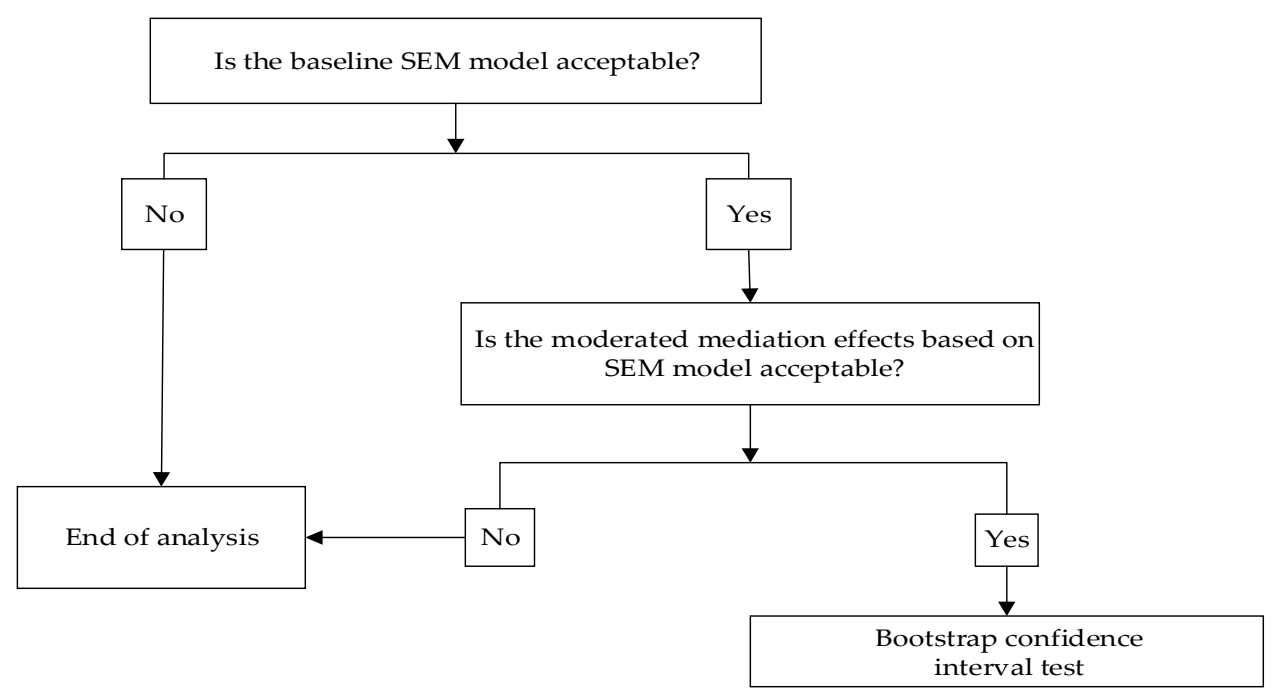

Figure 4. Flow chart of the analysis of moderated mediation effects based on SEM.

\section{Structural Equation Model and Result Analysis}

\subsection{Data Quality Analysis}

4.1.1. Reliability and Validity Analysis

Cronbach's alpha coefficient is the most common test index for internal consistency reliability; Kaiser-Meyer-Olkin Measure of Sampling Adequacy (KMO) test and Bartlett's spherical test are common validity tests. In this paper, the above tests were conducted by SPSS Statistics 24.0 software (International Business Machines Corporation, New York, NY, USA) to verify the suitability of the data for factor analysis. The Cronbach's alpha coefficients for each dimension are shown in Table 3; it can be seen that the Cronbach's alpha coefficients for each latent variable were greater than 0.7. This indicates that the scale has high reliability, dependability, and stability.

Table 3. Reliability test.

\begin{tabular}{ccc}
\hline Dimension & Variables & Cronbach's Alpha \\
\hline \multirow{2}{*}{ Performance expectancy } & PE1 & 0.871 \\
& PE2 & \\
Effort expectancy & PE3 & \multirow{2}{*}{0.791} \\
& EE1 & \\
Social impact & EE2 & \multirow{2}{*}{0.756} \\
& EE3 & \\
\hline \multirow{2}{*}{ Personal innovation } & SI1 & \multirow{2}{*}{0.865} \\
\hline \multirow{2}{*}{ Perceived risk } & SI3 & \multirow{2}{*}{ SI4 } \\
\hline \multirow{2}{*}{ Adoption intention } & PI1 & \\
& PI2 & 0.836 \\
\hline
\end{tabular}

In addition, the results of KMO and Bartlett's spherical test for the 16 measures of adoption willingness of IoT traceability technology in pig farming show that the KMO value is 0.807 . This indicates that the scale data has good validity. In the Bartlett's spherical test value, the approximate chi-square value was 2025.370 and the significance level was 0.000 . Thus, it was appropriate to conduct factor analysis on the data. 


\subsubsection{Model Simulation Test}

The following indexes were used in this study to measure the fitting of the measurement model: Chi-square/degree of freedom, the Root Mean Square Error of Approximation (RMSEA), Comparative Fit Index (CFI), Non-Normed Fit Index (NNFI), Tucker-Lewis Index (TLI), Incremental Fit Index (IFI), and Standardized Residual Mean Square (SRMR). It can be seen in Table 4 that the results of the model tests in this study satisfied the range of judgmental criteria values.

Table 4. Results of overall model fitness index values.

\begin{tabular}{cccccccccc}
\hline Indicators & $\chi^{2}$ & $\mathbf{d f}$ & $\chi^{2} / \mathbf{d f}$ & RMSEA & CFI & NNFI & TLI & IFI & SRMR \\
\hline $\begin{array}{l}\text { Criteria values } \\
\text { for judgement }\end{array}$ & & & $<3$ & $<0.10$ & $>0.9$ & $>0.9$ & $>0.9$ & $>0.9$ & $<0.1$ \\
$\quad$ Results & 152.338 & 69 & 2.208 & 0.068 & 0.952 & 0.916 & 0.936 & 0.952 & 0.028 \\
\hline
\end{tabular}

\subsection{Analysis and Discussion of the Model}

In this paper, the maximum likelihood estimate method was used to estimate the model parameters by using AMOS23 software (International Business Machines Corporation, New York, NY, USA), and the parameter estimation model is shown in Figure 5.

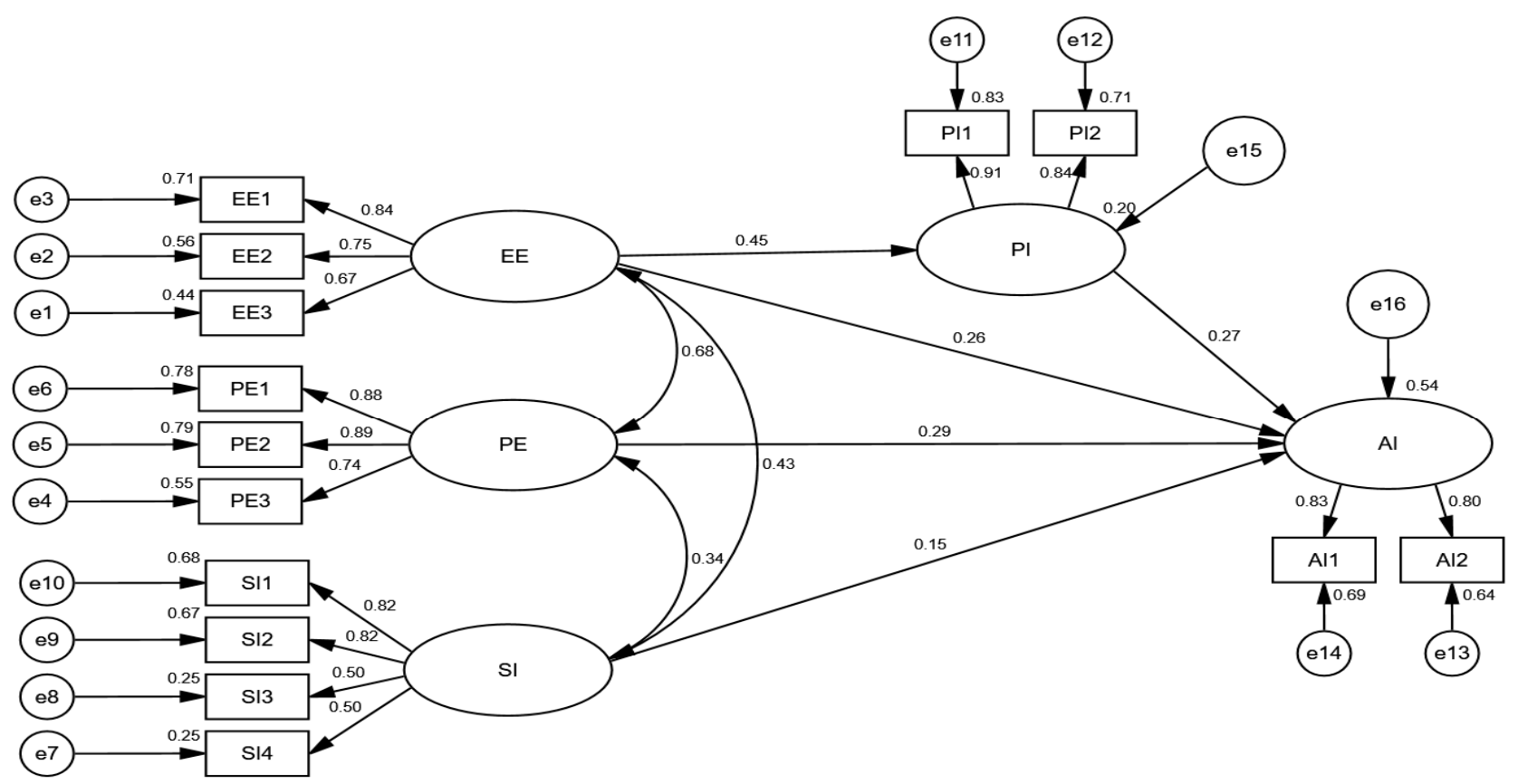

Figure 5. Structural equation model and normalized coefficients.

\subsubsection{Hypothesis Test and Results}

From the model estimation and hypothesis results (Table 5) as well as the structural equations and standardized path coefficients (Figure 6), it can be seen that the path coefficient of farmers' effort expectancy on their willingness to adopt IoT traceability technology was 0.262 and passed the significance test at the $5 \%$ level, indicating that farmers' effort expectancy significantly and positively affects their willingness to adopt IoT traceability technology. This means the hypothesis (H1) that farmers' effort expectancy has a positive influence on their willingness to adopt IoT traceability technology holds. The path coefficient of farmers' performance expectancy to farmers' adoption willingness of IoT traceability technology was 0.290 and passed the significance test at the $1 \%$ level, indicating that farmers' performance expectancy has a significant positive influence on their adoption willingness of IoT traceability technology. This means the hypothesis (H2) that farmers' performance expectancy has a positive influence on the adoption willingness of IoT trace- 
ability technology holds. The influence path coefficient of farmers' social environment on their adoption willingness was 0.146 and passed the significance test of $5 \%$, indicating that the social impact has a significant positive influence on farmers' adoption willingness of IoT traceability technology. This means the hypothesis (H3) that social impact has a positive influence on farmers' willingness to adopt IoT traceability technology holds.

Table 5. Model estimation and hypothesis results.

\begin{tabular}{ccccccc}
\hline Paths & Directions & $\begin{array}{c}\text { Standardized } \\
\text { Coefficients }\end{array}$ & S.E. & C.R. & P & Test Results \\
\hline $\mathrm{EE} \rightarrow \mathrm{AI}$ & + & 0.262 & 0.054 & 2.485 & 0.013 & H1 is established. \\
$\mathrm{PE} \rightarrow \mathrm{AI}$ & + & 0.290 & 0.051 & 3.199 & 0.001 & H2 is established. \\
$\mathrm{SI} \rightarrow \mathrm{AI}$ & + & 0.146 & 0.121 & 1.984 & 0.047 & H3 is established. \\
\hline
\end{tabular}

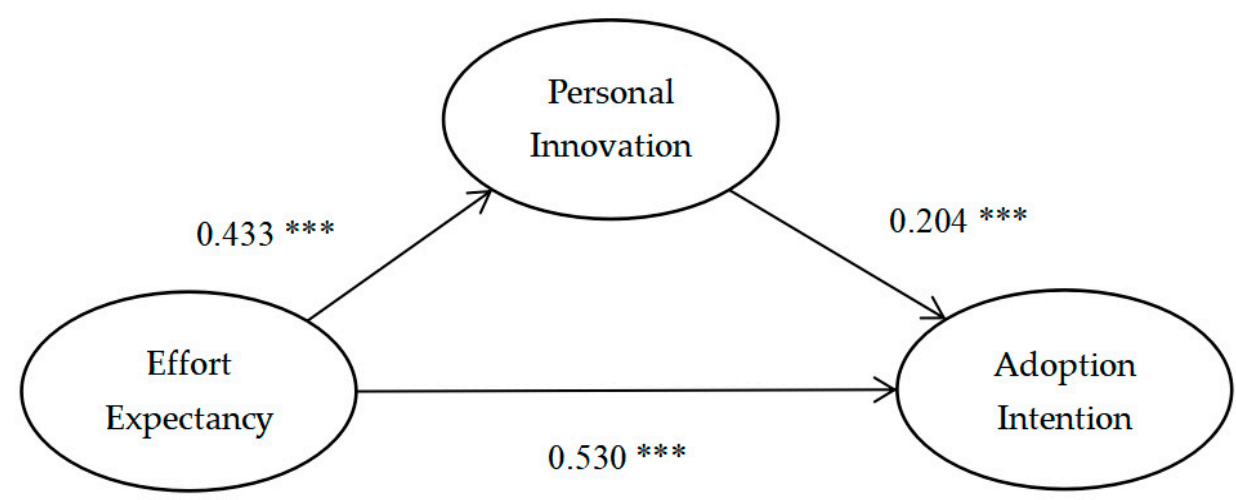

Figure 6. Mediation effect test. ${ }^{* * *}$ It indicates that the impact of the path is extremely significant.

\subsubsection{Test of Mediating Effect}

In this paper, we used the bootstrapping method to test the mediating effect of farmers' personal innovation by setting the sample number to 2000 and the confidence interval as $95 \%$, and judged the existence of the mediating effect by the test results. The results show (Figure 6) that the indirect effect of personal innovation on farmers' effort expectancy and adoption willingness was $0.088(0.433 \times 0.204)$, and the path coefficient was significant at the $1 \%$ level. The bias-corrected confidence interval of the mediating effect was [0.056, 0.22], indicating that the lower limit of the indirect effect of farmers' effort expectancy on the adoption willingness of IoT traceability technology was 0.056 and the upper limit was 0.22 , and the confidence interval excluded 0 . Therefore, personal innovation has a significant mediating effect on farmers' effort expectancy and adoption willingness, and the hypothesis (H4) that personal innovation is a mediating variable of farmers' effort expectancy and adoption intention is valid.

\subsubsection{Moderated Mediating Effect Test}

The mediating effect of personal innovation was tested above. Next, the mediator variable and regulated variable were included in the model at the same time, and the analysis of moderated mediating effect was carried out by the coefficient multiplication method. It can be seen from the path test results in Table 6 that when farmers were at high risk perception, the moderating effect of the path of perceived risk on farmers' personal innovation and adoption willingness of IoT traceability technology was significant when farmers with a path coefficient of 0.158 and a bootstrap confidence interval of $[0.09,0.268]$ at the $95 \%$ level excluding 0 , indicating that the moderating effect was significant. When farmers were at moderate risk perception, the moderating effect of the path of perceived risk on farmers' personal innovation and adoption willingness was significant with a path coefficient of 0.088 , and the bootstrap confidence interval was [0.026, 0.17] at the $95 \%$ level excluding 0 , indicating that the moderated mediating effect was significant. When farmers were at low risk perception, the path moderation effect of perceived risk on farmers' 
personal innovation and adoption willingness was not significant, and the bootstrap confidence interval was $[-0.086,0.137]$ at the $95 \%$ level including 0 , which indicates that the mediating effect with moderation was not significant. Therefore, the hypothesis (H5) is valid. The above results indicate that with the increase of the moderating variable (perceived risk), the mediating effect of farmers' personal innovation on the adoption willingness of IoT traceability technology increases significantly, which implies that the moderating variable significantly moderates the degree of the mediating effect.

Table 6. Tests of moderated mediating effects based on moderating path analysis.

\begin{tabular}{ccccc}
\hline \multirow{2}{*}{ Regulated Variables } & Path Coefficients & \multicolumn{3}{c}{ Bias-Corrected 95\% IC } \\
\cline { 3 - 5 } & & Lower & Upper & P \\
\hline High risk perception & 0.158 & 0.09 & 0.268 & 0 \\
Moderate risk perception & 0.088 & 0.026 & 0.17 & 0.009 \\
Low risk perception & 0.019 & -0.086 & 0.137 & 0.643 \\
\hline
\end{tabular}

\subsection{Analysis of Estimation Results}

\subsubsection{Effort Expectancy}

Effort expectancy is the degree to which farmers personally perceive whether IoT is easy to use and simple to master. Effort expectancy has a significant positive influence on farmers' adoption willingness of IoT traceability technology. It implies that the degree to which farmers subjectively perceive the technology to be easy to use in relation to their own reality through appropriate explanation by investigators under the existing degree of IoT technology popularity. Due to the intelligence of IOT devices and the learning ability of farmers, we found in the actual survey that when "IoT traceability technology" was first mentioned, most farmers had a low willingness to adopt IoT traceability technology because they had never heard of it and did not know anything about it. However, after listening to the brief explanation and examples given by investigators, farmers showed higher enthusiasm and willingness to adopt than before, which also conformed to the test results of the data. This was because when farmers felt that the technology was easy to master, the learning cost in practical application might decrease. From a profit perspective, farmers were more willing to adopt it. Therefore, the higher the effort expectancy of farmers for the technology, the stronger the willingness to adopt IoT traceability technology. In other words, effort expectancy positively influences farmers' adoption willingness of IoT traceability technology.

\subsubsection{Performance Expectancy}

In this study, performance expectancy represents the extent to which farmers subjectively believe that the adoption of IoT traceability technology will have benefits for their farming and marketing process. Performance expectancy has a significant positive effect on farmers' willingness to adopt IoT traceability technology. In the survey, it was found that most of the farmers' questions about IoT traceability technology focused on the cost and consumer acceptance. High performance expectancy means that farmers subjectively predict that IoT traceability technology will bring higher profits to their farming and marketing process. Except for a small number of family pig farmers, most farmers raise pigs to maintain living expenses, so as long as the technology can increase the existing profit amount, farmers will show a high willingness to adopt IoT traceability technology. This means that performance expectancy positively influences farmers' adoption willingness of IoT traceability technology.

\subsubsection{Social Influence}

Social influence refers to the extent to which farmers personally perceive whether others think they should adopt IoT traceability technology. For example, if someone among the farmers' relatives, friends, neighbors, intermediaries, or consumers they usually come into contact with recommended the farmers use IoT traceability technology or had used 
IoT traceability technology, then the social influence was at a high level. The more people recommended or used it, the greater the social influence. In the survey, it was found that most farmers usually use cell phones, computers, and other electronic products to access the Internet mainly for entertainment, so it is impossible for them to learn and understand IoT traceability technology from the Internet. News and mass media reports on IoT traceability technology are rare, so farmers' knowledge of IoT traceability technology mostly comes from the surrounding environment. When the social influence is greater, farmers will have more expectation and trust in the convenience or profit brought by IoT traceability technology. Moreover, due to the influence of conformity psychology, a higher level of social influence will also have a positive impact on farmers' adoption of IoT traceability technology. This means that social influence significantly and positively affects farmers' adoption of IoT traceability technology.

\subsubsection{Personal Innovation}

Personal innovation refers to the degree of farmers' personal acceptance of new things. When a farmer is willing to take the initiative to understand and learn more about emerging technologies, the degree of his personal innovation is higher. In addition, when a technology is easier for a farmer to master, which implies the farmer's effort expectancy is higher, he tends to be more willing to take the initiative to learn about it. Therefore, effort expectancy has a significant positive effect on personal innovation. Moreover, when the farmer's personal innovation is stronger, his enthusiasm for learning emerging technologies including IoT traceability technology is stronger, so his adoption willingness is higher. This means that farmers' personal innovation has a significant positive influence on their adoption of IoT traceability technologies. In summary, farmers effort expectancy significantly and positively influences farmers' adoption willingness through personal innovation as a mediating variable.

\subsubsection{Perceived Risk}

Perceived risk refers to the extent to which farmers personally believe that the use of IoT traceability technologies will likely have adverse consequences. The results of the data analysis show that the mediating effect of perceived risk on farmers' personal innovation and willingness to adopt plays a significant positive moderating role. In contrast, general research suggests that perceived risk negatively affects adoption willingness, which implies that the lower the perceived risk, the more significant the impact of farmers' personal innovation on adoption willingness should be. Wu et al. (2010) divided perceived risk into four aspects, which were technology, function, behavior, and economy, and found that perceived risk significantly and negatively affects users' willingness to use. However, through the data analysis, we found that the greater the perceived risk of farmers, the greater the impact of their personal innovation on adoption willingness. In fact, this is because the stronger perceived risk means that farmers are more active in learning the new technology and tend to make a prudent risk judgment after understanding a new technology, rather than unconditionally trusting new technology in order to complete the questionnaire. The author found in the communication with farmers during the field survey that the more skeptical farmers were about IoT traceability technology, the more willing they were to actively ask the investigators questions about the specific operation of IoT traceability technology and showed stronger initiative and enthusiasm. This means that personal innovation will significantly and positively affect their willingness to adopt IoT traceability technology. Thus, the effect of perceived risk on personal innovation has a significant positive moderating effect. 


\section{Conclusions}

There have been a series of food safety incidents that have brought great harm to people's health in China and the application of IoT traceability technology is conducive to ensuring food quality and safety, improving the public's awareness of traceability products, and promoting the steady development of social economy. However, farmers are affected by their own conditions, social environment, cultural beliefs, and other factors. Most of them have low willingness to adopt new technologies.

It is necessary to identify and classify the influencing factors that affect farmers' adoption of IoT traceability technology, which will have a positive impact on resilience in agri-food supply chains and sustainability. The higher willingness of farmers to adopt IoT traceability technology means higher agility in the agri-food supply chains. Supply chain agility positively contributes to supply chain resilience [33]. In other words, with more transparent information exchanges and better joint collaboration, supply chain members are able to prepare for, adapt to, and recover from the risks better, which means that supply chain agility positively contributes to supply chain resilience. Moreover, the willingness of farmers to adopt new technologies explored in this paper contributes to the application of circular principles in supply chain systems. Applying the principles of circularity to the supply chains allows new rules to be established with suppliers and customers. It increases the number of actors with an active role in greener operations. A long-term partnership between customers and suppliers is fundamental to achieve social and environmental solutions [34]. Finally, the digital technology studied in this paper improves the sustainability of agricultural production. Digital technologies increase the operational efficiency through the accessibility and collection of process data in real time, the management of energy and resource consumption, and knowledge of the entire life cycle (design, manufacturing, distribution, maintenance, and use) with the potential to eliminate discontinuities and inefficiencies [35].

In fact, both resilience and sustainability are viewed as distinct concepts, but are positively correlated [1]. On the one hand, resilience has a positive impact on sustainability. Giudice et al. [36] mentioned that achieving a sustainable food system means "increasing or maintaining agricultural yields and efficiency while decreasing the environmental burden on biodiversity, soils, water and air." Klumpp et al. [37] also pointed out that the efficiency reductions after IT disruptions occur at different levels and for diverse reasons, and successful preparation and contingency management could support improvements. Moreover, the pandemic period has caused severe socio-economic damage, but it is accompanied by environmental deterioration that can also affect economic opportunities and social equity. In the face of this double risk, future generations are ready to be resilient and make their contribution not only on the consumption side but also through their inclusion in companies by bringing green and circular principles with them [38]. These examples all show the positive effect of resilience on sustainability to some extent. On the other hand, sustainability has a positive effect on resilience. A profound and holistic discussion is emerging around the question of how sustainable the present food system is and how prepared it is to face the kind of shock posed by the COVID-19 pandemic. Fabio Giudice et al. pointed out that circular practices improved resilience of the entire value chain (from production to consumption and post-consumption) through the introduction of localized supply chains.

Therefore, based on research data from 264 pig farmers in Shaanxi Province, the innovative UTAUT model was established. The researchers verified the research hypothesis through empirical analysis and analyzed the factors that influence pig farmers' adoption willingness of traceability technology.

The contributions in this article can be differentiated between theoretical and practical contributions. 


\subsection{Theoretical Contribution}

First of all, the previous research on the IoT traceability technology was mainly about the innovation of the technology and the combination with blockchain. These studies regarded pig farmers' adoption intention as an exogenous variable and ignored its endogeneity. This article filled the gap in the research of IoT traceability technology.

Secondly, in the past, the Structural Equation Model was mostly used to study the adoption intention, ignoring the relationship between independent variables. The Structural Equation Model including the intermediate variable and the latent variable established in this study makes up for the shortcomings of previous studies and puts forward an impact path that is more in line with the actual situation.

\subsection{Practical Contribution}

First, the higher the expectancy of farmers' efforts, the stronger their willingness to adopt the IoT traceability technology. This shows that farmers are more willing to adopt the technology when the actual operation of the technology is easier than the farming methods they use at present. In fact, Fedushko et al. [39] pointed out that the developed machine learning model made a difference to improve transaction tracing. This helped identify errors, enhance operations, data pipelines to make a project requirement precise, identify use-cases, and apply monitoring for project improvement. Moreover, continuous real-time monitoring combined with machine learning for a certain industrial operational use-case allowed a system to increase availability which was one of the factors that led to higher user satisfaction levels. Second, when farmers predict that the technology will bring higher profits, their willingness to adopt it is stronger. Puriwat et al. [19] pointed out that when people knew that social media was useful for business purposes and using social media as an alternative business platform would enhance their business performance, they would be more willing to adopt social media for business purposes. Third, farmers tend to show higher adoption willingness of the technology when they are surrounded by people who have recommended the technology to them, especially when they have already used it for pig farming. The more people recommend and use the technology, the higher the farmers' adoption willingness. Wissal et al. [40] pointed out that one of the strongest predictors of patients' behavioral intention to use connected devices in healthcare was social influence. Health was a personal matter. However, as people were often not experts in many health-related issues, they were easily impacted by the important others in their social groups, such as their family physicians. Fourth, when the IoT traceability technology is simpler and easier for farmers, and farmers are more active in understanding it, they are more likely to adopt the technology. It means that personal innovation as a mediating variable of farmers' effort expectancy and adoption willingness has a significant positive effect on the results. Fifth, the personal characteristics of farmers when faced with a new technology have a significant effect on their adoption willingness, mainly because farmers are skeptical about new technology. This means that the farmers are more motivated to learn, thus the effect of personal innovation on adoption willingness is more significant.

Based on the above research conclusions, the following policy inspirations are obtained. First, the improvement of IoT traceability technology at the technical level plays an important role in its popularization, so the relevant departments should increase the investment in the research of this technology and strive to make the actual operation of farmers using this technology easier than the existing traditional farming methods. Second, for the farmers who have adopted the technology, government departments should give appropriate subsidies, which not only plays a role in the protection of farmers after the adoption of new technology risk, but also encourages more farmers to adopt the technology. Third, the government, village committees, and other relevant departments should increase the publicity of IoT traceability technology. These departments should not only carry out technical promotion and publicity work on the farmers themselves, but also on other environmental factors that may potentially affect the farmers, such as other villagers 
and intermediaries, so as to help improve the adoption willingness of IoT traceability technology as a whole.

\subsection{Limitation and Future Recommendation}

In the existing literature, we found that most researchers studied the farmers' adoption intention as an exogenous variable, so this paper tried to take the adoption intention as an important endogenous variable in the distribution and promotion of new technologies. However, in practice, we clearly perceived that the willingness to adopt new technologies was only one of many endogenous variables that had not been studied. There were many factors affecting it besides those listed in this paper. In other words, our research on the popularity of IoT traceability technology and the influencing factors of adoption intention are not complete.

In future research, we should continue to explore the endogenous variables that affect the adoption of new technologies. Exploring the impact of these factors on adoption intention by developing different theoretical models to improve the integration and resilience of the supply chains will contribute to the sustainability of agricultural development. In addition, the results of this article showed that the mediating effect of perceived risk on farmers' personal innovation and willingness to adopt played a significant positive moderating role. As the result is inconsistent with previous studies, we will continue further discussion regarding whether perceived risk has a significant impact on users in different variables (e.g., gender, education, usage experience, etc.) adopting new technologies.

Author Contributions: Conceptualization, R.S. and S.Z.; methodology, R.S. and J.H.; software, R.S.; formal analysis, R.S.; investigation, R.S. and S.Z.; writing-original draft preparation, R.S.; writingreview and editing, T.W. and J.R. (Junhu Ruan); supervision, J.R. (Junyong Ruan). All authors have read and agreed to the published version of the manuscript.

Funding: This research received no external funding.

Institutional Review Board Statement: Ethical review and approval were waived for this study, due to the fact that we used anonymous data that was not retraceable to individuals at any time.

Informed Consent Statement: Patient consent was waived due to the fact that we used anonymous data that was not retraceable to individuals at any time.

Data Availability Statement: The data are not publicly available due to confidentiality reasons.

Conflicts of Interest: The authors declare no conflict of interest.

\section{References}

1. D'Adamo, I.; Rosa, P. How Do You See Infrastructure? Green Energy to Provide Economic Growth after COVID-19. Sustainability 2020, 12, 4738. [CrossRef]

2. Thacker, S.; Adshead, D.; Fay, M.; Hallegatte, S.; Harvey, M.; Meller, H.; O’Regan, N.; Rozenberg, J.; Watkins, G.; Hall, J.W. Infrastructure for sustainable development. Nat. Sustain. 2019, 2, 324-331. [CrossRef]

3. Islam, S.; Cullen, J.M.; Manning, L. Visualising food traceability systems: A novel system architecture for mapping material and information flow. Trends Food Sci. Technol. 2021, 112, 708-719. [CrossRef]

4. Nurgazina, J.; Pakdeetrakulwong, U.; Moser, T.; Reiner, G. Distributed Ledger Technology Applications in Food Supply Chains: A Review of Challenges and Future Research Directions. Sustainability 2021, 13, 4206. [CrossRef]

5. Cheng, M.X.W.B.; Li, Q.F.; He, X.Y. Research on digital equipment technology and typical experience of intelligent pig farming. Pig Farm 2021, 2, 71-72.

6. Chai, J. The current situation and development trend of IOT pig farm application in China. Pig Sci. 2020, 37, 86-89.

7. Reyna, A.; Martín, C.; Chen, J.; Soler, E.; Díaz, M. On blockchain and its integration with IoT. Challenges and opportunities. Future Gener. Comput. Syst. 2018, 88, 173-190. [CrossRef]

8. Kamilaris, A.; Fonts, A.; Prenafeta-Bold v́, F.X. The rise of blockchain technology in agriculture and food supply chains. Trends Food Sci. Technol. 2019, 91, 640-652. [CrossRef]

9. Ma, P. Application Research on Traceability System of Plateau Summer Vegetables Based on Internet of Things Technology-A Case Study of Yuzhong County, Lanzhou City. Master Thesis, Lanzhou University, Lanzhou, China, 2019.

10. Jurgilevich, A.; Birge, T.; Kentala-Lehtonen, J.; Korhonen-Kurki, K.; Pietikäinen, J.; Saikku, L.; Schösler, H. Transition towards Circular Economy in the Food System. Sustainability 2016, 8, 69. [CrossRef] 
11. Davis, F.D.; Bagozzi, R.P.; Warshaw, P.R. User acceptance of computer technology: A comparison of two theoretical models. Manag. Sci. 1989, 35, 982-1003. [CrossRef]

12. Venkatesh, V.; Morris, M.G.; Davis, G.B. User acceptance of information technology: Toward a unifified view. MIS Q. 2003, 27, 425-478. [CrossRef]

13. Barber, $\mathrm{H}$. Intentions to participate in political crowdfunding- from the perspective of civic voluntarism model and theory of planned behavior. Technol. Soc. 2020, 63, 101435. [CrossRef]

14. Robertson, T.S. The Process of Innovation and the Diffusion of Innovation. J. Mark. 1967, 31, 14-19. [CrossRef]

15. Bandura, A. Social cognitive theory of self-regulation. Organ. Behav. Hum. Decis. Process. 1991, 50, 248-287. [CrossRef]

16. Zhang, L.; Guo, Q.; Li, W.J.; Zhang, J.B. Farmers' Willingness to Adopt Rice Low-Carbon Production Technology: Influencing Factors and Intervention Paths. J. Southwest Univ. 2018, 40, 94-103.

17. Hoque, R.; Sorwar, G. Understanding factors influencing the adoption of mHealth by the elderly: An extension of the UTAUT model. Int. J. Med. Inform. 2017, 101, 75-84. [CrossRef] [PubMed]

18. Akinnuwesi, B.A.; Uzoka, F.E.; Okwundu, O.S.; Fashoto, G. Exploring biometric technology adoption in a developing country context using the modified UTAUT. Int. J. Bus. Inf. Syst. 2016, 23, 482-521. [CrossRef]

19. Alalwan, A.A.; Dwivedi, Y.K.; Rana, N.P.; Algharabat, R. Examining factors influencing Jordanian customers' intentions and adoption of internet banking: Extending UTAUT2 with risk. J. Retail. Consum. Serv. 2018, 40, 125-138. [CrossRef]

20. Yassine, J. A meta-analysis of the UTAUT model in the mobile banking literature: The moderating role of sample size and culture. J. Bus. Res. 2021, 132, 19.

21. Janet, M.M.; Nele, V.; Juan, T.C.; Andrea, G.M.; Bram, G. Understanding Smallholder Farmers' Intention to Adopt Agricultural Apps: The Role of Mastery Approach and Innovation Hubs in Mexico. Agronomy 2021, 11, 1.

22. Antony, A.P.; Leith, K.; Jolley, C.; Lu, J.; Sweeney, D.J. A Review of Practice and Implementation of the Internet of Things (IoT) for Smallholder Agriculture. Sustainability 2020, 12, 3750. [CrossRef]

23. Hossein, R.M.; Amir, F. A contextualized study of the usage of the Internet of things (IoT) in smart farming in a typical Middle Eastern country within the context of Unified Theory of Acceptance and Use of Technology model (UTAUT). Technol. Soc. 2020, 63, 63.

24. Dewi, C.K.; Mohaidin, Z.; Murshid, M.A. Determinants of online purchase intention: A PLS-SEM approach: Evidence from Indonesia. J. Asia Bus. Stud. 2019, 14, 281-306. [CrossRef]

25. Wu, X.F.; Fan, J.H. An empirical study of mobile payment usage behavior based on perceived risk. Stat. Dec. Mak. 2010, 20, $145-148$.

26. Tang, K. Development of foreign agricultural Internet of things technology and its inspiration to China. Agric. Eng. Technol. 2017, 37, 11-17.

27. Dai, J.L. Research on Fresh Pork Quality Traceability System of DLS Company Based on Blockchain and Internet of Things Coupling. Master Thesis, Shandong University, Jinan China, 2019.

28. Wen, Z.L.; Ye, B.J. Mediated model testing methods with regulation: Competition or substitution? J. Psychol. 2014, 46, 714-726.

29. Fang, J.; Wen, Z.L.; Zhang, M.Q.; Ren, H. Analysis of multilayer mediating effects based on structural equation modeling. Adv. Psychol. Sci. 2014, 22, 530-539. [CrossRef]

30. Cheung, G.W.; Lau, R.S. Accuracy of parameter estimates and confidence intervals in moderated mediation models: A comparison of regression and latent moderated structural equations. Org. Res. Meth. 2017, 20, 746-769. [CrossRef]

31. Wang, M.C.; Ye, B.J. Calculating the reliability of several commonly used tests through Mplus. Psychol. Inq. New 2014, 34, 48-52.

32. Fang, J.; Wen, Z.L. Analysis of mediating effects with moderation based on structural equation modeling. Psychol. Sci. 2018, 41, 453-458.

33. Zhuo, N.; Ji, C.; Yin, N. Supply chain integration and resilience in China's pig sector: Case study evidences from emerging institutional arrangements. Environ. Sci. Pollut. Res. 2021, 28, 8310-8322. [CrossRef] [PubMed]

34. Alonso-Muñoz, S.; González-Sánchez, R.; Siligardi, C.; García-Muiña, F.E. New Circular Networks in Resilient Supply Chains: An External Capital Perspective. Sustainability 2021, 13, 6130. [CrossRef]

35. Miceli, A.; Hagen, B.; Riccardi, M.P.; Sotti, F.; Settembre-Blundo, D. Thriving, Not Just Surviving in Changing Times: How Sustainability, Agility and Digitalization Intertwine with Organizational Resilience. Sustainability 2021, 13, 2052. [CrossRef]

36. Giudice, F.; Caferra, R.; Morone, P. COVID-19, the Food System and the Circular Economy: Challenges and Opportunities. Sustainability 2020, 12, 7939. [CrossRef]

37. Klumpp, M.; Loske, D. Sustainability and Resilience Revisited: Impact of Information Technology Disruptions on Empirical Retail Logistics Efficiency. Sustainability 2021, 13, 5650. [CrossRef]

38. D'Adamo, I.; Lupi, G. Sustainability and Resilience after COVID-19: A Circular Premium in the Fashion Industry. Sustainability 2021, 13, 1861. [CrossRef]

39. Fedushko, S.; Ustyianovych, T.; Gregu, M. Real-Time High-Load Infrastructure Transaction Status Output Prediction Using Operational Intelligence and Big Data Technologies. Electronics 2020, 9, 668. [CrossRef]

40. Wissal, B.A.; Imed, B.B.; Galina, K.; Lubica, H. The role of trust in intention to use the IoT in eHealth: Application of the modified UTAUT in a consumer context. Technol. Forecast. Soc. Chang. 2021, 167, 120688. 



\title{
Solar Photovoltaic Architecture and Agronomic Management in Agrivoltaic System: A Review
}

\author{
Mohd Ashraf Zainol Abidin 1,2(D), Muhammad Nasiruddin Mahyuddin 2,*(i) \\ and Muhammad Ammirrul Atiqi Mohd Zainuri ${ }^{3}$ \\ 1 Faculty of Plantation and Agrotechnology, Universiti Teknologi MARA, Perlis Branch, Arau Campus, \\ Arau 02600, Perlis, Malaysia; ashrafzainol@uitm.edu.my \\ 2 School of Electrical and Electronic Engineering, Engineering Campus, Universiti Sains Malaysia, \\ Nibong Tebal 14300, Pulau Pinang, Malaysia \\ 3 Department of Electrical, Electronic and Systems Engineering, Faculty of Engineering and Built Environment, \\ Universiti Kebangsaan Malaysia, Bangi 43600 UKM, Selanor, Malaysia; ammirrulatiqi@ukm.edu.my \\ * Correspondence: nasiruddin@usm.my
}

Citation: Zainol Abidin, M.A.; Mahyuddin, M.N.; Mohd Zainuri, M.A.A. Solar Photovoltaic Architecture and Agronomic Management in Agrivoltaic System: A Review. Sustainability 2021, 13, 7846. https://doi.org/10.3390/su13147846

Academic Editor: Idiano D'Adamo

Received: 10 June 2021

Accepted: 29 June 2021

Published: 14 July 2021

Publisher's Note: MDPI stays neutral with regard to jurisdictional claims in published maps and institutional affiliations.

Copyright: (c) 2021 by the authors. Licensee MDPI, Basel, Switzerland. This article is an open access article distributed under the terms and conditions of the Creative Commons Attribution (CC BY) license (https:// creativecommons.org/licenses/by/ $4.0 /)$.

\begin{abstract}
Agrivoltaic systems (AVS) offer a symbiotic strategy for co-location sustainable renewable energy and agricultural production. This is particularly important in densely populated developing and developed countries, where renewable energy development is becoming more important; however, profitable farmland must be preserved. As emphasized in the Food-Energy-Water (FEW) nexus, AVS advancements should not only focus on energy management, but also agronomic management (crop and water management). Thus, we critically review the important factors that influence the decision of energy management (solar PV architecture) and agronomic management in AV systems. The outcomes show that solar PV architecture and agronomic management advancements are reliant on (1) solar radiation qualities in term of light intensity and photosynthetically activate radiation (PAR), (2) AVS categories such as energy-centric, agricultural-centric, and agricultural-energy-centric, and (3) shareholder perspective (especially farmers). Next, several adjustments for crop selection and management are needed due to light limitation, microclimate condition beneath the solar structure, and solar structure constraints. More importantly, a systematic irrigation system is required to prevent damage to the solar panel structure. To summarize, AVS advancements should be carefully planned to ensure the goals of reducing reliance on non-renewable sources, mitigating global warming effects, and meeting the FEW initiatives.
\end{abstract}

Keywords: agrivoltaic system; solar photovoltaics; agronomic management; crop production; FoodEnergy-Water nexus; sustainable integration

\section{Introduction}

The concept of integrating solar PV with agricultural produce, known as agrivoltaic system (AVS), was originally proposed by [1] back in 1982; however, this concept was rarely discussed until the beginning of the new millennium. This agrivoltaism approach is derived from the intercropping method applied in the agricultural sector to increase the land equivalent ratio and total revenue [2-6]. AVS technology is gaining popularity due to its dependability in variable-scale applications. The development of commercial and research facilities around the world demonstrates the potential of this technology $[5,7,8]$. The concept of the system utilizes the generation of electrical energy and the production of agricultural products in the same area of production [6,9-12]. In other words, beneficial interaction, or symbiosis between these two productions in the same area, is created in this way [2,3,8,13-16]. Solar production could also offset global energy demand if less than $1 \%$ of cropland were converted to an AV system [17]. The integration of both productions in the same area may reduce the efficiency of either solar energy generation, agricultural production, or both productions; however, the total revenue may be increased $[7,15,18-21]$ 
as illustrated in Figure 1. Additionally, crop production consumes less than $1 \%$ of the total energy generated by AVS [10].

\section{Separate Land Use On 2 Hectare Cropland}

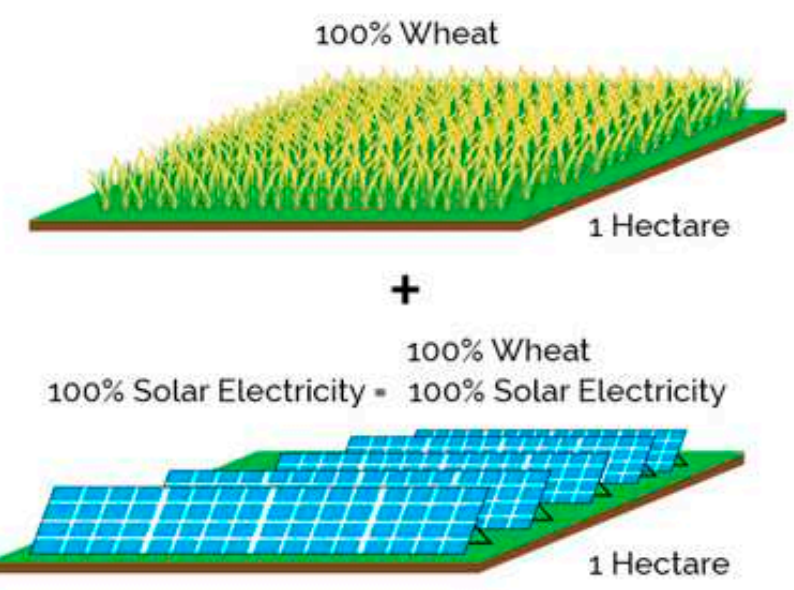

Combined Land Use On 2 Hectare Cropland: Efficiency Increases Over $60 \%$

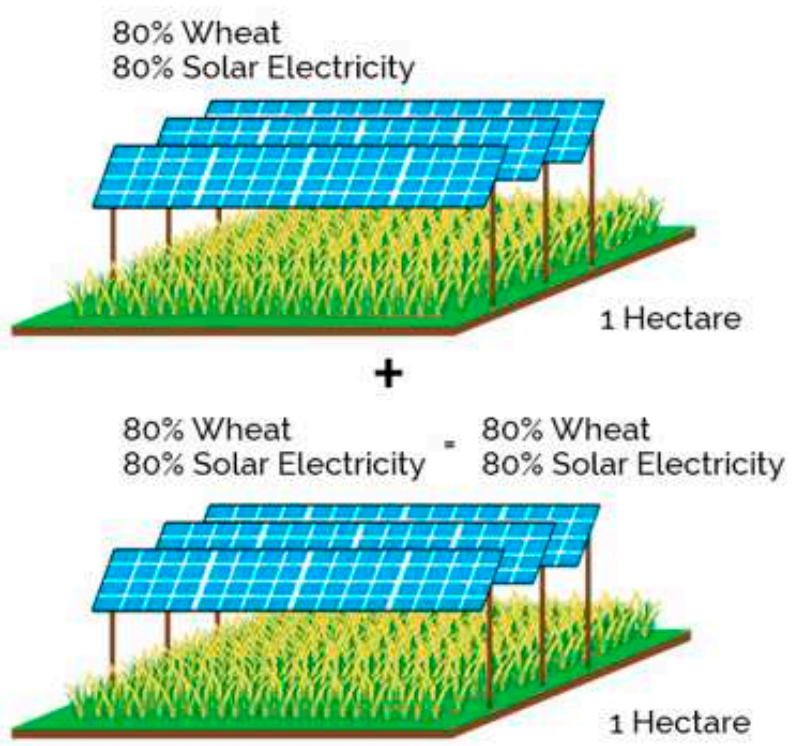

Figure 1. Comparison of efficiency traditional farming versus AVS. (Source: [22]).

\subsection{Motivation for the Development of Agrivoltaic System}

There is a connection between sustainability and resilience, and COVID-19 has illustrated how rapidly life can change. The work of [23] demonstrates that the deployment of new infrastructure lowers land-use availability, and this element must be managed properly. Despite the profound upheaval and uncertainty produced by the Covid-19 epidemic in the energy industry, which has forced global energy consumption to decline by $5 \%$ by 2020, renewables continue to play a critical part in all of our circumstances, with solar taking center stage [24]. The rapid development of solar farms raises a new threat and friction in terms of land-use for electricity production whilst satisfying increasing food demand. The amount of land required to establish large-scale solar farms has become a source of concern $[8,13,25-27]$.

Current studies also show that the environmental factors that may influence the efficiency of photovoltaic (PV) panels are sometimes ignored [12,17,28-31]; however, for AVS, these factors are very crucial [32,33]. For example, gravel underlay for solar PV sites may contribute to a heat island effect that increases the ambient temperature below the PV structure. This situation potentially reduces the efficiency and life span of the solar panel $[3,10,11,34,35]$. Simultaneously, agricultural lands are shrinking due to land reuse for new industry production, homes, and urban areas [8,10,36-39]. The summary report of World Agriculture: Towards 2015/2030, released by [40], has stated that the global demand for an agricultural product will keep on increasing every year. Developed countries will suffer from a high dependency on agricultural imports, and food insecurity in developing countries will persist without a significant increase in local production. These conflicts are compounded by the fact that the amount of arable land available per capita decreased by 48 percent between 1961 and 2016, owing to the global population increase [41]. Thus, it is believed that the solution of AVS technologies enables the possibility of resolving the competing interest between the two sectors' requirements while meeting the demand $[14,21,42-44]$. 


\subsection{Benefits of Agrivoltaic System}

There are a variety of fascinating details about AVS technology derived from previous studies such as: (1) increase in total revenue [7,15,19-21,45,46]; (2) crops cultivated beneath the solar structure help reduce the ambient air temperature by creating a cooler microclimate [7,11,47-49]; hence, indirectly reducing the solar panel temperature up to $1-2{ }^{\circ} \mathrm{C}$ and increasing the solar PV efficiency [21,50]; (3) solar PV panels must be washed regularly to maintain their solar radiation efficiency. The water used to clean them can be reused to irrigate the agriculture beneath the solar panel, resulting in increased water efficiency $[2,13,21,26,34,51]$; (4) emissions due to $\mathrm{CO}_{2}$ are also uptaken by crops, while low $\mathrm{CO}_{2}$ is produced by solar energy compared to fossil fuel-based power generation [2,20,43]; (5) solar PV provides a good shading effect on some plants that do not like direct sunlight [30,39,52-55]; (6) providing new jobs [12,21,27,51,56]; (7) raising taxes [20] and (8) the expansion of cleaner and renewable energies are necessary to reduce the fossil fuel dependency and global warming $[9,28,29,41,46,57-59]$. Furthermore, efforts to reduce $\mathrm{CO}_{2}$ emissions and promote sustainable energy are always gaining international support [60]. Thus, these AVS initiatives are highly in line with Food-Energy-Water (FEW) nexus $[9,13,14,34,61]$ and Sustainable Development Goals (SDGs) [28,32,56,62-64].

\subsection{Land Equivalent Ratio}

Land equivalent ratio (LER) is a method used for measuring the efficiency of land utilization for the simultaneous production of crops and electricity $[11,18,53,55,65,66]$. A similar technique is used in agroforestry systems, which integrate trees and food crops $[4,41,66,67]$. The $L E R$ can be extended to include the mixing of any two (or more) production systems in the same area. Numerous previous studies have also used this method to identify the possibility of integrating agriculture production with a solar farm. In general, $L E R$ is the ratio of the AVS installation's area to the total of the areas required to meet the AVS installation's agricultural and electric production [18]:

$$
L E R=\frac{F M_{A V S}}{F M_{C P}}+\frac{E_{A V S}}{E_{P V}}
$$

where $F M_{A V S}$ and $F M_{C P}$ denote fresh biomass in the $A V S$ installation and on the $C P$ control plot (agricultural mono-production), while $E_{A V S}$ and $E_{P V}$ denote the $A V S$ installation's and $\mathrm{PV}$ installation's electric production, respectively. $L E R$ values greater than one indicate that combining agriculture and solar $P V$ is more efficient than doing so separately $[11,37,68]$. To be noted, the efficiency of AVS technology is highly dependent on the solar PV architecture $[4,6,21,28,51,53,69,70]$ and agronomic management $[6,25,31,53,54,56]$. Numerous methods are constantly being developed to improve the effectiveness of this technology. However, AVS applications are still in their early stages, therefore there is plenty of room for technological advancements and expanded application fields [14,34,61,62,71-73]. Thus, this paper is written to discuss the consideration of $A V S$ architecture with design criteria for solar photovoltaic systems and agronomic managements to improve the AVS outputs.

\section{Design Consideration for Agrivoltaic System}

\subsection{The Importance of Solar Radiation for Energy Generation and Crop Cultivation}

Solar radiation is an important factor in photosynthesis, which is the process by which plants and other autotrophic organisms convert light energy from the sun into chemical energy that can be used to synthesize carbohydrates and power the organisms' activities [28,34,55,73-75]. At the same time, solar radiation can also be converted to electricity using solar power systems. Solar systems are classified into two types: photovoltaic (PV) systems that convert sunlight directly to electricity using semiconductor materials [36,76-79] and concentrated solar power (CSP) systems that convert sunlight to heat before using the heat to produce electricity $[30,41,46,51,80]$. Despite the fact that both processes need solar radiation to occur, the photosynthesis process is concerned more with 
the Photosynthesis Active Radiation (PAR) range [47,52,68,81-83] while electrical energy generation is dependent on solar irradiance or light intensity $[8,15,35,84,85]$.

\subsubsection{Photosynthetically Active Radiation}

Photosynthesis is a light-dependent process that occurs best in the visible light spectrum. As shown in Figure 2, visible light has a wavelength range of 400-700 nm. Ultraviolet cannot be used for photosynthesis because it has too much energy which can disrupt molecular bonding, and destroy DNA and other important organismal structures. On the other end of the spectrum, infrared light does not contain enough energy to sufficiently excite electrons in molecules for photosynthesis [86]. The range of light wavelengths that is most suitable for photosynthesis is called Photosynthetically Active Radiation (PAR) [87]. Since photosynthesis is a quantum process, PAR is expressed in terms of Photosynthetic Photon Flux Density (PPFD, $\mathrm{mol} \cdot \mathrm{m}^{-2} \cdot \mathrm{s}^{-1}$ ), or in terms of Photosynthetic Radiation Flux Density, $\mathrm{Q}_{\mathrm{p}}\left(\mathrm{PAR}\right.$ irradiance, $\left.\mathrm{W} \cdot \mathrm{m}^{-2}\right)$, which is more relevant to energy balance studies $[47,81,83]$.

\subsubsection{Light Intensity}

In contrast, the intensity with which radiation enters the atmosphere is the solar irradiation, $R_{s}[28,77]$. It is the amount of radiant flux incident on a surface and is expressed in watts per square meter $\left(\mathrm{W} \cdot \mathrm{m}^{-2}\right)$. The $\mathrm{R}_{\mathrm{s}}$ parameter is frequently integrated over time to determine the radiant energy emitted into the surrounding environment (joules per square meter, $\mathrm{J} \cdot \mathrm{m}^{-2}$ ) during that period $[88,89]$. This total solar irradiance is referred to as solar irradiation. The amount and intensity of $\mathrm{R}_{\mathrm{s}}$ that the earth's surface receives depends on a variety of factors such as cloud cover [81,90-93], latitude [6,73,80,94], altitude [77,78,90,95], season $[73,93,96-98]$, weather $[19,38,40,99,100]$, and daytime [34,46,48,76,101]. In addition, the distance that radiation has to travel is dependent on the angle of the sun $[88,93]$. The greater the angle, the lower the solar intensity, which is why the $\mathrm{R}_{\mathrm{s}}$ parameter is less intense in the morning and evening than at noon $[89,101]$. Furthermore, the annual net Rs is higher at the equator than at the northern and southern poles [28,102]. Also, as a hemisphere is tilted away from the sun, the $R_{s}$ value decreases $[68,96,103,104]$.

\subsubsection{Correlation between Photosynthetically Active Radiation and Light Intensity}

Though $R_{s}$ and $Q_{p}$ are correlated, photosynthetic and photovoltaic systems have distinctive requirements in solar radiation quality and quantity [74]. The quality of solar radiation absorbed by PV panels can be tailored to capture a specific solar spectrum or the entire solar spectrum (Figure 2A). In contrast, the absorption spectra of plants are determined by their photosynthetic pigments (Figure 2B). The amount of solar radiation absorbed and used to create products further distinguishes plants and solar panels. The electrical output of solar panels is usually linearly proportional to the intensity of incident light. While plants require radiation energy to generate biomass, this does not correlate linearly above a certain intensity because the rate is limited by numerous linked, complex metabolic steps [97]. Next, owing to the lack of ground measurements of the $Q_{p}$ parameter, the improvement of plant-growth models also necessitates precise estimations of the $Q_{p}$ values, which are also indirectly determined based on their interaction with the $R_{s}$ parameter [105]. Acting to balance the needs of solar radiation between PV panels and crops may be able to further improve the efficiency of electricity generation and agriculture yield production in AV systems. Thus, the AVS design should pay attention to how to maximize the exposure of high quality solar irradiation to the PV panels and the exposure of optimal PAR flux to the crop underneath the panel structures $[8,21,87]$. 


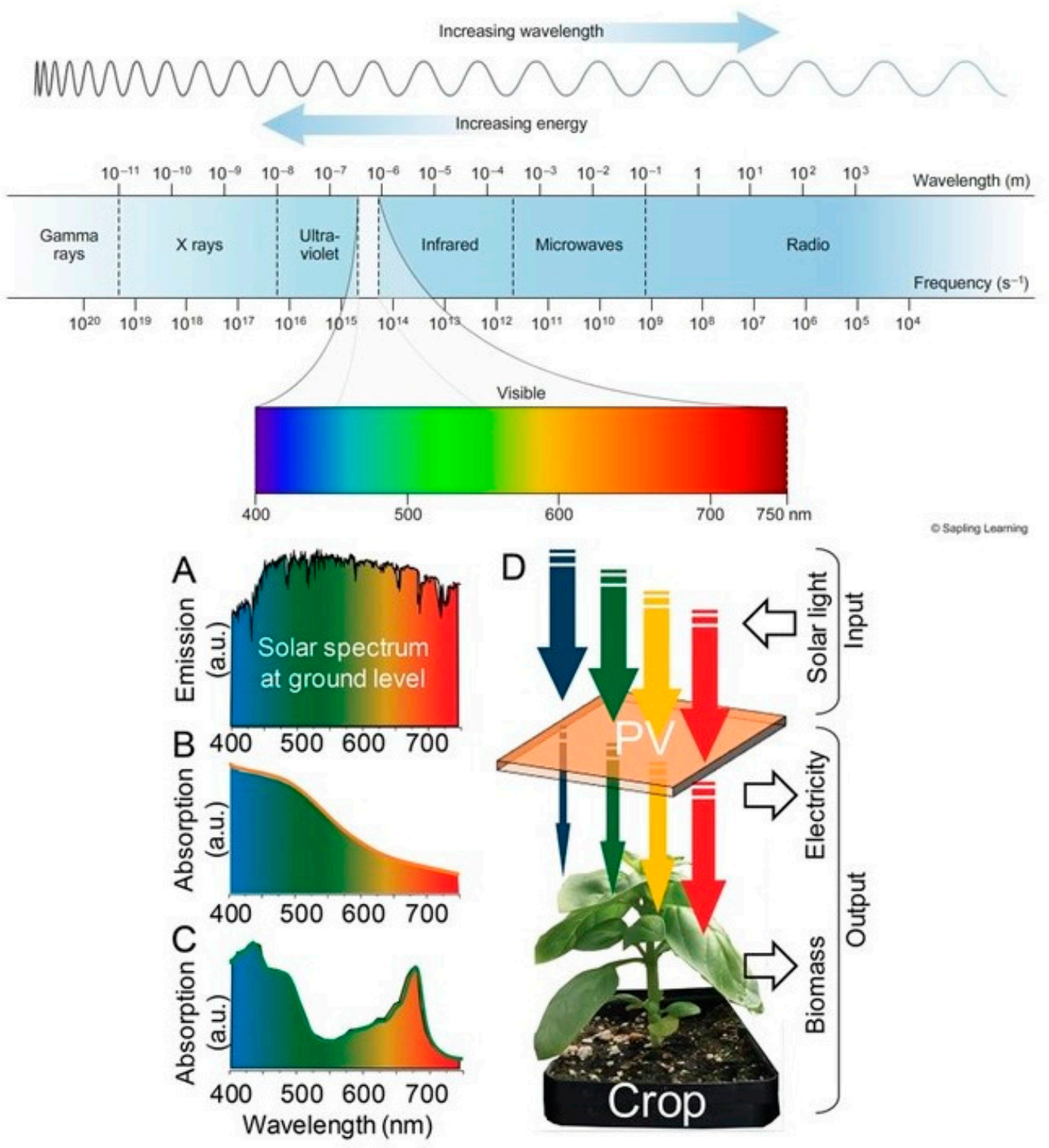

Figure 2. Electromagnetic spectrum. (A) Solar radiation spectrum in the visible range at the ground level. (B) Absorption spectrum for PV panel. (C) Absorption spectrum for a crop. (D) Schematic representation of the input (solar light) and the two contextual outputs of AVS (i.e., electricity and biomass). (Modified from original. The electromagnetic spectrum, source: [106]; The absorption spectrum, source: [97]).

\subsection{Integration of Solar Energy and Agriculture}

The integration of large-scale solar power and agriculture has the capacity to ensure efficient energy generation and to sustain agricultural production with minimal environmental impacts $[33,92,95]$. This act to co-locate is characterized as the intentional development of agriculture and energy in the same location $[17,34,63]$. Agriculture growth could occur beneath or near energy infrastructure $[6,9,16]$. There are three types of colocation opportunities and approaches: energy-centric, agricultural-centric, and integrated agricultural-energy-centric [44,107]. Energy-centric approaches to the co-location of solar energy developments and agriculture are marked by behaviours that optimize solar energy production while minimizing changes to solar construction best practices and fostering agriculture growth under and around the solar installation. Energy-centric methods could be better suited to areas with large amounts of land earmarked for solar energy production or areas where solar development has already taken place [107]. For example, when [1] first introduced the concept of agrivoltaic in 1982, the proposed design was focused on energy production. A minimal modification of the PV structure has been made to allow light to 
reach the ground at the gap between the rows of solar panels, allowing crops to be planted there. Next, Tenaga Nasional Berhad (TNB) has developed a 50 MW solar farm in Selangor, Malaysia, as part of the Large-Scale Solar (LSS) initiative led by the Malaysian government. The project was created to generate renewable energy [108], and the AVS concept was used indirectly in these solar farms by planting grass to help maintain soil stability.

Agricultural-centric approaches to the co-location of solar energy and agriculture are defined as actions that serve to optimize biomass production activities and mitigate alterations to current plant management activities, while still integrating solar energy production activities. In areas with limited land or that are already established agricultural areas, agriculture-centric methods could be a good fit [44,107]. For example, as illustrated in Table 1, Ref. [54] has conducted a study to assess the integration of different PV module arrangements (i.e., (a) straight pattern and (b) checkboard pattern) with different orientations (E-W orientation and N-S orientation) for greenhouse production in Decimomannu (Sardinia, Italy). The study reveals fundamental assumptions, stating that the agricultural efficiency of the land being used is not sacrificed for the sake of solar generation. Ideally, optimizing solar energy generation does not significantly alter standard agronomic management and has an insignificant impact on agriculture yields. Meanwhile, [63] highly recommends the model AVS of the Junagadh Agriculture University (JAU) to the farmer's community in India. The model is said to be self-sufficient in terms of energy for agricultural and other operations. The crop production has been recorded as $15 \%$ higher under this type of system than under open field conditions. The farmer can also make additional money by generating electricity revenue.

Table 1. Approaches of agrivoltaic system.

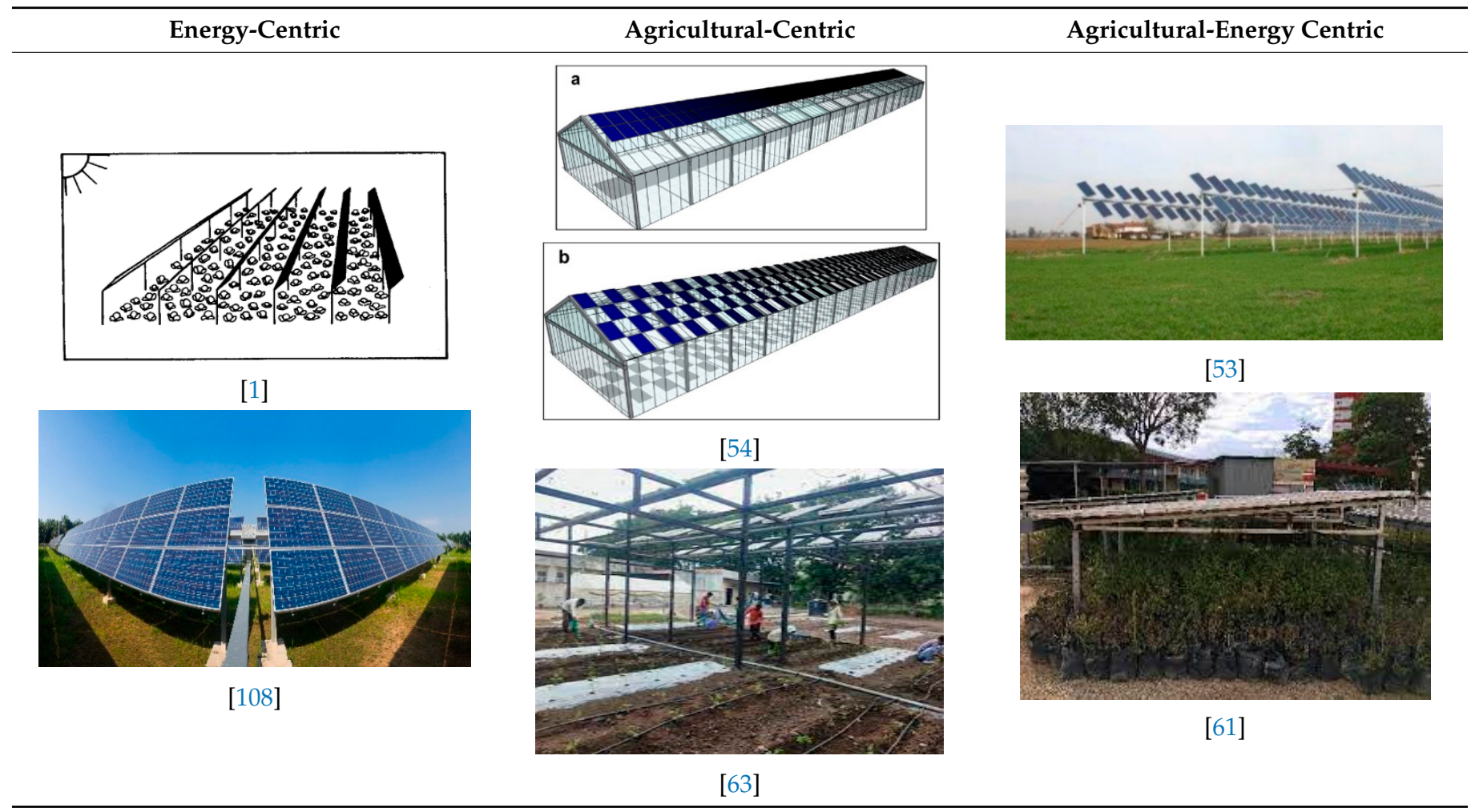

Next, there are hybrid methods that aim to combine both energy performance and agriculture production targets, in addition to agriculture-centric and energy-centric approaches to the co-location of solar energy technologies and agriculture. Known as agriculturalenergy-centric, these methods could result in lower agriculture and energy production; however, other advantages make co-location preferable, including additional revenue streams [107]. These methods are differentiated by the integration of both agriculture 
and energy priorities into system designs. Incorporating agriculture and energy into the same area of production could be suitable: (1) on an existing solar farm or farmland, and (2) on underdeveloped land [109]. However, hybrid approaches to entail alterations for both agriculture and solar energy production methods can range from major structural changes to minor tweaks [107]. Several prior experiments focused on these methods have been carried out. There are modifications to be made to the solar energy system, such as altering the solar panel's structure $[6,53]$ and using a specific algorithm for agricultural growth [4,53], but also in terms of using agronomic practices $[33,59]$ to maximize the output $[6,8,47,56]$. Table 1 shows several previous studies based on different approaches to the agrivoltaic system.

Based on the description of AVS approaches, the developers, energy providers, and farmers need to decide which AVS approach is the best suited to their interest. It is important to note that, although investing in AVS may be of benefit to them in the longterm, at the moment it is still an open debate as to whether the high capital cost in the installation of solar panel would be prudently compensated when farmers have a diverse financial profile and risk adversity $[41,44]$.

\subsection{Agricultural Sector Perspective on Agrivoltaic System}

Policymakers should conduct sector-wide social impact scoping (SSIS) for renewable energy technology (RET) to elicit diverse perspectives from previously reclusive stakeholders, and to gradually increase the positive impact and mitigate the negative impact of RET [110]. For agrivoltaic system technology (AVS), the fundamental focus in order to be successful is the farmers' perception of the application, challenges, and opportunities of this dual land-use system $[12,44]$. Experts in the agriculture sector believe that AVS installation seemed to be able to produce a positive impact for themselves, with several defining constraints to the acceptance of AV technology. Among the constraints are (1) the need for long-term land productivity. Farmers, for example, are concerned about the longevity of permanent solar panel systems and the limitation of agricultural maintenance due to the permanence of all solar panels and the scale of the plot [109-111]; (2) farmers are concerned about demand insecurity as a result of the many unknown procedures associated with this dual land-use technology $[2,9,44,46]$; (3) farmers are looking for compensation if a portion of their property is taken for the installation of solar panel structures [38,44,52,112]; (4) built-in versatility to accommodate different sizes and types of activities, as well as evolving farming practices [54,109]; (5) the implementation of specific AVS approaches that have both positive economic and no non-negative environmental effects $[62,110,113]$; (6) in some cases, design of PV infrastructure should prioritize potential reversibility [3]; (7) proactive awareness-building events for AVS promotion $[10,11,15,110]$.

Despite these constraints, there was a significant gap in the social impacts perceived by farmers who had already implemented AVS technology and those who had not [110]. Most farm operators who had already implemented AVS thought that the ultimate effect had been more positive than negative, including the positive impact of stable income and sustaining stable agricultural productivity as a result of electricity sales [21]. A considerable number of these interviewees were motivated by the increased revenue from selling AVSgenerated energy to keep their farms rather than to farm [14,110]. Income from electricity production was sufficient to pay for strenuous farming work and provided additional gratification due to the possibility of conserving farmlands. To their minds, this dualland technology was not a hindrance to growth; instead, they considered it an additional resource to work with [110]. However, in a case study in China, the act of agrivoltaism with an innovative business model shows a promising economic performance for both productions. The Annual Return on Investment (AROI) increased by about 9\% to 20\%, with a discounted payback period ranging from four to eight years depending on the crops produced in this integration scheme. According to the study, sensitivity and uncertainty analyses indicate that the most sensitive aspect is crop price. The significance of the electricity feed-in tariff is somewhat less than we expected [20]. In this case, the AVS firms 
are advised to prioritize crop planting [20] without neglecting energy production [44]. At the same time, policymakers should be more flexible in terms of solar energy and agricultural incentives [3,20], depending on AVS approaches [107].

Following that, based on the level of growth of the AVS industry, various government officials may be tasked with setting specific quantity targets, ranging from prototype installations to widespread adoption [2,34]. Either an AVS price control system could be used to grant an add-on to an already commissioned solely ground-mounted photovoltaic (PV-GM) development program, compensating for the cost of technology adaptation, or a separate AVS funding mechanism could be established [41]. Governments will be asked to legally identify AVS, adopting a nationwide AVS standard that will guarantee high-quality AVS execution, in order to prevent windfall gains [2,41], improve social recognition, and resolve land rivalry between solar PV and agriculture [44]. In comparison to PV-GM, AVS technology is still in its infancy, with a high learning curve but the scope for even more techno-environmental synergies. The dual role of AVS, which protects agricultural yields while also producing solar electricity $[7,27,51,54]$, boosts economic production per square meter $[13,46]$ and strengthens farmers' resistance to the effects of global warming by securing and diversifying their sources of income [14,59]. The implementation of financial support schemes to conserve cropland, diversify farmers' income sources, and counteract rural exodus in some countries was the driving political goal behind the introduction of AVS diffusion regulations $[38,41,114]$. With proper implementation, this technology is expected to accelerate development and lead to farmer yield increases, as well as environmental sustainability $[2,19,115]$.

\section{Solar Photovoltaic Architecture in Agrivoltaic System}

\subsection{Alteration and Modification of Solar Photovoltaic}

A solar photovoltaic (PV) system is a power generation unit made up of an electrically integrated assembly of a PV array, inverter, and other components. PV panels (also called $\mathrm{PV}$ modules) are composed of several photovoltaic cells that convert sunlight energy to electricity. The solar PV modules are wired together in series to form PV strings, which are then linked together in parallel to form a PV array (MS IEC 61836:2010) [116]. The process is simple as it is a direct conversion of sunlight to electricity without any complicated mechanical movement or release of waste to the surrounding environment [52,78,92]. The solar PV cells can absorb up to $80 \%$ of the incident solar radiation received from the solar band, but only a small amount of this absorbed energy is transformed into electricity, with the rest increasing the temperature of the cells $[89,117,118]$. Next, grid-connected and stand-alone solar systems are the two types of solar energy systems. These two systems are intended to offer a direct current or alternating current source for use with the utility grid, an independent storage system, or other electrical/electronic equipment $[77,93,119,120]$. Even though much of the photovoltaic system demand can be matched with aggressive building-integrated PV (BIPV) and rooftop PV [79,87,95,102], both systems cannot provide all the energy necessary, especially for regions with high population densities compared to land-based solar farms [8,95]. A solar farm is designed to generate enough energy [37] to power thousands of homes and business $[9,121]$. They are much like the solar panels you place on your roof to power your specific requirements [28]. Most energy generated by solar farms is sold to the grid. However, to sell power to the grid first you need approval from your country's power provider and authorities [32,122].

The AVS idea is mainly based on photovoltaic technology being adapted for agricultural use, considering space and wiring restrictions $[30,38,117,123,124]$. However, AVS is configured differently from typical ground-mounted photovoltaic systems (PV-GM), which are more prevalent because of their cheaper installation costs and higher panel density, which improve energy production $[3,25,36,62]$. The systems in traditional ground-mounted solar installations are immobile $[2,36,93]$. For this solar installation, the PV panels are around 1.6 feet $(0.5 \mathrm{~m})$ from the ground, and space between rows is kept to a minimum to avoid shade. Also, panel clusters do not have spacings since they abut [125]. Certain 
modifications of the solar PV structure to suit the agricultural production requirements are needed to allow optimum solar radiation to reach the ground under the solar PV structure. Among the modifications are: (1) elevation of solar PV structure [25,28,65,94,112]; (2) optimizing the distance between solar PV structure [15,25,68,95]; (3) configuring of the density of solar panels in one solar structure $[4,6,49,87]$; (4) optimization of the sloping angle of the solar panel $[4,6,113,115]$. Nevertheless, AVS developers should keep in mind that all modifications and alterations to solar photovoltaic structures would adhere to AVS approaches [91,107,109], geographical regions [19,65], crop selection $[33,65,69]$, and agronomic management $[18,19,33]$.

Several studies have been conducted at Montpellier Experimental Agrivoltaic Station since 2010 by $[4,6,18,69]$ to determine the characteristics of the photovoltaic structure to be integrated into the AV system. Full-density (FD) and Half-density (HD) AV systems have been developed and tested in this facility for almost 10 years. FD structure is designed for optimal solar energy production, thus only allowing around $50 \%$ of solar radiation to the crop below the panel. While HD structure is designed to balance between electric generation and agriculture production, thus allowing up to $70 \%$ of solar radiation to reach the crop level. The percentage of light transmission was determined under the FD and HD experimental panels. To obtain the percentage of targeted solar radiation, the FD and HD structures were designed at $5.0 \mathrm{ft}(1.6 \mathrm{~m})$ and $10.0 \mathrm{ft}(3.2 \mathrm{~m})$ in the panel row spacing, respectively. Moreover, both panels were mounted $13.0 \mathrm{ft}(4.0 \mathrm{~m})$ above the ground and tilted at an angle of 25 degrees. The elevated PV panels make the spatial distribution of radiation increase because light can penetrate underneath the panels from the sides, thus creating conditions where crops are able to grow below the panels [53]. In another study conducted at UMass Crop Research Farm (Massachusetts), the AVS structure without concrete bases was constructed with a height of $7.5 \mathrm{ft}(2.3 \mathrm{~m})$ from the ground to allow nearly $70 \%$ of solar radiation to reach the crop below the panel. The study found that $4.0 \mathrm{ft}(1.2 \mathrm{~m})$ and $5.0 \mathrm{ft}(1.5 \mathrm{~m})$ distances are optimal for the AVS plots for biomass production [125]. These conclude that the modification of panel rows spacing, the height of mounted panels, configuration of the density of the solar panel, and the tilting angle are variable and dependent on the specific geographic location. In addition, the growing season also contributed to AVS adjustment $[18,25,45]$.

In addition to the modification of the tilted monofacial PV structure, a vertical bifacial PV structure could be an alternative to AVS infrastructure [5,7,47,65]. According to [65], vertical bifacial PV provides several advantages, including less land coverage, less interference with agricultural machinery and rains, natural resilience to PV soiling, faster cleaning, and cost savings owing to a possible lower elevation. However, the combined PAR/energy yields for this type of arrangement may not always be superior [65]. Still, the trade-off between a higher capital expense and a low cost for frequent cleaning should be carefully considered when calculating the relative Levelized Cost of Energy (LCOE) [41,62,65].

Next, instead of modifying the PV structure and use of opaque photovoltaic (OPV) as modules, there is also a study conducted to determine the potential of using semitransparent photovoltaic (STPV) modules in AV system $[76,91,126]$. The idea is to turn the agrivoltaic principle from just solar sharing to the selective use of various light wavelengths $[97,126]$. The solar sharing concept is a common concept in conventional AVS that uses OPV modules and creates a shade to the portion of farm fields, throwing a shadow on the underlying plants $[97,127,128]$. However, The STPV's eclipsing frequency was 9.7 percent, and the cell shadow never completely covers the plants while the gap between the module and the crop is greater than $1 \mathrm{~m}$ [126]. Next, as illustrated in Figure 2, since the electrical energy generation capacity also varies depending on the light spectrum $[55,78,117,129]$, special attention should be paid to identify suitable types of crops that are able to carry out the photosynthesis process with the limitation of certain PAR wavelengths $[28,47,69]$ to be integrated with SPTV modules in agrivoltaic system $[97,126]$. In addition, Table 2 summarizes some of the studies that have been conducted worldwide to improve AVS technology. 
Table 2. Overview of Existing Agrivoltaic System Research Project.

\begin{tabular}{|c|c|c|c|c|c|c|c|c|}
\hline Location & $\begin{array}{l}\text { Electricity Yield } \\
\left(\mathrm{kWha}^{-1}\right)\end{array}$ & Capacity (kWp) & $\begin{array}{c}\text { Solar } \\
\text { Tracking }\end{array}$ & PV Specification & Cultivated Crops & Sub-Treatment & Highlights & Source \\
\hline $\begin{array}{l}\text { Oregon State } \\
\text { University, USA }\end{array}$ & unknown & 1435 & No & $\begin{array}{l}\text { Polycrystalline, east-west } \\
\text { oriented strips, } 1.65 \mathrm{~m} \\
\text { wide and inclined } \\
\text { southward with a tilt } \\
\text { angle of } 18^{\circ}, 1.1 \mathrm{~m} \text { above } \\
\text { ground (at lowest point) } \\
\text { and distance between } \\
\text { panel is } 6 \mathrm{~m}\end{array}$ & semi-arid pasture & $\mathrm{SFO}, \mathrm{SPO}, \mathrm{SFC}$ & $\begin{array}{l}\text { Extreme heterogeneity and spatial } \\
\text { gradients in biomass production } \\
\text { and soil moisture were observed as } \\
\text { a result of the heterogeneous shade } \\
\text { pattern of the PV array. }\end{array}$ & {$[5,44]$} \\
\hline Sardinia, Italy & $\begin{array}{l}\text { E-W } 1547 \text { N-S } 1330 \\
\text { (100\% Mono-pitched } \\
\text { roof), E-W 1562 N-S } \\
1290 \text { (60\% Venlo-type), } \\
\text { E-W 1553 N-S 1317 } \\
\text { (50\% Gable roof), E-W } \\
1523 \text { N-S } 1292 \text { (25\% } \\
\text { Gable roof) }\end{array}$ & $\begin{array}{c}71 \text { (100\% } \\
\text { Mono-pitched roof), } \\
47 \text { (60\% Venlo-type), } \\
35 \text { (50\% Gable roof), } \\
20 \text { (25\% Gable roof) }\end{array}$ & No & $\begin{array}{c}\text { Multicrystalline and } \\
\text { Monocrystalline, PV } \\
\text { greenhouse } \\
\text { (mono-pitched, } \\
\text { venlo-type, gable roof). } \\
\text { East-west and } \\
\text { north-south orientations. } \\
\text { PV cover ratios ranging } \\
\text { from } 25 \% \text { to } 100 \%\end{array}$ & Unknown & unknown & $\begin{array}{l}\text { (1) Both the checkerboard pattern } \\
\text { and the N-S orientation allowed to } \\
\text { improve the uniformity of } \\
\text { light distribution. } \\
\text { (2) A valid design criterion to } \\
\text { improve the agronomic } \\
\text { sustainability of next-generation } \\
\text { PV greenhouses }\end{array}$ & [54] \\
\hline Japan & unknown & Unknown & No & $\begin{array}{c}\text { Installing } \\
\text { semi-transparent PV } \\
\text { module (STM) on the } \\
\text { greenhouse roof }\end{array}$ & Unknown & unknown & $\begin{array}{l}\text { (1) The conversion efficiency of the } \\
\text { semi-transparent module (STM) } \\
\text { was stable at around } 0.2 \% \text { and was } \\
\text { not affected by the slope angle, } \\
\text { because of the isotropic } \\
\text { photoreception of the } \\
\text { spherical microcells. } \\
\text { (2) The eclipsing level of the STM } \\
\text { was } 9.7 \% \text { and the cell shadow never } \\
\text { covers the plants entirely when the } \\
\text { distance between the module and } \\
\text { the crop is greater than } 1 \mathrm{~m}\end{array}$ & [126] \\
\hline
\end{tabular}


Table 2. Cont.

\begin{tabular}{|c|c|c|c|c|c|c|c|c|}
\hline Location & $\begin{array}{l}\text { Electricity Yield } \\
\left(\mathrm{kWha}^{-1}\right)\end{array}$ & Capacity (kWp) & $\begin{array}{c}\text { Solar } \\
\text { Tracking }\end{array}$ & PV Specification & Cultivated Crops & Sub-Treatment & Highlights & Source \\
\hline \multirow[t]{2}{*}{$\begin{array}{c}\text { Montpellier } \\
\text { Experimental } \\
\text { Agrivoltaic Station, } \\
\text { France }\end{array}$} & \multirow[t]{2}{*}{ Unknown } & \multirow[t]{2}{*}{ Unknown } & No & $\begin{array}{l}\text { Monocrystalline, panels were } \\
\text { mounted } 13 \mathrm{ft}(4 \mathrm{~m}) \text { above } \\
\text { the ground, } 14 \text { degree aspect } \\
\text { angle orientation of the } \\
\text { panels towards East, tilted at } \\
\text { an angle of } 25 \text { degrees, space } \\
\text { every } 1.64 \mathrm{~m} \text { (distance } \\
\text { between panel structure) }\end{array}$ & $\begin{array}{l}\text { lettuces (short cycle } \\
\text { crop), cucumbers } \\
\text { (short cycle crop), } \\
\text { and durum wheat } \\
\text { (long cycle crop) }\end{array}$ & $\begin{array}{l}\text { FD (50\% light } \\
\text { allowable) } 1.6 \mathrm{~m} \\
\text { panel spacing, } \\
\text { HD (70\% light } \\
\text { allowable) } 3.2 \mathrm{~m} \\
\text { panel spacing }\end{array}$ & $\begin{array}{l}\text { (1) The study found that although the } \\
\text { FD plot had higher LER's than the } \\
\text { HD plot because of higher energy } \\
\text { production, the HD plot significantly } \\
\text { limited crop yield losses while also } \\
\text { maintaining an LER over } 1 \text {. } \\
\text { (2) AV system should be designed to } \\
\text { allow about } 70 \% \text { radiation to the crop } \\
\text { to prevent significant } \\
\text { restrictions in yields. } \\
\text { (3) Different varieties of certain crops } \\
\text { that can be chosen for AV systems } \\
\text { due to their adaptability to } \\
\text { shaded conditions. } \\
\text { (4) Shading in the AV systems saved } \\
\text { between } 14-29 \% \text { water depending on } \\
\text { the level of shade (FD or HD). }\end{array}$ & {$[4,21,22,45]$} \\
\hline & & & $\begin{array}{c}\text { Yes } \\
\text { (single-axis) }\end{array}$ & $\begin{array}{l}\text { Controlled-tracking }(\mathrm{CT}) \\
\text { system (Distance from the } \\
\text { ground: } 16.5 \mathrm{ft}(5 \mathrm{~m}), \text { Panel } \\
\text { rotation: } 50 \text { degrees E and } \\
50 \text { degrees } \mathrm{W}), \text { Sun-tracking } \\
\text { (ST) system (Distance from } \\
\text { the ground: } 16.5 \mathrm{ft}(5 \mathrm{~m}), \\
\text { Panel rotation: } 50 \text { degree E } \\
\text { and } 50 \text { degrees } \mathrm{W})\end{array}$ & & $\begin{array}{l}\mathrm{FD}, \mathrm{HD}, \mathrm{ST} \\
\text { and CT }\end{array}$ & $\begin{array}{l}\text { (1) ST AVS is the most effective design } \\
\text { to optimise AV outputs (LER 1.5), } \\
\text { while Fixed HD AVS and CT were the } \\
\text { most efficient in producing biomass. }\end{array}$ & {$[2,6,13]$} \\
\hline $\begin{array}{l}\text { Renewable Energy } \\
\text { Research Office } \\
\text { (RERO), Malaysia }\end{array}$ & unknown & 10 & No & Monocrystalline & Java Tea & FD & $\begin{array}{l}\text { (1) Strong justifications of sustainable } \\
\text { herbal plant growth, profitable } \\
\text { margin with short returns of the } \\
\text { initial investment is the backbone of } \\
\text { this work. } \\
\text { (2) It is observed that high humidity } \\
\text { level due to water evaporation } \\
\text { process with PV shading features } \\
\text { provides a good attraction for pests } \\
\text { which increases the risk of } \\
\text { attack to crop. }\end{array}$ & {$[14,17,33]$} \\
\hline
\end{tabular}


Table 2. Cont.

\begin{tabular}{|c|c|c|c|c|c|c|c|c|}
\hline Location & $\begin{array}{l}\text { Electricity Yield } \\
\left(\mathrm{kWha}^{-1}\right)\end{array}$ & Capacity $(\mathbf{k W p})$ & $\begin{array}{c}\text { Solar } \\
\text { Tracking }\end{array}$ & PV Specification & Cultivated Crops & Sub-Treatment & Highlights & Source \\
\hline $\begin{array}{l}\text { Demeter-certified } \\
\text { farm community } \\
\text { Heggelbach, } \\
\text { Germany }\end{array}$ & unknown & 194.4 & No & $\begin{array}{l}\text { Duo bi-facial PV, } \\
\text { clearance height: } 5 \mathrm{~m} \text {, } \\
\text { overall height: } 7.8 \mathrm{~m}, \\
\text { Unit width: } 19 \mathrm{~m}\end{array}$ & $\begin{array}{c}\text { Potato, winter } \\
\text { wheat }\end{array}$ & unknown & $\begin{array}{l}\text { (1) The maximum sunlight } \\
\text { reduction due to shading from the } \\
\text { PV panels on any square foot of } \\
\text { land under the dual-use system } \\
\text { may be no more than } 50 \% \text {. } \\
\text { (2) Beneficial price-performance } \\
\text { ratio of } 0.85 \text { for potato production } \\
\text { and a nonbeneficial } \\
\text { price-performance ratio of } 4.62 \text { for } \\
\text { winter wheat }\end{array}$ & [41] \\
\hline Zhangjiakou, China & unknown & $1500-1700$ & $\begin{array}{c}\text { Yes } \\
\text { (single-axis) }\end{array}$ & $\begin{array}{l}\text { Oblique PV, East-west } \\
\text { oriented and faces } \\
\text { towards the south, PV } \\
\text { height: } 2.5 \mathrm{~m} \text { from } \\
\text { ground, tilt angle } \\
39 \text { degree }\end{array}$ & unknown & unknown & $\begin{array}{l}\text { (1) By studying the tracking law of } \\
\text { oblique single-axis AV system, it } \\
\text { can be found that in the higher } \\
\text { latitude, variations in rotation angle } \\
\text { are approximately similar during } \\
\text { every day of the growth period } \\
\text { of plants. } \\
\text { (2) Light adaption point (LAP) and } \\
\text { required solar radiation time length } \\
\text { of crops can be regarded as two } \\
\text { indexes to select the right crop }\end{array}$ & [101] \\
\hline India & unknown & $200-250$ & No & $\begin{array}{l}\text { Ground clearance: } 0.5 \mathrm{~m} \text {, } \\
\text { structure width: } 2.95 \mathrm{~m} \text {, } \\
\text { structure heigh: } 1.94 \mathrm{~m}, \\
\text { row distance: } 6 \mathrm{~m}\end{array}$ & $*$ & $\mathrm{SFO}, \mathrm{SPC}$ & $\begin{array}{l}\text { Suitable crops for AVS suggested } \\
\text { here is applicable for arid western } \\
\text { India and for other regions } \\
\text { different crops need to be identified } \\
\text { as per prevailing rainfall and } \\
\text { weather conditions }\end{array}$ & [25] \\
\hline
\end{tabular}




\subsection{Solar Tracker for Agrivoltaic System}

Solar monitoring is a technique for increasing the amount of energy obtained by keeping a solar collector, either PV or photothermal, in an optimal location perpendicular to the sun during daylight hours $[7,76,77]$. A solar tracker aims to ensure that the panels achieve the greatest amount of solar irradiation possible during the day $[54,85,96,130]$. Solar tracking began in 1962 when Finster launched the world's first fully mechanical tracker. The following year, Saavedra demonstrated an electronic-controlled system for orienting an Eppleypyrheliometer [131]. Since then, the techniques of solar tracking were improved with the main purpose being to increase the total amount of irradiance to the maximum possible $[35,78,80]$. Despite that fixed solar PV configuration is preferable to be integrated with AV systems $[1,6,54]$ because fixed solar PV intercepts less solar radiation compared to single-axis and dual-axis solar trackers, there is an effort towards the integration of solar trackers into AV systems [8,30,53,101,127].

Refs. $[4,18]$ have developed an AV solar tracking system at Montpellier Experimental Agrivoltaic Station in their trials to increase the electricity generation without having detrimental effects on agricultural production. There are five plots set up in their experiments. The first plot is a fixed structure with full-density (FD) AVS and the second plot is a fixed plot with half-density (HD) and has the same specifications as the original AV systems developed by [6]. The two types of AV solar tracker system used in their studies are controlled-tracking (CT) system and sun-tracking (ST) system. Both systems were specifically designed by: (1) altering the density of the PV panel and height of the solar PV from the ground; (2) developing a specific solar tracking algorithm with the inclusion of the parameters for agricultural growth. The LER values obtained were more than one in all $\mathrm{AV}$ plots, indicating that the AV system is more efficient than the monosystem production. With LER values of 1.5 and above, the ST plot has proven to be the most successful method for optimizing AV outputs; the ST plot's high LER value is mostly due to electricity generation. It is critical to highlight that the CT layout was the most efficient in terms of agricultural production. Furthermore, the LER values for either the CT or ST plots were greater than the LER values for the HD plot.

In another study by [53], at Po Valley (Northern Italy), a new platform was developed and introduced to conduct simulations aimed at optimizing agrivoltaic systems, which combine the output of electrical energy and arable crops. There are four configurations of AVS set up in this study: (1) dual-axis, sun-tracking system equipped with 5 secondary axes and 10 solar panels (ST1); (2) dual-axis, sun-tracking system equipped with 4 secondary axes and 32 solar panels (ST2); (3) still unit equipped with 5 secondary axes and 10 solar panels (F1); (4) still unit equipped with 4 secondary axes and 32 solar panels (F2). All the $\mathrm{AV}$ systems were constructed by raising the panels and fixed to a rotating axis before being coupled with Agrovoltaico software. A radiation model was integrated with the Agrovoltaico programme (based on the shading conditions determined from the AVS structure set-up). A crop model known as GECROS was used to input AVS's modelled radiation and a 40-year temperature and environmental dataset from the site. Then, the software is used to measures radiation mitigation and its effect on simulated crop yields in aggregate. Based on the simulation, the highest electricity generation came from ST2, followed by F2, ST1, and F1. While, for biomass, even though F2 has the highest yield, the yield in all treatments ranges from 2202-2091 gm ${ }^{-3}$ only. Surprisingly, ST1 and ST2 have higher biomass yields compared to stand-alone agriculture production. Other summaries of studies that utilize solar trackers are mentioned in Table 2.

To the best of the understanding of the authors, there is a potential to integrate the solar tracking system into the agrivoltaic system $[4,5,30,53]$. According to $[77,88,130]$, a solar tracker system can be classified based on the techniques used to control the movement of the PV panels. The tracker system can be a passive, active [78,89], or chronological tracker system [77]. The operation, advantages, and disadvantages of each type of solar tracking system technology are summarized in Table 3 [77]. Even though an active solar tracker (also known as a dynamic solar tracker) is required, despite extra power consumption 
and not being very accurate under a cloudy day, the use of this type of solar tracking system contributed to the higher energy generation efficiency compared to a passive and chronological system $[77,132]$. For this reason, previous studies by $[4,18,53,101]$ also used this type of solar tracking system. The active solar tracker uses sensors and motors $[77,88]$ to control the rotational angle of the PV axis and allows them to follow the sun's trajectory directly $[4,78,101,132]$, or based on a tracking algorithm $[27,49,53,78,133]$. For AVS conditions, the integration of a solar tracker in the AVS is focused on creating an ingenious partnership between both parties to optimize the productions of electricity and agriculture $[28,30,61,87,110]$. A specific and improvised tracking algorithm can be developed to optimize light penetration beneath the solar structure to suit selected crops while balancing the energy production $[47,53,101]$.

Table 3. Passive, Active, and Chronological Solar Tracking System.

\begin{tabular}{|c|c|c|c|c|}
\hline Technology & Descriptions & Advantages & Disadvantages & AVS Preference \\
\hline Passive & $\begin{array}{l}\text { - thermal expansion of } \\
\text { the tracker's material } \\
\text { or an imbalance in } \\
\text { pressure between two } \\
\text { spots at the } \\
\text { tracker's ends }\end{array}$ & $\begin{array}{l}\text { - } \text { operate independently } \\
\text { of motors or actuators. } \\
\text { - } \text { quick and simple to } \\
\text { set up. } \\
\text { - } \text { minimal } \\
\text { maintenance cost. }\end{array}$ & $\begin{array}{l}\text { - a high level of } \\
\text { weather dependency. } \\
\text { - low precision. }\end{array}$ & - \\
\hline Active & $\begin{array}{l}\text { - design systems that } \\
\text { employ sensors } \\
\text { and motors. }\end{array}$ & $\begin{array}{l}\text { - } \text { more accurate. } \\
\text { - efficient at tracking } \\
\text { the sun's location. }\end{array}$ & $\begin{array}{l}\text { - } \text { requires additional } \\
\text { electricity usage. } \\
\text { - not very precise on an } \\
\text { overcast day. }\end{array}$ & $\begin{array}{c}\text { Single-axis: }[4] * \text { ST and } \\
* * \text { CT, }[18] * \text { ST and ** } \\
\text { CT, and }[101] * \text { ST } \\
\text { Dual-axis: }[53] * \text { ST }\end{array}$ \\
\hline Chronological & $\begin{array}{l}\text { rotate at a specific rate } \\
\text { of degrees each hour. }\end{array}$ & $\begin{array}{l}\text { - low energy losses. } \\
\text { - minimal } \\
\text { tracking error. }\end{array}$ & $\begin{array}{l}\text { - continuous rotation } \\
\text { is more } \\
\text { energy-intensive. } \\
\text { - irrational work on an } \\
\text { overcast day. }\end{array}$ & - \\
\hline
\end{tabular}

* ST $=$ Sun-tracking system, ${ }^{* *} \mathrm{CT}=$ Controlled-tracking system.

\section{Agronomic Management for Agrivoltaic System}

\subsection{Crop Selection}

The microclimate aspect under the solar PV structure should be taken into account in the selection of suitable crops to be cultivated in solar farms $[5,46,103,126]$. A setup by Refs. $[42,134]$ in particular divided the area beneath the solar panel into three subtreatments: (1) sky fully open area between panels (SFO); (2) Solar partially open between panels (SPO); (3) solar fully covered area under panels (SFC) as illustrated in Figure 3A. $\mathrm{SFO}$ zones are located between the edges of mounted photovoltaic panels and areas that have received full light [28]. No shade covers the SFO zone, according to the shadow length estimate [132], while SPO areas are situated in the penumbra and have been subjected to episodic shade [104]. SFC areas are located immediately under the photovoltaic panels and receive complete shade [73]. However, the division of the sub-treatments are subjective and subjected to the solar photovoltaic design $[5,19,38,91]$. For example, the AVS design at Montpellier Experimental Agrivoltaic Station by $[4,6,18,69]$ (as described on page 9) is high in length, and is thus less suitable to be divided into SFO, SPO, and SFC. Previous researchers at that station classified the area beneath the solar panel as FD and HD. The average proportion of daily radiation emitted below the solar panel (FD and HD treatments) relative to the FS treatment varies with the growing season. In general, AVS is not recommended in crop rotation systems [41]. However, crop rotation could increase 
the production of agriculture in AVS farms [69], especially in regions that experience different seasons throughout the year [128]. Furthermore, when used in conjunction with permanent cultures - such as berries, bananas, or wine grapes- the cost of these types of applications is smaller, thus delivering increased efficiency through the optimization of techno-ecological synergies [41].

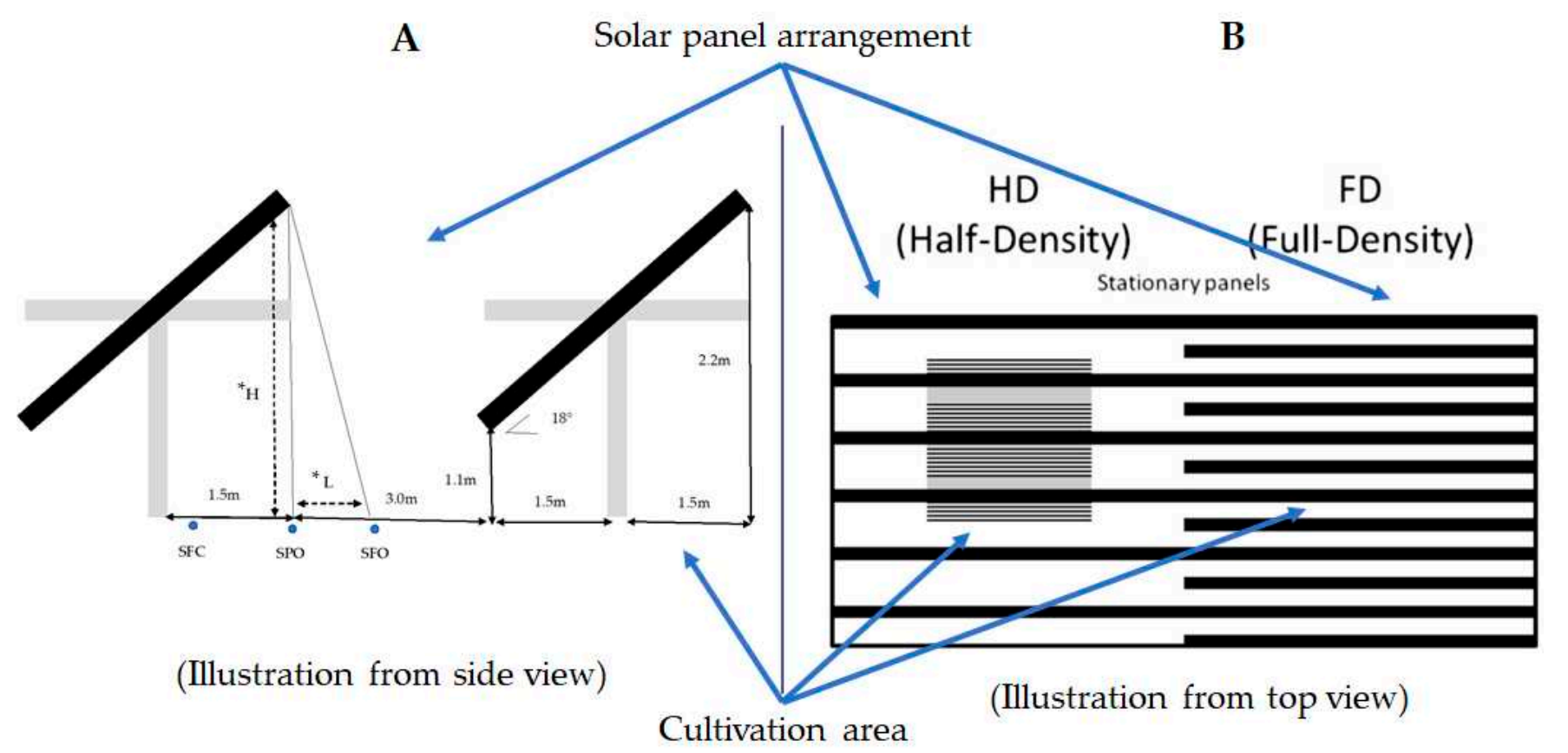

Figure 3. Schematic Drawing of Shade Zones. (A) SFO, SPO and SFC. (B) FD and HD. (Modified Figure 3A), source: [32]; The illustration 3(B), source: [4]). *H is object height and $\mathrm{L}$ is shadow length.

Next, the heat or thermal energy dissipated under the photovoltaic array is also a critical factor to consider in relation to the continuous development of the crops beneath $[19,54,111,123]$. In this case, the open field AVS is better than the closed greenhouse AVS [82], as its key characteristics are the mean daily reduction in light access for plants $[28,38]$ without major changes in other microclimate parameters such as relative humidity, wind speed and direction, and soil moisture $[26,84,132]$ at the level of the canopy $[69,127]$. If the AVS design were able to regulate adequate air circulation below the open structure, the air temperature, VPD [69], mean relative humidity, and wind speed [132] might be insignificantly different [98], or optimized [11], compared to the ambient surrounding; however, it depends on the structural design $[21,54,62]$ and regions $[10,103,115]$. Enclosed structures, on the other hand, offer the advantage of being able to regulate the temperature inside the structure to meet the demands of the crop [91,103,126]. Furthermore, in a study conducted by [31], it was found that reduced light is not often harmful to crop quality, as improved Radiation Interception Efficiency (RIE) has been shown in the shade; however, a specific arrangement of the solar panel is needed to compromise between agriculture production and electricity generation in a way that can improve the production of both. However, solar management is not amenable to all types of crops, and there is a need for further research before an economically viable approachable system using PV technology can be designed [7,12,26,132,135].

There are several factors suggested by the author, based on reviews, to facilitate the crop selection for AVS: (1) the design of solar PV structure [8,54,62,63]; (2) the location of sub-treatments $[6,69,132]$; (3) the approaches of AVS [44,107]. For the first factor, types of design considered for solar PV structure have been described in Section 3. In case the introduction of agriculture production is on the existing solar farms or an unaltered solar panel structure, the approach used by [50] could be the sustainable solution to combine both productions. They suggested the planting of high-value herbal crops in solar 
farms with zero or minimal modification of the solar PV structure. The authenticity of growing herbal crops under solar photovoltaic arrays is justified by the sustainability and morphological aspect of the arrangement as a way of using unused land. For example, the maximum height of the Java Tea Plant (high-value herbal crops), which is less than three feet (from the ground) and grows in a regulated manner, is considered suitable and will not interfere with the PV panel electricity generation operation. The chosen plant is also classified as a shade-loving herb, and the temperature beneath the solar PV structure measured is within an acceptable range for ornamental herbal plants. The solar farm project's maintenance requirements are met by field arrangements of herbal polybags and a manual irrigation solution [59].

For the location of sub-treatments which are SFO, SPO and SFC [42,132], or FD and $\mathrm{HD}[4,6,18,69]$, a wide range of crops can be selected to be planted based on their physiological and morphological traits $[4,28,71]$. Besides that, the selection of suitable crops for AVS should also be identified based on local climate and weather conditions $[5,12,21,28]$. In general, shade-loving plants are best suited for planting in less sunlit areas, while sun-loving plants are better suited to seeding in sunlight areas [64,107,112]. For the arid region, such spices may be successfully grown between two rows of solar PV, as these are short in nature, for example: Trigonella foenum-graecum Linn. ('methi'), Plantago ovata Forsk. ('isabgol'), Coriandrum sativum Linn. (coriander or 'dhania'), etc [25]. The following vegetable crops may also be grown: Brassica oleracea var. botrytis (cauliflower), Brassica oleracea var. capitate (cabbage), Allium cepa Linn. (onion), Allium sativum (garlic), Capsicum annum Linn. (chilli), etc. [25]. The land area beneath photovoltaic panels can also be used to grow vegetable crops of the Cucurbitaceae family, such as Cucurbita pepo Linn. ('kakri'), Lagenaria siceraria ('lauki'), Citrullus fistulosus Stock ('tinda'), etc. [25]. Cultivating crops in areas below the photovoltaic panel has the added benefit of reducing the heat load on the bottom surface of the photovoltaic panel by modifying the microclimate and thus assisting in generating the maximum amount of electricity [21,50]. Additionally, [59] also proposed that herbs could be planted in tropic areas using AVS applications with minor modification of the solar panel structures. Herbal plants such as Orthosiphon stamnieus is suitable in the tropical region [50], while Cassia angustifolia (senna), Aloe vera ('gwarpatha'), and others may also be considered as potential crops if the PV structure is in rocky scrubs or degraded lands, depending on the region [25]. Next, some studies performed in various regions of the world indicate different kinds of crops, such as semi-arid pasture (Oregon, USA; [132]), Maize (Po Valley, Northern Italy; [53]), lettuce (short cycle crop), cucumbers (short cycle crop), durum wheat (long cycle crop) cultivated at Montpellier Experimental Agrivoltaic Station, France [6,31], and potato and wheat (Demeter-certified farm community Heggelbach, Germany; [41]). In addition, [5] stated that, in some regions, certain crops such as fruit trees (i.e., kiwi, apple, pear, cherry), berries (i.e., raspberries, blackberries), tomatoes, sweet peppers, coffee, and ginseng, are among the crops that are also able to cope with a reduction of more than $50 \%$ in the light source. Based on these findings, it is possible to conclude that the selection of suitable crops for integration into the AV system is subjective, depending on local weather and the architecture of the PV structure $[8,28,33,87,103,136]$.

However, a suggestion from [107], as shown in the table below, to include the AVS approaches may be able to further facilitate the selection of suitable crops. The table illustrates how solar farms and crops can be combined according on the land-use type and AVS strategies. Next, several modifications of the solar PV structure and types of the crop cultivated will be recommended. For example, the suggestions for short crop planting area with agriculture centric approach are as follows: (1) plant mix of sun-loving and shade-tolerant crops, (2) raised solar PV structures, and (3) space solar PV structures. Other options are covered in Table 4. 
Table 4. Opportunities for Solar PV and Agricultural Integration by Land-Use Type. (Source: [107]).

\begin{tabular}{|c|c|c|c|}
\hline & Energy Centric & Agriculture Centric & Integrated Agriculture-Energy Centric \\
\hline $\begin{array}{l}\text { Grazing/ } \\
\text { un-used/scrub/ } \\
\text { desert land }\end{array}$ & $\begin{array}{l}\text { - Leave native } \\
\text { vegetation intact } \\
\text { - Plant short } \\
\text { shade-tolerant crops }\end{array}$ & $\begin{array}{l}\text { - Leave native } \\
\text { vegetation intact } \\
\text { - Plant mix of sun-loving and } \\
\text { shade-tolerant crops } \\
\text { - Elevate solar PV structure } \\
\text { - Space out solar PV structure } \\
\text { - Continue/initiate } \\
\text { grazing activities }\end{array}$ & $\begin{array}{l}\text { - Leave native vegetation intact } \\
\text { - Plant short shade-tolerant crops } \\
\text { - Elevate solar PV structure } \\
\text { - Continue/initiate grazing activities }\end{array}$ \\
\hline $\begin{array}{l}\text { Agriculture } \\
\text { (short crop) }\end{array}$ & $\begin{array}{l}\text { - Plant short } \\
\text { shade-tolerant crops } \\
\text { beneath and around } \\
\text { solar PV structure }\end{array}$ & $\begin{array}{l}\text { - Plant mix of sun-loving and } \\
\text { shade-tolerant crops } \\
\text { - Elevate solar PV structure } \\
\text { - Space-out solar PV structure }\end{array}$ & $\begin{array}{l}\text { - Plant mix of sun-loving and } \\
\text { shade-tolerant crops } \\
\text { - Elevate solar PV structure }\end{array}$ \\
\hline $\begin{array}{l}\text { Agriculture } \\
\text { (tall crop) }\end{array}$ & - Limited options & $\begin{array}{l}\text { - Plant mix of sun-loving and } \\
\text { shade-tolerant crops } \\
\text { - Elevate solar PV structure } \\
\text { - Space out solar PV structure }\end{array}$ & $\begin{array}{l}\text { - Place solar PV structure in } \\
\text { non-utilized parts of agricultural land } \\
\text { - Elevate solar PV structure }\end{array}$ \\
\hline
\end{tabular}

\subsection{Agronomic Practices}

Solar energy is the most plentiful and readily available source of energy $[25,28,47,98]$. The use of AVS technologies in areas where a solar farm and agriculture coexist $[51,75,109]$ could have synergistic effects that aid in the production of ecosystem services such as crop production $[9,20,38]$, local climate regulation $[34,115,137]$, water conservation $[13,18,56]$, and renewable energy production $[21,87,138]$; and it also aligns with food-energy-water (FEW) nexus [34,63,137].

Thus, the integration should potentially influence the microclimate and soil moisture [21,51,103]; hence, it may provide suitable environmental conditions [18,34,43] and increase the water-use efficiency for agricultural production $[3,82,97]$ while maintaining the renewable energy production $[41,44]$. As mentioned in 2.1, the photosynthesis process requires light, carbon dioxide, and water to produce glucose as the source of energy for plants. If the sources of light and carbon dioxide are not limited, an optimum amount of irrigation water is needed to enhance the photosynthesis rate. Thus, regions with insufficient water resources are most likely to benefit as solar management decreases potential evapotranspiration (PET) and water demand [26,51,132,137]. The reducing amount of irrigation water needed without compromising crop-water requirements can make a significant contribution to reducing agricultural production costs, making the industry more competitive and sustainable $[18,21,65]$. However, a systematic or proper irrigation schedule is a must in AVS sites [82,139] to minimize the environmental impacts caused by excess water and leaching of subsequent agrichemicals [140] that might affect the structure of solar PV. Water-use efficiency can be improved [3,8,82] by understanding the concept of evaporation, evapotranspiration (ET), and irrigation water requirements [141]. ET is the mechanism by which water originates from a wide range of sources such as soil compartment and/or layer of vegetation and is transferred to the atmosphere [100]. Also, ET involves evaporation from bodies of surface water, surface of land, sublimation of snow and ice, plant transpiration, and intercepted canopy water [82]. Besides that, the evaporation process that happens also significantly reduces the percentage of soil moisture content $[19,41,137]$. On the other hand, irrigation water requirements are defined as the quantity of water necessary for crop growth [141,142]. In addition, the loss of electrical output due to dust accumulation on the panel surface as a result of agricultural management, such as tillage and harvesting, is also a source of concern $[5,7,10,21]$. In regions with low precipitation or long stretches 
of dry weather (e.g., monsoon climates), periodic cleaning of the module surface should be considered to prevent decreasing electricity yields due to dust accumulation [87]. This could be done by combining irrigation systems and PV cleaning to reduce increased water use [51]; however, without a small water distributor under the panels, it may result in inconsistent watering of crops [111]. Hence, proper assessment of evapotranspiration [139], soil moisture content [140], and PV cleaning processes $[10,111]$ are needed before designing the irrigation system for agricultural production in AVS.

Another aspect is that extreme heterogeneity and spatial gradients in biomass production [5] and soil moisture [45,82] were observed as a result of the heterogeneous shade pattern of the PV array [132]. In the studies conducted at Montpellier Experimental Agrivoltaic Station by $[4,6,18,69]$, the shadow effect of the PV array can be seen from the agricultural yield, where the HD structure produces more yield than the FD structure. The results show that, with the improvement of PV panel arrangement, LER may potentially exceed 1 [38,87]. Next, a solar tracker controller developed by [53] found that maize grown under the AVS plots tended to have more stabilized and higher yields in drought stressors and rainfed conditions. Besides that, crop selection can also reduce the effect of the heterogeneous shade pattern of the PV array $[11,25,28]$. This can be seen in the experiment conducted by [61] using Java Plant Tea in Malaysia. The result obtained shows a good agreement between the selected crop and the PV panels above them that act as their artificial shading. To sum up, acts to reduce the extreme heterogeneity and spatial gradients in agricultural production are: (1) optimize PV array placement to create a spatially uniform shadow pattern $[4,10,75]$; (2) improve the solar tracker controller that considers the need for solar radiation for both productions (electricity and agriculture) $[53,91,101]$; (3) select a suitable crop to be planted with a minimal light source (due to shading effect of solar PV structure) $[25,54,87]$. Besides that, as suggested by $[5,112]$, the PV structure can be raised to reduce the heterogeneity effect, while allowing the conventional agricultural machines to pass $[4,28,38]$, and reducing the back pain [68] while doing agricultural work due to low PV structure [95]. The gap between the pillars also needs to be suitable for planting distances and working widths of the machinery to avoid the loss of utilizable land [5,41]. Careful planning is essential, since the space required for the machine to pass might restrict the amount of land available for solar panels $[19,68]$. Also, ram protection should be installed to avoid collisions between agricultural machines and the solar PV pillars [68].

Other than that, the agronomic practices for agricultural production at AVS, likely similar to standard and common agronomic practices [62,126], include the steps listed in Figure 4. More information on standard practices can be found in documents such as the ones written by [143], which specifically address cropping systems and agronomic management. However, precision agriculture methods such as site-specific crop management (SSCM), for which decisions on resource application and agronomic procedures are being improvised, can be developed to better meet crop requirements based on soil heterogeneity in the field [33].
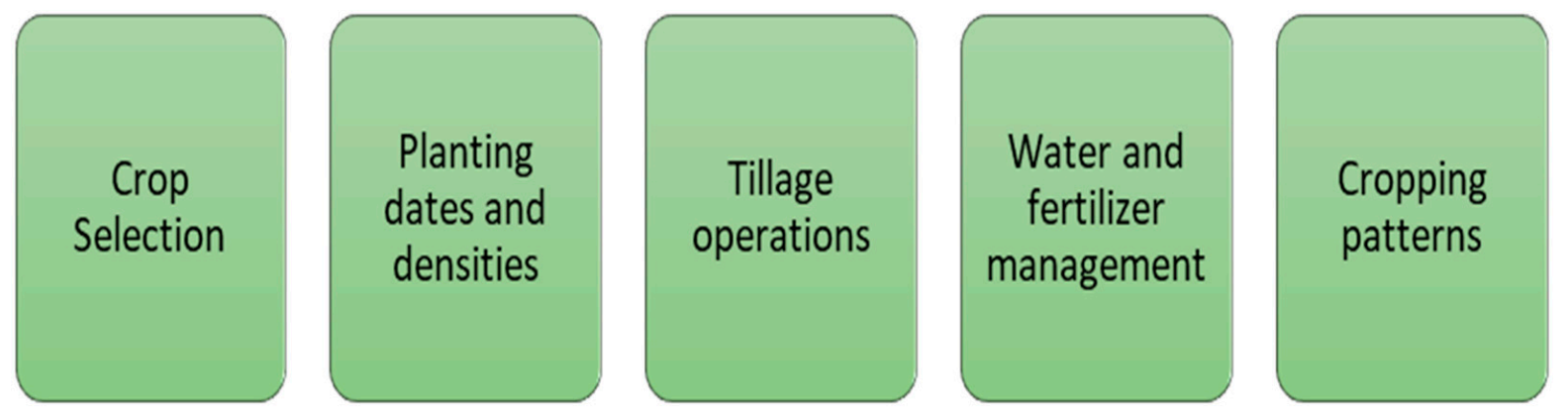

Figure 4. Common Agronomic Practices. 


\section{Outlook and Future Improvement}

AV systems are still in their infancy, and there is plenty of room for technical advancements and expanded application fields [7,95]. This paper is focused more on technical improvements that can be made to the solar PV architecture and agronomic management to improve the AVS outputs. As already described in Section 2.1 to Section 2.3, there are several factors such as (1) competition against solar radiation between solar PV and crop; (2) AVS approaches; (3) public's acceptance of AVS applications (especially farmers). The issues related to the first factor can be minimized by properly designing the PV structure $[6,31,54,62]$ and by efficiently managing the agricultural production $[33,64,87]$. In many cases, some alterations to PV architecture are required to balance the amount of solar radiation received by $\mathrm{PV}$ panels with the amount of light allowed to reach the crop beneath the solar structure $[5,6,28,87]$. Most importantly, future research should not overlook the fact that the photosynthesis process is primarily concerned with the Photosynthesis Active Radiation (PAR) range $[49,83,128,129]$, whereas electrical energy is dependent on solar irradiance or light intensity $[65,80,96]$. Therefore, as a future study for AV system technology, we suggest some areas that are deemed intriguing to be investigated.

\subsection{Guidelines for PV Architecture of Agrivoltaic System}

Solar farms and agricultural production can be integrated in a variety of ways, with small to large impacts on solar energy and vegetation best practices $[44,107]$. Solar PV structure installations, at one extreme, can incorporate low-lying crops beneath the installation to mitigate environmental impacts without modifying existing site preparation activities [50]. On the other end of the spectrum, certain agricultural areas can incorporate solar PV technologies in ways that preserve crop production or harvesting techniques while also providing an additional source of electricity [54]. Between these two extremes, solar farm developers and agricultural producers have a variety of options for modifying system designs to allow for greater levels of integration [2,4,6]. The preliminary AVS studies suggested that solar farming and agricultural productions integration is only feasible when a fixed solar structure is used [6]. Numerous alterations and modifications can be made to optimize the AVS integration, including optimizing the spacing between panel rows, adjusting the height of mounted panels, configuring the solar panel density, and adjusting the tilt angle; however, all the works are subjected to the type of AVS developed $[44,107]$, geographical condition, and crop growing season $[25,45]$. As no comprehensive guidelines and regulations exist for PV structure alterations for different climates at the moment, this allows for more research and testing on AVS technologies to take place [5].

\subsection{Solar Tracker Improvement for Agrivoltaic System}

Research conducted more recently has shown that solar trackers can also be sustainably used in AVS applications $[4,18,53,101]$. Based on the techniques used to control the movement of the PV panels, solar tracker systems are divided into passive, active [78,89], and chronological tracker systems [77]. However, previous studies indicate that the use of active solar trackers, as opposed to passive and chronological tracker systems, is gaining more traction either in mono PV systems $[77,80,88,93]$ or in AV systems $[5,8,30,53,101]$. The integration of direct sun-tracking (ST) with agricultural production in previous studies conducted by $[4,18]$ recorded an LER value of 1.5 and above, hence proving that the combinations effectively optimize the AV productions. The high LERs are mostly contributed by the PV production compared to agriculture production. Meanwhile, the use of a controlled-tracking (CT) system based on a tracking algorithm has been seen to be more reliable in creating an ingenious partnership between both parties in optimizing the productions of electricity and agriculture. The algorithm-based solar tracker pairing with a single-axis system $[4,18,101]$ or dual-axis system [53] is acceptable in the AV system. The most important thing is that a customized and adaptable tracking algorithm should be developed to optimize light penetration beneath the solar structure for specific crops while maintaining a balance in energy production $[53,101]$. 


\subsection{Guidelines for Agronomic Management of Agrivoltaic System}

Generally, crops that can withstand a reduction in solar radiation should be chosen to be planted using the AV system $[65,87,94]$; however, previous studies show some reduction in agriculture yields depending on light penetration, crop growing season, and climate $[5,28,87]$. Even for shade-loving plants, the allowable light penetration should not be less than $50 \%$; otherwise, the qualities of crop production could be significantly dampened [5]. In addition, according to [41], crop rotation systems are not recommended in AVS unless in conjunction with permanent cultures, due to cost efficiency. However, this recommendation should be validated because crop selection in some regions is different depending on crop growing season and climates. Moreover, efficient solar management has great potential to significantly improve land-use efficiency economically [17]. For the time being, there is not enough data on crop recommendation provided by the previous studies. Most of these studies evaluated the potential of the AV system by utilizing only one or a few crops in their research. Furthermore, fewer studies focus on water management $[18,132]$, even though water management is one of three critical components of AVS [34,137]. The agronomic practices for AV systems are generally similar to common agronomic practices $[62,126]$; however, precision agricultural methods such as site-specific crop management (SSCM) may be created to better match crop requirements in the field depending on soil heterogeneity [33]. Also, a systematic or proper irrigation schedule is critical in AVS sites to minimize environmental impacts caused by excess water and subsequent agrichemical leaching [140] that could affect the solar PV structure. To summarize, the discussions above provide opportunities for additional in-depth research to: (1) create more comprehensive crop recommendation guidelines [28,31]; (2) improvise existing crop management that can be used in various AVS circumstances [33]; (3) develop a systematic irrigation system for AVS systems [18,25].

\subsection{Farmer's Perspectives on Agrivoltaic System Design}

The adoption of AVS technology is likely to face some challenges [14,28]. There is always a certain amount of public controversy accompanying the introduction of new technologies, and this should not be underestimated in AV systems [5]. The barriers to agrivoltaic adoption include the following: (1) a demand for long-term production to be guaranteed [109-111]; (2) market potential [2,9,44,46]; (3) fair compensation [38,44,52,112]; (4) a need for predesigned system flexibility to suit varied sizes, types of activities, and evolving agricultural methods [54,109]; (5) the identification of AVS approaches that are both economically beneficial and have no adverse environmental consequences to them $[62,110,113]$; (6) the design of photovoltaic infrastructure should promote reversibility [3]; (7) less promotional activities aimed at increasing public knowledge about AVS [10,11,15,110]. All these obstacles can be overcome with ongoing public education and strong political will on the part of the authorities to ensure the effective implementation of AVS projects [41]. These AVS technology acceptance factors may be less relevant to technical improvements of AV systems; however, farmers' perspectives and acceptance must also be considered before any improvements are made, because they are the end-users of this technology [44].

\subsection{Food-Energy-Water Nexus in Agrovoltaic System}

Based on the FEW nexus concepts, improvement of the AV system should account for food (agriculture), energy, and water management as interdependent aspects of the integration system $[9,14,34,63]$. As agricultural and water management are parts of agronomic management, future studies should emphasize the importance of both managements in a balanced manner $[33,132,142]$. Also, to advance our understanding of complex interactions between food (agriculture), energy, and water in the AVS system, we require a mathematical framework that can adjust in response to new data and incorporates a wide range of interactions, including natural processes and anthropogenic inputs to sustain resources for future generations [137]. 


\section{Conclusions}

The deployment of agrivoltaic systems provides a variety of benefits that vary according to geographic and climatic conditions. The real added advantage of the AVS approach is that it is applicable to agriculture and sustainable energy production, allowing a unique combination of short-synergic results to sustain resources and give economic benefit to the farmers. This is especially relevant in heavily populated developing and developed countries, where renewable energy development is becoming increasingly necessary; however, profitable farmland must be maintained. The performance of the AV system is determined using LER, and if the value exceeds 1, the system is said to be optimal. A good deal of care and attention needs to be paid to the architecture of the solar PV structure and agronomic management for AVS to succeed. For instance, relevant parties such as AVS developers, researchers, government officials, etc., should consider and understand the energy and agricultural production systems' reliance on light sources, the approaches of AVS production combinations, and the public' acceptance towards the system. Once these three factors are grasped, the additional features of the solar panel structure and agronomic management become apparent. However, it is important to periodically review the AVS application's capability and employ dynamic actions to further improve its effectiveness. For instance, structural alterations to solar panels used to be primarily focused on fixed structural design; however, now the advancements include the use of vertical bifacial photovoltaic (PV) technology, semi-transparent photovoltaic (SPTV), and solar tracking systems. The goal is to keep competition for solar radiation between solar PV and agriculture to a minimum while maximizing energy generation and agricultural production. As addressed in the FEW nexus concepts, the advancements of AVS technologies should not only focus on energy management, but also food (agriculture) and water management, as these three factors are nexus domains. Since the management of agriculture (crop) and water are parts of agronomic management, future enhancements should emphasize the importance of balancing the two. The agronomic management in AV systems that requires improvement includes crop selection recommendations, improved crop management guidelines, and a systematic irrigation system that minimizes environmental impacts caused by excess water and subsequent agrichemical leaching that could affect the solar PV structure. In conclusion, the advancements of AVS technology are expected to reduce reliance on nonrenewable fuel sources and mitigate the effects of global warming, as well as addressing the food-energy-water nexus's demands.

Author Contributions: Conceptualization, M.A.Z.A., M.N.M. and M.A.A.M.Z.; methodology, M.A.Z.A. and M.N.M.; software, M.A.Z.A.; validation, formal analysis, and investigation, M.A.Z.A., M.N.M. and M.A.A.M.Z.; resources, M.N.M. and M.A.A.M.Z.; data curation, M.A.Z.A., M.N.M. and M.A.A.M.Z.; writing—original draft preparation, M.A.Z.A.; writing—review and editing, M.N.M. and M.A.A.M.Z.; visualization, M.A.Z.A.; supervision, M.N.M. and M.A.A.M.Z.; project administration, M.A.Z.A.; funding acquisition, M.N.M. All authors have read and agreed to the published version of the manuscript.

Funding: The first author's PhD study is sponsored by the Ministry of Higher Education Malaysia and Universiti Teknologi Mara Malaysia (UiTM). This research was funded by Universiti Sains Malaysia under Research University (Rui) Grant (1001/PELECT/8014088), the School of Electrical and Electronic Engineering KPI fund and the USM RCMO incentive fund.

Institutional Review Board Statement: Not applicable.

Informed Consent Statement: Not applicable.

Data Availability Statement: No new data were created or analyzed in this study. Data sharing is not applicable to this article.

Conflicts of Interest: The authors declare no conflict of interest. 


\section{References}

1. Goetzberger, A.; Zastrow, A. On the Coexistence of Solar-Energy Conversion and Plant Cultivation. Int. J. Sol. Energy 1982, 1, 55-69. [CrossRef]

2. Proctor, K.W.; Murthy, G.S.; Higgins, C.W. Agrivoltaics align with green new deal goals while supporting investment in the us' rural economy. Sustainability 2021, 13, 137. [CrossRef]

3. Hernandez, R.R.; Armstrong, A.; Burney, J.; Ryan, G.; Moore-O'Leary, K.; Diédhiou, I.; Grodsky, S.M.; Saul-Gershenz, L.; Davis, R.; Macknick, J.; et al. Techno-ecological synergies of solar energy for global sustainability. Nat. Sustain. 2019, 2, 560-568. [CrossRef]

4. Valle, B.; Simonneau, T.; Sourd, F.; Pechier, P.; Hamard, P.; Frisson, T.; Ryckewaert, M.; Christophe, A. Increasing the total productivity of a land by combining mobile photovoltaic panels and food crops. Appl. Energy 2017, 206, 1495-1507. [CrossRef]

5. Weselek, A.; Ehmann, A.; Zikeli, S.; Lewandowski, I.; Schindele, S.; Högy, P. Agrophotovoltaic systems: Applications, challenges, and opportunities. A review. Agron. Sustain. Dev. 2019, 39, 1-20. [CrossRef]

6. Dupraz, C.; Marrou, H.; Talbot, G.; Dufour, L.; Nogier, A.; Ferard, Y. Combining solar photovoltaic panels and food crops for optimising land use: Towards new agrivoltaic schemes. Renew. Energy 2011, 36, 2725-2732. [CrossRef]

7. Sekiyama, T.; Nagashima, A. Solar sharing for both food and clean energy production: Performance of agrivoltaic systems for corn, a typical shade-intolerant crop. Environments 2019, 6, 65. [CrossRef]

8. Mavani, D.D.; Chauhan, P.M.; Joshi, V. Beauty of Agrivoltaic System regarding double utilization of same piece of land for Generation of Electricity \& Food Production. Glob. Sci. J. 2019, 10, 118-148.

9. Babatunde, O.M.; Denwigwe, I.H.; Adedoja, O.S.; Babatunde, D.E.; Gbadamosi, S.L. Harnessing renewable energy for sustainable agricultural applications. Int. J. Energy Econ. Policy 2019, 9, 308-315. [CrossRef]

10. Majumdar, D.; Pasqualetti, M.J. Dual use of agricultural land: Introducing 'agrivoltaics' in Phoenix Metropolitan Statistical Area, USA. Landsc. Urban Plan. 2018, 170, 150-168. [CrossRef]

11. Kostik, N.; Bobyl, A.; Rud, V.; Salamov, I. The potential of agrivoltaic systems in the conditions of southern regions of Russian Federation. IOP Conf. Ser. Earth Environ. Sci. 2020, 578, 012047. [CrossRef]

12. Winkler, B.; Lewandowski, I.; Voss, A.; Lemke, S. Transition towards renewable energy production? Potential in smallholder agricultural systems in West Bengal, India. Sustainability 2018, 10, 801. [CrossRef]

13. Al-Saidi, M.; Lahham, N. Solar energy farming as a development innovation for vulnerable water basins. Dev. Pract. 2019, 29, 619-634. [CrossRef]

14. Miao, R.; Khanna, M. Harnessing Advances in Agricultural Technologies to Optimize Resource Utilization in the Food-EnergyWater Nexus. Annu. Rev. Resour. Econ. Forthcom. 2019, 12, 65-85. [CrossRef]

15. Lytle, W.; Meyer, T.K.; Tanikella, N.G.; Burnham, L.; Engel, J.; Schelly, C.; Pearce, J.M. Conceptual Design and Rationale for a New Agrivoltaics Concept: Pasture-Raised Rabbits and Solar Farming. J. Clean. Prod. 2021, 282, 124476. [CrossRef]

16. Othman, N.F.; Mat Su, A.S.; Ya'Acob, M.E. Promising Potentials of Agrivoltaic Systems for the Development of Malaysia Green Economy. IOP Conf. Ser. Earth Environ. Sci. 2018, 146, 012002. [CrossRef]

17. Adeh, E.H.; Good, S.P.; Calaf, M.; Higgins, C.W. Solar PV Power Potential is Greatest Over Croplands. Sci. Rep. 2019, 9, 1-6. [CrossRef]

18. Elamri, Y.; Cheviron, B.; Lopez, J.M.; Dejean, C.; Belaud, G. Water budget and crop modelling for agrivoltaic systems: Application to irrigated lettuces. Agric. Water Manag. 2018, 208, 440-453. [CrossRef]

19. Cuppari, R.I.; Higgins, C.W.; Characklis, G.W. Agrivoltaics and weather risk: A diversification strategy for landowners. Appl. Energy 2021, 291, 116809. [CrossRef]

20. Li, C.; Wang, H.; Miao, H.; Ye, B. The economic and social performance of integrated photovoltaic and agricultural greenhouses systems: Case study in China. Appl. Energy 2017, 190, 204-212. [CrossRef]

21. Patel, B.; Gami, B.; Baria, V.; Patel, A.; Patel, P. Co-generation of solar electricity and agriculture produce by photovoltaic and photosynthesis-dual model by Abellon, India. J. Sol. Energy Eng. 2019, 141, 031014. [CrossRef]

22. Metsolar What is Agrivoltaics? How Can Solar Energy and Agriculture Work Together? Available online: https://metsolar.eu/ blog/what-is-agrivoltaics-how-can-solar-energy-and-agriculture-work-together/ (accessed on 1 October 2020).

23. D'Adamo, I.; Rosa, P. How do you see infrastructure? Green energy to provide economic growth after COVID-19. Sustainability 2020, 12, 4738. [CrossRef]

24. IEA. World Energy Outlook 2020. Available online: https://www.iea.org/reports/world-energy-outlook-2019 (accessed on 21 March 2021).

25. Santra, P.; Pande, P.C.; Kumar, S.; Mishra, D.; Singh, R.K. Agri-voltaics or solar farming: The concept of integrating solar PV based electricity generation and crop production in a single land use system. Int. J. Renew. Energy Res. 2017, 7, 694-699.

26. Al-Agele, H.A.; Proctor, K.; Murthy, G.; Higgins, C. A case study of tomato (Solanum lycopersicon var. legend) production and water productivity in agrivoltaic systems. Sustainability 2021, 13, 2850. [CrossRef]

27. Kim, B.; Kim, C.; Han, S.U.; Bae, J.H.; Jung, J. Is it a good time to develop commercial photovoltaic systems on farmland? An American-style option with crop price risk. Renew. Sustain. Energy Rev. 2020, 125, 109827. [CrossRef]

28. Chamara, R.; Beneragama, C. Agrivoltaic systems and its potential to optimize agricultural land use for energy production in Sri Lanka: A Review. J. Sol. Energy Res. 2020, 5, 417-431. 
29. Li, P.C.; Ma, H. Evaluating the environmental impacts of the water-energy-food nexus with a life-cycle approach. Resour. Conserv. Recycl. 2020, 157, 104789. [CrossRef]

30. Kussul, E.; Baydyk, T.; García, N.; Herrera, G.V.; Department, A.V.C.L. Combinations of Solar Concentrators with Agricultural Plants. J. Environ. Sci. Eng. B 2020, 9, 168-181. [CrossRef]

31. Marrou, H.; Wery, J.; Dufour, L.; Dupraz, C. Productivity and radiation use efficiency of lettuces grown in the partial shade of photovoltaic panels. Eur. J. Agron. 2013, 44, 54-66. [CrossRef]

32. Othman, N.F.; Mohammad, E.; Suhaizi, A.; Su, M.; Jaafar, J.N.; Hizam, H.; Shahidan, M.F.; Jamaluddin, A.H.; Chen, G.; Jalaludin, A. Modeling of Stochastic Temperature and Heat Stress Directly Underneath Agrivoltaic Conditions with Orthosiphon Stamineus Crop Cultivation. Agronomy 2020, 10, 1472. [CrossRef]

33. Kumar, S.; Saravaiya, S.N.; Pandey, A.K. Precision Farming and Protected Cultivation: Concepts and Applications, 1st ed.; CRC Press: Oxon, UK, 2021; ISBN 9781032052762.

34. Barron-gafford, G.A.; Pavao-zuckerman, M.A.; Minor, R.L.; Sutter, L.F.; Barnett-moreno, I.; Blackett, D.T.; Thompson, M.; Dimond, K.; Gerlak, A.K.; Nabhan, G.P.; et al. Agrivoltaics provide mutual benefits across the food-energy-water nexus in drylands. Nat. Sustain. 2019, 2, 848-855. [CrossRef]

35. Skoplaki, E.; Palyvos, J.A. On the temperature dependence of photovoltaic module electrical performance: A review of efficiency/power correlations. Sol. Energy 2009, 83, 614-624. [CrossRef]

36. Kumar, N.M.; Kanchikere, J.; Mallikarjun, P. Floatovoltaics: Towards improved energy efficiency, land and water management. Int. J. Civ. Eng. Technol. 2018, 9, 1089-1096.

37. Guerin, T.F. Impacts and opportunities from large-scale solar photovoltaic (PV) electricity generation on agricultural production. Environ. Qual. Manag. 2019, 28, 7-14. [CrossRef]

38. Dos Santos, C.N.L. Agrivoltaic System: A Possible Synergy between Agriculture and Solar Energy; KTH Royal Institute of Technology: Stockholm, Sweden, 2020.

39. Marucci, A.; Zambon, I.; Colantoni, A.; Monarca, D. A combination of agricultural and energy purposes: Evaluation of a prototype of photovoltaic greenhouse tunnel. Renew. Sustain. Energy Rev. 2018, 82, 1178-1186. [CrossRef]

40. FOA. World Agriculture: Towards 2015/2030 Summary Report; FAO: Rome, Italy, 2002.

41. Schindele, S.; Trommsdorff, M.; Schlaak, A.; Obergfell, T.; Bopp, G.; Reise, C.; Braun, C.; Weselek, A.; Bauerle, A.; Högy, P.; et al. Implementation of agrophotovoltaics: Techno-economic analysis of the price-performance ratio and its policy implications. Appl. Energy 2020, 265, 114737. [CrossRef]

42. Andrew, A.C.; Higgins, C.W.; Smallman, M.A.; Graham, M.; Ates, S. Herbage Yield, Lamb Growth and Foraging Behavior in Agrivoltaic Production System. Front. Sustain. Food Syst. 2021, 5, 1-12. [CrossRef]

43. Leon, A.; Ishihara, K.N. Assessment of new functional units for agrivoltaic systems. J. Environ. Manage. 2018, 226, 493-498. [CrossRef]

44. Pascaris, A.S.; Schelly, C.; Pearce, J.M. A First Investigation of Agriculture Sector Perspectives on the Opportunities and Barriers for Agrivoltaics. Agronomy 2020, 10, 1885. [CrossRef]

45. Elamri, Y.; Cheviron, B.; Mange, A.; Dejean, C.; Liron, F.; Belaud, G. Rain concentration and sheltering effect of solar panels on cultivated plots. Hydrol. Earth Syst. Sci. 2018, 22, 1285-1298. [CrossRef]

46. Sani Ibrahim, M.; Kumari, R. Emerging Solar Energy Technologies for Sustainable Farming: A Review. J. Xi'an Univ. Archit. Technol. 2020, 12, 5328-5336.

47. Imran, H.; Riaz, M.H.; Butt, N.Z. Optimization of Single-Axis Tracking of Photovoltaic Modules for Agrivoltaic Systems. Conf. Rec. IEEE Photovolt. Spec. Conf. 2020, 2020, 1353-1356. [CrossRef]

48. Hassanien, R.H.E.; Li, M.; Yin, F. The integration of semi-transparent photovoltaics on greenhouse roof for energy and plant production. Renew. Energy 2018, 121, 377-388. [CrossRef]

49. Kumpanalaisatit, M.; Setthapun, W.; Sintuya, H.; Jansri, S.N. Design and Test of Agri-Voltaic System. Turk. J. Comput. Math. Educ. 2021, 12, 2395-2404.

50. Othman, N.F.; Ya'Acob, M.E.; Abdul-Rahim, A.S.; Hizam, H.; Farid, M.M.; Abd Aziz, S. Inculcating herbal plots as effective cooling mechanism in urban planning. Acta Hortic. 2017, 1152, 235-242. [CrossRef]

51. Ravi, S.; Macknick, J.; Lobell, D.; Field, C.; Ganesan, K.; Jain, R.; Elchinger, M.; Stoltenberg, B. Colocation opportunities for large solar infrastructures and agriculture in drylands. Appl. Energy 2016, 165, 383-392. [CrossRef]

52. Yano, A.; Onoe, M.; Nakata, J. Prototype semi-transparent photovoltaic modules for greenhouse roof applications. Biosyst. Eng. 2014, 122, 62-73. [CrossRef]

53. Amaducci, S.; Yin, X.; Colauzzi, M. Agrivoltaic systems to optimise land use for electric energy production. Appl. Energy 2018, 220, 545-561. [CrossRef]

54. Cossu, M.; Cossu, A.; Deligios, P.A.; Ledda, L.; Li, Z.; Fatnassi, H.; Poncet, C.; Yano, A. Assessment and comparison of the solar radiation distribution inside the main commercial photovoltaic greenhouse types in Europe. Renew. Sustain. Energy Rev. 2018, 94, 822-834. [CrossRef]

55. Allardyce, C.S.; Fankhauser, C.; Zakeeruddin, S.M.; Grätzel, M.; Dyson, P.J. The influence of greenhouse-integrated photovoltaics on crop production. Sol. Energy 2017, 155, 517-522. [CrossRef]

56. Chel, K. Renewable energy for sustainable agriculture. Agron. Sustain. Dev. 2011, 31, 91-118. [CrossRef] 
57. Ott, E.M.; Kabus, C.A.; Baxter, B.D.; Hannon, B.; Celik, I. Environmental Analysis of Agrivoltaic Systems. In Reference Module in Earth Systems and Environmental Sciences; Elsevier: Amsterdam, The Netherlands, 2020; ISBN 9780128197271.

58. Leon, A.; Ishihara, K.N. Influence of allocation methods on the LC-CO2 emission of an agrivoltaic system. Resour. Conserv. Recycl. 2018, 138, 110-117. [CrossRef]

59. Othman, N.F.; Ya'acob, M.E.; Abdul-Rahim, A.S.; Shahwahid Othman, M.; Radzi, M.A.M.; Hizam, H.; Wang, Y.D.; Ya'Acob, A.M.; Jaafar, H.Z.E. Embracing new agriculture commodity through integration of Java Tea as high Value Herbal crops in solar PV farms. J. Clean. Prod. 2015, 91, 71-77. [CrossRef]

60. Zhai, M.; Huang, G.; Liu, L.; Zheng, B.; Guan, Y. Inter-regional carbon flows embodied in electricity transmission: Network simulation for energy-carbon nexus. Renew. Sustain. Energy Rev. 2020, 118, 109511. [CrossRef]

61. Othman, N.F.; Yap, S.; Ya'Acob, M.E.; Hizam, H.; Su, A.S.M.; Iskandar, N. Performance evaluation for agrovoltaic DC generation in tropical climatic conditions. AIP Conf. Proc. 2019, 2129, 020006. [CrossRef]

62. Agostini, A.; Colauzzi, M.; Amaducci, S. Innovative agrivoltaic systems to produce sustainable energy: An economic and environmental assessment. Appl. Energy 2021, 281, 116102. [CrossRef]

63. Makavana, J.M.; Kalaiya, S.V.; Chauhan, P.M.; Dulawat, M.S. Advantage of Agrivoltaics Across the Food-Energy-Water Connection. ACTA Sci. Agric. 2020, 4, 15-17.

64. Santiteerakul, S.; Sopadang, A.; Tippayawong, K.Y.; Tamvimol, K. The role of smart technology in sustainable agriculture: A case study of wangree plant factory. Sustainability 2020, 12, 4640. [CrossRef]

65. Riaz, M.H.; Younas, R.; Imran, H.; Alam, M.A.; Butt, N.Z. Module Technology for Agrivoltaics: Vertical Bifacial vs. Tilted Monofacial Farms. EEE J. Photovolt. 2021, 11, 469-477. [CrossRef]

66. Burgess, P.; Graves, A.; de Jalón, S.G.; Palma, J.; Dupraz, C.; van Noordwijk, M. Modelling Agroforestry Systems. In Agroforestry for Sustainable Agriculture; Burleigh Dodds Science Publishing: Cambridge, UK, 2019; pp. 209-238.

67. Dupraz, C.; Talbot, G.; Marrou, H.; Wery, J.; Roux, S.; Liagre, F.; A., F.Y.N.; System, U.M.R.; Viala, P.; Cedex, M.; et al. To Mix or Not to Mix: Evidences for the Unexpected High Productivity of New Complex Agrivoltaic and Agroforestry Systems. In Proceedings of the 5th World Congress of Conservation Agriculture: Resilient Food Systems for a Changing World, Brisbane, Australia, 26-29 September 2011; pp. 5-7.

68. Trommsdorff, M.; Kang, J.; Reise, C.; Schindele, S.; Bopp, G.; Ehmann, A.; Weselek, A.; Högy, P.; Obergfell, T. Combining food and energy production: Design of an agrivoltaic system applied in arable and vegetable farming in Germany. Renew. Sustain. Energy Rev. 2021, 140, 110694. [CrossRef]

69. Marrou, H.; Guilioni, L.; Dufour, L.; Dupraz, C.; Wery, J. Microclimate under agrivoltaic systems: Is crop growth rate affected in the partial shade of solar panels? Agric. For. Meteorol. 2013, 177, 117-132. [CrossRef]

70. Cossu, M.; Ledda, L.; Urracci, G.; Sirigu, A.; Cossu, A.; Murgia, L.; Pazzona, A.; Yano, A. An algorithm for the calculation of the light distribution in photovoltaic greenhouses. Sol. Energy 2017, 141, 38-48. [CrossRef]

71. Jumali, S.; Ya'acob, M.E.; Shamsudin, R.; Othman, N.F. Field assessment for photovoltaic array as herbal plots based on bioactive compounds analysis. In Proceedings of the 2016 IEEE Industrial Electronics and Applications Conference, Kota Kinabalu, Malaysia, 20-22 November 2016; pp. 88-91. [CrossRef]

72. Groesbeck, J.G.; Pearce, J.M. Coal with Carbon Capture and Sequestration is not as Land Use Efficient as Solar Photovoltaic Technology for Climate Neutral Electricity Production. Sci. Rep. 2018, 8, 1-17. [CrossRef] [PubMed]

73. Barbera, E.; Sforza, E.; Vecchiato, L.; Bertucco, A. Energy and economic analysis of microalgae cultivation in a photovoltaic-assisted greenhouse: Scenedesmus obliquus as a case study. Energy 2017, 140, 116-124. [CrossRef]

74. Papaioannou, G.; Papanikolaou, N.; Retalis, D. Theoretical and Applied Climatology Relationships of Photosynthetically Active Radiation and Shortwave Irradiance. Theor. Appl. Climatol. 1993, 27, 23-27. [CrossRef]

75. Perna, A.; Grubbs, E.K.; Agrawal, R.; Bermel, P. Design Considerations for Agrophotovoltaic Systems: Maintaining PV Area with Increased Crop Yield. In Proceedings of the IEEE 46th Photovoltaic Specialists Conference (PVSC), Chicago, IL, USA, 16-21 June 2019; pp. 668-672. [CrossRef]

76. Tang, Y.; Li, M.; Ma, X. Study on Photovoltaic Modules on Greenhouse Roof for Energy and Strawberry Production. In Proceedings of the E3S Web of Conferences (ICAEER 2019), Shanghai, China, 16-18 August 2019; Volume 118, p. 03049.

77. Amelia, A.R.; Irwan, Y.M.; Leow, W.Z.; Mat, M.H.; Rahim, M.S.A.; Esa, S.M. Technologies of solar tracking systems: A review. IOP Conf. Ser. Mater. Sci. Eng. 2020, 767, 1-10. [CrossRef]

78. Seme, S.; Štumberger, B.; Hadžiselimović, M.; Sredenšek, K. Solar photovoltaic tracking systems for electricity generation: A review. Energies 2020, 13, 4224. [CrossRef]

79. Lim, J.R.; Shin, W.G.; Lee, C.G.; Lee, Y.G.; Ju, Y.C.; Ko, S.W.; Kim, J.D.; Kang, G.H.; Hwang, H. A Study of the Electrical Output and Reliability Characteristics of the Crystalline Photovoltaic Module According to the Front Materials. Energies 2021, 14, 163. [CrossRef]

80. Hafez, A.Z.; Soliman, A.; El-Metwally, K.A.; Ismail, I.M. Tilt and azimuth angles in solar energy applications-A review. Renew. Sustain. Energy Rev. 2017, 77, 147-168. [CrossRef]

81. Pashiardis, S.; Kalogirou, S.A.; Pelengaris, A. Characteristics of Photosynthetic Active Radiation (PAR) Through Statistical Analysis at Larnaca, Cyprus. SM J. Biometrics Biostat. 2017, 2, 1-16. [CrossRef] 
82. Marrou, H.; Dufour, L.; Wery, J. How does a shelter of solar panels influence water flows in a soil-crop system? Eur. J. Agron. 2013, 50, 38-51. [CrossRef]

83. Ren, X.; He, H.; Zhang, L.; Yu, G. Global radiation, photosynthetically active radiation, and the diffuse component dataset of China, 1981-2010. Earth Syst. Sci. Data 2018, 10, 1217-1226. [CrossRef]

84. Pérez-Alonso, J.; Pérez-García, M.; Pasamontes-Romera, M.; Callejón-Ferre, A.J. Performance analysis and neural modelling of a greenhouse integrated photovoltaic system. Renew. Sustain. Energy Rev. 2012, 16, 4675-4685. [CrossRef]

85. Ayush Das, S.D. Simulation and Implementation of Single Axis Solar Tracker Ayush. Int. Res. J. Eng. Technol. 2020, 7, 756-761.

86. Hohmann-Marriott, M.F.; Blankenship, R.E. Evolution of photosynthesis. Annu. Rev. Plant Biol. 2011, 515-548. [CrossRef] [PubMed]

87. Dinesh, H.; Pearce, J.M. The potential of agrivoltaic systems. Renew. Sustain. Energy Rev. 2016, 54, 299-308. [CrossRef]

88. Kuttybay, N.; Saymbetov, A.; Mekhilef, S.; Nurgaliyev, M. Optimized Single-Axis Schedule Solar Tracker. Energies 2020, $13,5226$. [CrossRef]

89. Chang, T.P. Output energy of a photovoltaic module mounted on a single-axis tracking system. Appl. Energy 2009, 86, 2071-2078. [CrossRef]

90. Gul, M.; Kotak, Y.; Muneer, T.; Ivanova, S. Enhancement of albedo for solar energy gain with particular emphasis on overcast skies. Energies 2018, 11, 2881. [CrossRef]

91. Moretti, S.; Marucci, A. A photovoltaic greenhouse with variable shading for the optimization of agricultural and energy production. Energies 2019, 12, 2589. [CrossRef]

92. Nakoul, Z.; Bibi-Triki, N.; Kherrous, A.; Bessenouci, M.Z.; Khelladi, S. Optimization of a solar photovoltaic applied to greenhouses. Phys. Procedia 2014, 55, 383-389. [CrossRef]

93. Yadav, A.K.; Chandel, S.S. Tilt angle optimization to maximize incident solar radiation: A review. Renew. Sustain. Energy Rev. 2013, 23, 503-513. [CrossRef]

94. Harinarayana, T.; Vasavi, K.S.V. Solar Energy Generation Using Agriculture Cultivated Lands. Smart Grid Renew. Energy 2014, 5, 31-42. [CrossRef]

95. Malu, P.R.; Sharma, U.S.; Pearce, J.M. Agrivoltaic potential on grape farms in India. Sustain. Energy Technol. Assess. 2017, 23, 104-110. [CrossRef]

96. Ozcelik, S.; Prakash, H.; Challoo, R. Two-axis solar tracker analysis and control for maximum power generation. Procedia Comput. Sci. 2011, 6, 457-462. [CrossRef]

97. Thompson, E.P.; Bombelli, E.L.; Shubham, S.; Watson, H.; Everard, A.; D’Ardes, V.; Schievano, A.; Bocchi, S.; Zand, N.; Howe, C.J.; et al. Tinted Semi-Transparent Solar Panels Allow Concurrent Production of Crops and Electricity on the Same Cropland. Adv. Energy Mater. 2020, 10, 1-9. [CrossRef]

98. Touil, S.; Richa, A.; Fizir, M.; Bingwa, B. Shading effect of photovoltaic panels on horticulture crops production: A mini review. Rev. Environ. Sci. Biotechnol. 2021, 20, 281-296. [CrossRef]

99. Gese, P.; Martínez-Conde, F.M.; Ramírez-Sagner, G.; Dinter, F. Agrivoltaic in Chile-Integrative solution to use efficiently land for food and energy production and generating potential synergy effects shown by a pilot plant in Metropolitan region. In Proceedings of the International Conference on Solar Heating and Cooling for Buildings and Industry (SHC), Santiago de Chile, Chile, 3-7 November 2019; pp. 1016-1024. [CrossRef]

100. Verstraeten, W.W.; Veroustraete, F.; Feyen, J. Assessment of evapotranspiration and soil moisture content across different scales of observation. Sensors 2008, 8, 70-117. [CrossRef]

101. Wang, D.; Sun, Y. Optimizing Light Environment of the Oblique Single-axis Tracking Agrivoltaic System. IOP Conf. Ser. Earth Environ. Sci. 2018, 170, 042069. [CrossRef]

102. Fadaeenejad, M.; Radzi, M.A.M.; Fadaeenejad, M.; Zarif, M.; Gandomi, Z. Optimization and comparison analysis for application of PV panels in three villages. Energy Sci. Eng. 2015, 3, 145-152. [CrossRef]

103. Marucci, A.; Cappuccini, A. Dynamic photovoltaic greenhouse: Energy balance in completely clear sky condition during the hot period. Energy 2016, 102, 302-312. [CrossRef]

104. Dufour, L.; Metay, A.; Talbot, G.; Dupraz, C. Assessing light competition for cereal production in temperate agroforestry systems using experimentation and crop modelling. J. Agron. Crop Sci. 2013, 199, 217-227. [CrossRef]

105. García-Rodríguez, A.; García-Rodríguez, S.; Díez-Mediavilla, M.; Alonso-Tristán, C. Photosynthetic active radiation, solar irradiance and the cie standard sky classification. Appl. Sci. 2020, 10, 8007. [CrossRef]

106. Macmillan Learning The Electromagnetic Spectrum. Available online: https://sites.google.com/site/chempendix/em-spectrum (accessed on 20 January 2001).

107. Macknick, J.; Beatty, B.; Hill, G. Overview of Opportunities for Co-Location of Solar Energy Technologies and Vegetation; National Renewable Energy Lab.: Golden, CO, USA, 2013.

108. Kenning, T. TNB connects first phase of Malaysia's largest solar project to the grid. Available online: https://www.pv-tech.org/ tnb-connects-malaysias-largest-solar-project-to-the-grid/ (accessed on 25 March 2021).

109. Pascaris, A.S.; Schelly, C.; Burnham, L.; Pearce, J.M. Integrating solar energy with agriculture: Industry perspectives on the market, community, and socio-political dimensions of agrivoltaics. Energy Res. Soc. Sci. 2021, 75, 102023. [CrossRef] 
110. Irie, N.; Kawahara, N.; Esteves, A.M. Sector-wide social impact scoping of agrivoltaic systems: A case study in Japan. Renew. Energy 2019, 139, 1463-1476. [CrossRef]

111. Yeongseo, Y.; Yekang, K. A Review of the Attributes of Successful Agriphotovoltaic Projects. In Proceedings of the APRU 2020 Sustainable Cities and Landscapes. PhD. Thesis, The University of Auckland, Auckland, Australia, 2020.

112. Kadowaki, M.; Yano, A.; Ishizu, F.; Tanaka, T.; Noda, S. Effects of greenhouse photovoltaic array shading on Welsh onion growth. Biosyst. Eng. 2012, 111, 290-297. [CrossRef]

113. Chen, N.; Wu, P.; Gao, Y.; Ma, X. Review on Photovoltaic Agriculture Application and Its Potential on Grape Farms in Xinjiang, China. Adv. Sci. Eng. 2018, 10, 73-81.

114. Kuemmel, B.; Langer, V.; Magid, J.; De Neergaard, A.; Porter, J.R. Energetic, economic and ecological balances of a combined food and energy system. Biomass Bioenergy 1998, 15, 407-416. [CrossRef]

115. Dias, L.; Gouveia, J.P.; Lourenço, P.; Seixas, J. Interplay between the potential of photovoltaic systems and agricultural land use. Land Policy 2019, 81, 725-735. [CrossRef]

116. Department of Standard Malaysia. Solar Photovoltaic Energy Systems_-Terms, Definition and Symbols; Department of Standard Malaysia: Cyberjaya, Malaysia, 2010.

117. Husain, A.A.F.; Hasan, W.Z.W.; Shafie, S.; Hamidon, M.N.; Pandey, S.S. A review of transparent solar photovoltaic technologies. Renew. Sustain. Energy Rev. 2018, 94, 779-791. [CrossRef]

118. Shukla, A.; Kant, K.; Sharma, A.; Biwole, P.H. Cooling methodologies of photovoltaic module for enhancing electrical efficiency: A review. Sol. Energy Mater. Sol. Cells 2017, 160, 275-286. [CrossRef]

119. Zaini, N.H.; Mohd Zainal, M.Z.A.; Radzi, M.A.M.; Izadi, M.; Azis, N.; Ahmad, N.I.; Nasir, M.S.M. Lightning surge analysis on a large scale grid-connected solar photovoltaic system. Energies 2017, 10, 2149. [CrossRef]

120. Wan Abdullah, W.S.; Osman, M.; Ab Kadir, M.Z.A.; Verayiah, R. The Potential and Status of Renewable Energy Development in Malaysia. Energies 2019, 12, 2437. [CrossRef]

121. Loik, M.E.; Carter, S.A.; Alers, G.; Wade, C.E.; Shugar, D.; Corrado, C.; Jokerst, D.; Kitayama, C. Wavelength-Selective Solar Photovoltaic Systems: Powering Greenhouses for Plant Growth at the Food-Energy-Water Nexus. Earth Future 2017, 5, $1044-1053$. [CrossRef]

122. REN21. Key Findings of The Renewables 2020 Global Status Report; REN21: Paris, France, 2020.

123. Othman, N.F.; Jamian, S.; Su, A.S.M.; Ya'Acob, M.E. Tropical field assessment on pests for Misai Kucing cultivation under agrivoltaics farming system. AIP Conf. Proc. 2019, 2129. [CrossRef]

124. Osterthun, N.; Neugebohrn, N.; Gehrke, K.; Vehse, M.; Agert, C. Spectral engineering of ultrathin germanium solar cells for combined photovoltaic and photosynthesis. Opt. Express 2021, 29, 938. [CrossRef] [PubMed]

125. Oleskewicz, K. The Effect of Gap Spacing Between Solar Panel Clusters on Crop Biomass Yields, Nutrients and The Microenvironment in a Dual-Use Agrivoltaic System; University of Massachusetts Amherst: Amherst, MA, USA, 2020.

126. Cossu, M.; Yano, A.; Li, Z.; Onoe, M.; Nakamura, H.; Matsumoto, T.; Nakata, J. Advances on the semi-transparent modules based on micro solar cells: First integration in a greenhouse system. Appl. Energy 2015, 162, 1042-1051. [CrossRef]

127. Weselek, A.; Bauerle, A.; Zikeli, S.; Lewandowski, I.; Högy, P. Effects on Crop Development, Yields and Chemical Composition of Celeriac (Apium graveolens L. var. rapaceum) Cultivated Underneath an Agrivoltaic System. Agronomy 2021, 11, 733. [CrossRef]

128. Zorz, J.; Richardson, W.D.L.; Laventure, A.; Haines, M.; Cieplechowicz, E.; Aslani, A.; Vadlamani, A.; Bergerson, J.; Welch, G.C.; Strous, M. Light manipulation using organic semiconducting materials for enhanced photosynthesis. Cell Reports Phys. Sci. 2021, 2, 100390. [CrossRef]

129. Daigle, Q.; Talebzadeh, N.; O’Brien, P.G.; Rauf, I.A. Spectral Splitting Luminescent Solar Concentrator Panels for Agrivoltaic Applications. Proc. 3rd Int. Conf. Energy Harvest. Storage Transf. 2019, 3, 132-133. [CrossRef]

130. Guo, L.; Han, J.; Otieno, A.W. Design and Simulation of a Sun Tracking Solar Power System. In Proceedings of the 120th ASEE Annual Conference and Exposition, Atlanta, GA, USA, 23-26 June 2013; p. 7854.

131. Roth, P.; Georgiev, A.; Boudinov, H. Cheap two axis sun following device. Energy Convers. Manag. 2005, 46, 1179-1192. [CrossRef]

132. Adeh, E.H.; Selker, J.S.; Higgins, C.W. Remarkable agrivoltaic influence on soil moisture, micrometeorology and water-use efficiency. PLoS ONE 2018, 13. [CrossRef]

133. Hubach, J.O. Solar Tracking Using a Parallel Manipulator Mechanism to Achieve Two-Axis Position Tracking; Rose-Hulman Institute of Technology: Terre Haute, IN, USA, 2019.

134. Kentli, F.; Yilmaz, M. Mathematical modelling of two-axis photovoltaic system with improved efficiency. Elektron. Elektrotechnika 2015, 21, 40-43. [CrossRef]

135. Saini, V.; Tiwari, S.; Tiwari, G.N. Environ economic analysis of various types of photovoltaic technologies integrated with greenhouse solar drying system. J. Clean. Prod. 2017, 156, 30-40. [CrossRef]

136. Sacchelli, S.; Garegnani, G.; Geri, F.; Grilli, G.; Paletto, A.; Zambelli, P.; Ciolli, M.; Vettorato, D. Trade-off between photovoltaic systems installation and agricultural practices on arable lands: An environmental and socio-economic impact analysis for Italy. Land Policy 2016, 56, 90-99. [CrossRef]

137. Higgins, C.W.; Najm, M.A. An Organizing Principle for the Water-Energy-Food Nexus. Sustainability 2020, 12, 8135. [CrossRef]

138. Nonhebel, S. Renewable energy and food supply: Will there be enough land? Renew. Sustain. Energy Rev. 2005, 9, 191-201. [CrossRef] 
139. Aguilar, J.; Rogers, D.; Kisekka, I. Irrigation Scheduling Based on Soil Moisture Sensors and Evapotranspiration. Kans. Agric. Exp. Stn. Res. Rep. 2015, 1. [CrossRef]

140. Muñoz-Carpena, R.; Dukes, M.D. Automatic Irrigation Based on Soil Moisture for Vegetable Crops; AE354: Gainesville, FL, USA, 2005.

141. Jensen, M.E.; Allen, R.G. Evaporation, Evapotranspiration and Irrigation Water Requirements, 2nd ed.; American Society of Civil Engineers: Reston, VA, USA, 2016; No.70; ISBN 9780784479209.

142. Sharu, E.H.; Ab Razak, M.S. Hydraulic performance and modelling of pressurized drip irrigation system. Water 2020, $12,2295$. [CrossRef]

143. Moswetsi, G.; Fanadzo, M.; Ncube, B. Review Article Cropping Systems and Agronomic Management Practices in Smallholder Farms in South Africa: Constraints, Challenges and Opportunities. J. Agron. 2017, 16, 51-67. [CrossRef] 



\title{
The Urban Characteristics of High Economic Resilient Neighborhoods during the COVID-19 Pandemic: A Case of Suwon, South Korea
}

\author{
Sungjo Hong ${ }^{1}$ id and Seok-Hwan Choi ${ }^{2, *}$ \\ 1 Department of Urban Engineering, Chungbuk National University, Cheongju 28644, Korea; \\ sjhong@chungbuk.ac.kr \\ 2 Department of Urban Space Research, Suwon Research Institute, Suwon 16429, Korea \\ * Correspondence: csh@suwon.re.kr; Tel.: +82-31-220-8041
}

check for updates

Citation: Hong, S.; Choi, S.-H. The Urban Characteristics of High Economic Resilient Neighborhoods during the COVID-19 Pandemic: A Case of Suwon, South Korea. Sustainability 2021, 13, 4679 https://doi.org/10.3390/su13094679

Academic Editor: Idiano D’Adamo

Received: 26 February 2021

Accepted: 20 April 2021

Published: 22 April 2021

Publisher's Note: MDPI stays neutral with regard to jurisdictional claims in published maps and institutional affiliations.

Copyright: (c) 2021 by the authors. Licensee MDPI, Basel, Switzerland. This article is an open access article distributed under the terms and conditions of the Creative Commons Attribution (CC BY) license (https:// creativecommons.org/licenses/by/ $4.0 /)$.

\begin{abstract}
Infectious diseases and pandemics, including the COVID-19 pandemic, have a huge economic impact on cities. However, few studies examine the economic resilience of small-scale regions within cities. Thus, this study derives neighborhoods with high economic resilience in a pandemic situation and reveals their urban characteristics. It evaluates economic resilience by analyzing changes in the amount of credit card payments in the neighborhood and classifying the types of neighborhoods therefrom. The study conducted the ANOVA, Kruskal-Wallis, and post hoc tests to analyze the difference in urban characteristics between neighborhood types. Accordingly, three neighborhood types emerged from the analysis: high-resilient neighborhood, low-resilient neighborhood, and neighborhood that benefited from the pandemic. The high-resilient neighborhood is a low-density residential area where many elderly people live. Neighborhoods that benefited are residential areas mainly located in high-density apartments where many families of parents and children live. The lowresilient neighborhood is an area with many young people and small households, many studio-type small houses, and a high degree of land-use mix.
\end{abstract}

Keywords: resilience of city; pandemic; infectious disease; COVID-19; urban planning

\section{Introduction \\ 1.1. Background and Purpose}

As of 15 February 2021, the number of confirmed Coronavirus disease (COVID-19) cases exceeded 100 million, and the number of deaths exceeded 2.3 million [1]. The damage caused by COVID-19 is global, with its effect especially being felt in large cities. Île-deFrance accounts for 34\% of French cases; New York, 14.6\% of American cases; Quebec, $61 \%$ of Canadian cases; Metropolitan Santiago, 70\% Chilean cases; and Sao Paulo, 25\% of Brazilian cases [2]. In Korea, as of 26 January 2021, cases in the Seoul Metropolitan Area accounted for $61.2 \%$ of all cases [3]. In large cities, both the number of infected people and fatalities are high. As of November 2020, several regions with the highest fatality rate are situated in metropolitan areas. Specifically, Île-de-France in France, Quebec in Canada, New Jersey in the United States (US), Stockholm in Sweden, and metropolitan Santiago in Chile recorded the highest fatality rates [2]. The fatality rates in these regions are often more than twice the national average [2]. The characteristic of large cities with many people and activities is generally advantageous. However, in a pandemic situation, it is a vulnerability. The COVID-19 pandemic is re-kindling the old debate over cities' vulnerabilities [4] to epidemics.

As of 2018, 55.2\% of the world's population lives in cities, and $23.3 \%$ live in large cities of over one million people [5]. Moreover, the city population is expected to exceed $60 \%$ by 2030 [5]. Regarding global urbanization, the vulnerability of cities to infectious diseases is a critical issue. In particular, the incidence of infectious diseases is expected to increase due 
to climate change [6-8]. Thus, it is necessary to examine cities' preparation, response, and adaptation methods for infectious diseases [7].

Infectious diseases affect cities in various ways. This study focuses on the economic impact of COVID-19 on cities. Unlike natural disasters such as earthquakes, floods, and typhoons that cause significant physical damage to cities, infectious diseases are well known historically for their significant social and economic impacts [9]. For instance, the Black Death completely changed the economic structure of the time [10]. Even in relatively modern times, the 1918-1920 Spanish flu had the fourth largest economic impact after World War II, World War I, and the Great Depression [11].

After 2000, the 2002-2003 SARS-COV, the 2004-2006 Avian Influenza (H5N1), and the 2012 MERS-COV had a major impact on the economy. According to studies in Hong Kong [12] and Taiwan [13], SARS-COV drastically reduced consumption demand, even in a short time. The gross domestic product (GDP) is expected to decrease by $0.2 \%$ in Korea, $0.14 \%$ in the US, and $2.42 \%$ in China due to SARS-COV [14]. H5N1, though unlikely to be transmitted to humans, poses a significant threat. The global GDP is expected to decrease by 0.7 to $4.8 \%$ or 0.1 to $0.7 \%$, depending on whether it is transmitted to humans or not [15] MERS-COV was mainly in Saudi Arabia, the United Arab Emirates, and Korea [16]. The economic loss due to MERS-COV in Korea was estimated to reach 11 trillion won (about USD 9.9 billion) [17].

Although the COVID-19 pandemic is ongoing, some studies examine its economic impact. Sharifi and Khavarian-Garmsir [18] found that research on the impact of COVID-19 on cities is mainly conducted in four areas: environmental quality, socio-economic impact, management and governance, transportation and urban design. Early studies on the economic impact of COVID-19 reported that city revenue, income, tourism, and small- and medium-sized businesses were significantly affected [18].

The most widely cited item for the economic impact of the epidemic is the decrease in GDP [15,19-22]. The decline in GDP can be considered to be due to the combined economic impact of the epidemic. Among the individual economic impacts of infectious diseases, the reduction in consumption is widely cited [12-14,23]. Temporary increases in consumption for stockpiling have been reported in the United States [24,25], but this is a limited result. Consumption declines have been reported in most countries. In EU countries, consumption expenditures declined in the second quarter of 2020 [23]. In particular, the service sector, tourism, catering, and leisure sectors are immediately affected by lockdown and social distancing [9]. Unlike past epidemics, the overall decline in consumption and the transfer of consumption to online shopping now appear together. Thus, local retailers suffer significantly. Moreover, reduced consumption and changes in consumption behavior may adversely affect tax revenue for central and local governments. In addition, the collapse of the supply chain should be noted as a major economic impact of the pandemic [26], which impacts many industries [27]. In particular, the collapse of the food supply chain has been raised at the local level as well [28]. In Korea, there were no major problems in terms of daily necessities, but a significant price increase due to the shortage of masks and hand sanitizers was evident.

Economic impacts from infectious diseases, such as the decline in consumption, are not the same regionally $[18,29]$. Infectious diseases inhibit the movement of people via social distancing and lockdowns, causing great damage in tourist destinations and large cities where the influx of people is vital $[2,29]$. Moreover, since the ratio of jobs that allow for remote work is also high in large cities [2], the economic damage to commercial business areas in large cities can be significant. Further, the negative economic impact of the epidemic is greater in areas where cities lack economic diversity [30]. Given that the impact of the epidemic varies from region to region, the OECD has also raised the importance of place-based policies [2].

Thus, to establish a place-based policy, information on regional differences in pandemic influence and characteristics of such regions should be provided. However, apart from studies on tourist cities, few studies focus on the regional effects of infectious diseases 
and regional characteristics. In particular, it is challenging to find studies that analyze microscopic regional units smaller than the city-scale.

Given the disastrous effects of a pandemic, post-disaster resilience in cities is garnering scholarly attention. Notably, economic resilience is crucial for cities to prepare against such black-swan events [31,32]. Despite much interest in urban resilience to COVID-19, there are few academic approaches to addressing the problem because empirical research on resilience is challenging in an ongoing pandemic. Therefore, studies mainly report or estimate the economic impact of the pandemic.

This study approaches the economic resilience of cities from the perspective of local consumption. As described above, the decrease in consumption is a common phenomenon due to infectious diseases. This study gauges regional economic resilience via how quickly the reduced regional consumption recovers.

The proportion of self-employed in Korea is $24.6 \%$, ranking sixth among the OECD, which is very high relative to the OECD average of $16.4 \%$ and the EU average of $15.3 \%$ [33]. They mainly engage in commercial activities, such as small retail, catering, and grocery, in neighborhoods. Therefore, the self-employed are directly affected by the decrease in local consumption due to the pandemic. The number of stores closed in the second quarter of 2020 in Korea reached 103,943. Thus, the number of self-employed having employees decreased by $11.5 \%$ [34]. They mainly operate small stores with part-time jobs. Their decline means the closing of small stores and unemployment for themselves and their employees.

Even after COVID-19, new pandemics are expected [6-8]. Even so, research on pandemics is insufficient because no infectious disease has reached pandemic status since the 1918 Spanish flu. Therefore, it is necessary to increase insight into city resilience in a pandemic situation through COVID-19 to draw implications for improving resilience in a future crisis. While pandemics do cause socio-economic damage, they also drive positive changes [35]. Therefore, this situation should be recognized as an opportunity to institute a sustainable economic system via a comprehensive understanding of all relevant changes.

Therefore, this study derived high economic resilient neighborhoods in the Covid-19 pandemic and analyzed the urban characteristics of these neighborhoods.

\subsection{Theoretical Perspective}

\subsubsection{Urban Economic Resilience}

The concept of resilience began in the fields of psychology and ecology, spreading to various fields [36]. Earlier concepts regarding resilience emphasized how quickly the system could return to an original equilibrium state when an external shock was applied [37]. This concept focused on recovery speed, and it is called "engineering resilience" [38]. Since then, the concept of resilience has expanded further. Even if the system fails to restore equilibrium, reaching a new state of equilibrium is being accepted as recovery as well. This concept concentrates on the attainment of "ecological resilience" [39]. Subsequently, studies have emerged that define the ability to adapt to continuous change as resilience, which is called "adaptive resilience" [40,41]. These concepts of resilience are related to the end goal, which can either be to "bounce back" or acquire a "new state of being" [42]. However, there are disagreements about which concept is appropriate depending on the practitioner and the discipline [42].

Since the early 2000s, urban resilience has emerged as a major issue in urban planning [42]; it has emerged as a concern of many researchers, given the spread of global urbanization trends [43]. Urban resilience is studied in various fields of urban planning. Leichenko [31] classified the literature on urban resilience into four categories: urban ecological resilience, urban hazards and disaster risk reduction, the resilience of urban and regional economies, and promoting resilience through urban governance and institutions. The OECD proposed to measure the resilience of cities in four areas: economy, society, governance, and the environment [32]. That is, studying economic resilience is a major means of understanding the resilience of a city. 
As mentioned above, the concept of resilience varies across academic domains. In this study, we measured the economic resilience of a neighborhood in terms of how quickly the reduced consumption amount in the neighborhood recovering to its pre-disaster level. In other words, we used the concept of bounce-back resilience (i.e., engineering resilience) because the declining sale of local stores is the biggest damage caused by COVID-19 in Korea. Since Korea has never implemented a forced lockdown, people have not experienced any difficulty in purchasing daily necessities. In other words, the damage to consumers caused by COVID-19 is not significant. Still, the decrease in sales and closure of businesses due to voluntary social distancing and changes in consumer preferences are serious [34]. As the proportion of self-employed individuals in Korea is high [33], the problem of diminishing sales has expanded to become an overall economic problem. Therefore, the return of local consumption to its previous level is essential in Korea.

After a sufficient period following the pandemic, it will be possible to analyze resilience from the standpoint of adaptation or achieving a new equilibrium. However, this study took the first wave and first stable period of Korea as the time range of the study. This period is short (i.e., 5 months); therefore, not enough time has passed to address ecological or adaptive resilience.

\subsubsection{Urban Resilience and Urban Sustainability}

Urban areas account for more than 75\% of global GDP and are responsible for most energy consumption and carbon emissions [44]. In this regard, urban sustainability is closely linked to global sustainability. It is also closely related to urban resilience [45], with past studies generally finding a positive correlation between them $[45,46]$. In the United Nation's New Urban Agenda [47], which discusses the future direction of cities, a positive correlation between urban sustainability and urban resilience is often mentioned. However, in an urban context, the concepts of resilience and sustainability are often misdefined, too narrowly defined, or used interchangeably [48]. In such cases, resilience and sustainability can be interpreted as having a negative correlation. For example, sustainability, narrowly interpreted as maximizing efficiency, can reduce resilience by removing redundancy [46]. Elmqvist et al. [44] proposed a new concept of urban sustainability and urban resilience to overcome these problems. According to these concepts, urban sustainability normatively sets the trajectory a city should take, and resilience implies the ability to absorb disturbances and remain functional to maintain this trajectory [44]. Therefore, urban resilience can be a means to achieve urban sustainability goals.

Regarding resilience and natural disasters, D'Adamo and Rosa [45] presented the need for research on the relationship between resilience and sustainability in natural disasters such as COVID-19. In particular, they insist that the resilience system can reduce damage from natural disasters and increase urban sustainability by investing in infrastructure to overcome such disasters [45]. In other words, with COVID-19, research on urban resilience should be used as an opportunity to increase the sustainability of both cities and the human race overall. In particular, external shocks such as pandemics can shift the trajectory toward sustainability [44] and should therefore be taken as an opening to transition to a more sustainable lifestyle [49].

\subsubsection{Concept of Neighborhood}

In this research, the neighborhood was considered to be a unit of study. Moreover, there is no single consensus regarding the definition of a neighborhood [50]. Baffoe [50] reviewed previous studies on neighborhood and categorized the definition of a neighborhood into three categories: neighborhood as a place, neighborhood as a community, and neighborhood as a policy unit. The neighborhood as a place denotes a locality where people reside and spend a lot of time. Consequently, people's quality of life is greatly affected by neighborhoods [51]. Neighborhood as a community takes into account the shared beliefs and interests of people living in the neighborhood [52]. Neighborhood as a policy unit refers to a unit for implementing policies at the local level [50]. 
In the field of planning, the concept of neighborhood, which began with Howard's garden city concept [53] and developed into Perry's neighborhood unit [54], is mainly employed. According to this concept, neighborhoods are small areas where people spend their daily lives, and their physical, social, and economic characteristics directly or indirectly affect others around them [55]. This definition of a neighborhood is closest to the concept of the neighborhood as a place. In the planning field, neighborhoods are also perceived as areas where essential services are provided [56,57]. This concept links to the neighborhood as a policy unit. This study deals with the neighborhood as an area where residents' daily consumption activities take place. Thus, the concept of a neighborhood as a place is most actively borrowed.

There have been many discussions concerning the criteria for classifying and categorizing neighborhoods, but no consensus has been achieved. The demographic composition (e.g., age, race, gender, etc.), housing-related factors (e.g., housing type, housing age, etc.), socio-economic class, physical environment, transportation, and health are frequently used as criteria for classifying neighborhoods [58,59]. For analysis, we selected related variables as urban characteristics.

\subsection{Case Context}

\subsubsection{Changes in the Number of COVID-19 Confirmed Cases in Korea}

Figure 1 shows the number of COVID-19 confirmed cases per month in 2020. In Korea, there were three waves after the first confirmed case. During the stable period after the first wave, the number of confirmed cases decreased sharply. In May, there were fewer than 25 confirmed cases per day nationwide. Accordingly, in Korea, the local economy recovered rapidly, and people's consumption also recovered. Given such rapid recovery, Korea's economic growth rate in the second quarter of 2020 was $-3.2 \%$, ranking first among OECD member countries. Further, this is very high relative to the OECD average $(-10.5 \%)$, the EU average (-11.4\%), and the US (-9\%) [60].

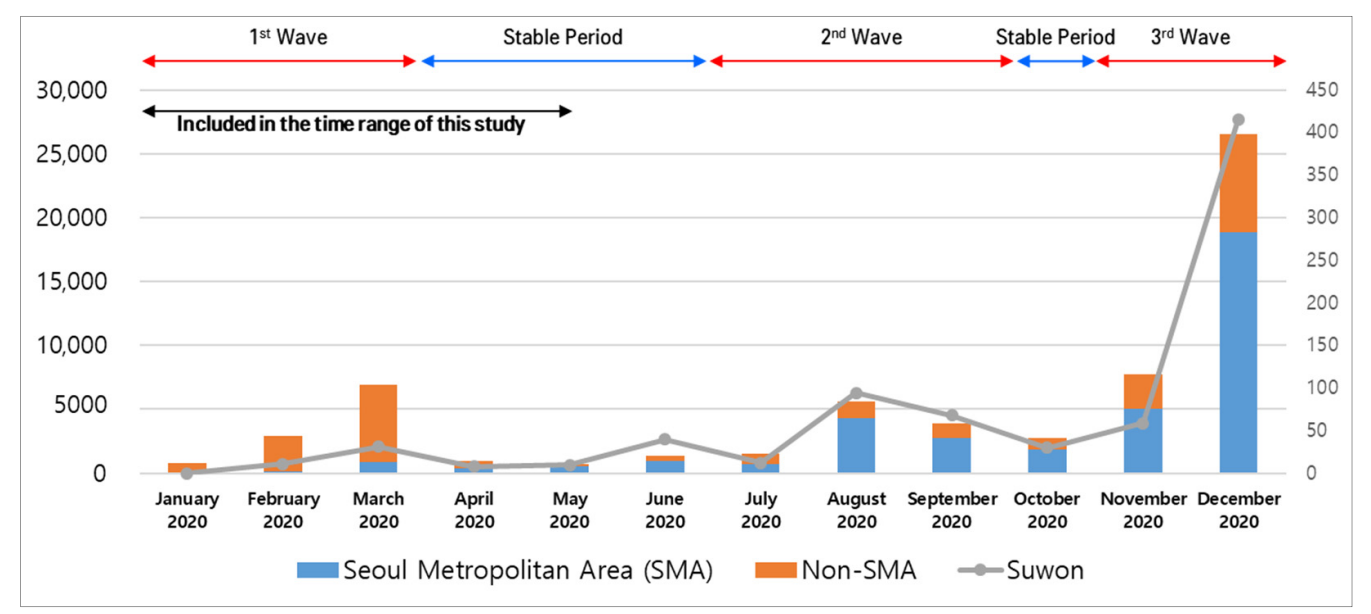

Figure 1. South Korea's COVID-19 new cases per month (data source: References [3,61]).

These changes make it possible to study the economic damage and recovery of cities in the situation where the COVID-19 pandemic is still ongoing. This study spanned June 2019 to May 2020, when the number of confirmed cases decreased the most, including the first wave. Moreover, the study employed consumption data for a year to confirm the usual consumption pattern.

\subsubsection{Status of Suwon City}

This study investigated Suwon. In Korea, cities are administratively divided into metropolitan cities and general cities. Since this study conducted a neighborhood-level analysis, we posited that metropolitan cities with large commercial catchment areas were 
not appropriate. Moreover, this study analyzed the economic impact of large cities vulnerable to pandemics. Suwon has a population of 1.23 million [62], making it the most populous among general cities in Korea.

Further, since this study employed credit card payments as consumption data, cities with online-shopping companies are unsuitable for analyses, because online shopping payments are counted as sales at such locations. Therefore, Seoul, with $83.1 \%$ of Korea's major online-shopping companies, and Seongnam, with 7.7\% [63], are unsuitable for the study. Suwon has no major online-shopping company; thus, the amount of card consumption in Suwon was used at offline stores.

Figure 2 shows the location and topography of Suwon City. It is located in Gyeonggido, with an area of $121.09 \mathrm{~km}^{2}$ and a population density of 10,115 people $/ \mathrm{km}^{2}$. A mountain is located in the north of Suwon, and agricultural land is preserved in the west. At the south, there is an airbase, which is expressed as agricultural land.

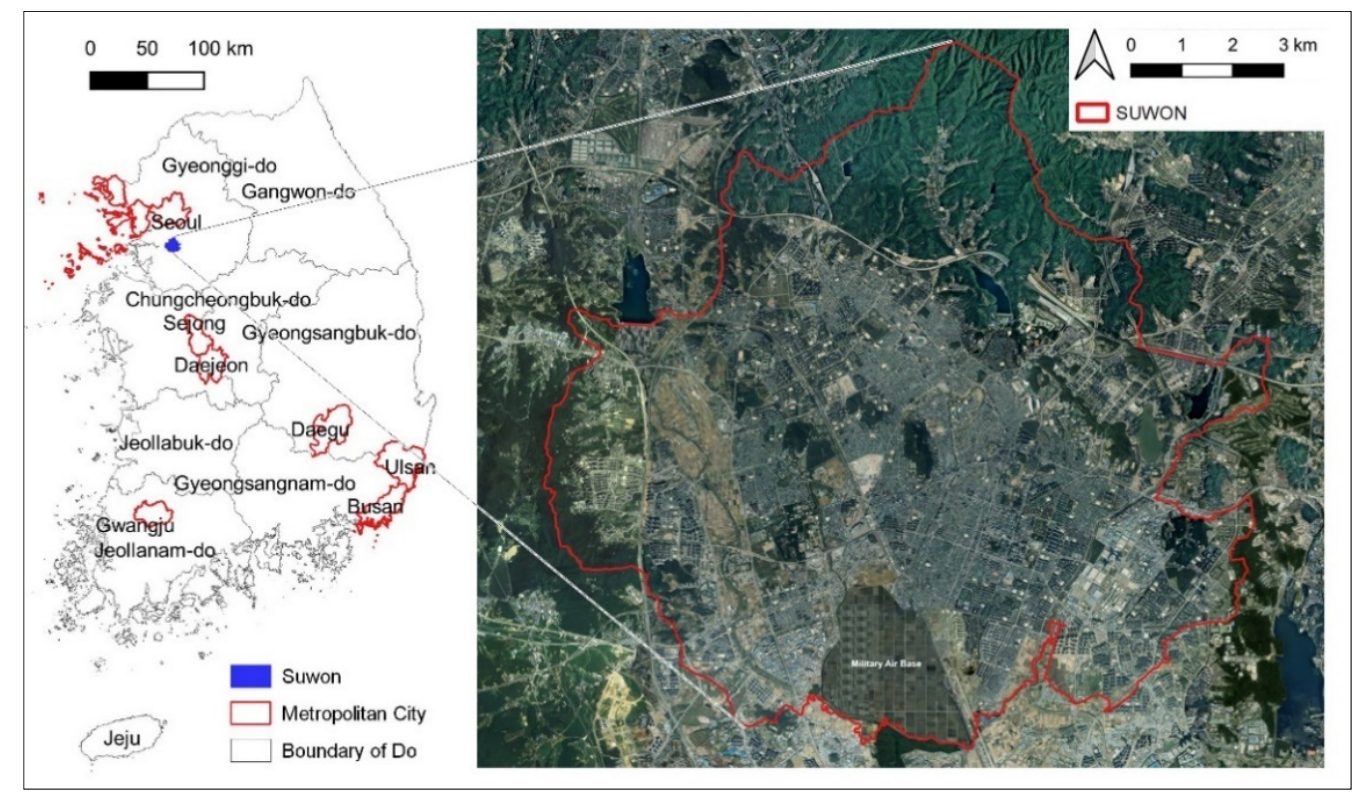

Figure 2. Location and satellite image of Suwon.

During the second half of 2019, the employment rate in Suwon city was $60.2 \%$, which is lower than the national average (i.e., 60.9\%) and the average employment rate of Gyeonggido (i.e., 61.9\%) [64]. Conversely, in 2018, the ratio of manufacturing workers in Suwon City was 10.7\%, which is less in comparison to Gyeonggi-do (25.5\%) [65]. On the other hand, the ratio of workers in the professional service industry was $12.4 \%$, which was larger in contrast to Gyeonggi-do (i.e., 5.0\%) [65]. In sum, Suwon is a city with a smaller manufacturing sector and a larger service sector, as opposed to other cities in Gyeonggi-do. Moreover, the ratio of accommodation and food service, which are industries vulnerable to COVID-19, is $11.2 \%$, which is greater than in Gyeonggi-do (i.e., 9.7\%) [65].

\subsubsection{Suwon's Consumption Reduction and Recovery in the COVID-19 Pandemic}

Consumption declined significantly due to the outbreak of COVID-19 in Korea but recovered rapidly after the first wave subsided. Figure 3 shows the change in credit card payments in Suwon and the number of COVID-19 confirmed cases in Korea within the study period. The line graph shows the amount of card payment in Suwon, and the bar graph shows the number of new COVID-19 cases. The gray dotted line represents the average value of the card payment for the seven months (June 2019 to December 2019) before the occurrence of COVID-19.

After the first case in January, the amount of credit card payments decreased sharply. In March, when the payment amount was the lowest, the number of cases was the highest, 
and intense social distancing began. In response to the rapid decline in consumption, the central and local governments began to pay the Disaster Relief Fund (DRF). In Suwon, from 9 April, the local government paid 100,000 won per person [66]. From 4 May, the central government's paid 1 million won for a family of four [67]. The DRF can be used within the local area and cannot be used for online shopping. Accordingly, local consumption recovered rapidly. Moreover, in May, the consumption of Suwon recovered to a normal level. On May 6, the Korean government lowered the level of social distancing, allowing citizens to return to their daily life. Such changes are typical shock-and-recovery patterns in disaster situations. Thus, the temporal range of this study is appropriate to analyze the resilience of the pandemic.

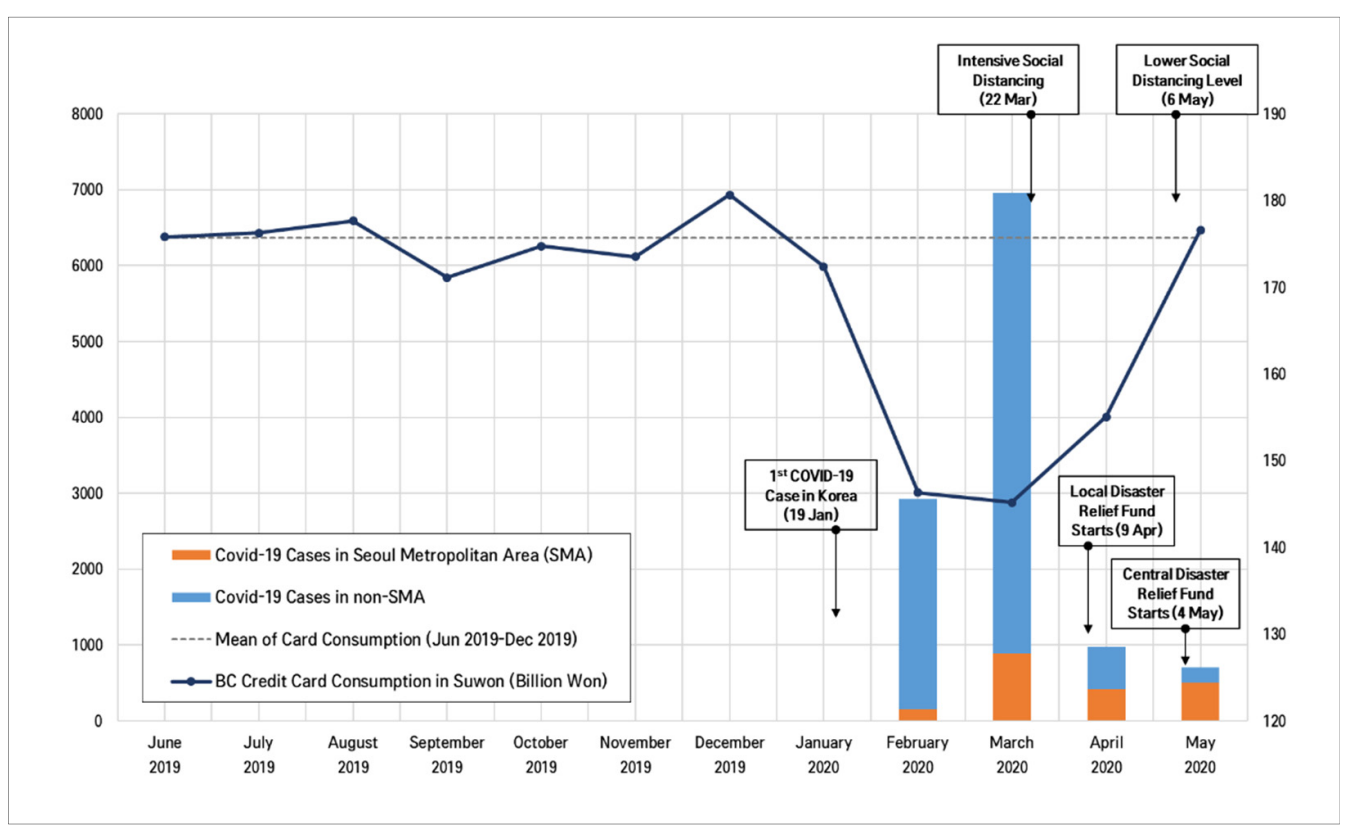

Figure 3. Korea's COVID-19 cases per month and credit card payment amount in Suwon (data source: References [3,68]).

\subsection{Research Question}

This study began with the awareness of the problem that consumption reduction and recovery are not the same in all neighborhoods. The overall consumption of Suwon showed a pattern of decreasing and recovering. However, there were different patterns for each neighborhood. In a pandemic situation, consumption in some neighborhoods recovers quickly (slowly). Some neighborhoods have high economic resilience relative to other neighborhoods. The first research question is as follows: what are the urban characteristics of high-economic-resilient neighborhoods in a pandemic situation?

Further, after the outbreak of COVID-19, consumption did not decrease but increases continuously in some neighborhoods. Such neighborhoods benefit economically in a pandemic situation. The second research question is as follows: what are the urban characteristics of neighborhoods that benefit economically in a pandemic situation?

\section{Materials and Methods}

\subsection{Research Method}

Figure 4 presents the analysis method. First, the neighborhood types are classified by analyzing the change in the credit card payment by neighborhood. The neighborhoods are classified into low-resilient neighborhoods, high-resilient neighborhoods, and benefited neighborhoods. The specific classification method is reported in Section 2.3. 


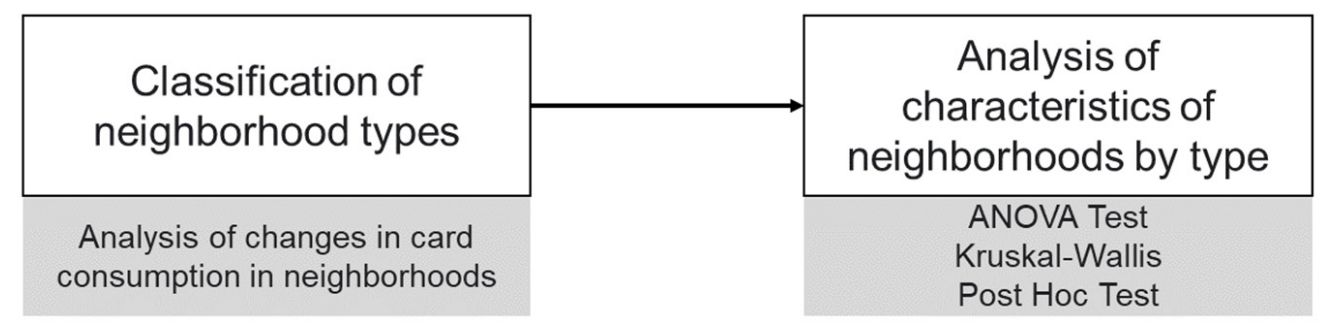

Figure 4. Research method.

ANOVA (Analysis of Variance) and Kruskal-Wallis (K-W) tests were utilized to assess urban characteristics for each classified neighborhood type. Thereafter, post hoc testing was performed to confirm the significance of the difference between each type. When a variable satisfied the normality, a parametric test method (i.e., ANOVA) was used, and when the normality was not satisfied, a non-parametric test method (i.e., $\mathrm{K}-\mathrm{W}$ ) was utilized. For variables using ANOVA, Levene's equal variance test was performed. For variables with equal variance, F statistic was used in the ANOVA, and the Scheffe method was used in the post hoc. For variables not satisfying the equal variance, the Welch statistic was used in the ANOVA, and the Games-Howell (GH) method was used in the post hoc. Further, for the variables using the K-W test, we adopted Dunn's method during post hoc analysis.

\subsection{Data Construction}

\subsubsection{Selection of Neighborhoods to Be Analyzed}

This study employed the Bank and Credit (BC) credit card payment data in the neighborhood to determine the consumption amount by neighborhood. In Korea, credit card payments represent local consumption [69]. The share of credit card usage in Korea's total consumption was $53.8 \%$ as of 2019 [70], and BC Card's credit card market share was $24 \%$ [66]. Therefore, the BC Card payment amount is expected to represent the overall consumption behavior. Nevertheless, if there is a large difference in user characteristics depending on the card company, there is a possibility that the payment amount data of the $\mathrm{BC}$ card may not represent the total consumption. Therefore, caution is mandated in the use of research results.

The card payment data used in this study were collected in units of Output Area $(\mathrm{OA})$. An OA is the smallest spatial unit for collecting statistical data in Korea and is set based on population size, socio-economic homogeneity, and boundary shape [71]. It is set per 500 people, and socio-economic homogeneity is set per housing type and land price [71]. An OA is a neighborhood where about 500 people with similar socio-economic characteristics live.

Since the population size is the most important criterion for setting an OA, the OA of mountains or farmlands is very wide. Figure 5 shows Suwon's OAs and land-use area. The OAs of green areas are vast relative to other OAs. It is challenging to consider such large OAs as neighborhoods. Thus, we excluded OAs with an area of $100,000 \mathrm{~m}^{2}$ or more from the analysis.

This study addresses consumption in neighborhoods. Therefore, OAs for which card payments do not exist were also excluded. If the card payment data do not exist, and there are no stores or restaurants, the OA can be considered as comprising only residential building. Further, even if card payment data exist, an OA with a small number of payments is considered a neighborhood consisting mostly of houses. Therefore, we also excluded OAs with less than 5000 payments per year from the analysis. This study uses buildingrelated data as a major urban characteristic variable. Therefore, OAs for which no building data exist were also excluded. 


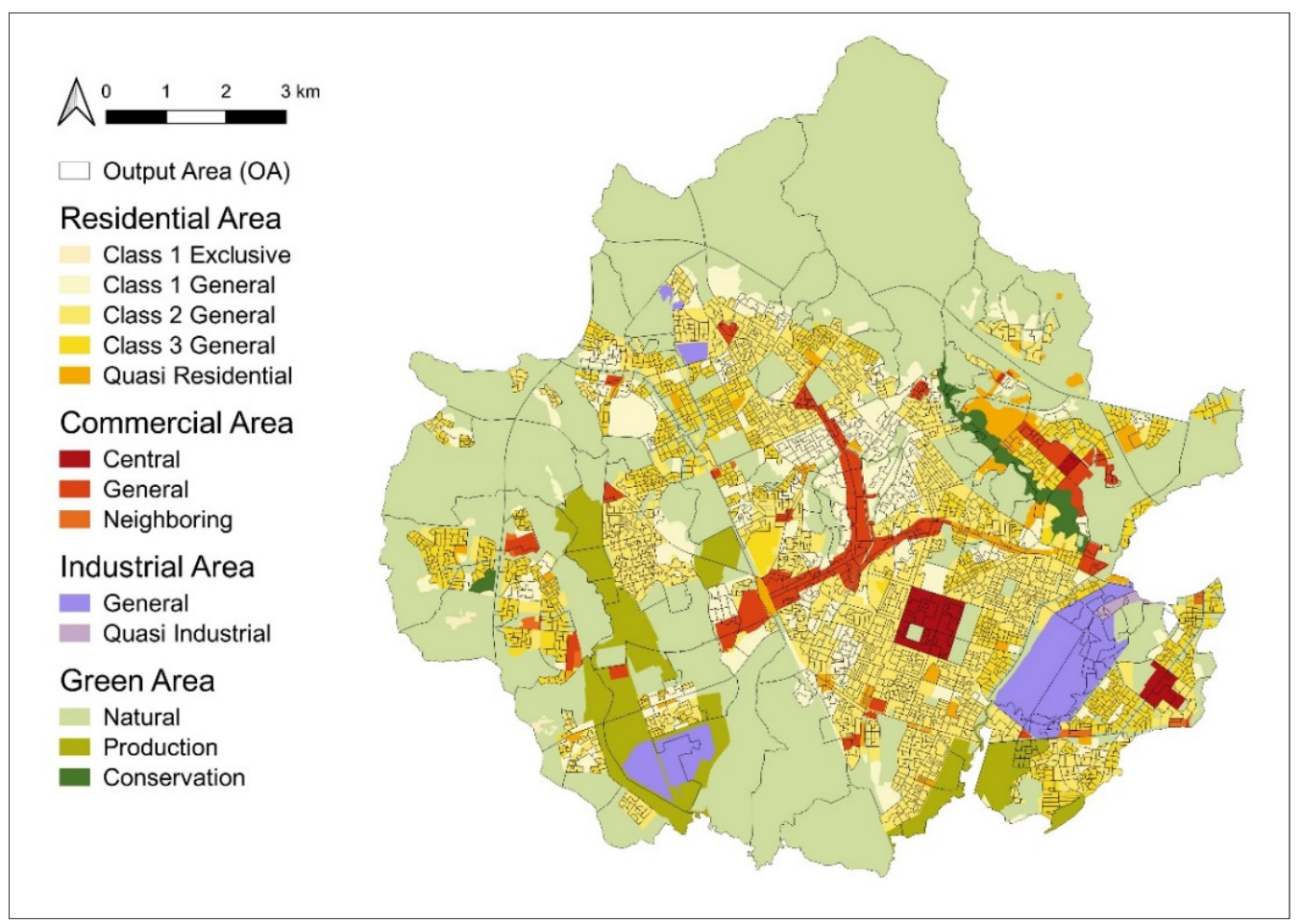

Figure 5. OA boundary and land-use zone of Suwon.

Hence, 846 OAs were included in this study. The OA derived through this process has a population of about 500, with residential and commercial facilities. Figure 6 reports the OAs included in the study. All large OAs, including green areas, were excluded, and some OAs in industrial and commercial areas were excluded. The area average of OA included in this study was $25,870 \mathrm{~m}^{2}$, and the population average was 483.6 .

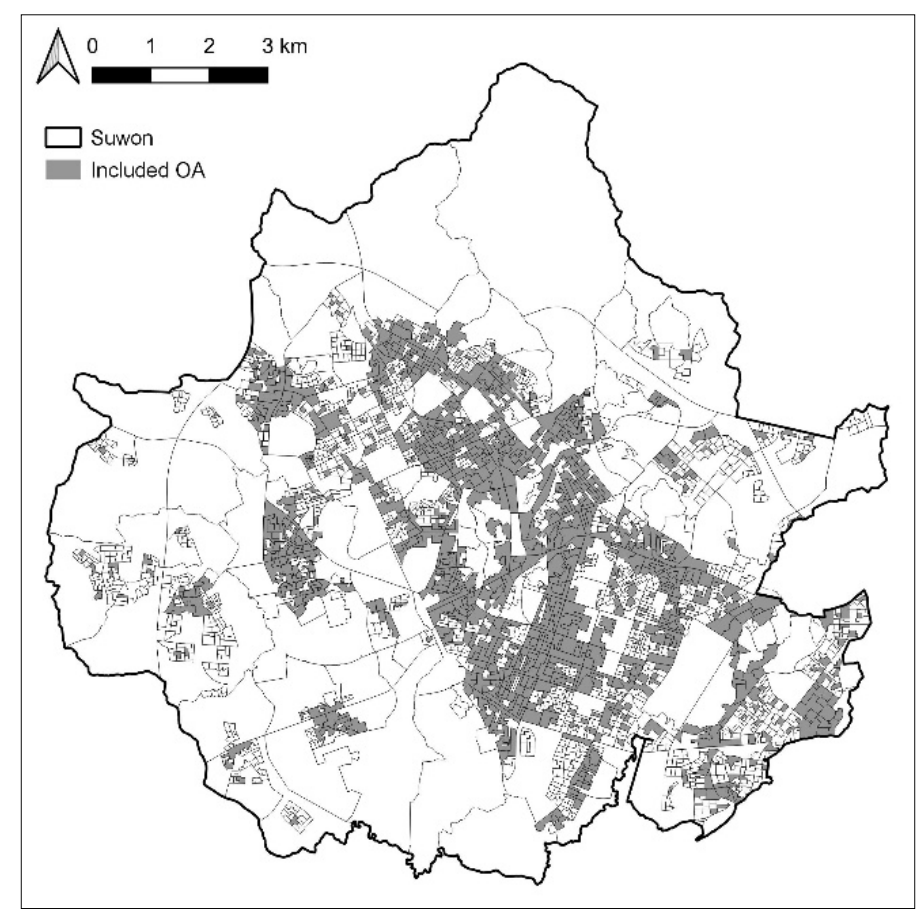

Figure 6. OAs included in this study. 


\subsubsection{Variables and Data Source}

Since the economic impact of the pandemic has been studied mainly on macroeconomic factors such as GDP, the urban characteristics of neighborhoods regarding the economic impact are not well known. Therefore, we selected basic variables representing city characteristics as analysis variables. Moreover, these variables are well-known criteria for the classification of neighborhoods [58,59]. Table 1 summarizes the study variables. In the process of data construction, when analysis and calculation of spatial data were required, the Quantum Geographic Information System (QGIS) 3.10 was used.

First, in the demographic category, the population ratio by age was selected to know the age groups that live in the neighborhood. Notably, the changes in behavior patterns in pandemic situations differ per age. Migration for those aged 20 and over 70 decreased significantly more than it did for those in their 30s and 50s [72]. Changes in consumption patterns also differ per age, with the smallest decrease among those in their 50s [72].

The household structure was employed to ascertain what household type lives in the neighborhood. It comprises variables that can determine the number of household members living in one house ( $\mathrm{HH}-\mathrm{M})$, variables that can determine the generation of family members living together ( $\mathrm{HH}-1 \mathrm{G}, \mathrm{HH}-2 \mathrm{G}$, and $\mathrm{HH}-3 \mathrm{G})$, and the ratio of non-related households (HH-NR) as the analysis variable.

Housing-related variables are divided into housing size and type. Through the size of the house, it is possible to check the income level in the area. The type of house is the main variable that determines the characteristics of the neighborhood. A specific housing type often dominates one OA.

Data provided by the Statistical Geographic Information System (SGIS) [73] operated by the Statistics Korea (national statistical agency) were used for the demographic, household structure, and housing-related variables. SGIS provides the population by age, the number of households by type, the number of houses by area, and the number of houses by type (i.e., aggregated in OA), which is the analysis unit of this study. We created proportion variables that divided these parameters according to the total population, the total number of households, and the total number of houses.

The proportions of building floor area by use and land-use mix variables were selected as land-use-related variables. The former was divided into residential, commercial, and educational or cultural. These uses are the most representative in the city.

Land-use mix notably affects the volume of pedestrians and vitality in a region. Several studies in urban planning have repeatedly confirmed that as the land-use mix increases, the volume of pedestrians increases [74-77], which leads to an increase in local consumption [78-80]. Among the various variables for measuring land-use mix, this study selected three variables. The number of uses (No-U) checks the diversity of uses. Diversity has been known to significantly influence regional vitality since Jacobs [81]. The Hirschman-Herfindahl Index (HHI) and the Residential and Non-Residential Balance Index (RNR) were included as indicators to determine the degree of the land-use mix. $\mathrm{HHI}$ is widely used to measure market concentration in the economic field $[82,83]$; it is also widely used as a variable to measure the land-use mix [84-87]. HHI is calculated via Equation (1). It is 1 for single-use and $1 / \mathrm{k}$ when $\mathrm{k}$ uses are uniformly mixed. Therefore, the smaller the HHI value, the greater the degree of the land-use mix.

$$
\mathrm{HHI}=\sum_{i=1}^{k} p_{i}^{2} .
$$

In the above equation, $\mathrm{K}$ is the number of use, and $p_{i}$ is the proportion of floor area of use (i).

RNR is measured by using Equation (2). It measures the degree of land-use mix simply by dividing the use of a building into two types: residential and non-residential. RNR is 0 
for single-use and closer to 1 when residential and non-residential are mixed. That is, the greater the RNR, the greater the degree of the land-use mix.

$$
\mathrm{RNR}=1-\left|\frac{R-N R}{R+N R}\right| .
$$

In the above equation, $\mathrm{R}$ is the floor area of residential buildings, and NR is the floor area of non-residential buildings.

The National Spatial Information Portal [88] provides shape (SHP) files that specify the location and shape of buildings. This file elucidates the use and floor area of each building. We utilized this data to calculate the total floor area according to use and the number of uses for each OA. The variables of the use of building and land-use mix categories were calculated by using this data.

Table 1. Urban characteristic variables.

\begin{tabular}{|c|c|c|c|}
\hline Category & Variables & Explanation & Reference \\
\hline \multirow{8}{*}{ Demographic } & $\mathrm{P}-00$ & Proportion of population $<10$ & \multirow{29}{*}{ [73] } \\
\hline & P-10 & Proportion of population 10 19 & \\
\hline & P-20 & Proportion of population $20 \sim 29$ & \\
\hline & $\mathrm{P}-30$ & Proportion of population 30 39 & \\
\hline & $\mathrm{P}-40$ & Proportion of population $40 \sim 49$ & \\
\hline & P-50 & Proportion of population 50 59 & \\
\hline & P-60 & Proportion of population $60 \sim 69$ & \\
\hline & P-70 & Proportion of population $>70$ & \\
\hline \multirow{6}{*}{ Household Structure } & $\mathrm{HH}-\mathrm{M}$ & Mean no. of household members & \\
\hline & HH-1G & Proportion of 1 generation household & \\
\hline & $\mathrm{HH}-2 \mathrm{G}$ & Proportion of 2 generation household & \\
\hline & $\mathrm{HH}-3 \mathrm{G}$ & Proportion of 3 generation household & \\
\hline & HH-1P & Proportion of 1-person household & \\
\hline & HH-NR & Proportion of non-relative household & \\
\hline \multirow{9}{*}{ Size of Houses } & HS-1 & Proportion of houses $<20 \mathrm{~m}^{2}$ & \\
\hline & HS-2 & Proportion of houses $20 \mathrm{~m}^{2} \sim 40 \mathrm{~m}^{2}$ & \\
\hline & HS-3 & Proportion of houses $40 \mathrm{~m}^{2} \sim 60 \mathrm{~m}^{2}$ & \\
\hline & HS-4 & Proportion of houses $60 \mathrm{~m}^{2} \sim 85 \mathrm{~m}^{2}$ & \\
\hline & HS-5 & Proportion of houses $85 \mathrm{~m}^{2} \sim 100 \mathrm{~m}^{2}$ & \\
\hline & HS-6 & Proportion of houses $100 \mathrm{~m}^{2} \sim 130 \mathrm{~m}^{2}$ & \\
\hline & HS-7 & Proportion of houses $130 \mathrm{~m}^{2} \sim 165 \mathrm{~m}^{2}$ & \\
\hline & HS-8 & Proportion of houses $165 \mathrm{~m}^{2} \sim 230 \mathrm{~m}^{2}$ & \\
\hline & HS-9 & Proportion of houses $>230 \mathrm{~m}^{2}$ & \\
\hline \multirow{6}{*}{ Type of Houses } & HT-M & Proportion of multifamily house & \\
\hline & HT-D & Proportion of detached house & \\
\hline & HT-A & Proportion of apartment & \\
\hline & HT-R & Proportion of row house & \\
\hline & HT-C & Proportion of houses in commercial building & \\
\hline & HT-NH & Proportion of non-housing residence & \\
\hline & FA-C & Proportion of commercial building floor area & \multirow{6}{*}{ [88] } \\
\hline Use of Building & FA-E & Proportion of educational and cultural building floor area & \\
\hline & FA-R & Proportion of residential building floor area & \\
\hline \multirow{3}{*}{ Land-Use Mix } & No-U & Number of building use & \\
\hline & HHI & Hirschman-Herfindahl Index & \\
\hline & RNR & Residential and non-residential balance index & \\
\hline \multirow{3}{*}{ Transportation } & $\mathrm{RD}$ & Area of road/OA area & \\
\hline & TRN & Distance to subway (or railway) station & [89] \\
\hline & BUS & Number of bus stops in OA & [90] \\
\hline \multirow{2}{*}{ Density } & DN-P & Population/OA area & [73] \\
\hline & DN-B & Total floor area/OA area & [88] \\
\hline
\end{tabular}


The transportation-related variables were selected as the ratio of road area (RD), distance to the nearest railway (or subway) station (TR), and bus stop density (BUS). RD captures the convenience of using a car. To formulate this variable, the road area was calculated by using the road SHP file provided in the National Spatial Information Portal [88]. Afterward, the road area was derived and divided by the OA.

TR is railroad or subway accessibility. For this variable, SHP files of subway stations and railway stations provided by the Ministry of the Interior and Safety [89] were selected. The straight-line distance from the center point of OA to the nearest subway station or railway station was calculated.

BUS addresses the convenience of using the bus. This variable used the bus stop coordinate data provided by Gyeonggi Data Dream [90]. The location of bus stops was geocoded, and the number of bus stops in OA units was counted.

Population density (DN-P) and building density (DN-B) variables were selected as density variables. DN-P used population density data of the OA provided by SGIS [73]. DN-B was calculated by dividing the total building area calculated earlier by the OA.

\subsection{Classification of Neighborhoods}

The resilience triangle is widely used to quantitatively measure engineering resilience. Bruneau et al. [91] presented a concept for quantitatively measuring the resilience of infrastructure against earthquakes. Figure 7 shows that after the earthquake, the performance of the infrastructure drops sharply relative to the normal and recovers after a certain period. Bruneau et al. [91] quantitatively defined the resilience, as shown in Equation (3) in this situation, which is the same as the area of the part shaded blue in Figure 7 . Thus, the part at the top of the graph can be judged as the degree of damage caused by the disaster; it is known as a resilience triangle.

$$
\text { Resilience }=\int_{t_{0}}^{t_{1}}[100-Q(t)] d t \text {. }
$$

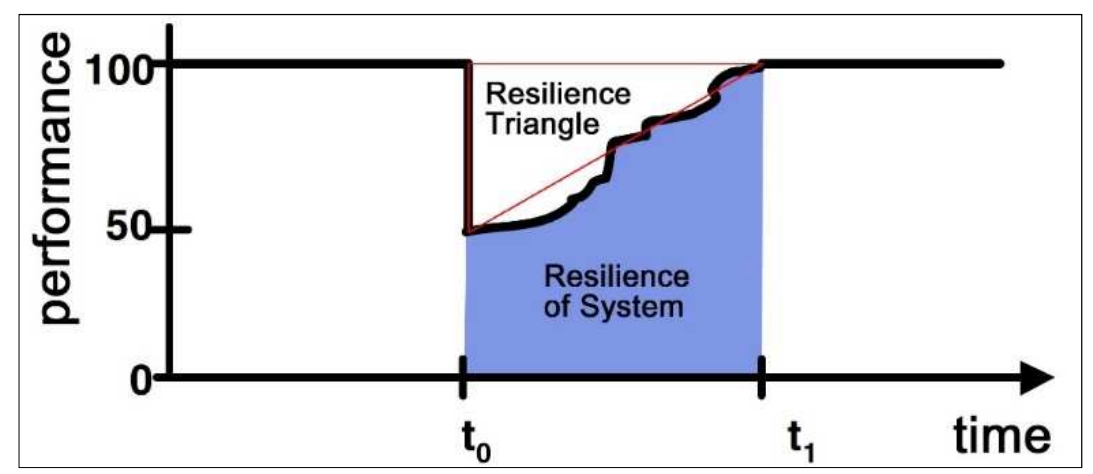

Figure 7. Conceptual definition of resilience (modified from Bruneau et al. [91]).

The concept of the resilience triangle was used to measure resilience in many prior studies [92-95]. The shape of the resilience triangle can vary depending on the analysis target and impact type. Balal et al. (2019) suggested the concept of a resilience polygon because the resilience triangle can appear in various shapes, such as right triangle, acute triangle, trapezoid, pentagon, and hexagon [92].

Following the concept of the resilience triangle, the concept of high resilience can be derived. Figure 8a shows that the size of the resilience triangle is determined by the degree of damage and the time it takes to recover. Moreover, the degree of damage is related to the system robustness, and the time taken to recover is related to the speed of recovery. A system with robustness and fast recovery has less disaster damage and a short recovery time. Such a system can be judged as a high-resilient system [91,96,97]. In other words, a system with a small resilience triangle has high resilience. 


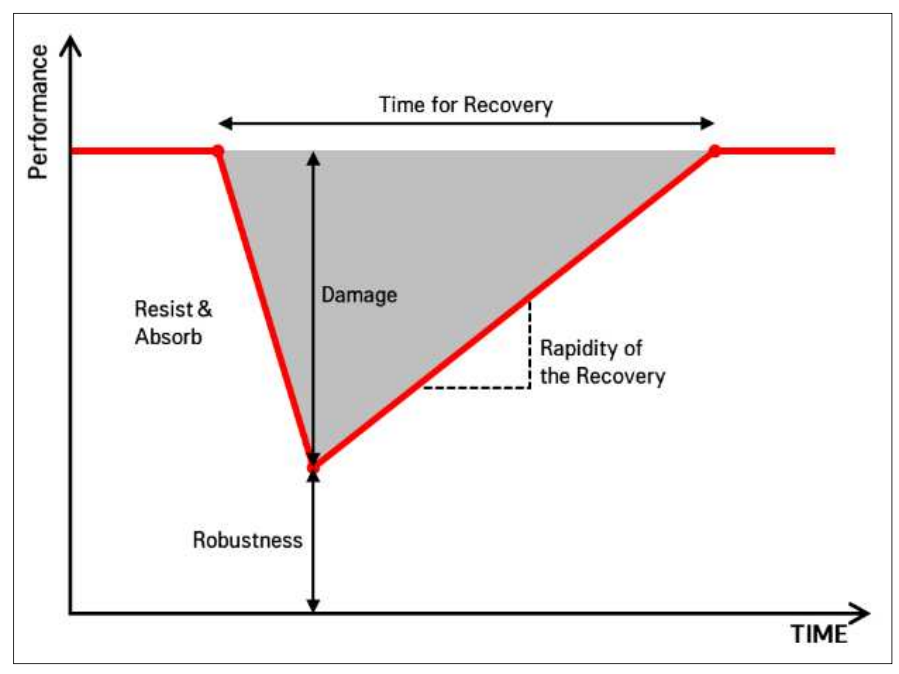

(a)

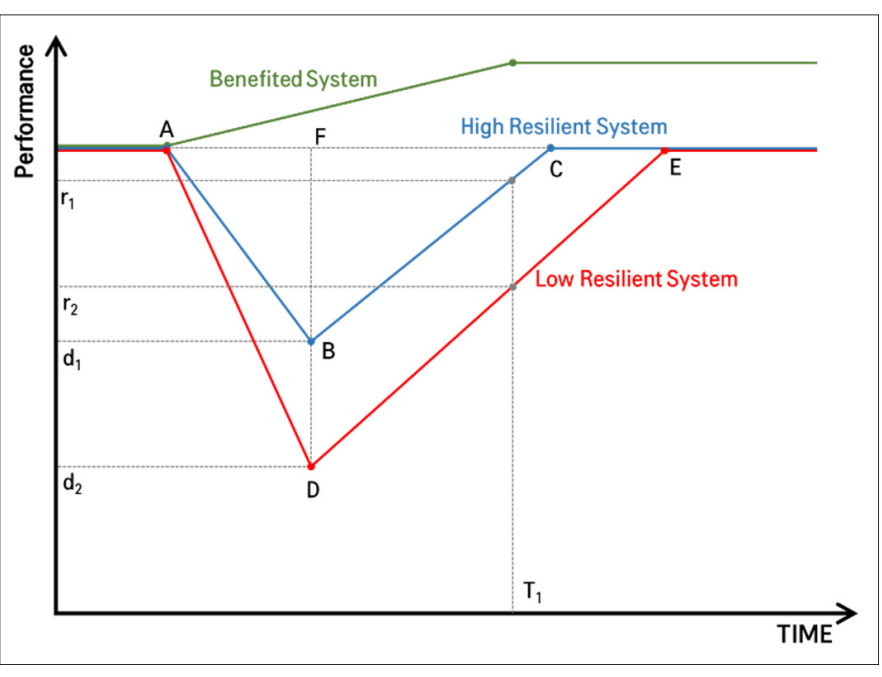

(b)

Figure 8. (a) Determinants of resilience triangle. (b) High- and low-resilient system.

Figure $8 \mathrm{~b}$ is a schematic diagram of this concept. The resilience triangle $\mathrm{ABC}$ of the high-resilient system, indicated in blue, is smaller than triangle ADE of the low-resilient system, indicated in red. The area of a triangle can be easily calculated by knowing the lengths of $\mathrm{AC}, \mathrm{AE}, \mathrm{FB}$, and FD. The length of FB and FD can be determined by the performance level at the time of the greatest damage. In other words, $d_{1}$, representing the robustness of the high-resilient system, is larger than $d_{2}$ of the low-resilient system. Thus, it maintains a relatively high performance level even in the event of a disaster, with minimal damage. Since the pandemic is not over, it is difficult to ascertain the length of AC and $\mathrm{AE}$, the time taken to recover to previous levels. Therefore, we judged the recovery speed through the degree of recovery at a specific time point after the COVID-19 outbreak. The recovery speed can be determined based on the performance level at the point $T_{1}$ after a certain time has passed since the disaster occurred. The recovery degree $\left(r_{1}\right)$ of the high-resilient system can be expected to be larger than that $\left(r_{2}\right)$ of the low-resilient system.

Unlike natural disasters, such as earthquakes, the economic shock caused by the pandemic did not damage all neighborhoods. In a pandemic situation, people are reluctant to use large stores or central commercial areas that are over-crowded, thereby reducing overall consumption. Simultaneously, essential consumption usually occurs in areas close to residences. Therefore, in certain neighborhoods, consumption may not decrease comparatively but does tend to increase. As shown by the green line in Figure $8 \mathbf{b}$, after a disaster, consumption in a neighborhood continues to increase more than the usual level. Such neighborhoods may be construed as having benefited economically from the pandemic. Accordingly, three neighborhood types emerge: high-resilient (HRN), lowresilient (LRN), and benefited (BFN) neighborhoods.

In this study, credit card payments in neighborhoods were used as variables to measure economic performance in a pandemic situation. However, the level of credit card payment varies per neighborhood. Therefore, a comparative indicator is necessary. From Equation (4), we used the mean value of card payments for each OA from June 2019 to December 2019 before the COVID-19 outbreak as the usual payment amount for the corresponding $\mathrm{OA}\left(U P_{i}\right)$. From Equation (5), the monthly card payment amount $\left(P_{i t}\right)$ was then divided by the usual payment amount $\left(U P_{i}\right)$ and used as an indicator $\left(I_{i t}\right)$ to determine the level of payment relative to the usual monthly payment amount. If the value of indicator $\left(I_{i t}\right)$ is less (greater) than 1, consumption decreased (increased) in the neighborhood than usual. If we can obtain the usual payment data for a specific month for each $\mathrm{OA}\left(U P_{i t}\right)$, it is ideal 
to standardize $P_{i t}$ by using this mechanism. Nonetheless, we used $U P_{i}$ because only one year's data can be used from June 2019 to May 2020.

$$
\begin{gathered}
U P_{i}=\frac{1}{7} \sum_{t=1}^{7} P_{i t} \\
I_{i t}=\frac{P_{i t}}{U P_{i}} .
\end{gathered}
$$

$I_{i 8}, I_{i 9}, I_{i 10}, I_{i 11}$, and $I_{i 12}$ indicate the consumption level in " $\mathrm{i}$ " neighborhood from January to May 2020 after the COVID-19 outbreak. We set $I_{i 8}, I_{i 9}, I_{i 10}, I_{i 11}$, and $I_{i 12}$ as greater than 1 regarding the BFN. They are neighborhoods where the level of consumption in the neighborhood has not decreased at all since the COVID-19 outbreak.

After classifying BFN, HRN and LRN were categorized for the remaining neighborhoods. The $d_{i}$, derived through Equation (6), represents the consumption level when the damage of COVID-19 was greatest in the neighborhood. This value has the same meaning as the " $\mathrm{d}$ " value in Figure 8b. Within the sample period, the consumption level in the last period of May 2020 is $I_{i 12}$, which has the same meaning as " $r$ " in Figure 8b.

$$
d_{i}=\min \left(I_{i 8}, I_{i 9}, I_{i 10}, I_{i 11}, I_{i 12}\right) .
$$

We classified the neighborhood in which the values of " $\mathrm{d}$ " for robustness and " $r$ " for the degree of recovery fall in the top 30\% as HRN. They are highly robust. Thus, the degree of damage is small, and the degree of recovery is high. Of course, these neighborhoods can be expected to have a small resilience triangle. Therefore, the low resilience of LRN is a relative concept to HRN.

\section{Result}

\subsection{Result of Neighborhoods Classification}

Based on the method described in the previous section, the OAs in Suwon were classified into three types: $130 \mathrm{BFN}, 111 \mathrm{HRN}$, and 605 LRN. Figure 9 shows the change in the mean value of the consumption indicator $\left(I_{i t}\right)$ for each neighborhood type. The results of all neighborhoods (shown in gray) show a pattern similar to the change in total sales (Figure 3). However, the results divided by type show very different patterns.

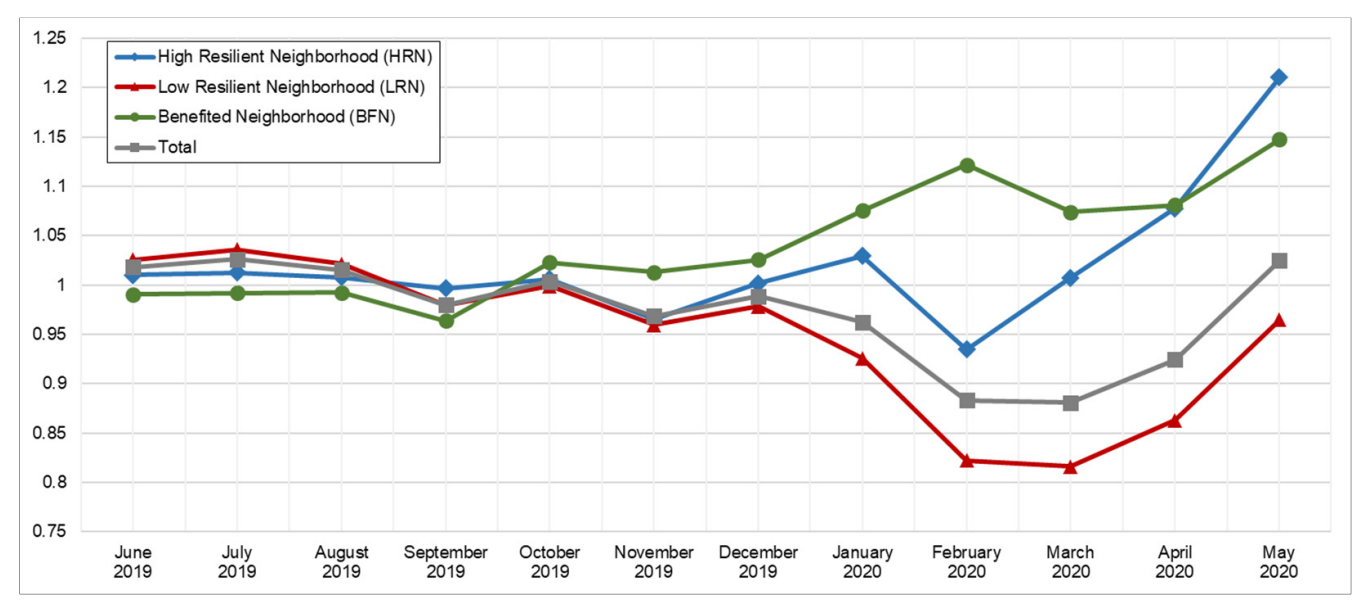

Figure 9. Changes in consumption indicator $\left(I_{i t}\right)$ by neighborhood type.

The BFN (the green line) increased after the COVID-19 outbreak. The HRN (the blue line) shows a decrease in February but starts to recover quickly, showing a higher recovery than BFN in May. Thus, resilience is high. The LRN (red) has a greater degree of damage and slower recovery than the HRN. This result conforms to the conceptual diagram in Figure $8 b$. 
Figure 10 shows the spatial distribution of divided neighborhoods. The BFN and HRN are not concentrated in a specific area and are distributed spatially evenly. Thus, the high resilience of neighborhoods is not due to locational features in the level of urban spatial structure. However, it can be understood that economic resilience varies per the internal characteristics of individual neighborhoods.

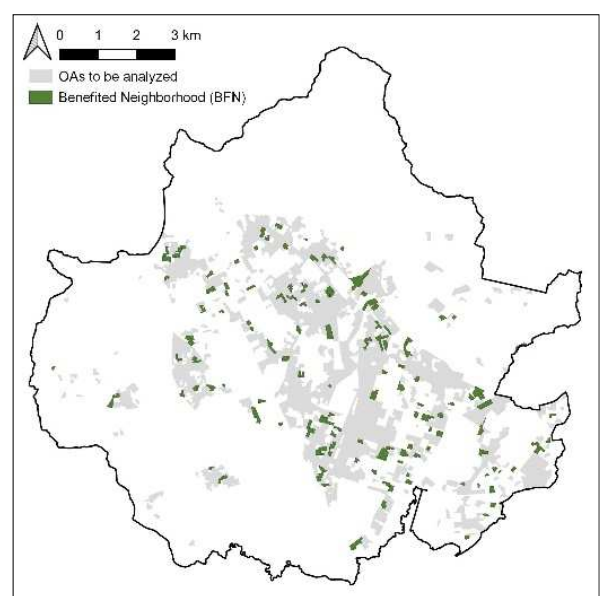

(a)

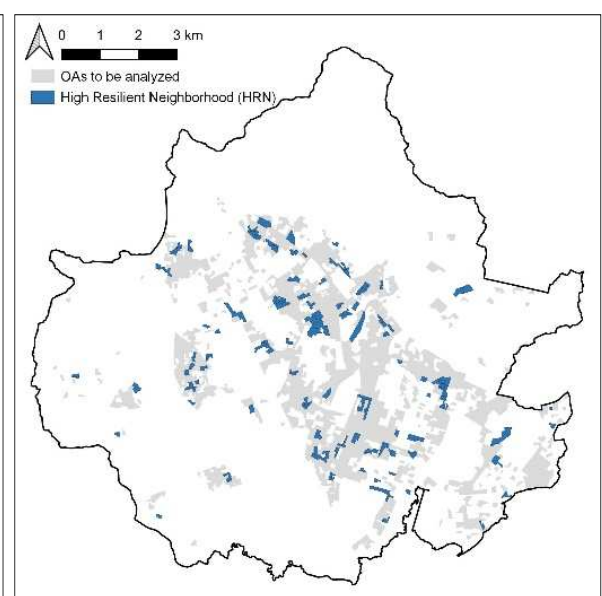

(b)

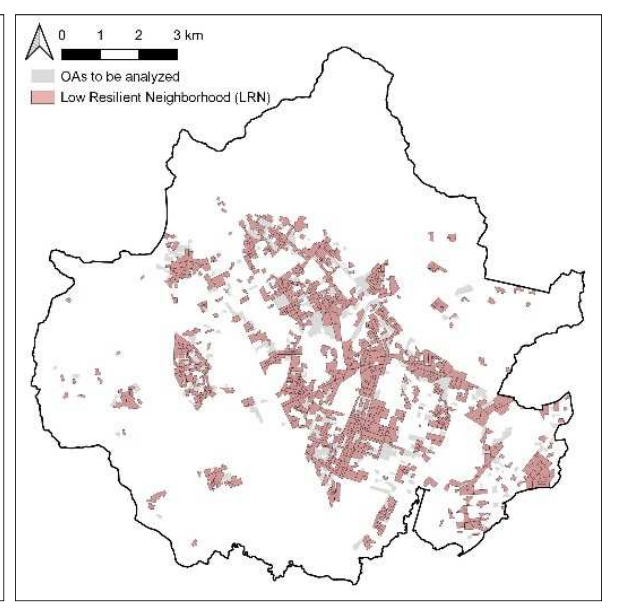

(c)

Figure 10. Spatial distribution of neighborhood by type: (a) benefited neighborhood (BFN), (b) high-resilient neighborhood (HRN), and (c) low-resilient neighborhood (LRN).

\subsection{Urban Characteristics by Neighborhood Type}

\subsubsection{Demographic Characteristics}

Table 2 shows the results of the ANOVA test for demographic variables. According to Levene's test result, if the variance was equal, the F statistic was reported. In the case of not equal variance, the Welch statistic that can be used when the assumption of equal variance is not satisfied is reported. For variables other than P-50, there is a statistically significant difference in mean values between neighborhood types.

Table 2. ANOVA test results of demographic variables.

\begin{tabular}{cccccc}
\hline \multirow{2}{*}{ Variables } & \multicolumn{2}{c}{ Levene's Test } & \multicolumn{3}{c}{ ANOVA Test } \\
\cline { 2 - 6 } & Levene's Statistic & Sig. & Statistic & Statistics Value & Sig. \\
\hline P-00 & 1.402 & 0.247 & F & $6.103^{* * *}$ & 0.002 \\
P-10 & $3.044^{* *}$ & 0.048 & Welch & $6.598^{* * *}$ & 0.002 \\
P-20 & $11.260^{* * *}$ & 0.000 & Welch & $5.789^{* * *}$ & 0.003 \\
P-30 & $3.950^{* *}$ & 0.020 & Welch & $3.851^{* *}$ & 0.023 \\
P-40 & 2.280 & 0.103 & F & $2.712^{*}$ & 0.067 \\
P-50 & $2.356^{*}$ & 0.095 & Welch & 1.086 & 0.339 \\
P-60 & $2.481^{*}$ & 0.084 & Welch & $6.472^{* * *}$ & 0.002 \\
P-70 & 1.623 & 0.198 & F & $4.100^{* *}$ & 0.017 \\
\hline Note: $^{* * *} P<0.01^{* *} p<0.05$, and & & & & &
\end{tabular}

Figure 11 reports the mean value of the variables by group. In the figure, the number of stars under the variable name indicates the statistical significance of the ANOVA or $\mathrm{K}-\mathrm{W}$ test. Table $\mathrm{A} 1$ reports descriptive statistics, including the mean value of each variable by type. Table A2 reports the results of the post hoc test, which can check the statistical significance of the difference in the mean value of the variable between each neighborhood type. Regarding (not) equal variance, the (GH) Scheffé method was used. Refer to the results of Tables A1 and A2 for the interpretation of the text. 


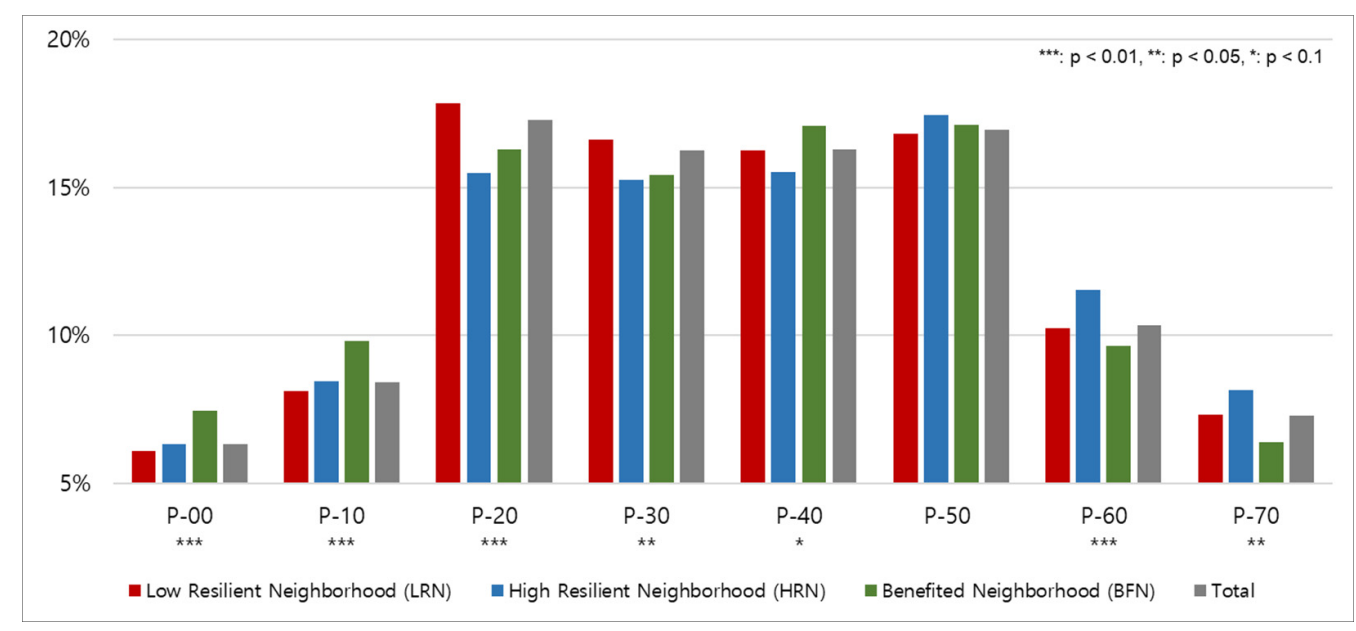

Figure 11. Mean difference of demographic variables by type.

P-00 mean value of the BFN is statistically larger than that of the LRN. In P-10, the value of the BFN is significantly greater than that of the LRN and HRN. Thus, more adolescents and children live in the BFN than in other neighborhoods. At P-20, the mean value of the LRN is statistically greater than the HRN. The mean P-30 of the LRN is statistically greater than that of the HRN and BFN. Thus, the LRN is a neighborhood where more young people in their 20s and 30s live than in other neighborhoods. Looking at P-40, the BFN has a larger value than the HRN, which is linked to the results of P-00 and P-10. In the BFN, children and adolescents often live with their parents. There was no statistically significant difference in P-50 between groups. P-60 represents a higher value for the HRN than for the LRN and BFN. In P-70, the HRN shows a higher value than the BFN. HRN is a neighborhood with more elderly aged 60 or older than other neighborhoods.

\subsubsection{Household Structure Characteristics}

Table 3 shows the results of the ANOVA test of variables related to household structure. The Welch statistic result was reported because all variables did not satisfy the assumption of equal variance. Except for $\mathrm{HH}-1 \mathrm{G}$, the other variables had statistically significant differences in mean values between neighborhood types.

Table 3. ANOVA test results of household structure variables.

\begin{tabular}{cccccc}
\hline \multirow{2}{*}{ Variables } & \multicolumn{2}{c}{ Levene's Test } & \multicolumn{3}{c}{ ANOVA Test } \\
\cline { 2 - 6 } & Levene's Statistic & Sig. & Statistic & Statistics Value & Sig. \\
\hline HH-M & $5.343^{* * *}$ & 0.005 & Welch & $10.825^{* * *}$ & 0.000 \\
HH-1G & $4.209^{* *}$ & 0.015 & Welch & 1.680 & 0.189 \\
HH-2G & $5.691^{* * *}$ & 0.004 & Welch & $13.063^{* * *}$ & 0.000 \\
HH-3G & $4.176^{* *}$ & 0.016 & Welch & $2.792^{*}$ & 0.063 \\
HH-1P & $6.694^{* * *}$ & 0.001 & Welch & $13.217^{* * *}$ & 0.000 \\
HH-NR & $8.842^{* * *}$ & 0.000 & Welch & $7.874^{* * *}$ & 0.000 \\
\hline
\end{tabular}

Note: ${ }^{* * *} p<0.01,{ }^{* *} p<0.05$, and ${ }^{*} p<0.1$.

Figure 12 reports the mean values for each type. The mean of HH-M was significantly greater in the BFN than in the HRN and LRN. That is, the number of member of households living in the BFN is higher. $\mathrm{HH}-1 \mathrm{G}$ and $\mathrm{HH}-3 \mathrm{G}$ had no significant difference between groups. In $\mathrm{HH}-2 \mathrm{G}$, the mean value of the $\mathrm{BFN}$ is significantly higher than that of the LRN and HRN. Overall, the BFN has more of two generations of parents and children than other neighborhoods. The HH-1P mean of the BFN is smaller than that of the LRN and HRN. Thus, there are fewer single-person households in the BFN. The mean value of HH-NR in the LRN is significantly larger than that of other types. Hence, in the LRN, 
many households comprise non-family people. Considering the results of the demographic variables, many young people live with roommates in the LRN.

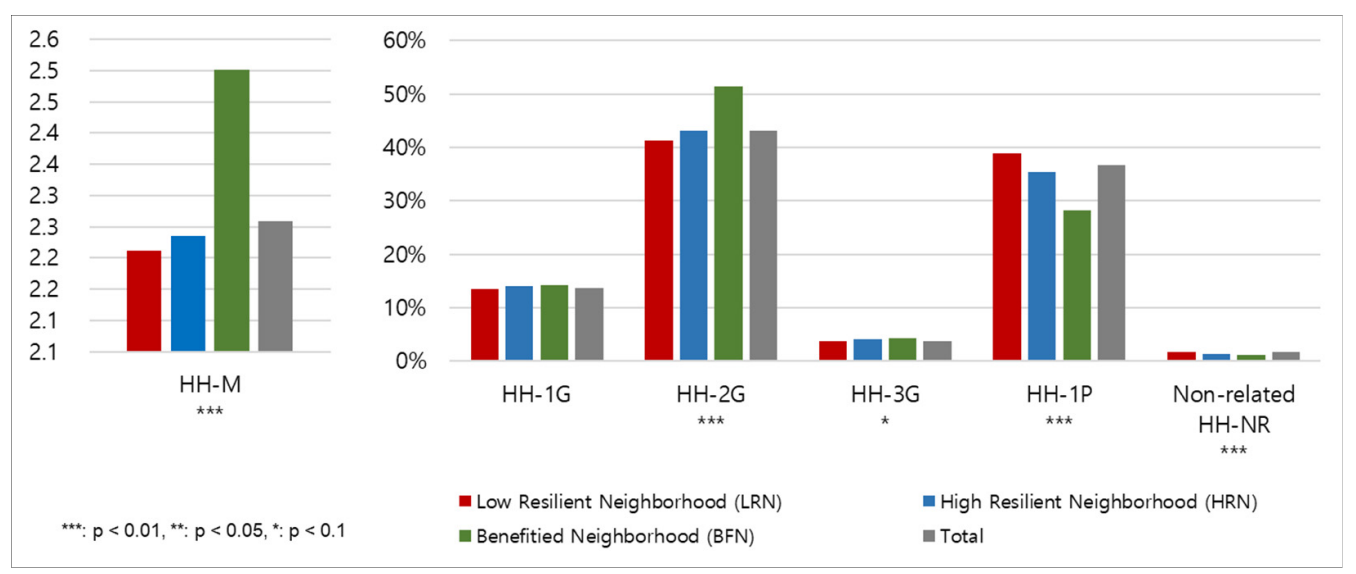

Figure 12. Mean difference of household structure variables by type.

\subsubsection{Housing-Related Characteristics}

By examining the normality of housing-related variables employing the $\mathrm{Q}-\mathrm{Q}$ plot, it is evaluated that the housing-related variables do not satisfy the normality. Correspondingly, $\mathrm{K}-\mathrm{W}$ analysis, a non-parametric test, was performed (refer to Table A3 for the Q-Q plot of the variables). Table 4 reports the results of the $\mathrm{K}-\mathrm{W}$ analysis of housing-related variables. It is divided into two categories: housing-area and housing-type variables. There were statistically significant differences between groups except for HS-4, HS-5, and HT-R. For HS-1, HS-3, and HT-M, the K-W test was significantly analyzed, but there was no significant difference between groups in the post hoc test.

Table 4. Kruskal-Wallis test results of housing-related variables.

\begin{tabular}{|c|c|c|c|}
\hline Category & Variables & Statistic & Sig. \\
\hline \multirow{9}{*}{ Area of Houses } & HS-1 & $5.618 *$ & 0.075 \\
\hline & HS-2 & $6.900^{* *}$ & 0.032 \\
\hline & HS-3 & $5.276^{*}$ & 0.072 \\
\hline & HS-4 & 2.931 & 0.231 \\
\hline & HS-5 & 1.005 & 0.605 \\
\hline & HS-6 & $5.187 *$ & 0.075 \\
\hline & HS-7 & $10.807^{* * *}$ & 0.005 \\
\hline & HS-8 & $14.737^{* * *}$ & 0.001 \\
\hline & HS-9 & $20.673^{* * *}$ & 0.000 \\
\hline \multirow{6}{*}{ Type of Houses } & HT-M & 4.905 * & 0.086 \\
\hline & HT-D & $29.701^{* * *}$ & 0.000 \\
\hline & HT-A & $24.163^{* * *}$ & 0.000 \\
\hline & HT-R & 1.489 & 0.475 \\
\hline & HT-C & $15.490 * * *$ & 0.000 \\
\hline & HT-NH & $25.981^{* * *}$ & 0.000 \\
\hline
\end{tabular}

Note: ${ }^{* *} p<0.01,{ }^{* *} p<0.05$, and ${ }^{*} p<0.1$

Figures 13 and 14 report the difference in the mean value of each type of housingrelated variable. The HS-2 mean value of the LRN is statistically larger than that of the BFN. In the case of HS-1, it is not statistically significant, but the value of LRN is the largest. That is, the proportion of small houses in the LRN is larger than that of other neighborhoods. 


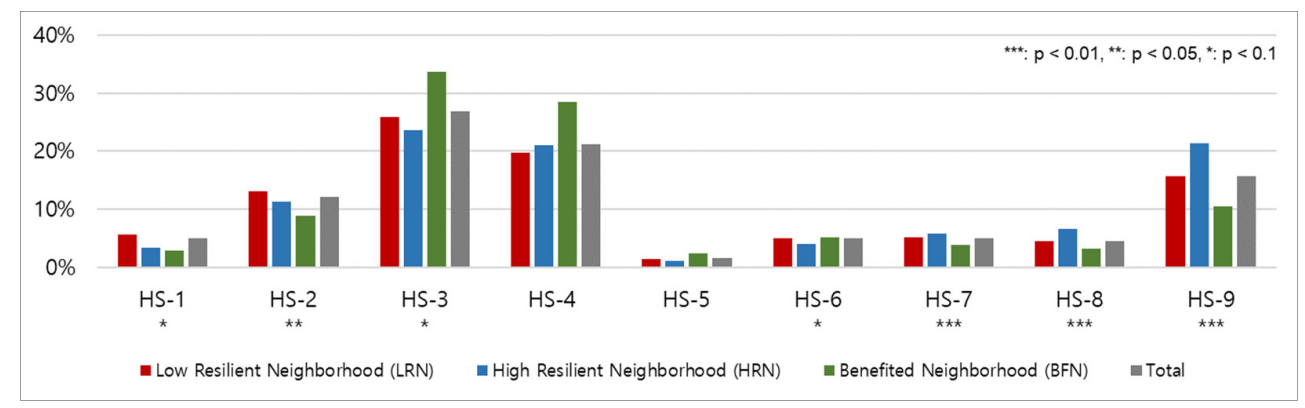

Figure 13. Mean difference of housing-size variables.

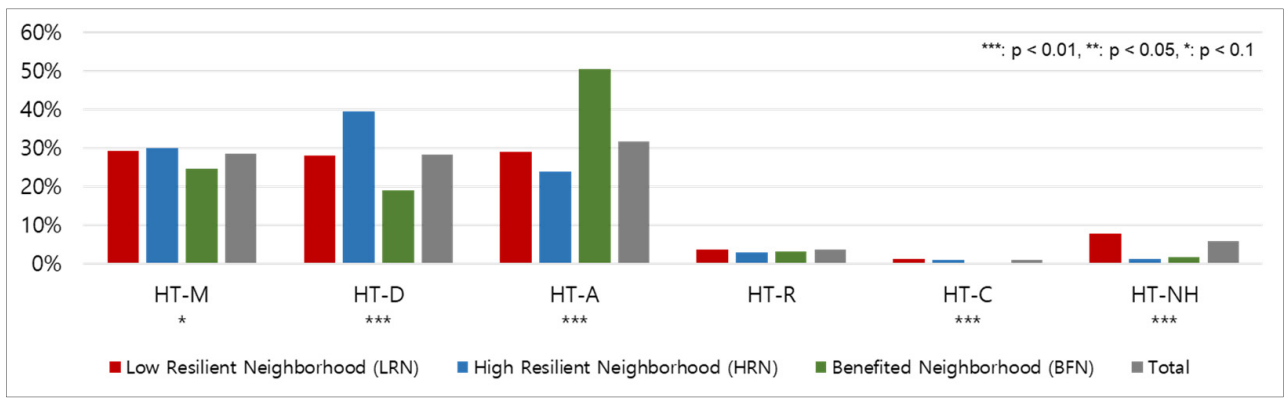

Figure 14. Mean difference of housing-type variables.

As for the values of HS-4 and HS-5, the BFN is very large. Therefore, BFN can be thought of as an area with many middle-sized houses, but it is not statistically significant; hence, the findings should be interpreted with care.

Regarding the mean value of the HS-7, HS-8, and HS-9 variables, representing the proportion of large-sized houses, the value of the $\mathrm{HRN}$ is significantly larger than that of the LRN and BFN. Hence, the HRN has more large-sized houses than in other neighborhoods.

In summary, there are more small (large) houses in the LRN (HRN) than in other neighborhoods. Further, in the BFN, there are more middle-sized houses relative to other neighborhoods.

The value of HT-D, the proportion of detached houses, is the highest in the order of HRN, LRN, and BFN. That is, the HRN is a neighborhood with many large detached houses.

The value of HT-A, the apartment ratio, is significantly larger in the BFN than in the HRN and LRN. That is, the BFN is an area with many apartments.

The values of HT-C and HT-NH are higher in the LRN than in other neighborhoods. It means many living quarters in the LRN use studio-type houses or commercial buildings.

\subsubsection{Land-Use Related Characteristics}

Table 5 shows the results of the ANOVA and K-W test of variables related to land-use. All variables have significant mean differences between groups. Figure 15 shows the mean difference between groups.

Table 5. ANOVA or K-W test results of land-use related variables.

\begin{tabular}{ccccccc}
\hline \multirow{2}{*}{ Category } & \multirow{2}{*}{ Variables } & \multicolumn{2}{c}{ Levene's Test } & \multicolumn{3}{c}{ ANOVA or K-W Test } \\
\cline { 3 - 7 } & & Levene's Statistic & Sig. & Statistic & Statistics Value & Sig. \\
\hline \multirow{3}{*}{ Use of Building } & FA-C & - & - & K-W & $33.029^{* * *}$ & 0.000 \\
& FA-E & - & - & K-W & $8.452^{* *}$ & 0.015 \\
& FA-R & - & - & K-W & $36.950^{* * *}$ & 0.000 \\
\hline \multirow{3}{*}{ Land-Use Mix } & No-U & 1.927 & 0.146 & F & $5.526^{* * *}$ & 0.004 \\
& HHI & 1.172 & 0.310 & F & $13.162^{* * *}$ & 0.000 \\
& RNR & $2.370^{*}$ & 0.094 & Welch & $9.447^{* * *}$ & 0.000 \\
\hline
\end{tabular}

Note: ${ }^{* * *} p<0.01,{ }^{* *} p<0.05$, and ${ }^{*} p<0.1$. 


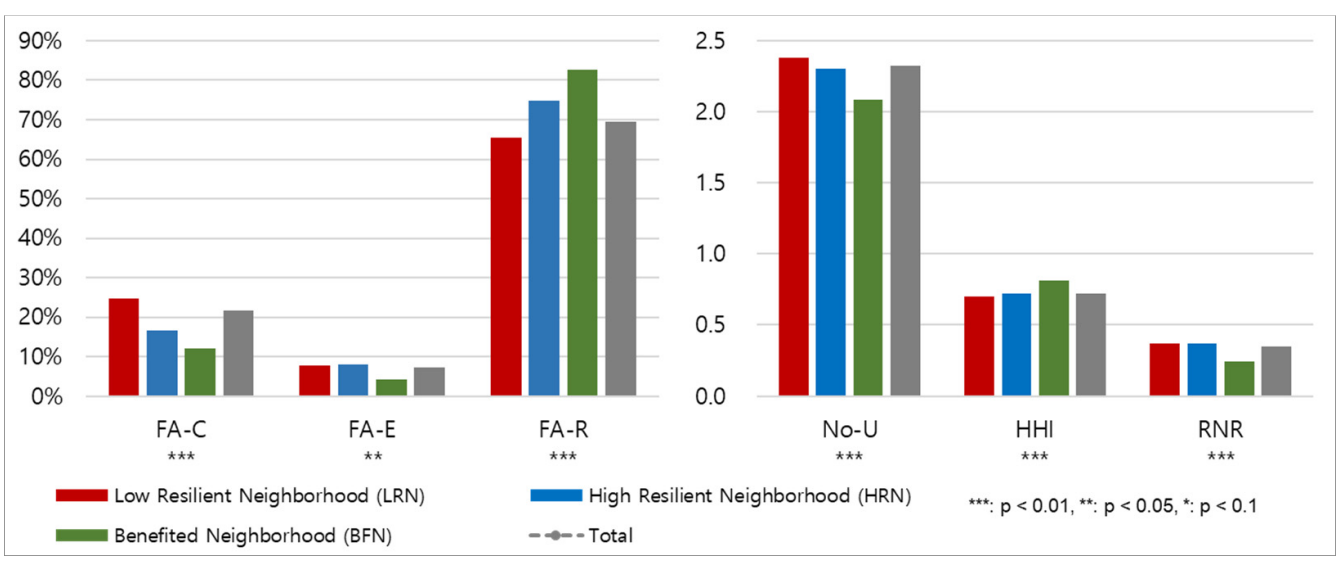

Figure 15. Mean difference of land-use related variables.

Regarding the floor area proportion of commercial buildings (FA-C), the LRN was the largest, followed by the HRN and BFN. However, for residential use (FA-R), BFN was the largest, followed by HRN and LRN. For educational and cultural uses (FA-E), the LRN value was larger than that of the BFN. The LRN (HRN and BFN) has (have) relatively many commercial (residential) buildings.

Similar results were also found for variables related to the land-use mix. In No-U, the value of the LRN is significantly larger than that of the BFN. The higher the HHI value, the more it is centered on single use. Further, the degree of the land-use mix is lower. The HHI value of the BFN was significantly higher than that of other neighborhoods. The smaller the RNR value, the smaller the degree of the land-use mix. The value of the BFN was also smaller than that of the other neighborhoods. Therefore, the BFN was mainly for residential purposes, with few other uses.

\subsubsection{Transportation Characteristics}

Table 6 reports the results of the ANOVA test for traffic-related variables. Figure 16 reports the mean value for each neighborhood type. There is a statistically significant difference between groups in RD and BUS, and there is no significant difference in TRN. The RD value of the BFN is statistically and significantly smaller than that of other neighborhoods. Hence, the proportion of apartments in the BFN is high. Since Korean apartment complexes are often large complexes that occupy the entire block, the road area ratio is considered low.

Table 6. ANOVA or K-W test results of transportation variables.

\begin{tabular}{cccccc}
\hline \multirow{2}{*}{ Variables } & \multicolumn{2}{c}{ Levene's Test } & \multicolumn{3}{c}{ ANOVA or K-W Test } \\
\cline { 2 - 6 } & Levene's Statistic & Sig. & Statistic & Statistics Value & Sig. \\
\hline RD & $4.980^{* * *}$ & 0.007 & Welch & $8.997^{* * *}$ & 0.000 \\
TRN & 0.201 & 0.818 & F & 1.795 & 0.167 \\
BUS & - & - & K-W & $12.432^{* * *}$ & 0.002 \\
\hline Note: ${ }^{* * *} p<0.011^{* *} p<0.05$, and ${ }^{*} p<0.1$. & & &
\end{tabular}

The BUS value of the LRN is significantly greater than that of other neighborhoods. There are relatively many commercial buildings in the LRN. Therefore, access to public transportation is considered better. 


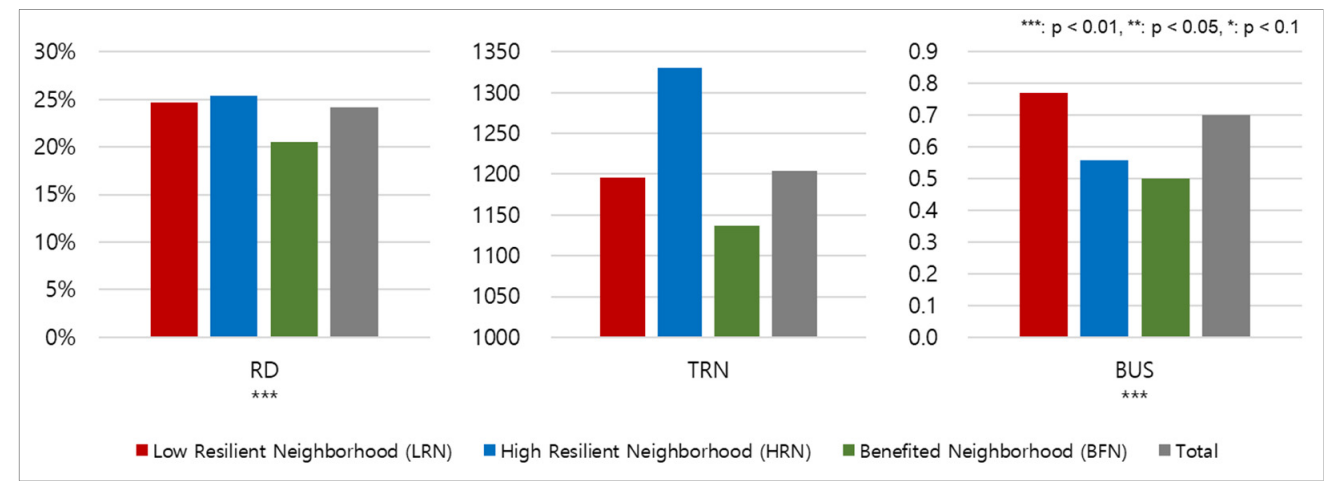

Figure 16. Mean difference of transportation variables.

\subsubsection{Density Characteristics}

Table 7 reports the results of the K-W test of the density-related variable. Figure 17 reports the mean value for each neighborhood type. There are differences between groups in both population density and building density. The population density is higher in the BFN than in other neighborhoods. Accordingly, many apartments are high-density houses in the BFN.

Table 7. Kruskal-Wallis test results of density variables.

\begin{tabular}{ccc}
\hline Variables & Statistic & Sig. \\
\hline DN-P & $38.662^{* * *}$ & 0.000 \\
DN-B & $10.003^{* * *}$ & 0.007 \\
\hline Note: $^{* * *} p<0.01^{* *} p<0.05$, and ${ }^{*} p<0.1$. & &
\end{tabular}

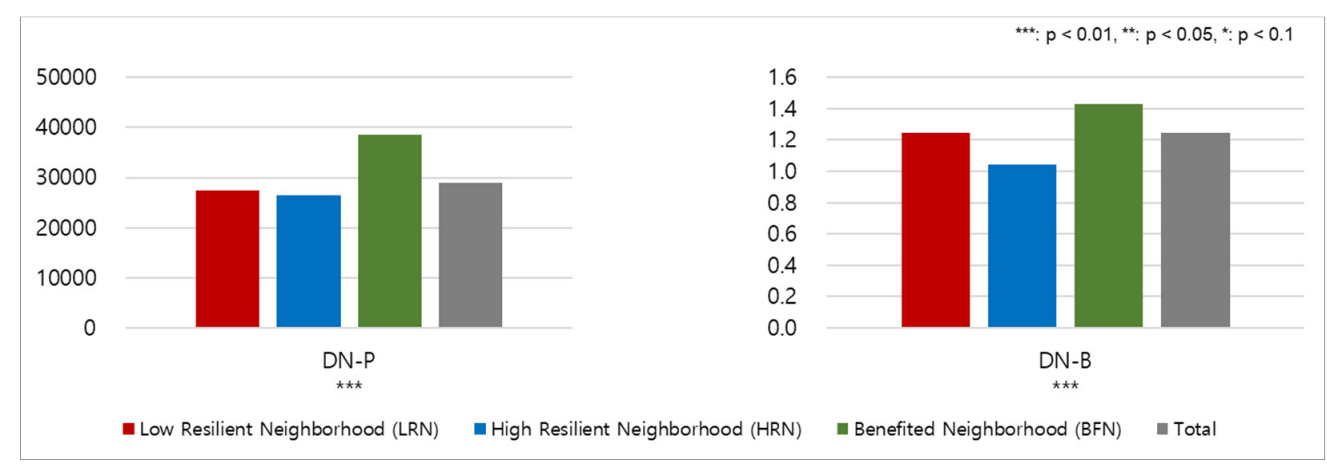

Figure 17. Mean difference of density variables.

The building density is statistically lower in the HRN than in other neighborhoods. The HRN result is similar to the previous analysis, which derived a low-density residential area mainly for detached houses.

\section{Discussion}

Table 8 summarizes urban characteristics by neighborhood type per the results of this study. Thus, it is possible to check the characteristics of the HRN and BFN in a pandemic. Moreover, the characteristics of the LRN, where recovery is slow, are evident.

First, the HRN is home to many senior citizens, with more large-scale detached houses than other areas. Regarding land-use, it is situated between the LRN and BFN. That is, commercial and residential are properly mixed. There are many low-rise detached houses in HRN, with low density. Hence, the HRN is a low-rise mixed-use area where many elderly people with relatively high incomes reside. Consumption in such neighborhoods decreased at the start of the COVID-19 outbreak but recovered rapidly after the initial shock due to the low online shopping accessibility and mobility among the elderly. The 
online-shopping usage rate among those in their 20s (96.9\%) and 30s (92.4\%) is very high compared to the overall average (i.e., 64.1\%) [98]. Contrariwise, online-shopping use rates of individuals in their 50s (44.1\%), 60s (20.8\%), and 70s (15.4\%) are very low [98]. During a pandemic, such a large difference in accessibility to online shopping seems to induce a difference in neighborhoods' consumption patterns.

Table 8. Urban characteristics according to neighborhood type.

\begin{tabular}{|c|c|c|c|}
\hline & $\begin{array}{l}\text { Low-Resilient Neighborhood } \\
\text { (LRN) }\end{array}$ & $\begin{array}{c}\text { High-Resilient Neighborhood } \\
\text { (HRN) }\end{array}$ & $\begin{array}{l}\text { Benefited Neighborhood } \\
\text { (BFN) }\end{array}$ \\
\hline Demographic Structure & $\begin{array}{l}\text { Many young people in their 20s } \\
\text { and 30s }\end{array}$ & Many elderly people over 60 & Many minors and $40 \mathrm{~s}$ \\
\hline Household Structure & $\begin{array}{l}\text { Many single-person households } \\
\text { and non-related households }\end{array}$ & & $\begin{array}{l}\text { Fewer single-person } \\
\text { households } \\
\text { Larger number of } \\
\text { household members } \\
\text { Many two-generation } \\
\text { households }\end{array}$ \\
\hline Size of Houses & Many small houses & Many large houses & Many medium-sized houses \\
\hline Type of Houses & $\begin{array}{l}\text { Many studio houses and } \\
\text { dwellings in } \\
\text { commercial buildings }\end{array}$ & Many detached houses & Many apartments \\
\hline Use of Building & Large commercial floor area & Intermediate characteristics & Large residential floor area \\
\hline Land-Use Mix & $\begin{array}{c}\text { Diversity of land-use } \\
\text { High degree of land-use mix }\end{array}$ & Intermediate characteristics & $\begin{array}{l}\text { Uniformity of land-use } \\
\text { Low degree of land-use mix }\end{array}$ \\
\hline Transportation & Many bus stops & & Low road area ratio \\
\hline Density & & Low building density & High population density \\
\hline
\end{tabular}

The BFN has a large population of minors, and many households comprised parents and children. Moreover, it has mainly high-density apartments, and the ratio of residential use is higher than in other neighborhoods. Such an area is a high-density residential area where large families, including children, live together. Residents of such neighborhoods normally shop in large commercial facilities away from their residence. However, during the pandemic, preference for large commercial facilities where many people gathered decreased. Accordingly, consumption near homes seems to have increased. Apparently, consumption did not decrease even in the early stages of the COVID-19 outbreak, as a large amount of essential consumption catered for children and adolescents. This change in consumption patterns can be viewed as positive: increasing consumption in small local shops can lead to higher consumption of locally produced goods, increasing the likelihood of a transition to a circular economy [35].

Relative to other neighborhoods, the LRN has more commercial buildings, a mixture of various uses, and many young people. There are many studio-type small houses, with many cases of non-family members living together. Such an area is usually the most vibrant in the city, given the many young people and commercial and social activities. However, during the pandemic, such regions were found to have relatively low resilience. Younger generations have quickly transitioned to online consumption due to the ease of access to (and comprehension of) online shopping. Further, the recovery in these regions was slow due to a significant decrease in social activities from social distancing. Since this study uses the concept of engineering resilience, this situation was deemed as low resilience. However, if we apply the concept of adaptive resilience, a new equilibrium is apparent. Additionally, the youth rapidly adopt new consumption behaviors to maintain their quality of life and are likely to maintain a new way of life even after the pandemic. Still, from the perspective of local merchants, it is unreasonable to see this situation as a new equilibrium. They suffer greatly from the decline in sales, and their survival is compromised. 
The study findings have the following implications for urban planning. First, urban characteristics that enhance urban vitality in general situations, such as youth population, various social activities, and land-use mix, can be disadvantageous in a pandemic because it is easy for young people to move their consumption online, and social activities are greatly restricted. The results are similar to those of city-scale studies that show that metropolitan areas and tourism-centered cities suffer a great deal of economic damage from infectious diseases. In a situation where the cycle of infectious diseases is expected to be short in the future, it is necessary to reexamine the existing common sense in urban planning. To overcome such a situation, urban planning with a reinforced social-mix concept should be implemented. In Korea, social-mix has aimed to mix income classes. However, considering the results of this study, a social mix wherein various ages and households can be grouped into neighborhood units should be conducted.

Second, experiences in pandemic situations may have a positive effect in the long term. In Korea, the problem of local retail stores closing due to their inability to compete with large commercial facilities has been noted. In a pandemic situation, as the preference for large-scale commercial facilities declines, consumption in local retail stores around residential areas is emerging. Accordingly, the BFN emerged. Simply put, the possibility of change to sustainable consumption behavior through experiences in pandemic situations is suggested. If the local consumption experience continues during the pandemic, longterm consumption preferences of consumers may change. Of course, local retail stores must also improve their competitiveness. To induce such a positive change, more active dissemination of local currency should be considered. In Korea, local currency can only be used in the local area but cannot be used in large commercial facilities. Hence, the spread of local currency can increase the positive local consumption experience. It is also possible to provide the DRF only in the local currency, and various incentives to facilitating the use of local currency can be presented in a pandemic situation.

Third, it is necessary to supply urban infrastructure for each neighborhood living area. As seen in the case of HRNs and BFNs, in a pandemic situation, people have the potential to shift their activities of everyday life, such as consumption, to the neighborhood around their homes, and there is a need for efforts from the public sector to sustain and strengthen such positive changes. Green spaces, such as small parks and children's playgrounds, can be important infrastructure for increasing activities in the neighborhood. In particular, it was confirmed that open space plays a key role as leisure space for citizens during a pandemic. The expansion of urban green spaces will strengthen urban resilience and sustainability [45] and increase local activities to help introduce sustainable consumption behaviors such as circular consumption [35].

Fourth, the elderly's digital literacy needs to be supported. In HRNs, consumption recovered rapidly, which could be due to the elderly's low accessibility to online shopping. However, in the long run, seniors' access to online shopping and delivery apps needs to be improved. D'Adamo and Rosa [45] proposed embracing digitalization for urban sustainability and resilience after the pandemic. In Korea, public support is needed to improve access to digital consumption, especially for the elderly. To this end, the Korean government recently launched a public delivery app [99] and is pursuing digitalization of infrastructure and strengthening of the digital access of vulnerable groups such as the elderly through the Digital New Deal policy $[100,101]$.

This study on the economic resilience of small regions in a pandemic is rare and significant. In particular, the urban characteristics of regions with high resilience can be used as basic data for future urban planning in preparation for the next potential pandemic. However, this study has limitations as follows. First, the long-term effects could not be analyzed. This study includes only the first wave and stable period in Korea. Since then, there have been second and third waves. Moreover, the pandemic is ongoing. It is challenging to understand the resilience and characteristics of neighborhoods in a situation where the pandemic and social distancing persist. Therefore, studies on the long-term impact and resilience should be considered in the future. 
Second, this study was performed by using only the engineering-resilience concept. The concept of resilience is diverse, and if different concepts of resilience are applied, the results of this study can be interpreted differently. Although the concept of engineering resilience was selected in consideration of the situation in Korea and the temporal range of the study, in the future, it will be necessary to apply the concept of adaptive resilience or evolutionary resilience.

Third, the effects of DRF could not be distinguished. DRF payment began within the analysis period of this study. Thus, it is considered to have affected the recovery of consumption in the neighborhood. However, the effects of DRF were not separately distinguished in this study. Further, the central government's DRF began to be paid in earnest in June. Therefore, even though the effect of DRF will likely be small, continuous monitoring is required.

Fourth, this study did not distinguish between the types of stores where credit card payment occurred. Given that the study is a pioneer in analyzing the resilience of neighborhoods in a pandemic situation, the total amount of credit card payments that did not differentiate between store types were analyzed. Future analyses can consider classification by consumption type to derive more implications.

Author Contributions: Conceptualization, S.H. and S.-H.C.; methodology, S.H.; resources, S.-H.C.; data curation, S.H.; writing—original draft preparation, S.H.; writing—review and editing, S.H. and S.-H.C.; project administration, S-H.C. Both authors have read and agreed to the published version of the manuscript.

Funding: This research was supported by Basic Science Research Program through the National Research Foundation of Korea(NRF) funded by the Ministry of Education(2020R111A3070154).

Institutional Review Board Statement: Not Applicable.

Informed Consent Statement: Not Applicable.

Data Availability Statement: Not Applicable.

Acknowledgments: The card payment data used in the study were supported by the Korea Data Agency's data voucher support project "Covid-19 response emergency support".

Conflicts of Interest: The authors declare no conflict of interest.

\section{Appendix A}

Table A1. Descriptive statistics of variables by neighborhood groups.

\begin{tabular}{|c|c|c|c|c|c|c|c|c|}
\hline Category & Variables & $\begin{array}{l}\text { Neighborhood } \\
\text { Groups }\end{array}$ & $\mathbf{N}$ & Mean & $\begin{array}{l}\text { Standard } \\
\text { Deviation }\end{array}$ & $\begin{array}{l}\text { Standard } \\
\text { Error }\end{array}$ & Min & $\operatorname{Max}$ \\
\hline \multirow{16}{*}{$\begin{array}{l}\text { Demographic } \\
\text { Structure }\end{array}$} & \multirow{4}{*}{ P-00 } & Low Resilient & 605 & 0.061 & 0.040 & 0.002 & 0.000 & 0.288 \\
\hline & & High Resilient & 111 & 0.063 & 0.038 & 0.004 & 0.000 & 0.204 \\
\hline & & Benefited & 130 & 0.074 & 0.039 & 0.003 & 0.000 & 0.204 \\
\hline & & Total & 846 & 0.063 & 0.040 & 0.001 & 0.000 & 0.288 \\
\hline & \multirow{4}{*}{ P-10 } & Low Resilient & 605 & 0.081 & 0.050 & 0.002 & 0.000 & 0.676 \\
\hline & & High Resilient & 111 & 0.084 & 0.034 & 0.003 & 0.000 & 0.177 \\
\hline & & Benefited & 130 & 0.098 & 0.048 & 0.004 & 0.000 & 0.256 \\
\hline & & Total & 846 & 0.084 & 0.049 & 0.002 & 0.000 & 0.676 \\
\hline & \multirow{4}{*}{ P-20 } & Low Resilient & 605 & 0.178 & 0.104 & 0.004 & 0.000 & 0.707 \\
\hline & & High Resilient & 111 & 0.155 & 0.060 & 0.006 & 0.000 & 0.473 \\
\hline & & Benefited & 130 & 0.163 & 0.093 & 0.008 & 0.000 & 0.764 \\
\hline & & Total & 846 & 0.173 & 0.098 & 0.003 & 0.000 & 0.764 \\
\hline & \multirow{4}{*}{ P-30 } & Low Resilient & 605 & 0.166 & 0.070 & 0.003 & 0.000 & 0.545 \\
\hline & & High Resilient & 111 & 0.153 & 0.058 & 0.005 & 0.000 & 0.350 \\
\hline & & Benefited & 130 & 0.154 & 0.057 & 0.005 & 0.000 & 0.358 \\
\hline & & Total & 846 & 0.163 & 0.067 & 0.002 & 0.000 & 0.545 \\
\hline
\end{tabular}


Table A1. Cont.

\begin{tabular}{|c|c|c|c|c|c|c|c|c|}
\hline Category & Variables & $\begin{array}{l}\text { Neighborhood } \\
\text { Groups }\end{array}$ & $\mathbf{N}$ & Mean & $\begin{array}{l}\text { Standard } \\
\text { Deviation }\end{array}$ & $\begin{array}{l}\text { Standard } \\
\text { Error }\end{array}$ & Min & $\operatorname{Max}$ \\
\hline \multirow{16}{*}{$\begin{array}{l}\text { Demographic } \\
\text { Structure }\end{array}$} & \multirow{4}{*}{ P-40 } & Low Resilient & 605 & 0.163 & 0.055 & 0.002 & 0.000 & 1.000 \\
\hline & & High Resilient & 111 & 0.155 & 0.034 & 0.003 & 0.000 & 0.232 \\
\hline & & Benefited & 130 & 0.171 & 0.047 & 0.004 & 0.000 & 0.308 \\
\hline & & Total & 846 & 0.163 & 0.052 & 0.002 & 0.000 & 1.000 \\
\hline & \multirow{4}{*}{ P-50 } & Low Resilient & 605 & 0.168 & 0.049 & 0.002 & 0.000 & 0.375 \\
\hline & & High Resilient & 111 & 0.174 & 0.043 & 0.004 & 0.000 & 0.252 \\
\hline & & Benefited & 130 & 0.171 & 0.043 & 0.004 & 0.000 & 0.284 \\
\hline & & Total & 846 & 0.169 & 0.047 & 0.002 & 0.000 & 0.375 \\
\hline & \multirow{4}{*}{ P-60 } & Low Resilient & 605 & 0.103 & 0.047 & 0.002 & 0.000 & 0.240 \\
\hline & & High Resilient & 111 & 0.115 & 0.041 & 0.004 & 0.000 & 0.201 \\
\hline & & Benefited & 130 & 0.096 & 0.045 & 0.004 & 0.000 & 0.232 \\
\hline & & Total & 846 & 0.103 & 0.046 & 0.002 & 0.000 & 0.240 \\
\hline & \multirow{4}{*}{ P-70 } & Low Resilient & 605 & 0.073 & 0.050 & 0.002 & 0.000 & 0.369 \\
\hline & & High Resilient & 111 & 0.081 & 0.043 & 0.004 & 0.000 & 0.197 \\
\hline & & Benefited & 130 & 0.064 & 0.041 & 0.004 & 0.000 & 0.165 \\
\hline & & Total & 846 & 0.073 & 0.048 & 0.002 & 0.000 & 0.369 \\
\hline \multirow{24}{*}{$\begin{array}{l}\text { Household } \\
\text { Structure }\end{array}$} & \multirow{4}{*}{ HH-M } & Low Resilient & 605 & 2.212 & 0.609 & 0.025 & 0.000 & 3.800 \\
\hline & & High Resilient & 111 & 2.235 & 0.536 & 0.051 & 0.000 & 3.500 \\
\hline & & Benefited & 130 & 2.501 & 0.652 & 0.057 & 0.000 & 3.600 \\
\hline & & Total & 846 & 2.259 & 0.615 & 0.021 & 0.000 & 3.800 \\
\hline & \multirow{4}{*}{ HH-1G } & Low Resilient & 605 & 0.135 & 0.048 & 0.002 & 0.000 & 0.256 \\
\hline & & High Resilient & 111 & 0.141 & 0.039 & 0.004 & 0.000 & 0.233 \\
\hline & & Benefited & 130 & 0.142 & 0.046 & 0.004 & 0.000 & 0.293 \\
\hline & & Total & 846 & 0.137 & 0.047 & 0.002 & 0.000 & 0.293 \\
\hline & \multirow{4}{*}{ HH-2G } & Low Resilient & 605 & 0.413 & 0.200 & 0.008 & 0.000 & 0.914 \\
\hline & & High Resilient & 111 & 0.431 & 0.160 & 0.015 & 0.000 & 0.789 \\
\hline & & Benefited & 130 & 0.515 & 0.207 & 0.018 & 0.000 & 0.944 \\
\hline & & Total & 846 & 0.431 & 0.199 & 0.007 & 0.000 & 0.944 \\
\hline & \multirow{4}{*}{ HH-3G } & Low Resilient & 605 & 0.037 & 0.028 & 0.001 & 0.000 & 0.130 \\
\hline & & High Resilient & 111 & 0.041 & 0.024 & 0.002 & 0.000 & 0.138 \\
\hline & & Benefited & 130 & 0.042 & 0.028 & 0.002 & 0.000 & 0.157 \\
\hline & & Total & 846 & 0.038 & 0.028 & 0.001 & 0.000 & 0.157 \\
\hline & \multirow{4}{*}{ HH-1P } & Low Resilient & 605 & 0.388 & 0.225 & 0.009 & 0.000 & 1.000 \\
\hline & & High Resilient & 111 & 0.355 & 0.169 & 0.016 & 0.000 & 0.802 \\
\hline & & Benefited & 130 & 0.281 & 0.215 & 0.019 & 0.000 & 0.933 \\
\hline & & Total & 846 & 0.367 & 0.220 & 0.008 & 0.000 & 1.000 \\
\hline & \multirow{4}{*}{ HH-NR } & Low Resilient & 605 & 0.018 & 0.022 & 0.001 & 0.000 & 0.107 \\
\hline & & High Resilient & 111 & 0.014 & 0.018 & 0.002 & 0.000 & 0.074 \\
\hline & & Benefited & 130 & 0.011 & 0.018 & 0.002 & 0.000 & 0.073 \\
\hline & & Total & 846 & 0.017 & 0.021 & 0.001 & 0.000 & 0.107 \\
\hline \multirow{12}{*}{ Size of Houses } & \multirow{4}{*}{ HS-1 } & Low Resilient & 605 & 0.057 & 0.134 & 0.005 & 0.000 & 1.000 \\
\hline & & High Resilient & 111 & 0.033 & 0.078 & 0.007 & 0.000 & 0.388 \\
\hline & & Benefited & 130 & 0.030 & 0.071 & 0.006 & 0.000 & 0.331 \\
\hline & & Total & 846 & 0.050 & 0.120 & 0.004 & 0.000 & 1.000 \\
\hline & \multirow{4}{*}{ HS-2 } & Low Resilient & 605 & 0.131 & 0.182 & 0.007 & 0.000 & 1.000 \\
\hline & & High Resilient & 111 & 0.114 & 0.154 & 0.015 & 0.000 & 0.783 \\
\hline & & Benefited & 130 & 0.089 & 0.145 & 0.013 & 0.000 & 0.877 \\
\hline & & Total & 846 & 0.122 & 0.174 & 0.006 & 0.000 & 1.000 \\
\hline & \multirow{4}{*}{ HS-3 } & Low Resilient & 605 & 0.259 & 0.279 & 0.011 & 0.000 & 1.000 \\
\hline & & High Resilient & 111 & 0.236 & 0.258 & 0.025 & 0.000 & 1.000 \\
\hline & & Benefited & 130 & 0.338 & 0.336 & 0.029 & 0.000 & 1.000 \\
\hline & & Total & 846 & 0.268 & 0.287 & 0.010 & 0.000 & 1.000 \\
\hline
\end{tabular}


Table A1. Cont.

\begin{tabular}{|c|c|c|c|c|c|c|c|c|}
\hline Category & Variables & $\begin{array}{l}\text { Neighborhood } \\
\text { Groups }\end{array}$ & $\mathbf{N}$ & Mean & $\begin{array}{l}\text { Standard } \\
\text { Deviation }\end{array}$ & $\begin{array}{l}\text { Standard } \\
\text { Error }\end{array}$ & Min & $\operatorname{Max}$ \\
\hline \multirow{24}{*}{ Size of Houses } & \multirow{4}{*}{ HS-4 } & Low Resilient & 605 & 0.197 & 0.269 & 0.011 & 0.000 & 1.000 \\
\hline & & High Resilient & 111 & 0.211 & 0.287 & 0.027 & 0.000 & 1.000 \\
\hline & & Benefited & 130 & 0.284 & 0.352 & 0.031 & 0.000 & 1.000 \\
\hline & & Total & 846 & 0.212 & 0.287 & 0.010 & 0.000 & 1.000 \\
\hline & \multirow{4}{*}{ HS-5 } & Low Resilient & 605 & 0.014 & 0.059 & 0.002 & 0.000 & 1.000 \\
\hline & & High Resilient & 111 & 0.011 & 0.034 & 0.003 & 0.000 & 0.278 \\
\hline & & Benefited & 130 & 0.024 & 0.120 & 0.010 & 0.000 & 1.000 \\
\hline & & Total & 846 & 0.015 & 0.069 & 0.002 & 0.000 & 1.000 \\
\hline & \multirow{4}{*}{ HS-6 } & Low Resilient & 605 & 0.051 & 0.143 & 0.006 & 0.000 & 1.000 \\
\hline & & High Resilient & 111 & 0.040 & 0.114 & 0.011 & 0.000 & 1.000 \\
\hline & & Benefited & 130 & 0.052 & 0.174 & 0.015 & 0.000 & 1.000 \\
\hline & & Total & 846 & 0.050 & 0.145 & 0.005 & 0.000 & 1.000 \\
\hline & \multirow{4}{*}{ HS-7 } & Low Resilient & 605 & 0.051 & 0.112 & 0.005 & 0.000 & 1.000 \\
\hline & & High Resilient & 111 & 0.058 & 0.078 & 0.007 & 0.000 & 0.485 \\
\hline & & Benefited & 130 & 0.039 & 0.109 & 0.010 & 0.000 & 1.000 \\
\hline & & Total & 846 & 0.050 & 0.108 & 0.004 & 0.000 & 1.000 \\
\hline & \multirow{4}{*}{ HS-8 } & Low Resilient & 605 & 0.045 & 0.073 & 0.003 & 0.000 & 0.493 \\
\hline & & High Resilient & 111 & 0.067 & 0.094 & 0.009 & 0.000 & 0.407 \\
\hline & & Benefited & 130 & 0.032 & 0.071 & 0.006 & 0.000 & 0.434 \\
\hline & & Total & 846 & 0.046 & 0.076 & 0.003 & 0.000 & 0.493 \\
\hline & \multirow{4}{*}{ HS-9 } & Low Resilient & 605 & 0.158 & 0.254 & 0.010 & 0.000 & 1.000 \\
\hline & & High Resilient & 111 & 0.213 & 0.291 & 0.028 & 0.000 & 1.000 \\
\hline & & Benefited & 130 & 0.105 & 0.217 & 0.019 & 0.000 & 1.000 \\
\hline & & Total & 846 & 0.157 & 0.255 & 0.009 & 0.000 & 1.000 \\
\hline \multirow{24}{*}{ Type of Houses } & \multirow{4}{*}{ HT-M } & Low Resilient & 605 & 0.292 & 0.299 & 0.012 & 0.000 & 1.000 \\
\hline & & High Resilient & 111 & 0.301 & 0.293 & 0.028 & 0.000 & 0.948 \\
\hline & & Benefited & 130 & 0.247 & 0.321 & 0.028 & 0.000 & 0.966 \\
\hline & & Total & 846 & 0.286 & 0.301 & 0.010 & 0.000 & 1.000 \\
\hline & \multirow{4}{*}{ HT-D } & Low Resilient & 605 & 0.282 & 0.305 & 0.012 & 0.000 & 1.000 \\
\hline & & High Resilient & 111 & 0.395 & 0.341 & 0.032 & 0.000 & 1.000 \\
\hline & & Benefited & 130 & 0.190 & 0.266 & 0.023 & 0.000 & 1.000 \\
\hline & & Total & 846 & 0.282 & 0.309 & 0.011 & 0.000 & 1.000 \\
\hline & \multirow{4}{*}{ HT-A } & Low Resilient & 605 & 0.291 & 0.420 & 0.017 & 0.000 & 1.000 \\
\hline & & High Resilient & 111 & 0.238 & 0.385 & 0.037 & 0.000 & 1.000 \\
\hline & & Benefited & 130 & 0.505 & 0.477 & 0.042 & 0.000 & 1.000 \\
\hline & & Total & 846 & 0.317 & 0.432 & 0.015 & 0.000 & 1.000 \\
\hline & \multirow{4}{*}{ HT-R } & Low Resilient & 605 & 0.037 & 0.109 & 0.004 & 0.000 & 1.000 \\
\hline & & High Resilient & 111 & 0.028 & 0.066 & 0.006 & 0.000 & 0.276 \\
\hline & & Benefited & 130 & 0.031 & 0.108 & 0.010 & 0.000 & 0.949 \\
\hline & & Total & 846 & 0.035 & 0.104 & 0.004 & 0.000 & 1.000 \\
\hline & \multirow{4}{*}{ HT-C } & Low Resilient & 605 & 0.012 & 0.031 & 0.001 & 0.000 & 0.255 \\
\hline & & High Resilient & 111 & 0.008 & 0.026 & 0.002 & 0.000 & 0.197 \\
\hline & & Benefited & 130 & 0.003 & 0.014 & 0.001 & 0.000 & 0.111 \\
\hline & & Total & 846 & 0.010 & 0.029 & 0.001 & 0.000 & 0.255 \\
\hline & \multirow{4}{*}{ HT-NH } & Low Resilient & 605 & 0.077 & 0.210 & 0.009 & 0.000 & 1.000 \\
\hline & & High Resilient & 111 & 0.012 & 0.040 & 0.004 & 0.000 & 0.310 \\
\hline & & Benefited & 130 & 0.017 & 0.068 & 0.006 & 0.000 & 0.413 \\
\hline & & Total & 846 & 0.059 & 0.182 & 0.006 & 0.000 & 1.000 \\
\hline
\end{tabular}


Table A1. Cont.

\begin{tabular}{|c|c|c|c|c|c|c|c|c|}
\hline Category & Variables & $\begin{array}{l}\text { Neighborhood } \\
\text { Groups }\end{array}$ & $\mathbf{N}$ & Mean & $\begin{array}{l}\text { Standard } \\
\text { Deviation }\end{array}$ & $\begin{array}{l}\text { Standard } \\
\text { Error }\end{array}$ & Min & Max \\
\hline \multirow{12}{*}{ Use of Buildings } & \multirow{4}{*}{ FA-C } & Low Resilient & 605 & 0.246 & 0.260 & 0.011 & 0.000 & 1.000 \\
\hline & & High Resilient & 111 & 0.167 & 0.169 & 0.016 & 0.000 & 0.709 \\
\hline & & Benefited & 130 & 0.120 & 0.177 & 0.016 & 0.000 & 1.000 \\
\hline & & Total & 846 & 0.216 & 0.243 & 0.008 & 0.000 & 1.000 \\
\hline & \multirow{4}{*}{ FA-E } & Low Resilient & 605 & 0.076 & 0.156 & 0.006 & 0.000 & 1.000 \\
\hline & & High Resilient & 111 & 0.079 & 0.188 & 0.018 & 0.000 & 1.000 \\
\hline & & Benefited & 130 & 0.043 & 0.131 & 0.011 & 0.000 & 1.000 \\
\hline & & Total & 846 & 0.072 & 0.157 & 0.005 & 0.000 & 1.000 \\
\hline & \multirow{4}{*}{ FA-R } & Low Resilient & 605 & 0.656 & 0.318 & 0.013 & 0.000 & 1.000 \\
\hline & & High Resilient & 111 & 0.748 & 0.249 & 0.024 & 0.000 & 1.000 \\
\hline & & Benefited & 130 & 0.825 & 0.246 & 0.022 & 0.000 & 1.000 \\
\hline & & Total & 846 & 0.694 & 0.306 & 0.011 & 0.000 & 1.000 \\
\hline \multirow{12}{*}{ Land-Use Mix } & \multirow{4}{*}{ No-U } & Low Resilient & 605 & 2.379 & 0.926 & 0.038 & 1.000 & 6.000 \\
\hline & & High Resilient & 111 & 2.306 & 0.840 & 0.080 & 1.000 & 5.000 \\
\hline & & Benefited & 130 & 2.085 & 0.932 & 0.082 & 1.000 & 4.000 \\
\hline & & Total & 846 & 2.324 & 0.921 & 0.032 & 1.000 & 6.000 \\
\hline & \multirow{4}{*}{ HHI } & Low Resilient & 605 & 0.700 & 0.223 & 0.009 & 0.302 & 1.000 \\
\hline & & High Resilient & 111 & 0.720 & 0.211 & 0.020 & 0.301 & 1.000 \\
\hline & & Benefited & 130 & 0.810 & 0.216 & 0.019 & 0.323 & 1.000 \\
\hline & & Total & 846 & 0.720 & 0.224 & 0.008 & 0.301 & 1.000 \\
\hline & \multirow{4}{*}{ RNR } & Low Resilient & 605 & 0.372 & 0.326 & 0.013 & 0.000 & 0.996 \\
\hline & & High Resilient & 111 & 0.372 & 0.313 & 0.030 & 0.000 & 0.990 \\
\hline & & Benefited & 130 & 0.244 & 0.305 & 0.027 & 0.000 & 0.988 \\
\hline & & Total & 846 & 0.352 & 0.324 & 0.011 & 0.000 & 0.996 \\
\hline \multirow{12}{*}{ Transportation } & \multirow{4}{*}{$\mathrm{RD}$} & Low Resilient & 605 & 0.247 & 0.117 & 0.005 & 0.000 & 0.686 \\
\hline & & High Resilient & 111 & 0.254 & 0.093 & 0.009 & 0.000 & 0.472 \\
\hline & & Benefited & 130 & 0.205 & 0.108 & 0.009 & 0.000 & 0.599 \\
\hline & & Total & 846 & 0.241 & 0.114 & 0.004 & 0.000 & 0.686 \\
\hline & \multirow{4}{*}{ TRN } & Low Resilient & 605 & 1195.6 & 814.4 & 33.1 & 0.0 & 4401.3 \\
\hline & & High Resilient & 111 & 1329.7 & 824.6 & 78.3 & 61.2 & 4112.5 \\
\hline & & Benefited & 130 & 1136.2 & 821.7 & 72.1 & 0.0 & 4125.0 \\
\hline & & Total & 846 & 1204.1 & 817.6 & 28.1 & 0.0 & 4401.3 \\
\hline & \multirow{4}{*}{ BUS } & Low Resilient & 605 & 0.770 & 1.079 & 0.044 & 0.000 & 8.000 \\
\hline & & High Resilient & 111 & 0.559 & 0.960 & 0.091 & 0.000 & 4.000 \\
\hline & & Benefited & 130 & 0.500 & 0.847 & 0.074 & 0.000 & 5.000 \\
\hline & & Total & 846 & 0.701 & 1.036 & 0.036 & 0.000 & 8.000 \\
\hline \multirow{8}{*}{ Density } & \multirow{4}{*}{ DN-P } & Low Resilient & 605 & $27,378.9$ & $21,641.2$ & 879.8 & 0.0 & $213,506.5$ \\
\hline & & High Resilient & 111 & $26,511.1$ & $14,285.2$ & 1355.9 & 0.0 & $85,463.1$ \\
\hline & & Benefited & 130 & $38,502.7$ & $22,566.6$ & 1979.2 & 0.0 & $120,166.2$ \\
\hline & & Total & 846 & $28,974.4$ & $21,346.3$ & 733.9 & 0.0 & $213,506.5$ \\
\hline & \multirow{4}{*}{ DN-B } & Low Resilient & 605 & 1.247 & 1.026 & 0.042 & 0.003 & 10.356 \\
\hline & & High Resilient & 111 & 1.043 & 0.560 & 0.053 & 0.202 & 4.303 \\
\hline & & Benefited & 130 & 1.427 & 1.005 & 0.088 & 0.003 & 6.213 \\
\hline & & Total & 846 & 1.248 & 0.979 & 0.034 & 0.003 & 10.356 \\
\hline
\end{tabular}


Table A2. Post hoc test results of variables.

\begin{tabular}{|c|c|c|c|c|c|c|c|c|c|c|c|c|}
\hline Category & Variable & Method & $\begin{array}{l}\text { Group } \\
\text { (I) }\end{array}$ & $\underset{(J)}{\text { Group }}$ & $\begin{array}{c}\text { Mean Difference } \\
\text { (I-J) }\end{array}$ & $\begin{array}{l}\text { Sig. or } \\
\text { Adj. Sig. }\end{array}$ & Variable & Method & $\begin{array}{l}\text { Group } \\
\text { (I) }\end{array}$ & $\begin{array}{c}\text { Group } \\
(\mathrm{J})\end{array}$ & $\begin{array}{c}\text { Mean Difference } \\
\text { (I-J) }\end{array}$ & $\begin{array}{c}\text { Sig. or } \\
\text { Adj. Sig }\end{array}$ \\
\hline \multirow{22}{*}{ Demographic Structure } & \multirow{5}{*}{ P-00 } & \multirow{5}{*}{$\mathrm{S}$} & \multirow{2}{*}{1} & 2 & -0.00238 & 0.846 & \multirow{5}{*}{$\mathrm{P}-40$} & \multirow{5}{*}{ S } & \multirow{2}{*}{1} & 2 & 0.00723 & 0.403 \\
\hline & & & & 3 & -0.01345 & 0.002 & & & & 3 & -0.00827 & 0.258 \\
\hline & & & 2 & 3 & -0.01108 & 0.099 & & & 2 & 3 & -0.01550 & 0.070 \\
\hline & & & \multirow{2}{*}{3} & 1 & 0.01345 & 0.002 & & & \multirow{2}{*}{3} & 1 & 0.00827 & 0.258 \\
\hline & & & & 2 & 0.01108 & 0.099 & & & & 2 & 0.01550 & 0.070 \\
\hline & \multirow{6}{*}{ P-10 } & \multirow{6}{*}{ GH } & \multirow{2}{*}{1} & 2 & -0.00313 & 0.696 & \multirow{6}{*}{ P-50 } & \multirow{6}{*}{$\mathrm{GH}$} & \multirow{2}{*}{1} & 2 & -0.00636 & 0.347 \\
\hline & & & & 3 & -0.01700 & 0.001 & & & & 3 & -0.00325 & 0.730 \\
\hline & & & & 1 & 0.00313 & 0.696 & & & \multirow{2}{*}{2} & 1 & 0.00636 & 0.347 \\
\hline & & & 2 & 3 & -0.01387 & 0.026 & & & & 3 & 0.00311 & 0.844 \\
\hline & & & \multirow{2}{*}{3} & 1 & 0.01700 & 0.001 & & & \multirow{2}{*}{3} & 1 & 0.00325 & 0.730 \\
\hline & & & & 2 & 0.01387 & 0.026 & & & & 2 & -0.00311 & 0.844 \\
\hline & \multirow{5}{*}{$\mathrm{P}-20$} & \multirow{5}{*}{$\mathrm{GH}$} & \multirow{2}{*}{1} & 2 & 0.02344 & 0.003 & \multirow{5}{*}{ P-60 } & \multirow{5}{*}{$\mathrm{GH}$} & \multirow{2}{*}{1} & 2 & -0.01284 & 0.009 \\
\hline & & & & 3 & 0.01545 & 0.213 & & & & 3 & 0.00618 & 0.339 \\
\hline & & & \multirow{2}{*}{2} & 1 & -0.02344 & 0.003 & & & \multirow[b]{2}{*}{2} & 1 & 0.01284 & 0.009 \\
\hline & & & & 3 & -0.00799 & 0.699 & & & & 3 & 0.01903 & 0.002 \\
\hline & & & 3 & 2 & 0.00799 & 0.699 & & & 3 & 2 & -0.01903 & 0.002 \\
\hline & & & & 2 & 0.01382 & 0.068 & & & & 2 & -0.00837 & 0.240 \\
\hline & & & 1 & 3 & 0.01216 & 0.089 & & & 1 & 3 & 0.00926 & 0.136 \\
\hline & & & & 1 & -0.01382 & 0.068 & & & 2 & 1 & 0.00837 & 0.240 \\
\hline & $\mathrm{P}-30$ & GH & 2 & 3 & -0.00166 & 0.973 & P-70 & $\mathrm{S}$ & 2 & 3 & 0.01763 & 0.018 \\
\hline & & & & 1 & -0.01216 & 0.089 & & & & 1 & -0.00926 & 0.136 \\
\hline & & & 3 & 2 & 0.00166 & 0.973 & & & 3 & 2 & -0.01763 & 0.018 \\
\hline & & & & 2 & -0.02356 & 0.909 & & & & 2 & -0.00390 & 0.269 \\
\hline & & & 1 & 3 & -0.28920 & 0.000 & & & 1 & 3 & -0.00556 & 0.104 \\
\hline & & & & 1 & 0.02356 & 0.909 & & & & 1 & 0.00390 & 0.269 \\
\hline Household Structure & HН-M & GH & 2 & 3 & -0.26560 & 0.002 & HH-3G & GH & 2 & 3 & -0.00166 & 0.871 \\
\hline & & & & 1 & 0.28920 & 0.000 & & & & 1 & 0.00556 & 0.104 \\
\hline & & & 3 & 2 & 0.26560 & 0.002 & & & 3 & 2 & 0.00166 & 0.871 \\
\hline
\end{tabular}


Table A2. Cont.

\begin{tabular}{|c|c|c|c|c|c|c|c|c|c|c|c|c|}
\hline Category & Variable & Method & $\begin{array}{l}\text { Group } \\
\text { (I) }\end{array}$ & $\begin{array}{c}\text { Group } \\
(\mathrm{J})\end{array}$ & $\begin{array}{c}\text { Mean Difference } \\
\text { (I-J) }\end{array}$ & $\begin{array}{l}\text { Sig. or } \\
\text { Adj. Sig. }\end{array}$ & Variable & Method & $\underset{\text { (I) }}{\text { Group }}$ & $\begin{array}{c}\text { Group } \\
(\mathrm{J})\end{array}$ & $\begin{array}{c}\text { Mean Difference } \\
\text { (I-J) }\end{array}$ & $\begin{array}{l}\text { Sig. or } \\
\text { Adj. Sig }\end{array}$ \\
\hline \multirow{11}{*}{ Household Structure } & \multirow{5}{*}{$\mathrm{HH}-1 \mathrm{G}$} & \multirow{5}{*}{$\mathrm{GH}$} & \multirow{2}{*}{1} & 2 & -0.00567 & 0.372 & \multirow{5}{*}{ HH-1P } & \multirow{5}{*}{$\mathrm{GH}$} & \multirow{2}{*}{1} & 2 & 0.03347 & 0.167 \\
\hline & & & & 3 & -0.00677 & 0.295 & & & & 3 & 0.10675 & 0.000 \\
\hline & & & 2 & 3 & -0.00110 & 0.978 & & & 2 & 3 & 0.07329 & 0.009 \\
\hline & & & \multirow{2}{*}{3} & 1 & 0.00677 & 0.295 & & & \multirow{2}{*}{3} & 1 & -0.10675 & 0.000 \\
\hline & & & & 2 & 0.00110 & 0.978 & & & & 2 & -0.07329 & 0.009 \\
\hline & \multirow{6}{*}{$\mathrm{HH}-2 \mathrm{G}$} & \multirow{6}{*}{ GH } & \multirow{2}{*}{1} & 2 & -0.01847 & 0.534 & \multirow{6}{*}{ HH-NR } & \multirow{6}{*}{$\mathrm{GH}$} & \multirow{2}{*}{1} & 2 & 0.00432 & 0.075 \\
\hline & & & & 3 & -0.10189 & 0.000 & & & & 3 & 0.00690 & 0.001 \\
\hline & & & \multirow[b]{2}{*}{2} & 1 & 0.01847 & 0.534 & & & \multirow{2}{*}{2} & 1 & -0.00432 & 0.075 \\
\hline & & & & 3 & -0.08342 & 0.002 & & & & 3 & 0.00257 & 0.527 \\
\hline & & & \multirow{2}{*}{3} & 1 & 0.10189 & 0.000 & & & \multirow{2}{*}{3} & 1 & -0.00690 & 0.001 \\
\hline & & & & 2 & 0.08342 & 0.002 & & & & 2 & -0.00257 & 0.527 \\
\hline \multirow{17}{*}{ Size of Houses } & \multirow{5}{*}{ HS-1 } & \multirow{5}{*}{ Dunn } & \multirow{2}{*}{1} & 2 & 0.02406 & 0.429 & \multirow{5}{*}{ HS-6 } & & \multirow{2}{*}{1} & 2 & 0.01106 & 1.000 \\
\hline & & & & 3 & 0.02777 & 0.151 & & & & 3 & -0.00128 & 0.071 \\
\hline & & & \multirow{2}{*}{2} & 1 & -0.02406 & 0.429 & & & & 1 & -0.01106 & 1.000 \\
\hline & & & & 3 & 0.00371 & 1.000 & & Dunn & 2 & 3 & -0.01233 & 0.341 \\
\hline & & & 3 & 2 & -0.00371 & 1.000 & & & 3 & 2 & 0.01233 & 0.341 \\
\hline & & & & 2 & 0.01709 & 1.000 & & & & 2 & -0.00606 & 0.079 \\
\hline & & & 1 & 3 & 0.04145 & 0.026 & & & 1 & 3 & 0.01241 & 0.130 \\
\hline & HS-2 & Dunn & 2 & 1 & -0.01709 & 1.000 & HS-7 & Dunn & 2 & 1 & 0.00606 & 0.079 \\
\hline & HS-2 & Dunn & 2 & 3 & 0.02435 & 0.324 & HS-I & Dunn & 2 & 3 & 0.01847 & 0.003 \\
\hline & & & & 1 & -0.04145 & 0.026 & & & & 1 & -0.01241 & 0.130 \\
\hline & & & 3 & 2 & -0.02435 & 0.324 & & & 3 & 2 & -0.01847 & 0.003 \\
\hline & & & & 2 & 0.02274 & 1.000 & & & & 2 & -0.02182 & 0.059 \\
\hline & & & 1 & 3 & -0.07838 & 0.121 & & & 1 & 3 & 0.01284 & 0.026 \\
\hline & & & & 1 & -0.02274 & 1.000 & & & & 1 & 0.02182 & 0.059 \\
\hline & HS-3 & Dunn & 2 & 3 & -0.10112 & 0.113 & HS-8 & Dunn & 2 & 3 & 0.03466 & 0.000 \\
\hline & & & & 1 & 0.07838 & 0.121 & & & 3 & 1 & -0.01284 & 0.026 \\
\hline & & & 3 & 2 & 0.10112 & 0.113 & & & 3 & 2 & -0.03466 & 0.000 \\
\hline
\end{tabular}


Table A2. Cont.

\begin{tabular}{|c|c|c|c|c|c|c|c|c|c|c|c|c|c|}
\hline & Category & Variable & Method & $\begin{array}{l}\text { Group } \\
\text { (I) }\end{array}$ & $\begin{array}{c}\text { Group } \\
(\mathrm{J})\end{array}$ & $\begin{array}{c}\text { Mean Difference } \\
\text { (I-J) }\end{array}$ & $\begin{array}{l}\text { Sig. or } \\
\text { Adj. Sig. }\end{array}$ & Variable & Method & $\underset{\text { (I) }}{\text { Group }}$ & $\begin{array}{c}\text { Group } \\
(\mathrm{J})\end{array}$ & $\begin{array}{c}\text { Mean Difference } \\
\text { (I-J) }\end{array}$ & $\begin{array}{l}\text { Sig. or } \\
\text { Adj. Sig }\end{array}$ \\
\hline & \multirow{11}{*}{ Size of Houses } & \multirow{5}{*}{ HS-4 } & \multirow{5}{*}{ Dunn } & \multirow{2}{*}{1} & 2 & -0.01387 & - & \multirow{5}{*}{ HS-9 } & \multirow{5}{*}{ Dunn } & \multirow{2}{*}{1} & 2 & -0.05546 & 0.042 \\
\hline & & & & & 3 & -0.08714 & - & & & & 3 & 0.05307 & 0.002 \\
\hline & & & & 2 & 3 & -0.07327 & - & & & 2 & 3 & 0.10853 & 0.000 \\
\hline & & & & \multirow{2}{*}{3} & 1 & 0.08714 & - & & & \multirow{2}{*}{3} & 1 & -0.05307 & 0.002 \\
\hline & & & & & 2 & 0.07327 & - & & & & 2 & -0.10853 & 0.000 \\
\hline & & \multirow{6}{*}{ HS-5 } & \multirow{6}{*}{ Dunn } & \multirow{2}{*}{1} & 2 & 0.00392 & - & & & & & & \\
\hline & & & & & 3 & -0.00941 & - & & & & & & \\
\hline & & & & \multirow{2}{*}{2} & 1 & -0.00392 & - & & & & & & \\
\hline & & & & & 3 & -0.01333 & - & & & & & & \\
\hline & & & & \multirow{2}{*}{3} & 1 & 0.00941 & - & & & & & & \\
\hline & & & & & 2 & 0.01333 & - & & & & & & \\
\hline \multirow{17}{*}{$\stackrel{\infty}{\infty}$} & \multirow{17}{*}{ Type of Houses } & \multirow{5}{*}{ HT-M } & \multirow{5}{*}{ Dunn } & \multirow{2}{*}{1} & 2 & -0.00893 & 1.000 & \multirow{5}{*}{ HT-R } & \multirow{5}{*}{ Dunn } & \multirow{2}{*}{1} & 2 & 0.00953 & - \\
\hline & & & & & 3 & 0.04510 & 0.149 & & & & 3 & 0.00631 & - \\
\hline & & & & & 1 & 0.00893 & 1.000 & & & \multirow{2}{*}{2} & 1 & -0.00953 & - \\
\hline & & & & 2 & 3 & 0.05402 & 0.131 & & & & 3 & -0.00323 & - \\
\hline & & & & 3 & 2 & -0.05402 & 0.131 & & & 3 & 2 & 0.00323 & - \\
\hline & & & & \multirow{2}{*}{1} & 2 & -0.11311 & 0.002 & & & 1 & 2 & 0.00401 & 0.405 \\
\hline & & & & & 3 & 0.09203 & 0.001 & & & 1 & 3 & 0.00930 & 0.000 \\
\hline & & HT-D & Dunn & 2 & 1 & 0.11311 & 0.002 & HT-C & Dunn & 2 & 1 & -0.00401 & 0.405 \\
\hline & & H1-D & Dunn & 2 & 3 & 0.20514 & 0.000 & $H 1-\mathrm{C}$ & Dunn & 2 & 3 & 0.00529 & 0.280 \\
\hline & & & & & 1 & -0.09203 & 0.001 & & & & 1 & -0.00930 & 0.000 \\
\hline & & & & 3 & 2 & -0.20514 & 0.000 & & & 3 & 2 & -0.00529 & 0.280 \\
\hline & & & & & 2 & 0.05331 & 0.574 & & & & 2 & 0.06494 & 0.006 \\
\hline & & & & 1 & 3 & -0.21335 & 0.000 & & & 1 & 3 & 0.06004 & 0.000 \\
\hline & & & & & 1 & -0.05331 & 0.574 & & & & 1 & -0.06494 & 0.006 \\
\hline & & HT-A & Dunn & 2 & 3 & -0.26666 & 0.000 & HT-NH & Dunn & 2 & 3 & -0.00489 & 1.000 \\
\hline & & & & 3 & 1 & 0.21335 & 0.000 & & & & 1 & -0.06004 & 0.000 \\
\hline & & & & 3 & 2 & 0.26666 & 0.000 & & & 3 & 2 & 0.00489 & 1.000 \\
\hline
\end{tabular}


Table A2. Cont.

\begin{tabular}{|c|c|c|c|c|c|c|c|c|c|c|c|c|c|}
\hline & Category & Variable & Method & $\begin{array}{l}\text { Group } \\
\text { (I) }\end{array}$ & $\begin{array}{l}\text { Group } \\
(\mathrm{J})\end{array}$ & $\begin{array}{l}\text { Mean Difference } \\
\text { (I-J) }\end{array}$ & $\begin{array}{l}\text { Sig. or } \\
\text { Adj. Sig. }\end{array}$ & Variable & Method & $\begin{array}{l}\text { Group } \\
\text { (I) }\end{array}$ & $\begin{array}{c}\text { Group } \\
(\mathrm{J})\end{array}$ & $\begin{array}{l}\text { Mean Difference } \\
\text { (I-J) }\end{array}$ & $\begin{array}{l}\text { Sig. or } \\
\text { Adj. Sig }\end{array}$ \\
\hline & \multirow{11}{*}{ Use of Buildings } & \multirow{5}{*}{ FA-C } & \multirow{5}{*}{ Dunn } & \multirow{2}{*}{1} & 2 & 0.07943 & 0.115 & \multirow{5}{*}{ FA-R } & \multirow{5}{*}{ Dunn } & \multirow{2}{*}{1} & 2 & -0.09246 & 0.125 \\
\hline & & & & & 3 & 0.12654 & 0.000 & & & & 3 & -0.16974 & 0.000 \\
\hline & & & & 2 & 3 & 0.04711 & 0.032 & & & 2 & 3 & -0.07728 & 0.013 \\
\hline & & & & \multirow{2}{*}{3} & 1 & -0.12654 & 0.000 & & & \multirow{2}{*}{3} & 1 & 0.16974 & 0.000 \\
\hline & & & & & 2 & -0.04711 & 0.032 & & & & 2 & 0.07728 & 0.013 \\
\hline & & \multirow{6}{*}{ FA-E } & \multirow{6}{*}{ Dunn } & \multirow{2}{*}{1} & 2 & -0.00280 & 1.000 & & & & & & \\
\hline & & & & & 3 & 0.03333 & 0.011 & & & & & & \\
\hline & & & & \multirow{2}{*}{2} & 1 & 0.00280 & 1.000 & & & & & & \\
\hline & & & & & 3 & 0.03613 & 0.213 & & & & & & \\
\hline & & & & \multirow{2}{*}{3} & 1 & -0.03333 & 0.011 & & & & & & \\
\hline & & & & & 2 & -0.03613 & 0.213 & & & & & & \\
\hline \multirow{11}{*}{$\begin{array}{l}W \\
N\end{array}$} & \multirow{11}{*}{ Land-Use Mix } & \multirow{5}{*}{ No-U } & \multirow{5}{*}{$S$} & \multirow{2}{*}{1} & 2 & 0.07221 & 0.747 & \multirow{5}{*}{ RNR } & \multirow{5}{*}{ GH } & \multirow{2}{*}{1} & 2 & 0.00001 & 1.000 \\
\hline & & & & & 3 & 0.29400 & 0.004 & & & & 3 & 0.12791 & 0.000 \\
\hline & & & & \multirow{2}{*}{2} & 1 & -0.07221 & 0.747 & & & \multirow{2}{*}{2} & 1 & -0.00001 & 1.000 \\
\hline & & & & & 3 & 0.22169 & 0.174 & & & & 3 & 0.12790 & 0.004 \\
\hline & & & & 3 & 2 & -0.22169 & 0.174 & & & 3 & 2 & -0.12790 & 0.004 \\
\hline & & \multirow{6}{*}{ HHI } & & \multirow[b]{2}{*}{1} & 2 & -0.02011 & 0.677 & & & & & & \\
\hline & & & & & 3 & -0.10940 & 0.000 & & & & & & \\
\hline & & & & \multirow[b]{2}{*}{2} & 1 & 0.02011 & 0.677 & & & & & & \\
\hline & & & S & & 3 & -0.08929 & 0.008 & & & & & & \\
\hline & & & & & 1 & 0.10940 & 0.000 & & & & & & \\
\hline & & & & 3 & 2 & 0.08929 & 0.008 & & & & & & \\
\hline
\end{tabular}


Table A2. Cont.

\begin{tabular}{|c|c|c|c|c|c|c|c|c|c|c|c|c|c|}
\hline & Category & Variable & Method & $\begin{array}{l}\text { Group } \\
\text { (I) }\end{array}$ & $\begin{array}{l}\text { Group } \\
(\mathrm{J})\end{array}$ & $\begin{array}{l}\text { Mean Difference } \\
\text { (I-J) }\end{array}$ & $\begin{array}{l}\text { Sig. or } \\
\text { Adj. Sig. }\end{array}$ & Variable & Method & $\begin{array}{l}\text { Group } \\
\text { (I) }\end{array}$ & $\underset{(J)}{\text { Group }}$ & $\begin{array}{l}\text { Mean Difference } \\
\text { (I-J) }\end{array}$ & $\begin{array}{l}\text { Sig. or } \\
\text { Adj. Sig }\end{array}$ \\
\hline & \multirow{11}{*}{ Transportation } & \multirow{5}{*}{$\mathrm{RD}$} & \multirow{5}{*}{ GH } & \multirow{2}{*}{1} & 2 & -0.00712 & 0.759 & \multirow{5}{*}{ BUS } & \multirow{5}{*}{ Dunn } & \multirow{2}{*}{1} & 2 & 0.22269 & 0.032 \\
\hline & & & & & 3 & 0.04170 & 0.000 & & & & 3 & 0.27025 & 0.014 \\
\hline & & & & 2 & 3 & 0.04882 & 0.001 & & & 2 & 3 & 0.05856 & 1.000 \\
\hline & & & & \multirow{2}{*}{3} & 1 & -0.04170 & 0.000 & & & \multirow{2}{*}{3} & 1 & -0.27025 & 0.014 \\
\hline & & & & & 2 & -0.04882 & 0.001 & & & & 2 & -0.05856 & 1.000 \\
\hline & & \multirow{6}{*}{ TRN } & \multirow{6}{*}{$S$} & \multirow{2}{*}{1} & 2 & -134.2 & 0.283 & & & & & & \\
\hline & & & & & 3 & 59.4 & 0.754 & & & & & & \\
\hline & & & & \multirow{2}{*}{2} & 1 & 134.2 & 0.283 & & & & & & \\
\hline & & & & & 3 & 193.5 & 0.187 & & & & & & \\
\hline & & & & \multirow{2}{*}{3} & 1 & -59.4 & 0.754 & & & & & & \\
\hline & & & & & 2 & -193.5 & 0.187 & & & & & & \\
\hline \multirow{5}{*}{ 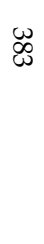 } & \multirow{5}{*}{ Density } & \multirow{5}{*}{ DN-P } & & \multirow[b]{2}{*}{1} & 2 & 867.9 & 0.668 & \multirow{5}{*}{ DN-B } & \multirow{5}{*}{ Dunn } & \multirow[b]{2}{*}{1} & 2 & 0.204 & 0.600 \\
\hline & & & & & 3 & $-11,123.7$ & 0.000 & & & & 3 & -0.180 & 0.025 \\
\hline & & & & & 1 & -867.9 & 0.668 & & & \multirow{2}{*}{2} & 1 & -0.204 & 0.600 \\
\hline & & & Dunn & 2 & 3 & $-11,991.6$ & 0.000 & & & & 3 & -0.384 & 0.008 \\
\hline & & & & 3 & 2 & $11,991.6$ & 0.000 & & & 3 & 2 & 0.384 & 0.008 \\
\hline
\end{tabular}


Table A3. Q-Q plot of variables.

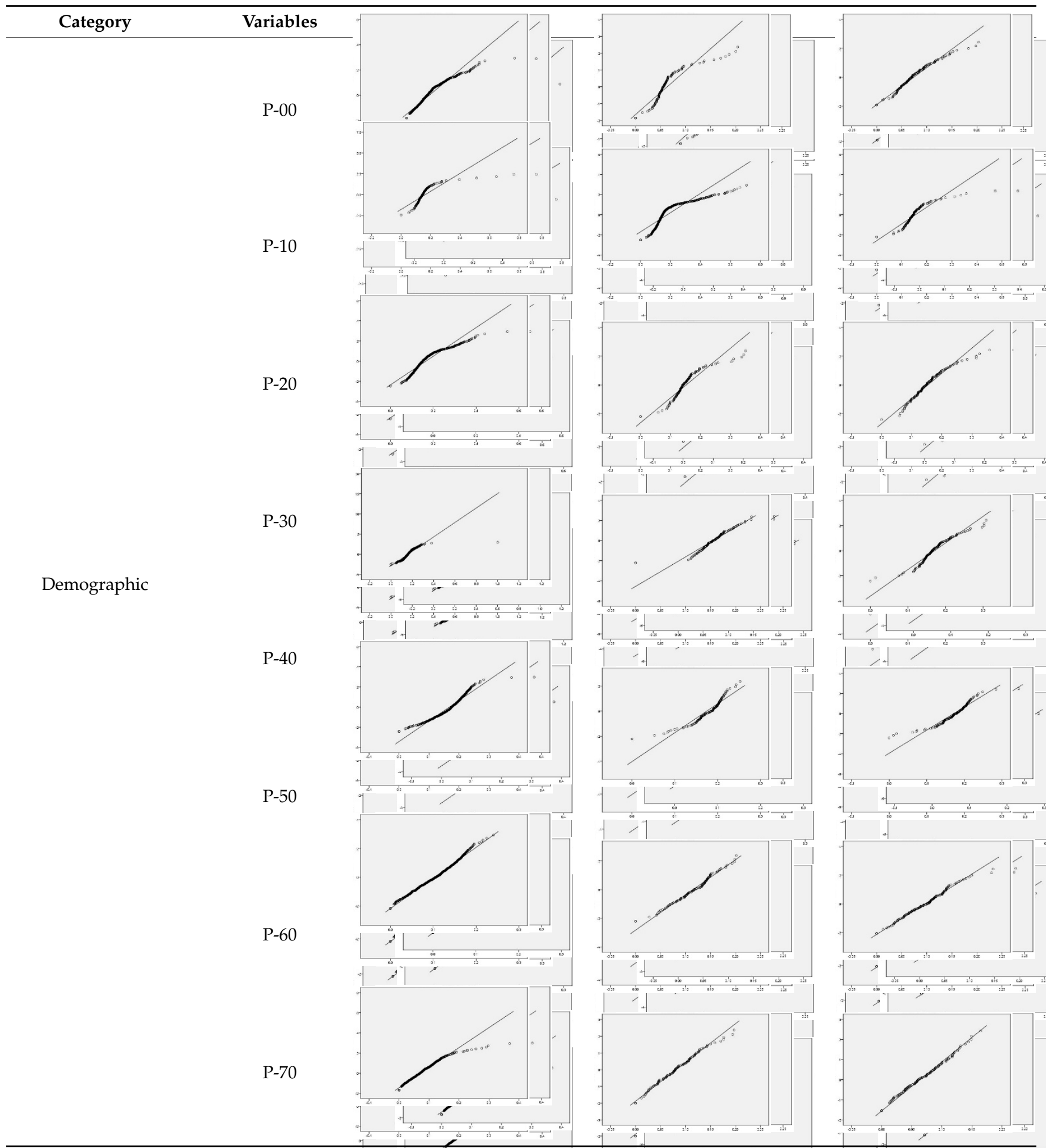


Table A3. Cont.

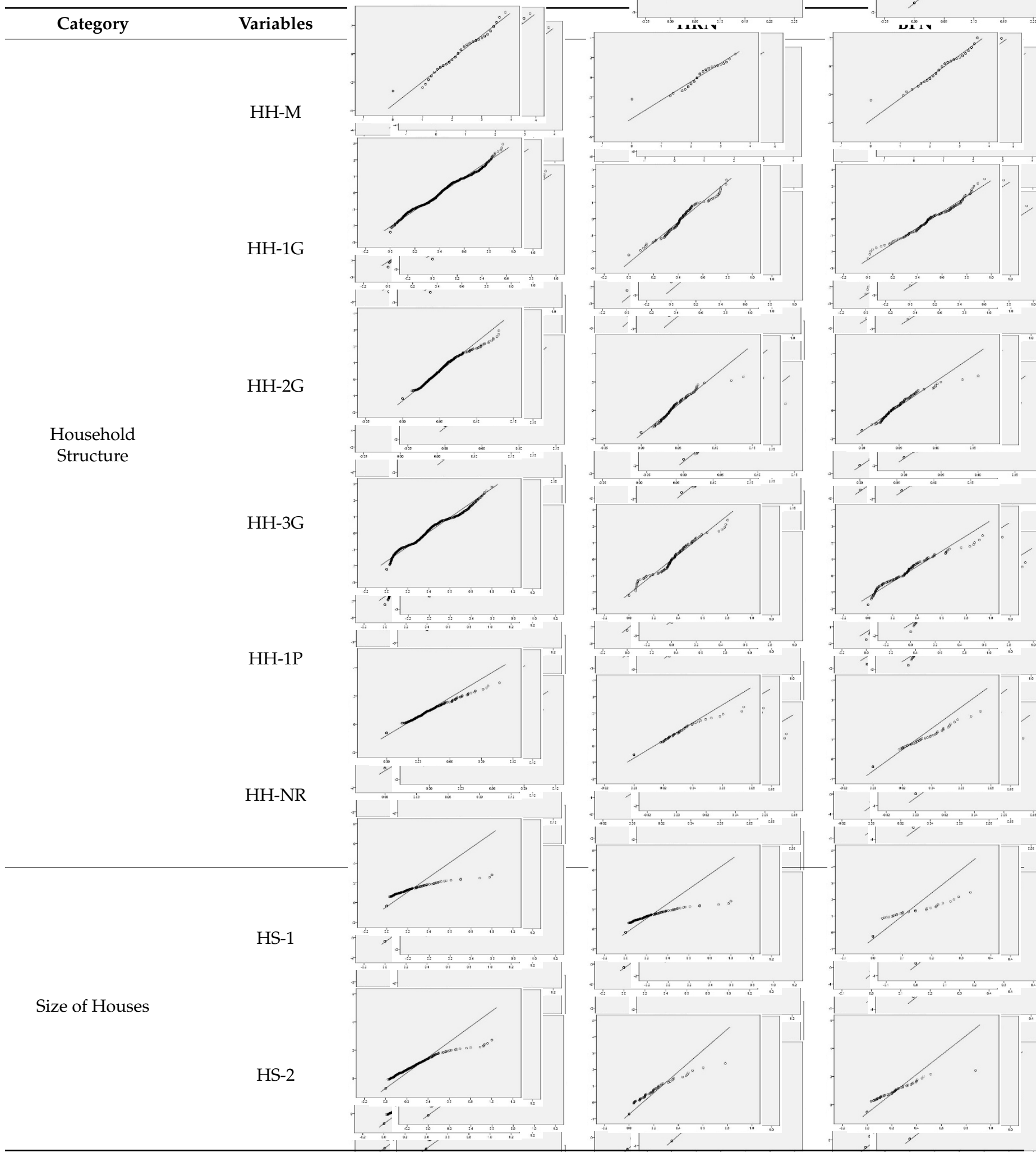


Table A3. Cont.

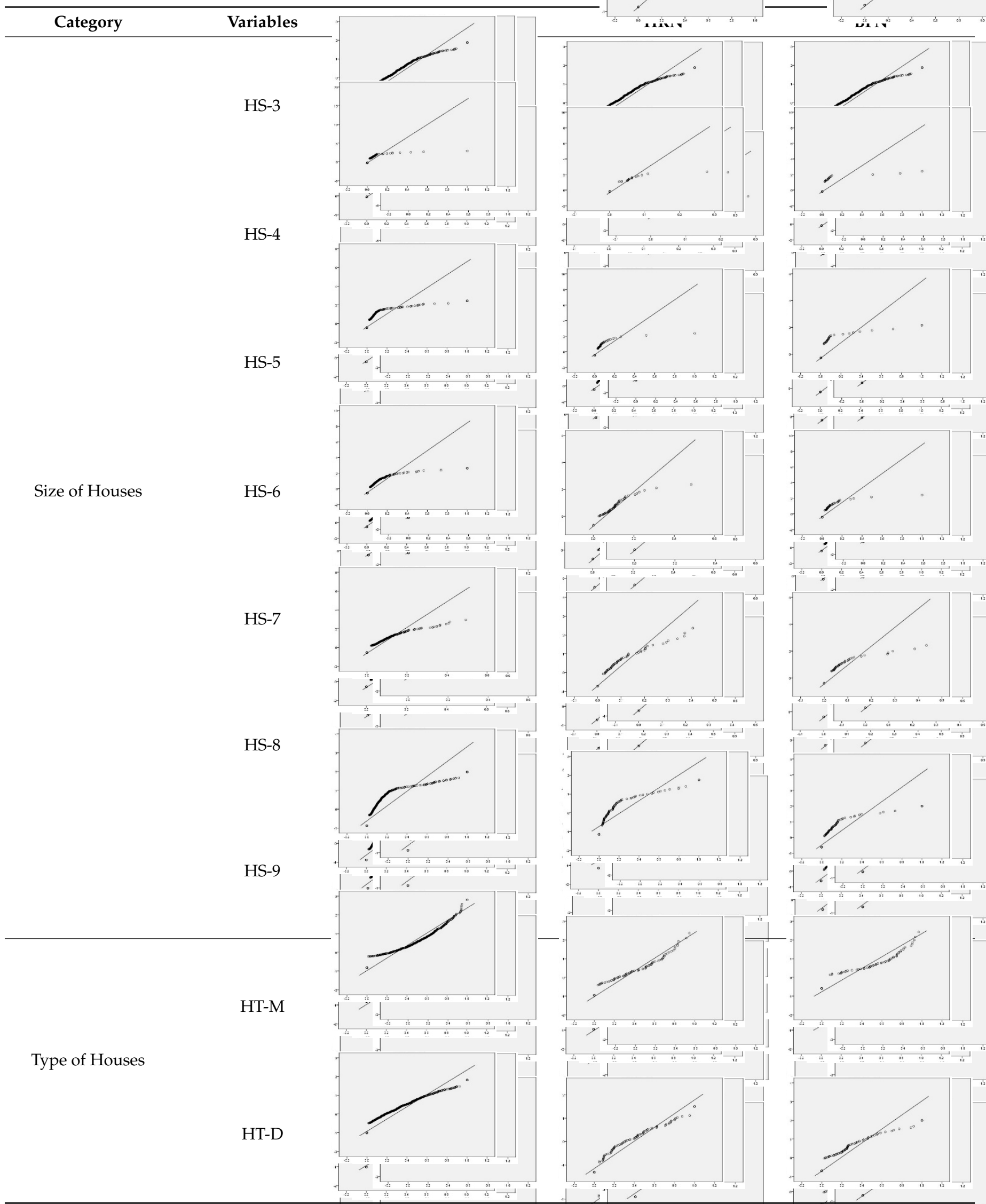


Table A3. Cont.

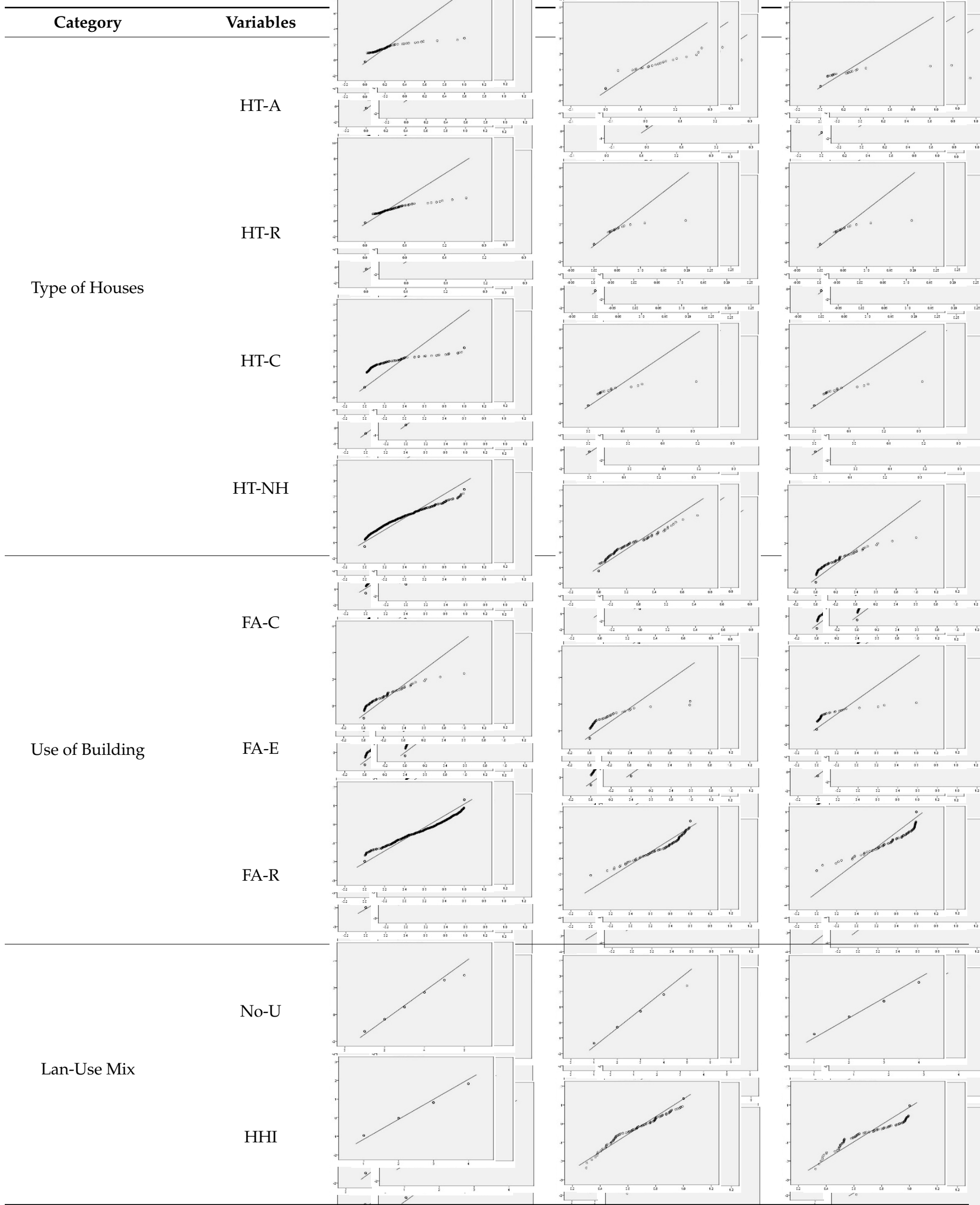


Table A3. Cont.

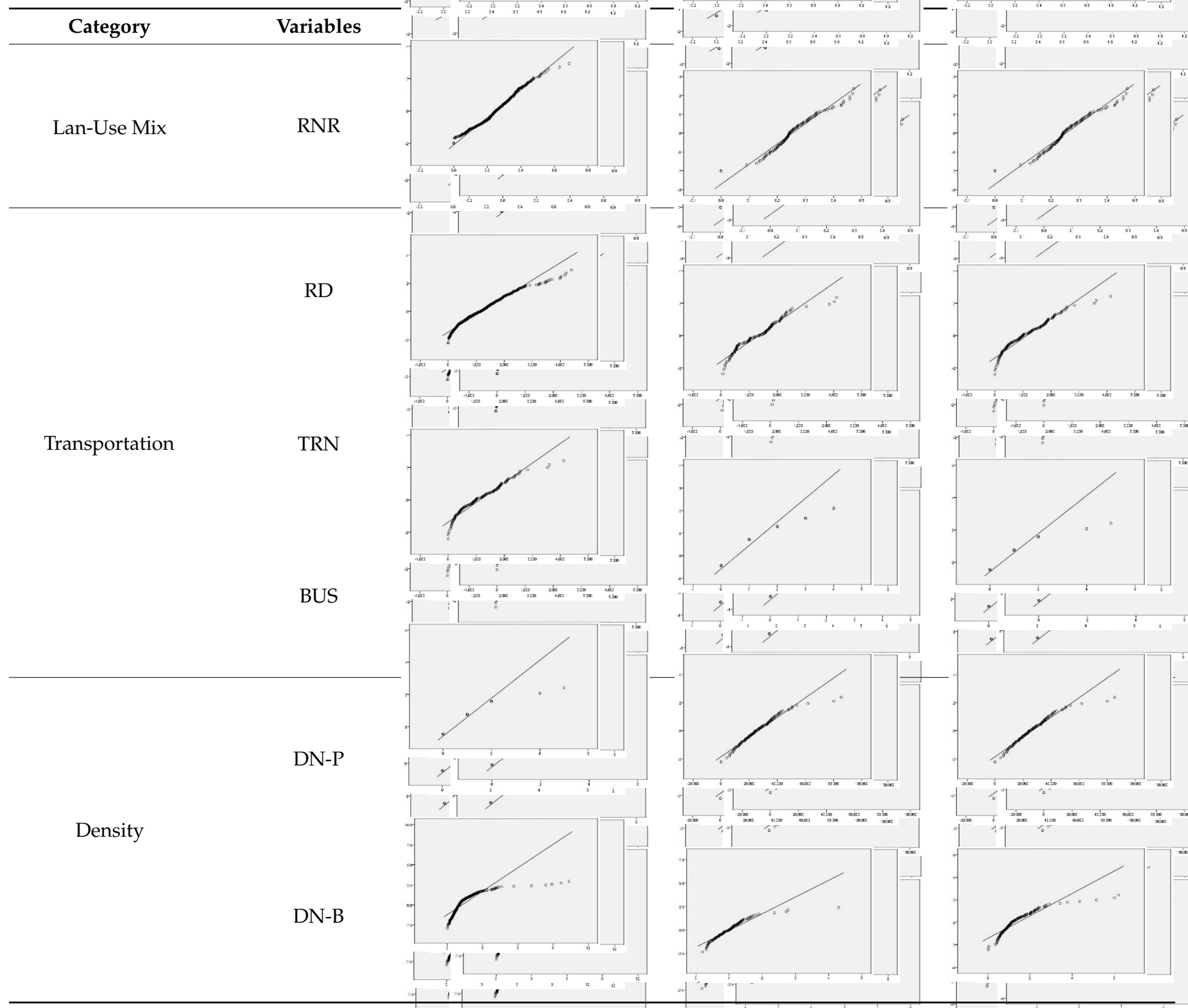

\section{References}

1. World Health Organization Coronavirus Disease (COVID-19) Pandemic. Available online: https://www.who.int/emergencies/ diseases/novel-coronavirus-2019 (accessed on 15 February 2021).

2. OECD. The Territorial Impact of COVID-19: Managing the Crisis across Levels of Government 2020. Available online: https: / / www.oecd.org/coronavirus/policy-responses/the-territorial-impact-of-covid-19-managing-the-crisis-across-levelsof-government-d3e314e1/ (accessed on 26 January 2021).

3. Central Disease Control Headquaters. Central Disaster Management Headquaters Coronavirus Disease 19(COVID-19). Available online: http:/ / ncov.mohw.go.kr/en/ (accessed on 17 February 2021).

4. Matthew, R.A.; McDonald, B. Cities under Siege: Urban planning and the threat of infectious disease. J. Am. Plann. Assoc. 2006, 72, 109-117. [CrossRef]

5. United Nations. Department of Economic and Social Affairs, Population Divisio; United Nations The World's Cities in 2018-Data Booklet 2018. Available online: https:/ / population.un.org/wup/Publications/ (accessed on 15 February 2021).

6. Thomas, M.B. Epidemics on the move: Climate change and infectious disease. PLoS Biol. 2020, 18, e3001013. [CrossRef] [PubMed]

7. Connolly, C.; Ali, S.H.; Keil, R. On the relationships between COVID-19 and extended urbanization. Dialog. Human Geogr. 2020, 10, 213-216. [CrossRef] 
8. Goodell, J. How climate change is ushering in a new pandemic era. Rolling Stone 2020. Available online: https://www.rollingstone. com/culture/culture-features/climate-change-risks-infectious-diseases-covid-19-ebola-dengue-1098923/ (accessed on 17 February 2021).

9. Ceylan, R.F.; Ozkan, B.; Mulazimogullari, E. Historical evidence for economic effects of COVID-19. Eur. J. Health Econ. 2020, 21, 817-823. [CrossRef] [PubMed]

10. Bell, C.; Lewis, M. The economic implications of epidemics old and new. World Econ. 2004, 5, 137-174.

11. Barro, R.J.; Ursua, J.F. Macroeconomic crises since 1870. Brook. Pap. Econ. Act. 2008, 39, 255-350. [CrossRef]

12. Siu, A.; Wong, Y.C.R. Economic impact of SARS: The case of Hong Kong. Asian Econ. Pap. 2004, 3, 62-83. [CrossRef]

13. Yang, H.Y.; Chen, K.H. A General equilibrium analysis of the economic impact of a tourism crisis: A case study of the SARS epidemic in Taiwan. J. Policy Res. Tour. Leisure Events 2009, 1, 37-60. [CrossRef]

14. Lee, J.W.; McKibbin, W.J. Globalization and Disease: The Case of SARS; The Australian National University: Canberra, Australia, 2003.

15. Burns, A.; Mensbrugghe, D.; Timmer, H. Evaluating the Economic Consequences of Avian Influenza; World Bank Group: Washington, DC, USA, 2006.

16. World Health Organization. Middle East Respiratory Syndrome Coronavirus (MERS-CoV). Available online: https://www.who. int/news-room/fact-sheets / detail/middle-east-respiratory-syndrome-coronavirus-(mers-cov) (accessed on 15 February 2021).

17. Jung, Y.H.; Ko, S.J.; Chae, S.M.; Chung, Y.; Bae, J.Y.; Kim, B.E. The Study on Estimating the Cost of Socio-Economic Damage Caused by Emerging Infectious Diseases and the Impact of Social Investment in Response to Emerging Infectious Diseases; Korea Institute for Health and Social Welfare: Sejong City, Korea, 2020.

18. Sharifi, A.; Khavarian-Garmsir, A.R. The COVID-19 pandemic: Impacts on cities and major lessons for urban planning, design, and management. Sci. Total Environ. 2020, 749, 142391. [CrossRef]

19. Douglas, J.; Szeto, K.; Buckle, B. Impacts of a potential influenza pandemic on New Zealand's macroeconomy. N. Z. Treas. Policy Perspect. Pap. 2006, 28, 1-21.

20. Barro, R.J.; Ursua, J.F.; Weng, J. The Coronavirus and the great influenza epidemic_-Lessons from the "Spanish Flu" for the Coronavirus's potential effects on mortality and economic activity. Natl. Bur. Econ. Res. 2020. [CrossRef]

21. Chang, C.C.; Lee, D.H.; Lin, H.C.; Hsu, S.S. The potential economic impact of avian flu pandemic on Taiwan. In Proceedings of the 2007 Annual Meeting, Portland, OR, USA, 29 July-1 August 2007.

22. Dixon, P.; Lee, B.; Muehlenbeck, T.; Rimmer, M.; Rose, A.; Verikios, G. Effects on the U.S. Of an H1N1 Epidemic: Analysis with a Quarterly CGE Model. J. Homel. Secur. Emerg. Manag. 2010, 7, 1-17. [CrossRef]

23. European Commision Impact of COVID-19 on Household Consumption and Savings. Available online: https://ec.europa.eu/ eurostat/web/products-eurostat-news/- /DDN-20201110-2 (accessed on 25 January 2021).

24. Baker, S.R.; Farrokhnia, R.A.; Meyer, S.; Pagel, M.; Yannelis, C. How does household spending respond to an epidemic? Consumption during the 2020 COVID-19 pandemic. Natl. Bur. Econ. Res. Work. Pap. Ser. 2020, 10, 834-862. [CrossRef]

25. Lusk, J. Retail Markets Get a Boost during COVID-19; Purdue University: West Lafayette, IN, USA, 2020; pp. 1-4.

26. Batty, M. The Coronavirus crisis: What will the post-pandemic city look like? Environ. Plann. B Urb. Anal. City Sci. 2020, 47, 547-552. [CrossRef]

27. Lightstone, A. What Will Be the "New Normal" for Global Supply Chains Post Covid-19? WSP: Bucharest, Romania, 2020.

28. Pulighe, G.; Lupia, F. Food first: COVID-19 outbreak and cities lockdown a booster for a wider vision on urban agriculture. Sustainability 2020, 12, 5012. [CrossRef]

29. Napierała, T.; Leśniewska-Napierała, K.; Burski, R. Impact of geographic distribution of COVID-19 cases on hotels' performances: Case of Polish cities. Sustainability 2020, 12, 4697. [CrossRef]

30. Krzysztofik, R.; Kantor-Pietraga, I.; Spórna, T. Spatial and functional dimensions of the COVID-19 epidemic in Poland. Eurasian Geogr. Econ. 2020, 61, 573-586. [CrossRef]

31. Leichenko, R. Climate change and urban resilience. Curr. Opin. Environ. Sustain. 2011, 3, 164-168. [CrossRef]

32. OECD. Resilient Cities. Available online: https://www.oecd.org/regional/resilient-cities.htm (accessed on 15 February 2021).

33. OECD. Self-Employment Rate. Available online: http://data.oecd.org/emp/self-employment-rate.htm (accessed on 27 January 2021).

34. Cheon, G.W. Self-employed with employees decreased by 170,000 in a year. Maeil Bus. News Korea 2020. Available online: https:/ / www.mk.co.kr/news/economy/view/2020/11/1133585/ (accessed on 17 February 2021).

35. D'Adamo, I.; Lupi, G. Sustainability and resilience after COVID-19: A circular premium in the fashion industry. Sustainability 2021, 13, 1861. [CrossRef]

36. Folke, C.; Carpenter, S.; Walker, B.; Scheffer, M.; Chapin, T.; Rockström, J. Resilience thinking: Integrating resilience, adaptability and transformability. Ecol. Soc. 2010, 15, 1-9. [CrossRef]

37. Pimm, S.L. The Balance of Nature?: Ecological Issues in the Conservation of Species and Communities; University of Chicago Press: Chicago, IL, USA, 1991; ISBN 978-0-226-66830-7.

38. Folke, C. Resilience: The emergence of a perspective for social-ecological systems analyses. Glob. Environ. Change 2006, 16, 253-267. [CrossRef]

39. Peterson, G.; Allen, C.R.; Holling, C.S. Ecological Resilience, Biodiversity, and Scale. Ecosystems 1998, 1, 6-18. [CrossRef]

40. Walker, B.; Holling, C.S.; Carpenter, S.; Kinzig, A. Resilience, Adaptability and transformability in social-ecological systems. Ecol. Soc. 2004, 9, 1-9. [CrossRef] 
41. Adger, W.N. Social capital, collective action, and adaptation to climate change. Econ. Geogr. 2003, 79, 387-404. [CrossRef]

42. Kim, J.G.; Im, J.H.; Lee, S.H. A Research on Urban Resilience for Urban Regeneration; Land \& Housing Instituition: Daejeon, Korea, 2016.

43. Yamagata, Y.; Sharifi, A. Resilience-Oriented Urban Planning: Theoretical and Empirical Insights; Springer International Publishing: Cham, Switzerlna, 2018; ISBN 978-3-319-75798-8.

44. Elmqvist, T.; Andersson, E.; Frantzeskaki, N.; McPhearson, T.; Olsson, P.; Gaffney, O.; Takeuchi, K.; Folke, C. Sustainability and resilience for transformation in the urban century. Nat. Sustain. 2019, 2, 267-273. [CrossRef]

45. D'Adamo, I.; Rosa, P. How do you see infrastructure? Green energy to provide economic growth after COVID-19. Sustainability 2020, 12, 4738. [CrossRef]

46. Elmqvist, T. Urban resilience thinking. Solutions 2014, 5, 26-30.

47. United Nations. The New Urban Agenda; United Nations: New York, NY, USA, 2017; ISBN 978-92-1-132731-1.

48. Elmqvist, T. Development: Sustainability and Resilience differ. Nature 2017, 546, 352. [CrossRef] [PubMed]

49. D'Adamo, I.; Falcone, P.M.; Martin, M.; Rosa, P. A sustainable revolution: Let's go sustainable to get our globe cleaner. Sustainability 2020, 12, 4387. [CrossRef]

50. Baffoe, G. Understanding the neighbourhood concept and its evolution: A Review. Environ. Urban. Asia 2019, 10, 393-402. [CrossRef]

51. Logan, J.R.; Molotch, H.L. Urban Fortunes: The Political Economy of Place; University of California Press: Oakland, CA, USA, 1987; ISBN 978-0-520-05577-3.

52. Chaskin, R.J. Perspectives on neighborhood and community: A review of the literature. Soc. Serv. Rev. 1997, 71, 521-547. [CrossRef]

53. Howard, E. To-Morrow: A Peaceful Path to Real Reform, 1st ed.; Cambridge University Press: Cambridge, UK, 2010; ISBN 978-1-108-02192-0.

54. Perry, C.A. The Neighborhood Unit, a Scheme of Arrangement for the Family-Life Community; Arno Press: New York, NY, USA, 1929.

55. Lee, D. A Study on neighborhood change in Seoul: Focused on changes in the officially assessed land value between 2005 and 2015. J. Korea Plann. Assoc. 2018, 53, 199-214. [CrossRef]

56. Castells, M. The Urban Question: A Marxist Approach; MIT Press: Cambridge, MA, USA, 1977; ISBN 978-0-262-03063-2.

57. Hunter, A. The urban neighborhood: Its analytical and social contexts. Urb. Aff. Quart. 1979, 14, 267-288. [CrossRef]

58. Hunter, A. Symbolic Communities: The Persistence and Change of Chicago's Local Communities; University of Chicago Press: Chicago, IL, USA, 1974; ISBN 978-0-226-36080-5.

59. Hopkins, E. Understanding the different types of low-income neighborhoods. Comm. Invest. 2010, 22, $13-1836$.

60. Quarterly National Accounts: Quarterly Growth Rates of Real GDP, Change over Previous Quarter. Available online: https://stats.oecd. org/index.aspx?queryid=350\# (accessed on 17 February 2021).

61. Suwon Government COVID-19 Comprehensive Information. Available online: https:/ /www.suwon.go.kr/web/safesuwon/ corona/PD_index.do (accessed on 27 March 2021).

62. Suwon Government Population of Suwon by Year. Available online: https://stat.suwon.go.kr/stat/stats/statsView.do? categorySeqNo=15 (accessed on 26 January 2021).

63. KOLSA. Status of Member Companies. Available online: http://www.kolsa.or.kr/neopress/index.php?mid=executive (accessed on 20 January 2021).

64. Korean Statistical Information Service. Available online: https:/ / kosis.kr/index/index.do (accessed on 28 March 2021).

65. Gyeonggi Statistics. Available online: https://stat.gg.go.kr/statgg/kr/dataMng/PublicationForm.html?pub_seq=568 (accessed on 27 March 2021).

66. Lee, S.; Choi, S.H. Analysis of the impact of COVID-19 on local market areas using credit card big data: A case of Suwon. Space Environ. 2020, 30, 167-208. [CrossRef]

67. Jun, J. South Korea begins offering coronavirus relief funds. Koreatimes 2020. Available online: http:/ / www.koreatimes.co.kr/ Www / nation/2021/01/119_288952.html (accessed on 27 January 2021).

68. Korea Data Agency. Available online: https:/ / global.kdata.or.kr/en/kdata/ (accessed on 27 March 2021).

69. Kim, K.-K.; Yeom, M. A Study of private expenditures and regional consumption by using credit card. J. Econ. Stud. 2015, 33, 121-142.

70. Bank of Korea, Bureau of Financial Settlements. 2019 Payment Method and Mobile Financial Service Usage Behavior Survey; Payment Survey Data; Bank of Korea. 2020. Available online: https://www.bok.or.kr/portal/bbs/B0000232/view.do?nttId=1005 $6898 \&$ menuNo=200706\&pageIndex $=1$ (accessed on 18 February 2021).

71. Statistics Korea Statistical Geographic Information Service. Available online: https://sgis.kostat.go.kr/view/common/ searchList?searchKeyword=\%EC \%A7\%91\%EA\%B3\%84\%EA\%B5\%AC (accessed on 18 February 2021).

72. Statistical Research Institute. Stat. Res. Instit. 2020, 10, 6-45.

73. Statistical Geographic Information System. Available online: https://sgis.kostat.go.kr/jsp/english/index.jsp (accessed on 18 February 2021).

74. Cervero, R.; Duncan, M. Walking, bicycling, and urban landscapes: Evidence from the San Francisco Bay Area. Am. J. Public Health 2003, 93, 1478-1483. [CrossRef] [PubMed]

75. Zhang, M. The role of land use in travel mode choice: Evidence from Boston and Hong Kong. J. Am. Plan. Assoc. 2004, 70, 344-360. [CrossRef]

76. Forsyth, A.; Hearst, M.; Oakes, J.M.; Schmitz, K.H. Design and destinations: Factors influencing walking and total physical activity. Urb. Stud. 2008, 45, 1973-1996. [CrossRef] 
77. Frank, L.D.; Pivo, G. Impacts of mixed use and density on utilization of three-modes of travel single-occupant vehicle, transit, and walking. Transp. Res. Record 1994, 1466, 44-52.

78. Choi, M.J.; Shin, S.M. An empirical analysis of the effect of pedestrian volume on retail sales. J. Korea Plan. Assoc. 2001, 36, 75-83.

79. Kim, S.H.; Kim, T.H.; Im, H.N.; Choi, C.G. Pedestrian volume and built environmental factors on sales of convenience stores, cosmetic shops and coffee shops in Seoul. J. Korea Plan. Assoc. 2015, 50, 299-318. [CrossRef]

80. Hong, S.; Lee, K.H.; Ahn, K.H. The effect of street environment on pedestrians' purchase in commercial street —Focused on Insa-dong and Munjeong-dong Commercial Street. J. Archit. Inst. Korea 2010, 26, 229-237.

81. Jacobs, J. The Death and Life of Great American Cities; Random House: New York, NY, USA, 1961; ISBN 978-0-394-42159-9.

82. Hirschman, A.O. The paternity of an index. Am. Econ. Rev. 1964, 54, 761.

83. Hirschman, A. National Power and the Structure of Foreign Trade; University of California Press: Berkeley, CA, USA, 1945.

84. Song, Y.; Merlin, L.; Rodriguez, D. Comparing Measures of Urban Land Use Mix. Comput. Environ. Urb. Syst. 2013, 42, 1-13. [CrossRef]

85. Song, Y.; Knaap, G.J. Measuring the effects of mixed land uses on housing values. Reg. Sci. Urb. Econ. 2004, 34, 663-680. [CrossRef]

86. Im, H.N. Developments and Useful Verification of land Use Mix (LUM) Entropy Index on the Effect of Pedestrian Volume. Ph.D. Thesis, Hanyang University, Seoul, Korea, 2015.

87. Jiao, J.; Rollo, J.; Fu, B. The hidden characteristics of land-use mix indices: An Overview and validity analysis based on the land use in Melbourne, Australia. Sustainability 2021, 13, 1898. [CrossRef]

88. National Spatial Information Portal. Available online: http:/ /www.nsdi.go.kr/lxportal/ (accessed on 18 February 2021).

89. Ministry of the Interior and Safety Road Name Address. Available online: https://www.juso.go.kr/openEngPage.do (accessed on 18 February 2021).

90. Gyeonggi Data Dream. Available online: https://data.gg.go.kr/portal/mainPage.do (accessed on 18 February 2021).

91. Bruneau, M.; Chang, S.; Eguchi, R.; Lee, G.; O’Rourke, T.; Reinhorn, A.; Shinozuka, M.; Tierney, K.; Wallace, W.; Winterfeldt, D. A Framework to quantitatively assess and enhance the seismic resilience of communities. Earthq. Spectra 2003, 19, 733-752. [CrossRef]

92. Balal, E.; Valdez, G.; Miramontes, J.; Cheu, R.L. Comparative evaluation of measures for urban highway network resilience due to traffic incidents. Int. J. Transp. Sci. Technol. 2019, 8, 304-317. [CrossRef]

93. Linkov, I.; Bridges, T.; Creutzig, F.; Decker, J.; Fox-Lent, C.; Kröger, W.; Lambert, J.H.; Levermann, A.; Montreuil, B.; Nathwani, J.; et al. Changing the resilience paradigm. Nat. Clim. Change 2014, 4, 407-409. [CrossRef]

94. Roy, K.C.; Cebrian, M.; Hasan, S. Quantifying human mobility resilience to extreme events using geo-located social media data. EPJ Data Sci. 2019, 8, 1-15. [CrossRef]

95. Han, F.; Bogus, S.M. Development of Resilience measures for assessing the performance of water infrastructure project delivery. J. Manag. Eng. 2020, 36, 04020035. [CrossRef]

96. Platt, S.; Brown, D.; Hughes, M. Measuring resilience and recovery. Int. J. Disaster Risk Reduct. 2016, 19, 447-460. [CrossRef]

97. Ravulakollu, A.; Urciuoli, L.; Rukanova, B.; Tan, Y.H.; Hakvoort, R. Risk based framework for assessing resilience in complex multi-actor supply chain domain. Supply Chain Forum 2018, 19, 266-281. [CrossRef]

98. Ministry of Science and ICT; National Information Society Agency. 2019 Survey on the Internet Use Statistical Table. Available online: https:/ / www.nia.or.kr/site/nia_kor/ex/bbs/View.do;jsessionid=8F7205BFD25A5E056A16750D171F9E90.cd4a8b7bbe0 a06361156?cbIdx=99870\&bcIdx=22082\&parentSeq=22082 (accessed on 24 March 2021).

99. Borowiec, S. South Korean Food Delivery Gets a Government-Backed Challenger. Nikkei Asia. 2020. Available online: https: / asia.nikkei. com/Business/Startups/South-Korean-food-delivery-gets-a-government-backed-challenger (accessed on 14 April 2021).

100. UNDP. Seoul Policy Centre for Knowledge Exchange through SDG Partnership Korean New Deal for the Post-COVID-19 Era. Available online: https://www.undp.org/content/seoul_policy_center/en/home/presscenter/articles/2019/Collection_of_ Examples_from_the_Republic_of_Korea/korean-new-deal-for-the-post-covid-19-era.html (accessed on 14 April 2021).

101. Ministry of Economy and Finance Government Releases an English Booklet on the Korean New Deal. Available online: https: / / english.moef.go.kr/pc/selectTbPressCenterDtl.do?boardCd=N0001\&seq=4948 (accessed on 14 April 2021). 



\title{
Does Corporate Financialization Have a Non-Linear Impact on Sustainable Total Factor Productivity? Perspectives of Cash Holdings and Technical Innovation
}

\author{
Hui Wang ${ }^{1, *}$, Qing Wang ${ }^{2}$ and Xia Sheng ${ }^{2}$ \\ 1 School of Business Administration, Southwestern University of Finance and Economics, \\ Chengdu 610031, China \\ 2 Institute of Chinese Financial Studies, Southwestern University of Finance and Economics, \\ Chengdu 610031, China; wqing@swufe.edu.cn (Q.W.); 1_leigh@126.com (X.S.) \\ * Correspondence: daisy8611@gmail.com
}

Citation: Wang, H.; Wang, Q.; Sheng, $X$. Does Corporate Financialization Have a Non-Linear Impact on Sustainable Total Factor Productivity? Perspectives of Cash Holdings and Technical Innovation. Sustainability 2021, 13, 2533. https://doi.org/ $10.3390 /$ su13052533

Academic Editor: Idiano D'Adamo

Received: 23 January 2021

Accepted: 22 February 2021

Published: 26 February 2021

Publisher's Note: MDPI stays neutral with regard to jurisdictional claims in published maps and institutional affiliations.

Copyright: (c) 2021 by the authors. Licensee MDPI, Basel, Switzerland. This article is an open access article distributed under the terms and conditions of the Creative Commons Attribution (CC BY) license (https:// creativecommons.org/licenses/by/ $4.0 /)$.

\begin{abstract}
This study explores the conditions under which financialization may foster sustainable total factor productivity (TFP). We examine the inverted U-shaped relationship between corporate financialization and TFP by employing a panel threshold model using microeconomic non-financial panel data from Chinese firms in the 2007 to 2018 period. Our results suggest that the turning point is more significant in holding short-term financial assets and state-owned enterprises. The threshold effect suggests that technical innovation determines the optimal threshold at which TFP is affected by financialization. Further, financialization is considered an alternative to cash in order to increase the value of capital, leading to a positive effect on TFP. Contrary to their positive effects below the optimal thresholds, financialization exceeds a certain level, displaces technical innovation, and becomes detrimental to TFP. Our analysis thus establishes the importance of sustainable growth of TFP and minimize the adverse effect of financialization.
\end{abstract}

Keywords: circular economy; sustainability strategy; resilience; financialization; TFP; innovation

\section{Introduction}

Following the widespread outbreak of Covid-19, China's the real economy is headed for downturn due to the low production efficiency. As the entity economy gradually shifts more from industrial sector to financial sector, productivity growth has been slowed down and there have been concerns about economic resilience. The need for strengthen the resilience related to the creation of sustainable growth is clearly identified by D'Adamo and Rosa [1]. One aspect of economic resilience that is often underappreciated concerns the sustainable productivity growth and the imbalance of reallocate resources. Notably, financial assets are a vital component of the capital composition, which have a significant impact on the aggregate productivity growth. As the pandemic spreads, given rapidly changing economic, competitive, and consumer trends. These trends presents the adoption of circular economy principles associated with sustainability have become relatively more significant [2]. A circular strategy gradually becomes a prime concern of corporate executives and policymakers [3]. Financialization is considered a key feature for capital extension and to reach higher production efficiency yields. Nevertheless, Over-financialization in economy may lead to a downward trend of the entity sector, yet that cannot be confirmed at this time. This paper aims to test this hypothesis by analyzing the potential nonlinear relationship between corporate financialization and TFP growth. Our analysis establishes the importance of sustained growth of TFP and minimize the adverse effect of financialization. More importantly, technologies, policies, and financial activities must consider the sustainability aspect [2].

China's economy growth has declined steadily in recent years. In fact, the decline in TFP explains most of the fall in economy growth since the global financial crisis exploded 
in 2008. In response, Chinese government has pushed expansionary fiscal and monetary policy to stimulate domestic economy growth while, stimulus policies have laid the key foundation for the increased development of financial market. The proportion of financial assets within the Chinese economy has skyrocketed, and the preference for corporate governance to hold financial assets continues to spread. A large share of financial investments is concentrated in firm portfolios, which grew from $\$ 39.3$ trillion to $\$ 141.2$ trillion from 2007 to 2018.

Growth in the financial development of non-financial corporations has received considerable attention in recent years. Several researchers and institutions have defined financialization as the proportion of financial assets held by non-financial corporations. It mainly implies that the financial assets contained in firm portfolios become a primary component of the capital expenditures highlighted in the analyses of the financialization trend [4]. On the other hand, since the 2008 United States subprime mortgage crisis, China's demographic dividend gradually disappeared, and the direct contribution of labor to gross domestic product (GDP) began to decline. The resource reallocation that China gained through the intersectoral transfer of labor and stable capital-return that resulted from the unlimited supply of labor will gradually disappear. For a long time, the catalyst of China's rapid GDP growth depended on the growth of total factor productivity (TFP), which means additional economic output is produced from a given amount of inputs. The driving forces of TFP have changed as capital plays a central role in the sustained economic growth of TFP. China's economy has promoted its shift in focus from high-speed growth to high-quality development in the midst of economic financialization, which means it has transitioned from factor-driven and investment-scale driven development to innovationdriven development. Meanwhile, the mutual integration of production efficiency, resource allocation efficiency, and technological innovation is particularly important. Therefore, the consequences of the corporate financialization for TFP are extensive.

There is an ambiguous relationship between financial development and TFP. The current studies on corporate financialization and TFP have not been agreed upon. Corporate financialization in terms of investment behavior is demonstrated by non-financial firms' frequent participation in financial markets and financial transactions [5]. It is also demonstrated by an increase in the contribution of financial gains to total corporate profits [6]. Studies have shown that firms hold financial assets as an expedient way to hedge liquidity risk [7-9] and reduce the risk of liquidity crunch by obtaining returns on financial investments [10], which can safeguard production and operations and contribute to high-quality development.

Based on summarizing existing research conclusions, we explore the impact of TFP on increased financial investments by non-financial firms and detect the dual mechanism through the following channels: (1) For cash holdings, in general, the level of cash holdings is considered an important basis for the allocation of production inputs and capital. Financial assets with low conversion costs not only manage liquidity shocks, but their excess returns can optimize profits and smooth the capital requirements of firms [11], which can effectively drive TFP growth from the accumulation of capital. (2) For technical innovation, research and development $(R \& D)$ investments play a key role in promoting the innovation initiative. The technology spillovers through openness are beneficial for TFP growth.

Unlike other works, standard regression models assuming a linear relationship between the two variables may have led to biased and misleading results. Our main assumption is that the distinct economic effects of financialization depend on the motives of holding financial assets and the degree of financialization. The objective of this paper is to examine whether the relationship between financialization and TFP is non-linear using samples of Chinese listed nonfinancial companies during the period from 2007 to 2018. By applying the threshold model introduced by Hansen [12], our empirical results confirm that the nexus between financialization and TFP is indeed non-linear. It shows that there exists a single significant threshold value of 0.13 above which the negative impact of financialization being found on TFP. The threshold model also allows us to measure the 
respective roles of cash holding and innovation. We therefore find a statistically significant threshold effect from the view of innovation, and cash holding only partly supported in the relationship between financialization and firms' TFP. Our empirical results indicate robust results that financialization does not always lead to TFP growth. Accordingly, corporate governance should undertake a substantial investment plan to generate sustained growth of TFP and minimize the adverse effect of financialization. The findings obtained from this research may offer meaningful policy implications and additional knowledge to this growing literature on financialization.

This study explores the conditions under which financialization may result in improved TFP, which is seldom discussed in previous literature. There are three major contributions. First, this study has the advantage of detecting potential nonlinearity in the relationship between financialization and TFP. In this sense, our paper contributes to the understanding of the impact of corporate financialization. Moreover, this study uses the panel threshold methodology to verify the dual effects of the financialization, where the threshold effect of financialization on TFP would differ above and below this level. To our knowledge, there are no published empirical studies that reveal the underlying mechanism by applying the threshold effects of cash holdings and the innovation initiative. The empirical analysis does emphasize the importance of innovation in determining the relationship between financialization and TFP. According to this finding, the nonfinancial corporates should review their allocation of production factors from the perspective of productivity, and pay keen attention to enhance their resilience in the face of shifting economic conditions. Furthermore, there is not enough study exploring the heterogeneous features of financialization. Our paper examined the various consequence of financialization based on the structures of financial assets and corporate ownership. Lastly, we reference the new capital management regulations issued in 2017 to address excessive investments in financial products as a quasi-natural experiment leading to a more extensive study.

The remainder of the paper is organized as follows. Section 2 presents a review of the relevant literature, emphasizing nonlinearities in the corporate financialization nexus. Section 3 is research design, which describes the samples, variables, and models. Section 4 presents empirical findings and detailed discussion, including baseline regression analysis, heterogeneity analysis, threshold regression analysis, and robustness test. Whereas the conclusion and summary of the findings are discussed in the final section.

\section{Literature Review and Research Hypothesis}

\subsection{The Impact of Financialization on Economy}

There is extensive theoretical literature on the impact of financialization on the economy from macroeconomic perspectives. Most economists generally view excessive financialization as a significant obstacle for economic development [4,13-15]. Several previous studies [16-18] empirically establish that excessive financialization has a negative effect on capital accumulation when resource and production input factors are established. The findings of Singh [19], Krugman and Anthony [20] and Orhangazi [4] indicate that financialization hinders economic growth by extracting additional profits from the economy into the financial sector; likewise, corporate expenditures are allocated from production activities to financial investments. Sweezy [21] argues that the dramatic expansion of the financial sector, high degree of independence within the financial sector, and gradual dominance of the real production system pose potential financial risks to the economy. The findings of Lazonick [22] demonstrate that the overspending of manufacturing firms leads to decreased investments in production and increased unemployment rates. The empirical result implies that over-financialization has a negative effect on unemployment. China's increasing capital flows into the stock market and real estate industries represent expanding financialization for the economy. Zhang et al. [23] examines the negative impact of excessive spending on the enterprise's physical investment ratio and indicates that these aspects play a significant role in weakening monetary policy. Wang et al. [24] also confirms that excessive corporate financialization exacerbates asset bubbles. In particular, Law and 
Singh [25] explore the possible asymmetric relationship between the extension of financial resources and growth, which indicates that the expansion of the financial system benefits growth to a certain extent. Sahay et al. [26] presents a similar argument by indicating that excessive financial resources increases economic risk and financial volatility.

\subsection{The Impact of Corporate Financialization}

Previous papers have emphasized that corporate investment behavior is associated with financialization. It is important to summarize the main findings of some influential studies. The increase in financial returns is related to a decrease in industrial returns, and corporate financialization makes non-financial firms hold less capital $[27,28]$. This indicates that high profits through financial channels are strongly associated with lower investments and capital accumulation. Significantly high financial assets are speculative and opportunities for operational growth are ignored. Krippner [29] studied the effects of financialization in the United States from 1950-2000 and found a negative correlation between manufacturing and the financial composition of corporate profits. Based on the historic performance of the Chinese economy, a recent study by Zhang and Zhang [30] found that pursuing high profits is one of the main reasons for corporate financialization at the expense of production inputs. High profits have ended investments in the economy. Investments resulting from increased profits might decreased operational spending in the manufacturing industry, which requires long term investment cycles and poses uncertainties in technology and risks. To maintain a stable profit, corporate managers seek opportunities to adjust their balance sheets and increase revenue from capital investments. The majority of the manufacturing industry has transitioned from traditional production activities to financial channels $[6,31]$. Since this transition, corporate financialization is defined as the increase in profits from unproductive business activities. In this case, corporate managers seek capital appreciation rather than operating profits. A significant portion of revenue is composed of profits [32], which is an example of corporate financialization.

\subsection{Nonlinear Effects of Financialization on Entrepreneurship}

There are two types of literature that cover the financial assets of firms and capital accumulation. Two main views exist, leading to ambiguous conclusions. One group of researchers believes that moderate financialization results in high capital gains and secures TFP. The second group of researchers believe that excessive financialization hinders the growth of TFP by inhibiting technological innovation and capital accumulation. However, both of these arguments are true to some extent. Thus, the extent to which TFP is affected depends on why financial assets are held. If motivated by speculation, firms will hold more financial assets considering the balance between risk and return.

When there has been a change in corporate management or corporate management is unable to meet the company's financial goals, they allocate financial assets $[10,33]$. This allocation of financial assets enhances capital liquidity, improves financing capacity, increases the return on assets [7,9] increases short-term shareholder value, and integrates production and finance [34], which contributes to the growth of TFP. Non-financial firms can capture higher returns during market booms by investing in diversified financial instruments, which provides a cushion and reduces risk during market downturns [8]. Arcand et al. [35] argue that the essential function of finance is to serve the entire economy. Only when financial development exceeds reasonable limits, does it shift from promoting economic growth to inhibiting economic growth. Again, as the return on investment of financial assets is higher than that of physical investments, enterprises will rely on financial investment income rather than working to improve operational efficiency. Liquidity plays a crucial role in investment decisions [36]. Financialization offers firms the flexible option of investing in reversible short-term financial assets instead of irreversible long-term physical assets; therefore, financial assets displace productivity accumulation as preferred by shareholders [37] mainly as a result of change in corporate management $[22,38]$. Therefore, this management leads to changes in decision-making related to capital structure and production alloca- 
tion. This evidence indicates that a reasonable level of financialization does not hinder production, but it may effectively promote technology upgrades and improvements of research and development (R\&D) [34]. Zhang and Luo [39] also argue that financialization of private firms contributes to the improvement of productivity improvement through actions such as reducing financing costs and easing financing constraints.

Bonfiglioli [7] identified that financialization could broaden finance options to allocate capital more efficiently. Adequate capital would give businesses and investors more choice and improve resource allocation; besides, financial constraints can limit the inputs in R\&D, which is a significant determinant of TFP [40]. More specifically, financial support is provided for firms' technological progress, upgrade of human capital, and productivity improvements. Overall, moderate financialization has a profound impact on enhancing the ability to create value, but excessive financialization is likely to lead to the misallocation of productivity factors, which ultimately affects the productive efficiency.

The growth theory suggests that the original driver of economic growth is productivity [41]. Economists generally agree that technology leads to productivity improvement; in other words, the increase in the growth of TFP is driven by technological innovation [42]. More recently, Seo et al. [13] examined nonfinancial Korean corporations from 1994 to 2009 and found that increased financial investment and profit opportunities displaced R\&D investment. Likewise, $\mathrm{Xu}$ and Liu [43] empirically examine the impact of financial asset allocations on R\&D activities in China from 2007 to 2015, and the results show evidence of a strong negative correlation between financial asset allocations and firms' innovations. From the empirical point of view, financialization may affect firms' productivity through technological improvement. This paradigm is based on the realization that technological innovation is a long-term capital input that contributes to growth through the reallocation of productive resources. Economic outcomes are difficult to determine because corporate investment decisions are complex due to the trade-off between short-term profits that are not guaranteed and sustainability strategies. Orhangazi [4] explains that a higher return from financial activities should drive a change in the priorities of strategies. A reasonable level of financialization plays an active role in generating new profit sources, thus improving liquidity. However, excessive financialization that replaces long-term R\&D investments with short-term profits that are not guaranteed may displace resources for economic development.

Obviously, the results derived from the above researches are not conclusive in matters of the exact relationship of financialization and TFP. Most of the empirical studies are based on ordinary least squares, which ignores the existence of asymmetries. Hence, based on the existing literature, we have assumed non-linearity in the relationship between financialization and TFP, to be empirically verified at a later stage of the study.

Following the arguments above, this study posits that holding excessive amounts of cash destroys the firm's value through maintenance costs. Therefore, in a comparable level of financialization, the effects of capital accumulation positively impact TFP. However, when the level of financialization exceeds a certain point, displacement, low resource allocation, and efficiency offset the positive effects of capital accumulation. This paper hypothesizes that the positive effects of financialization on innovation declines when a certain threshold is exceeded. Based on the above discussion, the paper hypothesizes the following:

Hypothesis (H1). The relationship between financialization and TFP is asymmetric.

The level of cash holding is not significant determinants of TFP in this sample because of coexistence of what I call "positive" and "crowding out channels" of effect running from technical innovation to factors that affect TFP in the growth prospects. Evidence of the existence of both types of channel will be presented.

Hypothesis (H2). The threshold effect between financialization and TFP depends on a certain level of cash holdings and innovation. 
As an alternative method, we propose a quadratic explanatory variable to examine the dual effect. To further analyze the channels through which financialization affects TFP, this paper applies the Hansen [12] fixed effect panel threshold model convey to explain non-line relationships.

\section{Empirical Methodology and Research Design}

\subsection{Basic Model Specification and Threshold Model Construction}

As aforementioned, the impact of financialization on TFP is not necessarily a simple linear relationship. To allow for nonlinearity in the relationship, we also include the quadratic term of the financialization $\left(\mathrm{Fin}_{i, t}{ }^{2}\right)$ in the model to examine test the research hypothesis proposed above:

$$
\operatorname{TFP}_{i, t}=\alpha_{0}+\alpha_{1} \text { Fin }_{i, t}+\alpha_{2} \text { Fin }_{i, t}^{2}+\alpha_{3} \sum \text { Control }_{i, t}+\mu_{i}+\sigma_{t}+\varepsilon_{i, t}
$$

where $T F P_{i, t}$ stands for the nonfinancial firms total factor productivity variable, for the measurement, it is important to notice that each of different estimates TFP measures may be affected by important statistical issues and limitations. This paper adopted the approach of Olley and Pakes [44]. Fin ${ }_{i, t}$ indicates the level of financialization; The variable Control $_{i, t}$ is a vector of control variables, we include several factors that potentially affect the level of TFP. The $i$ and $t$ indicate cross-section (nonfinancial firms) and time period (2007-2018), respectively.

To verify underlying mechanism, we apply the threshold regression model introduced by Hansen [12], which is widely used in economics. This threshold model allows to split the effects of a key independent variable on dependent variable into regimes based on the value of a threshold, which can be expressed as follows:

$T F P_{i, t}=\gamma_{0}+\gamma_{1} C I_{i, t} \mathrm{I}\left(\right.$ Fin $\left._{i, t} \leq \lambda\right)+\gamma_{2} C I_{i, t} \mathrm{I}\left(\right.$ Fin $\left._{i, t}>\lambda\right)+\gamma 3 \sum$ Control $_{i, t}+\mu_{i}+\sigma_{t}+\varepsilon_{i, t}$

where $\mathrm{I}(\cdot)$ is the indicator function representing the sample splitting. The above regression model describes the sample split by only one threshold level. Whereas the parameter $\lambda$ is the threshold value, which assumed unknown and needs to be estimated. Here we select the level of financialization as the threshold variable.

The panel threshold model is well suited for testing the possible nonlinearity between financialization and TFP for two reasons. Firstly, as illustrated in Equation (2), since the sample is endogenously split according to a threshold value, the sign and the magnitude of the key variable are separately determined by two different subsamples. This procedure thus permits a flexible way in modeling potential nonlinear relationship between two variables. Secondly, the threshold parameter is estimated simultaneously along with other parameters, this means the estimated nonlinear pattern is discovered by optimally fitting the underlying data features, which minimizes specification concerns.

This paper prefers to explore the response of cash holdings and R\&D investment channels to both appreciation and depreciation in the TFP, according to which we could then test the hypothesis to infer whether firm's financialization behavior is motivated by precautionary cash holdings or the crowding out of R\&D investment mechanism.

To identify the attributes of virtual and non-virtual Chinese real estate during periods of sustained housing price increases, we reveal investment restrictions for 16 cities in 2017. Comprehensive firm-level data and the restructure rule are used to determine the endogenous issue.

\subsection{Data and Empirical Strategy}

The financial data refer to China listed nonfinancial companies taken from CSMAR database, which contains standardized accounting information about not only investment, sales, profits, interest and dividend payments but also types of financial assets. The initial number of firms includes 3654 firms for the period 2007-2018. As for control of the financial development, we use data from CCER database. We exclude the sample of companies with missing main variables, Special treatment (ST)/*ST firms, and select firms that have at 
least three consecutive observations for the dependent variable, which is also required for econometric purposes we drop all the companies with a permanent negative total assets, an asset-liability ratio greater than one and negative owner's equity. Exclude Data anomalies and missing from such companies may affect the reliability of the results of this study. Finally, we exclude observations in the upper and lower $1 \%$ of each variable's distribution.

Interpreted variable: total factor productivity (TFP). This paper measures the TFP uses the semi-parametric approach which is initiated from Olley and Pakes [44]. Specifically, following by Xiao and Xue [45], the firm's current investment is proxied by the net cash for acquisition, construction of fixed assets, intangible assets, and other long-term assets.

Explanatory variable: Financialization. The absolute levels liquid financial holdings remain vast. Financialization is characterized by the expansion of financial assets relative to entity activity of nonfinancial firms [46]. This paper uses the ratio of financial asset to the total assets, reflecting the level of financialization. Following main categories of financial assets are identified: (1) trading financial assets, (2) available-for-sale financial assets, (3) held-to-maturity investments, (4) investment properties, (5) derivative financial instruments, (6) long-term equity investments. These assets are probably highly liquid and easy convertible, while the cash held by the company is excluded as the motives typically for operational reserve rather than speculative investment purposes.

Control variables: This paper selects the following variables to control the firm-level and macro-level factors that may affect the TFP, including firm size (Size), profitability (Roa), growth (Growth), and financial leverage (Lev); macro-level factors include financial deepening (M2/GDP). In Table 1, the data descriptions are given.

Table 1. Data description.

\begin{tabular}{|c|c|}
\hline Variable & Definition \\
\hline TFP & OP method \\
\hline Fin & $\begin{array}{c}\text { (Trading financial assets }+ \text { net held-to-maturity investments }+ \text { bought-back } \\
\text { financial assets }+ \text { available-for-sale financial assets }+ \text { derivative financial } \\
\text { assets }+ \text { investment properties)/Total assets }\end{array}$ \\
\hline Size & Logarithm of total assets \\
\hline Cashflow & Logarithm of net cash flows from operating activities \\
\hline Roe & Net profit/total assets \\
\hline Age & Current year -year of establishment of each company \\
\hline Growth & Annual growth rate of operating income \\
\hline Tng & Fixed assets/total assets \\
\hline Ltv & Total liabilities/total assets \\
\hline Top & Number of shares held by the largest shareholder/total share capital \\
\hline Capital & $\begin{array}{l}\text { Net expenditure on acquisition and disposal of fixed assets, intangible } \\
\text { assets and other long-term assets/total assets }\end{array}$ \\
\hline Cir & Total assets/operating income \\
\hline $\mathrm{RD}$ & R\&D investment/total assets \\
\hline $\mathrm{M} 2 / \mathrm{Gdp}$ & M2/GDP \\
\hline
\end{tabular}

\section{Results and Discussion}

Table 2 shows descriptive and normality statistics (mean, standard deviation, minimum, median, and maximum) of all variables of the study. The maximum value of Fin for nonfinancial enterprises in China is 43.01 , the minimum value is $2.71 \times 10^{-10}$, and the standard deviation is 0.962 , indicating that there are obvious differences in the level of financialization. 
Table 2. Descriptive statistics.

\begin{tabular}{ccccccc}
\hline Variable & Obs & Mean & Std. Dev. & Min & Median & Max \\
\hline TFP & 10243 & 19.210 & 1.363 & 10.909 & 19.203 & 24.490 \\
Fin & 10243 & 0.138 & 0.962 & $2.71 \times 10^{-10}$ & 0.0284 & 43.010 \\
Capital & 10243 & 0.0580 & 0.134 & -0.253 & 0.029 & 3.257 \\
Cashflow & 10243 & 18.270 & 1.929 & 7.409 & 18.415 & 25.396 \\
Size & 10243 & 21.090 & 1.239 & 13.680 & 21.770 & 28.060 \\
Ltv & 10243 & 0.397 & 0.205 & 0.007 & 0.385 & 0.984 \\
Roe & 10243 & 0.072 & 0.158 & -6.797 & 0.072 & 1.615 \\
Age & 10243 & 15.59 & 6.223 & 1 & 15 & 69 \\
Tng & 10243 & 1.426 & 1.972 & 0.007 & 0.798 & 19.96 \\
Top & 10243 & 33.656 & 14.722 & 3.00 & 31.050 & 89.99 \\
Tobinq & 10243 & 2.210 & 1.908 & 0.152 & 1.732 & 58.59 \\
Growth & 10243 & 0.171 & 0.376 & -0.988 & 0.121 & 4.792 \\
Cir & 10243 & 9.829 & 29.665 & 0.017 & 2.261 & 392.90 \\
Rd & 10243 & 0.055 & 0.361 & 0 & 0.014 & 11.11 \\
M2/Gdp & 10243 & 171.50 & 18.845 & 130.890 & 175.200 & 193.02 \\
\hline
\end{tabular}

As shown in Table 3, the absolute value of the correlation coefficient of the main variables is less than 0.5 , and the variance inflation factor VIF is less than 10 , indicating that there is basically no multicollinearity among variables, and the selection of each variable is reasonable.

Table 3. Correlation matrix of variables.

\begin{tabular}{|c|c|c|c|c|c|c|c|c|c|c|c|c|c|}
\hline & Fin & Capital & Cashflov & v Size & Ltv & Roe & Age & Tng & Top & Tobinq & Growth & Cir & Rd \\
\hline Fin & 1 & & & & & & & & & & & & \\
\hline Capital & -0.004 & 1 & & & & & & & & & & & \\
\hline Cashflow & -0.021 & -0.058 & 1 & & & & & & & & & & \\
\hline Size & -0.058 & -0.212 & 0.300 & 1 & & & & & & & & & \\
\hline Ltv & 0.011 & -0.059 & 0.198 & 0.088 & 1 & & & & & & & & \\
\hline Roe & 0.002 & 0.022 & 0.027 & -0.063 & -0.048 & 1 & & & & & & & \\
\hline Age & -0.014 & -0.102 & 0.272 & 0.308 & 0.125 & -0.070 & 1 & & & & & & \\
\hline Tng & 0.001 & -0.015 & 0.029 & 0.029 & 0.168 & -0.025 & 0.032 & 1 & & & & & \\
\hline Top & 0.038 & 0.014 & 0.005 & 0.064 & 0.044 & 0.017 & -0.029 & 0.010 & 1 & & & & \\
\hline Tobinq & -0.001 & -0.010 & -0.001 & -0.049 & 0.001 & 0.003 & 0.011 & -0.002 & -0.039 & 1 & & & \\
\hline Growth & 0.007 & 0.020 & -0.006 & -0.076 & 0.010 & 0.146 & -0.061 & -0.007 & -0.020 & 0.020 & 1 & & \\
\hline Cir & -0.005 & -0.086 & -0.051 & 0.319 & -0.021 & -0.021 & 0.017 & 0.001 & 0.034 & -0.017 & -0.029 & 1 & \\
\hline $\mathrm{Rd}$ & 0.000 & 0.010 & -0.041 & -0.094 & 0.011 & 0.008 & -0.028 & 0.001 & 0.003 & 0.005 & 0.007 & -0.009 & 1 \\
\hline
\end{tabular}

\subsection{Basic Regression Results}

Table 4 presents the results of specification, Column (1) focusing on only explanatory variables, as expected, the correlation between Fin and Fin2 are opposite statistically significant at the $1 \%$ statistical level. Column (2) shows that this dual relationship holds true after controlling for variables found to be important to TFP, such as firm characteristics, as well as controlling for industry characteristics. The results indicating that Fin has a positive impact, while Fin2 has a negative impact on TFP, which illustrates that an inverted U-shaped relationship between financialization and TFP. Columns (3) to (4), we include industry, year and city fixed effects respectively to control both heterogeneity in observable and unobservable characteristics and again find similar results. Column (4) illustrates the effects of the control variables, the nonlinear effects of Fin on TFP have a significance of 0.101 at the $1 \%$ statistical level, and those of Fin 2 on TFP have a significance of -0.002 at the $5 \%$ statistical level. 
Table 4. The U-shaped relationship between financialization and TFP.

\begin{tabular}{|c|c|c|c|c|}
\hline TFP & (1) & (2) & (3) & (4) \\
\hline Fin & $\begin{array}{c}0.096^{* * *} \\
(0.029)\end{array}$ & $\begin{array}{c}0.101 \text { *** } \\
(0.031)\end{array}$ & $\begin{array}{c}0.099 * * * \\
(0.029)\end{array}$ & $\begin{array}{c}0.101^{* * *} \\
(0.025)\end{array}$ \\
\hline Fin2 & $\begin{array}{c}-0.002 * * * \\
(0.001)\end{array}$ & $\begin{array}{c}-0.002 \text { ** } \\
(0.001)\end{array}$ & $\begin{array}{c}-0.002 \text { ** } \\
(0.001)\end{array}$ & $\begin{array}{c}-0.002 \text { ** } \\
(0.001)\end{array}$ \\
\hline Capital & & $\begin{array}{c}0.142 \\
(0.143)\end{array}$ & $\begin{array}{c}0.09 \\
(0.138)\end{array}$ & $\begin{array}{c}0.083 \\
(0.154)\end{array}$ \\
\hline Cashflow & & $\begin{array}{c}0.328^{* * *} \\
(0.013)\end{array}$ & $\begin{array}{c}0.327^{* * *} \\
(0.011)\end{array}$ & $\begin{array}{c}0.308^{* * *} \\
(0.012)\end{array}$ \\
\hline Size & & $\begin{array}{c}0.263^{* * *} \\
(0.018)\end{array}$ & $\begin{array}{c}0.264^{* * * *} \\
(0.018)\end{array}$ & $\begin{array}{c}0.260^{* * *} \\
(0.02)\end{array}$ \\
\hline Ltv & & $\begin{array}{c}0.695^{* * *} \\
(0.129)\end{array}$ & $\begin{array}{c}0.713^{* * *} \\
(0.129)\end{array}$ & $\begin{array}{c}0.660 * * * \\
(0.14)\end{array}$ \\
\hline Roe & & $\begin{array}{c}0.883 * * * \\
(0.076)\end{array}$ & $\begin{array}{c}0.910 * * * \\
(0.069)\end{array}$ & $\begin{array}{c}0.863^{* * *} \\
(0.061)\end{array}$ \\
\hline Age & & $\begin{array}{c}0.004 \\
(0.004)\end{array}$ & $\begin{array}{c}0.001 \\
(0.004)\end{array}$ & $\begin{array}{c}0.006 \\
(0.005)\end{array}$ \\
\hline Tng & & $\begin{array}{c}0.000 \\
(0.001)\end{array}$ & $\begin{array}{c}0.000 \\
(0.001)\end{array}$ & $\begin{array}{c}0.000 \\
(0.001)\end{array}$ \\
\hline Top & & $\begin{array}{c}0.001 \\
(0.007)\end{array}$ & $\begin{array}{c}0.001 \\
(0.001)\end{array}$ & $\begin{array}{c}0.001 \\
(0.001)\end{array}$ \\
\hline Tobinq & & $\begin{array}{c}0.011 \\
(0.008)\end{array}$ & $\begin{array}{l}0.024^{* *} \\
(0.010)\end{array}$ & $\begin{array}{c}0.030 * * * \\
(0.007)\end{array}$ \\
\hline Growth & & $\begin{array}{c}0.077^{* * *} \\
(0.017)\end{array}$ & $\begin{array}{c}0.051^{* * *} \\
(0.018)\end{array}$ & $\begin{array}{c}0.060^{* * *} \\
(0.015)\end{array}$ \\
\hline Cir & & $\begin{array}{c}-0.007^{* * *} \\
(0.001)\end{array}$ & $\begin{array}{c}-0.007^{* * *} \\
(0.001)\end{array}$ & $\begin{array}{c}-0.007^{* * * *} \\
(0.001)\end{array}$ \\
\hline $\mathrm{Rd}$ & & $\begin{array}{l}-0.042 \\
(0.102)\end{array}$ & $\begin{array}{l}-0.062 \\
(0.093)\end{array}$ & $\begin{array}{l}-0.082 \\
(0.081)\end{array}$ \\
\hline M2gdp & & $\begin{array}{c}0.009 * * * \\
(0.001)\end{array}$ & $\begin{array}{l}- \\
-\end{array}$ & - \\
\hline Constant & $\begin{array}{c}19.063^{* * *} \\
(0.044)\end{array}$ & $\begin{array}{c}5.284^{* * *} \\
(0.427)\end{array}$ & $\begin{array}{c}6.870 * * * \\
(0.546)\end{array}$ & $\begin{array}{c}7.240^{* * *} \\
(0.541)\end{array}$ \\
\hline IndustryFE & $\mathrm{No}$ & Yes & Yes & Yes \\
\hline YearFE & No & No & Yes & Yes \\
\hline CityFE & No & No & No & Yes \\
\hline Observations & 10,243 & 10,090 & 10,090 & 10,087 \\
\hline $\mathrm{R}$-squared & 0.002 & 0.32 & 0.329 & 0.389 \\
\hline
\end{tabular}

The coefficients of Fin are significant and positive, suggesting that an increase in financial assets level tends to improve TFP, but as financial assets proceeds further, it shows impediment. Moreover, the results of controlling variables are in line with the literature.

\subsection{Heterogeneity Studies}

The dual effect may vary for different types of financial assets and enterprises based on the asset structure and properties. Therefore, we explore heterogeneity by estimating separate regressions for the term structure of financial assets (Columns 1 and 2) and ownership structure of firms (Columns 3 and 4). This paper references Peng et al. [47] to categorize real estate and long-term equity investments as long-term financial assets based on the structure of financial assets, and the remaining category is short-term financial assets (total assets for standardized treatment). To study the effects of the financialization on different property rights enterprises, we categorize the samples according to the property rights and divide them into two sets of data: state-owned enterprises (SOE) and nonstateowned enterprises (non-SOE).

Previous studies have found that liquidity is important to ensure that firms are able to meet short-term obligations [48] and meet the needs of daily business operations [49]. 
However, too much liquidity can be detrimental to profits. A financial asset is considered liquid if it can be converted into cash immediately or reasonably soon without a loss of value, also known as a cash equivalent. Therefore, good management of liquidity requires establishing a balance between cash holding and financial assets in order to maximize the firm's value and meet short-term obligations. The liquidity of financial assets varies with different time periods. As expected, the empirical results in Columns (1) of Table 5 show that the regression coefficient of the Fin and Fin2 in the sample of short-term financial assets is 0.046 and -0.012 , respectively, which is significantly within the $1 \%$ confidence interval. The interaction between financialization and TFP turns out to be statistically significant only for short-term financial assets. Considering the short-term financial assets are characterized by high liquidity and low realization costs, enterprises have a stronger desire to seek financial profit through short term capital allocation and a higher degree of flexibility.

Table 5. Heterogeneity test.

\begin{tabular}{ccccc}
\hline TFP & $\mathbf{( 1 )}$ & $\mathbf{( 2 )}$ & $\mathbf{( 3 )}$ & (4) \\
\hline & $\begin{array}{c}\text { Short-term } \\
\text { financial assets }\end{array}$ & $\begin{array}{c}\text { Long-term } \\
\text { financial assets }\end{array}$ & SOE & Non-SOE \\
Fin & $0.046^{* * *}$ & -0.176 & $0.037^{* *}$ & 0.044 \\
& $(0.011)$ & $(0.811)$ & $(0.015)$ & $(0.291)$ \\
Fin2 & $-0.012^{* * *}$ & 0.284 & $-0.001^{* * *}$ & $(0.032$ \\
& $(0.0002)$ & $(1.235)$ & $(0.0002)$ & Yes \\
Controls & Yes & Yes & Yes & Yes \\
FirmFE & Yes & Yes & Yes & Yes \\
YearFE & Yes & Yes & 8386 & 1704 \\
Rservations & 10,090 & 10,090 & 0.302 & 0.288
\end{tabular}

Note: Standard errors in parentheses. ${ }^{* *} p<0.05,{ }^{* * *} p<0.01$.

Financial constrains is generally thought to be closely related to investment behavior. In general, there is a financialization behavioral difference between SOE and non-SOE. In Columns (3) and (4), we find that financialization at SOE is sensitive to TFP, but the dual affection is not statistically significant for non-SOE, mainly because the availability of internal funds adds constraints to the investment decision. As noted, financial constraints play an important role in determining the optimal cash level and investment, directly impact investment decisions, and restrict production expansion, which impedes sustainable development and value maximization. For $\mathrm{SOE}$, the degree of financing constraints is relatively low, and corporate management adjusts their management as needed. Corporate management depends on the principle of enterprise operations to maximize profits by optimizing input combinations. It seems that corporate governance may have incentives related to soft budget constraints to prefer the accumulation of financial assets over the creation of profit. On the other hand, large amounts of financing are channeled through SOE, which are much less efficient than China's private sector enterprises. The conclusion of existing studies shows that SOE are commonly perceived as performing poorly in TFP growth [50]. While SOE are unlikely to change their long-term production efficiency based on historical and policy factors, SOE could possibly gain a short-term profit. It implies that SOE have an advantage when involved in financial activities. Hence, they are prone to more financial assets when their low productivity revenue is adjusted due to the burden of excess capital. The increase in financial assets results from soft budget constraints, which is comparable to the fact that increased financialization in China is stronger in SOE. Compared to SOE, non-SOE have higher financial and budget constraints, which impose certain restrictions on capital use within the enterprises. Additionally, non-SOE are more likely to experience stagnant growth or bankruptcy due to financial constraints. However, 
production and operation requirements ultimately determine strategies. Non-SOE tend to focus on material input and output instead of financial activity to drive productive growth.

\subsection{Fixed-Effect Panel Threshold Estimation Results}

To provide further insights into the non-linear relationship between financialization and TFP, we need to estimate the turning points. Table 6 column (1) shows that below the identified threshold $\lambda 1=0.0032$ the financialization do have a positive but statistically insignificant effect. However, we find a significant negative coefficient of the cash holding if the threshold value above 0.0032 which indicates that a high level of substitution of financial assets crowds out cash resources, thereby inhibiting TFP growth. Financialization through cash holding has an adverse effect on TFP, Thus, cash holding channel is only partly supported in the relationship between financialization and firms' TFP.

Table 6. Threshold regression.

\begin{tabular}{ccc}
\hline TFP & $\mathbf{( 1 )}$ & $\mathbf{( 2 )}$ \\
\hline Fin & Threshold $\lambda 1=0.0032$ & Threshold $\lambda 2=0.13$ \\
\hline Cashd0 & 0.022 & \\
Cashd1 & $(0.109)$ & \\
In_ind0 & $-0.292^{* * *}$ & \\
& $(0.078)$ & $0.293^{* *}$ \\
In_ind1 & & $0.148)$ \\
& & $-0.365^{* * *}$ \\
FirmFE & & $(0.147)$ \\
YearFE & Yes & Yes \\
Observations & Yes & Yes \\
R-squared & 7758 & 7758 \\
\hline Sote: Standard errors in parentheses.** $p<0.05^{* * *} p<0.01$ & 0.291 \\
\hline
\end{tabular}

Note: Standard errors in parentheses. ${ }^{* *} p<0.05,{ }^{* * *} p<0.01$.

It indicates that financialization does not improve cash value, however the decrease in cash holding is attributed to high level of financial assets. Evidence shows that firms holds more cash with a lower financial development market. In other words, the amount of cash that firms can hold is limited by financial assets. This reduces the operational efficiency of SOE. Furthermore, overspending by SOEs leads the state to control prices and tighten monetary policies, which reduces the productivity of the non-state sector and reduces economic growth.

Column (2) incorporates the results of the single threshold estimation. We find a single significant threshold value of 0.13 above which the relationship of the financialization and innovation turns nonlinear. It is important to note that up to a threshold of 0.13 the coefficient is 0.293 , and above this threshold the coefficient declines slightly to -0.365 . It illustrates that the turning point is 0.13 , indicating that, before reaching this point, financialization has a positive relationship with TFP, while after this point, and the relationship becomes negative. From a theoretical perspective, the inverted U-shaped relationship shows that the impact of cash holding is bounded.

As discussed above, cash holdings have no significant effects on the firms' TFP, but innovation has an inverted U-shaped effect on TFP. Thus, it can be concluded that innovation has more significant effects on the firms' TFP. Innovation determines the growth of TFP in future sustainable development. This inconsistency can be attributed to the current stage of development in China, where policymakers use various policy instruments to promote the technological advances of firms. Particularly, it is important to note that most enterprises, especially private firms, face severe financial constraints in China. Therefore, these firms should rely on internal funding, which suggests that these firms are involved in activities that generate additional income. This can alleviate the pressure of external funding, which 
will relieve financing constraints and reduce financing costs. This means that financial assets and the improved efficiency of additional income will result in financialization and capital value. Until recently, anecdotal evidence suggests that the participation of non-financial firms in financial markets can help enterprises obtain substantial financial support for technological innovation, and capital markets can support the progress of technological innovation by providing long-term incentive capital, risk diversification, and sharing opportunities for investors [51]. The advantage of holding financial assets, highlighted by Ang [52] and Arizala et al. [53], is that it enables firms to ease financial constraints and accumulate high income, which may contribute to technical innovation. Therefore, a reasonable level of financial assets is more likely to broaden the capital value and secure long-term innovation. A lower level of financialization is more likely to improve the retention of capital. When income is retained more sufficiently, enterprise managers will be more willing and able to seek long-term development of technology innovation instead of focusing only short-term benefits. This will provide financial support for the technological innovation of enterprises, promote the participation of enterprises in technological innovation activities, and have a positive impact on the efficiency of technological innovation. Better technology innovation is associated with higher TFP.

However, excessive financialization of enterprises will gradually separate the compensation of employees, especially managers, from their long-term performance and establish a relatively close relationship with the short-term arbitrage behavior in the financial market [54]. The spread of uncertainty has a negative impact on the corporate governance structure; furthermore, the substitution of investment funds can be used for non-R\&D purposes, which results in the partial displacement of technological innovation.

The F-value and $p$-value obtained after 300 repeated samplings are presented in Table 7. The result shows that the single threshold effect of the model pass the test, it means a significant threshold effect of financialization exists, with the single threshold value of 0.13. Table 8 reports single threshold estimates and a $95 \%$ confidence interval. The LR value is less than 7.35 , which is the critical value at $5 \%$ significant level. Figure 1 shows the estimation and confidence interval for single threshold.

Table 7. Test result of threshold significance.

\begin{tabular}{cccccc}
\hline Threshold & F-Value & $p$-Value & $\mathbf{1 0} \%$ & $\mathbf{5 \%}$ & $\mathbf{1 \%}$ \\
\hline Single & $17.43^{* *}$ & 0.017 & 8.831 & 12.617 & 19.145 \\
\hline Note: Standard errors in parentheses. ${ }^{* *} p<0.05,{ }^{* * *} p<0.01$.
\end{tabular}

Table 8. Threshold values and confidence intervals.

\begin{tabular}{ccc}
\hline Model & Threshold Value & 95\% Confidence Intervals \\
\hline Single threshold & 0.1300 & $(0.0000,0.4600)$ \\
\hline
\end{tabular}

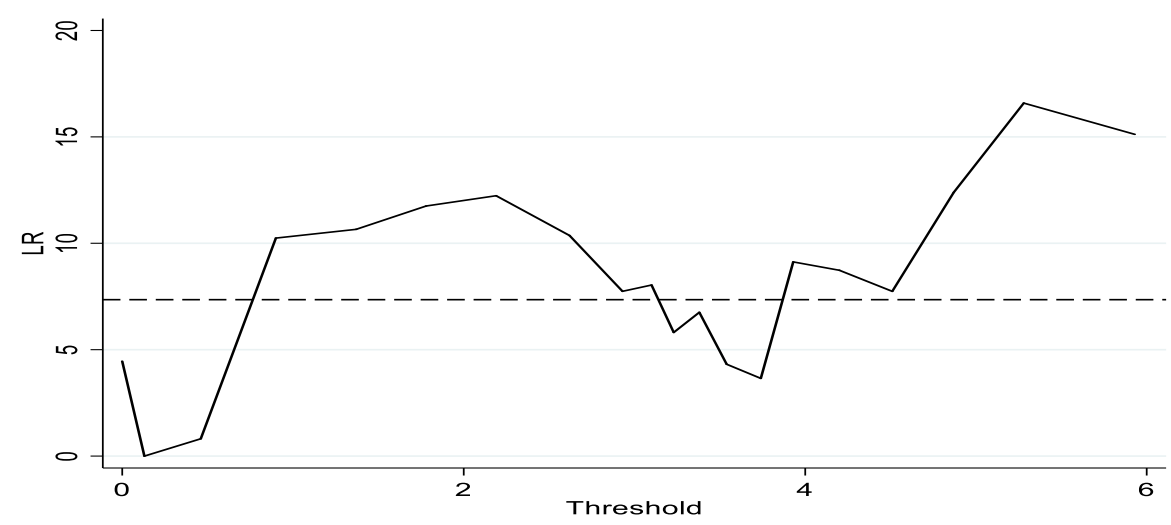

Figure 1. Estimation and confidence interval for single threshold. 


\subsection{Robustness Test}

The possibility of endogeneity is an issue that might affect our study of the relationship between financialization and TFP. To overcome the potential issues of omitted variables and reverse causality among variables that may cause parameter estimates to become biased and inconsistent, we construct the following difference-in-differences model to conduct a more extensive test.

Specifically, we reveal the limited investment regulations of private equity funds resulting from the unexpected systemic risk enforced in 16 cities with elevated housing prices starting in 2017. This regulation may discourage these nonfinancial firms to invest in property. It is unlikely that the regulation has a direct effect on nonfinancial firms outside these 16 cities, allowing us to construct a control group to examine the heterogeneous effects across cities. A natural question is why the regulation is related to corporate financialization. Two facts about Chinese housing prices and real estate are well-documented and largely agreed upon. Official statistics in China show that housing prices grew dramatically between 2007 and 2014 [55] and moderately in recent years. This is indeed a real estate boom with Chinese characteristics, which is typically related to government decisions. As housing prices increased, relevant investments increased also. Compared to the downward trend of the entity sector, the profitability of the real estate sector is associated with a higher probability. Chinese owners or investors either directly purchase real estate or invest in the form of financial derivatives. According to the National Bureau of Statistics, China's total real estate investment was 0.36 trillion yuan in 1998 and increased to 10.98 trillion yuan in 2017, which rose nearly 30 times within 20 years. Existing data (China Wealth Management Product Market Development and Evaluation) shows that nearly $25 \%$ of trust funds flow to real estate, and housing price fluctuations lead to the conversion of properties from real asset attributes to financial attributes. Real estate also accounts for a major part of financial assets within Chinese nonfinancial firms. Since the late 2000s, the government has increasingly shifted its focus to financial stability and the imbalances between finance and the economy. Under the central government's guidance, regulators have sought to stabilize housing prices through restrictions and limiting investments in real estate financial assets.

In China, holding financial assets is a crucial form of real estate investments fueled by funding non-financial firms because they are flexible and highly liquid. The portfolios of financial assets are highly skewed towards real estate. Due to the fact that the boom and burst of real estate markets are closely related corporate investments [56], the difference-indifferences approach is used to compare the TFP before and after the regulation became effective. If that were the case, financialization would not be binding, which means that the constraint on holding real estate financial assets has a positive effect on TFP. Summarizing our empirical strategy, we estimate the following DID equation:

$$
\text { TFP }_{i, t}=\lambda_{0}+\lambda_{1} \text { Treat }_{i}+\lambda_{2} \text { Time }_{t}+\lambda_{3} \text { Did }_{i, t}+\tau_{k} \sum \text { Control }_{i, t}+\mu_{i}+\sigma_{t}+\varepsilon_{i, t}
$$

where Treat $_{i}$ is a dummy variable taking value of 1 if firm $i$ is in the 16 cities affected by the regulation. Time $t$ takes value 1 if year is after 2017, and 0 otherwise. The regression controls for firm fixed effect, year fixed effects.

As it can be clearly seen from Table 9, once we include firm fixed effects, the absolute value of the coefficients decrease marginally, and hence accounting for the financialization quantitatively weakens the TFP, but economically the change is small. Whereas the results suggest that financialization was significantly affected by the regulation. This could potentially explain the increase in TFP reported in the period immediately after 2017 and why TFP was still negative before 2017, which could be driven by firms drawing on the level of financialization. Therefore, our main findings as above were confirmed. 
Table 9. Difference-in-differences regression.

\begin{tabular}{ccc}
\hline TFP & $\mathbf{( 1 )}$ & $\mathbf{( 2 )}$ \\
\hline Did & $0.107^{* *}$ & $0.059^{*}$ \\
& $(0.055)$ & $(0.036)$ \\
Controls & Yes & Yes \\
FirmFE & No & Yes \\
YearFE & No & Yes \\
Observations & 10,200 & 10,200 \\
R-squared & 0.30 & 0.268 \\
\hline Note: Standard errors in parentheses * $* 0.10^{* *} p<0.05$
\end{tabular}

Note: Standard errors in parentheses. ${ }^{*} p<0.10,{ }^{* *} p<0.05$.

It is worth discussing potential endogeneity concerns of our results. Firstly, all of our specifications, including the threshold regression model, have explicitly accounted for the individual fixed effects. These should eliminate endogenous bias caused by time invariant unobservable. Secondly, the remaining endogenous concern may come from reverse causality or simultaneously bias. Since the lagged explanatory variables tend to only be weakly correlated with current period's error in our main specification, we use lagged Fin to alleviate the potential endogeneity. Lastly, to overcome the potential bias caused by time-varying omitted variables, we re-estimated the quadratic regression and threshold regression by extensive control variables. Additional TFP determinant variables should be captured including industry and market characteristics. Specifically, Loan, the ratio of total loans to total debts, is used to control for the effect of lending capability. HHI, the Herfindahl-Hirschman index (HHI) as measured by the sum of the squared industry shares of each firm's assets for a given year, is used to control for the effects of industry concentration. Freturn, the financial return on investment is used to control for financial market return.

For the sake of brevity, we only report our main interest variables as shown in Tables 10 and 11, thus confirming the robustness of our previous findings.

Table 10. Lagged variables\& Additional control variables- quadratic regression.

\begin{tabular}{ccc}
\hline \multirow{2}{*}{ TFP } & $\mathbf{( 1 )}$ & $\mathbf{( 2 )}$ \\
\cline { 2 - 3 } & Lagged Fin & Fin \\
\hline Fin & $0.050^{*}$ & $0.038^{* *}$ \\
& $(0.029)$ & $(0.015)$ \\
Fin2 & $-0.002^{*}$ & $-0.0016^{* * *}$ \\
& $(0.001)$ & $(0.0004)$ \\
Loan & & -0.058 \\
& & $(0.046)$ \\
HHI & & 0.039 \\
& & $(0.079)$ \\
Freturn & & $0.337^{* * *}$ \\
& & $(0.081)$ \\
Controls & Yes & Yes \\
FirmFE & Yes & Yes \\
YbsearFE & Yes & Yes \\
R-squared & 9306 & 9747 \\
\hline
\end{tabular}

Note: This table column (1) shows the lagged explanatory variables in quadratic regression, column (2) presents the regression with additional control variables. Standard errors in parentheses. ${ }^{*} p<0.10,{ }^{* *} p<0.05,{ }^{* * *} p<0.01$. 
Table 11. Lagged variables\& Additional control variables- threshold regression.

\begin{tabular}{ccc}
\hline \multirow{2}{*}{ TFP } & (1) & (2) \\
\cline { 2 - 3 } & Lagged Fin & Fin \\
\hline In_ind0 & Threshold $\lambda 1=0.116$ & Threshold $\lambda 2=0.13$ \\
In_ind1 & $0.213^{*}$ & \\
& $(0.117)$ & \\
Loan & $-9.17^{*}$ & \\
& $(0.529)$ & 0.083 \\
HHI & & $(0.057)$ \\
& & 0.018 \\
Freturn & & $(0.814)$ \\
& & 0.154 \\
FirmFE & & $(0.219)$ \\
YearFE & Yes & Yes \\
Observations & Yes & Yes \\
R-squared & 5796 & 6680 \\
& 0.269 & 0.253 \\
\hline
\end{tabular}

Note: This table column (1) shows the lagged explanatory variables in threshold regression, column (2) presents the regression with additional control variables. Standard errors in parentheses. ${ }^{*} p<0.10$.

\section{Conclusions}

This paper presents new evidence regarding the effect of financialization on TFP in China. Our study contributes to the debate about the effect of holding financial assets of nonfinancial corporations, where no consensus emerges from prior literature. Our sample covered the period 2007-2018 for 3654 non-financial corporations, Through the use of a non-linear modeling strategy, we explore the relationship between financialization and TFP. Also we apply the threshold methodology to verify the mechanism between financialization and TFP from the perspectives of cash holdings and technological innovation. Through theoretical and empirical analysis, this paper concludes the following: Financialization, as measured by holding financial assets on total assets, has an inverted U-shaped relationship with the TFP. Taking heterogeneity into account, we find that the dual effect is more pronounced in short-term financial asset-holding and SOE. Furthermore, our findings indicate that there is a significant financialization threshold between technological innovation and TFP. In the low threshold interval $(\lambda<0.13)$, financialization can significantly promote TFP. Nevertheless, in the high threshold interval $((\lambda>0.13)$. Since change of innovation explains the non-linear relationship better than cash holding. Such a threshold method adds perspective to existing models, which demonstrates the key role of innovation switches the effect of financialization. And in this case, further analysis is still needed to suggest a policy for limiting over financialization. Several extensions of our research would be desirable, including optimal productivity factor allocation, maximization of financial assets profit as well as the sustainable growth when face the shifting economic conditions.

Technological innovation and capital upgrades can fundamentally drive productivity growth and accelerate the circular economy. Our findings emphasize the role of financilization to determine a sustainable competitive advantage of capital as part of resilient and sustainable systems. The nonfinancial corporates should review their allocation of production factors from the perspective of productivity, and pay keen attention to enhance their resilience in the face of shifting economic conditions. Besides, a broader policy framework is required to promote the rational allocation of resources. To successfully manage economic volatility created by the pandemic and foster sustainable economic growth the policymakers need to implement joint actions to support the development of circular economy. 
Author Contributions: Conceptualization, H.W. and Q.W.; methodology, H.W.; software, H.W.; validation, H.W., X.S.; formal analysis, H.W.; investigation, data curation, H.W., X.S.; writingoriginal draft preparation, H.W.; writing-review and editing, H.W.; supervision, Q.W.; project administration, Q.W.; funding acquisition, Q.W. All authors have read and agreed to the published version of the manuscript.

Funding: This research was funded by the National Natural Science Foundation of China [71950010].

Data Availability Statement: Not applicable.

Conflicts of Interest: The authors declare no conflict of interest.

\section{References}

1. D'Adamo, I.; Rosa, P. How do you see infrastructure? Green energy to provide economic growth after COVID-19. Sustainability 2020, 12, 4738. [CrossRef]

2. D'Adamo, I.; Falcone, P.M.; Martin, M.; Rosa, P. A Sustainable revolution: Let's go sustainable to get our globe cleaner. Sustainability 2020, 12, 4387. [CrossRef]

3. D'Adamo, I.; Lupi, G. Sustainability and resilience after COVID-19: A circular premium in the fashion industry. Sustainability 2021, 13, 1861. [CrossRef]

4. Orhangazi, Ö. Financialization and capital accumulation in the non-financial corporate sector: A theoretical and empirical investigation on the US economy: 1973-2003. Camb. J. Econ. 2008, 32, 863-886. [CrossRef]

5. Onaran, Ö.; Stockhammer, E.; Grafl, L. Financialization, income distribution and aggregate demand in the USA. Camb. J. Econ. 2010, 35, 637-661. [CrossRef]

6. Krippner, G. Capitalizing on crisis: The political origins of the rise of finance. Bus. Ethics Q. 2011, 21, 693-701.

7. Bonfiglioli, A. Financial integration, productivity and capital accumulation. J. Int. Econ. 2008, 76, 337-355. [CrossRef]

8. Demir, F. Financial liberalization, private investment and portfolio choice: Financialization of real sectors in emerging markets. J. Dev. Econ. 2009, 88, 314-324. [CrossRef]

9. Tornell, A. Real vs. financial investment can Tobin taxes eliminate the irreversibility distortion? J. Dev. Econ. 1990, 32, 419-444. [CrossRef]

10. Duchin, R.; Gilbert, T.; Harford, J.; Hrdlicka, C. Precautionary savings with risky assets: When cash is not cash. J. Financ. 2017, 72, 793-852. [CrossRef]

11. Corpataux, J.; Crevoisier, O.; Theurillat, T. The Expansion of the Finance Industry and Its Impact on the Economy: A Territorial Approach Based on Swiss Pension Funds. Econ. Geogr. 2009, 85, 313-334. [CrossRef]

12. Hansen, B.E. Threshold effects in no-dynamic panels: Estimation, testing, and inference. J. Econom. 1999, 93, 345-368. [CrossRef]

13. Seo, H.J.; Kim, H.S.; Kim, Y.C. Financialization and the Slowdown in Korean Firms' R\&D Investment. Asian Econ. Pap. 2012, 11, 35-49.

14. Shin, H.S. Global Banking Glut and Loan Risk Premium. Mundell-Fleming Lecture. IMF Econ. Rev. 2012, 60, 155-192. [CrossRef]

15. Akkemik, K.; Özen, S. Macroeconomic and institutional determinants of financialization of non-financial firms: Case study of Turkey. Socio-Econ. Rev. 2013, 12, 71-98. [CrossRef]

16. Gyimah-Brempong, K. Corruption, economic growth, and income inequality in Africa. Econ. Gov. 2002, 3, 183-209. [CrossRef]

17. Knack, S.; Keefer, P. Does Social Capital Have an Economic Payoff? A Cross-Country Investigation. Q. J. Econ. 1997, 112, 1251-1288. [CrossRef]

18. Sachs, J.; Warner, A. Natural Resource Abundance and Economic Growth. Nber Work. Pap. Ser. 1995, 5398, 1-47.

19. Singh, A. Financial liberalization, stock markets and economic development. Econ. J. 1997, 107, 771-782. [CrossRef]

20. Krugman, P.; Anthony, J.V. Globalization and the Inequality of Nations. Q. J. Econ. 1995, 110, 857-880. [CrossRef]

21. Sweezy, P. Economic reminiscences. Mon. Rev. 1995, 4, 1. [CrossRef]

22. Lazonick, W. The new economy business model and the crisis of US capitalism. Capital. Soc. 2009, 4, 2. [CrossRef]

23. Zhang, J.; Chen, S.Y.; Jefferson, G.H. Structural Reform and China's Industrial Growth. Chin. Econ. 2009, 3, $205-240$.

24. Wang, H.J.; Cao, T.Q.; Yang, Q.; Yang, Z. Financialization of Entities Promotes or Suppresses Corporate Innovation-An Empirical Study Based on Chinese Listed Manufacturing Companies. Nankai Manag. Rev. 2017, 20, 155-166.

25. Law, S.H.; Singh, N. Does too much finance harm economic growth? J. Bank. Financ. 2014, 41, 36-44. [CrossRef]

26. Sahay, R.; Čihák, M.; Papa N’Diaye, A.; Barajas, R.B.; Ayala, D.; Gao, Y.; Yousefi, S.R. Rethinking Financial Deepening: Stability and Growth in Emerging Markets. IMF Discuss. Note SDN 2015, 15, 8. [CrossRef]

27. Dumenil, G.; Levy, D. Capital Resurgent: Roots of the Neo-Liberal Revolution; Harvard University Press: Cambridge, MA, USA, 2004.

28. Palley, T.I. Financialization: What it is and Why it Matters, IMK Working Paper 04-2008, IMK at the Hans Boeckler Foundation; Macroeconomic Policy Institute: Washington, DC, USA, 2008.

29. Krippner, G.R. The Financialization of the American economy. Socio Econ. Rev. 2005, 3, 173-208. [CrossRef]

30. Zhang, C.S.; Zhang, B.T. The Mystery of China's Declining Industrial Investment Rate: The Financialization of the Economy Perspective. Econ. Res. 2016, 51, 32-46.

31. Sen, S.; Dasgupta, Z. Financializiton and corporate investments: The Indian case. Soc. Sci. Electron. Publ. 2015, 64, 844-853. 
32. Brenner, R. The Boom and Bubble: The US in the World Economy; Verso: New York, NY, USA, 2002; pp. $216-218$.

33. Campello, M.; Chen, L.; Yue, M.; Hong, Z. The Real and Financial Implications of Corporate Hedging. CFA Digest. 2012, 42, 64-66.

34. Gehringer, A. Growth, productivity and capital accumulation: The effects of financial liberalization in the case of European integration. Int. Rev. Econ. Financ. 2013, 25, 291-309. [CrossRef]

35. Arcand, J.L.; Berkes, E.; Panizza, U. Too much finance? J. Econ. Growth. 2015, 20, 105-148. [CrossRef]

36. Fazzari, S.M.; Petersen, B.C. Working capital and fixed investment: New evidence on financing constraints. RAND J. Econ. 1993, 24, 328-342. [CrossRef]

37. Dallery, T. Post-Keynesian theories of the firm under financialization. Rev. Radic. Political Econ. 2009, 41, 492-515. [CrossRef]

38. Lazonick, W.; O'sullivan, M. Maximizing shareholder value: A new ideology for corporate governance. Econ. Soc. $2000,29,13-35$. [CrossRef]

39. Zhang, M.; Luo, L. An Empirical Study of the Impact of Financialization on Productivity of Private Enterprises-An Empirical Analysis Based on Chinese A-Share Private Listed Companies. Econ. Syst. Reform. 2017, 5, 155-161.

40. Moreno-Badia, M.; Slootmaekers, V. The Missing Link between Financial Constraints and Productivity; International Monetary Fund: Washington, DC, USA, 2009.

41. Solow, R. A Contribution to the Theory of Economic Growth. Q. J. Econ. 1956, 70, 65-94. [CrossRef]

42. Talukder, D.; Chile, L. Technological innovation and total factor productivity growth of rice production in Bangladesh in the post-liberalization era. Glob. Econ. Obs. 2008, 6, 50-70.

43. Xu, S.; Liu, D.C. An empirical research on the effect of corporate financialization on technological innovation. Res. Manag. 2019, 10, 240-249.

44. Olley, G.S.; Pakes, A. The Dynamics of Productivity in the Telecommunications Equipment Industry. Econometrica 1996, 64, 1263-1297. [CrossRef]

45. Xiao, W.; Xue, T.H. Rising labour costs, financial constraints and changing TFP of enterprises in China. Glob. Econ. 2019, 1, 76-94.

46. Ashman, S.; Fine, B. Neo-liberalism, varieties of capitalism, and the shifting contours of South Africa's financial system. Crit. Perspect. South. Afr. 2013, 81, 144-178. [CrossRef]

47. Peng, Y.C.; Han, X.; Li, J.J. Economic policy uncertainty and corporate financialization. Chin. Ind. Econ. 2018, 1, 137-155.

48. Dunn, P.; Cheatham, L. Fundamentals of small business financial management for start-up, survival, growth, and changing economic circumstances. Manag. Financ. 1993, 19, 1-13. [CrossRef]

49. Niresh, J.A. Trade-off between liquidity and profitability, A Study of selected Manufacturing Firms in Sri Lanka. University of Jaffna. Res. Humanit. Social Sci. 2015, 5, 78-85.

50. Jefferson, G.H.; Rawski, T.G.; Wang, L.; Zheng, Y. Ownership, Productivity Change, and Financial Performance in Chinese Industry. J. Comp. Econ. 2000, 28, 786-813. [CrossRef]

51. Tadesse, D.T. Management practices, productive performances and egg quality traits of exotic chickens under village production system in east Shewa, Ethiopia. Appl. Energy 2012, 97, 5-15.

52. Ang, J. Finance and Inequality: The Case of India. South. Econ. J. 2010, 76, 738-761. [CrossRef]

53. Arizala, F.; Cavallo, E.; Calindo, A. Financial development and TFP growth: Cross country and industry level evidence. Appl. Financ. Econ. 2013, 23, 433-448. [CrossRef]

54. Montgomerie, J. Bridging the critical divide: Global finance, financialization and contemporary capitalism. Contemp. Politics. 2008, 14, 233-252. [CrossRef]

55. Anzoategui, D.; Chivakul, M.; Maliszewski, W. Financial Distortions in China: A General Equilibrium Approach; International Monetary Fund: Washington, DC, USA, 2015.

56. Liu, Z.; Wang, P.F.; Zha, T. Land Price Dynamics and Macroeconomic Fluctuations. Econometrica 2013, 81, 1147-1184. 

Article

\title{
Is Environmental Sustainability Taking a Backseat in China after COVID-19? The Perspective of Business Managers
}

\author{
Dongyong Zhang ${ }^{1, * \mathbb{D}}$, Mengge Hao ${ }^{1} \mathbb{( \mathbb { D }}$ and Stephen Morse ${ }^{2}$ \\ 1 College of Information and Management Science, Henan Agricultural University, Zhengzhou 450046, China; \\ haomengge99@foxmail.com \\ 2 Centre for Environment and Sustainability, University of Surrey, Surrey GU2 7XH, UK; s.morse@surrey.ac.uk \\ * Correspondence: dongyongzhang.cn@gmail.com; Tel.: +86-371-56990030
}

Received: 3 November 2020; Accepted: 10 December 2020; Published: 11 December 2020

check for updates

\begin{abstract}
China's quick economic recovery from COVID-19 has presented a narrow but vast opportunity to build an economy that is cleaner, fairer, and safer. Will China grab this opportunity? The answer rests with both business managers and the government. Based on a questionnaire survey of 1160 owners and managers of companies headquartered in 32 regions of China and covering 30 industries, this paper explores how COVID-19 has impacted Chinese business, especially with regard to the three dimensions of sustainability (economic, social, and environmental). The results suggest that Chinese companies' sustainability priorities have been shifted towards the social dimension both during COVID-19 and into the post-pandemic phase, regardless of the type of ownership, company size, or market focus (domestic, overseas, or mixture of the two). However, all types of company prioritize the need for economic sustainability in the post-pandemic phase and in relative terms the importance of the environmental dimension has been diminished. Hence the potential for a post-pandemic environmental rebound effect in China is clear. But it does not have to be the case if Chinese businesses and the government take actions to change its recovery plans to embrace the environmental dimension of sustainability. The paper puts forward some suggestions and recommendations for businesses and the government.
\end{abstract}

Keywords: social sustainability; economic sustainability; environmental sustainability; COVID-19; China; business

\section{Introduction}

The new coronavirus (COVID-19) has posed an unprecedented challenge to global sustainable development (SD). As of December 2020, more than 65 million people have the disease and more than 1.5 million have died from it [1]. Strategies such as large-scale coronavirus screening tests, stringent hygiene protocols, isolation, and social distancing have been adopted by governments worldwide to fight the virus, and these have resulted in significant negative economic impacts in many countries. The whole world has almost been brought to a standstill as economic growth faltered and international transport greatly reduced, although on the positive side these have resulted in a significant reduction in global carbon emissions [2]. However, some argue that the drop in $\mathrm{CO}_{2}$ emissions is just temporary as it does not reflect any significant structural change in the economic, transport, or energy system [3], and unless there is such change, it will be hard to avoid the environmental rebound effect when COVID-19 is over [4,5]. At the same time, other environmental issues such as waste recycling and water contamination have been neglected during the pandemic [2] and new types of pollution have originated from the large-scale manufacture and distribution of COVID-19 protective gear such as face masks [6]. 
In fact, COVID-19's unprecedented effect on most types of business operations may have already led to some sustainability initiatives being modified or cancelled [7]. A survey carried out in March 2020 by 101 UK-based energy and sustainability professionals found that $60 \%$ of the organizations had either confirmed their plans to pause investment in sustainability solutions or were considering doing so as a result of COVID-19 [8]. In an effort to avoid an environmental rebound effect, the Institute for Global Environmental Strategies [9] has been calling for a green/sustainable post-pandemic recovery.

In April 2020, Ursula von der Leyen-the president of the European Commission-pledged more than USD 800 billion to a European Green Deal which would turn the COVID-19 crisis into an opportunity to rebuild the EU economy in a different and more sustainable form [10]. EU leaders have agreed on an EU recovery plan which proposed to halve greenhouse gas emissions over the next 10 years by spending USD 28 billion to increase renewable energy capacity, USD 100 billion annually to improve home energy efficiency, and up to USD 67 billion to build zero-emission trains [11]. The International Energy Agency [12] has called for an annual one trillion USD investment in clean energy over the next three years. The South Korean government has promised a USD 10 billion Green New Deal to invest in renewable energy and an improvement in energy efficiency [13]. Costa Rica has become one of the few developing countries that has put in place a green recovery plan by introducing a new fee on gasoline as a way of funding social-welfare programs and is planning to issue new green bonds to fund the next stage of climate adaption programs [14]. The International Monetary Fund [15] has made climate resilience a key criterion for its lending and in June its 50 borrowing countries committed to include climate change in their COVID-19 recovery plans.

However, at the time of writing other major global players such as the US and China have no clear environmentally friendly recovery plans in place. Chinese leaders in May 2020 endorsed a proposal to spend USD 1.4 trillion on 'new infrastructure' including electric-vehicle charging stations, high-speed rail, and 5G technology which would stimulate economic growth with lower emissions, but there is no EU-type climate conditionality attached to its infrastructure projects. Despite the US's decision to withdraw from the Paris Agreement, President Xi Jinping pledged that China will fulfil its obligation under the UN emission treaty. At the 19th Congress of the Chinese Communist Party in October 2017, Xi claimed that China was in the 'driving seat' when it comes to international cooperation on climate change. In fact, as an increasingly influential global player and amid COVID-19, China showed leadership by clearly stating its commitment to increasing its contribution to WHO while US decided to withdraw funding from the agency. In a virtual UN General Assembly in New York in September 2020, Xi Jinping promised that China would go carbon neutral before 2060 [16]. However, $X^{\prime}$ 's pledge would need to be backed up with more detailed and concrete implementation plans which must reconcile carbon neutrality with China's ongoing support for the fossil fuel industry [17].

Once the COVID-19 hot spot of the world, China was the first country to shut down while the rest of the world, most notably the USA, still underestimated the risk posed by the virus. Shutting down the Chinese economy may have helped address the spread of the virus, but it did have severe consequences. China's economy suffered its first contraction in 28 years by shrinking $6.8 \%$ in the first quarter of 2020 compared to the same quarter in 2019. However, China has since then been on the path to recovery, and a year-on-year growth of $3.2 \%$ was achieved in the second quarter of 2020 [18]. As the first economy that is successfully (so far) recovering from COVID-19, China has presented itself a "narrow window, but vast opportunity" to build back its economy into something that is cleaner, fairer, and safer [19]. Based on a questionnaire survey of 1160 owners and managers of companies headquartered in mainland China as well as Hong Kong, Taiwan, and Macau and covering 30 industries, this paper explores how COVID-19 has impacted Chinese business, especially with regard to the three dimensions of sustainability (economic, social, and environmental) and provides an 'ex-post' assessment of the post-pandemic situation. It is assumed that China's experience with green/sustainable recovery would make it a useful case study to explore given that the shape of the recovery elsewhere in the world is uncertain. 
A number of theories helped inform the research, including the environmental rebound effect, slack resources theory, and the available funds hypothesis, and these are summarized in the following 'Theoretical Background' section. Previous literature on the impact of COVID-19 is also included in this section. This is followed by the hypotheses and the methodology employed in this study, including question design, data collection, and data analysis. Section four presents the results of the research, including the demographic information of the respondents, the impact that COVID-19 has had on the companies, coping strategies, and the current recovering situation, the changing sustainability priorities of the companies will also be explored in this section. Discussion of the results is also included in this section. The last section draws out some conclusions and suggests some implications for company mangers as well as theoretical implications and suggestions for future research.

\section{Theoretical Background}

\subsection{Environmental Rebound Effect}

The rebound effect is generally defined as the difference between the expected and actual environmental gains from an efficiency increase after the implementation of new technologies or other measures $[4,20]$. A good example of a rebound effect is found when a technological improvement in vehicle fuel efficiency makes driving cheaper but the saving is often offset by driving faster or further than before. Hence, resource efficiency may not reduce the use of resources and may even generate the contrary. Empirical rebound studies often try to capture the secondary effects of policies and behaviors. For example, although the use of information and communications technologies (ICT) was thought to be environmentally beneficial as it reduces road traffic and saves commuting time, Gossart [21] provided evidence of the rebound effect related to ICT and suggested ways of overcoming rebound. Of course, the magnitude of any rebound effect varies depending on cultural and structural context. For example, although the industrial Internet of things (IIoT) could potentially improve resource efficiency by establishing highly digitized, interconnected, self-regulating, and decentralized industrial value chains [22,23], it could rebound. By comparing China and Germany, Muller and Voigt [22] found the introduction of IIoT would cause more serious job loss problems in China than in Germany, and greater data security concerns in Germany than in China, while both countries would equally face a problem over skill shortages.

Various policies and measures taken by countries to contain the COVID-19 pandemic have caused abrupt changes in business operation, consumption, social interaction, and so on, and these changes have led to improvements in some environmental indicators such as air quality and greenhouse gas emissions. But will a rebound effect take place such that these changes are not permanent? Will the environmental improvement last in the future when the pandemic is over? A further question is whether the increased popularity of ICT and digitalization spurred by COVID-19 may backfire, as indicated by Muller and Voigt [22] and Gossart [21].

\subsection{Sustainable Development in China}

The principles of sustainable development have deep roots in China. The earliest ideas relating to sustainable development were recorded during the Xia dynasty (2070-1600 BC) in the Yellow River area in the form of religious beliefs. Back then, people respected the mountains and rivers in the same way they did their spiritual icons [24]. Chinese traditional philosophies such as Confucianism, Taoism, and Yin-Yang contain elements that are fundamental to sustainable development. For example, the basic foundation of Confucianism is the harmony between humans and nature while Taoism believes that all creatures are created by nature and advocates that following nature and taking no action is the best action to take [25]. Sustainable development also had an important place in Chairman Mao's administration. He called for green policies because they would benefit agriculture, industry, and other aspects of society [24]. In September 2015, President Xi Jinping attended the UN Sustainable Development Summit and joined other world leaders in adopting the program entitled Transforming 
Our World: the 2030 Agenda for Sustainable Development, which opened a new era for the undertaking of global sustainable development and charted the course for national development and international development cooperation. At his various speeches to international audiences, $\mathrm{Xi}$ said "green mountains and clear water are as good as mountains of gold and silver." Nonetheless, despite these historical roots and claims to be in the "driving seat" when it comes to tackling climate change, the question remains as to whether China will continue its leadership in sustainability during COVID-19; will climate change issues soon be forgotten after the pandemic as the emphasis shifts in favor of economic development?

\subsection{Ownership, Size, Slack Resources, and Sustainability}

Ownership has been widely discussed as a factor which could potentially influence a company's pursuit of social and environmental responsibility [26]. Based on the Shanghai National Accounting Institute (SNAI) CSR Index, Li and Zhang [27] explored whether and how ownership structure affects corporate social responsibility (CSR) in emerging markets, and found that the stronger the state's controlling of a firm is then the better the firm's social and environmental performances. Zhang et al. [28] investigated corporate social responsibility of a group of Chinese state-owned enterprises (SOEs) and private-owned firms and found that Chinese SOEs are more environmentally responsible than private owned firms. Oh et al. [29] employed a sample of 118 large Korean firms and found a significant and positive relationship between CSR ratings and ownership of institutions (which are under strong influence of the Korean government). However, Oh et al. [29] also found foreign invested firms have relatively high CSR ratings and this result was confirmed by Lee et al. [30] who conducted a study using panel data of the Korea Economic Justice Institute (KEJI) Index and found a positive correlation between CSR performance and foreign ownership.

Size has also been discussed as a factor that may influence a firm's social and environmental engagement. A positive relationship between firm size and social and environmental engagement has been found for firms in developed countries by Fry and Hock [31], Fombrun and Shanley [32], Pava and Krausz [33], McWilliams and Siegel [34], and Elsayed [35]. In recent years, this relationship has also been explored in emerging markets. For example, Zhang et al. [28] found that larger companies engage more in social and environmental responsibility in China than do smaller ones. The same conclusion was reached by Li and Zhang [27] in China and Muller and Kolk [36] in Mexico. Slack resources are the potentially utilizable resources that can be diverted or redeployed for the achievement of organizational goals. Slack resource theory suggests that because more profitable firms have more organizational slack (financial and other), they are likely to be more committed to CSR participation [37]. Based on slack resource theory, Preston and $\mathrm{O}^{\prime} \mathrm{bannon}$ [38] proposed an available funds hypothesis which suggests a positive relationship between a company's profitability and its social performance, i.e., companies with better financial performance would contribute more to fund discretionary projects. The available funds hypothesis was also found to be the case for companies in China [28].

\subsection{The Impact of COVID-19}

Understandably, recent publications on COVID-19 have focused mainly on medical and epidemiological dimensions of the pandemic, including treatments and vaccine development [39-41]. Some have noted the temporary improvement in environmental quality due to virus containment measures but have also expressed concerns about a possible rebound effect after the pandemic $[2-5,42,43]$. A few studies have looked at the economic and social impacts of COVID-19, especially on businesses. Barreiro-Gen et al. [7] surveyed 653 organizations internationally to analyze how the outbreak of COVID-19 had affected their sustainability priorities and they found that there had been a shift towards emphasizing the social dimension for most organizations while the environmental dimension had declined in importance. As a result, Barreiro-Gen et al. [7] suggested measures to avert the possible post-pandemic environmental rebound effect. Bartik et al. [44] explored the impact of COVID-19 on small businesses in the US and found that mass layoffs and closures occurred just a few weeks into the crisis and the risk of closure was negatively associated with the expected length of the crisis. 
Bartik et al. [44] also found that small businesses in the US are financially fragile and many of them anticipated problems such as bureaucratic hassles when seeking COVID-19 related funding.

Zabaniotou [45] considered COVID-19 as proof of an unsustainable human civilization and linked the pandemic to ecological sustainability and resilience. Zabaniotou [45] proposed a humanistic approach as a solution, based not only on virus containment strategies but also an ecological balance. A framework based on resilience was also proposed by D'Adamo and Rosa [46] as a post-pandemic recovery strategy.

However, few analyses to date have looked at the impacts of COVID-19 on sustainability in China especially from the point of view of businesses. This paper aims to explore the impacts COVID-19 may have had on sustainable development in China over both the short-term and long-term from the perspective of business. In particular, it sets out the results of research that sought to investigate whether and how priorities have changed among the three pillars of sustainable development during and post COVID-19 for companies with different ownerships, i.e.,:

- $\quad$ State-owned enterprise (SOE)

- Domestic private-owned company

- Foreign invested company (FIE)

- Domestic individual-owned company

\section{Hypothesis and Research Design}

\subsection{Hypothesis}

There are various types of businesses in China. Based on the ownership, they include mainly state-owned enterprises (SOEs), private-owned companies, foreign invested enterprise (FIE), and individual-owned companies. SOEs are companies that are owned and run by the Chinese government or stock companies having the state as the dominant stockholder. An FIE is a company that is invested solely by a foreign company (including companies based in Hong Kong, Macau, and Taiwan) or created through a partnership between foreign and Chinese investors. A Chinese domestic private-owned company is a Chinese company that is neither an SOE or an FIE and hires eight or more employees. An individual-owned company is a company that is neither an SOE nor an FIE and hires less than eight employees [47]. Both private-owned and individual-owned companies focus on domestic and overseas markets, with the larger companies being more orientated towards overseas. Because of the different stances of different types of companies in China, it is assumed that their sustainability priority changes would be different as a result of COVID-19. Based on the previous literature reviewed in Section 2.3, two hypotheses can be proposed:

Hypothesis 1 (H1). SOEs and FIEs prioritize social sustainability and environmental sustainability more than domestic private and individual owned companies after COVID-19.

Hypothesis 2 (H2). Bigger companies prioritize social and environmental sustainability more than small companies after COVID-19.

However, there could be a complication arising from the market focus of the company. Although the Chinese domestic market was hit hard at the beginning of the pandemic (February and March) when COVID-19 was mostly spreading within China, the overseas market was affected badly from March onward when the virus was spreading rapidly within Europe, US, and other parts of the world. Understandably, companies that have both domestic and overseas markets can avoid the negative economic impact better than companies that have a single market focus. Such companies with a diverse market focus could sell their products to overseas consumers in February and March while markets in China were subdued, but then switch to selling their produce/services in China after March once overseas markets had declined. Hence, based on slack resources theory and available funds hypothesis, a third hypothesis could be framed as: 
Hypothesis 3 (H3). Companies that enjoy both domestic and overseas markets prioritize social and environmental sustainability more than companies that focus solely on either domestic market or overseas market after COVID-19.

\subsection{Data Collection}

After a pilot study in June 2020, a questionnaire was finalized to explore if companies in China had changed their views of sustainable development. The questionnaire had a number of parts, including the demographic characterization of the surveyed companies, the general impact that COVID-19 had on them, how they coped with the impact, and the changing priority of the companies regarding the three-pillars of sustainable development. The targeted respondents were company owners and other managers across China, and the survey was designed to span all four types of company noted above as well as different sizes and market orientations. The survey was carried out online through a Chinese online survey platform Wenjuanxing in July 2020 with link to or QR code of the questionnaire being sent to potential respondents. A total of 1178 questionnaires were returned, of which 1160 (98\%) were considered valid in the sense that they came from different IP addresses, had a clear indication of their company ownership and the questionnaire had been completed in no less than $207 \mathrm{~s}$ (the shortest time tested by the authors to complete the questionnaire). There was no reward for completing the questionnaire, so respondents had little incentive to attempt multiple returns. While it was possible for a single respondent to complete multiple returns from a number of IP addresses, it would seem unlikely in practice.

Respondents' views of the importance of the three dimensions of SD (i.e., social, environmental, and economic) during three periods of COVID-19 (i.e., pre, during, and post) were assessed based on a five-point Likert scale with 1 being the least important, 5 being the most important, and the others are in the middle - the higher the score, the higher the perceived importance of the dimension. The average change (mean change) of the scores at later stages of COVID-19 compared to earlier stages (i.e., during vs. pre, post vs. during, and post vs. pre) were calculated using the following equation:

Mean change (environmental sustainability, post vs. pre) $=$ Mean score $($ environmental sustainability, post) - Mean score (environmental sustainability, pre).

Similarly, for other changes in mean score:

Mean change (environmental sustainability, during vs. pre), Mean change (environmental sustainability, post vs. during), Mean change (social sustainability, post vs. pre), Mean change (social sustainability, during vs. pre), Mean change (social sustainability, post vs. during), Mean change (economic sustainability, post vs. pre), Mean change (economic sustainability, during vs. pre), and Mean change (economic sustainability, post vs. during).

If the mean change (environmental sustainability, during vs. pre) was bigger than zero, then environmental sustainability was assumed to have been prioritized more during COVID-19 [7]. If the mean change (environmental sustainability, post vs. during) was bigger than zero, then environmental sustainability was assumed to have been prioritized immediately after the pandemic. If the mean change (environmental sustainability, post vs. pre) was bigger than zero, then environmental sustainability was assumed to have been prioritized over the longer term after the COVID-19 impact had ended. The bigger the mean change, the higher the level of the prioritization. The same interpretations were applied to the other two dimensions of SD.

\subsection{Data Analysis}

SPSS 24 was employed to store and analyze the data. Crosstab and Chi-square tests were employed to test the correlation between company ownership, size, age, and market focus. Welch's ANOVA was used to test changes in priority of sustainable development during and post COVID-19 for Chinese companies with different ownership, size, and market focus. As the data comprised scores, and these could not be assumed to be normally distributed, Welch's ANOVA was deemed to be the best alternative and does have some advantages compared to non-parametric tests such as Kruskall-Wallis. Also, Chi-square and Welch's ANOVA are relatively straightforward to implement and interpret compared to alternative approaches such as regression or factor analysis. 


\section{Results and Discussions}

\subsection{Company Profile}

Table 1 shows the profiles of the surveyed companies based upon the answers provided by respondents. Table 1a presents the distribution of the sample of companies based on four main company categories:

- Ownership of the company (domestic private-owned company, SOE, FIE, and domestic individual-owned company)

- Number of employees (1000 and above, 300-999, 20-299, and 1-19)

- Age (less than 4 years, 4-10 years, 11-20 years, and older than 20 years)

- Market focus (overseas-focused, domestic-focused, mixed but mostly domestic-focused, and mixed but mostly export-focused)

Also shown in Table 1 are the geographical distribution of the companies in China (Table 1b) and their main area of activity (manufacturing, wholesale/retail, etc.; Table 1c).

Out of 1160 companies, $70.5 \%$ of them (818) were private-owned, 195 (16.8\%) were SOEs, $76(6.5 \%)$ were FIEs, and 71 (6.1\%) were individually-owned. Half of the companies (588) had between 20 and 299 employees, a quarter (292) had between 300 and 999, 157 (13.5\%) had 1000 employees and more, while only $123(10.6 \%)$ had less than 20 employees. In terms of age, almost half of them (554) had existed for between 4 and 10 years, 262 (22.6\%) had been around for between 11 and 20 years, and $119(10.2 \%)$ was established within the past 4 years. Based on where the customers were mostly located, 65 (5.6\%) of the companies saw themselves as overseas-focused, 201 (17.3\%) were domestic-focused, one third of them (380) had both domestic and overseas customers with the domestic market being the main focus, while $514(44.3 \%)$ also enjoyed both markets but were mostly overseas-focused.

The surveyed companies were headquartered in 32 regions in China; $241(20.8 \%)$ were in Beijing, 155 (13.4\%) were in Shanghai, 84 (7.2\%) in Zhejiang, 79 (6.8\%) in Jiangsu, 76 (6.5\%) in Hebei, 70 (6.0\%) in Guangdong, 68 (5.9\%) in Henan, 55 (4.7\%) in Sichuan, 53 (4.6\%) in Hubei, 37 (3.2\%) in Fujian, 240 (20.8\%) were in other parts of China. The companies covered 30 industries, including IT/e-commerce/internet service (22.1\%), manufacturing (13.7\%), wholesale/retail (7.7\%), fast moving consumer goods, i.e., snacks, drinks, cosmetics (6.9\%), education/research/training $(6.8 \%)$, clothing/textile/leather (4.9\%), catering/entertainment/tourism $(4.6 \%)$, real estate/construction $(4.5 \%)$, communication/network equipment/value-added service $(2.8 \%)$, finance $(2.5 \%)$, and $23.6 \%$ other industries. 
Table 1. Profile of the companies who respondent to the online survey.

\begin{tabular}{|c|c|c|c|c|c|c|c|c|c|c|}
\hline \multicolumn{11}{|c|}{ (a) Ownership, size, age, and market focus } \\
\hline \multicolumn{3}{|c|}{ Ownership of the company } & \multicolumn{2}{|c|}{$\begin{array}{l}\text { Domestic private-owned } \\
\text { company } \\
818(70.5 \%)\end{array}$} & \multicolumn{2}{|c|}{$\begin{array}{c}\text { State-owned } \\
\text { enterprise (SOE) } \\
195(16.8 \%)\end{array}$} & \multicolumn{2}{|c|}{$\begin{array}{l}\text { Foreign invested } \\
\text { company (FIE) } \\
76(6.5 \%)\end{array}$} & \multicolumn{2}{|c|}{$\begin{array}{c}\text { Domestic individual-owned } \\
\text { company } \\
71(6.1 \%)\end{array}$} \\
\hline \multicolumn{3}{|c|}{$\begin{array}{c}\text { Company size }^{\text {a }} \text { (number of employees or } \\
\text { persons) }\end{array}$} & \multicolumn{2}{|c|}{$\begin{array}{c}1000 \text { and above } \\
157(13.5 \%)\end{array}$} & \multicolumn{2}{|c|}{$\begin{array}{c}300-999 \\
292(25.2 \%)\end{array}$} & \multicolumn{2}{|c|}{$\begin{array}{c}20-299 \\
588(50.7 \%)\end{array}$} & \multicolumn{2}{|c|}{$\begin{array}{c}1-19 \\
123(10.6 \%) \\
\end{array}$} \\
\hline \multicolumn{3}{|c|}{ Age of the company (year) } & \multicolumn{2}{|c|}{$\begin{array}{c}\text { Less than } 4 \text { years } \\
119(10.3 \%)\end{array}$} & \multicolumn{2}{|c|}{$\begin{array}{c}4-10 \text { (inc. 10) } \\
554(47.8 \%)\end{array}$} & \multicolumn{2}{|c|}{$\begin{array}{l}11-20 \text { (inc. } 20) \\
262(22.6 \%)\end{array}$} & \multicolumn{2}{|c|}{$\begin{array}{c}\text { Longer than } 20 \text { years } \\
225(19.4 \%)\end{array}$} \\
\hline \multicolumn{3}{|c|}{ Market focus of the company } & \multicolumn{2}{|c|}{$\begin{array}{l}\text { Overseas-focused } \\
\qquad 65(5.6 \%)\end{array}$} & \multicolumn{2}{|c|}{$\begin{array}{l}\text { Domestic-focused } \\
201(17.3 \%)\end{array}$} & \multicolumn{2}{|c|}{$\begin{array}{l}\text { Mixed but mostly } \\
\text { domestic-focused } \\
380(32.8 \%)\end{array}$} & \multicolumn{2}{|c|}{$\begin{array}{l}\text { Mixed but mostly } \\
\text { export-focused } \\
514(44.3 \%)\end{array}$} \\
\hline \multicolumn{11}{|c|}{ (b) Regional distribution (32 regions) } \\
\hline Beijing & Shanghai & Zhejiang & Jiangsu & Hebei & Guangdong & Henan & Sichuan & Hubei & Fujian & others \\
\hline $241(20.8 \%)$ & $155(13.4 \%)$ & $84(7.2 \%)$ & $79(6.8 \%)$ & $76(6.5 \%)$ & $70(6.0 \%)$ & $68(5.9 \%)$ & $55(4.7 \%)$ & $53(4.6 \%)$ & $37(3.2 \%)$ & $240(20.8 \%)$ \\
\hline \multicolumn{11}{|c|}{ (c) Industry distribution (30 industries) } \\
\hline $\begin{array}{l}\mathrm{IT} / \mathrm{e}-\text { commerce/ } \\
\text { internet service }\end{array}$ & Manufacturing & $\begin{array}{l}\text { Wholesale/ } \\
\text { retail }\end{array}$ & $\begin{array}{l}\text { Fast moving } \\
\text { consumer } \\
\text { goods }\end{array}$ & $\begin{array}{l}\text { Education/ } \\
\text { training/ } \\
\text { research }\end{array}$ & $\begin{array}{l}\text { Clothing/ } \\
\text { textile/ } \\
\text { leather }\end{array}$ & $\begin{array}{l}\text { Catering/ } \\
\text { entertainment/ } \\
\text { tourism }\end{array}$ & $\begin{array}{l}\text { Real estate/ } \\
\text { construction }\end{array}$ & $\begin{array}{l}\text { Commu } \\
\text { network } \\
\text { value-ado }\end{array}$ & $\begin{array}{l}\text { nication/ } \\
\text { quipment/ } \\
\text { ed service }\end{array}$ & Others \\
\hline $256(22.1 \%)$ & $159(13.7 \%)$ & $89(7.7 \%)$ & $80(6.9 \%)$ & $79(6.8 \%)$ & $57(4.9 \%)$ & $53(4.6 \%)$ & $52(4.5 \%)$ & 32( & $.8 \%)$ & $304(26.1 \%)$ \\
\hline
\end{tabular}

Note: The number of surveyed companies was 1160 and the figures are the number of companies that fall into that category (based upon answers provided by respondents) along with the percentage of the sample. a Company size is classified based on the official government document Notice of Issuing the Classification Criterion for SMEs (Ministry of Industry and Enterprises (2011) No. 300). 


\subsection{Ownership, Characterization, the General Impact of COVID-19, and Current Business Resumption}

Table 2 indicates that company characterization (size, age, and market focus) and company ownership are significantly correlated (Pearson Chi-square tests all statistically significant at $p<0.01$ ). As perhaps would be expected, it seems SOEs and FIEs are bigger in terms of number of employees, while private and individual owned companies, especially individual-owned, are smaller in size. SOEs and FIEs tend to have existed for longer than private and individual owned companies. In terms of the main market, SOEs tend to have mixed market, FIEs tend to have a more export focused market, private-owned companies tend to have a more domestic focused market, while individual-owned companies enjoy either overseas market or domestic market.

Generally speaking, companies with different ownerships have been impacted by COVID-19 to different extents. SOEs would seem to have been the least impacted with $28.7 \%$ of respondents in that category claiming that the company had been impacted in a 'major' way. The equivalent figure for FIEs was $31.6 \%$. Individual-owned and private-owned companies appear to have been hit much harder by the pandemic with $54.9 \%$ and $43.5 \%$ (respectively) of respondents from those categories claiming that their companies had been majorly impacted by the pandemic. This is perhaps to be expected given that SOEs are state-backed and perhaps have better access to bank loans and state support. However, private-owned companies resumed their business almost as fast as SOEs with $34.4 \%$ of private-owned companies recovering more than $75 \%$ of their production capacity ( $35.9 \%$ for SOEs), followed by FIEs $(30.3 \%)$. Individual-owned companies recovered the slowest with only $12.7 \%$ of them recovering more than $75 \%$ of their production capacity. Perhaps this is partly related to the speed at which China was able to bring the outbreak under control, but it may also be related to the smaller size of individually owned companies which means that they find it harder to lever support from banks and the state. 
Table 2. Crosstab and Chi-square test between company ownership and their characterization, general impact, and current business resumption.

\begin{tabular}{|c|c|c|c|c|c|c|c|}
\hline \multirow[t]{2}{*}{$\begin{array}{c}\text { Characterization } \\
\text { /Impact/Resumption }\end{array}$} & \multirow[t]{2}{*}{ Categories } & \multicolumn{4}{|c|}{$\begin{array}{c}\text { Ownership } \\
\text { Top Figures: Observed (Expected) Counts } \\
\text { Bottom Figures: \% within Column }\end{array}$} & \multirow[t]{2}{*}{ Total } & \multirow[t]{2}{*}{ Chi-Square Tests } \\
\hline & & SOE & Private-Owned & FIE & Individual-Owned & & \\
\hline \multirow{7}{*}{ Size (No. of employees) } & \multirow{2}{*}{$1-19$} & $3(20.7)$ & $70(86.7)$ & $2(8.1)$ & 48 (7.5) & 123 & \multirow{7}{*}{$\begin{array}{l}\text { Pearson Chi-Square: } 414.779 \\
\text { df: } 9 \\
\text { Sig. } \\
\text { Note: } 0 \text { cells have expected count less than } 5 . \\
\text { The minimum expected count is } 7.53\end{array}$} \\
\hline & & 1.5 & 8.6 & 2.6 & 67.6 & 10.6 & \\
\hline & $20-299$ & $53(98.8)$ & 494 (414.6) & $23(38.5)$ & $18(36.0)$ & 588 & \\
\hline & & 27.2 & 60.4 & 30.3 & 25.4 & 50.7 & \\
\hline & $300-999$ & $\begin{array}{c}79(49.1) \\
40.5\end{array}$ & $184(205.9)$ & $25(19.1)$ & $4(17.9)$ & 292 & \\
\hline & \multirow{2}{*}{1000 and above } & $60(26.4)$ & $70(110.7)$ & $\begin{array}{c}32.9 \\
26(10.3)\end{array}$ & $\begin{array}{c}5.6 \\
1(9.6)\end{array}$ & $\begin{array}{l}25.2 \\
157\end{array}$ & \\
\hline & & 30.8 & 8.6 & 34.2 & 1.4 & 13.5 & \\
\hline \multirow{8}{*}{ Age (No. of years established) } & \multirow{2}{*}{ Less than 4} & $5(20.0)$ & $88(83.9)$ & $2(7.8)$ & $24(7.3)$ & 119 & \multirow{8}{*}{$\begin{array}{c}\text { Pearson Chi-Square: } 239.951 \\
\text { df: } 9 \\
\text { Sig. (2-sided): } 0.000 \text { *** } \\
\text { Note: } 0 \text { cells have expected count less than } 5 . \\
\text { The minimum expected count is } 7.28 \text {. }\end{array}$} \\
\hline & & 2.6 & 10.8 & 2.6 & 33.8 & 10.3 & \\
\hline & $4-10$ & 40 (93.1) & 448 (390.7) & $25(36.3)$ & $41(33.9)$ & 554 & \\
\hline & \multirow{3}{*}{$11-20$} & 20.5 & 54.8 & 32.9 & 57.7 & 47.8 & \\
\hline & & $53(44.0)$ & 178 (184.8) & $26(17.2)$ & $5(16)$ & 262 & \\
\hline & & 27.2 & 21.8 & 34.2 & 7.0 & 22.6 & \\
\hline & \multirow{2}{*}{ Above 20} & $97(37.8)$ & 104 (158.7) & $23(14.7)$ & $1(13.8)$ & 225 & \\
\hline & & 49.7 & 12.7 & 30.3 & 1.4 & 19.4 & \\
\hline \multirow{7}{*}{ Market focus } & \multirow{2}{*}{ Overseas-focused } & $10(10.9)$ & $44(45.8)$ & $5(4.3)$ & $6(4.0)$ & 65 & \multirow{7}{*}{$\begin{array}{c}\text { Pearson Chi-Square: } 58.235 \\
\text { df: } 9 \\
\text { Sig. }(2 \text {-sided): } 0.000 \text { *** } \\
\text { Note: } 2 \text { cells (12.5\%) have expected count less than } 5 . \\
\text { The minimum expected count is } 3.98 .\end{array}$} \\
\hline & & 5.1 & 5.4 & 6.6 & 8.5 & 5.6 & \\
\hline & Mixed but mostly & $91(86.4)$ & $352(362.5)$ & $50(33.7)$ & $21(31.5)$ & 514 & \\
\hline & overseas focused & 46.7 & 43.0 & 65.8 & 29.6 & 44.3 & \\
\hline & $\begin{array}{l}\text { Mixed but mostly } \\
\text { domestic focused }\end{array}$ & $\begin{array}{c}74(63.9) \\
37.9\end{array}$ & $\begin{array}{c}276(268.0) \\
33.7\end{array}$ & $\begin{array}{c}16(24.9) \\
21.1\end{array}$ & $\begin{array}{c}14(23.3) \\
19.7\end{array}$ & $\begin{array}{l}380 \\
32.8\end{array}$ & \\
\hline & \multirow{2}{*}{ Domestic focused } & $20(33.8)$ & $146(141.7)$ & $5(13.2)$ & $30(12.3)$ & 201 & \\
\hline & & 10.3 & 17.8 & 6.6 & 42.3 & 17.3 & \\
\hline \multirow{6}{*}{$\begin{array}{l}\text { General impact from } \\
\text { COVID-19 }\end{array}$} & \multirow{2}{*}{ No } & $12(6.2)$ & $19(26.1)$ & $2(2.4)$ & $4(2.3)$ & 37 & \multirow{6}{*}{$\begin{array}{c}\text { Pearson Chi-Square: } 30.360 \\
\text { df: } 6 \\
\text { Sig. (2-sided): } 0.000^{* * *} \\
\text { Note: } 2 \text { cells (16.7\%) have expected count less than } 5 . \\
\text { The minimum expected count is } 2.26 \text {. }\end{array}$} \\
\hline & & 6.2 & 2.3 & 2.6 & 5.6 & 3.2 & \\
\hline & \multirow{2}{*}{ Yes, minor } & $127(108.9)$ & $443(457.0)$ & $50(42.5)$ & $28(39.7)$ & 648 & \\
\hline & & 65.1 & 54.2 & 65.8 & 39.4 & 55.9 & \\
\hline & \multirow{2}{*}{ Yes, major } & $56(79.8)$ & $356(335.0)$ & $24(31.1)$ & $39(29.1)$ & 475 & \\
\hline & & 28.7 & 43.5 & 31.6 & 54.9 & 40.9 & \\
\hline \multirow{8}{*}{$\begin{array}{l}\text { Business resumption (\% of } \\
\text { full production capacity) after } \\
\text { COVID-19 }\end{array}$} & \multirow{2}{*}{$25 \%$ and less } & $4(7.6)$ & $33(31.7)$ & $1(2.9)$ & $7(2.8)$ & 45 & \multirow{8}{*}{$\begin{array}{c}\text { Pearson Chi-Square: } 27.024 \\
\text { df: } 9 \\
\text { Sig. }\left(2 \text {-sided): } 0.0011^{* * *}\right. \\
\text { Note: } 2 \text { cells }(12.5 \%) \text { have expected count less than } 5 . \\
\text { The minimum expected count is } 2.75 .\end{array}$} \\
\hline & & 2.1 & 4.0 & 1.3 & 9.9 & 3.9 & \\
\hline & $26-50 \%$ & $30(31.9)$ & $125(134.0)$ & $15(12.4)$ & $20(11.6)$ & 190 & \\
\hline & $26-50 \%$ & 15.4 & 15.3 & 19.7 & 28.2 & 16.4 & \\
\hline & & $91(91.1)$ & $379(382.2)$ & $37(35.5)$ & $35(33.2)$ & 542 & \\
\hline & $51-75 \%$ & 46.7 & 46.3 & 48.7 & 49.3 & 46.7 & \\
\hline & Above $75 \%$ & $70(64.4)$ & $281(270.1)$ & $23(25.1)$ & $9(23.4)$ & 383 & \\
\hline & & 35.9 & 34.4 & 30.3 & 12.7 & 33.0 & \\
\hline
\end{tabular}

Note: $\mathrm{N}=1160,{ }^{* * *}:$ statistically significant at $p<0.01$. 


\subsection{Sustainability Priority Change for Companies Having Different Types of Ownership}

Table 3 indicates that companies with different ownerships have statistically significant different social, economic, and environmental priorities before, during, and after COVID-19 (all F-values from Welch's ANOVA were statistically significant at $p<0.01$ ), and there is an increasing trend of means in each of the three dimensions of SD following the stages of COVID-19. For example, for private-owned companies, the mean score for the social dimension of SD increased from 3.30 prior to COVID-19 to 4.09 when this research was conducted in July 2020 (during the pandemic), and to 4.34 after the pandemic had ended in China. Clearly, COVID-19 has made companies with all types of ownership realize the importance of all three dimensions of SD. However, the highest mean scores in the table across all four types of company are for the economic dimension of SD in the post-COVID-19 stage (shaded cells in Table 3), indicating the importance of regaining economic strength over the longer term after the pandemic.

Table 3. Welch's ANOVA test of sustainability priority change for Chinese companies.

\begin{tabular}{|c|c|c|c|c|c|}
\hline Ownership & $\begin{array}{l}\text { Dimension of } \\
\text { Sustainability }\end{array}$ & $\begin{array}{c}\text { Stage of } \\
\text { COVID-19 }\end{array}$ & $\mathbf{N}$ & $\begin{array}{l}\text { Mean } \\
\text { Score }\end{array}$ & Sig. \\
\hline \multirow{9}{*}{ SOE } & \multirow{3}{*}{$\begin{array}{c}\text { Social } \\
\text { sustainability }\end{array}$} & Before & 195 & 3.38 & \multirow[t]{3}{*}{$0.000^{* * *}$} \\
\hline & & During & 195 & 4.16 & \\
\hline & & Post & 195 & 4.44 & \\
\hline & \multirow{3}{*}{$\begin{array}{c}\text { Economic } \\
\text { sustainability }\end{array}$} & Before & 195 & 3.65 & \multirow[t]{3}{*}{$0.000^{* * *}$} \\
\hline & & During & 195 & 4.16 & \\
\hline & & Post & 195 & 4.47 & \\
\hline & \multirow{3}{*}{$\begin{array}{l}\text { Environmental } \\
\text { sustainability }\end{array}$} & Before & 195 & 3.33 & \multirow[t]{3}{*}{$0.000^{* * *}$} \\
\hline & & During & 195 & 3.99 & \\
\hline & & Post & 195 & 4.24 & \\
\hline \multirow{9}{*}{$\begin{array}{c}\text { Domestic } \\
\text { private-owned } \\
\text { company }\end{array}$} & \multirow{3}{*}{$\begin{array}{c}\text { Social } \\
\text { sustainability }\end{array}$} & Before & 818 & 3.30 & \multirow[t]{3}{*}{$0.000^{* * *}$} \\
\hline & & During & 818 & 4.09 & \\
\hline & & Post & 818 & 4.34 & \\
\hline & \multirow{3}{*}{$\begin{array}{c}\text { Economic } \\
\text { sustainability }\end{array}$} & Before & 818 & 3.63 & \multirow[t]{3}{*}{$0.000^{* * *}$} \\
\hline & & During & 818 & 4.25 & \\
\hline & & Post & 818 & 4.48 & \\
\hline & \multirow{3}{*}{$\begin{array}{l}\text { Environmental } \\
\text { sustainability }\end{array}$} & Before & 818 & 3.39 & \multirow[t]{3}{*}{$0.000^{* * *}$} \\
\hline & & During & 818 & 4.02 & \\
\hline & & Post & 818 & 4.30 & \\
\hline \multirow{9}{*}{ FIE } & \multirow{3}{*}{$\begin{array}{c}\text { Social } \\
\text { sustainability }\end{array}$} & Before & 76 & 3.38 & \multirow[t]{3}{*}{$0.000^{* * *}$} \\
\hline & & During & 76 & 4.04 & \\
\hline & & Post & 76 & 4.26 & \\
\hline & \multirow{3}{*}{$\begin{array}{c}\text { Economic } \\
\text { sustainability }\end{array}$} & Before & 76 & 3.87 & \multirow[t]{3}{*}{$0.001^{* * *}$} \\
\hline & & During & 76 & 4.13 & \\
\hline & & Post & 76 & 4.46 & \\
\hline & \multirow{3}{*}{$\begin{array}{l}\text { Environmental } \\
\text { sustainability }\end{array}$} & Before & 76 & 3.46 & \multirow[t]{3}{*}{$0.000^{* * *}$} \\
\hline & & During & 76 & 4.01 & \\
\hline & & Post & 76 & 4.24 & \\
\hline \multirow{9}{*}{$\begin{array}{c}\text { Domestic } \\
\text { individual-owned } \\
\text { company }\end{array}$} & \multirow{3}{*}{$\begin{array}{c}\text { Social } \\
\text { sustainability }\end{array}$} & Before & 71 & 3.30 & \multirow[t]{3}{*}{$0.000^{* * *}$} \\
\hline & & During & 71 & 3.94 & \\
\hline & & Post & 71 & 4.28 & \\
\hline & \multirow{3}{*}{$\begin{array}{c}\text { Economic } \\
\text { sustainability }\end{array}$} & Before & 71 & 3.56 & \multirow[t]{3}{*}{$0.000^{* * *}$} \\
\hline & & During & 71 & 4.11 & \\
\hline & & Post & 71 & 4.41 & \\
\hline & \multirow{3}{*}{$\begin{array}{l}\text { Environmental } \\
\text { sustainability }\end{array}$} & Before & 71 & 3.37 & \multirow[t]{3}{*}{$0.000^{* * *}$} \\
\hline & & During & 71 & 3.90 & \\
\hline & & Post & 71 & 4.32 & \\
\hline
\end{tabular}

Note: ***: statistically significant at $p<0.01$, the shaded cells are the highest mean scores in the post-COVID-19 stage. 
A Welch's ANOVA test was performed on the scores for the dimensions of SD over the three periods of COVID-19 (pre, during, and post) and the results are shown in Table 4 . In order to more clearly illustrate the trends in Table 4, Figure 1 presents the changes in the mean scores between the periods of COVID-19.

Table 4. Welch's ANOVA test of differences among dimensions of sustainability for each COVID-19 stage.

\begin{tabular}{|c|c|c|c|c|c|}
\hline Ownership & $\begin{array}{c}\text { Stage of } \\
\text { COVID-19 }\end{array}$ & $\begin{array}{l}\text { Dimension of } \\
\text { Sustainability }\end{array}$ & $\mathbf{N}$ & $\begin{array}{l}\text { Mean } \\
\text { Score }\end{array}$ & Sig. \\
\hline \multirow{9}{*}{ SOE } & \multirow{3}{*}{ Before } & Social & 195 & 3.38 & \multirow[t]{3}{*}{$0.000 * * *$} \\
\hline & & Economic & 195 & 3.65 & \\
\hline & & Environmental & 195 & 3.33 & \\
\hline & \multirow{3}{*}{ During } & Social & 195 & 4.16 & \multirow[t]{3}{*}{0.642} \\
\hline & & Economic & 195 & 4.16 & \\
\hline & & Environmental & 195 & 3.99 & \\
\hline & \multirow{3}{*}{ Post } & Social & 195 & 4.44 & \multirow[t]{3}{*}{$0.000^{* * *}$} \\
\hline & & Economic & 195 & 4.47 & \\
\hline & & Environmental & 195 & 4.24 & \\
\hline \multirow{9}{*}{$\begin{array}{l}\text { Domestic private } \\
\text { owned company }\end{array}$} & \multirow{3}{*}{ Before } & Social & 818 & 3.30 & \multirow[t]{3}{*}{$0.000^{* * *}$} \\
\hline & & Economic & 818 & 3.63 & \\
\hline & & Environmental & 818 & 3.39 & \\
\hline & \multirow{3}{*}{ During } & Social & 818 & 4.09 & \multirow[t]{3}{*}{$0.000 * * *$} \\
\hline & & Economic & 818 & 4.25 & \\
\hline & & Environmental & 818 & 4.02 & \\
\hline & \multirow{3}{*}{ Post } & Social & 818 & 4.34 & \multirow[t]{3}{*}{$0.000^{* * *}$} \\
\hline & & Economic & 818 & 4.48 & \\
\hline & & Environmental & 818 & 4.30 & \\
\hline \multirow{9}{*}{$\begin{array}{l}\text { Foreign invested } \\
\text { enterprise }\end{array}$} & \multirow{3}{*}{ Before } & Social & 76 & 3.38 & \multirow[t]{3}{*}{0.016 ** } \\
\hline & & Economic & 76 & 3.87 & \\
\hline & & Environmental & 76 & 3.46 & \\
\hline & \multirow{3}{*}{ During } & Social & 76 & 4.04 & \multirow[t]{3}{*}{0.734} \\
\hline & & Economic & 76 & 4.13 & \\
\hline & & Environmental & 76 & 4.01 & \\
\hline & \multirow{3}{*}{ Post } & Social & 76 & 4.26 & \multirow[t]{3}{*}{0.236} \\
\hline & & Economic & 76 & 4.46 & \\
\hline & & Environmental & 76 & 4.24 & \\
\hline \multirow{9}{*}{$\begin{array}{l}\text { Domestic individual } \\
\text { owned company }\end{array}$} & \multirow{3}{*}{ Before } & Social & 71 & 3.30 & \multirow[t]{3}{*}{0.344} \\
\hline & & Economic & 71 & 3.56 & \\
\hline & & Environmental & 71 & 3.37 & \\
\hline & \multirow{3}{*}{ During } & Social & 71 & 3.94 & \multirow[t]{3}{*}{0.459} \\
\hline & & Economic & 71 & 4.11 & \\
\hline & & Environmental & 71 & 3.90 & \\
\hline & \multirow{3}{*}{ Post } & Social & 71 & 4.28 & \multirow[t]{3}{*}{0.709} \\
\hline & & Economic & 71 & 4.41 & \\
\hline & & Environmental & 71 & 4.32 & \\
\hline
\end{tabular}

Note: ***: statistically significant at $p<0.01,{ }^{* *}$ : statistically significant at $p<0.05$, the shaded cells are the highest mean scores in the post-COVID-19 stage. 
(a) $\mathrm{SOE}$

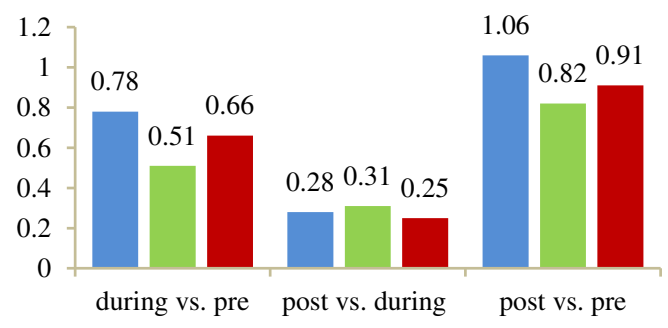

(c) Foreign invested enterprise

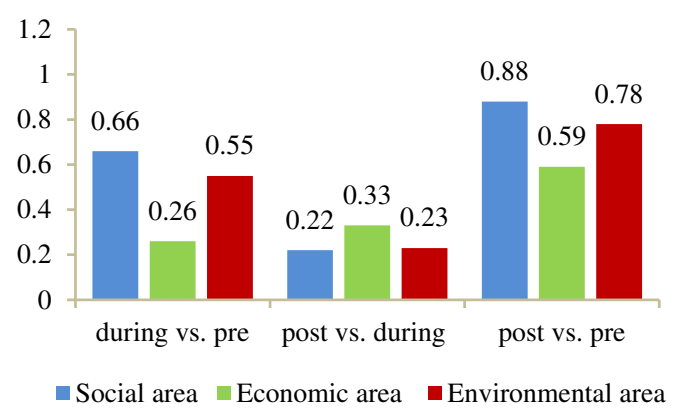

(b) Domestic private-owned company

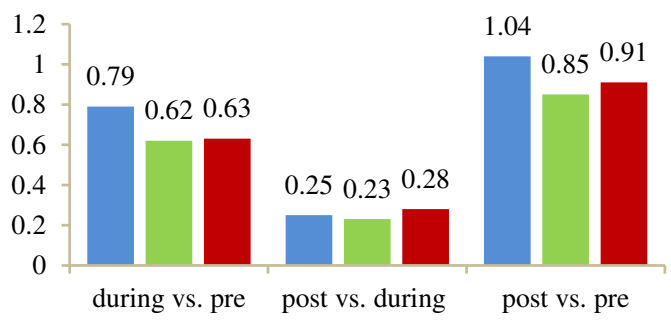

(d) Domestic individual-owned company

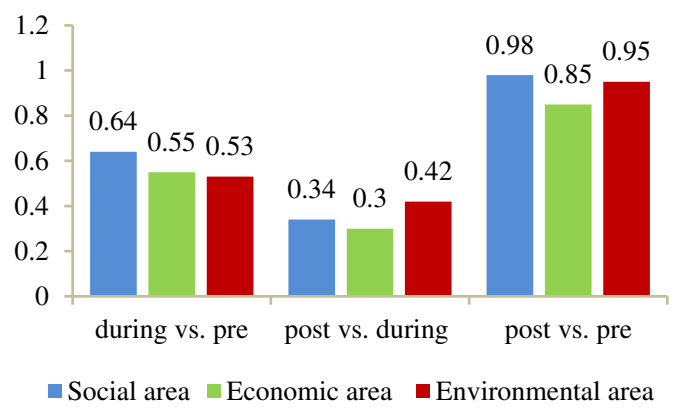

Figure 1. Mean change of the importance of each area of sustainable development at different stages of COVID-19 for companies with different ownership.

The results in Table 4 indicate that, for SOEs, there exists a significant difference among three dimensions of SD for pre- and post-COVID-19 periods, but there is no difference among dimensions of SD for the period during COVID-19, indicating the confusing state of Chinese SOEs during the pandemic. Figure 1a indicates that for Chinese SOEs, social sustainability was the most prioritized during the pandemic with a mean change (social responsibility, during vs. pre) in score of 0.78 , but it was less prioritized than economic sustainability immediately after the pandemic ( $0.28 \mathrm{vs.} 0.31)$; however, it was the most prioritized in a longer term after the pandemic comparing to economic and environmental sustainability (1.06 vs. 0.82 vs. 0.91). It would appear from Figure 1 that over the longer term SOEs prioritize social sustainability more than other companies (with a mean change of 1.06), followed by private-owned companies (1.04), individual-owned companies (0.98), and FIEs (0.88). It is not surprising given that Chinese SOEs have reportedly donated RMB 5.8 billion to help fight COVID-19, accounting for $47 \%$ of the total amount of corporate donations China received in response to COVID-19, while Chinese domestic private companies contributed $41 \%$ and FIEs only $12 \%$ [48]. Chinese SOEs' generosity in philanthropy has been well documented and the reason for their generosity is that they are highly integrated with the state and thus have looser budget constraints [49]. However, the motive of Chinese domestic private companies' donations, especially when facing a large-scale crisis, is mostly related to the protection of their property rights and nurturing of political connections, which in turn would lead to better profitability [50]. However, even with the long-term priority shift to the social dimension of SD after the pandemic, economic sustainability has remained absolutely essential post-COVID-19 for Chinese SOEs (4.47, Table 4), implying the falling importance of environmental sustainability at the post-COVID-19 era. Hence it would seem that Chinese SOEs are not prioritizing environmental sustainability as a result of COVID-19.

Table 4 also indicates that there exists a statistically significant difference among dimensions of SD for each stage of COVID-19 for Chinese domestic private-owned companies $(p<0.01)$, indicating a clear shift of priority of SD along with the development of COVID-19. In terms of the dynamics, Figure $1 \mathrm{~b}$ indicates that for Chinese domestic private-owned companies, social sustainability was the most prioritized during the pandemic, with a mean change (social sustainability, during vs. pre) equal to 0.79 , followed by environmental sustainability (0.63) and economic sustainability (0.62). But the priority shifted to environmental sustainability immediately after the pandemic $(0.28 \mathrm{vs} .0 .25 \mathrm{vs} .0 .23)$. 
However, the long-term priority after the pandemic shifted back to social sustainability, followed by environmental sustainability, then economic sustainability ( 1.04 vs. 0.91 vs. 0.85$)$. However, even with the priority shift to social sustainability after the pandemic, the absolute importance of the economic aspect of sustainability for this group of companies is clear with the highest mean score (4.48, Table 4). As with the SOEs, this indicates that environmental area of SD is also the least important dimension of sustainability for Chinese domestic private-owned companies.

The results in Table 4 for FIEs indicate that there is a statistically significant difference among three dimensions of SD for the pre-COVID-19 period. But such difference does not exist for periods amid and post the pandemic. Figure 1c presents the mean changes in score for the FIEs. Clearly, during the pandemic, social sustainability has gained priority $(0.66)$, more than environmental sustainability $(0.55)$, while the least prioritized one is economic sustainability (0.26). But immediately after the pandemic, the priority shifted to economic aspect of SD with the mean change (economic sustainability, post vs. during) being 0.33 , followed by environmental sustainability $(0.23)$ and social sustainability $(0.22)$. However, the long-term priority after the pandemic returned to the social area of sustainability with the mean change (social sustainability, post vs. pre) being 0.88 , followed by environmental sustainability (0.78) and economic sustainability (0.59). Hence it would seem that FIEs in China were uncertain what to prioritize during and post the pandemic. However, with the highest mean scores pre-, during, and post-COVID-19, economic sustainability remains the all-time essential for FIEs operating in China.

For the Chinese domestic individual-owned companies, Table 4 suggests that there is no statistically significant difference among the dimensions of SD for each COVID-19 stage. However, just like other types of companies, Chinese domestic individual-owned companies see economic sustainability as essential throughout the pandemic stages (pre, during, and post, Table 4). However, in terms of the changes in score, Figure 1d indicates that Chinese domestic individual-owned companies prioritized social sustainability the most during COVID-19 with mean change (social sustainability, during vs. pre) being 0.64 , followed by economic sustainability (0.55) and environmental sustainability $(0.53)$. The most prioritized immediately after COVID-19 is environmental sustainability $(0.42)$, followed by social sustainability (0.34) and economic sustainability (0.30). But over the longer term, the individual-owned companies prioritized social sustainability the most (0.98), followed by environmental sustainability (0.95) and economic sustainability (0.85).

Based on these results, Figure 2 provides a picture of the longer-term post-pandemic priority and post-pandemic business essential for companies with different ownership. It is clear that hypothesis 1 has failed. Chinese SOEs and private companies have responded to COVID-19 by prioritizing social sustainability more during the pandemic and also in the longer term following the pandemic. By way of contrast, FIEs and individual-owned companies have prioritized social sustainability less since the outbreak of COVID-19 (Figure 2a). This result is consistent with the findings of Li and Zhang [27], Zhang et al. [28], and Oh et al. [29] that state ownership has a positive relationship with social performance. However, the result is contrary to those of Oh et al. [29] and Lee et al. [30] who found that foreign invested firms are good CSR performers. It is a disappointing finding considering the large number of FIEs in China (960,725 by the end of 2018 [51]), whose contribution to social and environmental development would make a difference to China's long-term sustainability.

However, even with a priority for the social and environmental dimensions, economic sustainability is considered essential by all types of companies post-COVID-19, and this is more obvious for SOEs, domestic private-owned companies, and FIEs (Figure $2 b$ ), suggesting that environmental sustainability is the only one of the three dimensions that has been overlooked after the pandemic. This confirms the concerns of a post-pandemic environmental rebound effect as mentioned by Freire-Gonzalez and Vivanco [4], McCloskey and Heymann [5], Wang and Su [42], and Bao and Zhang [43]. It is even more worrying considering the bigger influence of SOEs, domestic private-owned companies, and FIEs in China's long-term sustainability. 
(a) Longer term post-pandemic priority

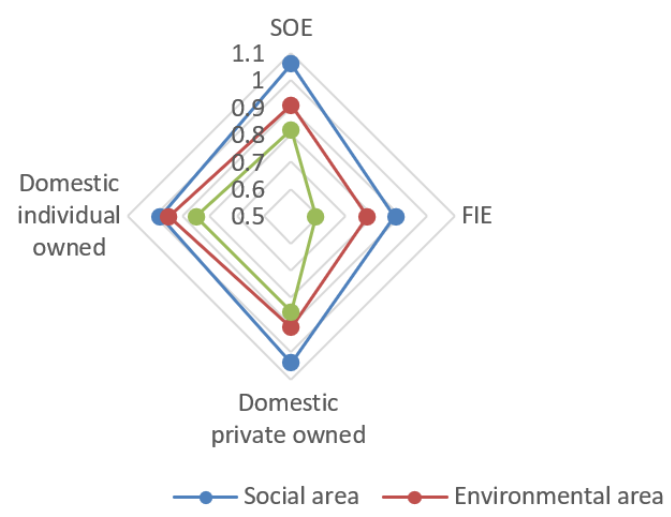

(b) Post-pandemic business essential

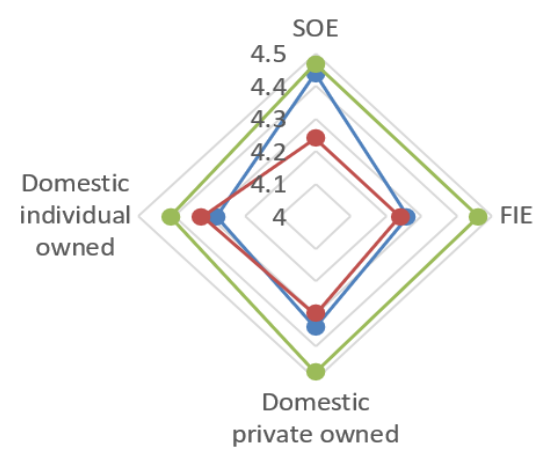

Figure 2. Longer term post-pandemic priority and post-pandemic business essential for companies with different ownership.

\subsection{Company Size Analysis}

As noted in Table 2, company size is related to company type, with SOEs and FIEs being larger in terms of number of employees compared to the domestic private companies. But in order to pick up on any effects of company size, Table 5 presents the results of a Welch's ANOVA test using four categories based on company size. Large companies (mostly SOEs and FIEs) are defined as those having 1000 or more employees, medium companies are those having 300 to 999 employees, small companies are those having 20 to 299 employees, and micro-companies are those having 1-19 employees.

The results in Table 5 indicate that no matter what size the companies are, their social, economic, and environmental aspects of sustainability are statistically different before, during, and post COVID-19, with a clear increasing trend of the importance of all of them as the pandemic progressed. Again, the highest values go to the post-pandemic economic sustainability for companies of all sizes (shaded cells in Table 5). This is further evidence that although companies of all sizes are devoted to all areas of sustainable development, economic sustainability will still be essential in the post-pandemic era.

Figure 3 indicates that comparing to pre-COVID-19, big-, medium-, and small-sized companies prioritized social sustainability the most during the pandemic among three areas of SD with the mean changes (social sustainability, during vs. pre) being $0.68,0.77$, and 0.82 , respectively (the left clusters of Figure 3), while micro-sized companies prioritized both social and economic sustainability during the same timeframe (both are 0.65 ). The priorities vary for the period between post and during COVID-19 (the middle clusters of Figure 3), while large companies prioritize economic area (with the mean change (economic sustainability, post vs. during) of 0.3 ), medium-sized companies prioritize social area (0.26), small and micro companies prioritize environmental area (0.29 and 0.41$)$. However, comparing to pre-COVID-19 period, the post pandemic priorities are still social issues for companies of all sizes (the right clusters of Figure 3). But surprisingly, small companies (1.06) appeared to emphasize social sustainability the most, followed by medium-sized companies (1.03) and micro companies (0.97). Big companies appeared to place relatively less emphasis on social issues after the pandemic (0.95) as illustrated in Figure 4a. Hence, hypothesis 2 is proved to be false. 
Table 5. Welch's ANOVA test of priority change for companies with different sizes.

\begin{tabular}{|c|c|c|c|c|c|}
\hline Size of Company & Sustainability & COVID-19 Stage & $\mathbf{N}$ & Mean & Sig. \\
\hline \multirow{9}{*}{ Large } & \multirow{3}{*}{$\begin{array}{c}\text { Social } \\
\text { sustainability }\end{array}$} & Before & 157 & 3.50 & \multirow[t]{3}{*}{$0.000 * * *$} \\
\hline & & During & 157 & 4.18 & \\
\hline & & Post & 157 & 4.45 & \\
\hline & \multirow{3}{*}{$\begin{array}{c}\text { Economic } \\
\text { sustainability }\end{array}$} & Before & 157 & 3.67 & \multirow[t]{3}{*}{$0.000 * * *$} \\
\hline & & During & 157 & 4.20 & \\
\hline & & Post & 157 & 4.50 & \\
\hline & \multirow{3}{*}{$\begin{array}{l}\text { Environmental } \\
\text { sustainability }\end{array}$} & Before & 157 & 3.29 & \multirow[t]{3}{*}{$0.000^{* * *}$} \\
\hline & & During & 157 & 3.95 & \\
\hline & & Post & 157 & 4.21 & \\
\hline \multirow{9}{*}{ medium } & \multirow{3}{*}{$\begin{array}{c}\text { Social } \\
\text { sustainability }\end{array}$} & Before & 292 & 3.35 & \multirow[t]{3}{*}{$0.000 * * *$} \\
\hline & & During & 292 & 4.12 & \\
\hline & & Post & 292 & 4.38 & \\
\hline & \multirow{3}{*}{$\begin{array}{c}\text { Economic } \\
\text { sustainability }\end{array}$} & Before & 292 & 3.68 & \multirow[t]{3}{*}{$0.000 * * *$} \\
\hline & & During & 292 & 4.28 & \\
\hline & & Post & 292 & 4.53 & \\
\hline & \multirow{3}{*}{$\begin{array}{l}\text { Environmental } \\
\text { sustainability }\end{array}$} & Before & 292 & 3.40 & \multirow[t]{3}{*}{$0.000^{* * *}$} \\
\hline & & During & 292 & 4.09 & \\
\hline & & Post & 292 & 4.30 & \\
\hline \multirow{9}{*}{ Small } & \multirow{3}{*}{$\begin{array}{c}\text { Social } \\
\text { sustainability }\end{array}$} & Before & 589 & 3.26 & \multirow[t]{3}{*}{$0.000^{* * *}$} \\
\hline & & During & 589 & 4.08 & \\
\hline & & Post & 589 & 4.32 & \\
\hline & \multirow{3}{*}{$\begin{array}{c}\text { Economic } \\
\text { sustainability }\end{array}$} & Before & 589 & 3.65 & \multirow[t]{3}{*}{$0.000 * * *$} \\
\hline & & During & 589 & 4.20 & \\
\hline & & Post & 589 & 4.46 & \\
\hline & \multirow{3}{*}{$\begin{array}{l}\text { Environmental } \\
\text { sustainability }\end{array}$} & Before & 589 & 3.40 & \multirow[t]{3}{*}{$0.000^{* * *}$} \\
\hline & & During & 589 & 4.01 & \\
\hline & & Post & 589 & 4.30 & \\
\hline \multirow{9}{*}{ Micro } & \multirow{3}{*}{$\begin{array}{c}\text { Social } \\
\text { sustainability }\end{array}$} & Before & 123 & 3.29 & \multirow[t]{3}{*}{$0.000^{* * *}$} \\
\hline & & During & 123 & 3.94 & \\
\hline & & Post & 123 & 4.26 & \\
\hline & \multirow{3}{*}{$\begin{array}{c}\text { Economic } \\
\text { sustainability }\end{array}$} & Before & 123 & 3.51 & \multirow[t]{3}{*}{$0.000^{* * *}$} \\
\hline & & During & 123 & 4.16 & \\
\hline & & Post & 123 & 4.33 & \\
\hline & \multirow{3}{*}{$\begin{array}{l}\text { Environmental } \\
\text { sustainability }\end{array}$} & Before & 123 & 3.34 & \multirow[t]{3}{*}{$0.000^{* * *}$} \\
\hline & & During & 123 & 3.84 & \\
\hline & & Post & 123 & 4.25 & \\
\hline
\end{tabular}

Note: ***: statistically significant at $p<0.01$, shade cells are the highest mean scores of companies of all sizes in the post-COVID-19 stage.

This result is contrary to previous study results which suggest that bigger companies are more socially responsible than smaller companies either because big companies have more resources which can be used for social issues or because large companies face more public social scrutiny, and therefore must be more responsive to social issues [24,34,52-54]. Maybe large companies were impacted so much by COVID-19 (97.5\% of the big companies in the survey were negatively affected by the pandemic and the number for the whole sample is $96.8 \%$ ) that they decided to withdraw from social responsibility.

Again, the economic dimension of SD remains essential for companies of all sizes, especially the medium and large companies (Figure 4b). This leaves the environmental side of SD to be the most vulnerable, another indication of post-pandemic environmental rebound effect. 
(a) Big

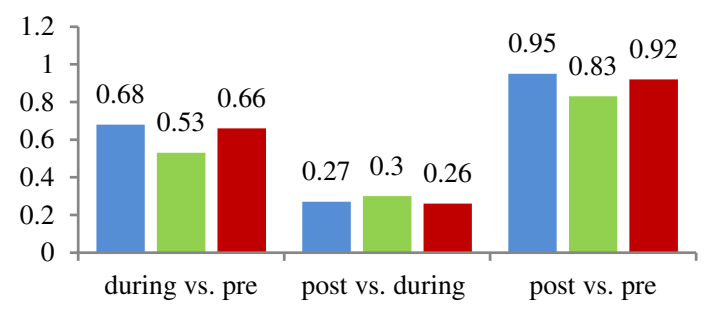

(c) Small

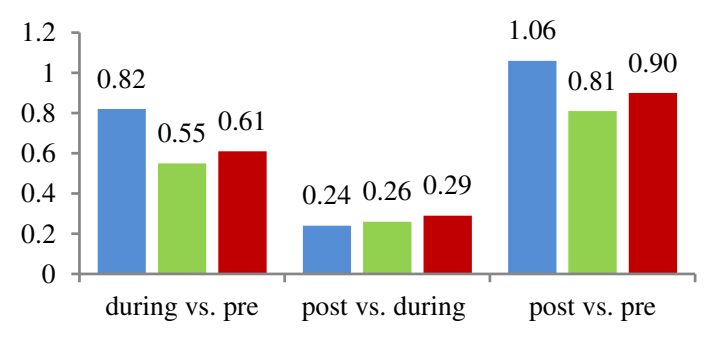

$\square$ Social area $\backsim$ Economic area Environmental area (b) Medium

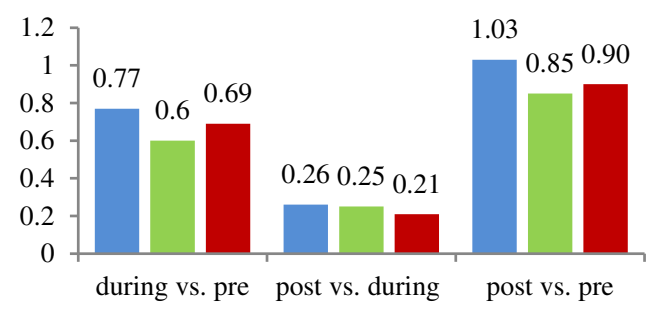

(d) Micro

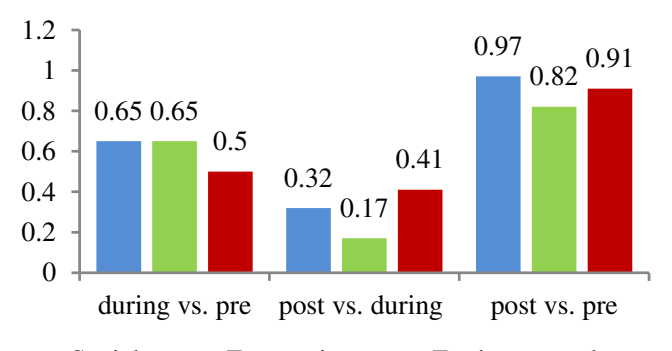

$\llbracket$ Social area $\llbracket$ Economic area $₫$ Environmental area

Figure 3. Mean change of each area of sustainable development for different sizes.

(a) Longer term post-pandemic priority

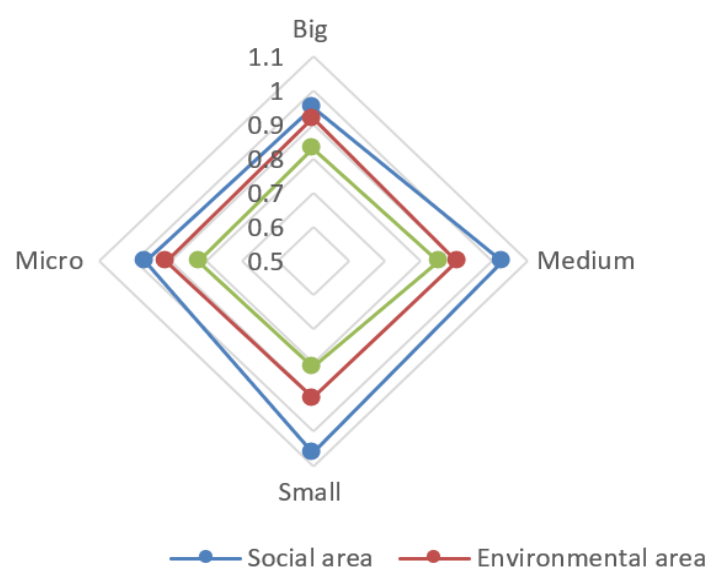

(b) Post-pandemic business essential

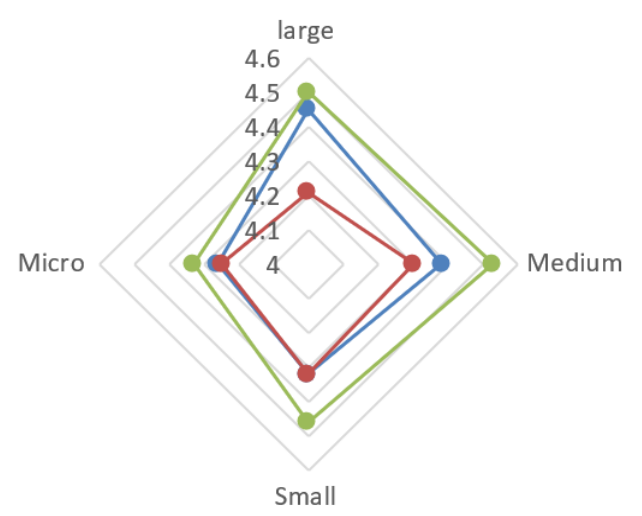

Figure 4. Longer term post-pandemic priority and post-pandemic business essential for companies with different size

\subsection{Market Focus Analysis}

Table 6 presents the score data based on the market focus (domestic, overseas, or mixtures of both) of the company. As noted earlier, the different types of company have different market focuses and there is a linkage (Table 2) between the two. The results in Table 6 suggest that for all types of market focus the companies have increased their concentration on the three dimensions of sustainability as the COVID-19 pandemic progressed. In line with the previous analyses, economic sustainability emerges as having the greatest emphasis once the pandemic ends (shaded cells in Table 6). 
Table 6. Welch's ANOVA test of priority change for companies with different market focus.

\begin{tabular}{|c|c|c|c|c|c|}
\hline Market Orientation & $\begin{array}{l}\text { Dimensions of } \\
\text { Sustainability }\end{array}$ & $\begin{array}{c}\text { COVID-19 } \\
\text { Stages }\end{array}$ & $\mathbf{N}$ & Mean & Sig. \\
\hline \multirow{9}{*}{ Overseas focused } & \multirow{3}{*}{$\begin{array}{c}\text { Social } \\
\text { sustainability }\end{array}$} & Before & 65 & 3.40 & \multirow[t]{3}{*}{$0.000^{* * *}$} \\
\hline & & During & 65 & 3.94 & \\
\hline & & Post & 65 & 4.28 & \\
\hline & \multirow{3}{*}{$\begin{array}{c}\text { Economic } \\
\text { sustainability }\end{array}$} & Before & 65 & 3.71 & \multirow[t]{3}{*}{$0.000^{* * *}$} \\
\hline & & During & 65 & 4.09 & \\
\hline & & Post & 65 & 4.58 & \\
\hline & \multirow{3}{*}{$\begin{array}{l}\text { Environmental } \\
\text { sustainability }\end{array}$} & Before & 65 & 3.57 & \multirow[t]{3}{*}{$0.000^{* * *}$} \\
\hline & & During & 65 & 4.09 & \\
\hline & & Post & 65 & 4.32 & \\
\hline \multirow{9}{*}{ Domestic orientated } & \multirow{3}{*}{$\begin{array}{c}\text { Social } \\
\text { sustainability }\end{array}$} & Before & 201 & 3.43 & \multirow[t]{3}{*}{$0.000^{* * *}$} \\
\hline & & During & 201 & 4.10 & \\
\hline & & Post & 201 & 4.29 & \\
\hline & \multirow{3}{*}{$\begin{array}{c}\text { Economic } \\
\text { sustainability }\end{array}$} & Before & 201 & 3.74 & \multirow[t]{3}{*}{$0.000^{* * *}$} \\
\hline & & During & 201 & 4.25 & \\
\hline & & Post & 201 & 4.48 & \\
\hline & \multirow{3}{*}{$\begin{array}{l}\text { Environmental } \\
\text { sustainability }\end{array}$} & Before & 201 & 3.57 & \multirow[t]{3}{*}{$0.000^{* * *}$} \\
\hline & & During & 201 & 4.09 & \\
\hline & & Post & 201 & 4.31 & \\
\hline \multirow{9}{*}{$\begin{array}{l}\text { Mixed but mostly } \\
\text { overseas focused }\end{array}$} & \multirow{3}{*}{$\begin{array}{c}\text { Social } \\
\text { sustainability }\end{array}$} & Before & 514 & 3.27 & \multirow[t]{3}{*}{$0.000^{* * *}$} \\
\hline & & During & 514 & 4.08 & \\
\hline & & Post & 514 & 4.32 & \\
\hline & \multirow{3}{*}{$\begin{array}{c}\text { Economic } \\
\text { sustainability }\end{array}$} & Before & 514 & 3.53 & \multirow[t]{3}{*}{$0.000^{* * *}$} \\
\hline & & During & 514 & 4.16 & \\
\hline & & Post & 514 & 4.39 & \\
\hline & \multirow{3}{*}{$\begin{array}{l}\text { Environmental } \\
\text { sustainability }\end{array}$} & Before & 514 & 3.37 & \multirow[t]{3}{*}{$0.000^{* * *}$} \\
\hline & & During & 514 & 3.96 & \\
\hline & & Post & 514 & 4.26 & \\
\hline \multirow{9}{*}{$\begin{array}{l}\text { Mixed but mostly } \\
\text { domestic orientated }\end{array}$} & \multirow{3}{*}{$\begin{array}{c}\text { Social } \\
\text { sustainability }\end{array}$} & Before & 381 & 3.31 & \multirow[t]{3}{*}{$0.000^{* * *}$} \\
\hline & & During & 381 & 4.12 & \\
\hline & & Post & 381 & 4.43 & \\
\hline & \multirow{3}{*}{$\begin{array}{c}\text { Economic } \\
\text { sustainability }\end{array}$} & Before & 381 & 3.75 & \multirow[t]{3}{*}{$0.000^{* * *}$} \\
\hline & & During & 381 & 4.30 & \\
\hline & & Post & 381 & 4.55 & \\
\hline & \multirow{3}{*}{$\begin{array}{l}\text { Environmental } \\
\text { sustainability }\end{array}$} & Before & 381 & 3.25 & \multirow[t]{3}{*}{$0.000^{* * *}$} \\
\hline & & During & 381 & 4.02 & \\
\hline & & Post & 381 & 4.29 & \\
\hline
\end{tabular}

Note: ${ }^{* * *}$ : statistically significant at $p<0.01$, shaded cells are the highest mean scores for companies with different market focus.

Figure 5 indicates that comparing to the pre-pandemic era, social sustainability has shown the largest change in priority during COVID-19 (the left clusters of Figure 5), especially for companies with mixed-market focuses (mean changes for both are 0.81 ), followed by domestic-focused companies $(0.67)$ and overseas-focused companies (0.54). Comparing to the time during the pandemic, the post-pandemic priorities vary (the middle clusters of Figure 5), with economic sustainability being prioritized by overseas-focused (0.49) and domestic-focused (0.23) companies, and environmental sustainability being prioritized by companies with mixed but mostly overseas market-focused companies $(0.30)$, and social sustainability being prioritized by overseas-focused companies (0.34). But the priority from pre- to post-pandemic is still social sustainability (the right clusters of Figure 5) with the lead being taken by the mixed but mostly domestic-focused companies (1.12), followed by mixed but mostly overseas-focused companies (1.05), solely overseas-focused companies (0.88), and then solely domestic-focused companies $(0.86)$. 
(a) Overseas focused

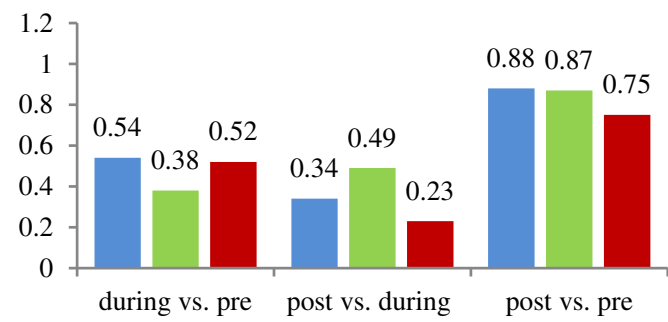

(c) Mixed but mostly overseas focused

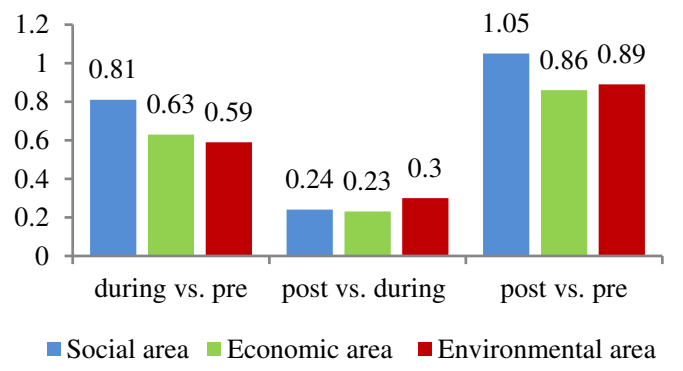

(b) Domestic focused

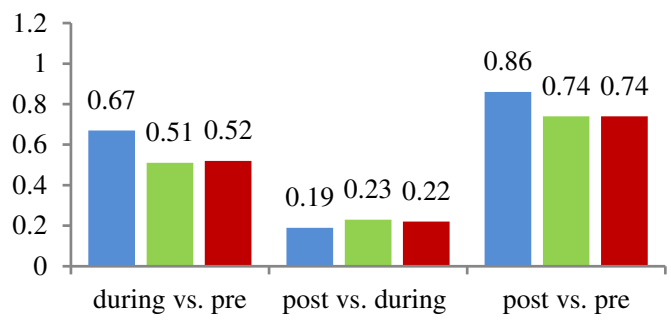

(d) Mixed but mostly domestic focused

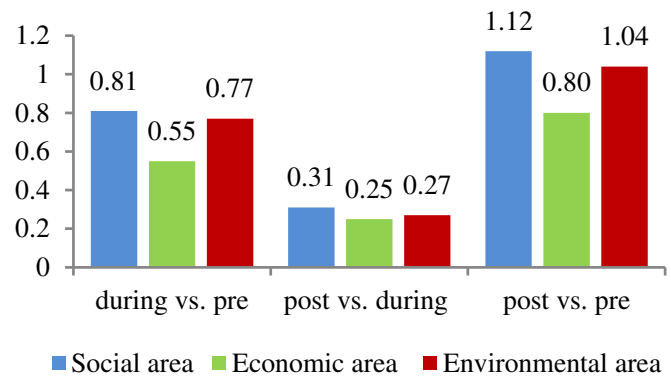

Figure 5. Mean change of each area of sustainable development for companies with different product markets.

Therefore, the test of hypothesis 3 is passed. Indeed, possibly because of their better financial situation during the pandemic ( $38 \%$ of them were impacted badly while the number for the whole sample was $41 \%$ ), companies with mixed markets prioritized social sustainability the most after the pandemic, followed by environmental sustainability. The levels of prioritization of mixed-market companies towards social and environmental sustainability are higher than companies that are solely overseas or domestic focused (Figure 6a). This finding may be explained by the slack resources theory [37] and available funds hypothesis [38]. However, all companies, regardless of their market focus, consider the economic dimension of sustainability as essential to their business. This is more obvious for overseas-focused companies and companies having a mixed market but more domestically-orientated (Figure 6b). Environmental sustainability is the least likely to receive attention during the pandemic and in the longer term following the pandemic.

(a) Longer term post-pandemic priority

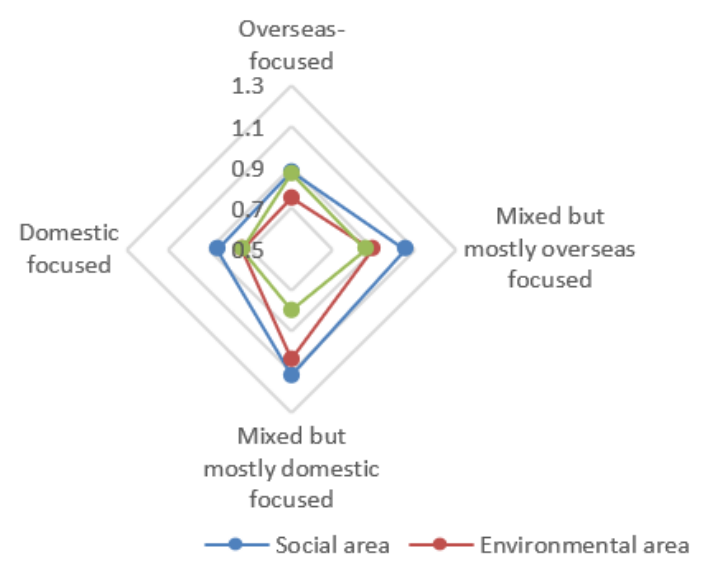

(b) Post-pandemic business essential

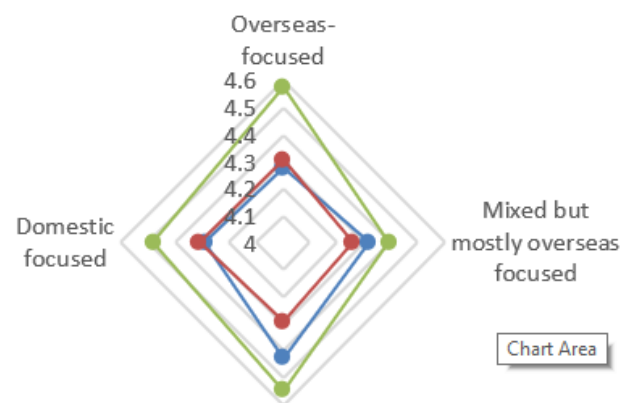

Mixed but mostly domestic

focused

Figure 6. Longer term post-pandemic priority and post-pandemic business essential for companies with different market focus. 


\section{Conclusions and Recommendations}

In the research reported here the short and long-term impact of COVID-19 on sustainability are looked at from the perspective of 1160 business managers by investigating the changes of sustainability priorities during and post the pandemic, and three hypotheses have been tested. The results failed hypothesis 1 as social and environmental dimensions of sustainability are prioritized more by SOEs and private-owned companies and less by FIEs and individual-owned companies. The results also failed hypothesis 2 in that the larger the company, the more they appear to emphasize social and environmental sustainability. It was found that the social dimension of sustainability was prioritized the most by medium- and small-sized companies during COVID-19 and in the longer term after the pandemic, followed by the environmental dimension of sustainability. The largest companies, instead, appeared to show the least interest in social and environmental sustainability, and individually owned companies are in the middle of the range. However, hypothesis 3 is proved to be true as companies with mixed markets did prioritize social sustainability (the first) and environmental sustainability (the second) during the pandemic and in the longer term following the pandemic.

However, it does have to be noted that economic sustainability clearly stood out as having the highest status among three aspects of sustainability post-COVID-19 for all types, sizes, and market focus of business. This leaves environmental sustainability to be the relatively neglected dimension and confirms the possibility of a post pandemic environmental rebound effect that some suggest is already happening in China [55].

\subsection{Managerial Implications}

The above findings and discussion have implications for company managers. To avoid the post-pandemic environmental rebound effect, Chinese companies need to act now. It should be noted that while COVID-19 may not change the global picture with regard to sustainability, the results suggest that sustainability priorities of companies have changed. Additional measures are needed to counteract a possible rebound effect so as to ensure the environmental dimension of sustainable development is not neglected in favor of the social and economic dimensions. In addition, it is important to motivate and empower employees and other stakeholders to pursue sustainability goals and help them integrate these goals into their daily work routines, thus making sustainability everybody's job rather than just the job of those who work in the sustainability department. This is especially important with the accelerating expansion of ICT and digitalization when company employees adapt to the 'new normal' way of working. Recent literature suggests that economic instruments like resources pricing or setting limits to resource use can be effective for this purpose [4]. Special attention should be paid to FIEs and Chinese individual-owned companies, companies larger in size and micro companies, and companies that sell products to only overseas markets or the domestic market.

Will China still be an important actor after COVID-19 in dealing with the issue of climate change? The answer is uncertain. Since government action and economic incentives post-pandemic would influence the global climate change path for decades [3], the opportunity to align long-term economic growth simultaneously with climate change objectives lies with the Chinese government and their decision-making in the coming months. The key is the balance of sustainability and resilience $[45,46]$. China's quick economic recovery from COVID-19 has presented such an opportunity. To do this, China needs to further confirm its commitment to 'new infrastructure' building. Second, China should not let the pandemic delay the start of the carbon market and move forward with its national emissions trading system (ETS) which aims to limit and reduce $\mathrm{CO}_{2}$ emissions in a cost-effective manner. In addition, it is crucial for China to stop relying on coal and accelerate the growth of renewable power. Furthermore, although the planned EU-China summit this autumn is postponed, China should see it as a new opportunity for both parties to cooperate on building a greener global economy by aligning their recoveries with the principles of a green transition and bringing other countries along with them. 


\subsection{Theoretical Implications}

This paper contributes to the scientific literature of the impact of COVID-19 by adding an empirical investigation of the sustainability priority change as the result of the pandemic in China. It also provides an empirical evidence of the possible rebound effect after the pandemic. Second, views of Chinese business owners and managers were considered in the research; this would help shed light on the business response to COVID-19. In addition, the predicted post-pandemic situation was assessed, which, given the high uncertainty of the possible impact of COVID-19 on the environment, provides an 'ex-post' perspective regarding the post-pandemic recovery worldwide.

\subsection{Limitations and Future Research}

The findings set out in the paper are subject to some limitations. First, only the views of business owners and managers were considered. Although it can be argued that these people are likely to have knowledge and be able to influence company sustainability policies to a greater extent than other employees, future research should garner the views of employee's as no sustainability policy can be implemented without their engagement. Second, this research was conducted in China, where there has not been a second or third wave of the pandemic and where people's lives have returned to being close to those they had before the pandemic. As all the respondents in the survey reside in China, they might not have experienced the consequences of the pandemic as much as people would have done in countries where the pandemic has lasted for much longer. This might influence the respondents' prediction of the post-pandemic sustainability priority, and indeed may be reflected in the relatively small deviations between some of the pre- and post-COVID-19 SD figures. Hence the results of this research might not be readily generalizable to countries that have been hit harder and longer by COVID-19. Nonetheless, it does shed light on the post-pandemic situation for countries/regions with a similar COVID-19 experience. In addition, this research did not involve a detailed investigation regarding the mechanism of the impact (direct and/or indirect) of the changes noted by respondents and future research could look at this for specific areas such as an increased use of ICT due to COVID-19. However, it is important to note that differences in cultural background (as indicated by Muller and Voigt [22]) and the COVID-19 experience should be considered in this kind of study.

Author Contributions: Conceptualization, D.Z.; data curation, M.H.; formal analysis, D.Z.; funding acquisition, D.Z.; investigation, D.Z.; methodology, D.Z.; project administration, D.Z.; supervision, D.Z.; writing-original draft, D.Z.; writing-review and editing, D.Z. and S.M. All authors have read and agreed to the published version of the manuscript.

Funding: This research was funded by the Department of Science and Technology of Henan Province, grant number: 202400410096.

Acknowledgments: The authors would like to thank everyone who offered help during the questionnaire survey.

Conflicts of Interest: The authors declare no conflict of interest.

\section{References}

1. World Health Organization (WHO). WHO Coronavirus Disease (COVID-19) Dashboard. Available online: https://covid19.who.int/?gclid=EAIaIQobChMIzqaup7iF6wIVibPtCh0s7AzuEAAYASAAEgLJ2fD_ BwE (accessed on 2 November 2020).

2. Zambrano-Monserrate, M.A.; Ruano, M.A.; Sanchez-Alcalde, L. Indirect effects of COVID-19 on the environment. Sci. Total Environ. 2020, 728, 138813. [CrossRef] [PubMed]

3. Le Quéré, C.; Jackson, R.B.; Jones, M.W.; Smith, A.J.P.; Abernethy, S.; Andrew, R.M.; De-Gol, A.J.; Willis, D.R.; Shan, Y.; Canadell, J.G.; et al. Temporary reduction in daily global $\mathrm{CO}_{2}$ emissions during the COVID-19 forced confinement. Nat. Clim. Chang. 2020, 10, 647-653. [CrossRef]

4. Freire-González, J.; Vivanco, D.F. Pandemics and the Environmental Rebound Effect: Reflections from COVID-19. Environ. Resour. Econ. 2020, 76, 447-517. [CrossRef]

5. McCloskey, B.; Heymann, D.L. SARS to novel coronavirus-Old lessons and new lessons. Epidemiol. Infect. 2020, 148, e22. [CrossRef] 
6. Huang, W.; Morawska, L. Face masks could raise pollution risks. Nature 2019, 574, 29-30. [CrossRef]

7. Barreiro-Gen, M.; Lozano, R.; Zafar, A. Changes in Sustainability Priorities in Organisations due to the COVID-19 Outbreak: Averting Environmental Rebound Effects on Society. Sustainability 2020, 12, 5031. [CrossRef]

8. McKinsey. COVID-19: Implications for Business. Available online: https://www.mckinsey.com/businessfunctions/risk/our-insights/covid-19-implications-for-business (accessed on 2 November 2020).

9. Institute for Global Environmental Strategies (IGES). Implications of COVID-19 for the Environment and Sustainability. Available online: https://www.iges.or.jp/en/news/20200514 (accessed on 2 November 2020).

10. European Commission. Europe's Moment: Repair and Prepare for the Next Generation. Available online: https://ec.europa.eu/commission/presscorner/detail/en/ip_20_940 (accessed on 2 November 2020).

11. European Council. A Recovery Plan for Europe. Available online: https://www.consilium.europa.eu/en/ policies/eu-recovery-plan/ (accessed on 2 November 2020).

12. International Energy Agency (IEA). Sustainable Recovery. Available online: https://www.iea.org/reports/ sustainable-recovery (accessed on 2 November 2020).

13. Bloomberg. South Korea's \$35 Billion Green Plan Skirts Zero-Carbon Target. Available online: https://www.bloomberg.com/news/articles/2020-07-14/green-new-deal-in-south-korea-stops-short-ofzero-carbon-target (accessed on 2 November 2020).

14. World Bank (WB). Costa Rica Receives World Bank Support for Economic Recovery and Promoting Low-Carbon Development. Available online: https:/www.worldbank.org/en/news/press-release/2020/06/25/ apoyo-del-banco-mundial-a-costa-rica-para-promover-la-recuperacion-economica-y-un-desarrollo-bajoen-carbono (accessed on 2 November 2020).

15. International Monetary Fund (IMF). Greening the Recovery. Available online: https://www.imf.org/en/ Topics/climate-change/green-recovery (accessed on 2 November 2020).

16. Xinhua. Xi Focus: Xi Announces China Aims to Achieve Carbon Neutrality before 2060. Available online: http://www.xinhuanet.com/english/2020-09/23/c_139388764.htm (accessed on 2 November 2020).

17. Financial Time (FT). China Pledges to Be 'Carbon-Neutral' by 2060. Available online: https://www.ft.com/ content/730e4f7d-3df0-45e4-91a5-db4b3571f353 (accessed on 2 November 2020).

18. National Bureau of Statistics of China (NBSC). Coordinative Efforts for Epidemic Control and Economic Development Delivered Notable Results with National Economic Recovered Gradually in the First Half of 2020. Available online: http://www.stats.gov.cn/english/PressRelease/202007/t20200716_1776211.html (accessed on 2 November 2020).

19. United Nations (UN). No Excuse Not to Meet Net-Zero Emission Target by 2050, Secretary-General Says in Global Lecture on Climate Change, Stressing Time for Small Steps Has Passed. Available online: https://www.un.org/press/en/2020/sgsm20183.doc.htm (accessed on 2 November 2020).

20. Vivanco, D.F.; McDowall, W.; Freire-González, J.; Kemp, R.; Van Der Voet, E. The foundations of the environmental rebound effect and its contribution towards a general framework. Ecol. Econ. 2016, 125, 60-69. [CrossRef]

21. Gossart, C. Rebound effects and ICT: A review of the literature. In ICT Innovations for Sustainability; Hilty, L.M., Aebischer, B., Eds.; Advances in Intelligent Systems and Computing; Springer: Cham, Switzerland, 2015; Volume 310, pp. 435-448. [CrossRef]

22. Müller, J.M.; Voigt, K.-I. Sustainable Industrial Value Creation in SMEs: A Comparison between Industry 4.0 and Made in China 2025. Int. J. Precis. Eng. Manuf. Green Technol. 2018, 5, 659-670. [CrossRef]

23. Kuo, C.-C.; Shyu, J.Z.; Ding, K. Industrial revitalization via industry 4.0-A comparative policy analysis among China, Germany and the USA. Glob. Transit. 2019, 1, 3-14. [CrossRef]

24. Zhang, D.; Morse, S.; Kambhampati, U. Sustainable Development and Corporate Social Responsibility, 1st ed.; Routledge: New York, NY, USA, 2018; p. 241.

25. Li, Y.; Cheng, H.; Beeton, R.J.S.; Sigler, T.; Halog, A. Sustainability from a Chinese cultural perspective: The implications of harmonious development in environmental management. Environ. Dev. Sustain. 2016, 18, 679-696. [CrossRef]

26. Peng, M.W. Institutional transitions and strategic choices. Acad. Manag. Rev. 2003, 28, 275-296. [CrossRef]

27. Li, W.; Zhang, R. Corporate Social Responsibility, Ownership Structure, and Political Interference: Evidence from China. J. Bus. Ethics 2010, 96, 631-645. [CrossRef] 
28. Zhang, D.; Morse, S.; Kambhampati, U.; Li, B. Evolving Corporate Social Responsibility in China. Sustainability 2014, 6, 7646-7665. [CrossRef]

29. Oh, W.Y.; Chang, Y.K.; Martynov, A. The Effect of Ownership Structure on Corporate Social Responsibility: Empirical Evidence from Korea. J. Bus. Ethics 2011, 104, 283-297. [CrossRef]

30. Lee, J.; Kim, S.-J.; Kwon, I. Corporate Social Responsibility as a Strategic Means to Attract Foreign Investment: Evidence from Korea. Sustainability 2017, 9, 2121. [CrossRef]

31. Fry, F.L.; Hock, R.J. Who claims corporate responsibility? The biggest and the worst. Bus. Soc. Rev. 1976, 18, 62-65.

32. Fombrun, C.; Shanley, M. What's in a name? Reputation, building and corporate strategy. Acad. Manag. J. 1990, 33, 233-258.

33. Pava, M.L.; Krausz, J. The association between corporate social-responsibility and financial performance: The paradox of social cost. J. Bus. Ethics 1996, 15, 321-357. [CrossRef]

34. McWilliams, A.; Siegel, D. Corporate Social Responsibility: A Theory of the Firm Perspective. Acad. Manag. Rev. 2001, 26, 117. [CrossRef]

35. Elsayed, K. Reexamining the Expected Effect of Available Resources and Firm Size on Firm Environmental Orientation: An Empirical Study of UK Firms. J. Bus. Ethics 2006, 65, 297-308. [CrossRef]

36. Muller, A.; Kolk, A. Extrinsic and Intrinsic Drivers of Corporate Social Performance: Evidence from Foreign and Domestic Firms in Mexico. J. Manag. Stud. 2010, 47, 1-26. [CrossRef]

37. Waddock, S.A.; Graves, S.B. The corporate social performance-Financial performance link. Strateg. Manag. J. 1997, 18, 303-319. [CrossRef]

38. Preston, L.E.; O'Bannon, D.P. The corporate social-financial performance relationship: A typology and analysis. Bus. Soc. 1997, 36, 419-429. [CrossRef]

39. Sun, J.; Zhuang, Z.; Zheng, J.; Li, K.; Wong, R.L.-Y.; Liu, D.; Huang, J.; He, J.; Zhu, A.; Zhao, J.; et al. Generation of a Broadly Useful Model for COVID-19 Pathogenesis, Vaccination, and Treatment. Cell 2020, 182, 734-743. [CrossRef]

40. Wiersinga, W.J.; Rhodes, A.; Cheng, A.C.; Peacock, S.J.; Prescott, H.C. Pathophysiology, Transmission, Diagnosis, and Treatment of Coronavirus Disease 2019 (COVID-19): A Review. JAMA 2020, 324, 782-793. [CrossRef]

41. Zhai, P.; Ding, Y.; Wu, X.; Long, J.; Zhong, Y.; Li, Y. The epidemiology, diagnosis and treatment of COVID-19. Int. J. Antimicrob. Agents 2020, 55, 105955. [CrossRef]

42. Wang, Q.; Su, M. A preliminary assessment of the impact of COVID-19 on environment-A case study of China. Sci. Total Environ. 2020, 728, 138915. [CrossRef]

43. Bao, R.; Zhang, A. Does lockdown reduce air pollution? Evidence from 44 cities in northern China. Sci. Total Environ. 2020, 731, 139052. [CrossRef]

44. Bartik, A.W.; Bertrand, M.; Cullen, Z.; Glaeser, E.L.; Luca, M.; Stanton, C. The impact of COVID-19 on small business outcomes and expectations. Proc. Natl. Acad. Sci. USA 2020, 117, 17656-17666. [CrossRef]

45. Zabaniotou, A. A systemic approach to resilience and ecological sustainability during the COVID-19 pandemic: Human, societal, and ecological health as a system-wide emergent property in the Anthropocene. Glob. Transit. 2020, 2, 116-126. [CrossRef]

46. D'Adamo, I.; Rosa, P. How Do You See Infrastructure? Green Energy to Provide Economic Growth after COVID-19. Sustainability 2020, 12, 4738. [CrossRef]

47. China State Council. The Labour Law of the People's Republic of China (2016). Available online: https: //www.chashebao.com/shebaotiaoli/16340.html (accessed on 2 November 2020).

48. Center for Strategic and International Studies (CSIS). Chinese Philanthropists Rush to Respond to COVID-19. Available online: https:/www.csis.org/blogs/trustee-china-hand/chinese-philanthropists-rush-respondcovid-19 (accessed on 2 November 2020).

49. Lin, K.J.; Lu, X.; Zhang, J.; Zheng, Y. State-owned enterprises in China: A review of 40 years of research and practice. China J. Account. Res. 2020, 13, 31-55. [CrossRef]

50. Su, J.; He, J. Does Giving Lead to Getting? Evidence from Chinese Private Enterprises. J. Bus. Ethics 2010, 93, 73-90. [CrossRef]

51. Ministry of Commerce of the People's Republic of China (MOC). Summary of the Direct Investment by Some Foreign Countries/Regions by the End of 2018. Available online: http://www.mofcom.gov.cn/article/ tongjiziliao/v/ (accessed on 3 December 2020). 
52. Henriques, I.; Sadorsky, P. The Determinants of an Environmentally Responsive Firm: An Empirical Approach. J. Environ. Econ. Manag. 1996, 30, 381-395. [CrossRef]

53. Russo, M.V.; Fouts, P.A. A resource-based perspective on corporate environmental performance and profitability. Acad. Manag. J. 1997, 40, 534-559.

54. Lepoutre, J.; Heene, A. Investigating the Impact of Firm Size on Small Business Social Responsibility: A Critical Review. J. Bus. Ethics 2006, 67, 257-273. [CrossRef]

55. Centre for Research on Energy and Clean Air (CREA). China's Air Pollution Overshoots Pre-Crisis Levels for the First Time. Available online: https://energyandcleanair.org/wp/wp-content/uploads/2020/05/China-airpollution-rebound-final.pdf (accessed on 2 November 2020).

Publisher's Note: MDPI stays neutral with regard to jurisdictional claims in published maps and institutional affiliations.

(C) 2020 by the authors. Licensee MDPI, Basel, Switzerland. This article is an open access article distributed under the terms and conditions of the Creative Commons Attribution (CC BY) license (http://creativecommons.org/licenses/by/4.0/). 


\title{
Multinomial Logistic Regression to Estimate and Predict the Perceptions of Individuals and Companies in the Face of the COVID-19 Pandemic in the Nuble Region, Chile
}

\author{
Benito Umaña-Hermosilla ${ }^{1}$, Hanns de la Fuente-Mella ${ }^{2, *} *$, Claudio Elórtegui-Gómez ${ }^{3}$ \\ and Marisela Fonseca-Fuentes ${ }^{1}$ \\ 1 Departamento de Gestión Empresarial, Facultad de Ciencias Empresariales, Universidad del Bío-Bío, \\ Chillán 2463334, Chile; benito@ubiobio.cl (B.U.-H.); mfonseca@ubiobio.cl (M.F.-F.) \\ 2 Escuela de Comercio, Facultad de Ciencias Económicas y Administrativas, \\ Pontificia Universidad Católica de Valparaíso, Valparaíso 2340025, Chile \\ 3 Escuela de Periodismo, Facultad de Ciencias Económicas y Administrativas, \\ Pontificia Universidad Católica de Valparaíso, Valparaíso 2373223, Chile; claudio.elortegui@pucv.cl \\ * Correspondence: hanns.delafuente@pucv.cl
}

Received: 16 October 2020; Accepted: 9 November 2020; Published: 17 November 2020

\begin{abstract}
The Coronavirus Disease 2019 (COVID-19) pandemic is transforming the world we live in, revealing our health, economic, and social weaknesses. In the local economy, the loss of job opportunities, the uncertainty about the future of small and medium-sized companies and the difficulties of families to face the effects of this crisis, invite us to investigate the perception of the local community. Based on a questionnaire applied to 313 citizens and 51 companies, this study explored the perception of these actors on the effects of the pandemic at the local level and determined the main factors that influenced their assessment using a multinomial logistic regression model. The results indicated a systematic concern for issues of employment, job security, and household debt. The variables of age and sex were significant when analyzing the vulnerability of certain groups, especially women and the elderly, to face the effects of the crisis and their role as citizens. At the business level, the focus was on economic policies that support its operational continuity and management capacity to face a changing scenario.
\end{abstract}

Keywords: COVID-19 pandemic; local community; perception analysis; econometric modeling; data science

\section{Introduction}

Local development studies have strongly encouraged interest to decipher, explain, and understand the role of the citizenry and local governments in promoting development $[1,2]$. The interaction between the economy, authorities, and the local citizenry is critical for dealing with crisis scenarios and accounts for the integration of the social paradigm in the context of economic policy formulation [3-5].

The cooperation and construction of the community are essential for economic, social, and environmental survival [6]. Likewise, it is considered that the community is the fabric for the collective action of individuals, a mediating structure between individuals/families and the social, economic, political, and environmental spheres, which are typical of local development [7]. As a result, the debate about local development not only involves experts, technocrats, and government authorities, but the assessment of the common citizen and the community also becomes more relevant to define political and social guidelines $[8,9]$. 
The vision of local stakeholders is fundamental to detecting gaps and trends in the local economic outlook. The collection of these visions using surveys allows local authorities to undertake efforts for strategic changes that promote local development processes and strengthen communication networks with citizens [10]. As part of a philosophy of community-based environmental monitoring, the survey can provide a viable solution and an attractive means of increasing legitimacy to implement measures that lead to improved quality of life [11,12].

Understanding that the effects of the COVID-19 pandemic have modified the daily dynamics of people, the way they relate to each other and the expectations of the local economy, it is necessary to investigate the social representations that arise in this context of uncertainty, where currently the business and social development of communities around the world is limited.

Following this approach, the socio-economic paradigms require new studies. A sustainable revolution is necessary to tackle climate change and these actions are globally required [13]. In addition, literature shows a positive relationship between sustainability and resilience [14,15].

This study investigates the perception of citizens and local companies about the effects that the COVID-19 pandemic has had on the development of their activities and lifestyle, seeking to understand how the current economic scenario is perceived in terms of employment, economy, and government performance, among others.

Special emphasis has been placed on the factors that influence the assessment of the challenges, risks and opportunities that this new and disruptive scenario offers to the region and its inhabitants, antecedents that allow the general vision of the population to be conceived according to the projection they can make based on their level of family income, supply, level of indebtedness, national and local economic situation.

The research will also allow to deepen the social resilience present in the population, where it has been observed that people and governments organize their efforts to face this global health crisis, thus trying to minimize the loss of human lives, help the population who are more vulnerable and adapt to better assimilate this new reality. In this framework, the study contributes to an emerging literature to assess the effects of the COVID-19 pandemic at the local scale.

This analysis adopts a quantitative approach, limiting the study area to Chile, specifically to the Nuble region. The following research question is established: What are the appreciations of the inhabitants and businesspeople about their reality in the COVID-19 pandemic? In relation to it, the objective is to identify the perception of citizens on various topics of interest and sensitivity, and their effect on the economic and financial aspects of the country, all marked by the uncertainty of COVID-19.

Regarding the problem, the need to have records related to the effects of the pandemic from social representations is identified, therefore it is established as a research question: What are the appreciations of the inhabitants and business owners about their reality in the COVID-19 pandemic? In relation to it, the objective is to identify the perception of citizens on various topics of interest and sensitivity, and its effect on the economic and financial aspects of the country, all marked by the uncertainty of COVID-19.

This article analyzes the results of the survey "Pulse of the economy in the Nuble region-Chile," where the perceptions of residents and business owners are evidenced in relation to the effects of the pandemic at the local level. The subject is approached from an analysis that adopts a quantitative approach and the results are presented in the following sections.

\section{World Context: COVID-19}

The SARS-CoV-2 virus is the cause of the infectious disease known as COVID-19; its most common symptoms are fever, muscle aches, cough, fatigue, and shortness of breath, and it can also cause pneumonia, sepsis, and acute respiratory distress in severe cases. The contagion of this disease is mainly through small drops expelled when talking, coughing or sneezing, which has caused an increase in patients in the world exceeding 30 million infections registered in 190 countries, with the United States, 
Brazil, and India being among the most affected countries, as their records exceed 100,000 deaths due to COVID-19.

In Chile, the first fatal victim of COVID-19 died on 17 March 2020; to date, according to information provided by the health ministry (until 14 October 2020), 13,415 deaths and 485,372 accumulated confirmed cases have been registered [16]. As in other countries, in Chile social distancing measures have been implemented that include confining families to their homes, in addition to activities such as work and study being carried out at a distance, in an attempt to reduce the number of infected people.

This situation is affecting the individual and social development of people, preventing physical contact, the operation of companies and free movement in the territory, which is generating negative emotional effects due to isolation and constant concern about contracting this disease. Among the most common alterations we find stress, depression, anxiety, insomnia, and dietary changes, among others, which will have a medium and long-term impact, weakening the mental health of the entire world.

Regarding the changes in people's dynamics, the role of the internet and social networks stands out, which currently allows them to carry out daily activities such as working and studying remotely. This new form has kept people connected and occupied from their homes, allowing them to advance and generate resources without exposing themselves to infection, but at the same time it has become a form of exclusion for the population of rural and vulnerable sectors $[17,18]$.

In relation to the effects on economic development, a decrease in the demand for goods and services derived from the confinement of people is identified; among the most affected sectors are tourism and recreation services. Regarding the labor market, although teleworking has been implemented to facilitate activities from home, it has also promoted the exclusion of the most vulnerable sectors derived from rurality, scarcity of resources and all kinds of limits that prevent access to the internet.

\section{Citizen Perception and Local Economic Development}

Based on different approaches, public opinion studies play a key role in the evaluation of economic scenarios at both the local and national levels [19-22]. Perception and expectations, as an individual and subjective element, are often built on trust in the performance of the economy and support to institutions [23].

The role of public opinion on various aspects of the economy has been the subject of intense debate [24] and growing interest to explore the micro and macro determinants of the social preferences of the citizenry [25-28]. Numerous studies have demonstrated that community participation and citizen empowerment are key instruments for building local capacity, improving quality of life, reducing poverty, and promoting economic development at the local level [29-31].

Empowerment of the citizenry is an increasingly important aspect as a starting point for local development [32] because it provides greater capabilities for communities, especially in the control and information flows of the territorial resources [33,34]. Higher levels of community involvement in the local development processes are associated with the extension of the citizenry and better levels of governance [35].

From the community perspective, citizens participate because they perceive themselves as part of an integrated body that has common goals in the socioeconomic, political, legal, and cultural spheres [36-38]. In the case of opinion polls, the greatest achievements in the participatory processes have been the creation of interactive spaces between civil society organizations and public institutions aimed at implementing development initiatives from a collaborative, dynamic, and multidimensional approach [39-41].

Different authors have emphasized the importance of examining the factors related to the perceptions of local development [42-44]. Some researchers highlight the analysis of the cultural and institutional dimensions to understand the determinants of successful regional processes [45-47]. A better understanding of the factors that influence perceptions is essential to improve communication 
strategies and the design of efficient local policies that facilitate development processes, especially in times of crisis, uncertainty, and instability.

To recognize their importance, it is necessary to comprehend that individual perceptions can be transformed into social representations that construct reality through language [48]. Likewise, admitting that cultural identity is established on the basis of the characteristics of a society and its social representations $[49,50]$ provides support for the role that the vision of the citizenry should play in local economic development policies.

Cultural identity can be associated with a sense of belonging inspired by a territory, its social group, and culture [51]. It is a dynamic process of permanent reconstruction that supports social interactions [52,53], and it is directly related to the people's sense of belonging, which is considered fundamental for implementing "social cooperation processes" [54,55]. Furthermore, it invites us to question the notion of territory, so as to review the reality of functional rural-urban territories [56].

"Territory not as an "objectively existing" physical space, but rather it exists as a set of social relations that give rise to, and at the same time express, an identity and a sense of purpose shared by multiple public and private agents (even though such a construction often involves going through processes of conflict and negotiation). It is this identity that gives meaning and content to a development project of a determined space based on the convergence of interests and intentions" [57].

Validating the existence of the territory as "a social construction" that emerges from the local identity $[58,59]$ allows us to explore population dynamics that are present in daily life, and which clearly influence perceptions and representations in the cultural identity of society. For example, special mobility is defined as an "internal, transitory, and frequent territorial movement of people without the need to establish a second residence" [60]; this phenomenon is present in functional territories where people travel to develop activities such as work, study, and have access to public and/or private goods and services. Such mobility constitutes interactions and allows different places to be part of a territory, which have, for example, a common labor market and supply of goods and services [61].

Some changes in the rural sector have been identified as a result of this interaction and influence from the urban sector, including an increase in non-agricultural occupations, higher participation by women in the labor force, and access to education and health, which directly promote economic development [62]. Moreover, the expectations of the rural population have shifted; for example, the educational attainment of the Chilean rural population has improved in recent years [63].

This reality is known as "urban living" [64] and allows people to maintain their residence in rural locations and pursue life goals in urban areas. They can therefore access goods and services not available in their community; this gradually modifies the expectations of new generations and, to some extent, modernizes rural life.

Given the background information and the current situation resulting from the COVID-19 pandemic, it is imperative to investigate the social perception of this "new normality", which has closed borders and paralyzed movement worldwide and activated quarantines and sanitary cordons to protect the population by lockdown and social distancing. This situation has also made the population aware of their responsibility for individual and social well-being [65].

Faced with this contingency that affects how people and organizations function, governments have implemented internal policies to mitigate the effects on economic well-being. Despite all efforts, we are facing a global economic crisis due to the pandemic. The results of the business survey on the impact of the COVID-19 crisis [66] indicate that most companies perceive decreased economic activity due to the health crisis, and smaller companies experience financing and liquidity problems. Another important aspect is how teleworking, production cost adjustment, and tax debt deferral are part of a strategy to continue their activities. As for expectations, there is quite a lot of pessimism as to the recovery of the economy.

Before projecting or comparing this reality, background information must be collected on what people and organizations are experiencing, and prepare representations based on the population's 
perceptions to identify vulnerability at the local level. It is truly important to know the expectations and emotions related to the effects of the COVID-19 in Chile and to consult the people's evaluation regarding such aspects as the implemented policies and the performance of the local and national governments, which provide an insight into the reality that the country is facing.

\section{Methodology}

\subsection{Data and Method}

To understand the perceptions and assessment of the citizens and companies in the face of the pandemic in the Nuble Region, Chile, a questionnaire was created and administered to residents and business owners of the region. The survey was administered in person and online. The sampling used to perform the measurement on this population was non-probabilistic and of convenience, where those interested in participating were contacted by an invitation. The online survey was circulated in the online version of a local daily newspaper. The survey included the Likert scale, multiple choice, open-ended, and demographic questions; it took approximately ten minutes to complete and respondents were not compensated.

To determine the factors that could predict the perceptions of people and companies as to how to face the pandemic in the Nuble Region, a multinomial logistic regression model was fitted to the responses, and was used to predict the probabilities of the different possible outcomes [67]. Multinomial logistic regression was used to predict categorical variables or the probability of category membership on a dependent variable based on multiple independent variables [68]. As in binary logistic regression, multinomial logistic regression uses maximum likelihood estimation to evaluate the probability of categorical membership. Thus, this type of model allowed us to characterize the probability of a respondent's decision for a particular multinomial discrete choice, conditional on the values of the explanatory variables [69]. The distribution functions that characterize explanatory variables are often nonlinear. Thus, once the multinomial regression model is created, the parameters are used to make predictions about the probability of an event occurring compared with the reference category.

In this particular case, we wanted to know how changes affected the abovementioned independent variables on the probabilities of the variable (infrastructure choice) in Equation (1) expressed as

$$
P\left(Y=j / X_{1}, X_{2}, \ldots, X_{k}\right)=P(Y=j / K) ; j=0,1, \ldots, J
$$

In the multinomial case, response probabilities were represented in Equations (2) and (3) as

$$
\begin{gathered}
P(Y=j / X)=\frac{\exp \left(X \beta_{j}\right)}{1+\sum_{h=1}^{J} \exp \left(X \beta_{h}\right)}=p_{j}(X, \beta) ; j=1, \ldots, J \\
P(Y=0 / X)=\frac{1}{1+\sum_{h=1}^{J} \exp \left(X \beta_{h}\right)}=p_{0}(X, \beta)
\end{gathered}
$$

We used maximum likelihood to estimate multinomial logit models in which the logarithm of the likelihood function that usually provides consistent and asymptotically normal estimators is expressed by Equation (4) as

$$
l(\beta)=\sum_{i=1}^{n} \sum_{j=0}^{J} 1\left[Y_{i}=j\right] \log \left[p_{j}\left(X_{i}, \beta\right)\right]
$$

\subsection{Study Area}

The study was conducted in the Nuble Region located in south-central Chile. This region covers an area of $13,178.5 \mathrm{~km}^{2}$ consisting of a diversity of landscapes ranging from mountainous areas to 
interior valleys. The region is divided into 3 provinces and 21 communes with a total population of 480,609 inhabitants, and 30.60\% live in rural areas, which places the region as the most rural in Chile, well above the national mean of $16.73 \%$ [70,71]. As for poverty, the region has the highest national indicators with $16.1 \%$ of households living in poverty, which is much higher than the national mean of 9.4\% [72]. There have been important demographic changes in recent years and the population has gradually decreased, reflecting the dynamics of the migration of the rural population to urbans spaces and new occupational patterns of the territory through activities linked to tourism in the mountains, nature, and/or agrotourism $[73,74]$.

Historically, the productive and economic base of the region is related to small- and medium-scale agricultural and forestry activity. Thus, the region shows a clear orientation towards agricultural production and this situation is not replicated in other sectors [75]. The seasonality of agricultural activities and the boom in vegetable and fruit export operations have a significant impact on job creation, accounting for $20.7 \%$ of jobs in the region. However, rural areas have undergone significant changes in the last 30 years, including the declining importance of agriculture in rural production and labor dynamics [76,77]. On many farms, owners and/or laborers spend part of their time in off-farm work. Rural modernization has changed the traditional ways of living and working. The services sector has become a source of employment and income [78]. In mountain areas, the diversification of activities has been a strategy to ensure the economic viability of farms by promoting various forms of rural tourism and protection and dissemination of both the natural and cultural heritage. Rurality is a strong component of the regional territorial identity [79].

\section{Results}

\subsection{Descriptive Analysis}

Within the framework of the complex world and Chilean pandemic scenario set in a historical context of great expectation for public opinion, research was carried out in the newly created Nuble Region, Chile.

Due to the quarantine as a result of the COVID-19 virus, the present study considered virtual data collection from 13 to 22 April 2020 using a survey in social networks and e-mail, in Chillán and its neighboring communities. A total of 313 valid responses were obtained for the citizenry (individuals) and 51 valid responses for companies (business owners) and two virtual instruments were used:

- The first instrument was applied to individuals; besides economic categories, it surveyed specifics related to the coronavirus context, such as sources of information for the citizenry in the face of the pandemic, assessment of national and regional media, importance of social networks, and emotions during quarantine.

- The second instrument was aimed at companies and surveyed the general background information of their owners as to the national and regional economy, employment, and investment. In addition, it included categories such as those directly related to the health emergency situation and assessment of crisis management by national and regional authorities.

Therefore, the present work is a quantitative and cross-sectional descriptive study [80] using the survey as its research strategy [81]. The subjects were the owners of regional businesses located in the municipalities of Chillán Viejo, San Carlos, Yungay, Coihueco, Pinto, Bulnes, San Ignacio, and Chillán. For the survey applied to the citizens, study subjects were adults residing in Chillán and other municipalities of the Nuble Region: Chillan Viejo, San Nicolás, Quillón, El Carmen, Quirihue, Yungay, Coihueco, Coelemu, Pemuco, Bulnes, San Carlos, San Ignacio, Portezuelo, Pinto, San Fabián, and Ranquil.

One of the main results described for individuals was the negative view of the existing economy $(47.9 \%)$. The surveyed citizens manifested that the economic situation of the country a year ago was better than at the present $(71.2 \%)$ and that 2021 would be worse than $2020(65.5 \%)$. 
Meanwhile, $64.2 \%$ of the sample maintained that the economic situation of the region in one more year would be worse than at the present. Some $48.3 \%$ of the surveyed individuals pointed out that the existing economic reality of the region was fair or bad.

The lack of employment (33.2\%), low salaries (25.9\%), and digital connectivity (12.5\%) were the three most important problems at the regional level that were identified by the sample in the context of the pandemic.

The majority of the sample indicated that their household income in the last 12 months had decreased (53\%), and they were pessimistic and predicted that it would continue to decrease $(46.6 \%)$. The population admitted to having fear of losing their jobs (74.4\%), even though teleworking had been introduced $(48.2 \%)$. Some $62 \%$ of the sample reported some degree of difficulty in their household due to debt. Meanwhile, $43.8 \%$ of the citizens indicated they did not feel economically prepared to face the pandemic. In addition, $69.3 \%$ of respondents stated that the work of the regional media in addressing the pandemic was "fair to very good" in contrast to the perception of $47.6 \%$ of individuals who negatively evaluated the work of the national media.

Regarding the main exploratory results for companies, $54.9 \%$ of businesspeople indicated that they were prepared to face the pandemic "only for a while". Some $41.2 \%$ positively evaluated the policies developed by the government to provide support to businesses. However, $90.2 \%$ were concerned for their levels of debt because they considered that the effects of the coronavirus, in addition to the social upheaval, had affected their commercial activity. Likewise, $64.7 \%$ of businesspeople specified that they considered that the economic situation of the country in 2021 would be the same or worse, and $62.7 \%$ pointed out that it would be the same or worse than the existing regional situation.

A positive perception was highlighted when associating the creation of the region with the management of future crises. However, there was uncertainty about the region's current preparedness and the responses of regional/community authorities to the COVID-19 crisis.

\subsection{Multinomial Logistic Regression}

The arrival of COVID-19 in Chile was set in a particular context and probably generated a number of characteristics prior to the emergence of the first case declared on 3 March 2020. The scenario immediately prior to the coronavirus outbreak is a relevant issue due to high sensitivity and concern for the immediate future of the country, both economically and institutionally, among citizens and business people.

This predisposition is very different to how citizens responded to the "subprime crisis" in 2008. At that time, trust in the political institutions and the security in the Chilean economic management during the first presidential term of Michelle Bachelet (2006-2010) and in the Minister of Finance Andrés Velasco were relevant to minimize the effects of such instability. Moreover, Chile was one of the countries in Latin America that most successfully overcame the crisis.

Since October 2019, Chile has experienced a social upheaval of a magnitude that was not on the research agenda, academic studies, or political surveys. In fact, the administration of President Sebastián Piñera was preparing to host two important global forums, APEC and COP25, events that were to strengthen the country's image, public diplomacy, and Chile's international standing vis-à-vis the world.

However, the social crisis became very difficult for the government and the political establishment to manage and those events were therefore cancelled, including visits by Donald Trump and Xi Jinping. This was due to a wave of demonstrations that affected the activities, movements, and routines of the population and which were only interrupted by the arrival of the COVID-19. Before the pandemic context, there was already talk in Chile of a "new normality" due to social indignation or simply the impossibility of returning to "normality."

It is therefore important to point out that the results reported in this study are on a continuum of tensions and concerns about what 2020 would be like for both the citizenry and entrepreneurs or business owners. The complexity of the health, economic, and psychological events related to the 
coronavirus and its rapid spread in Chile should be measured in the regions that were exposed to the severity of the pandemic.

To respond to the research objective, a multinomial logistic regression model was fitted to know the perceptions of individuals and businesses and quantify the effects of the COVID-19 pandemic in the Nuble Region. Table 1 summarizes the cases related to the citizens. A linear model was first run on the responses as a function of the predictors to ensure that there were no problems with multicollinearity; only predictors with variance inflation factors $(\mathrm{VIF})<2$ were included in these models [82].

Regarding the fitted model information, the chi-squared ratio test had a value of $317.910(p=0.000)$, indicating a good model fit. Acceptable values were also obtained for the pseudo R-squared (Cox and Snell: 0.638, Nagelkerke: 0.789). Table 2 shows that the power of our logistic multinomial model was suitable because it correctly classified $88.8 \%$ of the known observations and can be expected to project future estimates. Table 3 shows the likelihood ratio tests for the effects of the model and the partials whose low $p$-values show the high significance of the variables in the model.

The perception of the citizenry in the Nuble Region and the projections related to the health crisis are not encouraging, and it can be seen that there is a systemic concern. Assuming the slump in economic expectations prior to the coronavirus, the preparedness of the country to deal with the pandemic is associated with a number of aspects discussed below.

Age is an important issue in Chile because it has been permeating political and economic discussions over the last decade about the pension system, the fear of aging with low pensions, and the vulnerability of aging. It has also been a generational issue that transpired with the Chilean social upheaval because it established a perspective between the new emerging practices and old styles of leadership. The COVID-19 crisis cannot be excluded from the age-related aspects already incorporated in the perceptions of the population. Therefore, the interpretations of individuals in the face of the coronavirus also emerged from the diversified visions of the citizenry based on age group. The interest in greater social protagonism was found in the under-33 age group with its flexibility, vigor, and resilience to face the virus; this group has opened new spaces for discussion, voices, and empowerment never before observed in a country that shows a trend towards aging.

Gender was also important because the Chilean population has shifted the role of women. The feminine and masculine are perceived as differentiated styles of social and local problem solving. In addition, gender leadership tends to gain a greater presence in the regional social base. This should be considered when addressing such aspects as compliance with sanitary measures, responsibility for self-care to protect the community, or citizen support networks.

Closely related to the above, completion of schooling or educational level was relevant for the perceptions of the country's preparedness for the pandemic. The challenges that the disease poses in the analyzed Chilean reality have required individuals to meet the greatest instructional, technical, professional, or postgraduate demands as an efficient alternative to manage this complex situation. Knowledge, as a value of pandemic preparedness, dispels myths and insecurities and provides information and guidance. Moreover, in the midst of systemic insecurity, education has created new opportunities when faced with the lack or instability of employment generated by the COVID-19. 
Table 1. Summary of citizen responses.

\begin{tabular}{|c|c|c|c|c|}
\hline Questions & Variable & Alternatives & $n$ & Marginal Percentage \\
\hline \multirow{3}{*}{ How prepared is the country to face the pandemic? } & \multirow{3}{*}{ Country preparedness } & Well-prepared & 12 & $3.8 \%$ \\
\hline & & Moderately prepared & 160 & $51.1 \%$ \\
\hline & & Not at all prepared & 141 & $45.0 \%$ \\
\hline \multirow{3}{*}{ What is your sex? } & \multirow{3}{*}{ Sex } & Female & 177 & $56.5 \%$ \\
\hline & & Male & 135 & $43.1 \%$ \\
\hline & & Prefer not to say & 1 & $0.3 \%$ \\
\hline \multirow{6}{*}{ How old are you? } & \multirow{6}{*}{ Age } & $18-25$ years & 63 & $20.1 \%$ \\
\hline & & $26-33$ years & 46 & $14.7 \%$ \\
\hline & & $34-40$ years & 60 & $19.2 \%$ \\
\hline & & $41-50$ years & 74 & $23.6 \%$ \\
\hline & & $51-60$ years & 47 & $15.0 \%$ \\
\hline & & 61 years or more & 23 & $7.3 \%$ \\
\hline \multirow{5}{*}{ What is your educational attainment? } & \multirow{5}{*}{ Education } & Elementary & 1 & $0.3 \%$ \\
\hline & & High school & 96 & $30.7 \%$ \\
\hline & & Technical & 40 & $12.8 \%$ \\
\hline & & University & 46 & $14.7 \%$ \\
\hline & & Postgraduate studies & 130 & $41.5 \%$ \\
\hline \multirow{4}{*}{$\begin{array}{l}\text { What do you expect regarding household income in the } \\
\text { next } 12 \text { months? }\end{array}$} & \multirow{4}{*}{ Projected income } & Will increase & 28 & $8.9 \%$ \\
\hline & & Will remain the same & 94 & $30.0 \%$ \\
\hline & & Will decrease & 146 & $46.6 \%$ \\
\hline & & Does not know & 45 & $14.4 \%$ \\
\hline \multirow{4}{*}{ What is your household debt situation? } & \multirow{4}{*}{ Household debt } & Complicated & 72 & $23.0 \%$ \\
\hline & & Moderately complicated & 122 & $39.0 \%$ \\
\hline & & Without problems & 105 & $33.5 \%$ \\
\hline & & Does not know/Does not respond & 14 & $4.5 \%$ \\
\hline \multirow{3}{*}{ Has your household had any supply problems? } & \multirow{3}{*}{ Supplies } & Yes & 64 & $20.4 \%$ \\
\hline & & No & 246 & $78.6 \%$ \\
\hline & & Does not know/Does not respond & 3 & $1.0 \%$ \\
\hline
\end{tabular}


Table 1. Cont.

\begin{tabular}{|c|c|c|c|c|}
\hline Questions & Variable & Alternatives & $n$ & Marginal Percentage \\
\hline \multirow{12}{*}{ What is your political persuasion? } & \multirow{12}{*}{ Political persuasion } & Left & 16 & $5.1 \%$ \\
\hline & & 1 & 11 & $3.5 \%$ \\
\hline & & 2 & 12 & $3.8 \%$ \\
\hline & & 3 & 14 & $4.5 \%$ \\
\hline & & 4 & 8 & $2.6 \%$ \\
\hline & & Center & 21 & $6.7 \%$ \\
\hline & & 6 & 6 & $1.9 \%$ \\
\hline & & 7 & 7 & $2.2 \%$ \\
\hline & & 8 & 3 & $1.0 \%$ \\
\hline & & Right & 5 & $1.6 \%$ \\
\hline & & I have no political persuasion & 166 & $53.0 \%$ \\
\hline & & Does not know/Does not respond & 44 & $14.1 \%$ \\
\hline \multirow{5}{*}{$\begin{array}{l}\text { How do you evaluate the performance of the Chilean } \\
\text { government in the face of the pandemic? }\end{array}$} & \multirow{5}{*}{$\begin{array}{l}\text { Evaluation national } \\
\text { government for pandemic }\end{array}$} & Very bad & 68 & $21.7 \%$ \\
\hline & & Bad & 70 & $22.4 \%$ \\
\hline & & Fair & 103 & $32.9 \%$ \\
\hline & & Good & 58 & $18.5 \%$ \\
\hline & & Very good & 14 & $4.5 \%$ \\
\hline \multirow{5}{*}{$\begin{array}{l}\text { How do you evaluate the performance of the regional } \\
\text { government in the face of the pandemic? }\end{array}$} & \multirow{5}{*}{$\begin{array}{l}\text { Evaluation regional } \\
\text { government for pandemic }\end{array}$} & Very bad & 58 & $18.5 \%$ \\
\hline & & Bad & 70 & $22.4 \%$ \\
\hline & & Fair & 118 & $37.7 \%$ \\
\hline & & Good & 56 & $17.9 \%$ \\
\hline & & Very good & 11 & $3.5 \%$ \\
\hline \multirow{4}{*}{ How prepared are you financially to face the pandemic? } & \multirow{4}{*}{$\begin{array}{l}\text { Financial preparedness for } \\
\text { pandemic }\end{array}$} & Very bad & 60 & $19.2 \%$ \\
\hline & & Bad & 77 & $24.6 \%$ \\
\hline & & Fair & 129 & $41.2 \%$ \\
\hline & & Good & 47 & $15.0 \%$ \\
\hline \multirow{3}{*}{$\begin{array}{l}\text { Will the company where you work be able to financially } \\
\text { withstand the pandemic and not go bankrupt? }\end{array}$} & \multirow{3}{*}{$\begin{array}{l}\text { Company in the face of the } \\
\text { pandemic }\end{array}$} & Yes & 108 & $34.5 \%$ \\
\hline & & No & 43 & $13.7 \%$ \\
\hline & & Does not know & 162 & $51.8 \%$ \\
\hline \multirow{3}{*}{ What is the level of fear of losing your job? } & \multirow{3}{*}{ Fear of losing job } & High & 133 & $42.5 \%$ \\
\hline & & Moderate & 100 & $31.9 \%$ \\
\hline & & Low & 80 & $25.6 \%$ \\
\hline
\end{tabular}


Table 1. Cont.

\begin{tabular}{|c|c|c|c|c|}
\hline Questions & Variable & Alternatives & $n$ & Marginal Percentage \\
\hline \multirow{5}{*}{$\begin{array}{l}\text { How do you evaluate the work of the national media in } \\
\text { dealing with the pandemic? }\end{array}$} & \multirow{5}{*}{ National media } & Very bad, generate panic & 102 & $32.6 \%$ \\
\hline & & Bad & 47 & $15.0 \%$ \\
\hline & & Fair & 107 & $34.2 \%$ \\
\hline & & Good & 40 & $12.8 \%$ \\
\hline & & Very good, keep people informed & 17 & $5.4 \%$ \\
\hline \multirow{5}{*}{$\begin{array}{l}\text { How do you evaluate the work of the regional media in } \\
\text { dealing with the pandemic? }\end{array}$} & \multirow{5}{*}{ Regional media } & Very bad, generate panic & 43 & $13.7 \%$ \\
\hline & & Bad & 53 & $16.9 \%$ \\
\hline & & Fair & 125 & $39.9 \%$ \\
\hline & & Good & 67 & $21.4 \%$ \\
\hline & & Very good, keep people informed & 25 & $8.0 \%$ \\
\hline \multirow{6}{*}{$\begin{array}{l}\text { What were the social networks that provided you with } \\
\text { the most relevant information to make decisions or take } \\
\text { measures about the coronavirus? }\end{array}$} & \multirow{6}{*}{$\begin{array}{l}\text { Social networks for } \\
\text { pandemic }\end{array}$} & Facebook & 115 & $36.7 \%$ \\
\hline & & Twitter & 49 & $15.7 \%$ \\
\hline & & Instagram & 38 & $12.1 \%$ \\
\hline & & WhatsApp & 34 & $10.9 \%$ \\
\hline & & YouTube & 11 & $3.5 \%$ \\
\hline & & None & 66 & $21.1 \%$ \\
\hline
\end{tabular}


Table 2. Power of classification of citizen model.

\begin{tabular}{ccccc}
\hline \multirow{2}{*}{ Observed } & \multicolumn{4}{c}{ Predicted } \\
\cline { 2 - 5 } & Well-Prepared & Moderately Prepared & Not at All Prepared & Percent Correct \\
\hline Well-prepared & 12 & 0 & 0 & $100.0 \%$ \\
Moderately prepared & 0 & 142 & 18 & $88.8 \%$ \\
Not at all prepared & 0 & 17 & 124 & $87.9 \%$ \\
Overall percentage & $3.8 \%$ & $50.8 \%$ & $45.4 \%$ & $88.8 \%$ \\
\hline
\end{tabular}

Table 3. Multinomial logistic regression of citizen model.

\begin{tabular}{|c|c|c|c|c|}
\hline \multirow{2}{*}{ Effect } & \multirow{2}{*}{$\begin{array}{c}\text { Model Fitting Criteria } \\
-2 \text { Log Likelihood of } \\
\text { Reduced Model }\end{array}$} & \multicolumn{3}{|c|}{ Likelihood Ratio Tests } \\
\hline & & Chi-Squared & $\begin{array}{c}\text { Degrees of } \\
\text { Freedom }\end{array}$ & $p$-Value \\
\hline Intercept & 199.969 & 0.000 & 0 & - \\
\hline Sex & 370.670 & 170.701 & 4 & 0.000 \\
\hline Age & 2721.641 & 2521.672 & 10 & 0.000 \\
\hline Education & 385.351 & 185.382 & 8 & 0.000 \\
\hline Income projection & 951.248 & 751.278 & 6 & 0.000 \\
\hline Household debt & 457.733 & 257.764 & 6 & 0.000 \\
\hline Supplies & 204.003 & 4.034 & 4 & 0.401 \\
\hline Political persuasion & 216.214 & 16.245 & 22 & 0.804 \\
\hline Evaluation national government for pandemic & 250.876 & 50.907 & 8 & 0.000 \\
\hline Evaluation regional government for pandemic & 632.366 & 432.397 & 8 & 0.000 \\
\hline Financial preparedness for pandemic & 376.776 & 176.806 & 6 & 0.000 \\
\hline Company in the face of pandemic & 209.906 & 9.937 & 4 & 0.042 \\
\hline Fear of losing job & 204.415 & 4.446 & 4 & 0.349 \\
\hline National media & 423.152 & 223.183 & 8 & 0.000 \\
\hline Regional media & 416.013 & 216.044 & 8 & 0.000 \\
\hline Social networks for pandemic & 566.052 & 366.083 & 10 & 0.000 \\
\hline
\end{tabular}

From this perspective, household income was a critical measure of the country's preparedness. The cost of living in the regions was not an easy issue for residents: it was directly linked to employment and job security, which was seriously threatened by a standstill of activities caused by physical distancing and sanitary measures. To a large extent, the perceptions and confirmations of residents as to how the country would overcome the pandemic depended on how they felt their income would be affected.

For this reason, household debt was another important variable for the country in the face of the coronavirus. This situation was a matter of concern that has raised awareness, with or without the COVID-19, in both national and regional public discussions. The capacity to take on guaranteed debt in the face of job loss and the direct effects of the crisis or debt that continues to grow at an alarming rate to survive during these difficult circumstances are manifestations of another social aspect that define the preparedness of the country.

At the structural level, the figure of a government in any crisis management is relevant to face such events that impact the world. This was quickly perceived by the residents because the need for the protection of the state was assumed, which was represented by its political and immediate decision making and government response time in an emergency. Leadership, the ability of persuasion of effective and credible communication policies, and clarity in decision making in favor of citizens in the health, social, and economic spheres are essential to manage the risks to the population. Moreover, under the Chilean political presidential system in which the regions must assume that power is centralized, government efficiency is perceived as a matter of collective survival.

However, the conditions pointed out since the Chilean social upheaval in 2019 also highlighted the importance of having local authorities that could provide greater autonomy in their responses to people due to their proximity, knowledge of the territories, and empathy with regional and identity problems. The condition of the prior social crisis, intensified by the pandemic, further differentiates this aspect. Residents have perceived that the country's preparedness to face the coronavirus involves 
a more empowered and decentralized regional governance given that Nuble Region was recently established as a region in 2018.

The economic preparedness of the residents is another characteristic identified by the analysis to face the pandemic. The people of the region perceive what a crisis is and quickly assimilate that it will not only affect life but also material conditions. The protection of the most domestic and intimate limits for basic needs is threatened.

The economic preparation of the citizenry is another characteristic identified in the analysis to face the pandemic. People in the region gauged in their perceptions what constitutes a crisis and quickly assimilated that it would not exclusively affect the valued condition of life but also the material aspect. The protection of the most domestic and intimate boundaries in terms of basic needs has been threatened.

Linked to other previously mentioned aspects, the fragility of the regions could be reverted with stronger companies that have the capacity to protect themselves against these external threats. If these businesses could economically withstand the pandemic and manage to avoid bankruptcy, it would mean that the country was more prepared to face the disease.

From the perspective of the residents, media-related variables emerged that reflected the importance of information and journalism when facing pandemic risks. Given the political centralization of the country, but with a growing need for local empowerment, national media are necessary to provide the population with knowledge about the measures that have an impact on their territory from the decision-making core of power.

However, this information required an adequate local context that was provided by regional media. When the public is informed and interprets the national from the local, it can act more effectively and with greater certainty. This is also important in applying civic behavior, which is necessary in emergency situations.

Finally, the sphere of social networks demonstrated the coexistence with a different informational digital space that was more oriented to the emotions and immediate expectations of the residents along with interactions with many people, accounts, and entities that were important and oriented to the residents. The value of this as a social and supportive resource among residents is undeniably one of preparation in the face of pandemic instability.

As for the analysis of the perceptions of the businesspeople of Nuble Region, there is a series of other variables that complemented the preparation of the country for the coronavirus and others observed by the residents. Table 4 summarizes the cases related to the business owners. A linear model was first run on the responses as a function of the predictors to ensure that there were no problems with multicollinearity; only predictors with variance inflation factors (VIF) $<2$ were included in these models $[82,83]$.

Table 4. Summary of business owner responses.

\begin{tabular}{|c|c|c|c|c|}
\hline Questions & Variable & Alternatives & $n$ & $\begin{array}{c}\text { Marginal } \\
\text { Percentage }\end{array}$ \\
\hline \multirow{3}{*}{$\begin{array}{l}\text { In your opinion, the economic situation of the country in one } \\
\text { year will be: }\end{array}$} & \multirow{3}{*}{$\begin{array}{l}\text { Economic projection for } \\
\text { the country }\end{array}$} & Worse than it is now & 27 & $52.9 \%$ \\
\hline & & Same as it is now & 6 & $11.8 \%$ \\
\hline & & Better than it is now & 18 & $35.3 \%$ \\
\hline \multirow{3}{*}{ What is the sex of the owner? } & \multirow{3}{*}{ Sex } & Male & 41 & $80.4 \%$ \\
\hline & & Female & 8 & $15.7 \%$ \\
\hline & & Prefer not to say & 2 & $3.9 \%$ \\
\hline \multirow{5}{*}{$\begin{array}{l}\text { What do you think of the policies developed by the Chilean } \\
\text { government to support businesses? }\end{array}$} & \multirow{5}{*}{$\begin{array}{l}\text { Policies to support } \\
\text { businesses }\end{array}$} & Very bad & 7 & $13.7 \%$ \\
\hline & & Bad & 8 & $15.7 \%$ \\
\hline & & Fair & 15 & $29.4 \%$ \\
\hline & & Good & 19 & $37.3 \%$ \\
\hline & & Very good & 2 & $3.9 \%$ \\
\hline \multirow{3}{*}{ The debt situation of your company before the pandemic was? } & \multirow{3}{*}{ Pre-pandemic debt } & Complicated & 5 & $9.8 \%$ \\
\hline & & Moderately complicated & 17 & $33.3 \%$ \\
\hline & & Without problems & 29 & $56.9 \%$ \\
\hline \multirow{3}{*}{$\begin{array}{l}\text { What do you think will be the debt situation of your company } \\
\text { after the pandemic? }\end{array}$} & \multirow{3}{*}{ Post-pandemic debt } & Complicated & 27 & $52.9 \%$ \\
\hline & & Moderately complicated & 19 & $37.3 \%$ \\
\hline & & Without problems & 5 & $9.8 \%$ \\
\hline
\end{tabular}


Table 4. Cont.

\begin{tabular}{|c|c|c|c|c|}
\hline Questions & Variable & Alternatives & $n$ & $\begin{array}{l}\text { Marginal } \\
\text { Percentage }\end{array}$ \\
\hline $\begin{array}{l}\text { To support companies, the government should consider } \\
\text { privileging national over international companies }\end{array}$ & $\begin{array}{l}\text { Policy privileges for } \\
\text { national companies }\end{array}$ & $\begin{array}{l}\text { Not selected } \\
\text { Selected }\end{array}$ & $\begin{array}{l}34 \\
17\end{array}$ & $\begin{array}{l}66.7 \% \\
33.3 \%\end{array}$ \\
\hline $\begin{array}{l}\text { Which state economic stakeholders give you the most } \\
\text { guarantees or confidence to deal with the economic crisis } \\
\text { resulting from the social upheaval and COVID-19? }\end{array}$ & $\begin{array}{l}\text { Confidence in economic } \\
\text { stakeholders }\end{array}$ & $\begin{array}{l}\text { Ministry of Revenue } \\
\text { Ministry of Economy } \\
\text { SERNAC: National } \\
\text { Consumer Service } \\
\text { Central Bank } \\
\text { Superintendencies } \\
\text { SEREMIs: Regional } \\
\text { Ministerial Secretariats }\end{array}$ & $\begin{array}{c}28 \\
5 \\
2 \\
12 \\
1 \\
3\end{array}$ & $\begin{array}{c}54.9 \% \\
9.8 \% \\
3.9 \% \\
23.5 \% \\
2.0 \% \\
5.9 \%\end{array}$ \\
\hline $\begin{array}{l}\text { Do you think that the creation of the Nuble Region provides } \\
\text { better prospects for dealing with future global pandemics or } \\
\text { disasters in a decentralized manner? }\end{array}$ & Regional perspectives & $\begin{array}{l}\text { No } \\
\text { Yes }\end{array}$ & $\begin{array}{l}21 \\
30\end{array}$ & $\begin{array}{l}41.2 \% \\
58.8 \%\end{array}$ \\
\hline
\end{tabular}

Regarding the fitted model information, the chi-squared ratio test yielded a value of 87.472 $(p=0.000)$, indicating a good model fit. In addition, acceptable values were obtained for the pseudo R-squared (Cox and Snell: 0.820, Nagelkerke: 0.962, McFadden: 0.897). Table 5 shows that the power of the logistic multinomial model was suitable because it correctly classified $94.1 \%$ of the known observations and could be expected to project future estimates. Table 6 shows the likelihood ratio tests for the effects of the model and the partials whose low $p$-values show their high significance of the variables in the model.

Table 5. Power of classification of business owner model.

\begin{tabular}{ccccc}
\hline \multirow{2}{*}{ Observed } & \multicolumn{4}{c}{ Predicted } \\
\cline { 2 - 5 } & Worse than It Is Now & Same as It Is Now & Better than It Is Now & Percent Correct \\
\hline Worse than it is now & 25 & 1 & 1 & $92.6 \%$ \\
Same as it is now & 0 & 6 & 0 & $100.0 \%$ \\
Better than it is now & 1 & 0 & 17 & $94.4 \%$ \\
Overall percentage & $51.0 \%$ & $13.7 \%$ & $35.3 \%$ & $94.1 \%$ \\
\hline
\end{tabular}

Table 6. Multinomial logistic regression of business owner model.

\begin{tabular}{ccccc}
\hline Effect & Model Fitting Criteria & \multicolumn{2}{c}{ Likelihood Ratio Tests } \\
\cline { 2 - 5 } & $\begin{array}{c}\text {-2 Log Likelihood of } \\
\text { Reduced Model }\end{array}$ & Chi-Squared & $\begin{array}{c}\text { Degrees of } \\
\text { Freedom }\end{array}$ & $p$-Value \\
\hline Intercept & 4.500 & 0.000 & 0 & - \\
Sex & 18.962 & 14.463 & 4 & 0.006 \\
Policies to support businesses & 65.362 & 60.862 & 8 & 0.000 \\
Pre-pandemic debt & 38.162 & 33.663 & 4 & 0.000 \\
Post-pandemic debt & 38.890 & 34.390 & 4 & 0.000 \\
Policy privileges for national companies & 13.725 & 9.225 & 2 & 0.010 \\
Confidence in economic stakeholders & 64.045 & 59.545 & 10 & 0.000 \\
Regional perspectives & 13.398 & 8.898 & 2 & 0.012 \\
\hline
\end{tabular}

The results of the multinomial model applied to both businesspeople and residents of the Nuble Region highlighted the aspects of sex and age. However, they are associated with characteristics more typical of the elite, whose groups are historically considered to be "prepared" for crisis management; although things are changing, they maintain a symbolic and cultural weight because they are the owners of the companies. They are represented by adult men aged over 40 . These characteristics are also close to the classic patterns of the cultural stereotype of political leadership or personalized representation of power in Chile. They are also prominent figures in regional agendas and in local trade associations, and are economically legitimized to act.

A relevant variable to face COVID-19 in Chile was the policies developed by the government in favor of businesses. After months of growing concern due to the social upheaval in 2019, businesses experienced a crisis with effects that were more adverse than expected and caused 
by the coronavirus since March 2020. This quickly generated expectations from the owners for a relief package to provide sustainability to their respective businesses.

For the perceptions of the businesspeople, the types of economic policies the government has targeted to companies, as guarantor of the national budgets, are essential to determine the country's immediate economic success or failure in the crisis. Certain regions in Chile do not have a favorable situation for generating employment. In fact, the Nuble Region was one the regions with the highest unemployment rate before the pandemic, and this vulnerability is of great concern for business stakeholders.

Likewise, corporate debt issues are another sensitive and key point for the preparation of Chile to face the disease in two different situations. First, when the virus was not yet active in Chile and second, once the infectious outbreaks began to subside. The effects of indebtedness can be as devastating as the stopping of productive activities demonstrated under this scenario, although for many businesses they also provided options for survival, leading to the maintenance of regional employment.

The owners are aware that before any crisis arises, they should have a business without major debt problems; this is a necessary basis to face more complex times, which sooner or later come in inevitable cycles to countries open to the world. Indebtedness existing before the virus and the projected post-pandemic scenarios for regional businesses would mark a real level of country preparedness in this crisis, which is necessary when considering its success or failure.

Government measures such as those privileging national companies were appreciated in a context of increased global competition and the presence of international stakeholders in the regions. National companies, and those that were established in the same regions where they operate, tend to have a good relationship with the environments and communities that live there and have a positive impact on local economies. As key stakeholders that add cultural and identity value, they require greater protection from the weight of large foreign groups that have the capacity to protect themselves.

Meanwhile, for any government in times of crisis, confidence is essential in view of the social discipline required for the effectiveness of measures taken by the authorities and the persuasion of the policies they wish to promote in the economic sphere. The political and economic institutionality of the country leads to better preparedness in the face of destabilization by the pandemic.

On this level, business owners are aware that maintaining confidence in government authorities and state entities, especially the Ministry of Finance and the Central Bank which are responsible for overseeing the economic conditions of the country, minimizes uncertainty. This also places the state in its role as an essential coordination center to protect companies during the critical or recovery phases.

Finally, despite the recent creation of the Nuble Region, a decentralized territory with the capacity to uphold the demands of businesspeople to the national government and efficiently transfer aid measures to the productive activity of local areas is fundamental for an adequate preparedness that speeds up the timeframe and the concerns of the business stakeholders. The need has also been reestablished for a state that is present in the various regional realities to implement systems and early responses to human, material, health, and social catastrophes.

\section{Discussion and Conclusions}

These are very challenging times for Chile as for all other countries because of the situation caused by the COVID-19 world crisis. The pandemic is rapidly weakening economies and due to social isolation most productive activities have come to a standstill. According to the Economic Commission for Latin America and the Caribbean [84], the sectors strongly affected are tourism services, traditional cultural industry, commerce, repair of goods, hotels and restaurants, transport, fashion, and automobiles.

At the microeconomic level, the Chilean scenario is even more worrisome due to the consequences of the social upheaval that began on 18 October 2019. This is especially reflected in the precariousness of smaller companies, which have high unemployment rates and family debt. This is more complex at the regional level given the high degree of centralism in the country. Based on the results of the 
present study, it can be concluded that economic uncertainty and pessimism have increased in the Ñuble Region.

In this context, the present study addressed issues of great interest to the national and international community from a local and decentralized perspective, noting the serious economic and social conditions affecting both families and businesses. It also seeks to highlight the importance of generating local information that is relevant to the population and which can contribute to public policy and private investment decisions. In addition, the study emphasizes the need to strengthen local institutions, mainly universities and research centers.

The new scenario created by the COVID-19 pandemic has not only altered the "normality" of the inhabitants of the Nuble Region, but it has also increased uncertainty. It also has an impact on household incomes and the economy in general.

There is a high level of economic pessimism because of the health crisis. Results showed that the majority of respondents had a very poor perception of the country's level of preparedness to face the pandemic (only 3.8\% considered the country to be well prepared] and that their household income would continue to decrease $(46.6 \%)$. There is therefore pessimism about the economic situation of the country and the region in the upcoming 12 months. Likewise, it is concluded that there is deep concern about job loss $(74.4 \%)$, and $43.8 \%$ of the respondents indicated that they did not feel economically prepared to face the crisis. There was a negative evaluation $(44.1 \%)$ as to the efforts of the government to address the situation.

A similar level of pessimism and vulnerability to the effects of the crisis is also observed in the business environment of the Nuble Region, a territory made up mainly of smaller companies that are protagonists of the local economic development. This defines a complex future scenario generated by the global health crisis of COVID-19. Of the surveyed companies, $52.9 \%$ consider that the economic situation of the country in another year would be worse than the current one. Although the companies considered that their indebtedness was not for the most part complicated, they did believe that the situation would worsen significantly as a result of the pandemic.

Furthermore, it can be concluded that the health emergency, together with increased economic uncertainty and pessimism, has raised concerns about pending demands in the country. These are related to the quality of public health, employment, wages, and digital connectivity, which are determinants in the way families can face the challenges caused by this crisis.

Finally, it is necessary to remember that humanity has the ability to overcome and adapt to difficulties, but the pandemic is a global challenge and we do not know how long it will continue to threaten people's health. In this scenario, the concepts of resilience and sustainability are fundamental in the development of future social actions and will be the impulse that allows societies to restructure the lifestyle of families.

From now on, the most important challenge is to reduce the economic gap and for society to generate alternatives to include the most vulnerable in this new social dynamic, where access to technology and internet connection are elementary in "social isolation" and they are changing the way we communicate, learn, and work.

Regarding the limitations, we can mention that when carrying out the data collection through an online survey, an exclusion of the inhabitants who do not have internet access is generated due to the rurality of the town where they live or due to the lack of resources. In relation to this, it was not possible to obtain a proportional participation of all the communes of the Nuble region and therefore, the response rate was lower than expected. On the other hand, future research will consider other variables and relationships, such as the production linkages and employment effects of the business environment on the rest of the Nuble economy region [85-88].

Author Contributions: Formal analysis, H.d.1.F.-M., B.U.-H., M.F.-F., and C.E.-G.; funding acquisition, B.U.-H. and H.d.l.F.-M.; investigation, H.d.1.F.-M., B.U.-H., M.F.-F. and C.E.-G.; methodology, H.d.1.F.-M., M.F.-F.; project administration, B.U.-H.; software, H.d.1.F.-M.; supervision, B.U.-H. and M.F.-F.; validation, H.d.1.F.-M. and C.E.-G.; writing—original draft, B.U.-H. and M.F.-F.; writing—review \& editing, H.d.1.F.-M., C.E.-G. All authors have read and agreed to the published version of the manuscript. 
Funding: Hanns de la Fuente-Mella and Claudio Elórtegui-Gómez are supported by Grant Nucleo de Investigacion en Data Analytics/VRIEA/PUCV/039.432/2020.

Conflicts of Interest: The authors declare no conflict of interest.

\section{References and note}

1. Sánchez-Bernal, A.; Batiz, M.L.G. El desempeño económico de los gobiernos municipales mexicanos en los procesos de desarrollo a escala local. Econ. Soc. Territ. 2010, 22, 355-412. [CrossRef]

2. Salazar, C.; Jaime, M. Participación en organizaciones sociales en Chile. ¿Una alternativa para mejorar el bienestar económico de los hogares? Estud. Econ. 2009, 36, 191-215. [CrossRef]

3. Polanyi, K. The Great Transformation: The Political and Economic Origins of Our Time, 2nd ed.; Beacon Press: Boston, MA, USA, 2001.

4. Wright, A. Sustainable Development at the Community Level: An Analytical Framework for Embedding the Economy in Local Decision-Making. Ph.D. Thesis, Political Science, Northern Arizona University, Flagstaff, AZ, USA, 2017.

5. Hopkins, D.J.; Kim, E.; Kim, S. Does newspaper coverage influence or reflect public perceptions of the economy? Res. Polit. 2017, 1-7. [CrossRef]

6. Guillen, M.; Guardiola, J.; Garcia, F. Sustainable development in times of economic crisis: A needs-based illustration from Granada (Spain). J. Clean. Prod. 2017, 150, 267-276. [CrossRef]

7. Fawson, F. Local Economies, Local Governance and Community: Defining Community in Nova Scotia. Master's Thesis, Saint Mary's University, Halifax, NS, Canada, 2006.

8. Barrett, C. Rural poverty dynamics: Development policy implications. Agric. Econ. 2005, 32, 45-60. [CrossRef]

9. Vogt, C.; Polino, C. Percepción Pública de la Ciencia. Resultados de la Encuesta en Argentina, Brasil, España y Uruguay; Editora Unicamp: Sao Paulo, Brazil, 2003.

10. Rodríguez, C.; Padilla, G. Percepciones sobre ciencia y tecnología en Chile: Un análisis factorial exploratorio y confirmatorio para la primera versión de la Encuetas Nacional de Cultura Científica y Tecnológica. PAAKAT Rev. Tecnol. Soc. 2019, 8, 1-20.

11. Galardi, G. Exploring the Use of Citizen Satisfaction Survey Results in Municipal Policing. Ph.D. Thesis, Northcentral University, Prescott Valley, AZ, USA, 2008.

12. Miller, T.; Miller-Kobayashi, M. Citizen Surveys: How to Do Them; How to Use Them; What They Mean, 2nd ed.; International City Management Association: Washington, DC, USA, 2000.

13. Trojanowicz, R.; Kappeler, V.; Gaines, L. Community Policing: A Contemporary Perspective, 3rd ed.; Anderson: Cincinnati, OH, USA, 2002.

14. D'Adamo, I.; Falcone, P.M.; Martin, M.; Rosa, P.A. Sustainable revolution: Let's go sustainable to get our globe cleaner. Sustainability 2020, 12, 4387. [CrossRef]

15. D'Adamo, I.; Rosa, P. How Do You See Infrastructure? Green Energy to Provide Economic Growth after COVID-19. Sustainability 2020, 12, 4738. [CrossRef]

16. Elmqvist, T.; Andersson, E.; Frantzeskaki, N.; McPhearson, T.; Olsson, P.; Gaffney, O.; Takeuchi, K.; Folke, C. Sustainability and resilience for transformation in the urban century. Nat. Sustain. 2019, 2, 267-273. [CrossRef]

17. Ministerio de Salud. Reporte Diario COVID-19. 2020. Available online: https:/www.minsal.cl/wp-content/ uploads/2020/10/CP-REPORTE-COVID-19-Mi\%C3\%A9rcoles-14102020.pdf (accessed on 14 October 2020).

18. Huarcaya-Victoria, J. Consideraciones Sobre La Salud Mental En La Pandemia De Covid-19. Rev. Peru. Med. Exp. Salud Publica 2020, 37, 327-334. [CrossRef]

19. Vallina-Hernández, A.M.; Martínez, P.; González, C.; Fuentes, R.; De la Fuente-Mella, H. Gravity Models for Latin American Economies. In Advances in Human Factors, Business Management and Leadership, Proceedings of the International Conference on Applied Human Factors and Ergonomics, San Diego, CA, USA, 16-20 July 2020; Kantola, J., Nazir, S., Salminen, V., Eds.; Springer: Cham, Switzerland, 2020; pp. 495-501.

20. Rattliff, J.R. Economic News and Public Perceptions of the U.S. Economy. Ph.D. Thesis, Department of Political Science in the Graduate School of Duke University, Durham, NC, USA, 2001.

21. Lampis, A.; Rodríguez, L.K. Colombia, entre lo local y lo global: La inserción de las regiones en la nueva economía global. Soc. Econ. 2012, 22, 95-131.

22. Mildenberger, M.; Leiserowitz, A. Public opinion on climate change: Is there an economy-environment tradeoff? Environ. Polit. 2017, 26, 801-824. [CrossRef] 
23. Gómez, C.E.; Ruette, J.; de la Fuente-Mella, H. The Philanthropy of Chilean Citizenship: A Quantitative Data Science Study. In Advances in Human Factors, Business Management and Leadership, Proceedings of the International Conference on Applied Human Factors and Ergonomics, San Diego, CA, USA, 16-20 July 2020; Kantola, J., Nazir, S., Salminen, V., Eds.; Springer: Cham, Switzerland, 2020; pp. 509-515.

24. Schwarz, V. Citizen in the New Latin American State: Politics, the Economy and the Dynamics of Democratic Legitimacy in Bolivia and Latin America. Ph.D. Thesis, Vanderbilt University, Nashville, TN, USA, 2014.

25. Soroka, S.N.; Stecula, D.A.; Wlezien, C. It's (change in) the (future) economy, stupid: Economic indicators, the media, and the public opinion. Am. J. Polit. Sci. 2015, 59, 457-474. [CrossRef]

26. Svallfors, S. Contested Welfare States: Welfare Attitudes in Europe and Beyond; Stanford University Press: Redwood City, CA, USA, 2012.

27. Shapiro, R.Y. Public opinion and American democracy. Public Opin. Q. 2011, 75, 982-1017. [CrossRef]

28. Elórtegui-Gómez, C.; De la Fuente-Mella, H.; Alvarado, M.; Guajardo, M. Efficiency of the City Councils Using Cross-Sectional Model: Challenges in Times of Change and Political Tension. IntechOpen. Available online: https://www.intechopen.com/online-first/efficiency-of-the-city-councils-using-crosssectional-model-challenges-in-times-of-change-and-politi (accessed on 11 November 2020).

29. Mayeda, A.; Boyd, A. Factors influencing public perceptions of hydropower projects: A systematic literature review. Renew. Sustain. Energy Rev. 2020, 121, 109713. [CrossRef]

30. Ahmand, M.; Abu Talib, N. Empowering local communities: Decentralization, empowerment and community driven development. Qual. Quant. 2015, 49, 827-838. [CrossRef]

31. Duncana, S.; Goodwina, M. Local economic policies: Local regeneration or political mobilization. Local Gov. Stud. 2008, 11, 75-96. [CrossRef]

32. Agrawal, A.; Gupta, K. Decentralization and participation: The governance of common pool resources in Nepal's Terai. World Dev. 2005, 33, 1101-1114. [CrossRef]

33. Bennet, L. Using Empowerment and Social Inclusion for Pro-Poor Growth: A Theory of Social Change. Background Paper for the Social Development Sector Strategy; World Bank: Washington, DC, USA, 2002.

34. Corbett, J.; Keller, P. Empowerment and participatory geographic information and multimedia systems: Observations from two communities in Indonesia. Inf. Technol. Int. Dev. 2004, 2, 25-44. [CrossRef]

35. Salazar, I.; Caro, N.; Zegarra, E. Desempeño de la gerencia de desarrollo económico de la municipalidad de Leoncio Prado (Huanuco) y el desarrollo económico local. Investig. Amazon. 2016, 2, 11-19.

36. Fawaz, M.J.; Vallejos, R.; Tolón, A.; Lastra, X. Redefining local participation in sustainable rural development in Chile: The case of small farmers in Nuble Province. Int. Dev. Plan. Rev. 2012, 34, 295-318. [CrossRef]

37. Hickey, S.; Mohan, G. Towards participation as transformation: Critical themes and challenges. In Participation: From Tyranny to Transformation? Exploring New Approaches to Participation Development; Hickey, S., Mohan, G., Eds.; Zed Books: London UK; New York, NY, USA, 2004; pp. 3-24.

38. Stewart, A. Two conceptions of citizenship. Br. J. Sociol. 1995, 46, 63-79. [CrossRef]

39. Oldfield, A. Citizenship: An unnatural practice? Polit. Q. 1990, 61, 177-187. [CrossRef]

40. Cornwall, A. Making Spaces, Changing Spaces: Situating Participation in Development; IDS Working Paper; Institute for Development Studies: Sussex, UK, 2002; Volume 170.

41. Ramírez, R.; Quarry, W. Communication for Development. A Medium for Innovation in Natural Resource Management; IDRC/CRDI-FAO: Ottawa, ON, Canada, 2004; Available online: http://www.fao.org/waicent/ faoinfo/sustdev/Welcome_htm (accessed on 11 November 2020).

42. Gaventa, J. Exploring Citizenship, Participation and Accountability. IDS Bull. 2002, 33, 1-11. [CrossRef]

43. Wong, C. Determining factors for local economic development: The perception of practitioners in the North West and Eastern Regions of the UK. Reg. Stud. 1998, 32, 707-720. [CrossRef]

44. Peters, M.; Chan, C.; Legerer, A. Local Perception of Impact-Attitudes-Actions towards Tourism Development in the Urlaubsregion Murtal in Austria. Sustainability 2018, 10, 2360. [CrossRef]

45. Abukari, H.; Mwalyosi, R.B. Local communities' perceptions about the impact of protected areas on livelihoods and community development. Glob. Ecol. Conserv. 2020, 22, e00909. [CrossRef]

46. Rodrick, D. The New Global Economy and Developing Countries: Making Openness Work; Overseas Development Council: Washington, DC, USA, 1999.

47. Berdegué, J.; Schejtman, A. Desarrollo territorial rural. In Territorios Rurales. Movimientos Sociales y Desarrollo Territorial Rural en América Latina; Bengoa, J., Ed.; RIMISP: Santiago, Chile, 2006; pp. 45-83. 
48. Berninger, K.; Kneeshaw, D.; Messier, C. The role of cultural models in local perceptions of SFM-Differences and similarities of interest groups from three boreal regions. J. Environ. Manag. 2009, 90, 740-751. [CrossRef]

49. Cárcamo, H. Ciudadanía y formación inicial docente: Explorando las representaciones sociales de académicos y estudiantes. Rev. Pedag. 2008, 29, 245-268.

50. Martínez, A.G. La influencia de la cultura y las identidades en las relaciones interculturales. Kairos Rev. Temas Soc. 2008, 22, 3.

51. Castells, 1997 as cited in Balbontin, R.; Rojas, N. Percepciones sobre la identidad del profesor rural en la Región del Nuble, Chile. Rev. Educ. Las Am. 2020, 10, 1-14.

52. Vega, J. Geografía e identidad territorial. Notas Hist. Geogr. 2000, 11, 193-222.

53. Molina, J. Desarrollo, Identidad y Descentralización: El caso de la Sexta Región. 2005. Available online: http://repositorio.uchile.cl/bitstream/handle/2250/113368/cs39-molinaj38.pdf?sequence=1 (accessed on 11 November 2020).

54. Molano, O. Identidad cultural un concepto que evoluciona. Rev. Opera 2007, 7, 69-84.

55. Brea, L. Factores que determinan el sentido de pertenencia de los estudiantes de la PUCMM-CSTA. Cuad. Pedag. Univ. 2017, 12, 21-38. [CrossRef]

56. Giménez, G. Territorio e Identidad. Breve Introducción a la Geografía Cultural Trayectorias; Universidad Autónoma de Nuevo León: Monterrey, Nuevo León, México, 2005; Volume VII, Núm. 17; pp. 8-24. Available online: https://www.redalyc.org/pdf/607/60722197004.pdf (accessed on 11 November 2020).

57. Cazzuffi, C.; López, D.; Del Valle, V. Crecimiento e Inclusión en los Territorios Rurales-Urbanos de Chile; Serie documento de trabajo No 255, Jóvenes Rurales y Territorios: Una estrategia de Diálogos y Política; Rimisp: Santiago, Chile, 2019; Available online: https://rimisp.org/wp-content/files_mf/1570477913Crecimientoeinclusio\% CC\%81nenlosterritoriosruralesurbanosdeChile.pdf (accessed on 11 November 2020).

58. Schejtman, A.; Berdegué, J. Desarrollo territorial rural. Debat. Temas Rural. 2004, 1, 7-46.

59. Berdegué, J. ¿Qué Desarrollo Territorial? 2016. Available online: http://lasillavacia.com/silla-llena/red-rural/ historia/que-desarrollo-territorial-58748 (accessed on 24 July 2020).

60. Nogué i Font, J. Paisaje, Identidad y Globalización, Fabrikart: Arte, Tecnología, Industria, Sociedad. Available online: https://ojs.ehu.eus/index.php/Fabrikart/article/viewFile/2227/1843 (accessed on 11 November 2020).

61. Vallejos, R.; Fawas, J.; Fonseca, M. Análisis de la Movilidad Espacial Femenina en Territorio Funcional de la Provincia de Nuble: Una Caracterización de sus Efectos en los Ámbitos de Empleo, Educación, Acceso a Bienes y Estilos de vida en Mujeres Urbano-Rurales. 2015. Available online: http://repobib.ubiobio.cl/jspui/ handle/123456789/436 (accessed on 11 November 2020).

62. Berdegué, J.; Jara, B.; Fuentealba, R.; Tohá, J.; Modrego, F.; Schejtman, A.; Bro, N. Territorios Funcionales en Chile; Documento de trabajo No 102. Programa Dinámicas Territoriales Rurales; Rimisp: Santiago, Chile, 2011.

63. Salazar, X. El Estudio del Sistema de Asentamientos como parte del Diagnostico del Sistema Territorial Cantonal. Master's Thesis, University of Cuenca, Cuenca, Azuay, Ecuador, 2013.

64. Fawaz, M.; Vallejos, R. Calidad de vida, ocupación, participación y roles de género: Un sistema de indicadores sociales de sostenibilidad rural (Chile). Cuad. Desarro. Rural 2012, 8, 24.

65. Micheletti, S.; Cortés, F.S.; Troncoso, F.L. Los contenidos del habitar rurbano: Prácticas, movilidad e identidades en las ciudades intermedias del Maule, Chile. Cuad. Urban. 2020, 27, 111-131. [CrossRef]

66. Landa, C. Constitución y Emergencia Sanitaria. Palestra Editores. 2020. Available online: https://books. google.es/books?hl=es\&lr=\&id=icLsDwAAQBAJ\&oi=fnd\&pg=PT122\&dq=nueva+normalidad +\&ots= LKoFM36w6c\&sig=b1YVuyJOhzFv5B2n0KeR_HAuMLQ\#v=onepage\&q=nueva\%20normalidad\&f=false (accessed on 6 August 2020).

67. De España, B. Escenarios Macroeconómicos de Referencia Para la Economía Española Tras el Covid-19; Boletín económico/Banco de España (Artículos): Madrid, Spain, 2020.

68. Coughenour, C.; Paz, A.; De la Fuente-Mella, H.; Singh, A. Multinomial logistic regression to estimate and predict perceptions of bicycle and transportation infrastructure in a sprawling metropolitan area. J. Public Health 2016, 38, e401-e408. [CrossRef]

69. Clark, S.; Coughenour, C.; Bumgarner, K.; De la Fuente-Mella Reynolds, C.; Abelar, J. The impact of pedestrian crossing flags on driver yielding behavior in Las Vegas, NV. Sustainability 2019, 11, 4741. [CrossRef]

70. Coughenour, C.; De la Fuente-Mella, H.; Paz, A. Analysis of self-reported walking for transit in a sprawling urban metropolitan area in the western U.S. Sustainability 2019, 11, 852. [CrossRef] 
71. Subsecretaria de Desarrollo Regional y Administrativo. Observatorio Regional. Gobierno de Chile. 2020. Available online: http://www.observatorioregional.cl/ (accessed on 8 August 2020).

72. Instituto Nacional de Estadísticas. Síntesis de Resultados. Censo. Gobierno de Chile. 2017. Available online: https://www.censo2017.cl/descargas/home/sintesis-de-resultados-censo2017.pdf (accessed on 3 August 2020).

73. Ministerio de Desarrollo Social y Familia. Encuesta de Caracterización Socio-Económica. Gobierno de Chile, Santiago. 2017. Available online: http://observatorio.ministeriodesarrollosocial.gob.cl/casen-multidimensional/ casen/casen_2017.php (accessed on 24 July 2020).

74. De la Fuente-Mella, H.; Quezada, E.R.; Muñoz, C.G.; Lagos, M.; Inostroza, C. Probabilistic Models of Job Placement and Positioning for Students with a Career in Chile. In Advances in Human Factors, Business Management and Leadership, Proceedings of the International Conference on Applied Human Factors and Ergonomics, San Diego, CA, USA, 16-20 July 2020; Kantola, J., Nazir, S., Salminen, V., Eds.; Springer: Cham, Switzerland, 2020; pp. 516-522.

75. Umaña, B. Caracterización de la Provincia de Ñuble y una Propuesta Estratégica para el Desarrollo del Territorio; Ediciones Universidad del Bío-Bío: Concepcion, Chile, 2015.

76. Observatorio Laboral de Nuble. Reporte Sintesis Silvoagropecuario. OTIC SOFOFA, Universidad del Bío-Bío y Servicio de Capacitación y Empleo (SENCE), Ministerio del Trabajo, Gobierno de Chile. 2019. Available online: https://www.observatoriolaboralnuble.cl/wp-content/uploads/2019/pdf/Reporte\%20S\% C3\%ADntesis\%20Silvoagropecuario\%20[mayo\%202019).pdf (accessed on 5 August 2020).

77. De la Fuente-Mella, H.; Alvaro Martínez, M.; Elórtegui Gómez, C.; Calderón, M.G. Econometric Modeling of the Performance in the City Council of Chile. In Advances in Human Factors, Business Management and Leadership, Proceedings of the International Conference on Applied Human Factors and Ergonomics, San Diego, CA, USA, 16-20 July 2020; Kantola, J., Nazir, S., Salminen, V., Eds.; Springer: Cham, Switzerland, 2020; pp. 151-160.

78. Gobierno Regional/Agraria. Informe Política para el Sector Agroalimentario para la Región del Bío Bío; GORE: Concepción, Chile, 2010.

79. Fawaz, M.; Vallejos, R. Exploring the linkage between secondary technical and vocational education system, labor market and family setting. A prospective analysis from Central Chile. Educ. Stud. 2020, 56, 186-207. [CrossRef]

80. De la Fuente-Mella, H.; Vallina-Hernández, A.M.; Estay-Cornejo, R. Econometric modeling of the efficiency in the generation of electric power in Chile. In Advances in Human Factors, Business Management and Leadership, Proceedings of the International Conference on Applied Human Factors and Ergonomics, Washington, DC, USA, 24-28 July 2019; Kantola, J., Nazir, S., Eds.; Springer: Cham, Switzerland, 2019; pp. 352-360.

81. Hernández, R. Metodología de la Investigación; McGraw-Hill: Mexico City, Mexico, 2010.

82. Cea, M. Fundamentos y Aplicaciones en Metodología Cuantitativa; Síntesis: Madrid, Spain, 2012.

83. Paz, A.; De la Fuente-Mella, H.; Singh, A.; Conover, R.; Monteiro, H. Highway expenditures and associated customer satisfaction: A case study. Math. Probl. Eng. 2016, 2016. [CrossRef]

84. Naciones Unidas. Informe de Politicas: La COVID-19 y la Transformación del Turismo; UN: New York, NY, USA, 2020.

85. CEPAL. Sectores y Empresas Frente al COVID-19: Emergencia y Reactivación. 2020. Available online: https: //repositorio.cepal.org/bitstream/handle/11362/45734/4/S2000438_es.pdf (accessed on 11 November 2020).

86. Sadik-Zada, E.R.; Loewenstein, W.; Hasanli, Y. Production linkages and dynamic fiscal employment effects of the extractive industries: Input-output and nonlinear ARDL analyses of Azerbaijani economy. Miner. Econ. 2019. [CrossRef]

87. Sadik-Zada, E.R. Addressing the growth and employment effects of the extractive industries: White and black box illustrations from Kazakhstan. Post Commun. Econ. 2020. [CrossRef]

88. De la Fuente-Mella, H.; Vallina, A.M.; Solis, R. Stochastic analysis of the economic growth of OECD countries. Econ. Res. Ekon. Istraž. 2020, 33, 2189-2202. [CrossRef]

Publisher's Note: MDPI stays neutral with regard to jurisdictional claims in published maps and institutional affiliations. 


\title{
90 Days of COVID-19 Social Distancing and Its Impacts on Air Quality and Health in Sao Paulo, Brazil
}

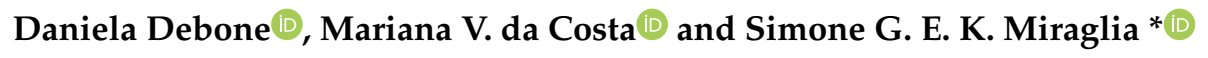 \\ Instituto de Ciências Ambientais, Químicas e Farmacêuticas, Universidade Federal de São Paulo (UNIFESP), \\ Diadema 09913-030, Brazil; d.debone@unifesp.br (D.D.); vieiramariana70@gmail.com (M.V.d.C.) \\ * Correspondence: simone.miraglia@unifesp.br
}

Received: 8 August 2020; Accepted: 8 September 2020; Published: 10 September 2020

\begin{abstract}
The COVID-19 pandemic has imposed a unique situation for humanity, reaching up to 5623 deaths in Sao Paulo city during the analyzed period of this study. Due to the measures for social distancing, an improvement of air quality was observed worldwide. In view of this scenario, we investigated the air quality improvement related to $\mathrm{PM}_{10}, \mathrm{PM}_{2.5}$, and $\mathrm{NO}_{2}$ concentrations during 90 days of quarantine compared to an equivalent period in 2019. We found a significant drop in air pollution of $45 \%$ of $\mathrm{PM}_{10}, 46 \%$ of $\mathrm{PM}_{2.5}$, and $58 \%$ of $\mathrm{NO}_{2}$, and using a relative-risk function, we estimated that this significant air quality improvement avoided, respectively, 78, 337, and 387 premature deaths, respectively, and prevented approximately US\$720 million on health costs. Moreover, we estimated that 5,623 deaths by COVID-19 represent an economic health loss of US\$ 10.5 billion. Both health and economic gains associated with air pollution reductions give a positive perspective of the efforts towards keeping air pollution reduced even after the pandemic, highlighting the importance of improving the strategies of air pollution mitigation actions, as well as the crucial role of adopting efficient measures to protect human health both during and after the COVID-19 global health crisis.
\end{abstract}

Keywords: COVID-19; air pollution; air quality; health effects; economic burden

\section{Introduction}

The coronavirus disease (COVID-19) pandemic caused by rapidly spreading a severe acute respiratory syndrome coronavirus 2 (SARS-CoV-2) has imposed a unique situation for humanity since the Spanish flu pandemic, transmitted by the $\mathrm{H}_{1} \mathrm{~N}_{1}$ influenza A virus, which devastated at least 50 million people in 1918. Thus far, COVID-19 is considered the deadliest pandemic in modern history, exceeding the outbreaks caused by $\mathrm{H}_{1} \mathrm{~N}_{1}$ virus, such as the most recent outbreak in 2009, formerly known as swine flu [1-8].

Likewise, in the past two decades, coronaviruses have also shown a continuing potential threat to public health since the emergence of severe acute respiratory syndrome coronavirus (SARS-CoV) and Middle East respiratory syndrome coronavirus (MERS-CoV). SARS-CoV and MERS-CoV can cause severe respiratory distress syndrome in humans, and despite SARS-CoV-2 being highly pathogenic and deadlier to humans, SARS epidemics (2003-2004) and MERS outbreaks (2012) were responsible for the deaths of thousands of people worldwide, as shown in Figure 1 [1-8]. 


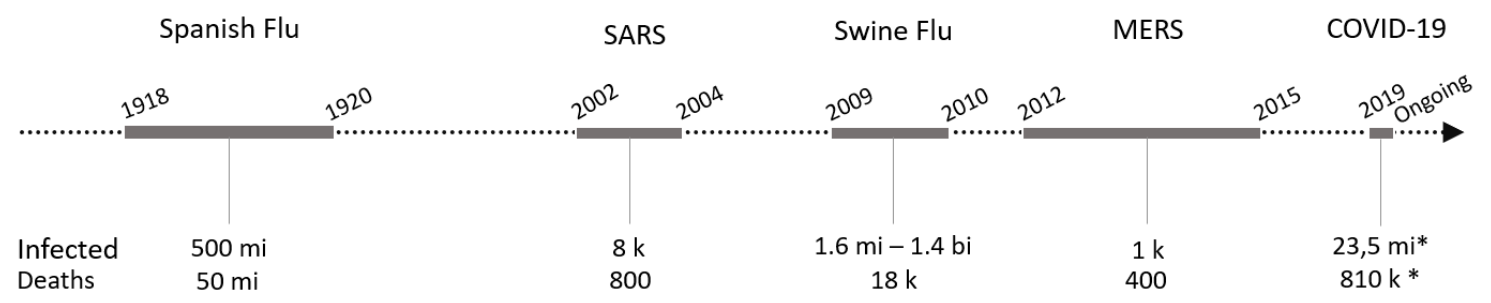

Figure 1. Worldwide pandemics' timeline. Estimated numbers of infected people and deaths to Spanish flu, severe acute respiratory syndrome (SARS), swine flu, Middle East respiratory syndrome (MERS), and COVID-19 between 1918 and 2020 * 25 August, according to WHO Coronavirus Disease (COVID-19) Dashboard [9].

Since its emergence in Wuhan, the capital of Hubei Province at the end of 2019, SARS-CoV-2 has quickly spread worldwide, characterizing a pandemic. On 30 January 2020, the Emergency Committee of the World Health Organization (WHO) declared a global health emergency based on the increasing rates of case reporting on different continents [10].

The first case in Latin America was confirmed in February 2020 in Sao Paulo city, Brazil. The confirmed patient had travelled to Lombardy in northern Italy, and the same origin was confirmed for the virus by genome sequencing [11]. Since then, an exponential increase in confirmed cases was observed in the following weeks. No specific drugs or vaccines are available, and health systems are overburdened in every city, especially in Sao Paulo city, which reported 91,198 confirmed cases of COVID-19 and 5623 deaths up to 14 June (last day of the analyzed period in this study), being considered the epicenter of the pandemic in Brazil and in South America. A follow-up on 25 August recorded 250,171 confirmed cases and 11,141 deaths.

In addition to the worrying symptoms of pneumonia, acute respiratory distress syndrome, (ARDS) and multiple organ dysfunction, which can lead to death, mainly in people considered to be at risk groups, such as the elderly and people with chronic noncommunicable diseases and respiratory diseases [12-15], some studies have suggested a higher susceptibility of COVID-19 infection of populations exposed to high concentrations of air pollution [16-18]. A recent study demonstrated that long-term average exposure to fine particulate matter $\left(\mathrm{PM}_{2.5}\right)$ is associated with an increased risk of COVID-19 death in the United States. They found that an increase of $1 \mu \mathrm{g} / \mathrm{m}^{3}$ in $\mathrm{PM}_{2.5}$ is related to a growth of $8 \%$ of COVID-19 death rate (95\% confidence interval [CI]: $2 \%, 15 \%$ ) [19]. Despite these evidences, much remains to be investigated to disentangle the relationship between high levels of air pollution and increasing susceptibility to SARS-CoV-2 infection. However, there is no doubt that in densely populated and polluted localities, such as Sao Paulo, strategies to prevent COVID-19 pandemic progression should be more severe [20].

Sao Paulo, one of the largest urban centers of the world, with a population of approximately 12,252,023 inhabitants in 2019 [21], stands out not only for its economic and industrial performance but also due to its high levels of atmospheric pollutants and greenhouse gas emissions, mainly from motor vehicles, responsible for air quality degradation and negative impacts on public health [21-23]. Considering the fleet of 9.1 million vehicles and 87.83 million $\mathrm{km}$ travelled daily by all vehicles in Sao Paulo, automobiles account for $69.5 \%$ and are responsible for $72.6 \%$ of greenhouse gas emissions per day $[24,25]$. According to measurements carried out by Sao Paulo's Environmental Agency (CETESB), vehicles are responsible for $97 \%$ of carbon monoxide (CO), $74 \%$ of hydrocarbons, $62 \%$ of nitrogen oxides $\left(\mathrm{NO}_{\mathrm{x}}\right)$, and $40 \%$ of particulate matter $\left(\mathrm{PM}_{10}\right.$ and $\left.\mathrm{PM}_{2.5}\right)$. Concerning the combustion of biofuels, ethanol does not contribute to the emission of particulate matter and releases pollutants not daily monitored by CETESB, such as formaldehyde and acetaldehyde [22]. Although Sao Paulo is one of the largest producers and consumers of biofuels in the world, emissions from fossil fuels are still prevalent, especially in the transport sector $[22,26]$.

Considering only particulate matter with a diameter less than 2.5 microns $\left(\mathrm{PM}_{2.5}\right), 5012$ premature deaths occur per year in Sao Paulo city [27]. In addition, a recent case study reported the strong 
influence of diesel-fueled heavy-duty vehicles on air quality. Merely during the Brazilian truck-driver strike in 2018, the reduction in the $\mathrm{PM}_{10}$ concentration resulted in the prevention of between six and eight deaths, which implies between 321 and 442 avoided deaths in a year scenario only in Sao Paulo [28].

Several factors, mainly of anthropogenic origin, are related to increased risk of disease outbreaks of vector-borne and zoonotic diseases, such as mega-trends in human population growth, ecosystem reduction and fragmentation, and land use modification and climate change [29-32]. Therefore, the relationship between a pandemic, such as the current COVID-19, global environmental change, and human health is overly complex and deep.

In this context, this ongoing pandemic has also been a great opportunity to discuss the effects of anthropogenic activities on air quality and its implications on public health, as well as highlight the need for a socioeconomic transformation regarding promoting environmentally friendly transport policies and a low-carbon economy towards a sustainable and resilient society [33].

Therefore, we aimed to investigate the air quality improvement during 90 days of social distancing, as well as to compare the associated avoided deaths to COVID-19 burden deaths, considering the relative risk and the economic outcomes in Sao Paulo megacity. The corresponding analysis was based on the comparison of $\mathrm{NO}_{2}, \mathrm{PM}_{10}$, and $\mathrm{PM}_{2.5}$ concentrations during quarantine and equivalent periods in 2019.

The paper is structured as follows: Section 2 introduces the method applied in the present study. Section 3 presents all performed analyses and their results. Section 4 presents the discussion of the outcomes. Finally, in Section 5, we finish the paper with concluding remarks and limitations.

\section{Materials and Methods}

On 11 March, the World Health Organization declared the new coronavirus a pandemic. In Brazil, on 16 March 2020, some activities started to cease, as universities, schools, and some companies imposed home offices, and the first death was reported in Sao Paulo city on 17 March. Thus, the Sao Paulo government officially decreed quarantine on 22 March.

According to these initial events, we chose to investigate the air quality in the city of Sao Paulo, starting on 16 March and dividing the analysis period into 13 weeks, ending on 14 June. As a reference for the comparison, the days in the equivalent period in 2019 (16 March-14 June 2019) were selected. This set of days was designated as the "control period".

Daily records of precipitation, wind speed, and means of air temperature were obtained from the Sao Paulo Environmental State Agency Air Quality Information System (QUALAR) [34] and from the Brazilian Agrometeorological Monitoring System [35]. These meteorological parameters were used as a criterion to exclude the days of the control period that differed from the range of the meteorological conditions of the quarantine period.

In Sao Paulo, the concentrations of atmospheric pollutants are higher in conditions of low ventilation, reduced relative humidity, and absence of precipitation, which occurs mainly in autumn and winter [36-38].

According to CETESB measurements, during the period between 16 March and 14 June 2020, the autumn season, the quarantine period presented an average daily temperature of approximately $25^{\circ} \mathrm{C}$ and a wind speed of $2 \mathrm{~m} / \mathrm{s}$. This period also presented 65 days with precipitation equal to zero or less than $0.5 \mathrm{~mm}$, equivalent to an average of $1.5 \mathrm{~mm}$ in total (Table 1). As a reference for the comparison, the control period, between 16 March and 14 June 2019, showed daily means of air temperature of approximately $26^{\circ} \mathrm{C}$, average wind speed of $1.9 \mathrm{~m} / \mathrm{s}$, and 52 days with precipitation equal to zero or less than $0.5 \mathrm{~mm}$, equivalent to an average of $1.5 \mathrm{~mm}$ in total (Table 1). In addition, both analyzed periods showed similarity in terms of atmospheric pressure (on average, approximately $927 \mathrm{hPa}$ ), global solar radiation (on average, approximately $677 \mathrm{~W} / \mathrm{m}^{2}$ ) and relative humidity (mean close to $47 \%)$ [34]. 
Table 1. Summary of the daily records of precipitation, wind speed, and mean temperature during quarantine and control periods.

\begin{tabular}{ccccccc}
\hline & \multicolumn{2}{c}{ Precipitation $(\mathbf{m m})$} & \multicolumn{2}{c}{ Wind $(\mathbf{m} / \mathbf{s})$} & \multicolumn{2}{c}{ Temperature $\left({ }^{\circ} \mathbf{C}\right)$} \\
\cline { 2 - 7 } & Control Period & Quarantine & Control Period & Quarantine & Control Period & Quarantine \\
\hline N & 91 & 91 & 91 & 91 & 91 & 91 \\
Mean (SD) & $3.7(8.1)$ & $1.5(4.3)$ & $1.8(0.4)$ & $1.9(0.4)$ & $26.4(3.3)$ & $25.3(3.2)$ \\
Median & 0.1 & 0 & 1.9 & 2.0 & 27.5 & 25.7 \\
Minimum & 0 & 0 & 1 & 1 & 17.8 & 15.8 \\
Maximum & 37.2 & 25.7 & 2.8 & 3.6 & 32.4 & 32.2 \\
\hline
\end{tabular}

Number of observations (N); Standard deviation (SD).

Therefore, based on these observations, the control period demonstrated meteorological conditions similar to those observed during the quarantine period, justifying the selection of 2019 as a control period, as previously reported in published studies related to the analysis of air pollution during the ongoing COVID-19 pandemic $[39,40]$.

The pollutants selected for analysis were nitrogen dioxide $\left(\mathrm{NO}_{2}\right)$, particulate matter with less than $10 \mu \mathrm{m}\left(\mathrm{PM}_{10}\right)$ and particulate matter with less than $2.5 \mu \mathrm{m}\left(\mathrm{PM}_{2.5}\right)$. Hourly atmospheric concentration data were obtained from records of QUALAR [34] for both periods. CETESB has 17 automatic monitoring stations in the municipality of Sao Paulo. Not all stations measure all pollutants. Therefore, $\mathrm{PM}_{10}, \mathrm{PM}_{2.5}$ and $\mathrm{NO}_{2}$ concentrations in both periods were collected, according to the availability of data from 15 monitoring stations (Figure 2), and compiled on their respective week, as previously established, to calculate the pollutants' weekly average.

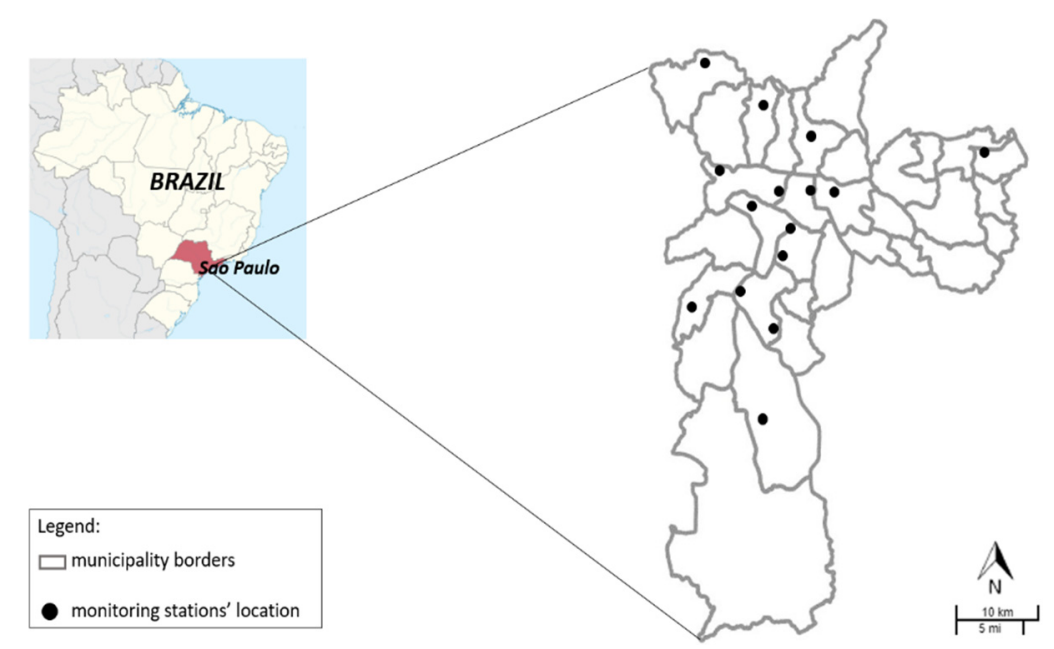

Figure 2. Location of the analyzed automatic monitoring stations in the municipality of Sao Paulo.

CETESB certifies that all monitoring systems of pollutants and meteorology strictly complied with the quality assurance/quality control (QA/QC) procedure established, as approved by the State Council of the Environment (CONSEMA) of Sao Paulo state [34].

The statistical analyses were performed using GraphPad Prism ${ }^{\circledR} 5.0$ software for analysis and graphing. The data were analyzed for normality by the D'Agostino-Pearson test. As the pollutants' concentrations behave as nonparametric variables, we used the Mann-Whitney test or Kruskal-Wallis test followed by Dunn's post hoc test to compare the weekly average of quarantine and control periods.

The images of $\mathrm{NO}_{2}$ were detected through the Tropospheric Monitoring Instruments (TROPOMI) on-board European Space Agency (ESA) Sentinel-5 satellite, based on the Differential Optical Absorption Spectroscopy (DOAS) retrieval method in the $405-465 \mathrm{~nm}$ spectral range [41].

To represent the reduction in $\mathrm{NO}_{2}$ during the quarantine period, two images were captured: 25 May 2020 (4:00 pm UTC) and 21 May 2019 (3:33 pm UTC), with minimal cloud cover. The predominant wind direction data were collected from CETESB and were estimated within the limits of the municipality for 
both analyzed years. The measurements were performed in the south $\left(23^{\circ} 40^{\prime} 56.921^{\prime \prime} \mathrm{S} 46^{\circ} 40^{\prime} 33.884^{\prime \prime} \mathrm{W}\right)$ and north $\left(23^{\circ} 32^{\prime} 57.48^{\prime \prime} \mathrm{S} 46^{\circ} 36^{\prime} 5.659^{\prime \prime} \mathrm{W}\right)$ regions of Sao Paulo.

The extraction and processing of the images were performed by Sentinel Hub EO Browser, Google Earth Pro, and ImageJ. This latter software was used to convert the images from RGB to 8-bit grayscale and to quantify the mean gray value as an approach to estimate the variation of $\mathrm{NO}_{2}$ concentration on the tropospheric vertical column [42,43].

The social distancing index was made available by the telecommunications service providers through a Big Data platform that is managed by the Brazilian Association of Telecommunications Resources (ABR Telecom). According to the telecommunication service providers, the social distancing index is based on the location obtained by cell phone antennas, which "mark" a reference for the place where the cell phone "slept" between $10 \mathrm{pm}$ and $2 \mathrm{am}$. During the day, a cell phone that has departed from this reference (which is variable but can reach approximately $200 \mathrm{~m}$ in the city of Sao Paulo) is considered out of isolation. All this processing is performed by the operator. The index is updated daily, and the operators aggregate and anonymize the data before the generation of the indexes with respect to privacy of the telephone mobile users. Thus, presenting grouped georeferenced data enables the elaboration of public policies that improve measures of social isolation to confront coronaviruses [44].

Based on air pollution reduction results for the quarantine period, the relative risk and avoided deaths attributed to each pollutant were estimated, adopting regression coefficients from epidemiological studies, a well-established method used to estimate the outcomes of air pollution on health [45-52]. The regression coefficients $(\beta)$ assumed in this study were 0.0008 for $\mathrm{PM}_{10}, 0.00405$ for $\mathrm{PM}_{2.5}$, and 0.00135 for $\mathrm{NO}_{2}$-related deaths, as previously stated [52-55]. The coefficients were used to estimate the probability of mortality associated with the exposure of the pollutants, named relative risk (RR), obtained by Equation (1)

$$
R R=\exp \left[{ }^{\beta}(\Delta x)\right],
$$

where RR is the relative risk of all-cause mortality due to air pollution; $\beta$ exposure-response coefficients are related to each pollutant; and $\Delta_{X}$ is the decrease in the pollutant concentrations $\left(\mu \mathrm{g} / \mathrm{m}^{3}\right)$, the difference in pollutant concentrations between the quarantine period and the equivalent period in 2018.

The RR values were used to calculate the attributable fraction (AF), the fraction of deaths attributable to the risk factor of $\mathrm{PM}_{10}, \mathrm{PM}_{2.5}$, or $\mathrm{NO}_{2}$ variation, defined in Equation (2), to estimate the avoided daily mortality during the quarantine period, based on the mean mortality in the control period.

$$
\mathrm{AF}=(\mathrm{RR}-1) / \mathrm{RR}
$$

The daily mean of the all-cause mortality in the control period was calculated and multiplied by the attributable factors to estimate the all-cause mortality avoided per day during the quarantine period.

The latest update of all cause daily mortality between March 2019 and June 2019 was collected from the Brazilian Health System Database [56]. For the effects of short-term exposure to $\mathrm{PM}_{2.5}$, only all-cause mortality in people older than 30 years old was considered. The health effects caused by this pollutant justified the selection of this age group. Exposure to $\mathrm{PM}_{2.5}$ is related to hypertension, lung cancer, type 2 diabetes, and other health conditions, which are mostly observed in this age group [57,58]. For $\mathrm{PM}_{10}$ and $\mathrm{NO}_{2}$, all ages were considered. The daily data of COVID-19 deaths were obtained from the State System for Data Analysis Foundation database from 16 March 2020 to 14 June 2020 [59].

The economic valuation of health outcomes was performed by the value of statistical life (VSL) estimation, a well-known approach [60-64]. Considering the avoided deaths related to air pollutant emissions reduction, an economic gain is expected. However, due to the deaths caused by COVID-19, a considerable economic loss is expected.

To estimate the economic impact of the quarantine and to calculate the trade-off between mortality and economic costs, data on confirmed deaths caused by COVID-19, collected from governmental 
records [59], were multiplied by the VSL established value. The same calculus was used to estimate avoided deaths related to air pollutant emissions.

VSL can be defined as a measure of willingness to pay for reducing the risk of dying and thus consists of the monetary value of postponed deaths $[60,64]$. For each avoided death and death caused by COVID-19, the VSL assumed was US\$ 1.88 million, previously proposed by the OECD [65].

\section{Results}

\subsection{Air Quality Improvement during 90 Days of COVID-19 Social Distancing}

As a first-step approach, we verified the monthly records of $\mathrm{PM}_{10}, \mathrm{PM}_{2.5}$, and $\mathrm{NO}_{2}$ concentrations. Except for March and May, $\mathrm{PM}_{10}$ showed a significant reduction in 2020. $\mathrm{PM}_{2.5}$ also showed a significant reduction in 2020, except in March. Finally, $\mathrm{NO}_{2}$ showed a significant decrease in April and June (Figure 3).

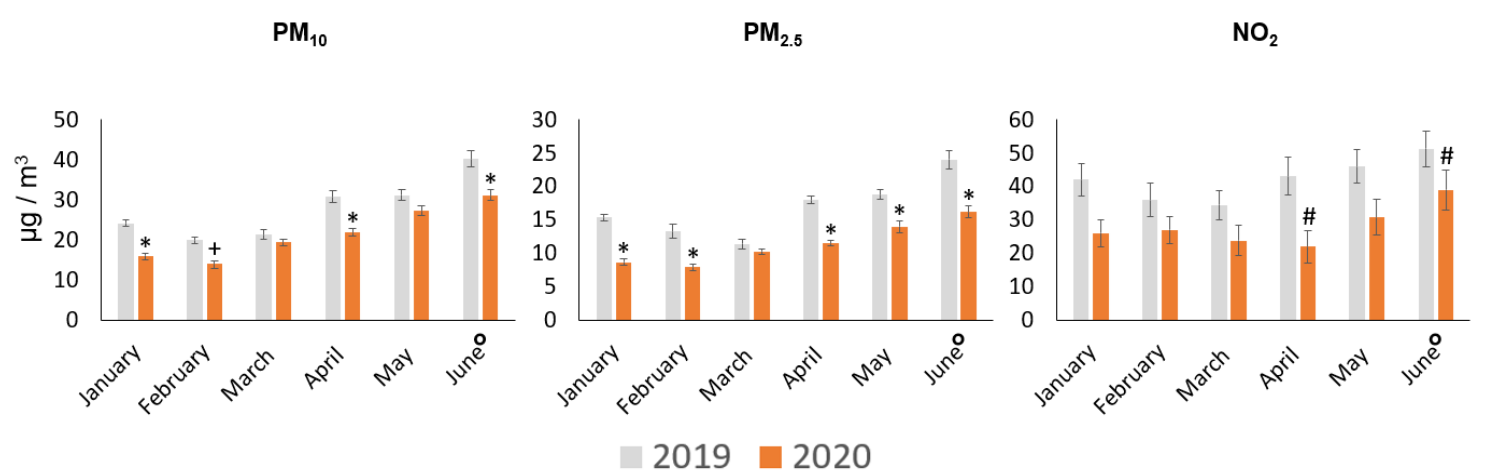

Figure 3. Monthly comparison of the analyzed pollutants. Bars represent the average \pm SEM. The analysis was conducted by Kruskal-Wallis test followed by Dunn's post hoc test (\# $p<0.05$; $\left.+p<0.01{ }^{*} p<0.001\right)$. Until 14 June.

Higher volumes of precipitation could explain the results observed for January, February, and March. In addition, the comparison between incompatible days, in terms of meteorological conditions, can affect the interpretation of the analysis. For instance, $\mathrm{NO}_{2}$ did not show a significant reduction in March and April (Figure 3). However, several studies have shown its reduction during the pandemic in different regions of the world [66-68].

From this initial analysis, we can conclude that the division of the analysis period on a weekly scale, considering meteorological conditions as an exclusion criterion, could provide more accurate information about the variation of pollutants.

We verified the daily records of precipitation, wind speed, and mean temperature. Comparing both periods (2019 and 2020), we observed that wind speed and mean temperature presented similar ranges. For precipitation, we detected a slight difference in a few days of the control period. Considering that precipitation is an important factor that influences the dilution and dispersion of pollutants [69-72], a 12-day control period with precipitation above $10 \mathrm{~mm}$ was not considered in this study. After excluding these outlier days, we observed that the means and ranges of meteorological variables were similar over the weeks of the quarantine and control periods (Table 2).

During 13 weeks of social distancing, we observed that the pollutants showed a gradual increase in concentrations over the weeks, similar to the monthly analysis. For $\mathrm{PM}_{10}$, the average concentrations ranged between 16.9 (week 2) and $32.38 \mu \mathrm{g} / \mathrm{m}^{3}$ (week 13). $\mathrm{PM}_{2.5}$ presented average concentrations varying between 9.05 (week 4) and $17.15 \mu \mathrm{g} / \mathrm{m}^{3}$ (week 12). For $\mathrm{NO}_{2}$, the average concentrations ranged between 17.21 (week 1) and $40.71 \mu \mathrm{g} / \mathrm{m}^{3}$ (week 11) (Figure 4). 
Table 2. Weekly averages of meteorological variables during quarantine and control periods.

\begin{tabular}{ccccccc}
\hline \multirow{2}{*}{ Weeks } & \multicolumn{2}{c}{ Precipitation $(\mathbf{m m})$} & \multicolumn{2}{c}{ Wind $(\mathbf{m} / \mathbf{s})$} & \multicolumn{2}{c}{ Temperature $\left({ }^{\circ} \mathbf{C}\right)$} \\
\cline { 2 - 6 } & $\begin{array}{c}\text { Control } \\
\text { Period }\end{array}$ & Quarantine & $\begin{array}{c}\text { Control } \\
\text { Period }\end{array}$ & Quarantine & $\begin{array}{c}\text { Control } \\
\text { Period }\end{array}$ & Quarantine \\
\hline 1 & $2.6(1.7)$ & $5.2(9.6)$ & $2.3(0.1)$ & $2.2(0.1)$ & $26.5(1.7)$ & $28.5(1.2)$ \\
2 & $0.8(1.6)$ & $2.6(6.9)$ & $2.1(0.2)$ & $2.3(0.2)$ & $28.6(0.9)$ & $26.9(0.6)$ \\
3 & $0.05(0.1)$ & $1.7(4.6)$ & $2.0(0.2)$ & $2.2(0.2)$ & $29.5(0.8)$ & $28.6(0.4)$ \\
4 & $1.9(3.4)$ & $0.5(1.2)$ & $1.9(0.1)$ & $2.1(0.1)$ & $26.3(1.9)$ & $24.3(1.2)$ \\
\hline 5 & $0.4(0.9)$ & $0.8(1.7)$ & $1.8(0.1)$ & $2.1(0.1)$ & $28.5(0.5)$ & $24.3(0.7)$ \\
6 & $0.4(0.7)$ & $0(0)$ & $1.8(0.1)$ & $1.7(0.1)$ & $28.7(0.3)$ & $26.9(0.6)$ \\
7 & $0.2(0.3)$ & $0.03(0.1)$ & $1.6(0.1)$ & $1.9(0.1)$ & $27.9(0.8)$ & $26.1(0.8)$ \\
8 & $0.5(0.7)$ & $0.5(1.2)$ & $1.8(0.2)$ & $1.9(0.1)$ & $26.7(1.1)$ & $23.4(1.4)$ \\
9 & $2.4(1.6)$ & $0.5(1)$ & $1.9(0.1)$ & $1.7(0.2)$ & $26.1(1.4)$ & $23.7(1.3)$ \\
10 & $0.9(1.3)$ & $1.2(2.6)$ & $1.9(0.2)$ & $2.3(0.3)$ & $24.9(1.2)$ & $23.9(1.5)$ \\
11 & $1.3(3.1)$ & $0.03(0.1)$ & $2.1(0.2)$ & $2.1(0.3)$ & $25.2(1.6)$ & $22.6(1.0)$ \\
12 & $0(7.1)$ & $5.5(7.4)$ & $2.1(0.2)$ & $1.6(0.1)$ & $20.4(1.3)$ & $23(1.0)$ \\
13 & $0.4(0.7)$ & $0.7(1.3)$ & $1.3(0.1)$ & $1.8(0.2)$ & $25.2(0.8)$ & $26.2(1.3)$ \\
\hline
\end{tabular}

Data are expressed as the mean \pm standard deviation (SD).

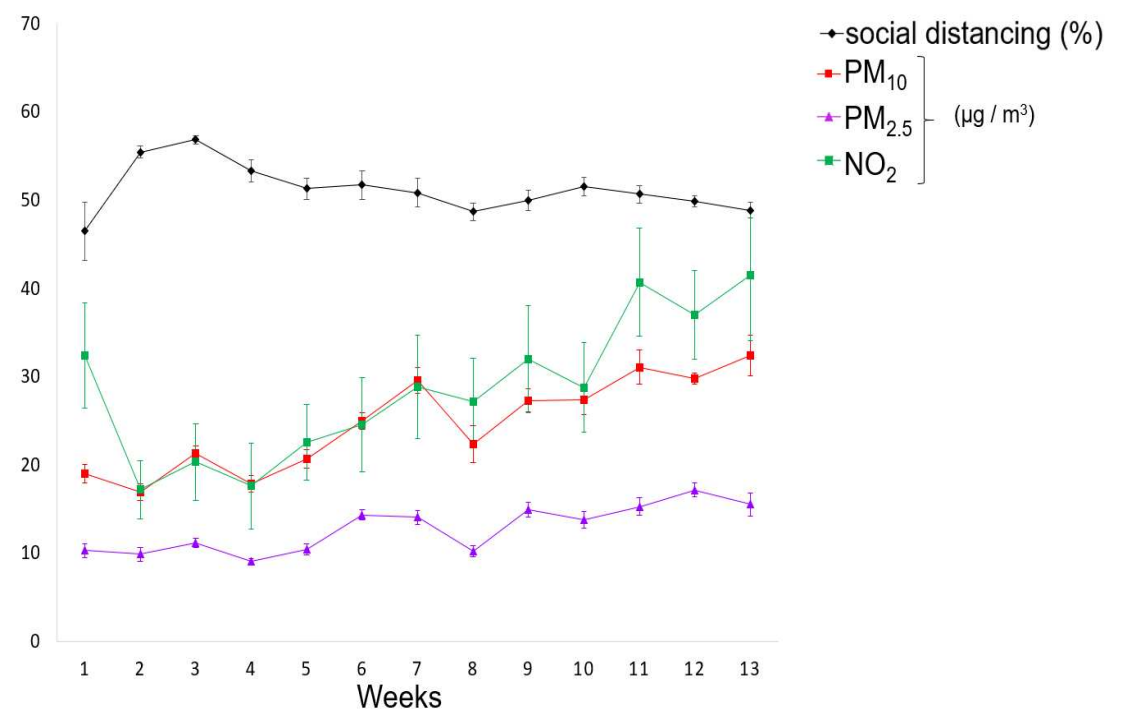

Figure 4. Averages of social distancing index and $\mathrm{PM}_{10}, \mathrm{PM}_{2.5}$, and $\mathrm{NO}_{2}$ concentrations during 13 weeks of the quarantine period in Sao Paulo city. Bars represent the average \pm SEM.

The average social distancing also fluctuated, presenting the lowest index at week 1 (46.5\%) and the highest between weeks 2 and 4 (55.4; 56.9 and 53.3\%, respectively). In the remaining weeks, the average social distancing ranged between 51.7 and $48.7 \%$.

The best air quality indexes for all pollutants were observed at weeks 2 and 4 , with social distances above $53.3 \%$, and the worst concentrations were detected at weeks 12 and 13, with social distances less than $50 \%$.

From week 4 on, we also found a slackening in the social distancing accompanied by an increase in the average pollutant concentrations, quite evident for $\mathrm{PM}_{10}$ and $\mathrm{NO}_{2}$. The remaining weeks recorded at least two days, reaching six consecutive days in week 13, with less than $50 \%$ social distancing. Some factors have contributed to the reduction in social distancing, such as popular manifestations against this measure that occurred in several days, differences in federal and local governments recommendations concerning COVID-19 prevention actions, and most importantly the poor and 
working class who do not have alternatives in this matter, can also explain the reduction in its index and consequently the fluctuation of pollutants' concentrations (Figure 4).

As shown in Figure 5, the comparison between the $\mathrm{PM}_{10}$ concentrations during quarantine and control periods revealed six weeks with a reduction of at least $25 \%$, the highest reaching $-45 \%$ in week $3(p<0.001)$. For $\mathrm{PM}_{2.5}$, we found eight weeks with a reduction of at least $29 \%$, reaching $-46 \%$ in week $13(p<0.001)$. The air quality improvement in terms of $\mathrm{NO}_{2}$ showed nine weeks with a reduction of at least $33 \%$, reaching $-58 \%$ in week $3(p<0.01)$.
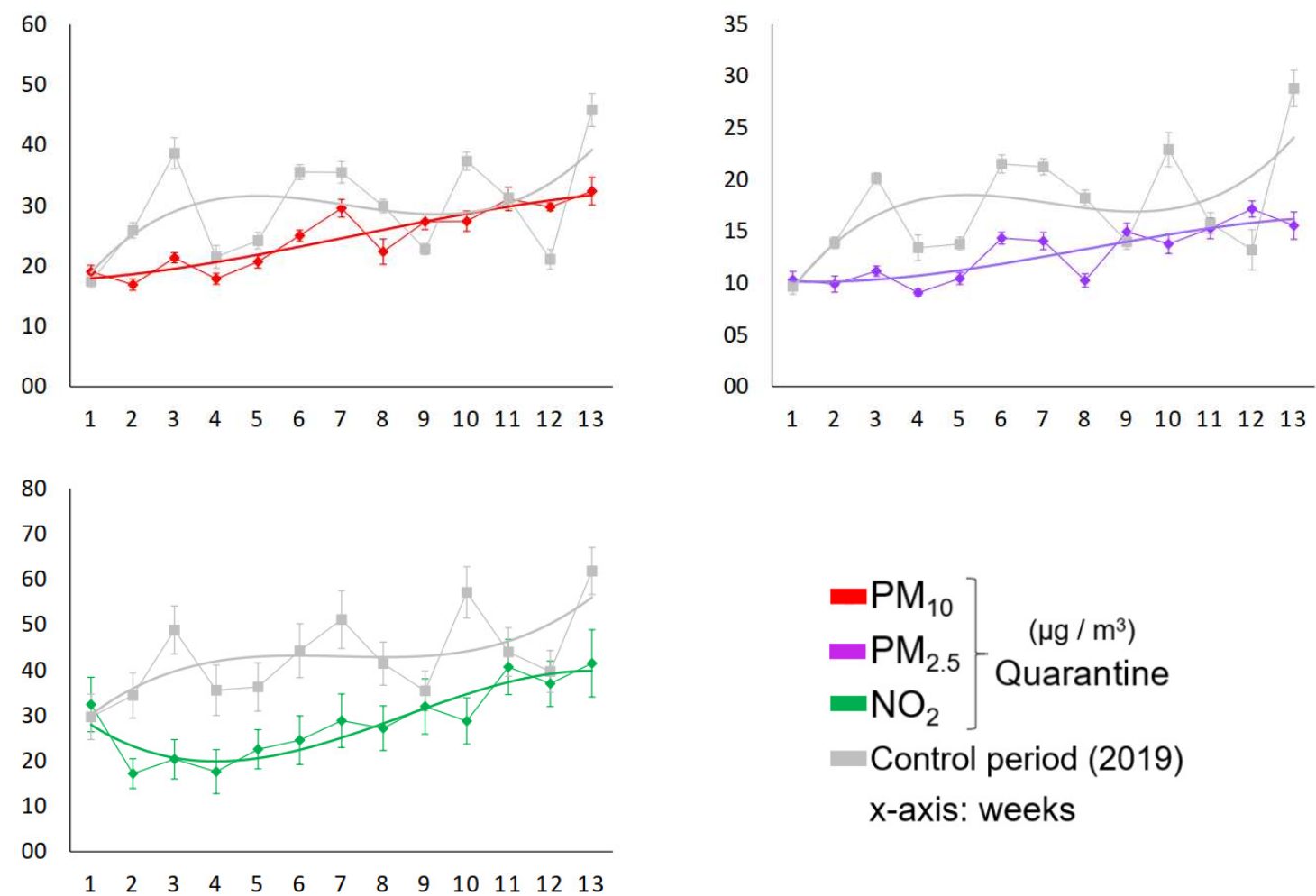

Figure 5. $\mathrm{PM}_{10}, \mathrm{PM}_{2.5}$, and $\mathrm{NO}_{2}$ concentrations and trend-line during 13 weeks of quarantine and control periods. Bars represent the average \pm SEM.

Following $\mathrm{PM}_{2.5}, \mathrm{NO}_{2}$ was the best indicator of air quality in the analyzed weeks. This finding could be confirmed from the satellite images detected on 21 May 2019, and 25 May 2020, which showed an abrupt decline of $62.8 \%$ in tropospheric $\mathrm{NO}_{2}$ concentrations, represented by the variation of the red pattern (Figure 6).

\subsection{Associated Health Economics Outcomes}

Based on the results presented in Figure 5, we estimated the potential health and economic benefits related to pollutant reductions achieved during the analyzed weeks of quarantine. As shown in Table 3, the relative risks (RRs) were estimated to be between 0.993 and 1.013 for $\mathrm{PM}_{10}, 0.996$ and 1.055 for $\mathrm{PM}_{2.5}$, and 0.996 and 1.039 for $\mathrm{NO}_{2}$. Thus, the attributable factors (AF) ranged $0.2-0.8 \%$ for $\mathrm{PM}_{10}$, 0.9-3.2\% for $\mathrm{PM}_{2.5}$, and 4-6.9\% for $\mathrm{NO}_{2}$. As expected, because $\mathrm{NO}_{2}$ presented the greatest levels of reduction compared to the control period, its weekly RR and AF values were also higher in relation to other analyzed pollutants. 

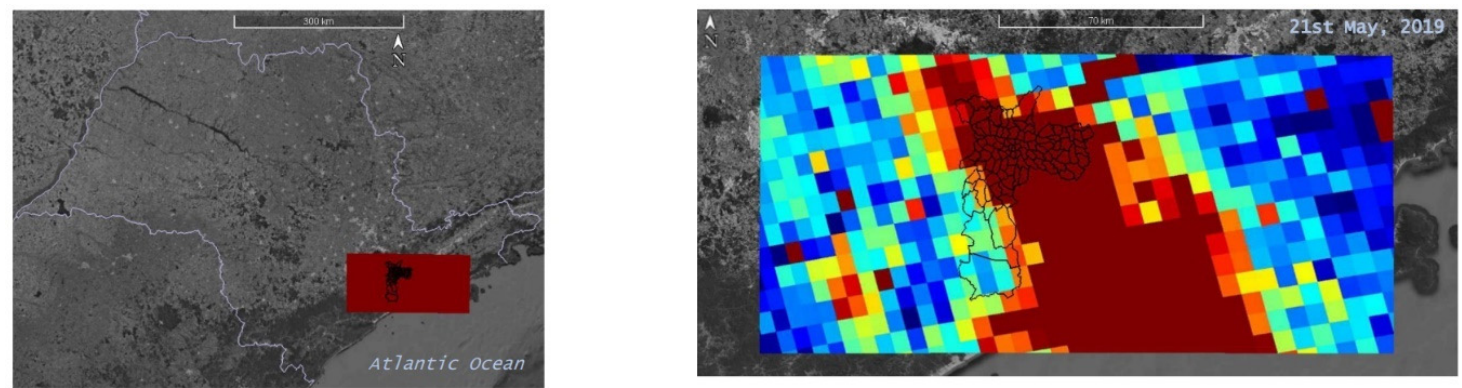

satellite image captured: Sentinel-5P TROPOMI

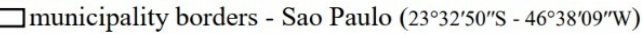

NNW predominant wind direction

$\mathrm{NO}_{2}$ tropospheric column
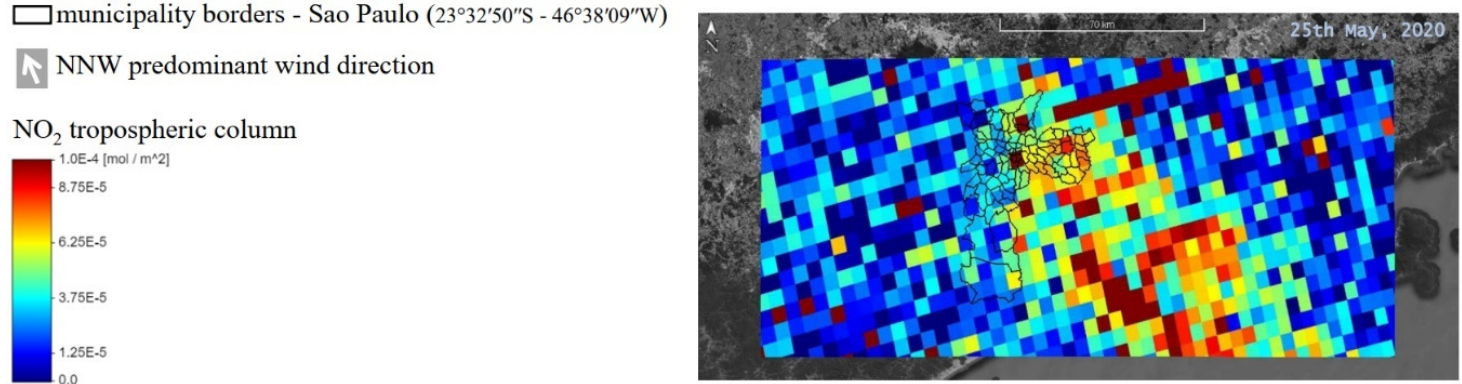

Figure 6. Tropospheric vertical column of $\mathrm{NO}_{2}$ detected on 21 May 2019, and 25 May 2020. Data from Tropospheric Monitoring Instrument (TROPOMI) on board Sentinel-5 Precursor, based on the Differential Optical Absorption (DOAS) retrieval method (405-465 nm spectral range). The north-northwest (NNW) predominant wind direction was estimated within the limits of the municipality for both analyzed years. The measurements were performed in the south $\left(23^{\circ} 40^{\prime} 56.921^{\prime \prime} \mathrm{S}\right.$ $\left.46^{\circ} 40^{\prime} 33.884^{\prime \prime} \mathrm{W}\right)$ and north $\left(23^{\circ} 32^{\prime} 57.48^{\prime \prime} \mathrm{S} 46^{\circ} 36^{\prime} 5.659^{\prime \prime} \mathrm{W}\right)$ regions of Sao Paulo.

Table 3. Relative risk (RR) and attributable factor (AF) related to PM10, PM2.5, and NO2 exposures.

\begin{tabular}{ccccccc}
\hline \multicolumn{7}{c}{ Relative Risks and Attributable Fractions } \\
\hline Weeks & \multicolumn{2}{c}{ PM $_{\mathbf{1 0}}$} & \multicolumn{2}{c}{ PM $_{\mathbf{2 . 5}}$} & \multicolumn{3}{c}{ NO $_{\mathbf{2}}$} \\
\hline & $\mathrm{RR}$ & $\mathrm{AF}(\%)$ & $\mathrm{RR}$ & $\mathrm{AF}(\%)$ & $\mathrm{RR}$ & $\mathrm{AF}(\%)$ \\
1 & 0.998 & -0.13 & 0.997 & -0.26 & 0.996 & -0.37 \\
2 & 1.007 & 0.72 & 1.016 & 1.60 & 1.023 & 2.30 \\
3 & 1.013 & 1.38 & 1.036 & 3.56 & 1.039 & 3.77 \\
4 & 1.002 & 0.29 & 1.017 & 1.74 & 1.024 & 2.39 \\
5 & 1.002 & 0.28 & 1.013 & 1.34 & 1.018 & 1.84 \\
6 & 1.008 & 0.84 & 1.029 & 2.86 & 1.026 & 2.63 \\
7 & 1.004 & 0.47 & 1.029 & 2.86 & 1.030 & 2.96 \\
8 & 1.006 & 0.60 & 1.032 & 3.18 & 1.019 & 1.90 \\
9 & 0.996 & -0.36 & 0.996 & -0.38 & 1.004 & 0.46 \\
10 & 1.007 & 0.79 & 1.037 & 3.62 & 1.039 & 3.75 \\
11 & 1.000 & 0.01 & 1.002 & 0.20 & 1.004 & 0.44 \\
12 & 0.993 & -0.70 & 0.984 & -1.61 & 1.003 & 0.37 \\
13 & 1.010 & 1.07 & 1.055 & 5.22 & 1.027 & 2.71 \\
\hline
\end{tabular}

Therefore, concomitant with the increase in the number of deaths from COVID-19 infection, which reached 5623 on the last day of this analysis, the decrease in $\mathrm{PM}_{10}, \mathrm{PM}_{2.5}$, and $\mathrm{NO}_{2}$ concentration levels prevented 78,337 and 387 premature deaths, respectively, in Sao Paulo city (Figure 7). 


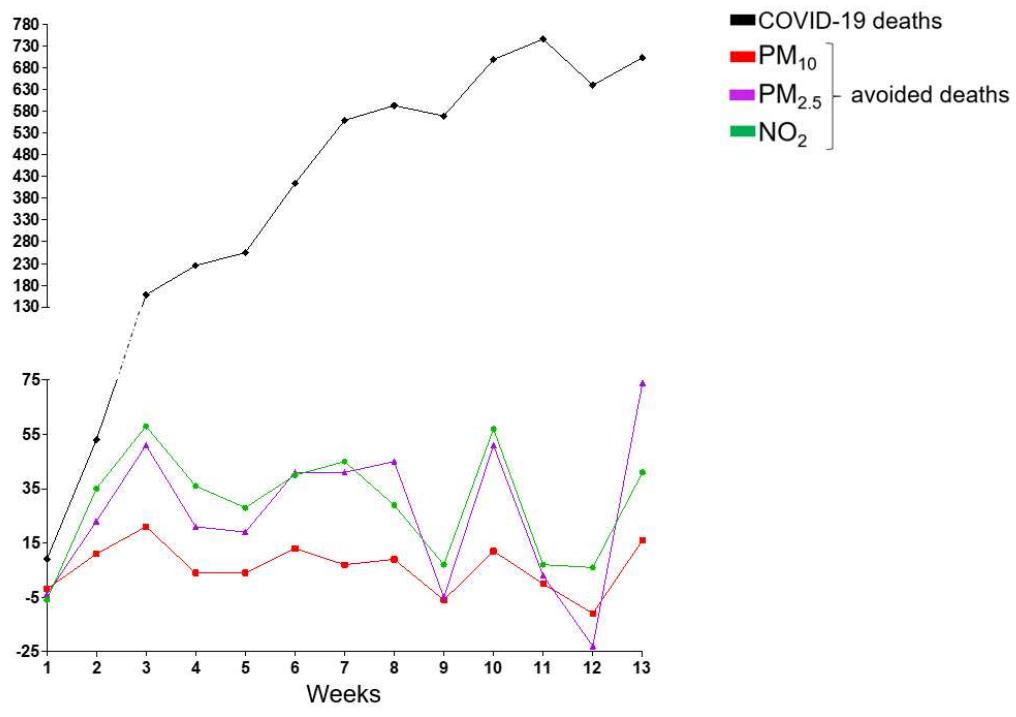

Figure 7. COVID-19 deaths, $\mathrm{PM}_{10}, \mathrm{PM}_{2.5}$, and $\mathrm{NO}_{2}$ avoided deaths during the weeks of the quarantine period in Sao Paulo city.

Given the monetary value for the observed health outcomes from the analyzed improvement of pollutant concentrations by the VSL approach, a well-known and widely used method [65,73], we applied the economic valuation of deaths caused by COVID-19 and of the avoided deaths due to the reduction in short-term exposure to $\mathrm{PM}_{10}, \mathrm{PM}_{2.5}$, and $\mathrm{NO}_{2}$ (Table 4).

Table 4. Economic valuation of deaths caused by COVID-19 and of avoided deaths due to the reductions of PM10, PM2.5, and NO2.

\begin{tabular}{ccc}
\hline & Deaths & Economic Outcome (US\$ million) * \\
\hline COVID-19 deaths & 5623 & $10571.2(-)$ \\
PM10 avoided deaths & 78 & $146.6(+)$ \\
PM2.5 avoided deaths & 337 & $633.6(+)$ \\
NO2 avoided deaths & 383 & $720.0(+)$ \\
\hline
\end{tabular}

* Based on the VSL value.

During the analyzed weeks of the quarantine period in Sao Paulo city, the deaths caused by COVID-19 accounted for an economic health loss of approximately US $\$ 10.5$ billion. On the other hand, we can consider a potential economic health benefit in terms of the prevented premature deaths related to the observed improvement in air quality. Thus, we estimated that the expressive number of avoided deaths due to $\mathrm{PM}_{10}, \mathrm{PM}_{2.5}$, and $\mathrm{NO}_{2}$ reductions can represent an economy of approximately US $\$ 720$ million, considering the results of $\mathrm{NO}_{2}$, the best air quality indicator observed.

\section{Discussion}

In a tightly connected and integrated world, the impacts of emerging infectious diseases can be devastating, causing large-scale mortality and morbidity, as we are observing over the days of this global outbreak. However, the adverse effects go beyond, and the size and persistence of the economic impacts of the ongoing COVID-19 pandemic are still uncertain, and our findings raise intriguing questions.

On the one hand, we estimated the economic health loss associated with deaths related to COVID-19 in Sao Paulo city, which was exceedingly raised, even before reaching the important goal of flattening the curve of coronavirus infections, which makes us wonder about the efficiency of the applied public policies and the commitment of the strategies of controlling the pandemic and population's commitment to comply with social distancing. On the other hand, we observed that 
the epidemic control actions were closely related to the improvement of air quality; due to the major source of air pollution in the region, the emissions from vehicle exhaust were significantly reduced.

The quarantine and cities in shutdown have also caused a supplementary effect, the improvement of air quality, which was observed in different regions of the world, such as India, China, Italy, France, and United States $[66,67,74]$. In Rio de Janeiro, Brazil, a study showed that the confinement of the population between March and April caused a reduction in road traffic and economic activities and led to a significant decrease in $\mathrm{CO}$ (between 37.0 and 43.6\%) and $\mathrm{NO}_{2}$ (between 24.1 and 32.9\%) levels and a non-significant reduction in $\mathrm{PM}_{10}$ concentrations [75].

Regarding $\mathrm{NO}_{2}$ and particulate matter, our findings showed that, in Sao Paulo, the impacts on its concentrations were more significant. During 16 March and 14 June 2020, $\mathrm{NO}_{2}$ decreased to 33\%, reaching $58 \%$, the decrease in $\mathrm{PM}_{10}$ ranged between 25 and $45 \%$, and the levels of $\mathrm{PM}_{2.5}$ decreased between 29 and $46 \%$ in comparison to the control period in 2019.

Air quality improvement could also be observed by satellite images worldwide. A dramatic reduction in air pollution over China during February 2020 accounted for over 30\% less in $\mathrm{NO}_{2}$ levels compared to early January when power plants were operating at normal levels. In addition, the study also estimated a reduction of $\mathrm{CO}_{2}$ emissions by $25 \%$ [66]. Xu et al. (2020) showed that from January to March 2020, the decrease in $\mathrm{NO}_{2}$ concentration was of great significance and much higher than other pollutants, such as $\mathrm{PM}_{10}$ and $\mathrm{PM}_{2.5}$ [68]. Moreover, Anjum (2020) also showed, from satellite images, a remarkable reduction of air pollution in Italy, France, and the USA [67].

Our results corroborate these findings since we also showed a significant reduction in $\mathrm{NO}_{2}$ concentration in Sao Paulo city, which was greater than the other analyzed pollutants, confirmed by the abrupt decline of $62.8 \%$ in tropospheric $\mathrm{NO}_{2}$ concentrations also observed by satellite images.

Human activities do not change only the atmosphere with the emission of pollutants or only the climate system with the emission of greenhouse gases; the consequences are systemic since they can directly affect human health, socioeconomic, and political stability. Emissions of air pollutants related to the exacerbation of the prevalence of cardiorespiratory diseases, type 2 diabetes, and mental disorders; land use modification associated with deforestation that modifies host/vector interactions, elevates disease risk, and drives wildlife zoonotic disease emergence; and mega-trends in climate accompanied by extreme weather conditions make the population increasingly vulnerable. These conditions can cause several socioeconomic impacts, such as morbidities, mortality, migration, poverty exacerbation, and violent conflict, affecting people of all ages and all nationalities [29,76,77].

In this context, the current global crisis we are experiencing goes beyond showing that environmental degradation is closely related to human resilience. In times of crisis, borders do not matter, showing the importance of cooperation and engagement of governments and all the people to work together for a common purpose and solution.

Foregone income related to laid-off workers or unable to work, disruption of supply chains, restrictions on international travel enforced by governments, and unprecedented consequences on stock markets are examples of already observed economic and social impacts. Individualities and structural differences of each country make forecasts difficult; however, there are some published estimates. According to Fernandes (2020), for Brazil, the GDP growth in 2020, assuming shutdown lasts three months, can reach $-4.0 \%$ [from $-5.9 \%$ to $-2.1 \%$ ]. Moreover, according to the OECD, global GDP growth is projected to slow from $2.9 \%$ in 2019 to $2.4 \%$ in 2020 [78-80]. The COVID-19 pandemic has hit underserved populations and communities of color particularly hard, exacerbating longstanding health disparities in Brazil. This fact can be observed in Sao Paulo municipality, which districts of poorer areas have been suffering most of the cases of COVID-19.

Politicians and some businessmen are against social distancing measures due to the impact on the economy, claiming that the virus is less harmful than presented; they do not value life. Life is more valuable than the economy-there is no need to have good economic indicators if the population dies. Nevertheless, there will be economic effects of the distance measures and the impacts on the mental 
health of the populations affected by them. In addition to anxiety due to fear of the disease, there are effects of prolonged quarantine for several weeks faced by a huge number of people around the world.

An impact assessment evaluation can consider the exposure to a multi-pollutant mixture. Adding pollutant-specific effects may be justified when levels of the specific pollutants are clearly not correlated. However, this approach would result in an overestimated analysis due to uncertainties about the contributions of each pollutant to the health effect $[81,82]$.

In this research, we estimated the health effects concerning a reduction in $\mathrm{PM}_{10}, \mathrm{PM}_{2.5}$ and $\mathrm{NO}_{2}$ concentrations. Thus, to avoid a biased analysis, we considered, in the health economic evaluation, the outcomes of $\mathrm{NO}_{2}$, the best air quality indicator observed. Accordingly, our results showed that air quality improvement contributed to a substantial fraction of the avoided deaths associated with significant monetized health co-benefits, signaling that governments have the capability to improve air quality through policy measures.

We found that the decrease in $\mathrm{PM}_{10}, \mathrm{PM}_{2.5}$, and $\mathrm{NO}_{2}$ concentration levels could prevent 78,337 and 387 premature deaths, respectively, corresponding to an economy of approximately US \$720 million and providing evidence for policy making by the quantification and monetization of air pollution-related health effects. This is a tool for environmental planning purposes since the monetized value of the avoided premature mortality typically dominates the calculated benefits of air pollution regulations.

The consideration of exclusively $\mathrm{NO}_{2}$ abatement in the quarantine period is a conservative simplification. This constraint may have biased our estimation downwards. If we add the effect of $\mathrm{PM}_{2.5}$ and $\mathrm{PM}_{10}$, we double the impact of prevented deaths due to air pollution, meaning a saving of US $\$ 1.5$ billion. Considering the maintenance of these mobility patterns in the new "business as usual" (BAU) COVID-19 era, in a 10-year period, this savings would achieve approximately US $\$ 60$ billion. Both health and economic gains associated with air pollution reductions give a positive perspective of the efforts towards keeping air pollution reduced even after the pandemic.

Unprecedented challenge demands an unprecedented response. Thus, shifting the BAU approach of life will be the most important and mandatory step to prevent future adverse events. For that, resilience is indispensable for facing new problems and challenges in our increasingly globalized world. The COVID-19 pandemic, forcing a drastic change in the BAU way of life, offers a unique scenario to take the opportunity during the crisis to move toward a more sustainable society and economy, in terms of speeding up the transition of make cities more inclusive, developing urban resilience, improving preventive health measures, and implementing green strategies for economics, especially for transport and energy sectors, since they are major contributors to the degradation of global air quality $[20,33,83,84]$.

In this sense, our analysis presents an estimate of what could be achieved if effective measures would be taken towards air pollution emissions control in an urban center of a developing country, such as Sao Paulo. The implementation of permanent or partial home-office jobs, dynamization of e-commerce, as well as improvement of energy efficiency, support of a low-carbon economy, and investments in cleaner transportation solutions are some recommendations for sustainable procedures that can diminish air pollution emissions in megacities, providing a transition to urban resilience and sustainability.

\section{Conclusions}

We found that, during 90 days of COVID-19 social distancing in Sao Paulo city, Brazil, 5623 deaths occurred due to this new disease estimated in an economic health loss of US $\$ 10.5$ billion. In contrast, we observed a significant air quality improvement. During the analyzed weeks, the decreases in $\mathrm{PM}_{2.5}$, $\mathrm{PM}_{10}$, and $\mathrm{NO}_{2}$ levels reached $45 \%, 46 \%$, and $58 \%$ reductions, respectively, in comparison to the control period in 2019. The positive impact on air quality in terms of $\mathrm{PM}_{2.5}, \mathrm{PM}_{10}$, and $\mathrm{NO}_{2}$ concentrations avoided 78,337 and 387 premature deaths, respectively, and prevented approximately US $\$ 720$ million on health costs, considering the results of $\mathrm{NO}_{2}$, the best air quality indicator observed. 
Our study faced some limitations. First, in addition to the reduced number of deaths due to air pollution, we should consider that there are other benefits attributable to the reduction in air pollution itself and could also have positive benefits in reducing preventable noncommunicable diseases $[85,86]$. Thus, a more comprehensive analysis should contemplate other health outcomes, such as the reductions in hospitalizations due to cardiorespiratory diseases. Second, meteorological data were collected and used as a criterion for selected days of the control period to ensure that the analysis was not carried out on incompatible days in terms of the meteorological similarity of both periods analyzed, minimizing the influence of these factors on pollutant dispersion. In this sense, an air quality dispersion modeling approach could be usefully explored in further future researches.

These findings have significant implications for understanding how important it is to improve the strategies for sustainable practices in urban centers, especially with regard to air pollution mitigation actions. Furthermore, we highlight the crucial role of appropriately allocating investments and adopting efficient measures to protect human health both during and after the COVID-19 global health crisis.

Author Contributions: Conceptualization, S.G.E.K.M. and D.D.; formal analysis, D.D., M.V.d.C., and S.G.E.K.M.; writing-original draft preparation, D.D., M.V.d.C., and S.G.E.K.M.; writing-review and editing, D.D., M.V.d.C., and S.G.E.K.M.; supervision, S.G.E.K.M. All authors have read and agreed to the published version of the manuscript.

Funding: Supported by Grant Number 1808529 from the Coordenação de Aperfeiçoamento de Pessoal de Nível Superior (CAPES). Supported by Grant Number 2018/26193-3 from the Fundação de Amparo à Pesquisa do Estado de São Paulo (FAPESP).

Acknowledgments: The authors are grateful to the Academic Editor Idiano D'Adamo and Etta Shen for all support provided.

Conflicts of Interest: The authors declare no conflict of interest.

\section{References}

1. Brida, M.; Chessa, M.; Gu, H.; Gatzoulis, M.A. The globe on the spotlight: Coronavirus disease 2019 (Covid-19). Int. J. Cardiol. 2020, 310, 170-172. [CrossRef] [PubMed]

2. Tu, H.; Tu, S.; Gao, S.; Shao, A.; Sheng, J. Current epidemiological and clinical features of COVID-19; a global perspective from China. J. Infect. 2020, 81, 1-9. [CrossRef] [PubMed]

3. Martini, M.; Gazzaniga, V.; Bragazzi, N.L.; Barberis, I. The Spanish Influenza Pandemic: A lesson from history 100 years after 1918. J. Prev. Med. Hyg. 2019, 60, E64-E67. [CrossRef]

4. Dhama, K.; Verma, A.K.; Rajagunala, S.; Deb, R.; Karthik, K.; Kapoor, S.M.; Tiwari, R.; Panwar, P.K.; Chakrabort, S. Swine flu is back again: A review. Pakistan J. Biol. Sci. 2012, 15, 1001-1009. [CrossRef]

5. World Health Organization. Influenza Updates; World Health Organization: Geneva, Switzerland, 2020. Available online: https://www.who.int/influenza/surveillance_monitoring/updates/en/ (accessed on 18 June 2020).

6. Centers for Disease Control and Prevention. Influenza (Flu). 2009 H1N1 Pandemic; Centers for Disease Control and Prevention: Atlanta, GA, USA, 2009. Available online: https://www.cdc.gov/flu/pandemic-resources/ 2009-h1n1-pandemic.html (accessed on 18 June 2020).

7. Bradley, K.C.; Jones, C.A.; Tompkins, S.M.; Tripp, R.A.; Russell, R.J.; Gramer, M.R.; Heimburg-Molinaro, J.; Smith, D.F.; Cummings, R.D.; Steinhauer, D.A. Comparison of the receptor binding properties of contemporary swine isolates and early human pandemic H1N1 isolates (Novel 2009 H1N1). Virology 2011, 413, 169-182. [CrossRef] [PubMed]

8. Chowell, G.; Ammon, C.E.; Hengartner, N.W.; Hyman, J.M. Estimation of the reproductive number of the Spanish flu epidemic in Geneva, Switzerland. Vaccine 2006, 24, 6747-6750. [CrossRef]

9. World Health Organization. Coronavirus Disease (COVID-19) Dashboard; World Health Organization: Geneva, Switzerland, 2020. Available online: https://covid19.who.int/ (accessed on 18 June 2020).

10. Velavan, T.P.; Meyer, C.G. The COVID-19 epidemic. Trop. Med. Int. Health 2020, 25, 278-280. [CrossRef]

11. Candido, D.D.S.; Watts, A.; Abade, L.; Kraemer, M.U.G.; Pybus, O.G.; Croda, J.; de Oliveira, W.; Khan, K.; Sabino, E.C.; Faria, N.R. Routes for COVID-19 importation in Brazil. J. Travel Med. 2020, 27. [CrossRef] [PubMed] 
12. Singhal, T. A review of coronavirus disease-2019 (COVID-19). Indian J. Pediatr. 2020, 87, 281-286. [CrossRef]

13. Ahmed, K.; Hayat, S.; Dasgupta, P. Global challenges to urology practice during the COVID-19 pandemic. BJU Int. 2020. [CrossRef]

14. Rothan, H.A.; Byrareddy, S.N. The epidemiology and pathogenesis of coronavirus disease (COVID-19) outbreak. J. Autoimmun. 2020, 109, 102433. [CrossRef] [PubMed]

15. Lake, M.A. What we know so far: COVID-19 current clinical knowledge and research. Clin. Med. (Northfield. Il) 2020, 20, 124-127. [CrossRef] [PubMed]

16. Conticini, E.; Frediani, B.; Caro, D. Can atmospheric pollution be considered a co-factor in extremely high level of SARS-CoV-2 lethality in northern Italy? Environ. Pollut. 2020, 261, 114465. [CrossRef] [PubMed]

17. Frontera, A.; Martin, C.; Vlachos, K.; Sgubin, G. Regional air pollution persistence links to COVID-19 infection zoning. J. Infect. 2020, 81, 318-356. [CrossRef]

18. Tavella, R.A.; da Silva Júnior, F.M. COVID-19 and air pollution: What do we know so far? Vittalle-Rev. Ciências da Saúde 2020, 1, 22-31. [CrossRef]

19. Wu, X.; Nethery, R.C.; Sabath, B.M.; Braun, D.; Dominici, F. Exposure to air pollution and COVID-19 mortality in the United States: A nationwide cross-sectional study. medRxiv 2020. [CrossRef]

20. Urrutia-Pereira, M.; Mello-da-Silva, C.A.; Solé, D. COVID-19 and air pollution: A dangerous association? Allergol. Immunopathol. (Madrid) 2020. [CrossRef]

21. Brazilian Institute of Statistics and Geography. Estimativa da População-Diretoria de Pesquisas, Coordenação de População e Indicadores Sociais; Brazilian Institute of Statistics and Geography: Rio de Janeiro, Brasil, 2018. Available online: https://www.ibge.gov.br/cidades-e-estados.html (accessed on 18 June 2020).

22. Companhia Ambiental do Estado de São Paulo. Qualidade do ar no Estado de São Paulo; Companhia Ambiental do Estado de São Paulo: São Paulo, Brasil, 2019. Available online: https://cetesb.sp.gov.br/ar/publicacoesrelatorios/ (accessed on 18 June 2020).

23. Andrade, M.F.; Kumar, P.; de Freitas, E.D.; Ynoue, R.Y.; Martins, J.; Martins, L.D.; Nogueira, T.; Perez-Martinez, P.; de Miranda, R.M.; Albuquerque, T.; et al. Air quality in the megacity of São Paulo: Evolution over the last 30 years and future perspectives. Atmos. Environ. 2017, 159, 66-82. [CrossRef]

24. Sao Paulo Transit State Department. São Paulo Vehicle Fleet; Sao Paulo Transit State Department: Sao Paulo, Brasil, 2011. Available online: https://www.detran.sp.gov.br/ (accessed on 18 June 2020).

25. IEMA. Inventário De Emissões Atmosféricas Do Transporte Rodoviário De Passageiros No Município De São Paulo; IEMA: Sao Paulo, Brasil, 2020. Available online: http://emissoes.energiaeambiente.org.br/graficos (accessed on 18 June 2020).

26. Scovronick, N.; França, D.; Alonso, M.; Almeida, C.; Longo, K.; Freitas, S.; Rudorff, B.; Wilkinson, P. Air quality and health impacts of future ethanol production and use in São Paulo State, Brazil. Int. J. Environ. Res. Public Health 2016, 13, 695. [CrossRef]

27. Abe, K.; Miraglia, S. Health Impact assessment of air pollution in São Paulo, Brazil. Int. J. Environ. Res. Public Health 2016, 13, 694. [CrossRef]

28. Leirião, L.F.L.; Debone, D.; Pauliquevis, T.; Rosário, N.M.É.; Miraglia, S.G.E.K. Environmental and public health effects of vehicle emissions in a large metropolis: Case study of a truck driver strike in Sao Paulo, Brazil. Atmos. Pollut. Res. 2020. [CrossRef]

29. Rizzoli, A.; Tagliapietra, V.; Cagnacci, F.; Marini, G.; Arnoldi, D.; Rosso, F.; Rosà, R. Parasites and wildlife in a changing world: The vector-host-pathogen interaction as a learning case. Int. J. Parasitol. Parasites Wildl. 2019, 9, 394-401. [CrossRef] [PubMed]

30. Allen, T.; Murray, K.A.; Zambrana-Torrelio, C.; Morse, S.S.; Rondinini, C.; Di Marco, M.; Breit, N.; Olival, K.J.; Daszak, P. Global hotspots and correlates of emerging zoonotic diseases. Nat. Commun. 2017, 8, 1124. [CrossRef]

31. Guo, F.; Bonebrake, T.C.; Gibson, L. Land-use change alters host and vector communities and may elevate disease risk. Ecohealth 2019, 16, 647-658. [CrossRef] [PubMed]

32. Loh, E.H.; Zambrana-Torrelio, C.; Olival, K.J.; Bogich, T.L.; Johnson, C.K.; Mazet, J.A.K.; Karesh, W.; Daszak, P. Targeting transmission pathways for emerging zoonotic disease surveillance and control. Vector Borne Zoonotic Dis. 2015, 15, 432-437. [CrossRef]

33. D'Adamo, I.; Falcone, P.M.; Martin, M.; Rosa, P. A Sustainable revolution: Let's go sustainable to get our globe cleaner. Sustainability 2020, 12, 4387. [CrossRef] 
34. Companhia Ambiental do Estado de São Paulo. QUALAR. Sistema de Informações da Qualidade do Ar; CETESB: São Paulo, Brasil, 2020. Available online: https://qualar.cetesb.sp.gov.br/qualar/home.do (accessed on 18 June 2020).

35. AGRITEMPO. Brazilian Agro-Meteorological Monitoring System; AGRITEMPO: Rio de Janeiro, Brasil, 2019. Available online: https://www.agritempo.gov.br/agritempo/index.jsp (accessed on 18 June 2020).

36. Sánchez-Ccoyllo, O.R.; de Fátima Andrade, M. The influence of meteorological conditions on the behavior of pollutants concentrations in São Paulo, Brazil. Environ. Pollut. 2002, 116, 257-263. [CrossRef]

37. Zeri, M.; Carvalho, V.S.B.; Cunha-Zeri, G.; Oliveira-Júnior, J.F.; Lyra, G.B.; Freitas, E.D. Assessment of the variability of pollutants concentration over the metropolitan area of São Paulo, Brazil, using the wavelet transform. Atmos. Sci. Lett. 2016, 17, 87-95. [CrossRef]

38. Carneseca, E.C.; Achcar, J.A.; Martinez, E.Z. Association between particulate matter air pollution and monthly inhalation and nebulization procedures in Ribeirão Preto, São Paulo State, Brazil. Cad. Saude Publica 2012, 28, 1591-1598. [CrossRef]

39. Tanzer-Gruener, R.; Li, J.; Eilenberg, S.R.; Robinson, A.L.; Presto, A.A. Impacts of modifiable factors on ambient air pollution: A case study of COVID-19 shutdowns. Environ. Sci. Technol. Lett. 2020, 7, 554-559. [CrossRef]

40. Freitas, E.D.; Ibarra-Espinosa, S.A.; Gavidia-Calderón, M.E.; Rehbein, A.; Abou Rafee, S.A.; Martins, J.A.; Martins, L.D.; Santos, U.P.; Ning, M.F.; Andrade, M.F. Mobility restrictions and air quality under COVID-19 pandemic in São Paulo, Brazil. Earth Sci. 2020. [CrossRef]

41. Wang, C.; Wang, T.; Wang, P.; Rakitin, V. Comparison and validation of TROPOMI and OMI NO2 Observations over China. Atmosphere 2020, 11, 636. [CrossRef]

42. Lopez, J.; Branch, J.W.; Chen, G. Line-based image segmentation method: A new approach to segment VHSR remote sensing images automatically. Eur. J. Remote Sens. 2019, 52, 613-631. [CrossRef]

43. Ciobotaru, A.-M.; Andronache, I.; Ahammer, H.; Radulovic, M.; Peptenatu, D.; Pintilii, R.-D.; Drăghici, C.-C.; Marin, M.; Carboni, D.; Mariotti, G.; et al. Application of Fractal and gray-level co-occurrence matrix indices to assess the forest dynamics in the curvature Carpathians—Romania. Sustainability 2019, 11, 6927. [CrossRef]

44. Sistema de Monitoramento Inteligente do Governo de São Paulo. Available online: https://www.saopaulo.sp. gov.br/coronavirus/isolamento/ (accessed on 27 June 2020).

45. Abe, K.C.; dos Santos, G.M.S.; Coêlho, M.; de Sousa, Z.S.; Miraglia, S.G.E.K. PM10 exposure and cardiorespiratory mortality-estimating the effects and economic losses in São Paulo, Brazil. Aerosol Air Qual. Res. 2018, 18, 3127-3133. [CrossRef]

46. Bell, M.L.; Ebisu, K.; Peng, R.D.; Samet, J.M.; Dominici, F. Hospital admissions and chemical composition of fine particle air pollution. Am. J. Respir. Crit. Care Med. 2009, 179, 1115-1120. [CrossRef]

47. Hemminki, K.; Pershagen, G. Cancer risk of air pollution: Epidemiological evidence. Environ. Health Perspect. 1994, 102, 187-192. [CrossRef] [PubMed]

48. Hoek, G.; Brunekreef, B.; Goldbohm, S.; Fischer, P.; van den Brandt, P.A. Association between mortality and indicators of traffic-related air pollution in the Netherlands: A cohort study. Lancet 2002, 360, 1203-1209. [CrossRef]

49. Jerrett, M.; Burnett, R.T.; Ma, R.; Pope, C.A.; Krewski, D.; Newbold, K.B.; Thurston, G.; Shi, Y.; Finkelstein, N.; Calle, E.E.; et al. Spatial analysis of air pollution and mortality in Los Angeles. Epidemiology 2005, 16, 727-736. [CrossRef] [PubMed]

50. Nyhan, M.M.; Kloog, I.; Britter, R.; Ratti, C.; Koutrakis, P. Quantifying population exposure to air pollution using individual mobility patterns inferred from mobile phone data. J. Expo. Sci. Environ. Epidemiol. 2019, 29, 238-247. [CrossRef] [PubMed]

51. Ostro, B.; Malig, B.; Broadwin, R.; Basu, R.; Gold, E.B.; Bromberger, J.T.; Derby, C.; Feinstein, S.; Greendale, G.A.; Jackson, E.A.; et al. Chronic PM2.5 exposure and inflammation: Determining sensitive subgroups in mid-life women. Environ. Res. 2014, 132, 168-175. [CrossRef] [PubMed]

52. Mudu, P.; Gapp, C.; Dunbar, M. AirQ+ 1.0 Example of Calculations; World Health Organization: Geneva, Switzerland, 2016.

53. Mead, R.W.; Brajer, V. Rise of the Automobiles: The costs of increased NO 2 pollution in China's changing urban environment. J. Contemp. China 2006, 15, 349-367. [CrossRef]

54. Ostro, B.; Prüss-Ustün, A.; Campbell-lendrum, D.; Corvalán, C.; Woodward, A. Outdoor Air Pollution: Assessing the Environmental Burden of Disease at National and Local Levels; World Health Organization: Geneva, Switzerland, 2004; pp. 1-54, ISBN 9241591463. 
55. Debone, D.; Leirião, L.F.L.; Miraglia, S.G.E.K. Air quality and health impact assessment of a truckers' strike in Sao Paulo state, Brazil: A case study. Urban. Clim. 2020, 34, 100687. [CrossRef]

56. DATASUS. Brazilian Health System Database (TABNET). Available online: http://datasus.saude.gov.br/ informacoes-de-saude/tabnet (accessed on 18 June 2020).

57. Coogan, P.F.; White, L.F.; Yu, J.; Burnett, R.T.; Seto, E.; Brook, R.D.; Palmer, J.R.; Rosenberg, L.; Jerrett, M. PM2. 5 and diabetes and hypertension incidence in the Black Women's Health Study. Epidemiology 2016, 27, 202. [PubMed]

58. Li, R.; Zhou, R.; Zhang, J. Function of PM2. 5 in the pathogenesis of lung cancer and chronic airway inflammatory diseases. Oncol. Lett. 2018, 15, 7506-7514. [PubMed]

59. SEADE. State Data Analysis System. Available online: https://www.seade.gov.br/institucional/ (accessed on 27 June 2020).

60. Alberini, A.; Hunt, A.; Markandya, A. Willingness to pay to reduce mortality risks: Evidence from a three-country contingent valuation study. Environ. Resour. Econ. 2006, 33, 251-264. [CrossRef]

61. Ding, D.; Xing, J.; Wang, S.; Liu, K.; Hao, J. Estimated contributions of emissions controls, meteorological factors, population growth, and changes in baseline mortality to reductions in ambient PM2.5 and PM2.5-related mortality in China, 2013-2017. Environ. Health Perspect. 2019, 127, 067009. [CrossRef]

62. Ran, T.; Nurmagambetov, T.; Sircar, K. Economic implications of unintentional carbon monoxide poisoning in the United States and the cost and benefit of CO detectors. Am. J. Emerg. Med. 2018, 36, 414-419. [CrossRef]

63. Robinson, L.A. Estimating the values of mortality risk reductions in low-and middle-income countries. J. Benefit Cost Anal. 2017, 8, 205-214. [CrossRef]

64. Wolfe, P.; Davidson, K.; Fulcher, C.; Fann, N.; Zawacki, M.; Baker, K.R. Monetized health benefits attributable to mobile source emission reductions across the United States in 2025. Sci. Total Environ. 2019, 650, 2490-2498. [CrossRef]

65. Roy, R.; Braathen, N.A. The Rising Cost of Ambient Air Pollution thus far in the 21st Century: Results from the BRIICS and the OECD Countries; OECD Environ. Working Paper; OECD: Paris, France, 2017. Available online: https://www.oecd-ilibrary.org/ (accessed on 27 June 2020).

66. Isaifan, R.J. The dramatic impact of Coronavirus outbreak on air quality: Has it saved as much as it has killed so far? Glob. J. Environ. Sci. Manag. 2020, 6, 275-288.

67. Anjum, N.A. Good in the worst: COVID-19 restrictions and ease in global air pollution. 2020; preprint. [CrossRef]

68. Xu, K.; Cui, K.; Young, L.-H.; Hsieh, Y.-K.; Wang, Y.-F.; Zhang, J.; Wan, S. Impact of the COVID-19 event on air quality in central China. Aerosol Air Qual. Res. 2020, 20, 915-929. [CrossRef]

69. Chou, S.C.; Bustamante, J.F.; Gomes, J.L. Evaluation of Eta Model seasonal precipitation forecasts over South America. Nonlinear Process. Geophys. 2005, 12, 537-555. [CrossRef]

70. Pellon de Miranda, F.; Marmol, A.M.Q.; Pedroso, E.C.; Beisl, C.H.; Welgan, P.; Morales, L.M. Analysis of RADARSAT-1 data for offshore monitoring activities in the Cantarell Complex, Gulf of Mexico, using the unsupervised semivariogram textural classifier (USTC). Can. J. Remote Sens. 2004, 30, 424-436. [CrossRef]

71. Ramis, J.E.; dos Santos, E.A. The impact of thermal comfort in the perceived level of service and energy costs of three Brazilian airports. J. Transp. Lit. 2013, 7, 192-206. [CrossRef]

72. Yu, K.-P. Enhancement of the deposition of ultrafine secondary organic aerosols by the negative air ion and the effect of relative humidity. J. Air Waste Manag. Assoc. 2012, 62, 1296-1304. [CrossRef]

73. Markandya, A.; Sampedro, J.; Smith, S.J.; Van Dingenen, R.; Pizarro-Irizar, C.; Arto, I.; González-Eguino, M. Health co-benefits from air pollution and mitigation costs of the Paris Agreement: A modelling study. Lancet Planet. Health 2018, 2, e126-e133. [CrossRef]

74. Sharma, S.; Zhang, M.; Anshika; Gao, J.; Zhang, H.; Kota, S.H. Effect of restricted emissions during COVID-19 on air quality in India. Sci. Total Environ. 2020, 728, 138878. [CrossRef]

75. Dantas, G.; Siciliano, B.; França, B.B.; da Silva, C.M.; Arbilla, G. The impact of COVID-19 partial lockdown on the air quality of the city of Rio de Janeiro, Brazil. Sci. Total Environ. 2020, 729, 139085. [CrossRef]

76. Grande, G.; Ljungman, P.L.S.; Eneroth, K.; Bellander, T.; Rizzuto, D. Association between cardiovascular disease and long-term exposure to air pollution with the risk of dementia. JAMA Neurol. 2020. [CrossRef]

77. Watts, N.; Amann, M.; Arnell, N.; Ayeb-Karlsson, S.; Belesova, K.; Berry, H.; Bouley, T.; Boykoff, M.; Byass, P.; Cai, W.; et al. The 2018 report of the Lancet Countdown on health and climate change: Shaping the health of nations for centuries to come. Lancet 2018, 392, 2479-2514. [CrossRef] 
78. Baldwin, R.E.; di Mauro, W. Mitigating the COVID Economic Crisis; CEPR Press: Washington, DC, USA, 2020. Available online: https://iheid.tind.io/record/298223 (accessed on 18 June 2020).

79. OECD. OECD Economic Outloo; OECD: Paris, France, 2020; Volume 2020, ISBN 9789264524156.

80. Fernandes, N. Economic effects of coronavirus outbreak (COVID-19) on the world economy. SSRN Electron. J. 2020. [CrossRef]

81. WHO. Quantification of Health Effects of Exposure to Air Pollution: Report on a WHO Working Group, Bilthoven, Netherlands 20-22 November 2000; World Health Organization: Geneva, Switzerland, 2000. Available online: https://apps.who.int/iris/handle/10665/108463 (accessed on 18 June 2020).

82. Blangiardo, M.; Pirani, M.; Kanapka, L.; Hansell, A.; Fuller, G. A hierarchical modelling approach to assess multi pollutant effects in time-series studies. PLoS ONE 2019, 14, e0212565. [CrossRef] [PubMed]

83. Koehl, A. Urban transport and COVID-19: Challenges and prospects in low-and middle-income countries. Cities Heath 2020, 4, 1-6.

84. D'Adamo, I.; Rosa, P. How do you see infrastructure? Green energy to provide economic growth after COVID-19. Sustainability 2020, 12, 4738. [CrossRef]

85. Chen, S.; Bloom, D.E. The macroeconomic burden of noncommunicable diseases associated with air pollution in China. PLoS ONE 2019, 14, e0215663. [CrossRef]

86. Neira, M.; Prüss-Ustün, A.; Mudu, P. Reduce air pollution to beat NCDs: From recognition to action. Lancet 2018, 392, 1178-1179. [CrossRef]

(C) 2020 by the authors. Licensee MDPI, Basel, Switzerland. This article is an open access article distributed under the terms and conditions of the Creative Commons Attribution (CC BY) license (http://creativecommons.org/licenses/by/4.0/). 

Article

\title{
Best-Worst Method for Modelling Mobility Choice after COVID-19: Evidence from Italy
}

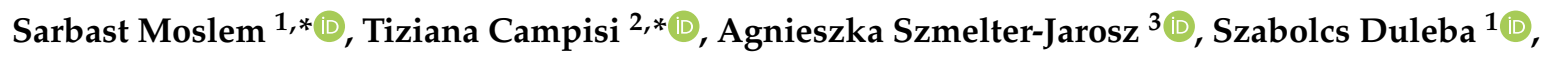 \\ Kh Md Nahiduzzaman ${ }^{4, *(D)}$ and Giovanni Tesoriere ${ }^{2}$ (D) \\ 1 Department of Transport Technology and Economics, Budapest University of Technology and Economics, \\ 1111 Budapest, Hungary; duleba.szabolcs@mail.bme.hu \\ 2 Faculty of Engineering and Architecture, Kore University of Enna, 94100 Enna, Italy; \\ giovanni.tesoriere@unikore.it \\ 3 Department of Logistics, Faculty of Economics, University of Gdansk, 80-309 Gdansk, Poland; \\ a.szmelter@ug.edu.pl \\ 4 School of Engineering, Faculty of Applied Science, Okanagan Campus, The University of British \\ Columbia (UBC), Kelowna, BC V1V 1V7, Canada \\ * Correspondence: moslem.sarbast@mail.bme.hu (S.M.); tiziana.campisi@unikore.it (T.C.); \\ kh.nahiduzzaman@ubc.ca (K.M.N.)
}

Received: 18 July 2020; Accepted: 19 August 2020; Published: 22 August 2020

\begin{abstract}
All countries have suffered from the COVID-19 crisis; the pandemic has adversely impacted all sectors. In this study, we examine the transport sector with a specific focus on the problem of commuting mode choice and propose a new decision-making approach for the alternative modes after synthesizing expert opinions. As a methodology, a customized model of the recently developed best-worst method (BWM) is used to evaluate mobility choice alternatives. The survey reflects citizens' opinions toward mobility choices in two Italian cities, Palermo and Catania, before and during the pandemic. BWM is a useful tool for examining mobility choice in big cities. The adopted model is easy to apply and capable of providing effective solutions for sustainable mode choice. The urban context is analyzed considering the importance of transport choices, evaluating the variation of resilience to the changing opinions of users.
\end{abstract}

Keywords: mobility choice; COVID-19; best-worst method; multi-criteria decision making

\section{Introduction}

The COVID-19 pandemic has produced several unprecedented effects around the world and has adversely affected the transport sector, which has experienced a drastic reduction in passenger traffic across all different modes of transport. With physical interaction being the key medium perpetuating the spread of the virus, government decisions have been pivotally centered on either discrete decisions or combinations of decisions to curtail or block mobility [1]. Therefore, during the outbreak, countries across the globe, including USA, Canada, Italy, and China, imposed different types of bans and restrictions on travel [2] and all types of mobility options that likely involve physical contact and implemented domestic emergency plans for medical response. According to the World Health Organization (WHO) [3], the virus is respiratory and spreads mainly through contact with an infected person. In particular, contact with droplets produced by infected subjects following sneezing or coughing is the key media spreading the virus. Transport modes are amongst the most critical platforms for the rapid spread of the infection in high-density and mixed-use urban environments. This aspect has manifested in the contemporary context where people move every day (on an average 2.5 journeys per day), covering an average distance of about $30 \mathrm{~km}$, for various reasons (e.g., work, study, 
shopping, entertainment) with different modes of transport, including on foot, bicycle, public transport, and personal vehicle [4].

During the second stage of restrictions, many countries addressed the challenge associated with conventional travel, asking for requalification of the road infrastructure and acquisition of electric micro-mobility through political will and endorsement, while promoting walking [4] and cycling [5-7]. Design of road infrastructures and the enhancement of pedestrian and bicycle lanes are highly encouraging avenues for increasing mobility after the pandemic, promoting integrated infrastructure design and control through intelligent transport system (ITS) technologies [8], building information modeling for infrastructure (I-BIM) design, and the mobility as a service (MaaS) concept [9]. To contain the spread of COVID-19 and in anticipation of the gradual resumption of economic activities, people's mobility was restricted using local and domestic restrictive regulations in various domains of mobility, such as halting domestic and international flights, banning movement between communities, and self-isolation in homes. Production systems and work environments were also adapted to the necessary safety conditions through intelligent work environments or increased social distance.

Social media reports and the literature suggest that people will have to live with COVID at least until an effective vaccine is produced. During this time, the most effective strategy has proved to be social distancing, which has reduced the spread of the virus and significantly limited the contagion. Conversely, this seems to have negatively impacted the economy and societal relationships.

Having overcome the peak of contagion, we now enter a phase 2 of coexistence with the virus, which, considering the direct and indirect risks of contagion as the restrictions regarding the more radical social spacing of phase 1 are relaxed, emphasizes the issue of the mobility of people. This phase represents an essential opportunity to build an urban resilience strategy based on necessarily anti-fragile scenarios around mobility policies [10], taking the opportunity during the crisis to instigate an urban and social transformation that is able to strengthen the balance of the complex city system.

Some cities in Europe and in particular in Italy are facing the complex challenge of the reorganization of mobility, where the partial reopening on 4 May 2020 involved the resumption of some productive activities and the consequent increase in related mobility flows. In agreement with Renaud et al. [11], the different forms of mobility must ensure that global mobility can promote local mobility, encouraging solutions that can manage the entire territory.

The COVID-19 pandemic has raised some questions about the vision of infrastructure, changing everyone's viewpoint. Through long-term planning, the acquisition of public funds, and public-private partnerships, the economic system can be improved and the demand for transport can be increased. The long-term consequences of the COVID-19 pandemic may support the creation of more permanent changes related to smart working and other daily activities, thus reducing mobility needs and overall fossil energy consumption. These developments can promote research and new practices arising from the COVID-19 pandemic to accelerate sustainability transitions, improving understanding of the role of governance in transitions and bringing to attention the ethical and policy implications of the shock effect on the various landscapes [12].

In the literature, several studies examined resilience and sustainability in the transport sector. D'Adamo et al. [13] reported that the implementation of circular and green strategies is not explicitly aimed at improving resilience. Still, their impacts are significant in terms of response and recovery, and one benefit is their positive effect on the environment and climate change, reducing the likelihood of environmental disasters.

The main challenge is adapting transport systems to ensure safe mobility for people who return to work from May 2020 onward without losing efficiency. The efficiency of the transport system is linked to its ability to carry a large volume of passengers in a small number of vehicles to increase load factors while reducing mileage and the associated impacts (pollution, greenhouse gases, energy consumption, accidents, congestion). This principle has been adopted by most of the transport companies during the pre-pandemic era, particularly for the popular urban transport modes, i.e., subways/metros, trams/streetcars, and buses. The minimum physical distancing being 
critical during the pandemic, the conventional measurements for the efficiency of the transport system are being severely challenged [14]. In this critical juncture during this transition, we addressed the emerging problem of mobility choice for daily commuting during and after the pandemic in metropolitan cities. As a methodology, the best-worst method (BWM) was applied to evaluate the mobility choice alternatives. Urban residents were the evaluators of the possible mobility choices during the outbreak. The data were collected through an online survey in two metropolitan cities, Palermo and Catania, of the region of Sicily in Italy.

The work focused on three steps related to (1) the selection of a random sample of inhabitants of two metropolises, (2) data processing using the BWM method, and (3) the evaluation of results for the assumption of future developments

We focused on the BWM methodology, which has been implemented in the literature in different sectors, as we considered it optimal for the analysis in the post-pandemic period in favor of the choice of optimal or strongly negative modes of transport in large cities. The data were directly derived from users of the different transport modes, which guarantees higher-accuracy data. The BWM is based on a systematic pairwise comparison of the decision criteria and lays the foundation for more in-depth studies.

\section{Multi-Criteria Decision-Making (MCDM) Methods in Examining Mobility Choices}

\subsection{Specifics of Mobility Choices}

Mobility is a natural part of human life. It is a result of lifestyles, infrastructure, climate, and multiple other factors widely presented in the literature [15]. Mobility can be described in many different dimensions, for example, as urban mobility (inter- and intra-urban mobility), for long and short distances, driving changes for a short or long term.

Mobility choices, even if a part of the mobility, are often capacious, and are mentioned in the scientific papers as travel behavior, transport choices, or mobility patterns [16]. They represent travel data and are defined by many socioeconomic attributes of travelers, enabling the identification of separable patterns [17], e.g., for younger and older cohorts, called generations, primarily mentioned only in the human resources literature, then adapted by transport- and mobility-related studies, among others [18]. Those criteria are not limited to age but also sex, family status, life stage, having a driving license, and access to a car $[19,20]$. Mobility choices involve decisions of passengers resulting from considering possible travel scenarios and priorities (criteria) [21]. Included in this set of criteria are, among others, accessibility, fares, travel time, comfort, safety, being on time, reliability, directness, and waiting time [22]. A strongly developing area of study is the multimodality of mobility, followed by choosing eco-friendly travel modes [23]. Usually, investigation is undertaken for policymakers [24] by evaluating the needs of different stakeholders [25]; these studies concern mobility management. The mentioned travel priorities and scenarios mainly result in the choice of means of transport (one or more, if a multimodal pattern). The most popular, comfortable, flexible, and convenient transport mode is a personal vehicle; although it is perceived as not eco-friendly, creating congestion and traffic, it decreases the popularity of public and active transport [26].

The car culture is still alive in many countries; in others, it is only in its infancy [27]. A number of studies focused on identifying the different aims of car users, e.g., by recommending the car-sharing initiatives [28]. The goal of many activities focused on evaluating the mobility patterns is promoting modes other than individual motorized transport, especially cars, e.g., by extensive use of park-and-ride stations or simply using a different mode of transport [29-31]. During the pandemic, a car can be perceived as one of the safest choices for mobility because of the low risk of being infected.

Mobility choices have been a popular area of studies over the last 10 years [32]. These choices can be measured and analyzed using surveys, interviews, travel diaries, and geographic information systems (GISs) [33]. Unfortunately, many results are biased [34] by evaluating the mobility choices of non-random samples or samples containing only chosen groups of respondents (usually students) [35]. 
Mobility management involves a set of rules for managing the travel demands of different users including residents and non-residents (e.g., guests, tourists, and workers living in other areas). Mobility management differs between weekdays and other dates (e.g., weekends, holidays, incidents). In urban areas, the primary tool for strategic mobility management should be a sustainable urban mobility plan [36], further implemented using forced and voluntary solutions [37], promoting the reduction of travel-trip substitution and travel distance, and promoting mode shift or low- and zero-emission mobility [29]. Solutions should influence changes in mobility choices, especially those of younger generations as they are more flexible than older adults [38]. The mobility patterns differ for the residents of big cities, smaller cities, and rural areas according to the characteristics of their surroundings and transport possibilities.

As a consequence, multiple studies analyzed mobility choices. The complexity of this issue and the wide range of significant criteria led to the dynamic development of multi-criteria decision-making (MCDM) methods for mobility investigation. The gaps appearing between strategic and operational levels in the decision-making process of travelers require further study [39], including:

- information gaps (differences in nature, kind, interpretation, and reliability of sources of information),

- methodological gaps (differences in complexity, transparency, and interpretation of approaches, and methods for choosing transport scenario),

- feedback/continuity gaps (gap resulting from the objective definition through solution selection to implementation),

- contextual gaps (differences in thinking models—rational and others-having some level of uncertainly of decision), and

- $\quad$ spatial vision gaps (differences in scales of decision and spatial dimension).

Therefore, multi-criteria approaches in the field of mobility choices indicate how to overcome these gaps and rationalize the assessment and decision-making processes.

\subsection{Examining Mobility Choices by MCDM Methods}

MCDM is defined as a set of different methods with various mathematical approaches used to identify the best solution among the proposed or possible group of solutions/scenarios. MCDM methods are used in research where a number of decision criteria are defined that they have different levels of importance for the decision-maker [40]. Sometimes they are also called multiple-attribute decision-making methods [41]. The number of these methods is extensive, as is that of customized MCDM approaches (e.g., fuzzy approaches). They differ from each other in terms of the aggregation, data (direct or indirect, like comparing the variables), kind of output, and ease of application by decision-makers less familiar with complex mathematical operations. A complex review of available MCDM methods was published [42,43]. In turn, the mode choice attributes were revised [21].

In the literature, multiple approaches were used, including both classical and customized as fuzzy-based methods, to explain the nature of mobility choices. Among the MCDM methods for describing the mobility choices are the multi-attribute utility technique [44]; spatial multi-criteria evaluation (SMCE) [25], which is a combination of spatial analysis and multi-criteria evaluation; analytic hierarchy process (AHP) [21,44,45]; preference ranking organization method for enrichment evaluation (PROMETHEE) [44,45] or a mix of previous methods (AHP-PROMETHEE) [46]; multi-actor multi-criteria Analysis (MAMCA) [47,48]; technique for order of preference by similarity to ideal solution (TOPSIS); complex proportional assessment (COPRAS), weighted aggregated sum product assessment (WASPAS) and evaluation based on distance from average solution EDAS [39]; best-worst Method (BWM) [38]; and maximum entropy multi-criteria user equilibrium (ME-MUE) [49]. For example, commuter mobility was evaluated using the TOPSIS method [28] for China (Beijing) using smart card data. BWM was found to be the best for the analysis of large amounts of data [45].

MCDM is the dominant method used for evaluating mobility choices in urban mobility studies [16,37], including those examining sustainable urban mobility plans [38,46-50]. Other researchers used MCDM 
in transport and mobility along with different approaches to compare public transport systems [51], to assess and add quality to transport nodes [32], to evaluate transport providers [34], and assess road projects [40]. Additionally, MCDM methods were used to evaluate scenarios in the fields similar to mobility, like neighborhood selection by the city newcomer (e.g., multi-attributive border approximation area comparison (MABAC) and Visekriterijumsko Kompromisno Rangiranje, (VIKOR), and combinative distance-based assessment (CODAS) [52].

\subsection{Best-Worst Method (BWM) for Examining Mobility Choices}

BWM was created by Rezaei [53] and is an element of an extensive set of MCDM methods. It is perceived as an efficient approach due to its data requirements, being well-structured, transparency, easy application, and reliable results [50]. It has attracted the attention of researchers from different fields. It has been used in research in business and non-business areas, like technological innovation [54], scientific activity assessment and relationships with industry [55,56], quality of life [39], consumer ethical beliefs [57], water resource management [58,59], and research and development performance [60]. A wide review of using BWM was published [38].

BWM has been applied to several transportation and logistics research areas like supplier selection [60,61], supply chain management (SCM) [62], and logistics performance [5]. BWM was also used to assess transport mode choice for freight transport [45], including combined transport [63]. BWM can be used as a stand-alone method and in a few derivative concepts, e.g., the new multiple-attribute decision-making method (MADM), which is a combination of BWM and multi-attributive ideal-real comparative analysis (MAIRCA) [52].

The usefulness of other MCDM methods is undeniable, but the main difference between the BWM method and the others based on pairwise comparisons is its main structure, which is based on the most and the least significant criteria [61,64]. The attractiveness of the BWM results from its advantages, including a number of features that facilitate calculations and their interpretation: a smaller number of pairwise comparisons than in other methods, higher reliability of calculated weight coefficients, consistency of output, and using only integer values when comparing criteria (in pairs) [33]. Additionally, BWM works well when comparing and considering many conflicting criteria and goals of decision-makers (tradeoffs). BWM by its nature-specific, structured pairwise comparison—solves the inconsistency issue with, e.g., in AHP. Compared to other methods, BWM requires fewer comparison data $[60,65]$ and provides better consistency ratio and lower minimum violation, total deviation, and conformity; therefore, BWM generates more reliable results [45].

BWM has not been used in any situation with a best/worst choice. The best-worst approach is misleading, and the description of the BWM is unclear in the scientific literature. Sometimes the literature on, e.g., general or specific consumer values in mobility or transport mentions the best-worst choices being unequal to the best-worst method, but best-worst scaling (BWS) is only an approach used to support, e.g., logit or probit models [66,67] or cluster analysis [68]. The same applies to best/worst values used in multiple logistics regression [69] or a best/worst rank [70,71].

\section{Changes in Mobility Choices during and after COVID-19}

\subsection{Impact of COVID-19 on Mobility Worldwide}

The COVID-19 pandemic has affected the lives of almost all countries, even if they did not confirm any cases of infected residents or visitors. Three countries were perceived as most affected by the COVID-19 epidemic: China (as the first country with many deceased infected patients), Italy (most affected in Europe), and the USA (with the highest number of infected people). Although the situation in Europe has yet to stabilize, Italy is attempting to recover from this crisis as soon as possible [72].

Mobility was partly responsible for the rapid development of the epidemic. Both shortand long-distance travel caused the virus spread to increase dynamically. Therefore, among others, all the planned massive gatherings, rallies, and concerts were banned. The lockdown caused a sharp reduction in the number of trips and distance traveled. As the COVID-19 epidemic is still ongoing, 
some initial research results on mobility changes are now available. However, they are based mostly on Google data [73-75], which are non-random data, as the data are shared only by some groups of individuals who agreed to share the data about their localization. This generated the need to evaluate the mobility changes using data gathered separately for different countries.

The basic restrictions imposed by governments across the world are a group of solutions focusing on social distancing. They resulted in a number of changes in mobility as well as in short-term lifestyles [76]. Since the beginning of the epidemic, a number of techniques attempted to predict the possible scenarios associated with mobility $[77,78]$. Given the restrictions, long-distance travel, especially international travel, were sharply curtailed or banned. As a result, the spread of the new coronavirus through international mobility was controlled, given the limited possibility of traveling. Passengers were also under strict surveillance during and after the journey. A number of non-clinical strategies appeared to minimize the risk of infection [79]. After some time, short-distance travel was also strictly controlled as the epidemic continued to spread through urban areas [80]. Essentially, individual mobility decisions were the key medium to spreading the disease in local and international locations.

A set of changes were implemented regarding mobility during COVID-19 pandemic. Whereas mobility is purpose-driven, the change in the purpose (leisure, work, education, shopping, etc.) alters the nature of individual mobility. At the outset, with home-based work being an immediate response to contain the spread of COVID-19, many workers converted part of their homes into workstations; while many lost their jobs, others had to take care of children alongside their regular job [75]. Due to the need for stay-at-home and restricted mobility, the total number of trips to schools and offices drastically reduced almost to zero [72]. People started to display a higher propensity for recreation, especially the use of green spaces (which increased by $291 \%$ in Oslo) [81] and active transport (cycling, walking). This was especially observed in urban areas, which sparked discussion about the true needs during a pandemic and how the future cities will be reshaped to meet these transformational needs [82].

Understanding COVID-19-induced changes in mobility is still developing as the epidemic continues. However, several studies only mentioned this topic in Japan [82], Canada [83], China [84], Italy [85], the USA [86], Bangladesh [87], India [88], Australia (but only a simulation) [89], France [90], and Sweden [91].

In Italy, the changes in mobility were mainly caused by government restrictions and society [92]; these changes intensified due to the rapid spread of the virus. The interventions regarding social distancing are a basic concept of regulating mobility. A simulation for Newcastle (Australia) showed that when $90 \%$ of people work from home, schools are closed, and social contact decreases by $70 \%$, the infection rate decreased from $66 \%$ to $1 \%$. Even when only $50 \%$ of the workers work from home and community interactions decrease by $30 \%$, the infection ratio would be less than $10 \%$ [89]. This, however, would impact the mobility and change in travel distances, purposes, and numbers. All the needed restrictions are not possible to implement, so social distancing became the fundamental element of governmental decisions. It was challenging to implement in countries with low access to all the needed facilities including sanitation, especially in high-density societies and lower-middle-income economies [87].

The most comprehensive study on mobility during the COVID-19 epidemic was conducted by Chan et al. [93]. They examined the data for risk preferences for 60 countries (concerning the individuals) between 15 February and 11 April 2020 independent of government lockdown measures. They discovered that risk of infection caused people to be less willing to take risks, which reduced their travel to shops (even groceries and pharmacy), retail, and recreation locations. The decline in mobility to all local shops and other destinations was substantial for high-population-density countries. The larger percentage was the share of subpopulation of age 65 years old or older; this part of the population has a high risk of infection, so they additionally limited their visits to grocery stores, pharmacies, and parks. After the official WHO announcement about the COVID-19 pandemic, retail-, 
recreation-, transit-, and work-related trips decreased. For recreation and retail, the decrease was $7.47 \%$ [93].

Another study divided the COVID-19 epidemic period into two: before and after officially announcing the pandemic [93]. In the first period, people were starting to reduce work trips (especially the use of public transport) and non-essential retail shopping. The second period produced the next behavioral shifts, enhancing the previous changes. Social distancing was a more robust guideline than the lockdown (considering the people aged 65 years or older), which was intensified by shorter working hours in most of the shops. However, social distancing protocols and travel restrictions in many countries, were difficult to maintain. Sometimes, the controlling administration agencies were not prepared for such a large increase in tasks and people to be controlled [88]. In some cases, military force was used to enforce mobility restrictions. Leisure-centered mobility, including sports activities, was drastically limited. A noticeable change in mobility was observed from motorized to soft modes or active (cycling, walking etc.) transport due to companies, schools, and shopping centers being closed. Having more free time, people wanted to go walking, and many quarantine restrictions were violated.

To summarize, mobility choices were drastically disturbed by the COVID-19 outbreak. During this period, the choices mostly depended on government enforcements and optional recommendations. The many COVID-19 effects on mobility include: changing modes for both more active and non-motorized, from public to individual transport, more time spent playing sports, changes in travel purpose, and limiting travel aimed at shopping. This also caused changes in freight transport, e.g., for courier express parcels (CEP) and service providers for online shops. However, the studies are lacking on the changes in mobility choices during the COVID-19 epidemic. Therefore, the review of these studies provided limited empirical results.

\subsection{Spread of COVID-19 in Italy and Effects on Mobility}

Italy was the first country in Europe to be infected. According to Murgante et al. [94], COVID-19 hit Italy in February 2020 after its outbreak in China in early January. By comparing the spatial distribution and mortality model associated with COVID-19 in Italy with various geographical, environmental, and socioeconomic variables at the provincial level, a correlation was found between the number of COVID-19 cases and the associated pollutants nitrogen and soil, especially in the Po Valley area. Using a historical data series on air pollution, human mobility, winter temperature, housing concentration, health care density, population size, and age, the epidemic risk was assessed by identifying the most vulnerable areas [95].

They found that the highest risk occurred in some northern regions compared to central and southern Italy. Although the COVID-19 epidemic started almost simultaneously in both the north (Lombardy and Veneto) and in Lazio (central Italy) when the first cases were officially certified in early 2020, the disease spread more rapidly and with more serious consequences in regions with a higher epidemic risk.

Using various methodologies, the evolution of travel during the pandemic period has been monitored, with particular attention to the reasons for traveling and using public transport. Various technological tools made it possible to follow the development of passenger movements in various states. Some applications on smartphones and tablets enabled the geo-localization of people and the obtaining of information on the distances traveled [96].

The use of traffic meters, public transport ITS [97], and recordings from traffic control cameras and environmental sensors facilitated comparisons between travel flows and times before and during the lockdown. The impact on externalities, such as $\mathrm{NO}_{2}$ emissions and road accidents, were also estimated [98]. Public transport users decreased by $93 \%, \mathrm{NO}_{2}$ concentration by $60 \%$, and road accidents by $67 \%$.

One out of four movements is provided by public transport in Italian cities with more than 250,000 inhabitants. A reduction or downsizing of the service provided was necessary 
The decrease in the use of public transport compared to use on a working day, due to the COVID-19 emergency, is shown in Figure 1, considering the red zone where all travel was limited implemented from 12 to 25 March 2020 in various regions of Italy, comparing the South Italy data represented by Palermo and Trapani (for Sicily) and Naples and Province (for Campania) with the data of Milan and Lombardy, where the first deaths were recorded.

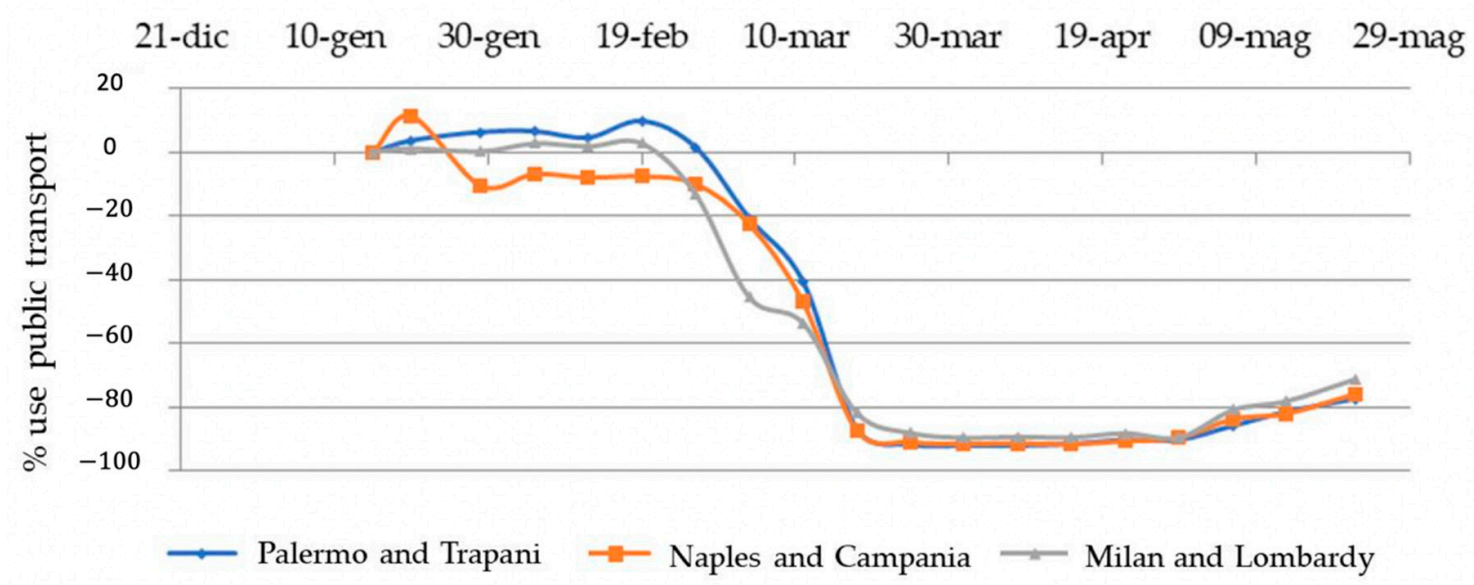

Figure 1. Public transport trend in South Italy

\section{Methodology}

For evaluating mobility choices, we adopted one of the most recently created MCDM techniques. BWM is similar to many other MCDM techniques, consisting of pairwise comparisons of the decision elements [53]. The main difference of BWM from the other techniques is that not all comparisons are completed; only the pairs with the previously selected best and worst alternatives are evaluated. In modelling and application, the following phases were followed (Figure 2).

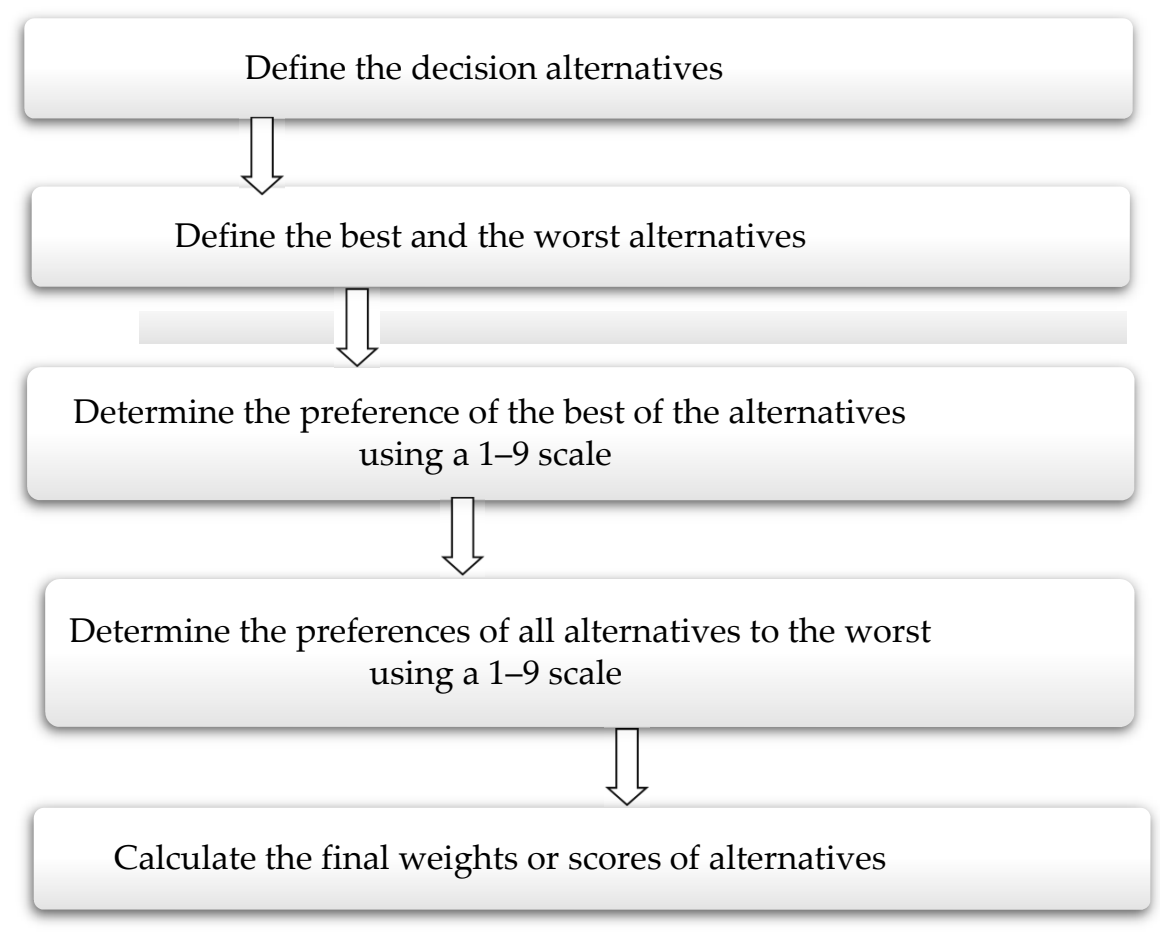

Figure 2. The main steps of the best-worst method to derive the weights of the alternatives. 
The first step was identifying the best and worst alternatives determined by the evaluators. Secondly, the pairwise comparisons were applied between the best and other alternatives, then between the worst alternative and other alternatives. After, the weights of all alternatives were calculated. The consistency ratio was used to test the reliability of the pairwise comparisons.

To provide an overview of all stages for the survey, we completed the phases in the following order:

- $\quad$ Step 1: Identifying the mobility types.

- Step 2: Defining the best and worst mobility types using simple scoring by expert participants.

- Step 3: Evaluating the pairwise comparisons between the best mobility type with the other types using a (1-9) scale, where 1 denotes equal importance and 9 means extremely important. The following set represents the results of the best to other types $\left(V_{B}\right)$ :

$$
V_{B}=\left(v_{B 1}, v_{B 2}, \ldots, v_{B n}\right),
$$

where $v_{B j}$ is the preference of criterion $B$ (the most important or the best) over all criteria $j$, and $v_{B B}=1$. In our model, $n=6$, as we have six alternatives to compare, $j=(1,2, \ldots, n)$.

- Step 4: Making the pairwise comparisons between the worst mobility type and all other types by using a (1-9) scale. The result of other to worst type $\left(V_{W}\right)$ is represented by the following set:

$$
V_{W}=\left(v_{1 W}, v_{2 W}, \ldots, v_{n W}\right)
$$

where $v_{j D}$ is the preference of criteria $\mathrm{j}$ (the most important or the best) over the criteria $\mathrm{D}$ and $v_{D D}=1 . \mathrm{n}=6$, as we have six alternatives to compare in our model, $j=(1,2, \ldots, 6)$.

- Step 5: Calculating the final optimal weights $\left(D_{1}^{*}, D_{2}^{*}, \ldots, D_{n}^{*}\right)$ of the mobility types, and the indicator of the optimal consistency of comparisons, $\xi^{*}$.

The maximum absolute difference has to be minimized by:

$$
\min _{\max }\left\{\left|\frac{D_{B}}{D_{j}}-v_{B j}\right|,\left|\frac{D_{j}}{D_{W}}-v_{j W}\right|\right\}, \sum_{j}^{\text {s.t. }} D_{j}=1, D_{j} \geq 0 \text {, for all } j .
$$

Then, the solution can be obtained by solving the following Linear programing (LP):

$$
\min \xi^{*} \text { s.t. }\left|\frac{D_{j}}{D_{W}}-v_{j w}\right| \leq \xi^{*} \text {, for all } \mathrm{j}, \sum_{j} D_{j}=1, D_{j} \geq 0 \text {, for all } j .
$$

The following formula computes the consistency ratio (CR) to check the consistency of the comparisons [65]:

$$
\text { Consistency Ratio }=\frac{\xi^{*}}{\text { Consistency Index }}
$$

where the consistency index (CI) is presented in Table 1 [70], which was determined by random experiments for a different number of comparisons.

Table 1. The consistency index (CI) values for computing the consistency ratio.

\begin{tabular}{cccccccccc}
\hline$v_{B D}$ & $\mathbf{1}$ & $\mathbf{2}$ & $\mathbf{3}$ & $\mathbf{4}$ & $\mathbf{5}$ & $\mathbf{6}$ & $\mathbf{7}$ & $\mathbf{8}$ & $\mathbf{9}$ \\
\hline $\mathrm{CI}$ & 0 & 0.44 & 1 & 1.63 & 2.3 & 3 & 3.73 & 4.47 & 5.23 \\
\hline
\end{tabular}

The CR is acceptable for the BWM methodology if its value is between 0 and 1.

This ratio can be calculated for individual evaluators or group-wise when aggregating the scores of the group by creating the geometric mean of the scores and then conducting the consistency check. In our case, the CR was checked individually. The survey was based on the BWM method and collected general information about participants' characteristics. 


\section{Results}

We defined the area of investigation to Sicily in southernmost Italy, including the metropolitan areas of Palermo and Catania. These cities are located in the north (Palermo), with approximately 674,000 inhabitants, and in the east (Catania), with approximately 314,000 inhabitants, respectively. The areas presented different forms of mobility, including shared and multimodal types.

We collected 400 surveys before the crisis (November and December 2019); we analyzed the data and did not publish the results.

However, the same sample size was used to collect data during the crisis (March and April 2020) to highlight the main differences in public preference toward mobility usage. The participants' characteristics are presented in Table 2.

Table 2. Participants' characteristics.

\begin{tabular}{ccc}
\hline & $n=400$ & \\
\hline \multirow{2}{*}{ Gender } & Male & $43 \%$ \\
& Female & $47 \%$ \\
\hline \multirow{2}{*}{ Marital Status } & Married/in a relationship & $27 \%$ \\
& Single & $73 \%$ \\
\hline \multirow{2}{*}{ Age } & $18-30$ years & $69 \%$ \\
& $31-50$ years & $17 \%$ \\
& $>50$ years & $14 \%$ \\
\hline
\end{tabular}

Before the pandemic, although the online survey was sent to 4000 respondents, only 487 responded, which were selected for analysis. However, during the pandemic, the survey was sent again to the 487 respondents, of which 400 surveys were received and selected for evaluation. The users who responded were controlled through identification by email address. Before the pandemic, $10 \%$ of the sample responded.

To conduct a longitudinal analysis and compare the responses of the users, we decided to limit the sample. This type of study design is mostly suitable for research regarding existing resources and constraints. We chose one longitudinal study (e.g., a panel survey), in which measurements over time were foreseen for each detection unit (repeated measurements) and specifically the variations in the choice of mobility.

The number of users, although small, was acquired randomly, guaranteeing the heterogeneity of the sample as hypothesized during the planning phase of the research. This approach enables estimates with variations over time and reduces related distortions of remembrance, especially for time intervals of the order used (a few months). We chose the variables to be investigated and administered an in-person questionnaire.

The transport choices are described in Figure 3. All the transport modes were available in the two cities except for the tram/streetcar, which was only available in Palermo.

Having selected the commuting alternatives (Figure 4), the following questions were created according to the BWM logic:

- $\quad$ Please select the most- and the least-used transport mode for commuting to work before COVID-19.

- Please select the most- and the least-used mobility option for commuting to work during COVID-19.

- Please evaluate the other types of transport with respect to the most used using a 1-9 scale.

- Please evaluate the least-used using a 1-9 scale.

where 1 represents equal importance between two alternatives, 9 represents the extreme importance of one alternative over another, and 2-8 are intermediate values [65]. 


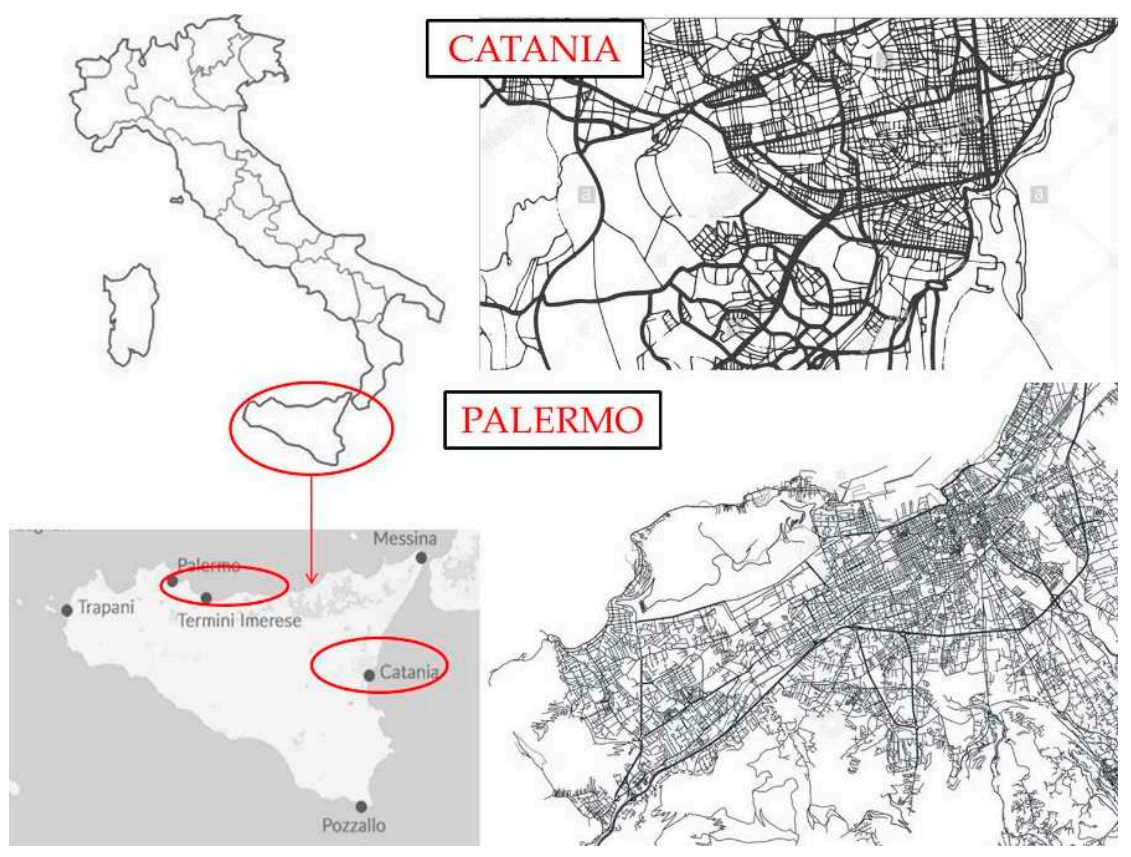

Figure 3. Location of case study.

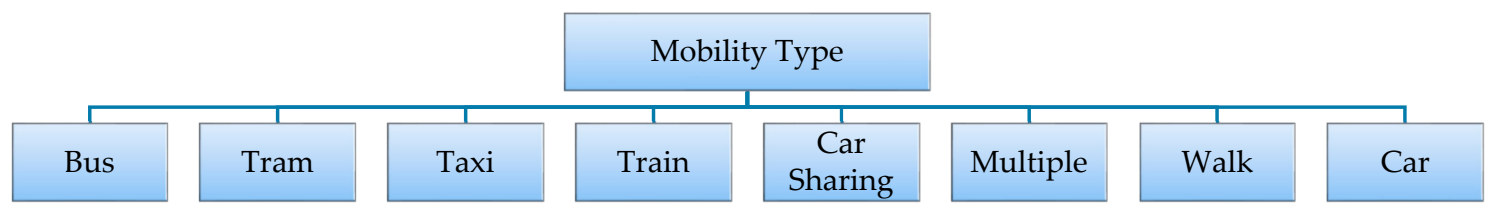

Figure 4. Transport modes in Sicily.

The example in Table 3 illustrates how the survey was conducted.

Table 3. Example of evaluating all mobility types compared to the most-used mobility type.

\begin{tabular}{ccccccccc}
\hline Mobility Type & Bus & Tram & Taxi & Train & Car Sharing & Multiple & Walk & Car \\
\hline Most used mobility choice: Tram & 2 & 1 & 5 & 4 & 6 & 6 & 7 & 3 \\
\hline
\end{tabular}

Table 3 shows the weights of an evaluator of the best mobility type, which, in this case, was tram. The lower the number, the closer a certain alternative to the best choice.

Table 4 shows the weights of an evaluator of the least-used mobility type, which was sharing. Lower numbers indicate closeness of a certain alternative to the least-used type.

Table 4. Example of evaluating the least used mobility type to all other mobility types.

\begin{tabular}{ccccccccc}
\hline Mobility Type & Bus & Tram & Taxi & Train & Car Sharing & Multiple & Walk & Car \\
\hline Least-used mobility choice: sharing & 3 & 6 & 2 & 5 & 1 & 4 & 7 & 6 \\
\hline
\end{tabular}

The consistency ratio (CR) was acceptable for all individual responses, as its value was between (0-1) in all cases. CR values were computed using Equation (5).

The final results before COVID-19 are presented in Table 5. The most-used mobility type was car, followed by walking; the least-used was car sharing, followed by multiple modes (Figure 5). 
Table 5. Final scores of the mobility types before COVID-19.

\begin{tabular}{ccccccccc}
\hline \multirow{2}{*}{ Weight Score } & Bus & Tram & Taxi & Train & Car Sharing & Multiple & Walk & Car \\
\cline { 2 - 9 } & 0.1319 & 0.079 & 0.0659 & 0.0989 & 0.0312 & 0.0565 & 0.1979 & 0.3385 \\
\hline
\end{tabular}

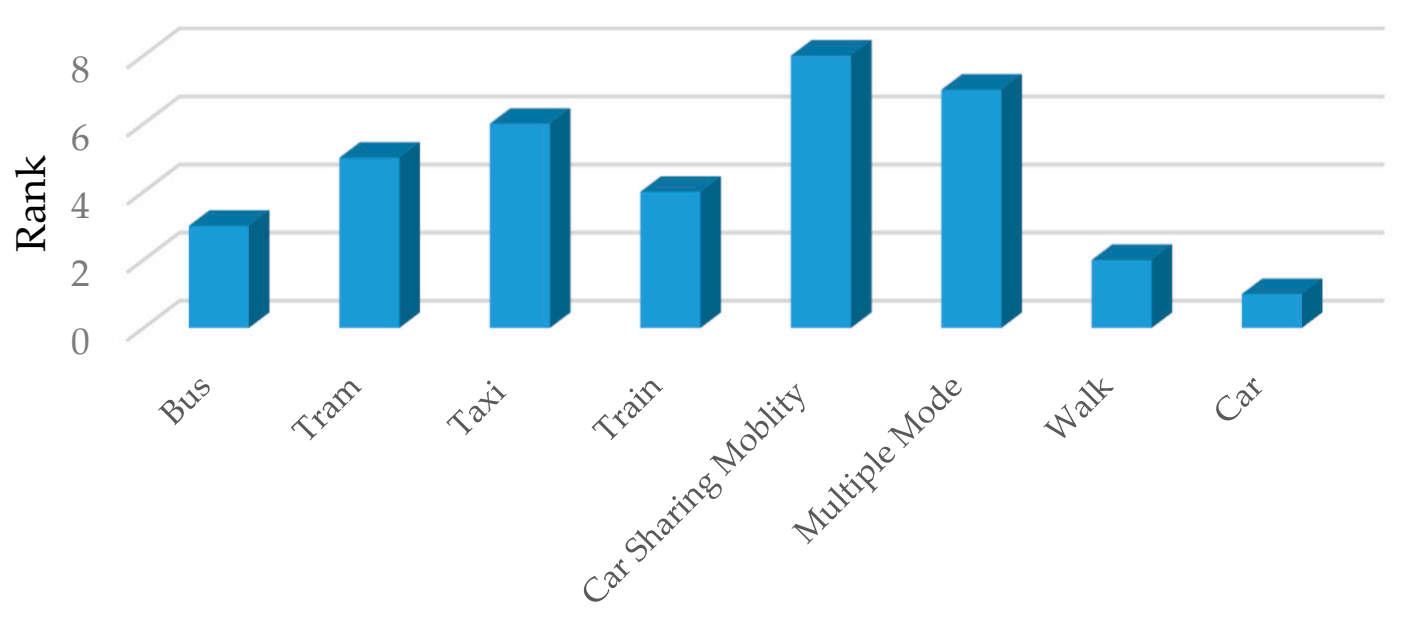

Figure 5. The final rank of the most used mobility types before COVID-19.

The final results of the surveys during the crisis are presented in Table 6. The most-used mobility type was walking, followed by car. The least-used mobility type was tram, followed by taxi (Figure 6).

Table 6. Final scores of mobility types during COVID-19.

\begin{tabular}{ccccccccc}
\hline \multirow{2}{*}{ Weight Score } & Bus & Tram & Taxi & Train & Car Sharing & Multiple & Walk & Car \\
\cline { 2 - 9 } & 0.1184 & 0.0338 & 0.0592 & 0.0789 & 0.0677 & 0.0947 & 0.3892 & 0.1579 \\
\hline
\end{tabular}

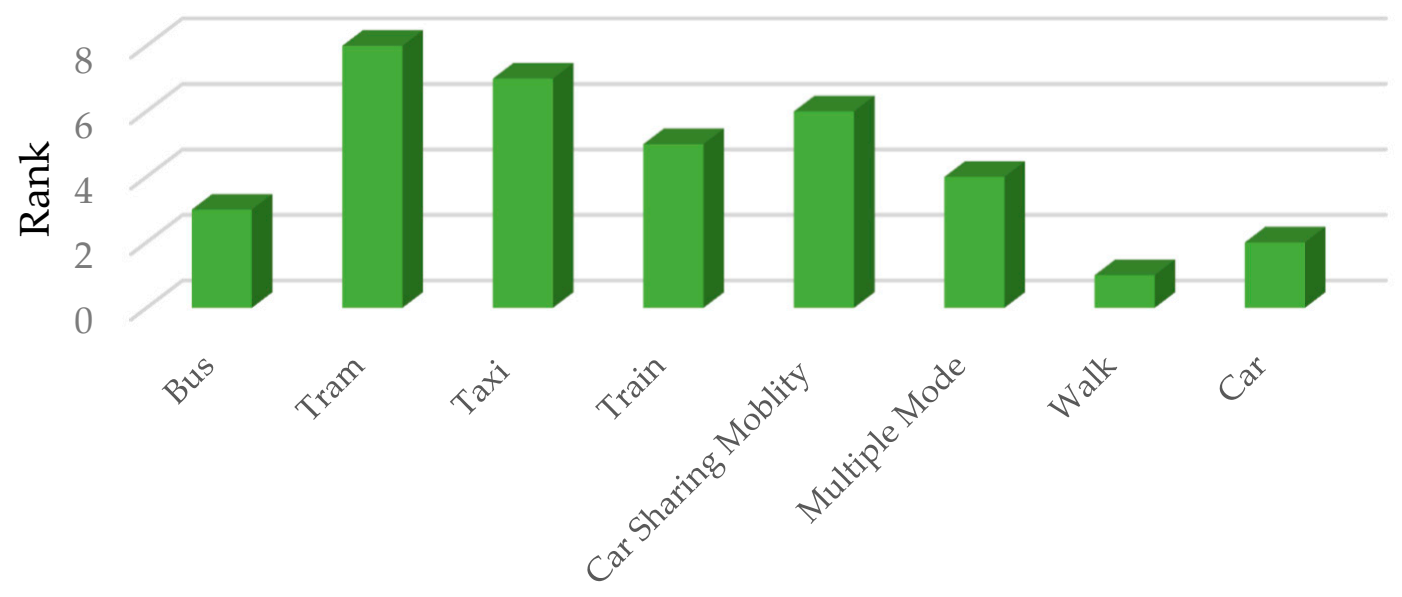

Figure 6. The final rank of the most used mobility types during COVID-19.

Table 7 illustrates the differences in preference before and during COVID-19 crisis based on the citizens' point of view. 
Table 7. Final scores and ranks of the mobility types before and during COVID-19.

\begin{tabular}{ccccc}
\hline Mobility Type & Score before COVID-19 & Rank & Score during COVID-19 & Rank \\
\hline Bus & 0.1319 & 3 & 0.1184 & 3 \\
Tram & 0.0791 & 5 & 0.0338 & 8 \\
Taxi & 0.0659 & 6 & 0.0592 & 7 \\
Train & 0.0989 & 4 & 0.0789 & 5 \\
Car Sharing & 0.0312 & 8 & 0.0677 & 6 \\
Multiple Modes & 0.0565 & 7 & 0.0947 & 4 \\
Walk & 0.1979 & 2 & 0.3892 & 1 \\
Car & 0.3384 & 1 & 0.1579 & 2 \\
\hline
\end{tabular}

The results showed that the transport mode split for Italians significantly changed during the pandemic. Especially observable was the shift from public into individual transport (including walking, car travel, and ride sharing). Bus remained the third choice of Italians, but the multimodality increased, which may influence the mobility choices even if the epidemic ends.

The variation in choice by users regarding mobility is linked to the reduction in the use of public transport and an increase in the number of individuals both in shared vehicles, which indicates some considerations in terms of the vulnerability and resilience of the city.

The increase in individual mobility will have to be supported by local and domestic strategies to encourage soft or shared mobility at the expense of private mobility, which could lead to high pollution rates as well as traffic congestion. The new transport choices should influence planners to rethink the use of open public spaces and roads, providing more services to the citizens and creating a resilient transformation of the city, i.e., by redesigning or adapting an urban system to climate change as well as social (including distance), cultural, economic, and structural changes.

\section{Discussion}

The study results suggested a greater tendency to walk for shorter journeys, in compliance with social distancing and other safety precautions during COVID-19. We identified differences in the transport mode split in Italy, similar to those observed in other countries, especially the increasing share of walking and car transport and the decreasing percentage of public transport use. As Italy was the country most affected by the pandemic in Europe, the changes in modal split were obvious, but the characteristics of those changes were unknown. The dynamic situation in many countries and the dynamics of the pandemic, combined with regulatory issues (restrictions), determined the changes in mobility choices.

A reduction in the use of public transport, a change in the reasons for moving due to confinement, and social distancing led to a sharp decrease in the use of some forms of mobility such as public transport, but also to a strong reduction in pollution. This finding brings into question the sustainability of urban mobility. Mass transit and shared transport might need to be re-designed in a way that respects the need for social distancing [98].

Mobility studies during the COVID-19 outbreak are scarce. Most of the published papers [72,79,82] were based on Google or Apple sources, where data were gathered from mobile phones. However, they do not reflect the real opinions of the citizens of particular regions, but instead offer valuable insights into the changes in mobility (mode and pattern) and demand.

In this study, we addressed the necessity of comparing mobility choices before and after the outbreak in Italy.

The government decisions on mobility restrictions aimed to reduce the risk of spreading COVID-19 consisted of multiple actions. However, the change in the mobility choices in Italy may be an effect of the temporary closure of schools and companies and therefore the lower travel demand [72]. This was especially observed in March and April 2020 (the worst periods for Italy in terms of coronavirus spread), when the primary survey of this study was conducted. 
During that peak time, public transport was justifiably perceived to be a potentially risky mode of transport in terms of infection spread [79]. Therefore, walking was found to be the first choice of transport mode [82]. The results of this study were similar to those previously reported for Italy [72], but those results were less complex and needed some more in-depth insight. Some additional information should be obtained about the socioeconomic characteristics of respondents to conduct a profound analysis of mobility choices [78]. The share of the real impact of government restrictions and personal decisions should be analyzed for Italy [92,93]. Although future studies are warranted to examine the changing nature of mode choices, our findings provide new insights into the current dynamics of mobility choices in the selected Italian regions. We identified a transformation not only resulting from governmental restrictions, but also from the choices of the individual residents [96].

We found that before COVID-19, the preferred form of movement was the car, followed by walking. This was due to the moderate frequencies of public transport and the relative delays, which prevent users from reaching their destination on time. Therefore, multiple modes were the least-used type due to the difficulty of synchronization and the lack of guarantees among the various modes of transport of delays of less than 5-10 min in the context examined. The development of mobility as a service (MaaS) could guarantee and manage this complex issue, but it requires further investigations.

\section{Conclusions}

The survey results presented in this paper suggest that the BWM method can act as a reference point and base for future studies. Firstly, our results can be compared with those for other countries. The simplicity of the BWM method combined with the usefulness of the results indicates that BWM could be perceived by other researchers as a valuable method for data analysis and an attractive alternative for the well-known multi-criteria decision-making methods used in mobility studies. The public stakeholders (public transportation companies, local authorities) can use the method for their own research and managerial purposes to strengthen the already-used methods. Secondly, the results provide a starting point for more in-depth analyses of mobility choices during the COVID-19 outbreak and the changes in mode choices after the epidemic, e.g., examining the long-term results in the mobility area, like walking or using active transport modes more than before the outbreak.

Despite the strengths of this study (including research about mobility changes not based on Google data [73-75]), it has a few limitations. Firstly, the sample size was small. The survey was simple and contained only a few questions, which prevented deep analysis of the causes of mobility choices of the respondents. Secondly, we included only two Italian cities, so the results from this limited area may not be necessarily extended to other areas. Thirdly, most of the outcomes resulted from obligatory changes. Many changes in the observed mobility resulted from the government restrictions and were not facultative for Italians. Fourthly, comparing the results with the studies for other countries with similar or different pandemic characteristics is difficult, because the results for those countries are not known. Regardless, this study contributes to mobility studies, especially those about the changes during the COVID-19 outbreak. We plan to conduct further studies focusing on passenger mobility.

The sample will be extended by implementing predictive market models based on the idea of crowdsourcing, i.e., on what collective information should be based to support the forecasting of transport demand $[99,100]$.

Author Contributions: Conceptualization, S.M. and T.C.; data curation, G.T.; formal analysis, S.M. and T.C.; funding acquisition, S.M. and T.C.; investigation, S.M., T.C. and K.M.N.; methodology, S.M. and T.C.; project administration, S.M. and T.C.; software, S.M. and T.C.; supervision, S.D., K.M.N. and G.T.; validation, S.M., T.C. and G.T.; writing—original draft, S.M., T.C. and A.S.-J.; writing—review and editing, S.M., T.C., A.S.-J. and G.T. All authors have read and agreed to the published version of the manuscript.

Funding: This research received no external funding.

Acknowledgments: The fourth author would like to acknowledge the support of the MTA Bolyai research scholarship.

Conflicts of Interest: The authors declare no conflict of interest. 


\section{References}

1. Colbourn, T. COVID-19: Extending or relaxing distancing control measures. Lancet Public Health 2020, 5, e236-e237. [CrossRef]

2. Tian, H.; Liu, Y.; Li, Y.; Wu, C.-H.; Chen, B.; Kraemer, M.U.G.; Li, B.; Cai, J.; Xu, B.; Yang, Q.; et al. An investigation of transmission control measures during the first 50 days of the COVID-19 epidemic in China. Science 2020, 368, 638-642. [CrossRef] [PubMed]

3. World Health Organization. Management of Ill Travellers at Points of Entry-International Airports, Seaports and Ground Crossings_-In the Context of COVID-19; WHO: Geneva, Switzerland, 2020.

4. Campisi, T.; Canale, A.; Tesoriere, G. The development of walkability in the historic centre of Enna: The case of the Saint Tommaso neighbourhood. Eur. Transp. Trasp. Eur. 2019, 73, e4.

5. Campisi, T.; Tibljaš, A.D.; Tesoriere, G.; Canale, A.; Rencelj, M.; Šurdonja, S. Cycling traffic at turbo roundabouts: Some considerations related to cyclist mobility and safety. Transp. Res. Procedia 2020, 45, 627-634. [CrossRef]

6. Campisi, T.; Acampa, G.; Marino, G.; Tesoriere, G. Cycling master plans in Italy: The I-BIM feasibility tool for cost and safety assessments. Sustainability 2020, 12, 4723. [CrossRef]

7. Campisi, T.; Akgün, N.; Ticali, D.; Tesoriere, G. Exploring public opinion on personal mobility vehicle use: A Case study in Palermo, Italy. Sustainability 2020, 12, 5460. [CrossRef]

8. Qi, X. How Next-Generation Information Technologies Tackled COVID-19 in China. Available online: https://www.weforum.org/agenda/2020/04/how-next-generation-information-technologies-tackled-covid19-in-china/ (accessed on 2 July 2020).

9. Canale, A.; Tesoriere, G.; Campisi, T. The MAAS development as a mobility solution based on the individual needs of transport users. In AIP Conference Proceedings; AIP Publishing LLC.: Melville, NY, USA, 2019; Volume 2186, p. 16005.

10. Blečić, I.; Cecchini, A. Verso una Pianificazione Antifragile. Come Pensare al Futuro Senza Prevederlo; FrancoAngeli: Milano, Italy, 2016; ISBN 978-88-917-2775-6.

11. Renaud, L. Reconsidering global mobility-Distancing from mass cruise tourism in the aftermath of COVID-19. Tour. Geogr. 2020, 22, 679-689. [CrossRef]

12. Kanda, W.; Kivimaa, P. What opportunities could the COVID-19 outbreak offer for sustainability transitions research on electricity and mobility? Energy Res. Soc. Sci. 2020, 68, 101666. [CrossRef]

13. D'Adamo, I.; Rosa, P. How do you see infrastructure? Green energy to provide economic growth after COVID-19. Sustainability 2020, 12, 4738. [CrossRef]

14. Nahiduzzaman, K.M.; Lai, S.K. Editorial: What Does the Global Pandemic COVID-19 Teach Us? Some Reflections. J. Urban Manag. 2020, 9, 3.

15. Birkin, M. Spatial data analytics of mobility with consumer data. J. Transp. Geogr. 2018, 76, $245-253$. [CrossRef]

16. Szmelter, A. Car-related mobility patterns of Polish Y generation-Implications for future urban transport. Transp. Res. Procedia 2019, 39, 514-524. [CrossRef]

17. Suchanek, M.; Szmelter-Jarosz, A. Environmental aspects of generation Y's sustainable mobility. Sustainability 2019, 11, 3204. [CrossRef]

18. Rześny-Cieplińska, J.; Szmelter-Jarosz, A. Assessment of the crowd logistics solutions-The stakeholders' analysis approach. Sustainability 2019, 11, 5361. [CrossRef]

19. Assi, K.J.; Shafiullah, M.; Nahiduzzaman, K.M.; Mansoor, U. Travel-to-school mode choice modelling employing artificial intelligence techniques: A comparative study. Sustainability 2019, 11, 4484. [CrossRef]

20. Assi, K.J.; Nahiduzzaman, K.M.; Ratrout, N.T.; Aldosary, A.S. Mode choice behavior of high school goers: Evaluating logistic regression and MLP neural networks. Case Stud. Transp. Policy 2018, 6, 225-230. [CrossRef]

21. Kumar, C.; Ganguly, A. Travelling together but differently: Comparing variations in public transit user mode choice attributes across New Delhi and New York. Theor. Empir. Res. Urban Manag. 2018, 13, 54-73.

22. Spickermann, A.; Grienitz, V.; Von Der Gracht, H.A.; Abbas, R.; Michael, K.; Michael, M.; Redman, L.; Friman, M.; Gärling, T.; Hartig, T. Quality attributes of public transport that attract car users: A research review. Inf. Technol. People 2014, 89, 2-20.

23. Ma, X.; Liu, C.; Wen, H.; Wang, Y.; Wu, Y.J. Understanding commuting patterns using transit smart card data. J. Transp. Geogr. 2017, 58, 135-145. [CrossRef] 
24. Macharis, C.; De Witte, A.; Ampe, J. The multi-actor, multi-criteria analysis methodology (MAMCA) for the evaluation of transport projects: Theory and practice. J. Adv. Transp. 2009, 43, 183-202. [CrossRef]

25. Mozos-Blanco, M.Á.; Pozo-Menéndez, E.; Arce-Ruiz, R.; Baucells-Aletà, N. The way to sustainable mobility. A comparative analysis of sustainable mobility plans in Spain. Transp. Policy 2018, 72, 45-54. [CrossRef]

26. Klinger, T. Moving from monomodality to multimodality? Changes in mode choice of new residents. Transp. Res. Part A Policy Pract. 2017, 104, 221-237. [CrossRef]

27. Grischkat, S.; Hunecke, M.; Böhler, S.; Haustein, S. Potential for the reduction of greenhouse gas emissions through the use of mobility services. Transp. Policy 2014, 35, 295-303. [CrossRef]

28. Diez, J.M.; Lopez-Lambas, M.E.; Gonzalo, H.; Rojo, M.; Garcia-Martinez, A. Methodology for assessing the cost effectiveness of Sustainable Urban Mobility Plans (SUMPs). The case of the city of Burgos. J. Transp. Geogr. 2018, 68, 22-30. [CrossRef]

29. Russo, F.; Rindone, C.; Panuccio, P.; May, A.D.; Russo, F.; Rindone, C.; Panuccio, P.; Bínová, H.; Endrizalová, E.; Heralová, D.; et al. European plans for the smart city: From theories and rules to logistics test case. Eur. Plan. Stud. 2016, 24, 1709-1726. [CrossRef]

30. Kamruzzaman, M.; Hine, J.; Gunay, B.; Blair, N. Using GIS to visualise and evaluate student travel behaviour. J. Transp. Geogr. 2011, 19, 13-32. [CrossRef]

31. Jabeen, F.; Olaru, D.; Smith, B. Combining samples to offset nonresponse and respondent biases. Case Stud. Transp. Policy 2018, 6, 190-199. [CrossRef]

32. Szmelter-Jarosz, A. Urban mobility of young adults-An example of Poland. Przedsiębiorczość $i$ Zarzadzanie 2020, 7, 271-284.

33. Arsenio, E.; Martens, K.; Di Ciommo, F. Sustainable urban mobility plans: Bridging climate change and equity targets? Res. Transp. Econ. 2016, 55, 30-39. [CrossRef]

34. Bos, R.; Temme, R. A roadmap towards sustainable mobility in Breda. Transp. Res. Procedia 2014, 4, 103-115. [CrossRef]

35. Tilley, S.; Houston, D. The gender turnaround: Young women now travelling more than young men. J. Transp. Geogr. 2016, 54, 349-358. [CrossRef]

36. Navarro-Ligero, M.L.; Valenzuela-Montes, L.M. A tool for the assessment of urban mobility scenarios in climate change mitigation: An Application to the granada's LRT project. Transp. Res. Procedia 2016, 19, 364-379. [CrossRef]

37. Groenendijk, L.; Rezaei, J.; Correia, G. Incorporating the travellers' experience value in assessing the quality of transit nodes: A Rotterdam case study. Case Stud. Transp. Policy 2018, 6, 564-576. [CrossRef]

38. Hashemkhani Zolfani, S.; Ecer, F.; Pamučar, D.; Raslanas, S. Neighborhood selection for a newcomer via a novel BWM-based revised mairca integrated model: A case from the coquimbo-la serena conurbation, Chile. Int. J. Strateg. Prop. Manag. 2020, 24, 102-118. [CrossRef]

39. Keshavarz Ghorabaee, M.; Amiri, M.; Zavadskas, E.K.; Turskis, Z.; Antucheviciene, J. A new hybrid simulation-based assignment approach for evaluating airlines with multiple service quality criteria. J. Air Transp. Manag. 2017, 63, 45-60. [CrossRef]

40. Mardani, A.; Jusoh, A.; Zavadskas, E.K. Fuzzy multiple criteria decision-making techniques and applications - Two decades review from 1994 to 2014. Expert Syst. Appl. 2015, 42, 4126-4148. [CrossRef]

41. Ghodmare, S.D.; Khode, B.V.; Bajaj, P. Application of the multi attribute utility technique with its for sustainability evaluation of emerging metropolitan city of Nagpur. Int. J. Civ. Eng. Technol. 2019, 10, 942-950.

42. Reveshty, M.A.; Vafaii, F. The Ranking of urban inner travel producing regions using Multi- criteria decision models ( A case study: Sanandaj city urban regions ). Urban Reg. Stud. Res. J. 2015, 6, 9-12.

43. Damidavičius, J.; Burinskienè, M.; Vitkūnienė, R.U. A monitoring system for Sustainable Urban Mobility Plans. Balt. J. Road Bridg. Eng. 2019, 12, 158-177. [CrossRef]

44. Kazan, H.; Çiftci, C. Transport path selection: Multi-criteria comparison. Int. J. Oper. Logist. Manag. 2013, 2, 33-48.

45. Bhandari, S.B.; Nalmpantis, D. Application of various multiple criteria analysis methods for the evaluation of rural road projects. Open Transp. J. 2018, 12, 57-76. [CrossRef]

46. Macharis, C.; Turcksin, L.; Lebeau, K. Multi actor multi criteria analysis (MAMCA) as a tool to support sustainable decisions: State of use. Decis. Support Syst. 2012, 54, 610-620. [CrossRef] 
47. Turcksin, L.; Bernardini, A.; Macharis, C. A combined AHP-PROMETHEE approach for selecting the most appropriate policy scenario to stimulate a clean vehicle fleet. Procedia Soc. Behav. Sci. 2011, 20, 954-965. [CrossRef]

48. Gagatsi, E.; Morfoulaki, M. MultiActors Multi-Criteria Analysis for supporting policy making in the Greek Coastal Transport System. In Proceedings of the 10th Multiicriteria Decision Analysis Metting-13th Special Conference of the HELOPS, Thessaloniki, Greece, 20-23 November 2013; pp. 1-7.

49. Sun, C.; Cheng, L.; Zhu, S.; Han, F.; Chu, Z. Multi-criteria user equilibrium model considering travel time, travel time reliability and distance. Transp. Res. Part D Transp. Environ. 2019, 66, 3-12. [CrossRef]

50. Liu, W. Determining the Importance of Factors for Transport Modes in Freight Transportation. Master's Thesis, Delft University of Technology, Delft, The Netherlands, 2016.

51. Duleba, S.; Moslem, S. Sustainable urban transport development with stakeholder participation, an AHP-Kendall model: A case study for Mersin. Sustainability 2018, 10, 3647. [CrossRef]

52. Ghorbanzadeh, O.; Moslem, S.; Blaschke, T.; Duleba, S. Sustainable urban transport planning considering different stakeholder groups by an interval-AHP decision support model. Sustainability 2018, 11,9. [CrossRef]

53. Rezaei, J. Best-worst multi-criteria decision-making method. Omega 2015, 53, 49-57. [CrossRef]

54. Alkharabsheh, A.; Moslem, S.; Duleba, S. Evaluating passenger demand for development of the urban transport system by an AHP model with the real-world application of Amman. Appl. Sci. 2019, 9, 4759. [CrossRef]

55. Duleba, S.; Moslem, S. Examining Pareto optimality in analytic hierarchy process on real Data: An application in public transport service development. Expert Syst. Appl. 2019, 116, 21-30. [CrossRef]

56. Moslem, S.; Ghorbanzadeh, O.; Blaschke, T.; Duleba, S. Analysing stakeholder consensus for a sustainable transport development decision by the fuzzy AHP and interval AHP. Sustainability 2019, 11, 3271. [CrossRef]

57. Goumi, B.E.L.; El Khomssi, M.; Chaibi, G. Comparative analysis multiple criteria for the choice of a common transport system in Rabat (Morocco). EuroEconomica 2015, 2, 1-14.

58. Zolfani, S.H.; Antucheviciene, J. Team member selecting based on AHP and TOPSIS grey. Eng. Econ. 2012, 23, 425-434. [CrossRef]

59. Gupta, H.; Barua, M.K. A framework to overcome barriers to green innovation in SMEs using BWM and Fuzzy TOPSIS. Sci. Total Environ. 2018, 633, 122-139. [CrossRef] [PubMed]

60. Salimi, N.; Rezaei, J. Evaluating firms' R \& D performance using best worst method. Eval. Program Plan. 2018, 66, 147-155.

61. Salimi, N.; Rezaei, J. Measuring efficiency of university-industry Ph.D. projects using best worst method. Scientometrics 2016, 109, 1911-1938. [CrossRef]

62. Auger, P.; Devinney, T.M.; Louviere, J.J. Using best-worst scaling methodology to investigate consumer ethical beliefs across countries. J. Bus. Ethics 2007, 70, 299-326. [CrossRef]

63. Chitsaz, N.; Azarnivand, A. Water Scarcity Management in Arid Regions Based on an Extended Multiple Criteria Technique. Water Resour. Manag. 2017, 31, 233-250. [CrossRef]

64. Ren, J.; Liang, H.; Chan, F.T.S. Urban sewage sludge, sustainability, and transition for Eco-City: Multi-criteria sustainability assessment of technologies based on best-worst method. Technol. Forecast. Soc. Chang. 2017, 116, 29-39. [CrossRef]

65. Rezaei, J.; Nispeling, T.; Sarkis, J.; Tavasszy, L. A supplier selection life cycle approach integrating traditional and environmental criteria using the best worst method. J. Clean. Prod. 2016, 135, 577-588. [CrossRef]

66. Mi, X.; Tang, M.; Liao, H.; Shen, W.; Lev, B. The state-of-the-art survey on integrations and applications of the best worst method in decision making: Why, what, what for and what's next? Omega 2019, 87, 205-225. [CrossRef]

67. Rezaei, J.; Wang, J.; Tavasszy, L. Linking supplier development to supplier segmentation using Best Worst Method. Expert Syst. Appl. 2015, 42, 9152-9164. [CrossRef]

68. Marjanović, S.; Radivojev, D. Application Method for Making Decision in Combined Transport: The Processing of the Case Studies. Horizons 2016, 53, 341-348.

69. Safarzadeh, S.; Khansefid, S.; Rasti-Barzoki, M. A group multi-criteria decision-making based on best-worst method. Comput. Ind. Eng. 2018, 126, 111-121. [CrossRef]

70. Killi, M.; Nossum, A.; Veisten, K. Lexicographic answering in travel choice: Insufficient scale extensions and steep indifference curves? Eur. J. Transp. Infrastruct. Res. 2007, 7, 39-62. 
71. Scarpa, R.; Notaro, S.; Louviere, J.; Raffaelli, R. Exploring scale effects of best/worst rank ordered choice data to estimate benefits of tourism in alpine grazing commons. Am. J. Agric. Econ. 2011, 93, 809-824. [CrossRef]

72. Xiao, H.; Cohen Eilon, Z.; Ji, C.; Tanimoto, T. COVID-19 societal response captured by seismic noise in China and Italy. Seism. Res. Lett. 2020. [CrossRef]

73. Aktay, A.; Bavadekar, S.; Cossoul, G.; Davis, J.; Desfontaines, D.; Fabrikant, A.; Gabrilovich, E.; Gadepalli, K.; Gipson, B.; Guevara, M.; et al. Google COVID-19 Community Mobility Reports: Anonymization Process Description (version 1.0). 2020. Available online: https://arxiv.org/abs/2004.04145 (accessed on 2 July 2020).

74. Luther, W.J. Behavioral and Policy Responses to COVID-19: Evidence from Google Mobility Data on State-Level Stay-at-Home Orders. SSRN Electron. J. 2020. [CrossRef]

75. Yilmazkuday, H. International Evidence from Google Mobility Data. SSRN Electron. J. 2020. [CrossRef]

76. Ganem, F.; Macedo Mendes, F.; de Oliveira, S.B.; Porto, V.B.G.; de Araújo, W.N.; Nakaya, H.I.; Diaz-Quijano, F.A.; Croda, J. The Impact of Early Social Distancing at COVID-19 Outbreak in the Largest Metropolitan Area of Brazil; MedRxiv, The Cold Spring Harbor Laboratory: Cold Spring Harbor, NY, USA, 2020.

77. Kaplan, E.H. Containing 2019-nCoV (Wuhan) coronavirus. Health Care Manag. Sci. 2020, 23, 311-314. [CrossRef]

78. Engle, S.; Stromme, J.; Zhou, A. Staying at Home: Mobility Effects of COVID-19. SSRN Electron. J. 2020. [CrossRef]

79. Biscayart, C.; Angeleri, P.; Lloveras, S.; Chaves, T.d.S.S.; Schlagenhauf, P.; Rodríguez-Morales, A.J. The next big threat to global health? 2019 novel coronavirus (2019-nCoV): What advice can we give to travellers?-Interim recommendations January 2020, from the Latin-American society for Travel Medicine (SLAMVI). Travel Med. Infect. Dis. 2020, 33, 17-20. [CrossRef] [PubMed]

80. Rubin, O.; Nikolaeva, A.; Nello-Deakin, S.; Te Brömmelstroet, M. What can we Learn from the COVID-19 Pandemic about how People Experience Working from Home and Commuting? 1; Centre for Urban Studies, University of Amsterdam: Amsterdam, The Netherlands, 2020.

81. Venter, Z.S.; Barton, D.N.; Gundersen, V.; Figari, H.; Nowell, M. Urban Nature in a Time of Crisis: Recreational Use of Green Space Increases during the COVID-19 Outbreak in Oslo, Norway; SocArXiv, The University of Maryland: College Park, MD, USA, 2020.

82. Morita, H.; Nakamura, S.; Hayashi, Y. Changes of urban activities and behaviors due to COVID-19 in Japan. SSRN Electron. J. 2020. [CrossRef]

83. Chan, J. Using Google Data to Understand Canadian Movement Reductions During the COVID-19 Pandemic. SSRN Electron. J. 2020. [CrossRef]

84. Kraemer, M.U.G.; Yang, C.H.; Gutierrez, B.; Wu, C.H.; Klein, B.; Pigott, D.M.; du Plessis, L.; Faria, N.R.; Li, R.; Hanage, W.P.; et al. The effect of human mobility and control measures on the COVID-19 epidemic in China. Science 2020, 368, 6146-6151. [CrossRef]

85. Pepe, E.; Bajardi, P.; Gauvin, L.; Privitera, F.; Lake, B.; Cattuto, C.; Tizzoni, M. COVID-19 Outbreak Response: A First Assessment of Mobility Changes in Italy following National Lockdown; MedRxiv, The Cold Spring Harbor Laboratory: Cold Spring Harbor, NY, USA, 2020.

86. de Paz, C.; Muller, M.; Munoz Boudet, A.M.; Gaddis, I. Gender Dimensions of the COVID-19 Pandemic; World Bank: Washington, DC, USA, 2020.

87. Anwar, S.; Nasrullah, M.; Hosen, M.J. COVID-19 and Bangladesh: Challenges and How to Address Them. Front. Public Health 2020, 8, 1-8. [CrossRef] [PubMed]

88. Gunthe, S.S.; Patra, S.S. Impact of international travel dynamics on domestic spread of 2019-nCoV in India: Origin-based risk assessment in importation of infected travelers. Global. Health 2020, 16, 45. [CrossRef] [PubMed]

89. Milne, G.J.; Xie, S. The Effectiveness of Social Distancing in Mitigating COVID-19 Spread: A Modelling Analysis; MedRxiv, The Cold Spring Harbor Laboratory: Cold Spring Harbor, NY, USA, 2020; pp. 1-16.

90. Bounie, D.; Camara, Y.; Galbraith, J.W. Consumers' Mobility, Expenditure and Online-Offline Substitution Response to COVID-19: Evidence from French Transaction Data. SSRN Electron. J. 2020. [CrossRef]

91. Dahlberg, M.; Edin, P.-A.; Grönqvist, E.; Lyhagen, J.; Östh, J.; Siretskiy, A.; Toger, M. Effects of the COVID-19 Pandemic on Population Mobility under Mild Policies: Causal Evidence from Sweden; arXiv, Cornell University: Ithaca, NY, USA, 2020; pp. 1-32. 
92. Gatto, M.; Bertuzzo, E.; Mari, L.; Miccoli, S.; Carraro, L.; Casagrandi, R. Spread and dynamics of the COVID-19 epidemic in Italy: Effects of emergency containment measures. Proc. Natl. Acad. Sci. USA 2020, 117, 10484-10491. [CrossRef]

93. Chan, H.F.; Skali, A.; Savage, D.; Stadelmann, D.; Torgler, B. Risk Attitudes and Human Mobility During the COVID-19 Pandemic; Center for Research in Economics, Management and the Arts (CREMA): Zurich, Switzerland, 2020; Volume 6.

94. Murgante, B.; Borruso, G.; Balletto, G.; Castiglia, P.; Dettori, M. Why Italy First ? Health, Geographical and Planning aspects of the Covid-19 outbreak. Sustainability 2020, 12, 5064. [CrossRef]

95. Pluchino, A.; Inturri, G.; Rapisarda, A.; Biondo, A.E.; Le Moli, R.; Zappala', C.; Giuffrida, N.; Russo, G.; Latora, V. A Novel Methodology for Epidemic Risk Assessment: The case of COVID-19 outbreak in Italy; arXiv, Cornell University: Ithaca, NY, USA, 2020; pp. 1-37.

96. Zhou, C.; Su, F.; Pei, T.; Zhang, A.; Du, Y.; Luo, B.; Cao, Z.; Wang, J.; Yuan, W.; Zhu, Y.; et al. COVID-19: Challenges to GIS with Big Data. Geogr. Sustainability 2020, 1, 77-87. [CrossRef]

97. Młyńczak, J. Analysis of Intelligent Transport Systems (ITS) in public transport of upper Silesia. In Modern Transport Telematics; Springer: Berlin/Heidelberg, Germany, 2011.

98. Aloi, A.; Alonso, B.; Benavente, J.; Cordera, R.; Echániz, E.; González, F.; Ladisa, C.; Lezama-Romanelli, R.; López-Parra, Á.; Mazzei, V.; et al. Effects of the COVID-19 Lockdown on Urban Mobility: Empirical Evidence from the City of Santander (Spain). Sustainability 2020, 12, 3870. [CrossRef]

99. Czwajda, L.; Kosacka-Olejnik, M.; Kudelska, I.; Kostrzewski, M.; Sethanan, K.; Pitakaso, R. Application of prediction markets phenomenon as decision support instrument in vehicle recycling sector. Logforum 2019, 15, 265-278. [CrossRef]

100. Snowberg, E.; Wolfers, J.; Zitzewitz, E. Prediction markets for economic forecasting. In Handbook of Economic Forecasting; Elsevier: Amsterdam, The Netherlands, 2013.

(C) 2020 by the authors. Licensee MDPI, Basel, Switzerland. This article is an open access article distributed under the terms and conditions of the Creative Commons Attribution (CC BY) license (http://creativecommons.org/licenses/by/4.0/). 



\title{
Resilience, Leadership and Female Entrepreneurship within the Context of SMEs: Evidence from Latin America
}

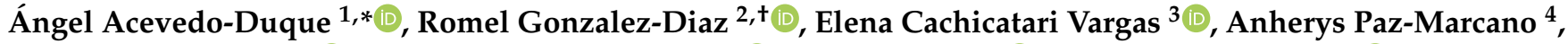

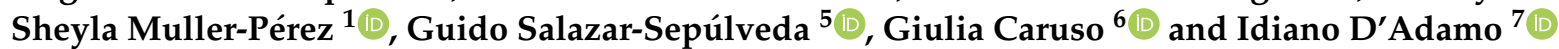

check for

updates

Citation: Acevedo-Duque, Á.; Gonzalez-Diaz, R.; Vargas, E.C.; Paz-Marcano, A.; Muller-Pérez, S.; Salazar-Sepúlveda, G.; Caruso, G.; D'Adamo, I. Resilience, Leadership and Female Entrepreneurship within the Context of SMEs: Evidence from Latin America. Sustainability 2021, 13, 8129. https://doi.org/10.3390/ su13158129

Academic Editor: Andrea Appolloni

Received: 15 June 2021

Accepted: 14 July 2021

Published: 21 July 2021

Publisher's Note: MDPI stays neutral with regard to jurisdictional claims in published maps and institutional affiliations.

Copyright: (c) 2021 by the authors. Licensee MDPI, Basel, Switzerland. This article is an open access article distributed under the terms and conditions of the Creative Commons Attribution (CC BY) license (https:// creativecommons.org/licenses/by/ $4.0 /)$.
1 Public Policy Observatory, Faculty of Business and Administration, Universidad Autónoma de Chile, Santiago 7500912, Chile; sheyla.muller@uautonoma.cl

2 Centro Internacional de Investigación y Desarrollo (CIID), Montería 230001, Colombia; director@ciid.com.co

3 Faculty of Health Sciences, Universidad Nacional Jorge Basadre Grohmann, Tacna 23000, Peru; ecachicatariv@unjbg.edu.pe

4 Programa de Administración de Empresas, Facultad de Ciencias Económicas y Administrativas, Universidad de La Guajira, Riohacha 440001, Colombia; aipaz@uniguajira.edu.co

5 Departamento de Ingeniería Industrial, Facultad de Ingeniería, Universidad Católica de la Santísima Concepción, Concepción 4090541, Chile; gsalazar@ucsc.cl

6 Department of Neuroscience, Imaging and Clinical Sciences, “G. d'Annunzio" University of Chieti-Pescara, 66100 Chieti, Italy; giulia.caruso@unich.it

7 Department of Computer, Control and Management Engineering, Sapienza University of Rome, Via Ariosto 25, 00185 Rome, Italy; idiano.dadamo@uniroma1.it

* Correspondence: angel.acevedo@uautonoma.cl

$+\quad$ This work is dedicated to the memory of a young and promising researcher Romel Gonzalez-Diaz. Leave it to us to promote sustainability in all parts of the world. We are grateful for your insights.

\begin{abstract}
The purpose of this article is to analyze resilient female leadership as a sustainable promoter of business excellence in small and medium-sized Wayuu handicraft marketing enterprises. The present study uses a quantitative methodology with a non-experimental cross-sectional field design, with an analysis and interpretation of the data provided by the surveyed subjects. A 33-item questionnaire with multiple response options is applied. The population consists of 110.012 eradicated women. A probabilistic sampling technique is applied with a margin of error of $5 \%$ and a confidence level of $95 \%$, for a total of 383 Wayuu women entrepreneurs in the Department of La Guajira, Colombia. Our findings explain that female leadership transcends the boundaries of business management, being present in both small and medium enterprises (SMEs). This study confirms the positive relationship between sustainability and resilience in the Wayuu handicrafts market, being women who turn their actions into success factors by working with women who show technical, conceptual, and human skills.
\end{abstract}

Keywords: women's leadership; America Latina; small and medium-sized enterprises; resilience; sustainability

\section{Introduction}

Ending all forms of discrimination against women and girls is a fundamental duty to preserve human rights and IT is also crucial for sustainable development [1]. According to the United Nations Development Programme (UNDP), gender equality is a central issue. Moreover, we have witnessed remarkable progress in the last 20 years. Female schooling has increased, compared to 15 years ago, and most regions have reached gender parity in primary education. However, although there are more women than ever before in the labor market, in some regions significant inequalities still persist and women's labor rights are still not the same as those of their male counterparts [2]. Violence and sexual exploitation, unequal sharing of unpaid work-both domestic and care work-and discrimination in decision-making remain major obstacles. 
Climate change and disasters continue to have a disproportionate impact on women and children, including conflict and migration [3]. Ensuring universal access to reproductive and sexual health and granting equal rights to women in accessing economic resources, such as land and property, are critical in order to achieve this goal [4]. Today, more women than ever are holding public office. However, encouraging more women to become leaders will help to achieve greater gender equality.

To achieve the intended objectives in organizations, it is necessary to consider some elements that are capable of producing business excellence, and which translate themselves into the coordination of various factors, such as human, financial, material, economic, and technological factors [5]. These factors promote the organization of work activities that are able to create value through the creation of teamworks, which allow one to unify efforts and apply strategies to achieve common objectives. In addition, they are able to facilitate the articulation of business functions and to strengthen the company's presence in the market, creating competitive advantages. Sustainability can create a competitive advantage when the human component plays a key role [6].

Organizations are subject to periods of transition due to external environment dynamics [7]. Consequently, they can adapt and take into consideration those changes and trends that are necessary to be competitive in the environment in which they exchange their goods and services. The management of entrepreneurial activities is inescapable [8-10], and it requires the coordination of individual efforts. These ones converge in shared commitments, i.e., company-collaborators and vice versa, which guide actions in order to achieve the desired objectives. However, the way to get the expected results remains a challenge in business management and in business organization. It finally results in corporate social responsibility (CSR) because of its impact on the society in which it allocates its goods and services [8].

The new organizations are the ones who should coordinate their missionary activities in various units and the essential functions of the organizational structure, minimizing the risk impact [11]. The global market requires changes in the managerial paradigm, in order to obtain new managerial approaches and to promote business cohesion, through the participation of all employees. For some authors, new managerial behavior in organizations must be different from the traditional behavior, more based on a transformational style, and it is necessary to move from hierarchical and rigid organizations to more flexible horizontal ones, which have a greater capacity to adapt to changes occurring in the working world [12].

Thus, business management has to look for qualified collaborators whose competencies are suitable for managerial positions, contributing to image, reputation, and profitability, in order to position companies in those markets where they are offering their products, goods, and services $[13,14]$. Managerial leadership contributes to the excellence of its missionary processes, leaving behind the old paradigms of exclusivity of organizational management, which are usually occupied by men. Thus, emerging female characteristics are dominance, strength, robustness, intelligence, perseverance, and responsibility. These characteristics create a sense of equality in business management [15].

However, in world business scenarios, this trend and change of paradigm of business management has led to some debates about which are the gender skills required to occupy management positions in organizations. It is essential to know the opinions of the specialists on this subject. They underline the importance of knowing which are the essential characteristics, skills, abilities, and competencies of a manager. They should take into account the qualities that inspire credibility, trust, and reputation in the exercise of their functions, and their projection and acceptance in the environment, both internally and externally of the market where they participate $[2,16]$.

According to some authors [17,18], the leader figure is crucial in any field, since his/her actions are an essential factor in guiding collaborators to achieve common objectives. These innovative trends of maintaining sustainability and stability in the market [19] also involve small and medium enterprises, mainly in Colombia. 
Therefore, we considered marketers or micro-entrepreneurs of Wayuu handicrafts in the unique tourist and cultural district of Riohacha, in Colombia. The focus is on those sectors where women's leadership enabled excellent performance from an economic perspective [20,21]. In particular, these works highlighted how women's rights, as well as important professional and managerial insight, made it possible to change for the better a working environment excessively characterized by male stereotypes. However, there are situations in which it is not difficult to avoid such kinds of characterizations that label the company's management, especially with regards to the current demands of adaptation in the world [20].

Since our objective is to understand how different markets are moving towards a sustainable revolution, the three hypotheses of this research, which will be answered through the literature and our results, are:

- Hypothesis 1 (H1). Leadership characteristics influence female leadership in those SMEs that are dedicated to the organization of Wayuu handicrafts.

- Hypothesis 2 (H2). Leadership styles are related to female leadership in those SMEs that are dedicated to Wayuu handicrafts.

- Hypothesis 3 (H3). Leadership skills influence the development of female leadership in Wayuu handicraft marketing SMEs.

We investigated how different stakeholder perspectives need to be combined [22] The focus is on how a company can create competitive advantage and on how human and organizational factors are able to influence this epochal change. In this context, the synergy between the concepts of sustainability and resilience represents an element of analysis to be investigated since it can harmonize the different factors that determine the success of a company in a market [23]. The literature shows a positive relationship between sustainability and resilience in the fashion industry [7]. Given the heterogeneity and diversity of Latin America as a geographical area [24,25], the interest of this research is to analyze resilient female leadership as a sustainable factor that promotes business excellence in small and medium-sized Wayuu handicraft co-marketing enterprises. We propose three study hypotheses, and we underline that the conclusions obtained in this paper can be applied to other regions and continents of the planet, based on grounded theory. The results are shown and discussed in the following sections.

\section{Background}

Hypothesis 1 (H1). Leadership characteristics influence female leadership in those SMEs dedicated to the organization of Wayuu handicrafts.

\subsection{Resilient Female Leadership and Its Distinctive Approach to Manage Small and Medium-Sized Wayuu Handicraft Enterprises}

Worldwide, the women inclusion in management positions has an important significance. The focus is on promoting sustainable leadership and human capital with competencies that can boost sustainable and resilient development in organizations [5]. However, there are still gaps in Latin America, specifically in Colombia, where women, despite being resilient and covering some senior management positions, still have little presence in the system. Some studies claim that when women cover management positions it is possible to increase the diversity of perspectives and opinions in companies [20,22]. According to the official World Bank data, in Colombia in the last 10 years the women labor participation rate ( $\%$ of the female population between $15-64$ years old) has been equal to $43.39 \%$, with only the $20 \%$ covering top management positions (see Figure 1 ). The estimate has been modeled by the ILO. 


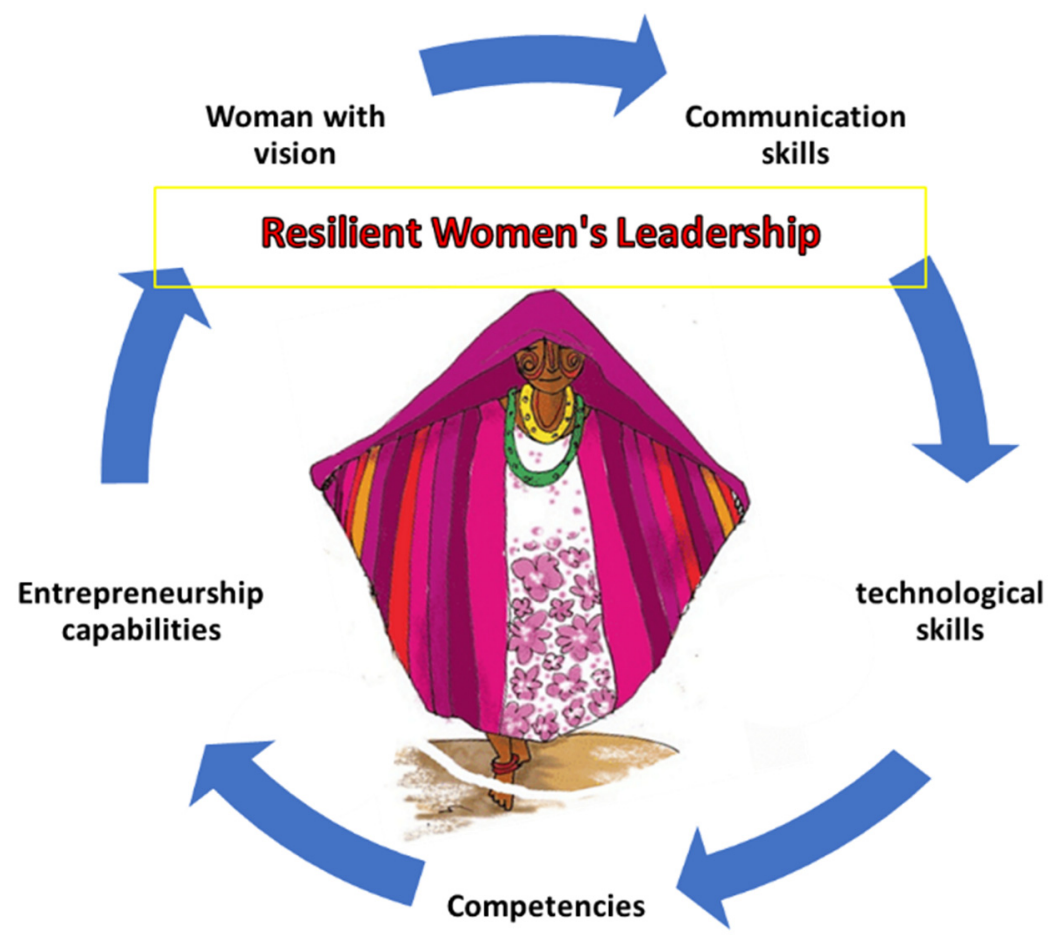

Figure 1. Resilient Women's Leadership. Source: own elaboration.

In those family businesses that are characterized by the Wayuu indigenous culture, women have been playing an invisible role for many years, as can be seen in the literature [26]. It has been shown that very few women reach high positions in the management bodies of these types of companies. However, the number of women in top management and board positions has been slowly increasing over the last decade [27]. Female leadership is a trending topic in business management and in those ventures that empower the sustainable education and culture. They became a focus of attention in the management of work processes, with the aim to promote shared performance and motivate collaborators in the development of their functions. It is possible, through open dialogue, effective communication, and the exposure of ideas in particular situations, to achieve teamwork of excellence and quality [28].

However, there is still a cultural barrier that leads one to encounter different inconveniences, which, in many cases, can become real problems, generating conflicts and wasting time, especially when it comes to breaking sociocultural patterns in which the male figure prevails as an icon of business management.

Leadership in Wayuu societies faces different situations associated with gender, which are based on a culture represented by ancestral teaching [29]. The person, as a sociable entity, desires impartiality and objectivity in acting, for him/herself, but also to relate positively with others, while, in reality, often there are attitudes of rivalry and low human sense in interactions with others.

From these perspectives, it seems that leadership implies intuition as an internal dynamic, which is useful to consolidate the relationship of people with themselves and with others. Also crucial is the ability to behave and interact in society [30], depending on gender typology. Leadership, in fact, has caused a division of labor, a separation of spaces, which has led to changes in certain expectations concerning characteristics, styles, skills, abilities, skills, resilience, and aptitudes in terms of management related to a certain gender (the female one) [31,32]. Female performance has been stereotyped by traditional roots that, until today, have been difficult to eradicate in daily practices and in the world vision. The current reality demands equality, commitment, and excellence at work where each human being can grow professionally without discrimination. 
It is worth mentioning that several authors agree on the praxis of resilient leadership as a business trend and as a critical factor to achieve success [33-35] and to obtain spaces in the international economy. This perspective considers resilient female leadership as a way to change the business paradigms rooted in the figure of male gender management, thus evidencing the position of taking women out of their role as being only dedicated to domestic life, to transform the changes as women with executive visions interested in solving the problems of business and social environments. In this way, they will play a key role, assuming from their ideas consonant answers, sustainable to the active reality of the environment, creating strategies to find solutions to elusive issues, so that their role gives way to the fulfillment of various functions in today's society (see Figure 1), being co-participants in the transformation of an egalitarian society.

In this framework, we highlight the evolution of women in society, from their work in business management, to being resilient, to having new approaches in leadership style $[8,36]$; women using this resilient approach assume various ways of managing to achieve their intended objectives and insert their values as people in a dynamic and proactive way, to ensure business competitiveness and increase the commitment and loyalty of their people [37]. In addition, we consider some authors who inserted the image of resilient female leadership in the business context, to break the limiting paradigm of the glass ceiling $[33,38]$. Women entrepreneurs are seen with leadership characteristics, styles, and skills that empower them on the road to success and in their lives. Thus, it has been possible to verify, from the research results, the role of women to exercise leadership in the management of companies.

Hypothesis 2 (H2). Leadership styles are related to female leadership in SMEs marketing Wayúu handicrafts.

\subsection{Characteristic Styles of Female Leadership in the Management of Small and Medium-Sized} Enterprises That Market Wayuu Handicrafts

Female leadership is characterized by initiative, by effectiveness in building work teams, and by the ability to make decisions in times of crisis. Furthermore, it acts with resilience, it is characterized by self-development and orientation to results, and it shows high integrity and honesty. It develops, inspires, and motivates others; it builds leadership and relationships; it sets ambitious goals, collaboration, and teamwork; it connects with the outside world; it communicates powerfully and prolifically; and it analyzes and solves problems. Moreover, it is characterized by speed of leadership and innovation [36]. Therefore, each company should have the priority to develop, train, and give the opportunity to a woman to lead employees. Thus, it is necessary to demonstrate that female leadership, in an everyday sense, is characterized by more than a domestic life and by more than a maternal role. On many occasions, women are seen as responsible human beings [39], committed to their domestic functions, transforming this vision with a managerial training as a manager, who plays a role of responsibility through various personal characteristics, such as organizing herself, the ability to plan family activities, and facing to adversity [40].

Women have the ability to plan and are able to preserve their balance in difficult situations, among other things. In addition, they have strong leadership skills, such as the ability to negotiate, supervise, teach, guide, direct, establish conflict control mechanisms, and show an objective and impartial stance, which makes them authentic human beings in society.

Some authors highlight the following leadership styles that influence the optimal development of organizational processes: coercive, participative, helmsman, coaching, visionary [41,42]. According to the criteria of another work [43], feminine characteristics at work can be related to leadership through the attitude of inclusion, and confidence in one's own charisma, capacity, and interpersonal skills to influence others. On the other hand, other authors define different attributes such as dexterity, capacity, expertise, art, mastery, techno-science, poise, and experience; the person with such attributes is a very committed guide, as they tend to increase the ability to handle situations or interact with people [44]. 
Hypothesis 3 (H3). Leadership skills influence the development of female leadership in Wayúu handicraft marketing SMEs.

2.3. Skills Developed by Women Entrepreneurs in the Management of Small and Medium-Sized Wayuu Handicraft Trading Companies

Despite social and business issues, women in their leading role and their working function apply their following skills, both conceptual, technical, and human [45], in order to contribute to an excellent performance in their working places:

- Conceptual ability: The leader is able to see the organization as a whole, in which the parts complement each other; in this case, it regards the relationship of the company with another one. According to authors' criteria, conceptual ability is the mental capacity to coordinate diverse interests and activities. It means having the capacity of abstract thinking, of analyzing information, and of establishing connections between data [43,46]; therefore, the manager must achieve critical thinking and conceptualize things with respect to how they could be. The authors highlight how the practice of the analyzed companies shows the relational abilities and the capacity of creating alliances, in order to work with strategies favoring the common good.

- Technical competencies: Technical competencies are the set of knowledge, experiences, and skills that are necessary to adequately fulfill the requirements of their positions [43,47]. The skills represented by technical competencies, which are associated with tools, procedures, and techniques specific to their situation of specialization, are necessary to master their work. In the analyzed companies, the technical competencies of female leadership derive from their practical experience and from the setting of strategies to carry out the work plan.

- Human competencies: Human skills that the leader has to master to perceive the strengths of the human talents in the organization. Some authors underline that human relations skills focus on the aptitude to work together, to understand, and to motivate people in the workplace [43,48]. The importance of assertive and effective communication is emphasized, through a vision shared with collaborators, by listening to what they have to say and by guiding, facilitating, and supporting people in the workplace.

\subsection{Leadership Styles in the Entrepreneurship of Women Marketers of Wayuu Handicrafts}

Industry and associated technological change result in modifications that are difficult to reach. They not only have an impact on the organization of a company but also on the people within it [49]. The dynamics of the business environment require the development of collective learning processes able to deploy actions aimed at achieving efficient leadership management in SMEs. They go beyond the search for adapting or managing leadership styles. Enterprises that identify with this behavior are the so-called "learning organizations", and they are recognized as entities that deliberately adopt structures and strategies to stimulate a helping and learning approach in their collaborators and that are continuously promoting actions aimed at improving their organizational learning capacity [4].

The end of the post-managerial era has given rise to leadership, the purpose of which, according to [50], relates to the "implementation of deliberate change" and is developed through influencing and motivating followers. In the case of Wayuu women craft entrepreneurs, they are characterized by assuming visionary leadership styles that inspire and help their generations to continue promoting their traditions in a sustained way, promoting resilient actions both in individual, team, and organizational learning, in order that such enhanced learning increases the performance of the women of this culture for generations [51].

Thus, Wayuu women are characterized by leadership styles such as Coercive Leadership, which develops a leadership style in which the informed Wayuu woman has a certain degree of coercive influence over her followers (family, collaborators, clients, among 
others) $[52,53]$. The participative leadership style is also characterized by Wayuu women involving subordinates in decision making, listening to their suggestions, and integrating them into the set of decisions that are daily taken in an organization [54].

Helping leadership is one of the most important leadership styles in the Wayuu culture as it is constantly looking for ways to integrate people into organizational processes [49]. This leader is convinced that the only way to achieve results is with the commitment to help people with the firm's activities. In terms of coaching style, the leader acts more like a counsellor than a traditional boss. He listens to people's concerns and hopes and shares his own [55]. Finally, visionary leadership is characterized by the construction of a company vision. It develops organizational cohesion in the pursuit of it. Leaders who use this style help followers to identify their strengths and weaknesses, adjusting them to be resilient agents of change in their professional and personal aspirations.

\section{Materials and Methods}

This article is based on scientific criteria, and on a quantitative paradigm, consisting in the analysis and the interpretation of female leadership data, elaborated from their competencies in Wayuu handicraft marketing companies [17,37].

This study uses a quantitative methodology, under a non-experimental and transactional field design, and a survey is applied (a 33-item questionnaire with a Likert scale and a Cronbach's Alpha coefficient of 0.906) taking into account some characteristics, styles, and competencies of Wayuu women [49].

These results have been collected on the basis of the opinion of the involved population and of their skills in small and medium-sized companies that trade Wayuu handicrafts in La Guajira Colombia. It is a field study since the data provided by the respondents express their perception about the female leadership variable (See Table 1).

Table 1. Data interpretation. Source: Own elaboration.

\begin{tabular}{cc}
\hline Rank & Interpretation \\
\hline Min-1.80 & Variable: Female Leadership \\
$1.81-2.60$ & Deficient presence of female leadership. \\
$2.61-3.40$ & Low presence of female leadership \\
$3.41-4.20$ & The regular presence of female leadership. \\
$4.21-$ Max & High presence of female leadership. \\
& Very high presence of female leadership. \\
Min-1.80 & Dimension: Characteristics \\
$1.81-2.60$ & Very few leadership characteristics. \\
$2.61-3.40$ & Few leadership characteristics \\
$3.41-4.20$ & Some leadership characteristics \\
$4.21-$ Max & Many leadership characteristics. \\
& Too many leadership characteristics. \\
Min-1.80 & Coercive \\
$1.81-2.60$ & Participatory \\
$2.61-3.40$ & Helmsman \\
$3.41-4.20$ & Coaching \\
$4.21-$ Max & Visionary \\
Min-1.80 & Dimension: Leadership Styles \\
$1.81-2.60$ & Dimension: Leadership Skills \\
$2.61-3.40$ & Deficient leadership skills. \\
$3.41-4.20$ & Low leadership skills. \\
& Regular leadership skills. \\
& High leadership skills. \\
\hline
\end{tabular}

On the other hand, this type of research is descriptive since it shows the information extracted from observable elements in a variable, the female leadership. The small and medium companies under study have begun to apply equality approaches with regards to women, recognizing their competences without gender discrimination. Thus, from a 
legal point of view, the principle of equality has gained space and it involves important changes in women rights and in their ability to assume challenges in the effective and efficient managerial performance of business.

For the above reasons, we have chosen to measure the data on the female leadership behavior variable, considering the following dimensions: styles, skills, and characteristics. The population is composed of 110,012 eradicated women, based on data from the National Administrative Department of Statistics (DANE-2019). A probabilistic sampling technique has been applied, with a margin of error of $5 \%$ and a confidence level of $95 \%$. The sample consists of a total of 383 Wayuu women entrepreneurs in the Department of La Guajira, and it includes criteria such as being located in the Department of La Guajira, having more than 5 years of experience, being older than 25 years, holding a high school level of study, and having had a formal job. This information has been collected from August 2020 to January 2021.

The data have been analyzed through a descriptive statistical analysis using SPSS software, in order to determine the behavior of the variables under study. To test the hypotheses related to frequency distributions (described in Figure 2), a Chi-square statistic has been used.

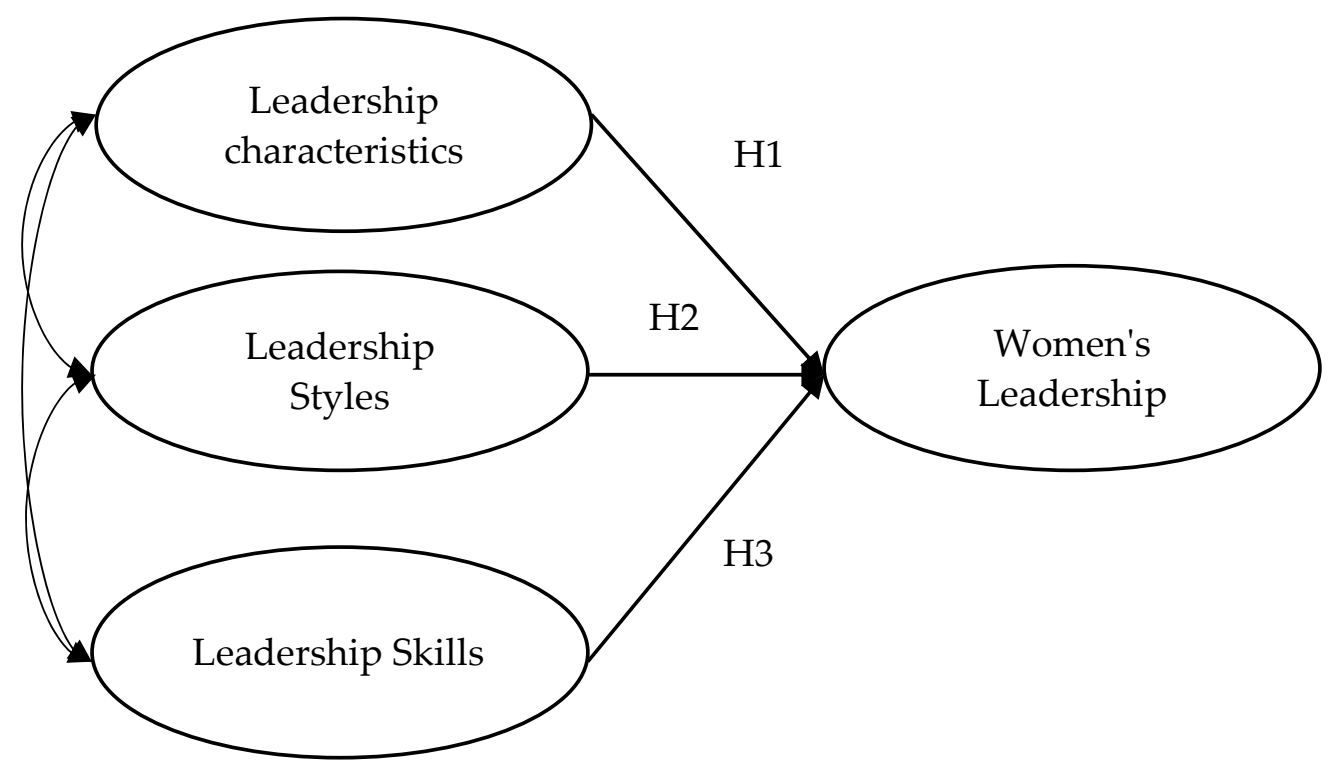

Figure 2. Research hypothesis. Source: own elaboration.

\section{Results}

The results of a research study are the product of a data analysis regarding the population under study. The interpretation of the questionnaire items has to be objective and impartial. Answers should indeed be read through a scale of interpretation of arithmetic averages (Table 2).

The female leadership variable presents a minimum of 3.48, a maximum of 4.85 , a mean of 4.179 , and a standard deviation of 0.386 . It means that for $53.3 \%$ of the 383 respondents, located in La Guajira, there is a very high presence of female leadership, while for $46.6 \%$ there is a high presence of female leadership. This implies that there are different leadership characteristics: in $39.7 \%$ of the cases, it is characterized by a coaching style based on driving processes, in $26.6 \%$ of them it is based on a participative leadership to manage SMEs, and in $23.5 \%$ of them it is characterized by Helmsman leadership. In addition, it turns out that there is a very high $(56.7 \%)$ and high $(43.3 \%)$ leadership ability. This data is high due to the answers given by the Wayuu entrepreneurs in the information exploration. 
Table 2. Descriptive statistics. Source: own elaboration.

\begin{tabular}{ccccc}
\hline & Women's Leadership & Characteristics of Leaders & Leadership Styles & Leader Skills \\
\hline & Valid & 179 & 179 & 179 \\
& Lost & 0 & 0 & 0 \\
High presence of N & Mean & 5.00 & 2.58 & 2.00 \\
female leadership & Median & 5.00 & 2 & 4.07 \\
& Mode & 5 & 0.733 & 0.538 \\
& Deviation & 0.000 & 204 & 0.068 \\
Variance & 0.000 & 0 & 204 \\
Very high presence of & Valid & 204 & 4.00 & 0 \\
female leadership. & Mean & 0 & 4.00 & 5.00 \\
& Median & 5.00 & 4 & 5.00 \\
& Mode & 5.00 & 0.620 & 5 \\
& Deviation & 5 & 0.384 & 0.000 \\
\end{tabular}

Figure 3 shows the existence of high leadership characteristics (above 4.0), in which there is a concentration of participative, coaching, and Helmsman leadership. The study discovers that there is a high presence of female leadership in the population under study, highlighting the rigorousness of the performance of resilient women, characterized by an empowered leadership in the management of Wayuu handicraft sales.

\section{Diagrama de cajas Agrupado de Caracteristicas de las lideres por Women's Leadership por Leadership styles}

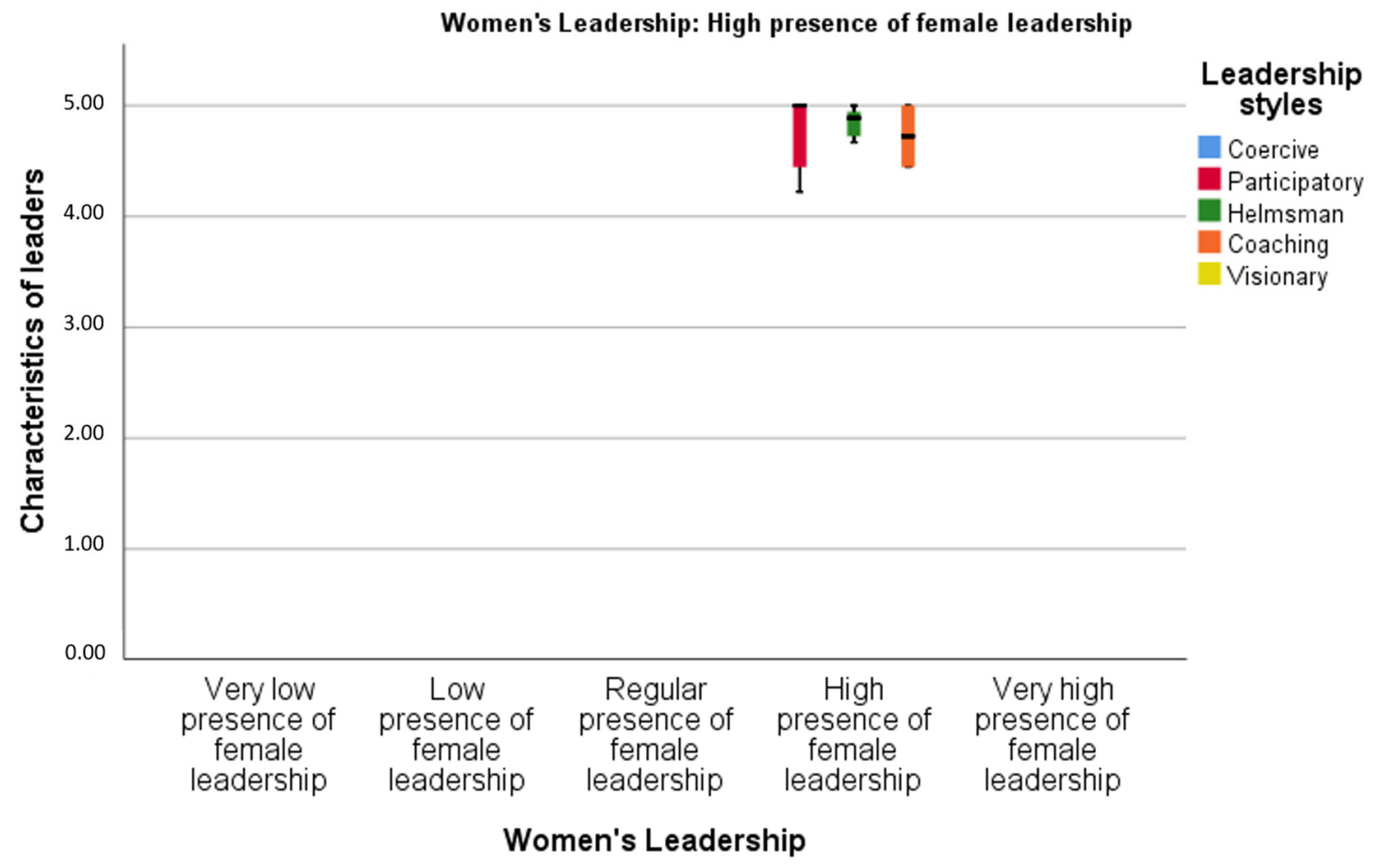

Figure 3. Women's Leadership. Source Own elaboration.

Figure 4 proposes a matrix dispersion of female leadership, on the basis of leadership styles and leader abilities (see H1). Leadership characteristics do not influence female leadership in those SMEs dedicated to the commercialization of Wayuu handicrafts. The Pearson's chi-square shows an asymptotic significance level of $0.00>0.05$; therefore, the null hypothesis is rejected, and the alternative hypothesis is accepted. In other words, it 
appears that, in the SMEs dedicated to the commercialization of Wayuu handicrafts, there is a relationship between leadership characteristics and female leadership. The intensity of the relationship through the V Cramer is 0.937 .

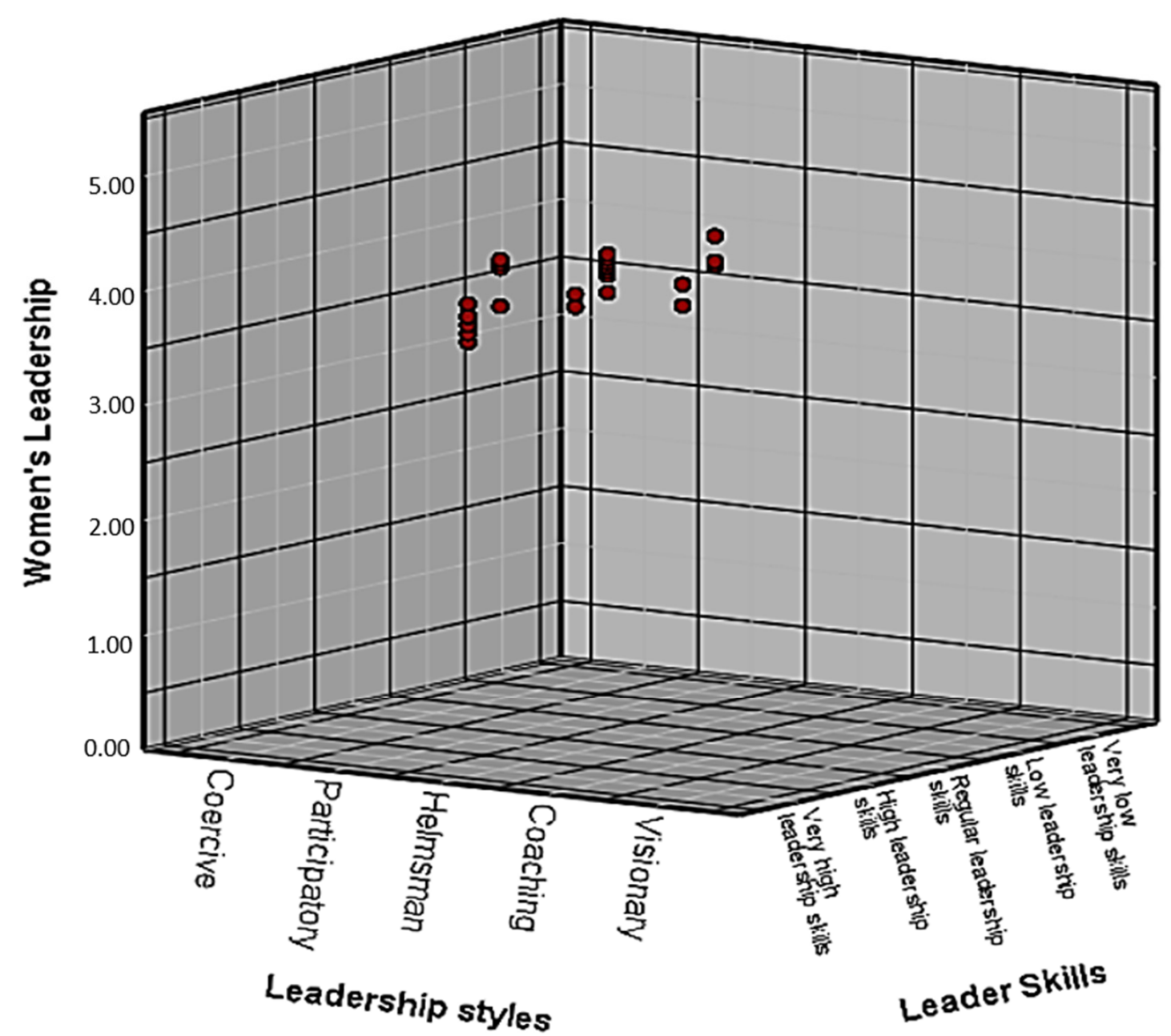

Figure 4. Filtering by variable characteristics of leaders. Source: own elaboration.

Figure 5 proposes different female leadership styles, on the basis of leadership styles and leader's capabilities (see H2). The Pearson's chi-square yields an asymptotic significance level of $0.00>0.05$; therefore, the null hypothesis is rejected, and the alternative hypothesis is accepted. In other words, in SMEs dedicated to the commercialization of Wayuu handicrafts, there is a relationship between leadership styles and female leadership. The intensity of the relationship, obtained through the V Cramer, is 0.736 (strong relationship).

Figure 6 evaluates women's leadership (H3). Leadership skills do not influence the development of female leadership in Wayuu handicraft marketing SMEs. The Pearson's chi-square yields an asymptotic significance level of $0.00>0.05$; therefore, the null hypothesis is rejected, and the alternative hypothesis is accepted. In other words, there is a relationship between leadership skills and female leadership in those SMEs related to the commercialization of Wayuu handicrafts. The intensity of the relationship through Cramer's V is 0.934 (strong relationship). 


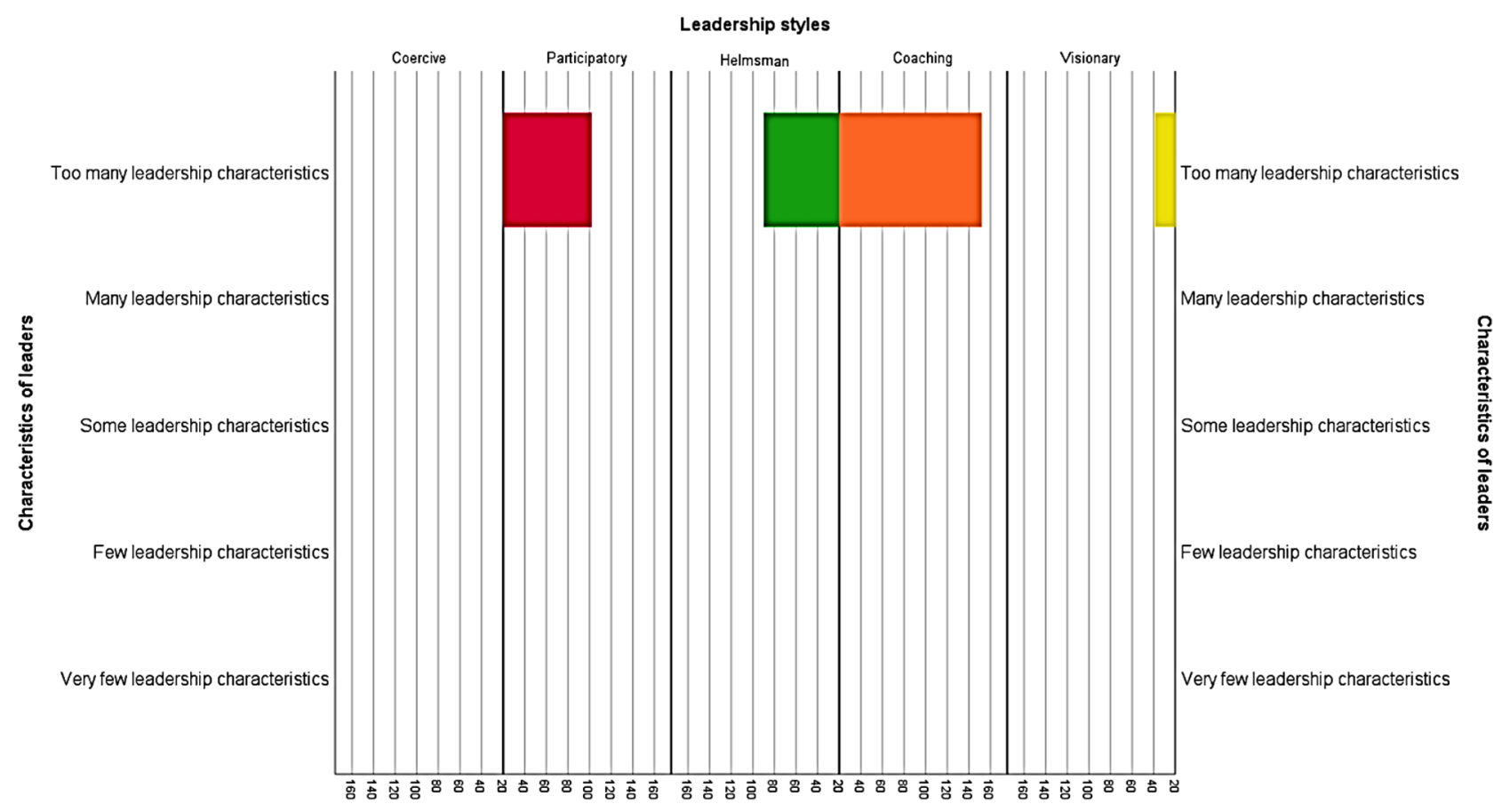

Figure 5. Leadership styles. Source: own elaboration.

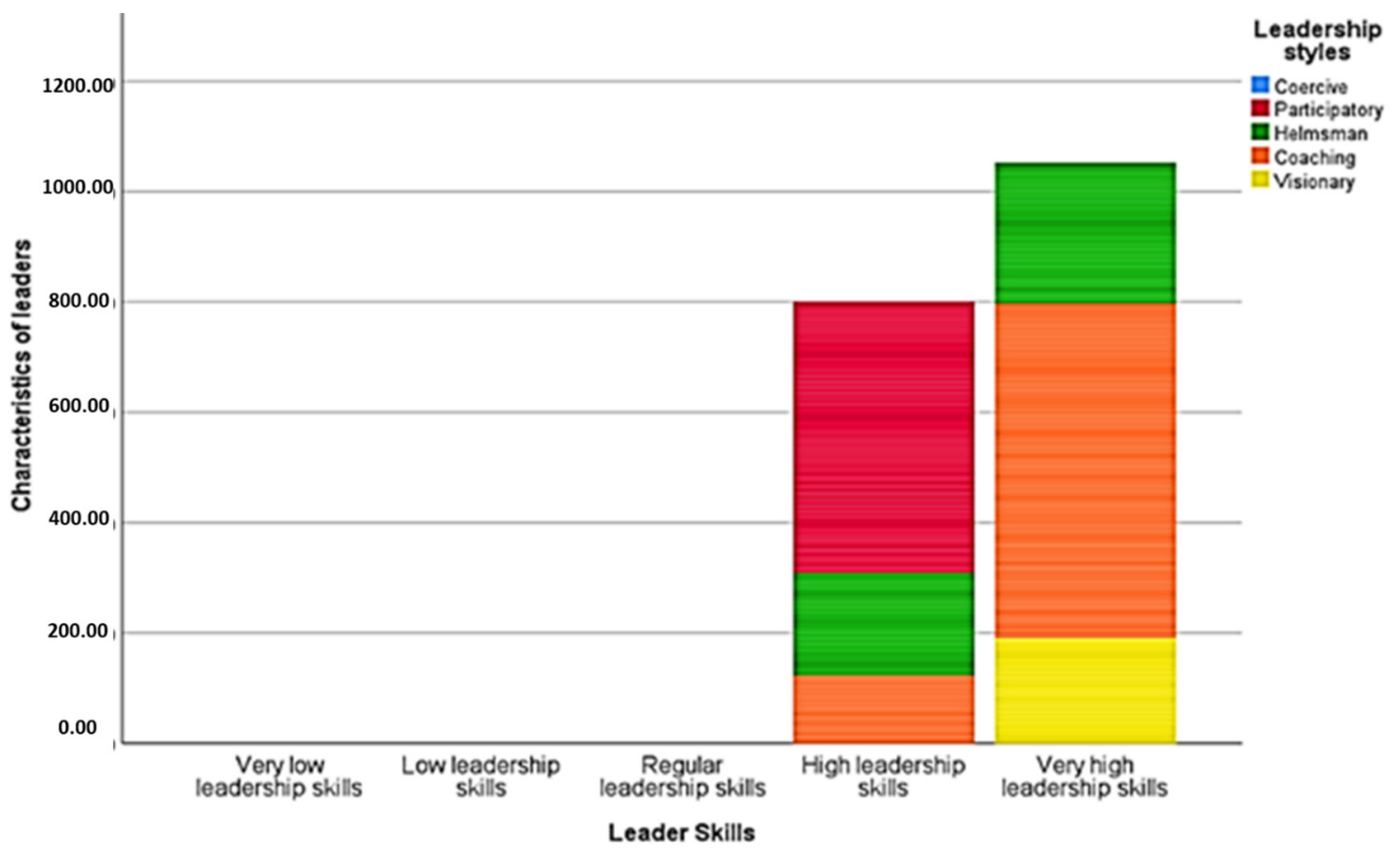

Figure 6. Filtering by variable Women's Leadership. Source: own elaboration.

\section{Discussion}

The above postulates are derived from business and academic research that analyzed women in their leadership role within companies. In this study, the female leadership variable presents a minimum and a maximum value and other statistical parameters (e.g., standard deviation). The 383 respondents are located in La Guajira, where there is a high presence of female leadership in the population under study. Leadership styles can be coercive, participative, helping, visionary, and coaching $[49,50,53]$. The rigorous performance of resilient women stands out, characterized by an empowered leadership in the management of the Wayuu handicrafts sale [56,57]. 
Women's way of thinking differs from men's on the basis of some distinctive characteristics, such as their more resilient, equanimous, consensual, and mediating sense of leadership [58]. In addition, they are characterized by their ability to propose strategies that enable them to significantly contribute to the achievement of management results in companies and to successfully contribute to the achievement of strategic and corporate sustainability objectives $[59,60]$.

Wayuu women have a receptive and participative attitude, and a multidirectional, multidimensional, more democratic, dialogic, and consensual approach to leadership, as shown in this research. In other words, this paper shows that in those SMEs dedicated to the commercialization of Wayuu handicrafts, there is a relationship between leadership characteristics and female leadership [43,46,47,61].

Many indigenous populations, such as the Wayuu one, are accustomed to humanize and identify male and female genders with meteorological phenomena, with the stars, the sun, the earth, and the moon, and with a number of elements of the native land where they coexist [62]. Thus, they assume that leadership positions are open to dialogue and to collaborative work [63], and their initiatives are characterized by respect for values and principles and by the dissemination of collective actions.

Moreover, the structures in which they act turn out to be less bureaucratic and hierarchical, characterized by the diffusion of creative and innovative initiatives. The female management style is characterized by being open, competitive, innovative, empowering, person-centered, flexible, communicative, persuasive, and with a strong perception of quality. In addition, it is less hierarchical, impersonal, and inflexible, thanks to its strong empathy and decisiveness in taking risks $[7,50]$.

Thanks to female emotional intelligence, which is an essential managerial competence $[17,61,64]$, female leadership enables the promotion of key competencies in organizations, such as skills development, cooperation, and participation. They are able to control their own emotions, and their distinctive characteristics are self-awareness, self-control, self-motivation, and empathy, which translate into a strong ability to establish positive relationships with others. In short, thanks to their intrapersonal and interpersonal intelligence, they have a great capacity to generate healthy working relationships [65].

On the other hand, some works underline that female leadership could be a limiting factor for the fulfillment of business objectives $[66,67]$ and that the exercise of their functions could assume negative traits. Other researchers, such as [68], emphasize instead the positive perception related to female leadership, which is characterized by personal security, independence, altruism, the entrepreneurial woman capable of creating change, and the ability to transform work into a vocation.

Traditionally, male leadership has been attributed to competencies that are opposed to feminine ones and to those of human talent $[69,70]$. This style of leadership is more conditioned to the lack of security and independence in the fulfillment of work processes. Attempts to devalue or even relativize the positive valuations of female leadership appear as a way of maintaining male privileges [71]. Women, in fact, are sometimes classified by society as a delicate and sensitive gender, when in fact they can assume models or patterns of behavior usually attributable to men that prove to be more than adequate to achieve success in the labor market.

Currently, some companies located in Latin America are adopting trends that denote a modern sense of business management [72]. Being inserted in an emerging economy, they must adopt approaches capable of ensuring, in various productive sectors, work models that are able to respond to the demands of a globalized economy. Several authors [73-78] point out that leadership involves some complementary competencies, such as intellectual capacity, which is the most important of all and represents the ability to create the strategic vision of the company, the culture, the organization, and all its intangible elements. This work confirms the positive relationship between sustainability and resilience already shown in other sectors, showing the key role of female leadership in reaching this goal [79-82]. 
Technical and human competencies are necessary to create excellent relations between managers and collaborators at lower management levels [83-86]. They refer to specific skills involved in the correct performance of a roles in a technical area or in a specific function and generally describe the ability to put into practice technical and specific knowledge that is closely linked to the success related to the technical execution of the role. Therefore, their definition is variable according to the technological segment of the organization.

\section{Conclusions}

An excellent leader must be able to manage appropriate communication processes, adequately combining his or her characteristics, style, and managerial skills. The latter can be summarized as technical, human, and conceptual, and are manifested as listening, observation, and expression skills. In this way, he/she can be able to relate effectively with his/her colleagues and collaborators in order to perform his/her functions.

Our findings highlight the vital importance of female leadership in Wayuu women, demonstrating to their collaborators the competencies necessary to achieve the common good, not only for economic well-being but also for their people. What distinguishes women coming from this Colombian region is that they seek to conceive companies in a more humanized way. Thus, thanks to the consistent harmonization of the work environment and their vision of SMEs companies, it is possible to achieve excellent results and promote the integration of people within work processes, promoting spaces of coexistence to achieve the common good.

However, the study is based on a very important tourism and commercial sector in Colombia, where women are often disadvantaged with regards to men. Wayuu men, indeed, have fulfilled a variety of gender roles, which have been historically inherited from one generation to another and are rooted in their culture and social organization. This can be controversial from the perspective of Western culture. For the indigenous people who have been surveyed, the gender approach is typical of Western culture; it is not a concern to talk about equality and equity, because the indigenous people understand themselves mainly from complementarity. The above shows that an investigative interest is in process, given the limitations in this first advance and also the phenomena associated with their culture and with ancestral formation, where the man, unlike the woman, is obliged to sustain his family.

This work pays attention to an issue that is underestimated by some. Sustainable development can only be achieved when resilience, leadership, and female entrepreneurship are linked. Therefore, the case study presented on Latin America shows a positive relationship between sustainability and resilience as a key to generating economic opportunities for people that today are being neglected. Moreover, it is able to favor the development of social equity.

The study has some limitations that can be overcame in future work. The first one concerns that the work is based on only one sector, and it would be interesting to study what would happen in other ones. The second is that it is based only on women, which is an added value of this paper but a comparison with the male gender could be useful. Thirdly, the methodology used is based on non-parametric tests, and therefore other methods could be proposed to make the results more robust.

In the meantime, through the results of this research, our objective is to answer other open questions in the future. The capacity of women could allow overcoming socio-cultural barriers, which is one of the factors limiting access and development in management positions. Resilient female leadership is expressed through distinctive traits, such as the ability to be effective, efficient, disciplined, and professionally ethical. In addition, it is characterized by their collaborative and consensual behavior, openness to dialogue, and participative managerial leadership. 
Author Contributions: Conceptualization, Á.A.-D., I.D., G.S.-S., R.G.-D. and E.C.V.; methodology, Á.A.-D. and R.G.-D.; software, Á.A.-D., A.P.-M., S.M.-P. and R.G.-D.; research, Á.A.-D. and A.P.-M.; data curation, A.P.-M.; writing-original draft preparation, Á.A.-D. and G.S.-S.; writing-revising and editing, Á.A.-D.; supervision, G.C., I.D., E.C.V. and Á.A.-D.; project management, Á.A.-D., A.P.-M. and E.C.V. All authors have read and agreed to the published version of the manuscript.

Funding: The APC was partially funded by Universidad Católica de la Santísima Concepción.

Institutional Review Board Statement: Not applicable.

Informed Consent Statement: Not applicable.

Data Availability Statement: Data are available on request from the authors.

Conflicts of Interest: The authors declare no conflict of interest.

\section{References}

1. Novalbos Ruiz, J. Las Bibliotecas Públicas Españolas ante los Objetivos de Desarrollo Sostenible de las Naciones Unidas «Agenda 2030». Master's Thesis, University of Extremadura, Badajoz, Spain, 2021. Available online: http://hdl.handle.net/10662/11761 (accessed on 26 June 2021).

2. González-Díaz, R.R.; Acevedo-Duque, Á.; Salazar-Sepúlveda, G.; Castillo, D. Contributions of Subjective Well-Being and Good Living to the Contemporary Development of the Notion of Sustainable Human Development. Sustainability 2021, 13, 3298. [CrossRef]

3. Giménez, T.V. Women, defenders of equality and care for nature. iQual. Revista Género Igualdad 2021, 4, 35-59. [CrossRef]

4. Peiró, J.M.; Ayala, Y.; Tordera, N.; Lorente, L.; Rodríguez, I. Bienestar sostenible en el trabajo: Revisión y reformulación [Sustainable wellness at work: Review and reformulation]. Papeles Psicólogo 2014, 35, 5-14. Available online: http://www. papelesdelpsicologo.es (accessed on 26 June 2021).

5. Di Fabio, A.; Peiró, J.M. Human Capital Sustainability Leadership to Promote Sustainable Development and Healthy Organizations: A New Scale. Sustainability 2018, 10, 2413. [CrossRef]

6. Di Fabio, A.; Bucci, O.; Gori, A. High Entrepreneurship, Leadership, and Professionalism (HELP): Towards an integrated, empirically based perspective. Front. Psychol. 2016, 7, 1842. [CrossRef]

7. D'Adamo, I.; Lupi, G. Sustainability and Resilience after COVID-19: A Circular Premium in the Fashion Industry. Sustainability 2021, 13, 1861. [CrossRef]

8. Benites, M.; González-Díaz, R.R.; Acevedo-Duque, Á.; Becerra-Pérez, L.A.; Tristancho Cediel, G. Latin American Microentrepreneurs: Trajectories and Meanings about Informal Work. Sustainability 2021, 13, 5449. [CrossRef]

9. Joga-Elvira, L.; Jacas, C.; Joga, M.L.; Roche-Martínez, A.; Brun-Gasca, C. Pilot study of socio-emotional factors and adaptive behavior in young females with fragile $X$ syndrome. Child Neuropsychol. 2021, 1-11. [CrossRef]

10. Lee, J.; Kim, E. Would Overconfident CEOs Engage More in Environment, Social, and Governance Investments? With a Focus on Female Representation on Boards. Sustainability 2021, 13, 3373. [CrossRef]

11. Ullah, F.; Wu, Y.; Mehmood, K.; Jabeen, F.; Iftikhar, Y.; Acevedo-Duque, Á.; Kwan, H.K. Impact of Spectators' Perceptions of Corporate Social Responsibility on Regional Attachment in Sports: Three-Wave Indirect Effects of Spectators' Pride and Team Identification. Sustainability 2021, 13, 597. [CrossRef]

12. Rolinek, L.; Kopta, D.; Plevny, M.; Rost, M.; Kubecova, J. Level of process management implementation in SMEs and some related implications. Transform. Bus. Econ. 2015, 14, 360-377.

13. Caruso, G.; Gattone, S.A.; Fortuna, F.; Di Battista, T. Cluster Analysis for mixed data: An application to credit risk evaluation. Socio Econ. Plan. Sci. 2021, 73, 100850. [CrossRef]

14. Mabula, J.B.; Dongping, H.; Mwakapala, L.Y. SME's use of I.C.T. and financial services on innovation performance: The mediating role of managers' experience. Hum. Syst. Manag. 2020, 39, 427-439. [CrossRef]

15. Bajrami, D.D.; Radosavac, A.; Cimbaljević, M.; Tretiakova, T.N.; Syromiatnikova, Y.A. Determinants of 580 residents' support for sustainable tourism development: Implications for rural communities. Sustainability 2020, 12, 9438. [CrossRef]

16. Acevedo, Á.E.; Vergara, O.; González, Y. Responsible Marketing: Distinctive Advantage in the Value Chain of Organizations. J. Manag. Bus. Stud. 2019, 1, 44-74. [CrossRef]

17. Díez-Gutiérrez, E.-J.; Valle Flórez, R.E.; Terrón Bañuelos, E.; Centeno Suárez, B. El liderazgo femenino y su ejercicio en las organizaciones educativas. Iberoam. J. Educ. 2003, 33, 1-19. [CrossRef]

18. Biswas, P.K.; Roberts, H.; Stainback, K. Does women's board representation affect non-managerial gender inequality? Hum. Resour. Manag. 2021. [CrossRef]

19. Thomas, G. A typology for the case study in social science following a review of definition, discourse, and structure. Qual. Inq. 2011, 17, 511-521. [CrossRef]

20. Kadi-Montiel, O.; Acevedo-Duque, A. Liderazgo ético frente a la diversidad cultural dentro de las organizaciones con régimen disciplinario. Económicas CUC 2014, 35, 75-88. Available online: https:/ / revistascientificas.cuc.edu.co/economicascuc/article/ view/522 (accessed on 10 July 2021). 
21. González-Díaz, R.R.; Guanilo-Gómez, S.L.; Acevedo-Duque, E.; Campos, J.S.; Cachicatari Vargas, E. Intrinsic alignment with strategy as a source of business sustainability in S.M.E.s. Entrep. Sustain. Issues 2021, 8, 377-388. [CrossRef]

22. Weiner, J.M.; Cyr, D.; Burton, L.J. Microaggressions in administrator preparation programs: How black female participants experienced discussions of identity, discrimination, and leadership. J. Res. Leadersh. Educ. 2019. [CrossRef]

23. Kawai, N.; Kazumi, T. Female entrepreneurs' cognitive attributes and venture growth in Japan: The moderating role of perceived social legitimacy. Int. J. Gend. Entrep. 2021, 13. [CrossRef]

24. Müller, J.; Acevedo-Duque, Á.; Müller, S.; Kalia, P.; Mehmood, K. Predictive Sustainability Model Based on the Theory of Planned Behavior Incorporating Ecological Conscience and Moral Obligation. Sustainability 2021, 13, 4248. [CrossRef]

25. Vigil, K.M. Warrior Women: Recovering Indigenous Visions across Film and Activism. JCMS J. Cine. Media Stud. 2021, 60, 169-174. [CrossRef]

26. Russomanno, J.; Tree, J.M.J.T.J. Assessing sense of community at farmers markets. J. Agric. Food Syst. Community Dev. 2021, 10, 1-18. [CrossRef]

27. Hallinger, P.; Chatpinyakoop, C. A Bibliometric Review of Research on Higher Education for Sustainable Development, 1998-2018. Sustainability 2019, 11, 2401. [CrossRef]

28. D'Adamo, I.; Falcone, P.M.; Imbert, E.; Morone, P. A Socio-economic Indicator for EoL Strategies for Bio-based Products. Ecol. Econ. 2020, 178, 106794. [CrossRef]

29. D'Adamo, I.; Rosa, P. How Do You See Infrastructure? Green Energy to Provide Economic Growth after COVID-19. Sustainability 2020, 12, 4738. [CrossRef]

30. Paolasso, P. Desigualdad y fragmentación territorial en América Latina. J. Lat. Am. Geogr. 2020, 19, 152-162. [CrossRef]

31. Saiz-Álvarez, J.M.; Vega-Muñoz, A.; Acevedo-Duque, Á.; Castillo, D. B Corps: A Socioeconomic Approach for the COVID-19 Post-crisis. Front. Psychol. 2020, 11, 1867. [CrossRef]

32. Macaulay, B.S.; Krishnamoorthy, S. (Re)imagined Possibilities: The Resilience of the Black Woman Griot, Zeinabu irene Davis in Conversation. Fem. Media Hist. 2021, 7, 81-114. [CrossRef]

33. Vrchota, J.; Ǩehoř, P.; Marikova, M.; Pech, M. Critical Success Factors of the Project Management in Relation to Industry 4.0 for Sustainability of Projects. Sustainability 2021, 13, 281. [CrossRef]

34. Cho, Y.; Kim, S.; You, J.; Han, H.; Kim, M.; Yoon, S. How South Korean women leaders respond to their token status: Assimilation and resistance. Hum. Resour. Dev. Int. 2021, 1-24. [CrossRef]

35. Wu, H.; Perez-Lugo, M.; Garcia, C.O.; Crespo, F.G.; Castillo, A. Empowered Stakeholders: Female University Students' Leadership During the COVID-19-Triggered On-campus Evictions in Canada and the United States. Int. J. Disaster Risk Sci. 2021, 1-12. [CrossRef]

36. Francis, B.B.; Hasan, I.; Shen, Y.V.; Wu, Q. Do activist hedge funds target female CEOs? The role of CEO gender in hedge fund activism. J. Financ. Econ. 2021, 141, 372-393. [CrossRef]

37. Pastor, R.; Verge, T. The symbolic representation of women's political firsts in editorial cartoons. Fem. Media Stud. 2021, 1-16. [CrossRef]

38. Šípová-Jungová, H.; Jurgová, L.; Hemmerová, E.; Homola, J. Interaction of Tris with D.N.A. molecules and carboxylic groups on self-assembled monolayers of alkanethiols measured with surface plasmon resonance. Appl. Surf. Sci. 2021, 546, 148984. [CrossRef]

39. Cherneski, J. Zebras showing their stripes: A critical sense-making study of women C.S.R. leaders. Qual. Res. Organ. Manag. Int. J. 2021. [CrossRef]

40. Vimal, K.E.K.; Kandasamy, J.; Duque, A.A. Integrating sustainability and remanufacturing strategies by remanufacturing quality function deployment (RQFD). Environ. Dev. Sustain. 2021, 1-33. [CrossRef]

41. Osi, E.C.; Teng-Calleja, M. Women on top: The career development journey of Filipina business executives in the Philippines. Career Dev. Int. 2021. [CrossRef]

42. Eckert, S.; Assmann, K. The "ProQuote" initiative: Women journalists in Germany push to revolutionise newsroom leadership. Fem. Media Stud. 2021, 1-18. [CrossRef]

43. Xu, H.; Luke, N.; Short, S.E. Women's Political Leadership and Adult Health: Evidence from Rural and Urban China. J. Health Soc. Behav. 2021, 62, 100-118. [CrossRef] [PubMed]

44. Shahin, M.; Ilic, O.; Gonsalvez, C.; Whittle, J. The impact of a STEM-based entrepreneurship program on the entrepreneurial intention of secondary school female students. Int. Entrep. Manag. J. 2021, 1-32. [CrossRef]

45. Leschber, G. From female surgical resident to academic leaders: Challenges and pathways forward. J. Thorac. Dis. 2021, 13, 480. [CrossRef]

46. Malloy, E.; Kavussanu, M. A comparison of authentic and transformational leadership in sport. J. Appl. Soc. Psychol. 2021. [CrossRef]

47. Kolpakov, A.; Boyer, E. Examining Gender Dimensions of Leadership in International Nonprofits. Public Integr. 2021, 23, 68-81. [CrossRef]

48. Thomas, G. Michelle Bachelet's Liderazgo Femenino (Feminine Leadership) redefining political leadership in Chile's 2005 presidential campaign. Int. Fem. J. Politics 2011, 13, 63-82. [CrossRef]

49. Helming, S.; Ungermann, F.; Hierath, N.; Stricker, N.; Lanza, G. Development of a training concept for leadership 4.0 in production environments. Procedia Manuf. 2019, 31, 38-44. [CrossRef] 
50. Kotter, J.P. La Verdadera Labor de un Líder. Ed. Norma SA. Colombia. 1999. Available online: https://books.google. $\mathrm{rs} /$ books?hl=sr\&lr=\&id=hcleWS-2kQgC\&oi=fnd\&pg=PA39\&dq=La+verdadera + labor + de + un $+1 \% C 3 \% A D d e r \& o t s=m i N e 5$ HnGWS\&sig=TotyQYwi6NW6Fe8pNbqEUnAVB40\&redir_esc=y\#v=onepage\&q=La\%20verdadera \%20labor\%20de\%20un\%20 $1 \%$ C3\%ADder\&f=false (accessed on 7 July 2021).

51. Fitzgerald, T. Changing the deafening silence of indigenous women's voices in educational leadership. J. Educ. Adm. 2003, 41, 9-23. [CrossRef]

52. Landa, D.; Tyson, S.A. Coercive leadership. Am. J. Political Sci. 2017, 61, 559-574. [CrossRef]

53. Ornelas, R. Water and the Indigenous Women's Leadership Project. 2011. Available online: https://digital.library.txstate.edu/ handle/10877/12841 (accessed on 7 July 2021).

54. Davies, B.; Brighouse, T. Passionate leadership. Manag. Educ. 2010, 24, 4-6. [CrossRef]

55. Berg, M.E.; Karlsen, J.T. A study of coaching leadership style practice in projects. Manag. Res. Rev. 2016, 39, 1122-1142. [CrossRef]

56. Patel, P.; Meagher, K.; El Achi, N.; Ekzayez, A.; Sullivan, R.; Bowsher, G. Having more women humanitarian leaders will help transform the humanitarian system: Challenges and opportunities for women leaders in conflict and humanitarian health. Confl. Health 2020, 14, 1-15. [CrossRef]

57. Jaffari, A.; Lee, J.; Kim, E. Variability Modeling in Software Product Line: A Systematic Literature Review. In Software Engineering in IoT, Big Data, Cloud and Mobile Computing. Studies in Computational Intelligence; Kim, H., Lee, R., Eds.; Springer: Cham, Switzerland, 2020; Volume 930. [CrossRef]

58. Acevedo-Duque, Á.; Gonzalez-Diaz, R.; Vega-Muñoz, A.; Fernández Mantilla, M.M.; Ovalles-Toledo, L.V.; Cachicatari-Vargas, E. The Role of B Companies in Tourism towards Recovery from the Crisis COVID-19 Inculcating Social Values and Responsible Entrepreneurship in Latin America. Sustainability 2021, 13, 7763. [CrossRef]

59. Lu, P.W.; Hill, S.S.; Fields, A.C.; Davids, J.S.; Melnitchouk, N. Factors associated with the professional success of female surgical department chairs: A qualitative study. JAMA Surg. 2020, 155, 1028-1033. [CrossRef]

60. Le Loarne-Lemaire, S.; Bertrand, G.; Razgallah, M.; Maalaoui, A.; Kallmuenzer, A. Women in innovation processes as a solution to climate change: A systematic literature review and an agenda for future research. Technol. Forecast. Soc. Chang. 2020, 120440. [CrossRef]

61. Flory, J.A.; Leibbrandt, A.; Rott, C.; Stoddard, O. Increasing Workplace Diversity Evidence from a Recruiting Experiment at a Fortune 500 Company. J. Hum. Resour. 2021, 56, 73-92. [CrossRef]

62. Bulmer, E.; Riera, M.; Rodríguez, R. The Importance of Sustainable Leadership amongst Female Managers in the Spanish Logistics Industry: A Cultural, Ethical and Legal Perspective. Sustainability 2021, 13, 6841. [CrossRef]

63. Burlea-Schiopoiu, A.; Mihai, L.S. An Integrated Framework on the Sustainability of SMEs. Sustainability 2019, 11, 6026. [CrossRef]

64. Miliopoulou, G.Z.; Kapareliotis, I. The toll of success: Female leaders in the "women-friendly" Greek advertising agencies. Gend. Work. Organ. 2021. [CrossRef]

65. Sterbenk, Y.; Champlin, S.; Windels, K.; Shelton, S. Is Femvertising the New Greenwashing? Examining Corporate Commitment to Gender Equality. J. Bus. Ethics 2021, 1-15. [CrossRef]

66. Bozhinov, V.; Joecks, J.; Scharfenkamp, K. Gender spillovers from supervisory boards to management boards. Manag. Decis. Econ. 2021. [CrossRef]

67. Reichert, P.; Bird, M.D.; Farber, V. Gender and entrepreneurial propensity: Risk-taking and prosocial preferences in labour market entry decisions. Soc. Enterp. J. 2021. [CrossRef]

68. Hossain, D.M.; Alam, M.S.; Mazumder, M.M.M.; Amin, A. Gender-related discourses in corporate annual reports: An exploratory study on the Bangladeshi companies. J. Account. Organ. Chang. 2021. [CrossRef]

69. Gigol, T. Gender Differences in Engagement in Unethical Pro-Organizational Behavior-Two Studies in Poland. Sustainability 2021, 13, 39. [CrossRef]

70. Schmitt, M. Women Engineers on Their Way to Leadership: The Role of Social Support Within Engineering Work Cultures. Eng. Stud. 2021, 13, 30-52. [CrossRef]

71. Siegel, R.; König, C.J.; Zobel, Y. Executive Search Consultants' Biases Against Women (or Men?). Front. Psychol. 2020, 11, 541766. [CrossRef]

72. Baldner, C.; Pierro, A. The trials of women leaders in the workforce: How a need for cognitive closure can influence acceptance of harmful gender stereotypes. Sex Roles 2019, 80, 565-577. [CrossRef]

73. Biswas, P.K.; Roberts, H.; Whiting, R.H. Female directors and C.S.R. disclosure in Bangladesh: The role of family affiliation. Meditari Account. Res. 2021. [CrossRef]

74. Alshmery, S.N.; Alqirnas, H.R.; Alyuosef, M.I. Influence of the social and economic characteristics of Saudi women on their attitudes toward empowering them in online labor market. J. Sustain. Financ. Invest. 2021, 1-15. [CrossRef]

75. Riner, A.N.; Herremans, K.M.; Neal, D.W.; Johnson-Mann, C.; Hughes, S.J.; McGuire, K.P.; Upchurch, G.R., Jr.; Trevino, J.G. Diversification of Academic Surgery, Its Leadership, and the Importance of Intersectionality. JAMA Surg. 2021. [CrossRef]

76. De Masi, S.; Słomka-Gołębiowska, A.; Paci, A. Women on boards and monitoring tasks: An empirical application of Kanter's theory. Manag. Decis. 2021. [CrossRef]

77. Uduji, J.I.; Okolo-Obasi, E.N.; Asongu, S.A. Sustaining cultural tourism through higher female participation in Nigeria: The role of corporate social responsibility in oil host communities. Int. J. Tour. Res. 2020, 22, 120-143. [CrossRef] 
78. Caruso, G.; Fortuna, F. Mediterranean diet Patterns in the Italian Population: A functional data analysis of Google Trends. Decis. Trends Soc. Syst. 2020, 63-72. [CrossRef]

79. Appolloni, A.; Colasanti, N.; Fantauzzi, C.; Fiorani, G.; Frondizi, R. Distance Learning as a Resilience Strategy during Covid-19: An Analysis of the Italian Context. Sustainability 2021, 13, 1388. [CrossRef]

80. D’Adamo, I.; González-Sánchez, R.; Medina-Salgado, M.S.; Settembre-Blundo, D. E-Commerce Calls for Cyber-Security and Sustainability: How European Citizens Look for a Trusted Online Environment. Sustainability 2021, 13, 6752. [CrossRef]

81. Giudice, F.; Caferra, R.; Morone, P. COVID-19, the Food System and the Circular Economy: Challenges and Opportunities. Sustainability 2020, 12, 7939. [CrossRef]

82. Mahmud, P.; Paul, S.K.; Azeem, A.; Chowdhury, P. Evaluating Supply Chain Collaboration Barriers in Small- and Medium-Sized Enterprises. Sustainability 2021, 13, 7449. [CrossRef]

83. Rzemieniak, M.; Wawer, M. Employer Branding in the Context of the Company's Sustainable Development Strategy from the Perspective of Gender Diversity of Generation Z. Sustainability 2021, 13, 828. [CrossRef]

84. Vega, A.; Bustamante, G.; Salazar, G. Orange Economy and Digital Entrepreneurship in Latin America: Creative Sparkles among Raw Materials, Chapter 10. In Handbook of Research on Digital Marketing Innovations in Social Entrepreneurship and Solidarity Economics; Saiz-Alvarez, J.M., Ed.; I.G.I. Global: Hershey, PA, USA, 2019; pp. 182-203. [CrossRef]

85. Molina-Ramírez, E.; Barba-Sánchez, V. Embeddedness as a Differentiating Element of Indigenous Entrepreneurship: Insights from Mexico. Sustainability 2021, 13, 2117. [CrossRef]

86. Hristov, I.; Appolloni, A.; Chirico, A.; Cheng, W. The role of the environmental dimension in the performance management system: A systematic review and conceptual framework. J. Clean. Prod. 2021, 293, 126075. [CrossRef] 

MDPI

St. Alban-Anlage 66 4052 Basel

Switzerland

Tel. +41616837734

Fax +41 613028918

www.mdpi.com

Sustainability Editorial Office

E-mail: sustainability@mdpi.com

www.mdpi.com/journal/sustainability

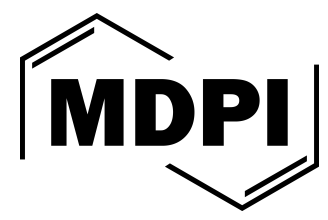



MDPI

St. Alban-Anlage 66

4052 Basel

Switzerland

Tel: +41 616837734

Fax: +41 613028918 